NATIONAL LABORATORY

FOR THE DEPARTMENT OF ENERGY

\title{
Irradiation Effects for the Pulsed Fast Neutron Analysis (PFNA) Cargo Interrogation System
}

\author{
C. O. Slater \\ J. V. Pace III \\ R. T. Santoro
}




\section{DOCUMENT AVAILABILITY}

Reports produced after January 1, 1996, are generally available free via the U.S. Department of Energy (DOE) Information Bridge.

Web site http://www.osti.gov/bridge

Reports produced before January 1, 1996, may be purchased by members of the public from the following source.

National Technical Information Service

5285 Port Royal Road

Springfield, VA 22161

Telephone 703-605-6000 (1-800-553-6847)

TDD 703-487-4639

Fax 703-605-6900

E-mail info@ntis.fedworld.gov

Web site http://www.ntis.gov/support/ordernowabout.htm

Reports are available to DOE employees, DOE contractors, Energy Technology Data Exchange (ETDE) representatives, and International Nuclear Information System (INIS)

representatives from the following source.

Office of Scientific and Technical Information

P.O. Box 62

Oak Ridge, TN 37831

Telephone 865-576-8401

Fax 865-576-5728

E-mail reports@adonis.osti.gov

Web site http://www.osti.gov/contact.html

This report was prepared as an account of work sponsored by an agency of the United States Government. Neither the United States Government nor any agency thereof, nor any of their employees, makes any warranty, express or implied, or assumes any legal liability or responsibility for the accuracy, completeness, or usefulness of any information, apparatus, product, or process disclosed, or represents that its use would not infringe privately owned rights. Reference herein to any specific commercial product, process, or service by trade name, trademark, manufacturer, or otherwise, does not necessarily constitute or imply its endorsement, recommendation, or favoring by the United States Government or any agency thereof. The views and opinions of authors expressed herein do not necessarily state or reflect those of the United States Government or any agency thereof. 
Computational Physics and Engineering Division

\title{
Irradiation Effects for the Pulsed Fast Neutron Analysis (PFNA) Cargo Interrogation System
}

\author{
C. O. Slater \\ J. V. Pace III \\ R. T. Santoro
}

Date published: February 2001

Prepared by the

OAK RIDGE NATIONAL LABORATORY

Oak Ridge, TN 37831-6363

Managed by

UT-BATTELLE, LLC,

for the

U.S. DEPARTMENT OF ENERGY

under contract DE-AC05-00OR22725 
$\rightarrow$

7 


\section{CONTENTS}

Page

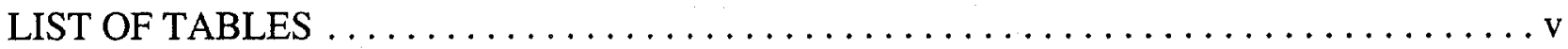

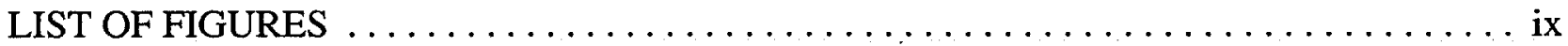

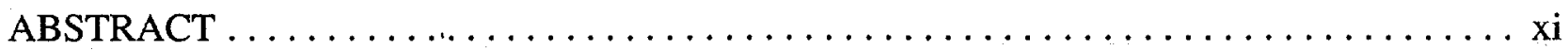

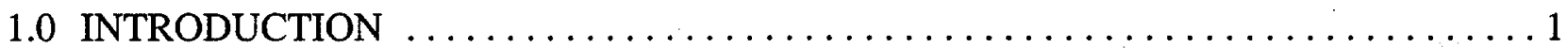

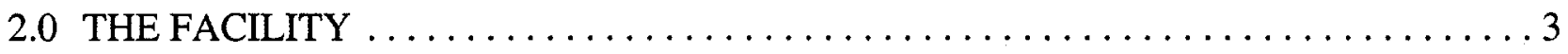

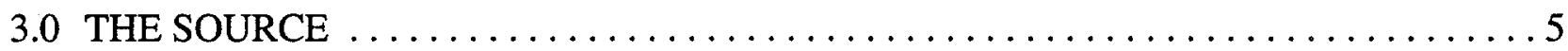

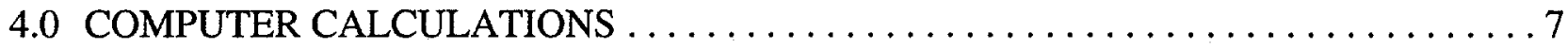

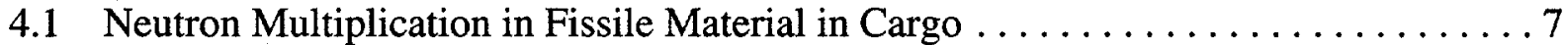

4.2 Dose Rates Within and Around the Facility ..................... 9

4.3 Neutron Activation of Structural Materials and Stream-of-Commerce Cargoes . . . 11

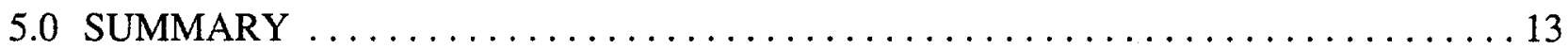

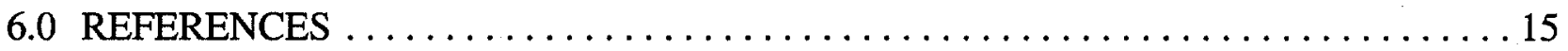

APPENDIX A Listings of Input Files MCNP-X Criticality Calculations $\ldots \ldots \ldots \ldots \ldots 53$

APPENDIX B Listings of Input Files MCNP-4B Fissile Material Dose-Rate Calculations . . 61

APPENDIX C Plots of Plane Slices Through the PFNA Facility Calculational Geometry .. 153

APPENDIX D Listings of Codes and Input Files Dose-Rate Averaging $\ldots \ldots \ldots \ldots \ldots$

APPENDIX E Listings of Input Files MCNP-4B Calculations of Dose Rates Within and Around the PFNA Facility $\ldots \ldots \ldots \ldots \ldots \ldots \ldots \ldots \ldots \ldots \ldots \ldots \ldots \ldots$

APPENDIX F Tables of ORIGEN-Calculated Radioactivities of Selected Cargoes and Structures Irradiated in the PFNA Facility . . . . . . . . . . . . . 375

APPENDIX G Tables of ORIGEN-Calculated Decay Photon Spectra for Selected Cargoes and Structures Irradiated in the PFNA Facility 
APPENDIX $H$ Listings of the MCNP-4B Input Files Used to Calculate Neutron Spectra for the ORIGEN Activation Calculations ...............4 437

APPENDIX I Listings of the ORIGEN Input Files for Calculating Neutron Activation of Selected Materials . ............................ 499 


\section{LIST OF TABLES}

Table

Page

1. Angle- and Energy-Dependent Neutron Source Spectrum ${ }^{a}$ for the PFNA Facility . . . . . 16

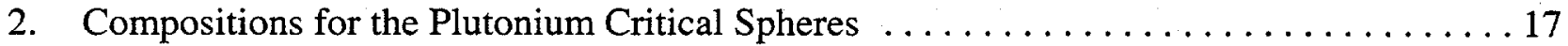

3. Compositions of hydrogenous materials used in the PFNA calculations $\ldots \ldots \ldots \ldots \ldots$

4. Compositions of Metal Materials Used in the PFNA Calculations $\ldots \ldots \ldots \ldots \ldots \ldots 19$

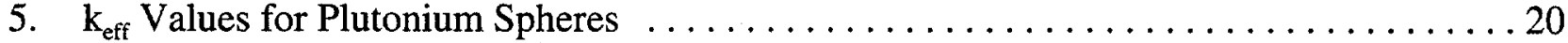

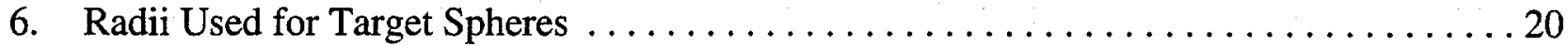

7. Neutron Dose Factors from the VELM61 Cross-Section Library $\ldots \ldots \ldots \ldots \ldots \ldots 21$

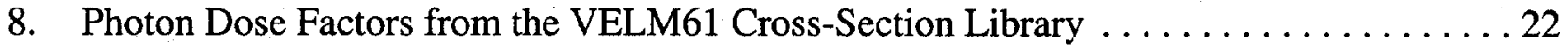

9. Comparison of Target Dose Rates with Background Dose Rates for a $6.32-\mathrm{cm}-$ Radius

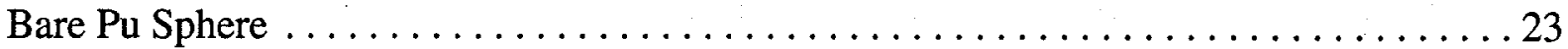

10. Comparison of Target Dose Rates with Background Dose Rates for a Bare Pu Sphere

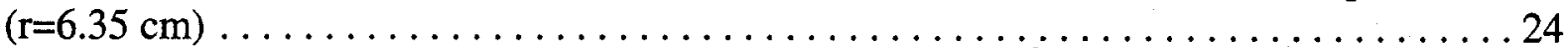

11. Comparison of Target Dose Rates with Background Dose Rates for a Bare Pu Sphere

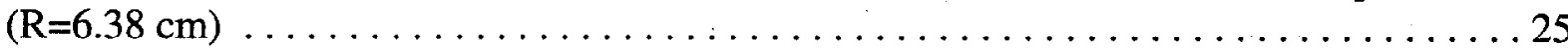

12. Comparison of Target Dose Rates with Background Dose Rates for a TungstenReflected Pu Sphere .................................... 26

13. Comparison of Target Dose Rates with Background Dose Rates for a Water-Reflected

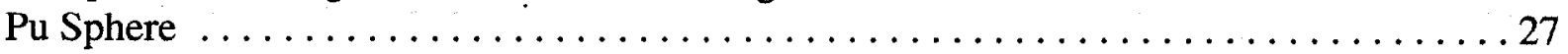

14. Changes in the Bare Pu Sphere $(r=6.38 \mathrm{~cm})$ Detector Dose Rates with Changes in the Number of Histories . . . . . . . . . . . . . . . . . . . . . . . . . . . . 28

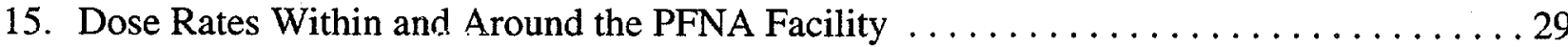

16. Neutron Dose Rates Aiong the Centerline of the Vehicle Lane of the PFNA Facility . . . . 30

17. Photon Dose Rates Along the Centerline of the Vehicle Lane of the PFNA Facility . . . . . 31

18. Total Dose Rates Along the Centerline of the Vehicle Lane of the PFNA Facility . . . . . 32

19. Dose Rates in Beam Line at Various Distances from the PFNA Truck Lane Centerline . . 33

20. Neutron Dose Rates at Locations Below the Source Overhead Shield and Slightly

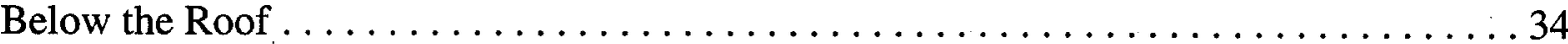

21. Distribution of the Neutron and Photon Dose Rates Along the Centerline of the Truck Lane and Perpendicular to the Beam for Detectors Inside a Salted Beef Cargo ........ 35

22. Compositions (weight percent) of Cargo Materials Irradiated .................. 36

23. Compositions (weight percent) of the Structural Materials Irradiated ............. 37

24. ORIGEN Flux Parameters for the PFNA Facility Activation Calculations ............. 38

F1. Radioactivity (Ci) After 8-s Irradiation of 1-kg Salted Beef Cargo (Flux Parameter Set 1) ............................................ 377

F2. Radioactivity (Ci) After 16-s Irradiation of 1-kg Salted Beef Cargo (Flux Parameter Set 1) .......................................... 378

F3. Radioactivity (Ci) After 24-s Irradiation of 1-kg Salted Beef Cargo (Flux Parameter

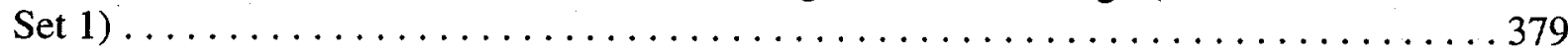


F4. Radioactivity (Ci) After 8-s Irradiation of 1-kg Ball Bearings Cargo (Flux Parameter Set 1) . . . . . . . . . . . . . . . . . . . . . . . . . . . . . . . . . . 380

F5. Radioactivity (Ci) After 16-s Irradiation of 1-kg Ball Bearings Cargo (Flux Parameter

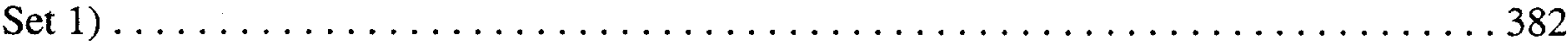

F6. Radioactivity (Ci) After 24-s Irradiation of 1-kg Ball Bearings Cargo (Flux Parameter

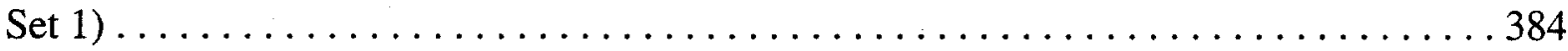

F7. Radioactivity (Ci) After 8-s Irradiation of 1-kg Surgical Implant Composition A Cargo (Flux Parameter Set 1) . . . . . . . . . . . . . . . . . . . . . . . . 386

F8. Radioactivity (Ci) After 16-s Irradiation of 1-kg Surgical Implant Composition A Cargo (Flux Parameter Set 1) . . . . . . . . . . . . . . . . . . . . . . . . . 388

F9. Radioactivity (Ci) After 24-s Irradiation of 1-kg Surgical Implant Composition A Cargo (Flux Parameter Set 1) . . . . . . . . . . . . . . . . . . . . . . . . . 390

F10. Radioactivity (Ci) After 8-s Irradiation of 1-kg Surgical Implant Composition B Cargo (Flux Parameter Set 1) . . . . . . . . . . . . . . . . . . . . . . 392

F11. Radioactivity (Ci) After 16-s Irradiation of 1-kg Surgical Implant Composition B Cargo (Flux Parameter Set 1) . . . . . . . . . . . . . . . . . . . . . . . . 393

F12. Radioactivity (Ci) After 24-s Irradiation of 1-kg Surgical Implant Composition B Cargo (Flux Parameter Set 1) . . . . . . . . . . . . . . . . . . . . . . . . . . . . 394

F13. Radioactivity (Ci) After 8-s Irradiation of 1-kg 16-16-16 Fertilizer Cargo (Flux Parameter Set 1) . . ................................... 395

F14. Radioactivity (Ci) After 16-s Irradiation of 1-kg 16-16-16 Fertilizer Cargo (Flux

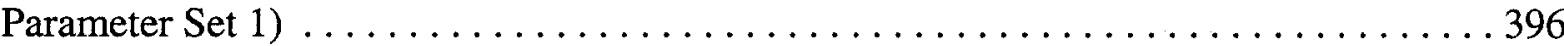

F15. Radioactivity (Ci) After 24-s Irradiation of 1-kg 16-16-16 Fertilizer Cargo (Flux Parameter Set 1) . . .................................... 397

F16. Radioactivity (Ci) After One-Day Irradiation of 1-kg Concrete (Flux Parameter

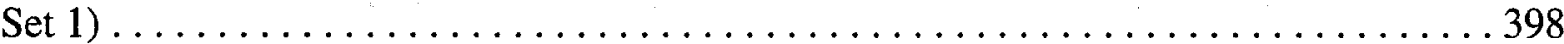

F17. Radioactivity (Ci) After Ten-Day Irradiation of 1-kg Concrete (Flux Parameter Set 1) . . . . . . . . . . . . . . . . . . . . . . . . . . . . . . . . 399

F18. Radioactivity (Ci) After One-Day Irradiation of 1-kg Concrete (Flux Parameter

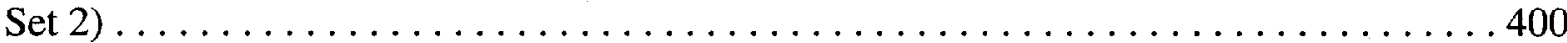

F19. Radioactivity (Ci) After Ten-Day Irradiation of 1-kg Concrete (Flux Parameter

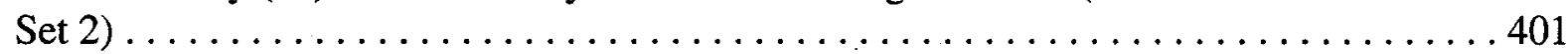

F20. Radioactivity (Ci) After One-Day Irradiation of 1-kg Aluminum 6061-T6 (Flux

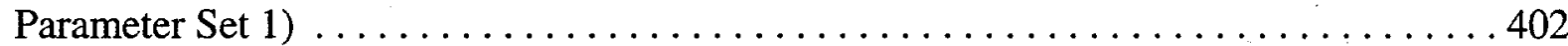

F21. Radioactivity (Ci) After Ten-Day Irradiation of 1-kg Aluminum 6061-T6 (Flux Parameter Set 1) . ................................... 403

F22. Radioactivity (Ci) After One-Day Irradiation of 1-kg Aluminum 6061-T6 (Flux Parameter Set 2) . . . . . . . . . . . . . . . . . . . . . . . . . . . . . 404

F23. Radioactivity (Ci) After Ten-Day Irradiation of 1-kg Aluminum 6061-T6 (Flux Parameter Set 2) .................................... 405

F24. Radioactivity (Ci) After One-Day Irradiation of 1-kg Steel Plate (Flux Parameter

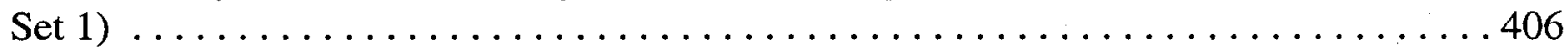


F25. Radioactivity (Ci) After Ten-Day Irradiation of 1-kg Steel Plate (Flux Parameter

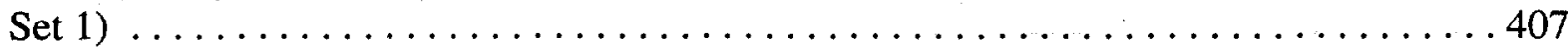

F26. Radioactivity (Ci) After One-Day Irradiation of 1-kg Steel Plate (Flux Parameter

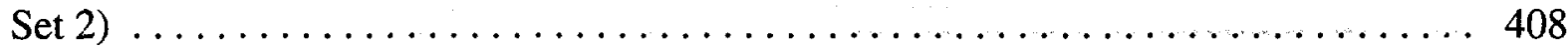

F27. Radioactivity (Ci) After Ten-Day Irradiation of 1-kg Steel Plate (Flux Parameter

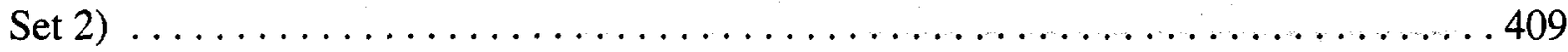

F28. Radioactivity (Ci) After One-Day Irradiation of 1-kg Havar Foil (Flux Parameter

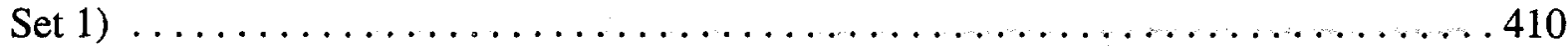

F29. Radioactivity (Ci) After Ten-Day Irradiation of 1-kg Havar Foil (Flux Parameter

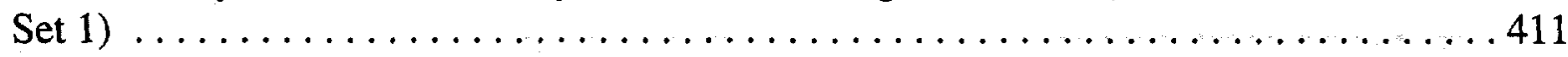

F30. Radioactivity (Ci) After One-Day Irradiation of 1-kg Havar Foil (Flux Parameter

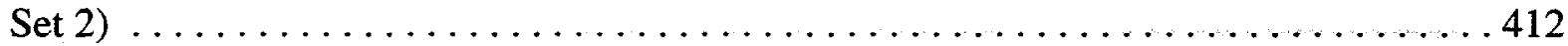

F31. Radioactivity (Ci) After Ten-Day Irradiation of 1-kg Havar Foil (Flux Parameter

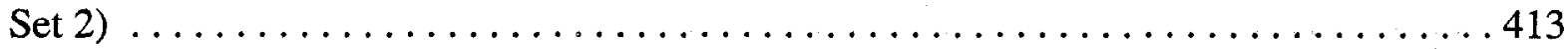

F32. Radioactivity (Ci) After One-Day Irradiation of 1-kg Concrete (Flux Parameter Set 3) .......................................... 415

F33. Radioactivity (Ci) After Ten-Day Irradiation of 1-kg Concrete (Flux Parameter

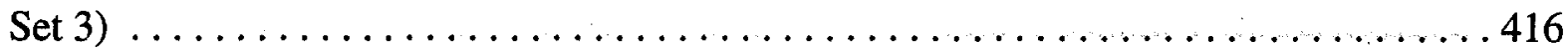

G1. Decay Photon Spectrum (/kg.s) After 8-s Irradiation of 1-kg Salted Beef Cargo

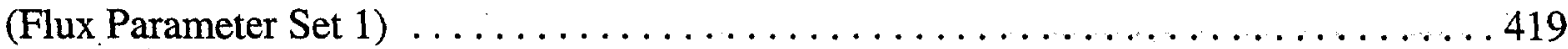

G2. Decay Photon Spectrum (/kg.s) After 16-s Irradiation of 1-kg Salted Beef Cargo

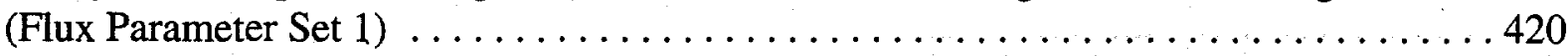

G3. Decay Photon Spectrum (/kg.s) After 24-s Irradiation of 1-kg Salted Beef Cargo (Flux Parameter Set 1) ................................. 420

G4. Decay Photon Spectrum (/kg.s) After 8-s Irradiation of 1-kg Ball Bearings Cargo (Flux Parameter Set 1) ................................ 421

G5. Decay Photon Spectrum (/kg.s) After 16-s Irradiation of 1-kg Ball Bearings Cargo (Flux Parameter Set 1)

G6. Decay Photon Spectrum (/kg.s) After 24-s Irradiation of 1-kg Ball Bearings Cargo

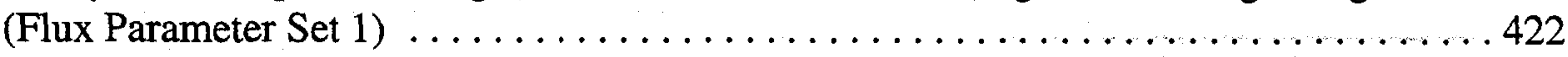

G7. Decay Photon Spectrum (/kg.s) After 8-s Irradiation of 1-kg Surgical Implant Composition A Cargo (Flux Parameter Set 1) . ..................... 422

G8. Decay Photon Spectrum (/kg.s) After 16-s Irradiation of 1-kg Surgical Implant Composition A Cargo (Flux Prameter Set 1) . ..................... 423

G9. Decay Photon Spectrum ( $/ \mathrm{kg}$. After 24-s Irradiation of 1-kg Surgical Implant Composition A Cargo (Flux Parameter Set 1) . ...................... 423

G10. Decay Photon Spectrum (/kg.s) After 8-s Irradiation of 1-kg Surgical Implant Composition B Cargo (Flux Parameter Set 1) ...................... 424

G11. Decay Photon Spectrum ( $/ \mathrm{kg} . s)$ After 16-s Irradiation of 1-kg Surgical Implant Composition B Cargo (Flux Parameter Set 1) . ..................... 424

G12. Decay Photon Spectrum ( $/ \mathrm{kg} . \mathrm{s})$ After 24-s Irradiation of 1-kg Surgical Implant Composition B Cargo (Flux Parameter Set 1) 
G13. Decay Photon Spectrum (/kg.s) After 8-s Irradiation of 1-kg 16-16-16

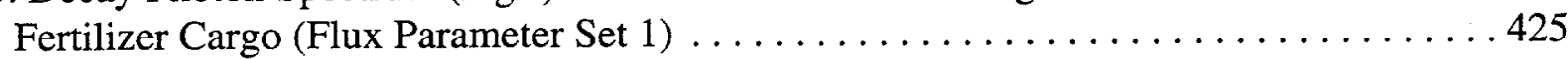

G14. Decay Photon Spectrum (/kg.s) After 16-s Irradiation of 1-kg 16-16-16

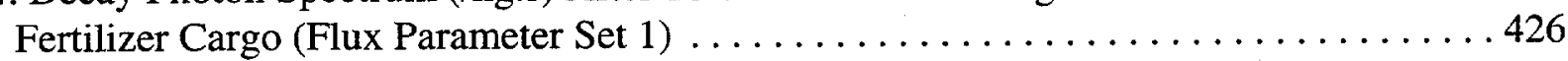

G15. Decay Photon Spectrum ( $/ \mathrm{kg} . \mathrm{s})$ After 24-s Irradiation of 1-kg 16-16-16

Fertilizer Cargo (Flux Parameter Set 1) ........................ 426

G16. Decay Photon Spectrum ( $/ \mathrm{kg} . \mathrm{s})$ After One-Day Irradiation of 1-kg Concrete

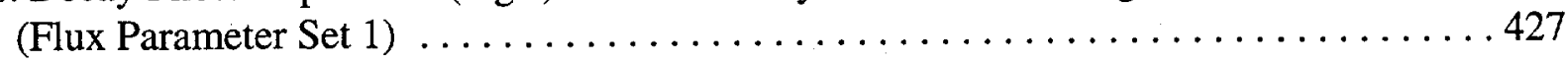

G17. Decay Photon Spectrum (/kg.s) After Ten-Day Irradiation of 1-kg Concrete

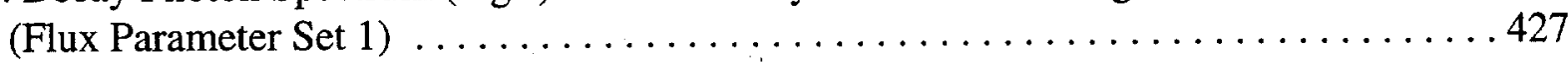

G18. Decay Photon Spectrum ( $/ \mathrm{kg} . \mathrm{s})$ After One-Day Irradiation of $1-\mathrm{kg}$ Concrete

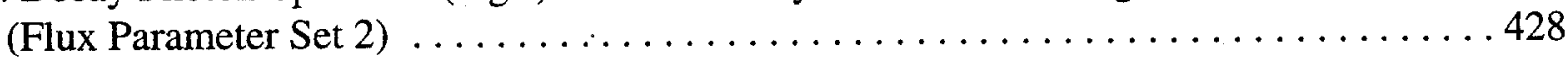

G19. Decay Photon Spectrum (/kg.s) After Ten-Day Irradiation of 1-kg Concrete

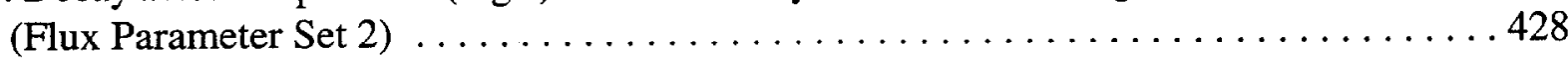

G20. Decay Photon Spectrum (/kg.s) After One-Day Irradiation of 1-kg Aluminum

6061-T6 (Flux Parameter Set 1) . . . . . . . . . . . . . . . . . . . . . . . 429

G21. Decay Photon Spectrum ( $/ \mathrm{kg} . \mathrm{s})$ After Ten-Day Irradiation of 1-kg Aluminum

6061-T6 (Flux Parameter Set 1) . . . . . . . . . . . . . . . . . . . . . . . . 429

G22. Decay Photon Spectrum ( $/ \mathrm{kg} .3$ ) After One-Day Irradiation of $1-\mathrm{kg}$ Aluminum

6061-T6 (Flux Parameter Set 2) . . . . . . . . . . . . . . . . . . . . . 430

G23. Decay Photon Spectrum (/kg.s) After Ten-Day Irradiation of 1-kg Aluminum

6061-T6 (Flux Parameter Set 2) ............................. 430

G24. Decay Photon Spectrum (/kg.s) After One-Day Irradiation of 1-kg Steel Plate

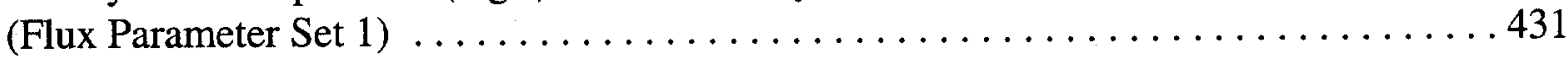

G25. Decay Photon Spectrum (/kg.s) After Ten-Day Irradiation of 1-kg Steel Plate

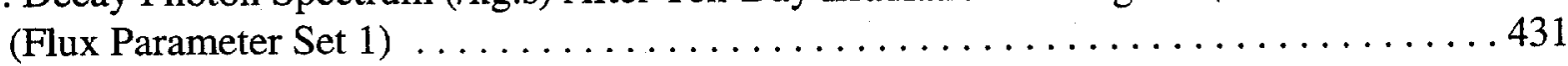

G26. Decay Photon Spectrum (/kg.s) After One-Day Irradiation of 1-kg Steel Plate

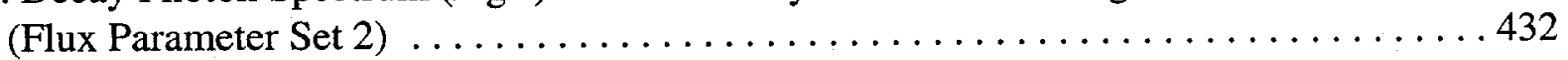

G27. Decay Photon Spectrum (/kg.s) After Ten-Day Irradiation of 1-kg Steel Plate

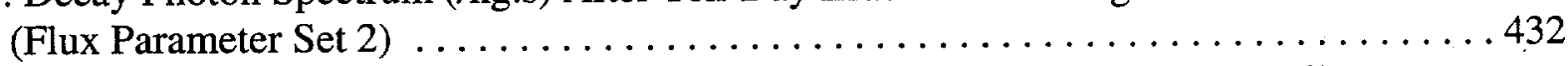

G28. Decay Photon Spectrum (/kg.s) After One-Day Irradiation of 1-kg Havar Foil

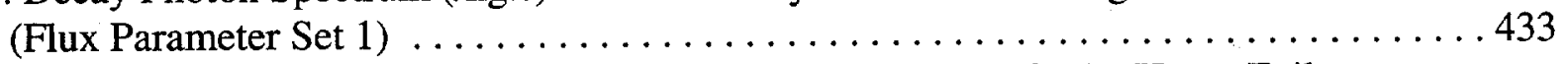

G29. Decay Photon Spectrum (/kg.s) After Ten-Day Irradiation of 1-kg Havar Foil

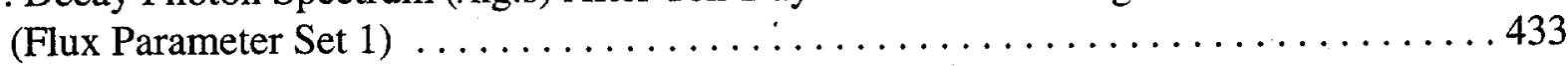

G30. Decay Photon Spectrum (/kg.s) After One-Day Irradiation of 1-kg Havar Foil

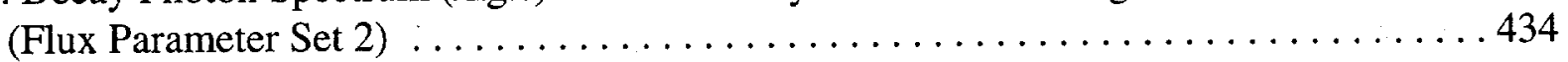

G31. Decay Photon Spectrum (/kg.s) After Ten-Day Irradiation of 1-kg Havar Foil

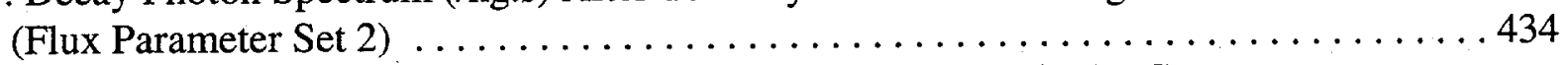

G32. Decay Photon Spectrum (/kg.s) After One-Day Irradiation of 1-kg Concrete

(Flux Parameter Set 3) .................................. 435

G33. Decay Photon Spectrum (/kg.s) After Ten-Day Irradiation of 1-kg Concrete

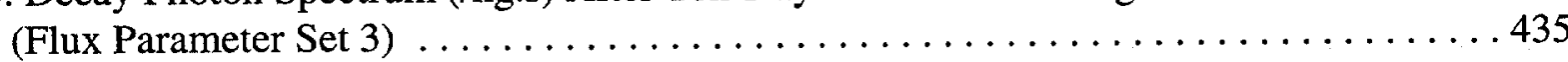

viii 


\section{LIST OF FIGURES}

Figure $\quad$ Page

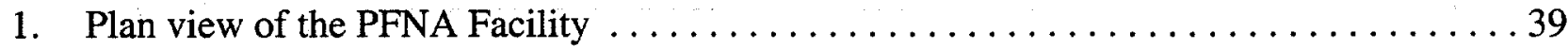

2. Elevation view of the PFNA Facility at the beam line plane $\ldots \ldots \ldots \ldots \ldots \ldots \ldots \ldots$

3. Sketch of the vertical and horizontal polyethylene beam stops $\ldots \ldots \ldots \ldots \ldots \ldots \ldots$

4. Head-on and side views of the polyethylene beam stop $\ldots \ldots \ldots \ldots \ldots \ldots \ldots \ldots \ldots$

5. Plan view of the stationary collimator and vertical collimator detailing most exterior

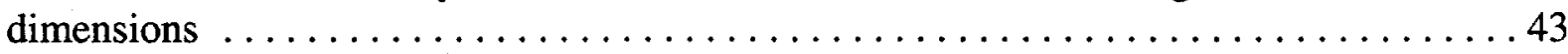

6. Plan view of the stationary collimator and vertical collimator detailing most interior

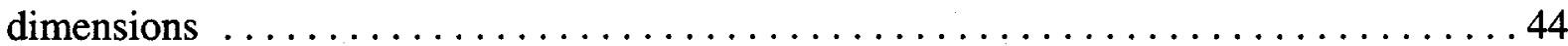

7. Elevation view of the vertical and stationary collimators with the vertical collimator

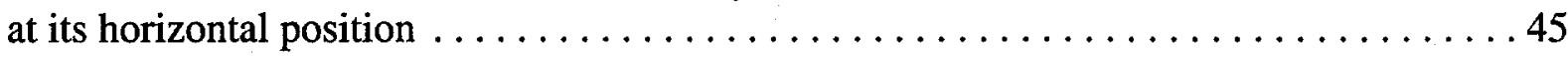

8. Vertical slice through the collimator and the bare plutonium sphere mockup used in the MCNP-4B calculations of dose multiplication by fissile targets $\ldots \ldots \ldots \ldots \ldots \ldots$

9. Horizontal slice through the collimator and the bare plutonium sphere mockup used in the MCNP-4B calculations of dose multiplication by fissile targets $\ldots \ldots \ldots \ldots \ldots 4$

10. Closeup horizontal slice through the bare plutonium sphere mockup used in the MCNP-4B calculations of dose multiplication by fissile targets. The numbered planes 611,614 , and 624 are three of the surface flux detectors used in the problem.

The fourth surface, 634 , is above the sphere $\ldots \ldots \ldots \ldots \ldots \ldots \ldots \ldots \ldots$

11. Closeup horizontal slice through the tungsten-reflected plutonium sphere mockup used in the NCNP-4B calculations of dose multiplication by fissile targets. The numbered planes 611,614 , and 624 are three of the surface flux detectors used in the problem. The fourth surface, 634 , is above the sphere $\ldots \ldots \ldots \ldots \ldots \ldots \ldots \ldots$

12. Closeup horizontal slice through the water-reflected plutonium sphere mockup used in the MCNP-4B calculations of dose multiplication by fissile targets. The numbered planes 611,614 , and 624 are three of the surface flux detectors used in the problem.

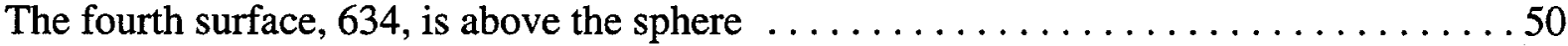

13. Approximate detector locations for dose rates shown in Table $15 \ldots \ldots \ldots \ldots \ldots$

C1. Plan view at $\mathrm{z}=0 \mathrm{~cm}$ through the MCNP geometry mockup of the PFNA Facility . . . 155

C2. Plan view at $\mathrm{z}=0 \mathrm{~cm}$ through the $\mathrm{MCNP}$ geometry mockup of the PFNA Facility. Closeup view of regions around the stationary collimator $\ldots \ldots \ldots \ldots \ldots \ldots \ldots$

C3. Plan view at $\mathrm{z}=0 \mathrm{~cm}$ through the MCNP geometry mockup of the PFNA Facility. Closeup view of regions around the stationary collimator $\ldots \ldots \ldots \ldots \ldots \ldots 7$

C4. Elevation view at $\mathrm{y}=0 \mathrm{~cm}$ through the MCNP geometry mockup of the PFNA Facility for the vertical collimator in the horizontal position $\ldots \ldots \ldots \ldots \ldots \ldots \ldots \ldots$

C5. Elevation view at $y=0 \mathrm{~cm}$ through the MCNP geometry mockup of the PFNA Facility for the vertical collimator in the horizontal position. Closeup view of the vertical

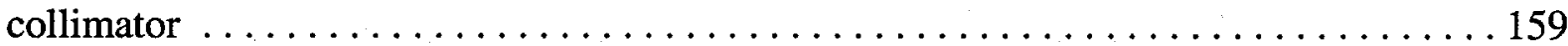

C6. Elevation view at $y=30 \mathrm{~cm}$ through the MCNP geometry mockup of the PFNA Facility .160 
C7. Elevation view at $y=0 \mathrm{~cm}$ through the MCNP geometry mockup of the PFNA Facility for the vertical collimator in the maximum up position . . . . . . . . . . . . 161

C8. Elevation view at $y=0 \mathrm{~cm}$ through the MCNP geometry mockup of the PFNA Facility for the vertical collimator in the maximum up position. Closeup view of the vertical

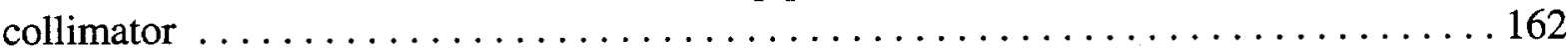

C9. Elevation view at $y=0 \mathrm{~cm}$ through the MCNP geometry mockup of the PFNA Facility for the vertical collimator in the maximum down position $\ldots \ldots \ldots \ldots \ldots \ldots$

C10.Elevation view at $y=0 \mathrm{~cm}$ through the MCNP geometry mockup of the PFNA Facility for the vertical collimator in the maximum down position. Closeup view of the vertical collimator . . . . . . . . . . . . . . . . . . . . . . . . . . 164 


\begin{abstract}
At the request of Safety and Ecology Corporation of Tennessee, radiation effects of the proposed Pulsed Fast Neutron Analysis (PFNA) Cargo Interrogation System have been examined. First, fissile cargo were examined to determine if a significant neutron signal would be observable during interrogation. Results indicated that ample multiplication would be seen for near critical bare targets. The water-reflected sphere showed relatively little multiplication. By implication, a fissile target shielded by hydrogenous cargo might not be detectable by neutron interrogation, particularly if reliance is placed on the neutron signal. The cargo may be detectable if use can be made of the ample increase in the photon signal. Second, dose rates were calculated at various locations within and just outside the facility building. These results showed that some dose rates may be higher than the target dose rate of $0.05 \mathrm{mrem} / \mathrm{h}$. However, with limited exposure time, the total dose may be well below the allowed total dose. Lastly, estimates were made of the activation of structures and typical cargo. Most cargo will not be exposed long enough to be activated to levels of concern. On the other hand, portions of the structure may experience buildup of some radionuclides to levels of concern.
\end{abstract}




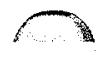




\subsection{INTRODUCTION}

The proposed Pulsed Fast Neutron Analysis (PFNA) Cargo Interrogation System is designed to scan large transport vehicles to determine if the vehicles are transporting illegal cargo (such as personnel and special nuclear materials) across U. S. borders. The scanning is accomplished by sweeping the towed, slow-moving vehicle with a fast-neutron beam (having a nominal energy of 9 $\mathrm{MeV}$ in the forward cone) and analyzing the emerging radiation signatures to ascertain the cargo. ORNL analysts at the request of Safety and Ecology Corporation (SEC) of Tennessee have analyzed the facility for (1) neutron multiplication in fissile cargo, (2) dose rates at various locations within and around the facility, and (3) neutron activation of the facility structure and selected cargoes. The facility, the calculational models, and the calculational procedures are described and the calculational results are presented. 


\subsection{THE FACILITY}

The PFNA facility consists of a source, a source collimator, several banks of radiation detectors, a vehicle lane, overhead shielding, and concrete walls for structural support and shielding. Plan and elevation views of the facility are shown in Figures 1 and 2. Figures 3 and 4 show details of the vertical and overhead horizontal beam stops, and Figures 5-7 show details of the source collimator. 
$\curvearrowright$

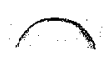




\subsection{THE SOURCE}

The neutron source consisting of nominally 9-MeV neutrons in the forward cone is produced by an accelerator via a D-D reaction in a target material. The resulting angle- and energy-dependent source spectrum is presented in Table 1 . The source is collimated such that the illuminated area of the beam at the center of the interrogation region measures $45 \mathrm{~cm}$ by $45 \mathrm{~cm}$ in terms of full width at half maximum. A travelling vertical collimator allows the movement of the source in a vertical arc to allow full vertical interrogation of the cargo. The mechanics of the collimator movement are such that the rate of movement is about the same for all angles in the sweep, thus uniformly irradiating the cargo vertically. Calculations must be performed on snapshots of the facility at selected angles of the sweep, since the sweep alters the geometry and present calculational procedures only allow for fixed geometries throughout a given calculation. Along with the geometry change, one must also change the source emission location and the reference vector for defining the source angular emission. 


\subsection{COMPUTER CALCULATIONS}

\subsection{Neutron Multiplication in Fissile Material in Cargo}

It is expected that fissile material in the cargo can be detected because of the increased radiation signal generated by neutron multiplication. Therefore, the first calculational task was the determination of the dose-rate multiplication for several near critical plutonium spheres, including bare and tungsten- and water-reflected spheres. Data on the critical radii and the compositions of the plutonium spheres were supplied by SEC. These were based on data from Ref. 1. Compositions of the plutonium spheres are given in Table 2.

For these calculations, only the plutonium sphere and the sourcc collimator were modeled. The distance from the source to the plutonium sphere was supplied by SEC. Figures 5-7, which show some dimensions, were used to model the collimator. The collimator includes a stationary part and a moving vertical part (or vertica! collimator). The vertical collimator is a half-cylinder disk with regions of two different thickness and a tapered slot to allow desired portions of the neutron beam to pass through. It is made of boraied polyethylene. The stationary portion of the collimator consists of borated polyethylene, borated paraffin, aluminum, and lead in two mirror-image, shaped aluminum containers resting on a steel plate and a raised concrete block. The small regions of regular polyethylene in the stationary collimator were missed because of the similar shading to the borated paraffin and because they were not labeled on each drawing. These regions were modeled as borated paraffin. Compositions of the collimator materials, the plutonium sphere reflector materials, and of materials used in other PFNA facility calculations are given in Tables 3 and 4. While some dimensions were given on the drawings, some of the missing dimensions were determined by scaling from dimensions given on the drawings. An attempt was made to size the openings through the collimators by extrapolating from the specified $45-\mathrm{cm}$ by $45-\mathrm{cm}$ beam at the center of the interrogation region. However, the taper so computed for the stationary collimator was inconsistent with that shown in Figure 5 and 6 . Therefore, the taper for the stationary collimator was determined by measurements of the starting and ending points of the taper on Figure 5 or 6 . For the vertical collimator, the $4^{\circ}$ overtravel shown on Figure 2 was mistakenly assumed to be the angle of the opening in the vertical collimator, since the lines appeared to follow the taper of the collimator opening. A later design was based on measurements on Figure 7. The differences between the two models didn't appear to be too great and should not have a big impact on the calculated results obtained using the first model. Calculations were performed using the MCNP-4B Monte Carlo radiation transport code ${ }^{2}$ and ENDF/B-IV cross-section data. The calculations were run in parallel mode to condense the duration of each calculation from days to a few hours.

Because the plutonium spheres were supposed to be critical and calculations using a fixed source would be impossible to perform, we opted to perform the calculations with slightly subcritical spheres. Since the measured critical radii might be different from the calculated ones, it was necessary that the effective multiplication factor $\left(\mathrm{k}_{\text {eff }}\right)$ of the critical spheres be calculated in order to assure configurations would be subcritical for the codes and dat used. Therefore, criticality

calculations were performed using the MCNP-X Monte Carlo radiation transport code. ${ }^{3}$ While this 
code is designed to perform calculations for many species of particles with energies in the very high to very low range, it performs calculations the same as $\mathrm{MCNP}-4 \mathrm{~B}$ for low-energy $(\mathrm{E}<20 \mathrm{MeV})$ neutron, photon, and electron transport. MCNP-X was used because the $\mathrm{k}_{\text {eff }}$ calculations could be run within a reasonable time on a single computer node and several calculations could be run simultaneously on different single nodes without competing for parallel resources with other parallel calculations. The calculated $\mathrm{k}_{\text {eff }}$ values for the plutonium spheres having the specified critical radii and compositions and for two subcritical bare plutonium spheres are shown in Table 5 along with the standard deviations of the results. For the critical spheres (the last three in the table), the $\mathbf{k}_{\text {eff }}$ values vary from $0.3 \%$ below critical to $1.05 \%$ above critical. Even the 6.35 -cm-radius bare sphere at $99.4 \%$ of the critical radius and $98.4 \%$ of the critical volume has a $\mathrm{k}_{\text {eff }}$ that is only $0.7 \%$ below critical or only $0.4 \%$ more subcritical than the critical sphere. Listings of input files for the MCNP-X criticality calculations are given in Appendix A.

After the $\mathrm{k}_{\text {eff }}$ values were determined, dose rates were then calculated for subcritical spheres irradiated by the neutron source. The calculations were performed using the MCNP-4B computer code. Calculations were performed without a target sphere in the mockup (void replaces plutonium in the spheres) to get a background dose rate due to direct beam and scattering from the collimator. No consideration was given to scattering from the concrete floor and the walls of the facility. Dose rates with the sphere present are compared to the background dose rates to give an indication of the multiplication of the detector signal when fissile material is present. Because of the large multiplication that results for a near-critical sphere and the calculational difficulties that would result, the calculations were performed initially for spheres having about $97 \%$ of the critical mass or about $99 \%$ of the critical radius. The values used for the radii of the plutonium spheres and their reflectors if any are shown in Table 6 . For the bare sphere, dose rates were calculated at detectors $10 \mathrm{~cm}$ in front of, behind, to the side of, and above the center of the sphere. Since initial calculations seemed to indicate excessive computer time when point detectors were used ${ }^{2}$, dose rates were computed using surface detectors around the spheres. Figure 8 shows a vertical slice through the collimator and the bare plutonium sphere mockup, and Figure 9 shows a horizontal slice through the collimator and the bare plutonium sphere mockup along with several surfaces around the sphere. Figures 10-12 are closeup views of respectively the bare, tungsten-reflected, and water-reflected spheres and the surface detectors surrounding them. One can also see that the spheres are subdivided into two or three regions, mainly in anticipation of the reflected sphere calculations, but also to allow for different importance regions for the bare sphere. Some calculations were also performed using point detectors. The flux-to-dose conversion factors used were obtained from the tissue dose response function in the VELM61 cross-section library ${ }^{4}$ and are shown in Tables 7 and 8 . Only source in the forward directions (Table 1) was used because the backward-directed particles could not contribute to the dose since there was no material to scatter the beam back toward the detectors. These particles would simply escape the system, increasing the computing time per contributing particle and reducing the calculational efficiency.

${ }^{a}$ In reality, the code for unknown reasons ceased to run in parallel mode and began to run on a single node, thus greatly increasing the calculation time. 
The calculated dose rates and the dose multiplication are shown in Tables 9-13. These results were obtained from calculations that each followed 400,000 neutron histories. The statistical uncertainties on the foreground results (with a plutonium sphere in the model) are larger than desired for reliable results. While these results may change considerably with large increases in the number of histories, the background results should change less since most statistics are reasonable. The results show large increases in the neutron dose rate in the presence of the bare spheres and a significant increase for the tungsten-reflected sphere. Little multiplication is seen for the water-reflected sphere. All cases show substantial increases in the photon dose rate above the background levels, with factors above 20 for the water-reflected sphere and factors of hundreds and thousands for the others. Finally, calculations were performed using many more histories. Dose rates calculated for a 6.38 -cm-radius bare plutonium sphere with 400,000 histories are compared in Table 14 to those calculated using 16,000,000 histories for both point and surface detectors. The statistics for the 16-million-history results are reasonable. Note that the point detector results start out low and increase with the number of histories while the surface detector results start out high and decrease with the number of histories. The point and surface detector results for the 16-million-history cases are essentially in agreement. One might expect the dose rate to be higher at the center of the $10-\mathrm{cm} \times 10-\mathrm{cm}$ surface detectors and an average over the surface to be somewhat lower as the Table 14 results indicate. Listings of input files for the MCNP-4B calculations of dose rates may be found in Appendix B.

\subsection{Dose Rates Within and Around the Facility}

The facility was modeled from the views shown in Figures 1-7. Except for the use of borated polyethylene instead of regular polyethylene in the hut surrounding the source and the small region of regular polyethylene in the stationary collimator (previously mentioned), the materials were modeled as specified. The use of borated polyethylene should result in lower calculated dose rates in regions to the side of or behind the hut than one would calculate using regular polyethylene. While no material was included for the $\mathrm{NaI}$ detectors and their associated shielding, the shielding preceding the regions should be sufficient enough that dose rates behind these regions will be due mainly to source coming through the center of the collimator and scattering from walls surrounding the truck lane. Material compositions are given in Tables 3 and 4 . While Figure 1 shows possibly two different concrete shield walls, an August 29, 2000 memorandum from Peter Ryge of Ancore Corporation stated in reference to Concrete-2 that "no specific composition data has been available for earth blocks or other materials, so the same concrete is assumed as a conservative approach." Therefore, a density of $2.0 \mathrm{~g} / \mathrm{cm}^{3}$ was used for both Concrete- 1 and Concrete- 2 . Because the end walls of the facility measured about $20 \mathrm{~cm}$, the outer facility walls were assumed to be composed of masonry block at half the concrete shield wall density and were assumed to be stacked $609.6 \mathrm{~cm}$ $(20 \mathrm{ft})$ high. In addition, a thin steel roof $(0.318 \mathrm{~cm}$ or $0.125 \mathrm{in}$.) was assumed. Plots of plan and elevation plane slices through the PFNA facility calculational geometry are given in Appendix C.

Neutron and photon dose rates were calculated using the point detector estimator in the MCNP-4B computer code, first for locations around the facility and then for locations along the centerline of the truck lane and across the truck lane along the centerline of the neutron beam. All 
these dose rates were calculated for three source positions: (a) a maximum down position of $8.6^{\circ}$ below the horizontal, (b) a horizontal position of $0^{\circ}$, and (c) a maximum up position of $34.8^{\circ}$ above the horizontal, and all were calculated at a height of $z=0.0 \mathrm{~cm}$, which in the calculational model is $125.095 \mathrm{~cm}$ above the floor and at the horizontal source elevation. Two million neutron source histories were followed in all calculations, resulting in reasonable statistics for most detector positions. Total dose rates were obtained by summing the neutron and photon dose rates. Then the neutron, photon, and total dose rates were averaged for the vertical sweep by assuming exponential variation of the dose rate between the three angles at which the dose rate was calculated as given in the equations below. Listings of the simple Fortran computer codes used to do the averaging and the input files for the codes are given in Appendix D. The calculated dose rates for the three source angles as well as the average dose rates are shown in Tables 15-19. The dose rates are specified per

$$
\begin{aligned}
& \bar{f}=\frac{\sum_{i=1}^{2} \int_{\mu_{i}}^{\mu_{i+1}} f\left(\mu_{i}\right) e^{\lambda_{i}\left(\mu-\mu_{i}\right)} d \mu}{\left(\mu_{3}-\mu_{1}\right)} \\
& \bar{f}=\frac{\sum_{i=1}^{2} \frac{f\left(\mu_{i}\right)}{\lambda_{i}}\left(e^{\lambda_{i}\left(\mu_{i+1}-\mu_{i}\right)}-1\right)}{\left(\mu_{3}-\mu_{1}\right)}
\end{aligned}
$$

where $\mu_{i}=\theta_{i}$ was used here, but one might also consider $\mu_{i}=\sin \theta_{i}$. In addition,

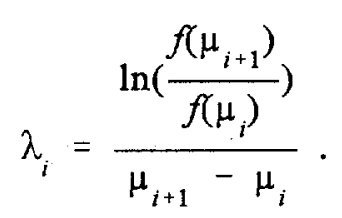

Now since

$$
f\left(\mu_{i+1}\right)=f\left(\mu_{i}\right) \quad e^{\lambda_{i}\left(\mu_{i+1}-\mu_{i}\right)}
$$

then

$$
\bar{f}=\sum_{i=1}^{2} \frac{f\left(\mu_{i+1}\right)-f\left(\mu_{i}\right)}{\lambda_{i}}
$$

hour, while dose limits may be time-integrated. Therefore, these calculated dose rates must be multiplied by the exposure time to determine if the dose limit is exceeded at a given location. Additionally, dose rates were calculated below and above the top source shield at a range of 
locations across the truck lane with the source in its maximum up position. These results are presented in Table 20. Note that for the $X$ locations, the center of the truck lane is at $X=298.958 \mathrm{~cm}$. The $\mathrm{Z}=0 \mathrm{~cm}$ location is about $125 \mathrm{~cm}$ above the floor. For the lower elevation $(374 \mathrm{~cm})$ in the table, a detector X location $(332.7 \mathrm{~cm})$ that corresponds to the intersection of the source vector with the bottom surface of the source top shield was included as the possible location for the maximum dose rate at that height in the plane of the source sweep. A detector with the same $\mathrm{X}$ coordinate was included for the upper elevation. No maximum dose location is evident for the lower elevation (beneath the source top shield bottom surface). In fact, the slope of a dose-rate curve plotted through the calculated points would indicate further increases in the dose rate toward the source. These locations are probably receiving uncollided dose from the relatively large and reasonably energetic neutron source emitted at angles outside the forward source cone even though they may have to pass through part of the vertical collimator. The dose rate above the shield does peak closer to the $\mathrm{X}$ coordinate $(484.3 \mathrm{~cm})$ that is along the straight-ahead source vector. The dose rate doesn't peak there because (1) the particles have to travel through $35.6 \mathrm{~cm}$ of polyethylene travelling straight but through as little as $20.3 \mathrm{~cm}$ if scattered and (2) the localized scurce created by scattering in the shield is closer to the source and thus shifts the peak dose rate position toward the source. The overhead dose rates were calculated mainly as an indicator of skyshine dose rates. Based on the maximum calculated roof dose rate from the table $(1020 \mathrm{mrem} / \mathrm{h})$ and a rule-of-thumb skyshine dose rate estimate of $2 \%$ of the roof dose rate, one would anticipate a maximum skyshine dose rate of about $20 \mathrm{mrem} / \mathrm{h}$ if the source were stuck at its maximum up position. Photons should contribute less than $10 \%$ additional to the dose rate. Input files for the calculations are in Appendix E.

\subsection{Neutron Activation of Structural Materials and Stream-of-Commerce Cargoes}

The presence of certain elements (such as cobalt, iron, nitrogen, nickel, etc.) in materials undergoing neutron irradiation can be of concern due to the possible activation of certain isotopes and the nontrivial residual activity minutes, hours, or even days following irradiation. Therefore, an attempt was made to quantify the activity levels in selected stream-of-commerce cargoes that pass through the main beam in less than eight seconds and in structural materials that are irradiated for hours day after day. First, flux levels in the regions of interest had to be determined. These flux levels were calculated in a broad three-energy-group structure by the MCNP-4B computer code. The three-group structure is that used for ascii cross-section data in the ORIGEN $^{5}$ isotope generation and depletion code. Three flux spectra were used in the calculations: (a) one at the center of the truck lane that was used for activation calculations for cargo and structural materials, (b) one within the stationary collimator that was used for activation calculations for structural materials, and (c) one at the front of the beam stop concrete that was used for activation calculations for the beam stop concrete. In addition to the three-group flux spectra, with user input flux factors, MCNP-4B calculated the integral of $1 / \mathrm{v}$ times the thermal-neutron flux over the interval from 0 to $0.5 \mathrm{eV}$. This value along with the other flux data were used to produce spectral parameters (THERM, RES, and FAST) used by ORIGEN.

For the cargoes, the cargo material was irradiated for 8,16 , and $24 \mathrm{~s}$ time periods and the induced activity was allowed to decay for up to $1200 \mathrm{~s}$. The 8-s irradiation period was based on a point being in the main beam area about $8 \mathrm{~s}$. The other two irradiation times attempt to account for 
the point being in a higher flux field for a longer period of time because of neutron scattering within the cargo material. Table 21 shows the neutron and photon dose rate distribution at the center of the truck lane and perpendicular to the beam line for detectors in salted beef cargo. While the statistics on the neutron dose rates are large and make the results inconclusive, the results do tend to indicate that the main beam is confined to the $45-\mathrm{cm} \times 45-\mathrm{cm}$ square mentioned earlier. The photon dose rates with their acceptable statistics indicate a significant thermal-neutron flux $50 \mathrm{~cm}$ either side of the beam line (the dose rate is $1 / 4$ to $1 / 3$ of the centerline value). Thus a particular cargo location may be exposed to significant thermal-neutron flux for up to $16 \mathrm{~s}$, although at lower flux levels than those calculated in the absence of the nonfissile cargo. The dose distribution may be different near the source edge of the cargo from that calculated at the center of the cargo, and the flux levels should be much higher. Compositions of the cargoes by weight percent are given in Table 22.

For the structural materials, since twenty trucks per day can be processed through the facility and each truck would be allotted about 24 minutes per 8-h workday, it was assumed that the structural material would be irradiated continuously for eight hours each day. The facility would then be shut down 16 hours each day. Therefore, ORIGEN calculations were performed for ten days in cycles consisting of eight hours irradiation followed by 16 hours decay. Compositions of the structural materials per kilogram are given in Table 23.

Results obtained from the ORIGEN calculations are tables of the radioactivity of various radionuclides at various times following irradiation and the corresponding decay photon spectra. For the radioactivities, results for a given radionuclide were printed only if the radioactivity at the initial decay time step were greater than $10^{-20} \mathrm{Ci} / \mathrm{kg}$. A lower $10^{-12} \mathrm{Ci} / \mathrm{kg}$ cutoff caused results for some long-lived species such as ${ }^{60} \mathrm{Co}$ and ${ }^{59} \mathrm{Fe}$ not to be printed. Table 24 shows along with their definitions the ORIGEN parameters (THERM, RES, FAST, and the thermal-neutron flux) that were used in the various calculations. The associated tabular radioactivity results are presented in tables in Appendix $F$ and the decay photon spectra are presented in tables in Appendix G. The activities presented are probably conservative, and even then most are not at levels of concern. Thermal-neutron flux levels are too low to produce significant activity over short irradiation periods. There may be some activity buildup in regions of structural materials that are irradiated for much longer periods of time. Listings of the MCNP-4B input files used to calculate the ORIGEN flux spectra are given in Appendix H, while listings of the input files for the ORIGEN calculations are given in Appendix I. 


\subsection{SUMMARY}

MCNP-4B calculations have been performed on models of the PFNA facility to analyze (1) dose signal increase in the presence of fissile cargo, (2) dose rates in and around the facility absent any cargo, and (3) neutron activation of cargo and structures as a result of interrogation with a neutron beam. The results indicate first that there could be detectable enhancement of the dose rate or some other detector signal in the presence of some near-critical fissile cargo. Other fissile cargo may escape detection if shielded by hydrogenous cargo or if the cargo is too subcritical. Second, the dose rates at some peripheral locations may be at levels of concern for long periods of operation because the accumulated dose may exceed regulatory limits. Third, cargo will generally be irradiated over too short a time period to experience appreciable buildup of radioactivity. Structures, however, may become activated because of the many irradiation/decay cycles experienced over long periods of time. Portions of the structure lying within the path swept by the vertical collimator will experience the highest activation levels. 
$\curvearrowright$

$\cap$ 


\subsection{REFERENCES}

1. NEA Nuclear Science Committee, International Handbook of Evaluated Criticality Safety Benchmark Experiments: Plutonium Systems, Volume I.a, Nuclear Energy Agency, Paris, 1995 (entries dated March 31, 1995 and August 31, 1996).

2. J. F. Briesmeister, Editor, MCNP - A General Monte Carlo N-Particle Transport Code, Version 4B, Los Alamos National Laboratory Report LA-12625-M, March 1997.

3. L. S. Wäters, Editor, MCNPX User's Manual, Version 2.1.5, Accelerator Production of Tritium Program Report TPO-E83-G-UG-X-00001, Revision 0, November 14, 1999.

4. C. Y. Fu and D. T. Ingersoll, VELM61 and VELM22: Multigroup Cross-Section Libraries for Sodium-Cooled Reactor Shield Analysis, Oak Ridge National Laboratory Report ORNL/TM-10302, April 1987.

5. O. W. Hermann and R. M. Westfall, "ORIGEN-S: SCALE System Module to Calculate Fuel Depletion, Actinide Transmutation, Fission Product Buildup and Decay, And Associated Radiation Source Terms," Section F7 in SCALE: A Modular Code System for Performing Standardized Computer Analyses for Licensing Evaluation, Book III, Volume 2, Oak Ridge National Laboratory Report ORNL/NUREG/CSD-2 (NUREG/CR-0200), December 1984. 
Table 1. Angle- and Energy-Dependent Neutron Source Spectrum ${ }^{a}$ for the PFNA Facility.

\begin{tabular}{||c|c|c|c|}
\hline $\begin{array}{c}\text { Angle Range } \\
\text { (degrees) }\end{array}$ & $\begin{array}{c}\text { Cross Section } \\
(\mathrm{mb})\end{array}$ & $\begin{array}{c}\text { Neutron Yield } \\
\left(\mathrm{s}^{-1}\right)\end{array}$ & $\begin{array}{c}\text { Mean Energy } \\
(\mathrm{MeV})\end{array}$ \\
\hline 0 to 5 & 1.83 & $1.09+09^{\mathrm{b}}$ & 8.537 \\
\hline 5 to 30 & 24.72 & $1.47+10$ & 8.105 \\
\hline 30 to 60 & 14.88 & $8.82+09$ & 6.668 \\
\hline 60 to 90 & 20.36 & $1.21+10$ & 4.682 \\
\hline 90 to 135 & 14.51 & $8.60+09$ & 2.847 \\
\hline 135 to 180 & 19.67 & $1.17+10$ & 1.872 \\
\hline
\end{tabular}

${ }^{2}$ The values were calculated using the tabulated cross sections and energies for the $\mathrm{D}(\mathrm{d}, \mathrm{n})^{3} \mathrm{He}$ reaction given in the article by H. Liskien and A. Paulsen, "Neutron Production Cross Sections and Energies for the Reactions $\mathrm{T}(\mathrm{p}, \mathrm{n})^{3} \mathrm{He}, \mathrm{D}(\mathrm{d}, \mathrm{n})^{3} \mathrm{He}$, and $\mathrm{T}(\mathrm{d}, \mathrm{n})^{4} \mathrm{He}$ in Nuclear Data Tables 11, 569-619 (1973).

${ }^{\mathrm{b}}$ Read as $1.09 \times 10^{9}$. 
Table 2. Compositions for the Plutonium Critical Spheres.

\begin{tabular}{||l|c|c|c||}
\hline \multirow{2}{*}{\begin{tabular}{l} 
Nuclide \\
\cline { 2 - 4 }
\end{tabular}} & Bare & Tungsten-Reflected & Water-Reflected \\
\cline { 2 - 4 } & $1.3752-3^{\mathrm{a}}$ & $1.3628-3$ & \\
\hline $\mathrm{Ga}$ & $3.7047-2$ & $3.7291-2$ & $4.6982-2$ \\
\hline${ }^{239} \mathrm{Pu}$ & $1.7512-3$ & $1.9277-3$ & $2.5852-3$ \\
\hline${ }^{240} \mathrm{Pu}$ & $1.1674-4$ & $1.2196-4$ & $1.4915-4$ \\
\hline${ }^{241} \mathrm{Pu}$ & & & $9.9432-6$ \\
\hline${ }^{242} \mathrm{Pu}$ & & & \\
\hline
\end{tabular}

${ }^{\mathrm{a}}$ Read as $1.3752 \times 10^{-3}$. 
Table 3. Compositions of hydrogenous materials used in the PFNA calculations.

\begin{tabular}{||l|l|l|l|l||}
\hline \multirow{2}{*}{ Nuclide } & \multicolumn{4}{|c|}{ Composition $\left(\mathrm{b}^{-1} \cdot \mathrm{cm}^{-1}\right.$ unless otherwise noted) } \\
\cline { 2 - 5 } & $\begin{array}{c}\text { borated } \\
\text { polyethylene }\end{array}$ & borated paraffin & concrete $^{\mathrm{a}}$ & water \\
\hline $\mathrm{H}$ & $6.5800-2^{\mathrm{b}}$ & $7.83500-2$ & 1.0 & $6.6766-2$ \\
\hline${ }^{10} \mathrm{~B}$ & $5.1940-4$ & $4.34260-4$ & & \\
\hline${ }^{11} \mathrm{~B}$ & $2.1306-3$ & $1.78134-3$ & & \\
\hline $\mathrm{C}$ & $2.9100-2$ & $3.82300-2$ & 0.1 & $3.3383-2$ \\
\hline $\mathrm{O}$ & $7.9400-3$ & & 52.9 & \\
\hline $\mathrm{Na}$ & & & 1.6 & \\
\hline $\mathrm{Mg}$ & & & 0.2 & \\
\hline $\mathrm{Al}$ & & & 3.4 & \\
\hline $\mathrm{Si}$ & & & 33.7 & \\
\hline $\mathrm{K}$ & & & 1.3 & \\
\hline $\mathrm{Ca}$ & & & 4.4 & \\
\hline${ }^{54} \mathrm{Fe}$ & & & $7.91056-2$ & \\
\hline${ }^{56} \mathrm{Fe}$ & & & 1.28657 & \\
\hline${ }^{57} \mathrm{Fe}$ & & & $3.02595-2$ & \\
\hline${ }^{58} \mathrm{Fc}$ & & & $4.06658-3$ & \\
\hline
\end{tabular}

${ }^{\mathrm{a}}$ Weight percent (Concretes 1 and 2 are $2.0 \mathrm{~g} / \mathrm{cm}^{3}$ and block walls are $1.0 \mathrm{~g} / \mathrm{cm}^{3}$ ).

${ }^{\mathrm{b}}$ Read as $6.5800 \times 10^{-2}$. 
Table 4. Compositions of metal materials used in the PFNA calculations.

\begin{tabular}{|c|c|c|c|c|c|}
\hline \multirow{2}{*}{ Nuclide } & \multicolumn{5}{|c|}{ Composition $\left(\mathrm{b}^{-1} \cdot \mathrm{cm}^{-1}\right.$ unless otherwise noted) } \\
\hline & Lead & $\mathrm{Al} 6061-\mathrm{T} 6$ & Steel & Tungsten & Iron $^{a}$ \\
\hline $\mathrm{C}$ & & & $3.16929-4$ & & \\
\hline $\mathrm{N}$ & & & $5.43529-4$ & & \\
\hline $\mathrm{Mg}$ & & $6.68900-4$ & & & \\
\hline $\mathrm{Al}$ & & $5.82700-2$ & & & \\
\hline $\mathrm{Si}$ & & $3.47400-4$ & $1.27063-3$ & & \\
\hline $\mathrm{P}$ & & & $6.91287-5$ & & \\
\hline$S$ & & & $4.45160-5$ & & \\
\hline$\overline{\mathrm{Ti}}$ & & $5.09300-5$ & & & \\
\hline${ }^{50} \mathrm{Cr}$ & & $2.72300-6$ & $7.96600-4$ & & \\
\hline${ }^{52} \mathrm{Cr}$ & & $5.24440-5$ & $1.53441-2$ & & \\
\hline${ }^{53} \mathrm{Cr}$ & & $5.94600-6$ & $1.73970-3$ & & \\
\hline${ }^{54} \mathrm{Cr}$ & & $1.47700-6$ & $4.32200-4$ & 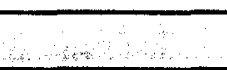 & \\
\hline${ }^{55} \mathrm{Mn}$ & & $4.44000-5$ & $1.73219-3$ & & \\
\hline${ }^{54} \mathrm{Fe}$ & & $1.19200-5$ & $3.23670-3$ & & 5.85 \\
\hline${ }^{56} \mathrm{Fe}$ & & $1.86990-4$ & $5,07622-2$ & & 91.75 \\
\hline${ }^{57} \mathrm{Fe}$ & & $4.32100-6$ & $1.17290-3$ & & 2.12 \\
\hline${ }^{58} \mathrm{Fe}$ & & $5.07600-7$ & $1.54900-4$ & & 0.28 \\
\hline${ }^{58} \mathrm{Ni}$ & & & $6.62295-3$ & $6.61220-3$ & \\
\hline$\overline{{ }^{60} \mathrm{Ni}}$ & & & $2.55073-3$ & $2.54659-3$ & \\
\hline${ }^{61} \mathrm{Ni}$ & & & $1.10900-4$ & $1.10721-4$ & \\
\hline${ }^{62} \mathrm{Ni}$ & & & $3.53130-4$ & $3.52560-4$ & \\
\hline${ }^{64} \mathrm{Ni}$ & & & $9.04600-5$ & $9.03250-5$ & \\
\hline${ }^{63} \mathrm{Cu}$ & & $9.08133-5$ & & $2.82034-3$ & \\
\hline${ }^{65} \mathrm{Cu}$ & & $4.04767-5$ & & $1.25706-3$ & \\
\hline $\mathrm{Zr}$ & & & . & $7.95280-4$ & \\
\hline${ }^{182} \mathrm{~W}$ & & & & $1.36998-2$ & \\
\hline${ }^{183} \mathrm{~W}$ & & & & $7.36713-3$ & \\
\hline${ }^{184} \mathrm{~W}$ & & & & $1.57708-2$ & \\
\hline${ }^{186} \mathrm{~W}$ & & & & $1.46303-2$ & \\
\hline${ }^{206} \mathrm{~Pb}$ & $8.37420-3^{b}$ & & & & \\
\hline${ }^{207} \mathrm{~Pb}$ & $7.25764-3$ & & & & \\
\hline${ }^{208} \mathrm{~Pb}$ & $1.72082-2$ & & & & \\
\hline
\end{tabular}

${ }^{\text {a }}$ Composition by atom percent. The density used was $7.86 \mathrm{~g} / \mathrm{cm}^{3}$.

${ }^{\mathrm{b}} \mathrm{Read}$ as $8.37420 \times 10^{-3}$. The atom density includes that for ${ }^{204} \mathrm{~Pb}$ for which no cross sections are available. 
Table 5. $\mathbf{k}_{\text {eff }}$ Values for Plutonium Spheres

\begin{tabular}{||l|c|c|c||}
\hline Sphere & Radius $(\mathrm{cm})$ & $\mathrm{k}_{\text {eff }}$ & Standard Deviation \\
\hline Bare & 6.32 & 0.98902 & 0.00057 \\
\hline Bare & 6.35 & 0.99319 & 0.00059 \\
\hline Bare & $6.3849^{\mathrm{a}}$ & 0.99699 & 0.00058 \\
\hline Tungsten Reflected & $5.0419^{\mathrm{a}}$ & 1.01053 & 0.00065 \\
\hline Water Reflected & $4.1217^{\mathrm{a}}$ & 0.99830 & 0.00075 \\
\hline
\end{tabular}

${ }^{\mathrm{a}}$ Specified critical radii.

Table 6. Radii Used for Target Spheres. .

\begin{tabular}{|l|c|c|c|c||}
\hline Sphere & $\begin{array}{c}\text { Critical Radius } \\
(\mathrm{cm})\end{array}$ & $\begin{array}{c}\text { Reflector Radius } \\
(\mathrm{cm})\end{array}$ & Radius Used (cm) & $\begin{array}{c}\text { Reflector Radius } \\
\text { Used (cm) }\end{array}$ \\
\hline Bare & 6.3849 & 6.3849 & 6.32 & 6.32 \\
\hline Tungsten-Reflected & 5.0419 & 9.7409 & 4.9910 & 9.69 \\
\hline Water-Reflected & 4.1217 & 29.5217 & 4.0801 & 29.4801 \\
\hline
\end{tabular}


Table 7. Neutron Dose Factors ${ }^{\mathrm{a}}$ from the VELM61 Cross-Section Library.

\begin{tabular}{|c|c|c|c|c|c|}
\hline Group & Avg. Energy ${ }^{b}$ & Dose Factor & Group & Avg. Energy & Dose Factor \\
\hline 1 & $2.07002-7^{\mathrm{c}}$ & $3.70370-3$ & 32 & $1.36365-1$ & $2.86610-2$ \\
\hline 2 & $7.69672-7$ & $4.37060-3$ & 33 & $1.66556-1$ & $3.43150-2$ \\
\hline 3 & $1.75386-6$ & $4.52500-3$ & 34 & $2.03432-1$ & $4.10860-2$ \\
\hline 4 & $3.71293-6$ & $4.57410-3$ & 35 & $2.48472-1$ & $4.92110-2$ \\
\hline 5 & $7.86024-6$ & $4.55830-3$ & 36 & $2.83877-1$ & $5.56070-2$ \\
\hline 6 & $1.66401-5$ & $4.48970-3$ & 37 & 2.95864-1 & $5.77300-2$ \\
\hline 7 & $3.52273-5$ & $4.38170-3$ & 38 & $2.97851-1$ & $5.80800-2$ \\
\hline 8 & $7.45761-5$ & $4.24920-3$ & 39 & $3.00232-1$ & $5.84980-2$ \\
\hline 9 & $1.34159-4$ & $4.12890-3$ & 40 & $3.44858-1$ & $6.59980-2$ \\
\hline 10 & $2.21191-4$ & $4.03110-3$ & 41 & $4.42806-1$ & $8.26560-2$ \\
\hline 11 & $3.64682-4$ & $3.93550-3$ & 42 & $5.10634-1$ & $9.04890-2$ \\
\hline 12 & $6.01258-4$ & $3.84530-3$ & 43 & $5.65749-1$ & $9.54810-2$ \\
\hline 13 & $9.91309-4$ & $3.76290-3$ & 44 & $6.75418-1$ & $1.05360-1$ \\
\hline 14 & $1.63439-3$ & $3.69080-3$ & 45 & $8.24957-1$ & $1.17860-1$ \\
\hline 15 & $2.14167-3$ & $3.65280-3$ & 46 & $1.00760+0$ & $1.29670-1$ \\
\hline 16 & $2.43063-3$ & $3.63870-3$ & 47 & $1.23069+0$ & $1.30100-1$ \\
\hline 17 & $2.82399-3$ & $3.62290-3$ & 48 & $1.50317+0$ & $1.28650-1$ \\
\hline 18 & $3.37141-3$ & $3.60620-3$ & 49 & $1.83598+0$ & $1.27220-1$ \\
\hline 19 & 4.61914-3 & $3.58250-3$ & 50 & $2.12513+0$ & $1.26160-1$ \\
\hline 20 & $7.32483-3$ & $3.55850-3$ & 51 & $2.28850+0$ & $1.25620-1$ \\
\hline 21 & $1.20766-2$ & $4.08750-3$ & 52 & $2.40583+0$ & $1.25270-1$ \\
\hline 22 & $1.93065-2$ & $5.89750-3$ & 53 & $2.73896+0$ & $1.28500-1$ \\
\hline 23 & $2.41830-2$ & $7.13870-3$ & 54 & $3.34537+0$ & $1.36960-1$ \\
\hline 24 & $2.54229-2$ & $7.42410-3$ & 55 & $4.08604+0$ & $1.45980-1$ \\
\hline 25 & 2.65293-2 & $7.67700-3$ & 56 & $4.99070+0$ & $1.54120-1$ \\
\hline 26 & $2.77506-2$ & $7.95180-3$ & 57 & $6.09565+0$ & $1.51160-1$ \\
\hline 27 & $3.14039-2$ & $8.74210-3$ & 58 & $7.44525+0$ & $1.47220-1$ \\
\hline 28 & $4.33909-2$ & $1.11490-2$ & 59 & $9.09365+0$ & $1.47060-1$ \\
\hline 29 & $5.45187-2$ & $1.35030-2$ & 60 & $1.11070+1$ & $1.63200-1$ \\
\hline 30 & $7.15396-2$ & $1.65080-2$ & 61 & $1.35661+1$ & $2.00340-1$ \\
\hline 31 & $1.04645-1$ & $2.25270-2$ & & & \\
\hline
\end{tabular}


Table 8. Photon Dose Factors ${ }^{\mathrm{a}}$ from the VELM61 Cross-Section Library.

\begin{tabular}{|c|c|c|c|c|c|}
\hline Group & Avg. Energy & Dose Factor & Group & Avg. Energy $^{\mathrm{a}}$ & Dose Factor $^{\mathrm{b}}$ \\
\hline 1 & $1.50000-2^{c}$ & $2.14390-3$ & 13 & $1.75000+0$ & $2.92700-3$ \\
\hline 2 & $3.25000-2$ & $5.77600-4$ & 14 & $2.25000+0$ & $3.46860-3$ \\
\hline 3 & $5.75000-2$ & $2.71850-4$ & 15 & $2.75000+0$ & $3.95960-3$ \\
\hline 4 & $8.50000-2$ & $2.68170-4$ & 16 & $3.50000+0$ & $4.62210-3$ \\
\hline 5 & $1.25000-1$ & $3.27670-4$ & 17 & $4.50000+0$ & $5.41370-3$ \\
\hline 6 & $2.25000-1$ & $5.66760-4$ & 18 & $5.50000+0$ & $6.19090-3$ \\
\hline 7 & $3.50000-1$ & $8.75940-4$ & 19 & $6.50000+0$ & $6.92650-3$ \\
\hline 8 & $4.55000-1$ & $1.08450-3$ & 20 & $7.25000+0$ & $7.47830-3$ \\
\hline 9 & $5.55000-1$ & $1.27970-3$ & 21 & $7.75000+0$ & $7.84680-3$ \\
\hline 10 & $6.50000-1$ & $1.44170-3$ & 22 & $9.00000+0$ & $8.77160-3$ \\
\hline 11 & $8.50000-1$ & 1.756303 & 23 & $1.20000+1$ & $1.10200-2$ \\
\hline 12 & $1.25000+0$ & $2.31560-3$ & & & \\
\hline
\end{tabular}

${ }^{2} \mathrm{mrem} / \mathrm{h}$ per unit flux.

${ }^{b} \mathrm{MeV}$.

${ }^{c}$ Read as $1.50000 \times 10^{-2}$. 
Table 9. Comparison of Target Dose Rates with Background Dose Rates for a 6.32-cm-Radius Bare Pu Sphere.

\begin{tabular}{|c|c|c|c|c|c|c|}
\hline \multirow[b]{2}{*}{ Detector $^{\mathrm{a}}$} & \multicolumn{3}{|c|}{ Neutron Dose Rate $(\mathrm{mrem} / \mathrm{h})$} & \multicolumn{3}{|c|}{ Photon Dose Rate (mrem/h) } \\
\hline & Background & With Target & Ratio $^{b}$ & Background & With Target & Ratio $^{b}$ \\
\hline 1 & $\begin{array}{c}8.5821+4 \\
(.0177)^{\mathrm{c}} \\
\end{array}$ & $\begin{array}{l}6.1555+5 \\
(.261) \\
\end{array}$ & 7.17 & $\begin{array}{l}1.0877+1 \\
(.208)\end{array}$ & $\begin{array}{l}3.2786+3 \\
(.276) \\
\end{array}$ & 301. \\
\hline 2 & $\begin{array}{l}7.5584+4 \\
(.0189) \\
\end{array}$ & $\begin{array}{l}5.4393+5 \\
(.293) \\
\end{array}$ & 7.20 & $\begin{array}{l}8.3045+0 \\
(.210) \\
\end{array}$ & $\begin{array}{l}3.3571+3 \\
(.306)\end{array}$ & 404. \\
\hline 3 & $\begin{array}{l}5.6137+4 \\
(.0981) \\
\end{array}$ & $\begin{array}{l}5.7529+5 \\
(.267) \\
\end{array}$ & 10.2 & $\begin{array}{l}2.7385+0 \\
(.708) \\
\end{array}$ & $\begin{array}{l}3.1099+3 \\
(.267) \\
\end{array}$ & 1136. \\
\hline 4 & $\begin{array}{l}5.9108+4 \\
(.0954)\end{array}$ & $\begin{array}{l}5.7128+5 \\
(.269)\end{array}$ & 9.67 & $\begin{array}{l}2.6507+0 \\
(.499)\end{array}$ & $\begin{array}{l}3.5566+3 \\
(.291)\end{array}$ & 1342. \\
\hline
\end{tabular}

${ }^{\mathrm{a}}$ These are $10-\mathrm{cm} \times 10-\mathrm{cm}$ surface detectors located $10 \mathrm{~cm}$ in front of (detector 1), behind (detector 2), to the side of (detector 3) and above (detector 4) the target.

${ }^{b}$ Dose rate with target to dose rate without target (background).

${ }^{\mathrm{c}}$ Read as $8.5821 \times 10^{4}$ with a fractional standard deviation of 0.0177 . 
Table 10. Comparison of Target Dose Rates with Background Dose Rates for a Bare Pu Sphere $(r=6.35 \mathrm{~cm})$.

\begin{tabular}{|c|c|c|c|c|c|c|}
\hline \multirow[b]{2}{*}{ Detector $^{\mathrm{a}}$} & \multicolumn{3}{|c|}{ Neutron Dose Rate $(\mathrm{mrem} / \mathrm{h})$} & \multicolumn{3}{|c|}{ Photon Dose Rate $(\mathrm{mrem} / \mathrm{h})$} \\
\hline & Background & With Target & Ratio $^{b}$ & Background & With Target & Ratio $^{\mathrm{b}}$ \\
\hline 1 & $\begin{array}{l}8.5821+4 \\
(.0177)^{\mathrm{c}} \\
\end{array}$ & $\begin{array}{l}1.0163+6 \\
(.0517) \\
\end{array}$ & 11.8 & $\begin{array}{l}1.0877+1 \\
(.208) \\
\end{array}$ & $\begin{array}{c}5.8412+3 \\
(.0560) \\
\end{array}$ & 537.0 \\
\hline 2 & $\begin{array}{l}7.5584+4 \\
(.0189) \\
\end{array}$ & $\begin{array}{l}9.2495+5 \\
(.0572) \\
\end{array}$ & 12.2 & $\begin{array}{l}8.3045+0 \\
(.210) \\
\end{array}$ & $\begin{array}{l}5.7662+3 \\
(.0646) \\
\end{array}$ & 694.3 \\
\hline 3 & $\begin{array}{l}5.6137+4 \\
(.0981) \\
\end{array}$ & $\begin{array}{l}1.0037+6 \\
(.0526)\end{array}$ & 17.9 & $\begin{array}{l}2.7385+0 \\
(.708)\end{array}$ & $\begin{array}{l}5.7923+3 \\
(.0597) \\
\end{array}$ & 2115. \\
\hline
\end{tabular}

${ }^{a}$ For background, these are $10-\mathrm{cm} \times 10-\mathrm{cm}$ surface detectors located $10 \mathrm{~cm}$ in front of (detector 1), behind (detector 2), and to the side of (detector 3). Dose rates with the target present were obtained using point detectors at the center of the $10-\mathrm{cm} \times 10$-cm surface detectors.

${ }^{\mathrm{b}}$ Dose rate with target to dose rate without target (background).

${ }^{c}$ Read as $8.5821 \times 10^{4}$ with a fractional standard deviation of 0.0177 . 
Table 11. Comparison of Target Dose Rates with Background Dose Rates for a Bare Pu Sphere $(\mathrm{r}=6.38 \mathrm{~cm})$.

\begin{tabular}{|c|c|c|c|c|c|c|}
\hline \multirow[b]{2}{*}{ Detector $^{a}$} & \multicolumn{3}{|c|}{ Neutron Dose Rate $(\mathrm{mrem} / \mathrm{h})$} & \multicolumn{3}{|c|}{ Photon Dose Rate $(\mathrm{mrem} / \mathrm{h})$} \\
\hline & Background & With Target & Ratio $^{\mathrm{b}}$ & Background & With Target & Ratio $^{b}$ \\
\hline 1 & $\begin{array}{c}8.5821+4 \\
(.0177)^{\mathrm{c}} \\
\end{array}$ & $\begin{array}{l}2.2441+6 \\
(.405) \\
\end{array}$ & 26.1 & $\begin{array}{l}1.0877+1 \\
(.208)\end{array}$ & $\begin{array}{l}1.3301+4 \\
(.419) \\
\end{array}$ & 1223. \\
\hline 2 & $\begin{array}{l}7.5584+4 \\
(.0189) \\
\end{array}$ & $\begin{array}{l}2.1332+6 \\
(.415) \\
\end{array}$ & 28.2 & $\begin{array}{l}8.3045+0 \\
(.210) \\
\end{array}$ & $\begin{array}{l}1.3372+4 \\
(.419) \\
\end{array}$ & 1610. \\
\hline 3 & $\begin{array}{l}5.6137+4 \\
(.0981) \\
\end{array}$ & $\begin{array}{l}2.1870+6 \\
(.404) \\
\end{array}$ & 39.0 & $\begin{array}{l}2.7385+0 \\
(.708) \\
\end{array}$ & $\begin{array}{l}1.3023+4 \\
(.413) \\
\end{array}$ & 4756. \\
\hline 4 & $\begin{array}{l}5.9108+4 \\
(.0954) \\
\end{array}$ & $\begin{array}{l}2.2000+6 \\
(.412) \\
\end{array}$ & 37.2 & $\begin{array}{l}2.6507+0 \\
(.499) \\
\end{array}$ & $\begin{array}{l}1.3426+4 \\
(.420) \\
\end{array}$ & 5065 \\
\hline
\end{tabular}

${ }^{\mathrm{a}}$ These are $10-\mathrm{cm} \times 10-\mathrm{cm}$ surface detectors located $10 \mathrm{~cm}$ in front of (detector 1), behind (detector 2), to the side of (detector 3 ) and above (detector 4) the target.

${ }^{b}$ Dose rate with target to dose rate without target (background).

${ }^{c}$ Read as $8.5821 \times 10^{4}$ with a fractional standard deviation of 0.0177 . 
Table 12. Comparison of Target Dose Rates with Background Dose Rates for a Tungsten-Reflected Pu Sphere.

\begin{tabular}{|c|c|c|c|c|c|c|}
\hline \multirow[b]{2}{*}{ Detcctor $^{\mathbf{a}}$} & \multicolumn{3}{|c|}{ Neutron Dose Rate $(\mathrm{mrem} / \mathrm{h})$} & \multicolumn{3}{|c|}{ Photon Dose Rate $(\mathrm{mrem} / \mathrm{h})$} \\
\hline & Background & With Target & Ratio $^{b}$ & Background & With Target & Ratio $^{\text {b }}$ \\
\hline 1 & $\begin{array}{l}8.9557+4 \\
(.0173)^{c} \\
\end{array}$ & $\begin{array}{l}5.3636+5 \\
(.414) \\
\end{array}$ & 5.99 & $\begin{array}{l}1.0864+1 \\
(.207) \\
\end{array}$ & $\begin{array}{l}1.0560+3 \\
(.464) \\
\end{array}$ & 97.2 \\
\hline 2 & $\begin{array}{l}7.2801+4 \\
(.0192) \\
\end{array}$ & $\begin{array}{l}4.4615+5 \\
(.497) \\
\end{array}$ & 6.13 & $\begin{array}{l}7.8226+0 \\
(.209) \\
\end{array}$ & $\begin{array}{l}9.2852+2 \\
(.500) \\
\end{array}$ & 118.7 \\
\hline 3 & $\begin{array}{l}7.9935+4 \\
(.0822) \\
\end{array}$ & $\begin{array}{l}5.1176+5 \\
(.436) \\
\end{array}$ & 6.40 & 0.0 & $\begin{array}{l}9.8202+2 \\
(.445) \\
\end{array}$ & - \\
\hline 4 & $\begin{array}{l}8.0522+4 \\
(.0817)\end{array}$ & $\begin{array}{l}5.2799+5 \\
(.418)\end{array}$ & 6.56 & $\begin{array}{l}3.3315+0 \\
(.648)\end{array}$ & $\begin{array}{l}9.3721+2 \\
(.480)\end{array}$ & 281.3 \\
\hline
\end{tabular}

${ }^{a}$ These are $10-\mathrm{cm} \times 10-\mathrm{cm}$ surface detectors located $15 \mathrm{~cm}$ in front of (detector 1), behind (detector 2), to the side of (detector 3 ) and above (detector 4) the target.

${ }^{\mathrm{b}}$ Dose rate with target to dose rate without target (background).

${ }^{c}$ Read as $8.9557 \times 10^{4}$ with a fractional standard deviation of 0.0173 . 
Table 13. Comparison of Target Dose Rates with Background Dose Rates for a Water-Reflected Pu Sphere.

\begin{tabular}{|c|c|c|c|c|c|c|}
\hline \multirow[b]{2}{*}{ Detector $^{\mathrm{a}}$} & \multicolumn{3}{|c|}{ Neutron Dose Rate $(\mathrm{mrem} / \mathrm{h})$} & \multicolumn{3}{|c|}{ Photon Dose Rate $(\mathrm{mrem} / \mathrm{h})$} \\
\hline & Background & With Target & Ratio $^{b}$ & Background & With Target & Ratio $^{b}$ \\
\hline 1 & $\begin{array}{l}1.0371+5 \\
(.0081)^{c}\end{array}$ & $\begin{array}{l}1.0962+5 \\
(.0178) \\
\end{array}$ & 1.057 & $\begin{array}{l}1.3880+1 \\
(.102)\end{array}$ & $\begin{array}{l}5.0402+2 \\
(.0898) \\
\end{array}$ & 36.3 \\
\hline 2 & $\begin{array}{l}6.3409+4 \\
(.0103) \\
\end{array}$ & $\begin{array}{l}8.3543+2 \\
(.137) \\
\end{array}$ & 0.0132 & $\begin{array}{l}8.6057+0 \\
(.134) \\
\end{array}$ & $\begin{array}{l}2.2645+2 \\
(.178) \\
\end{array}$ & 26.3 \\
\hline 3 & $\begin{array}{l}1.1727+3 \\
(.185) \\
\end{array}$ & $\begin{array}{l}2.8291+3 \\
(.172) \\
\end{array}$ & 2.412 & $\begin{array}{l}1.3205+1 \\
(.309)\end{array}$ & $\begin{array}{l}3.0421+2 \\
(.135)\end{array}$ & 23.0 \\
\hline 4 & $\begin{array}{c}3.1183+4 \\
(.0428)\end{array}$ & $\begin{array}{l}4.3438+4 \\
(.0790)\end{array}$ & 1.393 & $\begin{array}{l}1.1609+1 \\
(.354)\end{array}$ & $\begin{array}{l}2.9943+2 \\
(.137)\end{array}$ & 25.8 \\
\hline
\end{tabular}

${ }^{\mathrm{a}}$ These are $20-\mathrm{cm} \times 20-\mathrm{cm}$ surface detectors located $35 \mathrm{~cm}$ in front of (detector 1), behind (detector 2), to the side of (detector 3 ) and above (detector 4) the target.

${ }^{b}$ Dose rate with target to dose rate without target (background).

${ }^{\mathrm{c}}$ Read as $1.0371 \times 10^{5}$ with a fractional standard deviation of 0.0081 . 
Table 14. Changes in the Bare Pu Sphere $(r=6.38 \mathrm{~cm})$ Detector Dose Rates with Changes in the Number of Histories.

\begin{tabular}{|c|c|c|c|c|c|}
\hline \multirow[b]{2}{*}{ Detector } & \multicolumn{2}{|c|}{ Surface Detectors } & \multicolumn{3}{|c|}{ Point Detectors } \\
\hline & 400,000 histories & $16,000,000$ histories & 400,000 histories & $2,000,000$ histories & $16,000,000$ histories \\
\hline \multicolumn{6}{|c|}{ Neutron Dose Rates $(\mathrm{mrem} / \mathrm{h})$} \\
\hline 1 & $\begin{array}{l}2.2441+6 \\
(.405)^{\mathrm{a}} \\
\end{array}$ & $\begin{array}{l}1.5660+6 \\
(.0615) \\
\end{array}$ & $\begin{array}{ll} & 1.1536+6 \\
\cdot(.313) \\
\end{array}$ & $\begin{array}{l}1.4986+6 \\
(.159) \\
\end{array}$ & $\begin{array}{l}1.6645+6 \\
(.0639) \\
\end{array}$ \\
\hline 2 & $\begin{array}{l}2.1332+6 \\
(.415) \\
\end{array}$ & $\begin{array}{c}1.4798+6 \\
(.0651) \\
\end{array}$ & $\begin{array}{l}1.0834+6 \\
(.333) \\
\end{array}$ & $\begin{array}{l}1.4133+6 \\
(.169) \\
\end{array}$ & $\begin{array}{l}1.5770+6 \\
(.0676)\end{array}$ \\
\hline 3 & $\begin{array}{l}2.1870+6 \\
(.404) \\
\end{array}$ & $\begin{array}{l}1.5308+6 \\
(.0629) \\
\end{array}$ & $\begin{array}{l}1.1592+6 \\
(.316) \\
\end{array}$ & $\begin{array}{l}1.4993+6 \\
(.160) \\
\end{array}$ & $\begin{array}{l}1.6509+6 \\
(.0641) \\
\end{array}$ \\
\hline \multicolumn{6}{|c|}{ Photon Dose Rates (mrem/h) } \\
\hline 1 & $\begin{array}{l}1.3301+4 \\
(.419) \\
\end{array}$ & $\begin{array}{c}9.2353+3 \\
(.0648) \\
\end{array}$ & $\begin{array}{l}6.8100+3 \\
(.326) \\
\end{array}$ & $\begin{array}{l}8.7138+3 \\
(.166) \\
\end{array}$ & $\begin{array}{l}9.6998+3 \\
(.0662) \\
\end{array}$ \\
\hline 2 & $\begin{array}{l}1.3372+4 \\
(.419) \\
\end{array}$ & $\begin{array}{l}8.9836+3 \\
(.0661) \\
\end{array}$ & $\begin{array}{l}6.6875+3 \\
(.333) \\
\end{array}$ & $\begin{array}{l}8.5810+3 \\
(.171) \\
\end{array}$ & $\begin{array}{l}9.5791+3 \\
(.0685) \\
\end{array}$ \\
\hline 3 & $\begin{array}{l}1.3023+4 \\
(.413) \\
\end{array}$ & $\begin{array}{l}9.1137+3 \\
(.0654) \\
\end{array}$ & $\begin{array}{l}6.6108+3 \\
(.344) \\
\end{array}$ & $\begin{array}{l}8.7588+3 \\
(.171) \\
\end{array}$ & $\begin{array}{l}9.6407+3 \\
(.0669)\end{array}$ \\
\hline
\end{tabular}

${ }^{a}$ Read as $2.2441 \times 10^{6}$ with a fractional standard deviation of 0.405 . 
Table 15. Dose Rates Within and Around the PFNA Facility.

\begin{tabular}{|c|c|c|c|c|}
\hline \multirow{2}{*}{ Detector } & \multicolumn{4}{|c|}{ Dose Rates $(\mathrm{mrem} / \mathrm{h})$} \\
\hline & Max. Down & Horizontal & Max. Up & Average $^{a}$ \\
\hline \multicolumn{5}{|c|}{ Neutron } \\
\hline 1 & $5.58024-1^{\mathrm{b}}$ & $4.70220+0$ & $2.39937-1$ & $1.58782+0$ \\
\hline 2 & $6.10697-1$ & $5.47704-1$ & $1.25419-1$ & $3.44367-1$ \\
\hline 3 & $5.87105+2$ & $1.49350+4$ & $7.00707+1$ & $3.10147+3$ \\
\hline 4 & $1.81381+0$ & $3.90125-1$ & $4.05931-1$ & $5.02696-1$ \\
\hline 5 & $2.28500+0$ & $8.29166-1$ & $9.49863+0$ & $3.13535+0$ \\
\hline 6 & $1.16587+3$ & $7.67914+4$ & $1.63541+2$ & $1.35665+4$ \\
\hline 7 & $6.21285+0$ & $4.34500+0$ & $7.28572+0$ & $5.59694+0$ \\
\hline 8 & $2.26431-5$ & $4.08722-3$ & $2.34775-4$ & $1.23624-3$ \\
\hline 9 & $1.46130-1$ & $5.60654-1$ & $8.76999-2$ & $2.65508-1$ \\
\hline 10 & $1.99241-1$ & $2.23255-1$ & $4.66521-1 \cdot$ & $3.06488-1$ \\
\hline \multicolumn{5}{|c|}{ Photons } \\
\hline 1 & $5.56058-2$ & $1.30955+0$ & $9.76129-3$ & $2.91395-1$ \\
\hline 2 & $1.62714-1$ & $1.45858-1$ & $2.94213-2$ & $8.88615-2$ \\
\hline 3 & $1.25930+1$ & $8.43161+1$ & $2.27785+0$ & $2.56899+1$ \\
\hline 4 & $3.56180-2$ & $1.43302-2$ & $2.55025-2$ & $201749-2$ \\
\hline 5 & $9.11276-2$ & $3.90251-2$ & $5.37723-2$ & $4.90634-2$ \\
\hline 6 & $1.70391+1$ & $1.39578+1$ & $5.39429+0$ & $1.02837+1$ \\
\hline 7 & $1.83418+0$ & $1.67950+0$ & $2.31934+0$ & $1.93735+0$ \\
\hline 8 & $1.14903-2$ & $4.35777-2$ & $1.36479-2$ & $2.54415-2$ \\
\hline 9 & $5.30021-2$ & $1.11127-1$ & $7.83837-3$ & 4.67914-2 \\
\hline 10 & $6.01096+0$ & $5.54711+0$ & $6.62591+0$ & $6.01216+0$ \\
\hline \multicolumn{5}{|c|}{ Total } \\
\hline 1 & $6.13630-1$ & $6.01175+0$ & $2.49698-1$ & $1.92108+0$ \\
\hline 2 & $7.73411-1$ & $6.93562-1$ & $1.54840-1$ & $4.33288-1$ \\
\hline 3 & $5.99698+2$ & $1.50193+4$ & $7.23485+1$ & $3.13345+3$ \\
\hline 4 & $1.84943+0$ & $4.04455-1$ & $4.31433-1$ & $5.23374-1$ \\
\hline 5 & $2.37613+0$ & $8.68191-1$ & $9.55240+0$ & $3.20045+0$ \\
\hline 6 & $1.18291+3$ & $7.68054+4$ & $1.68935+2$ & $1.36324+4$ \\
\hline 7 & $8.04703+0$ & $6.02450+0$ & $9.60506+0$ & $7.53956+0$ \\
\hline 8 & $1.15129-2$ & $4.76649-2$ & $1.38827-2$ & $2.70017-2$ \\
\hline 9 & $1.99132-1$ & $6.71781-1$ & $9.55383-2$ & $3.13927-1$ \\
\hline 10 & $6.21020+0$ & $5.77037+0$ & $7.09243+0$ & $6.32524+0$ \\
\hline
\end{tabular}

${ }^{2}$ Averaged by assuming exponential variation of the dose rate with the collimator angle.

${ }^{\mathrm{b}}$ Read as $5.58024 \times 10^{-1}$. 
Table 16. Neutron Dose Rates Along the Centerline of the Vehicle Lane of the PFNA Facility.

\begin{tabular}{|c|c|c|c|c|c|}
\hline \multirow[b]{2}{*}{ Detector } & \multirow{2}{*}{$\begin{array}{l}\text { Location } \\
\qquad(\mathrm{cm})\end{array}$} & \multicolumn{4}{|c|}{ Dose Rates (mrem/h) } \\
\hline & & $\begin{array}{l}\text { Maximum Down } \\
\text { Source Position }\end{array}$ & $\begin{array}{c}\text { Horizontal Source } \\
\text { Position } \\
\end{array}$ & $\begin{array}{l}\text { Maximum Up Source } \\
\text { Position } \\
\end{array}$ & Average $^{\mathrm{a}}$ \\
\hline 1 & -1710.0 & $1.81381+0^{\mathrm{b}}$ & $3.90125-1$ & $4.05931-1$ & $5.02696-1$ \\
\hline 2 & -1524.0 & $1.45005+0$ & $5.09248-1$ & $6.68034-1$ & $6.47278-1$ \\
\hline 3 & -1371.6 & $1.84981+0$ & $6.24773-1$ & $7.66245-1$ & $7.79400-1$ \\
\hline 4 & -1219.2 & $2.51946+0$ & $8.40224-1$ & $1.08589+0$ & $1.07103+0$ \\
\hline 5 & -1066.8 & $3.58813+0$ & $1.22390+0$ & $1.50473+0$ & $1.52566+0$ \\
\hline 6 & -914.4 & $5.30024+0$ & $1.83703+0$ & $2.28986+0$ & $2.29555+0$ \\
\hline 7 & -762.0 & $8.48255+0$ & $3.05426+0$ & $3.77451+0$ & $3.78066+0$ \\
\hline 8 & -609.6 & $1.48590+1$ & $5.49383+0$ & $6.45836+0$ & $6.64661+0$ \\
\hline 9 & -457.2 & $2.91353+1$ & $1.06697+1$ & $1.76760+1$ & $1.47716+1$ \\
\hline 10 & -304.8 & $7.20955+1$ & $3.57631+1$ & $2.99380+1$ & $3.65411+1$ \\
\hline 11 & -152.4 & $1.77789+2$ & $9.28149+1$ & $5.16757+1$ & $8.22340+1$ \\
\hline 12 & -76.2 & $3.20803+2$ & $2.13007+2$ & $7.27455+1$ & $1.56846+2$ \\
\hline 13 & -30.5 & $8.00332+2$ & $1.15811+3$ & $1.36333+2$ & $5.74813+2$ \\
\hline 14 & 0.0 & $1.16687+3$ & $7.67914+4$ & $1.63541+2$ & $1.35672+4$ \\
\hline 15 & 30.5 & $8.00332+2$ & $1.15811+3$ & $1.36333+2$ & $5.74813+2$ \\
\hline 16 & 76.2 & $3.20803+2$ & $2.13007+2$ & $7.27455+1$ & $1.56846+2$ \\
\hline 17 & 152.4 & $1.81242+2$ & $9.43788+1$ & $5.21843+1$ & $8.34781+1$ \\
\hline 18 & 304.8 & $7.28867+1$ & $3.59743+1$ & $3.02640+1$ & $3.68494+1$ \\
\hline 19 & 457.2 & $2.95375+1$ & $1.08823+1$ & $1.77131+1$ & $1.49452+1$ \\
\hline 20 & 609.6 & $1.53587+1$ & $5.56264+0$ & $6.13926+0$ & $6.59906+0$ \\
\hline 21 & 762.0 & $8.95194+0$ & $3.29045+0$ & $3.34407+0$ & $3.78079+0$ \\
\hline 22 & 914.4 & $5.68971+0$ & $2.08403+0$ & $2.12947+0$ & $2.40061+0$ \\
\hline 23 & 1066.8 & $3.93217+0$ & $1.44988+0$ & $1.48254+0$ & $1.66863+0$ \\
\hline 24 & 1296.0 & $2.28500+0$ & $8.29166-1$ & $9.49863+0^{\circ}$ & $3.13535+0$ \\
\hline
\end{tabular}

${ }^{a}$ Averaged by assuming exponential variation of the dose rate with the collimator angle.

${ }^{\mathrm{b}}$ Read as $1.81381 \times 10^{0}$. 
Table 17. Photon Dose Rates Along the Centerline of the Vehicle Lane of the PFNA Facility.

\begin{tabular}{|c|c|c|c|c|c|}
\hline \multirow[b]{2}{*}{ Detector } & \multirow{2}{*}{$\begin{array}{l}\text { Location } \\
(\mathrm{cm})\end{array}$} & \multicolumn{4}{|c|}{ Dose Rates (mrem/h) } \\
\hline & & $\begin{array}{c}\text { Maximum Down } \\
\text { Source Position }\end{array}$ & $\begin{array}{c}\text { Horizontal Source } \\
\text { Position } \\
\end{array}$ & $\begin{array}{l}\text { Maximum Up } \\
\text { Source Position }\end{array}$ & Average $^{a}$ \\
\hline 1 & -1710.0 & $3.56180-2^{b}$ & $1.43302-2$ & $2.55025-2$ & $2.01749-2$ \\
\hline 2 & -1524.0 & $5.39488-2$ & $2.43626-2$ & $3.71394-2$ & $3.16732-2$ \\
\hline 3 & -1371.6 & $6.46439-2$ & $2.74815-2$ & $4.31652-2$ & $3.64612-2$ \\
\hline 4 & -1219.2 & $8.69921-2$ & $3.69437-2$ & $5.14859-2$ & $4.67115-2$ \\
\hline 5 & -1066.8 & $1.25492-1$ & $4.82811-2$ & $7.10139-2$ & $6.32607-2$ \\
\hline 6 & -914.4 & $1.84925-1$ & $6.76465-2$ & $1.01122-1$ & $8.98749-2$ \\
\hline 7 & -762.0 & 2.88393-1 & $1.07229-1$ & $1.61069-1$ & $1.42392-1$ \\
\hline 8 & -609.6 & $4.83245-1$ & $1.73985-1$ & $2.32722-1$ & $2.21906-1$ \\
\hline 9 & -457.2 & $9.01676-1$ & $3.19502-1$ & $4.74318-1$ & $4.25376-1$ \\
\hline 10 & -304.8 & $2.17521+0$ & $1.12029+0$ & $7.88501-1$ & $1.07254+0$ \\
\hline 11 & -152.4 & $4.72385+0$ & $2.50499+0$ & $1.38074+0$ & $2.20652+0$ \\
\hline 12 & -76.2 & $7.33929+0$ & $4.02129+0$ & $2.08345+0$ & $3.45580+0$ \\
\hline 13 & -30.5 & $1.37813+1$ & $1.08577+1$ & $3.52223+0$ & $7.65442+0$ \\
\hline 14 & 0.0 & $1.70391+1$ & $1.39578+1$ & $5.39429+0$ & $1.02837+1$ \\
\hline 15 & 30.5 & $1.37813+1$ & $1.08577+1$ & $3.52223+0$ & $7.65442+0$ \\
\hline 16 & 76.2 & $7.33929+0$ & $4.02129+0$ & $2.08345+0$ & $3.45580+0$ \\
\hline 17 & 152.4 & $4.76635+0$ & $2.39828+0$ & $1.46280+0$ & $2,20042+0$ \\
\hline 18 & 304.8 & $2.25568+0$ & $1.19457+0$ & $9.42471-1$ & $1.18358+0$ \\
\hline 19 & 457.2 & $9.48638-1$ & $3.53345-1$ & $5.41115-1$ & $4.72722-1$ \\
\hline 20 & 609.6 & $4.94655-1$ & $1.85464-1$ & $2.59925-1$ & $2.39345-1$ \\
\hline 21 & 762.0 & $3.00849-1$ & $1.22278-1$ & $1.61437-1$ & $1.52325-1$ \\
\hline 22 & 914.4 & 2.01295-1 & $8.19611-2$ & $1.15803-1$ & $1.04825-1$ \\
\hline 23 & 1066.8 & $1.52244-1$ & $5.45651-2$ & 8.09399-2 & 7.24973-2 \\
\hline 24 & 1296.0 & $9.11276-2$ & $3.90251-2$ & $5.37723-2$ & $4.90634-2$ \\
\hline
\end{tabular}

${ }^{a}$ Averaged by assuming exponential variation of the dose rate with the collimator angle.

${ }^{\mathrm{b}}$ Read as $3.56180 \times 10^{-2}$. 
Table 18. Total Dose Rates Along the Centerline of the Vehicle Lane of the PFNA Facility.

\begin{tabular}{|c|c|c|c|c|c|}
\hline \multirow[b]{2}{*}{ Detector } & \multirow{2}{*}{$\begin{array}{l}\text { Location } \\
\quad(\mathrm{cm})\end{array}$} & \multicolumn{4}{|c|}{ Dose Rates $(\mathrm{mrem} / \mathrm{h})$} \\
\hline & & $\begin{array}{c}\text { Maximum Down } \\
\text { Source Position }\end{array}$ & $\begin{array}{c}\text { Horizontal Source } \\
\text { Position }\end{array}$ & $\begin{array}{c}\text { Maximum Up } \\
\text { Source Position }\end{array}$ & Average $^{a}$ \\
\hline 1 & -1710.0 & $1.84943+0^{\mathrm{b}}$ & $4.04455-1$ & $4.31433-1$ & $5.23374-1$ \\
\hline 2 & -1524.0 & $1.50400+0$ & $5.33611-1$ & $7.05173-1$ & $6.79032-1$ \\
\hline 3 & -1371.6 & $1.91445+0$ & $6.52255-1$ & $8.09410-1$ & $8.16033-1$ \\
\hline 4 & -1219.2 & $2.60645+0$ & $8.77168-1$ & $1.13738+0$ & $1.11781+0$ \\
\hline 5 & -1066.8 & $3.71362+0$ & $1.27218+0$ & $1.57574+0$ & $1.58906+0$ \\
\hline 6 & -914.4 & $5.48517+0$ & $1.90468+0$ & $2.39098+0$ & $2.38561+0$ \\
\hline 7 & -762.0 & $8.77094+0$ & $3.16149+0$ & $3.93558+0$ & $3.92338+0$ \\
\hline 8 & -609.6 & $1.53422+1$ & $5.66782+0$ & $6.69108+0$ & $6.86873+0$ \\
\hline 9 & -457.2 & $3.00370+1$ & $1.09892+1$ & $1.81503+1$ & $1.51973+1$ \\
\hline 10 & -304.8 & $7.42707+1$ & $3.68834+1$ & $3.07265+1$ & $3.76155+1$ \\
\hline 11 & -152.4 & $1.82513+2$ & $9.53199+1$ & $5.30564+1$ & $8.44405+1$ \\
\hline 12 & -76.2 & $3.28142+2$ & $2.17028+2$ & $7.48289+1$ & $1.60339+2$ \\
\hline 13 & -30.5 & $8.14113+2$ & $1.16897+3$ & $1.39855+2$ & $5.83005+2$ \\
\hline 14 & 0.0 & $1.18391+3$ & $7.68054+4$ & $1.68935+2$ & $1.36331+4$ \\
\hline 15 & 30.5 & $8.14113+2$ & $1.16897+3$ & $1.39855+2$ & $5.83005+2$ \\
\hline 16 & 76.2 & $3.28142+2$ & $2.17028+2$ & $7.48289+1$ & $1.60339+2$ \\
\hline 17 & 152.4 & $1.86008+2$ & $9.67771+1$ & $5.36471+1$ & $8.56797+1$ \\
\hline 18 & 304.8 & $7.51424+1$ & $3.71689+1$ & $3.12065+1$ & $3.80333+1$ \\
\hline 19 & 457.2 & $3.04861+1$ & $1.12356+1$ & $1.82542+1$ & $1.54180+1$ \\
\hline 20 & 609.6 & $1.58534+1$ & $5.74810+0$ & $6.39918+0$ & $6.83923+0$ \\
\hline 21 & 762.0 & $9.25279+0$ & $3.41273+0$ & $3.50551+0$ & $3.93375+0$ \\
\hline 22 & 914.4 & $5.89101+0$ & $2.16599+0$ & $2.24527+0$ & $2.50611+0$ \\
\hline 23 & 1066.8 & $4.08441+0$ & $1.50445+0$ & $1.56348+0$ & $1.74172+0$ \\
\hline 24 & 1296.0 & $2.37613+0$ & $8.68191-1$ & $9.55240+0$ & $3.20045+0$ \\
\hline
\end{tabular}

${ }^{2}$ Averaged by assuming exponential variation of the dose rate with the collimator angle.

${ }^{\mathrm{b}}$ Read as $1.84943 \times 10^{\circ}$. 
Table 19. Dose Rates in Beam Line at Various Distances from the PFNA Truck Lane Centerline.

\begin{tabular}{|c|c|c|c|c|c|}
\hline \multirow[b]{2}{*}{ Detector } & \multirow{2}{*}{$\begin{array}{c}\text { Distance }{ }^{\mathrm{a}} \text { from } \\
\text { Center of Truck } \\
\text { Lane } \\
(\mathrm{cm})\end{array}$} & \multicolumn{4}{|c|}{ Dose Rates $(\mathrm{mrem} / \mathrm{h})$} \\
\hline & & $\begin{array}{l}\text { Maximum Down } \\
\text { Source Position }\end{array}$ & $\begin{array}{c}\text { Horizontal Source } \\
\text { Position }\end{array}$ & $\begin{array}{l}\text { Maximum Up } \\
\text { Source Position }\end{array}$ & Average $^{b}$ \\
\hline \multicolumn{6}{|c|}{ Neutrons } \\
\hline 1 & -182.88 & $5.27242+3^{c}$ & $5.38063+5$ & $8.05332+2$ & $8.90556+4$ \\
\hline 2 & -121.92 & $2.64372+3$ & $2.21584+5$ & $2.96955+2$ & $3.66200+4$ \\
\hline 3 & -60.96 & $1.57531+3$ & $1.21564+5$ & $2.17530+2$ & $2.08524+4$ \\
\hline 4 & 60.96 & $9.88388+2$ & $5.28997+4$ & $1.31665+2$ & $9.64130+3$ \\
\hline 5 & 121.92 & $9.24120+2$ & $3.86709+4$ & $1.11483+2$ & $7.28932+3$ \\
\hline 6 & 182.88 & $8.85173+2$ & $2.95280+4$ & $9.70457+1$ & $5.74548+3$ \\
\hline \multicolumn{6}{|c|}{ Photons } \\
\hline 1 & -182.88 & $1.61237+2$ & $2.94997+2$ & $2.00615+1$ & $1.25886+2$ \\
\hline 2 & -121.92 & $4.49017+1$ & $5.27021+1$ & $1.03627+1$ & $3.05233+1$ \\
\hline 3 & -60.96 & $2.36999+1$ & $2.33104+1$ & $7.18505+0$ & $1.56441+1$ \\
\hline 4 & 60.96 & $1.48370+1$ & $9.98272+0$ & $4.26452+0$ & $7.81832+0$ \\
\hline 5 & 121.92 & $1.47948+1$ & $8.24923+0$ & $3.49869+0$ & $6.66139+0$ \\
\hline 6 & 182.88 & $1.50075+1$ & $7.79060+0$ & $2.96020+0$ & $6.18388+0$ \\
\hline \multicolumn{5}{|c|}{ Total } & - \\
\hline 1 & -182.88 & $5.43366+3$ & $5.38358+5$ & $8.25393+2$ & $8.94882+4$ \\
\hline 2 & -121.92 & $2.68862+3$ & $2.21637+5$ & $3.07318+2$ & $3.68012+4$ \\
\hline 3 & -60.96 & $1.59901+3$ & $1.21587+5$ & $2.24715+2$ & $2.09520+4$ \\
\hline 4 & 60.96 & $1.00322+3$ & $5.29097+4$ & $1.35930+2$ & $9.68890+3$ \\
\hline 5 & 121.92 & $9.38915+2$ & $3.86791+4$ & $1.14982+2$ & $7.32594+3$ \\
\hline 6 & 182.88 & $9.00180+2$ & $2.95358+4$ & $1.00006+2$ & $5.77503+3$ \\
\hline
\end{tabular}

${ }^{a}$ Negative distances are toward the source.

${ }^{\mathrm{b}}$ Averaged by assuming exponential variation of the dose rate with the collimator angle.

${ }^{c}$ Read as $5.27242 \times 10^{3}$. 
Table 20. Neutron Dose Rates at Locations Below the Source Overhead Shield and Slightly Below the Roof.

\begin{tabular}{||c|c|c|c||}
\hline \multicolumn{2}{|c|}{ Detector Coordinates $(\mathrm{cm})^{\mathrm{a}}$} & \multirow{2}{*}{$\begin{array}{c}\text { Dose Rate } \\
(\mathrm{mrem} / \mathrm{h})\end{array}$} & $\begin{array}{c}\text { Fractional } \\
\text { Standard } \\
\text { Deviation }\end{array}$ \\
\hline $\mathrm{X}^{\mathrm{b}}$ & $\mathrm{Z}^{\mathrm{c}}$ & $4.5358+4^{\mathrm{d}}$ & 0.0076 \\
\hline 275.0 & 374.0 & $4.4357+4$ & 0.0115 \\
\hline 300.0 & 374.0 & $4.2029+4$ & 0.0102 \\
\hline 315.0 & 374.0 & $3.9848+4$ & 0.0106 \\
\hline 332.7 & 374.0 & $3.8785+4$ & 0.0153 \\
\hline 345.0 & 374.0 & $3.6839+4$ & 0.0142 \\
\hline 360.0 & 374.0 & $3.4177+4$ & 0.0111 \\
\hline 375.0 & 374.0 & $3.2073+4$ & 0.0120 \\
\hline 400.0 & 374.0 & $3.9278+2$ & 0.0227 \\
\hline 332.7 & 480.0 & $4.3514+2$ & 0.0178 \\
\hline 350.0 & 480.0 & $1.0195+3$ & 0.0294 \\
\hline 400.0 & 480.0 & $7.2460+2$ & 0.0241 \\
\hline 450.0 & 480.0 & $5.0524+2$ & 0.0189 \\
\hline 485.0 & 480.0 & & \\
\hline
\end{tabular}

${ }^{\mathrm{a} T}$ The $\mathrm{Y}$ coordinate of all detector locations is $0 \mathrm{~cm}$ (i.e. along the beam line).

${ }^{\mathrm{b}}$ The centerline of the truck lane is at $\mathrm{X}=298.958 \mathrm{~cm}$.

${ }^{c}$ The $\mathrm{Z}=0 \mathrm{~cm}$ location is $125.095 \mathrm{~cm}$ (49.25 in.) above the concrete floor. The top of the roof is at $\mathrm{Z}=484.23 \mathrm{~cm}$ or about $5 \mathrm{~cm}$ above the detector locations (thus the dose is not attenuated by the roof, the thickness and composition of which was assumed).

${ }^{\mathrm{d}}$ Read as $4.5358 \times 10^{4}$. 
Table 21. Distribution of the Neutron and Photon Dose Rates Along the Centerline of the Truck Lane and Perpendicular to the Beam for Detectors Inside a Salted Beef Cargo.

\begin{tabular}{|c|c|c|c|}
\hline \multicolumn{2}{|c|}{ Detector Coordinates $(\mathrm{cm})^{\mathrm{a}}$} & \multicolumn{2}{|c|}{ Dose Rate (mrem/h) and f.s.d. } \\
\hline $\mathrm{X}$ & Y & Neutron & Photon \\
\hline 298.958 & -200.0 & $\begin{array}{c}3.748-3^{c} \\
(0.3933) \\
\end{array}$ & $\begin{array}{c}3.2154-1 \\
(0.1767) \\
\end{array}$ \\
\hline 298.958 & -100.0 & $\begin{array}{c}8.445-1 \\
(0.4777) \\
\end{array}$ & $\begin{array}{c}4.0864+0 \\
(0.0578) \\
\end{array}$ \\
\hline 298.958 & -50.0 & $\begin{array}{c}4.532+1 \\
(0.1547) \\
\end{array}$ & $\begin{array}{c}2.6093+1 \\
(0.0520) \\
\end{array}$ \\
\hline 298.958 & -25.0 & $\begin{array}{c}8.956+2 \\
(0.2095) \\
\end{array}$ & $\begin{array}{c}6.3449+1 \\
(0.0535) \\
\end{array}$ \\
\hline 298.958 & 0.0 & $\begin{array}{c}1.972+3 \\
(0.1012) \\
\end{array}$ & $\begin{array}{c}9.4700+1 \\
(0.0548) \\
\end{array}$ \\
\hline 298.958 & 25.0 & $\begin{array}{c}2.128+3 \\
(0.4463) \\
\end{array}$ & $\begin{array}{c}6.8711+1 \\
(0.0758) \\
\end{array}$ \\
\hline 298.958 & 50.0 & $\begin{array}{r}9.320+1 \\
(0.2339) \\
\end{array}$ & $\begin{array}{c}3.0167+1 \\
(0.1148) \\
\end{array}$ \\
\hline 298.958 & 100.0 & $\begin{array}{l}1.5366+0 \\
(0.7563) \\
\end{array}$ & $\begin{array}{l}4.6223+0 \\
(0.1108) \\
\end{array}$ \\
\hline 298.958 & 200.0 & $\begin{array}{c}3.0223-3 \\
(0.2878) \\
\end{array}$ & $\begin{array}{c}4.4565-1 \\
(0.3352) \\
\end{array}$ \\
\hline 298.958 & 250.0 & $\begin{array}{c}5.4126-4 \\
(0.1351) \\
\end{array}$ & $\begin{array}{c}1.7967-1 \\
(0.4295)\end{array}$ \\
\hline
\end{tabular}

${ }^{a}$ Detectors are centered in the cargo at the center of the truck lane about $125 \mathrm{~cm}$ above the floor.

${ }^{\mathrm{b}}$ Fractional standard deviations in parentheses.

${ }^{c} \operatorname{Read}$ as $3.748 \times 10^{-3}$ with a fractional standard deviation of 0.3933 . 
Table 22. Compositions (weight percent) of Cargo Materials Irradiated.

\begin{tabular}{|c|c|c|c|c|c|}
\hline \multirow[b]{2}{*}{ Element } & \multicolumn{5}{|c|}{ Cargo Material } \\
\hline & Salted Beef ${ }^{a}$ & Ball Bearings $^{b}$ & $\begin{array}{c}\text { Surgical } \\
\text { Implant A } \\
\end{array}$ & $\begin{array}{c}\text { Surgical } \\
\text { Implant B }\end{array}$ & $\begin{array}{l}16-16-16 \\
\text { Fertilizer } \\
\end{array}$ \\
\hline $\mathrm{H}$ & 10.11 & & & & 3.81 \\
\hline $\mathrm{B}$ & & & 0.01 & & \\
\hline C & 26.02 & 0.98 & 0.35 & & 6.363 \\
\hline $\mathrm{N}$ & 1.22 & & 0.25 & & 17.247 \\
\hline $\mathrm{O}$ & 56.54 & 0.0015 & & & 30.915 \\
\hline $\mathrm{F}$ & 0.0036 & & & & \\
\hline $\mathrm{Na}$ & 1.76 & & & & 0.203 \\
\hline $\mathrm{Mg}$ & 0.0164 & & & & \\
\hline $\mathrm{Al}$ & 0.0086 & 0.05 & 0.3 & & \\
\hline$\underline{\mathrm{Si}}$ & 0.0273 & 0.25 & 1.0 & & \\
\hline $\mathrm{P}$ & 0.157 & 0.025 & 0.02 & & 8.167 \\
\hline$S$ & & 0.015 & 0.01 & & \\
\hline $\mathrm{Cl}$ & & & & & 12.662 \\
\hline$\underline{K}$ & 0.2 & & & & 13.619 \\
\hline $\mathrm{Ca}$ & & & & & 7.014 \\
\hline $\mathrm{Cr}$ & & 1.5 & 30.0 & 27.0 & \\
\hline $\mathrm{Mn}$ & & 0.35 & 1.0 & & \\
\hline $\mathrm{Fe}$ & & 96.1785 & 0.75 & & \\
\hline $\mathrm{Co}$ & & & 58.11 & 68.0 & \\
\hline $\mathrm{Ni}$ & & 0.25 & 1.0 & & \\
\hline $\mathrm{Cu}$ & & 0.3 & & & \\
\hline \multicolumn{6}{|l|}{$\mathrm{Zn}$} \\
\hline Mo & & 0.1 & 7.0 & 5.0 & \\
\hline W & & & 0.2 & & \\
\hline
\end{tabular}

${ }^{\mathrm{a}}$ Sums to $96.0629 \%$.

${ }^{\mathrm{b}}$ ASTM A295 (52100).

${ }^{c}$ Compositions from Material Safety Data Sheets for 19-19-19 fertilizer with N-P-K component fractions scaled by $16 / 19$ and the remaining fraction allocated $\mathrm{CaCO}_{3}$. Note that the N-P-K percentages are not equal at $16 \%$ (the $P$ percentage is about half this value). 
Table 23. Compositions (weight percent) of the Structural Materials Irradiated.

\begin{tabular}{|c|c|c|c|c|}
\hline \multirow[b]{2}{*}{ Element } & \multicolumn{4}{|c|}{ Structural Material } \\
\hline & Concrete & $\mathrm{Al} \mathrm{6061-T6}$ & Steel Plate & Havar Foil \\
\hline $\mathrm{H}$ & 1.0 & & & \\
\hline $\mathrm{C}$ & 0.1 & & 0.08 & 0.2 \\
\hline $\mathrm{N}$ & & & 0.16 & \\
\hline $\mathrm{O}$ & 52.9 & & & \\
\hline $\mathrm{Na}$ & 1.6 & & & \\
\hline $\mathrm{Mg}$ & 0.2 & 1.0 & & \\
\hline $\mathrm{Al}$ & 3.4 & 96.68 & & \\
\hline $\mathrm{Si}$ & 33.7 & 0.6 & 0.75 & \\
\hline$P$ & & 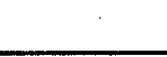 & 0.045 & \\
\hline$S$ & & & 0.03 & \\
\hline$\underline{K}$ & 1.3 & & & \\
\hline $\mathrm{Ca}$ & 4.4 & & & \\
\hline $\mathrm{Ti}$ & & 0.15 & & \\
\hline $\mathrm{Cr}$ & & 0.2 & 20.0 & 19.5 \\
\hline $\mathrm{Mn}$ & & 0.15 & 2.0 & 1.6 \\
\hline $\mathrm{Fe}$ & 1.4 & 0.7 & 64.935 & 19.1 \\
\hline Co & & & & 42.0 \\
\hline $\mathrm{Ni}$ & & & 12.0 & 12.7 \\
\hline $\mathrm{Cu}$ & & 0.27 & & \\
\hline $\mathrm{Zn}$ & & 0.25 & & \\
\hline Mo & & & & 2.2 \\
\hline w & & & & 2.7 \\
\hline
\end{tabular}


Table 24. ORIGEN Flux Parameters for the PFNA Facility Activation Calculations.

\begin{tabular}{|c|c|c|c|c|}
\hline Tables & $\begin{array}{l}\text { Thermal-Neutron } \\
\text { Flux }\left(\mathrm{cm}^{-2} \cdot \mathrm{s}^{-1}\right)\end{array}$ & THERM & RES & FAST \\
\hline $\begin{array}{l}\text { F1-F17, F20, } \\
\text { F21, F24, F25, } \\
\text { F28, F29 } \\
\text { G1-G17, G20, } \\
\text { G21, G24, } \\
\text { G25, G28, G29 }\end{array}$ & $451.24^{\mathrm{a}}$ & 0.7171 & 0.3522 & 1675.8 \\
\hline $\begin{array}{l}\text { F22, F23, F26, } \\
\text { F27, F30, F31 } \\
\text { G22, G23, G26 } \\
\text { G27, G30, G31 }\end{array}$ & $3227.31^{b}$ & 0.5089 & 1.4711 & 1637.5 \\
\hline $\begin{array}{l}\text { F32, F33, } \\
\text { G32, G33 }\end{array}$ & $2801.82^{\mathrm{c}}$ & 0.6192 & 0.56447 & 23.505 \\
\hline
\end{tabular}

${ }^{\mathrm{a}}$ At centerline of truck lane.

${ }^{\mathrm{b}}$ Inside the collimator $182.88 \mathrm{~cm}$ from the centerline of the truck lane.

${ }^{c}$ At inside face of backstop concrete.

THERM $=\frac{\int_{0}^{0.5}{ }^{e V} \phi(E) \sqrt{\frac{E_{0}}{E}} d E}{\phi_{t h}}$

$R E S=\frac{\int_{0.5 \mathrm{eV}}^{1.0 \mathrm{MeV}} \phi(E) d E}{\ln \left(\frac{10^{6}}{0.5}\right) \phi_{t h}}$

$F A S T=\frac{1.45}{\Phi_{t h}} \int_{1.0 \mathrm{MeV}}^{\infty} \phi(E) d E$

$\phi_{t h}=\int_{0}^{0.5^{e V}} \phi(E) d E$ 


\section{PFNA NOTIONAL BUILDING - PLAN VIEW}

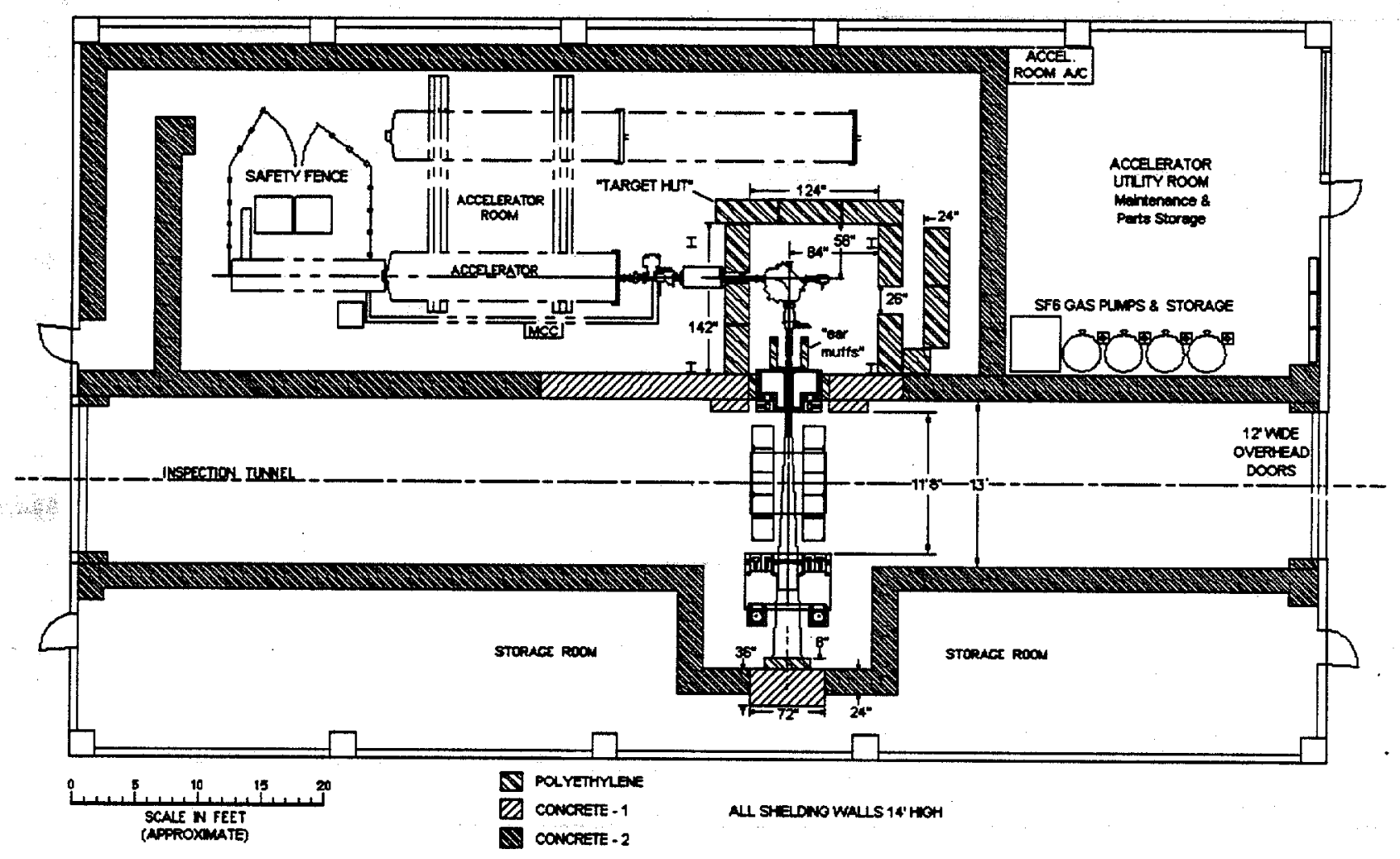

Figure 1. Plan view of the PFNA Facility. 


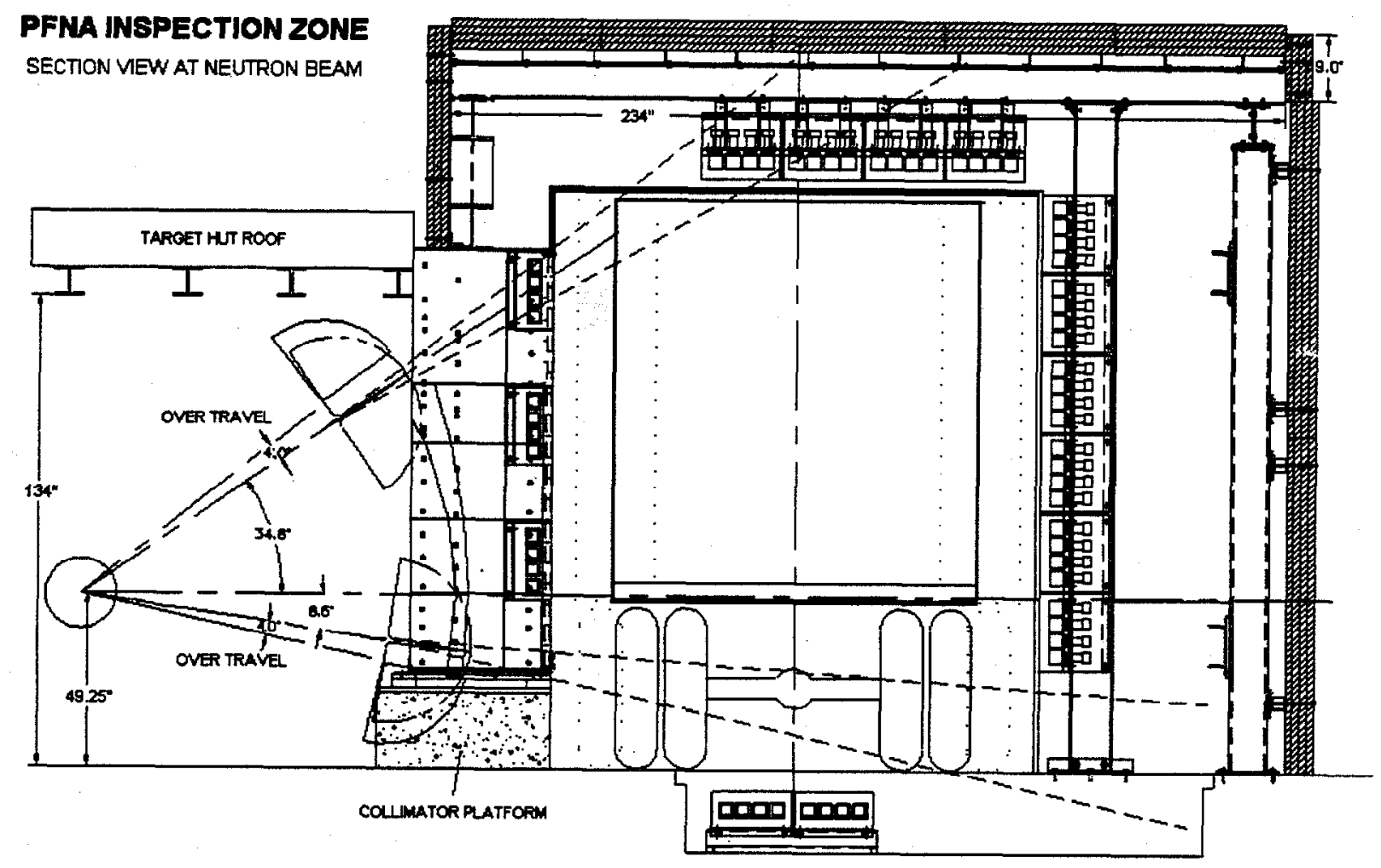

Figure 2. Elevation view of the PFNA Facility at the beam line plane. 


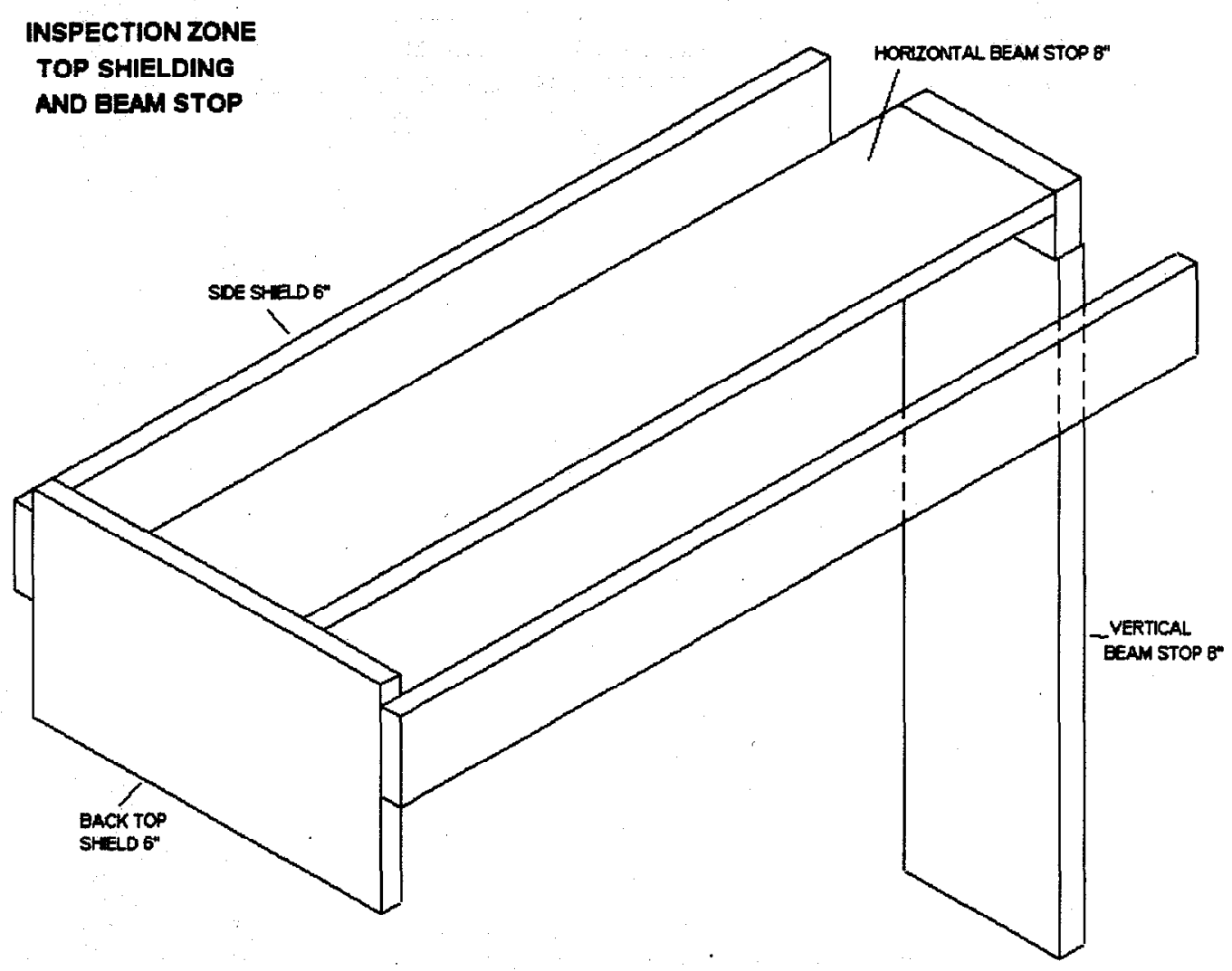

Figure 3. Sketch of the vertical and horizontal polyethylene beam stops. 


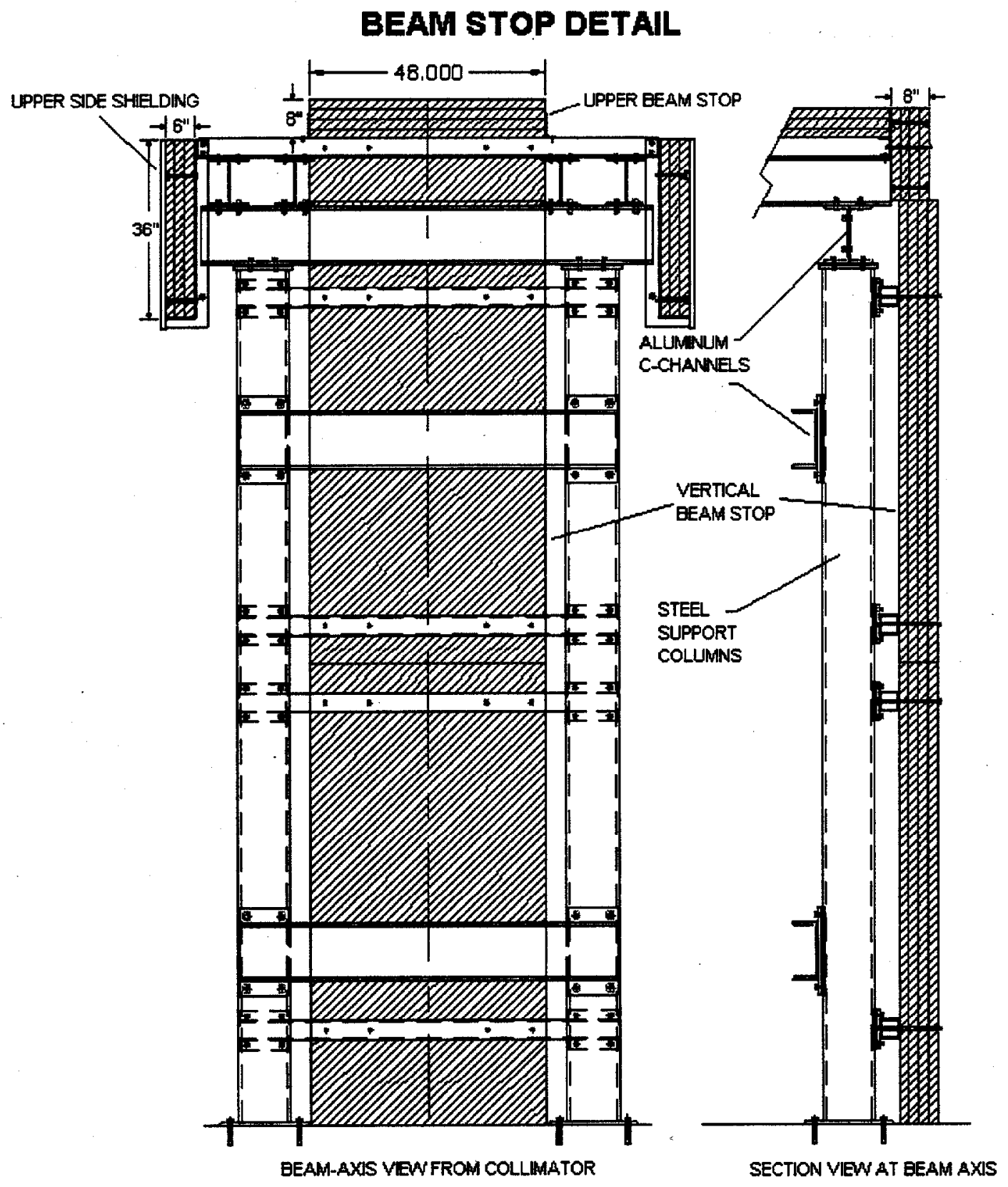

Figure 4. Head-on and side views of the polyethylene beam stop. 


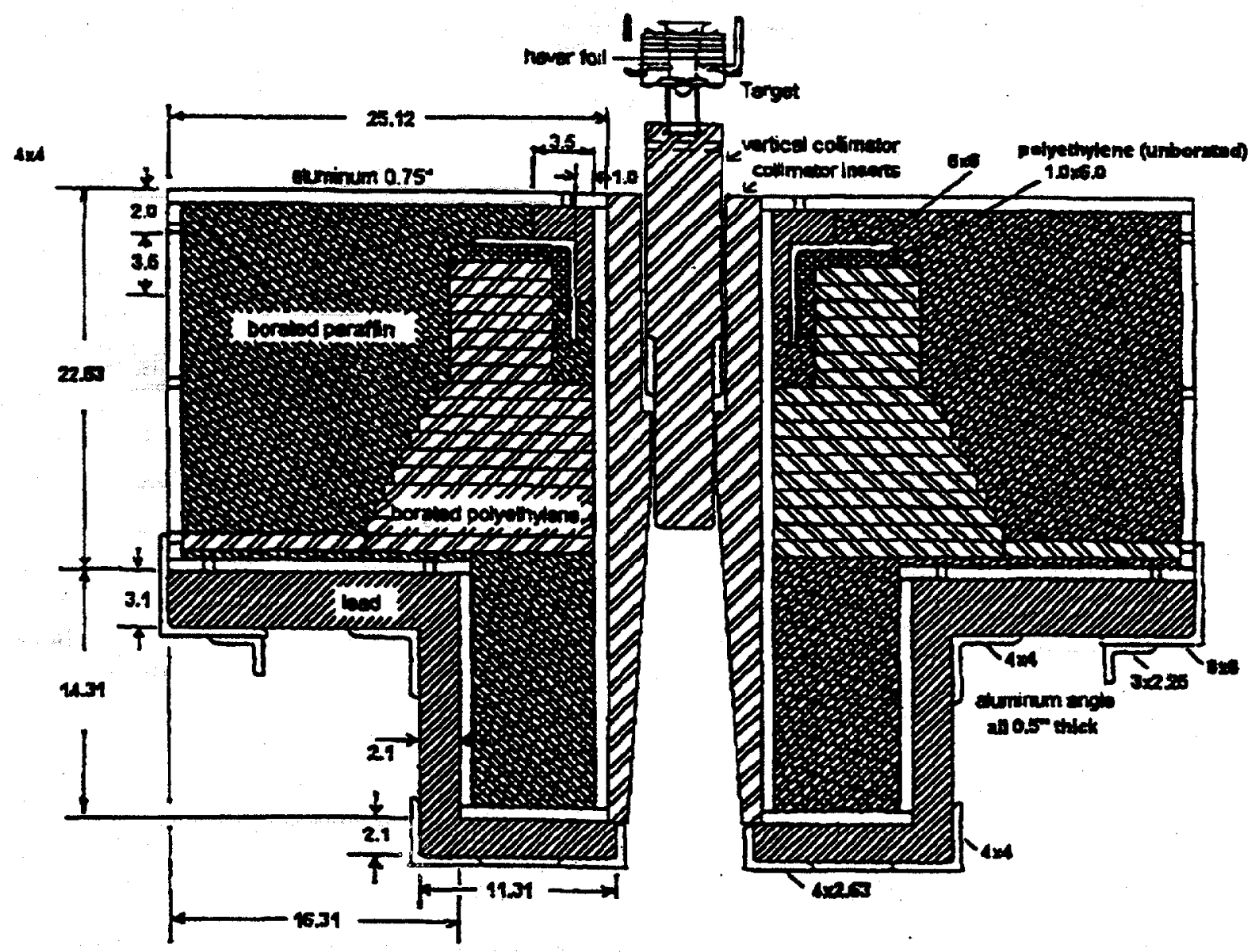

Figure 5. Plan view of the stationary collimator and vertical collimator detailing most exterior dimensions. 


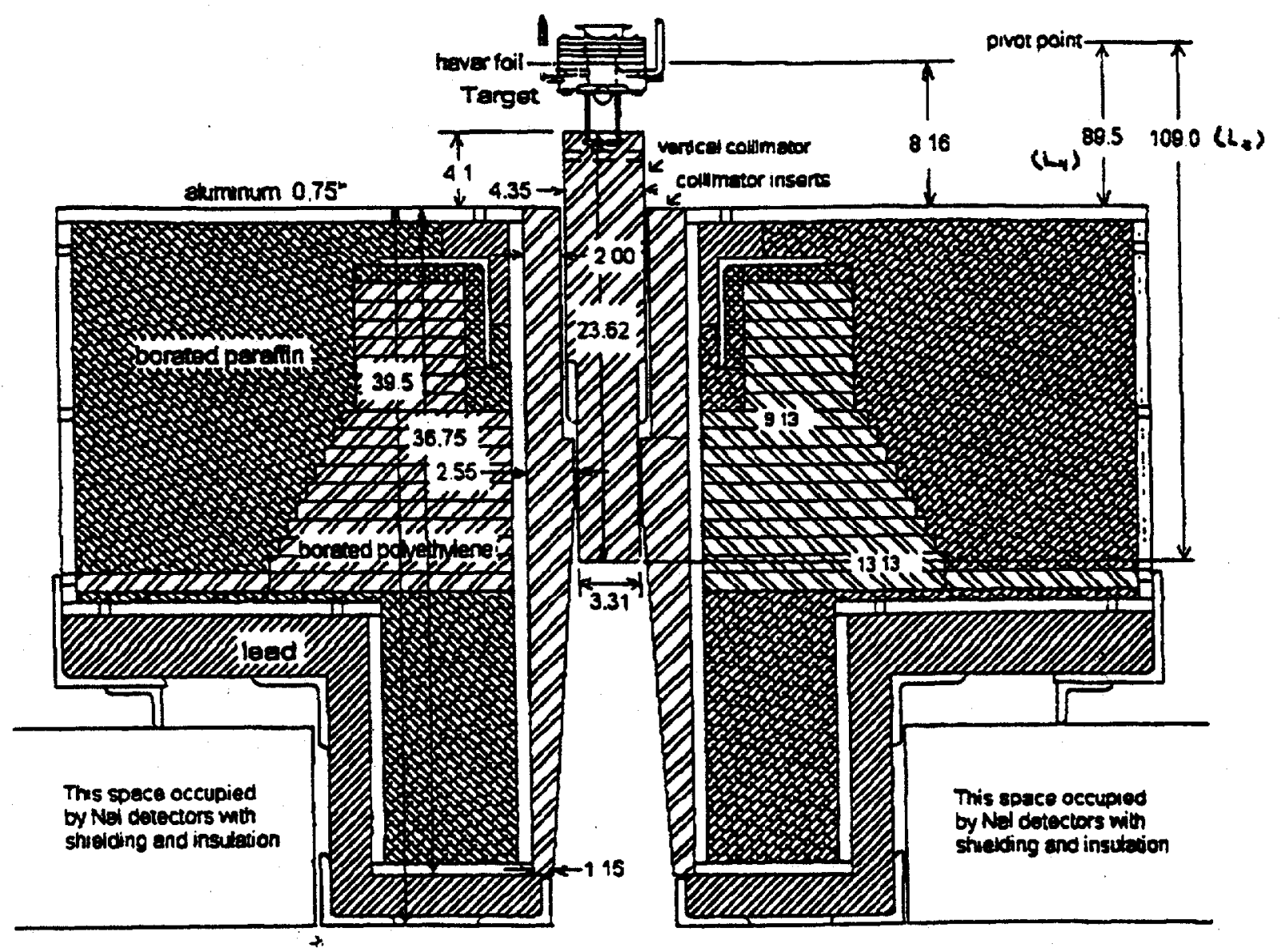

Figure 6. Plan view of the stationary collimator and vertical collimator detailing most interior dimensions. 


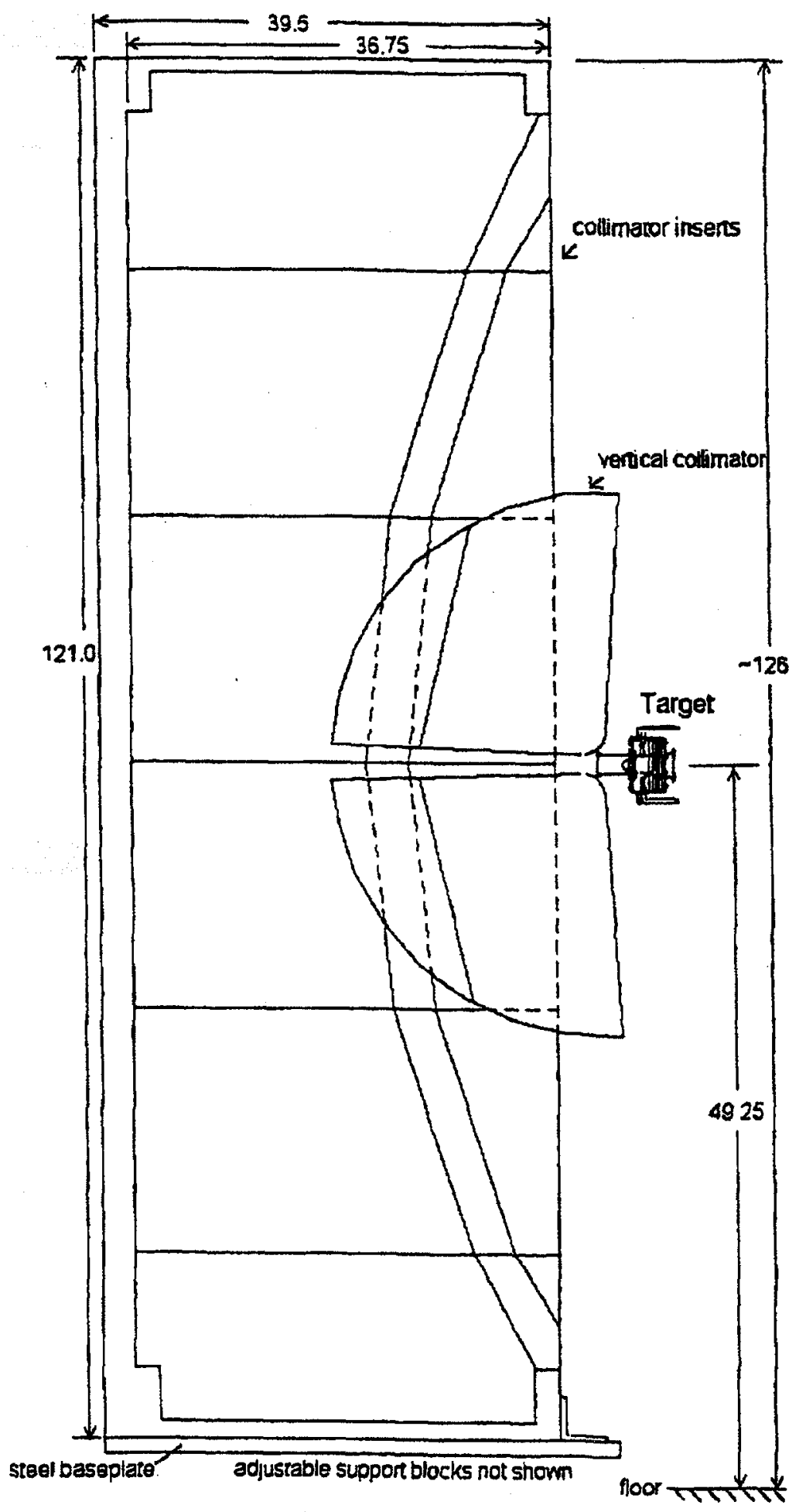

Figure 7. Elevation view of the vertical and stationary collimators with the vertical collimator at its horizontal position. 


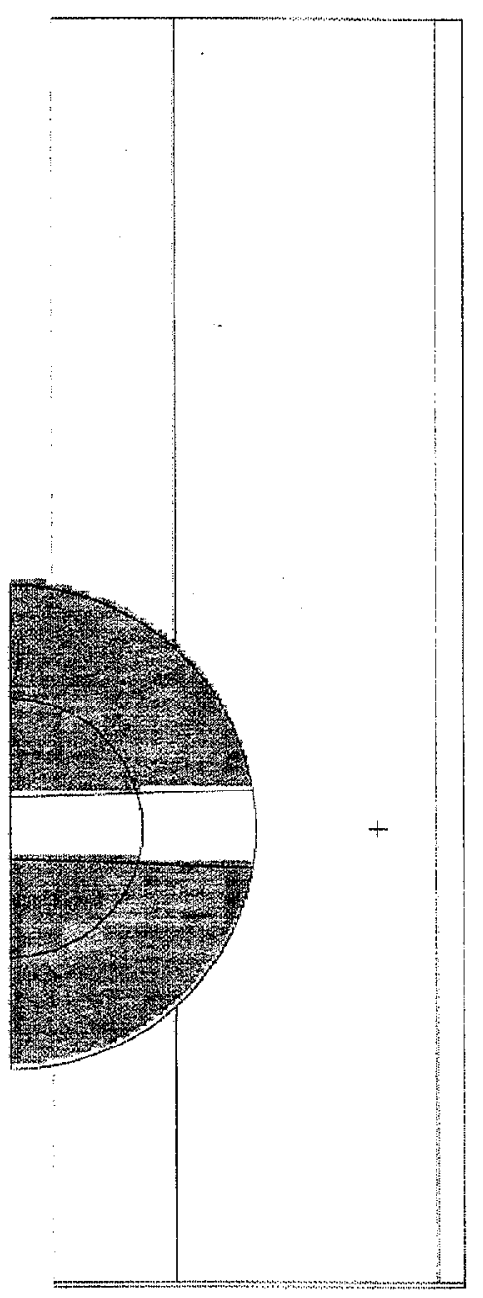

Figure 8. Vertical slice through the collimator and the bare plutonium sphere mockup used in the MCNP-4B calculations of dose multiplication by fissile targets. 


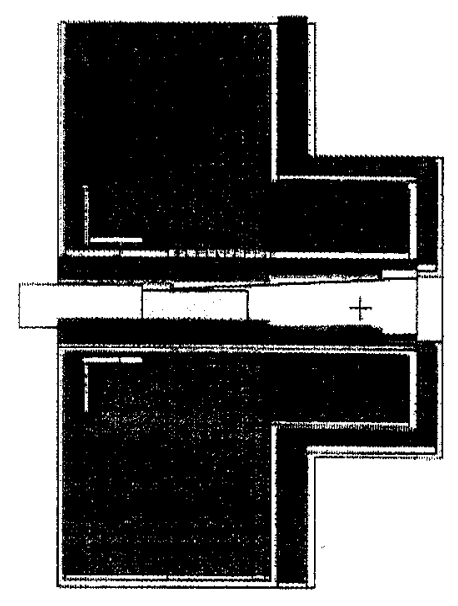

Figure 9. Horizontal slice through the collimator and the bare plutonium sphere mockup used in the MCNP-4B calculations of dose multiplication by fissile targets. 


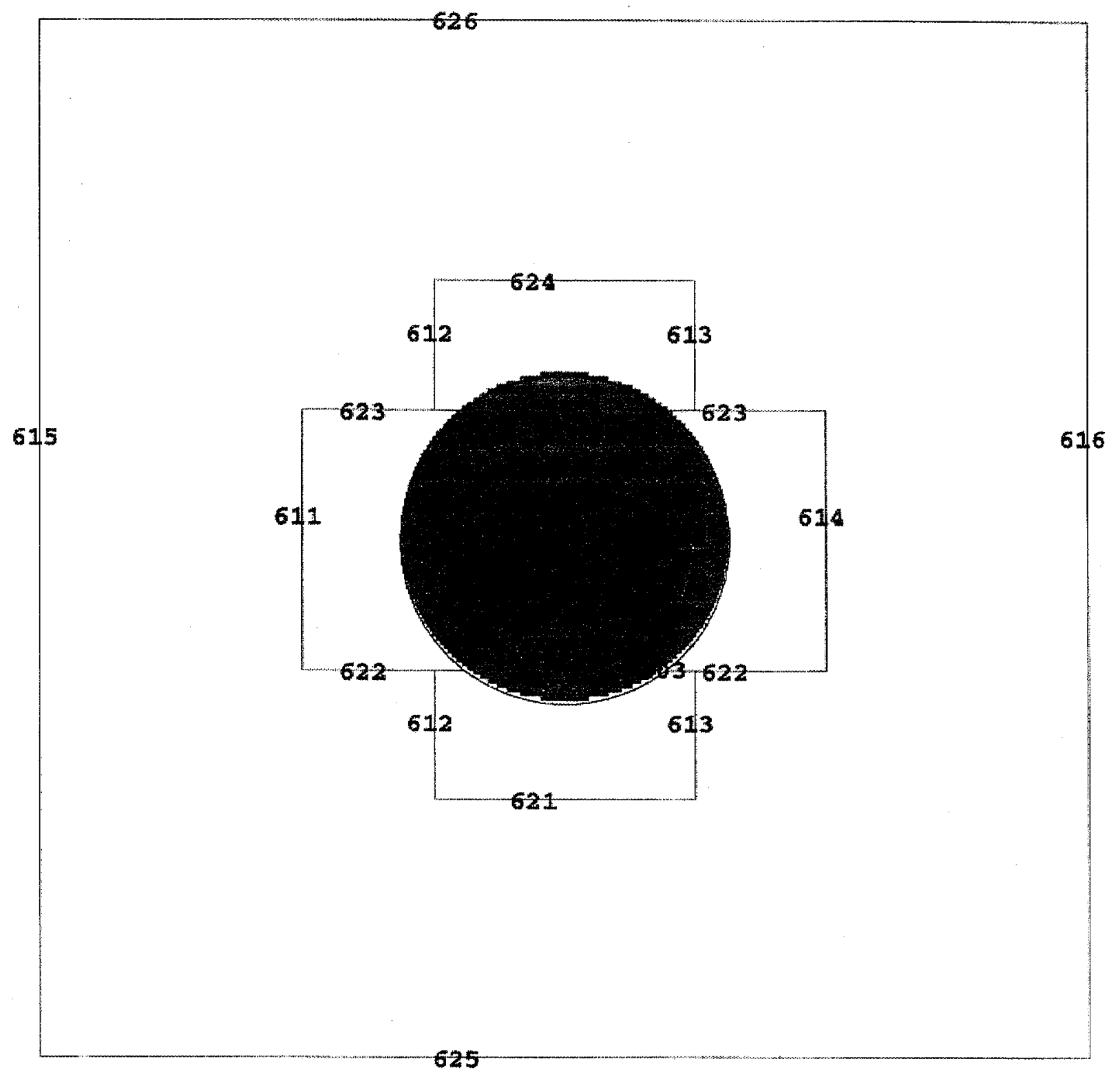

Figure 10. Closeup horizontal slice through the bare plutonium sphere mockup used in the MCNP-4B calculations of dose multiplication by fissile targets. The numbered planes 611, 614 , and 624 are three of the surface flux detectors used in the problem. The fourth surface, 634 , is above the sphere. 


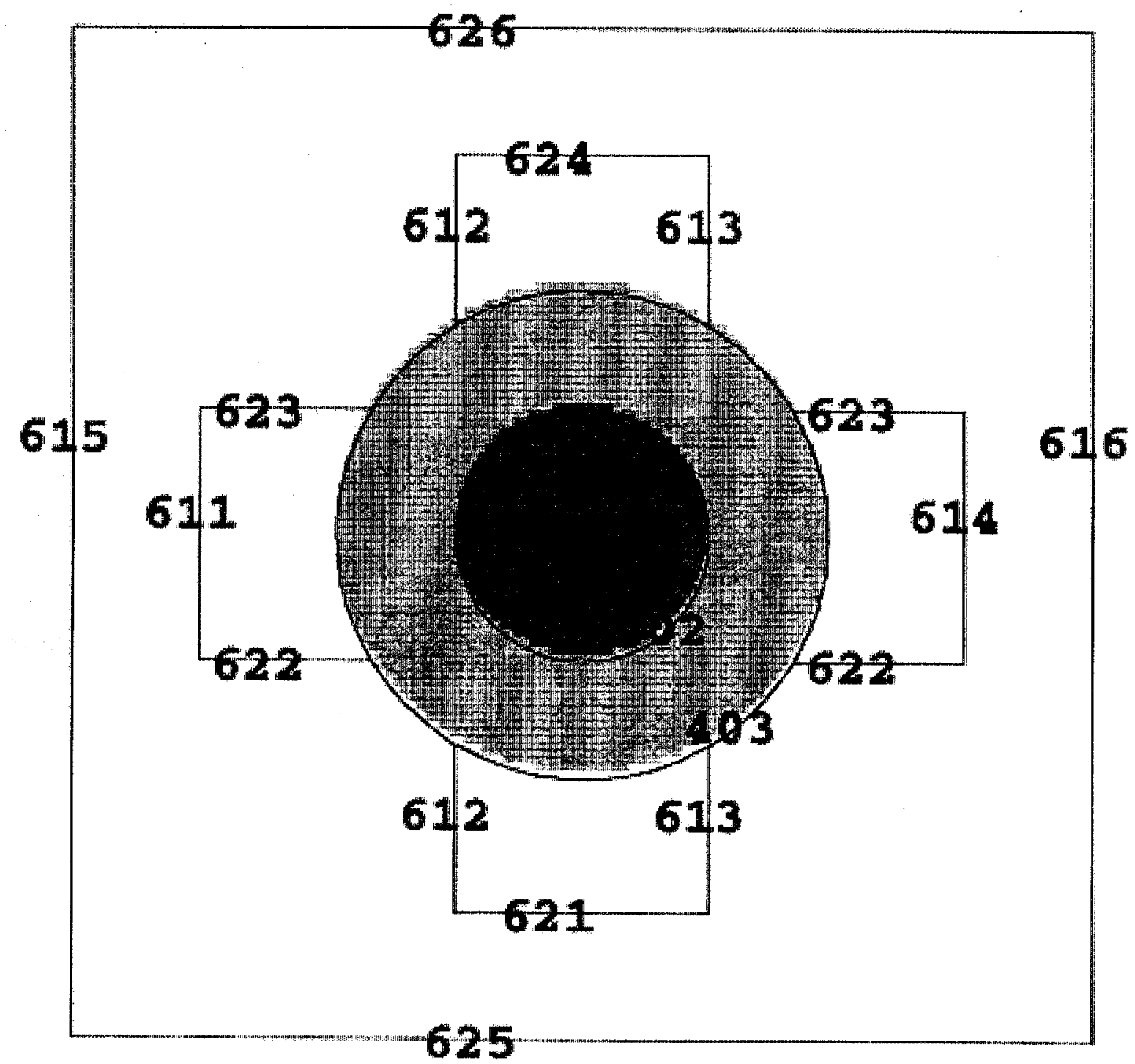

Figure 11. Closeup horizontal slice through the tungsten-reflected plutonium sphere mockup used in the MCNP-4B calculations of dose multiplication by fissile targets. The numbered planes 611,614 , and 624 are three of the surface flux detectors used in the problem. The fourth surface, 634 , is above the sphere. 


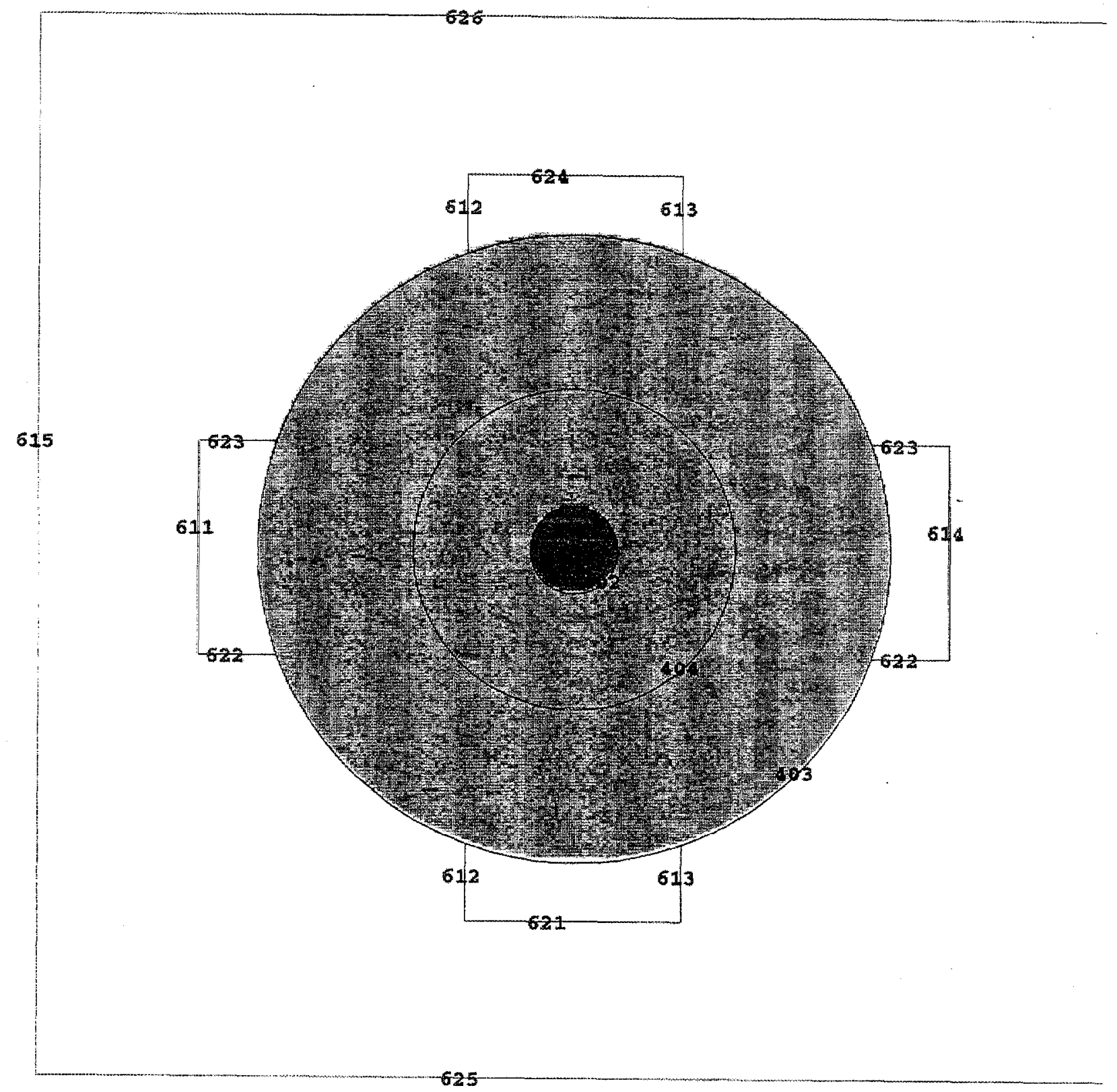

Figure 12. Closeup horizontal slice through the water-reflected plutonium sphere mockup used in the MCNP-4B calculations of dose multiplication by fissile targets. The numbered planes 611,614 , and 624 are three of the surface flux detectors used in the problem. The fourth surface, 634 , is above the sphere. 
Detectors at $\mathrm{z}=0.0$ or $1.25 \mathrm{~m}$ above floor

\section{PFNA NOTIONAL BUILDING - PLAN VIEW}

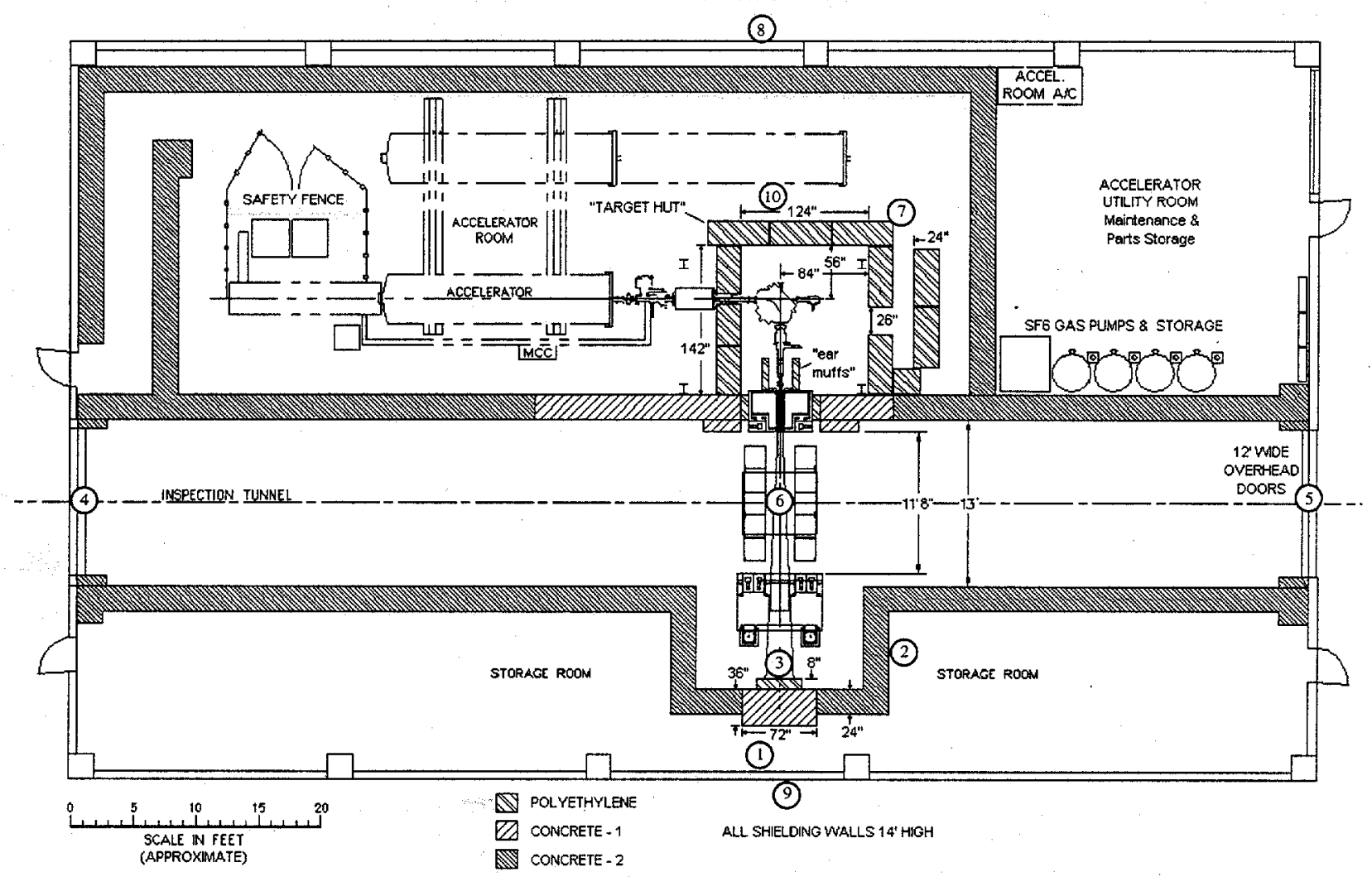

Figure 13. Approximate detector locations for dose rates shown in Table 15. 


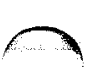

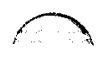

$a$ 


\section{APPENDIX A}

\section{LISTINGS OF INPUT FILES MCNP-X CRITICALITY CALCULATIONS}


$\curvearrowright$

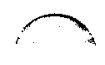




\section{APPENDIX A}

\section{LISTINGS OF INPUT FILES MCNP-X CRITICALITY CALCULATIONS}

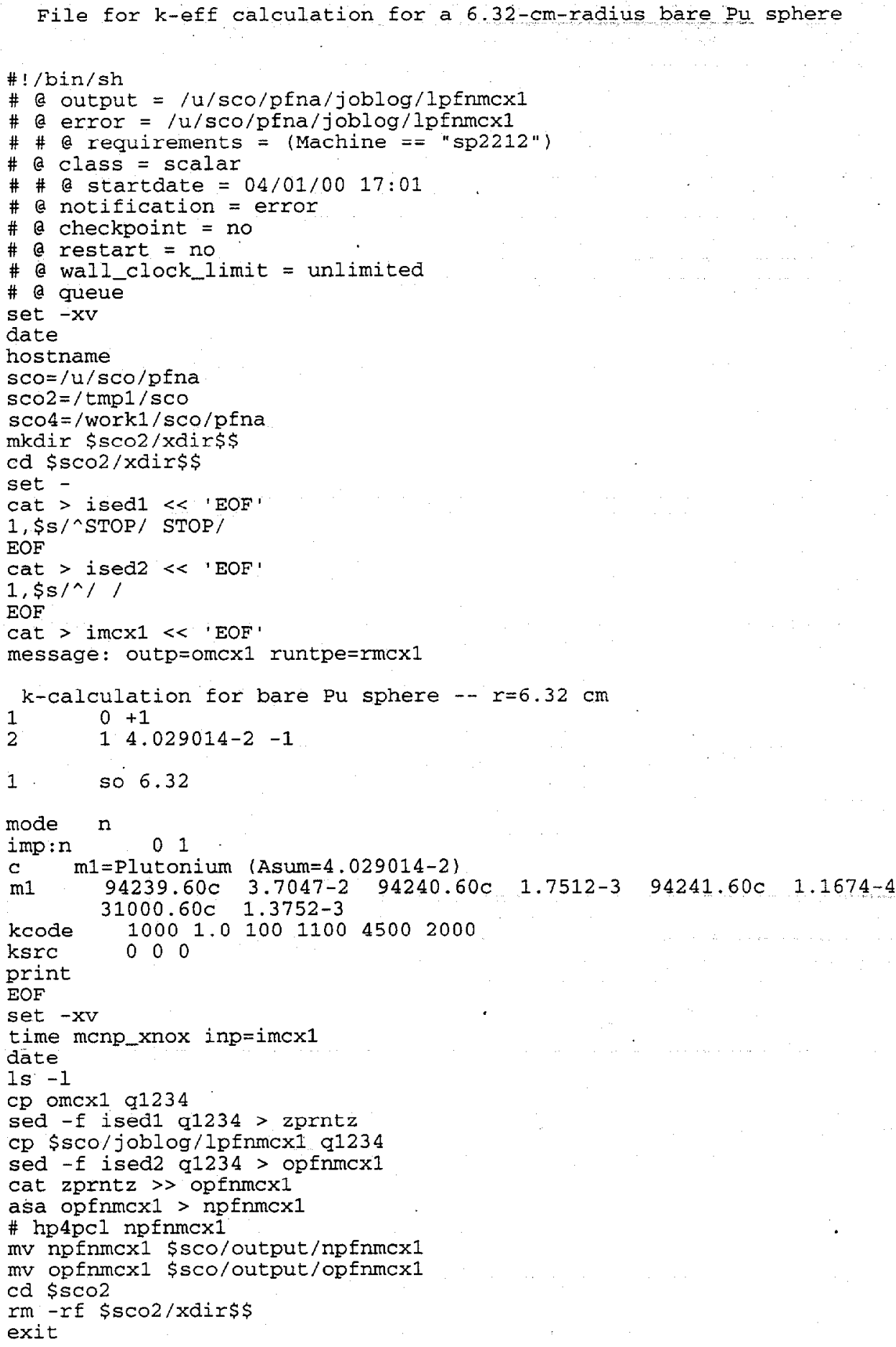




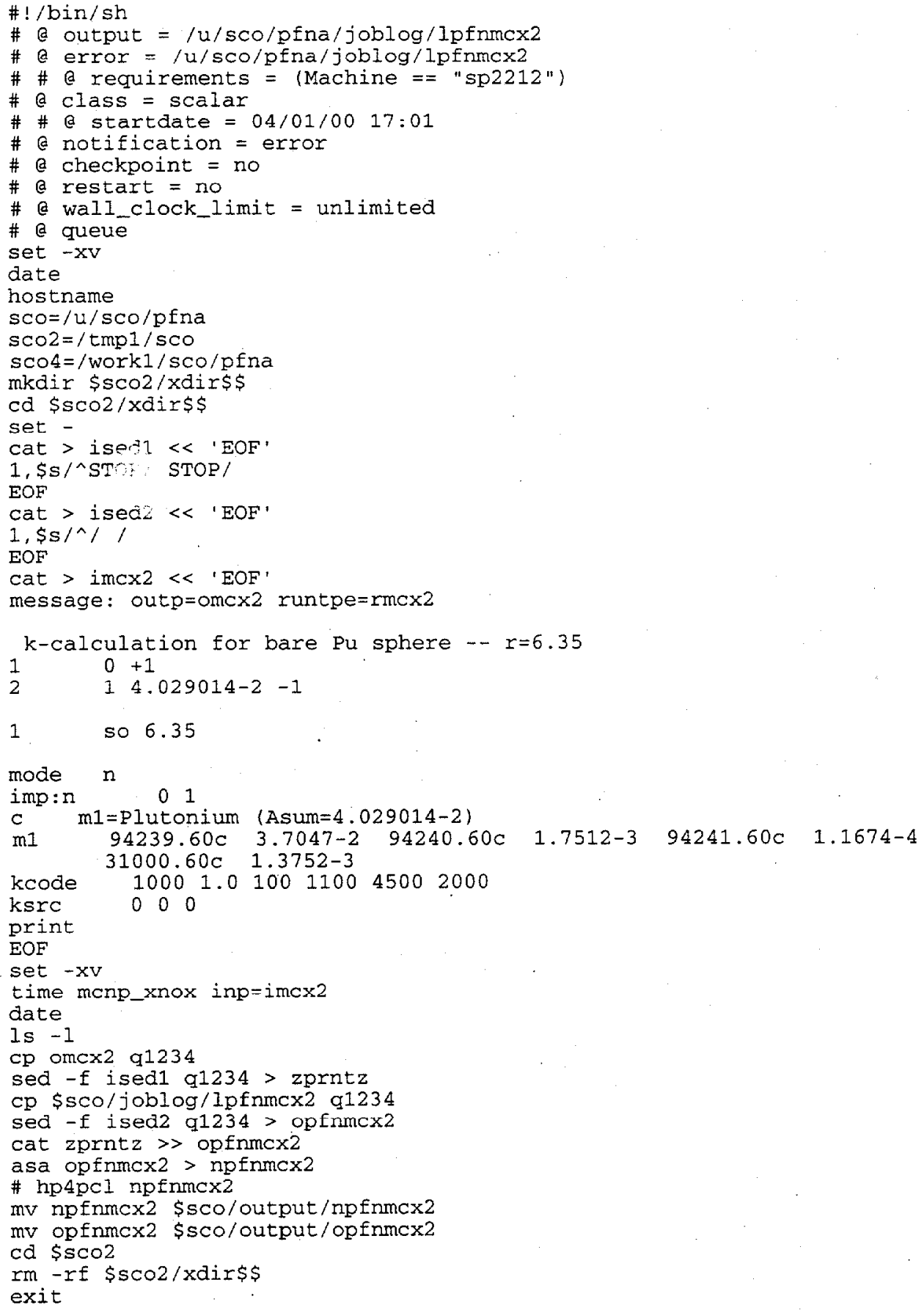


File for k-eff calculation for a 6.3849 -cm-radius critical bare Pu sphere

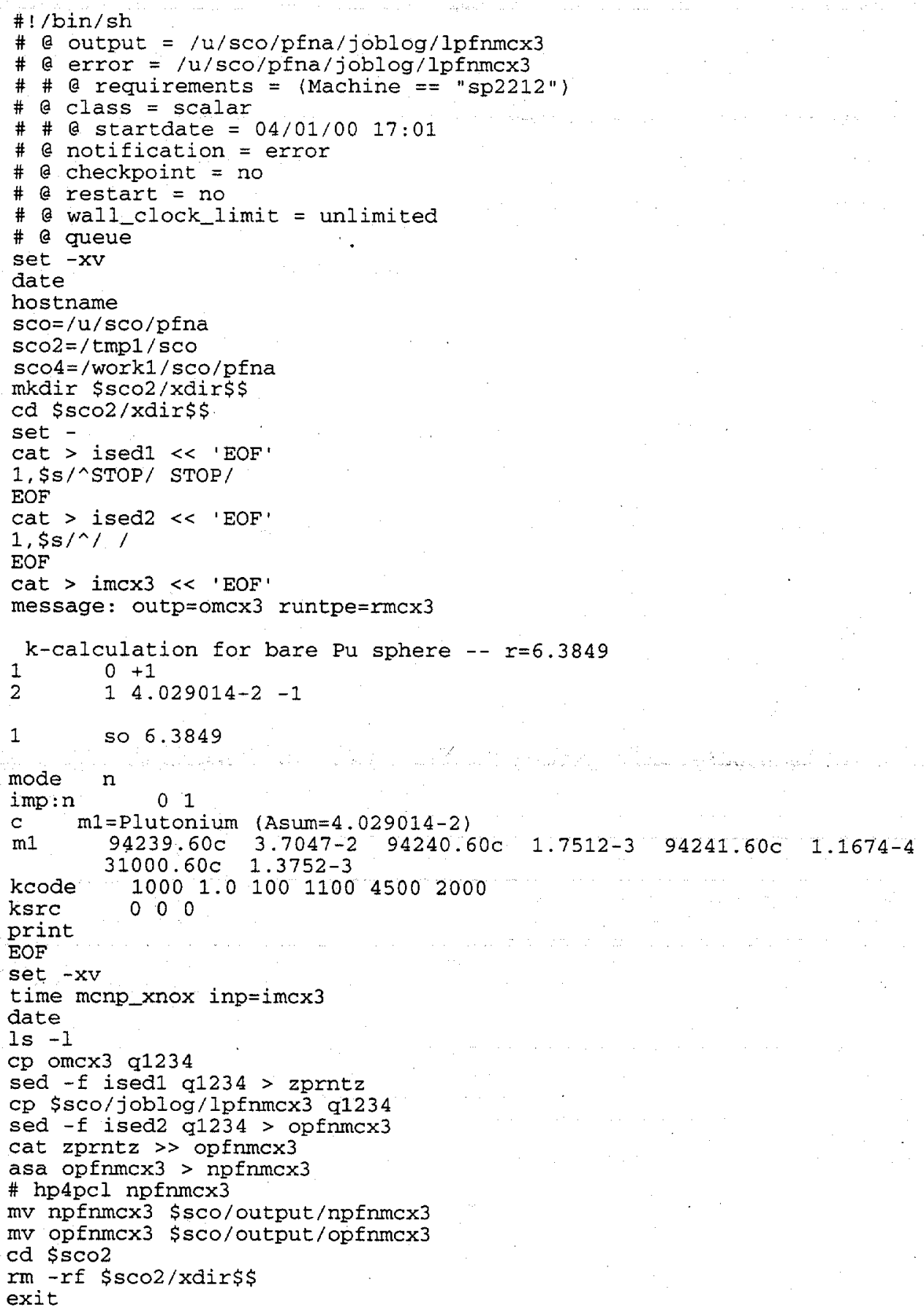


File for k-eff calculation for a tungsten-reflected critical Pu shpere

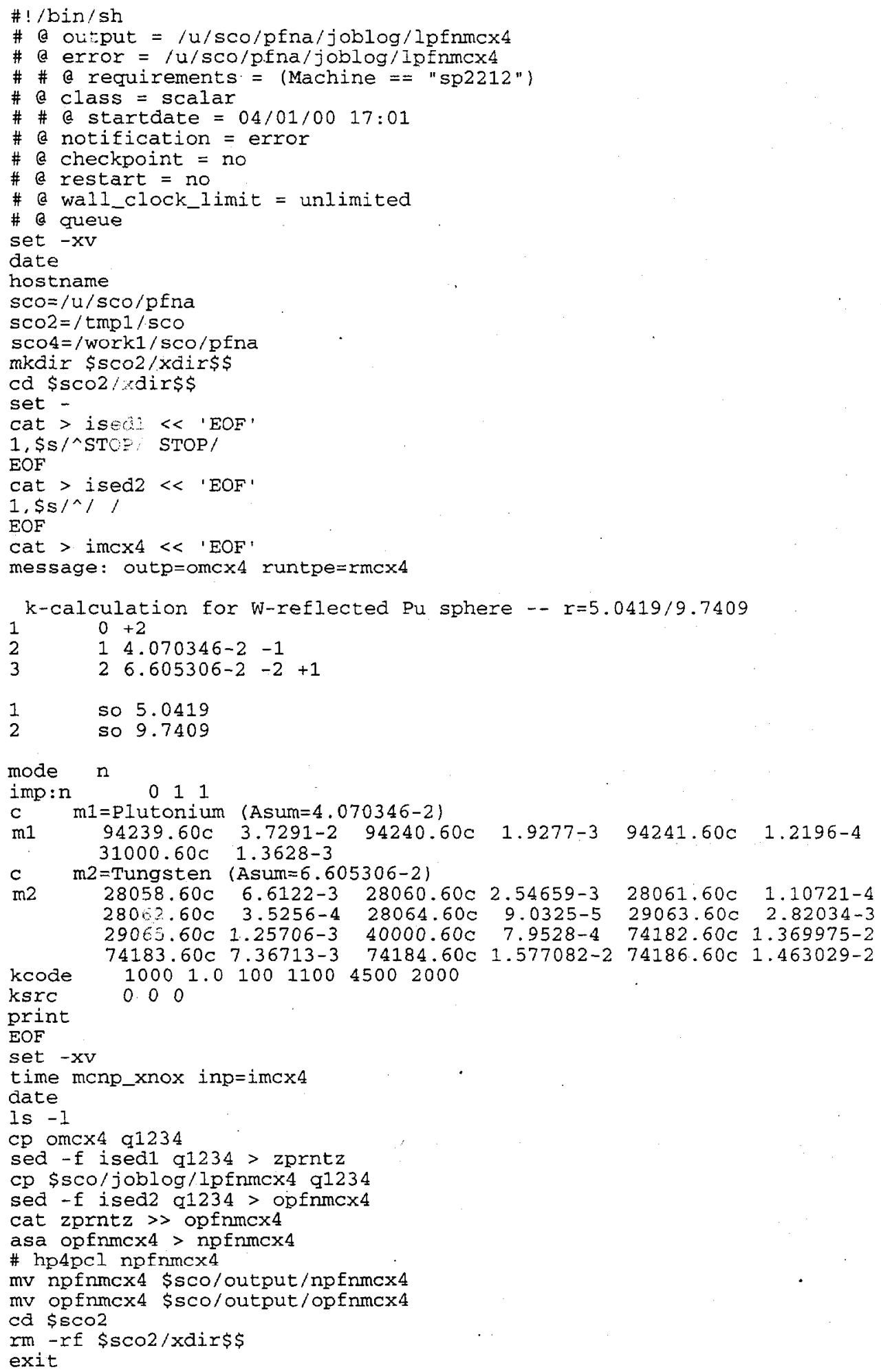


File for k-eff calculation for a water-reflected critical pu shpere

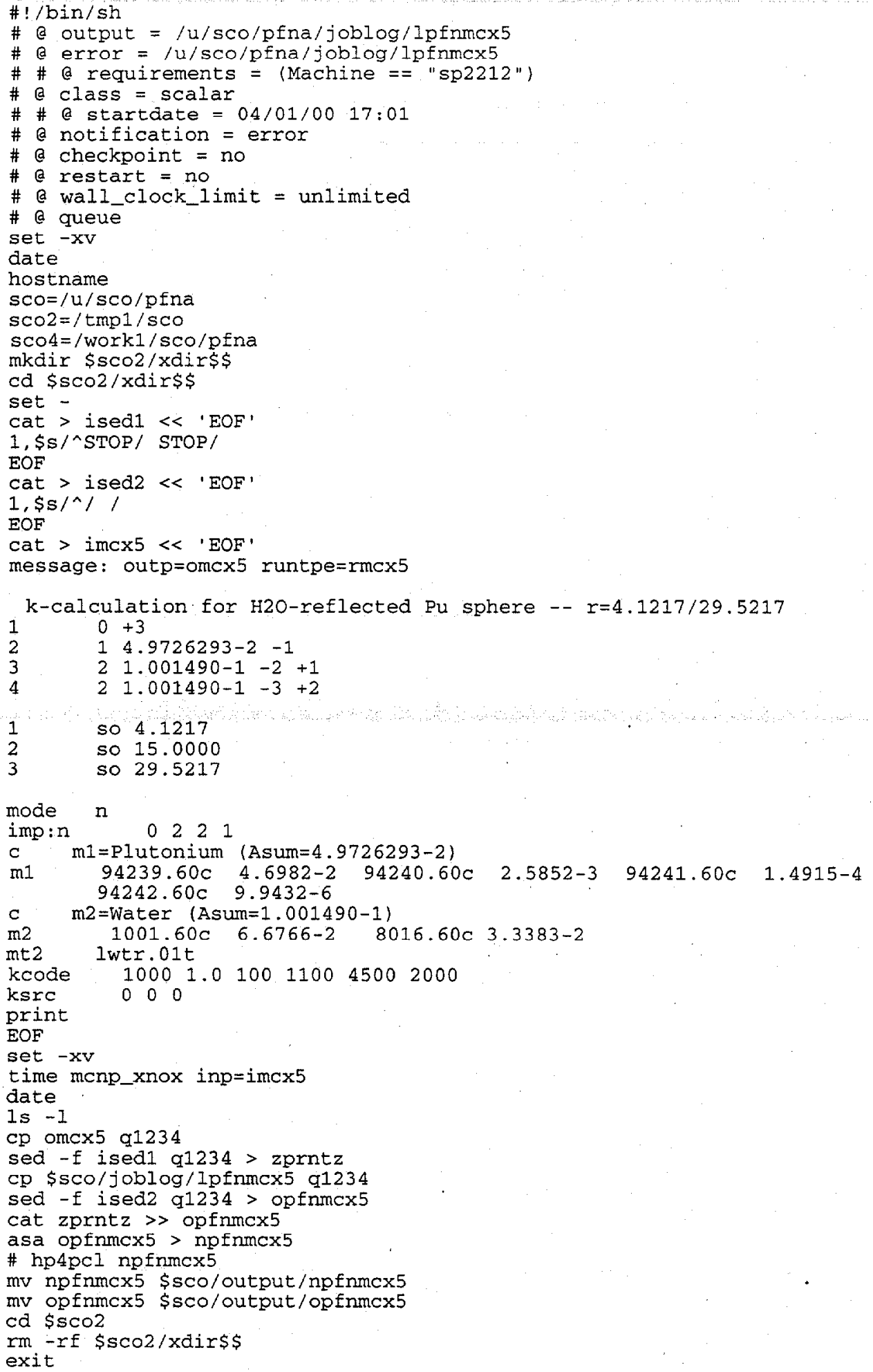


D

$\curvearrowright$ 


\section{APPENDIX B}

\section{LISTINGS OF INPUT FILES MCNP-4B FISSILE MATERIAL}

DOSE-RATE CALCULATIONS 
$\curvearrowright$

$\curvearrowright$ 
APPENDIX B

\title{
LISTINGS OF INPUT FILES MCNP-4B FISSILE MATERIAL DOSE-RATE CALCULATIONS
}

\author{
File for MCNP-4B point-detector dose-rate calculations for a 6.38-cm-radius \\ bare Pu sphere in the PFNA beam at the center of the truck lane \\ $(2,000,000$ histories $)$
}

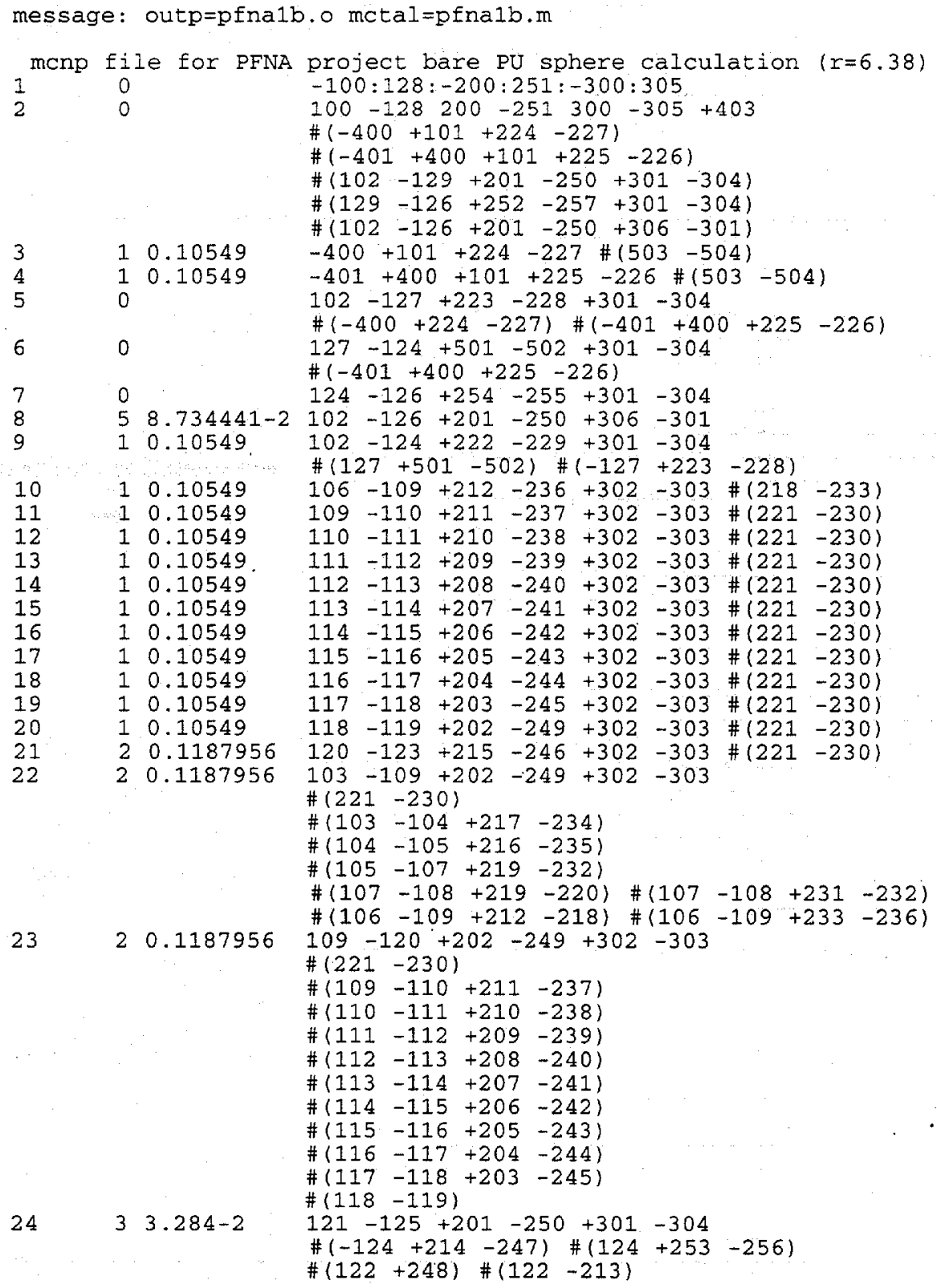




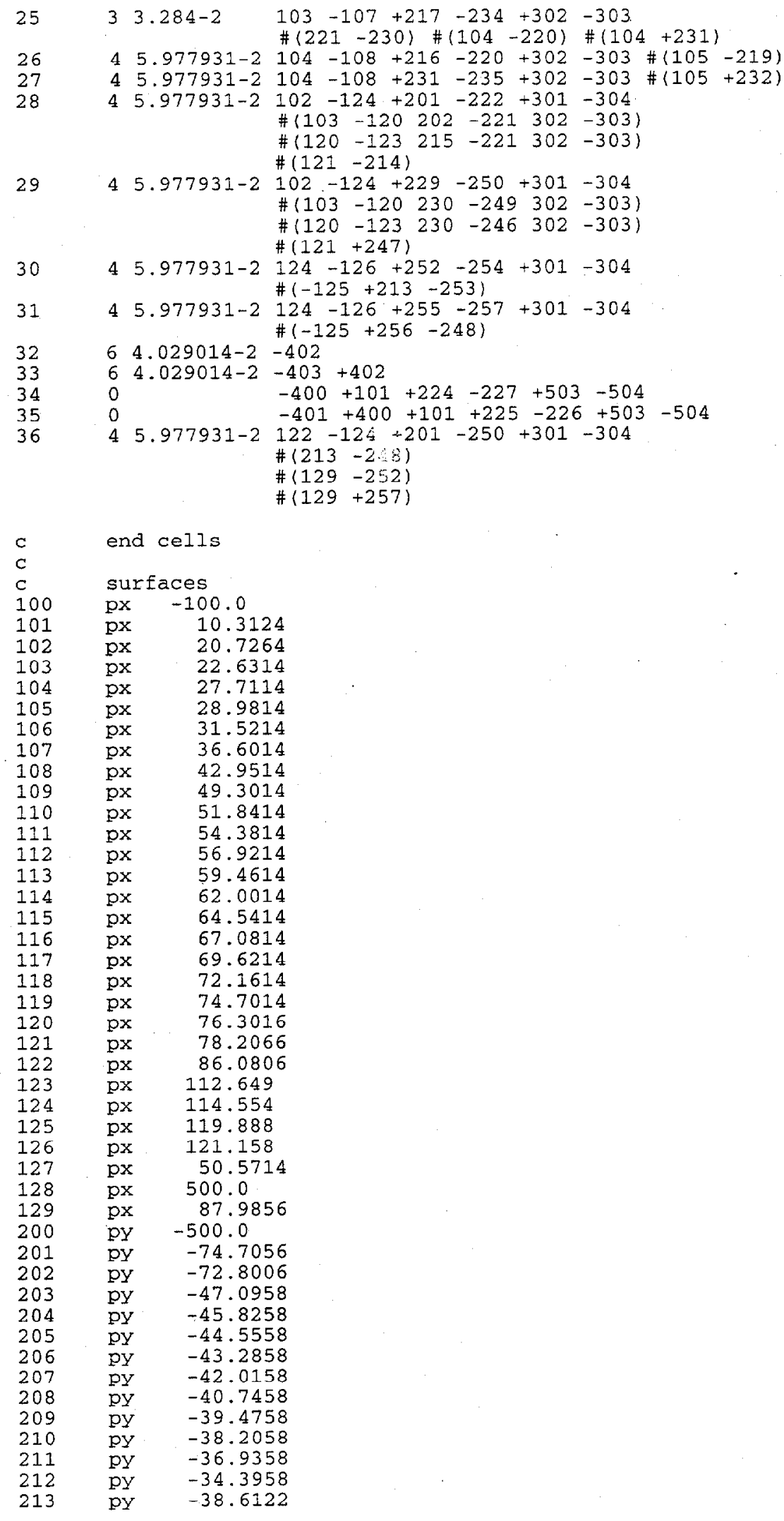

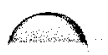




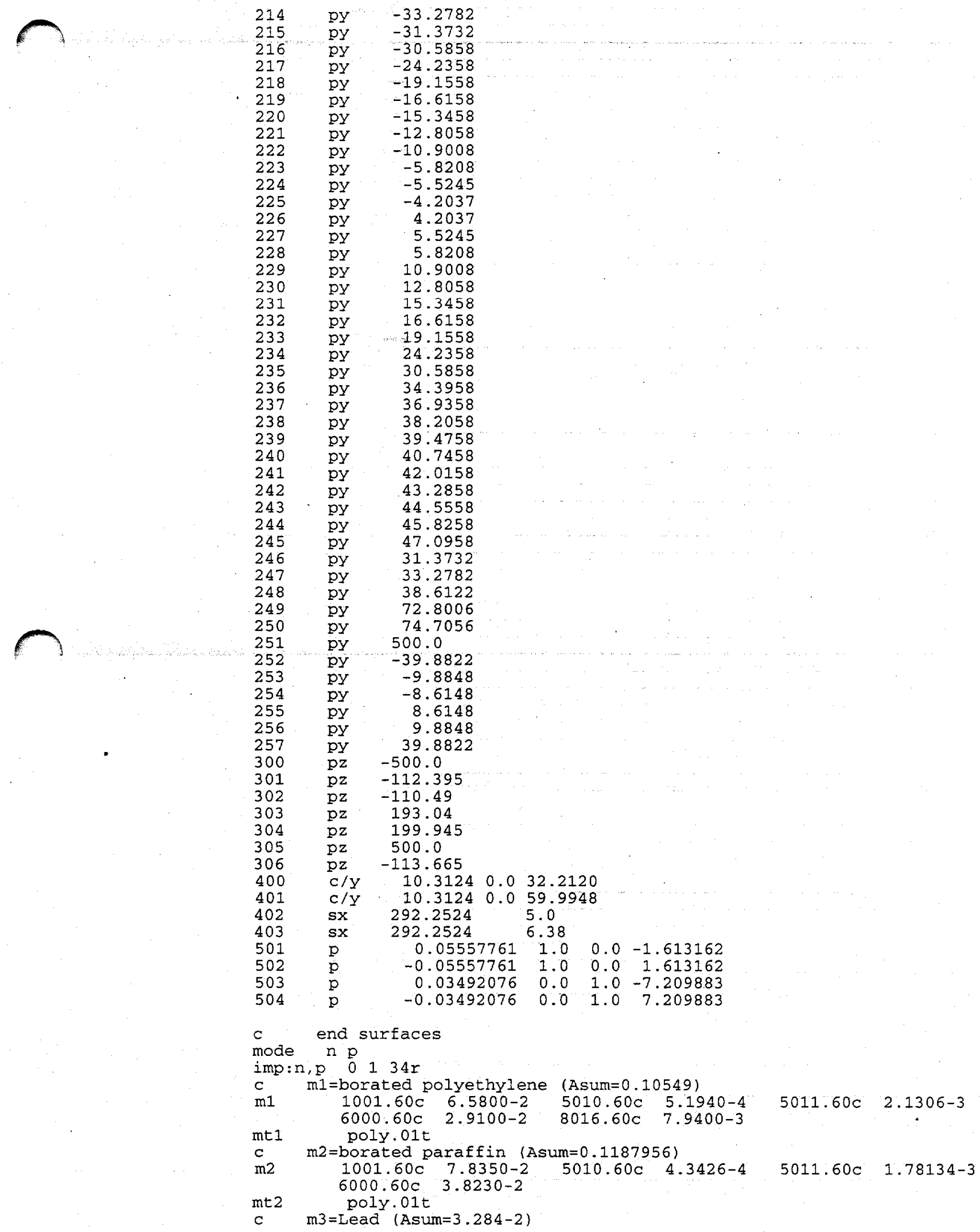




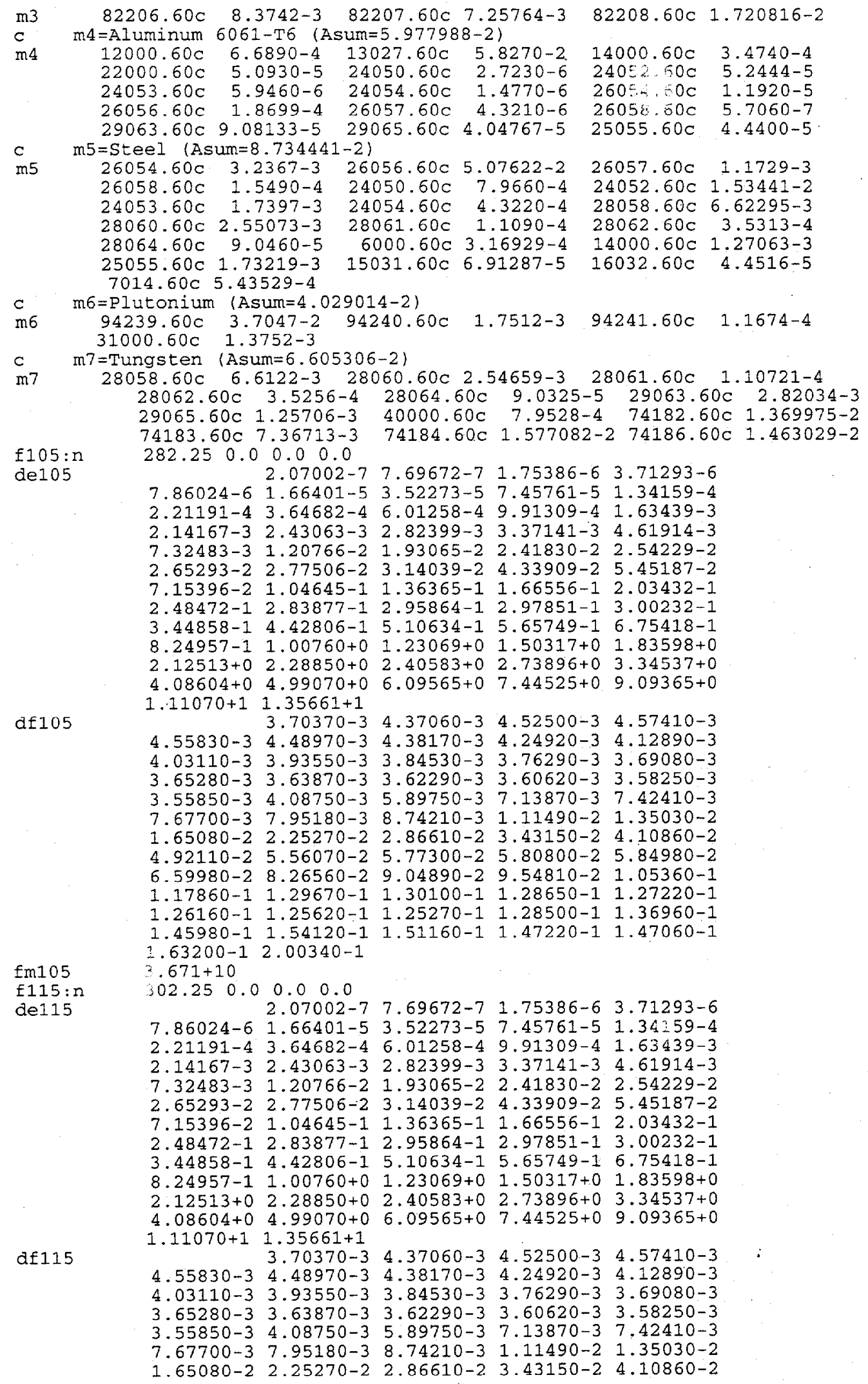


$4.92110-2 \quad 5.56070-2 \quad 5.77300-2 \quad 5.80800-2 \quad 5.84980-2$ $6.59980-2 \quad 8.26560-2 \quad 9.04890-2 \quad 9.54810-2 \quad 1.05360-1$ 1. $17860-1$ 1.29670-1 $1.30100-1$ 1.28650-1 $1.27220-1$ $1.26160-1 \quad 1.25620-1 \quad 1.25270-1 \quad 1.28500-11.36960-1$ $1.45980-1 \quad 1.54120-1 \quad 1.51160-1 \quad 1.47220-1 \quad 1.47060-1$ 1. $63200-12.00340-1$

$\operatorname{fm} 115$ $3.671+10$

f125: $292.2510 .0 \quad 0.0 \quad 0.0$

de125

df125

$2.07002-7 \quad 7.69672-7 \quad 1.75386-6 \quad 3.71293-6$

7.86024-6 $1.66401-5 \quad 3.52273-5 \quad 7.45761-5 \quad 1.34159-4$

2.21191-4 3.64682-4 6.01258-4 9.91309-4 $1.63439-3$

$2.14167-3 \quad 2.43063-3 \quad 2.82399-3 \quad 3.37141-3 \quad 4.61914-3$

7.32483-3 $1.20766-2 \quad 1.93065-2 \quad 2.41830-2.2 .54229-2$

2.65293-2 2.77506-2 $3.14039-2 \quad 4.33909-2 \quad 5.45187-2$

7.15396-2 $1.04645-1 \quad 1.36365-1 \quad 1.66556-1 \quad 2.03432-1$

2.48472-1 $2.83877-1 \quad 2.95864-1 \quad 2.97851-1 \quad 3.00232-1$

3.44858-1 4.42806-1 $5.10634-1 \quad 5.65749-1 \quad 6.75418-1$

8.24957-1 $1.00760+0 \quad 1.23069+0 \quad 1.50317+0 \quad 1.83598+0$

$2.12513+0 \quad 2.28850+0 \quad 2.40583+0 \quad 2.73896+0 \quad 3.34537+0$ $4.08604+0 \quad 4.99070+0 \quad 6.09565+0 \quad 7.44525+0 \quad 9.09365+0$ $1.11070+11.35661+1$

$\begin{array}{rllll}3.70370-3 & 4.37060-3 & 4.52500-3 & 4.57410-3\end{array}$

$4.55830-3 \quad 4.48970-3.4 .38170-3 \quad 4.24920-3 \quad 4.12890-3$

$4.03110-3 \quad 3.93550-3 \quad 3.84530-3 \quad 3.76290-3 \quad 3.69080-3$

$3.65280-3 \quad 3.63870-3 \quad 3.62290-3 \quad 3.60620-3 \quad 3.58250-3$

$3.55850-3 \quad 4.08750-3 \quad 5.89750-3 \quad 7.13870-3 \quad 7.42410-3$

$\begin{array}{lllll}7.67700-3 & 7.95180-3 & 8.74210-3 & 1.11490-2 & 1.35030-2\end{array}$

$\begin{array}{lllll}1.65080-2 & 2.25270-2 & 2.86610-2 & 3.43150-2 & 4.10860-2\end{array}$

$4.92110-2 \quad 5.56070-2 \quad 5.77300-2 \quad 5.80800-2 \quad 5.84980-2$

$6.59980-2 \quad 8.26560-2 \quad 9.04890-2 \quad 9.54810-2 \quad 1.05360-1$

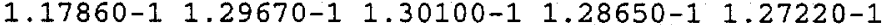

$1.26160-1 \quad 1.25620-1 \quad 1.25270-1.28500-1 \quad 1.36960-1$

$\begin{array}{llllll}1.45980-1 & 1.54120-1 & 1.51160-1 & 1.47220-1 & 1.47060-1\end{array}$ $1.63200-12.00340-1$

$\operatorname{fm} 125 \quad 3.671+10$

f205:D $\quad 282.250 .0 \quad 0.0 \quad 0.0$

de205 $1.50000-2 \quad 3.25000-2 \quad 5.75000-28.50000-2$

1.25000-1 $2.25000-1 \quad 3.50000-1 \quad 4.55000-1 \quad 5.55000-1$ $6.50000-1 \quad 8.50000-1 \quad 1.25000+0 \quad 1.75000+02.25000+0$ $2.75000+0 \quad 3.50000+0 \quad 4.50000+0 \quad 5.50000+0 \quad 6.50000+0$ $7.25000+07.75000+0 \quad 9.00000+0 \quad 1.20000+1$

$\begin{array}{llllll}2.14390-3 & 5.77600-4 & 2.71850-4 & 2.68170-4\end{array}$ $3.27670-4 \quad 5.66760-4 \quad 8.75940-4 \quad 1.08450-3 \quad 1.27970-3$ $1.44170-3 \quad 1.75630-3 \quad 2.31560-3 \quad 2.92700-3 \quad 3.46860-3$ 3.95960-3 $4.62210-3 \quad 5.41370-3 \quad 6.19090-3 \quad 6.92650-3$ $7.47830-3 \quad 7.84680-3 \quad 8.77160-3 \quad 1.10200-2$

Em205 $\quad 3.671+10$

$f 215: p \quad 302.25 \quad 0.0 \quad 0.0 \quad 0.0$

de215 $1.50000-2 \quad 3.25000-2 \quad 5.75000-2 \quad 8.50000-2$ $1.25000-1 \quad 2.25000-1 \quad 3.50000-1 \quad 4.55000-1 \quad 5.55000-1$ $6.50000-1 \quad 8.50000-1 \quad 1.25000+0 \quad 1.75000+0 \quad 2.25000+0$ $2.75000+0 \quad 3.50000+0 \quad 4.50000+0 \quad 5.50000+0 \quad 6.50000+0$ $7.25000+0 \quad 7.75000+0 \quad 9.00000+0 \quad 1.20000+1$

$\begin{array}{llllll}\text { df215 } 2.14390-3 & 5.77600-4 & 2.71850-4 & 2.68170-4\end{array}$ $3.27670-4 \quad 5.66760-4 \quad 8.75940-4 \quad 1.08450-3 \quad 1.27970-3$ $1.44170-3 \quad 1.75630-3 \quad 2.31560-3 \quad 2.92700-3 \quad 3.46860-3$ $3.95960-3.4 .62210-3 \quad 5.41370-3 \quad 6.19090-36.92650-3$ $\begin{array}{llll}7.47830-3 & 7.84680-3 & 8.77160-3 & 1.10200-2\end{array}$

fm215 $\quad 3.671+10$

f225:p $\quad 292.25 \quad 10.0 \quad 0.0 \quad 0.0$

de225 $1.50000-2 \quad 3.25000-2 \quad 5.75000-2 \quad 8.50000-2$ $1.25000-12.25000-1 \quad 3.50000-1 \quad 4.55000-1 \quad 5.55000-1$ $6.50000-1 \quad 8.50000-1 \quad 1.25000+0 \quad 1.75000+0 \quad 2.25000+0$ $2.75000+0 \quad 3.50000+0 \quad 4.50000+0 \quad 5.50000+0 \quad 6.50000+0$ $7.25000+0 \quad 7.75000+0 \quad 9.00000+0 \quad 1.20000+1$

df225 $2.14390-3 \quad 5.77600-4 \quad 2.71850-4 \quad 2.68170-4$ $\begin{array}{llllll}3.27670-4 & 5.66760-4 & 8.75940-4 & 1.08450-3 & 1.27970-3\end{array}$ $\begin{array}{llllll}1.44170-3 & 1.75630-3 & 2.31560-3 & 2.92700-3 & 3.46860-3\end{array}$ $3.95960-3 \quad 4.62210-3 \quad 5.41370-3 \quad 6.19090-3 \quad 6.92650-3$ $7.47830-3 \quad 7.84680-3 \quad 8.77160-3 \quad 1.10200-2$

$\operatorname{tm} 225 \quad 3.671+10$

sdef pos $=0$ o 0 vec=1 0 o dir=d1 erg=fdir=d2 


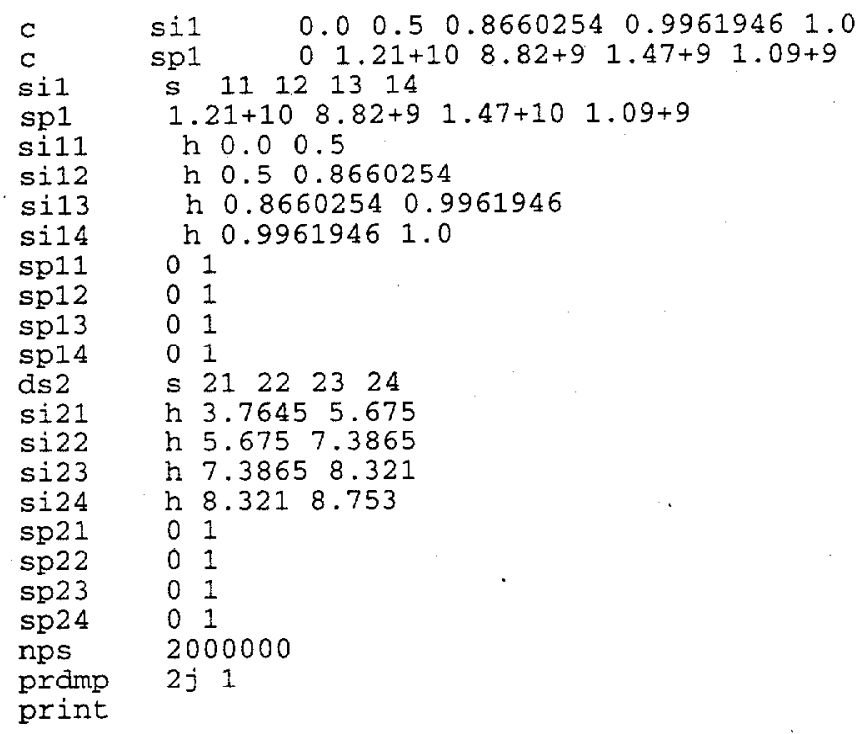


File for MCNP-4B point-detector dose-rate calculations for a 6.38 -cm-radius bare Pu sphere in the PFNA beam at the center of the truck lane (16,000,000 histories)

message: outp=pfnalc.o mctal=pfnalc.$m$

mcnp file for PFNA project bare PU sphere calculation ( $r=6.38$ )

$1 \quad-100: 128:-200: 251:-300: 305$

$2 \quad 100-128200-251300-305+403$

$\#(-400+101+224-227)$

$\#(-401+400+101+225-226)$

\# $(102-129+201-250+301-304)$

$\#(129-126+252-257+301-304)$

$\#(102-126+201-250+306-301)$

$\begin{array}{lll}3 & 1 & 0.10549 \\ 4 & 1 & 0.10549\end{array}$

$-400+101+224-227 \#(503-504)$

$-401+400+101+225-226 \#(503-504)$

$102-127+223-228+301-304$

$\#(-400+224-227) \#(-401+400+225-226)$

$6 \quad 0$

$127-124+501-502+301-304$

$\#(-401+400+225-226)$

$\begin{array}{llllll}7 & 0 & 124 & -126+254 & -255+301 & -304 \\ 8 & 5 & 8.734441-2 & 102 & -126+201-250+306 & -301\end{array}$

$9 \quad 10.10549 \quad 102-124+222-229+301-304$

$\begin{array}{llllllll} & & & \#(127+501-502) \#(-127+223-228) & \\ 10 & 1 & 0.10549 & 106-109+212-236+302-303 & \#(218-233)\end{array}$

$\begin{array}{lllllllll}11 & 1 & 0.10549 & 109 & -110+211 & -237+302 & -303 & \#(221 & -230) \\ 12 & 1 & 0.10549 & 110 & -111+210 & -238+302 & -303 & \#(221 & -230)\end{array}$

$13110.10549 \quad 111-112+209-239+302-303 \#(221-230)$

$14110.10549 \quad 112-113+208-240+302-303 \#(221-230)$

$15110.10549 \quad 113-114+207-241+302-303 \#(221-230)$

$16 \quad 1 \quad 0.10549 \quad 114-115+206-242+302-303$ \# $(221-230)$

$171 \quad 10.10549 \quad 115-116+205-243+302-303 \#(221-230)$

$18110.10549 \quad 116-117+204-244+302-303 \#(221-230)$

$19110.10549 \quad 117-118+203-245+302-303$ \# $(221-230)$

$20 \quad 10.10549 \quad 118-119+202-249+302-303 \#(221-230)$

$212 \quad 2 \quad 0.1187956 \quad 120-123+215-246+302-303 \#(221-230)$

$22 \quad 20.1187956103-109+202-249+302-303$

\#(22i -230$)$

$\#(103-104+217-234)$

$\#(104-105+216-235)$

$\#(105-107+219-232)$

$\#(107-108+219-220) \quad \#(107-108+231-232)$

23

20.1187956

$\#(106-109+212-218)$ \# $(106-109+233-236)$

23

(10 $-120+202-249+302-303$

\# $(221-230)$

$\#(109-110+211-237)$

$\#(110-111+210-238)$

$\#(111-112+209-239)$

$\#(112-113+208-240)$

\# $(113-114+207-241)$

$\#(114-115+206-242)$

$\#(115-116+205-243)$

\#( $(116-117+204-244)$

$\#(117-118+203-245)$

\# $(118-119\}$

$2433.284-2$

$121-125+201-250+301-304$

$\#(-124+214-247) \#(124+253-256)$

$\#(122+248)$ \# $(122-213)$

$25 \quad 3 \quad 3.284-2 \quad 103-107+217-234+302-303$

$\#(221-230) \#(104-220) \#(104+231)$

26

26
27

28

$45.977931-2 \quad 104-108+216-220+302-303 \#(105-219)$

$45.977931-2 \quad 104-108+231-235+302-303 \#(105+232)$

$45.977931-2102-124+201-222+301-304$

\#( $\left.\begin{array}{llllll}103 & -120 & 202 & -221 & 302 & -303\end{array}\right)$

$\#\left(\begin{array}{llllll}120 & -123 & 215 & -221 & 302 & -303\end{array}\right)$

$\#(121-214)$

29

$45.977931-2102-124+229-250+301-304$

$\#\left(\begin{array}{llllll}103 & -120 & 230 & -249 & 302 & -303\end{array}\right)$

$\#\left(\begin{array}{llllll}120 & -123 & 230 & -246 & 302 & -303\end{array}\right)$ 


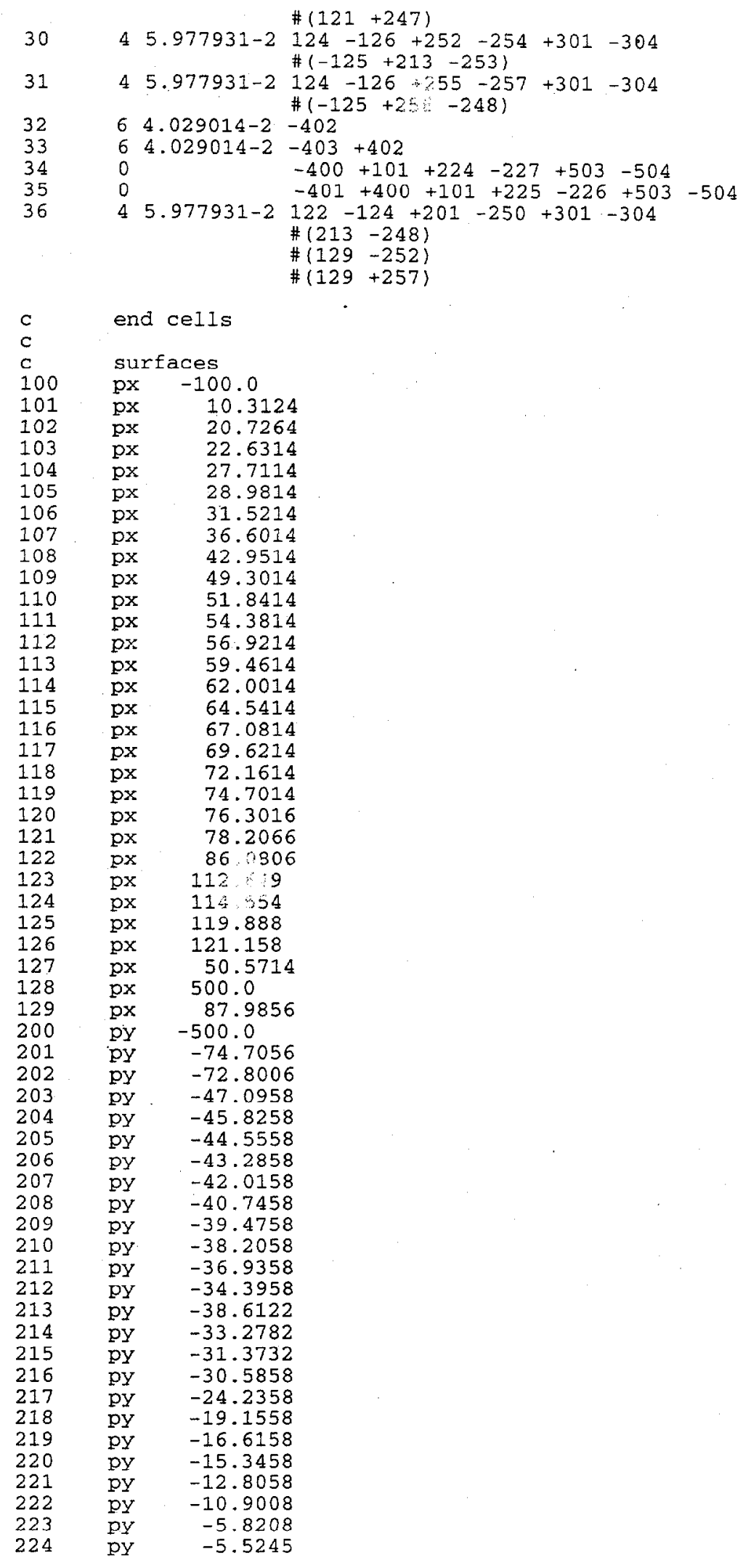



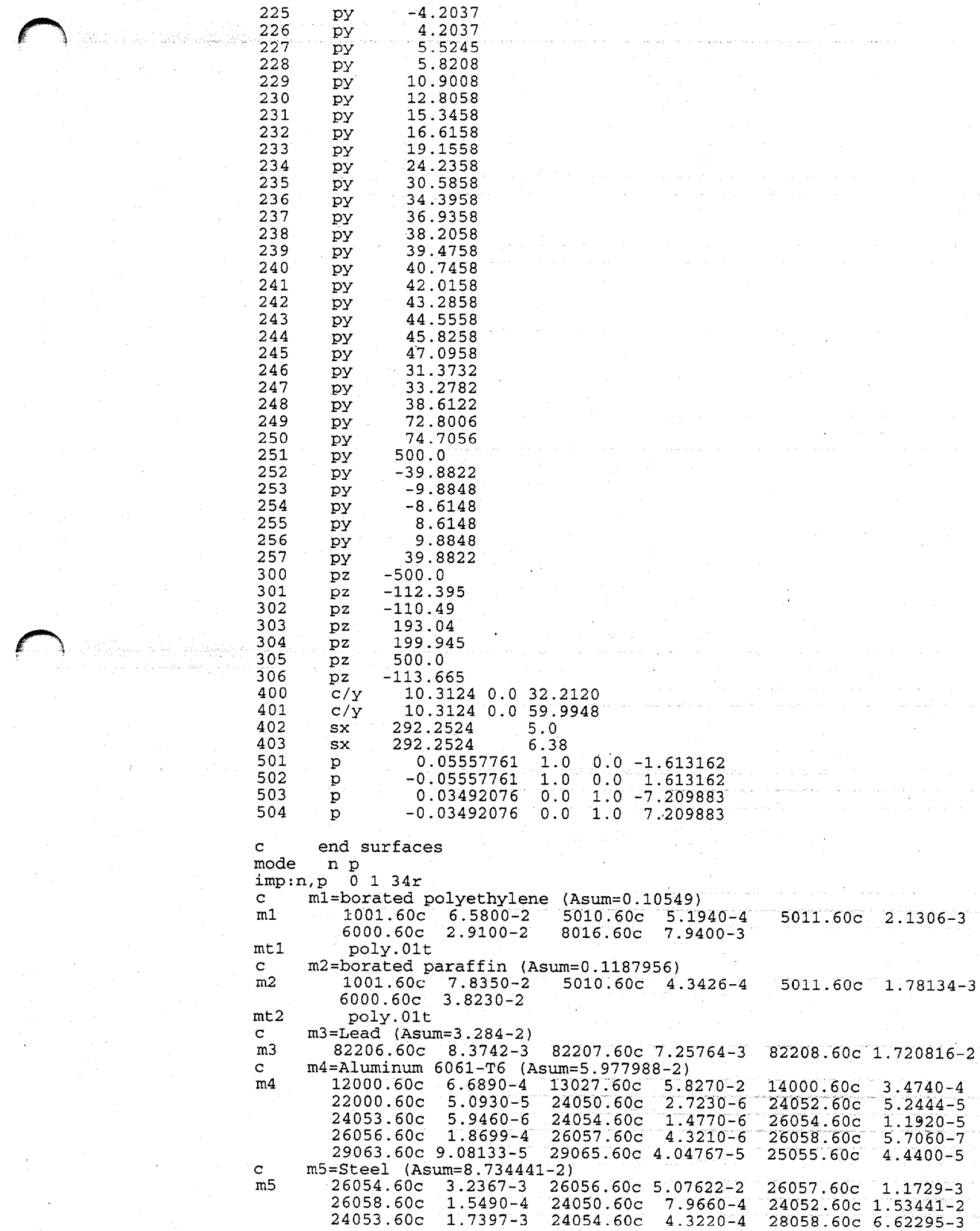


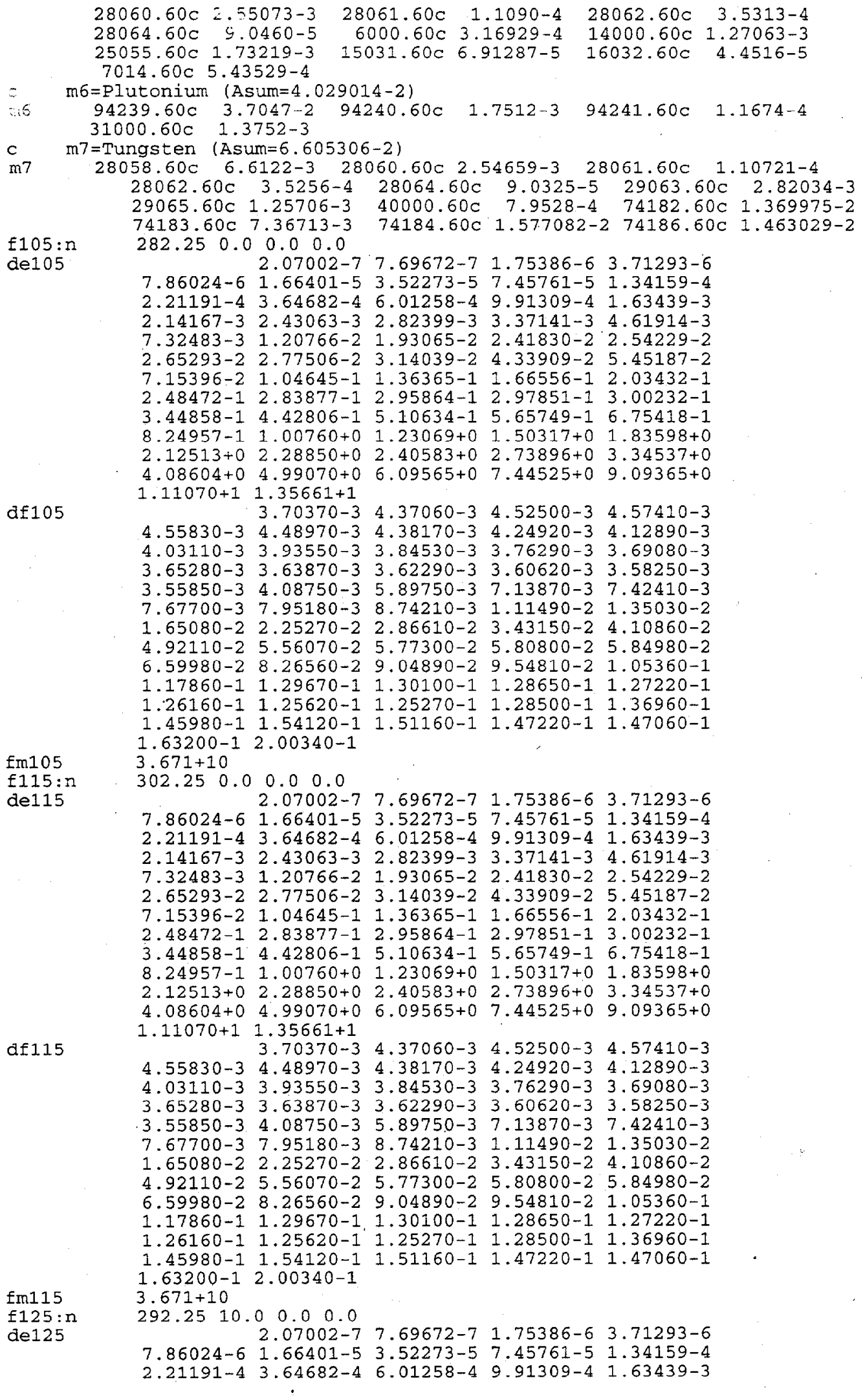


2.14167-3 $2.43063-3 \quad 2.82399-3 \quad 3.37141-3 \quad 4.61914-3$

$7.32483-3 \quad 1.20766-2 \quad 1.93065-2 \quad 2.41830-2 \quad 2.54229-2$

2.65293-2 2.77506-2 3.14039-2 4.33909-2 5. $45187-2$

7.15396-2 1.04645-1 1.36365-1 1.66556-1 2.03432-1

$2.48472-1 \quad 2.83877-1 \quad 2.95864-1 \quad 2.97851-1 \quad 3.00232-1$

3.44858-1 4.42806-1 5.10634-1 $5.65749-1 \quad 6.75418-1$

$8.24957-1 \quad 1.00760+0 \quad 1.23069+0 \quad 1.50317+0 \quad 1.83598+0$

$\begin{array}{llllll}2.12513+0 & 2.28850+0 & 2.40583+0 & 2.73896+0 & 3.34537+0\end{array}$

$4.08604+0 \quad 4.99070+0 \quad 6.09565+0 \quad 7.44525+0 \quad 9.09365+0$

$1.11070+1 \quad 1.35661+1$

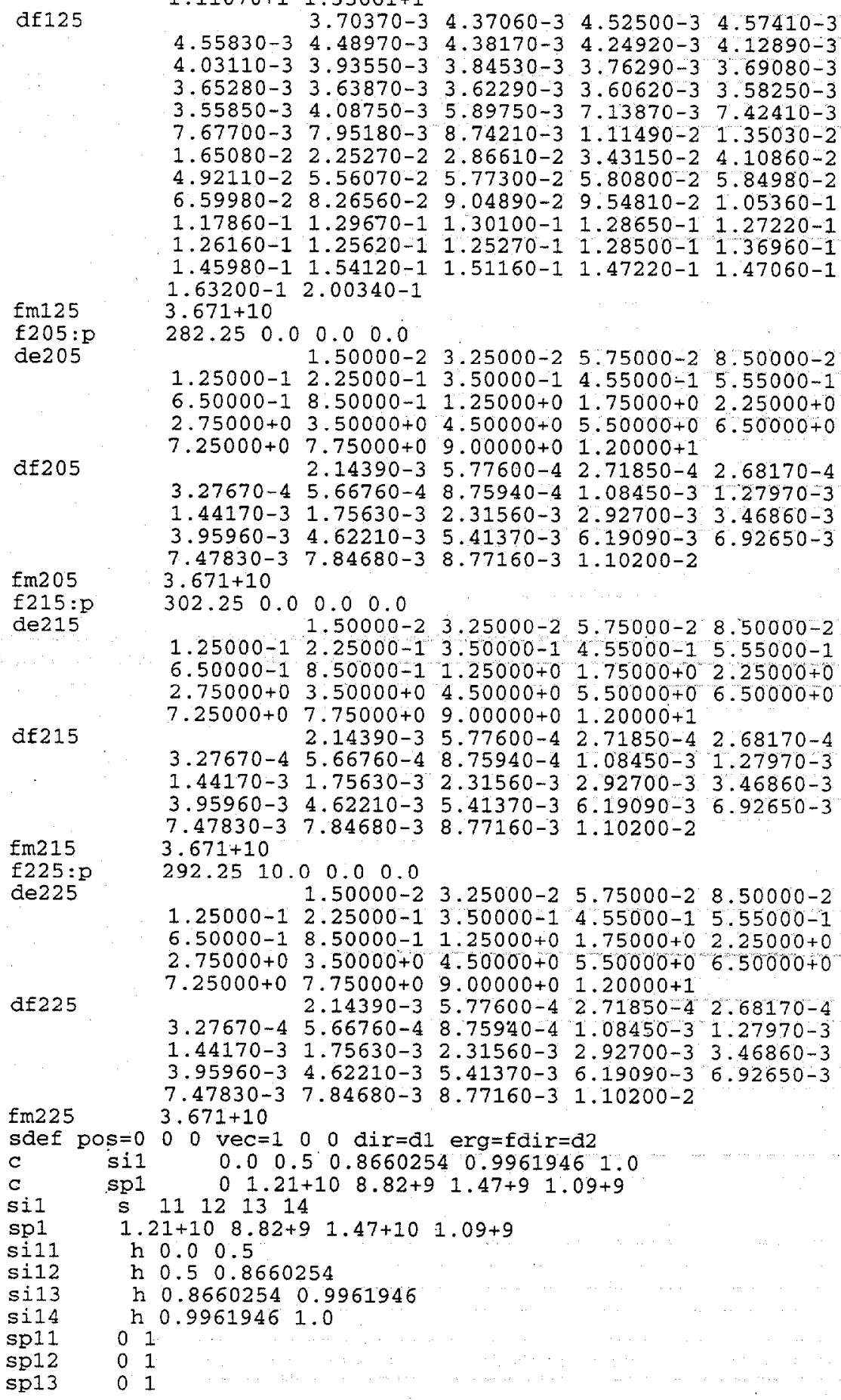




\begin{tabular}{|c|c|}
\hline \multirow{2}{*}{\multicolumn{2}{|c|}{ sp14 }} \\
\hline & \\
\hline $\begin{array}{l}\text { ds } 2 \\
\text { si2 } 21\end{array}$ & $\begin{array}{lllll}s & 21 & 22 & 23 & 24 \\
\text { h } & 3.7645 & 5.675\end{array}$ \\
\hline $\operatorname{si22}$ & h $5.675 \quad 7.3865$ \\
\hline si23 & h $7.3865 \quad 8.321$ \\
\hline si24 & h 8.3218 .753 \\
\hline $\operatorname{sp} 21$ & 01 \\
\hline $\operatorname{sp} 22$ & 01 \\
\hline $\operatorname{sp} 23$ & 01 \\
\hline $\operatorname{sp} 24$ & $\begin{array}{ll}0 & 1\end{array}$ \\
\hline nps & 16000000 \\
\hline prdmp & $2 j 1$ \\
\hline print & \\
\hline
\end{tabular}


File for MCNP-4B point-detector dose-rate calculations for a 6.35 -cm-radius bare Pu sphere in the PFNA beam at the center of the truck lane $(16,000,000$ histories)

message: outp=pfnale. 0 motal=pfnale $\cdot m$

monp file for PFNA project bare PU sphere calculation ( $r=6.38$ )

$1 \quad 0 \quad-100: 128:-200: 251:-300: 305$

$100-128 \quad 200-251 \quad 300-305+403$

$\#(-400+101+224-227)$

$\#(-401+400+101+225-226)$

$\#(102-129+201-250+301-304)$

$\#(129-126+252-257+301-304)$

$\#(102-126+201-250+306-301)$

$\begin{array}{lll}3 & 1 & 0.10549 \\ 4 & 1 & 0.10549\end{array}$

$-400+101+224-227 \#(503-504)$

$-401+400+101+225-226 \#(503-504)$

$102-127+223-228+301-304$

$\#(-400+224-227) \#(-401+400+225-226)$

6.0

$127-124+501-502+301-304$

$(-401+400+225-226)$

$7 \quad 0 \quad 124-126+254-255+301-304$

$8558.734441-2102-126+201-250+306-301$

$91 \quad 1 \quad 0.10549 \quad 102-124+222-229+301-304$

$10 \quad \#(127+501-502) \#(-127+223-228)$

$10110.10549 \quad 106-109+212-236+302-303 \#(218-233)$

$11110.10549 \quad 109-110+211-237+302-303 \#(221-230)$

$12 \quad 1 \quad 0.10549 \quad 110-111+210-238+302-303 \#(221-230)$

$111-112+209-239+302-303 \#(221-230)$

$14 \quad \begin{array}{lllllll}1 & 0.10549 & 112 & -113+208-240+302 & -303 \#(221-230)\end{array}$

$15110.10549 \quad 113-114+207-241+302-303 \#(221-230)$

$16110.10549 \quad 114-115+206-242+302-303 \#(221-230)$

$17 \quad 1 \quad 0.10549 \quad 115-116+205-243+302-303 \#(221-230)$

$181 \quad 1 \quad 0.10549 \quad 116-117+204-244+302-303 \#(221-230)$

$19 \quad \begin{array}{llllllll}1 & 0.10549 & 117 & -118+203 & -245+302 & -303 \#(221-230)\end{array}$

$20 \quad 1 \quad 0.10549 \quad 118-119+202-249+302-303 \#(221-230)$

$120-123+215-246+302-303 \#(221-230)$

$20.1187956 \quad 103-109+202-249+302-303$

$\#(221-230)$

$\#(103-104+217-234)$

$\#(104-105+216-235)$

$\#(105-107+219-232)$

$\#(107-108+219-220) \quad \#(107-108+231-232)$

$\#(106-109+212-218)$ \# $(106-109+233-236)$

$109-120+202-249+302-303$

\# $(221-230)$

$\#(109-110+211-237)$

$\#(110-111+210-238)$

$\#(111-112+209-239)$

$\#(112-113+208-240)$

$\#(113-114+207-241)$

$\#(114-115+206-242)$

$\#(115-116+205-243)$

$\#(116-117+204-244)$

$\#(117-118+203-245)$

\# (118-119)

$2433.284-2 \quad 121-125+201-250+301-304$

$\#(-124+214-247) \#(124+253-256)$

\#(122+248) \#(122-213)

$25 \quad 3 \quad 3.284-2 \quad 103-107+217-234+302-303$

$\#(221-230) \#(104-220) \#(104+231)$

$2645.977931-2 \quad 104-108+216-220+302-303 \#(105-219)$

$2745.977931-2 \quad 104-108+231-235+302-303 \#(105+232)$

$2845.977931-2 \quad 102-124+201-222+301-304$

\#(103 $-120 \quad 202 \quad-221 \quad 302-303)$

\#( $\left.\begin{array}{llllll}120 & -123 & 215 & -221 & 302 & -303\end{array}\right)$

\# $(121-214)$

$2945.977931-2102-124+229-250+301-304$

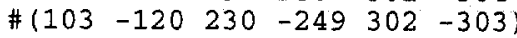

\#( $\left(\begin{array}{llllll}120 & -123 & 230 & -246 & 302 & -303\end{array}\right)$ 


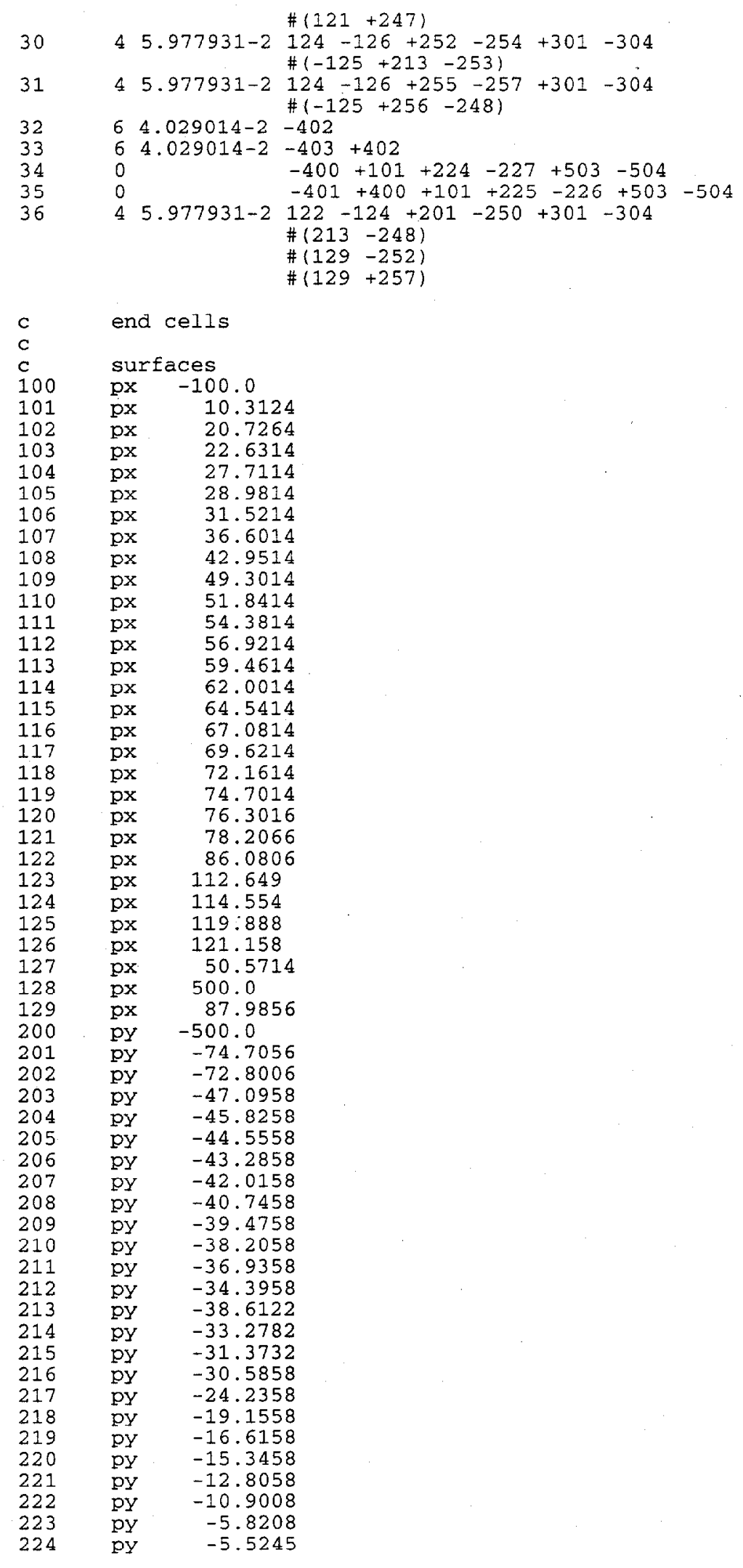




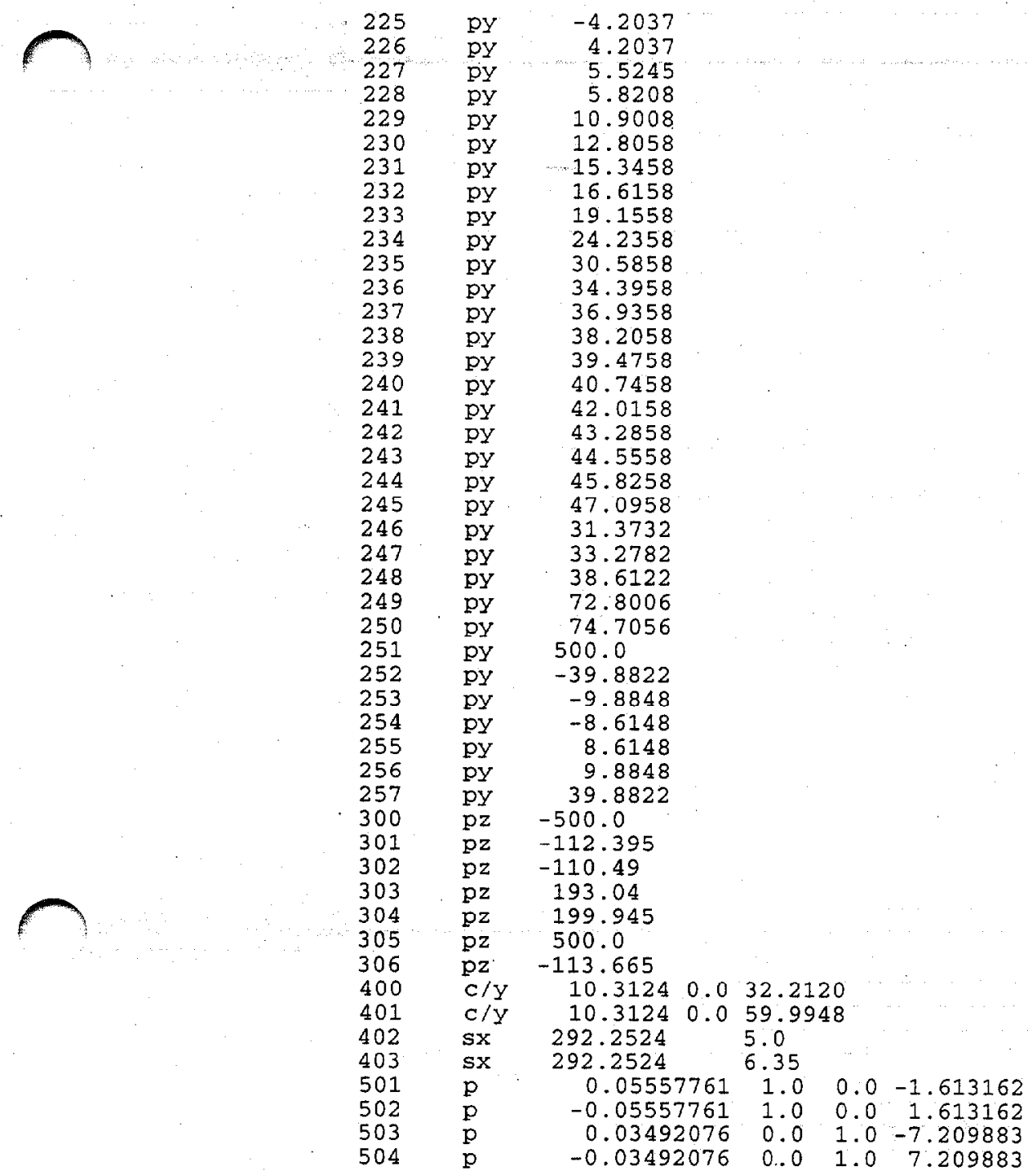

$\mathrm{c}$

end surfaces

mode

imp: $n, p$ o $134 r$

c $\mathrm{ml}=$ borated polyethylene (Asum $=0.10549$ )

$\begin{array}{lllllll}\mathrm{m} 1 & 1001.60 \mathrm{C} & 6.5800-2 & 5010.60 \mathrm{C} & 5.1940-4 & 5011.60 \mathrm{C} & 2.1306-3\end{array}$

mt1 poly.01t

c $\mathrm{m} 2$ =borated paraffin (Asum=0.1187956)

$\mathrm{m} 2 \quad 1001.60 \mathrm{c} \quad 7.8350-2 \quad 5010.60 \mathrm{c} \quad 4.3426-4 \quad 5011.60 \mathrm{c} \quad 1.78134-3$ $6000.60 \mathrm{C} \quad 3.8230-2$

mt2 poly.01t

c $\mathrm{m} 3=$ Lead (Asum $=3.284-2$ )

$\mathrm{m} 3 \quad 82206.60 \mathrm{c} \quad 8.3742-3 \quad 82207.60 \mathrm{c} \quad 7.25764-3 \quad 82208.60 \mathrm{c} \quad 1.720816-2$

C m4=Aluminum 6061-T6 (Asum=5.977988-2)

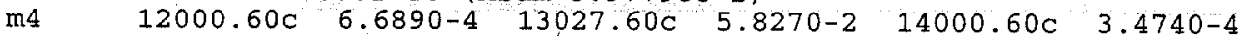

$22000.60 \mathrm{C} \quad 5.0930-5 \quad 24050.60 \mathrm{c} \quad 2.7230-6 \quad 24052.60 \mathrm{c} \quad 5.2444-5$

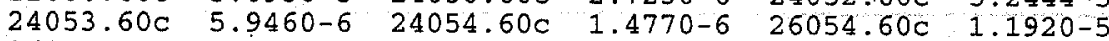

$26056.60 \mathrm{c} \quad 1.8699-4 \quad 26057.60 \mathrm{c} \quad 4.3210-6 \quad 26058.60 \mathrm{c} \quad 5.7060-7$

C m5=Steel. (Asum $=8.734441-2$ )

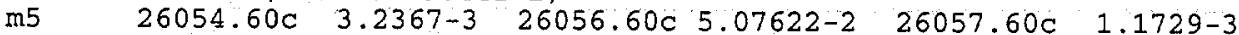

$26058.60 \mathrm{c} \quad 1.5490-4 \quad 24050.60 \mathrm{c} \quad 7.9660-4 \quad 24052.60 \mathrm{c} \quad 1.53441-2$

$24053.60 \mathrm{c} \quad 1.7397-3 \quad 24054.60 \mathrm{c} \quad 4.3220-4 \quad 28058.60 \mathrm{c} \quad 6.62295-3$ 


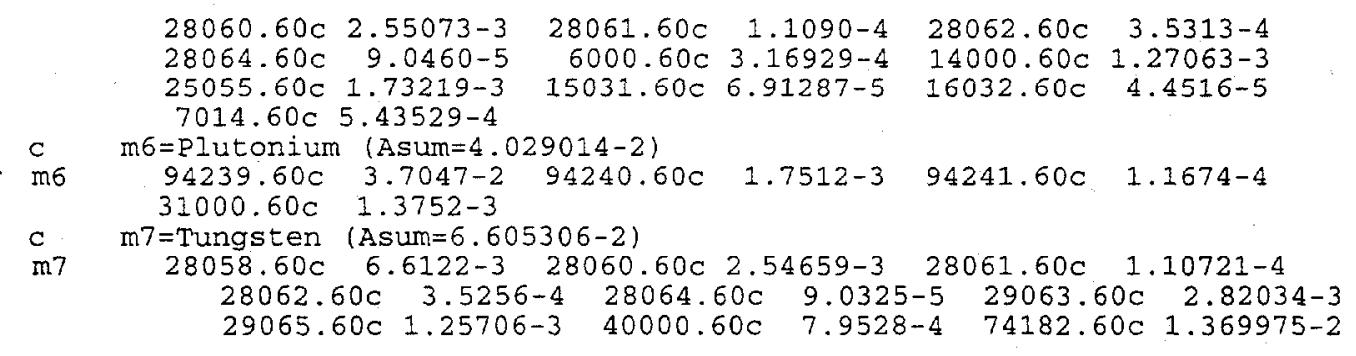


$74183.60 \mathrm{C} \quad 7.36713-3 \quad 74184.60 \mathrm{C} \quad 1.577082-2 \quad 74186.60 \mathrm{C} \quad 1.463029-2$ 282.250 .00 .00 .0

f $105: n$ de 105

df105

fm105 f115:n de115

$\operatorname{df1} 15$

$\mathrm{fm} 115$ f125:n de125

df125 $\begin{array}{rllll}2.07002-7 & 7.69672-7 & 1.75386-6 & 3.71293-6\end{array}$

$7.86024-6 \quad 1.66401-5 \quad 3.52273-5 \quad 7.45761-5 \quad 1.34159-4$

2.21191-4 $3.64682-4 \quad 6.01258-4 \quad 9.91309-4 \quad 1.63439-3$

$2.14167-3 \quad 2.43063-3 \quad 2.82399-3 \quad 3.37141-3 \quad 4.61914-3$

$7.32483-3 \quad 1.20766-2 \quad 1.93065-2 \quad 2.41830-2 \quad 2.54229-2$

$2.65293-2 \quad 2.77506-2 \quad 3.14039-2 \quad 4.33909-2 \quad 5.45187-2$

7.15396-2 $1.04645-1 \quad 1.36365-1 \quad 1.66556-1 \quad 2.03432-1$

$\begin{array}{lllllll}2.48472-1 & 2.83877-1 & 2.95864-1 & 2.97851-1 & 3.00232-1\end{array}$

3.44858-1 4.42806-1 5.10634-1 $5.65749-1 \quad 6.75418-1$

$8.24957-1 \quad 1.00760+0 \quad 1.23069+0 \quad 1.50317+0 \quad 1.83598+0$

$2.12513+0 \quad 2.28850+0 \quad 2.40583+0 \quad 2.73896+0 \quad 3.34537+0$

$4.08604+0 \quad 4.99070+0 \quad 6.09565+0 \quad 7.44525+0 \quad 9.09365+0$

$1.11070+11.35661+1$

$3.70370-3 \quad 4.37060-3 \quad 4.52500-3 \quad 4.57410-3$

$4.55830-3 \quad 4.48970-3 \quad 4.38170-3 \quad 4.24920-3 \quad 4.12890-3$

$4.03110-3 \quad 3.93550-3 \quad 3.84530-3 \quad 3.76290-3 \quad 3.69080-3$

$3.65280-3 \quad 3.63870-3 \quad 3.62290-3 \quad 3.60620-3 \quad 3.58250-3$

$\begin{array}{llllll}3.55850-3 & 4.08750-3 & 5.89750-3 & 7.13870-3 & 7.42410-3\end{array}$

$\begin{array}{llllll}7.67700-3 & 7.95180-3 & 8.74210-3 & 1.11490-2 & 1.35030-2\end{array}$

$1.65080-2 \quad 2.25270-2 \quad 2.86610-2 \quad 3.43150-2 \quad 4.10860-2$

4.92110-2 5.56070-2 $5.77300-2 \quad 5.80800-2 \quad 5.84980-2$

$6.59980-2 \quad 8.26560-2 \quad 9.04890-2 \quad 9.54810-2 \quad 1.05360-1$

1.17860-1 $1.29670-1 \quad 1.30100-1 \quad 1.28650-1 \quad 1.27220-1$

$1.26160-1$ 1.25620-1 $1.25270-1 \quad 1.28500-1 \quad 1.36960-1$

$1.45980-1$ 1.54120-1 $1.51160-1$ 1.47220-1 1.47060-1

$1.63200-12.00340-1$

$3.671+10$

$302.25 \quad 0.0 \quad 0.0 \quad 0.0$

$2.07002-7 \quad 7.69672-7 \quad 1.75386-6 \quad 3.71293-6$

$\begin{array}{llllll}7.86024-6 & 1.66401-5 & 3.52273-5 & 7.45761-5 & 1.34159-4\end{array}$

$\begin{array}{llllll}2.21191-4 & 3.64682-4 & 6.01258-4 & 9.91309-4 & 1.63439-3\end{array}$

2.14167-3 2.43063-3 2.82399-3 $3.37141-3 \quad 4.61914-3$

$7.32483-3$ 1.20766-2 $1.93065-2 \quad 2.41830-2 \quad 2.54229-2$

2.65293-2 2.77506-2 $3.14039-2 \quad 4.33909-2 \quad 5.45187-2$

7.15396-2 1.04645-1 $1.36365-1 \quad 1.66556-1 \quad 2.03432-1$

$2.48472-1 \quad 2.83877-1 \quad 2.95864-1 \quad 2.97851-1.3 .00232-1$

3.44858-1 4.42806-1 $5.10634-1 \quad 5.65749-1 \quad 6.75418-1$

$8.24957-1 \quad 1.00760+0 \quad 1.23069+0 \quad 1.50317+0 \quad 1.83598+0$

$2.12513+0 \quad 2.28850+0 \quad 2.40583+0 \quad 2.73896+0 \quad 3.34537+0$

$4.08604+0 \quad 4.99070+0 \quad 6.09565+0 \quad 7.44525+0 \quad 9.09365+0$ $1.11070+11.35661+1$

3.70370-3 $4.37060-3 \quad 4.52500-3 \quad 4.57410-3$

4.55830-3 4.48970-3 $4.38170-3 \quad 4.24920-3 \quad 4.12890-3$

$4.03110-3 \quad 3.93550-3 \quad 3.84530-3 \quad 3.76290-3 \quad 3.69080-3$

$3.65280-3 \quad 3.63870-3 \quad 3.62290-3 \quad 3.60620-3 \quad 3.58250-3$

$\begin{array}{llllll}3.55850-3 & 4.08750-3 & 5.89750-3 & 7.13870-3 & 7.42410-3\end{array}$

$7.67700-3 \quad 7.95180-3 \quad 8.74210-3 \quad 1.11490-2 \quad 1.35030-2$

$1.65080-2 \quad 2.25270-2 \quad 2.86610-2 \quad 3.43150-2 \quad 4.10860-2$

$4.92110-2 \quad 5.56070-2 \quad 5.77300-2 \quad 5.80800-2 \quad 5.84980-2$

$6.59980-2 \quad 8.26560-2 \cdot 9.04890-2 \quad 9.54810-2 \quad 1.05360-1$

$1.17860-1 \quad 1.29670-1 \quad 1.30100-1 \quad 1.28650-1 \quad 1.27220-1$

$1.26160-1$ 1.25620-1 $1.25270-1 \quad 1.28500-1$ 1.36960-1

$1.45980-1 \quad 1.54120-1 \quad 1.51160-1 \quad 1.47220-1 \quad 1.47060-1$ 1.63200-1 2.00340-1

$3.671+10$

$292.2510 .0 \quad 0.0 \quad 0.0$

$2.07002-7 \quad 7.69672-7 \quad 1.75386-6 \quad 3.71293-6$

$7.86024-6 \quad 1.66401-5 \quad 3.52273-5 \quad 7.45761-5 \quad 1.34159-4$

2.21191-4 $3.64682-4 \quad 6.01258-4 \quad 9.91309-4 \quad 1.63439-3$

$2.14167-3 \quad 2.43063-32.82399-3 \quad 3.37141-3 \quad 4.61914-3$

7.32483-3 $1.20766-2$ 1.93065-2 $2.41830-2 \quad 2.54229-2$

2.65293-2 2.77506-2 3.14039-2 $4.33909-2$ 5.45187-2

$7.15396-2$ 1.04645-1 $1.36365-1 \quad 1.66556-1 \quad 2.03432-1$

2.48472-1 $2.83877-1 \quad 2.95864-1 \quad 2.97851-1 \quad 3.00232-1$

3.44858-1 4.42806-1 5.10634-1 $5.65749-1 \quad 6.75418-1$

$8.24957-1 \quad 1.00760+0.1 .23069+0 \quad 1.50317+0.1 .83598+0$

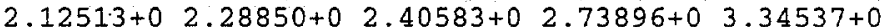

$4.08604+0 \quad 4.99070+0 \quad 6.09565+0 \quad 7.44525+0 \quad 9.09365+0$

$1.11070+1 \quad 1.35661+1$

$3.70370-3 \quad 4.37060-3 \quad 4.52500-3 \quad 4.57410-3$ 
$4.55830-3 \quad 4.48970-3 \quad 4.38170-3 \quad 4.24920-3 \quad 4.12890-3$ $4.03110-3 \quad 3.93550-3 \quad 3.84530-3 \quad 3.76290-3 \quad 3.69080-3$ $3.65280-3 \quad 3.63870-3 \quad 3.62290-3 \quad 3.60620-3 \quad 3.58250-3$ $3.55850-3 \quad 4.08750-3 \quad 5.89750-3 \quad 7.13870-3 \quad 7.42410-3$ $\begin{array}{llllll}7.67700-3 & 7.95180-3 & 8.74210-3 & 1.11490-2 & 1.35030-2\end{array}$ $1.65080-2 \quad 2.25270-2 \quad 2.86610-2 \quad 3.43150-2 \quad 4.10860-2$ $4.92110-2 \quad 5.56070-2 \quad 5.77300-2 \quad 5.80800-2 \quad 5.84980-2$ $6.59980-2 \quad 8.26560-2 \quad 9.04890-2 \quad 9.54810-2 \quad 1.05360-1$ $1.17860-1 \quad 1.29670-1 \quad 1.30100-1 \quad 1.28650-1 \quad 1.27220-1$ $1.26160-1$ 1.25620-1 $1.25270-1$ 1.28500-1 $1.36960-1$ $1.45980-1$ 1.54120-1 1.51160-1 $1.47220-1 \quad 1.47060-1$ $1.63200-12.00340-1$

$\operatorname{fm} 125$ 3. $671+10$

f205:p

$282.250 .0 \quad 0.0 \quad 0.0$

de 205 $\begin{array}{llllll}1.25000-1 & 2.25000-1 & 3.50000-1 & 4.55000-1 & 5.55000-1\end{array}$ $6.50000-1 \quad 8.50000-1 \quad 1.25000+01.75000+02.25000+0$ $2.75000+0 \quad 3.50000+0 \quad 4.50000+0 \quad 5.50000+0 \quad 6.50000+0$ $7.25000+0 \quad 7.75000+09.00000+0 \quad 1.20000+1$

df 205 $2.14390-3 \quad 5.77600-4 \quad 2.71850-4 \quad 2.68170-4$ $3.27670-4 \quad 5.66760-4 \quad 8.75940-4 \quad 1.08450-3 \quad 1.27970-3$ $\begin{array}{llllll}1.44170-3 & 1.75630-3 & 2.31560-3 & 2.92700-3 & 3.46860-3\end{array}$ $3.95960-3 \quad 4.62210-3 \cdot 5.41370-3 \quad 6.19090-3 \quad 6.92650-3$ $7.47830-3 \quad 7.84680-3 \quad 8.77160-3 \quad 1.10200-2$

fm205 $\quad 3.671+10$

$£ 215: p$ $302.250 .0 \quad 0.0 \quad 0.0$

de 215 $1.50000-2 \quad 3.25000-2 \quad 5.75000-2 \quad 8.50000-2$ $1.25000-1 \quad 2.25000-1 \quad 3.50000-1 \quad 4.55000-1 \quad 5.55000-1$ $6.50000-1 \quad 8.50000-1 \quad 1.25000+0 \quad 1.75000+0 \quad 2.25000+0$ $2.75000+0 \quad 3.50000+0 \quad 4.50000+0 \quad 5.50000+06.50000+0$ $7.25000+07.75000+0 \quad 9.00000+0 \quad 1.20000+1$

$\operatorname{dE} 215$ $2.14390-3 \quad 5.77600-4 \quad 2.71850-4 \quad 2.68170-4$ $3.27670-4 \quad 5.66760-4 \quad 8.75940-4 \quad 1.08450-3 \quad 1.27970-3$ $1.44170-3 \quad 1.75630-3 \quad 2.31560-3 \quad 2.92700-3 \quad 3.46860-3$ $3.95960-3 \quad 4.62210-3 \quad 5.41370-3 \quad 6.19090-3 \quad 6.92650-3$ $7.47830-3 \quad 7.84680-3 \quad 8.77160-3 \quad 1.10200-2$

$\operatorname{fm} 215 \quad 3.671+10$

f225:p 292.25 10.00 .00 .0

de225 $1.50000-2 \quad 3.25000-2 \quad 5.75000-2 \quad 8.50000-2$ $1.25000-1 \quad 2.25000-1 \quad 3.50000-1 \quad 4.55000-1 \quad 5.55000-1$ $6.50000-1 \quad 8.50000-1 \quad 1.25000+0 \quad 1.75000+02.25000+0$ $2.75000+0 \quad 3.50000+0 \quad 4.50000+0 \quad 5.50000+0 \quad 6.50000+0$ $7.25000+0 \quad 7.75000+0 \quad 9.00000+0 \quad 1.20000+1$ $2.14390-3 \quad 5.77600-4 \quad 2.71850-4 \quad 2.68170-4$

df 225

$3.27670-4 \quad 5.66760-4 \quad 8.75940-4 \quad 1.08450-3 \quad 1.27970-3$ $\begin{array}{lllll}1.44170-3 & 1.75630-3 & 2.31560-3 & 2.92700-3 & 3.46860-3\end{array}$ $3.95960-3 \quad 4.62210-3 \quad 5.41370-3 \quad 6.19090-3 \quad 6.92650-3$ $7.47830-37.84680-38.77160-3 \quad 1.10200-2$

$\operatorname{sm} 225 \quad 3.671+10$

sdef pos $=0$ o 0 vec $=1 \quad 0 \quad 0$ dir $=d 1$ erg=fdir=d2

c sil $\quad 0.00 .50 .86602540 .99619461 .0$

C spl $\quad 01.21+108.82+91.47+91.09+9$

sil s $11 \quad 12 \quad 1314$

sp1 $1.21+108.82+9 \quad 1.47+10 \quad 1.09+9$

si11 h 0.00 .5

$\begin{array}{llll}\text { si12 h } & 0.5 & 0.8660254\end{array}$

si13 h 0.86602540 .9961946

sii4 h $0.9961946 \quad 1.0$

$\operatorname{sp} 11 \quad 0$

sp12 01

$\operatorname{sp} 13 \quad 0 \quad 1$

$\operatorname{sp} 14 \quad 0$

$\begin{array}{llllll}\text { ds } 2 & s & 21 & 22 & 23 & 24\end{array}$

si21 h $3.7645 \quad 5.675$

si22 h $5.675 \quad 7.3865$

si23 h 7.38658 .321

si24 h 8.3218 .753

$\operatorname{sp2} 1001$

$\operatorname{sp} 22 \quad 01$

$\operatorname{sp23} \quad 01$

$\operatorname{sp} 24 \quad 01$

nps 16000000 
prdmp $2 j 1$

print

$\bigcap$ 
File for MCNP-4B point-detector dose-rate background calculations for the bare Pu spheres in the PFNA beam at the center of the truck lane $(400,000$ histories)

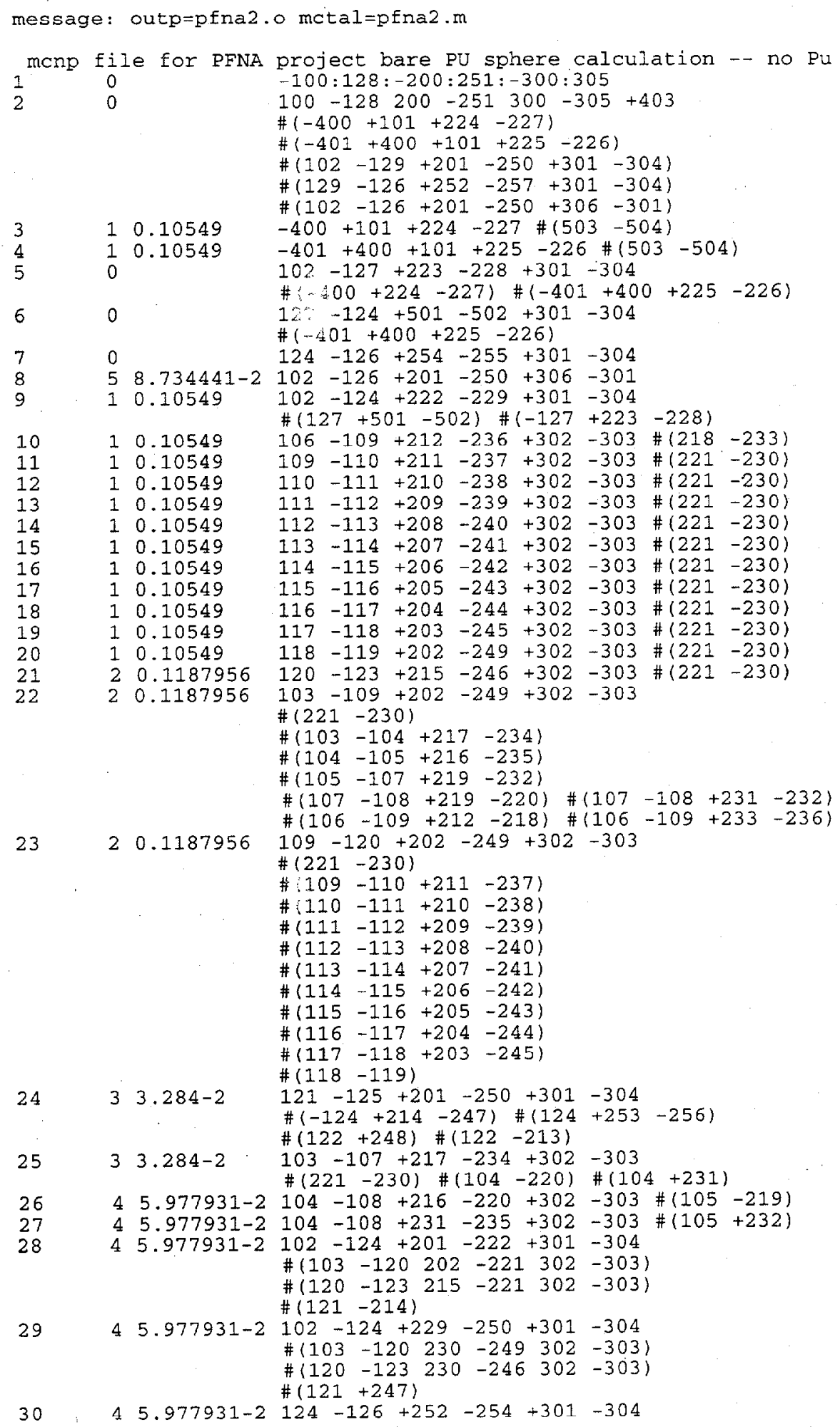




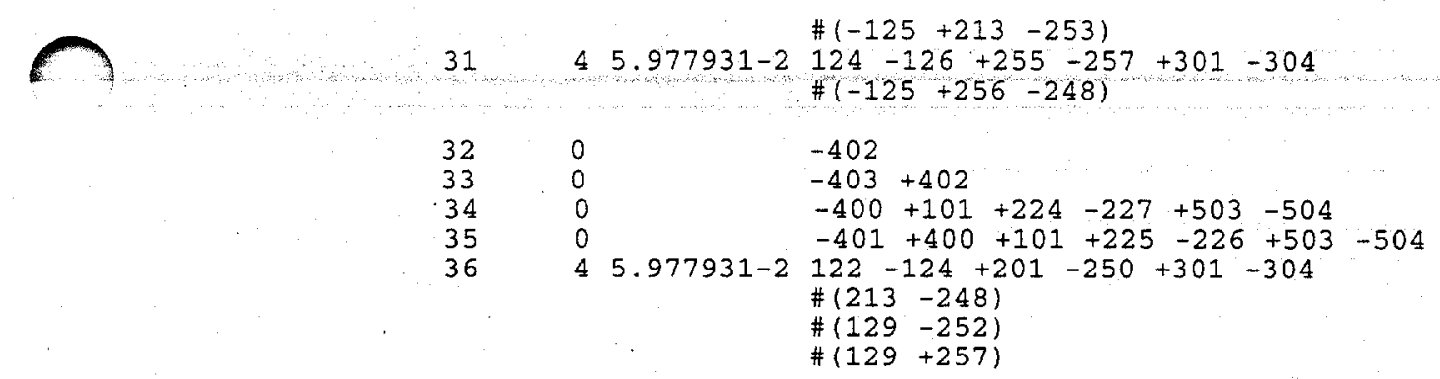

\begin{tabular}{|c|c|c|}
\hline $\begin{array}{l}c \\
c\end{array}$ & \multicolumn{2}{|c|}{ end cells } \\
\hline c & \multicolumn{2}{|c|}{ surfaces } \\
\hline 100 & $\mathrm{px}$ & -100.0 \\
\hline 101 & $\mathrm{px}$ & 10.3124 \\
\hline 102 & $\mathrm{px}$ & 20.7264 \\
\hline 103 & $p x$ & 22.6314 \\
\hline 104 & $\mathrm{px}$ & 27.7114 \\
\hline 105 & $\mathrm{px}$ & 28.9814 \\
\hline 106 & $\mathrm{px}$ & 31.5214 \\
\hline 107 & $\mathrm{px}$ & 36.6014 \\
\hline 108 & $\mathrm{px}$ & 42.9514 \\
\hline 109 & $p x$ & 49.3014 \\
\hline 110 & $\mathrm{px}$ & 51.8414 \\
\hline 111 & $\mathrm{px}$ & 54.3814 \\
\hline 112 & $\mathrm{px}$ & 56.9214 \\
\hline 113 & $\mathrm{px}$ & 59.4614 \\
\hline 114 & $\mathrm{px}$ & 62.0014 \\
\hline 115 & $\mathrm{px}$ & 64.5414 \\
\hline 116 & $\mathrm{px}$ & 67.0814 \\
\hline 117 & $\mathrm{px}$ & 69.6214 \\
\hline 118 & $p x$ & 72.1614 \\
\hline 119 & $\mathrm{px}$ & 74.7014 \\
\hline 120 & $\mathrm{px}$ & 76.3016 \\
\hline 121 & $\mathrm{px}$ & 78.2066 \\
\hline 122 & $\mathrm{px}$ & 86.0806 \\
\hline 123 & $\mathrm{px}$ & 112.649 \\
\hline 124 & $\mathrm{px}$ & 114.554 \\
\hline 125 & $\mathrm{px}$ & 119.888 \\
\hline 126 & $\mathrm{px}$ & 121.158 \\
\hline 127 & $\mathrm{px}$. & 50.5714 \\
\hline 128 & $\mathrm{px}$ & 500.0 \\
\hline 129 & $\mathrm{px}$ & 87.9856 \\
\hline 200 & py & -500.0 \\
\hline 201 & py & -74.7056 \\
\hline 202 & py & -72.8006 \\
\hline 203 & py & -47.0958 \\
\hline 204 & DY & -45.8258 \\
\hline 205 & PY & -44.5558 \\
\hline 206 & py & -43.2858 \\
\hline 207 & py & -42.0158 \\
\hline 208 & py & -40.7458 \\
\hline 209 & DY & -39.4758 \\
\hline 210 & py & -38.2058 \\
\hline 211 & PY & -36.9358 \\
\hline 212 & pY & -34.3958 \\
\hline 213 & py & -38.6122 \\
\hline 214 & py & -33.2782 \\
\hline 215 & py & -31.3732 \\
\hline 216 & py & -30.5858 \\
\hline 217 & py & -24.2358 \\
\hline 218 & py & -19.1558 \\
\hline 219 & py & -16.6158 \\
\hline 220 & py & -15.3458 \\
\hline 221 & py & -12.8058 \\
\hline 222 & PY & $-10,9008$ \\
\hline 223 & py & -5.8208 \\
\hline 224 & py & -5.5245 \\
\hline 225 & py & -4.2037 \\
\hline
\end{tabular}




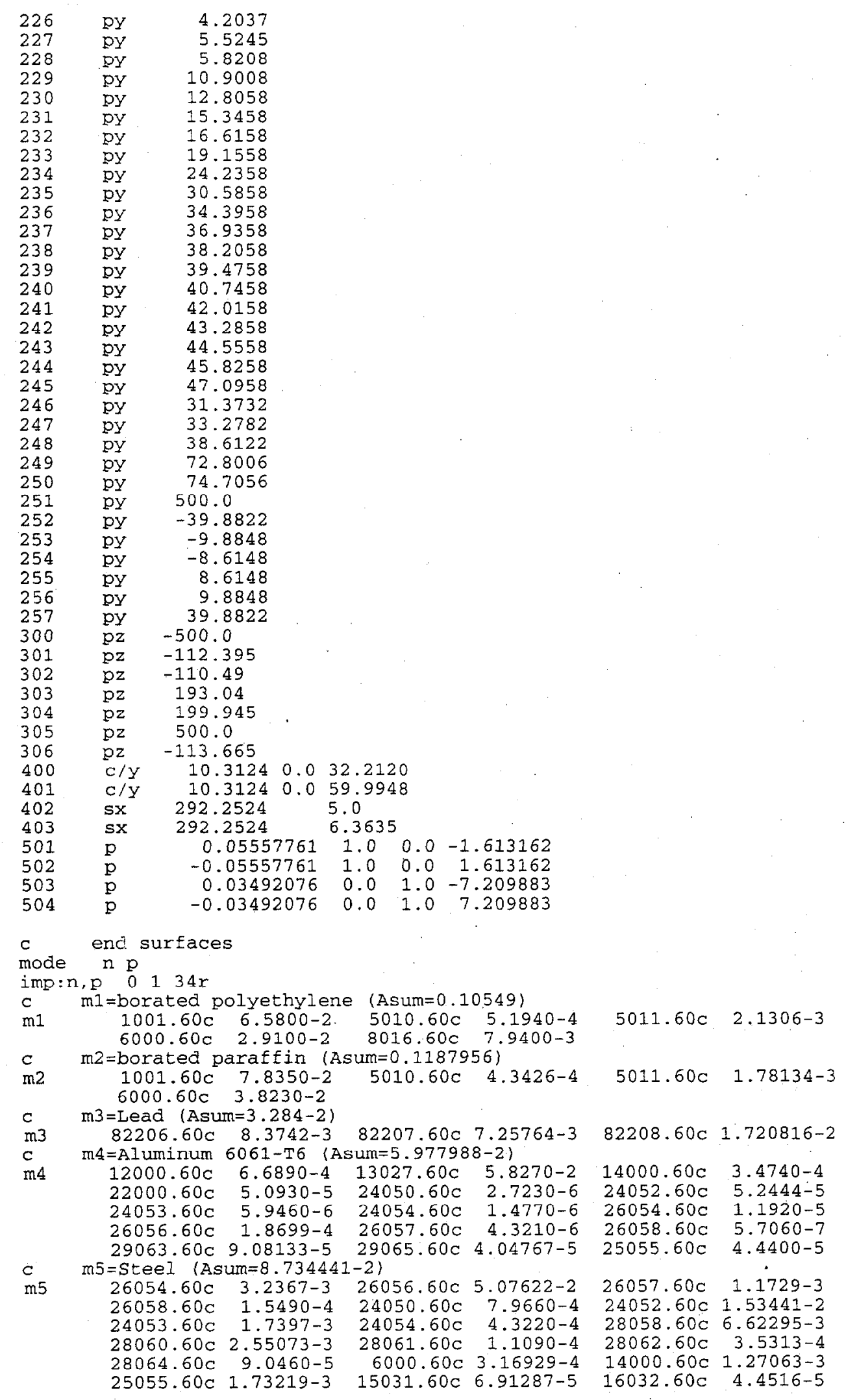


$7014.60 C^{2} 5.43529-4$

$c$

$\mathrm{m} 6=$ Plutonium (Asum $=4.070346-2$ )

m6 $94239.60 \mathrm{c} \quad 3.7291-2 \quad 94240.60 \mathrm{c} \quad 1.9277-3 \quad 94241.60 \mathrm{c} \quad 1.2196-4$ $31000.60 \mathrm{C} \quad 1.3628-3$

c m7=Tungsten (Asum $=6.605306-2$ )

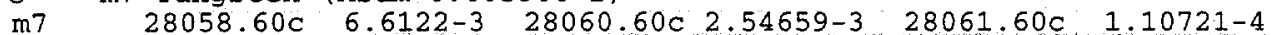

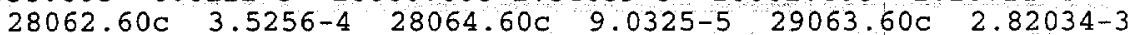
$29065.60 \mathrm{c} 1.25706-3 \quad 40000.60 \mathrm{c} \quad 7.9528-4 \quad 74182.60 \mathrm{c} \quad 1.369975-2$ $74183.60 c 7.36713-3 \quad 74184.60 \mathrm{c} \quad 1.577082-2 \quad 74186.60 \mathrm{c} \quad 1.463029-2$

f105: $282.250 .0 \quad 0.00 .0$

de105

df 105 $\begin{array}{rlllll}2.07002-7 & 7.69672-7 & 1.75386-6 & 3.71293-6\end{array}$ $7.86024-6 \quad 1.66401-5 \quad 3.52273-5 \quad 7.45761-5 \quad 1.34159-4$ 2.21191-4 $3.64682-4 \quad 6.01258-4 \quad 9.91309-4 \quad 1.63439-3$ 2.14167-3 2.43063-3 2.82399-3 $3.37141-3 \quad 4.61914-3$ $7.32483-3 \quad 1.20766-2 \quad 1.93065-2 \quad 2.41830-2 \quad 2.54229-2$ $2.65293-2 \quad 2.77506-2$ 3.14039-2 4.33909-2 5.45187-2 7.15396-2 $1.04645-1 \quad 1.36365-1 \quad 1.66556-1 \quad 2.03432-1$ $2.48472-1 \quad 2.83877-1 \quad 2.95864-1 \quad 2.97851-1 \quad 3.00232-1$ 3.44858-1 4.42806-1 5.10634-1 $5.65749-1 \quad 6.75418-1$ $\begin{array}{llllll}8.24957-1 & 1.00760+0 & 1.23069+0 & 1.50317+0 & 1.83598+0\end{array}$ $2.12513+0 \quad 2.28850+0 \quad 2.40583+0 \quad 2.73896+0 \quad 3.34537+0$ $4.08604+0 \quad 4.99070+0 \quad 6.09565+0 \quad 7.44525+0 \quad 9.09365+0$ $1.11070+1 \quad 1.35661+1$

$\begin{array}{rrrr}3.70370-3 & 4.37060-3 & 4.52500-3 & 4.57410-3\end{array}$ $4.03110-3 \quad 3.93550-3 \quad 3.84530-3 \quad 3.76290-3.3 .69080-3$ $3.65280-3 \quad 3.63870-3 \quad 3.62290-3 \quad 3.60620-3 \quad 3.58250-3$ $3.55850-3 \quad 4.08750-3 \quad 5.89750-3 \quad 7.13870-3 \quad 7.42410-3$ $7.67700-3 \quad 7.95180-3 \quad 8.74210-3 \quad 1.11490-2 \quad 1.35030-2$ $1.65080-22.25270-22.86610-2 \quad 3.43150-2 \quad 4.10860-2$ $4.92110-2 \quad 5.56070-2 \quad 5.77300-2 \quad 5.80800-2 \quad 5.84980-2$ $6.59980-28.26560-2 \quad 9.04890-2.9 .54810-2 \quad 1.05360-1$ $1.17860-1 \quad 1.29670-1 \quad 1.30100-1 \quad 1.28650-1 \quad 1.27220-1$ $1.26160-1$ 1.25620-1 1.25270-1 $1.28500-1 \quad 1.36960-1$ $1.45980-1$ 1.54120-1 1.51160-1 1.47220-1 1.47060-1 $1.63200-12.00340-1$

fm105 $\quad 2.348+10$

f115:n $\quad 302.250 .00 .00 .0$

de115

$2.07002-7 \quad 7.69672-7 \quad 1.75386-6 \quad 3.71293-6$

$7.86024-6 \quad 1.66401-5 \quad 3.52273-5 \quad 7.45761-5 \quad 1.34159-4$ $2.21191-4 \quad 3.64682-4 \quad 6.01258-4 \quad 9.91309-4 \quad 1.63439-3$ 2.14167-3 $2.43063-3 \quad 2.82399-3 \quad 3.37141-3 \quad 4.61914-3$ $7.32483-3 \quad 1.20766-2 \quad 1.93065-2 \quad 2.41830-2 \quad 2.54229-2$ 2.65293-2 2.77506-2 $3.14039-2 \quad 4.33909-2 \quad 5.45187-2$ 7.15396-2 1.04645-1 $1.36365-1$ 1.66556-1 2.03432-1 $2.48472-1 \quad 2.83877-1 \quad 2.95864-1 \quad 2.97851-1 \quad 3.00232-1$ $3.44858-1 \quad 4.42806-1 \quad 5.10634-1 \quad 5.65749-1 \quad 6.75418-1$ $8.24957-1 \quad 1.00760+0 \quad 1.23069+0 \quad 1.50317+0 \quad 1.83598+0$ $2.12513+0 \quad 2.28850+0 \quad 2.40583+0 \quad 2.73896+0 \quad 3.34537+0$ $4.08604+0 \quad 4.99070+0 \quad 6.09565+0 \quad 7.44525+0 \quad 9.09365+0$ $1.11070+1 \quad 1.35661+1$

df115 $\quad 3.70370-3 \quad 4.37060-3 \quad 4.52500-3 \quad 4.57410-3$ $4.55830-3 \quad 4.48970-3 \quad 4.38170-3 \quad 4.24920-3 \quad 4.12890-3$ $4.03110-3 \quad 3.93550-3 \quad 3.84530-3 \quad 3.76290-3 \quad 3.69080-3$ $3.65280-3 \quad 3.63870-3 \quad 3.62290-3 \quad 3.60620-3 \quad 3.58250-3$ $3.55850-3 \quad 4.08750-3 \quad 5.89750-3 \quad 7.13870-3 \quad 7.42410-3$ $\begin{array}{lllll}7.67700-3 & 7.95180-3 & 8.74210-3 & 1.11490-2 & 1.35030-2\end{array}$ $1.65080-2 \quad 2.25270-2 \quad 2.86610-2 \quad 3.43150-2 \quad 4.10860-2$ $4.92110-2 \quad 5.56070-2 \quad 5.77300-2 \quad 5.80800-2.5 .84980-2$ $6.59980-2 \quad 8.26560-2 \quad 9.04890-2 \quad 9.54810-2 \quad 1.05360-1$ $1.17860-1 \quad 1.29670-1 \quad 1.30100-1 \quad 1.28650-1 \quad 1.27220-1$ $1.26160-1 \quad 1.25620-1 \quad 1.25270-1 \quad 1.28500-1 \quad 1.36960-1$ 1.45980-1 1.54120-1 1.51160-1 1.47220-1 1.47060-1 $1.63200-12.00340-1$

fm115 $2.348+10$

f125:n $\quad 292.2510 .0 \quad 0.00 .0$

de 125

$2.07002-7 \quad 7.69672-7,1.75386-6 \quad 3.71293-6$ 7.86024-6 $1.66401-5 \quad 3.52273-5 \quad 7.45761-5 \quad 1.34159-4$ $\begin{array}{llllll}2.21191-4 & 3.64682-4 & 6.01258-4 & 9.91309-4 & 1.63439-3\end{array}$ $2.14167-3 \quad 2.43063-3 \quad 2.82399-3 \quad 3.37141-3 \quad 4.61914-3$ $7.32483-3 \quad 1.20766-2$ 1.93065-2 $2.41830-2 \quad 2.54229-2$ 2.65293-2 2.77506-2 3.14039-2 4.33909-2 5.45187-2 


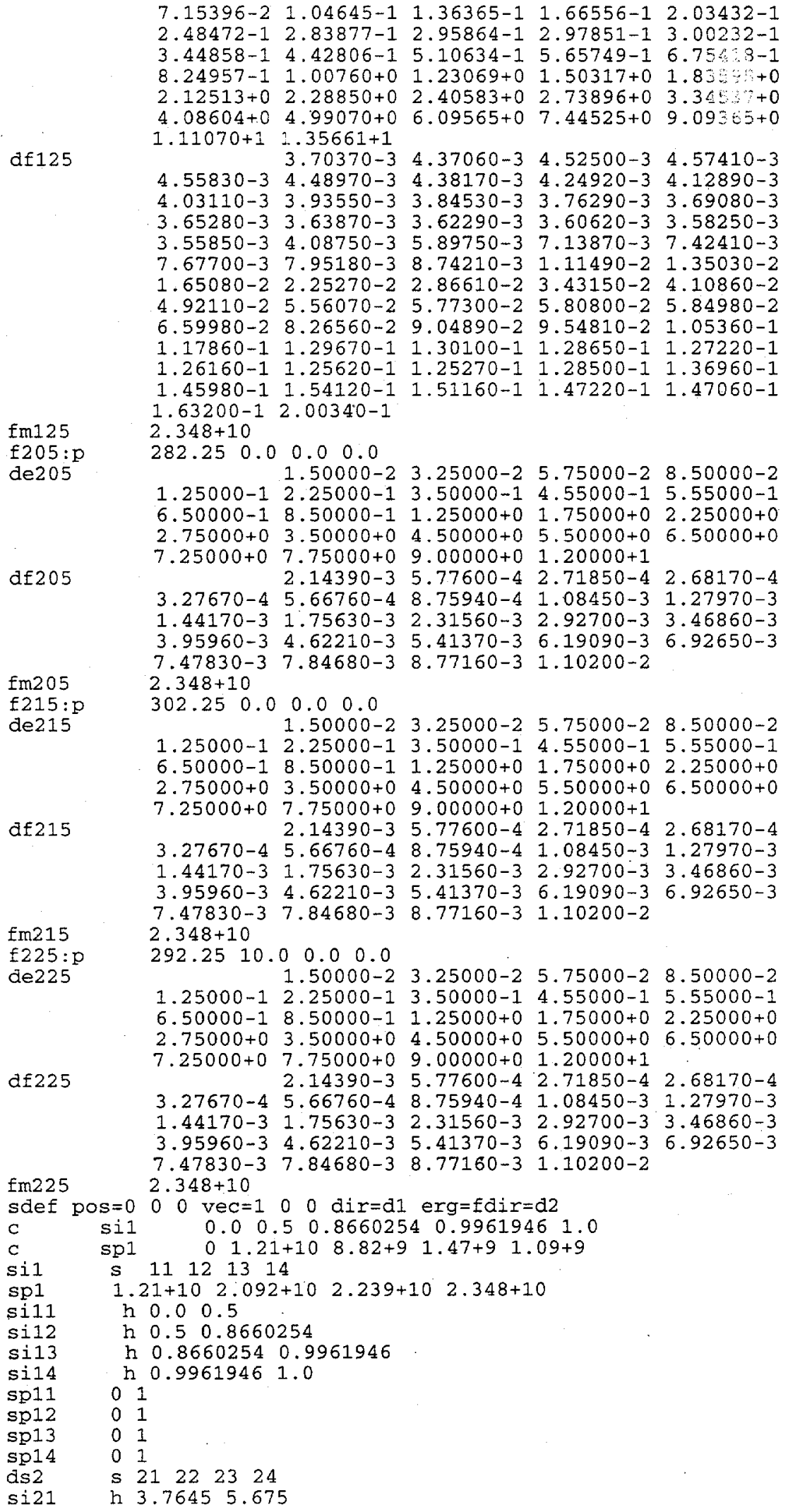


si22 h 5.6757 .3865

si23 h 7.38658 .321

si 24 h 8.3218 .753

$\operatorname{sp} 21 \quad 0 \quad 1$

$\operatorname{sp} 22 \quad 01$

$\operatorname{sp} 23 \quad 01$

$\operatorname{sp24} \quad 0 \quad 1$

nps $\quad 400000$

prdmp $2 j 1$ 
File for MCNP-4B surface-detector dose-rate calculations for a 6.32 -cm-radius bare Pu sphere in the PFNA beam at the center of the truck lane $(400,000$ histories)

message: outp=pina5.o mctal=pfna5.m

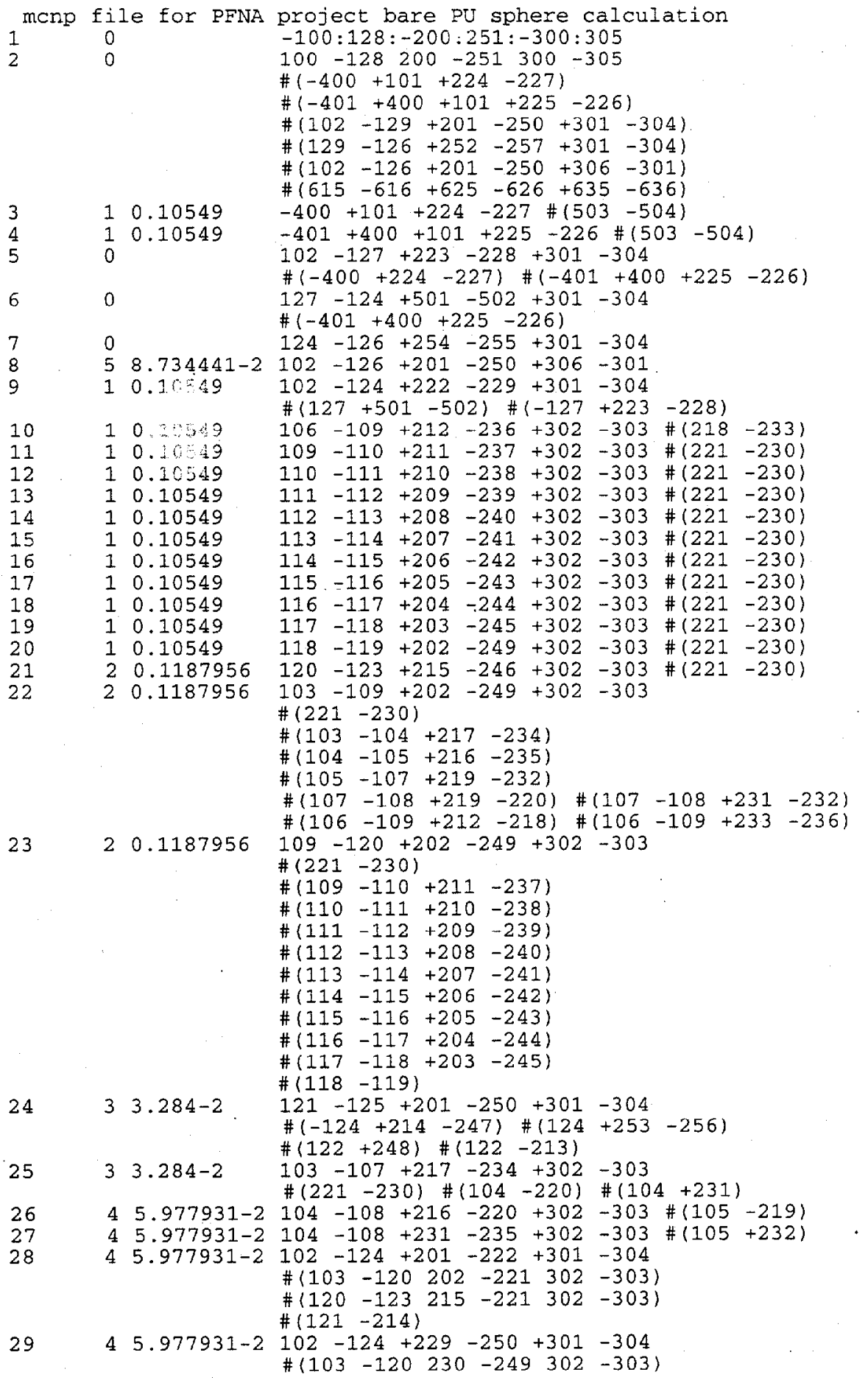




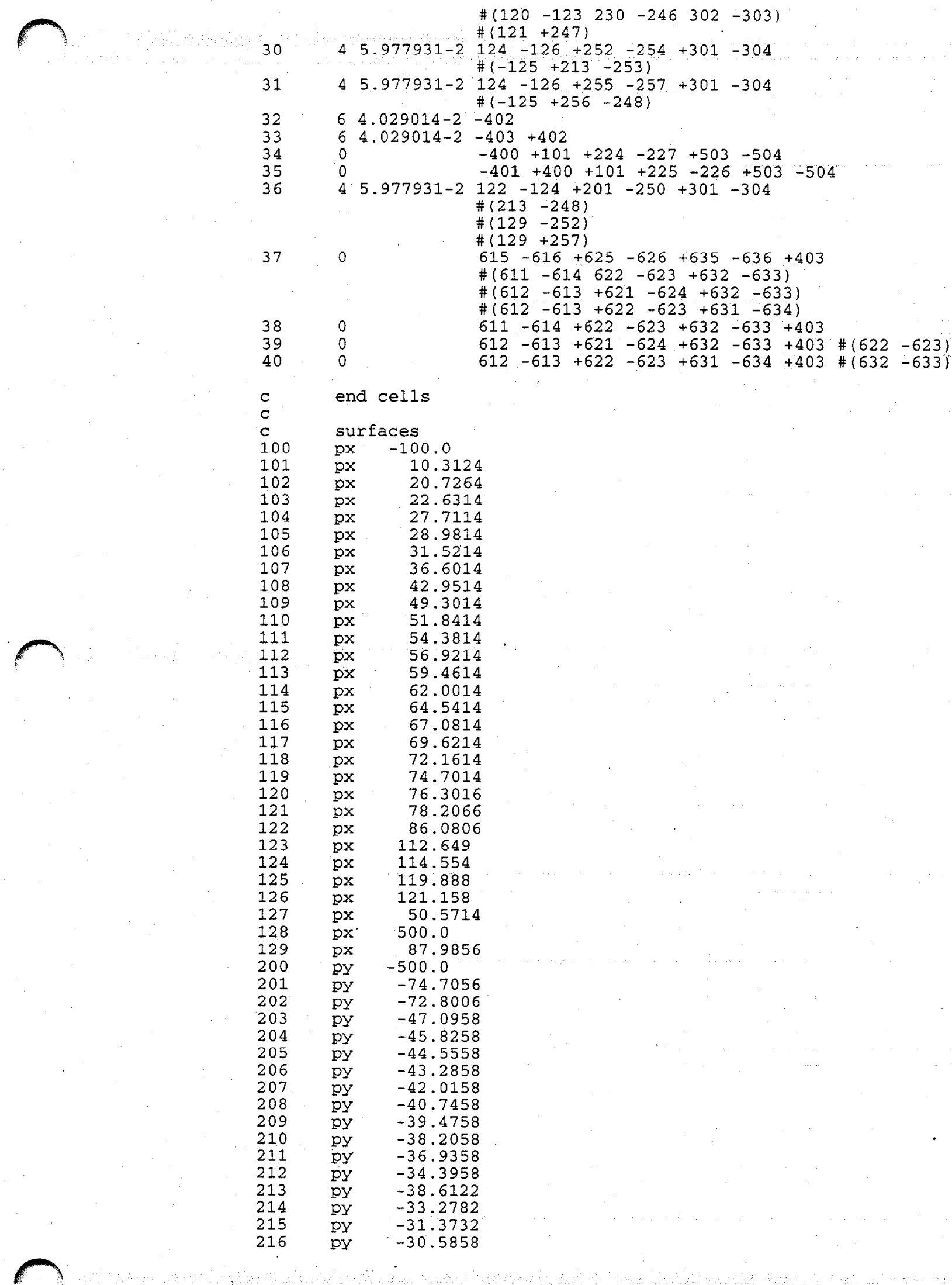




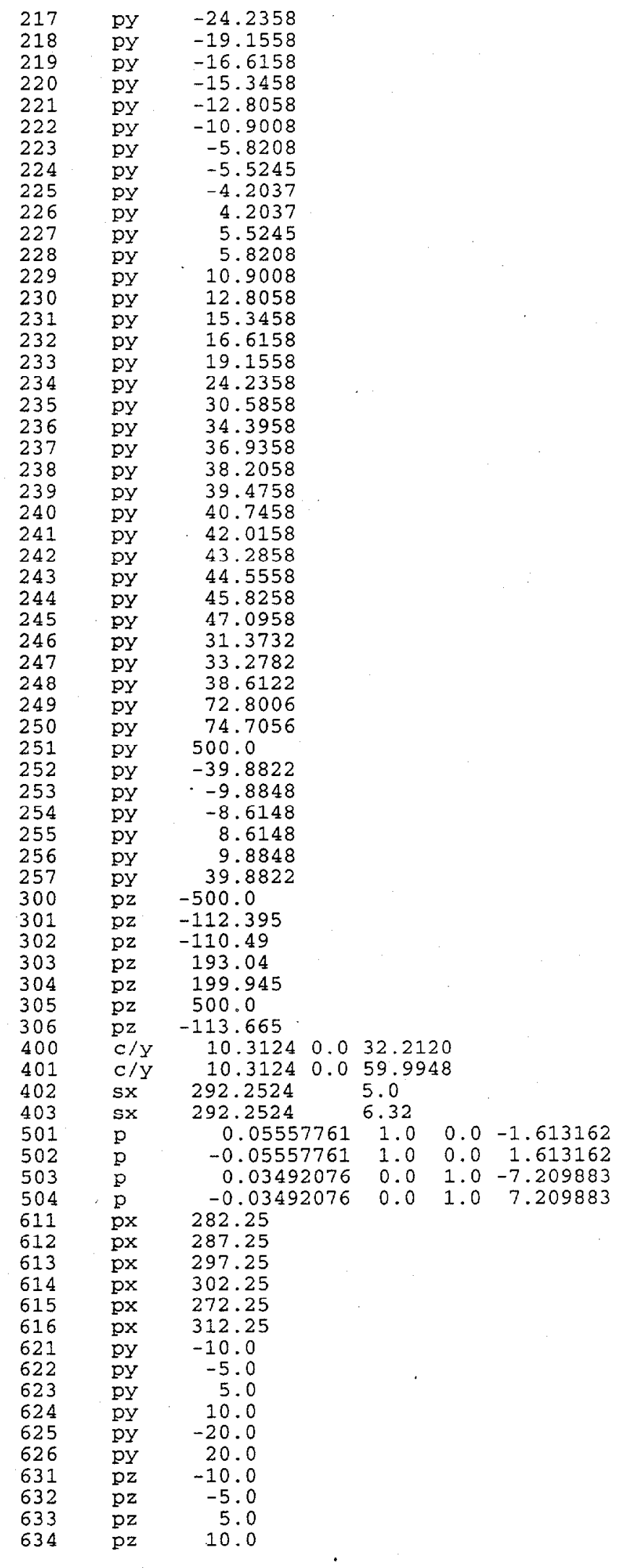




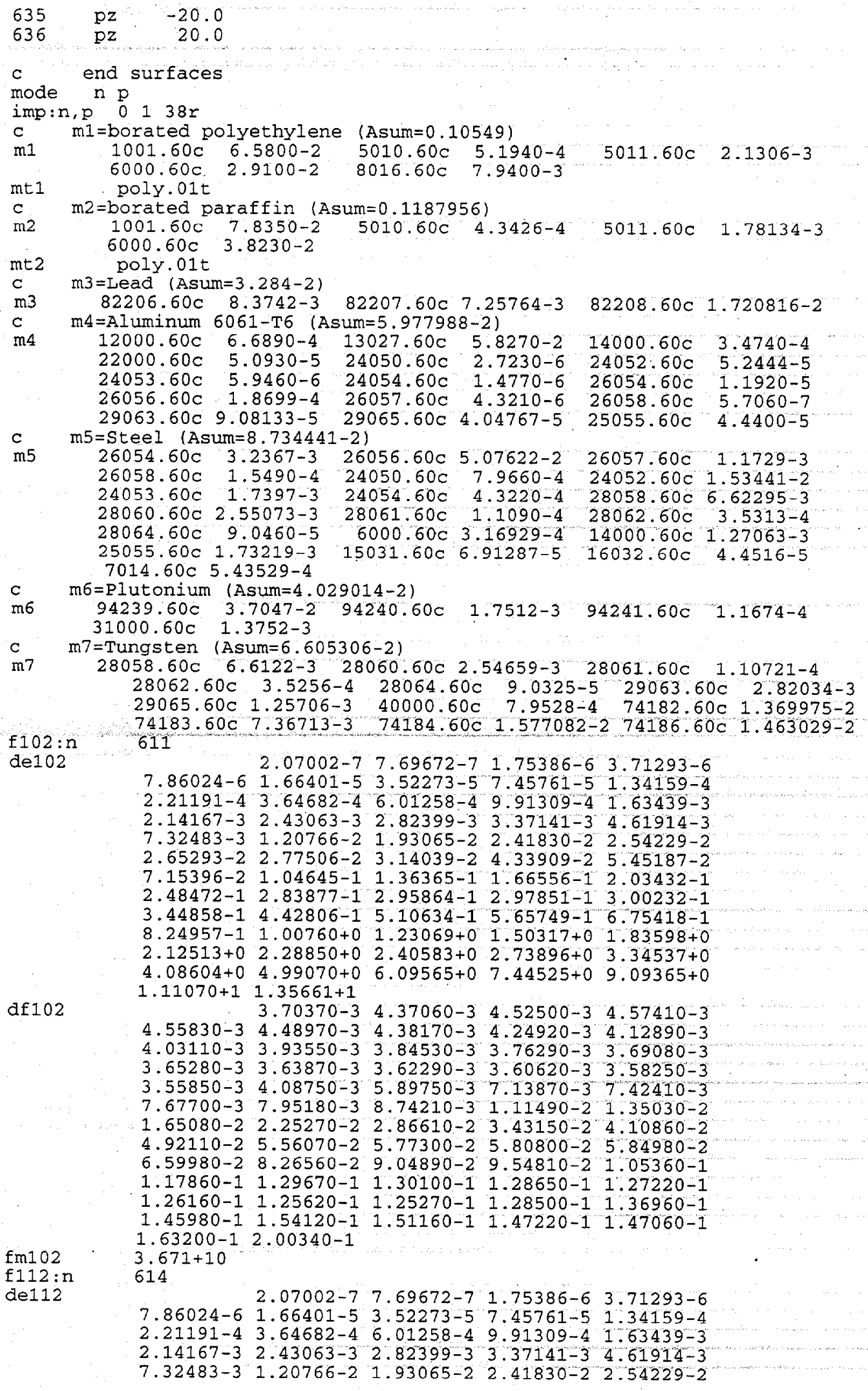


$\operatorname{df} 112$

Im112

E122:n

de122

af122

$\operatorname{fm} 122$

f132:n

$\operatorname{de} 132$

df132
2.65293-2 2.77506-2 $3.14039-2 \quad 4.33909-2 \quad 5.45187-2$ 7.15396-2 $1.04645-1 \quad 1.36365-1 \quad 1.66556-1 \quad 2.03432-1$ $\begin{array}{lllll}2.48472-1 & 2.83877-1 & 2.95864-1 & 2.97851-1 & 3.00232-1\end{array}$ 3.44858-1 4.42806-1. 5.10634-1 5.65749-1 $6.7 .418-1$ $8.24957-1 \quad 1.00760+0 \quad 1.23069+0 \quad 1.50317+0 \quad 1.8 .98+0$ $\begin{array}{lllll}2.12513+0 & 2.28850+0 & 2.40583+0 & 2.73896+0 & 3.34537+0\end{array}$ $4.08604+0 \quad 4.99070+0 \quad 6.09565+0 \quad 7.44525+0 \quad 9.09365+0$ $1.11070+1.1 .35661+1$

$3.70370-3 \quad 4.37060-3 \quad 4.52500-3 \quad 4.57410-3$ $4.55830-3 \quad 4.48970-3 \quad 4.38170-3 \quad 4.24920-3 \quad 4.12890-3$ $\begin{array}{llllll}4.03110-3 & 3.93550-3 & 3.84530-3 & 3.76290-3 & 3.69080-3\end{array}$ $3.65280-3 \quad 3.63870-3 \quad 3.62290-3 \quad 3.60620-3 \quad 3.58250-3$ $\begin{array}{llllll}3.55850-3 & 4.08750-3 & 5.89750-3 & 7.13870-3 & 7.42410-3\end{array}$ $7.67700-3 \quad 7.95180-3 \quad 8.74210-3 \quad 1.11490-2 \quad 1.35030-2$ 1.65080-2 2.25270-2 2.86610-2 3.43150-2 4.10860-2 $4.92110-2 \quad 5.56070-2 \quad 5.77300-2 \quad 5.80800-2 \quad 5.84980-2$ $6.59980-2 \quad 8.26560-2 \quad 9.04890-2 \quad 9.54810-2 \quad 1.05360-1$ $1.17860-1 \quad 1.29670-1 \quad 1.30100-1 \quad 1.28650-1 \quad 1.27220-1$ $1.26160-1 \quad 1.25620-1 \quad 1.25270-1 \quad 1.28500-1 \quad 1.36960-1$ $1.45980-1$ 1.54120-1 $1.51160-1$ 1.47220-1 1.47060-1 $1.63200-12.00340-1$

$3.671+10$

624

$\begin{array}{lllll}2.07002-7 & 7.69672-7 & 1.75386-6 & 3.71293-6\end{array}$ 7.86024-6 $1.66401-5 \quad 3.52273-5 \quad 7.45761-5 \quad 1.34159-4$ 2.21191-4 3.64682-4 $6.01258-4 \quad 9.91309-4 \quad 1.63439-3$ $2.14167-3 \quad 2.43063-3 \quad 2.82399-3 \quad 3.37141-3 \quad 4.61914-3$ $\begin{array}{llllll}7.32483-3 & 1.20766-2 & 1.93065-2 & 2.41830-2 & 2.54229-2\end{array}$ 2.65293-2 2.77506-2 3.14039-2 4.33909-2 5.45187-2 7.15396-2 $1.04645-1 \quad 1.36365-1 \quad 1.66556-1 \quad 2.03432-1$ $\begin{array}{lllll}2.48472-1 & 2.83877-1 & 2.95864-1 & 2.97851-1 & 3.00232-1\end{array}$ 3.44858-1 $4.42806-1 \quad 5.10634-1 \quad 5.65749-1 \quad 6.75418-1$ $8.24957-1 \quad 1.00760+0 \quad 1.23069+0 \quad 1.50317+0 \quad 1.83598+0$ $\begin{array}{lllll}2.12513+0 & 2.28850+0 & 2.40583+0 & 2.73896+0 & 3.34537+0\end{array}$ $4.08604+0 \quad 4.99070+0 \quad 6.09565+0 \quad 7.44525+0 \quad 9.09365+0$ $1.11070+11.35661+1$

$3.70370-3 \quad 4.37060-3 \quad 4.52500-3 \quad 4.57410-3$ $4.55830-3 \quad 4.48970-3 \quad 4.38170-3 \quad 4.24920-3 \quad 4.12890-3$ $\begin{array}{llllll}4.03110-3 & 3.93550-3 & 3.84530-3 & 3.76290-3 & 3.69080-3\end{array}$ $3.65280-3 \quad 3.63870-3 \quad 3.62290-3 \quad 3.60620-3 \quad 3.58250-3$ $3.55850-3 \quad 4.08750-3 \quad 5.89750-3 \quad 7.13870-3 \quad 7.42410-3$ $7.67700-3 \quad 7.95180-3 \quad 8.74210-3 \quad 1.11490-2 \quad 1.35030-2$ $1.65080-2 \quad 2.25270-2 \quad 2.86610-2 \quad 3.43150-2 \quad 4.10860-2$ $4.92110-2 \quad 5.56070-2 \quad 5.77300-2 \quad 5.80800-2 \quad 5.84980-2$ $6.59980-2 \quad 8.26560-2 \quad 9.04890-2 \quad 9.54810-2 \quad 1.05360-1$ 1.17860-1 $1.29670-1 \quad 1.30100-1 \quad 1.28650-1 \quad 1.27220-1$ $1.26160-1 \quad 1.25620-1 \quad 1.25270-1 \quad 1.28500-1 \quad 1.36960-1$ $1.45980-1 \quad 1.54120-1 \quad 1.51160-1 \quad 1.47220-1 \quad 1.47060-1$ $1.63200-12.00340-1$

$3.671+10$

634

$2.07002-7 \quad 7.69672-7 \quad 1.75386-6 \quad 3.71293-6$ 7.86024-6 $1.66401-5 \quad 3.52273-5 \quad 7.45761-5 \quad 1.34159-4$ 2.21191-4 $3.64682-4 \quad 6.01258-4 \quad 9.91309-4 \quad 1.63439-3$ 2.14167-3 $2.43063-3 \quad 2.82399-3 \quad 3.37141-3 \quad 4.61914-3$ 7.32483-3 $1.20766-2 \quad 1.93065-2 \quad 2.41830-2 \quad 2.54229-2$ $\begin{array}{lllll}2.65293-2 & 2.77505-2 & 3.14039-2 & 4.33909-2 & 5.45187-2\end{array}$ 7.15396-2 $1.04645-1 \quad 1.36365-1 \quad 1.66556-1 \quad 2.03432-1$ $\begin{array}{llllll}2.48472-1 & 2.83877-1 & 2.95864-1 & 2.97851-1 & 3.00232-1\end{array}$ 3.44858-1 $4.42806-1 \quad 5.10634-1 \quad 5.65749-1 \quad 6.75418-1$ $8.24957-1 \quad 1.00760+0 \quad 1.23069+0 \quad 1.50317+0 \quad 1.83598+0$ $\begin{array}{lllll}2.12513+0 & 2.28850+0 & 2.40583+0 & 2.73896+0 & 3.34537+0\end{array}$ $4.08604+0 \quad 4.99070+0 \quad 6.09565+0 \quad 7.44525+0 \quad 9.09365+0$ $1.11070+1 \quad 1.35661+1$

$3.70370-3 \quad 4.37060-3 \quad 4.52500-3 \quad 4.57410-3$ $\begin{array}{llllll}4.55830-3 & 4.48970-3 & 4.38170-3 & 4.24920-3 & 4.12890-3\end{array}$ $4.03110-3 \quad 3.93550-3 \quad 3.84530-3 \quad 3.76290-3 \quad 3.69080-3$ $\begin{array}{llllll}3.65280-3 & 3.63870-3 & 3.62290-3 & 3.60620-3 & 3.58250-3\end{array}$ $3.55850-3 \quad 4.08750-3 \quad 5.89750-3 \quad 7.13870-3 \quad 7.42410-3$ $7.67700-3 \quad 7.95180-3 \quad 8.7 .010-3 \quad 1.11490-2 \quad 1.35030-2$ $1.65080-2 \quad 2.25270-2 \quad 2.56 \cdots-2 \quad 3.43150-2 \quad 4.10860-2$ $4.92110-25.56070-2 \quad 5.7 .319-2 \quad 5.80800-2 \quad 5.84980-2$ 
6.59980-2 8.26560-2 $9.04890-2 \quad 9.54810-2 \quad 1.05360-1$ 1.17860-1 $1.29670-1 \quad 1.30100-1 \quad 1.28650-1 \quad 1.27220-1$ $1.26160-1$ 1.25620-1 1.25270-1 1.28500-1 1.36960-1 $1.45980-1$ 1.54120-1 1.51160-1 1.47220-1 1.47060-1 $1.63200-12.00340-1$

fm132 $3.671+10$

f202:p

611

de 202

df202

f212:p

(1)

$1.50000-2 \quad 3.25000-2 \quad 5.75000-2 \quad 8.50000-2$ $1.25000-1 \quad 2.25000-1 \quad 3.50000-1 \quad 4.55000-1 \quad 5.55000-1$ 6.50000-1 $8.50000-1 \quad 1.25000+0 \quad 1.75000+0 \quad 2.25000+0$ $2.75000+0 \quad 3.50000+0 \quad 4.50000+0 \quad 5.50000+0.6 .50000+0$ $7.25000+0 \quad 7.75000+09.00000+01.20000+1$

$2.14390-3 \quad 5.77600-4 \quad 2.71850-4 \quad 2.68170-4$ $3.27670-4 \quad 5.66760-4 \quad 8.75940-4 \quad 1.08450-3 \quad 1.27970-3$ $1.44170-3 \quad 1.75630-3 \quad 2.31560-32.92700-3 \quad 3.46850-3$ $\begin{array}{llllll}3.95960-3 & 4.62210-3 & 5.41370-3 & 6.19090-3 & 6.92650-3\end{array}$ $7.47830-3 \quad 7.84680-3 \quad 8.77160-3 \quad 1.10200-2$

$3.671+10$

de 212

df 212 614

(0.7. $3.25000-2 \quad 5.75000-28.50000-2$ $1.25000-1 \quad 2.25000-1 \quad 3.50000-1 \quad 4.55000-1 \quad 5.55000-1$ $6.50000-1 \quad 8.50000-1 \quad 1.25000+01.75000+02.25000+0$ $2.75000+0 \quad 3.50000+0 \quad 4.50000+0 \quad 5.50000+0.6 .50000+0$ $7.25000+0 \quad 7.75000+0 \quad 9.00000+01.20000+1$

$2.14390-3 \quad 5.77600-4 \quad 2.71850-4 \quad 2.68170-4$ $3.27670-4 \quad 5.66760-4 \quad 8.75940-4 \quad 1.08450-3 \quad 1.27970-3$ $1.44170-3 \quad 1.75630-32.31560-32.92700-3 \quad 3.46860-3$ $3.95960-3 \quad 4.62210-3 \quad 5.41370-3 \quad 6.19090-3 \quad 6.92650-3$ $7.47830-3 \quad 7.84680-38.77160-3 \cdots 1.10200-2$

$\operatorname{tm} 212$ $3.671+10$

$f 222: \mathrm{p} \quad 624$

de 222

$\operatorname{df} 222$

(25000-1 2.25000-1 $3.50000-1 \quad 4.55000-1 \quad 5.55000-1$

$6.50000-1 \quad 8.50000-1 \quad 1.25000+0.1 .75000+02.25000+0$

$2.75000+0.3 .50000+0 \quad 4.50000+0 \quad 5.50000+0 \quad 6.50000+0$ $7.25000+0 \quad 7.75000+0 \quad 9.00000+01.20000+1$

$2.14390-3 \quad 5.77600-4 \quad 2.71850-4 \quad 2.68170-4$ $3.27670-4 \quad 5.66760-4 \quad 8.75940-4 \quad 1.08450-31.27970-3$ $1.44170-31.75630-32.31560-32.92700-3 \quad 3.46860-3$ $3.95960-3 \quad 4.62210-3 \quad 5.41370-3 \quad 6.19090-3 \quad 6.92650-3$ $7.47830-37.84680-38.77160-31.10200-2$

fm222

f232:p $3.671+10$

de232

634

$1.50000-2 \quad 3.25000-2 \quad 5.75000-2 \times 8.50000-2$ $2.25000-1 \quad 3.50000-1 \quad 4.55000-1 \quad 5.55000-1$ $6.50000-1 \quad 8.50000-1 \quad 1.25000+0 \quad 1.75000+02.25000+0$ $2.75000+0 \quad 3.50000+0 \quad 4.50000+0 \quad 5.50000+0 \quad 6.50000+0$ $7.25000+0 \quad 7.75000+0 \quad 9.00000+0.1 .20000+1$ $2.14390-3 \quad 5.77600-4 \quad 2.71850-4 \quad 2.68170-4$ $3.27670-4 \quad 5.66760-4 \quad 8.75940-4 \quad 1.08450-3 \quad 1.27970-3$ $1.44170-3 \quad 1.75630-32.31560-3.2 .92700-3 \quad 3.46860-3$

$3.95960-3 \quad 4.62210-3 \quad 5.41370-3 \quad 6.19090-3 \quad 6.92650-3$

$7.47830-37.84680-38.77160-31.10200-2$

$\operatorname{fm} 232 \quad 3.671+10$

$\mathrm{eIO} 2$

4.13994-7 $1.12535-6 \quad 2.38237-6 \quad 5.04348-6 \quad 1.06770-5$ 2.26033-5 4.78512-5 $1.01301-4 \quad 1.67017-4$ 2.75364-4 4.53999-4 $7.48518-4 \quad 1.23410-3 \quad 2.03468-3 \quad 2.24867-3$

$\begin{array}{llllll}2.61259-3 & 3.03539-3 & 3.70744-3 & 5.53084-3 & 9.11882-3\end{array}$

1.50344-2 2.35786-2 2.47875-2 2.60584-2 2.70001-2

2.85011-2 $3.43067-2 \quad 5.24752-2 \quad 5.65622-2 \quad 8.65169-2$

1.22773-1 1.49956-1 1.83156-1 2.23708-1 2.73237-1

$2.94518-1$ 2.97211-1 2.98491-1 $3.01974-1 \quad 3.87742-1$

4.97870-1 5.23397-1 $6.08101-1 \quad 7.42735-1 \quad 9.07180-1$

$1.10803+0 \quad 1.35335+0 \quad 1.65299+0 \quad 2.01897+0 \quad 2.23130+0$

$2.34570+0 \quad 2.46597+0 \quad 3.01194+0 \quad 3.67879+0 \quad 4.49329+0$

$5.48811+0 \quad 6.70320+0 \quad 8.18730+0 \quad 1.00000+11.22140+1$

e202

$1.49183+1$

$2.00000-24.50000-2 \quad 7.00000-21.00000-1 \quad 1.50000-1$

$3.00000-1 \quad 4.00000-15.10000-1 \quad 6.00000-1 \quad 7.00000-1$

$1.00000+01.50000+02.00000+02.50000+03.00000+0$ 
$4.00000+0 \quad 5.00000+0 \quad 6.00000+0 \quad 7.00000+0 \quad 7.50000+0$ $8.00000+0 ; 1.000+11.40000+1$

e112

4.13994-7 :.335-6 2.38237-6 5.04348-6 1.06770-5 $2.26033-5 \quad \cdots \quad .312-5 \quad 1.01301-4 \quad 1.67017-4 \quad 2.75364-4$ $4.53999-4 \quad 3518-4.1 .23410-3 \quad 2.03468-3 \quad 2.24867-3$ $\begin{array}{llllll}2.61259-3 & 3.03539-3 & 3.70744-3 & 5.53084-3 & 9.11882-3\end{array}$ $\begin{array}{lllll}1.50344-2 & 2.35786-2 & 2.47875-2 & 2.60584-2 & 2.70001-2\end{array}$ $2.85011-2 \quad 3.43067-2 \quad 5.24752-2 \quad 5.65622-2 \quad 8.65169-2$ $1.22773-1$ 1.49956-1 $1.83156-1 \quad 2.23708-1 \quad 2.73237-1$ $2.94518-1 \quad 2.97211-1 \quad 2.98491-1 \quad 3.01974-1 \quad 3.87742-1$ 4.97870-1 $5.23397-1 \quad 6.08101-1 \quad 7.42735-1 \quad 9.07180-1$ $1.10803+0 \quad 1.35335+0 \quad 1.65299+0 \quad 2.01897+0 \quad 2.23130+0$ $2.34570+0 \quad 2.46597+0 \quad 3.01194+0 \quad 3.67879+0 \quad 4.49329+0$ b. $48811+0 \quad 6.70320+08.18730+01.00000+11.22140+1$ $1.49183+1$

$\mathrm{e} 212$

2.00000-2 4.50000-2 7.00000-2 1.00000-1 1.50000-1 3.00000-1 4.00000-1 5.10000-1 6.00000-1 7.00000-1. $1.00000+0 \quad 1.50000+0 \quad 2.00000+0 \quad 2.50000+0 \quad 3.00000+0$ $4.00000+0 \quad 5.00000+0 \quad 6.00000+0 \quad 7.00000+0 \quad 7.50000+0$ $8.00000+01.00000+1 \quad 1.40000+1$

e122

e222

4.13994-7 $1.12535-6 \quad 2.38237-6 \quad 5.04348-6 \quad 1.06770-5$ 2.26033-5 $4.78512-5 \quad 1.01301-4 \quad 1.67017-4 \quad 2.75364-4$ 4.53999-4 7.48518-4 $1.23410-3 \quad 2.03468-3 \quad 2.24867-3$ $\begin{array}{llllll}2.61259-3 & 3.03539-3 & 3.70744-3 & 5.53084-3 & 9.11882-3\end{array}$ 1.50344-2 2.35786-2 2.47875-2 $2.60584-2 \quad 2.70001-2$ $\begin{array}{lllll}2.85011 \% 2 & 3.43067-2 & 5.24752-2 & 5.65622-2 & 8.65169-2\end{array}$ $\begin{array}{llllll}1.22773-1 & 1.49956-1 & 1.83156-1 & 2.23708-1 & 2.73237-1\end{array}$ 2.94518-1 2.97211-1 $2.98491-1 \quad 3.01974-1 \quad 3.87742-1$ $\begin{array}{lllll}4.97870-1 & 5.23397-1 & 6.08101-1 & 7.42735-1 & 9.07180-1\end{array}$ $1.10803+0 \quad 1.35335+0 \quad 1.65299+0 \quad 2.01897+0 \quad 2.23130+0$ $2.34570+0 \quad 2.46597+0 \quad 3.01194+0 \quad 3.67879+0 \quad 4.49329+0$ $5.48811+0 \quad 6.70320+0 \quad 8.18730+0 \quad 1.00000+1 \quad 1.22140+1$ $1.49183+1$

$2.00000-2 \quad 4.50000-2 \quad 7.00000-2 \quad 1.00000-1 \quad 1.50000-1$ $3.00000-1 \quad 4.00000-1 \quad 5.10000-1 \quad 6.00000-1 \quad 7.00000-1$ $1.00000+0 \quad 1.50000+0 \quad 2.00000+0 \quad 2.50000+0 \quad 3.00000+0$ $4.00000+0 \quad 5.00000+0 \quad 6.00000+0 \quad 7.00000+0 \quad 7.50000+0$

e132 $8.00000+01.00000+11.40000+1$

4.13994-7 1.12535-6 2.38237-6 5.04348-6 $1.06770-5$ 2.26033-5 $4.78512-5 \quad 1.01301-4 \quad 1.67017-4 \quad 2.75364-4$ $\begin{array}{llllll}4.53999-4 & 7.48518-4 & 1.23410-3 & 2.03468-3 & 2.24867-3\end{array}$ 2.61259-3 $3.03539-3 \quad 3.70744-3 \quad 5.53084-3 \quad 9.11882-3$ $1.50344-2 \quad 2.35786-2 \quad 2.47875-2 \quad 2.60584-2 \quad 2.70001-2$ $\begin{array}{llllll}2.85011-2 & 3.43067-2 & 5.24752-2 & 5.65622-2 & 8.65169-2\end{array}$ $\begin{array}{llllll}1.22773-1 & 1.49956-1 & 1.83156-1 & 2.23708-1 & 2.73237-1\end{array}$ 2.94518-1 $2.97211-1 \quad 2.98491-1 \quad 3.01974-1 \quad 3.87742-1$ $\begin{array}{llllll}4.97870-1 & 5.23397-1 & 6.08101-1 & 7.42735-1 & 9.07180-1\end{array}$ $1.10803+0 \quad 1.35335+0 \quad 1.65299+0 \quad 2.01897+0 \quad 2.23130+0$ $2.34570+0 \quad 2.46597+0 \quad 3.01194+0 \quad 3.67879+0 \quad 4.49329+0$ $5.48811+0 \quad 6.70320+0 \quad 8.18730+0 \quad 1.00000+1 \quad 1.22140+1$. $1.49183+1$

e232

$2.00000-2 \quad 4.50000-2 \quad 7.00000-2 \quad 1.00000-1 \quad 1.50000-1$ $3.00000-1.4 .00000-1 \quad 5.10000-1 \quad 6.00000-1 \quad 7.00000-1$ $1.00000+0 \quad 1.50000+0 \quad 2.00000+0 \quad 2.50000+0 \quad 3.00000+0$ $4.00000+0 \quad 5.00000+0 \quad 6.00000+0 \quad 7.00000+0 \quad 7.50000+0$ $8.00000+01.00000+11.40000+1$

sd102 100.0

sd112 100.0

sd122 100.0

sd132 100.0

sd202 100.0

sd212 100.0

sd222 100.0

sd232 100.0

sdef pos $=0$ o 0 vec $=1 \quad 0 \quad 0$ dir $=$ d 1 erg=fdir $=d 2$

$c \quad$ sil $\quad 0.0 \quad 0.50 .86602540 .99619461 .0$ 


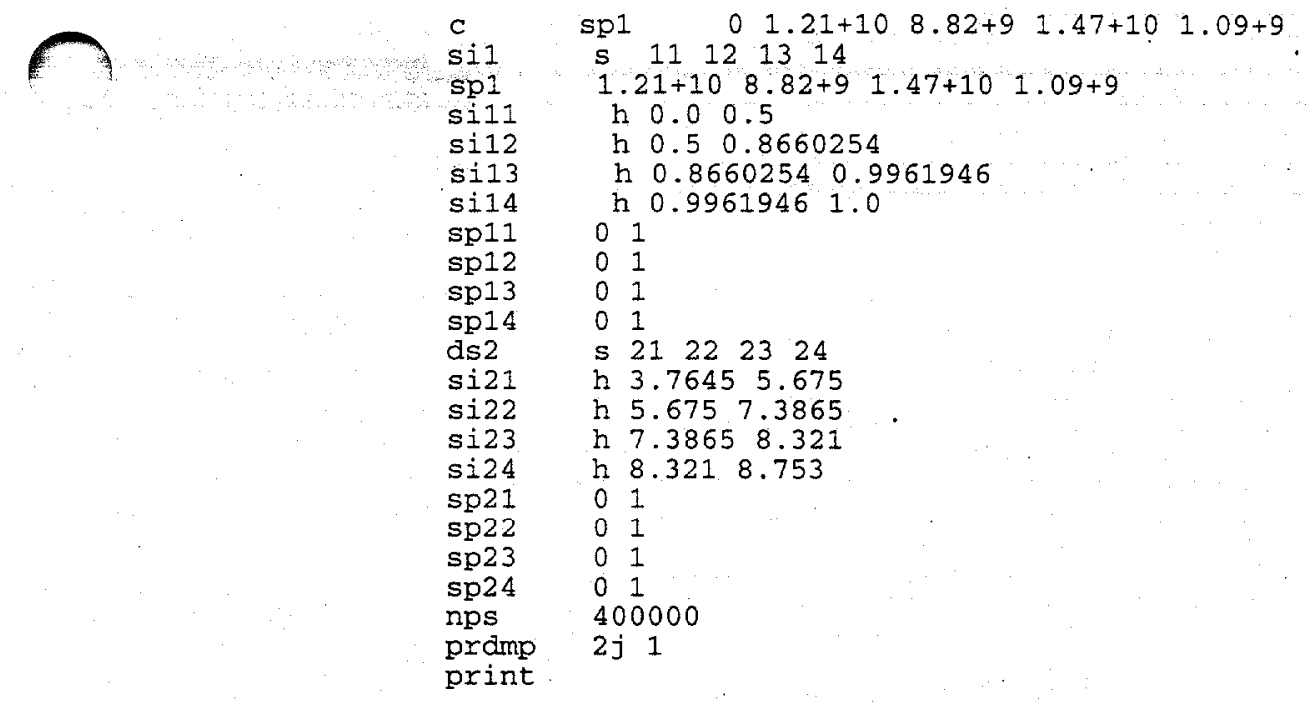


File for MCNP-4B surface-detector dose-rate background calculations for the bare Pu spheres in the PFNA beam at the center of the truck lane $(2,000,000$ histories $)$

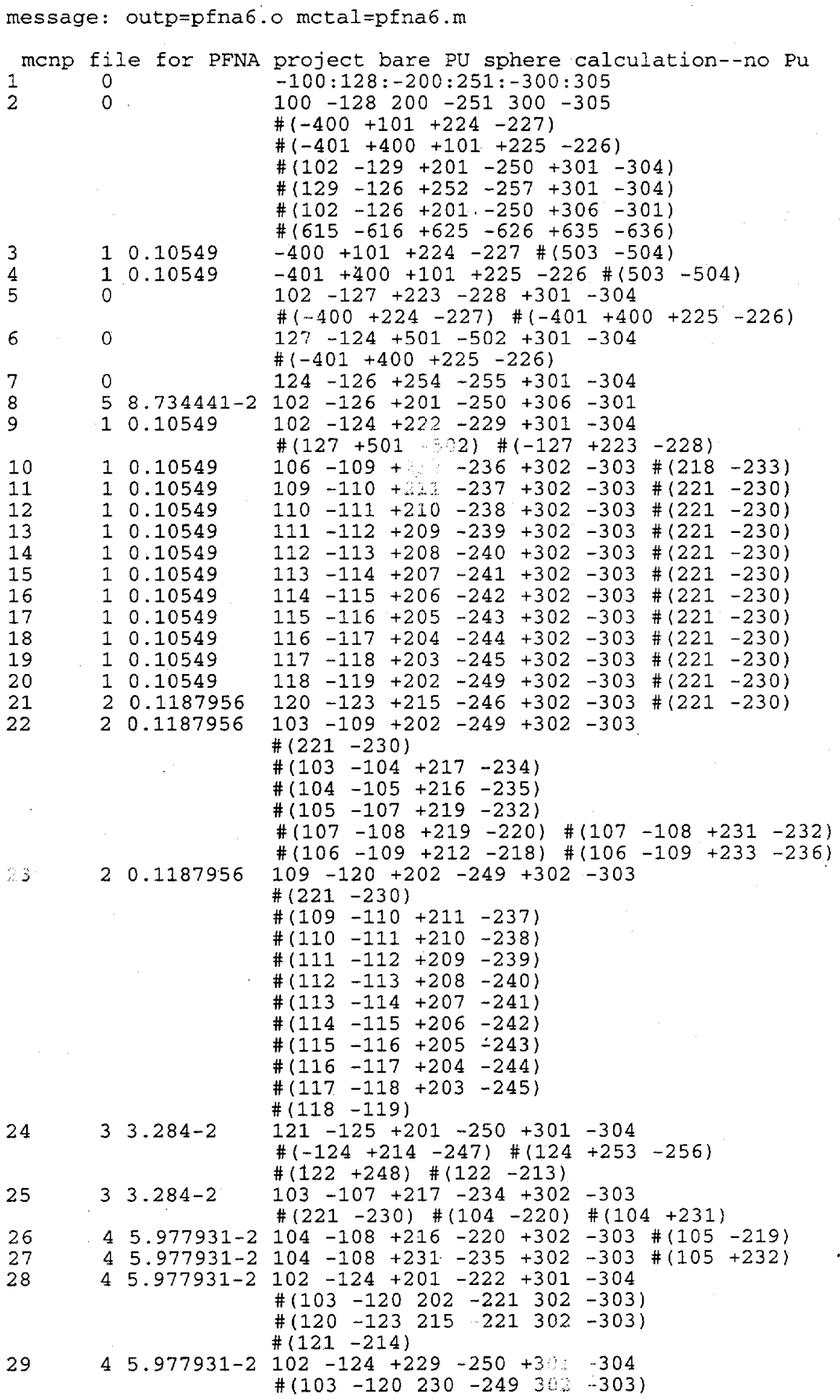




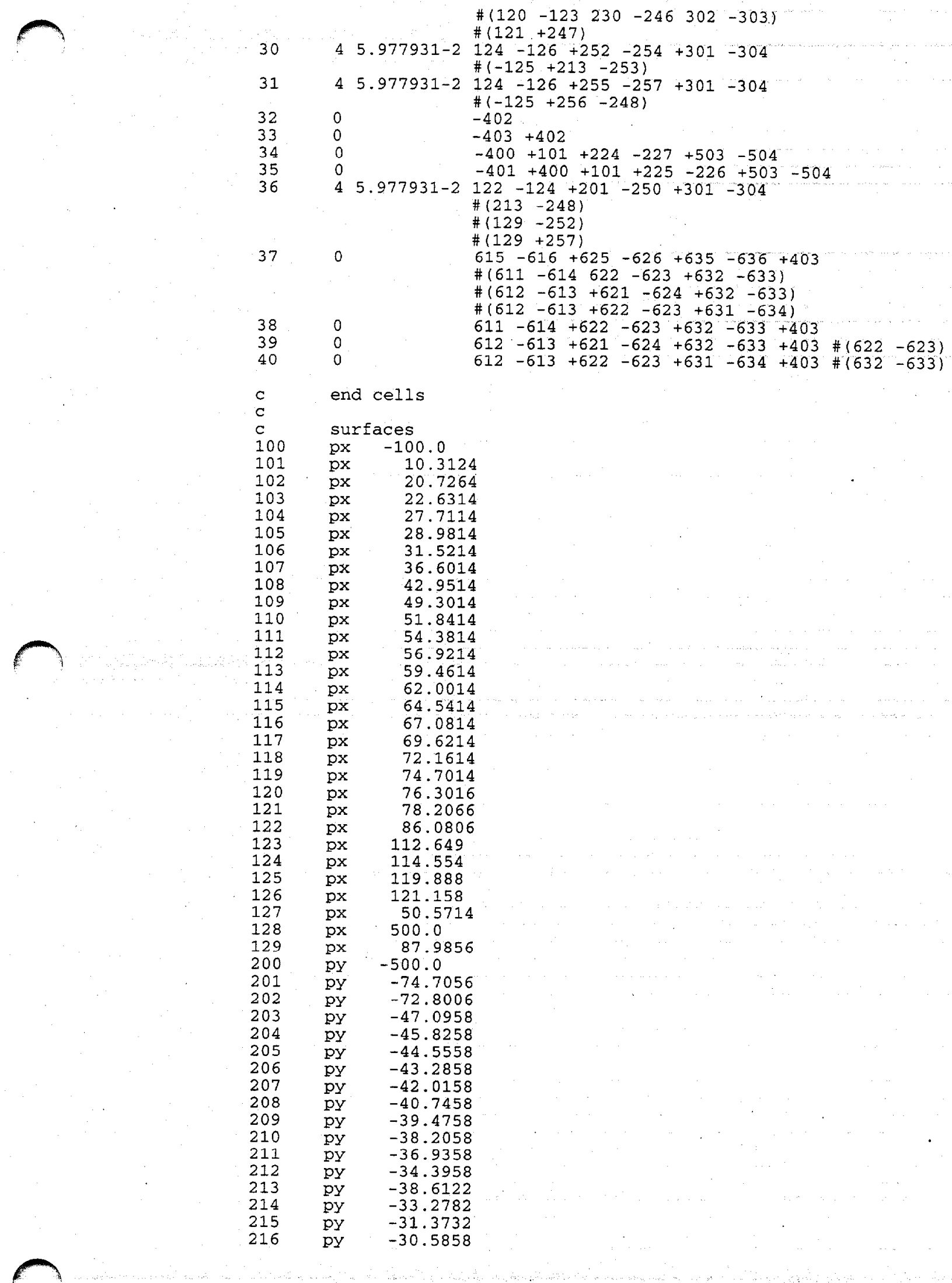




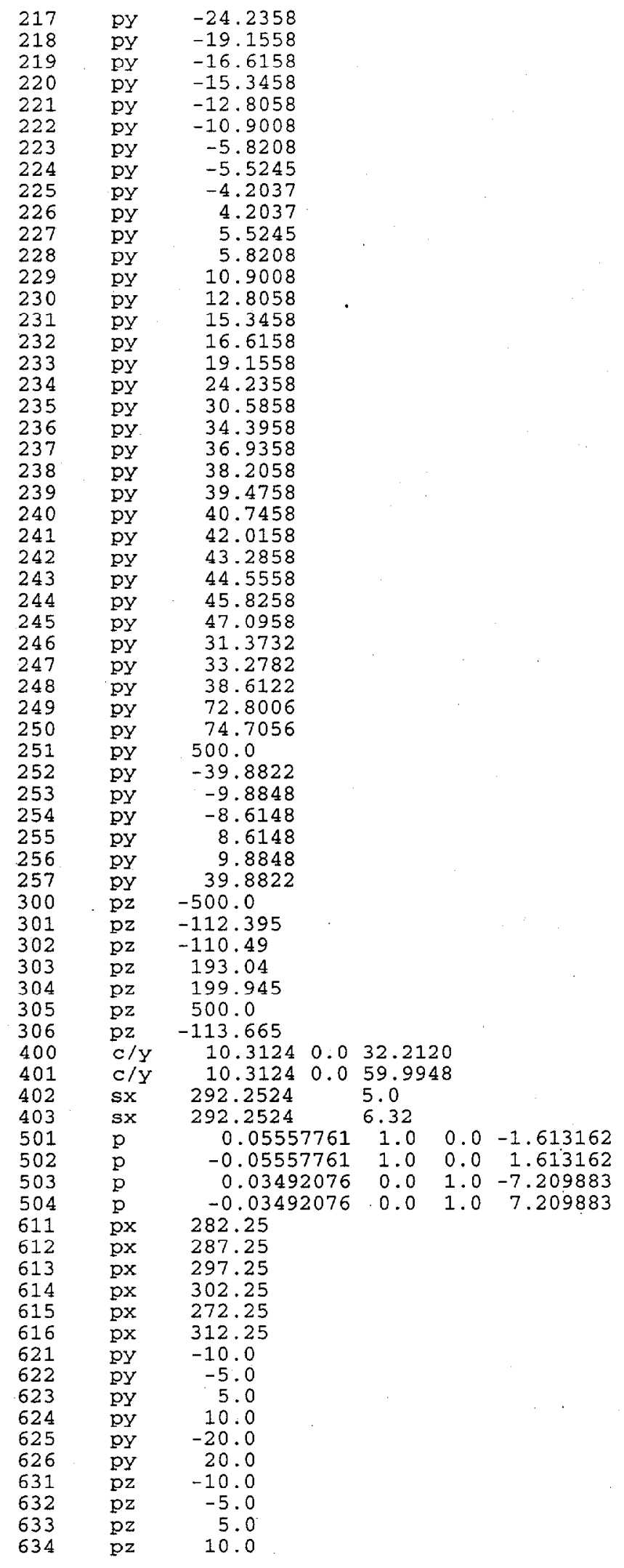


$\begin{array}{rrrr}635 & \mathrm{pz} & -20.0 \\ 636 & \mathrm{pz} & 20.0\end{array}$

c end surfaces

mode $n$ p

imp:n,p $0138 x$

c $\quad \mathrm{m} 1$ =borated polyethylene (Asum=0.10549)

m1 $\quad 1001.60 \mathrm{c} \quad 6.5800-2 \quad 5010.60 \mathrm{c} \quad 5.1940-4 \quad 5011.60 \mathrm{c} \quad 2.1306-3$

$\quad 6000.60 \mathrm{c}$

me1 poly.01t

$c \mathrm{~m} 2=$ borated paraffin (Asum $=0.1187956$ )

m2 $\begin{array}{lllllll}1001.60 \mathrm{C} & 7.8350-2 & 5010.60 \mathrm{C} & 4.3426-4 & 5011.60 \mathrm{C} & 1.78134-3 \\ 6000.60 \mathrm{C} & 3.8230-2 & & & & \end{array}$

mt2 poly.01t

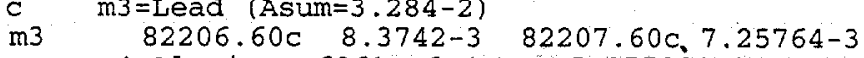

$\mathrm{m} 4=\mathrm{A}$ luminum $6061-\mathrm{T} 6 \quad$ (Asum $=5.977988-2$ )

m4

$\begin{array}{llll}12000.60 \mathrm{C} & 6.6890-4 & 13027.60 \mathrm{C} & 5 . \\ 22000.60 \mathrm{C} & 5.0930-5 & 24050.60 \mathrm{C} & 2.72\end{array}$

24053.600

$\begin{array}{llll}29063.60 \mathrm{C} & 9.08133-5 & 29065.60 \mathrm{C} & 4.04767-5\end{array}$

c $\quad \mathrm{m} 5=$ Steel (Asum=8.734441-2)

m5

$26054.60 \mathrm{C} \quad 3.2367-3 \quad 26056.60 \mathrm{c} \quad 5.07622-2$

$26058.60 \mathrm{c} \quad 1.5490-4 \quad 24050.60 \mathrm{c} \quad 7.9660-4$

$24053.60 \mathrm{c} \quad 1.7397-3 \quad 24054.60 \mathrm{c} \quad 4.3220-4$

$28060.60 \mathrm{C} 2.55073-3 \quad 28061.60 \mathrm{C} \quad 1.1090-4$

$28064.60 \mathrm{c} \quad 9.0460-5 \quad 6000.60 \mathrm{c} \quad 3.16929-4$

$25055.60 \mathrm{C} \quad 1.73219-3 \quad 15031.60 \mathrm{C} \quad 6.91287-5$

$7014.60 \mathrm{C} \quad 5.43529-4$

c m6=Plutonium (Asum $=4.070346-2$ )

m6 $\begin{array}{rrrrrr}94239.60 c & 3.7291-2 & 94240.60 \mathrm{c} & 1.9277-3 & 94241.60 \mathrm{c} & 1.2196-4 \\ & 31000.60 \mathrm{c} & 1.3628-3 & & 1.36\end{array}$

C $m 7=$ Tungsten (Asum $=6.605306-2$ )

m7 $28058.60 \mathrm{C} \quad 6.6122-3 \quad 28060.60 \mathrm{C} \quad 2.54659-3 \quad 28061.60 \mathrm{C} \quad 1.10721-4$ $28062.60 \mathrm{c} \quad 3.5256-4 \quad 28064.60 \mathrm{c} \quad 9.0325-5 \quad 29063.60 \mathrm{c} \quad 2.82034-3$ $29065.60 \mathrm{c} \quad 1.25706-3 \quad 40000.60 \mathrm{c} \quad 7.9528-4 \quad 74182.60 \mathrm{c} \quad 1.369975-2$

f102: $\quad 74183.60 c \quad 7.36713-3 \quad 74184.60 \mathrm{c} \quad 1.577082-2 \quad 74186.60 \mathrm{c} \quad 1.463029-2$

de102 $2.07002-7 \quad 7.69672-7 \quad 1.75386-63.71293-6$ $7.86024-6 \quad 1.66401-5 \quad 3.52273-5 \quad 7.45761-5 \quad 1.34159-4$ $\begin{array}{llllll}2.21191-4 & 3.64682-4 & 6.01258-4 & 9.91309-4 & 1.63439-3\end{array}$ $\begin{array}{llllll}2.14167-3 & 2.43063-3 & 2.82399-3 & 3.37141-3 & 4.61914-3\end{array}$ $7.32483-3 \quad 1.20766-2 \quad 1.93065-2 \quad 2.41830-2 \quad 2.54229-2$ 2.65293-2 2.77506-2 $3.14039-2 \quad 4.33909-2 \quad 5.45187-2$ 7.15396-2 1.04645-1 1.36365-1 1.66556-1 2.03432-1 $2.48472-1 \quad 2.83877-12.95864-1 \quad 2.97851-1 \quad 3.00232-1$ 3.44858-1 $4.42806-1 \quad 5.10634-1 \quad 5.65749-1 \quad 6.75418-1$ $8.24957-1 \quad 1.00760+0 \quad 1.23069+0.1 .50317+0 \quad 1.83598+0$ $2.12513+0 \quad 2.28850+0 \quad 2.40583+0 \quad 2.73896+0 \quad 3.34537+0$ $4.08604+0 \quad 4.99070+0 \quad 6.09565+0 \quad 7.44525+0 \quad 9.09365+0$ $1.11070+1 \quad 1.35661+1$

df102 $3.70370-3 \quad 4.37060-3 \quad 4.52500-3 \quad 4.57410-3$ $4.55830-3 \quad 4.48970-3 \quad 4.38170-3 \quad 4.24920-3 \quad 4.12890-3$ $4.03110-3 \quad 3.93550-3 \quad 3.84530-3 \quad 3.76290-3 \quad 3.69080-3$ $3.65280-3 \quad 3.63870-3 \quad 3.62290-3 \quad 3.60620-3 \quad 3.58250-3$ $3.55850-3 \quad 4.08750-3 \quad 5.89750-3 \quad 7.13870-3 \quad 7.42410-3$ $7.67700-3 \quad 7.95180-38.74210-31.11490-2 \quad 1.35030-2$ $1.65080-2 \quad 2.25270-2 \quad 2.86610-2 \quad 3.43150-2 \quad 4.10860-2$ $4.92110-2 \quad 5.56070-2 \quad 5.77300-2 \quad 5.80800-2 \quad 5.84980-2$ $6.59980-2 \quad 8.26560-2 \quad 9.04890-2 \quad 9.54810-2 \quad 1.05360-1$ $1.17860-1 \quad 1.29670-1 \quad 1.30100-1 \quad 1.28650-1 \quad 1.27220-1$ $1.26160-1 \quad 1.25620-1 \quad 1.25270-1 \quad 1.28500-1 \quad 1.36960-1$ $1.45980-1 \quad 1.54120-1 \quad 1.51160-1$ 1.47220-1 1.47060-1 $1.63200-12.00340-1$

fm102 $\quad 3.671+10$

E112:n $\quad 614$

de112

$2.07002-7 \quad 7.69672-7 \quad 1.75386-6 \quad 3.71293-6$ 7.86024-6 $1.66401-5 \quad 3.52273-5 \quad 7.45761-5 \quad 1.34159-4$ 2.21191-4 $3.64682-4 \quad 6.01258-4 \quad 9.91309-4 \quad 1.63439-3$ 2.14167-3 2.43063-3 2.82399-3 $3.37141-3 \quad 4.61914-3$ $7.32483-31.20766-2 \quad 1.93065-2 \quad 2.41830-2 \quad 2.54229-2$ 
$\operatorname{df} 112$

$\operatorname{fm} 112$

$\pm 122: \mathrm{n}$

de122

$\operatorname{df} 122$

$\operatorname{fm} 122$

f132:n

de132

df132
$2.65293-2 \quad 2.77506-2 \quad 3.14039-2 \quad 4.33909-2 \quad 5.45187-2$ 7.15396-2 1.04645-1 $1.36365-1 \quad 1.66556-1 \quad 2.03432-1$ $2.48472-1 \quad 2.83877-1 \quad 2.95864-1 \quad 2.97851-1 \quad 3.00232-1$ 3.44858-1 4.42806-1 5.10634-1 $5.65749-1 \quad 6.75418-1$ $8.24957-1 \quad 1.00760+0 \quad 1.23069+0 \quad 1.50317+0 \quad 1.83598+0$ $2.12513+0 \quad 2.28850+0 \quad 2.40583+0 \quad 2.73896+0 \quad 3.34537+0$ $4.08604+0 \quad 4.99070+0 \quad 6.09565+0 \quad 7.44525+0 \quad 9.09365+0$ $1.11070+11.35661+1$

$3.70370-3 \quad 4.37060-3 \quad 4.52500-3 \quad 4.57410-3$ $4.55830-3 \quad 4.48970-3 \quad 4.38170-3 \quad 4.24920-3 \quad 4.12890-3$ $\begin{array}{llllll}4.03110-3 & 3.93550-3 & 3.84530-3 & 3.76290-3 & 3.69080-3\end{array}$ $3.65280-3 \quad 3.63870-3 \quad 3.62290-3 \quad 3.60620-3 \quad 3.58250-3$ $\begin{array}{llllll}3.55850-3 & 4.08750-3 & 5.89750-3 & 7.13870-3 & 7.42410-3\end{array}$ $7.67700-3 \quad 7.95180-3 \quad 8.74210-3 \quad 1.11490-2 \quad 1.35030-2$ $1.65080-2 \quad 2.25270-2 \quad 2.86610-2 \quad 3.43150-2 \quad 4.10860-2$ $4.92110-2 \quad 5.56070-2 \quad 5.77300-2 \quad 5.80800-2 \quad 5.84980-2$ $6.59980-2 \quad 8.26560-2 \quad 9.04890-2 \quad 9.54810-2 \quad 1.05360-1$ 1.17860-1 $1.29670-1 \quad 1.30100-1 \quad 1.28650-1 \quad 1.27220-1$ $1.26160-1$ 1.25620-1 $1.2527 \ldots 1.28500-11.36960-1$ $1.45980-11.54120-11.511 \ldots .47220-11.47060-1$ 1. 63200-1 2.00340-1

$3.671+10$

624

$2.07002-7 \quad 7.6967 \ldots \quad \ldots .75386-6 \quad 3.71293-6$ 7.86024-6 $1.66401-5 \quad 3.52273 \ldots 7.45761-5 \quad 1.34159-4$ 2.21191-4 $3.64682-4 \quad 6.01258-4 \quad 9.91309-4 \quad 1.63439-3$ 2.14167-3 $2.43063-3 \quad 2.82399-3 \quad 3.37141-3 \quad 4.61914-3$ $\begin{array}{llllll}7.32483-3 & 1.20766-2 & 1.93065-2 & 2.41830-2 & 2.54229-2\end{array}$ 2.65293-2 2.77506-2 3.14039-2 4.33909-2 5.45187-2 7.15396-2 $1.04645-1 \quad 1.36365-1 \quad 1.66556-1 \quad 2.03432-1$ $\begin{array}{llllll}2.48472-1 & 2.83877-1 & 2.95864-1 & 2.97851-1 & 3.00232-1\end{array}$ 3.44858-1 4.42806-1 $5.10634-1 \quad 5.65749-1 \quad 6.75418-1$ 8.24957-1 $1.00760+01.23069+0 \quad 1.50317+0 \quad 1.83598+0$ $\begin{array}{lllll}2.12513+0 & 2.28850+0 & 2.40583+0 & 2.73896+0 & 3.34537+0\end{array}$ $4.08604+0 \quad 4.99070+0 \quad 6.09565+0 \quad 7.44525+0 \quad 9.09365+0$ $1.11070+1 \quad 1.35661+1$

$\begin{array}{lllll}1.70370-3 & 4.37060-3 & 4.52500-3 & 4.57410-3\end{array}$ 4.55830-3 $4.48970-3 \quad 4.38170-3 \quad 4.24920-3 \quad 4.12890-3$ $4.03110-3 \quad 3.93550-3 \quad 3.84530-3 \quad 3.76200-3 \quad 3.69080-3$ $3.65280-3 \quad 3.63870-3 \quad 3.62290-3 \quad 3.60620-3 \quad 3.58250-3$ $3.55850-3 \quad 4.08750-3 \quad 5.89750-3 \quad 7.13870-3 \quad 7.42410-3$ $7.67700-3 \quad 7.95180-3 \quad 8.74210-3.1 .11490-2 \quad 1.35030-2$ $1.65080-2 \quad 2.25270-2 \quad 2.86610-2 \quad 3.43150-2 \quad 4.10860-2$ 4.92110-2 5.56070-2 5.77300-2 $5.80800-2 \quad 5.84980-2$ $6.59980-2 \quad 8.26560-2 \quad 9.04890-2 \quad 9.54810-2 \quad 1.05360-1$

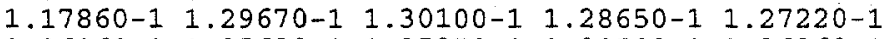

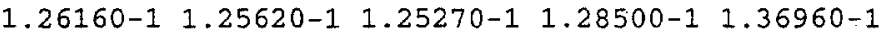
$1.45980-1 \quad 1.54120-1 \quad 1.51160-1 \quad 1.47220-1 \quad 1.47060-1$ $1.63200-12.00340-1$

$3 \cdot 671+10$

634

$\begin{array}{lllll}2.07002-7 & 7.69672-7 & 1.75386-6 & 3.71293-6\end{array}$ 7.86024-6 $1.66401-5 \quad 3.52273-5 \quad 7.45761-5 \quad 1.34159-4$ $\begin{array}{llllll}2.21191-1 & 3.64682-4 & 6.01258-1 & 9.91309-4 & 1.63439-3\end{array}$ 2.14167-3 $2.43063-3 \quad 2.82399-3 \quad 3.37141-3 \quad 4.61914-3$ $7.32483-3 \quad 1.20766-2 \quad 1.93065-2 \quad 2.41830-2 \quad 2.54229-2$ 2.65293-2 $2.77506-2 \quad 3.14039-2 \quad 4.33909-2 \quad 5.45187-2$ 7.15396-2 1.04645-1 $1.36365-1 \quad 1.56556-1 \quad 2.03432-1$ $\begin{array}{llllll}2.48472-1 & 2.83877-1 & 2.95864-1 & 2.97851-1 & 3.00232-1\end{array}$ 3.44858-1 4.42806-1 $5.10634-1 \quad 5.65749-1 \quad 6.75418-1$ $8.24957-11.00760+0 \quad 1.23069+0 \quad 1.50317+0 \quad 1.83598+0$ $2.12513+0 \quad 2.28850+0 \quad 2.40583+0 \quad 2.73896+0 \quad 3.34537+0$ $4.08604+0 \quad 4.99070+0 \quad 6.09565+0 \quad 7.44525+0 \quad 9.09365+0$ $1.11070+1 \quad 1.35661+1$

$3.70370-3 \quad 4.37060-3 \quad 4.52500-3 \quad 4.57410-3$ 4.55830-3 $4.48970-3 \quad 4.38170-3 \quad 4.24920-3 \quad 4.12890-3$ $\begin{array}{llllll}4.03110-3 & 3.93550-3 & 3.84530-3 & 3.76290-3 & 3.69080-3\end{array}$ $\begin{array}{llllll}3.65280-3 & 3.63870-3 & 3.62290-3 & 3.60620-3 & 3.58250-3\end{array}$ $\begin{array}{llllll}3.55850-3 & 4.08750-3 & 5.89750-3 & 7.13870-3 & 7.42410-3\end{array}$ $\begin{array}{lllll}7.67700-3 & 7.95180-3 & 8.74210-3 & 1.11490-2 & 1.35030-2\end{array}$ $\begin{array}{lllll}1.65080-2 & 2.25270-2 & 2.86610-2 & 3.43150-2 & 4.10851-2\end{array}$ $4.92110-2 \quad 5.56070-2 \quad 5.77300-2 \quad 5.80800-2 \quad 5.8496 \% ?$ 
$6.59980-2 \quad 8.26560-2 \quad 9.04890-2 \quad 9.54810-2 \quad 1.05360-1$

$1.17860-1 \quad 1.29670-1 \quad 1.30100-1 \quad 1.28650-1 \quad 1.27220-1$

$1.26160-1 \quad 1.25620-1$ 1.25270-1 $1.28500-1$ 1.36960-1

$1.45980-1.1 .54120-1 \quad 1.51160-11.47220-1 \% 1.47060-1$

$1.63200-12.00340-1$

fm1.32 $3.671+10$

$£ 202: \mathrm{p} \quad 611$

de202 $1.50000-2 \quad 3.25000-2 \quad 5.75000-28.50000-2$

$\begin{array}{lllllll}1.25000-1 & 2.25000-1 & 3.50000-1 & 4.55000-1 & 5.55000-1\end{array}$

$6.50000-18.50000-11.25000+01.75000+02.25000+0$

$2.75000+0 \quad 3.50000+0 \quad 4.50000+0 \quad 5.50000+0 \quad 6.50000+0$

$7.25000+07.75000+0 \quad 9.00000+01.20000+1$

df202

$\begin{array}{llll}2.14390-3 & 5.77600-4 & 2.71850-4 & 2.68170-4\end{array}$

$3.27670-4 \quad 5.66760-4 \quad 8.75940-4 \quad 1.08450-3 \quad 1.27970-3$

$\begin{array}{lllll}1.44170-3 & 1.75630-3 & 2.31560-3 & 2.92700-3 & 3.46860-3\end{array}$

$3.95960-3 \quad 4.62210-3 \quad 5.41370-3 \quad 6.19090-3 \quad 6.92650-3$

$7.47830-3 \quad 7.84680-3 \quad 8.77160-3 \quad 1.10200-2$ 


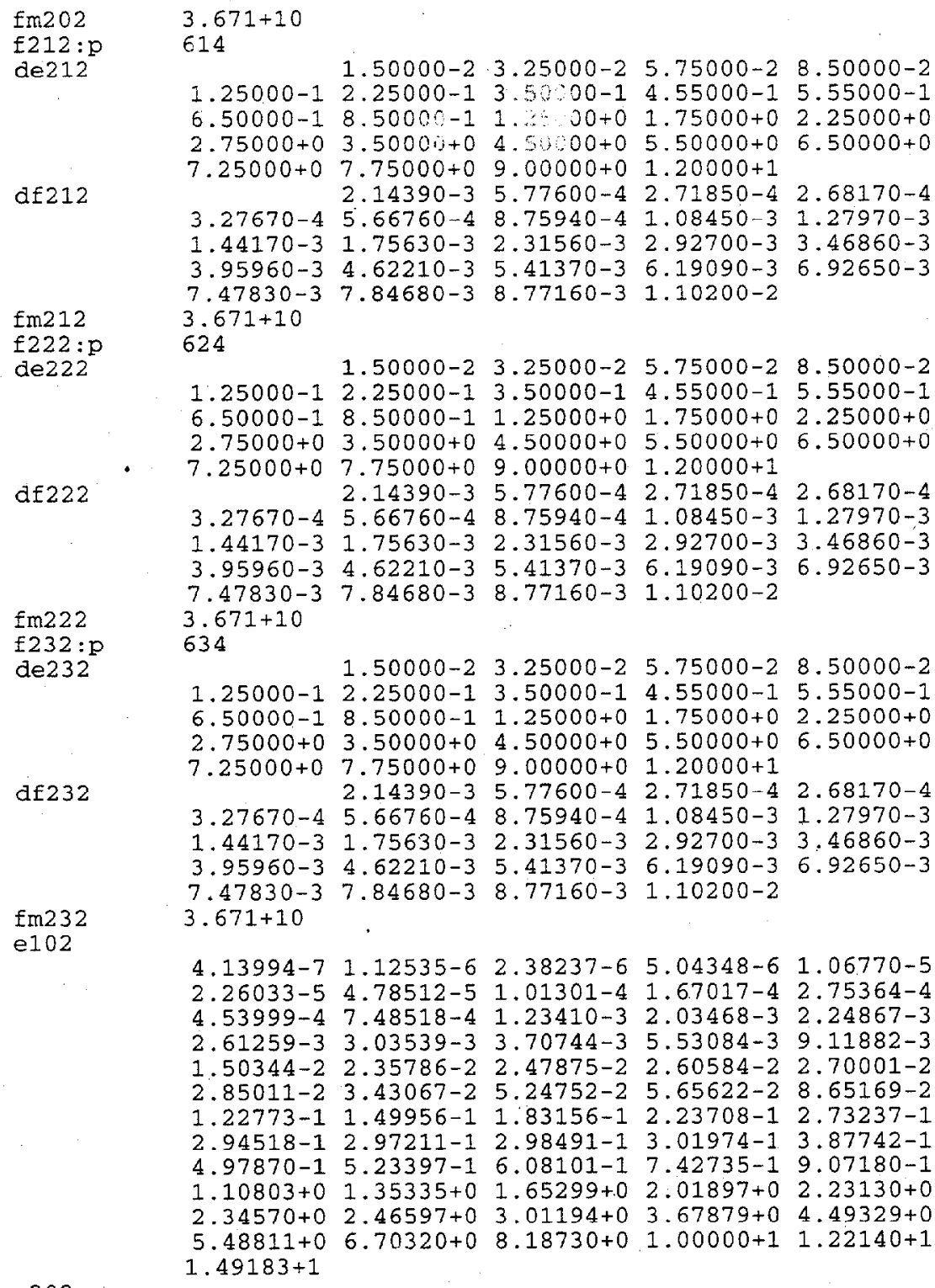

4.13994-7 $1.12535-6 \quad 2.38237-6 \quad 5.04348-6 \quad 1.06770-5$ 2.26033-5 $4.78512-5 \quad 1.01301-4 \quad 1.67017-4 \quad 2.75364-4$ 4.53999-4 $7.48518-4 \quad 1.23410-3 \quad 2.03468-3 \quad 2.24867-3$ $\begin{array}{lllllll}2.61259-3 & 3.03539-3 & 3.70744-3 & 5.53084-3 & 9.11882-3\end{array}$ $1.50344-2 \quad 2.35786-2 \quad 2.47875-2 \quad 2.60584-2 \quad 2.70001-2$ 2.85011-2 $3.43067-2 \quad 5.24752-2 \quad 5.65622-2 \quad 8.65169-2$ $\begin{array}{llllll}1.22773-1 & 1.49956-1 & 1.83156-1 & 2.23708-1 & 2.73237-1\end{array}$ 2.94518-1 $2.97211-1 \quad 2.98491-1 \quad 3.01974-1 \quad 3.87742-1$ $\begin{array}{lllll}4.97870-1 & 5.23397-1 & 6.08101-1 & 7.42735-1 & 9.07180-1\end{array}$ $\begin{array}{llllll}1.10803+0 & 1.35335+0 & 1.65299+0 & 2.01897+0 & 2.23130+0\end{array}$ $2.34570+0 \quad 2.46597+0 \quad 3.01194+0 \quad 3.67879+0 \quad 4.49329+0$ $\begin{array}{llllll}5.48811+0 & 6.70320+0 & 8.18730+0 & 1.00000+1 & 1.22140+1\end{array}$ $1.49183+1$

e202

$2.00000-2 \quad 4.50000-2 \quad 7.00000-2 \quad 1.00000-1 \quad 1.50000-1$ 3.00000-1 4.00000-1 $5.10000-1 \quad 6.00000-1 \quad 7.00000-1$ $1.00000+01.50000+0 \quad 2.00000+0 \quad 2.50000+0 \quad 3.00000+0$ $4.00000+0 \quad 5.00000+0 \quad 6.00000+0 \quad 7.00000+0 \quad 7.50000+0$ $8.00000+01.00000+1 \quad 1.40000+1$

$\mathrm{e} 112$

4.13994-7 $1.12535-6 \quad 2.38237-6 \quad 5.04348-6 \quad 1.06770-5$ 2.26033-5 $4.78512-5$ 1.01301-4 $1.67017-4 \quad 2.75364-4$ $\begin{array}{llllll}4.53999-4 & 7.48518-4 & 1.23410-3 & 2.03468-3 & 2.24867-3\end{array}$ $\begin{array}{llllll}2.61259-3 & 3.03539-3 & 3.70744-3 & 5.53084-3 & 9.11882-3\end{array}$ 1.50344-2 2.35786-2 2.47875-2 2.60584-2 2.70001-2 $\begin{array}{llllll}2.85011-2 & 3.43067-2 & 5.24752-2 & 5.65622-2 & 8.65169-2\end{array}$ 1.22773-1 $1.49956-1 \quad 1.83156-1 \quad 2.23708-1 \quad 2.73237-1$ 2.94518-1 2.97211-1 $2.98491-1 \quad 3.01974-1 \quad 3.87742-1$ $\begin{array}{lllll}4.97870-1 & 5.23397-1 & 6.08101-1 & 7.42735-1 & 9.07180-1\end{array}$ $1.10803+0 \quad 1.35335+0 \quad 1.65299+0 \quad 2.01897+0 \quad 2.23130+0$ $2.34570+0 \quad 2.46597+0 \quad 3.01194+0 \quad 3.67879+0 \quad 4.49329+0$ $5.48811+0 \quad 6.70320+0 \quad 8.18730+0 \quad 1.00000+1 \quad 1.22140+1$ 1. $49183+1$

e212 
$2.00000-2 \quad 4.50000-2 \quad 7.00000-2 \quad 1.00000-1 \quad 1.50000-1$

3.00000-1 4.00000-1 $5.10000-1 \quad 6.00000-1 \quad 7.00000-1$

$1.00000+01.50000+02.00000+02.50000+03.00000+0$

$4.00000+0 \quad 5.00000+0 \quad 6.00000+0 \quad 7.00000+0 \quad 7.50000+0$

$\mathrm{e} 122$

$8.00000+01.00000+11.40000+1$

4.13994-7 $1.12535-6 \quad 2.38237-6 \quad 5.04348-6 \quad 1.06770-5$ $2.26033-5 \quad 4.78512-5 \quad 1.01301-4 \quad 1.67017-4 \quad 2.75364-4$ $\begin{array}{llllll}4.53999-4 & 7.48518-4 & 1.23410-3 & 2.03468-3 & 2.24867-3\end{array}$

$\begin{array}{llllll}2.61259-3 & 3.03539-3 & 3.70744-3 & 5.53084-3 & 9.11882-3\end{array}$

$1.50344-2 \quad 2.35786-2 \quad 2.47875-2 \quad 2.60584-2 \quad 2.70001-2$

2.85011-2 $3.43067-2 \quad 5.24752-2 \quad 5.65622-2 \quad 8.65169-2$

1.22773-1 1.49956-1 $1.83156-1$ 2.23708-1 $2.73237-1$

2.94518-1 2.97211-1 2.98491-1 3.01974-1 3.87742-1

$4.97870-1 \quad 5.23397-1 \quad 6.08101-1 " 7.42735-1 \quad 9.07180-1$

$1.10803+0 \quad 1.35335+0 \quad 1.65299+0 \quad 2.01897+0 \quad 2.23130+0$

$2.34570+0 \quad 2.46597+0 \quad 3.01194+0 \quad 3.67879+0 \quad 4.49329+0$

$5.48811+0 \quad 6.70320+0 \quad 8.18730+0 \quad 1.00000+1 \quad 1.22140+1$

$\mathrm{e} 222$ $1.49183+1$

$2.00000-2 \quad 4.50000-27.00000-21.00000-11.50000-1$ 3.00000-1 4.00000-1 5.10000-1 6.00000-1 7.00000-1

$1.00000+0 \quad 1.50000+0 \quad 2.00000+0 \quad 2.50000+0 \quad 3.00000+0$ $4.00000+0 \quad 5.00000+0 \quad 6.00000+0 \quad 7.00000+0 \quad 7.50000+0$ $8.00000+01.00000+11.40000+1$

e132

4.13994-7 $1.12535-6 \quad 2.38237-6 \quad 5.04348-6 \quad 1.06770-5$ $2.26033-5 \quad 4.78512-5 \quad 1.01301-4 \quad 1.67017-4 \quad 2.75364-4$ 4.53999-4 7.48518-4 $1.23410-3 \quad 2.03468-3 \quad 2.24867-3$

$\begin{array}{llllll}2.61259-3 & 3.03539-3 & 3.70744-3 & 5.53084-3 & 9.11882-3\end{array}$

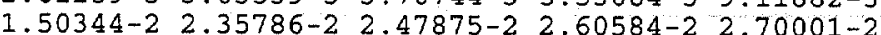

2.85011-2 3.43067-2 $5.24752-2 \quad 5.65622-2 \quad 8.65169-2$

1.22773-1 1.49956-1 $1.83156-1 \quad 2.23708-1 \quad 2.73237-1$

2.94518-1 2.97211-1 2.98491-1 3.01974-1 3.87742-1

4.97870-1 $5.23397-1 \quad 6.08101-1 \quad 7.42735-1 \quad 9.07180-1$

$1.10803+0 \quad 1.35335+0 \quad 1.65299+0 \quad 2.01897+0 \quad 2.23130+0$

$2.34570+0 \quad 2.46597+0 \quad 3.01194+0 \quad 3.67879+0 \quad 4.49329+0$

$5.48811+0 \quad 6.70320+0 \quad 8.18730+0 \quad 1.00000+11.22140+1$ $1.49183+1$

e232

$2.00000-2 \quad 4.50000-2 \quad 7.00000-2 \quad 1.00000-1 \quad 1.50000-1$

$3.00000-1 \quad 4.00000-1 \quad 5.10000-1 \quad 6.00000-1 \quad 7.00000-1$

$1.00000+0 \quad 1.50000+0.2 .00000+0 \quad 2.50000+0 \quad 3.00000+0$

$4.00000+0 \quad 5.00000+0 \quad 6.00000+0 \quad 7.00000+0 \quad 7.50000+0$

$\begin{array}{ll}\operatorname{sd} 102 & 100.0 \\ \text { sd112 } & 100.0\end{array}$

$\operatorname{sd} 122 \quad 100.0$

sd132 100.0

sd202 100.0

sd212 100.0

sd222 100.0

sd232 100.0

sdef pos $=0$ o 0 vec $=1$ o 0 dir=d1 erg=fdir $=d 2$

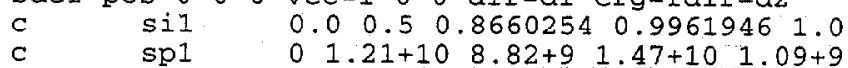

si1 s $11 \quad 12 \quad 13.14$

$\begin{array}{lllll}\operatorname{sp} 1 & 1.21+10 & 8.82+9 & 1.47+10 & 1.09+9\end{array}$

sil1 h $0.0 \quad 0.5$

sil2 h $0.5 \quad 0.8660254$

si13 h 0.86602540 .9961946

si14 h 0.99619461 .0

$\operatorname{sp11} \quad 0 \quad 1$

sp12 01

$\operatorname{sp13} \cdot 01$

sp14 01

$\begin{array}{lllll}\text { ds2 } 2 & 21 & 22 & 23 & 24\end{array}$

si21 h $3.7645 \quad 5.675$

si22 h 5.6757 .3865

si23 h 7.38658 .321

si24 h $8.321 \quad 8.753$

sp21 01 


$$
\begin{aligned}
& \text { sp22 } 01 \\
& \begin{array}{lll}
\operatorname{sp} 23 & 0 & 1 \\
\operatorname{sp} 24 & 0 & 1
\end{array} \\
& \text { nps } 2000000 \\
& \text { pramp } 2 \mathrm{j} 1
\end{aligned}
$$


File for MCNP-4B surface-detector dose-rate calculations for a tungstenreflected Pu sphere in the PFNA beam at the center of the truck lane $(400,000$ histories $)$

message: outp=pfna 7.0 mctal=pfna $7 . m$

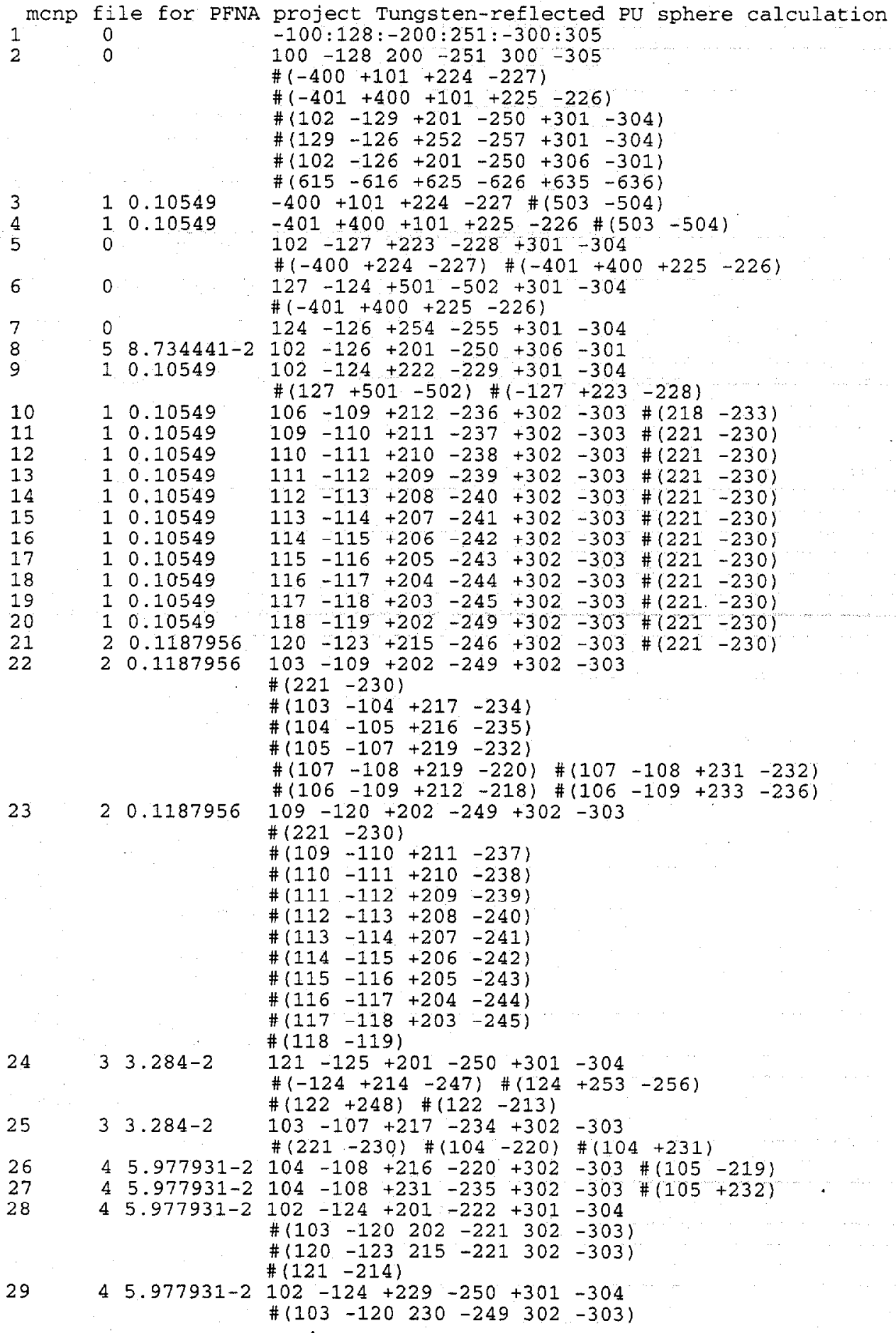




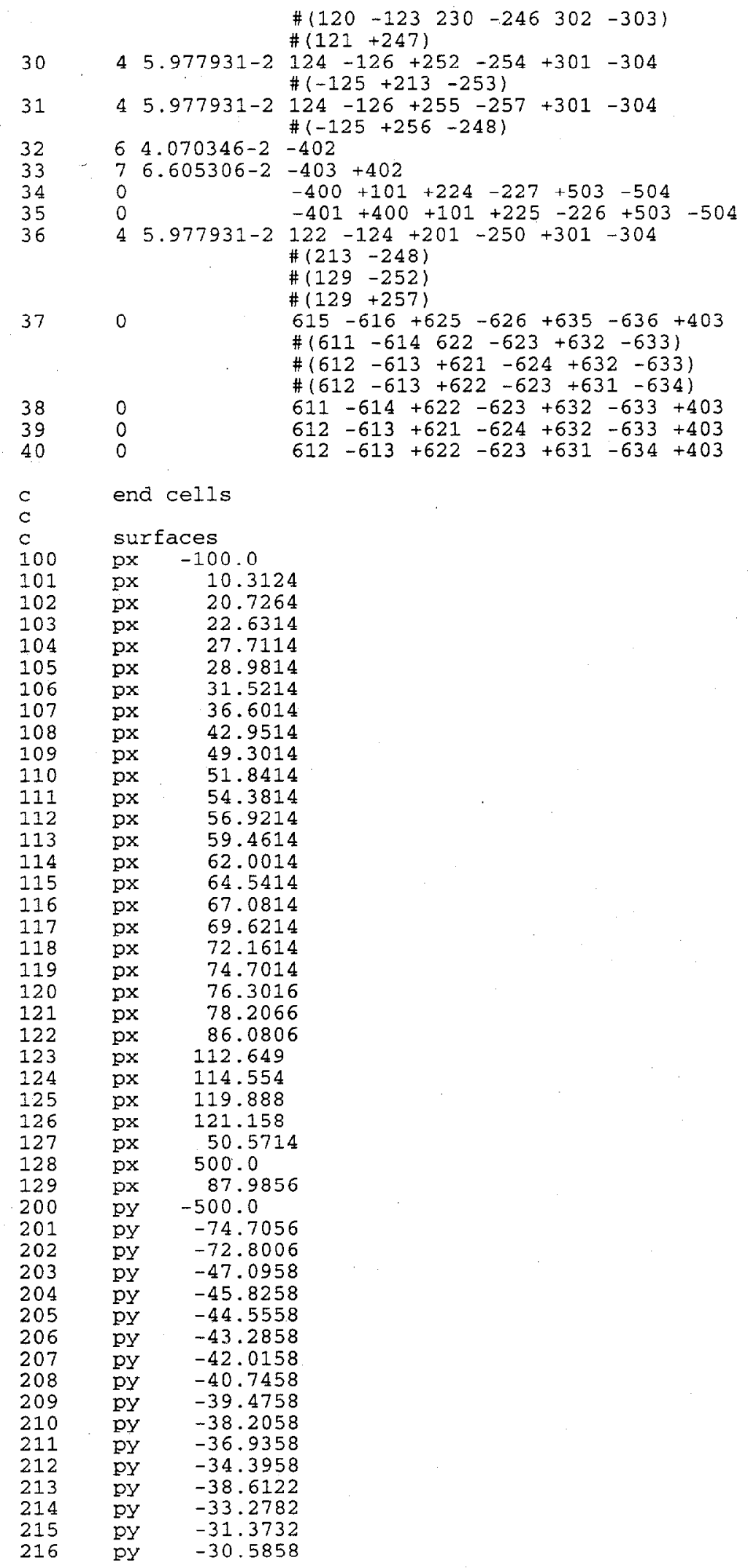




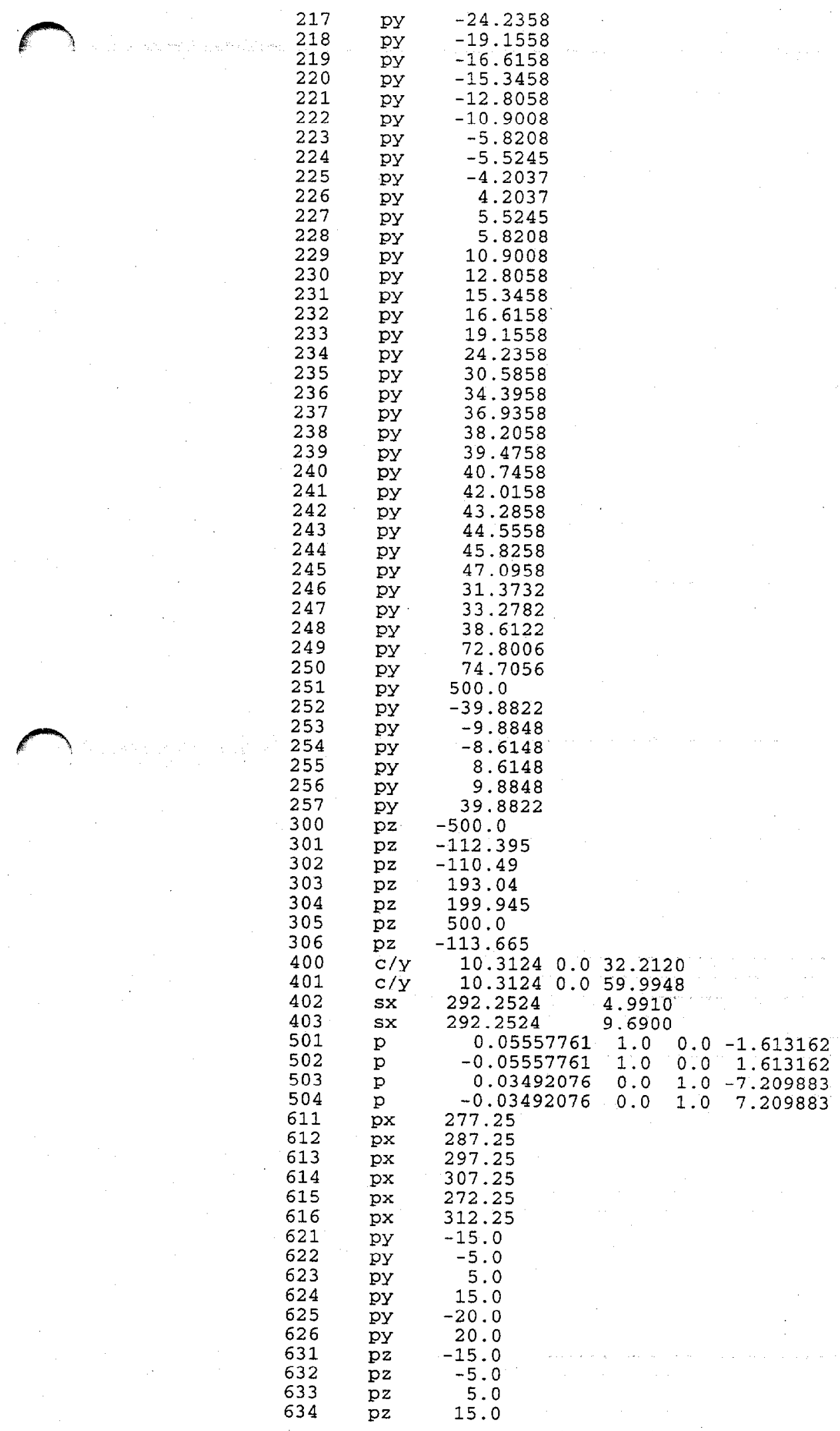




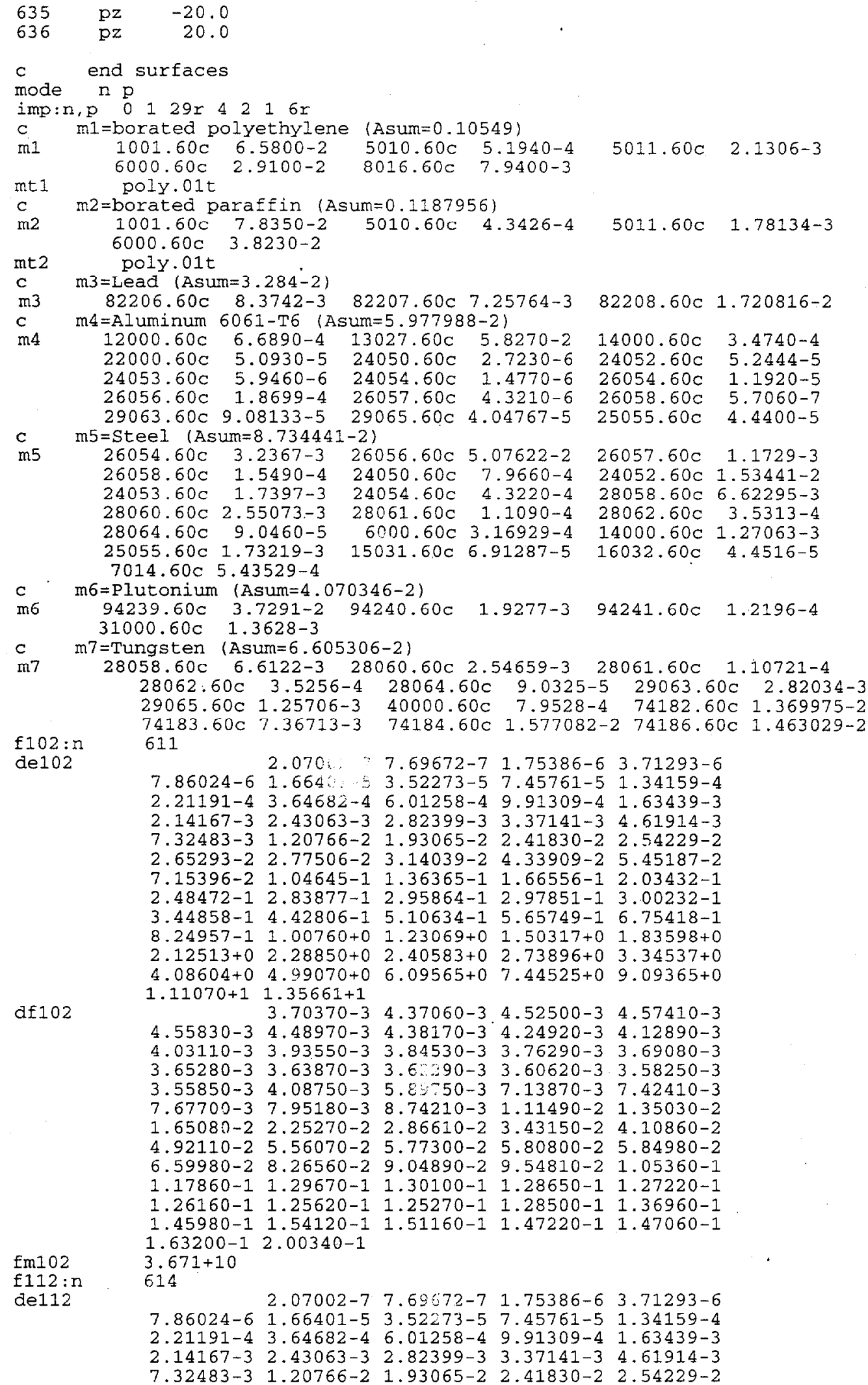


$2.65293-2 \quad 2.77506-2 \quad 3.14039-2 \quad 4.33909-2 \quad 5.45187-2$

$7.15396-2 \quad 1.04645-1 \quad 1.36365-1 \quad 1.66556-1 \quad 2.03432-1$

$2.48472-1$ 2.83877-1 $2.95864-1$ 2.97851-1 $3.00232-1$

3.44858-1 $4.42806-1 \quad 5.10634-1 \quad 5.65749-1 \quad 6.75418-1$ $8.24957-1 \quad 1.00760+0 \quad 1.230 .69+0 \quad 1.50317+0 \quad 1.83598+0$ $2.12513+0 \quad 2.28850+0 \quad 2.40583+0 \quad 2.73896+0 \quad 3.34537+0$ $4.08604+0.4 .99070+0 \quad 6.09565+0 \quad 7.44525+0 \quad 9.09365+0$ $1.11070+11.35661+1$

df112 $3.70370-3 \quad 4.37060-3 \quad 4.52500-3 \quad 4.57410-3$ 4.55830-3 $4.48970-3 \quad 4.38170-3 \quad 4.24920-3 \quad 4.12890-3$ $4.03110-3 \quad 3.93550-3 \quad 3.84530-3 \quad 3.76290-3 \quad 3.69080-3$ $3.65280-3 \quad 3.63870-3 \quad 3.62290-3 \quad 3.60620-3 \quad 3.58250-3$ $3.55850-3 \quad 4.08750-3 \quad 5.89750-3 \quad 7.13870-3 \quad 7.42410-3$ $7.67700-3 \quad 7.95180-3 \quad 8.74210-3 \quad 1.11490-2 \quad 1.35030-2$ $1.65080-2 \quad 2.25270-2 \quad 2.86610-2 \quad 3.43150-2 \quad 4.10860-2$ $4.92110-2 \quad 5.56070-2 \quad 5.77300-2 \quad 5.80800-2 \quad 5.84980-2$ 6.59980-2 $8.26560-2 \quad 9.04890-2 \quad 9.54810-2 \quad 1.05360-1$ $1.17860-1.1 .29670-1 \quad 1.30100-1 \quad 1.28650-11.27220-1$ $1.26160-1 \quad 1.25620-1 \quad 1.25270-1 \quad 1.28500-1 \quad 1.36960-1$ $1.45980-1 \quad 1.54120-1 \quad 1.51160-1 \quad 1.47220-1 \quad 1.47060-1$ $1.63200-12.00340-1$

$\operatorname{fm} 112 \quad 3.671+10$

$\begin{array}{lllllll}\text { f122:n } & 624 & 2.07002-7 & 7.69672-7 & 1.75386-6 & 3.71293-6\end{array}$ $7: 86024-6 \quad 1.66401-5 \quad 3.52273-5 \quad 7.45761-5 \quad 1.34159-4$ $2.21191-4 \quad 3.64682-4 \quad 6.01258-4 \quad 9.91309-4 \quad 1.63439-3$ $\begin{array}{llllll}2.14167-3 & 2.43063-3 & 2.82399-3 & 3.37141-3 & 4.61914-3\end{array}$ $7.32483-3$ 1.20766-2 $1.93065-2 \quad 2.41830-2 \quad 2.54229-2$ 2.65293-2 $2.77506-2 \quad 3.14039-2 \quad 4.33909-2 \quad 5.45187-2$ 7.15396-2 1.04645-1 $1.36365-1 \quad 1.66556-12.03432-1$ $\begin{array}{llllll}2.48472-1 & 2.83877-1 & 2.95864-1 & 2.97851-1 & 3.00232-1\end{array}$ 3.44858-1 $4.42806-1 \quad 5.10634-1 \quad 5.65749-1 \quad 6.75418-1$ $8.24957-11.00760+0 \quad 1.23069+0 \quad 1.50317+0 \quad 1.83598+0$ $\begin{array}{llllll}2.12513+0 & 2.28850+0 & 2.40583+0 & 2.73896+0 & 3.34537+0\end{array}$ $4.08604+0 \quad 4.99070+0 \quad 6.09565+0 \quad 7.44525+0 \quad 9.09365+0$ $1.11070+1 \quad 1.35661+1$

df122 $\quad 3.70370-3 \quad 4.37060-3 \quad 4.52500-3 \quad 4.57410-3$ $4.55830-3 \quad 4.48970-3 \quad 4.38170-3 \quad 4.24920-3$ 4.12890-3 $4.03110-3 \quad 3.93550-3 \quad 3.84530-3 \quad 3.76290-3 \quad 3.69080-3$ $3.65280-3.3 .63870-3 \quad 3.62290-3 \quad 3.60620-3 \quad 3.58250-3$ $3.55850-3 \quad 4.08750-3 \quad 5.89750-3 \quad 7.13870-3 \quad 7.42410-3$ $7.67700-3 \quad 7.95180-3 \quad 8.74210-3 \quad 1.11490-2 \quad 1.35030-2$ $1.65080-2 \quad 2.25270-2 \quad 2.86610-2 \quad 3.43150-2 \quad 4.10860-2$ $4.92110-2 \quad 5.56070-2 \quad 5.77300-2 \quad 5.80800-2 \quad 5.84980-2$ $6.59980-2 \quad 8.26560-2 \quad 9.04890-2 \quad 9.54810-2 \quad 1.05360-1$ $1.17860-1 \quad 1.29670-1 \quad 1.30100-1 \quad 1.28650-1 \cdot 1.27220-1$ $1.26160-1 \quad 1.25620-1 \quad 1.25270-1 \quad 1.28500-1 \quad 1.36960-1$ $1.45980-1 \quad 1.54120-1.1 .51160-1 \quad 1.47220-1 \quad 1.47060-1$

fm122

f132:n 1. $63200-1 \quad 2.00340-1$

de 132 $3.671+10$

634

$\begin{array}{llllll}2.07002-7 & 7.69672-7 & 1.75386-6 & 3.71293-6\end{array}$ 7.86024-6 $1.66401-5 \quad 3.52273-5 \quad 7.45761-5 \quad 1.34159-4$ 2.21191-4 $3.64682-4 \quad 6.01258-4 \quad 9.91309-4 \quad 1.63439-3$ 2.14167-3 2.43063-3 2.82399-3 $3.37141-3$ 4.61914-3 7.32483-3 1.20766-2 $1.93065-2 \quad 2.41830-2 \quad 2.54229-2$ $2.65293-2 \quad 2.77506-2 \quad 3.14039-2 \quad 4.33909-2 \quad 5.45187-2$ 7.15396-2 $1.04645-1 \quad 1.36365-1 \quad 1.66556-1 \quad 2.03432-1$ 2.48472-1 2.83877-1 2.95864-1 2.97851-1 3.00232-1 3.44858-1 4.42806-1 $5.10634-1 \quad 5.65749-1 \quad 6.75418-1$ $8.24957-1 \quad 1.00760+0 \quad 1.23069+0 \quad 1.50317+0 \quad 1.83598+0$ $\begin{array}{llllll}2.12513+0 & 2.28850+0 & 2.40583+0 & 2.73896+0 & 3.34537+0\end{array}$ $4.08604+0 \quad 4.99070+0 \quad 6.09565+0 \quad 7.44525+0 \quad 9.09365+0$ $1.11070+1 \quad 1.35661+1$

df132 $3.70370-3 \quad 4.37060-3 \quad 4.52500-3 \quad 4.57410-3$ $\begin{array}{lllll}4.55830-3 & 4.48970-3 & 4.38170-3 & 4.24920-3 & 4.12890-3\end{array}$ $4.03110-3 \quad 3.93550-3 \quad 3.84530-3 \quad 3.76290-3.3 .69080-3$ $\begin{array}{llllll}3.65280-3 & 3.63870-3 & 3.62290-3 & 3.60620-3 & 3.58250-3\end{array}$ $\begin{array}{llllll}3.55850-3 & 4.08750-3 & 5.89750-3 & 7.13870-3 & 7.42410-3\end{array}$ $\begin{array}{llllll}7.67700-3 & 7.95180-3 & 8.74210-3 & 1.11490-2 & 1.35030-2\end{array}$ $1.65080-2 \quad 2.25270-2 \quad 2.86610-2 \quad 3.43150-2 \quad 4.10860-2$ $4.92110-2 \quad 5.56070-2 \quad 5.77300-2 \quad 5.80800-2 \quad 5.84980-2$ 
$6.59980-2 \quad 8.26560-2 \quad 9.04890-2 \quad 9.54810-2 \quad 1.05360-1$ $1.17860-1 \quad 1.29670-1 \quad 1.30100-1 \quad 1.28650-1 \quad 1.27220-1$ $1.26160-1 \quad 1.25620-1 \quad 1.25270-1 \quad 1.28500-1 \quad 1.36960-1$ $1.45980-1 \quad 1.54120-1 \quad 1.51160-1 \quad 1.47220-1 \quad 1.47060-1$ $1.63200-12.00340-1$

$f \mathrm{~m} 132$

$\mathrm{f} 202: \mathrm{p}$

de202

df202

fm202

$\mp 212: p$

de 212

$\operatorname{df} 212$

fm2 12

$\mathrm{f} 222: \mathrm{p}$

de 222

df222

fm222

f232:p

de232

$\mathrm{df} 232$

$\operatorname{fm} 232$

$\mathrm{e} 102$

$\mathrm{e} 202$
$1.50000-2 \quad 3.25000-2 \quad 5.75000-2 \quad 8.50000-2$

611

$1.25000-12.55000-13.50000-15.75000-2.8 .50000-2$ $6.50000-1 \quad 8.50000-1 \quad 1.25000+0 \quad 1.75000+0 \quad 2.25000+0$ $2.75000+0 \quad 3.50000+0 \quad 4.50000+0 \quad 5.50000+0 \quad 6.50000+0$ $7.25000+0 \quad 7.75000+0 \quad 9.00000+0 \quad 1.20000+1$ $\begin{array}{llll}2.14390-3 & 5.77600-4 & 2.71850-4 & 2.68170-4\end{array}$ $3.27670-4 \quad 5.66760-4 \quad 8.75940-4 \quad 1.08450-3 \quad 1.27970-3$ $1.44170-3 \quad 1.75630-3 \quad 2.31560-3 \quad 2.92700-3 \quad 3.46860-3$

$\begin{array}{llllll}3.95960-3 & 4.62210-3 & 5.41370-3 & 6.19090-3 & 6.92650-3\end{array}$ $7.47830-3 \quad 7.84680-3 \quad 8.77160-3 \quad 1.10200-2$ $3.671+10$ 614

$1.50000-2 \quad 3.25000-2 \quad 5.75000-2 \quad 8.50000-2$ $\begin{array}{lllll}1.25000-1 & 2.25000-1 & 3.50000-1 & 4.55000-1 & 5.55000-1\end{array}$ $6.50000-18.50000-1 \quad 1.25000+0 \quad 1.75000+02.25000+0$ $2.75000+0 \quad 3.50000+0 \quad 4.50000+0 \quad 5.50000+0 \quad 6.50000+0$ $7.25000+0 \quad 7.75000+0 \quad 9.00000+0 \quad 1.20000+1$ $2.14390-3 \quad 5.77600-4 \quad 2.71850-4 \quad 2.68170-4$ $\begin{array}{llllll}3.27670-4 & 5.66760-4 & 8.75940-4 & 1.08450-3 & 1.27970-3\end{array}$ $1.44170-3 \quad 1.75630-3 \quad 2.31560-3 \quad 2.92700-3 \quad 3.46860-3$ $3.95960-3 \quad 4.62210-3 \quad 5.41370-3 \quad 6.19090-3 \quad 6.92650-3$ $7.47830-3 \quad 7.84680-3 \quad 8.77160-3 \quad 1.10200-2$ $3.671+10$

624

$1.50000-2 \quad 3.25000-2 \quad 5.75000-2 \quad 8.50000-2$ $1.25000-1 \quad 2.25000-1 \quad 3.50000-14.55000-1 \quad 5.55000-1$ $6.50000-1 \quad 8.50000-1 \quad 1.25000+0 \quad 1.75000+0 \quad 2.25000+0$ $2.75000+0 \quad 3.50000+0 \quad 4.50000+0 \quad 5.50000+0 \quad 6.50000+0$ $7.25000+0 \quad 7.75000+0 \quad 9.00000+0 \quad 1.20000+1$ $\begin{array}{lllll}2.14390-3 & 5.77600-4 & 2.71850-4 & 2.68170-4\end{array}$ $3.27670-4 \quad 5.66760-4 \quad 8.75940-4 \quad 1.08450-3 \quad 1.27970-3$ $1.44170-3 \quad 1.75630-3 \quad 2.31560-3 \quad 2.92700-3 \quad 3.46860-3$ $3.95960-3 \quad 4.62210-3 \quad 5.41370-3 \quad 6.19090-3 \quad 6.92650-3$ $7.47830-3 \quad 7.84680-3 \quad 8.77160-3 \quad 1.10200-2$

$3.671+10$ 634

$1.50000-2 \quad 3.25000-2 \quad 5.75000-2 \quad 8.50000-2$ $1.25000-1 \quad 2.25000-1 \quad 3.50000-1 \quad 4.55000-1 \quad 5.55000-1$ $\begin{array}{lllll}6.50000-1 & 8.50000-1 & 1.25000+0 & 1.75000+0 & 2.25000+0\end{array}$ $2.75000+0 \quad 3.50000+0 \quad 4.50000+0 \quad 5.50000+0 \quad 6.50000+0$ $7.25000+0 \quad 7.75000+0 \quad 9.00000+01.20000+1$ $2.14390-3 \quad 5.77600-4 \quad 2.71850-4 \quad 2.68170-4$ $3.27670-4 \quad 5.66760-4 \quad 8.75940-4 \quad 1.08450-3 \quad 1.27970-3$ $1.44170-3 \quad 1.75630-3 \quad 2.31560-3 \quad 2.92700-3 \quad 3.46860-3$ $\begin{array}{llllll}3.95960-3 & 4.62210-3 & 5.41370-3 & 6.19090 & 3 & 6.92650-3\end{array}$ $7.47830-3 \quad 7.84680-3 \quad 8.77160-3 \quad 1.10200-2$

$3.671+10$

4.13994-7 $1.12535-6 \quad 2.38237-6 \quad 5.04348-6 \quad 1.06770-5$ $2.26033-5 \quad 4.78512-5.1 .01301-4 \quad 1.67017-4 \quad 2.75364-4$ $\begin{array}{llllll}4.53999-4 & 7.48518-4 & 1.23410-3 & 2.03468-3 & 2.24867-3\end{array}$ $\begin{array}{llllll}2.61259-3 & 3.03539-3 & 3.70744-3 & 5.53084-3 & 9.11882-3\end{array}$ $1.50344-22.35786-2 \cdot 2.47875-2 \quad 2.60584-2 \quad 2.70001-2$ $2.85011-2 \quad 3.43067-2 \quad 5.24752-2 \quad 5.65622-2 \quad 8.65169-2$ 1.22773-1 $1.49956-1 \quad 1.83156-1 \quad 2.23708-1 \quad 2.73237-1$ 2.94518-1 $2.97211-1 \quad 2.98491-1 \quad 3.01974-1 \quad 3.87742-1$ $\begin{array}{lllll}4.97870-1 & 5.23397-1 & 6.08101-1 & 7.42735-1 & 9.07180-1\end{array}$ $1.10803+0 \quad 1.35335+0 \quad 1.65299+0 \quad 2.01897+0 \quad 2.23130+0$ $2.34570+0 \quad 2.46597+0 \quad 3.01194+0 \quad 3.67879+0 \quad 4.49329+0$ $5.48811+0 \quad 6.70320+0 \quad 8.18730+0 \quad 1.00000+1 \quad 1.22140+1$ $1.49183+1$

$2.00000-2 \quad 4.50000-2 \quad 7.00000-2 \quad 1.00000-1 \quad 1.50000-1$ $3.00000-1 \quad 4.00000-1 \quad 5.10000-1 \quad 6.00000-1 \quad 7.00000-1$ $1.00000+0 \quad 1.50000+0 \quad 2.00000+0 \quad 2.50000+0 \quad 3.00000+0$ 
$4.00000+0 \quad 5.00000+0 \quad 6.00000+0 \quad 7.00000+0 \quad 7.50000+0$ $8.00000+0 \quad 1.00000+1 \quad 1.40000+1$

e112

4.13994-7 1.12535-6 2.38237-6 5.04348-6 $1.06770-5$ $2.26033-5 \quad 4.78512-5 \quad 1.01301-4 \quad 1.67017-4 \quad 2.75364-4$ 4.53999-4 7.48518-4 1.23410-3 $2.03468-32.24867-3$ $2.61259-3 \quad 3.03539-3 \quad 3.70744-3 \quad 5.53084-3 \quad 9.11882-3$ 1.50344-2 2.35786-2 2.47875-2 $2.60584-2 \quad 2.70001-2$ $\begin{array}{lllll}2.85011-2 & 3.43067-2 & 5.21752-2 & 5.65622-2 & 8.65169-2\end{array}$ $\begin{array}{lllll}1.22773-1 & 1.49956-1 & 1.83156-1 & 2.23708-1 & 2.73237-1\end{array}$ 2.94518-1 2.97211-1 2.98491-1 3.01974-1 3.87742-1 $4.97870-1 \quad 5.23397-1 \quad 6.08101-1 \quad 7.42735-1 \quad 9.07180-1$ $\begin{array}{lllll}1.10803+0 & 1.35335+0 & 1.65299+0 & 2.01897+0 & 2.23130+0\end{array}$ $2.34570+0 \quad 2.46597+0 \quad 3.01194+0 \quad 3.67879+0 \quad 4.49329+0$ $5.48811+0 \quad 6.70320+0 \quad 8.18730+0 \quad 1.00000+1 \quad 1.22140+1$ $1.49183+1$

e212

$2.00000-2 \quad 4.50000-2 \quad 7.00000-2 \quad 1.00000-1 \quad 1.50000-1$ 3.00000-1 4.00000-1 5.10000-1 6.00000-1 7.00000-1 $1.00000+0 \quad 1.50000+0 \quad 2.00000+0 \quad 2.50000+0 \quad 3.00000+0$ $4.00000+0 \quad 5.00000+0 \quad 6.00000+0 \quad 7.00000+0 \quad 7.50000+0$ $8.00000+01.00000+11.40000+1$

e122

4.13994-7 $1.12535-6 \quad 2.38237-6 \quad 5.04348-6 \quad 1.06770-5$ 2.26033-5 $4.78512-5$ 1.01301-4 $1.67017-4$ 2.75364-4 $\begin{array}{llllll}4.53999-4 & 7.48518-4 & 1.23410-3 & 2.03468-3 & 2.24867-3\end{array}$ $2.61259-3 \quad 3.03539-3 \quad 3.70744-3 \quad 5.53084-3 \quad 9.11882-3$ 1.50344-2 2.35786-2 2.47875-2 2.60584-2 2.70001-2 $2.85011-2 \quad 3.43067-2 \quad 5.24752-2 \quad 5.65622-2 \quad 8.65169-2$ 1.22773-1 $1.49956-1 \quad 1.83156-1 \quad 2.23708-1 \quad 2.73237-1$ 2.94518-1 2.97211-1 $2.98491-1 \quad 3.01974-1 \quad 3.87742-1$ 4.97870-1 $5.23397-1 \quad 6.08101-1 \quad 7.42735-1 \quad 9.07180-1$ $1.10803+0 \quad 1.35335+0 \quad 1.65299+0 \quad 2.01897+0 \quad 2.23130+0$ $2.34570+0 \quad 2.46597+0 \quad 3.01194+0 \quad 3.67879+0 \quad 4.49329+0$ $5.48811+0 \quad 6.70320+0 \quad 8.18730+0 \quad 1.00000+1 \quad 1.22140+1$ $1.49183+1$

e222

$2.00000-2 \quad 4.50000-27.00000-21.00000-1 \quad 1.50000-1$ $3.00000-1 \quad 4.00000-1 \quad 5.10000-1 \quad 6.00000-1 \quad 7.00000-1$ $1.00000+0 \quad 1.50000+0 \quad 2.00000+0 \quad 2.50000+0 \quad 3.00000+0$ $4.00000+0 \quad 5.00000+0 \quad 6.00000+0 \quad 7.00000+0 \quad 7.50000+0$ $8.00000+01.00000+1 \quad 1.40000+1$

e132

4.13994-7 $1.12535-6 \quad 2.38237-6 \quad 5.04348-6 \quad 1.06770-5$ $2.26033-5 \quad 4.78512-5 \quad 1.01301-4 \quad 1.67017-4 \quad 2.75364-4$ 4.53999-4 7.48518-4 $1.23410-3 \quad 2.03468-3 \quad 2.24867-3$ $\begin{array}{llllll}2.61259-3 & 3.03539-3 & 3.70744-3 & 5.53084-3 & 9.11882-3\end{array}$ $\begin{array}{llllll}1.50344-2 & 2.35786-2 & 2.47875-2 & 2.60584-2 & 2.70001-2\end{array}$ 2.85011-2 $3.43067-2 \quad 5.24752-2 \quad 5.65622-2 \quad 8.65169-2$ 1.22773-1 1.49956-1 $1.83156-1 \quad 2.23708-1 \quad 2.73237-1$ 2.94518-1 2.97211-1 $2.98491-1 \quad 3.01974-1 \quad 3.87742-1$ 4.97870-1 5.23397-1 $6.08101-1 \quad 7.42735-1 \quad 9.07180-1$ $1.10803+0 \quad 1.35335+0 \quad 1.65299+0 \quad 2.01897+0 \quad 2.23130+0$ $2.34570+0 \quad 2.46597+0 \quad 3.01194+0 \quad 3.67879+0 \quad 4.49329+0$ $5.48811+0 \quad 6.70320+0 \quad 8.18730+0 \quad 1.00000+1 \quad 1.22140+1$ $1.49183+1$

e232

$2.00000-2 \quad 4.50000-2 \quad 7.00000-2 \quad 1.00000-1 \quad 1.50000-1$ $3.00000-1 \quad 4.00000-1 \quad 5.10000-1 \quad 6.00000-1 \quad 7.00000-1$ $1.00000+0 \quad 1.50000+0 \quad 2.00000+0 \quad 2.50000+0 \quad 3.00000+0$ $4.00000+05.00000+06.00000+07.00000+0 \quad 7.50000+0$ $8.00000+01.00000+11.40000+1$

sd102 100.0

$\operatorname{sa112} 100.0$

$\operatorname{sd122} 100.0$

sd132 100.0

sd202 100.0

$\operatorname{sd} 212 \quad 100.0$

$\begin{array}{ll}\text { sd222 } & 100.0\end{array}$

sd232 100.0

sdef pos $=0$ 0 0 vec $=1 \quad 0 \quad 0$ dir $=$ d1 erg $=$ fdir $=d 2$

c si1 $\quad 0.0 \quad 0.50 .86602540 .99619461 .0$ 


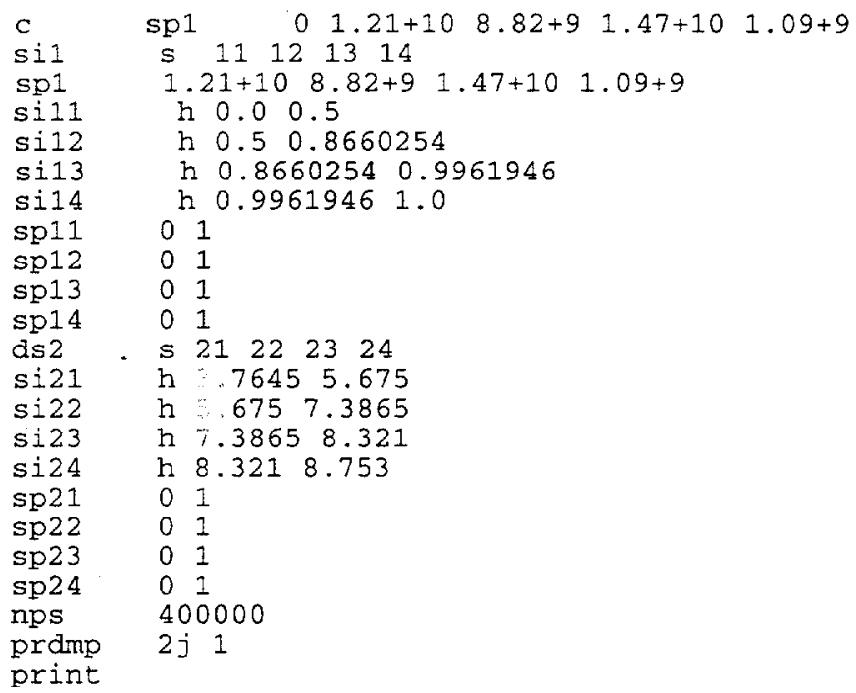


File for MCNP-4B surface-detector dose-rate calculations for a waterreflected PU sphere in the PFNA beam at the center of the truck lane $(400,000$ histories)

message: outp $=$ fna $8 . \circ$ mctal $=p f n a 8 \cdot m$

monp file for PFNA project water-reflected PU sphere calculation $1 \quad-100: 128:-200: 251:-300: 305$

$2 \quad 0 \quad 100-128 \quad 200-251300-305$

$\#(-400+101+224-227)$

$\#(-4.01+400+101+225-226)$

$\#(102-129+201-250+301-304)$

$\#(129-126+252-257+301-304)$

$\#(102-126+201-250+306-301)$

\#(615-616+625-626+635-636)

$3 \quad 10.10549 \quad-400+101+224-227 \#(503-504)$

$41 \quad 10.10549-401+400+101+225-226 \#(503-504)$

$102-127+223-228+301-304$

$6 \quad 0 \quad 127-124+501-502+301-304$

$\#(-401+400+225-226)$

$7 \quad 0 \quad 124-126+254-255+301-304$

$8 \quad 5 \quad 8.734441-2 \quad 102-126+201-250+306-301$

$9 \quad 10.10549 \quad 102-124+222-229+301-304$

$\begin{array}{lllllllll}10 & 1 & 0.10549 & \#(127+501-502) & \#(-127 & +223 & -228) & \\ 106 & -109+212 & -236 & +302 & -303 & \#(218 & -233)\end{array}$

$11110.10549 \quad 109-110+211-237+302-303 \#(221-230)$

$12110.10549 \quad 110-111+210-238+302-303 \#(221-230)$

$13110.10549 \quad 111-112+209-239+302-303 \quad \#(221-230)$

$14110.10549 \quad 112-113+208-240+302-303 \#(221-230)$

$151 \quad 1 \quad 0.10549 \quad 113-114+207-241+302-303 \#(221-230)$

$16 \quad 1 \quad 0.10549 \quad 114-115+206-242+302-303 \#(221-230)$

$171 \frac{1}{17} 0.10549 \quad 115-116+205-243+302-303 \#(221-230)$

$18 \quad 1 \quad 0.10549 \quad 116-117+204-244+302-303 \quad \#(221-230)$

$19110.10549 \quad 117-118+203-245+302-303 \#(221-230)$

$20 \quad 1 \quad 0.10549 \quad 118-119+202-249+302-303$ \# $(221-230)$

$\begin{array}{lll}21 & 2 & 0.1187956\end{array}$

$120-123+215-246+302-303 \#(221-230)$

20.1187956

$1.03-109+202-249+302-303$

$\#(221-230)$

$\#(103-104+217-234)$

$\#(104-105+216-235)$

\#(105-107+219-232)

$\#(107-108+219-220) \#(107-108+231-232)$

$\#(106-109+212-218) \#(106-109+233-236)$

$\#(221-230)$

$\#(109-110+211-237)$

$\#(110-111+210-238)$

$\#(111-112+209-239)$

$\#(112-113+208-240)$

$\#(113-114+207-241)$

$\#(114-115+206-242)$

$\#(115-116+205-243)$

$\#(116-117+204-244)$

$\#(117-118+203-245)$

\# $\{118-119\}$

$243 \quad 3.284-2$

$121-125+201-250+301-304$

$\#(-124+214-247) \#(124+253-256)$

$\#(122+248) \#(122-213)$

$25 \quad 33.284-2 \quad 103-107+217-234+302-303$

$\#(221-230) \#(104-220) \#(104+231)$

26

27

$45.977931-2 \quad 104-108+216-220+302-303 \quad \#(105-219)$

4 5.977931-2 $104-108+231-235+302-303 \#(105+232)$

$45.977931-2 \quad 102-124+201-222+301-304$

$\#\left(\begin{array}{llllll}103 & -120 & 202 & -221 & 302 & -303\end{array}\right)$

\#( $\left.\begin{array}{llllll}120 & -123 & 215 & -221 & 302 & -303\end{array}\right)$

\# $(121-214)$

29

$45.977931-2102-124+229-250+301-304$

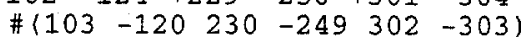




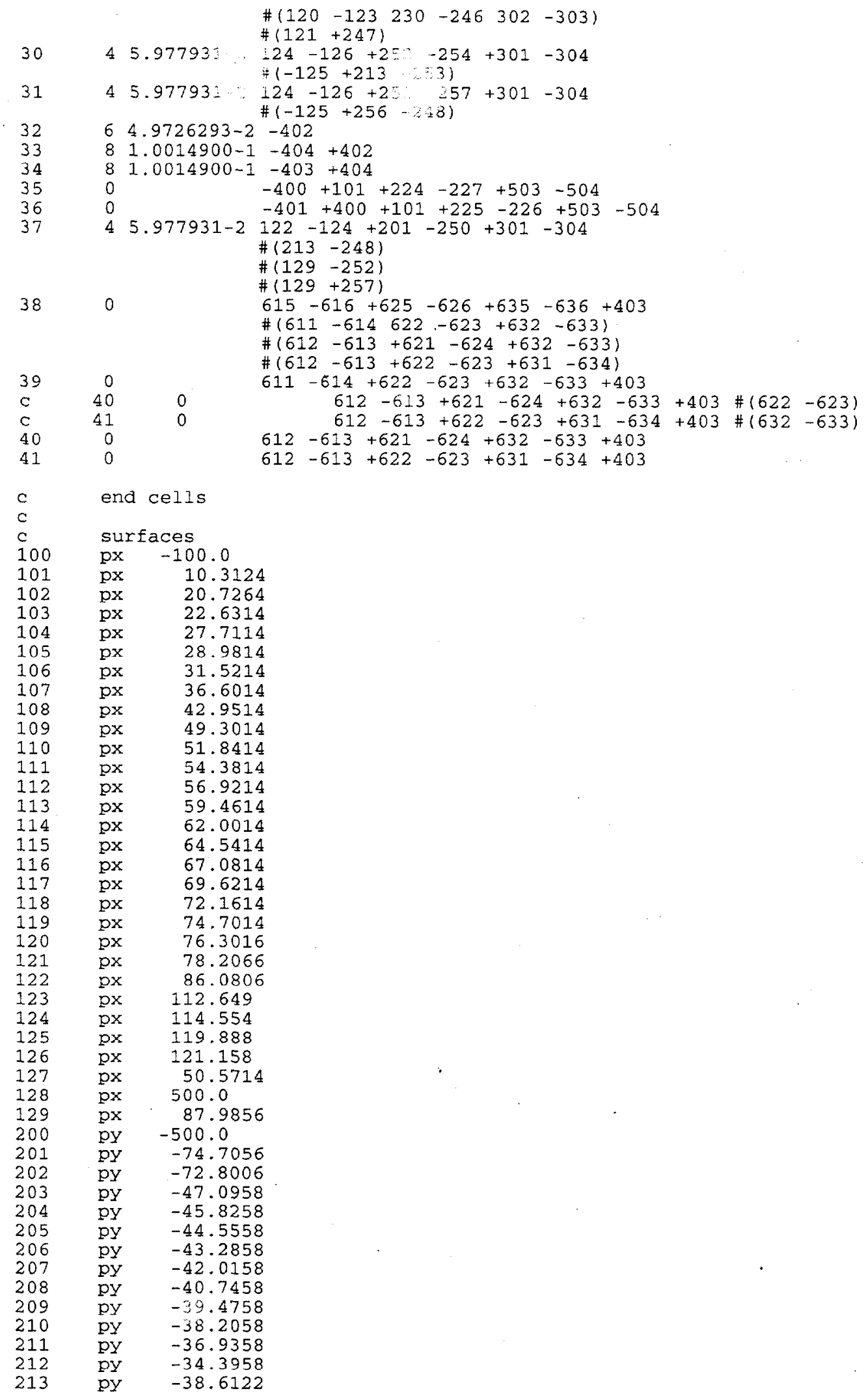




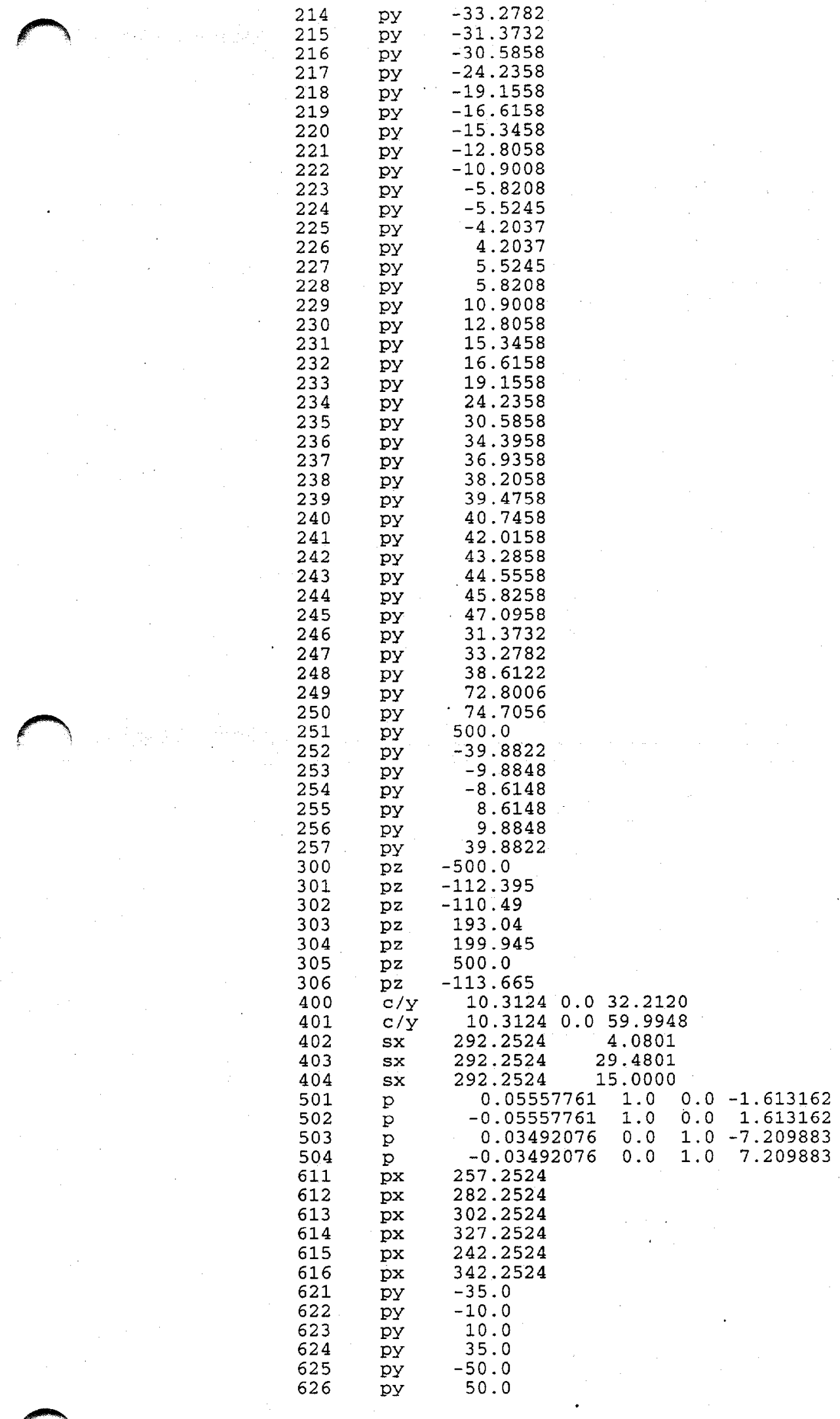




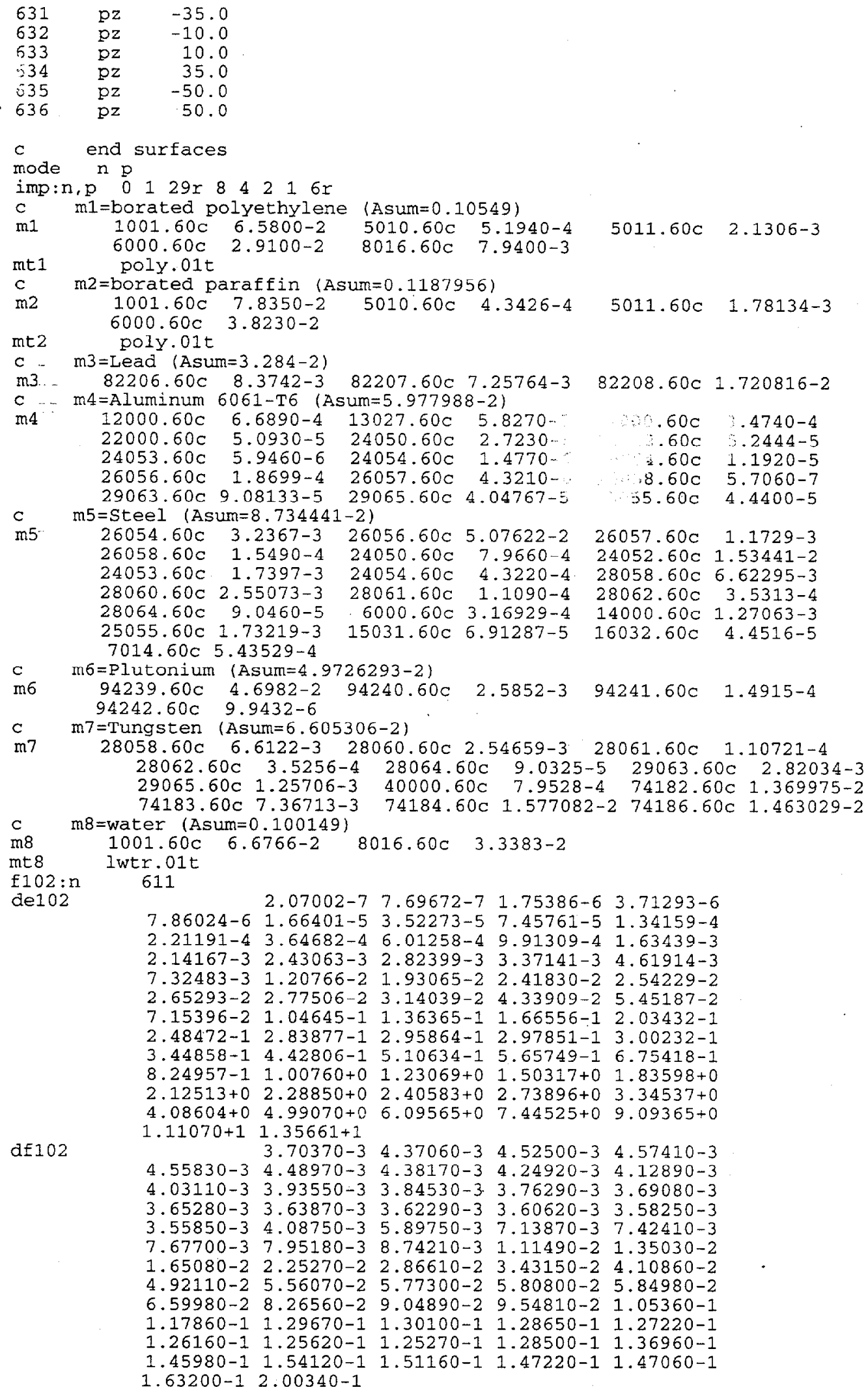




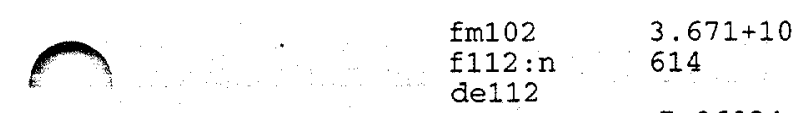

$2.07002-7 \quad 7.69672-7 \quad 1.75386-6 \quad 3.71293-6$ $\begin{array}{llllll}7.86024-6 & 1.66401-5 & 3.52273-5 & 7.45761-5 & 1.34159-4\end{array}$ $\begin{array}{llllll}2.21191-4 & 3.64682-4 & 6.01258-4 & 9.91309-4 & 1.63439-3\end{array}$ $2.14167-3 \quad 2.43063-3 \quad 2.82399-3 \quad 3.37141-3 \quad 4.61914-3$ $7.32483-3 \quad 1.20766-2 \quad 1.93065-2 \quad 2.41830-2 \quad 2.54229-2$ $2.65293-2 \quad 2.77506-2 \quad 3.14039-24.33909-2 \quad 5.45187-2$ 7.15396-2 $1.04645-1 \quad 1.36365-1 \quad 1.66556-1 \quad 2.03432-1$ $\begin{array}{llllll}2.48472-1 & 2.83877-1 & 2.95864-1 & 2.97851-1 & 3.00232-1\end{array}$ 3.44858-1 4.42806-1 $5.10634-1 \quad 5.65749-1 \quad 6.75418-1$ $8.24957-1 \quad 1.00760+0 \quad 1.23069+0 \quad 1.50317+0 \quad 1.83598+0$ $\begin{array}{lllll}2.12513+0 & 2.28850+0 & 2.40583+0 & 2.73896+0 & 3.34537+0\end{array}$ $4.08604+0 \quad 4.99070+0 \quad 6.09565+0 \quad 7.44525+0 \quad 9.09365+0$ $1.11070+1 \quad 1.35661+1$

df112 $\quad 3.70370-3 \quad 4.37060-3 \quad 4.52500-3 \quad 4.57410-3$ $4.55830-3 \quad 4.48970-3 \quad 4.38170-3 \quad 4.24920-3 \quad 4.12890-3$ $\begin{array}{llllll}4.03110-3 & 3.93550-3 & 3.84530-3 & 3.76290-3 & 3.69080-3\end{array}$ $3.65280-3 \quad 3.63870-3 \quad 3.62290-3 \quad 3.60620-3.3 .58250-3$ $\begin{array}{llllll}3.55850-3 & 4.08750-3 & 5.89750-3 & 7.13870-3 & 7.42410-3\end{array}$ $7.67700-3 \quad 7.95180-3 \quad 8.74210-3 \quad 1.11490-2 \quad 1.35030-2$ $1.65080-2 \quad 2.25270-2 \quad 2.86610-2 \quad 3.43150-2 \quad 4.10860-2$ $4.92110-2 \quad 5.56070-2 \quad 5.77300-2 \quad 5.80800-2 \quad 5.84980-2$ $6.59980-2 \quad 8.26560-2 \quad 9.04890-2 \quad 9.54810-2 \quad 1.05360-1$ $1.17860-11.29670-1 \quad 1.30100-1 \quad 1.28650-1 \quad 1.27220-1$ $1.26160-1 \quad 1.25620-1 \quad 1.25270-1 \quad 1.28500-1 \quad 1.36960-1$ $1.45980-1$ 1.54120-1 $1.51160-1$ 1.47220-1 $1.47060-1$ $1.63200-12.00340-1$

$\operatorname{fm} 112$ $£ 122: \mathrm{n}$ $3.671+10$

de122

$\mathrm{df} 122$ 624

$\begin{array}{lllll}2.07002-7 & 7.69672-7 & 1.75386-6 & 3.71293-6\end{array}$ 7.86024-6 $1.66401-5 \quad 3.52273-5 \quad 7.45761-5 \quad 1.34159-4$ 2.21191-4 3.64682-4 $6.01258-4 \quad 9.91309-4 \quad 1.63439-3$ $2.14167-3 \quad 2.43063-3.2 .82399-3 \quad 3.37141-3 \quad 4.61914-3$ 7.32483-3 $1.20766-2 \quad 1.93065-2 \quad 2.41830-2 \quad 2.54229-2$ 2.65293-2 2.77506-2 3.14039-2 4.33909-2 5.45187-2 7.15396-2 $1.04645-1 \quad 1.36365-1 \quad 1.66556-1 \quad 2.03432-1$ 2.48472-1 2.83877-1 2.95864-1 2.97851-1 3.00232-1 3.44858-1 $4.42806-1 \quad 5.10634-1 \quad 5.65749-1 \quad 6.75418-1$ $8.24957-1 \quad 1.00760+0 \quad 1.23069+0 \quad 1.50317+0 \quad 1.83598+0$ $2.12513+0 \quad 2.28850+0 \quad 2.40583+0 \quad 2.73896+0 \quad 3.34537+0$ $4.08604+0 \quad 4.99070+0 \quad 6.09565+0 \quad 7.44525+0 \quad 9.09365+0$ $1.11070+11.35661+1$

$\begin{array}{lllll}3.70370-3 & 4.37060-3 & 4.52500-3 & 4.57410-3\end{array}$ $4.55830-3 \quad 4.48970-3 \quad 4.38170-3 \quad 4.24920-3 \quad 4.12890-3$ $\begin{array}{llllll}4.03110-3 & 3.93550-3 & 3.84530-3 & 3.76290-3 & 3.69080-3\end{array}$ $3.65280-3 \quad 3.63870-3 \quad 3.62290-3 \quad 3.60620-3 \quad 3.58250-3$ $3.55850-3 \quad 4.08750-3 \quad 5.89750-3 \cdot 7.13870-3.7 .42410-3$ $7.67700-3 \quad 7.95180-3 \quad 8.74210-3 \quad 1.11490-2 \quad 1.35030-2$ $1.65080-2 \quad 2.25270-2 \quad 2.86610-2 \quad 3.43150-2 \quad 4.10860-2$ $4.92110-2 \quad 5.56070-2 \quad 5.77300-2 \quad 5.80800-2 \quad 5.84980-2$ $6.59980-2 \quad 8.26560-2 \quad 9.04890-2 \quad 9.54810-2 \quad 1.05360-1$ 1.17860-1 $1.29670-1 \quad 1.30100-1 \quad 1.28650-1 \quad 1.27220-1$ 1.26160-1 $1.25620-1 \quad 1.25270-1 \quad 1.28500-1 \quad 1.36960-1$ $1.45980-1$ 1.54120-1 $1.51160-1$ 1.47220-1 $1.47060 \cdots 1$ $1.63200-12.00340-1$

$\operatorname{fm} 122$

$1132: n$

de132 $3.671+10$

634 2.21191-4 $3.64682-4 \quad 6.01258-4 \quad 9.91309-4 \quad 1.63439-3$ 2.14167-3 $2.43063-3 \quad 2.82399-3 \quad 3.37141-3 \quad 4.61914-3$ 7.32483-3 1.20766-2 $1.93065-2 \quad 2.41830-2 \quad 2.54229-2$ 2.65293-2 $2.77506-2 \quad 3.14039-2 \quad 4.33909-2 \quad 5.45187-2$ 7.15396-2 $1.04645-1 \quad 1.36365-1 \quad 1.66556-1 \quad 2.03432-1$ 2.48472-1 $2.83877-1 \quad 2.95864-1 \quad 2.97851-1 \quad 3.00232-1$ 3.44858-1 4.42806-1 $5.10634-1 \quad 5.65749-1 \quad 6.75418-1$ $8.24957-1 \quad 1.00760+0 \quad 1.23069+0 \quad 1.50317+0 \quad 1.83598+0$ $2.12513+0 \quad 2.28850+0 \quad 2.40583+0 \quad 2.73896+0 \quad 3.34537+0$ $4.08604+0 \quad 4.99070+0 \quad 6.09565+0 \quad 7.44525+0 \quad 9.09365+0$ $1.11070+1 \quad 1.35661+1$ 


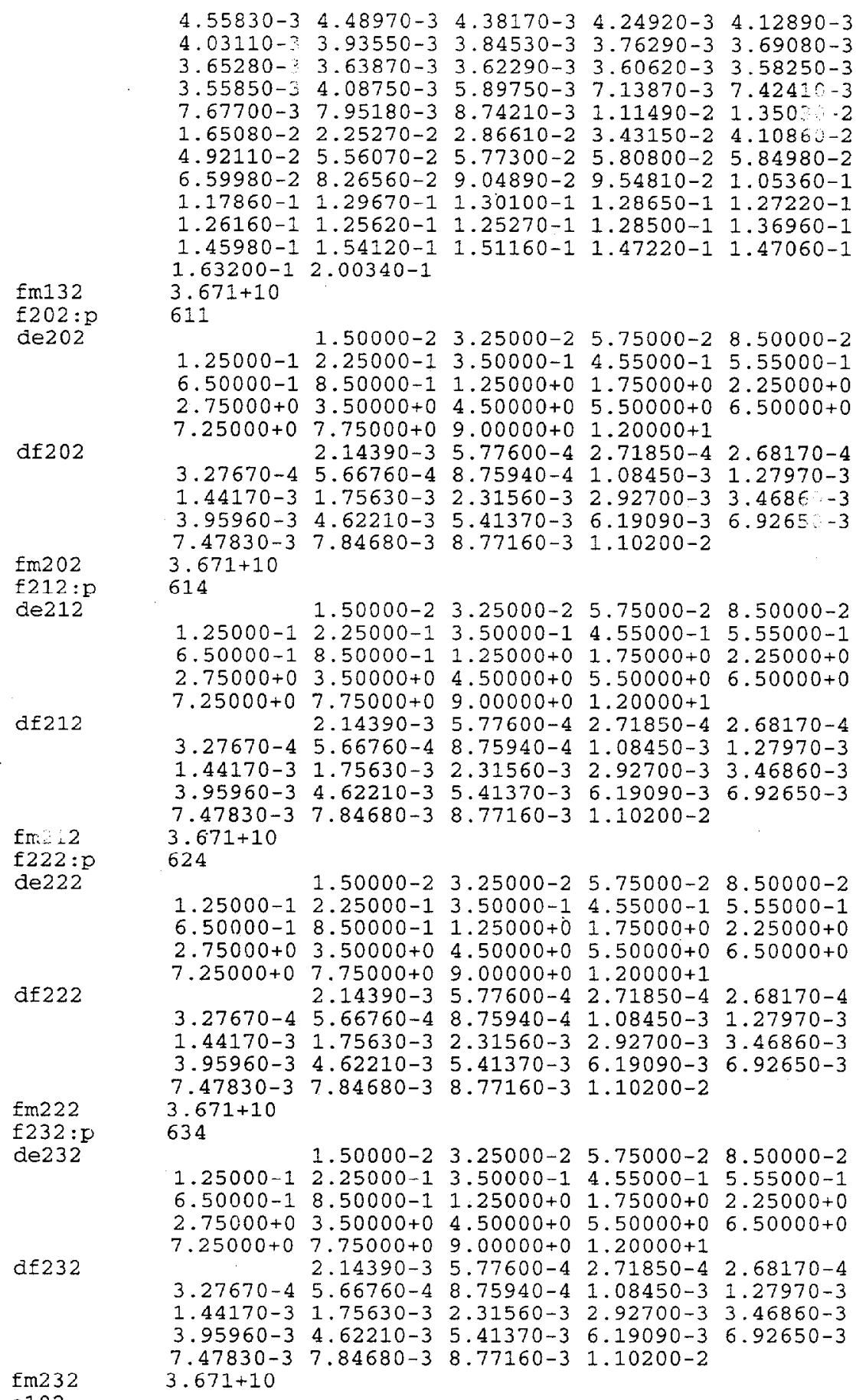

4.13994-7 $1.12535-6 \quad 2.38237-6 \quad 5.04348-6 \quad 1.06770-5$ $2.26033-5 \quad 4.78512-5 \quad 1.01301-4 \quad 1.67017-4 \quad 2.75364-4$ 4.53999-4 7.48518-4 $1.23410-3 \quad 2.03468-3 \quad 2.24867-3$ $2.61259-3 \quad 3.03539-3 \quad 3.70744-3 \quad 5.53084-3 \quad 9.11882-3$ $\begin{array}{lllll}1.50344-2 & 2.35786-2 & 2.47875-2 & 2.60584-2 & 2.70001-2\end{array}$ $2.85011-2 \quad 3.43067-2 \quad 5.24752-2 \quad 5.65622-2 \quad 8.65169-2$ 1.22773-1 $1.49956-1 \quad 1.83156-1 \quad 2.23708-1 \quad 2.73237-1$ 2.94518-1 2.97211-1 $2.98491-1 \quad 3.01974-1 \quad 3.87742-1$ $\begin{array}{lllll}4.97870-1 & 5.23397-1 & 6.08101-1 & 7.42735-1 & 9.07180-1\end{array}$ $1.10803+0 \quad 1.35335+0 \quad 1.65299+0 \quad 2.01897+0 \quad 2.23130+0$ 
$2.34570+0 \quad 2.46597+0 \quad 3.01194+0 \quad 3.67879+0 \quad 4.49329+0$ $5.48811+0 \quad 6.70320+0 \quad 8.18730+0 \quad 1.00000+1 \quad 1.22140+1$ $1.49183+1$

e202

2.00000-2 4.50000-2 7.00000-2 1.00000-1 1.50000-1 $3.00000-1 \quad 4.00000-1 \quad 5.10000-1 \quad 6.00000-1 \quad 7.00000-1$ $1.00000+0 \quad 1.50000+0 \quad 2.00000+0 \quad 2.50000+0 \quad 3.00000+0$ $4.00000+0 \quad 5.00000+0 \quad 6.00000+0 \quad 7.00000+0 \quad 7.50000+0$ $8.00000+01.00000+11.40000+1$

e112

4.13994-7 $1.12535-6 \quad 2.38237-6 \quad 5.04348-6 \quad 1.06770-5$ $2.26033-5 \quad 4.78512-5 \quad 1.01301-4 \quad 1.67017-4 \quad 2.75364-4$ $4.53999-4 \quad 7.48518-4 \quad 1.23410-3 \quad 2.03468-3 \quad 2.24867-3$ $2.61259-3 \quad 3.03539-3 \quad 3.70744-3 \quad 5.53084-3 \quad 9.11882-3$ $\begin{array}{lllll}1.50344-2 & 2.35786-2 & 2.47875-2 & 2.60584-2 & 2.70001-2\end{array}$ 2.85011-2 $3.43067-2 \quad 5.24752-2 \quad 5.65622-2 \quad 8.65169-2$ 1.22773-1 $1.49956-1 \quad 1.83156-1 \quad 2.23708-1 \quad 2.73237-1$ 2.94518-1 $2.97211-1 \quad 2.98491-1 \quad 3.01974-1 \quad 3.87742-1$ 4.97870-1 5.23397-1 6.08101-1 $7.42735-1 \quad 9.07180-1$ $1.10803+0 \quad 1.35335+0 \quad 1.65299+0 \quad 2.01897+0 \quad 2.23130+0$ $2.34570+0 \quad 2.46597+0 \quad 3.01194+0 \quad 3.67879+0 \quad 4.49329+0$ $5.48811+0 \quad 6.70320+0 \quad 8.18730+0 \quad 1.00000+11.22140+1$ $1.49183+1$

e212

$2.00000-24.50000-2 \quad 7.00000-2 \quad 1.00000-1 \quad 1.50000-1$ 3.00000-1 $4.00000-1 \quad 5.10000-1 \quad 6.00000-1 \quad 7.00000-1$ $1.00000+0 \quad 1.50000+0 \quad 2.00000+0 \quad 2.50000+0 \quad 3.00000+0$ $4.00000+05.00000+06.00000+0 \quad 7.00000+0 \quad 7.50000+0$ $8.00000+01.00000+1 \quad 1.40000+1$

e122

e222

4.13994-7 $1.12535-6 \quad 2.38237-6 \quad 5.04348-6 \quad 1.06770-5$ 2.26033-5 $4.78512-5 \quad 1.01301-4 \quad 1.67017-4 \quad 2.75364-4$ $\begin{array}{llllll}4.53999-4 & 7.48518-4 & 1.23410-3 & 2.03468-3 & 2.24867-3\end{array}$ $\begin{array}{lllllll}2.61259-3 & 3.03539-3 & 3.70744-3 & 5.53084-3 & 9.11882-3\end{array}$ $1.50344-2 \quad 2.35786-2.2 .47875-2 \quad 2.60584-2 \quad 2.70001-2$ 2.85011-2 $3.43067-2 \quad 5.24752-2 \quad 5.65622-2 \quad 8.65169-2$ $\begin{array}{llllll}1.22773-1 & 1.49956-1 & 1.83156-1 & 2.23708-1 & 2.73237-1\end{array}$ 2.94518-1 2.97211-1 2.98491-1 3.01974-1 3.87742-1 $4.97870-1 \quad 5.23397-1 \quad 6.08101-1 \quad 7.42735-1 \quad 9.07180-1$ $\begin{array}{lllll}1.10803+0 & 1.35335+0 & 1.65299+0 & 2.01897+0 & 2.23130+0\end{array}$ $2.34570+0 \quad 2.46597+0 \quad 3.01194+0 \quad 3.67879+0 \quad 4.49329+0$ $5.48811+0 \quad 6.70320+0 \quad 8.18730+0 \quad 1.00000+1 \quad 1.22140+1$ $1.49183+1$

2.00000-2 4.50000-2 7.00000-2 $1.00000-1 \quad 1.50000-1$ $3.00000-1 \quad 4.00000-1 \quad 5.10000-1 \quad 6.00000-1 \quad 7.00000-1$ $1.00000+0 \quad 1.50000+0 \quad 2.00000+0 \quad 2.50000+0 \quad 3.00000+0$ $4.00000+0 \quad 5.00000+0 \quad 6.00000+0 \quad 7.00000+0 \quad 7.50000+0$ e132

$8.00000+01.00000+11.40000+1$

4.13994-7 $1.12535-6 \quad 2.38237-6 \quad 5.04348-6 \quad 1.06770-5$ 2.26033-5 $4.78512-5 \quad 1.01301-4 \quad 1.67017-4 \quad 2.75364-4$ $4.53999-4 \quad 7.48518-4 \quad 1.23410-3 \quad 2.03468-3 \quad 2.24867-3$ $\begin{array}{llllll}2.61259-3 & 3.03539-3 & 3.70744-3 & 5.53084-3 & 9.11882-3\end{array}$ $1.50344-2 \quad 2.35786-2 \quad 2.47875-2 \quad 2.60584-2 \quad 2.70001-2$ $\begin{array}{llllll}2.85011-2 & 3.43067-2 & 5.24752-2 & 5.65622-2 & 8.65169-2\end{array}$ $\begin{array}{llllll}1.22773-1 & 1.49956-1 & 1.83156-1 & 2.23708-1 & 2.73237-1\end{array}$ 2.94518-1 $2.97211-1 \quad 2.98491-1 \quad 3.01974-1 \quad 3.87742-1$ $\begin{array}{llllll}4.97870-1 & 5.23397-1 & 6.08101-1 & 7.42735-1 & 9.07180-1\end{array}$ $\begin{array}{lllll}1.10803+0 & 1.35335+0 & 1.65299+0 & 2.01897+0 & 2.23130+0\end{array}$ $2.34570+0 \quad 2.46597+0 \quad 3.01194+0 \quad 3.67879+0 \quad 4.49329+0$ $\begin{array}{lllll}5.48811+0 & 6.70320+0 & 8.18730+0 & 1.00000+1 & 1.22140+1\end{array}$ $1.49183+1$

e232

sd102 400.0

2.00000-2 $4.50000-2 \quad 7.00000-2 \quad 1.00000-1 \quad 1.50000-1$ $3.00000-1 \quad 4.00000-1 \quad 5.10000-1 \quad 6.00000-1 \quad 7.00000-1$ $1.00000+0 \quad 1.50000+0 \quad 2.00000+0 \quad 2.50000+0 \quad 3.00000+0$ $4.00000+0 \quad 5.00000+0 \quad 6.00000+0 \quad 7.00000+0 \quad 7.50000+0$ $8.00000+01.00000+1 \quad 1.40000+1$

$\operatorname{sd112} 400.0$

$\operatorname{sd} 122 \quad 400.0$ 


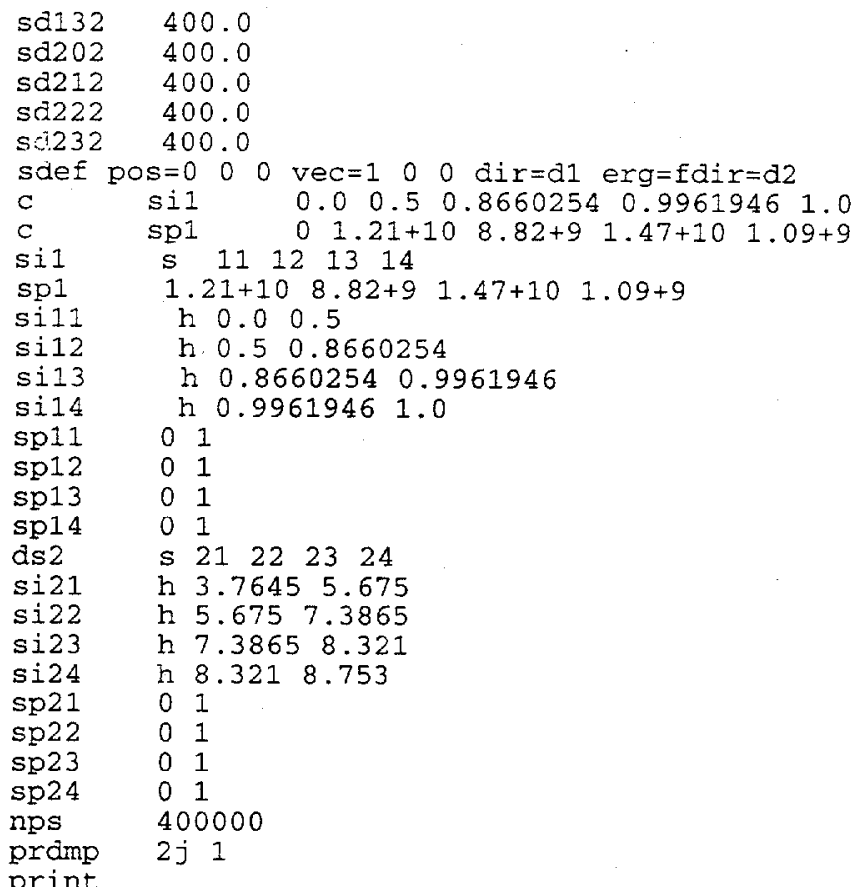

pramp 
File for MCNP-4B surface-detector dose-rate background calculations for the tungsten-reflected Pu spheres in the PFNA beam at the center of the truck lane $(2,000,000$ histories $)$

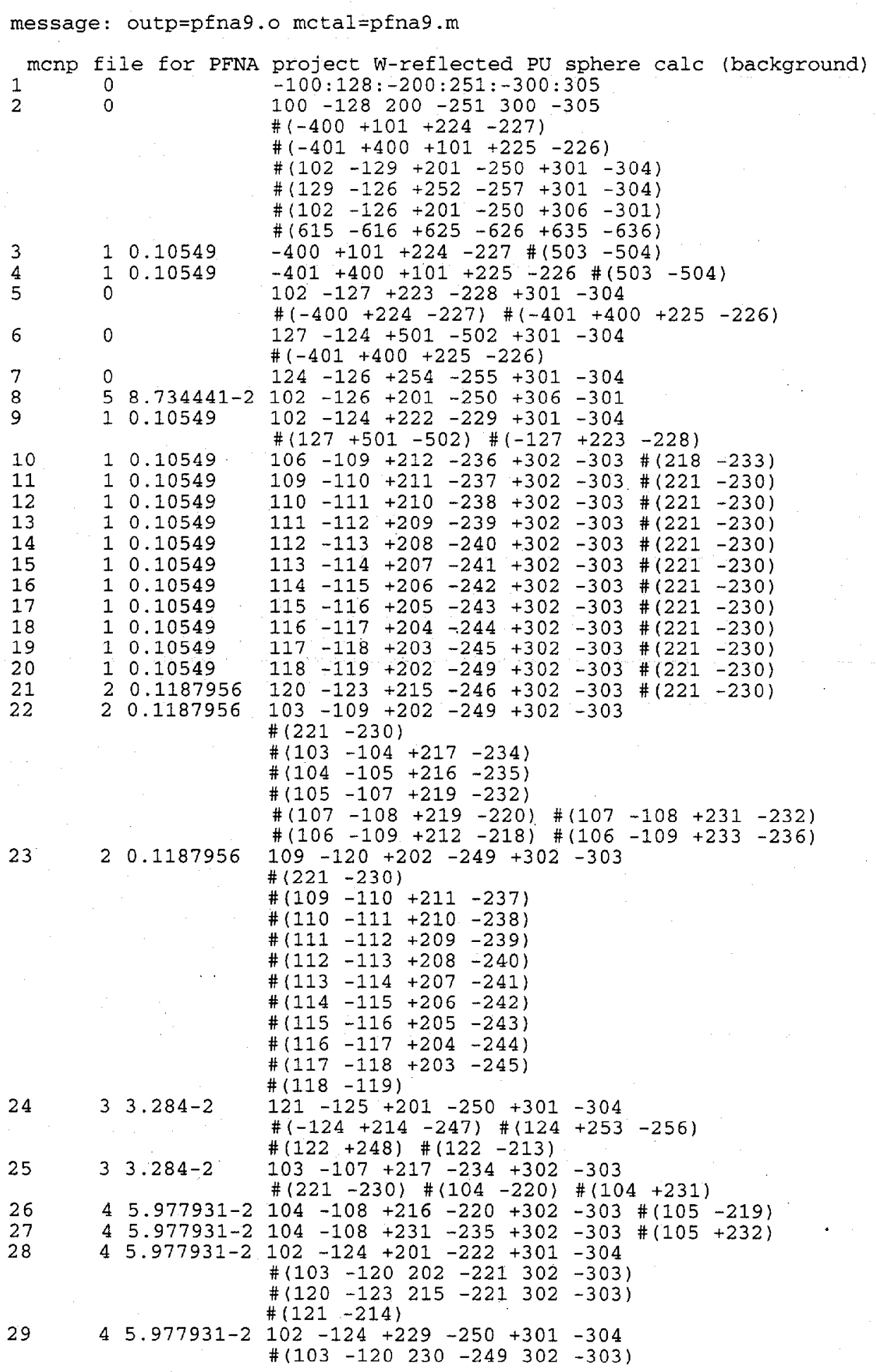




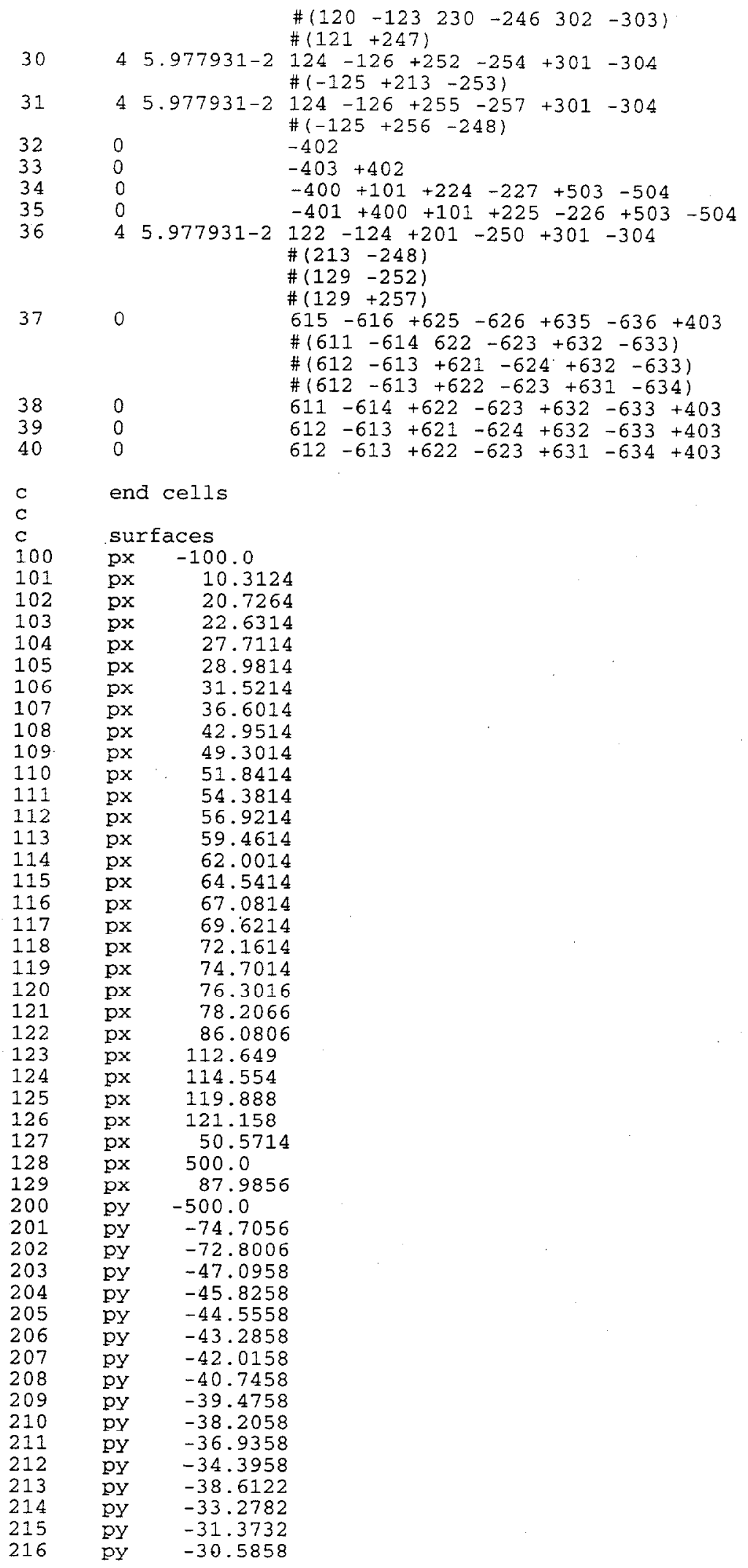




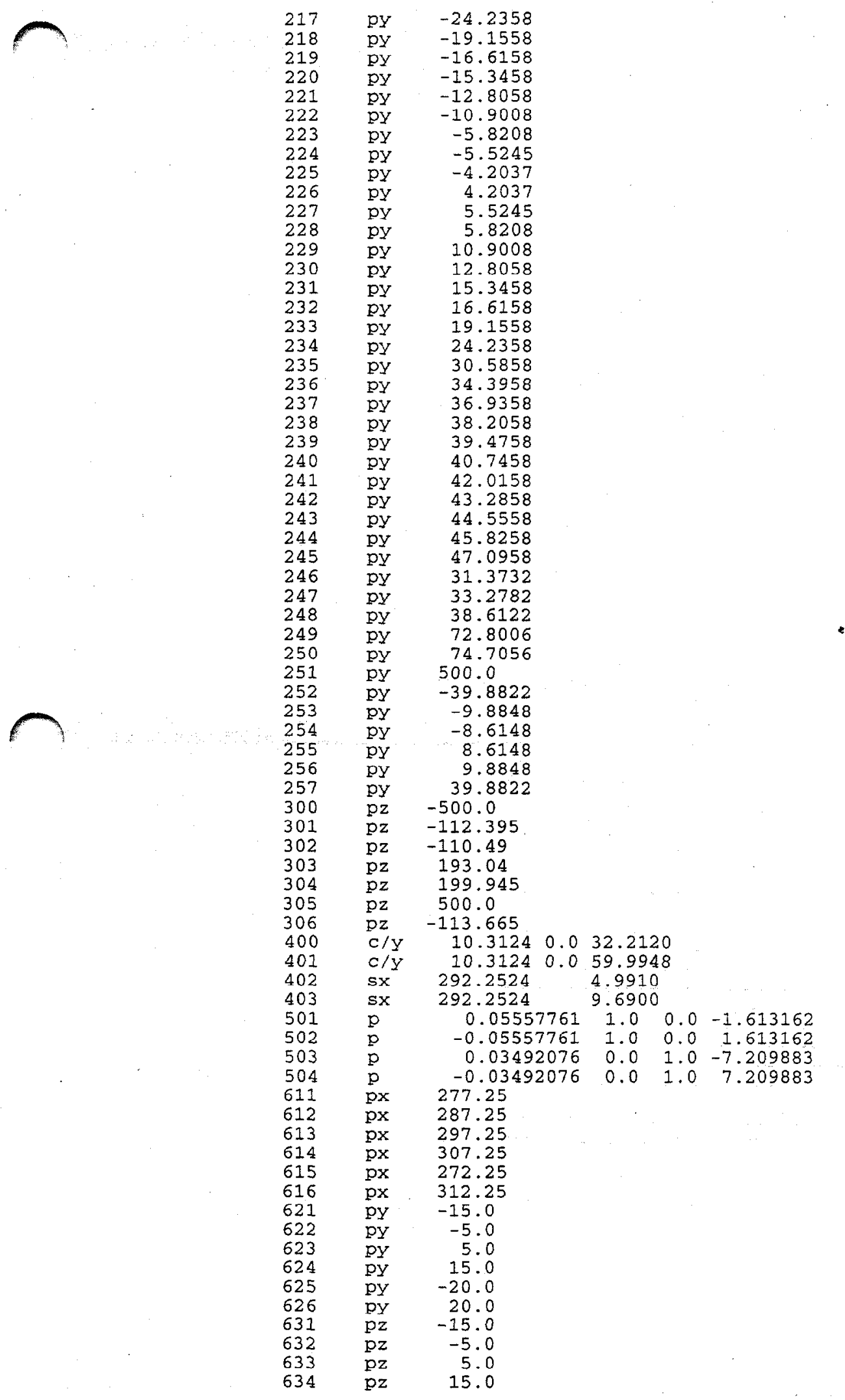




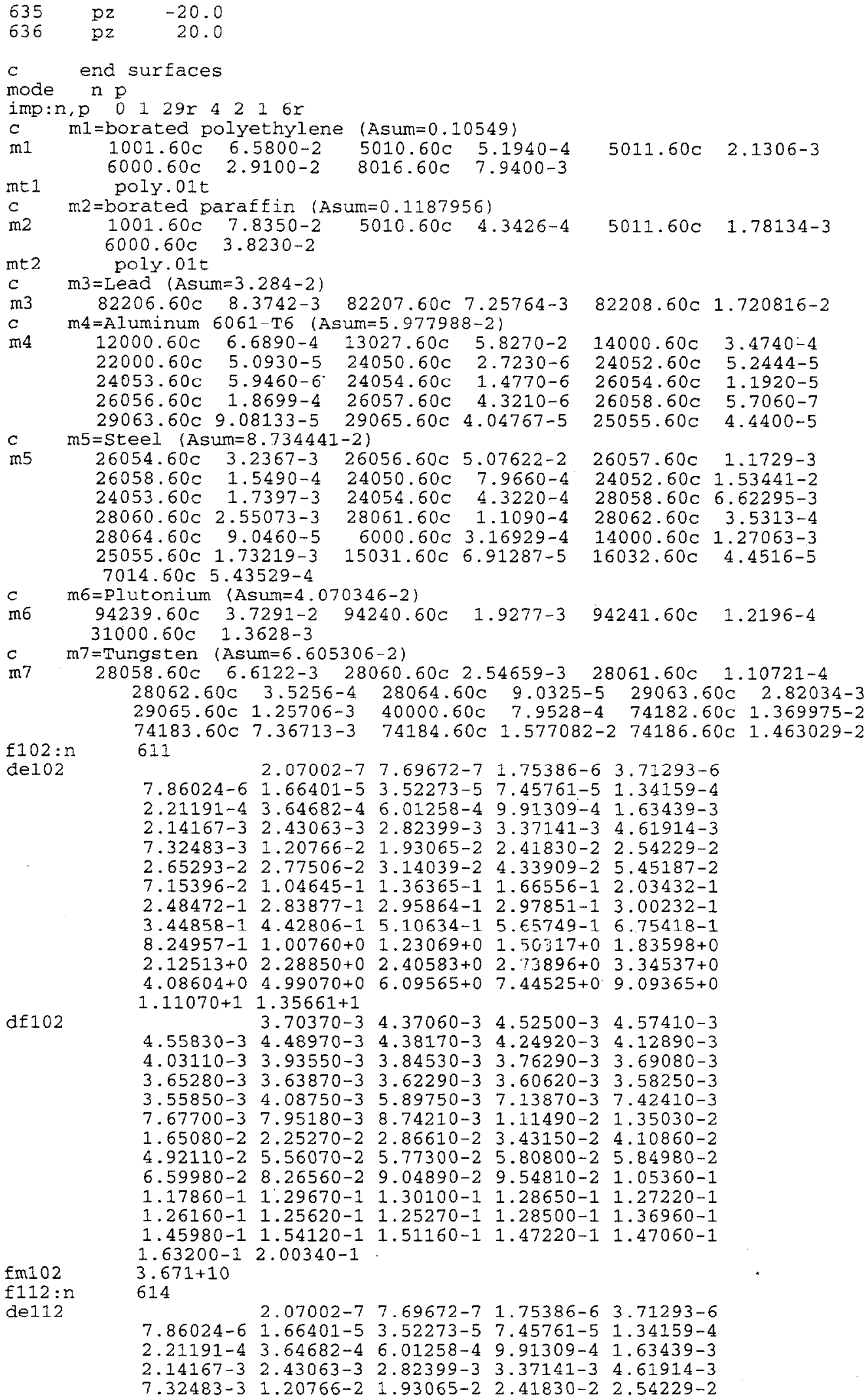


$2.65293-2 \quad 2.77506-2 \quad 3.14039-2 \quad 4.33909-2 \quad 5.45187-2$ $7.15396-2 \quad 1.04645-1 \quad 1.36365-1 \quad 1.66556-1 \quad 2.03432-1$ $\begin{array}{llllll}2.48472-1 & 2.83877-1 & 2.95864-1 & 2.97851-1 & 3.00232-1\end{array}$ $3.44858-1 \quad 4.42806-1 \quad 5.10634-1 \quad 5.65749-16.75418-1$ $8.24957-11.00760+01.23069+0 \quad 1.50317+0 \quad 1.83598+0$ $2.12513+0 \quad 2.28850+0 \quad 2.40583+0 \quad 2.73895+0 \quad 3.34537+0$ $4.08604+0 \quad 4.99070+0 \quad 6.09565+0 \quad 7.44525+0 \quad 9.09365+0$ $1.11070+1 \quad 1.35661+1$

$\operatorname{df112}$

$3.70370-3$

$3.70370-3 \quad 4.37060-3 \quad 4.52500-3 \quad 4.57410-3$

4.55830-3 $4.48970-3 \quad 4.38170-3 \quad 4.24920-3 \quad 4.12890-3$ $\begin{array}{llllll}4.03110-3 & 3.93550-3 & 3.84530-3 & 3.76290-3 & 3.69080-3\end{array}$ $\begin{array}{llllll}3.65280-3 & 3.63870-3 & 3.62290-3 & 3.60620-3 & 3.58250-3\end{array}$ $\begin{array}{llllll}3.55850-3 & 4.08750-3 & 5.89750-3 & 7.13870-3 & 7.42410-3\end{array}$ $7.67700-3 \quad 7.95180-3 \quad 8.74210-3 \quad 1.11490-2 \quad 1.35030-2$ $1.65080-2 \quad 2.25270-2 \quad 2.86510-2 \quad 3.43150-2 \quad 4.10860-2$ $4.92110-2$ 5.56070-2 5.77300-2 5.80800-2 5.84980-2 $6.59980-2 \quad 8.26560-2 \quad 9.04890-2 \quad 9.54810-2 \quad 1.05360-1$ $1.17860-1 \quad 1.29670-1 \quad 1.30100-1 \quad 1.28650-1 \quad 1.27220-1$ $1.26160-1 \quad 1.25620-1 \quad 1.25270-1 \quad 1.28500-1 \quad 1.36960-1$ $1.45980-1$ 1.54120-1 1.51160-1 1.47220-1 1.47060-1 1.63200-1 2.00340-1

$\operatorname{fm} 112$ f122:n $3.671+10$

de122 624

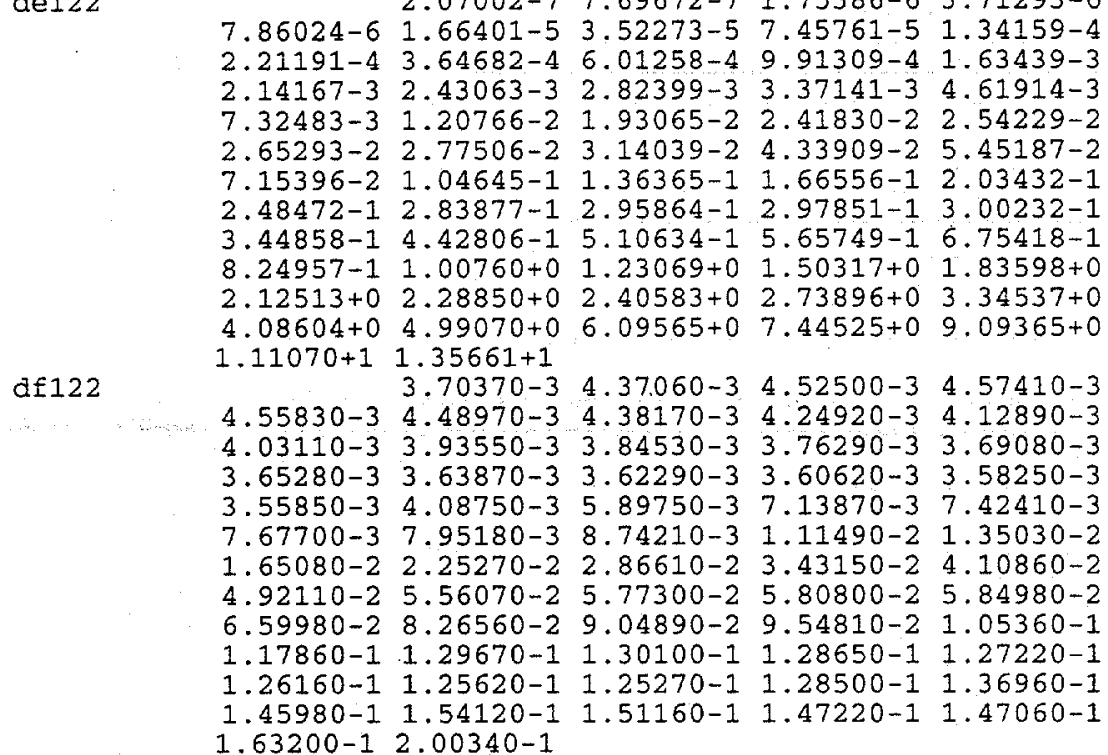

fim 122 E132:n

de132 634 7.86024-6 $1.66401-5 \quad 3.52273-5 \quad 7.45761-5 \quad 1.34159-4$ 2.21191-4 $3.64682-4 \quad 6.01258-4 \quad 9.91309-4 \quad 1.63439-3$ 2.14167-3 $2.43063-3 \quad 2.82399-3 \quad 3.37141-3 \quad 4.61914-3$ $7.32483-3 \quad 1.20766-2 \quad 1.93065-2 \quad 2.41830-2 \quad 2.54229-2$ $2.65293-2 \quad 2.77506-2 \quad 3.14039-2 \quad 4.33909-2 \quad 5.45187-2$

7.15396-2 $1.04645-1 \quad 1.36365-1 \quad 1.66556-1 \quad 2.03432-1$ $2.48472-1 \quad 2.83877-1 \quad 2.95864-1 \quad 2.97851-1 \quad 3.00232-1$ $3.44858-1 \quad 4.42806-1 \quad 5.10634-1 \quad 5.65749-1 \quad 6.75418-1$ $8.24957-1 \quad 1.00760+0 \quad 1.23069+0 \quad 1.50317+0 \quad 1.83598+0$ $2.12513+0 \quad 2.28850+0 \quad 2.40583+0 \quad 2.73896+0 \quad 3.34537+0$ $\begin{array}{lllll}4.08604+0 & 4.99070+0 & 6.09565+0 & 7.44525+0 & 9.09365+0\end{array}$ $1.11070+11.35661+1$

df132 $3.70370-3 \quad 4.37060-3 \quad 4.52500-3 \quad 4.57410-3$ $4.55830-3 \quad 4.48970-3 \quad 4.38170-3 \quad 4.24920-3 \quad 4.12890-3$ $4.03110-3 \quad 3.93550-3 \quad 3.84530-3 \quad 3.76290-3 \quad 3.69080-3$ $3.65280-3 \quad 3.63870-3 \quad 3.62290-3 \quad 3.60620-3 \quad 3.58250-3$ $3.55850-3 \quad 4.08750-3 \quad 5.89750-3 \quad 7.13870-3 \quad 7.42410-3$

$7.67700-3 \quad 7.95180-3 \quad 8.74210-3 \quad 1.11490-2 \quad 1.35030-2$

$\begin{array}{lllll}1.65080-2 & 2.25270-2 & 2.86610-2 & 3.43150-2 & 4.10860-2\end{array}$ $4.92110-2.5 .56070-2 \quad 5.77300-2 \quad 5.80800-2 \quad 5.81980-2$ 
fm132

$£ 202: p$

de 202

$d f 202$

fm202

f212:p

de 212

df 212

$\operatorname{fm} 212$

$\mathrm{f} 222: \mathrm{p}$

de 222

df 222

fm 222

f $232: p$

de232

df232

f 232

e102

e202
6.59980-2 $8.26560-2 \quad 9.04890-2 \quad 9.54810-2 \quad 1.05360-1$ $1.17860-1 \quad 1.29670-1 \quad 1.30100-1 \quad 1.28650-11.27220-1$ $1.26160-1 \quad 1.25620-1 \quad 1.25270-1 \quad 1.28500-1 \quad 1.36960-1$ $1.45980-1$ 1.54120-1 $1.51160-1$ 1.47220-1 1.47060-1 $1.63200-12.00340-1$

$3.671+10$

611

$1.50000-2 \quad 3.25000-2 \quad 5.75000-2 \quad 8.50000-2$ $\begin{array}{lllll}1.25000-1 & 2.25000-1 & 3.50000-1 & 4.55000-1 & 5.55000-1\end{array}$ $6.50000-1 \quad 8.50000-1 \quad 1.25000+0 \quad 1.75000+0 \quad 2.25000+0$ $2.75000+0 \quad 3.50000+0 \quad 4.50000+0 \quad 5.50000+0 \quad 6.50000+0$ $7.25000+0 \quad 7.75000+0 \quad 9.00000+0 \quad 1.20000+1$ $\begin{array}{llll}2.14390-3 & 5.77600-4 & 2.71850-4 & 2.68170-4\end{array}$ $3.27670-4 \quad 5.66760-4 \quad 8.75940-4 \quad 1.08450-3 \quad 1.27970-3$ $1.44170-3 \quad 1.75630-3 \quad 2.31560-3 \quad 2.92700-3 \quad 3.46860-3$ $3.95960-3 \quad 4.62210-3 \quad 5.41370-3 \quad 6.19090-36.92650-3$ $7.47830-3 \quad 7.84680-3 \quad 8.77160-3 \quad 1.10200-2$

$3.671+10$

614

$1.50000-2 \quad 3.25000-2 \quad 5.75000-2 \quad 8.50000-2$ $1.25000-1 \quad 2.25000-1 \quad 3.50000-1 \quad 4.55000-1 \quad 5.55000-1$ $\begin{array}{llllll}6.50000-1 & 8.50000-1 & 1.25000+0 & 1.75000+0 & 2.25000+0\end{array}$ $2.75000+0 \quad 3.50000+0.4 .50000+0 \quad 5.50000+0 \quad 6.50000+0$ $7.25000+0 \quad 7.75000+09.00000+01.20000+1$

$2.14390-3 \quad 5.77600-4 \quad 2.71850-4 \quad 2.68170-4$ $3.27670-4 \quad 5.66760-4 \quad 8.75940-4 \quad 1.08450-3 \quad 1.27970-3$ $1.44170-3 \quad 1.75630-3 \quad 2.31560-3 \quad 2.92700-3 \quad 3.46860-3$ $3.95960-3 \quad 4.62210-3 \quad 5.41370-3 \quad 6.19090-3 \quad 6.92650-3$ $\begin{array}{llll}7.47830-3 & 7.84680-3 & 8.77160-3 & 1.10200-2\end{array}$ $3.671+10$

624

$1.50000-2 \quad 3.25000-2 \quad 5.75000-2 \quad 8.50000-2$ $1.25000-1 \quad 2.25000-1 \quad 3.50000-1 \quad 4.55000-1 \quad 5.55000-1$ $6.50000-1 \quad 8.50000-1 \quad 1.25000+0 \quad 1.75000+0 \quad 2.25000+0$ $2.75000+0 \quad 3.50000+0 \quad 4.50000+0 \quad 5.50000+0 \quad 6.50000+0$ $7.25000+0 \quad 7.75000+0 \quad 9.00000+0 \quad 1.20000+1$

$2.14390-3 \quad 5.77600-4 \quad 2.71850-4 \quad 2.68170-4$ $3.27670-4 \quad 5.66760-4 \quad 8.75940-4 \quad 1.08450-3 \quad 1.27970-3$ $1.44170-3 \quad 1.75630-3 \quad 2.31560-3 \quad 2.92700-3 \quad 3.46860-3$ $\begin{array}{llllll}3.95960-3 & 4.62210-3 & 5.41370-3 & 6.19090-3 & 6.92650-3\end{array}$ $7.47830-3 \quad 7.84680-3 \quad 8.77160-3 \quad 1.10200-2$ $3.671+10$

634

$1.50000-2 \quad 3.25000-2 \quad 5.75000-2 \quad 8.50000-2$ $\begin{array}{llllll}1.25000-1 & 2.25000-1 & 3.50000-1 & 4.55000-1 & 5.55000-1\end{array}$ $6.50000-1 \quad 8.50000-1 \quad 1.25000+0 \quad 1.75000+0 \quad 2.25000+0$ $2.75000+0 \quad 3.50000+0 \quad 4.50000+0 \quad 5.50000+0 \quad 6.50000+0$ $7.25000+0 \quad 7.75000+0 \quad 9.00000+0 \quad 1.20000+1$ $\begin{array}{llll}2.14390-3 & 5.77600-4 & 2.71850-4 & 2.68170-4\end{array}$ $3.27670-4 \quad 5.66760-4 \quad 8.75940-4 \quad 1.08450-3 \quad 1.27970-3$ $\begin{array}{llllll}1.44170-3 & 1.75630-3 & 2.31560-3 & 2.92700-3 & 3.46860-3\end{array}$ $\begin{array}{llllll}3.95960-3 & 4.62210-3 & 5.41370-3 & 6.19090-3 & 6.92650-3\end{array}$ $7.47830-3 \quad 7.84680-3 \quad 8.77160-3 \quad 1.10200-2$ $3.671+10$

4.13994-7 $1.12535-6 \quad 2.38237-6 \quad 5.04348-6 \quad 1.06770-5$ 2.26033-5 $4.78512-5 \quad 1.01301-4 \quad 1.67017-4 \quad 2.75364-4$ 4.53999-4 7.48518-4 $1.23410-3 \quad 2.03468-3 \quad 2.24867-3$ $\begin{array}{lllll}2.61259-3 & 3.03539-3 & 3.70744-3 & 5.53084-3 & 9.11882-3\end{array}$ $\begin{array}{lllll}1.50344-2 & 2.35786-2 & 2.47875-2 & 2.60584-2 & 2.70001-2\end{array}$ 2.85011-2 $3.43067-2 \quad 5.24752-2 \quad 5.65622-2 \quad 8.65169-2$ $\begin{array}{lllll}1.22773-1 & 1.49956-1 & 3.83156-1 & 2.23708-1 & 2.73237-1\end{array}$ 2.94518-1 $2.97211-1 \quad 2.98491-1 \quad 3.01974-1 \quad 3.87742-1$ 4.97870-1 $5.23397-1 \quad 6.08101-1 \quad 7.42735-1 \quad 9.07180-1$ $1.10803+0 \quad 1.35335+0 \quad 1.65299+0 \quad 2.01897+0 \quad 2.23130+0$ $2.34570+0 \quad 2.46597+0 \quad 3.01194+0 \quad 3.67879+0 \quad 4.49329+0$ $5.48811+0 \quad 6.70320+0 \quad 8.18730+0 \quad 1.00000+1 \quad 1.22140+1$ $1.49183+1$

$2.00000-2 \quad 4.50000-2 \quad 7.00000-2 \quad 1.00000-1 \quad 1.50000-1$ 3.00000-1 $4.00000-1 \quad 5.10000-1 \quad 6.00000-1 \quad 7.00000-1$ $1.00000+0 \quad 1.50000+0 \quad 2.00000+0 \quad 2.50000+0 \quad 3.00000+0$ 
$4.00000+0 \quad 5.00000+0 \quad 6.00000+07.00000+0 \quad 7.50000+0$ $8.00000+0 \quad 1.00000+11.40000+1$

0112

4.13994-7 $1.12535-6 \quad 2.38237-6 \quad 5.04348-6 \quad 1.06770-5$ 2.26033-5 $4.78512-5 \quad 1.01301-4 \quad 1.67017-4 \quad 2.75364-4$ $\begin{array}{llllll}4.53999-4 & 7.48518-4 & 1.23410-3 & 2.03468-3 & 2.24867-3\end{array}$ $\begin{array}{llllll}2.61259-3 & 3.03539-3 & 3.70744-3 & 5.53084-3 & 9.11882-3\end{array}$ 1.50344-2 2.35786-2 2.47875-2 $2.60584-2 \quad 2.70001-2$ $2.85011-2 \quad 3.43067-2 \quad 5.24752-2 \quad 5.65622-2 \quad 8.65169-2$ 1.22773-1 $1.49956-1 \quad 1.83156-1 \quad 2.23708-1 \quad 2.73237-1$ 2.94518-1 $2.97211-1 \quad 2.98491-1 \quad 3.01974-1 \quad 3.87742-1$ $\begin{array}{lllll}4.97870-1 & 5.23397-1 & 6.08101-1 & 7.42735-1 & 9.07180-1\end{array}$ $1.10803+0.1 .35335+0 \quad 1.65299+0 \quad 2.01897+0 \quad 2.23130+0$ $2.34570+0 \quad 2.46597+0 \quad 3.01194+0 \quad 3.67879+0 \quad 4.49329+0$ $5.48811+0 \quad 6.70320+0 \quad 8.18730+0 \cdot 1.00000+1 \quad 1.22140+1$ $1.49183+1$

e212

2.00000-2 4.50000-2 7.00000-2 1.00000-1 1.50000-1 $3.00000-1 \quad 4.00000-1 \quad 5.10000-1 \quad 6.00000-1 \quad 7.00000-1$ $1.00000+0 \quad 1.50000+0 \quad 2.00000+0 \quad 2.50000+0 \quad 3.00000+0$ $4.00000+0 \quad 5.00000+0 \quad 6.00000+0 \quad 7.00000+0 \quad 7.50000+0$ $8.00000+01.00000+11.40000+1$

e122

4.13994-7 $1.12535-6 \quad 2.38237-6 \quad 5.04348-6 \quad 1.06770-5$ $2.26033-5 \quad 4.78512-5 \quad 1.01301-4 \quad 1.67017-4 \quad 2.75364-4$ $4.53999-4 \quad 7.48518-4 \quad 1.23410-3 \quad 2.03468-3 \quad 2.24867-3$ 2.61259-3 $3.03539-3.3 .70744-3 \quad 5.53084-3 \quad 9.11882-3$ $\begin{array}{lllll}1.50344-2 & 2.35786-2 & 2.47875-2 & 2.60584-2 & 2.70001-2\end{array}$ 2.85011-2 $3.43067-2 \quad 5.24752-2 \quad 5.65622-2.8 .65169-2$ $1.22773-1 \quad 1.49956-1 \quad 1.83156-1 \quad 2.23708-1 \quad 2.73237-1$ 2.94518-1 2.97211-1 $2.98491-1 \quad 3.01974-1 \quad 3.87742-1$ 4.97870-1 $5.23397-1 \quad 6.08101-1 \quad 7.42735-1 \quad 9.07180-1$ $1.10803+0 \quad 1.35335+0 \quad 1.65299+0 \quad 2.01897+0 \quad 2.23130+0$ $2.34570+0 \quad 2.46597+0 \quad 3.01194+0 \quad 3.67879+0 \quad 4.49329+0$ $5.48811+0 \quad 6,70320+0 \quad 8.18730+0 \quad 1.00000+1 \quad 1.22140+1$ $1.49183+1$

$e 222$

2.00000-2 4.50000-2 $7.00000-2 \quad 1.00000-1 \quad 1.50000-1$ $3.00000-1 \quad 4.00000-1 \quad 5.10000-1 \quad 6.00000-1 \quad 7.00000-1$ $1.00000+0 \quad 1.50000+0 \quad 2.00000+0 \quad 2.50000+0 \quad 3.00000+0$ $4.00000+0 \quad 5.00000+0 \quad 6.00000+0 \quad 7.00000+0.7 .50000+0$ $8.00000+01.00000+11.40000+1$

e132

4.13994-7 $1.12535-6 \quad 2.38237-6 \quad 5.04348-6 \quad 1.06770-5$ $2.26033-5 \quad 4.78512-5 \quad 1.01301-4 \quad 1.67017-4 \quad 2.75364-4$ $4.53999-4 \quad 7.48518-4 \quad 1.23410-3 \quad 2.03468-3 \quad 2.24867-3$ $2.61259-3 \quad 3.03539-3 \quad 3.70744-3 \quad 5.53084-3 \quad 9.11882-3$ $\begin{array}{llllll}1.50344-2 & 2.35786-2 & 2.47875-2 & 2.60584-2 & 2.70001-2\end{array}$ 2.85011-2 $3.43067-2 \quad 5.24752-2 \quad 5.65622-2 \quad 8.65169-2$ $\begin{array}{llllll}1.22773-1 & 1.49956-1 & 1.83156-1 & 2.23708-1 & 2.73237-1\end{array}$ 2.94518-1 2.97211-1 $2.98491-1 \quad 3.01974-1 \quad 3.87742-1$ 4.97870-1 $5.23397-1 \quad 6.08101-1 \quad 7.42735-1 \quad 9.07180-1$ $\begin{array}{llllll}1.10803+0 & 1.35335+0 & 1.65299+0 & 2.01897+0 & 2.23130+0\end{array}$ $2.34570+0 \quad 2.46597+0 \quad 3.01194+0 \quad 3.67879+0 \quad 4.49329+0$ $5.48811+0 \quad 6.70320+0 \quad 8.18730+0 \quad 1.00000+1 \quad 1.22140+1$ $1.49183+1$

e232

$2.00000-2 \quad 4.50000-2 \quad 7.00000-2 \quad 1.00000-1 \quad 1.50000-1$ $3.00000-1 \quad 4.00000-1 \quad 5.10000-1 \quad 6.00000-17.00000-1$

$1.00000+0 \quad 1.50000+02.00000+0 \quad 2.50000+0 \quad 3.00000+0$

$4.00000+0 \quad 5.00000+0 \quad 6.00000+0 \quad 7.00000+0 \quad 7.50000+0$ sd102 100.0 $8.00000+01.00000+11.40000+1$

sd112 100.0

sd122 100.0

$\operatorname{sd132} 100.0$

sd202 100.0

sd212 100.0

sđ222 100.0

sd232 100.0

sdef pos $=0$ o 0 vec=1 0 o dir=d1 erg=fdir=d2

$\begin{array}{lllllll}c & \text { si1 } & 0.0 & 0.5 & 0.8660254 & 0.9961946 & 1.0\end{array}$ 


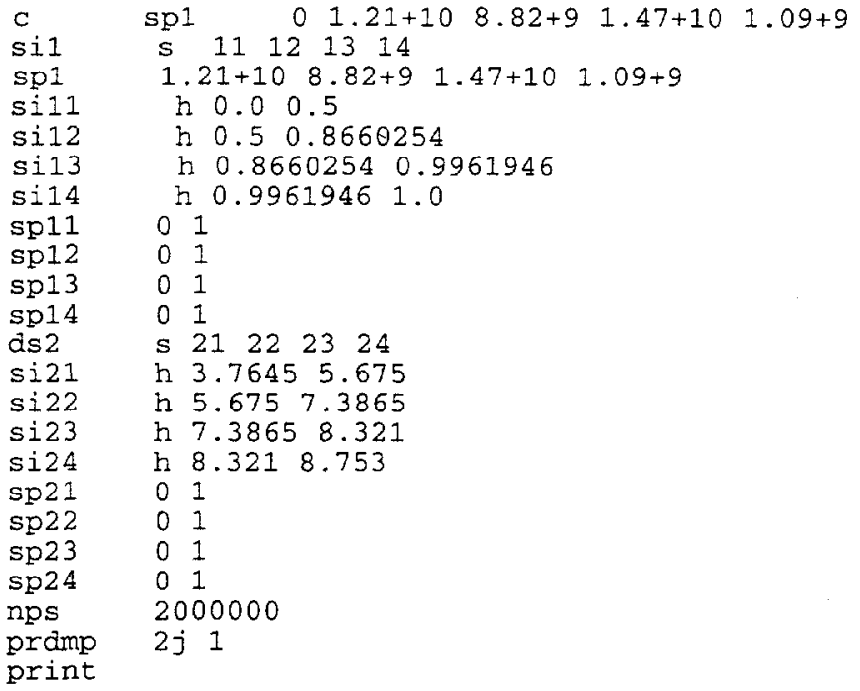


File for MCNP-4B surface-detector dose-rate background calculations for the water-reflected Pu spheres in the PFNA beam at the center of the truck lane $(2,000,000$ histories $)$

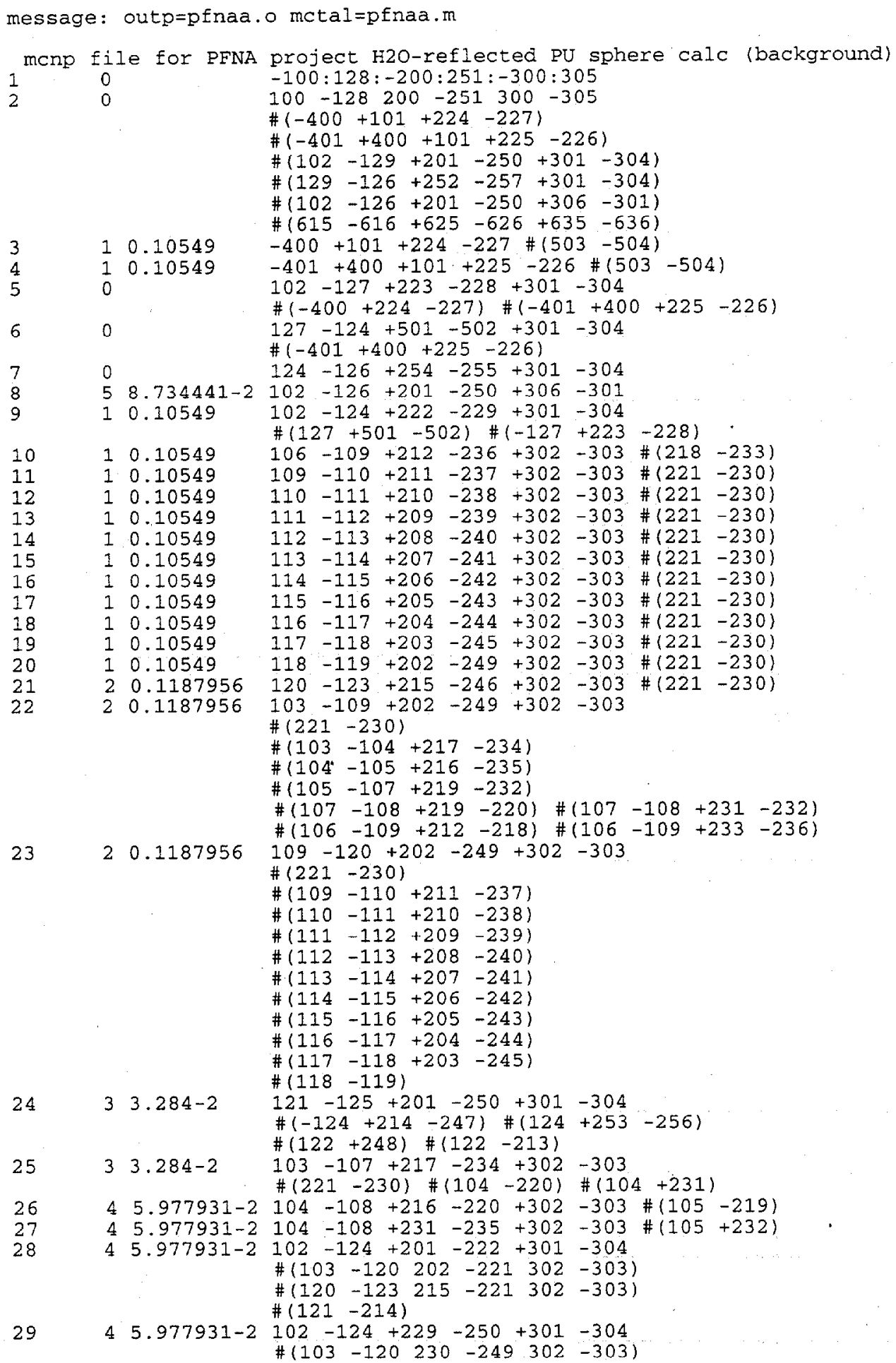




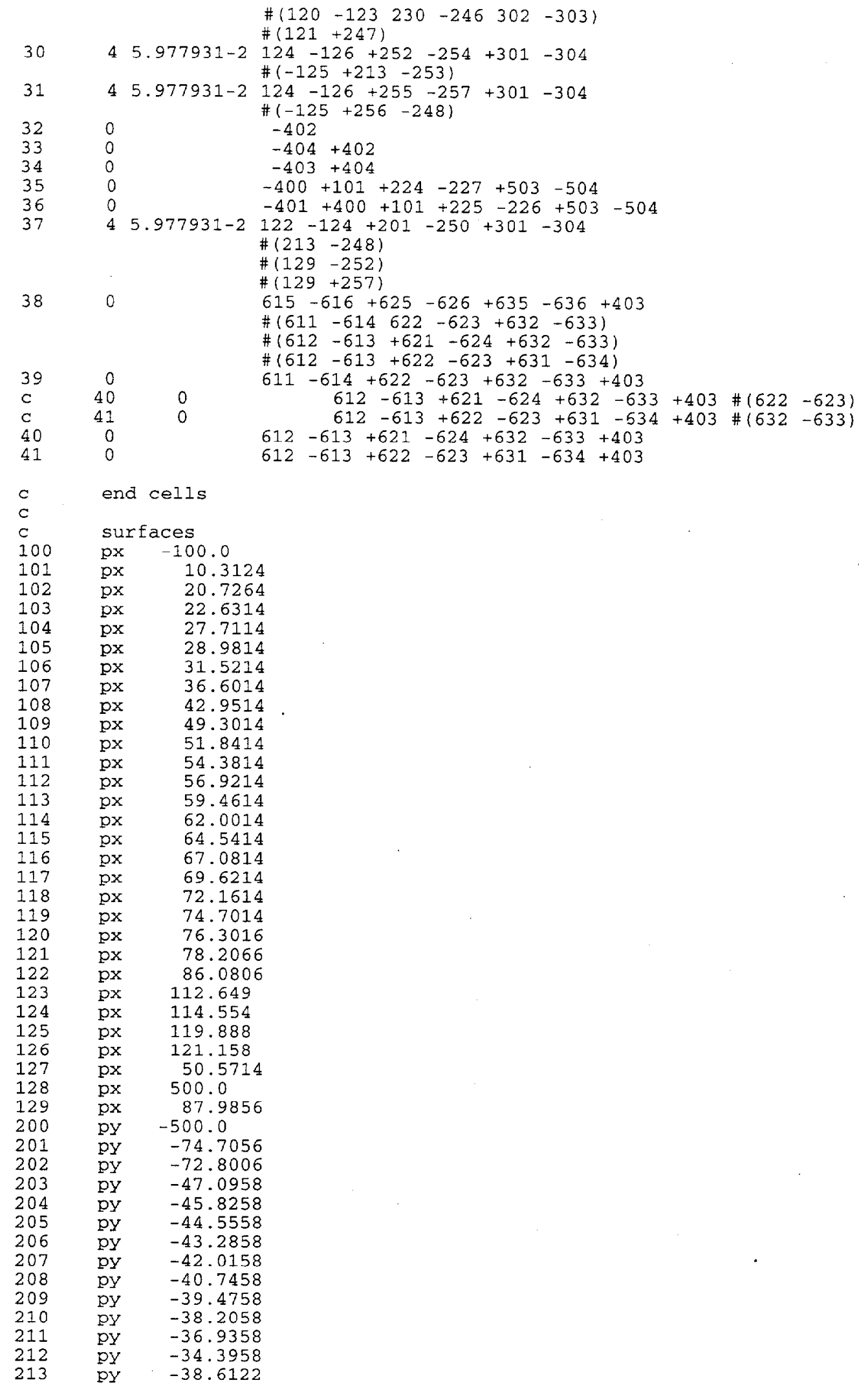




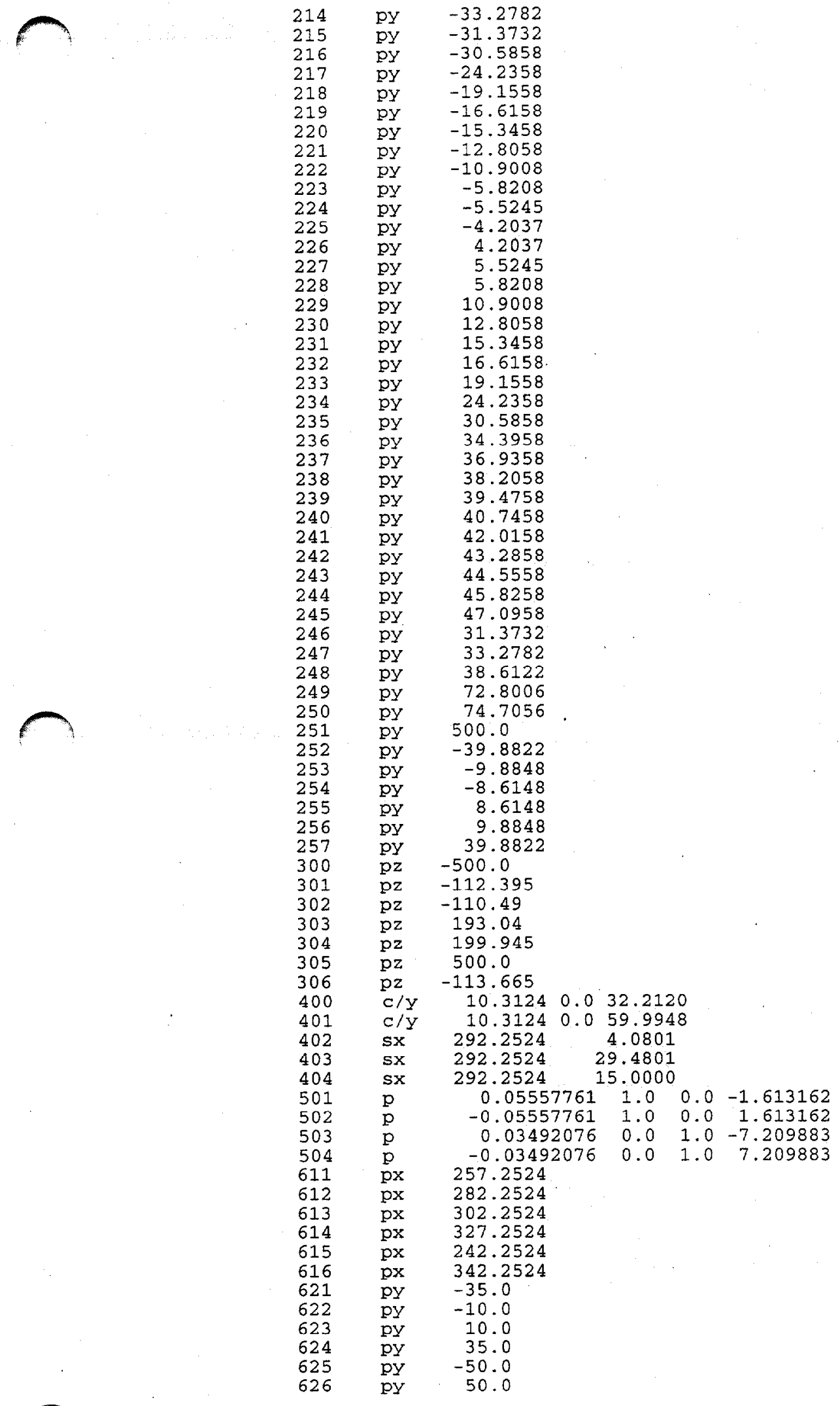




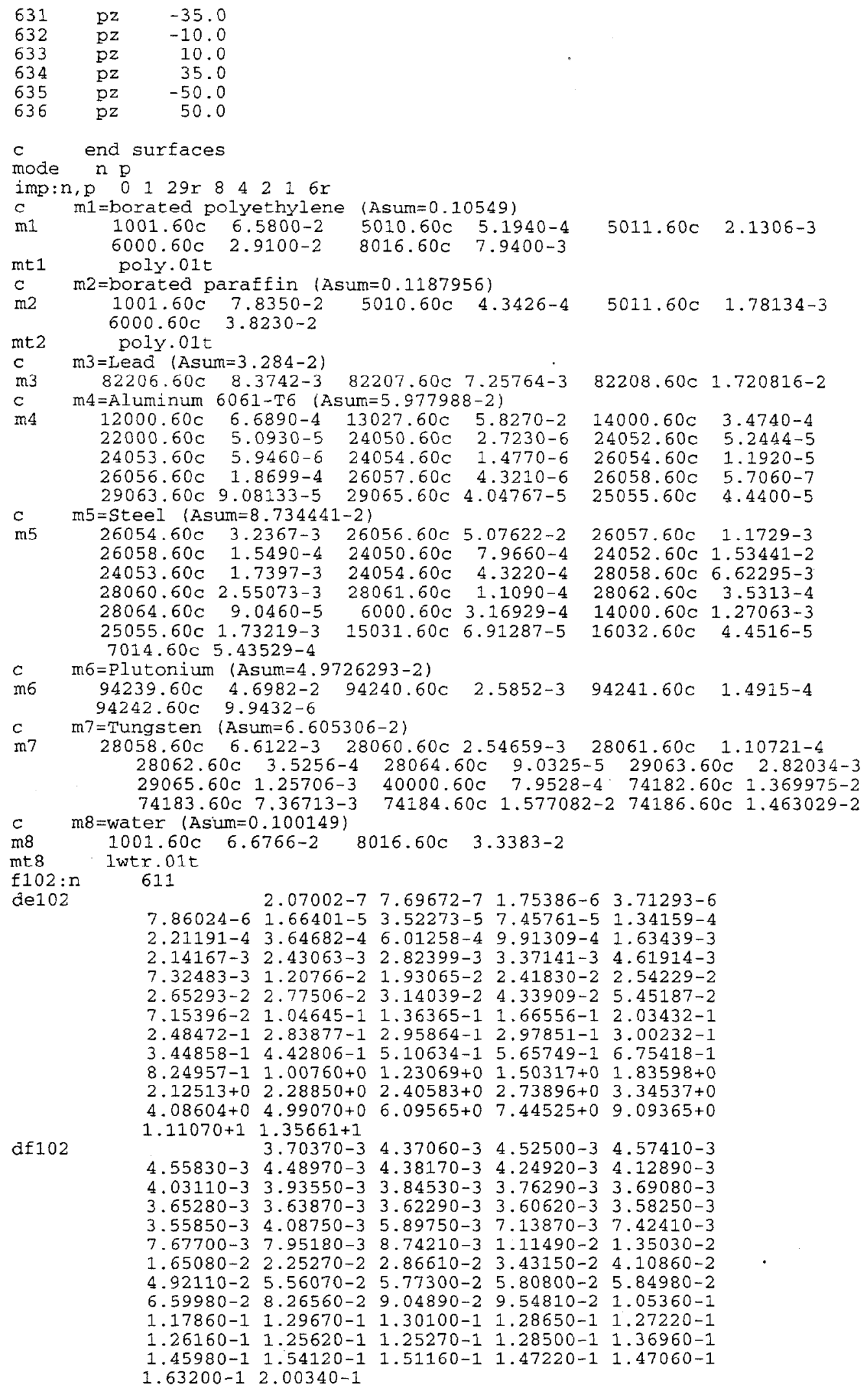


$\begin{array}{ll}\operatorname{fm} 102 & 3.671+10 \\ \mathrm{f} 112: \mathrm{n} & 614\end{array}$

$2.07002-77.69672-7 \quad 1.75386-6 \quad 3.71293-6$ $7.86024-6 \quad 1.66401-5 \quad 3.52273-5 \quad 7.45761-5 \quad 1.34159-4$ 2.21191-4 3.64682-4 $6.01258-4 \quad 9.91309-4 \quad 1.63439-3$ $2.14167-3 \quad 2.43063-3 \quad 2.82399-3 \quad 3.37141-3 \quad 4.61914-3$ 7.32483-3 $1.20766-2 \quad 1.93065-2 \quad 2.41830-2 \quad 2.54229-2$ 2.65293-2 2.77506-2 3.14039-2 $4.33909-2$ 5.45187-2 $7.15396-2 \quad 1.04645-1 \quad 1.36365-1 \quad 1.66556-1 \quad 2.03432-1$ 2.48472-1 $2.83877-1 \quad 2.95864-1 \quad 2.97851-1 \quad 3.00232-1$ 3.44858-1 $4.42806-1 \quad 5.10634-1 \quad 5.65749-1 \quad 6.75418-1$ 8.24957-1 $1.00760+0 \quad 1.23069+0 \quad 1.50317+0 \quad 1.83598+0$ $\begin{array}{lllll}2.12513+0 & 2.28850+0 & 2.40583+0 & 2.73896+0 & 3.34537+0\end{array}$ $4.08604+0 \quad 4.99070+0 \quad 6.09565+0 \quad 7.44525+0 \quad 9.09365+0$ $1.11070+1 \quad 1.35661+1$

df112 $\quad 3.70370-3 \quad 4.37060-3 \quad 4.52500-3 \quad 4.57410-3$ $4.55830-3 \quad 4.48970-3 \quad 4.38170-3 \quad 4.24920-3 \quad 4.12890-3$ $4.03110-3 \quad 3.93550-3 \quad 3.84530-3 \quad 3.76290-3 \quad 3.69080-3$ $3.65280-3 \quad 3.63870-3 \quad 3.62290-3 \quad 3.60620-3 \quad 3.58250-3$ $3.55850-3 \quad 4.08750-3 \quad 5.89750-3 \quad 7.13870-3 \quad 7.42410-3$ $7.67700-3 \quad 7.95180-3 \quad 8.74210-3 \quad 1.11490-2 \quad 1.35030-2$ $1.65080-22.25270-2 \quad 2.86610-2 \quad 3.43150-2$ 4.10860-2 $4.92110-2 \quad 5.56070-2 \quad 5.77300-2 \quad 5.80800-2 \quad 5.84980-2$ $6.59980-2 \quad 8.26560-2 \quad 9.04890-29.54810-2 \quad 1.05360-1$

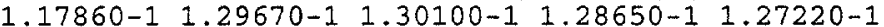
$1.26160-1 \quad 1.25620-1 \quad 1.25270-1 \quad 1.28500-1 \quad 1.36960-1$ $1.45980-1 \quad 1.54120-1$ 1.51160-1 $1.47220-1 \quad 1.47060-1$ $1.63200-12.00340-1$

$\operatorname{fm} 112$ $3.671+10$

$\mathrm{f} 122: \mathrm{n} \quad 624$

de122 $2.07002-7 \quad 7.69672-7 \quad 1.75386-6 \quad 3.71293-6$ $7.86024-6 \quad 1.66401-5 \quad 3.52273-5 \quad 7.45761-5 \quad 1.34159-4$ $2.21191-4 \quad 3.64682-4 \quad 6.01258-4 \quad 9.91309-4 \quad 1.63439-3$ $2.14167-3 \quad 2.43063-3 \quad 2.82399-3 \quad 3.37141-3 \quad 4.61914-3$ $7.32483-3 \quad 1.20766-2 \quad 1.93065-2 \quad 2.41830-2 \quad 2.54229-2$ $2.65293-2 \quad 2.77506-2 \quad 3.14039-2 \quad 4.33909-2 \quad 5.45187-2$ 7.15396-2 1.04645-1 $1.36365-1 \quad 1.66556-1 \quad 2.03432-1$ $2.48472-1 \quad 2.83877-12.95864-1 \quad 2.97851-1 \quad 3.00232-1$ $3.44858-1 \quad 4.42806-1 \quad 5.10634-1 \quad 5.65749-1 \quad 6.75418-1$ $8.24957-1 \quad 1.00760+0.1 .23069+0 \quad 1.50317+0 \quad 1.83598+0$ $2.12513+0 \quad 2.28850+0 \quad 2.40583+0 \quad 2.73896+0 \quad 3.34537+0$ $4.08604+0 \quad 4.99070+0 \quad 6.09565+0.7 .44525+0 \quad 9.09365+0$ $1.11070+1 \quad 1.35661+1$

df122 $3.70370-3 \quad 4.37060-3 \quad 4.52500-3 \quad 4.57410-3$ $4.55830-3 \quad 4.48970-3 \quad 4.38170-3 \quad 4.24920-3 \quad 4.12890-3$ $4.03110-3 \quad 3.93550-3 \quad 3.84530-3 \quad 3.76290-3 \quad 3.69080-3$ $3.65280-3 \quad 3.63870-3 \quad 3.62290-3 \quad 3.60620-3 \quad 3.58250-3$ $\begin{array}{lllll}3.55850-3 & 4.08750-3 & 5.89750-3 & 7.13870-3 & 7.42410-3\end{array}$ $7.67700-3 \quad 7.95180-3 \quad 8.74210-3 \quad 1.11490-2 \quad 1.35030-2$ $1.65080-2 \quad 2.25270-2 \quad 2.86610-2 \quad 3.43150-2 \quad 4.10860-2$ $4.92110-2 \quad 5.56070-2 \quad 5.77300-2 \quad 5.80800-2 \quad 5.84980-2$ $\begin{array}{llllll}6.59980-2 & 8.26560-2 & 9.04890-2 & 9.54810-2 & 1.05360-1\end{array}$

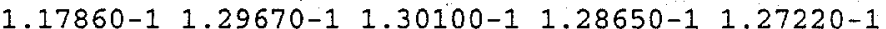
$1.26160-1 \quad 1.25620-1 \quad 1.25270-1 \quad 1.28500-1 \quad 1.36960-1$ $1.45980-1 \quad 1.54120-11.51160-1 \quad 1.47220-11.47060-1$ $1.63200-12.00340-1$

$\operatorname{tm} 122$ $3.671+10$

f132: 1 634

de132

$\operatorname{df132}$

$2.07002-7 \quad 7.69672-7 \quad 1.75386-6 \quad 3.71293-6$ $7.86024-6 \quad 1.66401-5 \quad 3.52273-5 \quad 7.45761-5 \quad 1.34159-4$ $2.21191-4 \quad 3.64682-4 \quad 6.01258-4 \quad 9.91309-4 \quad 1.63439-3$ $2.14167-3 \quad 2.43063-3 \quad 2.82399-3 \quad 3.37141-3 \quad 4.61914-3$ $\begin{array}{llllll}7.32483-3 & 1.20766-2 & 1.93065-2 & 2.41830-2 & 2.54229-2\end{array}$ $2.65293-2$ 2.77506-2 $3.14039-2 \quad 4.33909-2 \quad 5.45187-2$ 7.15396-2 1.04645-1 1.36365-1 1.66556-1 2.03432-1. $2.48472-1 \quad 2.83877-1 \quad 2.95864-1 \quad 2.97851-1 \quad 3.00232-1$ 3.44858-1 $4.42806-1 \quad 5.10634-1 \quad 5.65749-1 \quad 6.75418-1$ $8.24957-1.1 .00760+01.23069+01.50317+01.83598+0$ $2.12513+0 \quad 2.28850+0 \quad 2.40583+0 \quad 2.73896+0 \quad 3.34537+0$ $4.08604+0 \quad 4.99070+0 \quad 6.09565+0 \quad 7.44525+0 \quad 9.09365+0$ $1.11070+1 \quad 1.35661+1$

$3.70370-3 \quad 4.37060-3 \quad 4.52500-3 \quad 4.57410-3$ 
$\begin{array}{llllllll}4.55830-3 & 4.48970-3 & 4.38170-3 & 4.24920-3 & 4.12890-3\end{array}$ $4.03110-3 \quad 3.93550-3 \quad 3.84530-3 \quad 3.76290-3 \quad 3.69080-3$ $\begin{array}{llllll}3.65280-3 & 3.63870-3 & 3.62290-3 & 3.60620-3 & 3.58250-3\end{array}$ $\begin{array}{llllll}3.55850-3 & 4.08750-3 & 5.89750-3 & 7.13870-3 & 7.42410-3\end{array}$ $7.67700-3 \quad 7.95180-3 \quad 8.74210-3 \quad 1.11490-2 \quad 1.35030-2$ $1.65080-2 \quad 2.25270-2 \quad 2.86610-2 \quad 3.43150-2 \quad 4.10860-2$ $4.92110-2 \quad 5.56070-2 \quad 5.77300-2 \quad 5.80800-2 \quad 5.84980-2$ $6.59980-2 \quad 8.26560-2 \quad 9.04890-2 \quad 9.54810-2 \quad 1.05360-1$ $1.17860-1$ 1.29670-1 $1.30100-1 \quad 1.28650-1 \quad 1.27220-1$ 1. $26160-1 \quad 1.25620-1 \quad 1.25270-1 \quad 1.28500-1 \quad 1.36960-1$ $1.45980-1$ 1.54120-1 1.51160-1 1.47220-1 1.47060-1 1.63200-1 2.00340-1

$\operatorname{fm} 132$

$\mathrm{E} 202: \mathrm{p}$

3. $671+10$

611

1 1.50000-2 3.25000-2 5.75000-2 8.50000-2

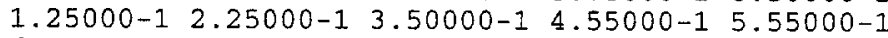
$6.50000-1 \quad 8.50000-1 \quad 1.25000+0 \quad 1.75000+0 \quad 2.25000+0$ $2.75000+0 \quad 3.50000+0 \quad 4.50000+0 \quad 5.50000+0 \quad 6.50000+0$ $7.25000+0 \quad 7.75000+0 \quad 9.00000+0 \quad 1.20000+1$

d=202 $2.14390-3 \quad 5.77600-4 \quad 2.71850-4 \quad 2.68170-4$

$3.27670-4 \quad 5.66760-4 \quad 8.75940-4 \quad 1.08450-3 \quad 1.27970-3$

$\begin{array}{lllll}1.44170-3 & 1.75630-3 & 2.31560-3 & 2.92700-3 & 3.46860-3\end{array}$

$\begin{array}{llllll}3.95960-3 & 4.62210-3 & 5.41370-3 & 6.19090-3 & 6.92650-3\end{array}$

$7.47830-3 \quad 7.84680-3 \quad 8.77160-3 \quad 1.10200-2$

fm202

£212:p 3. $671+10$

de 212

614

$1.50000-2 \quad 3.25000-2 \quad 5.75000-2 \quad 8.50000-2$ $1.25000-1 \quad 2.25000-1 \quad 3.50000-1 \quad 4.55000-1 \quad 5.55000-1$ $6.50000-1 \quad 8.50000-1 \quad 1.25000+0 \quad 1.75000+0 \quad 2.25000+0$ $2.75000+0 \quad 3.50000+0 \quad 4.50000+0 \quad 5.50000+06.50000+0$ $7.25000+0 \quad 7.75000+0 \quad 9.00000+0 \quad 1.20000+1$

$\mathrm{d} £ 212$ $2.14390-3 \quad 5.77600-4 \quad 2.71850-4 \quad 2.68170-4$ $3.27670-4 \quad 5.66760-4 \quad 8.75940-4 \quad 1.08450-3 \quad 1.27970-3$ $1.44170-3 \quad 1.75630-3 \quad 2.31560-3 \quad 2.92700-3 \quad 3.46860-3$ $\begin{array}{lllll}3.95960-3 & 4.62210-3 & 5.41370-3 & 6.19090-3 & 6.92650-3\end{array}$ $7.47830-3.7 .84680-3 \quad 8.77160-3 \quad 1.10200-2$

$\operatorname{fm} 212 \quad 3.671+10$

$1222: \mathrm{p} \quad 624$

de 222

$1.50000-2 \quad 3.25000-2 \quad 5.75000-2 \quad 8.50000-2$

$\begin{array}{llllll}1.25000-1 & 2.25000-1 & 3.50000-1 & 4.55000-1 & 5.55000-1\end{array}$

$6.50000-18.50000-1 \quad 1.25000+0 \quad 1.75000+02.25000+0$

$2.75000+0 \quad 3.50000+0 \quad 4.50000+0 \quad 5.50000+0 \quad 6.50000+0$

$7.25000+0 \quad 7.75000+0 \quad 9.00000+0 \quad 1.20000+1$

df222 $2.14390-3 \quad 5.77600-4 \quad 2.71850-4 \quad 2.68170-4$

$3.27670-4 \quad 5.66760-4 \quad 8.75940-4 \quad 1.08450-3 \quad 1.27970-3$

$1.44170-3 \quad 1.75630-3 \quad 2.31560-3 \quad 2.92700-3 \quad 3.46860-3$

$\begin{array}{llllll}3.95960-3 & 4.62210-3 & 5.41370-3 & 6.19090-3 & 6.92650-3\end{array}$

$7.47830-3 \quad 7.84680-3 \quad 8.77160-3 \quad 1.10200-2$

$\operatorname{fm} 222$

$\mathrm{f} 232: \mathrm{p}$ $3.671+10$

$1.50000-2 \quad 3.25000-2 \quad 5.75000-2 \quad 8.50000-2$ $1.25000-1 \quad 2.25000-1 \quad 3.50000-1 \quad 4.55000-1 \quad 5.55000-1$ $6.50000-1 \quad 8.50000-1 \quad 1.25000+0 \quad 1.75000+0 \quad 2.25000+0$ $2.75000+0 \quad 3.50000+0 \quad 4.50000+0 \quad 5.50000+0 \quad 6.50000+0$ $7.25000+0 \quad 7.75000+0 \quad 9.00000+0 \quad 1.20000+1$

df 232 $2.14390-3 \quad 5.77600-4 \quad 2.71850-4 \quad 2.68170-4$ $3.27670-4 \quad 5.66760-4 \quad 8.75940-4 \quad 1.08450-3 \quad 1.27970-3$ $1.44170-3 \quad 1.75630-3 \quad 2.31560-3 \quad 2.92700-3 \quad 3.46860-3$ $3.95960-3 \quad 4.62210-3 \quad 5.41370-3 \quad 6.19090-3 \quad 6.92650-3$ $7.47830-3 \quad 7.84680-3 \quad 8.77160-3 \quad 1.10200-2$

$\operatorname{Im} 232$ $3.671+10$

e102

$4.13994-7 \quad 1.12535-6 \quad 2.38237-6 \quad 5.04348-6 \quad 1.06770-5$ $2.26033-5 \quad 4.78512-5 \quad 1.01301-4 \quad 1.67017-4 \quad 2.75364-4$ $\begin{array}{lllll}4.53999-4 & 7.48518-4 & 1.23410-3 & 2.03468-3 & 2.24867-3\end{array}$ $2.61259-3 \quad 3.03539-3 \quad 3.70744-3 \quad 5.53084-3 \quad 9.11882-3$ $1.50344-2 \quad 2.35786-2 \quad 2.47875-2 \quad 2.60584-2 \quad 2.70001-2$ $2.85011-2 \quad 3.43067-2 \quad 5.24752-2 \quad 5.65622-2 \quad 8.65169-2$ $1.22773-1 \quad 1.49956-1 \quad 1.83156-1 \quad 2.23708-1 \quad 2.73237-1$ $2.94518-1 \quad 2.97211-1 \quad 2.98491-1 \quad 3.01974-1 \quad 3.87742-1$ $4.97870-1 \quad 5.23397-1 \quad 6.08101-1 \quad 7.42735-1 \quad 9.07180-1$ $1.10803+0 \quad 1.35335+0 \quad 1.65299+0 \quad 2.01897+0 \quad 2.23130+0$ 
$2.34570+0 \quad 2.46597+0 \quad 3.01194+0 \quad 3.67879+0 \quad 4.49329+0$ $5.48811+0 \quad 6.70320+0 \quad 8.18730+0 \quad 1.00000+1 \quad 1.22140+1$ $1.49183+1$

$\mathrm{e} 202$

e212

$e 122$

$\mathrm{e} 222$

e132

e232

sd102 sd112 sd122
$2.00000-2 \quad 4.50000-2 \quad 7.00000-2 \quad 1.00000-1 \quad 1.50000-1$ $3.00000-1 \quad 4.00000-1 \quad 5.10000-1 \quad 6.00000-1 \quad 7.00000-1$ $1.00000+01.50000+02.00000+02.50000+0 \quad 3.00000+0$ $4.00000+0 \quad 5.00000+06.00000+07.00000+07.50000+0$ $8.00000+01.00000+11.40000+1$

4.13994-7 $1.12535-6 \quad 2.38237-6 \quad 5.04348-6 \quad 1.06770-5$ $2.26033-5 \quad 4.78512-5 \quad 1.01301-4 \quad 1.67017-4 \quad 2.75364-4$ 4.53999-4 7.48518-4 $1.23410-3 \quad 2.03468-3 \quad 2.24867-3$ $\begin{array}{llllll}2.61259-3 & 3.03539-3 & 3.70744-3 & 5.53084-3 & 9.11882-3\end{array}$ $\begin{array}{llllll}1.50344-2 & 2.35786-2 & 2.47875-2 & 2.60584-2 & 2.70001-2\end{array}$ $2.85011-2 \quad 3.43067-2 \quad 5.24752-2 \quad 5.65622-2 \quad 8.65169-2$ $\begin{array}{lllll}1.22773-1 & 1.49956-1 & 1.83156-1 & 2.23708-1 & 2.73237-1\end{array}$ 2.94518-1 $2.97211-1 \quad 2.98491-1 \quad 3.01974-1 \quad 3.87742-1$ 4.97870-1 $5.23397-1 \quad 6.08101-1 \quad 7.42735-1 \quad 9.07180-1$ $1.10803+0 \quad 1.35335+0 \quad 1.65299+0 \quad 2.01897+0.2 .23130+0$ $2.34570+0 \quad 2.46597+0 \quad 3.01194+0 \quad 3.67879+0 \quad 4.49329+0$ $5.48811+0 \quad 6.70320+0 \quad 8.18730+0 \quad 1.00000+1 \quad 1.22140+1$ $1.49183+1$

$2.00000-2 \quad 4.50000-2 \quad 7.00000-2 \quad 1.00000-1 \quad 1.50000-1$ $3.00000-1 \quad 4.00000-1 \quad 5.10000-1 \quad 6.00000-1 \quad 7.00000-1$ $1.00000+0 \quad 1.50000+0 \quad 2.00000+0 \quad 2.50000+0 \quad 3.00000+0$ $4.00000+0 \quad 5.00000+0 \quad 6.00000+0 \quad 7.00000+0 \quad 7.50000+0$ $8.00000+01.00000+1 \quad 1.40000+1$

4.13994-7 $1.12535-6 \quad 2.38237-6 \quad 5.04348-6 \quad 1.06770-5$ $2.26033-5$ 4.78512-5 $1.01301-4 \quad 1.67017-4 \quad 2.75364-4$ 4.53999-4 7.48518-4 $1.23410-3 \quad 2.03468-3 \quad 2.24867-3$ $\begin{array}{llllll}2.61259-3 & 3.03539-3 & 3.70744-3 & 5.53084-3 & 9.11882-3\end{array}$ $\begin{array}{lllll}1.50344-2 & 2.35786-2 & 2.47875-2 & 2.60584-2 & 2.70001-2\end{array}$ 2.85011-2 $3.43067-2 \quad 5.24752-2 \quad 5.65622-2 \quad 8.65169-2$ $\begin{array}{llllll}1.22773-1 & 1.49956-1 & 1.83156-1 & 2.23708-1 & 2.73237-1\end{array}$ 2.94518-1 2.97211-1 2.98491-1 $3.01974-1 \quad 3.87742-1$ 4.97870-1 5.23397-1 $6.08101-1 \quad 7.42735-1 \quad 9.07180-1$ $\begin{array}{lllll}1.10803+0 & 1.35335+0 & 1.65299+0 & 2.01897+0 & 2.23130+0\end{array}$ $2.34570+0 \quad 2.46597+0 \quad 3.01194+0 \quad 3.67879+0 \quad 4.49329+0$ $5.48811+0 \quad 6.70320+0 \quad 8.18730+0 \quad 1.00000+1 \quad 1.22140+1$ $1.49183+1$

$2.00000-2 \quad 4.50000-2 \quad 7.00000-2 \quad 1.00000-1 \quad 1.50000-1$ $3.00000-1 \quad 4.00000-1 \quad 5.10000-1 \quad 6.00000-1 \quad 7.00000-1$ $\begin{array}{lllll}1.00000+0 & 1.50000+0 & 2.00000+0 & 2.50000+0 & 3.00000+0\end{array}$ $4.00000+0 \quad 5.00000+0 \quad 6.00000+0 \quad 7.00000+0 \quad 7.50000+0$ $8.00000+01.00000+11.40000+1$

4.13994-7 $1.12535-6 \quad 2.38237-6 \quad 5.04348-6 \quad 1.06770-5$ 2.26033-5 $4.78512-5 \quad 1.01301-4 \quad 1.67017-4 \quad 2.75364-4$ $4.53999-4 \quad 7.48518-4 \quad 1.23410-3 \quad 2.03468-3 \quad 2.24867-3$ $\begin{array}{lllllll}2.61259-3 & 3.03539-3 & 3.70744-3 & 5.53084-3 & 9.11882-3\end{array}$ $\begin{array}{llllll}1.50344-2 & 2.35786-2 & 2.47875-2 & 2.60584-2 & 2.70001-2\end{array}$ $2.85011-2 \quad 3.43067-2 \quad 5.24752-2 \quad 5.65622-2 \quad 8.65169-2$ $\begin{array}{llllll}1.22773-1 & 1.49956-1 & 1.83156-1 & 2.23708-1 & 2.73237-1\end{array}$ 2.94518-1 $2.97211-1 \quad 2.98491-1 \quad 3.01974-1 \quad 3.87742-1$ 4.97870-1 $5.23397-1 \quad 6.08101-1 \quad 7.42735-1 \quad 9.07180-1$ $\begin{array}{llllll}1.10803+0 & 1.35335+0 & 1.65299+0 & 2.01897+0 & 2.23130+0\end{array}$ $2.34570+0 \quad 2.46597+0 \quad 3.01194+0 \quad 3.67879+0.4 .49329+0$ $5.48811+0 \quad 6.70320+0 \quad 8.18730+0 \quad 1.00000+1 \quad 1.22140+1$ $1.49183+1$

$2.00000-2 \quad 4.50000-2 \quad 7.00000-2 \quad 1.00000-1 \quad 1.50000-1$ $3.00000-1 \quad 4.00000-1 \quad 5.10000-1 \quad 6.00000-1 \quad 7.00000-1$ $1.00000+0 \quad 1.50000+0 \quad 2.00000+0 \quad 2.50000+0 \quad 3.00000+0$ $4.00000+0 \quad 5.00000+0 \quad 6.00000+0 \quad 7.00000+0 \quad 7.50000+0$ 400.0

$8.00000+0 \quad 1.00000+11.40000+1$

400.0

400.0 


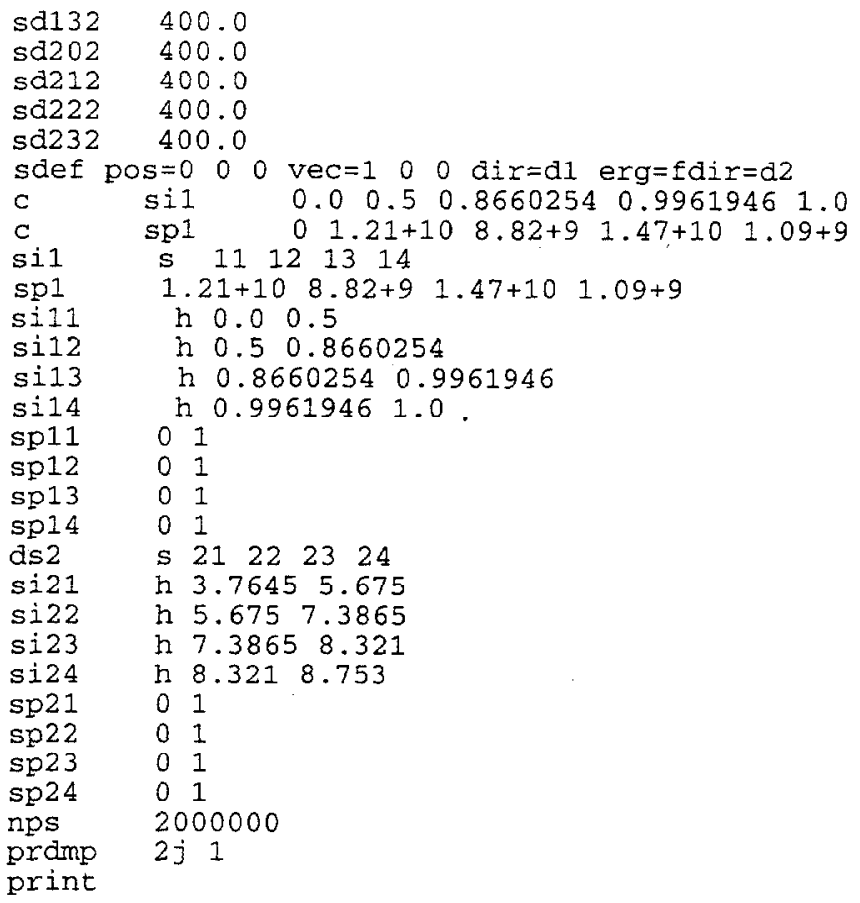


File for MCNP-4B surface-detector dose-rate calculations for a 6.38 -cm-radius bare Pu sphere in the PFNA heam at the center of the truck lane $(400,000$ histories)

message: outp=pfnall.o mctal=pfna11.m

menp file for PFNA project bare PU sphere calculation ( $r=6.38)$

$1 \quad-100: 128:-200: 251:-300: 305$

$100-128 \quad 200-251 \quad 300-305$

$\#(-400+101+224-227)$

$\#(-401+400+101+225-226)$

$\#(102-129+201-250+301-304)$

$\#(129-126+252-257+301-304)$

$\#(102-126+201-250+306-301)$

$\#(615-616+625-626+635-636)$

$3 \quad 10.10549-400+101+224-227 \#(503-504)$

$41 \quad 10.10549 \quad-401+400+101+225-226 \#(503-504)$

$5 \quad 0 \quad 102-127+223-228+301-304$

$\#(-400+224-227) \#(-401+400+225-226)$

$6 \quad 0 \quad 127-124+501-502+301-304$

$\#(-401+400+225-226)$

$7 \quad 0 \quad 124-126+254-255+301-304$

$\begin{array}{llllllll}8 & 5 & 8.734441-2 & 102 & -126+201 & -250+306 & -301 \\ 9 & 1 & 0.10549 & 102 & -124 & +222 & -229+301 & -304\end{array}$

$\#(127+501-502) \#(-127+223-228)$

$10 \quad 1 \quad 0.10549 \quad 106-109+212-236+302-303 \#(218-233)$

$1110.10549 \quad 109-110+211-237+302-303 \#(221-230)$

$12110.10549 \quad 110-111+210-238+302-303 \#(221-230)$

$13110.10549 \quad 111-112+209-239+302-303 \#(221-230)$

$14110.10549 \quad 112-113+208-240+302-303 \#(221-230)$

$15110.10549 \quad 113-114+207-241+302-303 \#(221-230)$

$16110.10549 \quad 114-115+206-242+302-303 \#(221-230)$

$171 \quad 1 \quad 0.10549 \quad 115-116+205-243+302-303 \quad \#(221-230)$

$18110.10549 \quad 116-117+204-244+302-303 \quad \#(221-230)$

$19 \quad 1 \quad 0.10549 \quad 117-118+203-245+302-303 \#(221-230)$

$20110.10549 \quad 118-119+202-249+302-303 \#(221-230)$

$21220.1187956 \quad 120-123+215-246+302-303 \#(221-230)$

$22 \quad 2 \quad 0.1187956103-109+202-249+302-303$

\# $(221-230)$

$\#(103-104+217-234)$

$\#(104-105+216-235)$

$\#(105-107+219-232)$

$\#(107-108+219-220)$ \# $(107-108+231-232)$

$23220.1187956 \quad 109-120+202-249+302-303$

$\#(221-230)$

$\#(109-110+211-237)$

$\#(110-111+210-238)$

$\#(111-112+209-239)$

$\#(112-113+208-240)$

$\#(113-114+207-241)$

$\#(114-115+206-242)$

$\#(115-116+205-243)$

$\#(116-117+204-244)$

$\#(117-118+203-245)$

$2433.284-2 \quad 121-125+201-250+301-304$

$\#(-124+214-247) \#(124+253-256)$

\# $(122+248) \#(122-213)$

$25 \quad 3 \quad 3.284-2 \quad 103-107+217-234+302-303$

\#(221 -230) \#(104-220) \#(104+231)

$26445.977931-2 \quad 104-108+216-220+302-303 \#(105-219)$

$2745.977931-2 \quad 104-108+231-235+302-303 \#(105+232)$

$28445.977931-2 \quad 102-124^{\circ}+201-222+301-304$

\#( $\left(\begin{array}{llllll}103 & -120 & 202 & -221 & 302 & -303\end{array}\right)$

\#( $\left(\begin{array}{llllll}120 & -123 & 215 & -221 & 302 & -303\end{array}\right)$

\# $(121-214)$

29

$45.977931-2102-124+229-250+301-304$

\#( $\left(\begin{array}{llllll}103 & -120 & 230 & -249 & 302 & -303\end{array}\right)$ 


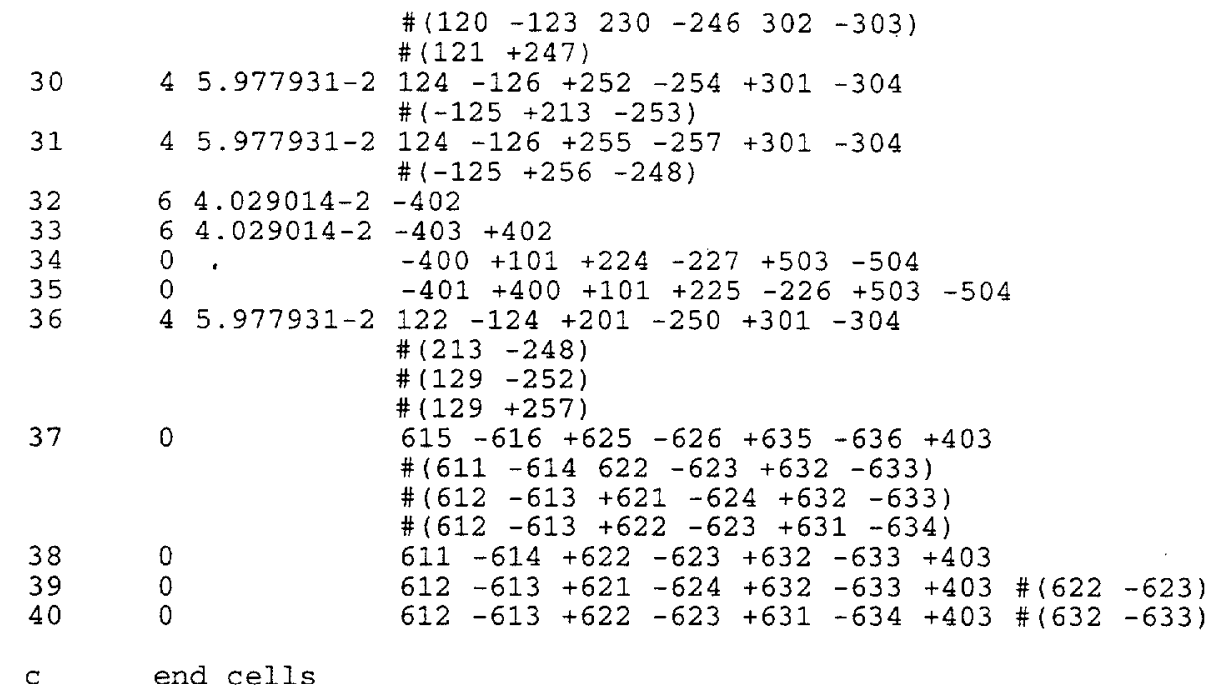

surfaces
px -100

100 px -100.0

101 px $\quad 10.3124$

102 px 20.7264

103 px 22.6314

$\begin{array}{lll}104 & \mathrm{px} & 27.7114 \\ 105 & \mathrm{px} & 28.9814\end{array}$

$106 \quad \mathrm{px} \quad 31.5214$

107 px $\quad 36.6014$

108 px 42.9514

109 px 49.3014

110 px 51.8414

111 px 54.3814

113 px 59.4614

114 px 62.0014

$115 \mathrm{px} \quad 64.5414$

116 px 67.0814

117 px 69.6214

118 px 72.1614

119 px 74.7014

120 px $\quad 76.3016$

121 px 78.2066

122 px 86.0806

123 px 112.649

124 px 114.554

125 px 119.888

126 px 121.158

127 px 50.5714

128 px 500.0

129 px 87.9856

200 py -500.0

201 py $\quad-74.7056$

202 py $\quad-72.8006$

203 py -47.0958

204 py $\quad-45.8258$

205 py -44.5558

206 py -43.2858

207 py -42.0158

208 py -40.7458

209 py $\quad-39.4758$

210 py -38.2058

211 py -36.9358

212 py -34.3958

213 py -38.6122

214 py -33.2782

215 py -31.3732

216 py -30.5858 


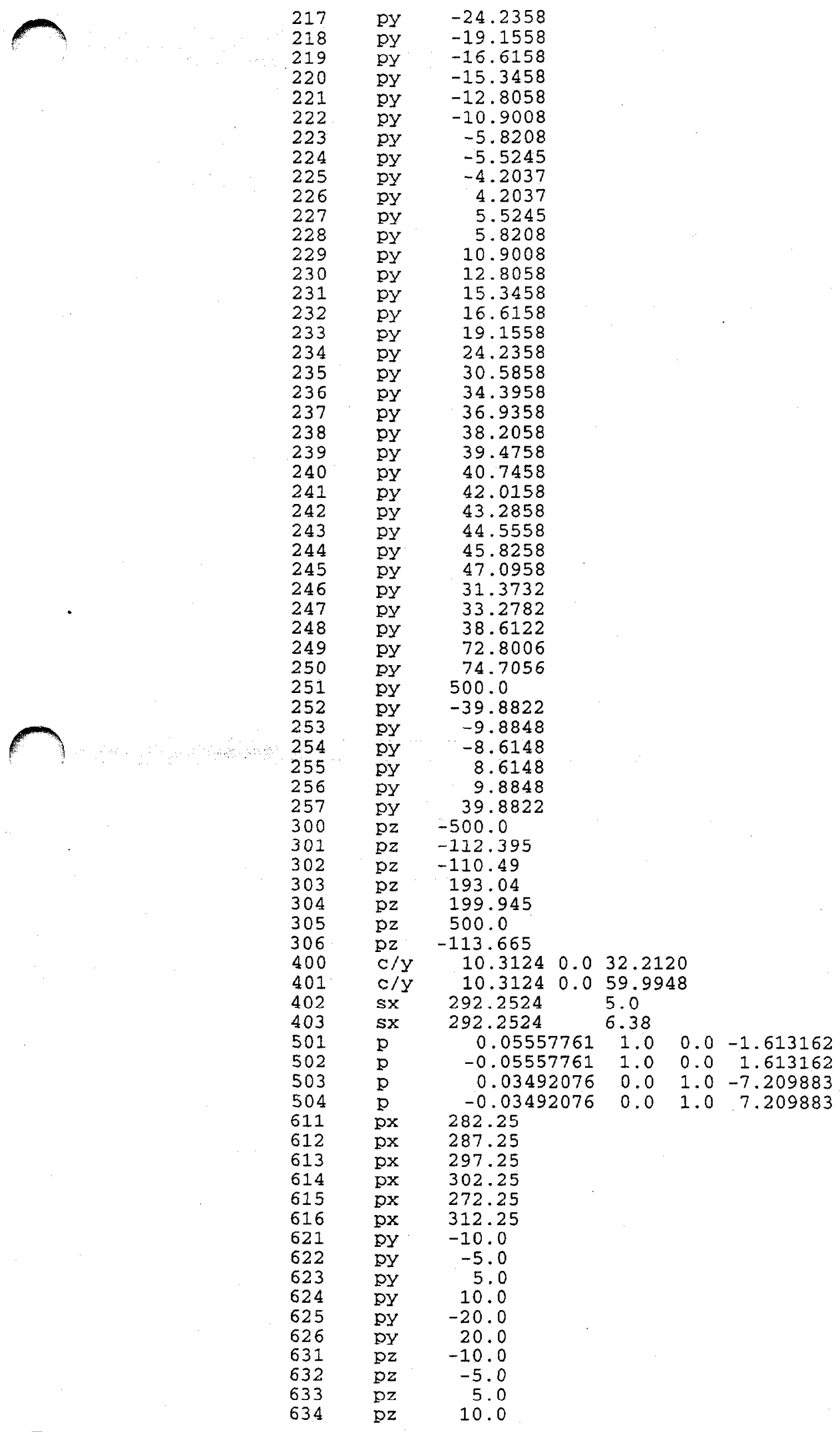




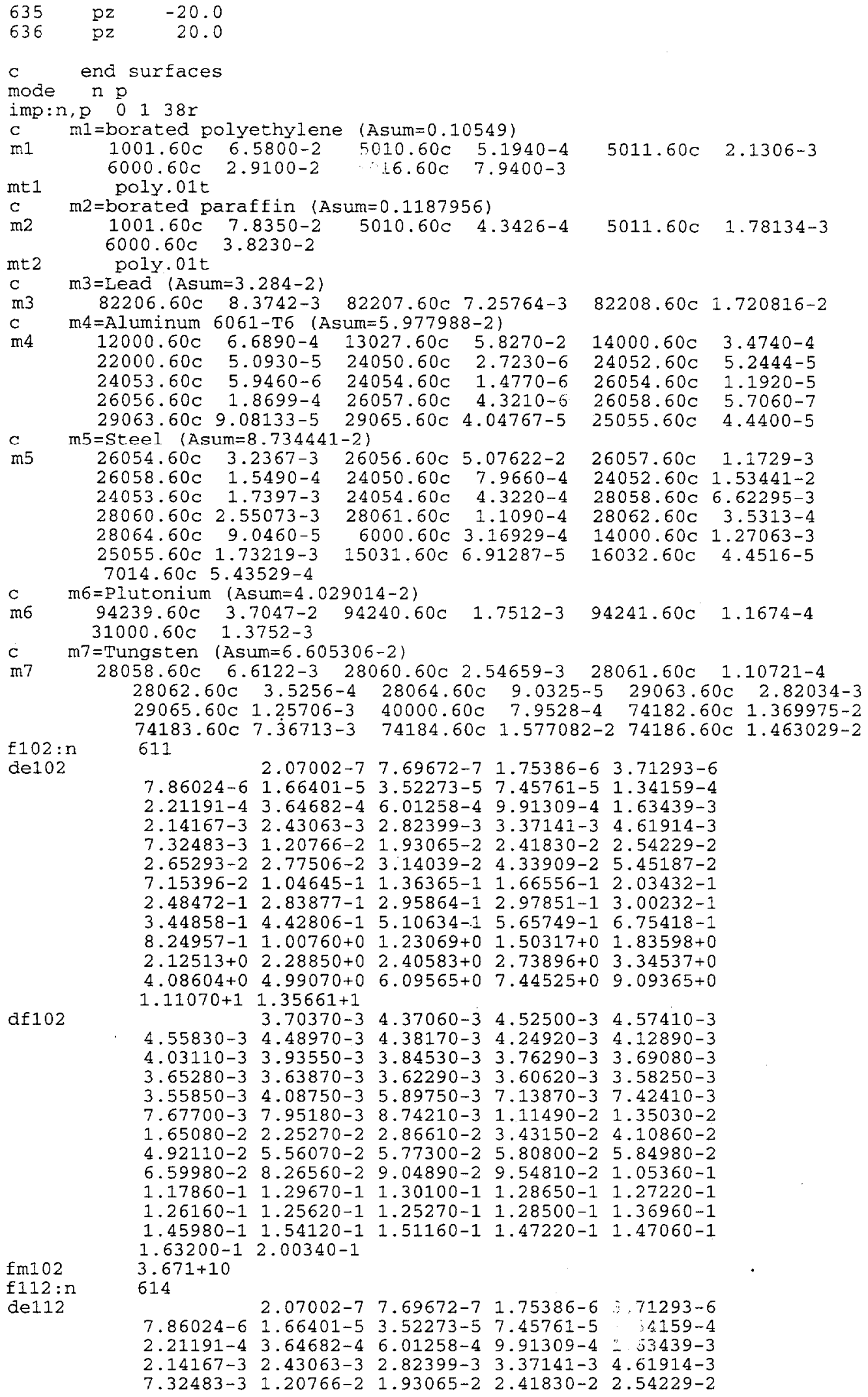


2.65293-2 2.77506-2 3.14039-2 $4.33909-2 \quad 5.45187-2$

7.15396-2 $1.04645-1 \quad 1.36365-1 \quad 1.66556-1 \quad 2.03432-1$

2.48172-1 2.83877-1 2.95864-1 2.97851-1 3.00232-1

3.44858-1 $4.42806-1 \quad 5.10634-1 \quad 5.65749-1 \quad 6.75418-1$

$8.24957-11.00760+01.23069+01.50317+0.1 .83598+0$

$2.12513+0 \quad 2.28850+0 \quad 2.40583+0 \quad 2.73896+0 \quad 3.34537+0$

$4.08604+0 \quad 4.99070+0 \quad 6.09565+0 \quad 7.44525+0 \quad 9.09365+0$

$1.11070+11.35661+1$

$\operatorname{df} 112$

$3.70370-3 \quad 4.37060-3 \quad 4.52500-3 \quad 4.57410-3$

$4.55830-3 \quad 4.48970-3 \quad 4.38170-3 \quad 4.24920-3 \quad 4.12890-3$

$4.03110-3 \quad 3.93550-3 \quad 3.84530-3 \quad 3.76290-3 \quad 3.69080-3$

$\begin{array}{lllll}3.65280-3 & 3.63870-3 & 3.62290-3 & 3.60620-3 & 3.58250-3\end{array}$

$3.55850-3 \quad 4.08750-3 \quad 5.89750-3 \quad 7.13870-3 \quad 7.42410-3$

$7.67700-3 \quad 7.95180-3 \quad 8.74210-3 \quad 1.11490-2 \quad 1.35030-2$

$1.65080-2 \quad 2.25270-2 \quad 2.86610-2 \quad 3.43150-2 \quad 4.10860-2$

$4.92110-2 \quad 5.56070-2 \quad 5.77300-2 \quad 5.80800-2 \quad 5.84980-2$

$\begin{array}{llllll}6.59980-2 & 8.26560-2 & 9.04890-2 & 9.54810-2 & 1.05360-1\end{array}$

$1.17860-1 \quad 1.29670-1 \quad 1.30100-1 \quad 1.28650-1 \quad 1.27220-1$

$1.26160-1 \quad 1.25620-1 \quad 1.25270-1 \quad 1.28500-1 \quad 1.36960-1$

$1.45980-1 \quad 1.54120-1 \quad 1.51160-1 \quad 1.47220-1 \quad 1.47060-1$

$1.63200-12.00340-1$

fm112 $\quad 3.671+10$

$\begin{array}{lllllll}\text { E122:n } & 624 & 2.07002-7 & 7.69672-7 & 1.75386-6 & 3.71293-6\end{array}$

$\begin{array}{llllll}7.86024-6 & 1.66401-5 & 3.52273-5 & 7.45761-5 & 1.34159-4\end{array}$

2.21191-4 $3.64682-4 \quad 6.01258-4 \quad 9.91309-4 \quad 1.63439-3$

$\begin{array}{llllll}2.14167-3 & 2.43063-3 & 2.82399-3 & 3.37141-3 & 4.61914-3\end{array}$

7. $32483-3 \quad 1.20766-2 \quad 1.93065-2 \quad 2.41830-2 \quad 2.54229-2$

2.65293-2 2.77506-2 $3.14039-2 \quad 4.33909-2 \quad 5.45187-2$

7.15396-2 $1.04645-1 \quad 1.36365-1 \quad 1.66556-1 \quad 2.03432-1$

$\begin{array}{llllll}2.48472-1 & 2.83877-1 & 2.95864-1 & 2.97851-1 & 3.00232-1\end{array}$

3.44858-1 $4.42806-1 \quad 5.10634-1.5 .65749-1 \quad 6.75418-1$ $8.24957-1 \quad 1.00760+0 \quad 1.23069+0 \quad 1.50317+0 \quad 1.83598+0$

$2.12513+0 \quad 2.28850+0 \quad 2.40583+0 \quad 2.73896+0 \quad 3.34537+0$

$4.08604+0 \quad 4.99070+0 \quad 6.09565+0 \quad 7.44525+0 \quad 9.09365+0$

$1.11070+1 \quad 1.35661+1$

df122 $3.70370-3 \quad 4.37060-3 \quad 4.52500-3 \quad 4.57410-3$ $4.55830-3 \quad 4.48970-3 \quad 4.38170-3 \quad 4.24920-3 \quad 4.12890-3$ $\begin{array}{llllll}4.03110-3 & 3.93550-3 & 3.84530-3 & 3.76290-3 & 3.69080-3\end{array}$ $\begin{array}{llllll}3.65280-3 & 3.63870-3 & 3.62290-3 & 3.60620-3 & 3.58250-3\end{array}$ $\begin{array}{llllll}3.55850-3 & 4.08750-3 & 5.89750-3 & 7.13870-3 & 7.42410-3\end{array}$ $\begin{array}{lllll}7.67700-3 & 7.95180-3 & 8.74210-3 & 1.11490-2 & 1.35030-2\end{array}$ $1.65080-2 \quad 2.25270-2 \quad 2.86610-2 \quad 3.43150-2 \quad 4.10860-2$ $4.92110-2 \quad 5.56070-2 \quad 5.77300-2 \quad 5.80800-2 \quad 5.84980-2$ 6.59980-2 $8.26560-2 \quad 9.04890-2 \quad 9.54810-2 \quad 1.05360-1$ $1.17860-1 \quad 1.29670-1 \quad 1.30100-1 \quad 1.28650-1 \quad 1.27220-1$ 1.26160-1 $1.25620-1 \quad 1.25270-1 \quad 1.28500-1 \quad 1.36960-1$ $1.45980-1$ 1.54120-1 $1.51160-1$ 1.47220-1 $1.47060-1$ $1.63200-12.00340-1$

$\mathrm{fm} 122 \quad 3.671+10$

$\pm 132: \mathrm{n} \quad 634$

de132

$\begin{array}{llllll}2.07002-7 & 7.69672-7 & 1.75386-6 & 3.71293-6\end{array}$ 7.86024-6 $1.66401-5 \quad 3.52273-5 \quad 7.45761-5 \quad 1.34159-4$ 2.21191-4 3.64682-4 6.01258-4 9.91309-4 $1.63439-3$ $\begin{array}{llllll}2.14167-3 & 2.43063-3 & 2.82399-3 & 3.37141-3 & 4.61914-3\end{array}$ 7.32483-3 $1.20766-2 \quad 1.93065-2 \quad 2.41830-2 \quad 2.54229-2$ 2.65293-2 2.77506-2 3.14039-2 4.33909-2 5.45187-2 $\begin{array}{llllll}7.15396-2 & 1.04645-1 & 1.36365-1 & 1.66556-1 & 2.03432-1\end{array}$ 2.48472-1 $2.83877-1 \quad 2.95864-1 \quad 2.97851-1 \quad 3.00232-1$ 3.44858-1 $4.42806-1 \quad 5.10634-1 \quad 5.65749-1 \quad 6.75418-1$ $8.24957-1 \quad 1.00760+0 \quad 1.23069+0 \quad 1.50317+0 \quad 1.83598+0$ $2.12513+0 \quad 2.28850+0 \quad 2.40583+0 \quad 2.73896+0 \quad 3.34537+0$ $4.08604+0 \quad 4.99070+0 \quad 6.09565+0 \quad 7.44525+0 \quad 9.09365+0$ $1.11070+11.35661+1$ $3.70370-3 \quad 4.37060-3 \quad 4.52500-3 \quad 4.57410-3$

$\operatorname{df132}$

$\begin{array}{llllll}4.55830-3 & 4.70370-3 & 4.37060-3 & 4.52500-3 & 4.57410-3 \\ 4 & 4.3970-3 & 4.38170-3 & 4.24920-3 & 4.12890-3\end{array}$ $4.03110-3 \quad 3.93550-3 \quad 3.84530-3 \quad 3.76290-3 \quad 3.69080-3$ $3.65280-3 \quad 3.63870-3 \quad 3.62290-3 \quad 3.60620-3 \quad 3.58250-3$ $\begin{array}{llllll}3.55850-3 & 4.08750-3 & 5.89750-3 & 7.13870-3 & 7.42410-3\end{array}$ $\begin{array}{lllll}7.67700-3 & 7.95180-3 & 8.74210-3 & 1.11490-2 & 1.35030-2\end{array}$

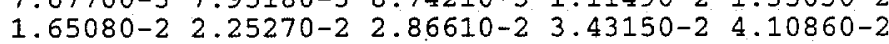
$4.92110-2 \quad 5.56070-2 \quad 5.77300-2 \quad 5.80800-2 \quad 5.84980-2$ 
fm. 132

f202:

de 202

df 202

fm 202

f212:p

de212

df212

fm 212

f $222: p$

de 222

df222

fm222

£232:p

de 232

df 232

fm 232

e102

e202

6.59980-2 $8.26560-2 \quad 9.04890-2 \quad 9.54810-2 \quad 1.05360-1$ $1.17860-1 \quad 1.29670-1 \quad 1.30100-1 \quad 1.28650-11.27220-1$ $1.26160-1 \quad 1.25620-1 \quad 1.25270-1 \quad 1.28500-1 \quad 1.36960-1$ $1.45980-1$ 1.54120-1 $1.51160-1 \quad 1.47220-1 \quad 1.47060-1$ $1.63200-12.00340-1$

$3.671+10$

611

1.50000-2 3.25000-2 $5.75000-28.50000-2$ $1.25000-1 \quad 2.25000-1 \quad 3.50000-1 \quad 4.55000-1 \quad 5.55000-1$ $6.50000-1 \quad 8.50000-1 \quad 1.25000+0 \quad 1.75000+0 \quad 2.25000+0$ $2.75000+0 \quad 3.50000+0 \quad 4.50000+0 \quad 5.50000+0 \quad 6.50000+0$ $7.25000+0 \quad 7.75000+0 \quad 9.00000+01.20000+1$ $\begin{array}{llll}2.14390-3 & 5.77600-4 & 2.71850-4 & 2.68170-4\end{array}$ $3.27670-4 \quad 5.66760-4 \quad 8.75940-4 \quad 1.08450-3 \quad 1.27970-3$ $1.44170-3 \quad 1.75630-3 \quad 2.31560-3 \quad 2.92700-3 \quad 3.46860-3$ $\begin{array}{llllll}3.95960-3 & 4.62210-3 & 5.41370-3 & 6.19090-3 & 6.92650-3\end{array}$ $7.17830-3 \quad 7.84680-3 \quad 8.77160-3 \quad 1.10200-2$ $3.671+10$ 614

1.50000-2 $3.25000-2 \quad 5.75000-2 \quad 8.50000-2$ $1.25000-1 \quad 2.25000-1 \quad 3.50000-1 \quad 4.55000-1 \quad 5.55000-1$ $6.50000-1 \quad 8.50000-1 \quad 1.25000+0 \quad 1.75000+0 \quad 2.25000+0$ $2.75000+0 \quad 3.50000+0 \quad 4.50000+0 \quad 5.50000+0 \quad 6.50000+0$ $7.25000+0 \quad 7.75000+0 \quad 9.00000+01.20000+1$

$2.14390-3 \quad 5.77600-4 \quad 2.71850-42.68170-4$

$3.27670-4 \quad 5.66760-4 \quad 8.75940-4 \quad 1.08450-3 \quad 1.27970-3$ $1.44170-3 \quad 1.75630-3 \quad 2.31560-3 \quad 2.92700-3 \quad 3.46860-3$ $3.95960-3 \quad 4.62210-3 \quad 5.41370-3 \quad 6.19090-3 \quad 6.92650-3$ $7.47830-3 \quad 7.84680-3 \quad 8.77160-3 \quad 1.10200-2$ $3.671+10$ 624

$1.50000-2 \quad 3.25000-2 \quad 5.75000-2 \quad 8.50000-2$ $\begin{array}{llllll}1.25000-1 & 2.25000-1 & 3.50000-1 & 4.55000-1 & 5.55000-1\end{array}$ $6.50000-18.50000-1 \quad 1.25000+0 \quad 1.75000+0 \quad 2.25000+0$ $2.75000+0 \quad 3.50000+0 \quad 4.50000+0 \quad 5.50000+0 \quad 6.50000+0$ $7.25000+07.75000+09.00000+01.20000+1$

$\begin{array}{llll}2.14390-3 & 5.77600-4 & 2.71850-4 & 2.68170-4\end{array}$ $\begin{array}{lllll}3.27670-4 & 5.66760-4 & 8.75940-4 & 1.08450-3 & 1.27970-3\end{array}$ $\begin{array}{llllll}1.44170-3 & 1.75630-3 & 2.31560-3 & 2.92700-3 & 3.46860-3\end{array}$ $\begin{array}{llllll}3.95960-3 & 4.62210-3 & 5.41370-3 & 6.19090-3 & 6.92650-3\end{array}$ $7.47830-3 \quad 7.84680-3 \quad 8.77160-31.10200-2$ $3.671+10$ 634

$1.50000-2 \quad 3.25000-2 \quad 5.75000-2 \quad 8.50000-2$ $\begin{array}{llllll}1.25000-1 & 2.25000-1 & 3.50000-1 & 4.55000-1 & 5.55000-1\end{array}$ $6.50000-1 \quad 8.50000-1 \quad 1.25000+0 \quad 1.75000+0 \quad 2.25000+0$ $2.75000+0 \quad 3.50000+0 \quad 4.50000+0 \quad 5.50000+0 \quad 6.50000+0$ $7.25000+0.75000+0 \quad 9.00000+0 \quad 1.20000+1$ $\begin{array}{llll}2.14390-3 & 5.77600-4 & 2.71850-4 & 2.68170-4\end{array}$ $3.27670-4 \quad 5.66760-4 \quad 8.75940-4 \quad 1.08450-3 \quad 1.27970-3$ $1.44170-3 \quad 1.75630-3 \quad 2.31560-3 \quad 2.92700-3 \quad 3.46860-3$ $\begin{array}{lllll}3.95960-3 & 4.62210-3 & 5.41370-3 & 6.19090-3 & 6.92650-3\end{array}$ $7.47830-3 \quad 7.84680-3 \quad 8.77160-3 \quad 1.10200-2$ $3.671+10$

4.13994-7 $1.12535-6 \quad 2.38237-6 \quad 5.04348-6 \quad 1.06770-5$ 2.26033-5 4.78512-5 $1.01301-4 \quad 1.67017-4 \quad 2.75364-4$ $\begin{array}{llllll}4.53999-4 & 7.48518-4 & 1.23410-3 & 2.03468-3 & 2.24867-3\end{array}$ $\begin{array}{llllll}2.61259-3 & 3.03539-3 & 3.70744-3 & 5.53084-3 & 9.11882-3\end{array}$ $1.50344-2$ 2.35786-2 $2.47875-2 \quad 2.60584-2 \quad 2.70001-2$ $2.85011-2 \quad 3.43067-2 \quad 5.24752-2 \quad 5.65622-2 \quad 8.65169-2$ 1.22773-1 $1.49956-1 \quad 1.83156-1 \quad 2.23708-1 \quad 2.73237-1$ 2.94518-1 2.97211-1 $2.98491-1 \quad 3.01974-1 \quad 3.87742-1$ $\begin{array}{lllll}4.97870-1 & 5.23397-1 & 6.08101-1 & 7.42735-1 & 9.07180-1\end{array}$ $\begin{array}{lllll}1.10803+0 & 1.35335+0 & 1.65299+0 & 2.01897+0 & 2.23130+0\end{array}$ $2.34570+0 \quad 2.46597+0 \quad 3.01194+0 \quad 3.67879+0 \quad 4.49329+0$ $\begin{array}{llllll}5.48811+0 & 6.70320+0 & 8.18730+0 & 1.00000+1 & 1.22140+1\end{array}$ $1.49183+1$

$2.00000-2 \quad 4.50000-2 \quad 7.00000-2 \quad 1.00000-1 \quad 1.50000-1$ $3.00000-1 \quad 4.00000-1 \quad 5.10000-1 \quad 6.00000-1 \quad 7.00000-1$ $1.00000+01.50000+0 \quad 2.00000+0 \quad 2.50000+0 \quad 3.00000+0$ 
$4.00000+0 \quad 5.00000+0 \quad 6.00000+07.00000+0 \quad 7.50000+0$ $8.00000+01.00000+11.40000+1$

e112

4.13994-7 $1.12535-6 \quad 2.38237-6 \quad 5.04348-6 \quad 1.06770-5$ $2.26033-5 \quad 4.78512-5 \quad 1.01301-4 \quad 1.67017-4 \quad 2.75364-4$ 4.53999-4 7.48518-4 $1.23410-3 \quad 2.03468-3 \quad 2.24867-3$ $2.61259-3 \quad 3.03539-3.3 .70744-3 \quad 5.53084-3 \quad 9.11882-3$ $\begin{array}{llllll}1.50344-2 & 2.35786-2 & 2.47875-2 & 2.60584-2 & 2.70001-2\end{array}$ $2.85011-2 \quad 3.43067-2 \quad 5.24752-2 \quad 5.65622-2 \quad 8.65169-2$ $1.22773-1$ 1.49956-1 $1.83156-1 \quad 2.23708-1 \quad 2.73237-1$ $2.94518-1 \quad 2.97211-1.2 .98491-1 \quad 3.01974-1 \quad 3.87742-1$ $4.97870-1 \quad 5.23397-1 \quad 6.08101-1 \quad 7.42735-1 \quad 9.07180-1$ $1.10803+0 \quad 1.35335+0 \quad 1.65299+0 \quad 2.01897+0 \quad 2.23130+0$ $2.34570+0 \quad 2.46597+0 \quad 3.01194+0 \quad 3.67879+0 \quad 4.49329+0$ $5.48811+0 \quad 6.70320+0 \quad 8.18730+0 \quad 1.00000+1 \quad 1.22140+1$ 1. $49183+1$

e212

el22

e222

e132

e232

sd102

sd112

sd122

sd132

sd202

sd212

sd222

$\operatorname{sd} 232$

sil
$2.00000-2 \quad 4.50000-2 \quad 7.00000-2 \quad 1.00000-1 \quad 1.50000-1$ $3.00000-1 \quad 4.00000-1 \quad 5.10000-1 \quad 6.00000-17.00000-1$ $1.00000+0 \quad 1.50000+0 \quad 2.00000+0 \quad 2.50000+0 \quad 3.00000+0$ $4.00000+0 \quad 5.00000+0 \quad 6.00000+0 \quad 7.00000+0 \quad 7.50000+0$ $8.00000+01.00000+1 \quad 1.40000+1$

4.13994-7 $1.12535-6 \quad 2.38237-6 \quad 5.04348-6 \quad 1.06770-5$ 2.26033-5 $4.78512-5 \quad 1.01301-4 \quad 1.67017-4 \quad 2.75364-4$ $4.53999-4$ 7.48518-4 $1.23410-32.03468-32.24867-3$ 2.61259-3 $3.03539-3 \quad 3.70744-3 \quad 5.53084-3 \quad 9.11882-3$ $\begin{array}{lllll}1.50344-2 & 2.35786-2 & 2.47875-2 & 2.60584-2 & 2.70001-2\end{array}$ $2.85011-2 \quad 3.43067-2 \quad 5.24752-2 \quad 5.65622-2 \quad 8.65169-2$ $1.22773-1 \quad 1.49956-1 \quad 1.83156-1 \quad 2.23708-1 \quad 2.73237-1$ $2.94518-1 \quad 2.97211-1 \quad 2.98491-1 \quad 3.01974-1 \quad 3.87742-1$ 4.97870-1 $5.23397-1 \quad 6.08101-1 \quad 7.42735-1 \quad 9.07180-1$ $1.10803+0 \quad 1.35335+0 \quad 1.65299+0 \quad 2.01897+0 \quad 2.23130+0$ $2.34570+0 \quad 2.46597+0 \quad 3.01194+0 \quad 3.67879+0 \quad 1.49329+0$ $5.48811+0 \quad 6.70320+0 \quad 8.18730+0 \quad 1.00000+1 \quad 1.22140+1$ $1.49183+1$

2.00000-2 4.50000-2 7.00000-2 $1.00000-1 \quad 1.50000-1$ $3.00000-1 \quad 4.00000-1 \quad 5.10000-1 \quad 6.00000-1 \quad 7.00000-1$ $1.00000+0 \quad 1.50000+0 \quad 2.00000+0 \quad 2.50000+0 \quad 3.00000+0$ $4.00000+0 \quad 5.00000+0 \quad 6.00000+07.00000+0 \quad 7.50000+0$ $8.00000+01.00000+11.40000+1$

4.13994-7 $1.12535-6 \quad 2.38237-6 \quad 5.04348-6 \quad 1.06770-5$ $2.26033-5 \quad 4.78512-5 \quad 1.01301-4 \quad 1.67017-4 \quad 2.75364-4$ $4.53999-4 \quad 7.48518-4 \quad 1.23410-3 \quad 2.03468-3 \quad 2.24867-3$ $2.61259-3 \quad 3.03539-3 \quad 3.70744-3 \quad 5.53084-3 \quad 9.11882-3$ $1.50344-2 \quad 2.35786-2 \quad 2.47875-2 \quad 2.60584-2 \% 2.70001-2$ $2.85011-2 \quad 3.43067-2 \quad 5.24752-2 \quad 5.65622-2 \quad 8.65169-2$ $1.22773-1$ 1.49956-1 $1.83156-1 \quad 2.23708-1 \quad 2.73237-1$ $2.94518-1 \quad 2.97211-1 \quad 2.98491-1 \quad 3.01974-1 \quad 3.87742-1$ 4.97870-1 $5.23397-1 \quad 6.08101-1 \quad 7.42735-1 \quad 9.07180-1$ $1.10803+0 \quad 1.35335+0 \quad 1.65299+0 \quad 2.01897+0 \quad 2.23130+0$ $2.34570+0 \quad 2.46597+0 \quad 3.01194+0 \quad 3.67879+0 \quad 4.49329+0$ $5.48811+0 \quad 6.70320+0 \quad 8.18730+0 \quad 1.00000+1 \quad 1.22140+1$ $1.49183+1$

2.00000-2 $4.50000-2 \quad 7.00000-2 \quad 1.00000-1 \quad 1.50000-1$ $3.00000-1 \quad 4.00000-1 \quad 5.10000-1 \quad 6.00000-1 \quad 7.00000-1$ $1.00000+0 \quad 1.50000+0 \quad 2.00000+0 \quad 2.50000+0 \quad 3.00000+0$ $4.00000+0 \quad 5.00000+0 \quad 6.00000+0 \quad 7.00000+0 \quad 7.50000+0$ 100.0

$8.00000+01.00000+1 \quad 1.40000+1$

100.0

100.0

100.0

100.0

100.0

100.0

0 o 0 vec $=1$ a 0 dir $=d 1$ erg $=f d i r=d 2$

$\begin{array}{lllll}0.0 & 0.5 & 0.8660254 & 0.9961946 & 1.0\end{array}$ 


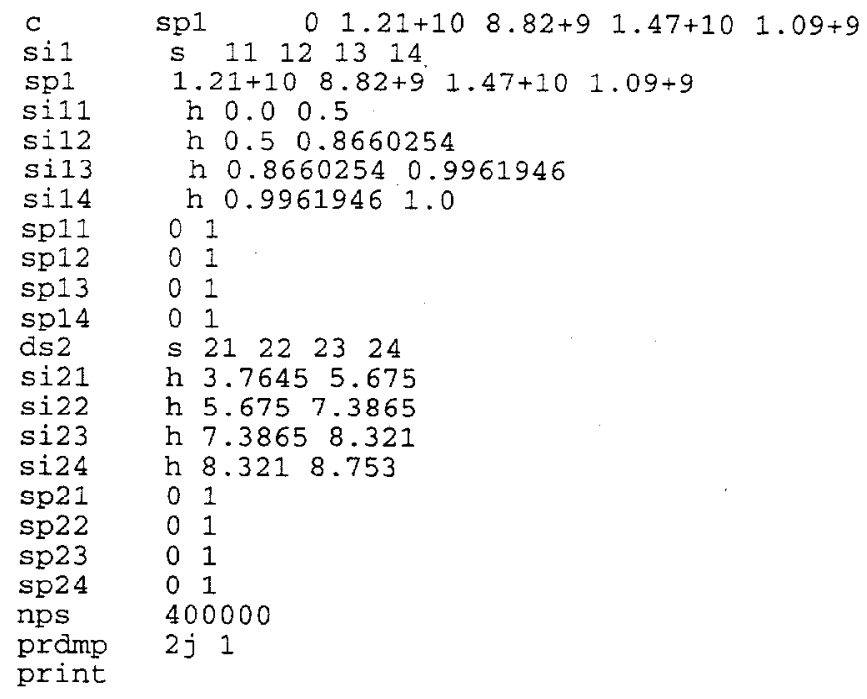


File for MCNP-4B surface-detector dose-rate calculations for a 6.38-cm-radius bare Pu sphere in the PFNA beam at the center of the truck lane $(16,000,000$ histories $)$

message: outp=pfna12. o metal=pfnal2.m monp file for PFNA project bare PU sphere calculation ( $x=6.38)$

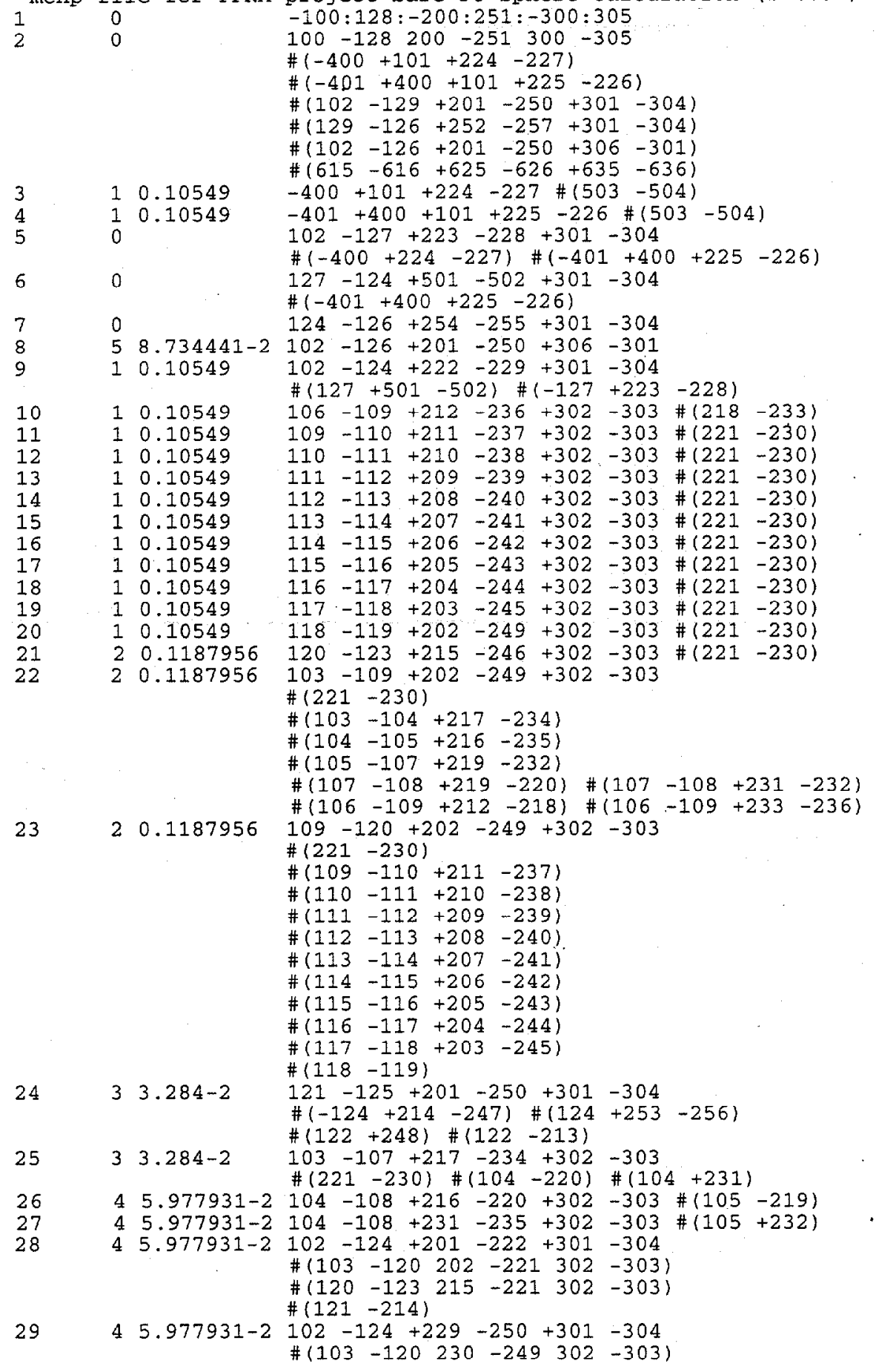




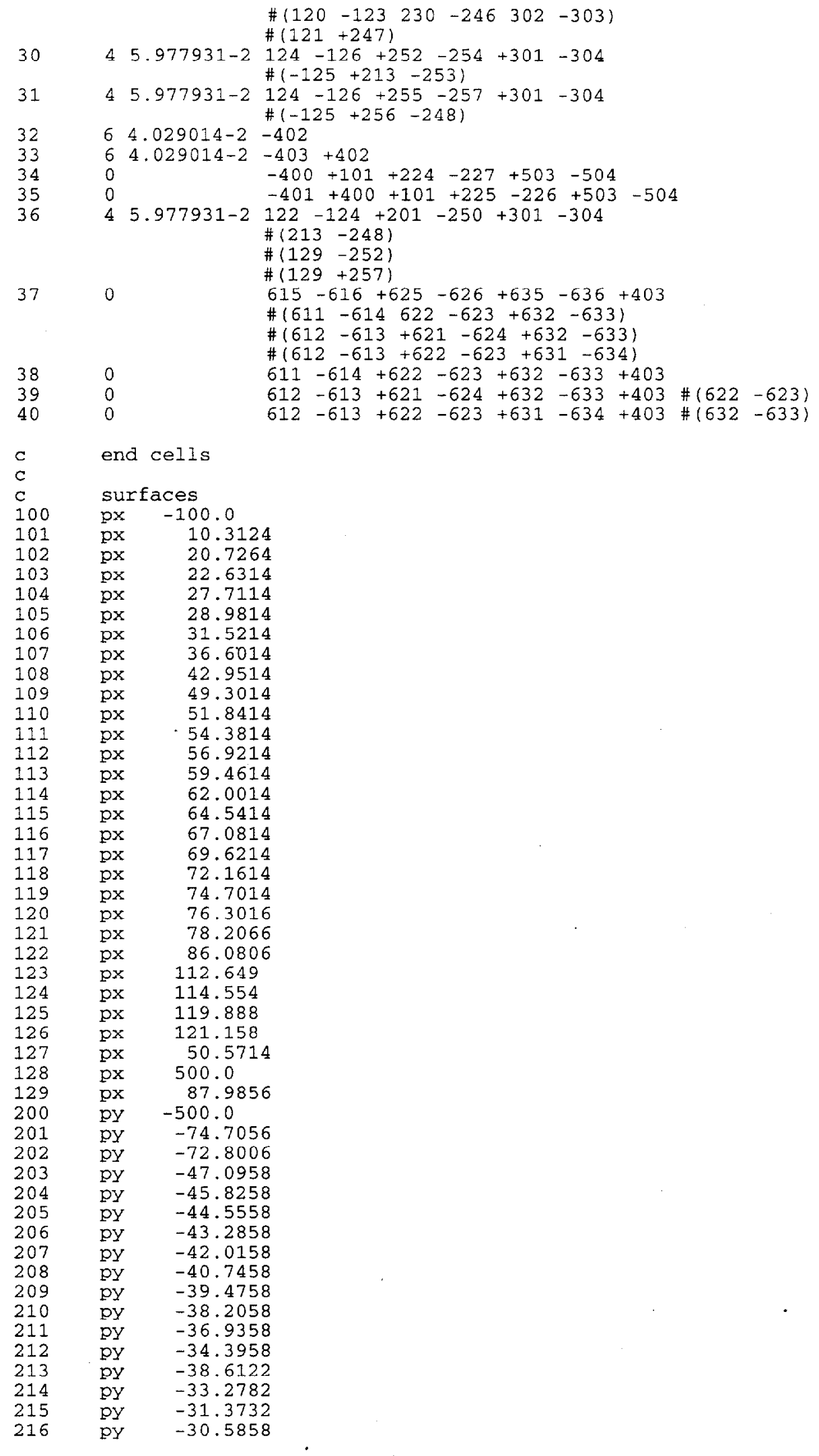




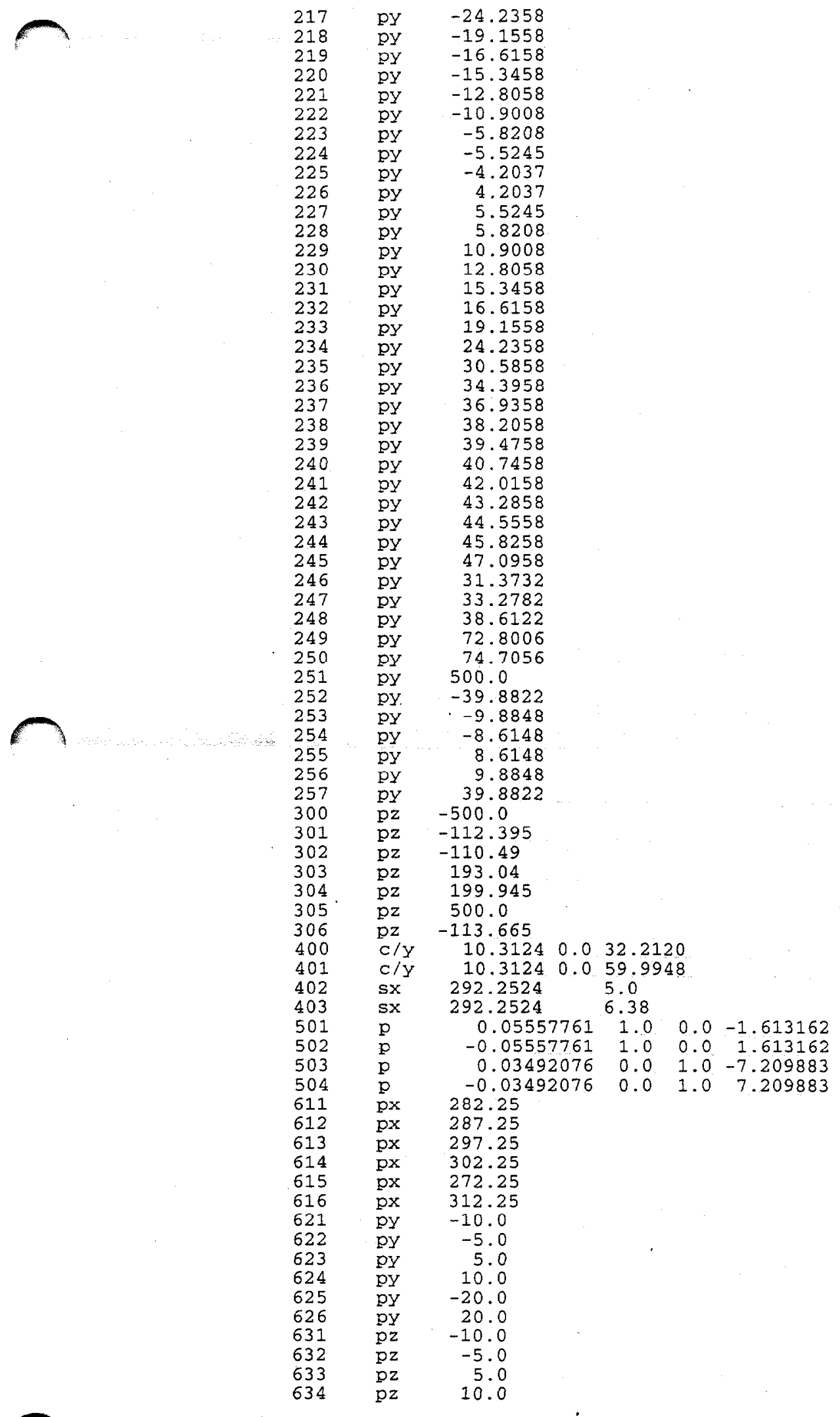




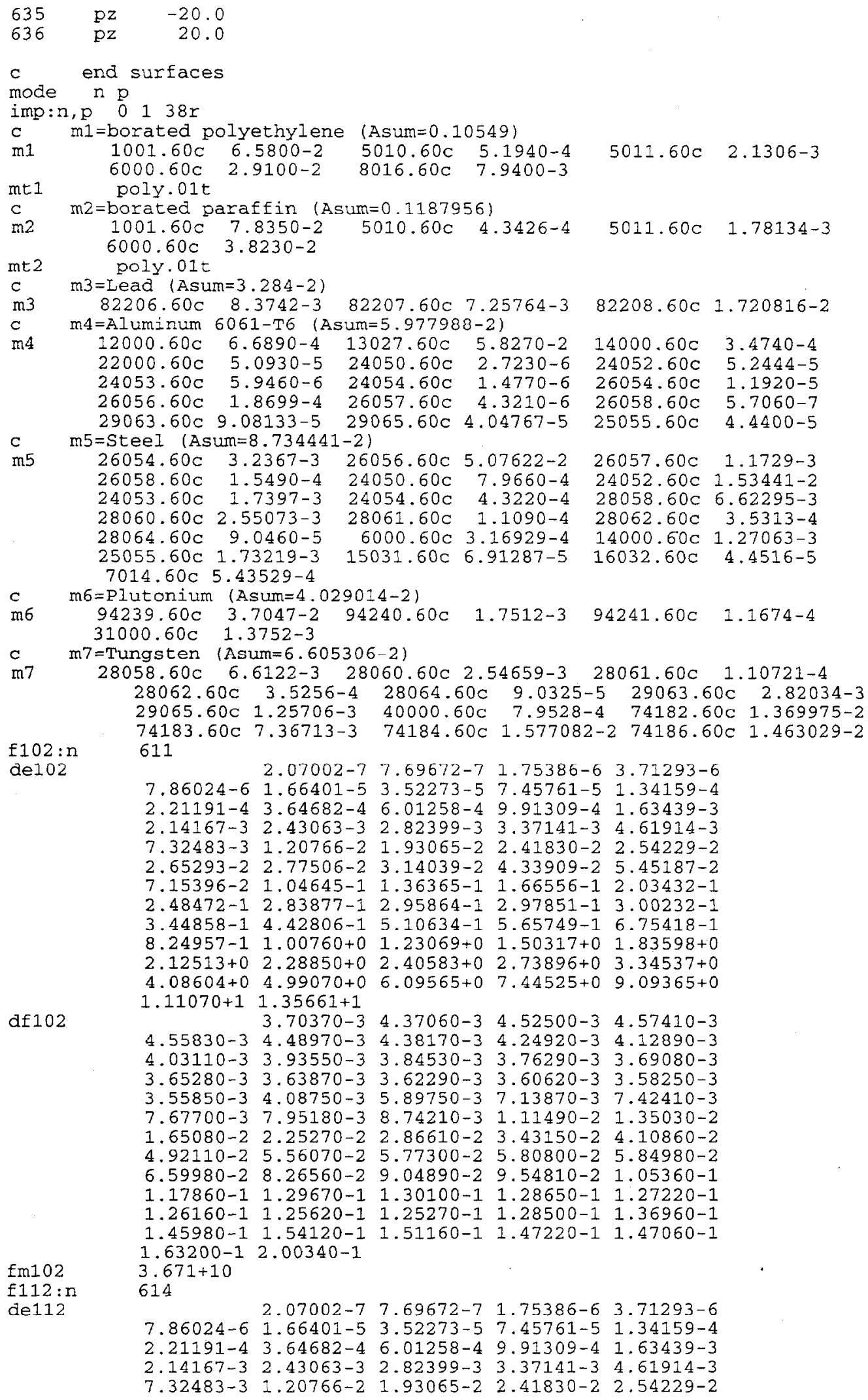


$2.65293-2 \quad 2.77506-2 \quad 3.14039-2 \quad 4.33909-2 \quad 5.45187-2$ $7.15396-2 \quad 1.04645-1 \quad 1.36365-1 \quad 1.66556-1 \quad 2.03432-1$ $2.48472-1 \quad 2.83877-1 \quad 2.95864-1 \quad 2.97851-1 \quad 3.00232-1$ 3.44858-1 4.42806-1 5.10634-1 5.65749-1 6.75418-1 $8.24957-1 \quad 1.00760+0 \quad 1.23069+0 \quad 1.50317+0 \quad 1.83598+0$ $2.12513+0 \quad 2.28850+0 \quad 2.40583+0 \quad 2.73896+0 \quad 3.34537+0$ $4.08604+0 \quad 4.99070+0 \quad 6.09565+0 \quad 7.44525+0 \quad 9.09365+0$ $1.11070+1 \quad 1.35661+1$

$3.70370-3 \quad 4.37060-3 \quad 4.52500-34.57410-3$ $4.55830-3 \quad 4.48970-3 \quad 4.38170-3 \quad 4.24920-3 \quad 4.12890-3$ $4.03110-3 \quad 3.93550-3 \quad 3.84530-3 \quad 3.76290-3 \quad 3.69080-3$ $3.65280-3 \quad 3.63870-3 \quad 3.62290-3 \quad 3.60620-3 \quad 3.58250-3$

$3.55850-3 \quad 4.08750-3 \quad 5.89750-3 \quad 7.13870-3 \quad 7.42410-3$

$7.67700-3 \quad 7.95180-3 \quad 8.74210-3 \quad 1.11490-2 \quad 1.35030-2$

$1.65080-2 \quad 2.25270-2 \quad 2.86610-2 \quad 3.43150-2 \quad 4.10860-2$

$4.92110-2 \quad 5.56070-2 \quad 5.77300-2 \quad 5.80800-2 \quad 5.84980-2$ $6.59980-2 \quad 8.26560-2 \quad 9.04890-2 \quad 9.54810-2 \quad 1.05360-1$ 1.17860-1 $1.29670-1 \quad 1.30100-1 \quad 1.28650-1 \quad 1.27220-1$ $1.26160-1 \quad 1.25620-1 \quad 1.25270-1 \quad 1.28500-1 \quad 1.36960-1$ $1.45980-1$ 1.54120-1 $1.51160-1 \quad 1.47220-1 \quad 1.47060-1$ $1.63200-12.00340-1$

$\mathrm{fm} 112$

$\mathrm{f} 122: \mathrm{n}$ de 122

$\operatorname{df} 122$

$\operatorname{fm} 122$ $\mathrm{f} 132: \mathrm{n}$ de 132

$\operatorname{df} 132$ $.671+10$

624

$2.07002-7 \quad 7.69672-7 \quad 1.75386-6 \quad 3.71293-6$ $7.86024-6 \quad 1.66401-5 \quad 3.52273-5 \quad 7.45761-5 \quad 1.34159-4$ $2.21191-4 \quad 3.64682-4 \quad 6.01258-4 \quad 9.91309-4 \quad 1.63439-3$ $2.14167-3 \quad 2.43063-3 \quad 2.82399-3 \quad 3.37141-3 \quad 4.61914-3$ $7.32483-3 \quad 1.20766-2 \quad 1.93065-2 \quad 2.41830-2 \quad 2.54229-2$ $2.65293-2 \quad 2.77506-2 \quad 3.14039-2 \quad 4.33909-2 \quad 5.45187-2$ $7.15396-2 \quad 1.04645-1 \quad 1.36365-1 \quad 1.66556-1 \quad 2.03432-1$ $2.48472-1 \quad 2.83877-1 \quad 2.95864-1 \quad 2.97851-1 \quad 3.00232-1$ $3.44858-1 \quad 4.42806-1 \quad 5.10634-1 \quad 5.65749-1 \quad 6.75418-1$ $8.24957-1 \quad 1.00760+0 \quad 1.23069+0 \quad 1.50317+0 \quad 1.83598+0$ $2.12513+0 \quad 2.28850+0 \quad 2.40583+0 \quad 2.73896+0 \quad 3.34537+0$ $4.08604+0 \quad 4.99070+0 \quad 6.09565+0 \quad 7.44525+0 \quad 9.09365+0$ $1.11070+1 \quad 1.35661+1$

$3.70370-3 \quad 4.37060-3 \quad 4.52500-3 \quad 4.57410-3$ $\begin{array}{llllll}4.55830-3 & 4.48970-3 & 4.38170-3 & 4.24920-3 & 4.12890-3\end{array}$ 4.03110-3 3.93550-3 3.84530-3 3.76290-3.3.69080-3 $\begin{array}{llllll}3.65280-3 & 3.63870-3 & 3.62290-3 & 3.60620-3 & 3.58250-3\end{array}$ $3.55850-3 \quad 4.08750-3 \quad 5.89750-3 \quad 7.13870-3 \quad 7.42410-3$ $7.67700-3 \quad 7.95180-3 \quad 8.74210-3 \quad 1.11490-2 \quad 1.35030-2$ $1.65080-2 \quad 2.25270-2 \quad 2.86610-2 \quad 3.43150-2 \quad 4.10860-2$ $4.92110-2 \quad 5.56070-2 \quad 5.77300-2 \quad 5.80800-2 \quad 5.84980-2$ $6.59980-2 \quad 8.26560-2 \quad 9.04890-2 \quad 9.54810-2 \quad 1.05360-1$ $1.17860-1 \quad 1.29670-1 \quad 1.30100-1 \quad 1.28650-11.27220-1$ $1.26160-1$ 1.25620-1 $1.25270-1$ 1.28500-1 $1.36960-1$ $1.45980-1$ 1.54120-1 1.51160-1 1.47220-1 1.47060-1 $1.63200-12.00340-1$

$3.671+10$ 634

$2.07002-7 \quad 7.69672-7 \quad 1.75386-6 \quad 3.71293-6$ $7.86024-6 \quad 1.66401-5 \quad 3.52273-5 \quad 7.45761-5 \quad 1.34159-4$ $2.21191-4 \quad 3.64682-4 \quad 6.01258-4 \quad 9.91309-4 \quad 1.63439-3$ $2.14167-3 \quad 2.43063-3 \quad 2.82399-3 \quad 3.37141-3 \quad 4.61914-3$ $7.32483-3 \quad 1.20766-2 \quad 1.93065-2 \quad 2.41830-2 \quad 2.54229-2$ $2.65293-2 \quad 2.77506-2 \quad 3.14039-2 \quad 4.33909-2 \quad 5.45187-2$ $7.15396-2$ 1.04645-1 $1.36365-1 \quad 1.66556-1 \quad 2.03432-1$ $2.48472-1 \quad 2.83877-1 \quad 2.95864-1 \quad 2.97851-1 \quad 3.00232-1$ 3.44858-1 4.42806-1 $5.10634-1 \quad 5.65749-1 \quad 6.75418-1$ 8. 24957-1 $1.00760+0 \quad 1.23069+0 \quad 1.50317+01.83598+0$ $2.12513+0 \quad 2.28850+0 \quad 2.40583+0 \quad 2.73896+0 \quad 3.34537+0$ $4.08604+0 \quad 4.99070+0 \quad 6.09565+0 \quad 7.44525+0 \quad 9.09365+0$ $1.11070+11.35661+1$ $3.70370-3 \quad 4.37060-3 \quad 4.52500-3 \quad 4.57410-3$ $4.55830-3 \quad 4.48970-3 \quad 4.38170-3 \quad 4.24920-3 \quad 4.12890-3$ $4.03110-3 \quad 3.93550-3 \quad 3.84530-3 \quad 3.76290-3 \quad 3.69080-3$ $3.65280-3 \quad 3.63870-3 \quad 3.62290-3 \quad 3.60620-3 \quad 3.58250-3$ $3.55850-3 \quad 4.08750-3 \quad 5.89750-3 \quad 7.13870-3 \quad 7.42410-3$ $\begin{array}{lllll}7.67700-3 & 7.95180-3 & 8.74210-3 & 1.11490-2 & 1.35030-2\end{array}$

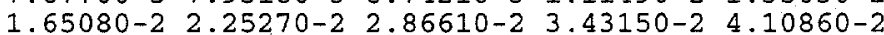

$4: 92110-2 \quad 5.56070-2 \quad 5.77300-2 \quad 5.80800-2 \quad 5.84980-2$ 
$\begin{array}{llllll}6.59980-2 & 8.26560-2 & 9.04890-2 & 9.54810-2 & 1.05360-1\end{array}$ $1.17860-1 \quad 1.29670-1 \quad 1.30100-1 \quad 1.28650-1 \quad 1.27220-1$

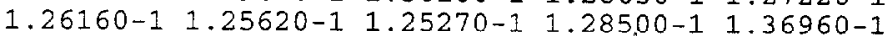
$1.45980-1 \quad 1.54120-1 \quad 1.51160-1 \quad 1.47220-1 \quad 1.47060-1$

fm 132

f202:p de 202

df 202

$\operatorname{fm} 202$

$\mathrm{f} 212: \mathrm{p}$ de212

$\operatorname{de} 212$

$\mathrm{fm} 212$

$f 222: p$

de222

df 222

fm222

f232:p

de2 32

df232

$\operatorname{fm} 232$

e102

e202

$1.63200-12.00340-1$

$3.671+10$

611

$1.50000-2 \quad 3.25000-2 \quad 5.75000-2 \quad 8.50000-2$ $\begin{array}{llllll}1.25000-1 & 2.25000-1 & 3.50000-1 & 4.55000-1 & 5.55000-1\end{array}$ $6.50000-1 \quad 8.50000-1 \quad 1.25000+0 \quad 1.75000+0 \quad 2.25000+0$ $2.75000+0 \quad 3.50000+0 \quad 4.50000+0 \quad 5.50000+0 \quad 6.50000+0$ $7.25000+0 \quad 7.75000+0 \quad 9.00000+0 \quad 1.20000+1$ $\begin{array}{llll}2.14390-3 & 5.77600-4 & 2.71850-4 & 2.68170-4\end{array}$ $3.27670-4 \quad 5.66760-4 \quad 8.75940-4 \quad 1.08450-3 \quad 1.27970-3$ $\begin{array}{lllll}1.44170-3 & 1.75630-3 & 2.31560-3 & 2.92700-3 & 3.46860-3\end{array}$ $\begin{array}{llllll}3.95960-3 & 4.62210-3 & 5.41370-3 & 6.19090-3 & 6.92650-3\end{array}$ $7.47830-3 \quad 7.84680-3 \quad 8.77160-3 \quad 1.10200-2$ $3.671+10$

614

$1.50000-2 \quad 3.25000-2 \quad 5.75000-2 \quad 8.50000-2$ $1.25000-1 \quad 2.25000-1 \quad 3.50000-1 \quad 4.55000-1 \quad 5.55000-1$ $6.50000-18.50000-1 \quad 1.25000+01.75000+02.25000+0$ $2.75000+0 \quad 3.50000+0 \quad 4.50000+0 \quad 5.50000+0 \quad 6.50000+0$ $7.25000+0 \quad 7.75000+09.00000+01.20000+1$

$\begin{array}{lllll}2.14390-3 & 5.77600-4 & 2.71850-4 & 2.68170-4\end{array}$ $3.27670-4 \quad 5.66760-4 \quad 8.75940-4 \quad 1.08450-3 \quad 1.27970-3$ $\begin{array}{lllll}1.44170-3 & 1.75630-3 & 2.31560-3 & 2.92700-3 & 3.46860-3\end{array}$ $\begin{array}{lllll}3.95960-3 & 4.62210-3 & 5.41370-3 & 6.19090-3 & 6.92650-3\end{array}$ $\begin{array}{llll}7.47830-3 & 7.84680-3 & 8.77160-3 & 1.10200-2\end{array}$ $3.671+10$ 624

$1.50000-2 \quad 3.25000-2 \quad 5.75000-2 \quad 8.50000-2$ $\begin{array}{lllll}1.25000-1 & 2.25000-1 & 3.50000-1 & 4.55000-1 & 5.55000-1\end{array}$ $\begin{array}{llllll}6.50000-1 & 8.50000-1 & 1.25000+0 & 1.75000+0 & 2.25000+0\end{array}$ $2.75000+0 \quad 3.50000+0 \quad 4.50000+0 \quad 5.50000+0 \quad 6.50000+0$ $7.25000+0 \quad 7.75000+0 \quad 9.00000+01.20000+1$

$2.14390-3 \quad 5.77600-4 \quad 2.71850-4 \quad 2.68170-4$ $3.27670-4 \quad 5.66760-4 \quad 8.75940-4 \quad 1.08450-3.1 .27970-3$ $1.44170-3 \quad 1.75630-3 \quad 2.31560-3 \quad 2.92700-3 \quad 3.46860-3$ $3.95960-3 \quad 4.62210-3 \quad 5.41370-3 \quad 6.19090-3 \quad 6.92650-3$ $7.47830-3 \quad 7.84680-3 \quad 8.77160-3 \quad 1.10200-2$ $3.671+10$

634

$1.50000-2 \quad 3.25000-2 \quad 5.75000-2 \quad 8.50000-2$ $\begin{array}{llllll}1.25000-1 & 2.25000-1 & 3.50000-1 & 4.55000-1 & 5.55000-1\end{array}$ $6.50000-18.50000-1 \quad 1.25000+0 \quad 1.75000+02.25000+0$ $2.75000+0 \quad 3.50000+0 \quad 4.50000+0 \quad 5.50000+0 \quad 6.50000+0$ $7.25000+0 \quad 7.75000+0 \quad 9.00000+0 \quad 1.20000+1$ $\begin{array}{llll}2.14390-3 & 5.77600-4 & 2.71850-4 & 2.68170-4\end{array}$ $3.27670-4 \quad 5.66760-4 \quad 8.75940-4 \quad 1.08450-3 \quad 1.27970-3$ $1.44170-3 \quad 1.75630-3 \quad 2.31560-3 \quad 2.92700-3 \quad 3.46860-3$ $\begin{array}{lllll}3.95960-3 & 4.62210-3 & 5.41370-3 & 6.19090-3 & 6.92650-3\end{array}$ $\begin{array}{llll}7.47830-3 & 7.84680-3 & 8.77160-3 & 1.10200-2\end{array}$

$3.671+10$

4.13994-7 $1.12535-6 \quad 2.38237-6 \quad 5.04348-6 \quad 1.06770-5$ $2.26033-5 \quad 4.78512-5 \quad 1.01301-4 \quad 1.67017-4 \quad 2.75364-4$ $4.53999-4 \quad 7.48518-4 \quad 1.23410-3 \quad 2.03468-3 \quad 2.24867-3$ $\begin{array}{llllll}2.61259-3 & 3.03539-3 & 3.70744-3 & 5.53084-3 & 9.11882-3\end{array}$ $\begin{array}{lllll}1.50344-2 & 2.35786-2 & 2.47875-2 & 2.60584-2 & 2.70001-2\end{array}$ $2.85011-2 \quad 3.43067-2 \quad 5.24752-2 \quad 5.65622-2 \quad 8.65169-2$ 1.22773-1 1.49956-1 $1.83156-1 \quad 2.23708-1 \quad 2.73237-1$ $\begin{array}{lllll}2.94513-1 & 2.97211-1 & 2.98491-1 & 3.01974-1 & 3.87742-1\end{array}$ 4.97870-1 $5.23397-1 \quad 6.08101-1 \quad 7.42735-1 \quad 9.07180-1$ $\begin{array}{lllll}1.10803+0 & 1.35335+0 & 1.65299+0 & 2.01897+0 & 2.23130+0\end{array}$ $2.34570+0 \quad 2.46597+0 \quad 3.01194+0 \quad 3.67879+0 \quad 4.49329+0$ $5.48811+0 \quad 6.70320+0 \quad 8.18730+0 \quad 1.00000+1 \quad 1.22140+1$ $1.49183+1$

$2.00000-2 \quad 4.50000-2 \quad 7.00000-2 \quad 1.00000-1 \quad 1.50000-1$ $3.00000-14.00000-15.10000-16.00000-17.00000-1$ $1.00000+01.50000+02.00000+02.50000+0 \quad 3.00000+0$ 
$4.00000+0 \quad 5.00000+0 \quad 6.00000+0 \quad 7.00000+07.50000+0$ $8.00000+0 \quad 1.00000+1 \quad 1.40000+1$

e112

4.13994-7 $1.12535-6 \quad 2.38237-6 \quad 5.04348-6 \quad 1.06770-5$ $2.26033-5$ 4.78512-5 $1.01301-4 \quad 1.67017-4.2 .75364-4$ $4.53999-4 \quad 7.48518-4 \quad 1.23410-3 \quad 2.03468-3 \quad 2.24867-3$ $2.61259-3 \quad 3.03539-3 \quad 3.70744-3 \quad 5.53084-3 \quad 9.11882-3$ $1.50344-2$ 2.35786-2 2.47875-2 2.60584-2 2.70001-2 $2.85011-2 \quad 3.43067-2 \quad 5.24752-2 \quad 5.65622-2 \quad 8.65169-2$ 1.22773-1 $1.49956-1 \quad 1.83156-1 \quad 2.23708-1 \quad 2.73237-1$ 2.94518-1 2.97211-1 $2.98491-1 \quad 3.01974-1 \quad 3.87742-1$ $\begin{array}{lllll}4.97870-1 & 5.23397-1 & 6.08101-1 & 7.42735-1 & 9.07180-1\end{array}$ $1.10803+0 \quad 1.35335+0 \quad 1.65299+0 \quad 2.01897+0 \quad 2.23130+0$ $2.34570+0 \quad 2.46597+0 \quad 3.01194+0 \quad 3.67879+0.4 .49329+0$ $5.48811+0 \quad 6.70320+0 \quad 8.18730+0 \quad 1.00000+1 \quad 1.22140+1$ $1.49183+1$

e212

$2.00000-2 \quad 4.50000-2 \quad 7.00000-2 \quad 1.00000-1 \quad 1.50000-1$ $3.00000-1 \quad 4.00000-1 \quad 5.10000-1 \quad 6.00000-1 \quad 7.00000-1$ $1.00000+0 \quad 1.50000+0 \quad 2.00000+0 \quad 2.50000+0 \quad 3.00000+0$ $4.00000+0 \quad 5.00000+0 \quad 6.00000+0 \quad 7.00000+0 \quad 7.50000+0$ $8.00000+01.00000+11.40000+1$

e122

$\mathrm{e} 222$

4.13994-7 $1.12535-6 \quad 2.38237-6 \quad 5.04348-6 \quad 1.06770-5$ $2.26033-5.4 .78512-5 \quad 1.01301-4 \quad 1.67017-4 \quad 2.75364-4$ 4.53999-4 7.48518-4 $1.23410-3 \quad 2.03468-3 \quad 2.24867-3$ $\begin{array}{llllll}2.61259-3 & 3.03539-3 & 3.70744-3 & 5.53084-3 & 9.11882-3\end{array}$ $\begin{array}{lllll}1.50344-2 & 2.35786-2 & 2.47875-2 & 2.60584-2 & 2.70001-2\end{array}$ 2.85011-2 $3.43067-2 \quad 5.24752-2 \quad 5.65622-2 \quad 8.65169-2$ 1.22773-1 1.49956-1 $1.83156-1 \quad 2.23708-1 \quad 2.73237-1$ 2.94518-1 $2.97211-1 \quad 2.98491-1 \quad 3.01974-1 \quad 3.87742-1$ 4.97870-1 5.23397-1 6.08101-1 7.42735-1 9.07180-1 $1.10803+0 \quad 1.35335+0 \quad 1.65299+0 \quad 2.01897+0 \quad 2.23130+0$ $2.34570+0 \quad 2.46597+0 \quad 3.01194+0 \quad 3.67879+0 \quad 4.49329+0$ $5.48811+0 \quad 6.70320+0 \quad 8.18730+0 \quad 1.00000+1 \quad 1.22140+1$ $1.49183+1$

2.00000-2 4.50000-2 7.00000-2 $1.00000-1 \quad 1.50000-1$ $3.00000-1 \quad 4.00000-1 \quad 5.10000-1 \quad 6.00000-1 \quad 7.00000-1$ $1.00000+0 \quad 1.50000+0 \quad 2.00000+0 \quad 2.50000+0 \quad 3.00000+0$ $4.00000+0 \quad 5.00000+0 \quad 6.00000+0 \quad 7.00000+0 \quad 7.50000+0$ $8.00000+01.00000+1$ 1. $40000+1$

e132

e232

$\begin{array}{llllll}4.13994-7 & 1.12535-6 & 2.38237-6 & 5.04348-6 & 1.06770-5\end{array}$ $2.26033-5 \quad 4.78512-5 \quad 1.01301-4 \quad 1.67017-4 \quad 2.75364-4$ $\begin{array}{lllll}4.53999-4 & 7.48518-4 & 1.23410-3 & 2.03468-3 & 2.24867-3\end{array}$ $\begin{array}{llllll}2.61259-3 & 3.03539-3 & 3.70744-3 & 5.53084-3 & 9.11882-3\end{array}$ $\begin{array}{llllll}1.50344-2 & 2.35786-2 & 2.47875-2 & 2.60584-2 & 2.70001-2\end{array}$ 2.85011-2 $3.43067-2 \quad 5.24752-2 \quad 5.65622-2 \quad 8.65169-2$ $\begin{array}{llllll}1.22773-1 & 1.49956-1 & 1.83156-1 & 2.23708-1 & 2.73237-1\end{array}$ 2.94518-1 2.97211-1 2.98491-1 3.01974-1 3.87742-1 $4.97870-1 \quad 5.23397-1 \quad 6.08101-1 \quad 7.42735-1 \quad 9.07180-1$ $\begin{array}{lllll}1.10803+0 & 1.35335+0 & 1.65299+0 & 2.01897+0 & 2.23130+0\end{array}$ $2.34570+0 \quad 2.46597+0 \quad 3.01194+0 \quad 3.67879+0 \quad 4.49329+0$ $5.48811+0 \quad 6.70320+0 \quad 8.18730+0 \quad 1.00000+1 \quad 1.22140+1$ $1.49183+1$

$2.00000-2 \quad 4.50000-2 \quad 7.00000-2 \quad 1.00000-1 \quad 1.50000-1$ $3.00000-1 \quad 4.00000-1 \quad 5.10000-1 \quad 6.00000-1 \quad 7.00000-1$

$\begin{array}{lllll}1.00000+0 & 1.50000+0 & 2.00000+0 & 2.50000+0 & 3.00000+0\end{array}$ $4.00000+0 \quad 5.00000+0 \quad 6.00000+0 \quad 7.00000+0 \quad 7.50000+0$ $\operatorname{sd102} 100.0$ $8.00000+01.00000+11.40000+1$

sd112 100.0

sd122 100.0

sd132 100.0

sd202 100.0

sd212 100.0

$\operatorname{sd222} 100.0$

$\operatorname{sd232} 100.0$

sdef pos $=0$ 0 0 vec $=1$ o 0 dir $=d 1$ erg=fdir $=d 2$

$\begin{array}{llllllll}c & \text { si1 } & 0.0 & 0.5 & 0.8660254 & 0.9961946 & 1.0\end{array}$ 


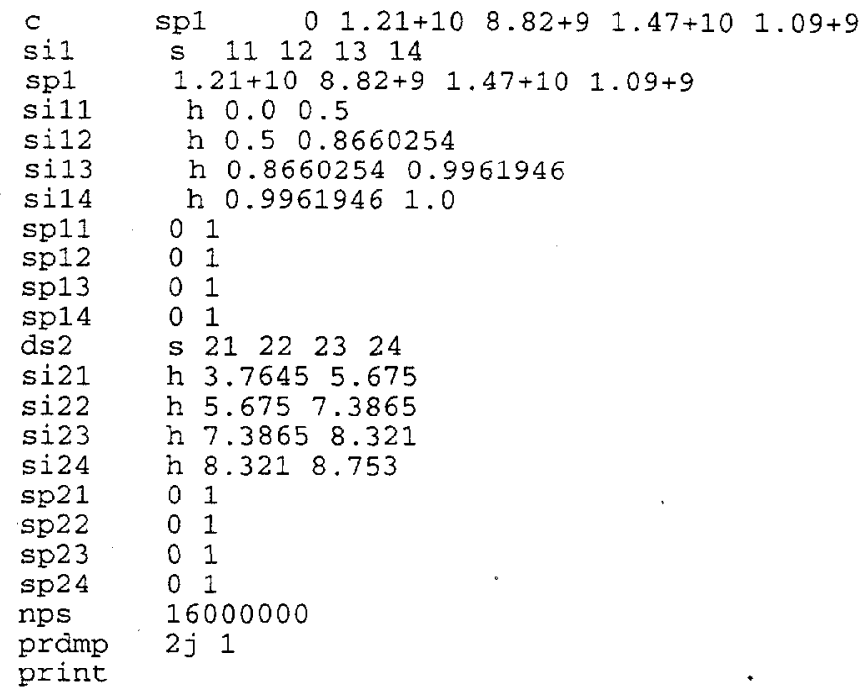




\section{APPENDIX C}

\section{PLOTS OF PLANE SLICES THROUGH THE PFNA FACILITY}

CALCULATIONAL GEOMETRY. 


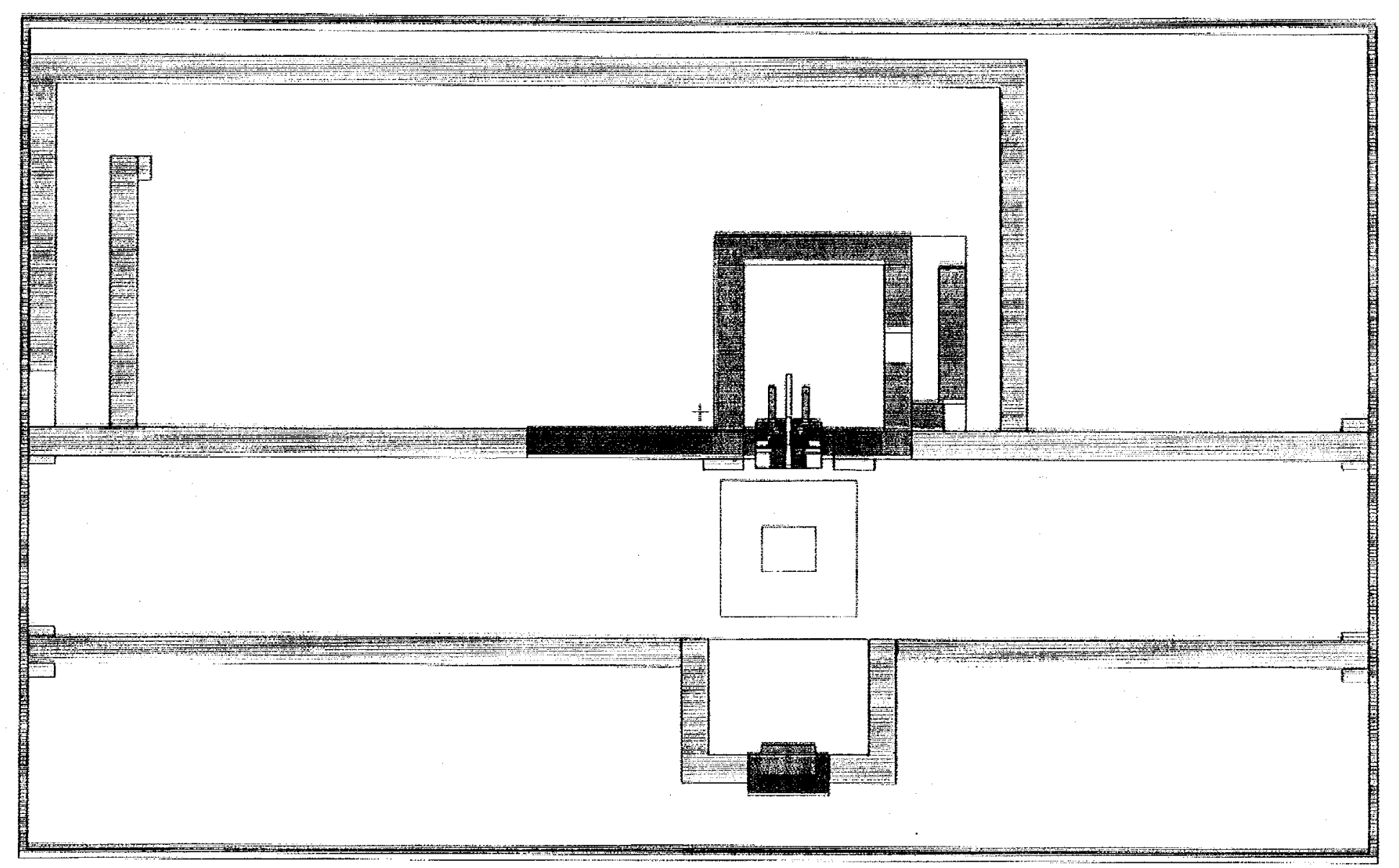

Figure C1. Plan view at $\mathrm{z}=0 \mathrm{~cm}$ through the MCNP geometry mockup of the PFNA Facility. 


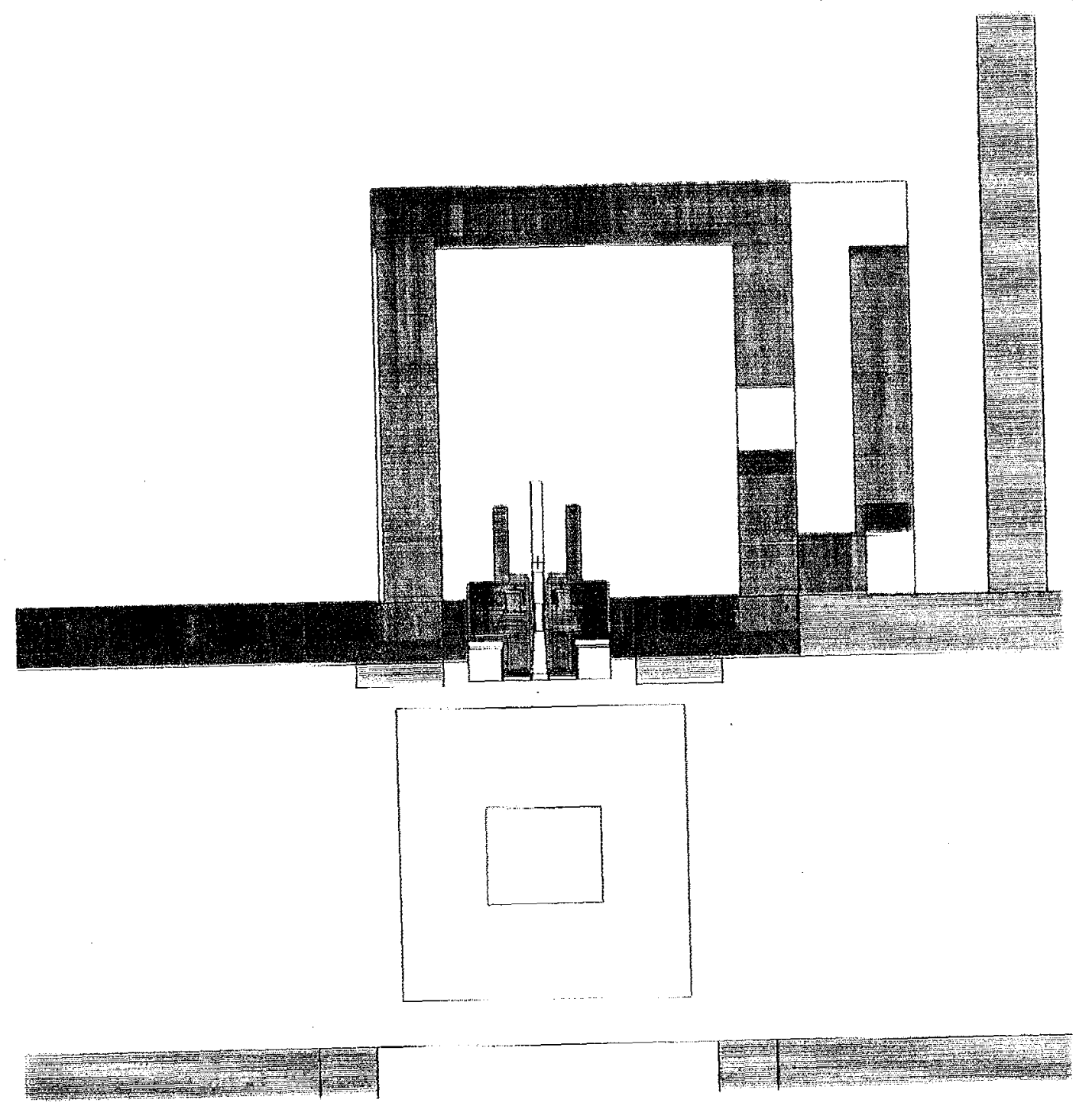

Figure C2. Plan view at $\mathrm{z}=0 \mathrm{~cm}$ through the MCNP geometry mockup of the PFNA Facility. Closeup view of regions around the stationary collimator. 

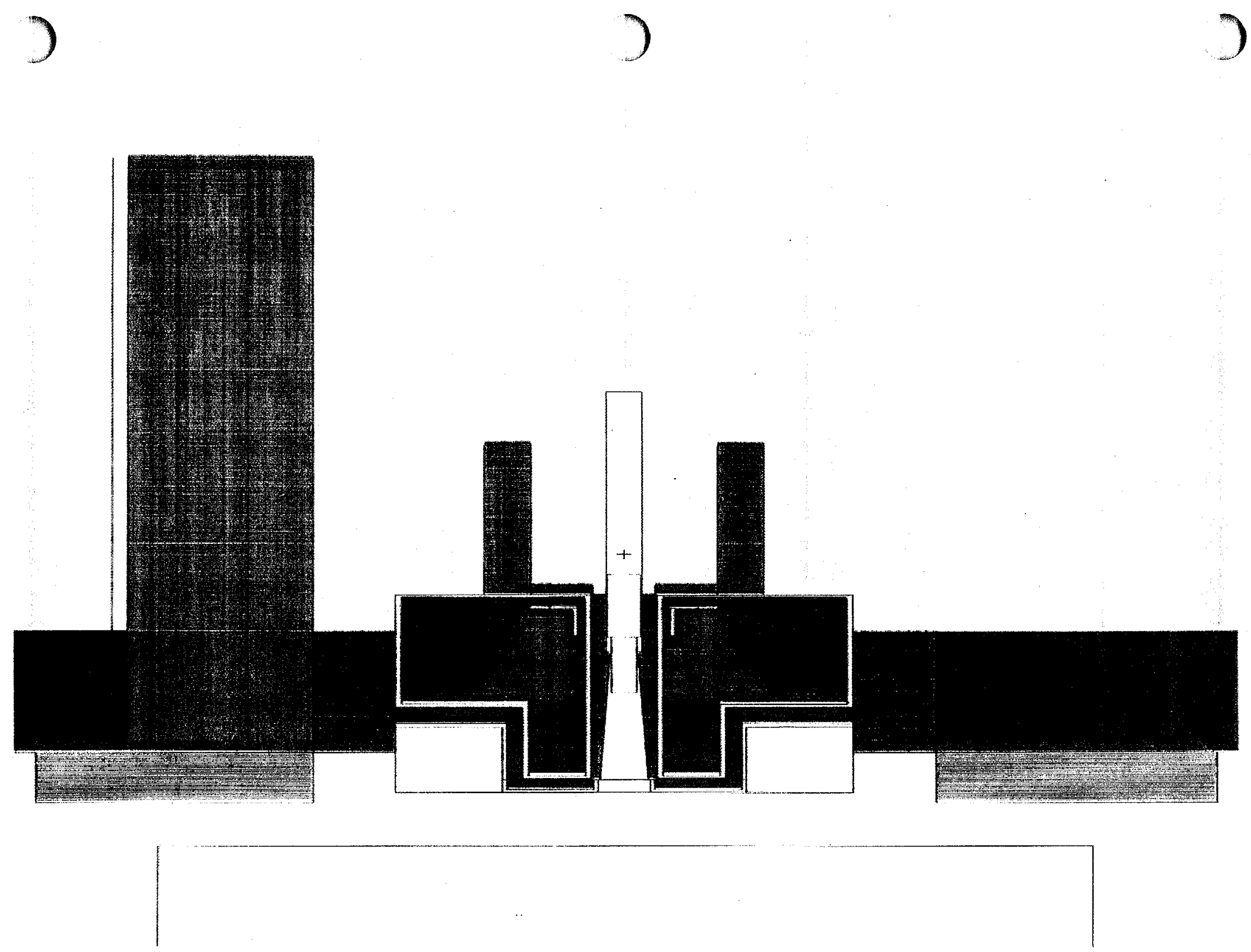

Figure C3. Plan view at $\mathrm{z}=0 \mathrm{~cm}$ through the MCNP geometry mockup of the PFNA Facility. Closeup view of regions around the stationary collimator. 


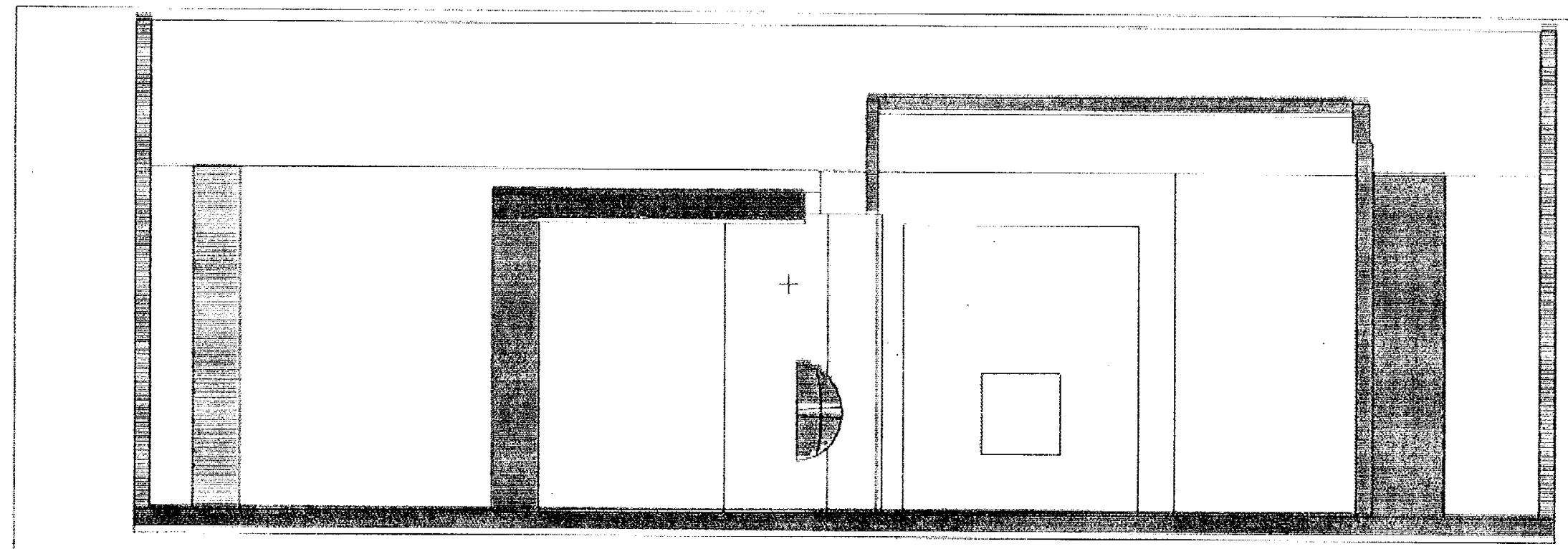

Figure C4. Elevation view at $y=0 \mathrm{~cm}$ through the MCNP geometry mockup of the PFNA Facility for the vertical collimator in the horizontal position. 


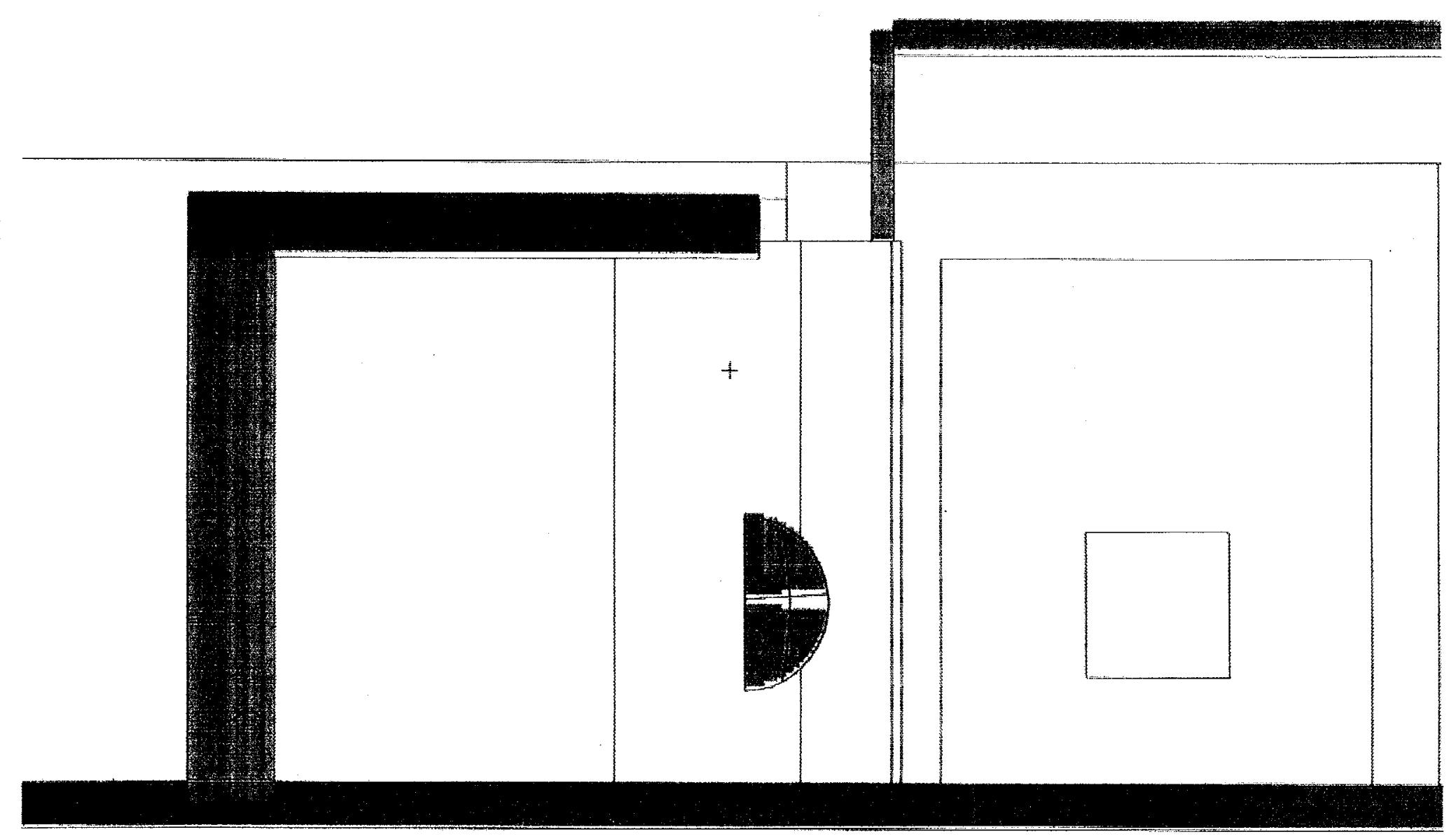

Figure C5. Elevation view at $y=0 \mathrm{~cm}$ through the MCNP geometry mockup of the PFNA Facility for the vertical collimator in the horizontal position. Closeup view of the vertical collimator. 


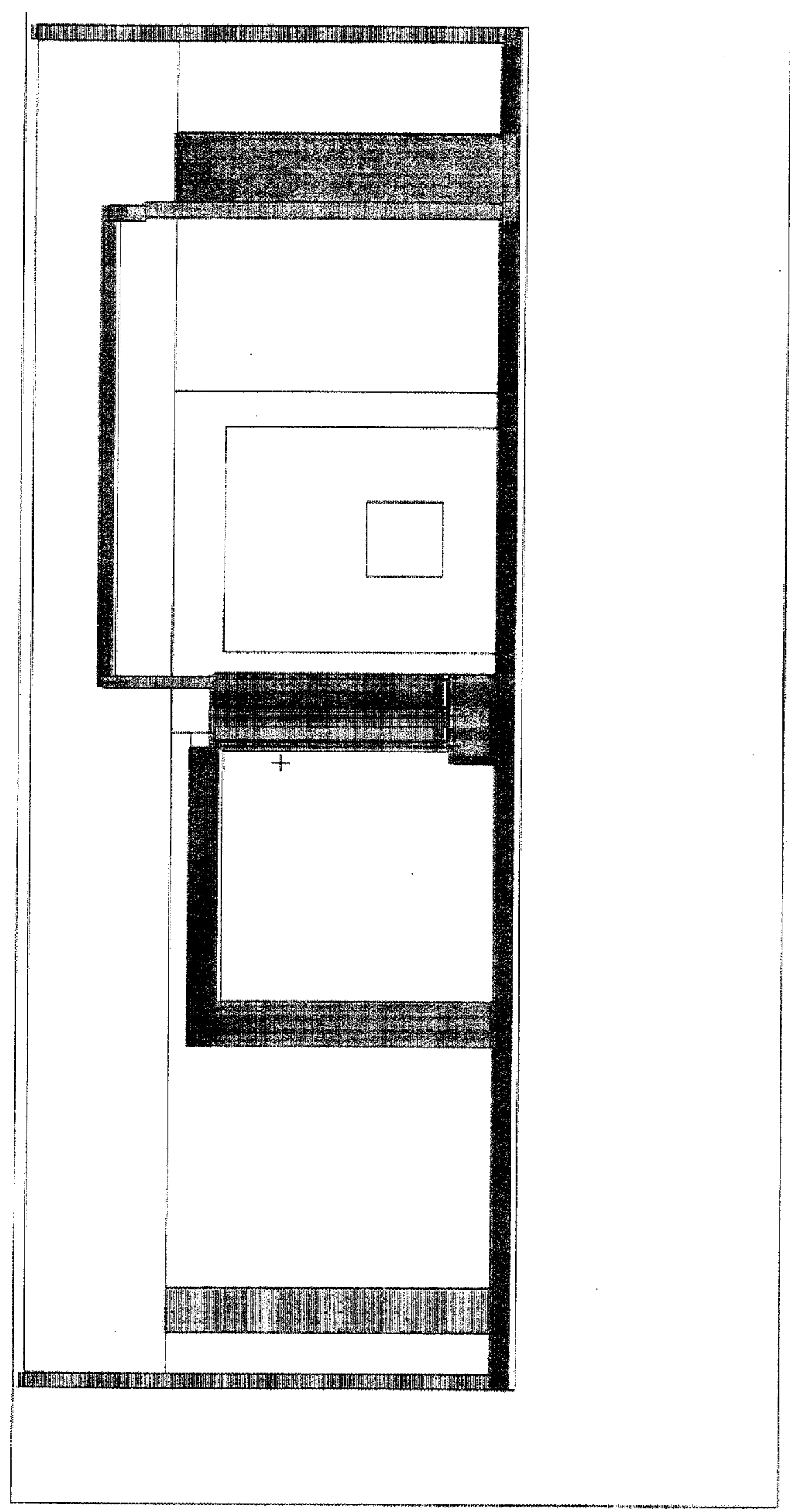

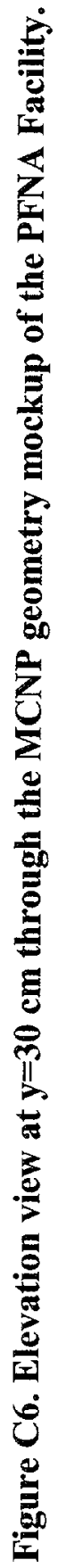




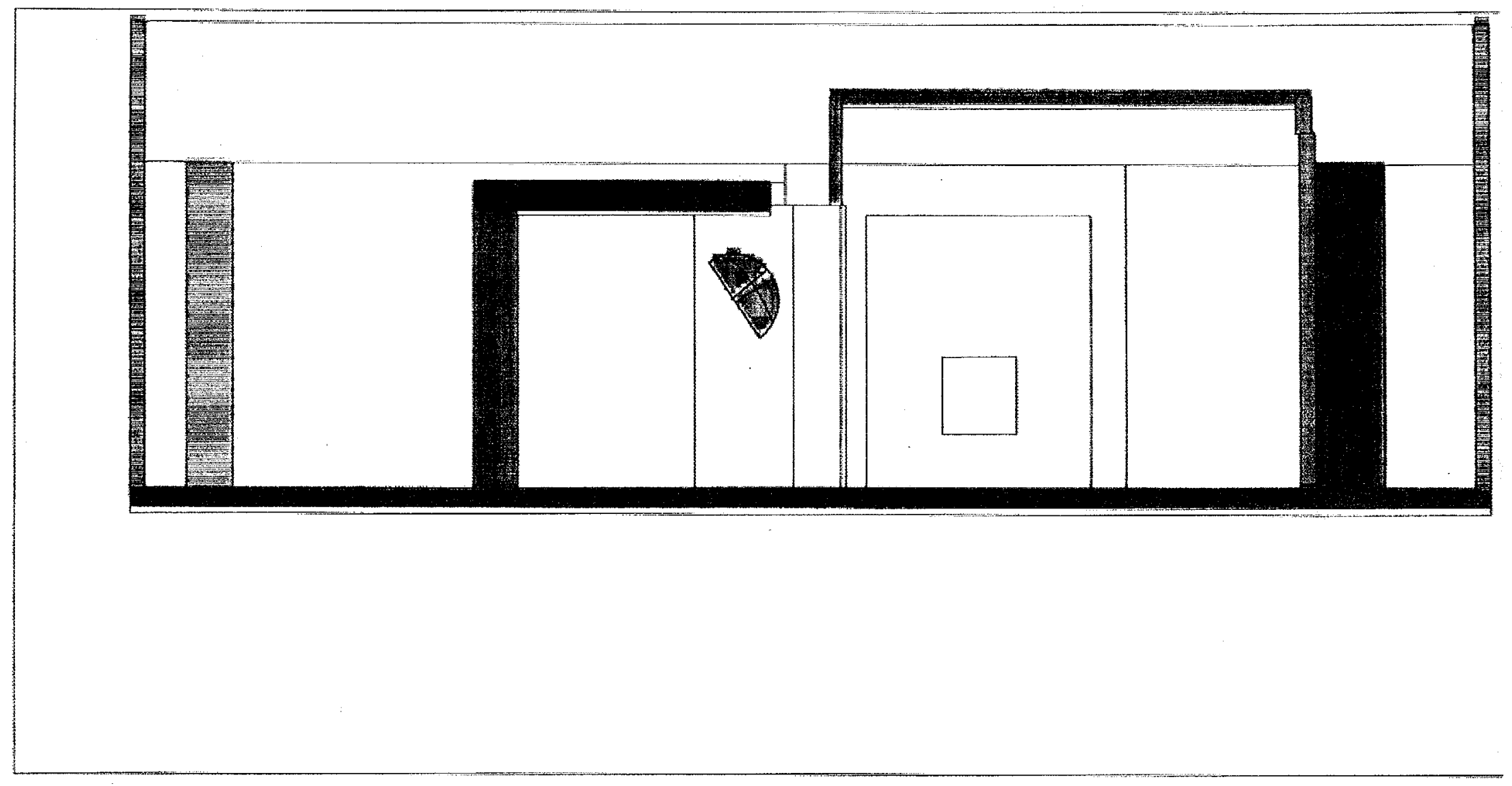

Figure C7. Elevation view at $y=0 \mathrm{~cm}$ through the MCNP geometry mockup of the PFNA Facility for the vertical collimator in the maximum up position. 


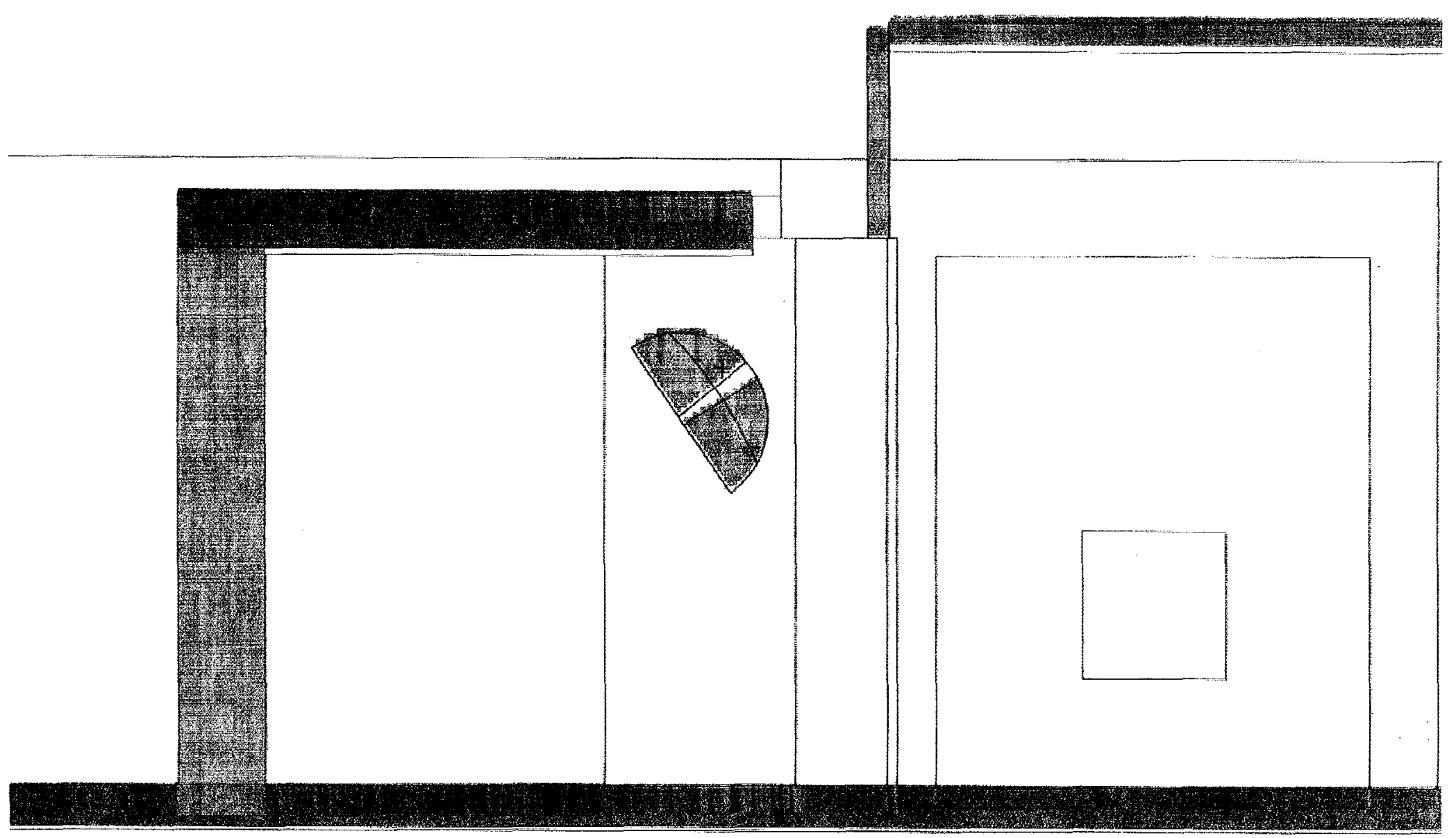

Figure C8. Elevation view at $\mathrm{y}=0 \mathrm{~cm}$ through the MCNP geometry mockup of the PFNA Facility for the vertical collimator in the maximum up position. Closeup view of the vertical collimator. 


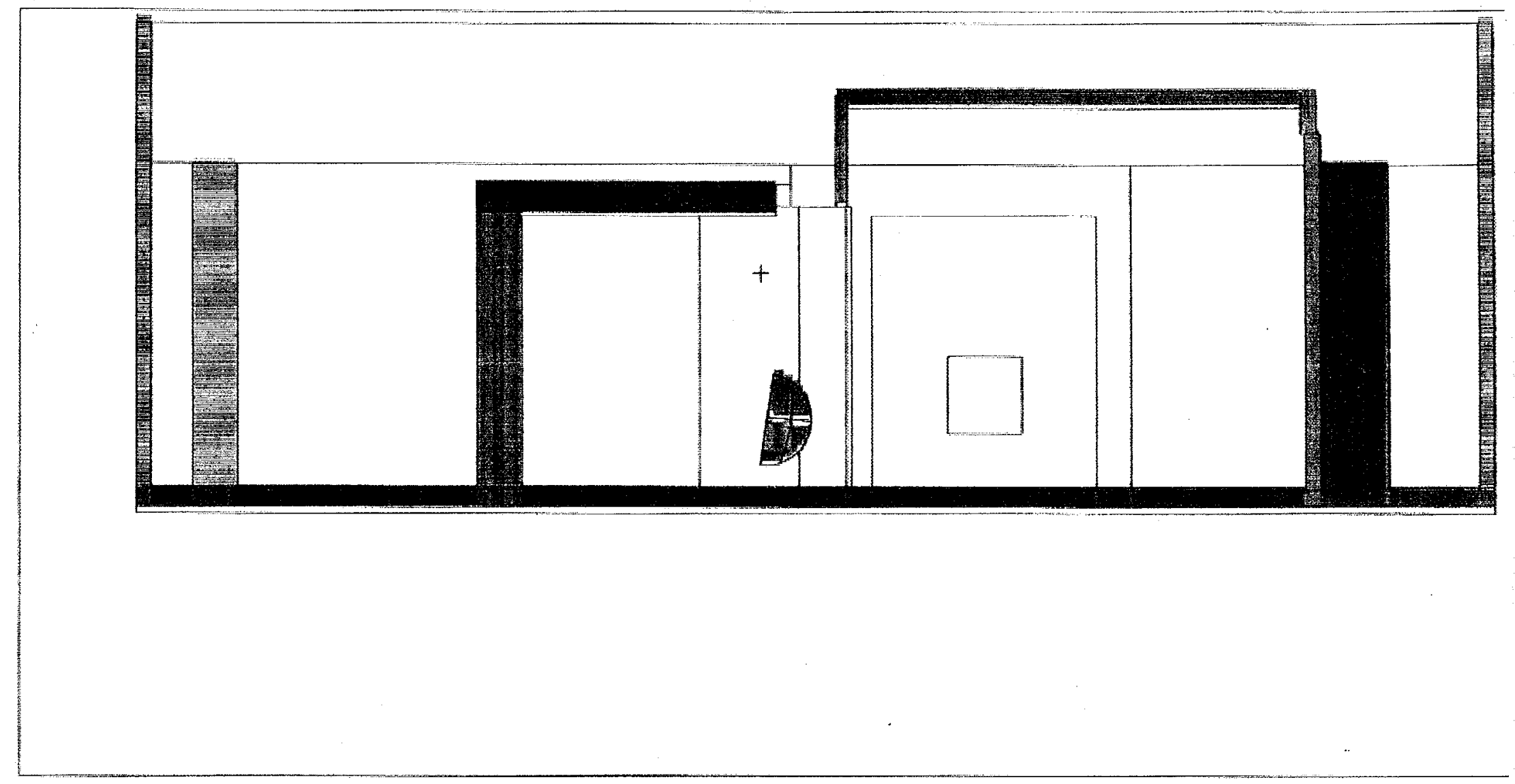

Figure C9. Elevation view at $y=0 \mathrm{~cm}$ through the MCNP geometry mockup of the PFNA Facility for the vertical collimator in the maximum down position. 


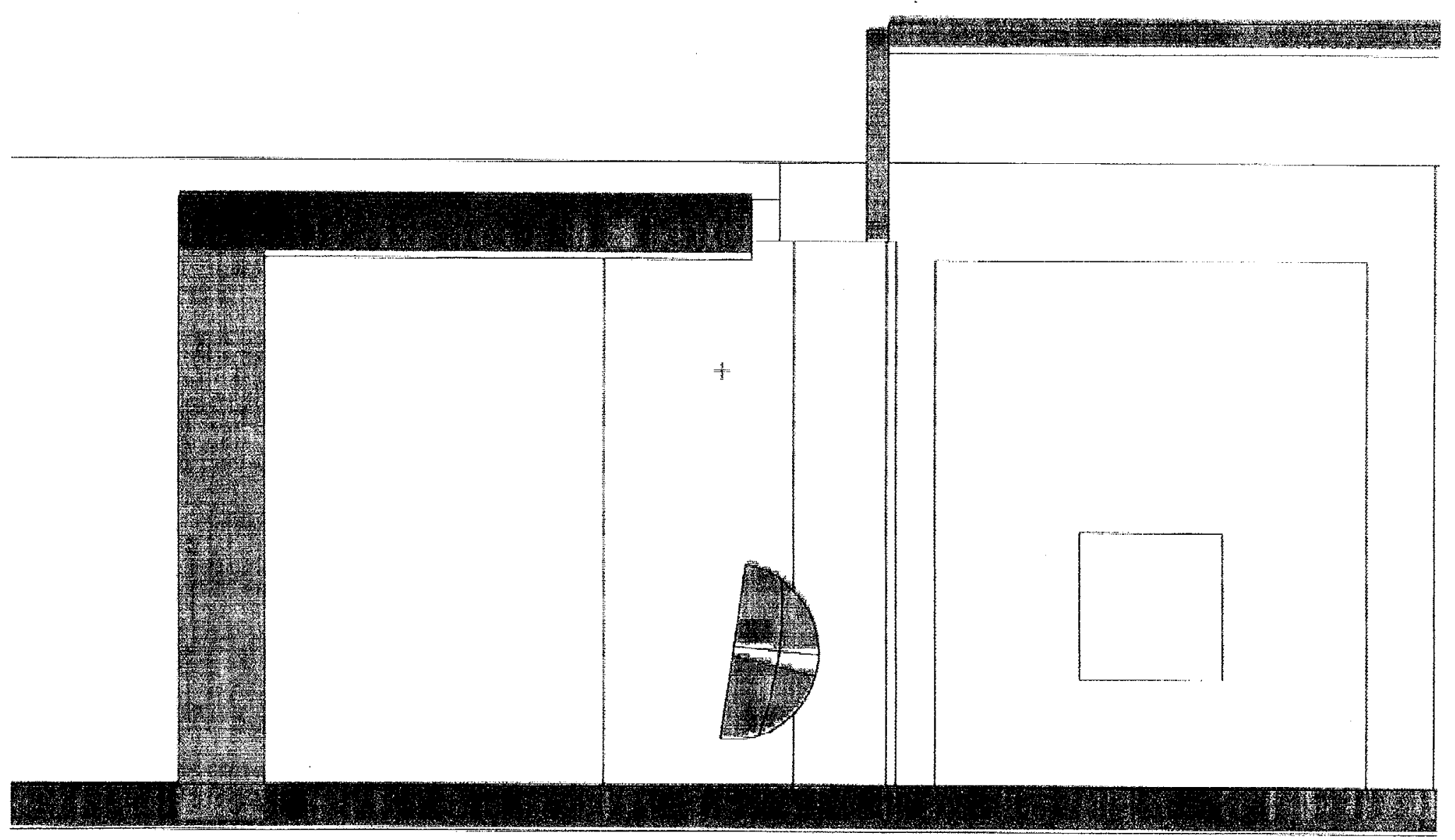

Figure C10. Elevation view at $y=0 \mathrm{~cm}$ through the MCNP geometry mockup of the PFNA Facility for the vertical collimator in the maximum down position. Closeup view of the vertical collimator. 


\section{APPENDIX D}

\section{LISTINGS OF CODES AND INPUT FILES DOSE-RATE AVERAGING}




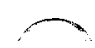

2 


\section{APPENDIX D}

\section{LISTINGS OF CODES AND INPUT FILES DOSE-RATE AVERAGING}

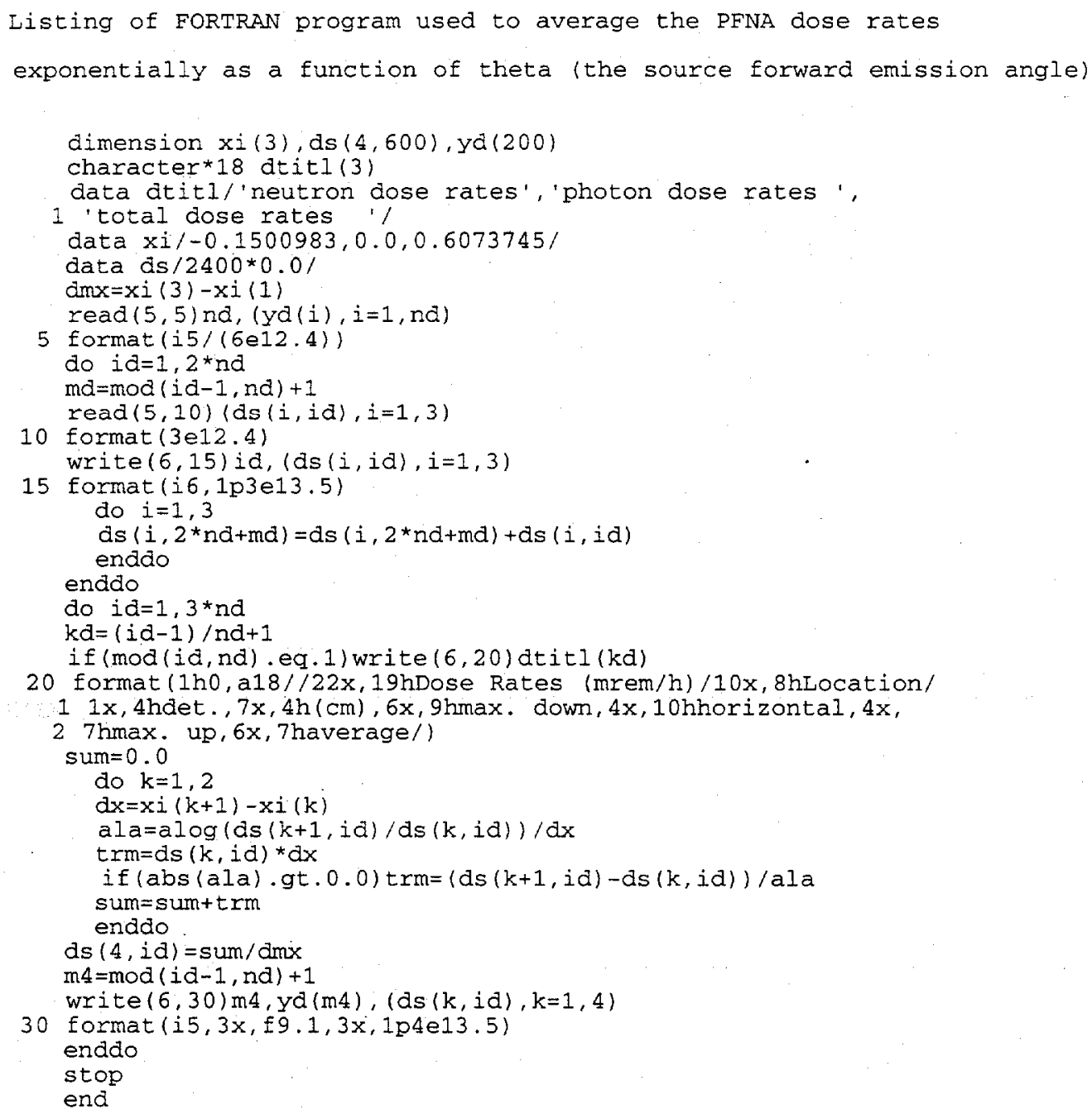


Listing of input file for averaging dose rates calculated at various

locations around the PFNA facility as a furlction of source angle

10

$\begin{array}{rrrrrr}0.0 & 245.0 & 0.0 & -1710.0 & 1296.0 & 0.0 \\ 300.0 & 0.0 & 0.0 & 0.0 & & \end{array}$

$5.58024-1$

0.0
$4.70220+0$

$2.39937-1$

$6.10697-1$

$5.87105+2$

$1.81381+0$

$5.47704-1$

1. $25419-1$

$1.49350+4 \quad 7.00707+1$

$1.16587+3 \quad 7.67914+4 \quad 1.63541+2$

$6.21285+0 \quad 4.34500+0 \quad 7.28572+0$

$2.26431-5 \quad 4.08722-3 \quad 2.34775-4$

$1.46130-1 \quad 5.60654-1 \quad 8.76999-2$

1.99241-1 $2.23255-1 \quad 4.66521-1$

$5.56058-2 \quad 1.30955+0 \quad 9.76129-3$

$\begin{array}{lll}1.62714-1 & 1.45858-1 & 2.94213-2\end{array}$

$\begin{array}{lll}1.25930+1 & 8.43161+1 & 2.27785+0\end{array}$

3.56180-2 $1.43302-2 \quad 2.55025-2$

$9.11276-2 \quad 3.90251-2 \quad 5.37723-2$

$1.70391+1 \quad 1.39578+1 \quad 5.39429+0$

$\begin{array}{lll}1.83418+0 & 1.67950+0 & 2.31934+0\end{array}$

$\begin{array}{lll}1.14903-2 & 4.35777-2 & 1.36479-2\end{array}$

$5.30021-2 \quad 1.11127-1 \quad 7.83837-3$

$6.01096+0 \quad 5.54711+0 \quad 6.62591+0$ 
Listing of input file to average PFNA dose rates calculated at various locations along the centerline of the truck lane as a function of source angle

24

$\begin{array}{rrrrrr}-1710.0 & -1524.0 & -1371.6 & -1219.2 & -1066.8 & -914.4 \\ -762.0 & -609.6 & -457.2 & -304.8 & -152.4 & -76.2 \\ -30.48 & 0.0 & 30.48 & 76.2 & 152.4 & 304.8 \\ 457.2 & 609.6 & 762.0 & 914.4 & 1066.8 & 1296.0 \\ 81381+0 & 3.90125-1 & 4.05931-1 & & & \end{array}$

$1.81381+0$

$1.84981+0$

$2.51946+0$

$3.58813+0$

$5.30024+0$

$8.48255+0$

1. $48590+1$

$2.91353+1$

7. $20955+1$

$1.77789+2$

3. $20803+2$

$8.00332+2$

1. $16687+3$

$8.00332+2$

3. $20803+2$

1. $81242+2$

$7.28867+1$

$2.95375+1$

1. $53587+1$

$8.95194+0$

5. $68971+0$

$3.93217+0$

$2.28500+0$

3. $56180-2$

5.39488-2

6. $46439-2$

8.69921-2

1. 25492-1

1. 84925-1

2. 88393-1

4.83245-1

9.01676-1

$2.17521+0$

$4.72385+0$

$7.33929+0$

$1.37813+1$

$1.70391+1$

1. $37813+1$

$7.33929+0$

$4.76635+0$

2. $25568+0$

9.48638-1

4. $94655-1$

3. $00849-1$

2.01295-1

1. 5224.4-1

9.11276-2
5. $09248-1$

6.24773-1

8.40224-1

$1.22390+0$

$1.83703+0$

$3.05426+0$

$5.49383+0$

$1.06697+1$

$9.28149+1$

$2.13007+2$

$1.15811+3$

$7.67914+4$

$1.15811+3$

$2.13007+2$

$9.43788+1$

$3.59743+1$

$1.08823+1$

$5.56264+0$

$3.29045+0$

$2.08403+0$

1. $44988+0$

8.29166-1

1. $43302-2$

2. $43626-2$

2. 74815-2

3. 69437-2

4.82811-2

$6.76465-2$

1.07229-1

1.73985-1

$3.19502-1$

$1.12029+0$

$2.50499+0$

$4.02129+0$

$1.08577+1$

$1.39578+1$

$1.08577+1$

$4.02129+0$

$2.39828+0$

$1.19457+0$

3. $53345-1$

1. 85464-1

1. 22278-1

8.19611-2

$5.45651-2$

3. $90251-2$
$3.57631+1$
4.05931-1

$6.68034-1$

7. $66245-1$

$1.08589+0$

1. $50473+0$

$2.28986+0$

3. $77451+0$

$6.45836+0$

1. $76760+1$

2. $99380+1$

5. $16757+1$

$7.27455+1$

1. $36333+2$

1. $63541+2$

1. $36333+2$

$7.27455+1$

$5.21843+1$

3. $02640+1$

$1.77131+1$

$6.13926+0$

3. $34407+0$

$2.12947+0$

1. $48254+0$

$9.49863+0$

2. 55025-2

3. $71394-2$

4. 31652-2

5. 14859-2

7.10139-2

1. 01122-1

1. 61069-1

2. 32722-1

4.74318-1

7.88501-1

$1.38074+0$

$2.08345+0$

$5.39429+0$

3. $52223+0$

2. $08345+0$

$1.46280+0$

9. $42471-1$

5.41115-1

2. 59925-1

1. 61437-1

1.15803-1

8.09399-2

5. 37723-2
3. $52223+0$ 
Listing of input file to average PFNA dose rates calculated at locations in the beam line and across the truck lane as a function of source angle

06

$\begin{array}{rrrrrr}116.078 & 177.038 & 237.998 & 359.918 & 420.878 & 481.838 \\ 5.27242+3 & 5.38063+5 & 8.05332+2 & & \\ 2.64372+3 & 2.21584+5 & 2.96955+2 & & \\ 1.57531+3 & 1.21564+5 & 2.17530+2 & & \\ 9.88388+2 & 5.28997+4 & 1.31665+2 & & \\ 9.24120+2 & 3.86709+4 & 1.11483+2 & & \\ 8.85173+2 & 2.95280+4 & 9.70157+1 & & \\ 1.61237+2 & 2.94997+2 & 2.00615+1 & & \\ 4.49017+1 & 5.27021+1 & 1.03627+1 & & \\ 2.36999+1 & 2.33104+1 & 7.18505+0 & & \\ 1.48370+1 & 9.98272+0 & 4.26452+0 & & \\ 1.47948+1 & 8.24923+0 & 3.49869+0 & & \\ 1.50075+1 & 7.79060+0 & 2.96020+0 & & \end{array}$




\section{APPENDIX E}

LISTINGS OF INPUT FILES MCNP-4B CALCULATIONS OF DOSE RATES WITHIN AND AROUND THE PFNA FACILITY 


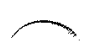

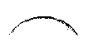

2 


\section{LISTINGS OF INPUT FILES MCNP-4B CALCULATIONS OF DOSE RATES WITHIN AND AROUND THE PFNA FACILITY}

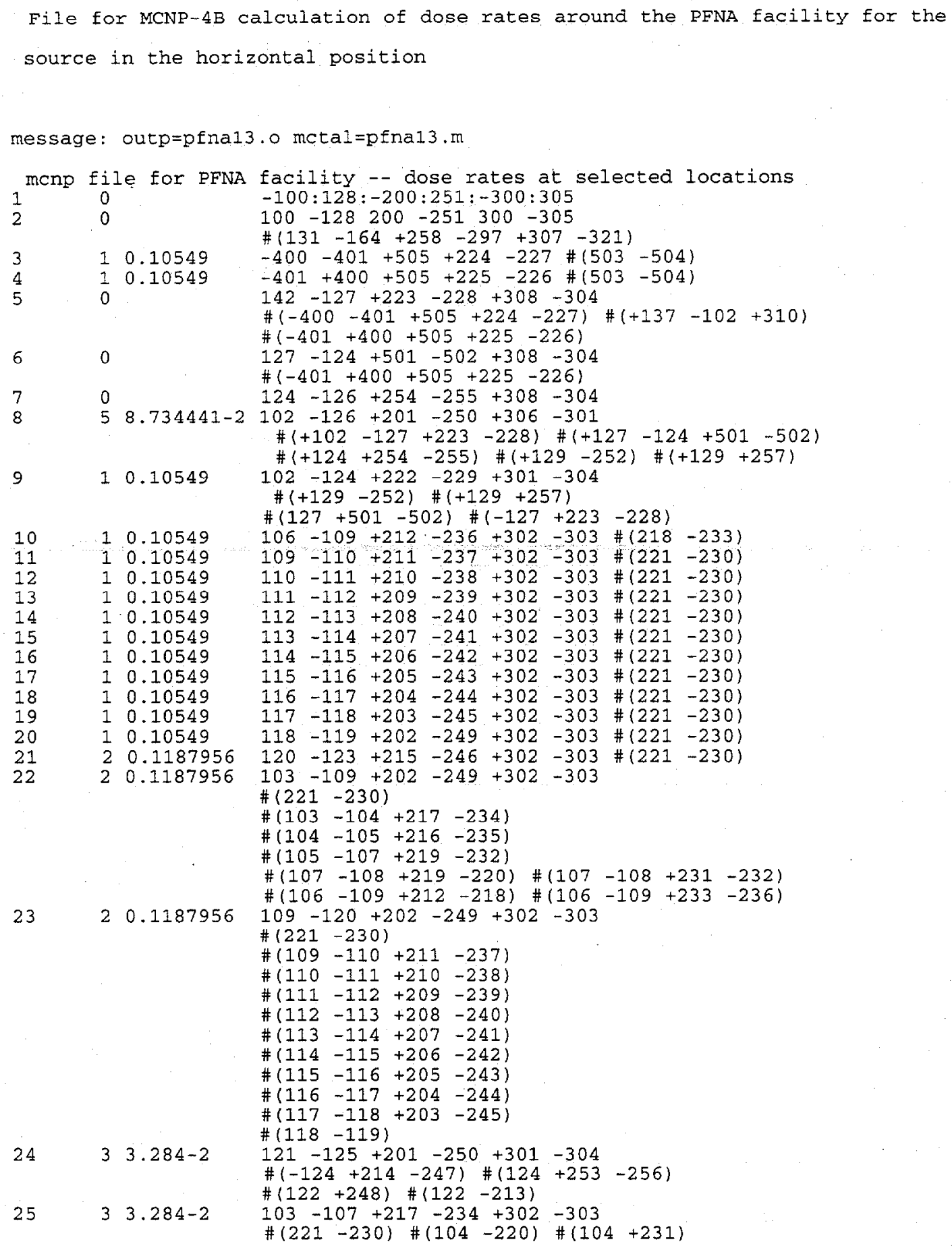




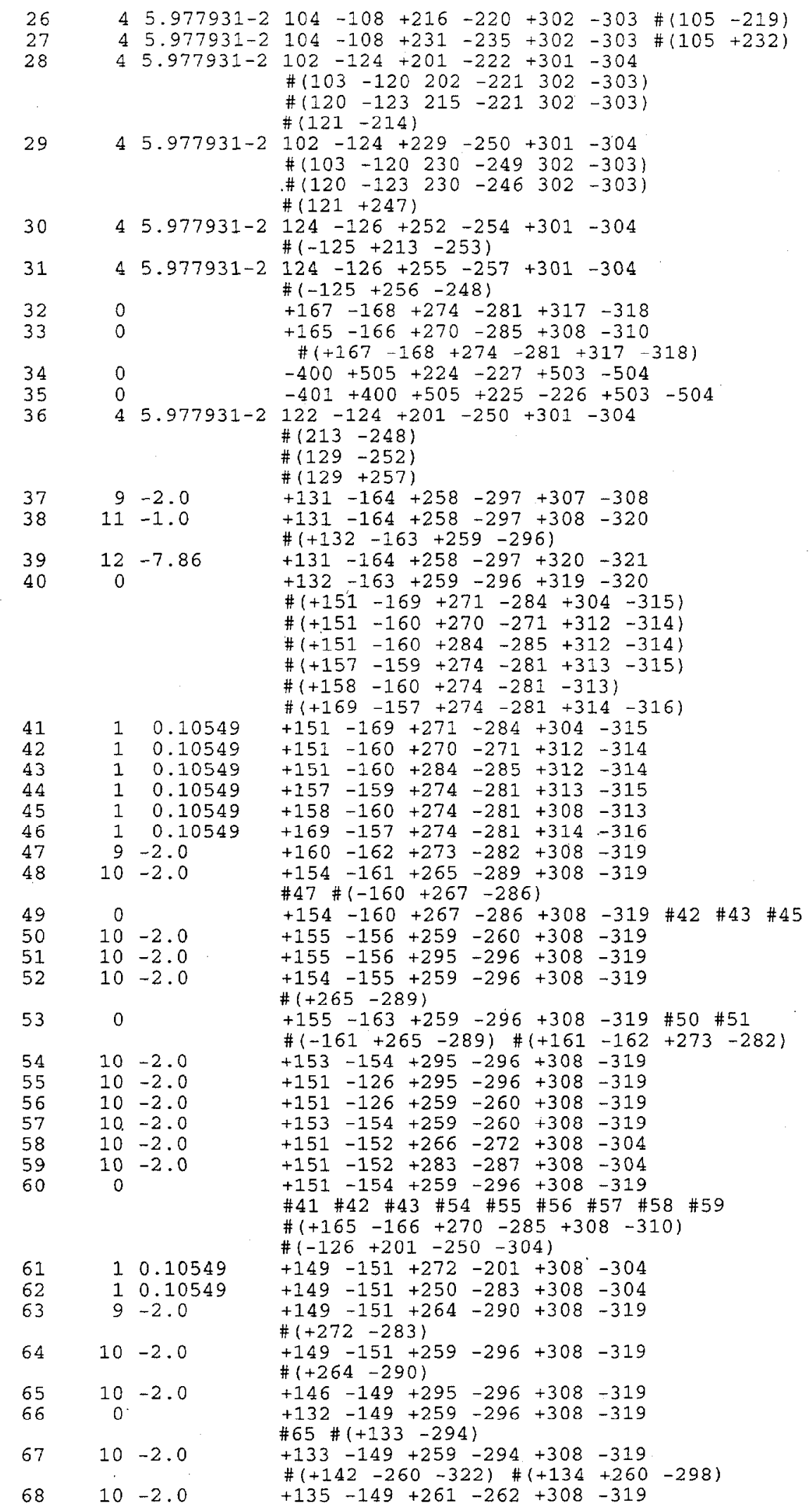




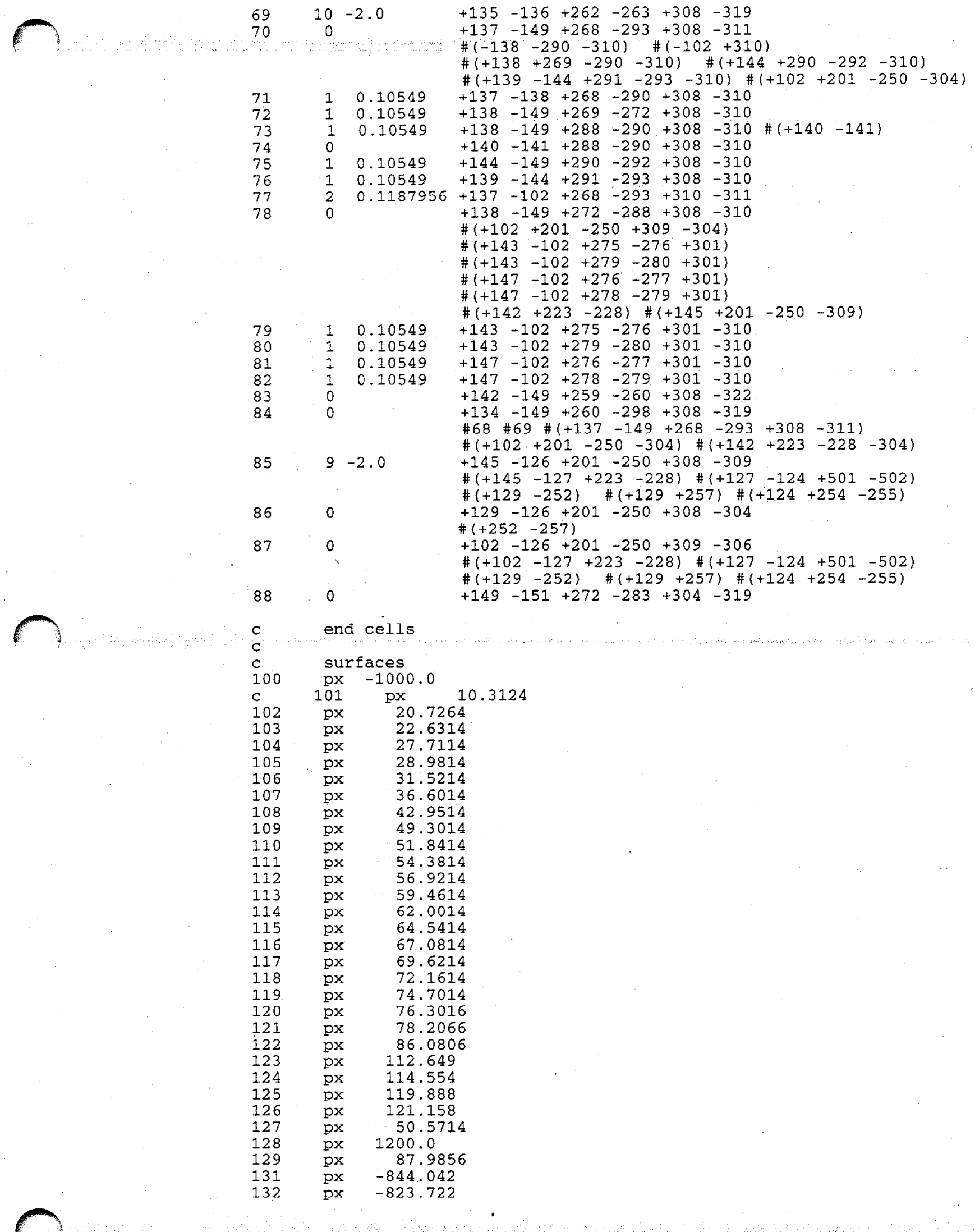




\begin{tabular}{|c|c|c|}
\hline 133 & $\mathrm{px}$ & -767.842 \\
\hline 134 & $\mathrm{px}$ & -706.882 \\
\hline 135 & $p x$ & -548.962 \\
\hline 136 & $p x$ & -493.522 \\
\hline 137 & $\mathrm{px}$ & -381.762 \\
\hline 138 & $\mathrm{p} x$ & -320.802 \\
\hline 139 & $\mathrm{px}$ & -315.722 \\
\hline 140 & $\mathrm{px}$ & -173.482 \\
\hline 141 & $\mathrm{px}$ & -107.442 \\
\hline 142 & $p x$ & -82.042 \\
\hline 143 & $p x$ & -55.4736 \\
\hline 144 & $\mathrm{px}$ & -21.082 \\
\hline 145 & $p x$ & -0.762 \\
\hline 146 & $p x$ & 9.398 \\
\hline $14 \%$ & $p x$ & 15.6464 \\
\hline 148 & $p x$ & 30.1103 \\
\hline 149 & $p x$ & 39.878 \\
\hline 150 & $\mathrm{px}$ & 45.3503 \\
\hline 151 & $\mathrm{px}$ & 100.838 \\
\hline 152 & $p x$ & 126.238 \\
\hline 153 & $p x$ & 476.758 \\
\hline 154 & $\mathrm{px}$ & 497.078 \\
\hline 155 & $\mathrm{px}$ & 558.038 \\
\hline 156 & $\mathrm{px}$ & 588.518 \\
\hline 157 & $\mathrm{px}$ & 725.678 \\
\hline 158 & $p x$ & 730.758 \\
\hline 159 & $\mathrm{px}$ & 745.998 \\
\hline 160 & $\mathrm{px}$ & 751.078 \\
\hline 161 & $\mathrm{px}$ & 812.038 \\
\hline 162 & $\mathrm{px}$ & 842.518 \\
\hline 163 & $\mathrm{px}$ & 964.438 \\
\hline 164 & $\mathrm{px}$ & 984.758 \\
\hline 165 & $\mathrm{px}$ & 148.387 \\
\hline 166 & $\mathrm{px}$ & 449.529 \\
\hline 167 & $\mathrm{px}$ & 250.0 \\
\hline 168 & $\mathrm{px}$ & 350.0 \\
\hline 169 & $\mathrm{px}$ & 116.078 \\
\hline 200 & py & -2000.0 \\
\hline 201 & py & -74.7056 \\
\hline 202 & py & -72.8006 \\
\hline 203 & py & -47.0958 \\
\hline 204 & py & -45.8258 \\
\hline 205 & py & -44.5558 \\
\hline 206 & PY & -43.2858 \\
\hline 207 & $\overrightarrow{p y}$ & -42.0158 \\
\hline 208 & py & -40.7458 \\
\hline 209 & py & -39.4758 \\
\hline 210 & py & -38.2058 \\
\hline 211 & py & -36.9358 \\
\hline 212 & py & -34.3958 \\
\hline 213 & py & -38.6122 \\
\hline 214 & py & -33.2782 \\
\hline 215 & py & -31.3732 \\
\hline 216 & py & -30.5858 \\
\hline 217 & $p Y$ & -24.2358 \\
\hline 218 & py & -19.1558 \\
\hline 219 & py & -16.6158 \\
\hline 220 & py & -15.3458 \\
\hline 221 & py & -12.8058 \\
\hline 222 & py & -10.9008 \\
\hline 223 & pY & -5.8208 \\
\hline 224 & py & -5.5245 \\
\hline 225 & pY & -4.2037 \\
\hline 226 & PY & 4.2037 \\
\hline 227 & py & 5.5245 \\
\hline 228 & PY & 5.8208 \\
\hline 229 & py & 10.9008 \\
\hline 230 & py & 12.8058 \\
\hline & PY & 15.3458 \\
\hline & $\mathrm{pY}$ & 16.6158 \\
\hline & PY & 19.1558 \\
\hline 23 & py & 24.2358 \\
\hline
\end{tabular}




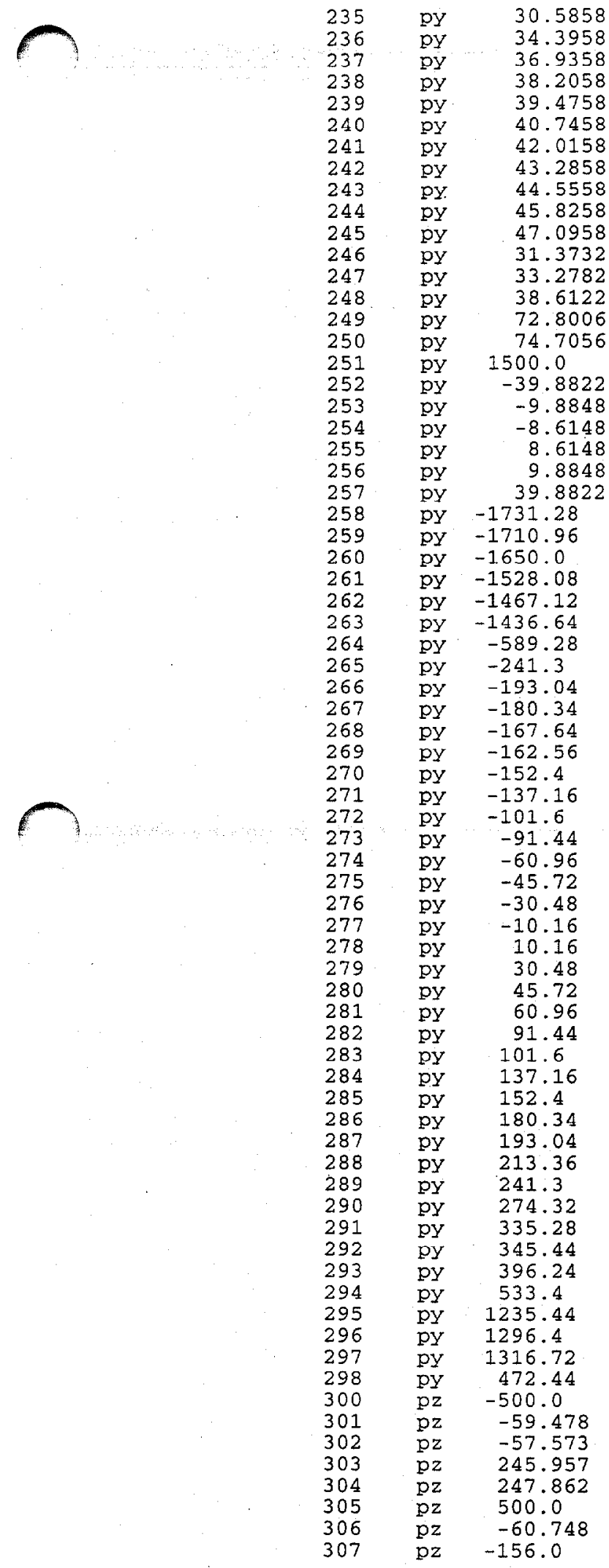




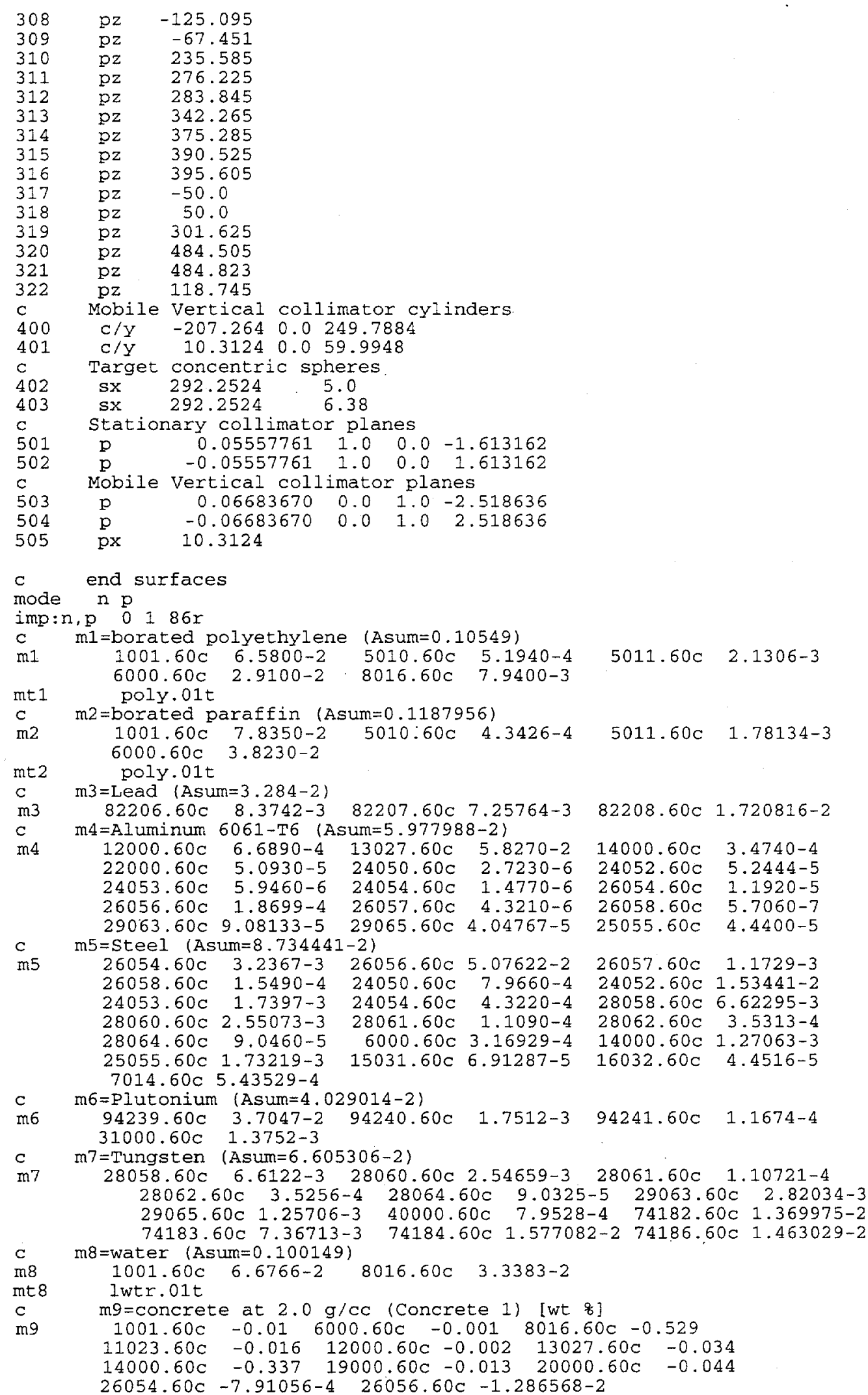




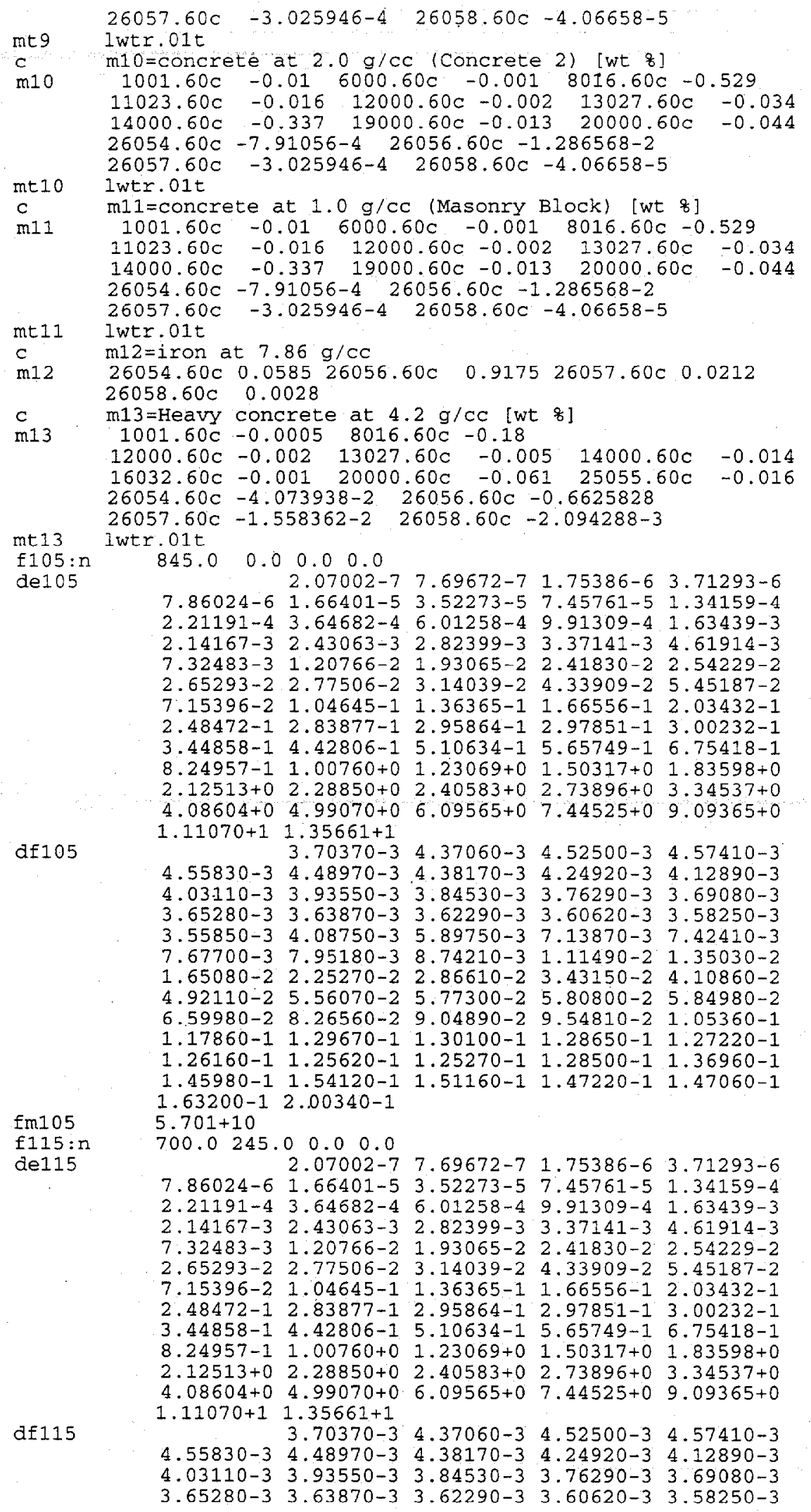




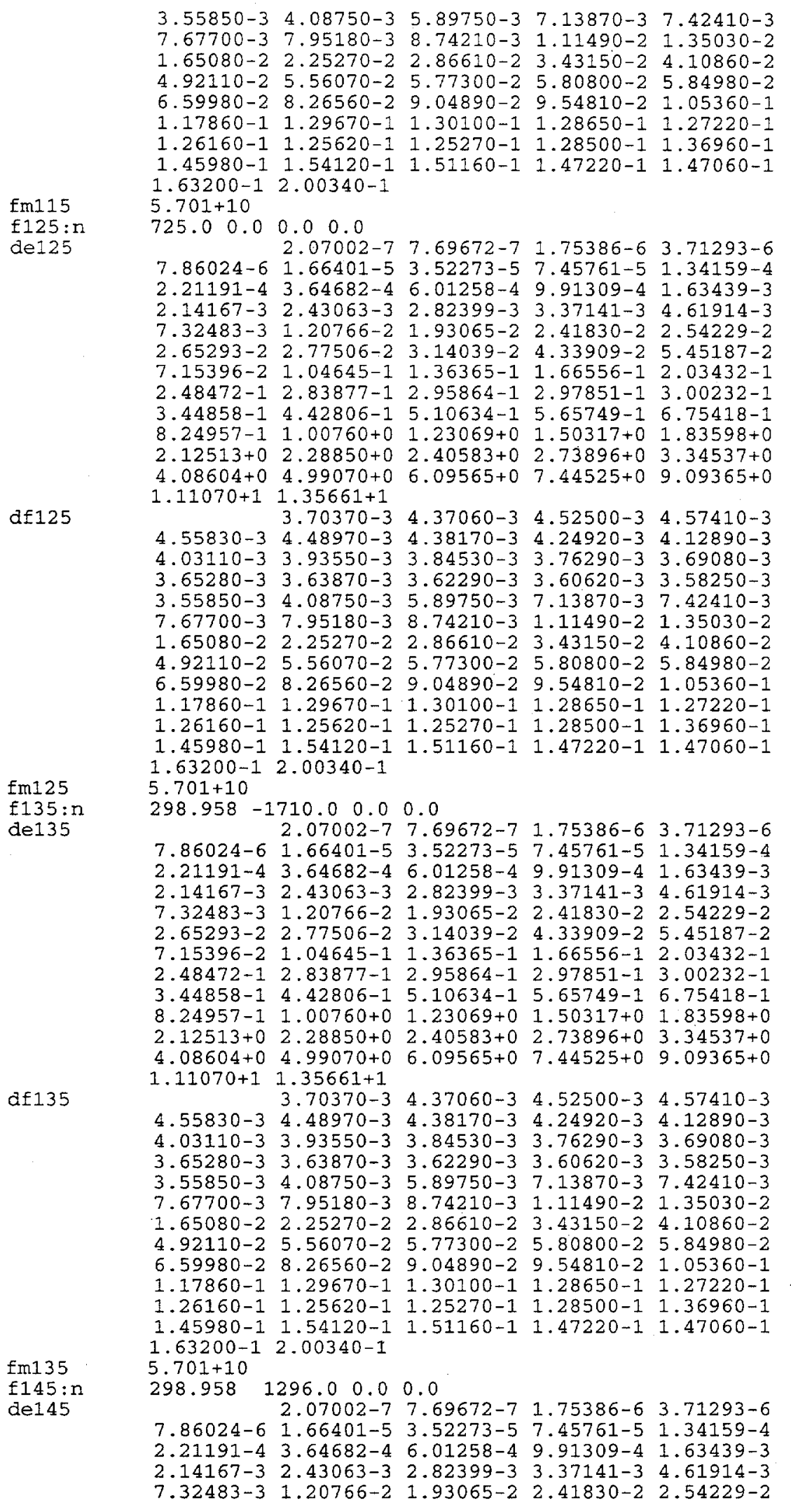


2.65293-2 2.77506-2 3.14039-2 $4.33909-2 \quad 5.45187-2$

$7.15396-2 \quad 1.04645-1 \quad 1.36365-1 \quad 1.66556-1 \quad 2.03432-1$

2.48472-1 2.83877-1 2.95864-1 2.97851-1 3.00232-1

$\begin{array}{lllllll}3.44858-1 & 4.42806-1 & 5.10634-1 & 5.65749-1 & 6.75418-1\end{array}$

$8.24957-1 \quad 1.00760+0 \quad 1.23069+0 \quad 1.50317+0 \quad 1.83598+0$

$\begin{array}{lllll}2.12513+0 & 2.28850+0 & 2.40583+0 & 2.73896+0 & 3.34537+0\end{array}$

$\begin{array}{lllll}4.08604+0 & 4.99070+0 & 6.09565+0 & 7.44525+0 & 9.09365+0\end{array}$ $1.11070+11.35661+1$

$\operatorname{df} 145$

$3.70370-3 \quad 4.37060-3 \quad 4.52500-3 \quad 4.57410-3$

4.55830-3 $4.48970-3 \quad 4.38170-3 \quad 4.24920-3 \quad 4.12890-3$

$4.03110-3 \quad 3.93550-3 \quad 3.84530-3 \quad 3.76290-3 \quad 3.69080-3$

$\begin{array}{llllll}3.65280-3 & 3.63870-3 & 3.62290-3 & 3.60620-3 & 3.58250-3\end{array}$

$\begin{array}{llllll}3.55850-3 & 4.08750-3 & 5.89750-3 & 7.13870-3 & 7.42410-3\end{array}$

$7.67700-3 \quad 7.95180-3 \quad 8.74210-3 \quad 1.11490-2 \quad 1.35030-2$

$1.65080-2 \quad 2.25270-2 \quad 2.86610-2 \quad 3.43150-2 \quad 4.10860-2$

$4.92110-25.56070-2 \quad 5.77300-2 \quad 5.80800-2 \quad 5.84980-2$

$\begin{array}{lllll}6.59980-2 & 8.26560-2 & 9.04890-2 & 9.54810-2 & 1.05360-1\end{array}$

$1.17860-1 \quad 1.29670-1.1 .30100-1 \quad 1.28650-11.27220-1$

$1.26160-1$ 1.25620-1 $1.25270-1$ 1.28500-1 $1.36960-1$

1.45980-1 1.54120-1 1.51160-1 1.47220-1 1.47060-1

1. $63200-12.00340-1$

fm1 $45 \quad 5,701+10$

$f 155: \mathrm{n} \quad 298.958 \quad 0.0 \quad 0.0 \quad 0.0$

$\begin{array}{llllll}\text { de155 } & 2.07002-7 & 7.69672-7 & 1.75386-6 & 3.71293-6\end{array}$

7.86024-6 $1.66401-5 \quad 3.52273-5 \quad 7.45761-5 \quad 1.34159-4$

$2.21191-4 \quad 3.64682-4 \quad 6.01258-4 \quad 9.91309-4 \quad 1.63439-3$

$\begin{array}{llllll}2.14167-3 & 2.43063-3 & 2.82399-3 & 3.37141-3 & 4.61914-3\end{array}$

$7.32483-3 \quad 1.20766-2 \quad 1.93065-2 \quad 2.41830-2 \quad 2.54229-2$

2.65293-2 2.77506-2 $3.14039-2 \quad 4.33909-2 \quad 5.45187-2$

$7.15396-2$ 1.04645-1 $1.36365-1.1 .66556-12.03432-1$

$\begin{array}{lllll}2.48472-1 & 2.83877-1 & 2.95864-1 & 2.97851-1 & 3.00232-1\end{array}$

3.44858-1 4.42806-1 $5.10634-1 \quad 5.65749-1 \quad 6.75418-1$

$8.24957-1 \quad 1.00760+0 \quad 1.23069+0 \quad 1.50317+0 \quad 1.83598+0$

$2.12513+0 \quad 2.28850+0 \quad 2.40583+0 \quad 2.73896+0 \quad 3.34537+0$

$4.08604+0 \quad 4.99070+0 \quad 6.09565+0 \quad 7.44525+0 \quad 9.09365+0$

$1.11070+1 \quad 1.35661+1$

df155 $3.70370-3 \quad 4.37060-3 \quad 4.52500-3 \quad 4.57410-3$

$4.55830-3 \quad 4.48970-3 \quad 4.38170-3 \quad 4.24920-3 \quad 4.12890-3$

$4.03110-3 \quad 3.93550-3 \quad 3.84530-3 \quad 3.76290-3 \quad 3.69080-3$

$3.65280-3 \quad 3.63870-3.3 .62290-3 \quad 3.60620-3 \quad 3.58250-3$

$\begin{array}{lllll}3.55850-3 & 4.08750-3 & 5.89750-3 & 7.13870-3 & 7.42410-3\end{array}$

$7.67700-3 \quad 7.95180-3 \quad 8.74210-3 \quad 1.11490-2 \quad 1.35030-2$

$1.65080-2 \quad 2.25270-2 \quad 2.86610-2 \quad 3.43150-2 \quad 4.10860-2$

$4.92110-2 \quad 5.56070-2 \quad 5.77300-2.5 .80800-2 \quad 5.84980-2$

$6.59980-2 \quad 8.26560-2 \quad 9.04890-2 \quad 9.54810-2 \quad 1.05360-1$

$\begin{array}{llllll}1.17860-1 & 1.29670-1 & 1.30100-1 & 1.28650-1 & 1.27220-1\end{array}$

$1.26160-1 \quad 1.25620-1 \quad 1.25270-1.1 .28500-1 \quad 1.36960-1$

$1.45980-1 \quad 1.54120-1 \quad 1.51160-1 \quad 1.47220-1 \quad 1.47060-1$

$1.63200-1 \quad 2.00340-1$

$\operatorname{fm} 155 \quad 5.701+10$

f165:n $\quad-385.0 \quad 300.0 \quad 0.0 \quad 0.0$

$\begin{array}{lllllll}\operatorname{de} 165 & 2.07002-7 & 7.69672-7 & 1.75386-6 & 3.71293-6\end{array}$

7.86024-6 $1.66401-5 \quad 3.52273-5 \quad 7.45761-5 \quad 1.34159-4$

$2.21191-4 \quad 3.64682-4 \quad 6.01258-4 \quad 9.91309-4 \quad 1.63439-3$

$2.14167-3 \quad 2.43063-3 \quad 2.82399-3 \quad 3.37141-3 \quad 4.61914-3$

7.32483-3 $1.20766-2 \quad 1.93065-2 \quad 2.41830-2 \quad 2.54229-2$

2.65293-2 $2.77506-2 \quad 3.14039-2 \quad 4.33909-2 \quad 5.45187-2$

7.15396-2 $1.04645-1 \quad 1.36365-1 \quad 1.66556-1 \quad 2.03432-1$

2.48472-1 $2.83877-1 \quad 2.95864-1 \quad 2.97851-1 \quad 3.00232-1$

$3.44858-1$ 4.42806-1 $5.10634-1 \quad 5.65749-1 \quad 6.75418-1$

$8.24957-1 \quad 1.00760+0 \quad 1.23069+0 \quad 1.50317+0 \quad 1.83598+0$

$2.12513+0 \quad 2.28850+0 \quad 2.40583+0 \quad 2.73896+0 \quad 3.34537+0$

$4.08604+0 \quad 4.99070+0 \quad 6.09565+0 \quad 7.44525+0 \quad 9.09365+0$

1. $11070+1 \quad 1.35661+1$

df165 $\quad 3.70370-3 \quad 4.37060-3 \quad 4.52500-3 \quad 4.57410-3$ $4.55830-3 \quad 4.48970-3 \quad 4.38170-3 \quad 4.24920-3 \quad 4.12890-3$ $\begin{array}{llllll}4.03110-3 & 3.93550-3 & 3.84530-3 & 3.76290-3 & 3.69080-3\end{array}$ $\begin{array}{lllll}3.65280-3 & 3.63870-3 & 3.62290-3 & 3.60620-3 & 3.58250-3\end{array}$ $\begin{array}{lllll}3.55850-3 & 4.08750-3 & 5.89750-3 & 7.13870-3 & 7.42410-3\end{array}$ $\begin{array}{llllll}7.67700-3 & 7.95180-3 & 8.74210-3 & 1.11490-2 & 1.35030-2\end{array}$

$1.65080-2 \quad 2.25270-2 \quad 2.86610-2 \quad 3.43150-2 \quad 4.10860-2$

$4.92110-2 \quad 5.56070-2 \quad 5.77300-2 \quad 5.80800-2 \quad 5.84980-2$ 


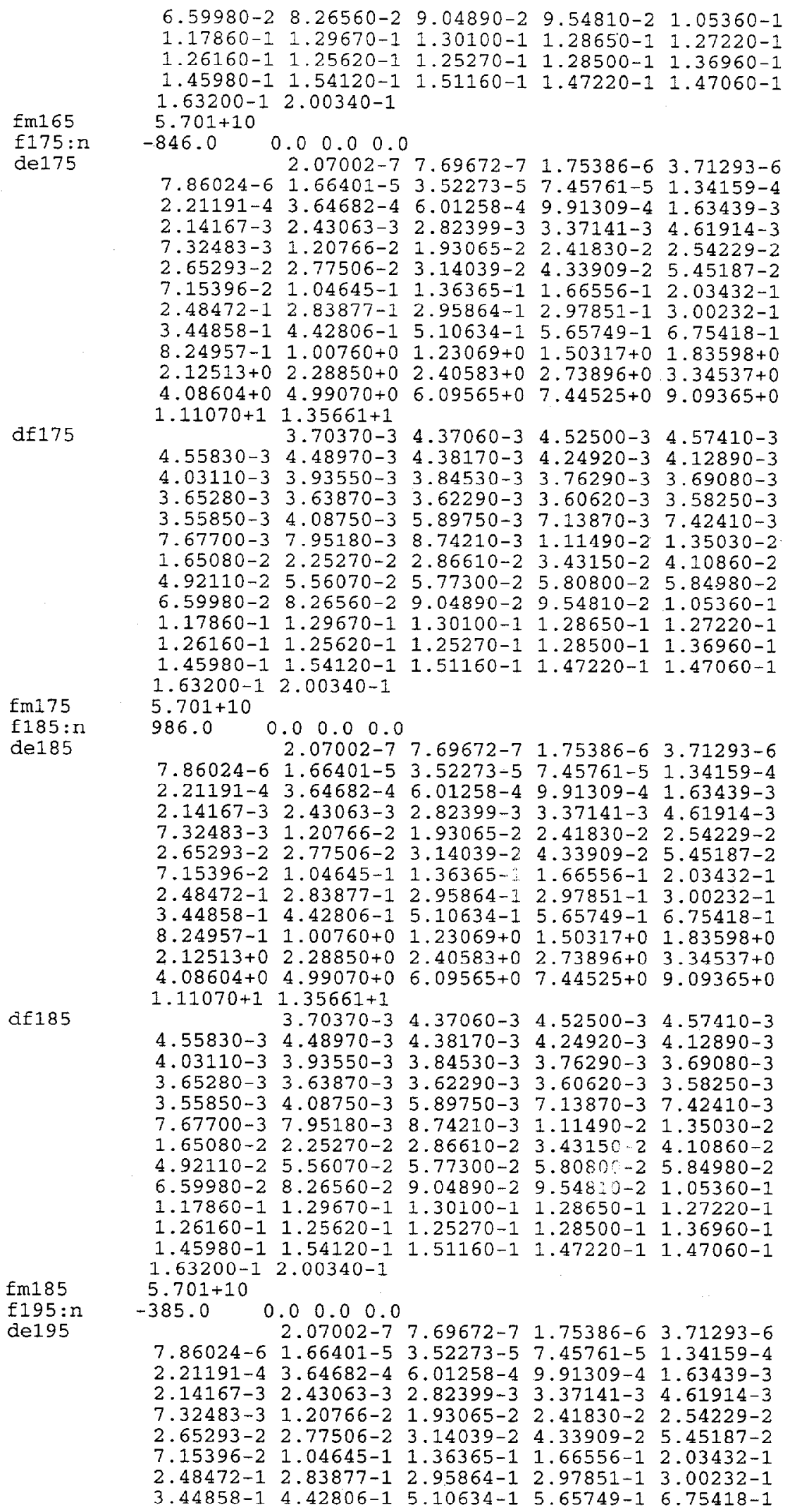


$8.24957-1 \quad 1.00760+0 \quad 1.23069+0 \quad 1.50317+0 \quad 1.83598+0$ $2.12513+0 \quad 2.28850+0 \quad 2.40583+0 \quad 2.73896+0 \quad 3.34537+0$ $4.08604+0 \quad 4.99070+0 \quad 6.09565+0 \quad 7.44525+0 \quad 9.09365+0$ $1.11070+1 \quad 1.35661+1$

df 195 $3.70370-3 \quad 4.37060-3 \quad 4.52500-3 \quad 4.57410-3$ $4.55830-3 \quad 4.48970-3 \quad 4.38170-3 \cdot 4.24920-3 \quad 4.12890-3$ $\begin{array}{lllll}4.03110-3 & 3.93550-3 & 3.84530-3 & 3.76290-3 & 3.69080-3\end{array}$ $3.65280-3 \quad 3.63870-3 \quad 3.62290-3 \quad 3.60620-3 \quad 3.58250-3$ $3.55850-3 \quad 4.08750-3 \quad 5.89750-3 \quad 7.13870-3 \quad 7.42410-3$ $7.67700-3 \quad 7.95180-3 \quad 8.74210-3 \quad 1.11490-2 \quad 1.35030-2$ $1.65080-2 \quad 2.25270-2 \quad 2.86610-2 \quad 3.43150-2 \quad 4.10860-2$ $4.92110-2 \quad 5.56070-2 \quad 5.77300-2 \quad 5.80800-2 \quad 5.84980-2$ $6.59980-2 \quad 8.26560-2 \quad 9.04890-2 \quad 9.54810-2 \quad 1.05360-1$ 1.17860-1 $1.29670-1 \quad 1.30100-1 \quad 1.28650-1 \quad 1.27220-1$ $1.26160-1 \quad 1.25620-1 \quad 1.25270-1 \quad 1.28500-11.36960-1$ $1.45980-1$ 1.54120-1 $1.51160-1$ 1.47220-1 $1.47060-1$ $1.63200-12.00340-1$

Em195 $5.701+10$

f205:p $8451+10$

de 205

df 205

0.00 .00 .0

$1.50000-2 \quad 3.25000-2 \quad 5.75000-2 \quad 8.50000-2$

$\begin{array}{lllll}1.25000-1 & 2.25000-1 & 3.50000-1 & 4.55000-1 & 5.55000-1\end{array}$

$6.50000-18.50000-1 \quad 1.25000+0 \quad 1.75000+0 \quad 2.25000+0$

$2.75000+0 \quad 3.50000+0 \quad 4.50000+0 \quad 5.5 .0000+0 \quad 6.50000+0$

$7.25000+0 \quad 7.75000+0 \quad 9.00000+0 \quad 1.20000+1$

$2.14390-3 \quad 5.77600-4 \quad 2.71850-4 \quad 2.68170-4$

$3.27670-4 \quad 5.66760-4 \quad 8.75940-4 \quad 1.08450-3 \quad 1.27970-3$

$\begin{array}{lllll}1.44170-3 & 1.75630-3 & 2.31560-3 & 2.92700-3 & 3.46860-3\end{array}$

$\begin{array}{llllll}3.95960-3 & 4.62210-3 & 5.41370-3 & 6.19090-3 & 6.92650-3\end{array}$ $7.47830-3 \quad 7.84680-3.8 .77160-3 \quad 1.10200-2$

fm205 $\quad 5.701+10$

f215:p $\quad 700.0245 .0 \quad 0.0 \quad 0.0$

de215

$1.50000-2 \quad 3.25000-2 \quad 5.75000-2 \quad 8.50000-2$

$\begin{array}{llllll}1.25000-1 & 2.25000-1 & 3.50000-1 & 4.55000-1 & 5.55000-1\end{array}$

$6.50000-1 \quad 8.50000-1 \quad 1.25000+0 \quad 1.75000+0 \quad 2.25000+0$

$2.75000+0 \quad 3.50000+0 \quad 4.50000+0 \quad 5.50000+0.6 .50000+0$

$7.25000+0 \quad 7.75000+0 \quad 9.00000+0 \quad 1.20000+1$

$\begin{array}{llllll}\mathrm{d} f 215 \quad 2.14390-3 & 5.77600-4 & 2.71850-4 & 2.68170-4\end{array}$ $3.27670-4 \quad 5.66760-4 \quad 8.75940-4$ 1.08450-3 $1.27970-3$ $1.44170-3 \quad 1.75630-32.31560-32.92700-3 \quad 3.46860-3$ $\begin{array}{llllll}3.95960-3 & 4.62210-3 & 5.41370-3 & 6.19090-3 & 6.92650-3\end{array}$ $\begin{array}{llll}7.47830-3 & 7.84680-3 & 8.77160-3 & 1.10200-2\end{array}$

$\mathrm{fm} 215 \quad 5.701+10$

f225:p $\quad 725.00 .0 \quad 0.0 \quad 0.0$

de225 $1.50000-2 \quad 3.25000-2 \quad 5.75000-2 \quad 8.50000-2$

$\begin{array}{llllll}1.25000-1 & 2.25000-1 & 3.50000-1 & 4.55000-1 & 5.55000-1\end{array}$

$6.50000-1 \quad 8.50000-1 \quad 1.25000+0 \quad 1.75000+02.25000+0$ $2.75000+0 \quad 3.50000+0 \quad 4.50000+0 \quad 5.50000+0 \quad 6.50000+0$ $7.25000+0 \quad 7.75000+0 \quad 9.00000+01.20000+1$

$\begin{array}{llllll}\text { df225 } 2.14390-3 & 5.77600-4 & 2.71850-4 & 2.68170-4\end{array}$

$3.27670-4 \quad 5.66760-4 \quad 8.75940-4 \quad 1.08450-3 \quad 1.27970-3$

$\begin{array}{lllll}1.44170-3 & 1.75630-3 & 2.31560-3 & 2.92700-3 & 3.46860-3\end{array}$ $3.95960-3 \quad 4.62210-3 \quad 5.41370-3 \quad 6.19090-3 \quad 6.92650-3$ $\begin{array}{llll}7.47830-3 & 7.84680-3 & 8.77160-3 & 1.10200-2\end{array}$

fm225 $5.701+10$

f235:p 298.958-1710.0 $0.0-0.0$

de235

df 235

$1.50000-2 \quad 3.25000-2 \quad 5.75000-2 \quad 8.50000-2$

$1.25000-1 \quad 2.25000-1 \quad 3.50000-1 \quad 4.55000-1 \quad 5.55000-1$

$6.50000-1 \quad 8.50000-1 \quad 1.25000+0 \quad 1.75000+0 \quad 2.25000+0$

$2.75000+0 \quad 3.50000+0 \quad 4.50000+0 \quad 5.50000+0 \quad 6.50000+0$

$7.25000+0 \quad 7.75000+0 \quad 9.00000+01.20000+1$

$2.14390-3 \quad 5.77600-4 \quad 2.71850-4 \quad 2.68170-4$

$3.27670-4 \quad 5.66760-4 \quad 8.75940-4 \quad 1.08450-3 \quad 1.27970-3$

$1.44170-3 \quad 1.75630-3 \quad 2.31560-3 \quad 2.92700-3 \quad 3.46860-3$

$\begin{array}{lllll}3.95960-3 & 4.62210-3 & 5.41370-3 & 6.19090-3 & 6.92650-3\end{array}$ $7.47830-3 \quad 7.84680-3 \quad 8.77160-3 \quad 1.10200-2$

fm235 $\quad 5.701+10$

f245:p $298.958 \quad 1296.0 \quad 0.0 \quad 0.0$

de245 $1.50000-2 \quad 3.25000-2 \quad 5.75000-2 \quad 8.50000-2$ $1.25000-1 \quad 2.25000-1 \quad 3.50000-1 \quad 4.55000-1 \quad 5.55000-1$ $6.50000-1 \quad 8.50000-1 \quad 1.25000+0 \quad 1.75000+0 \quad 2.25000+0$ $2.75000+0 \quad 3.50000+0 \quad 4.50000+0 \quad 5.50000+0 \quad 6.50000+0$ $7.25000+0 \quad 7.75000+09.00000+01.20000+1$ 
$\operatorname{df} 285$

$\begin{array}{lllll}1.44170-3 & 1.75630-3 & 2.31560-3 & 2.92700-3 & 3.46860-3\end{array}$

$\begin{array}{llllll}3.95960-3 & 4.62210-3 & 5.41370-3 & 6.19090-3 & 6.92650-3\end{array}$

$7.47830-3 \quad 7.84680-3 \quad 8.77160-3 \quad 1.10200-2$

$\mathrm{fm} 285 \quad 5.701+10$

f $295: p \quad-385.0 \quad 0.0 \quad 0.0 \quad 0.0$

de295 $1.50000-2 \quad 3.25000-2 \quad 5.75000-2 \quad 8.50000-2$ $\begin{array}{lllllll}1.25000-1 & 2.25000-1 & 3.50000-1 & 4.55000-1 & 5.55000-1\end{array}$ $6.50000-18.50000-1 \quad 1.25000+0 \quad 1.75000+02.25000+0$ $2.75000+0 \quad 3.50000+0 \quad 4.50000+0 \quad 5.50000+0 \quad 6.50000+0$

$7.25000+0.7 .75000+0 \quad 9.00000+0 \quad 1.20000+1$

df295 $2.14390-3 \quad 5.77600-4 \quad 2.71850-4 \quad 2.68170-4$

$3.27670-4 \quad 5.66760-4 \quad 8.75940-4 \quad 1.08450-3 \quad 1.27970-3$ $1.44170-3 \quad 1.75630-3 \quad 2.31560-3 \quad 2.92700-3 \quad 3.46860-3$

$3.95960-3 \quad 4.62210-3 \quad 5.41370-3 \quad 6.19090-3 \quad 6.92650-3$

$7.47830-3 \quad 7.84680-3 \quad 8.77160-3 \quad 1.10200-2$

$\operatorname{fm} 295 \quad 5.701+10$

sdef pos $=0$ o 0 vec=1 0 o dir=d1 erg=fdir $=d 2$

si1 s $111213 \quad 141516$

$\begin{array}{lllllll}\operatorname{sp} 1 & 1.17+10 & 8.60+9 & 1.21+10 & 8.82+9 & 1.47+10 & 1.09+9\end{array}$

si11 $h-1.0 \quad-0.7071067$

si12 $h-0.70710670 .0$

si13 h 0.00 .5 


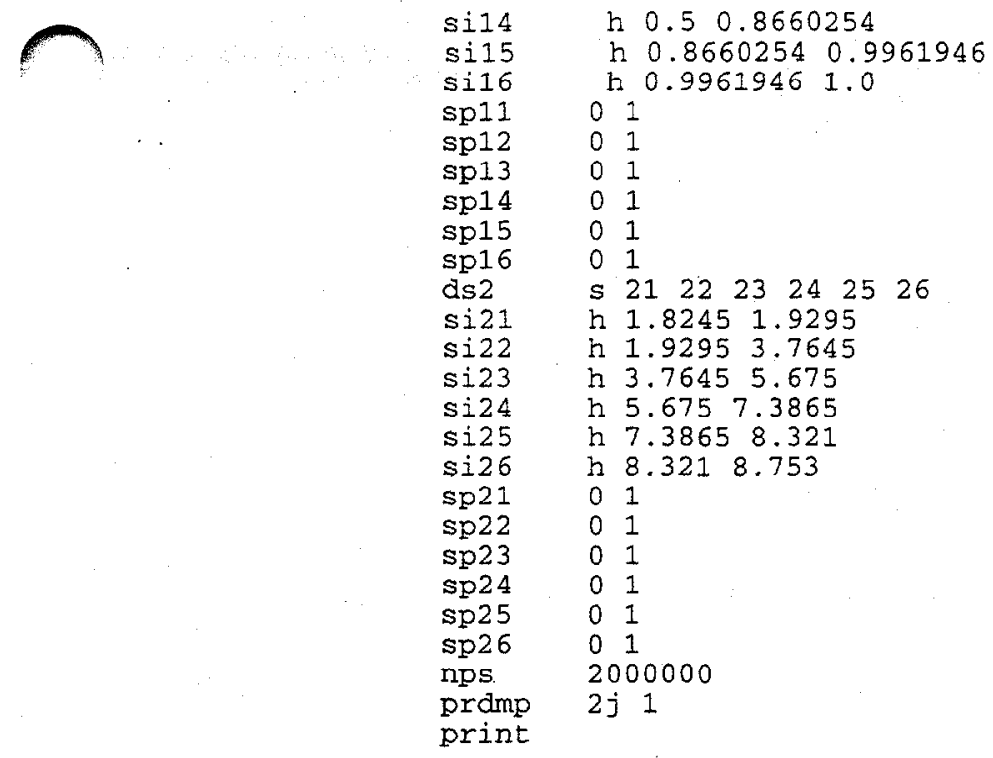


File for MCNP-4B Calculation of dose rates around the PFNA facility for the source in the maximum up position

message: outp=pfna14.o mctal=pfna14.m

mcnp file for PFNA facility -- dose rates at selected locations

c source in nominal maximum upward position ( $34.8 \mathrm{deg}$.) of the

c movable vertical collimator. check sdef, cells 3-6, surfaces

c 401 and 503-505, and the sign on surface 505 .

$-100: 128:-200: 251:-300: 305$

$2 \quad 0 \quad 100-128200-251300-305$

\#(131-164+258-297+307-321)$$
4
$$

$\begin{array}{ll}1 & 0.10549\end{array}$

$-400-401+505+224-227 \#(503-504)$

$-401+400+505+225-226 \#(503-504)$

$142-127+223-228+308-304$

$\#(-400-401+505+224-227) \#(+137-102+310)$

$\#(-401+400+505+225-226)$

$60127-124+501-502+308-304$

$\#(-401+400+505+225-226)$

$\begin{array}{lllllll}7 & 0 & & 124 & -126+254 & -255+308 & -304 \\ 8 & 5 & 8.734441-2 & 102 & -126+201 & -250+306 & -301\end{array}$

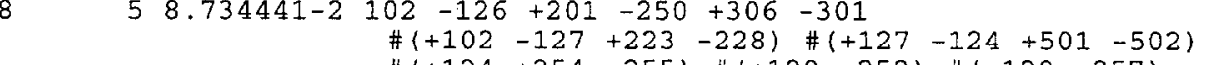

$9 \quad 10.10549$

$\#(+124+254-255) \quad \#(+129-252) \quad \#(+129+257)$

$10 \quad 10.10549$

$102-124+222-229+301-304$

$\#(+129-252) \quad \#(+129+257)$

$\#(127+501-502) \#(-127+223-228)$

$\begin{array}{ll}1 & 0.10549 \\ 1 & 0.10549\end{array}$

10.10549

10.10549

10.10549

10.10549

10.10549

10.10549

10.10549

10.10549

10.10549

20.1187956

20.1187956

$106-109+212-236+302-303$ \# $(218$

$109-110+211-237+302-303 \#(221-230)$

$110-111+210-238+302-303 \#(221-230)$

$111-112+209-239+302-303 \#(221-230)$

$112-113+208-240+302-303$ \# $221-230)$

$113-114+207-241+302-303$ \#(221-230)

$114-115+206-242+302-303$ \# $(221-230)$

$115-116+205-243+302-303 \#(221-230)$

$116-117+204-244+302-303 \#(221-230)$

$117-118+203-245+302-303 \#(221-230)$

$118-119+202-249+302-303 \#(221-230)$

$120-123+215-246+302-303 \#(221-230)$

$103-109+202-249+302-303$

\# $(221-230)$

$\#(103-104+217-234)$

$\#(104-105+216-235)$

$\#(105-107+219-232)$

$\#(107-108+219-220) \quad \#(107-108+231-232)$

20.1187956

$109-120+202-249+302-303$

\# $(221-230)$

$\#(109-110+211-237)$

$\#(110-111+210-238)$

$\#(111-112+209-239)$

$\#(112-113+208-240)$

$\#(113-114+207 \cdot-241)$

$\#(114-115+206-242)$

\#(115-116 +205-243)

$\#(116-117+204-244)$

$\#(117-118+203-245)$

\#(118-119)

$24 \quad 3 \quad 3.284-2$

$121-125+201-250+301-304$

$\#(-124+214-247) \quad \#(124+253-256)$

$\#(122+248) \#(122-213)$

$\begin{array}{llllll}25 & 3 & 3.284-2 & 103-107+217-234+302 & -303 \\ & & & \#(221-230) \#(104-220) & \#(104+231) \\ 26 & 4 & 5.977931-2 & 104-108+216 & -220+302-303 \#(105\end{array}$

$\begin{array}{llllll}25 & 3 & 3.284-2 & 103-107+217-234+302 & -303 \\ & & & \#(221-230) \#(104-220) & \#(104+231) \\ 26 & 4 & 5.977931-2 & 104-108+216 & -220+302-303 \#(105\end{array}$

$26445.977931-2 \quad 104-108+216-220+302-303 \quad \#(105-219)$

$\begin{array}{lllllllll}27 & 4 & 5.977931-2 & 104 & -108 & +231 & -235 & +302 & -303 \\ 28 & 4 & 5.977931-2 & 102 & -124 & +201 & -222 & +301 & -304\end{array}$

$\#\left(\begin{array}{llllll}103 & -120 & 202 & -221 & 302 & -303\end{array}\right)$

$\#\left(\begin{array}{llllll}120 & -123 & 215 & -221 & 302 & -303\end{array}\right)$

\# $(121-214)$ 


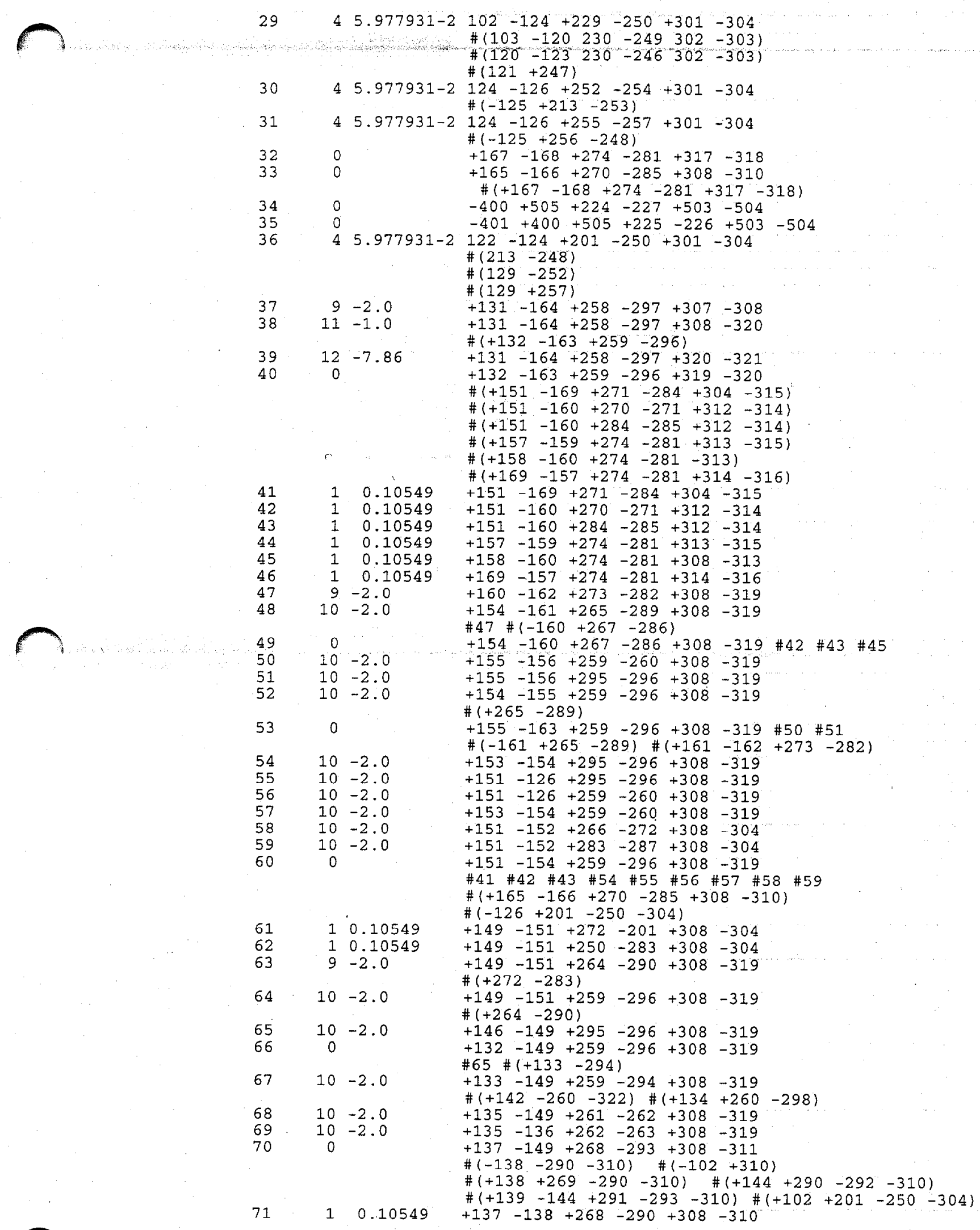




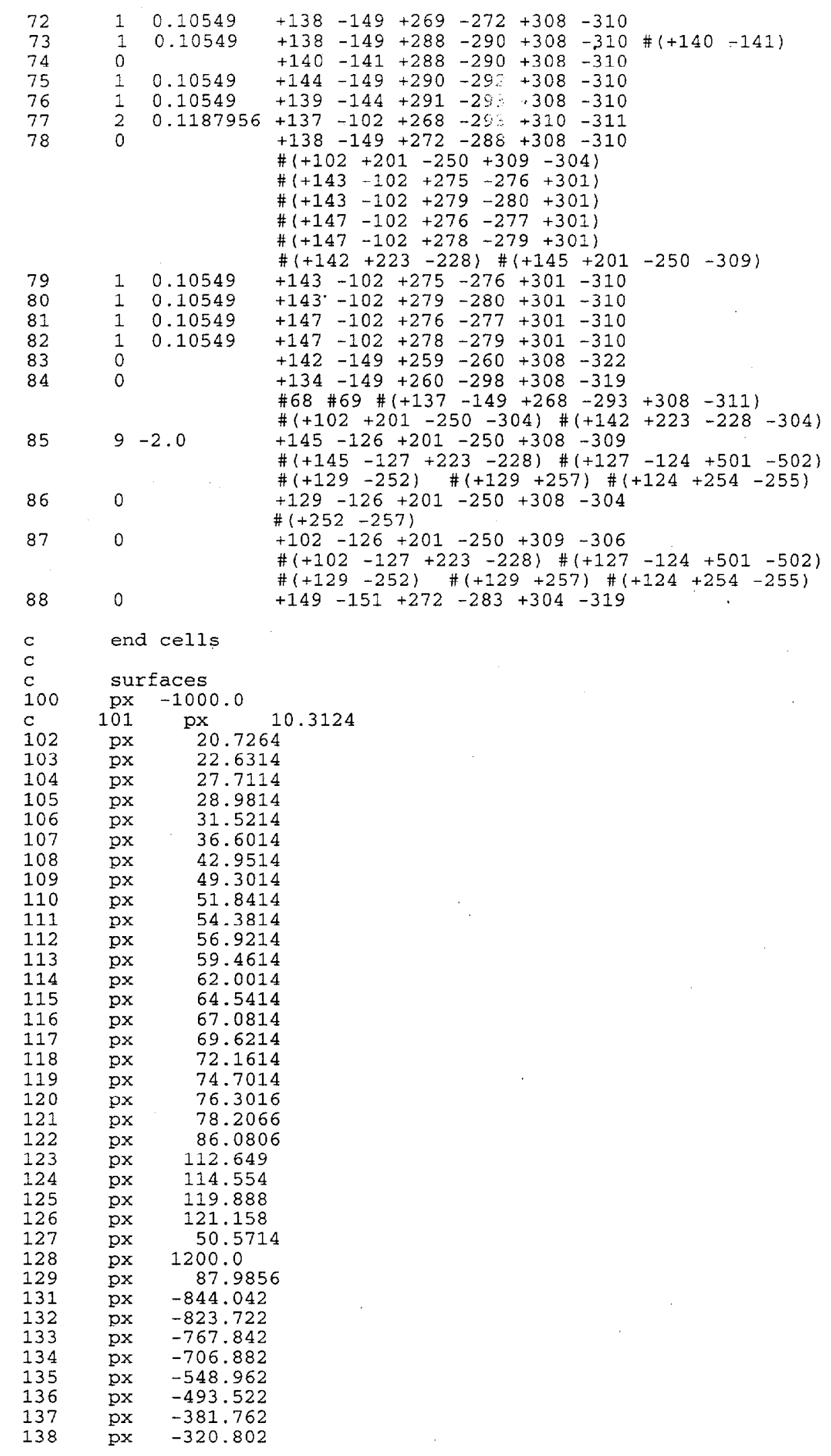




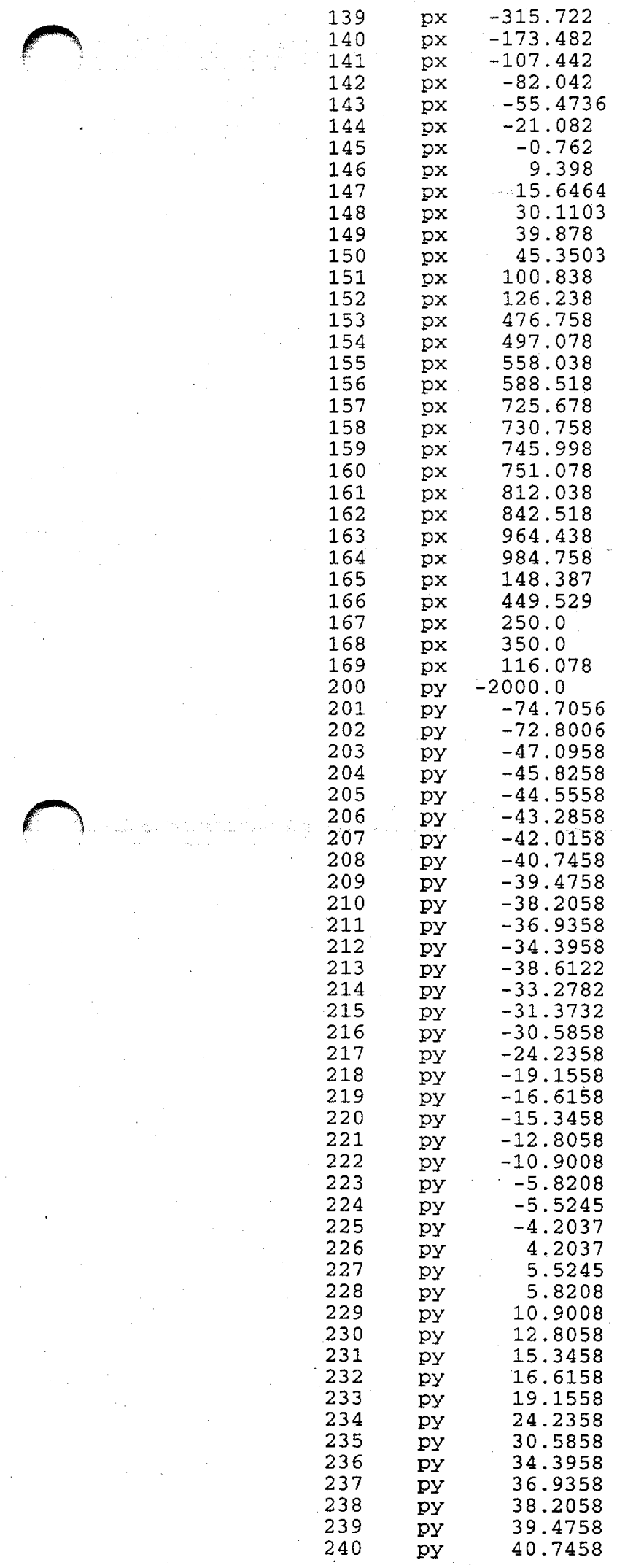




\begin{tabular}{|c|c|c|}
\hline 241 & py & 42.0158 \\
\hline 242 & py & 43.2858 \\
\hline 243 & py & 44.5558 \\
\hline 244 & py & 45.8258 \\
\hline 245 & py & 47.0958 \\
\hline 246 & py & 31.3732 \\
\hline 247 & py & 33.2782 \\
\hline 248 & py & 38.6122 \\
\hline 249 & py & 72.8006 \\
\hline 250 & py & 74.7056 \\
\hline 251 & PY & 1500.0 \\
\hline 252 & py & -39.8822 \\
\hline 253 & py & -9.8848 \\
\hline 254 & $\mathrm{pY}$ & -8.6148 \\
\hline לb & py & 8.6148 \\
\hline 256 & py & 9.8848 \\
\hline 257 & pY & 39.8822 \\
\hline 258 & py & -1731.28 \\
\hline 259 & pY & -1710.96 \\
\hline 260 & py & -1650.0 \\
\hline 261 & py & -1528.08 \\
\hline 262 & py & -1467.12 \\
\hline 263 & py & -1436.64 \\
\hline 264 & py & -589.28 \\
\hline 265 & py & -241.3 \\
\hline 266 & py & -193.04 \\
\hline 267 & py & -180.34 \\
\hline 268 & py & -167.64 \\
\hline 269 & pY & -162.56 \\
\hline 270 & py & -152.4 \\
\hline 271 & py & -137.16 \\
\hline 272 & py & -101.6 \\
\hline 273 & py & -91.44 \\
\hline 274 & py & -60.96 \\
\hline 275 & PY & -45.72 \\
\hline 276 & py & -30.48 \\
\hline 277 & py & -10.16 \\
\hline 278 & PY & 10.16 \\
\hline 279 & py & 30.48 \\
\hline 280 & py & 45.72 \\
\hline 281 & $\mathrm{PY}$ & 60.96 \\
\hline 282 & py & 91.44 \\
\hline 283 & py & 101.6 \\
\hline 284 & py & 137.16 \\
\hline 285 & pY & 152.4 \\
\hline 286 & $\mathrm{pY}$ & 180.34 \\
\hline 287 & py & 193.04 \\
\hline 288 & PY & 213.36 \\
\hline 289 & $\mathrm{PY}$ & 241.3 \\
\hline 290 & py & 274.32 \\
\hline 291 & py & 335.28 \\
\hline 292 & PY & 345.44 \\
\hline 293 & py & 396.24 \\
\hline 294 & py & 533.4 \\
\hline 295 & py & 1235.44 \\
\hline 296 & py & 1296.4 \\
\hline 297 & py & 1316.72 \\
\hline 298 & pY & 472.44 \\
\hline 300 & $\mathrm{pz}$ & -500.0 \\
\hline 301 & $\mathrm{pz}$ & -59.478 \\
\hline 302 & $\mathrm{pz}$ & -57.573 \\
\hline 303 & $\mathrm{pz}$ & 245.957 \\
\hline 304 & $p z$ & 247.862 \\
\hline 305 & $p z$ & 500.0 \\
\hline 306 & $\mathrm{pz}$ & -60.748 \\
\hline 307 & $\mathrm{pz}$ & -156.0 \\
\hline 308 & $p z$ & -125.095 \\
\hline 309 & $p z$ & -67.451 \\
\hline 310 & $p z$ & 235.585 \\
\hline 311 & $p z$ & 276.225 \\
\hline 312 & $p z$ & 283.845 \\
\hline 313 & $p z$ & 342.265 \\
\hline
\end{tabular}




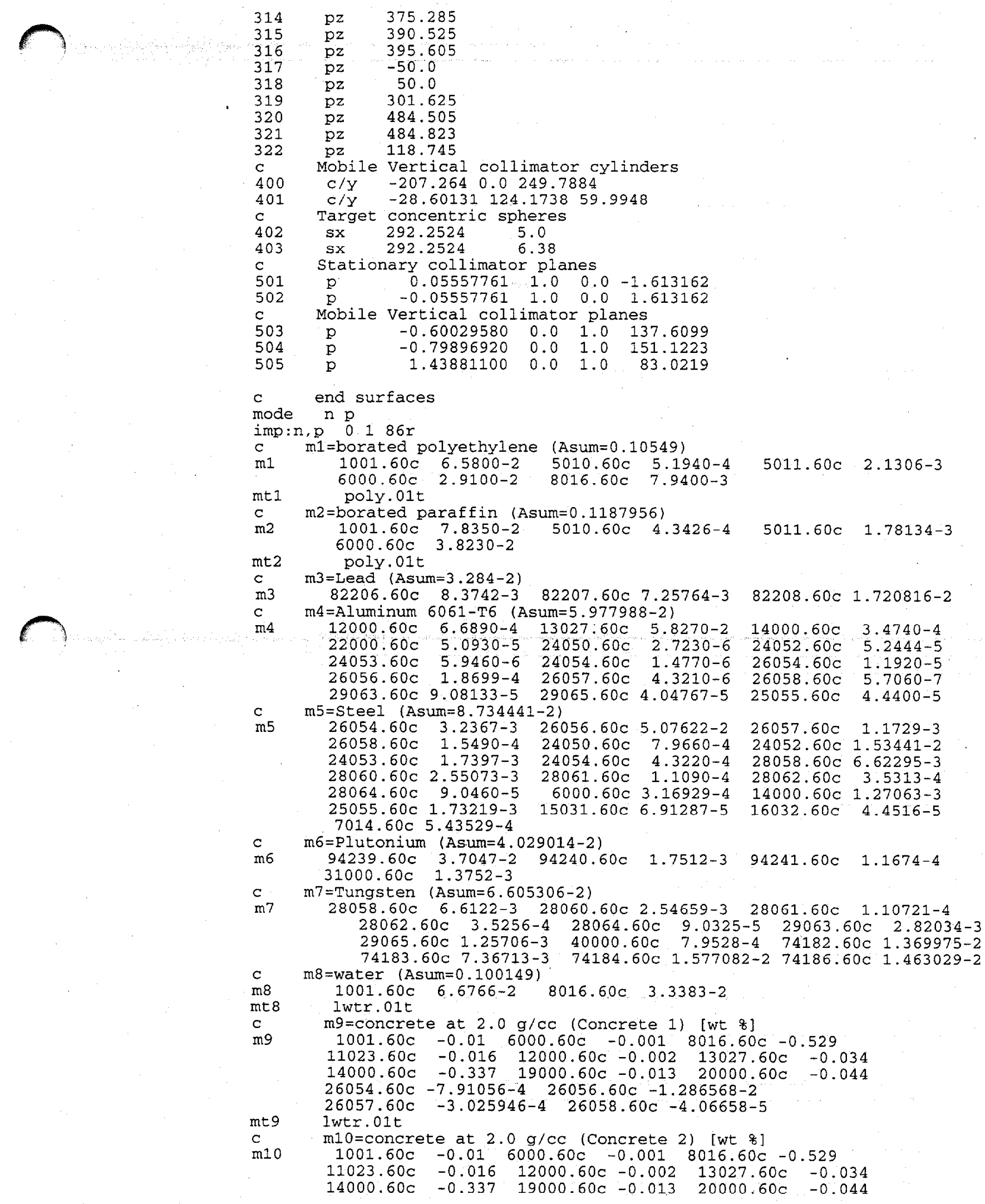




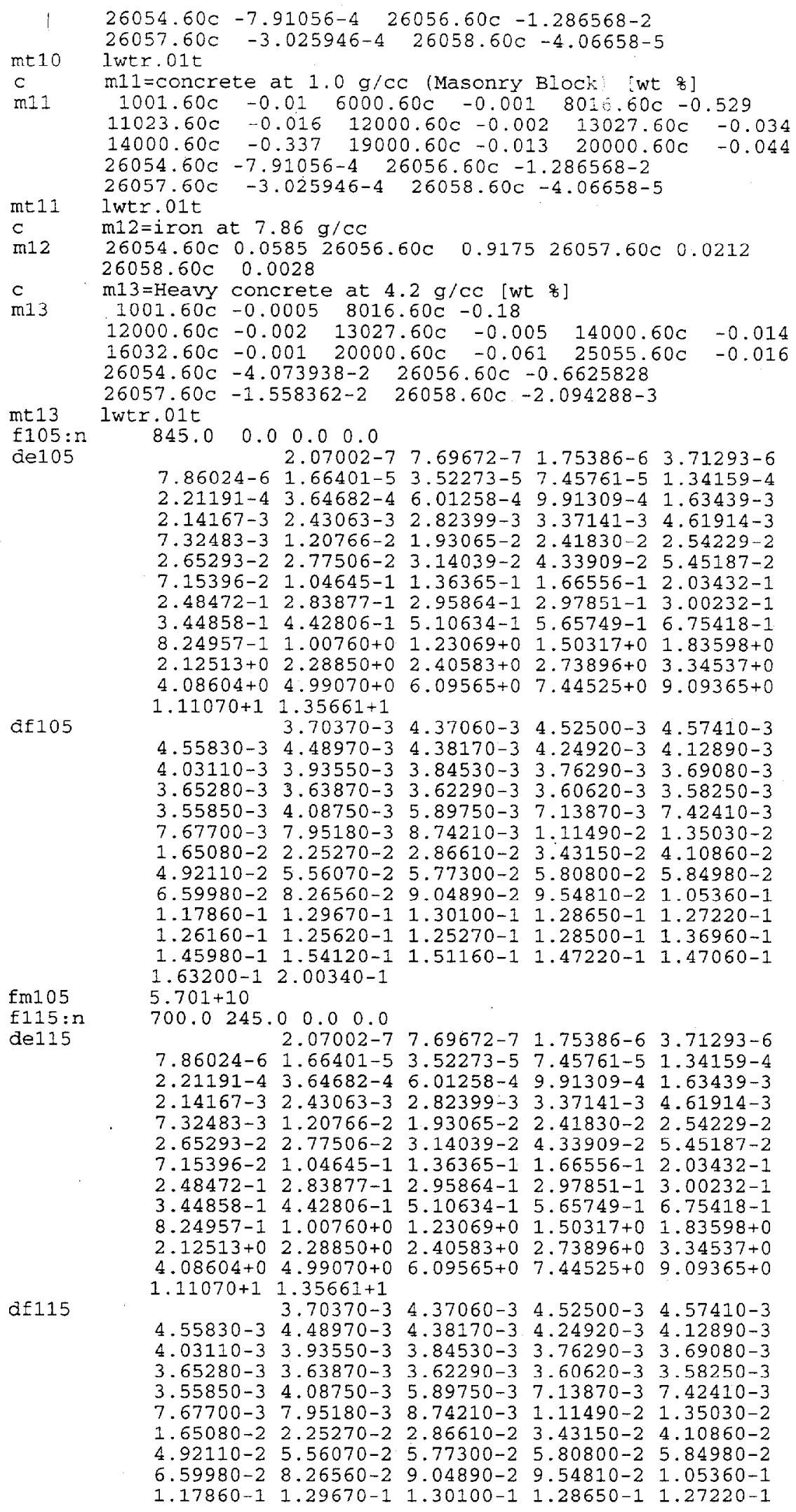




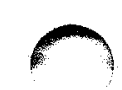

$1.26160-1 \quad 1.25620-1 \quad 1.25270-1 \quad 1.28500-1 \quad 1.36960-1$ $1.45980-1$ 1.54120-1 $1.51160-1 \quad 1.47220-1 \quad 1.47060-1$ $1.63200-1.2 .00340-1$.

fm115 $\quad 5.701+10$

f125:n $\quad 725.0 \quad 0.0 \quad 0.0 \quad 0.0$

de125 $2.07002-7 \quad 7.69672-7 \quad 1.75386-6 \quad 3.71293 \ldots 6$

$7.86024-6 \quad 1.66401-5 \quad 3.52273-5 \quad 7.45761-5 \quad 1.34159-4$

$2.21191-4 \quad 3.64682-4 \quad 6.01258-4 \quad 9.91309-4 \quad 1.63439-3$

$2.14167-3 \quad 2.43063-3 \quad 2.82399-3 \quad 3.37141-3 \quad 4.61914-3$

$7.32483-3 \quad 1.20766-2 \quad 1.93065-2 \quad 2.41830-2 \quad 2.54229-2$

$2.65293-2 \quad 2.77506-2 \quad 3.14039-2 \quad 4.33909-2.5 .45187-2$

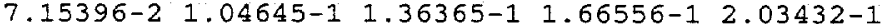

$2.48472-1 \quad 2.83877-1 \quad 2.95864-1 \quad 2.97851-1 \quad 3.00232-1$

3.44858-1 4.42806-1 $5.10634-1 \quad 5.65749-1 \quad 6.75418-1$

$8.24957-1 \quad 1.00760+0 \quad 1.23069+0 \quad 1.50317+0 \quad 1.83598+0$

$\begin{array}{lllll}2.12513+0 & 2.28850+0 & 2.40583+0 & 2.73896+0 & 3.34537+0\end{array}$

$4.08604+0 \quad 4.99070+0 \quad 6.09565+0 \quad 7.44525+0 \quad 9.09365+0$

$1.11070+11.35661+1$

df125 $3.70370-3 \quad 4.37060-3 \quad 4.52500-3 \quad 4.57410-3$

$4.55830-3 \quad 4.48970-3 \quad 4.38170-3 \quad 4.24920-3 \quad 4.12890-3$

$4.03110-3 \quad 3.93550-3 \quad 3.84530-3 \quad 3.76290-3 \quad 3.69080-3$

$3.65280-3 \quad 3.63870-3 \quad 3.62290-3 \quad 3.60620-3 \quad 3.58250-3$

$3.55850-3 \quad 4.08750-3 \quad 5.89750-3 \quad 7.13870-3 \quad 7.42410-3$

$7.67700-3 \quad 7.95180-3 \quad 8.74210-3.1 .11490-2 \quad 1.35030-2$

$1.65080-2 \quad 2.25270-2 \quad 2.86610-2 \quad 3.43150-2 \quad 4.10860-2$

$4.92110-2 \quad 5.56070-2 \quad 5.77300-2 \quad 5.80800-2 \quad 5.84980-2$

$6.59980-2 \quad 8.26560-2 \quad 9.04890-2 \quad 9.54810-2 \quad 1.05360-1$

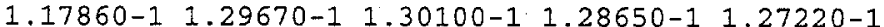

$1.26160-1$ 1.25620-1 $1.25270-1 \quad 1.28500-1 \quad 1.36960-1$

$1.45980-1$ 1.54120-1 $1.51160-1 \quad 1.47220-1 \quad 1.47060-1$ $1.63200-12.00340-1$

fm.125 $\quad 5.701+10$

f135:n $298.958-1710.00 .00 .0$

de135 $2.07002-7 \quad 7.69672-7 \quad 1.75386-6 \quad 3.71293-6$

$7.86024-6 \quad 1.66401-5 \quad 3.52273-5 \quad 7.45761-5 \quad 1.34159-4$

$2.21191-4 \quad 3.64682-4 \quad 6.01258-4 \quad 9.91309-4 \quad 1.63439-3$

$2.14167-3 \quad 2.43063-3 \quad 2.82399-3 \quad 3.37141-3 \quad 4.61914-3$

$7.32483-3 \quad 1.20766-2 \quad 1.93065-2 \quad 2.41830-2 \quad 2.54229-2$ $2.65293-2 \quad 2.77506-2 \quad 3.14039-2 \quad 4.33909-2.5 .45187-2$ 7.15396-2 1.04645-1 $1.36365-1 \quad 1.66556-1 \quad 2.03432-1$ $2.48472-1 \quad 2.83877-1 \quad 2.95864-1 \quad 2.97851-1 \quad 3.00232-1$ 3.44858-1 $4.42806-1 \quad 5.10634-1 \quad 5.65749-1 \quad 6.75418-1$ 8.24957-1 $1.00760+0 \quad 1.23069+0 \quad 1.50317+0 \quad 1.83598+0$ $2.12513+0.2 .28850+0 \quad 2.40583+0 \quad 2.73896+0 \quad 3.34537+0$ $4.08604+0 \quad 4.99070+0 \quad 6.09565+0 \quad 7.44525+0 \quad 9.09365+0$ $1.11070+1 \quad 1.35661+1$

df135 $3.70370-3 \quad 4.37060-3.4 .52500-34.57410-3$ $4.55830-3 \quad 4.48970-3 \quad 4.38170-3 \quad 4.24920-3 \quad 4.12890-3$ $4.03110-3 \quad 3.93550-3 \quad 3.84530-3 \quad 3.76290-3 \quad 3.69080-3$ $\begin{array}{llllll}3.65280-3 & 3.63870-3 & 3.62290-3 & 3.60620-3 & 3.58250-3\end{array}$ $3.55850-3 \quad 4.08750-3 \quad 5.89750-3 \quad 7.13870-3 \quad 7.42410-3$ $7.67700-3 \quad 7.95180-3 \quad 8.74210-3 \quad 1.11490-2 \quad 1.35030-2$ $1.65080-2 \quad 2.25270-2 \quad 2.86610-2 \quad 3.43150-2 \quad 4.10860-2$ $4.92110-25.56070-25.77300-2 \cdot 5.80800-2 \quad 5.84980-2$ $6.59980-2 \quad 8.26560-2 \quad 9.04890-2 \quad 9.54810-2 \quad 1.05360-1$ 1.17860-1 $1.29670-1 \quad 1.30100-1 \quad 1.28650-1 \quad 1.27220-1$ $\begin{array}{lllll}1.26160-1 & 1.25620-1 & 1.25270-1 & 1.28500-1 & 1.36960-1\end{array}$ $1.45980-1$ 1.54120-1 $1.51160-1$ 1.47220-1 1.47060-1 $1.63200-1 \quad 2.00340-1$

fm135 $\quad 5.701+10$

f145:n $298.958 \quad 1296.0 \quad 0.00 .0$

$\begin{array}{lrlllll}\text { del45 } & 2.07002-7 & 7.69672-7 & 1.75386-6 & 3.71293-6\end{array}$ $7.86024-6 \quad 1.66401-5 \quad 3.52273-5 \quad 7.45761-5 \quad 1.34159-4$ $\begin{array}{lllllll}2.21191-4 & 3.64682-4 & 6.01258-4 & 9.91309-4 & 1.63439-3\end{array}$ $\begin{array}{lllllll}2.14167-3 & 2.43063-3 & 2.82399-3 & 3.37141-3 & 4.61914-3\end{array}$ $\begin{array}{lllll}7.32483-3 & 1.20766-2 & 1.93065-2 & 2.41830-2 & 2.54229-2\end{array}$ 2.65293-2 2.77506-2 $3.14039-2 \quad 4.33909-2 \quad 5.45187-2$ 7.15396-2 $1.04645-1 \quad 1.36365-1 \quad 1.66556-1$ 2.03432-1 $2.48472-1 \quad 2.83877-1 \quad 2.95864-1 \quad 2.97851-1.3 .00232-1$ 3.44858-1 4.42806-1 5.10634-1 5.657449-1 $6.75418-1$ $8.24957-1 \quad 1.00760+01.23069+0 \quad 1.50317+0 \quad 1.83598+0$ $\begin{array}{lllll}2.12513+0 & 2.28850+0 & 2.40583+0 & 2.73896+0 & 3.34537+0\end{array}$ 
$4.08604+0 \quad 4.99070+0 \quad 6.09565+0 \quad 7.44525+0 \quad 9.09365+0$ $1.11070+1 \quad 1.35661+1$

df] $45 \quad 3.70370-3 \quad 4.37060-3 \quad 4.52500-3 \quad 4.57410-3$ $4.55830-3 \quad 4.48970-3 \quad 4.38170-3 \quad 4.24920-3 \quad 4.12890-3$ $\begin{array}{lllll}4.03110-3 & 3.93550-3 & 3.84530-3 & 3.76290-3 & 3.69080-3\end{array}$ $3.65280-3 \quad 3.63870-3 \quad 3.62290-3 \quad 3.60620-3.3 .58250-3$ $3.55850-3 \quad 4.08750-3 \quad 5.89750-3 \quad 7.13870-3 \cdot 7.42410-3$ $\begin{array}{lllll}7.67700-3 & 7.95180-3 & 8.74210-3 & 1.11490-2 & 1.35030-2\end{array}$ $1.65080-2 \quad 2.25270-2 \quad 2.86610-2 \quad 3.43150-2 \quad 4.10860-2$ $4.92110-2 \quad 5.56070-2 \quad 5.77300-2 \quad 5.80800-2 \quad 5.84980-2$ $6.59980-2 \quad 8.26560-2 \quad 9.04890-2 \quad 9.54810-2 \quad 1.05360-1$

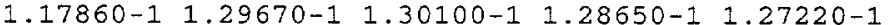
$1.26160-1 \quad 1.25620-1 \quad 1.25270-1 \quad 1.28500-1 \quad 1.36960-1$ $1.45980-1 \quad 1.54120-1 \quad 1.51160-1 \quad 1.47220-1 \quad 1.47060-1$

$\operatorname{fm} 145$ f155: $\mathrm{n}$ de155

dfI55

fm155

f165:n

de165

df165

$\operatorname{fm} 165$
$1.63200-12.00340-1$

$5.701+10$

$298.958 \quad 0.0 \quad 0.0 \quad 0.0$

$2.07002-7 \quad 7.69672-7 \quad 1.75386-6 \quad 3.71293-6$

7.86024-6 $1.66401-5 \quad 3.52273-5 \quad 7.45761-5 \quad 1.34159-4$ $2.21191-4 \quad 3.64682-4 \quad 6.01258-4 \quad 9.91309-4 \quad 1.63439-3$ $2.14167-3 \quad 2.43063-3 \quad 2.82399-3 \quad 3.37141-3 \quad 4.61914-3$ 7.32483-3 1.20766-2 $1.93065-2 \quad 2.41830-2 \quad 2.54229-2$ $2.65293-2 \quad 2.77506-2 \quad 3.14039-2 \quad 4.33909-2 \quad 5.45187-2$ 7.15396-2 1.04645-1 $1.36365-1 \quad 1.66556-1 \quad 2.03432-1$ $\begin{array}{llllll}2.48472-1 & 2.83877-1 & 2.95864-1 & 2.97851-1 & 3.00232-1\end{array}$ $3.44858-1 \quad 4.42806-1 \quad 5.10634-1 \quad 5.65749-1 \quad 6.75418-1$ $8.24957-1 \quad 1.00760+0 \quad 1.23069+0 \quad 1.50317+0 \quad 1.83598+0$ $2.12513+0 \quad 2.28850+0 \quad 2.40583+0 \quad 2.73896+0 \quad 3.34537+0$ $4.08604+0 \quad 4.99070+0 \quad 6.09565+0 \quad 7.44525+0 \quad 9.09365+0$ $1.11070+11.35661+1$

3. $70370-3 \quad 4.37060-3 \quad 4.52500-3 \quad 4.57410-3$ $4.55830-3 \quad 4.48970-3 \quad 4.38170-3 \quad 4.24920-3 \quad 4.12890-3$ $\begin{array}{llllll}4.03110-3 & 3.93550-3 & 3.84530-3 & 3.76290-3 & 3.69080-3\end{array}$ $\begin{array}{llllll}3.65280-3 & 3.63870-3 & 3.62290-3 & 3.60620-3 & 3.58250-3\end{array}$ $3.55850-3 \quad 4.08750-3 \quad 5.89750-3 \quad 7.13870-3 \quad 7.42410-3$ $\begin{array}{llllll}7.67700-3 & 7.95180-3 & 8.74210-3 & 1.11490-2 & 1.35030-2\end{array}$ $1.65080-2 \quad 2.25270-2 \quad 2.86610-2 \quad 3.43150-2 \quad 4.10860-2$ $4.92110-2 \quad 5.56070-2 \quad 5.77300-2 \quad 5.80800-2 \quad 5.84980-2$ $6.59980-2 \quad 8.26560-2 \quad 9.04890-2 \quad 9.54810-2 \quad 1.05360-1$ $1.17860-1 \quad 1.29670-1 \quad 1.30100-1 \quad 1.28650-11.27220-1$ $1.26160-11.25620-1 \quad 1.25270-1 \quad 1.28500-11.36960-1$ $1.45980-1 \quad 1.54120-1 \quad 1.51160-1 \quad 1.47220-1 \quad 1.47060-1$ $1.63200-12.00340-1$

$5.701+10$

$\begin{array}{lllll}-385.0 & 300.0 & 0.0 & 0.0\end{array}$

$2.07002-7 \quad 7.69672-7 \quad 1.75386-6 \quad 3.71293-6$

7.86024-6 $1.66401-5 \quad 3.52273-5 \quad 7.45761-5 \quad 1.34159-4$

2.21191-4 $3.64682-4 \quad 6.01258-4 \quad 9.91309-4 \quad 1.63439-3$ 2.14167-3 2.43063-3 $2.82399-3 \quad 3.37141-3.4 .61914-3$ $\begin{array}{llllll}7.32483-3 & 1.20766-2 & 1.93065-2 & 2.41830-2 & 2.54229-2\end{array}$ 2.65293-2 2.77506-2 3.14039-2 4.33909-2 5.45187-2 7.15396-2 $1.04645-1 \quad 1.36365-1 \quad 1.66556-1 \quad 2.03432-1$ 2.48472-1 $2.83877-1 \quad 2.95864-1 \quad 2.97851-1 \quad 3.00232-1$

3.44858-1 $4.42806-1 \quad 5.10634-1 \quad 5.65749-1 \quad 6.75418-1$ $\begin{array}{lllll}8.24957-1 & 1.00760+0 & 1.23069+0 & 1.50317+0 & 1.83598+0\end{array}$ $2.12513+0 \quad 2.28850+0 \quad 2.40583+0 \quad 2.73896+0 \quad 3.34537+0$ $4.08604+0 \quad 4.99070+0 \quad 6.09565+0 \quad 7.44525+0 \quad 9.09365+0$ $1.11070+1 \quad 1.35661+1$ $3.70370-3 \quad 4.37060-3 \quad 4.52500-3 \quad 4.57410-3$ $4.55830-3 \quad 4.48970-3 \quad 4.38170-3 \quad 4.24920-3 \quad 4.12890-3$ $\begin{array}{llllll}4.03110-3 & 3.93550-3 & 3.84530-3 & 3.76290-3 & 3.69080-3\end{array}$ $3.65280-3 \quad 3.63870-3 \quad 3.62290-3 \quad 3.60620-3 \quad 3.58250-3$ $\begin{array}{llllll}3.55850-3 & 4.08750-3 & 5.89750-3 & 7.13870-3 & 7.42410-3\end{array}$ $7.67700-3 \quad 7.95180-3 \quad 8.74210-3 \quad 1.11490-2 \quad 1.35030-2$ $1.65080-2 \quad 2.25270-2 \quad 2.86610-2 \quad 3.43150-2 \quad 4.10860-2$ $\begin{array}{llllll}4.92110-2 & 5.56070-2 & 5.77300-2 & 5.80800-2 & 5.84980-2\end{array}$ $6.59980-2 \quad 8.26560-2 \quad 9.04890-2 \quad 9.54810-2 \quad 1.05360-1$ $1.17860-1 \quad 1.29670-1 \quad 1.30100-1 \quad 1.28650-11.27220-1$ $\begin{array}{llllll}1.26160-1 & 1.25620-1 & 1.25270-1 & 1.28500-1 & 1.36960-1\end{array}$ $1.45980-1 \quad 1.54120-1 \quad 1.51160-1 \quad 1.47220-1 \quad 1.47060-1$ $1.63200-12.00340-1$

$5.701+10$ 


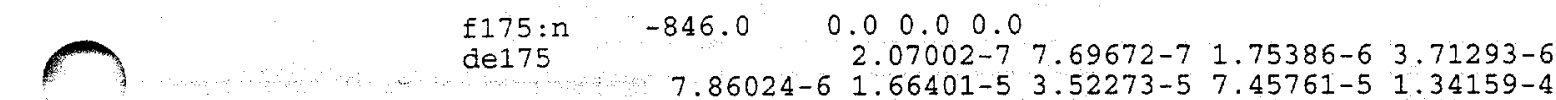

2.21191-4 $3.64682-4 \quad 6.01258-4 \quad 9.91309-4 \quad 1.63439-3$

$\begin{array}{llllll}2.14167-3 & 2.43063-3 & 2.82399-3 & 3.37141-3 & 4.61914-3\end{array}$

$7.32483-3 \quad 1.20766-2 \quad 1.93065-2 \quad 2.41830-2 \quad 2.54229-2$

2.65293-2 2.77506-2 3.14039-2 $4.33909-2 \quad 5.45187-2$

$7.15396-2$ 1.04645-1 $1.36365-1$ 1.66556-1 $2.03432-1$

$\begin{array}{llllll}2.48472-1 & 2.83877-1 & 2.95864-1 & 2.97851-1 & 3.00232-1\end{array}$

3.44858-1 4.42806-1 $5.10634-1 \quad 5.65749-1 \quad 6.75418-1$

$8.24957-1 \quad 1.00760+0 \quad 1.23069+0 \quad 1.50317+0 \quad 1.83598+0$

$2.12513+0 \quad 2.28850+0 \quad 2.40583+0 \quad 2.73896+0 \quad 3.34537+0$

$4.08604+0 \quad 4.99070+0 \quad 6.09565+0 \quad 7.44525+0 \quad 9.09365+0$

$1.11070+1 \quad 1.35661+1$

df175 $3.70370-3 \quad 4.37060-3 \quad 4.52500-3 \quad 4.57410-3$

$\begin{array}{llllll}4.55830-3 & 4.48970-3 & 4.38170-3 & 4.24920-3 & 4.12890-3\end{array}$

$4.03110-3 \quad 3.93550-3 \quad 3.84530-3 \quad 3.76290-3 \quad 3.69080-3$

$3.65280-3 \quad 3.63870-3 \quad 3.62290-3 \quad 3.60620-3 \quad 3.58250-3$

$3.55850-3 \quad 4.08750-3 \quad 5.89750-3 \quad 7.13870-3 \quad 7.42410-3$

$7.67700-3 \quad 7.95180-3 \quad 8.74210-3 \quad 1.11490-2 \quad 1.35030-2$

$1.65080-2 \quad 2.25270-2 \quad 2.86610-2 \quad 3.43150-2 \quad 4.10860-2$

$4.92110-25.56070-2.5 .77300-2 \quad 5.80800-2 \quad 5.84980-2$

$6.59980-2 \quad 8.26560-2 \quad 9.04890-2 \quad 9.54810-2 \quad 1.05360-1$

1.17860-1 $1.29670-1 \quad 1.30100-1 \quad 1.28650-1 \quad 1.27220-1$

$1.26160-1$ 1.25620-1 $1.25270-1$ 1.28500-1 $1.36960-1$

$1.45980-1$ 1.54120-1 $1.51160-1 \quad 1.47220-1 \quad 1.47060-1$ $1.63200-12.00340-1$

$\operatorname{fm} 175 \quad 5.701+10$

f185:n $\quad 986.0 \quad 0.0 \quad 0.0 .0 .0$

$\begin{array}{llllll}\text { de185 } & 2.07002-7 & 7.69672-7 & 1.75386-6 & 3.71293-6\end{array}$

7.86024-6 $1.66401-5 \quad 3.52273-5 \quad 7.45761-5 \quad 1.34159-4$

$2.21191-4 \quad 3.64682-4 \quad 6.01258-4 \quad 9.91309-4 \quad 1.63439-3$

2.14167-3 $2.43063-3 \quad 2.82399-3 \quad 3.37141-3 \quad 4.61914-3$

$7.32483-3.1 .20766-2 \quad 1.93065-2 \quad 2.41830-2 \quad 2.54229-2$

$2.65293-2$ 2.77506-2 $3.14039-2$ 4.33909-2 $5.45187-2$

7.15396-2 $1.04645-1 \quad 1.36365-1 \quad 1.66556-1 \quad 2.03432-1$

2.48472-1 $2.83877-1 \quad 2.95864-1 \quad 2.97851-1 \quad 3.00232-1$

$3.44858-1 \quad 4.42806-1 \quad 5.10634-1 \quad 5.65749-1 \quad 6.75418-1$

$8.24957-1 \quad 1.00760+0 \quad 1.23069+0 \quad 1.50317+0 \quad 1.83598+0$

$\begin{array}{llllll}2.12513+0 & 2.28850+0 & 2.40583+0 & 2.73896+0 & 3.34537+0\end{array}$

$4.08604+0 \quad 4.99070+0 \quad 6.09565+0 \quad 7.44525+0 \quad 9.09365+0$

$1.11070+1 \quad 1.35661+1$

df185 $\quad 3.70370-3 \quad 4.37060-3 \quad 4.52500-3 \quad 4.57410-3$

$4.55830-3 \quad 4.48970-3 \quad 4.38170-3 \quad 4.24920-3 \quad 4.12890-3$

$4.03110-3 \quad 3.93550-3 \quad 3.84530-3 \quad 3.76290-3 \quad 3.69080-3$

$3.65280-3 \quad 3.63870-3 \quad 3.62290-3 \quad 3.60620-3.3 .58250-3$

$3.55850-3 \quad 4.08750-3 \quad 5.89750-3 \quad 7.13870-3 \quad 7.42410-3$

$7.67700-3 \quad 7.95180-3 \quad 8.74210-3 \quad 1.11490-2 \quad 1.35030-2$

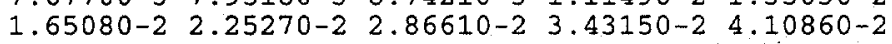

$4.92110-2 \quad 5.56070-2 \quad 5.77300-2 \quad 5.80800-2 \quad 5.84980-2$

$6.59980-2 \quad 8.26560-2 \quad 9.04890-2 \quad 9.54810-2 \quad 1.05360-1$

$1.17860-1 \quad 1.29670-1 \quad 1.30100-1 \quad 1.28650-1 \quad 1.27220-1$

$1.26160-1 \quad 1.25620-1 \quad 1.25270-1 \quad 1.28500-1 \quad 1.36960-1$

$1.45980-1$ 1.54120-1 $1.51160-1$ 1.47220-1 1.47060-1 $1.63200-12.00340-1$

fm $185 \quad 5.701+10$

f195:n $\quad-385.0 \quad 0.0 \quad 0.0 \quad 0.0$

de195 $2.07002-7 \quad 7.69672-7 \quad 1.75386-6 \quad 3.71293-6$

$\begin{array}{lllll}7.86024-6 & 1.66401-5 & 3.52273-5 & 7.45761-5 & 1.34159-4\end{array}$

$2.21191-4 \quad 3.64682-4 \quad 6.01258-4 \quad 9.91309-4 \quad 1.63439-3$

$2.14167-3 \quad 2.43063-3 \quad 2.82399-3 \quad 3.37141-3 \quad 4.61914-3$

$7.32483-3$ 1.20766-2 $1.93065-2 \quad 2.41830-2 \quad 2.54229-2$

$2.65293-2 \quad 2.77506-2 \quad 3.14039-2 \quad 4.33909-2 \quad 5.45187-2$

$7.15396-2$ 1.04645-1 $1.36365-1 \quad 1.66556-1 \quad 2.03432-1$

$\begin{array}{lllll}2.48472-1 & 2.83877-1 & 2.95864-1 & 2.97851-1 & 3.00232-1\end{array}$

3.44858-1 $4.42806-1 \quad 5.10634-1 \quad 5.65749-1 \quad 6.75418-1$

$8.24957-1 \quad 1.00760+0 \quad 1.23069+0 \quad 1.50317+0 \quad 1.83598+0$

$2.12513+0 \quad 2.28850+0 \quad 2.40583+0 \quad 2.73896+0 \quad 3.34537+0$

$4.08604+0 \quad 4.99070+0 \quad 6.09565+0 \quad 7.44525+0 \quad 9.09365+0$

$1.11070+11.35661+1$

df195 $3.70370-3 \quad 4.37060-3 \quad 4.52500-3 \quad 4.57410-3$

$4.55830-3 \quad 4.48970-3 \quad 4.38170-3 \quad 4.24920-3 \quad 4.12890-3$ 
$\begin{array}{llllll}4.03110-3 & 3.93550-3 & 3.84530-3 & 3.76290-3 & 3.69080-3\end{array}$ $3.65280-3 \quad 3.63870-3 \quad 3.62290-3 \quad 3.60620-3 \quad 3.58250-3$ $\begin{array}{llllll}3.55850-3 & 4.08750-3 & 5.89750-3 & 7.13870-3 & 7.42410-3\end{array}$ $\begin{array}{llllll}7.67700-3 & 7.95180-3 & 8.74210-3 & 1.11490-2 & 1.35030-2\end{array}$

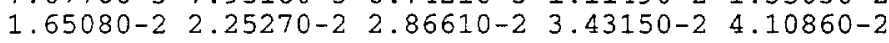
$\begin{array}{lllll}1.92110-2 & 5.56070-2 & 5.77300-2 & 5.80800-2 & 5.84980-2\end{array}$ $6.59980-2 \quad 8.26560-2 \quad 9.04890-2 \quad 9.54810-2 \quad 1.05360-1$ $1.17860-1 \quad 1.29670-1 \quad 1.30100-1 \quad 1.28650-11.27220-1$

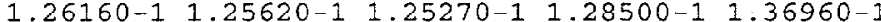
$1.45980-1$ 1.54120-1 $1.51160-1 \quad 1.47220-1 \quad 1.47060-1$ $1.63200-12.00340-1$

fm195 $5.701+10$ f205:p $\quad 845.0 \quad 0.0 \quad 0.0 \quad 0.0$

de205

$1.50000-2 \quad 3.25000-2 \quad 5.75000-2 \quad 8.50000-2$

$1.25000-1 \quad 2.25000-1 \quad 3.50000-1 \quad 4.55000-1 \quad 5.55000-1$

$6.50000-1 \quad 8.50000-1 \quad 1.25000+0 \quad 1.75000+0 \quad 2.25000+0$

$2.75000+0 \quad 3.50000+0 \quad 4.50000+0 \quad 5.50000+0 \quad 6.50000+0$

$7.25000+0 \quad 7.75000+0 \quad 9.00000+0 \quad 1.20000+1$ $\begin{array}{llll}2.14390-3 & 5.77600-4 & 2.71850-4 & 2.68170-4\end{array}$

df205

$3.27670-4 \quad 5.66760-4 \quad 8.75940-4 \quad 1.08450-3 \quad 1.27970-3$

$1.44170-3 \quad 1.75630-3 \quad 2.31560-3 \quad 2.92700-3 \quad 3.46860-3$

$3.95960-3 \quad 4.62210-3 \quad 5.41370-3 \quad 6.19090-3 \quad 6.92650-3$

$7.47830-3 \quad 7.84680-3 \quad 8.77160-3 \quad 1.10200-2$

$\mathrm{fm} 205 \quad 5.701+10$

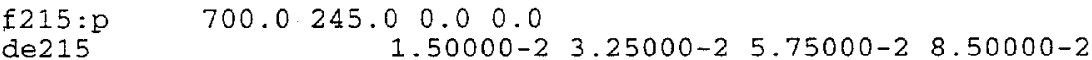

$1.25000-1 \quad 2.25000-1 \cdot 3.50000-1 \quad 4.55000-1 \quad 5.55000-1$

$6.50000-1 \quad 8.50000-1 \quad 1.25000+0 \quad 1.75000+0 \quad 2.25000+0$

$2.75000+0 \quad 3.50000+0 \quad 4.50000+0 \quad 5.50000+0 \quad 6.50000+0$

$7.25000+0 \quad 7.75000+0 \quad 9.00000+01.20000+1$

$2.14390-3 \quad 5.77600-4 \quad 2.71850-4 \quad 2.68170-4$

$3.27670-4 \quad 5.66760-4 \quad 8.75940-4 \quad 1.08450-3 \quad 1.27970-3$

$1.44170-3 \quad 1.75630-3 \quad 2.31560-3 \quad 2.92700-3 \quad 3.46860-3$

$3.95960-3 \quad 4.62210-3 \quad 5.41370-3 \quad 6.19090-3 \quad 6.92650-3$

$7.47830-3 \quad 7.84680-38.77160-3 \quad 1.10200-2$

$\operatorname{fm} 215$

$5.701+10$

$\mathrm{f} 225: \mathrm{p}$ $725.0 \quad 0.0 \quad 0.0 \quad 0.0$

de 225

$1.50000-2 \quad 3.25000-2 \quad 5.75000-2 \quad 8.50000-2$

$1.25000-1 \quad 2.25000-1 \quad 3.50000-1 \quad 4.55000-1 \quad 5.55000-1$

$6.50000-1 \quad 8.50000-1 \quad 1.25000+0 \quad 1.75000+0 \quad 2.25000+0$

$2.75000+0 \quad 3.50000+0 \quad 4.50000+0 \quad 5.50000+0 \quad 6.50000+0$

$7.25000+0 \quad 7.75000+0 \quad 9.00000+0 \quad 1.20000+1$

df225

$\begin{array}{llll}2.14390-3 & 5.77600-4 & 2.71850-4 & 2.68170-4\end{array}$

$3.27670-4 \quad 5.66760-4 \quad 8.75940-4 \quad 1.08450-3 \quad 1.27970-3$

$1.44170-3 \quad 1.75630-3 \quad 2.31560-3 \quad 2.92700-3 \quad 3.46860-3$

$\begin{array}{lllll}3.95960-3 & 4.62210-3 & 5.41370-3 & 6.19090-3 & 6.92650-3\end{array}$

$7.47830-3 \quad 7.84680-3 \quad 8.77160-3 \quad 1.10200-2$

fm225

f $235: p$

de235

$5.701+10$

$298.958-1710.0 \quad 0.0 \quad 0.0$

$1.50000-2 \quad 3.25000-2 \quad 5.75000-2 \quad 8.50000-2$

$\begin{array}{llllll}1.25000-1 & 2.25000-1 & 3.50000-1 & 4.55000-1 & 5.55000-1\end{array}$

$6.50000-1 \quad 8.50000-1 \quad 1.25000+0 \quad 1.75000+0 \quad 2.25000+0$

$2.75000+0 \quad 3.50000+0 \quad 4.50000+0 \quad 5.50000+0 \quad 6.50000+0$

$7.25000+0 \quad 7.75000+0 \quad 9.00000+0 \quad 1.20000+1$

$\begin{array}{lllllll}\text { df235 } & 2.14390-3 & 5.77600-4 & 2.71850-4 & 2.68170-4\end{array}$

$3.27670-4 \quad 5.66760-4 \quad 8.75940-4 \quad 1.08450-3 \quad 1.27970-3$

$\begin{array}{llllll}1.44170-3 & 1.75630-3 & 2.31560-3 & 2.92700-3 & 3.46860-3\end{array}$

$\begin{array}{llllll}3.95960-3 & 4.62210-3 & 5.41370-3 & 6.19090-3 & 6.92650-3\end{array}$

$\begin{array}{llll}7.47830-3 & 7.84680-3 & 8.77160-3 & 1.10200-2\end{array}$

$\operatorname{fm} 235 \quad 5.701+10$

f $245: \mathrm{p} \quad 298.958 \quad 1296.0 \quad 0.0 \quad 0.0$

de245 $1.50000-2 \quad 3.25000-2 \quad 5.75000-28.50000-2$ $\begin{array}{llllll}1.25000-1 & 2.25000-1 & 3.50000-1 & 4.55000-1 & 5.55000-1\end{array}$ $6.50000-1 \quad 8.50000-1 \quad 1.25000+0 \quad 1.75000+0 \quad 2.25000+0$ $2.75000+0 \quad 3.50000+0 \quad 4.50000+0 \quad 5.50000+0 \quad 6.50000+0$ $7.25000+0 \quad 7.75000+0 \quad 9.00000+0 \quad 1.20000+1$

$\operatorname{df} 245$ $\begin{array}{llll}2.14390-3 & 5.77600-4 & 2.71850-4 & 2.68170-4\end{array}$

$3.27670-4 \quad 5.66760-4 \quad 8.75940-4 \quad 1.08450-3 \quad 1.27970-3$

$\begin{array}{llllll}1.44170-3 & 1.75630-3 & 2.31560-3 & 2.92700-3 & 3.46860-3\end{array}$

$\begin{array}{llllll}3.95960-3 & 4.62210-3 & 5.41370-3 & 6.19090-3 & 6.92650-3\end{array}$

$7.47830-3 \quad 7.84680-3 \quad 8.77160-3 \quad 1.10200-2$

$\operatorname{fm} 245$

$5.701+10$ 


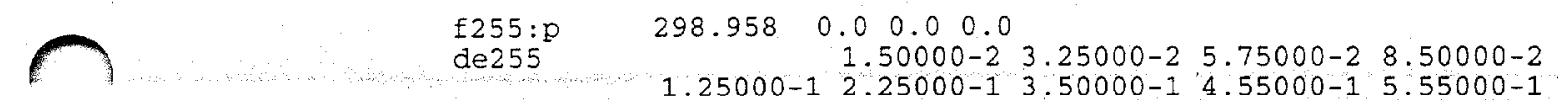
$6.50000-1 \quad 8.50000-1 \quad 1.25000+0 \quad 1.75000+0 \quad 2.25000+0$ $2.75000+0 \quad 3.50000+0 \quad 4.50000+0 \quad 5.50000+0 \quad 6.50000+0$ $7.25000+0 \quad 7.75000+0 \quad 9.00000+0 \quad 1.20000+1$

df255 2.14390-3 $5.77600-4 \quad 2.71850-4 \quad 2.68170-4$ $3.27670-4 \quad 5.66760-4 \quad 8.75940-4 \quad 1.08450-3 \quad 1.27970-3$ $\begin{array}{lllll}1.44170-3 & 1.75630-3 & 2.31560-3 & 2.92700-3 & 3.46860-3\end{array}$ $\begin{array}{llllll}3.95960-3 & 4.62210-3 & 5.41370-3 & 6.19090-3 & 6.92650-3\end{array}$ $7.47830-3 \quad 7.84680-3 \quad 8.77160-3 \quad 1.10200-2$

fIn255 $\quad 5.701+10$

f265:p $\quad-385.0 \quad 300.0 \quad 0.0 \quad 0.0$

de265 $1.50000-2 \quad 3.25000-2 \quad 5.75000-2 \quad 8.50000-2$

$\begin{array}{lllll}1.25000-1 & 2.25000-1 & 3.50000-1 & 4.55000-1 & 5.55000-1\end{array}$

$6.50000-18.50000-1 \quad 1.25000+0 \quad 1.75000+02.25000+0$

$2.75000+0 \quad 3.50000+0 \quad 4.50000+0 \quad 5.50000+0 \quad 6.50000+0$

$7.25000+0 \quad 7.75000+0 \quad 9.00000+0 \quad 1.20000+1$

df265 $\quad 2.14390-3 \quad 5.77600-4 \quad 2.71850-4 \quad 2.68170-4$

$\begin{array}{llllll}3.27670-4 & 5.66760-4 & 8.75940-4 & 1.08450-3 & 1.27970-3\end{array}$

$\begin{array}{lllll}1.44170-3 & 1.75630-3 & 2.31560-3 & 2.92700-3 & 3.46860-3\end{array}$

$\begin{array}{lllll}3.95960-3 & 4.62210-3 & 5.41370-3 & 6.19090-3 & 6.92650-3\end{array}$

$7.47830-3 \quad 7.84680-3 \quad 8.77160-3 \quad 1.10200-2$

fm265. $\quad 5.701+10$

f275:p $\quad-846.0 \quad 0.0 \quad 0.0 \quad 0.0$

de275 $1.50000-2 \quad 3.25000-2 \quad 5.75000-2 \quad 8.50000-2$

$\begin{array}{lllll}1.25000-1 & 2.25000-1 & 3.50000-1 & 4.55000-1 & 5.55000-1\end{array}$

$6.50000-1 \quad 8.50000-1 \quad 1.25000+0 \quad 1.75000+0 \quad 2.25000+0$

$2.75000+0 \quad 3.50000+0 \quad 4.50000+0 \quad 5.50000+0 \quad 6.50000+0$

$7.25000+07.75000+0 \quad 9.00000+0 \quad 1.20000+1$

$2.14390-3 \quad 5.77600-4 \quad 2.71850-4 \quad 2.68170-4$

$3.27670-4 \quad 5.66760-4 \quad 8.75940-4 \quad 1.08450-3 \quad 1.27970-3$

$1.44170-3 \quad 1.75630-3 \quad 2.31560-3 \quad 2.92700-3 \quad 3.46860-3$

$\begin{array}{llllll}3.95960-3 & 4.62210-3 & 5.41370-3 & 6.19090-3 & 6.92650-3\end{array}$

$7.47830-3 \quad 7.84680-3 \quad 8.77160-3 \quad 1.10200-2$

$\operatorname{tm} 275 \quad 5.701+10$

f285:p $\quad 986.0 \quad 0.0 \quad 0.0 \quad 0.0$

de285 $1.50000-2 \quad 3.25000-2 \quad 5.75000-2 \quad 8.50000-2$

$\begin{array}{lllllll}1.25000-1 & 2.25000-1 & 3.50000-1 & 4.55000-1 & 5.55000-1\end{array}$

$\begin{array}{lllll}6.50000-1 & 8.50000-1 & 1.25000+0 & 1.75000+0 & 2.25000+0\end{array}$

$2.75000+0 \quad 3.50000+0 \quad 4.50000+0 \quad 5.50000+0 \quad 6.50000+0$

$7.25000+07.75000+0 \quad 9.00000+0 \quad 1.20000+1$

dE285 $\quad 3.27670-4 \quad 2.14390-3 \quad 5.77600-4 \quad 2.71850-4 \quad 2.68170-4$

$\begin{array}{llllll}1.44170-3 & 1.75630-3 & 2.31560-3 & 2.92700-3 & 3.46860-3\end{array}$

$\begin{array}{lllll}3.95960-3 & 4.62210-3 & 5.41370-3 & 6.1 .9090-3 & 6.92650-3\end{array}$

$\begin{array}{llll}7.47830-3 & 7.84680-3 & 8.77160-3 & 1.10200-2\end{array}$

fm285 $\quad 5.701+10$

f295:p $\quad-385.00 .0 \quad 0.00 .0$

de295 $1.50000-2 \quad 3.25000-2 \quad 5.75000-2 \quad 8.50000-2$

$\begin{array}{lllll}1.25000-1 & 2.25000-1 & 3.50000-1 & 4.55000-1 & 5.55000-1\end{array}$

$6.50000-18.50000-1 \quad 1.25000+0 \quad 1.75000+02.25000+0$

$2.75000+0 \quad 3.50000+0 \quad 4.50000+0 \quad 5.50000+0 \quad 6.50000+0$

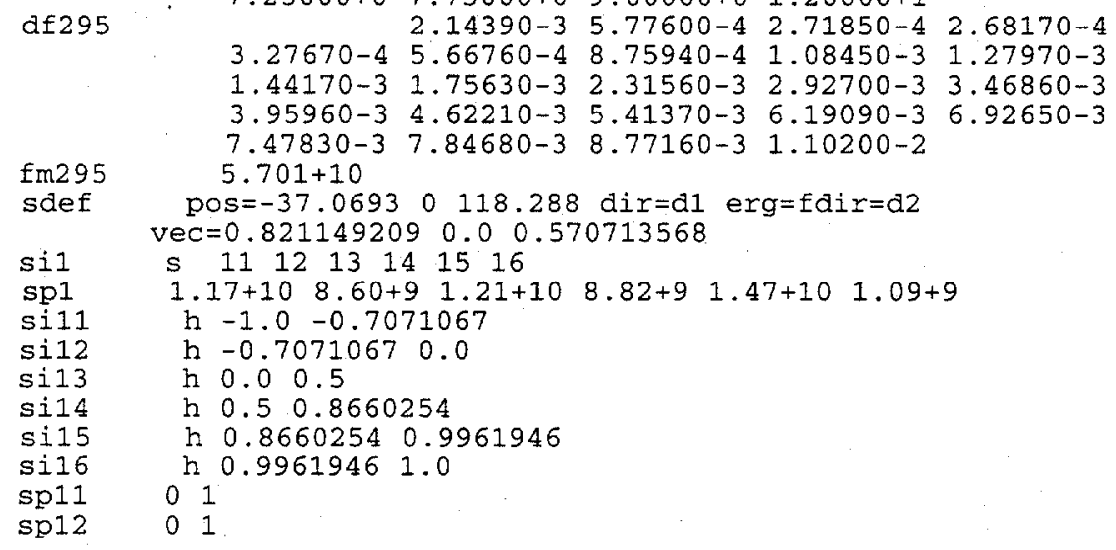

$7.25000+0 \quad 7.75000+0 \quad 9.00000+0 \quad 1.20000+1$ 


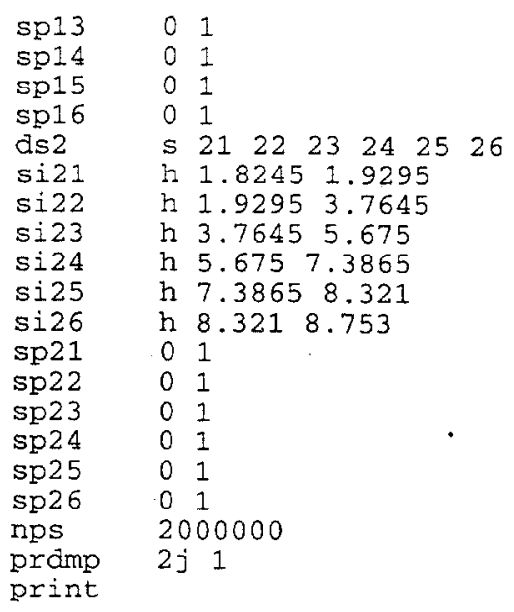


File for MCNP-4B calculation of dose rates around the PFNA facility for the source in the maximum down position

message: outp=pfna15.0 metal $=$ pfna15. $\mathrm{m}$ monp file for PFNA facility - dose rates at selected locations
c source in nominal minimum downward position $(-8.6$ deg.) of the
c movable vertical collimator. check sdef, cells $3-6$, surfaces
$c \quad 401$ and $503-505$, and the sign on surface 505.

0

$-100: 128:-200: 251:-300: 305$

$100-128 \quad 200-251 \quad 300-305$

$\#(131-164+258-297+307-321)$

$\begin{array}{lll}3 & 1 & 0.10549 \\ 4 & 1 & 0.10549\end{array}$

$-400-401-505+224-227 \#(503-504)$

50

$-401+400-505+225-226 \#(503-504)$

$142-127+223-228+308-304$

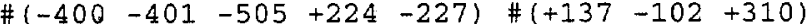

$\#(-401+400-505+225-226)$

$6 \quad 0 \quad 127-124+501-502+308-304$

$\#(-401+400-505+225-226)$

$\begin{array}{llllllll}7 & 0 & 124 & -126+254 & -255+308 & -304 \\ 8 & 5 & 8.734441-2 & 102 & -126+201 & -250+306 & -301\end{array}$

$\#(+102-127+223-228) \quad \#(+127-124+501-502)$

$\#(+124+254-255) \quad \#(+129-252) \quad \#(+129+257)$

9

10.10549

$102-124+222-229+301-304$

$\#(+129-252) \quad \#(+129+257)$

$\#(127+501-502)$ \# $(-127+223-228)$

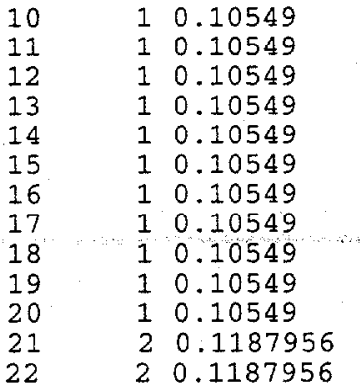

$106-109+212-236+302-303 \#(218-233)$

$109-110+211-237+302-303 \#(221-230)$

$110-111+210-238+302-303 \#(221-230)$

$111-112+209-239+302-303 \#(221-230)$

$112-113+208-240+302-303 \#(221-230)$

$113-114+207-241+302-303 \#(221-230)$

$114-115+206-242+302-303 \#(221-230)$

$115-116+205-243+302-303 \#(221-230)$

$116-117+204-244+302-303 \#(221-230)$

$117-118+203-245+302-303 \#(221-230)$

$118-119+202-249+302-303 \#(221-230)$

$120-123+215-246+302-303 \#(221-230)$

$103-109+202-249+302-303$

\#(221 -230)

$\#(103-104+217-234)$

$\#(104-105+216-235)$

$\#(105-107+219-232)$

$\#(107-108+219-220) \quad \#(107-108+231-232)$

$2 \quad 0.1187956$

$109-120+202-249+302-303$

$\#(221-230)$

$\#(109-110+211-237)$

$\#(110-111+210-238)$

$\#(111-112+209-239)$

$\#(112-113+208-240)$

$\#(113-114+207,-241)$

$\#(114-115+206-242)$

$\#(115-116+205-243)$

$\#(116-117+204-244)$

$\#(117-118+203-245)$

\# $(118-119)$

$24 \quad 3 \quad 3.284-2$

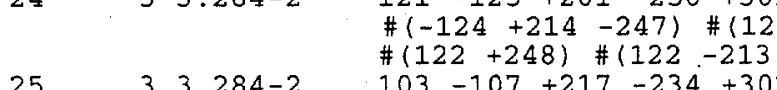

$25 \quad 3 \quad 3.284-2 \quad 103-107+217-234+302-303$

$\#(221-230) \#(104-220) \#(104+231)$

$2645.977931-2 \quad 104-108+216-220+302-303$ \# $(105-219)$

$2745.977931-2104-108+231-235+302-303 \#(105+232)$

$2845.977931-2 \quad 102-124+201-222+301-304$

\#( $\left(\begin{array}{llllll}103 & -120 & 202 & -221 & 302 & -303\end{array}\right)$

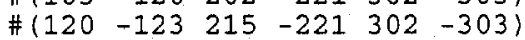

\#(121 -214) 


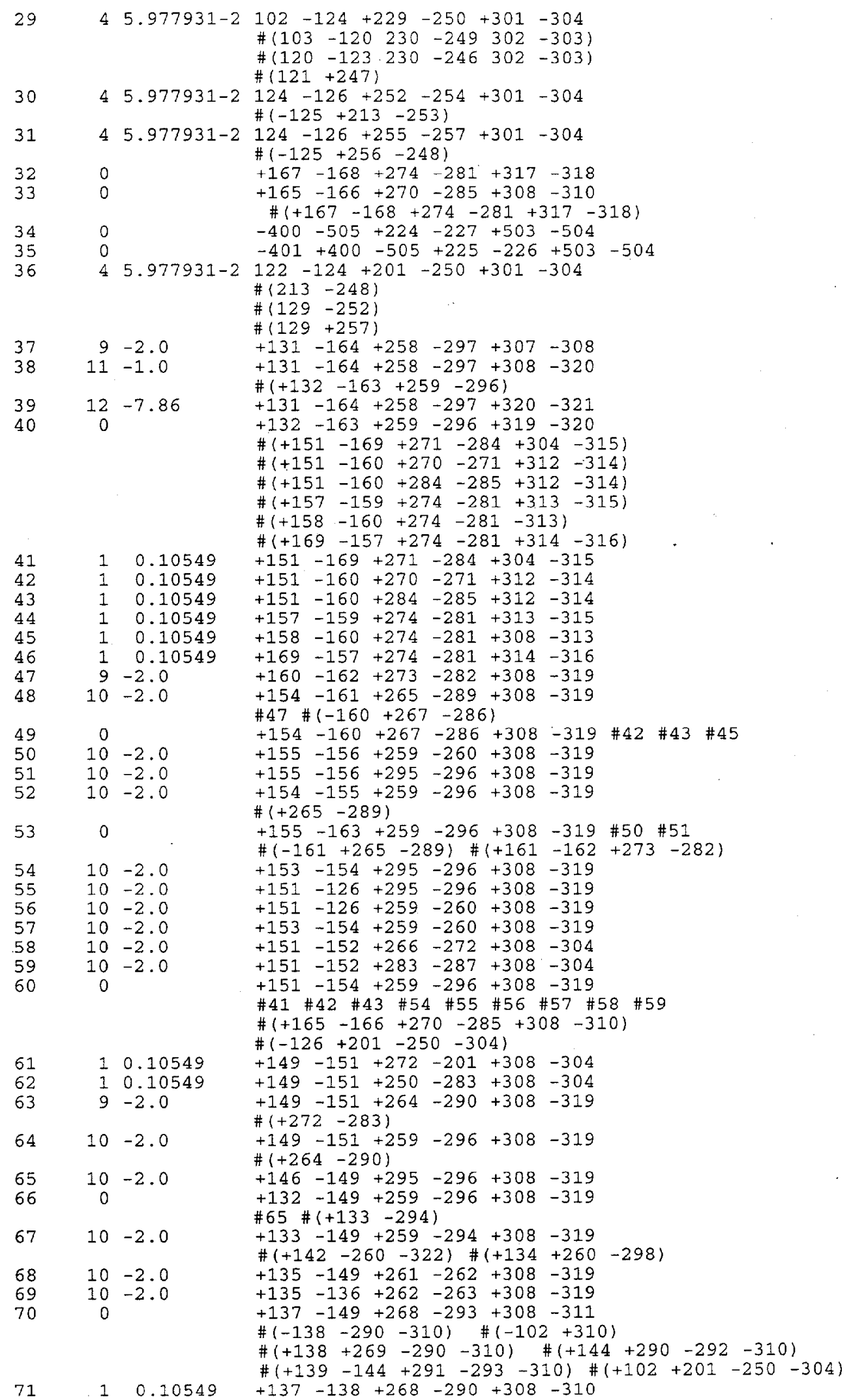




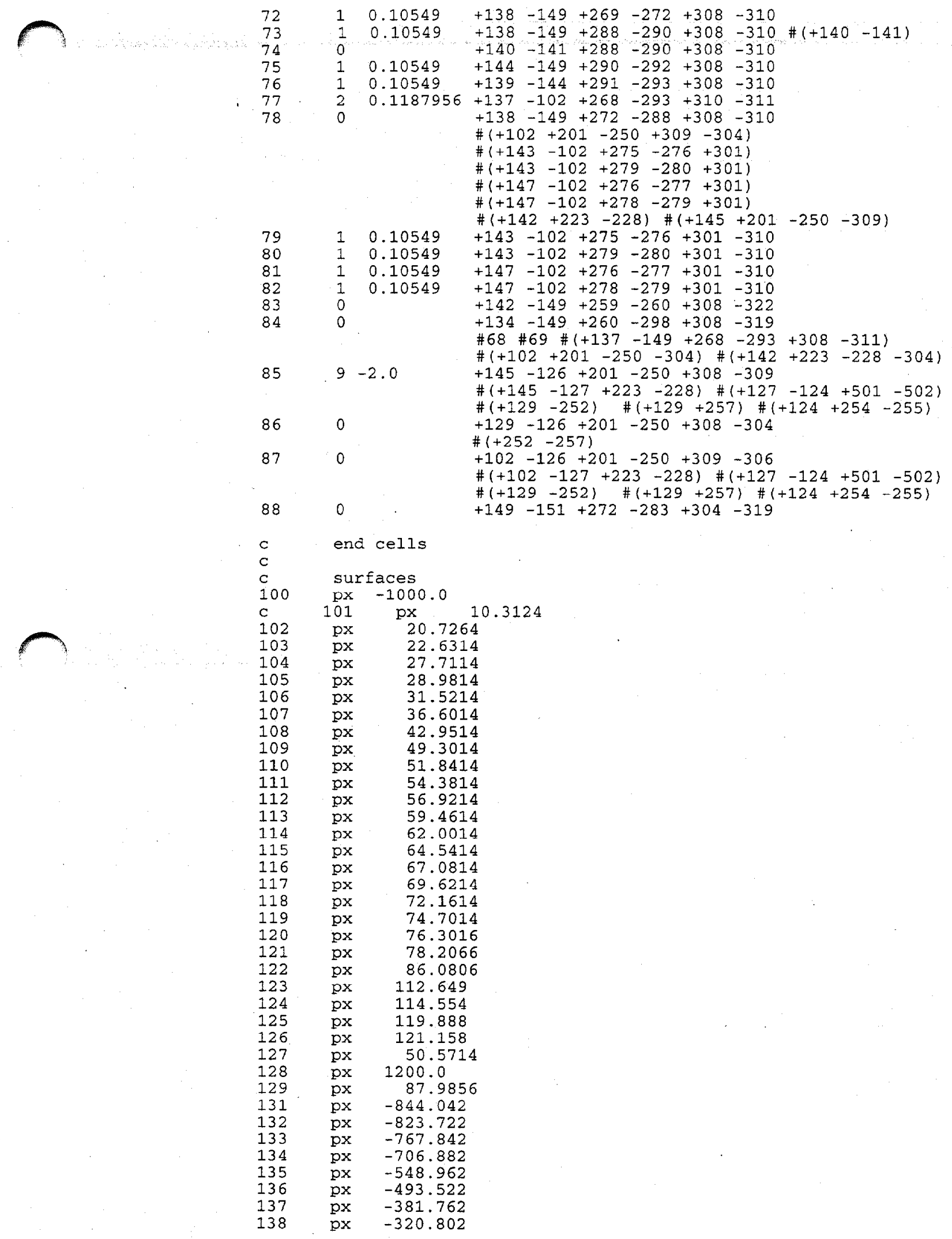




\begin{tabular}{|c|c|c|}
\hline 139 & $p x$ & -315.722 \\
\hline 140 & $\mathrm{px}$ & -173.482 \\
\hline 141 & $p x$ & -107.442 \\
\hline 142 & $\mathrm{px}$ & -82.042 \\
\hline 143 & $\mathrm{px}$ & -55.4736 \\
\hline 144 & $p x$ & -21.082 \\
\hline 145 & $p x$ & -0.762 \\
\hline 146 & $\mathrm{px}$ & 9.398 \\
\hline 147 & $\mathrm{px}$ & 15.6464 \\
\hline 148 & $\mathrm{px}$ & 30.1103 \\
\hline 149 & $\mathrm{px}$ & 39.878 \\
\hline 150 & $\mathrm{px}$ & 45.3503 \\
\hline 151 & $p x$ & 100.838 \\
\hline 152 & $\mathrm{px}$ & 126.238 \\
\hline 153 & $\mathrm{px}$ & 476.758 \\
\hline 154 & $\mathrm{px}$ & 497.078 \\
\hline 155 & $\mathrm{px}$ & 558.038 \\
\hline 156 & $\mathrm{px}$ & 588.518 \\
\hline 157 & $\mathrm{px}$ & 725.678 \\
\hline 158 & $p x$ & 730.758 \\
\hline 159. & $\mathrm{px}$ & 745.998 \\
\hline $160^{\circ}$ & $p x$ & 751.078 \\
\hline 161 & $p x$ & 812.038 \\
\hline 162 & $p x$ & 842.518 \\
\hline 163 & $p x$ & 964.438 \\
\hline 164 & $\mathrm{px}$ & 984.758 \\
\hline 165 & $p x$ & 148.387 \\
\hline 166 & $p x$ & 449.529 \\
\hline 167 & $p x$ & 250.0 \\
\hline 168 & $\mathrm{px}$ & 350.0 \\
\hline 169 & $p x$ & 116.078 \\
\hline 200 & py & -2000.0 \\
\hline 201 & py & -74.7056 \\
\hline 202 & py & -72.8006 \\
\hline 203 & py & -47.0958 \\
\hline 204 & DY & -45.8258 \\
\hline 205 & py & -44.5558 \\
\hline 206 & py & -43.2858 \\
\hline 207 & py & -42.0158 \\
\hline 208 & py & -40.7458 \\
\hline 209 & py & -39.4758 \\
\hline 210 & py & -38.2058 \\
\hline 211 & py & -36.9358 \\
\hline 212 & py & -34.3958 \\
\hline 213 & py & -38.6122 \\
\hline 214 & PY & -33.2782 \\
\hline 215 & py & -31.3732 \\
\hline 216 & py & -30.5858 \\
\hline 217 & $\overrightarrow{P Y}$ & -24.2358 \\
\hline 218 & py & -19.1558 \\
\hline 219 & $\overrightarrow{\mathrm{pY}}$ & -16.6158 \\
\hline 220 & py & -15.3458 \\
\hline 221 & PY & -12.8058 \\
\hline 222 & py & -10.9008 \\
\hline 223 & py & -5.8208 \\
\hline 224 & py & -5.5245 \\
\hline 225 & py & -4.2037 \\
\hline 226 & py & 4.2037 \\
\hline 227 & py & 5.5245 \\
\hline 228 & py & 5.8208 \\
\hline 229 & py & 10.9008 \\
\hline 230 & py & 12.8058 \\
\hline 231 & py & 15.3458 \\
\hline 232 & py & 16.6158 \\
\hline 233 & py & 19.1558 \\
\hline 234 & py & 24.2358 \\
\hline 235 & py & 30.5858 \\
\hline 236 & py & 34.3958 \\
\hline 237 & py & 36.9358 \\
\hline 238 & py & 38.2058 \\
\hline 239 & pY & 39.4758 \\
\hline 240 & PY & 40.7458 \\
\hline
\end{tabular}




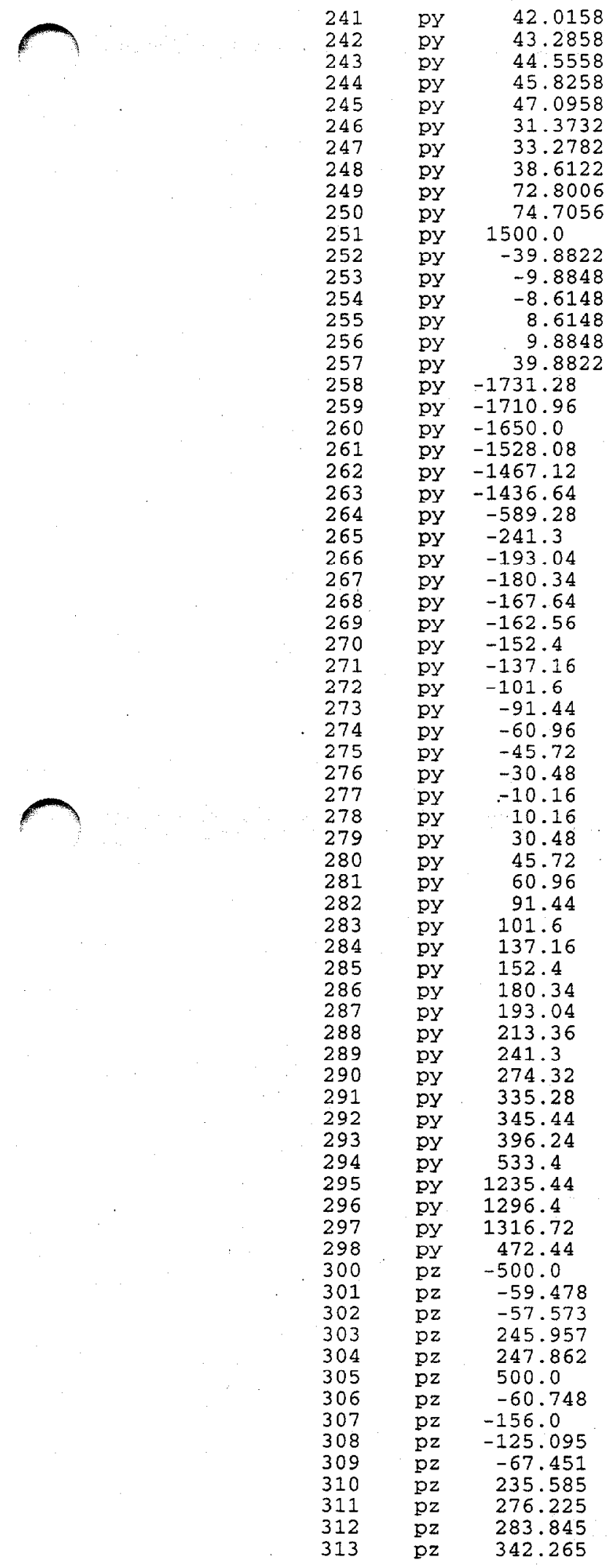




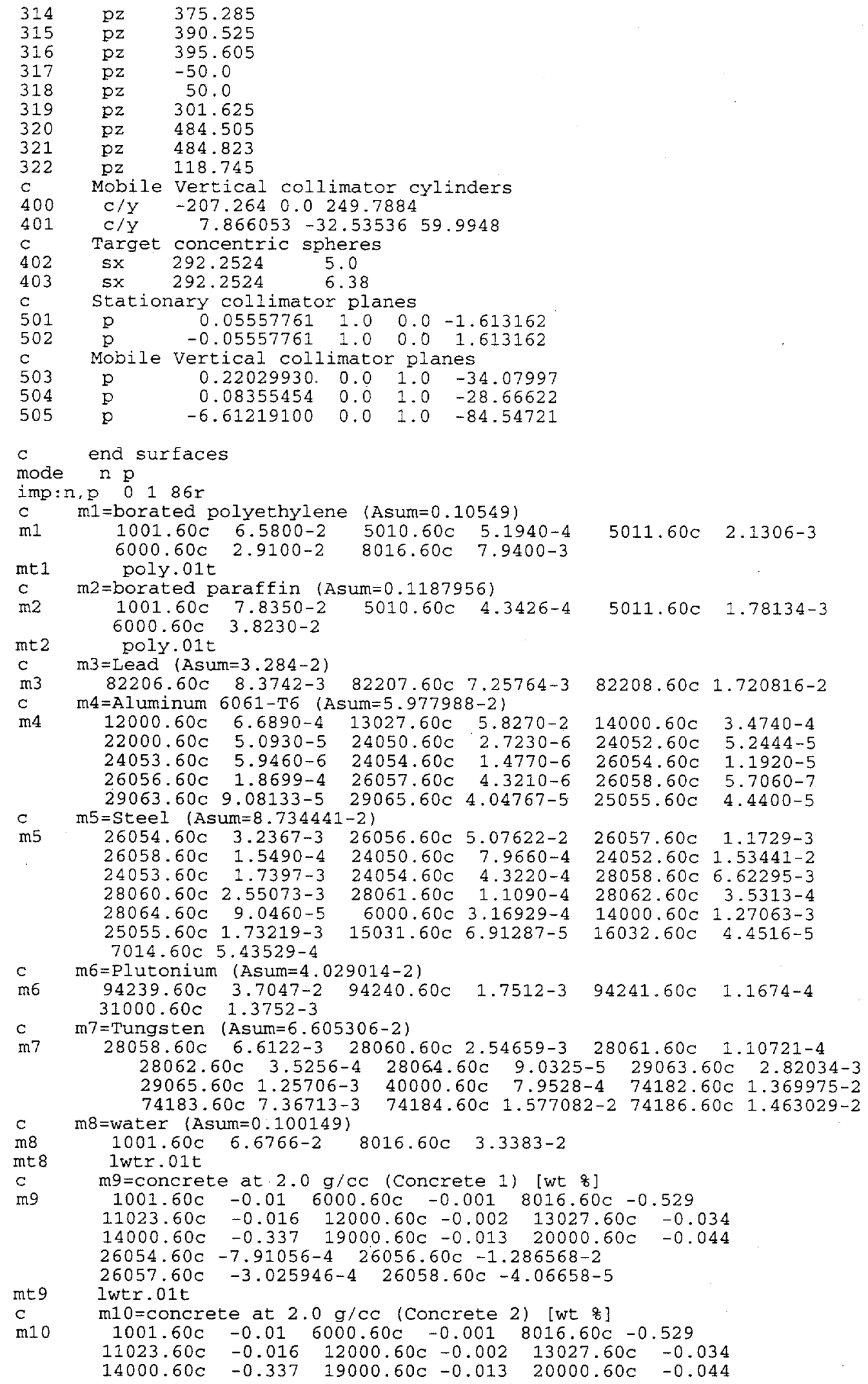


$26054.60 c-7.91056-4 \quad 26056.60 c-1.286568-2$

$26057.60 \mathrm{C} \quad-3.025946-4 \quad 26058.60 \mathrm{c}-4.06658-5$

mt10 lwtr.01t

c m11=concrete at $1.0 \mathrm{~g} / \mathrm{cc}$ (Masonry Block) [wt 8 ]

m11. $\quad 1001.60 c \quad-0.01 \quad 6000.60 c-0.001 \quad 8016.60 c-0.529$

$\begin{array}{lllllll}11023.60 c & -0.016 & 12000.60 c & -0.002 & 13027.60 c & -0.034\end{array}$

$\begin{array}{lllllll}14000.60 \mathrm{C} & -0.337 & 19000.60 \mathrm{c} & -0.013 & 20000.60 \mathrm{C} & -0.044\end{array}$

$26054.60 c-7.91056-4 \quad 26056.60 c-1.286568-2$

mt11 $26057.60 \mathrm{C}-3.025946-4 \quad 26058.60 \mathrm{c}-4.06658-5$

c m12=iron at $7.86 \mathrm{~g} / \mathrm{cc}$

$\begin{array}{llllllll}\mathrm{m} 12 & 26054.60 \mathrm{c} & 0.0585 & 26056.60 \mathrm{C} & 0.9175 & 26057.60 \mathrm{c} & 0.0212\end{array}$

$26058.60 \mathrm{c} \quad 0.0028$

c m13=Heavy concrete at $4.2 \mathrm{~g} / \mathrm{CC}$ [wt 8 ]

m13 $1001.60 \mathrm{C}-0.00058016 .60 \mathrm{c}-0.18$

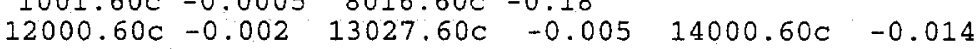
$\begin{array}{llllll}16032.60 \mathrm{c} & -0.001 & 20000.60 \mathrm{c} & -0.061 & 25055.60 \mathrm{c} & -0.016\end{array}$ $26054.60 \mathrm{C}-4.073938-2 \quad 26056.60 \mathrm{C}-0.6625828$ $26057.60 \mathrm{C}-1.558362-2 \quad 26058.60 \mathrm{C}-2.094288-3$

\section{mt 13} lwtr. $01 t$

f105:n $\quad 845.0 \quad 0.0 \quad 0.0 \quad 0.0$

de105 $\quad 2.07002-7 \quad 7.69672-7 \quad 1.75386-6 \quad 3.71293-6$

$7.86024-6 \quad 1.66401-5 \quad 3.52273-5 \quad 7.45761-5 \quad 1.34159-4$ 2.21191-4 $3.64682-4 \quad 6.01258-4 \quad 9.91309-4 \quad 1.63439-3$ $\begin{array}{llllll}2.14167-3 & 2.43063-3 & 2.82399-3 & 3.37141-3 & 4.61914-3\end{array}$ $\begin{array}{lllll}7.32483-3 & 1.20766-2 & 1.93065-2 & 2.41830-2 & 2.54229-2\end{array}$ 2.65293-2 2.77506-2 3.14039-2 $4.33909-2$ 5.45187-2 7.15396-2 $1.04645-1.1 .36365-1 \quad 1.66556-1.2 .03432-1$ 2.48472-1 2.83877-1 2.95864-1 $2.97851-1 \quad 3.00232-1$ 3.44858-1 4.42806-1 $5.10634-1 \quad 5.65749-1 \quad 6.75418-1$ 8.24957-1 $1.00760+0 \quad 1.23069+0 \quad 1.50317+0 \quad 1.83598+0$ $\begin{array}{lllll}2.12513+0 & 2.28850+0 & 2.40583+0 & 2.73896+0 & 3.34537+0\end{array}$ $4.08604+0 \quad 4.99070+0 \quad 6.09565+0 \quad 7.44525+0 \quad 9.09365+0$ $1.11070+1 \quad 1.35661+1$

$\begin{array}{lllllll}\text { df105 } 105 & 3.70370-3 & 4.37060-3 & 4.52500-3 & 4.57410-3 \\ & 4.55830-3 & 4.48970-3 & 4.38170-3 & 4.24920-3 & 4.12890-3\end{array}$ $4.03110-3 \quad 3.93550-3 \quad 3.84530-3 \quad 3.76290-3 \quad 3.69080-3$ $3.65280-3 \quad 3.63870-3 \quad 3.62290-3 \quad 3.60620-3 \quad 3.58250-3$ $3.55850-3 \quad 4.08750-3 \quad 5.89750-3 \quad 7.13870-3 \quad 7.42410-3$ $\begin{array}{lllll}7.67700-3 & 7.95180-3 & 8.74210-3 & 1.11490-2 & 1.35030-2\end{array}$ $1.65080-2 \quad 2.25270-2 \quad 2.86610-2 \quad 3.43150-2 \quad 4.10860-2$ $4.92110-2 \quad 5.56070-2 \quad 5.77300-2 \quad 5.80800-2 \quad 5.84980-2$ $6.59980-28.26560-2 \quad 9.04890-2 \quad 9.54810-21.05360-1$

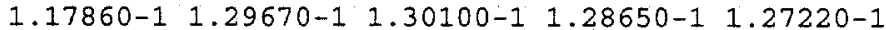
$1.26160-1 \quad 1.25620-1 \quad 1.25270-1 \quad 1.28500-1 \quad 1.36960-1$ $1.45980-1$ 1.54120-1 $1.51160-1$ 1.47220-1 $1.47060-1$ $1.63200-12.00340-1$

fm105 $5.701+10$

f115:n $\quad 700.0245 .0 \quad 0.0 \quad 0.0$

$\begin{array}{lllllll}\text { de115 } & 2.07002-7 & 7.69672-7 & 1.75386-6 & 3.71293-6\end{array}$

7.86024-6 $1.66401-5 \quad 3.52273-5 \quad 7.45761-5 \quad 1.34159-4$

$2.21191-4 \quad 3.64682-4 \quad 6.01258-4 \quad 9.91309-4 \quad 1.63439-3$

2.14167-3 2.43063-3 $2.82399-3 \quad 3.37141-3 \quad 4.61914-3$

$7.32483-3 \quad 1.20766-2 \quad 1.93065-2 \quad 2.41830-2 \quad 2.54229-2$

2.65293-2 2.77506-2 $3.14039-2 \quad 4.33909-2 \quad 5.45187-2$

7.15396-2 $1.04645-1 \quad 1.36365-1 \quad 1.66556-1 \quad 2.03432-1$

2.48472-1 2.83877-1 2.95864-1 2.97851-1 $3.00232-1$

$\begin{array}{llllll}3.44858-1 & 4.42806-1 & 5.10634-1 & 5.65749-1 & 6.75418-1\end{array}$

8.24957-1 $1.00760+0 \quad 1.23069+0 \quad 1.50317+0 \quad 1.83598+0$

$2.12513+0 \quad 2.28850+0 \quad 2.40583+0 \quad 2.73896+0 \quad 3.34537+0$ $4.08604+0 \quad 4.99070+0 \quad 6.09565+0 \quad 7.44525+0 \quad 9.09365+0$

df 115

$1.11070+11.35661+1$ $\begin{array}{llll}3.70370-3 & 4.37060-3 & 4.52500-3 & 4.57410-3\end{array}$ $4.55830-3 \quad 4.48970-3 \quad 4.38170-3 \quad 4.24920-3 \quad 4.12890-3$ $\begin{array}{lllllll}4.03110-3 & 3.93550-3 & 3.84530-3 & 3.76290-3 & 3.69080-3\end{array}$ $\begin{array}{llllll}3.65280-3 & 3.63870-3 & 3.62290-3 & 3.60620-3 & 3.58250-3\end{array}$ $3.55850-3 \quad 4.08750-3 \quad 5.89750-3 \quad 7.13870-3 \quad 7.42410-3$

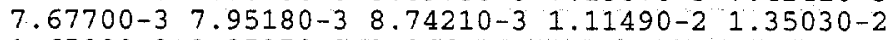
$1.65080-2 \quad 2.25270-2 \quad 2.86610-2 \quad 3.43150-2.4 .10860-2$ $4.92110-2 \quad 5.56070-2 \quad 5.77300-2 \quad 5.80800-2 \quad 5.84980-2$ $6.59980-2 \quad 8.26560-2 \quad 9.04890-2 \quad 9.54810-2 \quad 1.05360-1$ $1.17860-1 \quad 1.29670-1 \quad 1.30100-11.28650-1 \quad 1.27220-1$ 


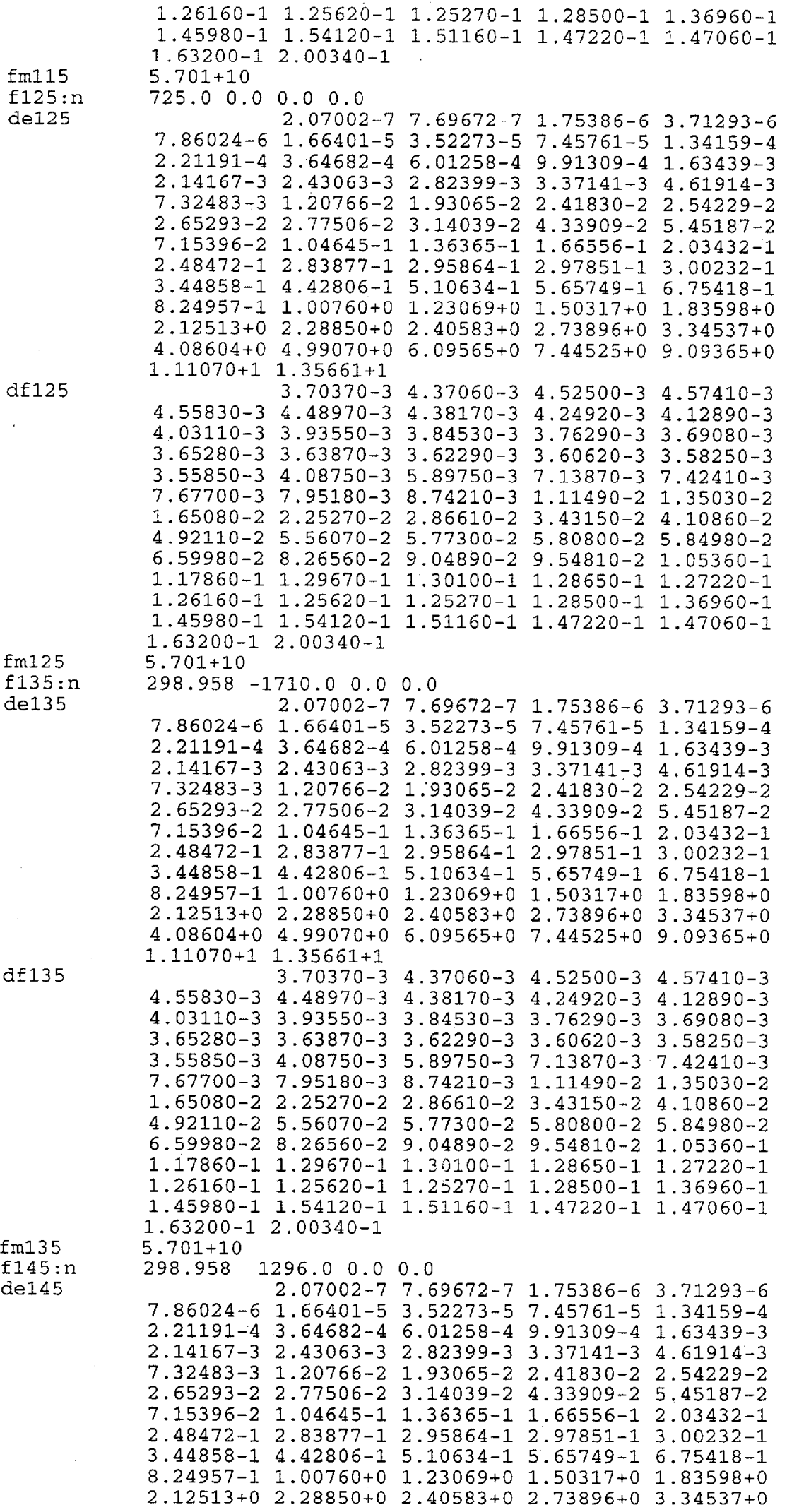


$4.08604+0 \quad 4.99070+0 \quad 6.09565+0 \quad 7.44525+0 \quad 9.09365+0$ $1.11070+11.35661+1$

df145 $3.70370-3 \quad 4.37060-3 \quad 4.52500-3 \quad 4.57410-3$ $\begin{array}{lllllll}4.55830-3 & 4.48970-3 & 4.38170-3 & 4.24920-3 & 4.12890-3\end{array}$ $4.03110-3 \quad 3.93550-3 \quad 3.84530-3 \quad 3.76290-3 \quad 3.69080-3$ $3.65280-3 \quad 3.63870-3 \quad 3.62290-3 \quad 3.60620-3 \quad 3.58250-3$ $\begin{array}{llllll}3.55850-3 & 4.08750-3 & 5.89750-3 & 7.13870-3 & 7.42410-3\end{array}$ $\begin{array}{lllll}7.67700-3 & 7.95180-3 & 8.74210-3 & 1.11490-2 & 1.35030-2\end{array}$ $\begin{array}{lllll}1.55080-2 & 2.25270 \cdots 2 & 2.86610-2 & 3.43150-2 & 4.10860-2\end{array}$ $4.92110-2 \quad 5.56070-2 \quad 5.77300-2 \quad 5.80800-2 \quad 5.84980-2$ $6.59980-2 \quad 8.26560-2 \quad 9.04890-2 \quad 9.54810-2 \quad 1.05360-1$

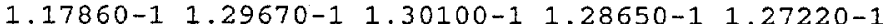
1.26160-1 $1.25620-1 \quad 1.25270-1 \quad 1.28500-1 \quad 1.36960-1$ $1.45980-1$ 1.54120-1 $1.51160-1$ 1.47220-1 $1.47060-1$ 1.63200-1 2.00340-1

fm145 $\quad 5.701+10$

f155:n $\quad 298.958 \quad 0.0 \quad 0.0 \quad 0.0$

de155 $\quad 2.07002-7 \quad 7.69672-7 \quad 1.75386-6 \quad 3.71293-6$

7.86024-6 $1: 66401-5 \quad 3.52273-5 \quad 7.45761-5 \quad 1.34159-4$ $2.21191-4 \quad 3.64682-4 \quad 6.01258-4 \quad 9.91309-4 \quad 1.63439-3$ $2.14167-3 \quad 2.43063-3 \quad 2.82399-3 \quad 3.37141-3 \quad 4.61914-3$ $7.32483-3 \quad 1.20766-2 \quad 1.93065-2 \quad 2.41830-2 \quad 2.54229-2$ $2.65293-2 \quad 2.77506-2 \quad 3.14039-2 \quad 4.33909-2 \quad 5.45187-2$ 7.15396-2 $1.04645-1 \quad 1.36365-1 \quad 1.66556-1 \quad 2.03432-1$ $2.48472-1 \quad 2.83877-1 \quad 2.95864-1 \quad 2.97851-1 \quad 3.00232-1$ $3.44858-1 \quad 4.42806-1 \quad 5.10634-1 \quad 5.65749-1 \quad 6.75418-1$ $8.24957-1 \quad 1.00760+0 \quad 1.23069+0 \quad 1.50317+0 \quad 1.83598+0$ $2.12513+0 \quad 2.28850+0 \quad 2.40583+0 \quad 2.73896+0 \quad 3.34537+0$ $4.08604+0 \quad 4.99070+0 \quad 6.09565+0 \quad 7.44525+0 \quad 9.09365+0$ $1.11070+11.35661+1$

df155 $\quad 3.70370-3 \quad 4.37060-3 \quad 4.52500-3 \quad 4.57410-3$ $4.55830-3 \quad 4.48970-3 \quad 4.38170-3 \quad 4.24920-3 \quad 4.12890-3$ $4.03110-3 \quad 3.93550-3 \quad 3.84530-3 \quad 3.76290-3 \quad 3.69080-3$ $\begin{array}{llllll}3.65280-3 & 3.63870-3 & 3.62290-3 & 3.60620-3 & 3.58250-3\end{array}$ $\begin{array}{llllll}3.55850-3 & 4.08750-3 & 5.89750-3 & 7.13870-3 & 7.42410-3\end{array}$ $\begin{array}{lllll}7.67700-3 & 7.95180-3 & 8.74210-3 & 1.11490-2 & 1.35030-2\end{array}$ $1.65080-2 \quad 2.25270-2 \quad 2.86610-2 \quad 3.43150-2 \quad 4.10860-2$ $\begin{array}{lllll}4.92110-2 & 5.56070-2 & 5.77300-2 & 5.80800-2 & 5.84980-2\end{array}$ $6.59980-2 \quad 8.26560-29.04890-2 \quad 9.54810-2 \quad 1.05360-1$ $1.17860-1 \quad 1.29670-1 \quad 1.30100-1 \quad 1.28650-1.1 .27220-1$ $1.26160-1$ 1.25620-1 $1.25270-1 \quad 1.28500-1$ 1.36960-1. $1.45980-1 \quad 1.54120-1 \quad 1.51160-1 \quad 1.47220-1 \quad 1.47060-1$ $1.632 .00-12.00340-1$

fm155 $\quad 5.701+10$

E165:n $\quad-385.0 \quad 300.0 \quad 0.0 \quad 0.0$

$\begin{array}{lrrrrr}\text { de165 } & 2.07002-7 & 7.69672-7 & 1.75386-6 & 3.71293-6\end{array}$ $7.86024-61.66401-5 \quad 3.52273-5 \quad 7.45761-51.34159-4$ $2.21191-4 \quad 3.64682-4 \quad 6.01258-4 \quad 9.91309-4 \quad 1.63439-3$ $2.14167-3 \quad 2.43063-3 \quad 2.82399-3 \quad 3.37141-3$ 4.61914-3 $7.32483-3$ 1.20766-2 $1.93065-2 \quad 2.41830-2 \quad 2.54229-2$ $\begin{array}{llllll}2.65293-2 & 2.77506-2 & 3.14039-2 & 4.33909-2 & 5.45187-2\end{array}$ 7.15396-2 $1.04645-1 \quad 1.36365-1 \quad 1.66556-1 \quad 2.03432-1$ 2.48472-1 $2.83877-1 \quad 2.95864-1 \quad 2.97851-1 \quad 3.00232-1$ 3.44858-1 4.42806-1 $5.10634-1 \quad 5.65749-1 \quad 6.75418-1$ $8.24957-11.00760+0 \quad 1.23069+01.50317+01.83598+0$ $\begin{array}{lllll}2.12513+0 & 2.28850+0 & 2.40583+0 & 2.73896+0 & 3.34537+0\end{array}$ $4.08604+0 \quad 4.99070+0 \quad 6.09565+0 \quad 7.44525+0 \quad 9.09365+0$ $1.11070+1 \quad 1.35661+1$

df165 $\quad 3.70370-3 \quad 4.37060-3 \quad 4.52500-3 \quad 4.57410-3$ $4.55830-3 \quad 4.48970-3 \quad 4.38170-3 \quad 4.24920-3 \quad 4.12890-3$ $\begin{array}{llllll}4.03110-3 & 3.93550-3 & 3.84530-3 & 3.76290-3 & 3.69080-3\end{array}$ $3.65280-3 \quad 3.63870-3 \quad 3.62290-3 \quad 3.60620-3 \quad 3.58250-3$ $\begin{array}{lllll}3.55850-3 & 4.08750-3 & 5.89750-3 & 7.13870-3 & 7.42410-3\end{array}$ $7.67700-3 \quad 7.95180-3 \quad 8.74210-3 \quad 1.11490-2 \quad 1.35030-2$ $1.65080-2 \quad 2.25270-2 \quad 2.86610-2 \quad 3.43150-2 \quad 4.10860-2$ $4.92110-25.56070-25.77300-2 \quad 5.80800-2 \quad 5.84980-2$ $6.59980-2 \quad 8.26560-2 \quad 9.04890-2 \quad 9.54810-2 \quad 1.05360-1$ $1.17860-1 \quad 1.29670-1 \quad 1.30100-1 \quad 1.28650-1 \quad 1.27220-1$ $1.26160-1$ 1.25620-1 $1.25270-1$ 1.28500-1 $1.36960-1$ 1.45980-1 1.54120-1 1.51160-1 1.47220-1 1.47060-1 fm165 $\quad \begin{aligned} & 1.63200-1 \\ & 5.701+10\end{aligned}$ 


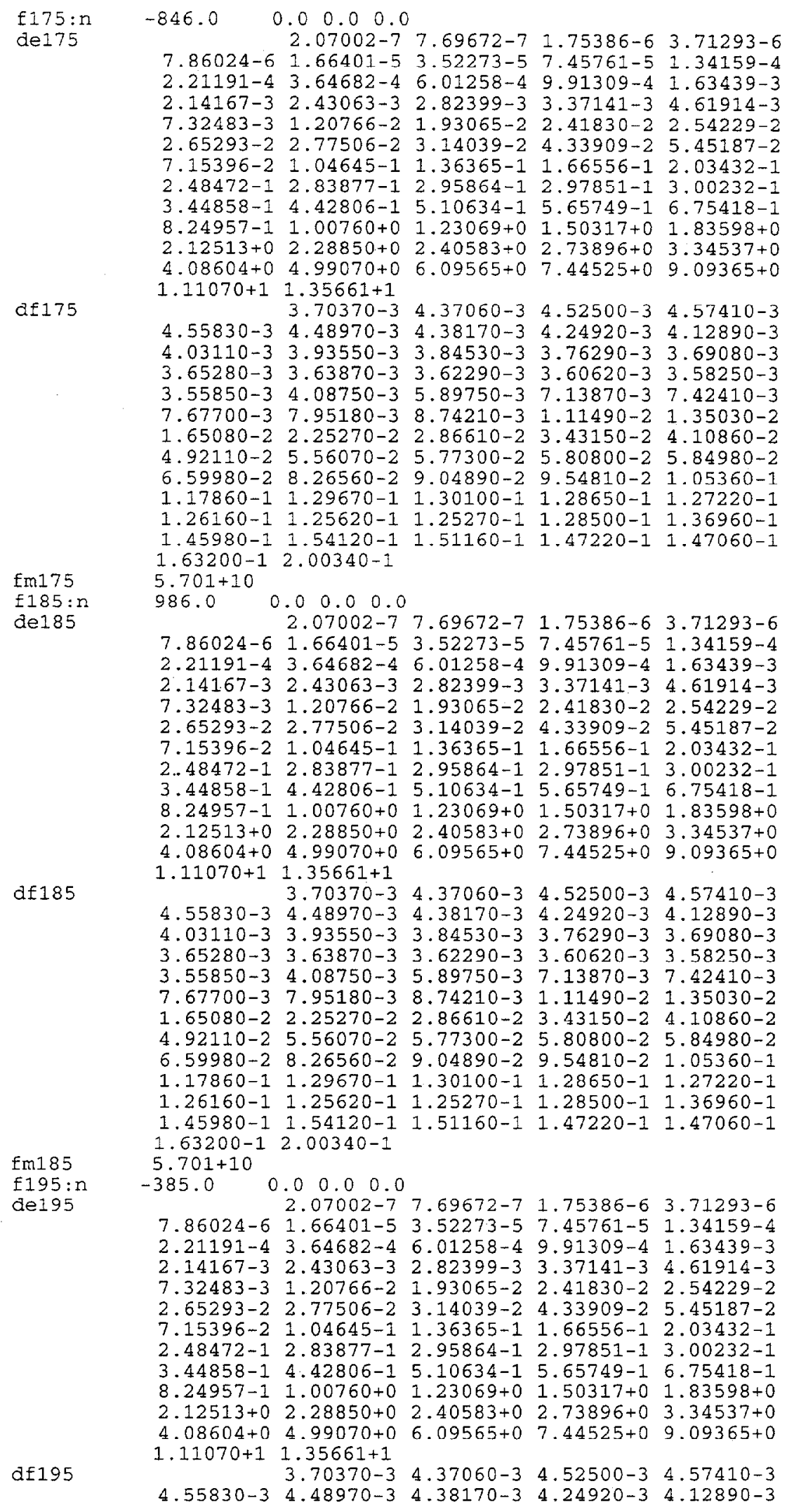


$\begin{array}{llllll}4.03110-3 & 3.93550-3 & 3.84530-3 & 3.76290-3 & 3.69080-3\end{array}$

$\begin{array}{llllll}3.65280-3 & 3.63870-3 & 3.62290-3 & 3.60620-3 & 3.58250-3\end{array}$

$3.55850-3 \quad 4.08750-3 \quad 5.89750-3 \quad 7.13870-3 \quad 7.42410-3$

$7.67700-3 \quad 7.95180-3 \quad 8.74210-3 \quad 1.11490-2 \quad 1.35030-2$

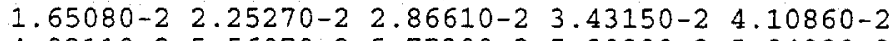

$4.92110-2 \quad 5.56070-2 \quad 5.77300-2 \quad 5.80800-2 \quad 5.84980-2$

$6.59980-2 \quad 8.26560-2 \quad 9.04890-2 \quad 9.54810-2 \quad 1.05360-1$

$1.17860-1 \quad 1.29670-1 \quad 1.30100-1 \quad 1.28650-1 \quad 1.27220-1$

$1.26160-11.25620-1 \quad 1.25270-1 \quad 1.28500-1 \quad 1.36960-1$

1.45980-1 1.54120-1 $1.51160-1 \quad 1.47220-1 \quad 1.47060-1$

$1.63200-12.00340-1$

fm195 $\quad 5.701+10$

f $205: \mathrm{p} \quad 845.0 \quad 0.0 \quad 0.00 .0$

de205 $1.50000-2 \quad 3.25000-2 \quad 5.75000-2 \quad 8.50000-2$

$1.25000-1 \quad 2.25000-1 \quad 3.50000-1 \quad 4.55000-1 \quad 5.55000-1$

$6.50000-18.50000-1 \quad 1.25000+0 \quad 1.75000+0 \quad 2.25000+0$

$2.75000+0 \quad 3.50000+0 \quad 4.50000+0 \quad 5.50000+0 \quad 6.50000+0$

$7.25000+0 \quad 7.75000+09.00000+01.20000+1$

df205 $\quad 2.14390-3 \quad 5.77600-4 \quad 2.71850-4 \quad 2.68170-4$

$3.27670-4 \quad 5.667 .60-4 \quad 8.75940-4 \quad 1.08450-3 \quad 1.27970-3$

$\begin{array}{lllll}1.44170-3 & 1.75630-3 & 2.31560-3 & 2.92700-3 & 3.46860-3\end{array}$

$\begin{array}{lllll}3.95960-3 & 4.62210-3 & 5.41370-3 & 6.19090-3 & 6.92650-3\end{array}$

$7.47830-3 \quad 7.84680-3 \quad 8.77160-3 \quad 1.10200-2$

fm 205

$\begin{array}{lrrrr}\text { f215:p } & 700.0245 .00 .00 .0 \\ \text { de215 } & 1.50000-2 & 3.25000-2 & 5.75000-2 & 8.50000-2\end{array}$

$5.701+10$

$\begin{array}{lllll}1.25000-1 & 2.25000-1 & 3.50000-1 & 4.55000-1 & 5.55000-1\end{array}$

$6.50000-18.50000-1 \quad 1.25000+0 \quad 1.75000+02.25000+0$

$2.75000+0 \quad 3.50000+0 \quad 4.50000+0 \quad 5.50000+0 \quad 6.50000+0$

$7.25000+07.75000+09.00000+01.20000+1$

df215 $2.14390-3 \quad 5.77600-4 \quad 2.71850-4 \quad 2.68170-4$

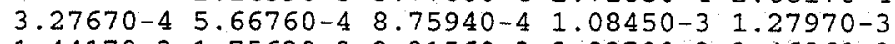

$1.44170-3 \quad 1.75630-3 \quad 2.31560-3 \quad 2.92700-3 \quad 3.46860-3$

$\begin{array}{llllll}3.95960-3 & 4.62210-3 & 5.41370-3 & 6.19090-3 & 6.92650-3\end{array}$ $7.47830-3 \quad 7.84680-3 \quad 8.77160-3 \quad 1.10200-2$

fm215 $\quad 5.701+10$

$f 225: p \quad 725.0 \quad 0.0 \quad 0.0 \quad 0.0$

de225 $1.50000-2 \quad 3.25000-2 \quad 5.75000-2 \quad 8.50000-2$ $1.25000-1 \quad 2.25000-1 \quad 3.50000-1 \quad 4.55000-1 \quad 5.55000-1$ 6.50000-1 8.50000-1 $1.25000+0 \quad 1.75000+0 \quad 2.25000+0$ $2.75000+0 \quad 3.50000+0 \quad 4.50000+0 \quad 5.50000+0 \quad 6.50000+0$ $7.25000+0 \quad 7.75000+0 \quad 9.00000+01.20000+1$

$\begin{array}{llllll}\text { df225 } 2.14390-3 & 5.77600-4 & 2.71850-4 & 2.68170-4\end{array}$ $3.27670-4 \quad 5.66760-4 \quad 8.75940-4 \quad 1.08450-3 \quad 1.27970-3$ $1.44170-3 \quad 1.75630-3 \quad 2.31560-3 \quad 2.92700-3 \quad 3.46860-3$ $\begin{array}{lllll}3.95960-3 & 4.62210-3 & 5.41370-3 & 6.19090-3 & 6.92650-3\end{array}$ $7.47830-37.84680-3 \quad 8.77160-3 \quad 1.10200-2$

fm225 $5.701+10$

f235:D $298.958-1710.00 .00 .0$

de235 $1.50000-2 \quad 3.25000-2 \quad 5.75000-2 \quad 8.50000-2$ $1.25000-12.25000-1 \quad 3.50000-1 \quad 4.55000-1 \quad 5.55000-1$ $6.50000-18.50000-1 \quad 1.25000+01.75000+0 \quad 2.25000+0$ $2.75000+0 \quad 3.50000+0 \quad 4.50000+0 \quad 5.50000+0 \quad 6.50000+0$ $7.25000+0 \quad 7.75000+0 \quad 9.00000+01.20000+1$

df235 $2.14390-3 \quad 5.77600-4 \quad 2.71850-4 \quad 2.68170-4$ $3.27670-4 \quad 5.66760-4 \quad 8.75940-4 \quad 1.08450-3 \quad 1.27970-3$ $\begin{array}{lllll}1.44170-3 & 1.75630-3 & 2.31560-3 & 2.92700-3 & 3.46860-3\end{array}$ $3.95960-3 \quad 4.62210-3 \quad 5.41370-3 \quad 6.19090-3 \quad 6.92650-3$ $\begin{array}{llll}7.47830-3 & 7.84680-3 & 8.77160-3 & 1.10200-2\end{array}$

$\operatorname{fm} 235 \quad 5.701+10$

f245:p $\quad 298.958 \quad 1296.0 \quad 0.0 \quad 0.0$

de245 $1.50000-2 \quad 3.25000-2 \quad 5.75000-2 \quad 8.50000-2$ $1.25000-1 \quad 2.25000-1 \quad 3.50000-1 \quad 4.55000-1 \quad 5.55000-1$ $6.50000-18.50000-1 \quad 1.25000+01.75000+0 \quad 2.25000+0$ $2.75000+0 \quad 3.50000+0 \quad 4.50000+0 \quad 5.50000+0 \quad 6.50000+0$ $7.25000+0 \quad 7.75000+09.00000+0 \quad 1.20000+1$

df245 $2.14390-3 \quad 5.77600-4 \quad 2.71850-4 \quad 2.68170-4$ $3.27670-4 \quad 5.66760-4 \quad 8.75940-4 \quad 1.08450-3 \quad 1.27970-3$ $\begin{array}{lllll}1.44170-3 & 1.75630-3 & 2.31560-3 & 2.92700-3 & 3.46860-3\end{array}$ $\begin{array}{lllll}3.95960-3 & 4.62210-3 & 5.41370-3 & 6.19090-3 & 6.92650-3\end{array}$ $\begin{array}{lllll}\operatorname{fm} 245 & 7.47830-3 & 7.84680-3 & 8.77160-3 & 1.10200-2\end{array}$ 


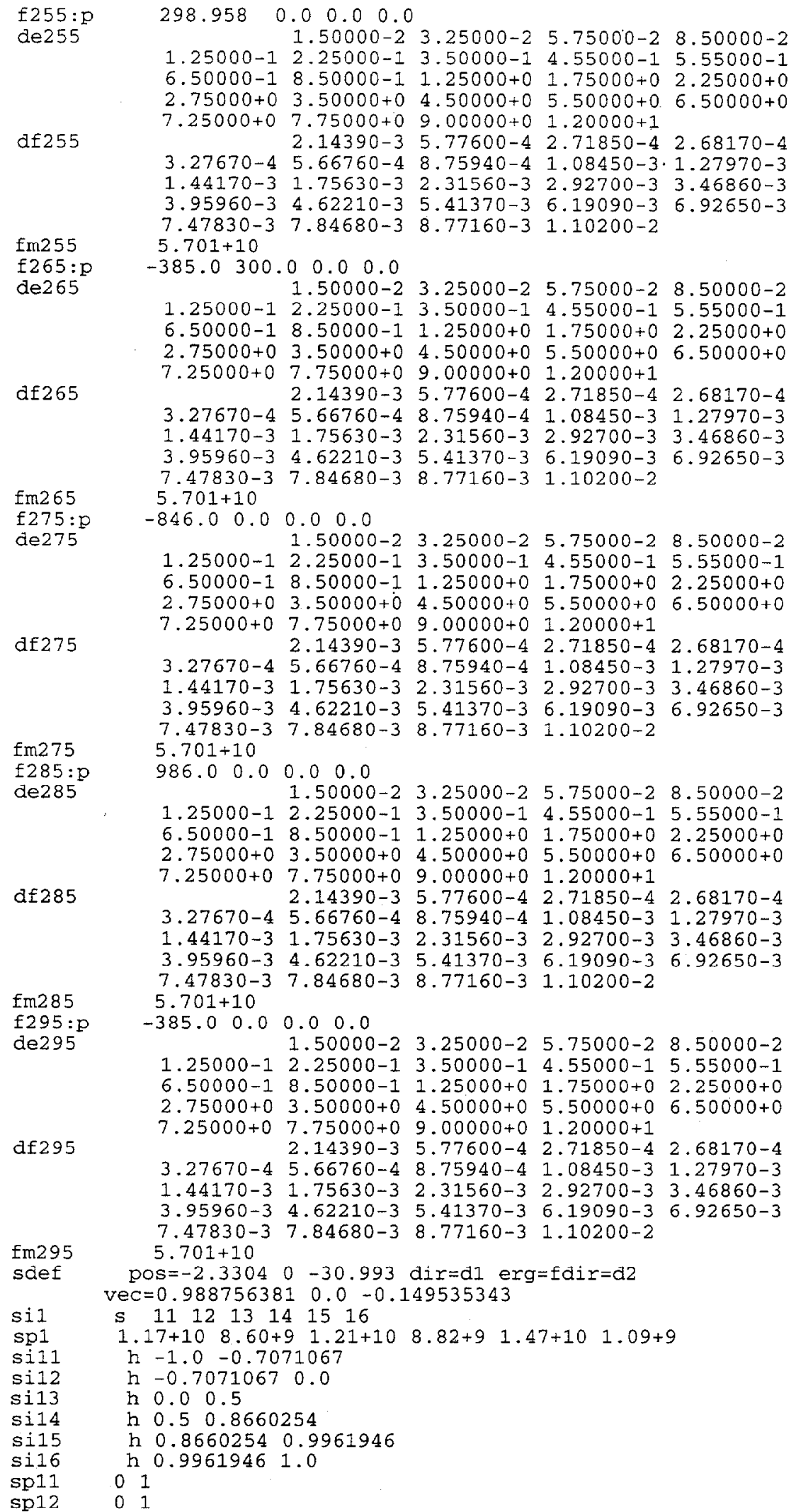




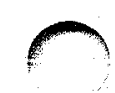

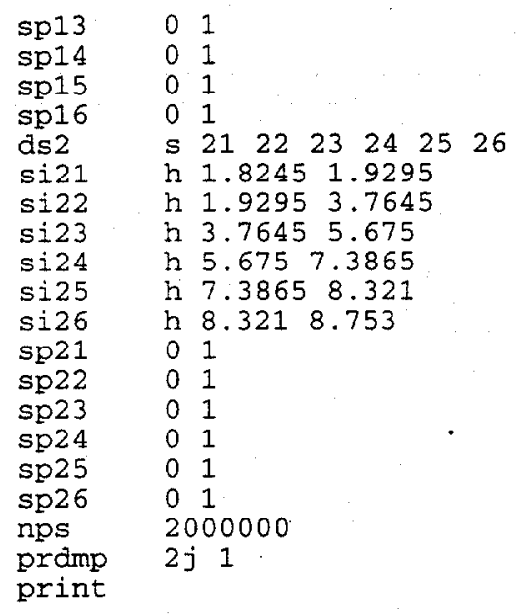

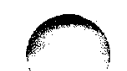


File for MCNP-4B Calculation of neutron dose rates along the truck lane of the PFNA facility for the source in the horizontal position

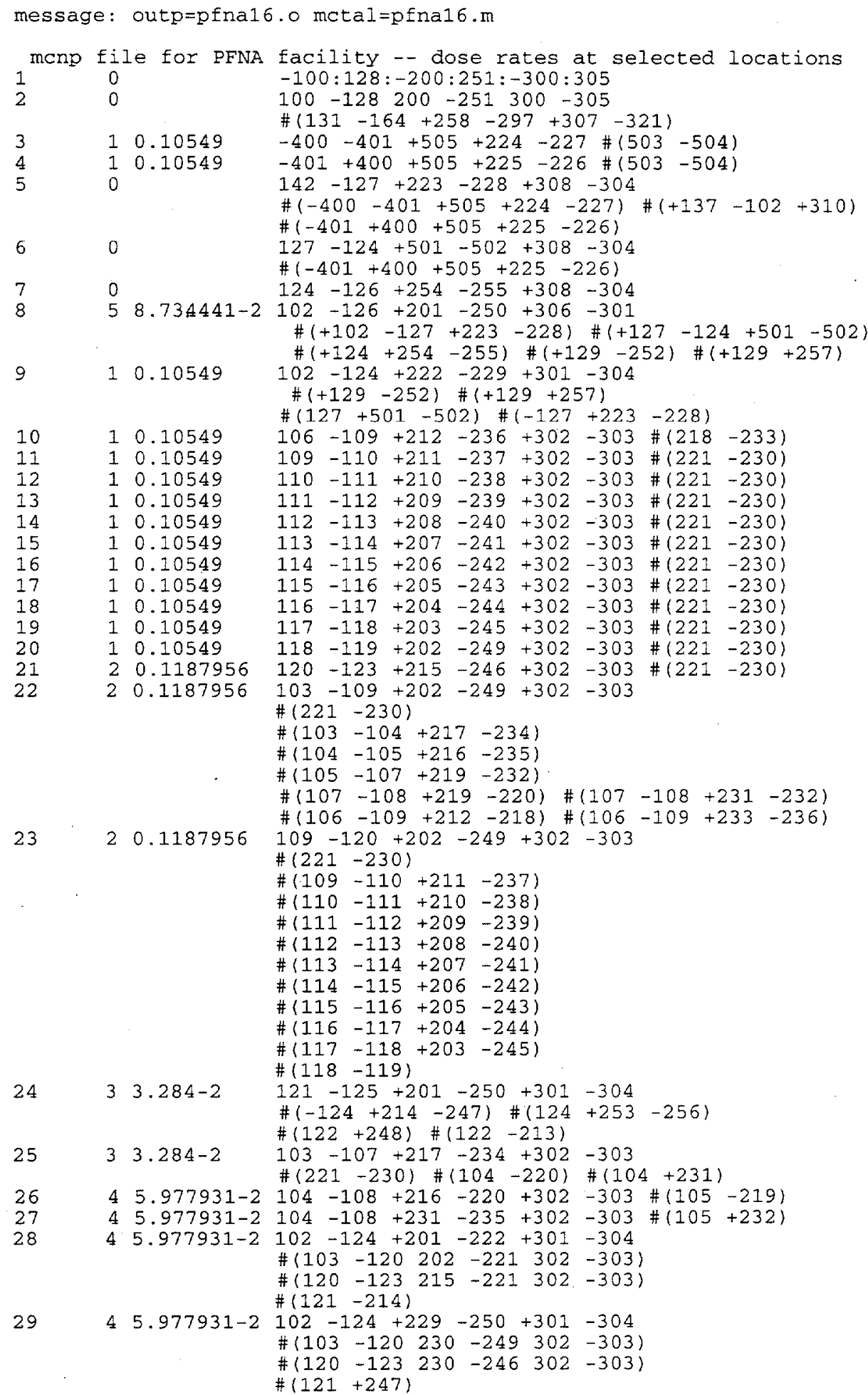




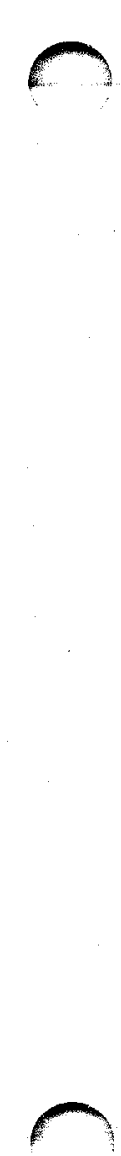

41
42
43
44
45
46
47
48

49

50

51

52

53

54

55

56

57

58
59

60

61

61
62
63

64

65

66

67

$\begin{array}{lll}68 & 10 & -2.0\end{array}$

$\begin{array}{rrr}69 & 10 & -2.0 \\ 70 & 0 & \end{array}$

$9-2.0$

$11-1.0$

$12-7.86$
0

$9-2.0$

$\begin{array}{ll}10 & -2.0\end{array}$

0

$10-2.0$

$10-2.0$

$10-2.0$.

0

$10-2.0$

$10-2.0$

$10-2.0$

$10-2.0$

$\begin{array}{ll}10 & -2.0\end{array}$

$10-2.0$

$9-2.0$

$10-2.0$

$10 .-2.0$.

$10-2.0$

$$
\#(-125+213-253)
$$

$45.977931-2 \quad 124-126+252-254+301-304$

$45.977931-2124-126+255-257+301-304$

$\#(-125+256-248)$

$+167-168+274-281+317-318$

$+165-166+270-285+308-310$

$\#(+167-168+274-281+317-318)$

$-400+505+224-227+503-504$

$-401+400+505+225-226+503-504$

$45.977931-2122-124+201-250+301-304$

\# $(213-248)$

$\#(129-252)$

$\#(129+257)$

$+131-164+258-297+307-308$

$+131-164+258-297+308-320$

$\#(+132-163+259-296)$

$+131-164+258-297+320-321$

$+132-163+259-296+319-320$

$\#(+151-169+271-284+304-315)$

$\#(+151-160+270-271+312-314)$

$\#(+151-160+284-285+312-314)$

$\#(+157-159+274-281+313-315)$

$\#(+158-160+274-281-313)$

$\#(+169-157+274-281+314-316)$

$10.10549+151-169+271-284+304-315$

$0.10549+151-160+270-271+312-314$

$0.10549+151-160+284-285+312-314$

$0.10549+157-159+274-281+313-315$

$0.10549+158-160+274-281+308-313$

$0.10549+169-157+274-281+314-316$

10.10549

10.10549

$+160-162+273-282+308-319$

$+154-161+265-289+308-319$

$\# 47 \#(-160+267-286)$

$+154-160+267-286+308-319 \# 42$ \#43 \#45

$+155-156+259-260+308-319$

$+155-156+295-296+308-319$

$+154-155+259-296+308-319$

\# ( $+265-289)$

$+155-163+259-296+308-319$ \#50 \#51

$\#(-161+265-289) \#(+161-162+273-282)$

$+153-154+295-296+308-319$

$+151-126+295-296+308-319$

$+151-126+259-260+308-319$

$+153-154+259-260+308-319$

$+151-152+266-272+308-304$

$+151-152+283-287+308-304$

$+151-154+259-296+308-319$

\#41 \#42 \#43 \#54 \#55 \#56 \#57 \#58 \#59

$\#(+165-166+270-285+308-310)$

$\#(-126+201-250-304)$

$+149-151+272-201+308-304$

$+149-151+250-283+308-304$

$+149-151+264-290+308-319$

$\#(+272-283)$

$+149-151+259-296+308-319$

\# $(+264-290)$

$+146-149+295-296+308-319$

$+132-149+259-296+308-319$

\#65 \# $(+133-294)$

$+133-149+259-294+308-319$

$\#(+142-260-322) \#(+134+260-298)$

$+135-149+261-262+308-319$

$+135-136+262-263+308-319$

$+137-149+268-293+308-311$

$\#(-138-290-310) \quad \#(-102+310)$

$\#(+138+269-290-310) \#(+144+290-292-310)$

$\left.\begin{array}{lllllll}\#+139 & -144 & +291 & -293 & -310\end{array}\right) \quad \#(+102+201-250-304)$

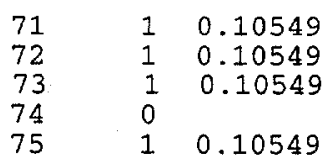

$+137-138+268-290+308-310$

$+138-149+269-272+308-310$

$+138-149+288-290+308-310 \#(+140-141)$

$+140-141+288-290+308-310$

$+144-149+290-292+308-310$ 


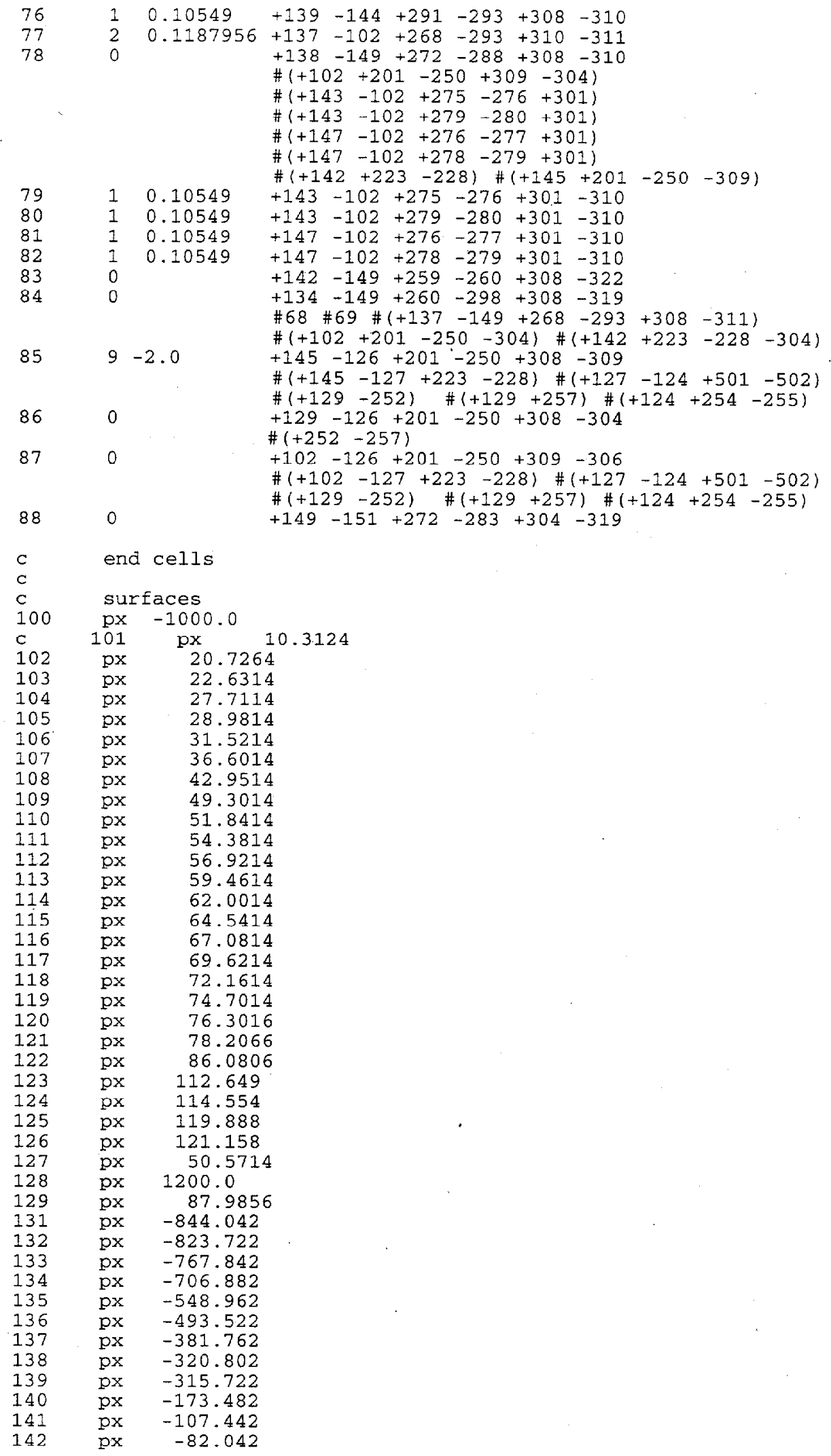




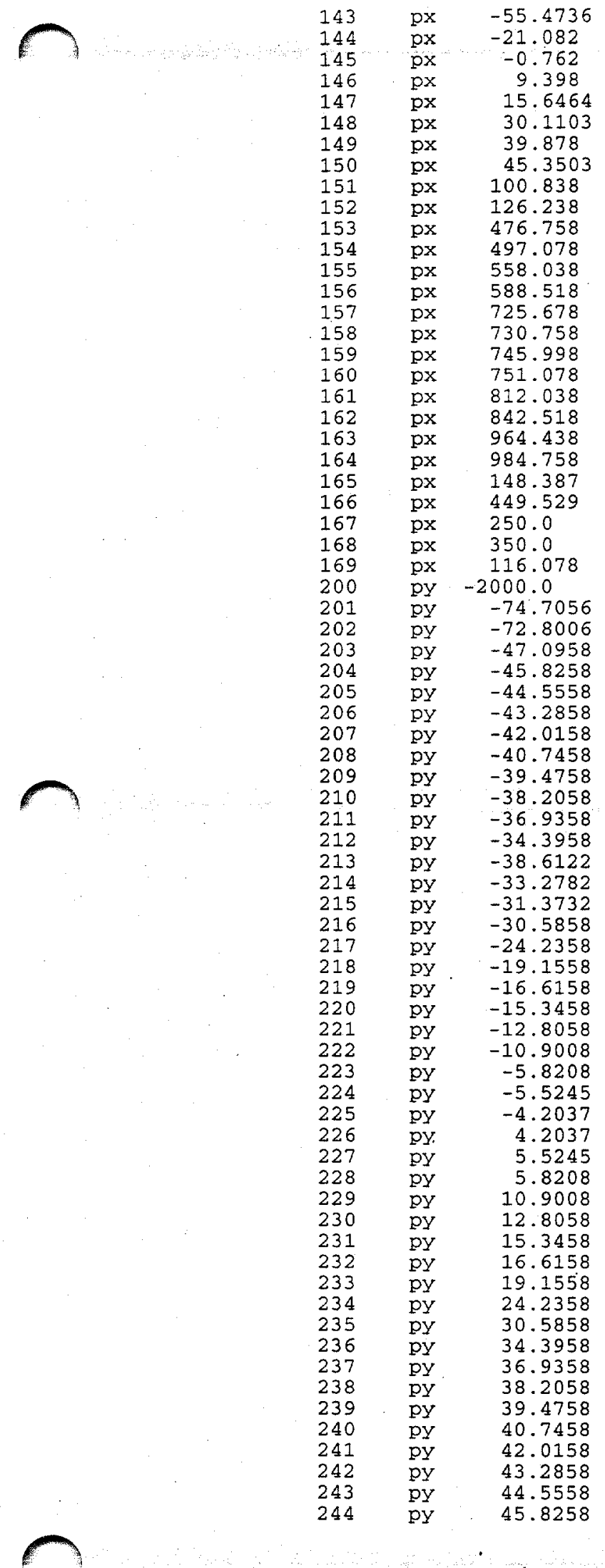




\begin{tabular}{|c|c|c|}
\hline 245 & py & 47.0958 \\
\hline 246 & py & 31.3732 \\
\hline 247 & py & 33.2782 \\
\hline 248 & py & 38.6122 \\
\hline 249 & py & 72.8006 \\
\hline 250 & py & 74.7056 \\
\hline 251 & py & 1500.0 \\
\hline 252 & py & -39.8822 \\
\hline 253 & py & -9.8848 \\
\hline 254 & py & -8.6148 \\
\hline 255 & py & 8.6148 \\
\hline 256 & py & 9.8848 \\
\hline 257 & py & 39.8822 \\
\hline 258 & py & -1731.28 \\
\hline 259 & py & -1710.96 \\
\hline 260 & py & -1650.0 \\
\hline 261 & py & -1528.08 \\
\hline 262 & py & $-146 \% \cdot 12$ \\
\hline 263 & py & -1436.64 \\
\hline 264 & py & -589.28 \\
\hline 265 & py & -241.3 \\
\hline 266 & py & -193.04 \\
\hline 267 & py & -180.34 \\
\hline 268 & py & -167.64 \\
\hline 269 & py & $-162 \cdot 56$ \\
\hline 270 & Dy & -152.4 \\
\hline 271 & py & $-137 \cdot 16$ \\
\hline 272 & py & -101.6 \\
\hline 273 & py & $-91 \cdot 44$ \\
\hline 274 & py & -60.96 \\
\hline 275 & py & -45.72 \\
\hline 276 & py & -30.48 \\
\hline 277 & py & -10.16 \\
\hline 278 & py & 10.16 \\
\hline 279 & py & 30.48 \\
\hline 280 & py & 45.72 \\
\hline 281 & py & 60.96 \\
\hline 282 & py & 91.44 \\
\hline 283 & py & 101.6 \\
\hline 284 & py & 137.16 \\
\hline 285 & py & 152.4 \\
\hline 286 & py & 180.34 \\
\hline 287 & py & 193.04 \\
\hline 288 & py & 213.36 \\
\hline 289 & py & 241.3 \\
\hline 290 & py & 274.32 \\
\hline 291 & py & 335.28 \\
\hline 292 & py & 345.44 \\
\hline 293 & py & 396.24 \\
\hline 294 & py & 533.4 \\
\hline 295 & py & 1235.44 \\
\hline 296 & py & 1296.4 \\
\hline 297 & py & 1316.72 \\
\hline 298 & py & 472.44 \\
\hline 300 & $\mathrm{pz}$ & -500.0 \\
\hline 301 & $\mathrm{pz}$ & -59.478 \\
\hline 302 & $p z$ & -57.573 \\
\hline 303 & $p z$ & 245.957 \\
\hline 304 & $\mathrm{pz}$ & 247.862 \\
\hline 305 & $p z$ & 500.0 \\
\hline 306 & $\mathrm{pz}$ & -60.748 \\
\hline 307 & $p z$ & -156.0 \\
\hline 308 & $\mathrm{pz}$ & -125.095 \\
\hline 309 & $p z$ & -67.451 \\
\hline 310 & $\mathrm{pz}$ & 235.585 \\
\hline 311 & $\mathrm{pz}$ & 276.225 \\
\hline 312 & $\mathrm{pz}$ & 283.845 \\
\hline 313 & $\mathrm{pz}$ & 342.265 \\
\hline 314 & $\mathrm{pz}$ & 375.285 \\
\hline 315 & $\mathrm{pz}$ & 390.525 \\
\hline 316 & $\mathrm{pz}$ & 395.605 \\
\hline 317 & $\mathrm{pz}$ & -50.0 \\
\hline
\end{tabular}



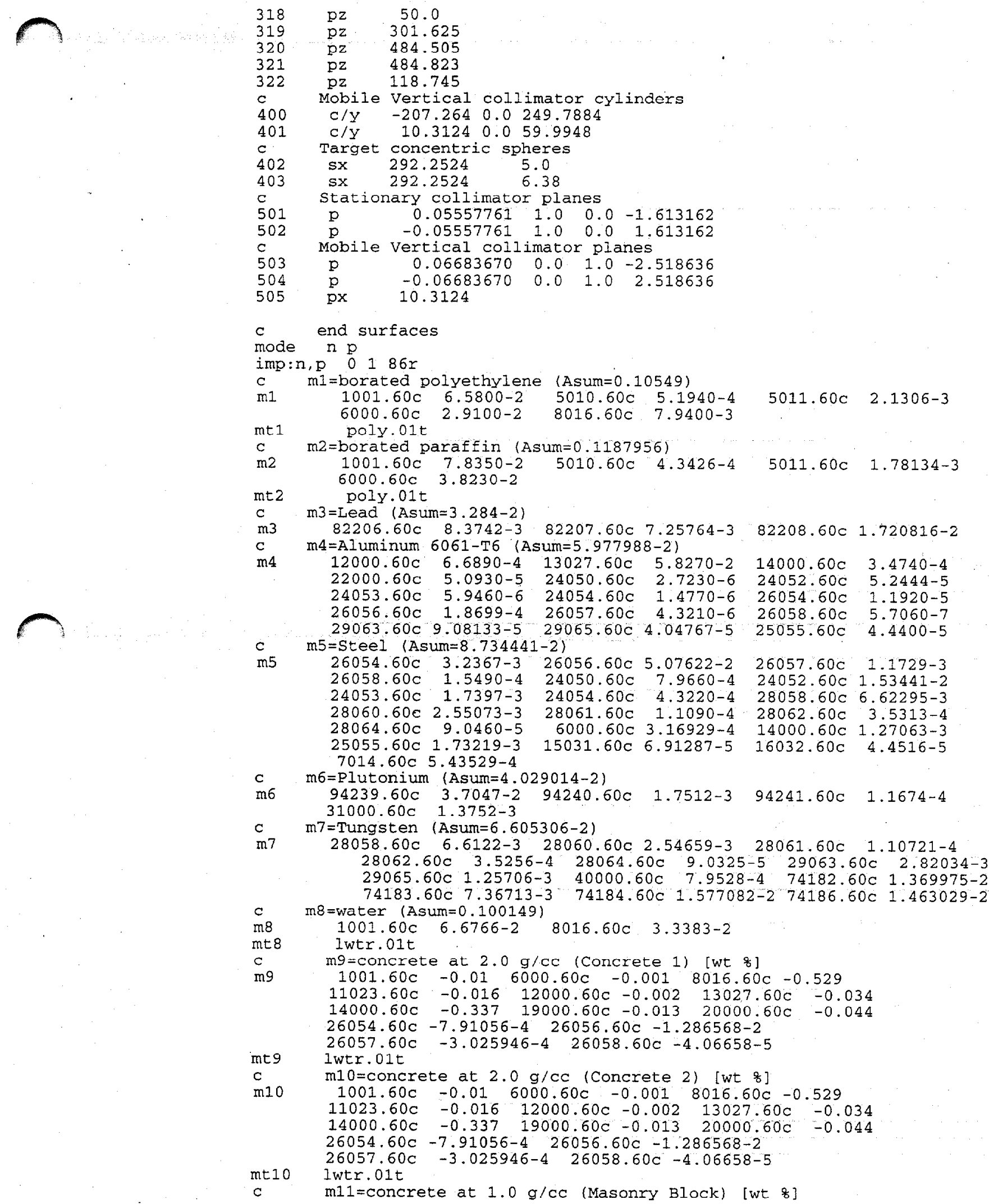


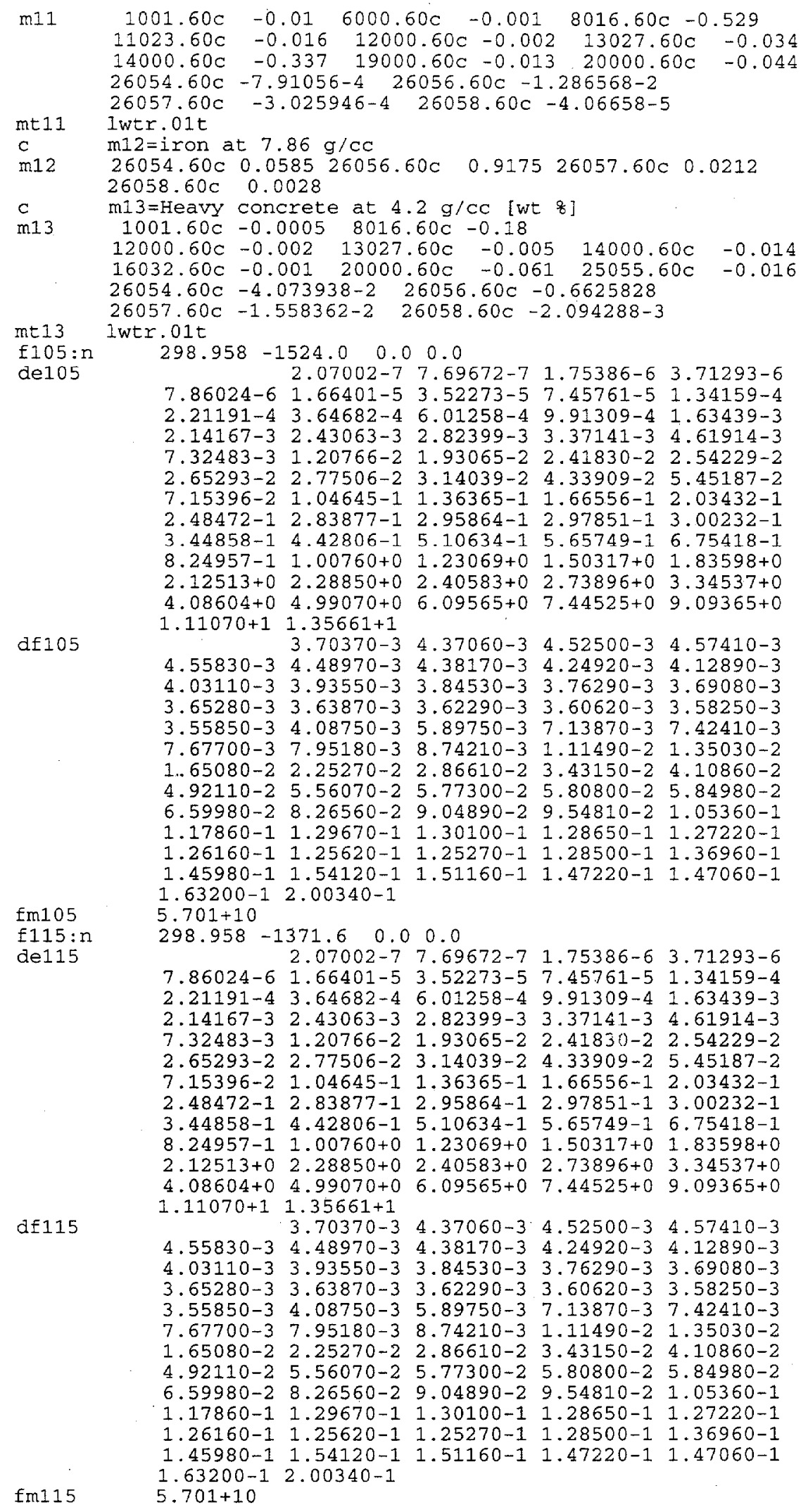




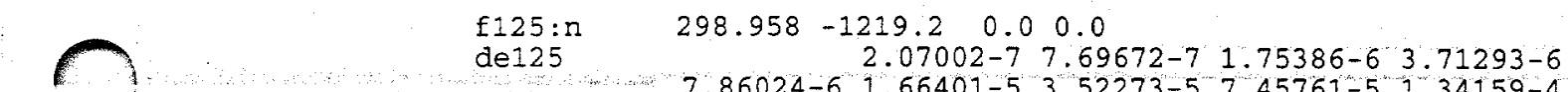
2.21191-4 $3.64682-4 \quad 6.01258-4 \quad 9.91309-4 \quad 1.63439-3$ $\begin{array}{llllll}2.14167-3 & 2.43063-3 & 2.82399-3 & 3.37141-3 & 4.61914-3\end{array}$ $7.32483-3 \quad 1.20766-2 \quad 1.93065-2 \quad 2.41830-2 \% 2.54229-2$ 2.65293-2 2.77506-2 3.14039-2 4.33909-2 5.45187-2 $7.15396-2$ 1.04645-1 $1.36365-1$ 1.66556-1 2.03432-1 $\begin{array}{lllll}2.48472-1 & 2.83877-1 & 2.95864-1 & 2.97851-1 & 3.00232-1\end{array}$ 3.44858-1 $4.42806-1 \quad 5.10634-1 \quad 5.65749-1 \quad 6.75418-1$ $8.24957-1 \quad 1.00760+0 \quad 1.23069+0 \quad 1.50317+0 \quad 1.83598+0$ $2.12513+0 \quad 2.28850+0 \quad 2.40583+0 \quad 2.73896+0 \quad 3.34537+0$ $4.08604+0 \quad 4.99070+0 \quad 6.09565+0 \quad 7.44525+0 \quad 9.09365+0$ $1.11070+1 \quad 1.35661+1$

$\begin{array}{llllll}3.70370-3 & 4.37060-3 & 4.52500-3 & 4.57410-3\end{array}$ $\begin{array}{lllll}4.55830-3 & 4.48970-3 & 4.38170-3 & 4.24920-3 & 4.12890-3\end{array}$ $\begin{array}{llllll}4.03110-3 & 3.93550-3 & 3.84530-3 & 3.76290-3 & 3.69080-3\end{array}$ $\begin{array}{llllll}3.65280-3 & 3.63870-3 & 3.62290-3 & 3.60620-3 & 3.58250-3\end{array}$ $\begin{array}{llllll}3.55850-3 & 4.08750-3 & 5.89750-3 & 7.13870-3 & 7.42410-3\end{array}$ $7.67700-3 \quad 7.95180-3 \quad 8.74210-3 \quad 1.11490-21.35030-2$ $1.65080-2 \quad 2.25270-2 \quad 2.86610-2 \quad 3.43150-2 \cdot 4.10860-2$ $4.92110-2 \quad 5.56070-2 \quad 5.77300-2 \quad 5.80800-2 \quad 5.84980-2$ $6.59980-2 \quad 8.26560-2 \quad 9.04890-2 \quad 9.54810-2 \cdots 1.05360-1$ $1.17860-1 \quad 1.29670-1 \quad 1.30100-1 \quad 1.28650-1 \quad 1.27220-1$ $1.26160-1 \quad 1.25620-1 \quad 1.25270-1 \quad 1.28500-1 \quad 1.36960-1$ $1.45980-1$ 1.54120-1 1.51160-1 1.47220-1 1.47060-1 $1.63200-12.00340-1$

fm125 $\quad 5.701+10$

f135:n $\quad 298.958-1066.8 \quad 0.0 \quad 0.0$

$\begin{array}{lllllll}\text { de135 } & 2.07002-7 & 7.69672-7 & 1.75386-6 & 3.71293-6\end{array}$ $7.86024-6 \quad 1.66401-5 \quad 3.52273-5 \quad 7.45761-5 \quad 1.34159-4$ $2.21191-4 \quad 3.64682-4 \quad 6.01258-4 \quad 9.91309-4 \quad 1.63439-3$ $2.14167-3 \quad 2.43063-3 \quad 2.82399-3 \quad 3.37141-3 \quad 4.61914-3$ $7.32483-3 \quad 1.20766-2 \quad 1.93065-2 \quad 2.41830-2 \quad 2.54229-2$ 2.65293-2 2.77506-2 3.14039-2 $4.33909-2 \quad 5.45187-2$ 7.15396-2 $1.04645-1 \quad 1.36365-1 \quad 1.66556-1 \quad 2.03432-1$ $2.48472-1 \quad 2.83877-1 \quad 2.95864-1 \quad 2.97851-1 \quad 3.00232-1$ 3.44858-1 $4.42806-1 \quad 5.10634-1 \quad 5.65749-1 \quad 6.75418-1$ $8.24957-1 \quad 1.00760+0 \quad 1.23069+01.50317+01.83598+0$ $2.12513+0 \quad 2.28850+0 \quad 2.40583+0 \quad 2.73896+0 \quad 3.34537+0$ $4.08604+0 \quad 4.99070+0 \quad 6.09565+0 \quad 7.44525+0 \quad 9.09365+0$ $1.11070+1 \quad 1.35661+1$

df135 $\quad 3.70370-3 \quad 4.37060-3 \quad 4.52500-3 \quad 4.57410-3$ $4.55830-3 \quad 4.48970-3 \quad 4.38170-3 \quad 4.24920-3 \quad 4.12890-3$ $\begin{array}{llllll}4.03110-3 & 3.93550-3 & 3.84530-3 & 3.76290-3 & 3.69080-3\end{array}$ $3.65280-3 \quad 3.63870-3 \quad 3.62290-3 \quad 3.60620-3 \quad 3.58250-3$ $3.55850-3 \quad 4.08750-3 \quad 5.89750-3 \quad 7.13870-3 \quad 7.42410-3$ $\begin{array}{lllll}7.67700-3 & 7.95180-3 & 8.74210-3 & 1.11490-2 & 1.35030-2\end{array}$ 1.65080-2 2.25270-2 2.86610-2 3.43150-2 4.10860-2 $4.92110-2 \quad 5.56070-2 \quad 5.77300-2 \quad 5.80800-2 \quad 5.84980-2$ 6.59980-2 8.26560-2 $9.04890-2 \quad 9.54810-21.05360-1$ $1.17860-1 \quad 1.29670-1 \quad 1.30100-1 \quad 1.28650-1 \quad 1.27220-1$ 1.26160-1 $1.25620-1 \quad 1.25270-1 \quad 1.28500-1 \quad 1.36960-1$ 1.45980-1 1.54120-1 $1.51160-1 \quad 1.47220-1 \quad 1.47060-1$ $1.63200-12.00340-1$

$f \operatorname{m135} \quad 5.701+10$

f145:n $\quad 298.958 \quad-914.4 \quad 0.00 .0$

$\begin{array}{llllll}\operatorname{de} 145 & 2.07002-7 & 7.69572-7 & 1.75386-6 & 3.71293-6\end{array}$ 7.86024-6 $1.66401-5 \quad 3.52273-5 \quad 7.45761-5 \quad 1.34159-4$ $\begin{array}{llllll}2.21191-4 & 3.64682-4 & 6.01258-4 & 9.91309-4 & 1.63439-3\end{array}$ $2.14167-3 \quad 2.43063-3 \quad 2.82399-3 \quad 3.37141-3 \quad 4.61914-3$ 7.32483-3 $1.20766-2 \quad 1.93065-2 \quad 2.41830-2 \quad 2.54229-2$ $2.65293-2 \quad 2.77506-2 \quad 3.14039-2 \quad 4.33909-2 \quad 5.45187-2$ 7.15396-2 1.04645-1 $1.36365-1 \quad 1.66556-1 \quad 2.03432-1$ 2.48472-1 2.83877-1 2.95864-1 2.97851-1 3.00232-1 3.44858-1 4.42806-1 5.10634-1 $5.65749-1 \quad 6.75418-1$ $8.24957-1 \quad 1.00760+0 \quad 1.23069+0 \quad 1.50317+0 \quad 1.83598+0$ $\begin{array}{llllll}2.12513+0 & 2.28850+0 & 2.40583+0 & 2.73896+0 & 3.34537+0\end{array}$ $4.08604+0 \quad 4.99070+0 \quad 6.09565+0 \quad 7.44525+0 \quad 9.09365+0$ $1.11070+1 \quad 1.35661+1$

df145 $3.70370-3 \quad 4.37060-3 \quad 4.52500-3 \quad 4.57410-3$ $4.55830-3 \quad 4.48970-3 \quad 4.38170-3 \quad 4.24920-3 \quad 4.12890-3$ 
$\begin{array}{llllll}4.03110-3 & 3.93550-3 & 3.84530-3 & 3.76290-3 & 3.69080-3\end{array}$ $\begin{array}{lllll}3.65280-3 & 3.63870-3 & 3.62290-3 & 3.60620-3 & 3.58250-3\end{array}$ $3.55850-3 \quad 4.08750-3 \quad 5.89750-3 \quad 7.13870-3 \quad 7.42410-3$ $\begin{array}{lllll}7.67700-3 & 7.95180-3 & 8.74210-3 & 1.11490-2 & 1.35030-2\end{array}$ $1.65080-2 \quad 2.25270-2 \quad 2.86610-2 \quad 3.43150-2 \quad 4.10860-2$ $4.92110-2 \quad 5.56070-2 \quad 5.77300-2 \quad 5.80800-2 \quad 5.84980-2$ $6.59980-2 \quad 8.26560-2 \quad 9.04890-2 \quad 9.54810-2 \quad 1.05360-1$ $1.17860-1 \quad 1.29670-1 \quad 1.30100-1 \quad 1.28650-11.27220-1$

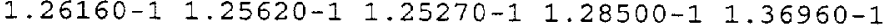
$1.45980-1$ 1.54120-1 $1.51160-1 \quad 1.47220-11.47060-1$ $1.63200-12.00340-1$

$\operatorname{fm} 145$ $5.701+10$

f155: $\mathrm{n}$ de 155 298.958 $\begin{array}{lll}-762.0 & 0.0 & 0.0\end{array}$ $2.07002-7 \quad 7.69672-7 \quad 1.75386-6 \quad 3.71293-6$ $7.86024-6 \quad 1.66401-5 \quad 3.52273-5 \quad 7.45761-5 \quad 1.34159-4$ $2.21191-4 \quad 3.64682-4 \quad 6.01258-4 \quad 9.91309-41.63439-3$ $2.14167-3 \quad 2.43063-3 \quad 2.82399-3.3 .37141-3 \quad 4.61914-3$ $\begin{array}{lllll}7.32483-3 & 1.20766-2 & 1.93065-2 & 2.41830-2 & 2.54229-2\end{array}$ 2.65293-2 2.77506-2 3.14039-2 4.33909-2 5.45187-2 7.15396-2 1.04645-1 $1.36365-1 \quad 1.66556-1 \quad 2.03432-1$ 2.48472-1 2.83877-1 $2.95864-1 \quad 2.97851-1 \quad 3.00232-1$ 3.44858-1 4.42806-1 $5.10634-1 \quad 5.65749-1 \quad 6.75418-1$ 8.24957-1 $1.00760+0 \quad 1.23069+0 \quad 1.50317+0 \quad 1.83598+0$ $2.12513+0 \quad 2.28850+0 \quad 2.40583+0 \quad 2.73896+0 \quad 3.34537+0$ $4.08604+0 \quad 4.99070+0 \quad 6.09565+0 \quad 7.44525+0 \quad 9.09365+0$ $1.11070+11.35661+1$

df155

fm155 $3.70370-3$
$3.11070+1$

$4.55830-3.4$. $4.38170-3 \quad 4.24920-3 \quad 4.12890-3$ $\begin{array}{lllll}4.03110-3 & 3.93550-3 & 3.84530-3 & 3.76290-3 & 3.69080-3\end{array}$ $\begin{array}{llllll}3.65280-3 & 3.63870-3 & 3.62290-3 & 3.60620-3 & 3.58250-3\end{array}$ $3.55850-3 \quad 4.08750-3 \quad 5.89750-3 \quad 7.13870-3 \quad 7.42410-3$ $\begin{array}{lllll}7.67700-3 & 7.95180-3 & 8.74210-3 & 1.11490-2 & 1.35030-2\end{array}$ $1.65080-2 \quad 2.25270-2 \quad 2.86610-2 \quad 3.43150-2 \quad 4.10860-2$ $4.92110-2 \quad 5.56070-2 \quad 5.77300-2 \quad 5.80800-2 \quad 5.84980-2$ $6.59980-2 \quad 8.26560-2 \quad 9.04890-2 \quad 9.54810-2 \quad 1.05360-1$ $1.17860-1 \quad 1.29670-1 \quad 1.30100-1 \quad 1.28650-11.27220-1$ $1.26160-1 \quad 1.25620-1 \quad 1.25270-1 \quad 1.28500-1 \quad 1.36960-1$ 1.45980-1 1.54120-1 1.51160-1 $1.47220-1$ 1.47060-1 $1.63200-12.00340-1$

$5.701+10$

f165:n $5.701+10$
298.958

de165

a 165

$2.07002-7 \quad 7.69672-7 \quad 1.75386-6 \quad 3.71293-6$ $66401-5 \quad 3.52273-5 \quad 7.45761-5 \quad 1.34159-4$ 2.21191-4 3.64682-4 6.01258-4 9.91309-4 $1.63439-3$ 2.14167-3 $2.43063-3 \quad 2.82399-3 \quad 3.37141-3 \quad 4.61914-3$ 7.32.483-3 1.20766-2 $1.93065-2 \quad 2.41830-2 \quad 2.54229-2$ 2.65293-2 2.77506-2 $3.14039-2 \quad 4.33909-2 \quad 5.45187-2$ 7.15396-2 $1.04645-1 \quad 1.36365-1 \quad 1.66556-1 \quad 2.03432-1$ 2.48472-1 $2.83877-1 \quad 2.95864-1 \quad 2.97851-1 \quad 3.00232-1$ 3.44858-1 $4.42806-1 \quad 5.10634-1 \quad 5.65749-1 \quad 6.75418-1$ $\begin{array}{llllll}8.24957-1 & 1.00760+0 & 1.23069+0 & 1.50317+0 & 1.83598+0\end{array}$ $2.12513+0 \quad 2.28850+0 \quad 2.40583+0 \quad 2.73896+0 \quad 3.34537+0$ $4.08604+0 \quad 4.99070+0 \quad 6.09565+0 \quad 7.44525+0 \quad 9.09365+0$ $1.11070+11.35661+1$ $3.70370-3 \quad 4.37060-3 \quad 4.52500-3 \quad 4.57410-3$ $4.55830-3 \quad 4.48970-3 \quad 4.38170-3 \quad 4.24920-3 \quad 4.12890-3$ $\begin{array}{llllll}4.03110-3 & 3.93550-3 & 3.84530-3 & 3.76290-3 & 3.69080-3\end{array}$ $3.65280-3 \quad 3.63870-3 \quad 3.62290-3 \quad 3.60620-3 \quad 3.58250-3$ $\begin{array}{llllll}3.55850-3 & 4.08750-3 & 5.89750-3 & 7.13870-3 & 7.42410-3\end{array}$ $7.67700-3 \quad 7.95180-3 \quad 8.74210-3 \quad 1.11490-2 \quad 1.35030-2$ $1.65080-2 \quad 2.25270-2 \quad 2.86610-2 \quad 3.43150-2 \quad 4.10860-2$ 4.92110-2 5.56070-2 5.77300-2 5.80800-2 5.84980-2 $6.59980-2 \quad 8.26560-2 \quad 9.04890-2 \quad 9.54810-2 \quad 1.05360-1$ $1.17860-1 \quad 1.29670-1 \quad 1.30100-1 \quad 1.28650-1 \quad 1.27220-1$ $1.26160-1$ 1.25620-1 $1.25270-1$ 1.28500-1 $1.36960-1$ 1.45980-1 1.54120-1 1.51160-1 1.47220-1 1.47060-1. $1.63200-1 \quad 2.00340-1$

$\operatorname{fm} 165 \quad 5.701+10$

$\begin{array}{llllll}\mathrm{f} 175: \mathrm{n} & 298.958 & -457.2 & 0.0 & 0.0\end{array}$

de175 $2.07002-7 \quad 7.69672-7 \quad 1.75386-6 \quad 3.71293-6$

$7.86024-6 \quad 1.66401-5 \quad 3.52273-5 \quad 7.45761-5 \quad 1.34159-4$

$2.21191-4 \quad 3.61682-4 \quad 6.01258-4 \quad 9.91309-4 \quad 1.63439 \cdots 3$ 
$2.14167-3 \quad 2.43063-3 \quad 2.82399-3 \quad 3.37141-3 \quad 4.61914-3$ $7.32483-3 \quad 1.20766-2 \quad 1.93065-2 \quad 2.41830-2 \quad 2.54229-2$ $2.65293-2$ 2.77506-2 3.14039-2 4.33909-2 5.45187-2 7.15396-2 $1.04645-1 \quad 1.36365-1 \quad 1.66556-1 \quad 2.03432-1$ 2.48472-1 2.83877-1 2.95864-1 $2.97851-1 \quad 3.00232-1$ $\begin{array}{lllllll}3.44858-1 & 4.42806-1 & 5.10634-1 & 5.65749-1 & 6.75418-1\end{array}$ $8.24957-1 \quad 1.00760+0 \quad 1.23069+0 \quad 1.50317+0 \quad 1.83598+0$ $2.12513+0 \quad 2.28850+0 \quad 2.40583+0 \quad 2.73896+0 \quad 3.34537+0$ $\begin{array}{lllll}4.08604+0 & 4.99070+0 & 6.09565+0 & 7.44525+0 & 9.09365+0\end{array}$ $1.11070+11.35661+1$

df175 $3.70370-3 \quad 4.37060-3 \quad 4.52500-3 \quad 4.57410-3$ $\begin{array}{lllllll}4.55830-3 & 4.48970-3 & 4.38170-3 & 4.24920-3 & 4.12890-3\end{array}$ $4.03110-3 \quad 3.93550-3 \quad 3.84530-3 \quad 3.76290-3 \quad 3.69080-3$ $\begin{array}{llllll}3.65280-3 & 3.63870-3 & 3.62290-3 & 3.60620-3 & 3.58250-3\end{array}$ $\begin{array}{llllll}3.55850-3 & 4.08750-3 & 5.89750-3 & 7.13870-3 & 7.42410-3\end{array}$ $7.67700-3.7 .95180-3 \quad 8.74210-3 \quad 1.11490-2 \quad 1.35030-2$ $1.65080-2 \quad 2.25270-2 \quad 2.86610-2 \quad 3.43150-2 \quad 4.10860-2$ 4.92110-2 5.56070-2 $5.77300-2 \quad 5.80800-2 \quad 5.84980-2$ $6.59980-2 \quad 8.26560-2 \quad 9.04890-2 \quad 9.54810-2 \quad 1.05360-1$

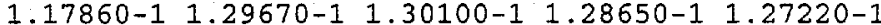
$1.26160-1$ 1.25620-1 $1.25270-1$ 1.28500-1 $1.36960-1$ 1.45980-1 1.54120-1 $1.51160-1 \quad 1.47220-1 \quad 1.47060-1$ $1.63200-12.00340-1$

$\operatorname{fm} 175$ $5.701+10$ $\begin{array}{llllll} & 185: n & 298.958 & -304.8 & 0.0 & 0.0\end{array}$

$\begin{array}{lllllll}\text { de185 } & 2.07002-7 & 7.69672-7 & 1.75386-6 & 3.71293-6\end{array}$ $7.86024-6 \quad 1.66401-5 \quad 3.52273-5 \quad 7.45761-5 \quad 1.34159-4$ $2.21191-4 \quad 3.64682-4 \quad 6.01258-4 \quad 9.91309-4 \quad 1.63439-3$ 2.14167-3 $2.43063-3 \quad 2.82399-3 \quad 3.37141-3 \quad 4.61914-3$ $\begin{array}{lllll}7.32483-3 & 1.20766-2 & 1.93065-2 & 2.41830-2 & 2.54229-2\end{array}$ $2.65293-2 \quad 2.77506-2 \cdot 3.14039-2 \quad 4.33909-2 \quad 5.45187-2$ $7.15396-2 \quad 1.04645-1 \quad 1.36365-1 \quad 1.66556-1 \quad 2.03432-1$ $\begin{array}{llllll}2.48472-1 & 2.83877-1 & 2.95864-1 & 2.97851-1 & 3.00232-1\end{array}$

3.44858-1 $4.42806-1 \quad 5.10634-1 \quad 5.65749-1 \quad 6.75418-1$ $8.24957-11.00760+0 \quad 1.23069+01.50317+0 \quad 1.83598+0$ $2.12513+0 \quad 2.28850+0 \quad 2.40583+0 \quad 2.73896+0 \quad 3.34537+0$ $4.08604+0 \quad 4.99070+0 \quad 6.09565+0 \quad 7.44525+0 \quad 9.09365+0$ $1.11070+11.35661+1$

$3.70370-3 \quad 4.37060-3 \quad 4.52500-3 \quad 4.57410-3$ $4.55830-3 \quad 4.48970-3 \quad 4.38170-3 \quad 4.24920-3 \quad 4.12890-3$ $\begin{array}{llllll}4.03110-3 & 3.93550-3 & 3.84530-3 & 3.76290-3 & 3.69080-3\end{array}$ $\begin{array}{llllll}3.65280-3 & 3.63870-3 & 3.62290-3 & 3.60620-3 & 3.58250-3\end{array}$ $\begin{array}{llllll}3.55850-3 & 4.08750-3 & 5.89750-3 & 7.13870-3 & 7.42410-3\end{array}$ $7.67700-3 \quad 7.95180-3 \quad 8.74210-3 \quad 1.11490-2 \quad 1.35030-2$

1.65080-2 2.25270-2 $2.86610-2 \quad 3.43150-2 \quad 4.10860-2$ 4.92110-2 $5.56070-2 \quad 5.77300-2 \quad 5.80800-2 \quad 5.84980-2$ $6.59980-2 \quad 8.26560-2 \quad 9.04890-2 \quad 9.54810-2 \quad 1.05360-1$ $\begin{array}{llllll}1.17860-1 & 1.29670-1 & 1.30100-1 & 1.28650-1 & 1.27220-1\end{array}$ $1.26160-1$ 1.25620-1 $1.25270-1$ 1.28500-1 $1.36960-1$ $1.45980-1 \quad 1.54120-1 \quad 1.51160-1 \quad 1.47220-1 \quad 1.47060-1$ $1.63200-12.00340-1$

fm185 $\quad 5.701+10$

f195:n $\quad 298.958 \quad-152.4 \quad 0.0 \quad 0.0$

$\begin{array}{lllllll}\text { de195 } & 2.07002-7 & 7.69672-7 & 1.75386-6 & 3.71293-6\end{array}$

$7.86024-6 \quad 1.66401-5 \quad 3.52273-5 \quad 7.45761-5 \quad 1.34159-4$

2.21191-4 $3.64682-4 \quad 6.01258-4 \quad 9.91309-4$ 1.63439-3

$2.14167-3 \quad 2.43063-32.82399-3 \quad 3.37141-3 \quad 4.61914-3$

7.32483-3 1.20766-2 $1.93065-2 \quad 2.41830-2 \quad 2.54229-2$

2.65293-2 2.77506-2 $3.14039-2 \quad 4.33909-2 \quad 5.45187-2$

7.15396-2 1.04645-1 $1.36365-1$ 1.66556-1 2.03432-1

$\begin{array}{llllll}2.48472-1 & 2.83877-1 & 2.95864-1 & 2.97851-1 & 3.00232-1\end{array}$

3.44858-1 $4.42806-1 \quad 5.10634-1 \quad 5.65749-1 \quad 6.75418-1$ $8.24957-1 \quad 1.00760+0 \quad 1.23069+0 \quad 1.50317+0 \quad 1.83598+0$ $\begin{array}{lllll}2.12513+0 & 2.28850+0 & 2.40583+0 & 2.73896+0 & 3.34537+0\end{array}$ $4.08604+0 \quad 4.99070+0 \quad 6.09565+0 \quad 7.44525+0 \quad 9.09365+0$ $1.11070+11.35661+1$

$\operatorname{df} 195$ $3.70370-3 \quad 4.37060-3 \quad 4.52500-3 \quad 4.57410-3$ $4.55830-3 \quad 4.48970-3 \quad 4.38170-3 \quad 4.24920-3 \quad 4.12890-3$ $\begin{array}{llllll}4.03110-3 & 3.93550-3 & 3.84530-3 & 3.76290-3 & 3.69080-3\end{array}$ $3.65280-3 \quad 3.63870-3 \quad 3.62290-3 \quad 3.60620-3 \quad 3.58250-3$

$3.55850-3 \quad 4.08750-3 \quad 5.89750-3 \quad 7.13870-3 \quad 7.42410-3$ $\begin{array}{lllll}7.67700-3 & 7.95180-3 & 8.74210-3 & 1.11490-2 & 1.35030-2\end{array}$ 


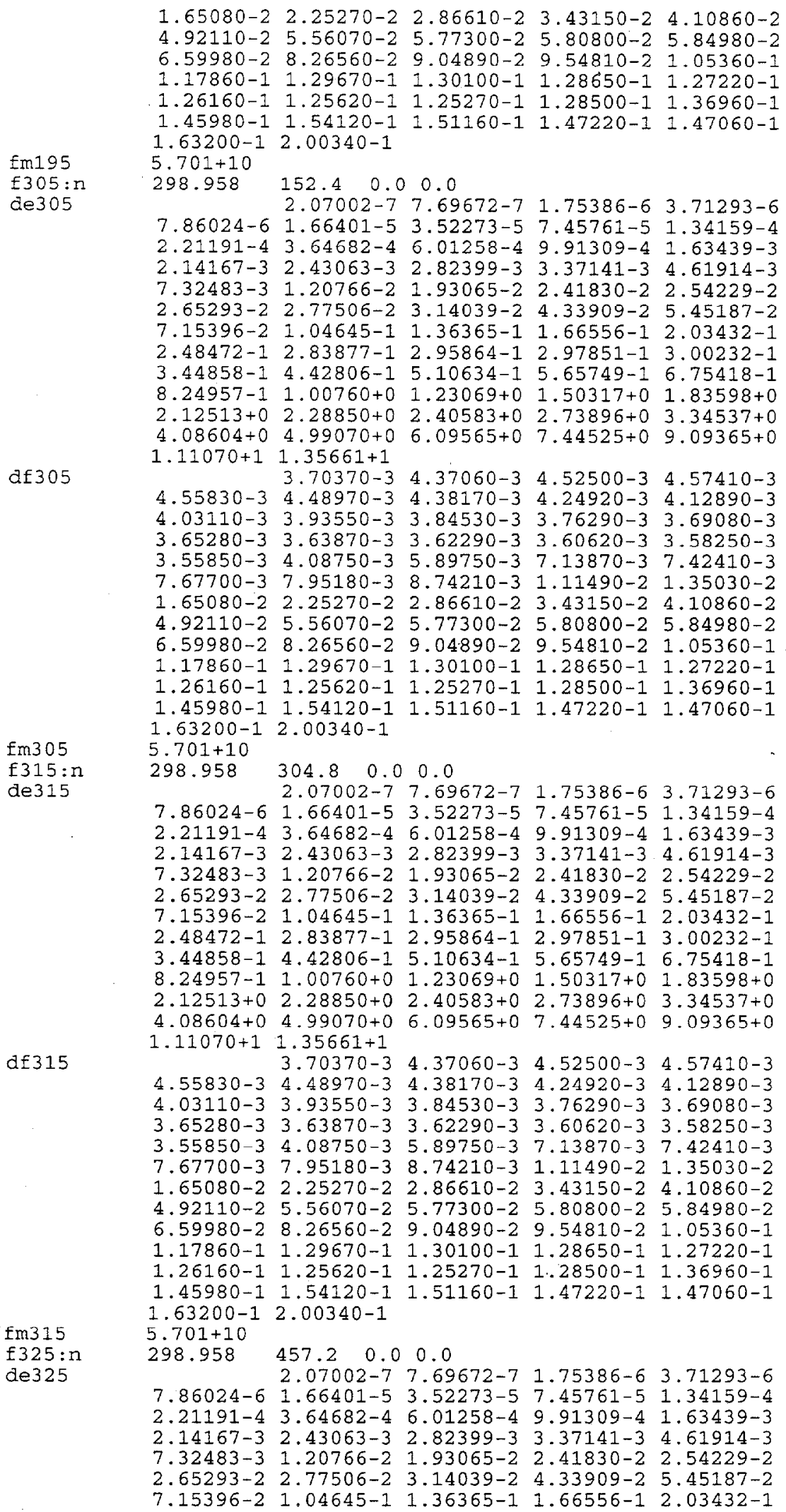


$\begin{array}{lllllll}2.48472-1 & 2.83877-1 & 2.95864-1 & 2.97851-1 & 3.00232-1\end{array}$ $3.44858-1 \quad 4.42806-1 \quad 5.10634-1 \quad 5.65749-1 \quad 6.75418-1$ $8.24957-1 \quad 1.00760+0 \quad 1.23069+0 \quad 1.50317+0 \quad 1.83598+0$ $\begin{array}{llllll}2.12513+0 & 2.28850+0 & 2.40583+0 & 2.73896+0 & 3.34537+0\end{array}$ $\begin{array}{lllll}4.08604+0 & 4.99070+0 & 6.09565+0 & 7.44525+0 & 9.09365+0\end{array}$ $1.11070+1 \quad 1.35661+1$

$3.70370-3 \quad 4.37060-3 \quad 4.52500-3 \quad 4.57410-3$ $4.55830-3 \quad 4.48970-3 \quad 4.38170-3 \quad 4.24920-3 \quad 4.12890-3$ $\begin{array}{llllll}4.03110-3 & 3.93550-3 & 3.84530-3 & 3.76290-3 & 3.69080-3\end{array}$ $3.65280-3 \quad 3.63870-3 \quad 3.62290-3 \quad 3.60620-3 \quad 3.58250-3$ $\begin{array}{llllll}3.55850-3 & 4.08750-3 & 5.89750-3 & 7.13870-3 & 7.42410-3\end{array}$ $7.67700-3 \quad 7.95180-3 \quad 8.74210-3 \quad 1.11490-2 \quad 1.35030-2$ $1.65080-2 \quad 2.25270-2 \quad 2.86610-2 \quad 3.43150-2 \quad 4.10860-2$ $4.92110-2 \quad 5.56070-2 \quad 5.77300-2 \quad 5.80800-2 \quad 5.84980-2$ $6.59980-2 \quad 8.26560-2 \quad 9.04890-2 \quad 9.54810-2 \quad 1.05360-1$ $\begin{array}{llllll}1.17860-1 & 1.29670-1 & 1.30100-1 & 1.28650-1 & 1.27220-1\end{array}$ $1.26160-1 \quad 1.25620-1 \quad 1.25270-1 \quad 1.28500-1 \quad 1.36960-1$ $1.45980-1$ 1.54120-1 $1.51160-1 \quad 1.47220-1 \quad 1.47060-1$ $1.63200-12.00340-1$

$\operatorname{fm} 325 \quad 5.701+10$

$\begin{array}{lllll}\mathrm{f} 335: \mathrm{n} & 298.958 & 609.6 & 0.0 & 0.0\end{array}$

$\begin{array}{llllll}\text { de335 } & 2.07002-7 & 7.69672-7 & 1.75386-6 & 3.71293-6\end{array}$

$7.86024-6 \quad 1.66401-5 \quad 3.52273-5 \quad 7.45761-5 \quad 1.34159-4$ $\begin{array}{llllll}2.21191-4 & 3.64682-4 & 6.01258-4 & 9.91309-4 & 1.63439-3\end{array}$ $2.14167-3 \quad 2.43063-3 \quad 2.82399-3 \quad 3.37141-3 \quad 4.61914-3$ $7.32483-3 \quad 1.20766-2 \quad 1.93065-2 \quad 2.41830-2 \quad 2.54229-2$ 2.65293-2 2.77506-2 3.14039-2 4.33909-2 5.45187-2 7.15396-2 $1.04645-1 \quad 1.36365-1 \quad 1.66556-12.03432-1$ $\begin{array}{llllll}2.48472-1 & 2.83877-1 & 2.95864-1 & 2.97851-1 & 3.00232-1\end{array}$ 3.44858-1 $4.42806-1 \quad 5.10634-1 \quad 5.65749-1 \quad 6.75418-1$ $8.24957-1 \quad 1.00760+0 \quad 1.23069+0 \quad 1.50317+0 \quad 1.83598+0$ $2.12513+0 \quad 2.28850+0 \quad 2.40583+0.2 .73896+0 \quad 3.34537+0$ $4.08604+0 \quad 4.99070+0 \quad 6.09565+0 \quad 7.44525+0 \quad 9.09365+0$ $1.11070+11.35661+1$

df335 $3.70370-3 \quad 4.37060-34.52500-34.57410-3$ $4.55830-3 \quad 4.48970-3 \quad 4.38170-3 \quad 4.24920-3 \quad 4.12890-3$ $4.03110-3 \quad 3.93550-3 \quad 3.84530-3 \quad 3.76290-3 \quad 3.69080-3$

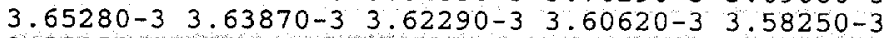
$3.55850-34.08750-3 \quad 5.89750-3 \quad 7.13870-37.42410-3$ $7.67700-3 \quad 7.95180-3 \quad 8.74210-3 \quad 1.11490-2 \quad 1.35030-2$ $1.65080-2 \quad 2.25270-2 \quad 2.86610-2 \quad 3.43150-2 \quad 4.10860-2$ $4.92110-2 \quad 5.56070-2 \quad 5.77300-2 \quad 5.80800-2 \quad 5.84980-2$ $6.59980-2 \quad 8.26560-2 \quad 9.04890-2 \quad 9.54810-2 \quad 1.05360-1$ $1.17860-1 \quad 1.29670-1 \quad 1.30100-1 \quad 1.28650-11.27220-1$ $1.26160-1 \quad 1.25620-1 \quad 1.25270-1 \quad 1.28500-1 \quad 1.36960-1$ 1.45980-1 1.54120-1 $1.51160-1 \quad 1.47220-1 \quad 1.47060-1$ $1.63200-12.00340-1$

fm335

f345:n $5.701+10$

de 345 298.958

$762.0 \div 0.0 \quad 0.0$ $2.07002-7 \quad 7.69672-7 \quad 1.75386-6 \quad 3.71293-6$ $7.86024-6 \quad 1.66401-5 \quad 3.52273-5 \quad 7.45761-5 \quad 1.34159-4$ $2.21191-4 \quad 3.64682-4 \quad 6.01258-4 \quad 9.91309-4 \quad 1.63439-3$ $\begin{array}{lllllll}2.14167-3 & 2.43063-3 & 2.82399-3 & 3.37141-3 & 4.61914-3\end{array}$ 7.32483-3 1.20766-2 $1.93065-2 \quad 2.41830-2 \quad 2.54229-2$ $2.65293-2 \quad 2.77506-2 \quad 3.14039-2 \quad 4.33909-2 \quad 5.45187-2$ $\begin{array}{llllll}7.15396-2 & 1.04645-1 & 1.36365-1 & 1.66556-1 & 2.03432-1\end{array}$ 2.48472-1 2.83877-1 $2.95864-1 \quad 2.97851-1 \quad 3.00232-1$ 3.44858-1 4.42806-1 5.10634-1 5.65749-1 6.75418-1 8.24957-1 $1.00760+0 \quad 1.23069+0 \quad 1.50317+0 \quad 1.83598+0$ $2.12513+0 \quad 2.28850+0 \quad 2.40583+0 \quad 2.73896+0.3 .34537+0$ $4.08604+0 \quad 4.99070+0 \quad 6.09565+0 \quad 7.44525+0 \quad 9.09365+0$ $1.11070+1 \quad 1.35661+1$

$\operatorname{df} 345$ $3.70370-3 \quad 4.37060-3 \quad 4.52500-3 \quad 4.57410-3$ $4.55830-3 \quad 4.48970-3 \quad 4.38170-3 \quad 4.24920-3 \quad 4.12890-3$ $\begin{array}{llllll}4.03110-3 & 3.93550-3 & 3.84530-3 & 3.76290-3 & 3.69080-3\end{array}$ $3.65280-3 \quad 3.63870-3 \quad 3.62290-3 \quad 3.60620-3 \quad 3.58250-3$ $3.55850-3 \quad 4.08750-3 \quad 5.89750-3 \quad 7.13870-3 \quad 7.42410-3$ $\begin{array}{lllll}7.67700-3 & 7.95180-3 & 8.74210-3 & 1.11490-2 & 1.35030-2\end{array}$ $\begin{array}{lllll}1.65080-2 & 2.25270-2 & 2.86610-2 & 3.43150-2 & 4.10860-2\end{array}$ $4.92110-2 \quad 5.56070-2 \quad 5.77300-2 \quad 5.80800-2 \quad 5.84980-2$ $6.59980-2 \quad 8.26560-2 \quad 9.04890-2 \quad 9.54810-2 \quad 1.05360-1$ $1.17860-11.29670-1$ 1.30100-1 $1.28650-1$ 1.27220-1 
1.26160-1 1.25620-1 $1.25270-1 \quad 1.28500-11.36960-1$ $1.45980-1 \quad 1.54120-1 \quad 1.51160-1 \quad 1.47220-1 \quad 1.47060-1$ $1.63200-12.00340-1$

$\operatorname{fm} 345$

f355:n

de 355

298.958

$914.4 \quad 0.0 \quad 0.0$

$2.07002-7 \quad 7.69672-7 \quad 1.75386-6 \quad 3.71293-6$

7.86024-6 $1.66401-5 \quad 3.52273-5 \quad 7.45761-5 \quad 1.34159-4$

2.21191-4 $3.64682-4 \quad 6.01258-4 \quad 9.91309-4 \quad 1.63439-3$ $2.14167-3 \quad 2.43063-3 \quad 2.82399-3 \quad 3.37141-3 \quad 4.61914-3$ 7.32483-3 $1.20766-2$ 1.93065-2 $2.41830-2 \quad 2.54229-2$ 2.65293-2 2.77506-2 $3.14039-2 \quad 4.33909-2 \quad 5.45187-2$ 7.15396-2 $1.04645-1 \quad 1.36365-1 \quad 1.66556-1 \quad 2.03432-1$ $\begin{array}{lllll}2.48472-1 & 2.83877-1 & 2.95864-1 & 2.97851-1 & 3.00232-1\end{array}$ 3.44858-1 4.42806-1 $5.10634-1 \quad 5.65749-1 \quad 6.75418-1$ $8.24957-1 \quad 1.00760+0 \quad 1.23069+0 \quad 1.50317+0 \quad 1.83598+0$ $\begin{array}{llllll}2.12513+0 & 2.28850+0 & 2.40583+0 & 2.73896+0 & 3.34537+0\end{array}$ $4.08604+0 \quad 4.99070+0 \quad 6.09565+0 \quad 7.44525+0 \quad 9.09365+0$ $1.11070+11.35661+1$

$3.70370-3 \quad 4.37060-3 \quad 4.52500-3 \quad 4.57410-3$

df355 $4.55830-3 \quad 4.48970-3 \quad 4.38170-3 \quad 4.24920-3 \quad 4.12890-3$ $4.03110-3 \quad 3.93550-3 \quad 3.84530-3 \quad 3.76290-3 \quad 3.69080-3$ $3.65280-3 \cdot 3.63870-3 \quad 3.62290-3 \quad 3.60620-3 \quad 3.58250-3$ $3.55850-3 \quad 4.08750-3 \quad 5.89750-3 \quad 7.13870-3 \quad 7.42410-3$ $\begin{array}{lllll}7.67700-3 & 7.95180-3 & 8.74210-3 & 1.11490-2 & 1.35030-2\end{array}$ $1.65080-2 \quad 2.25270-2 \quad 2.86610-2 \quad 3.43150-2 \quad 4.10860-2$ $4.92110-2 \quad 5.56070-2 \quad 5.77300-2 \quad 5.80800-2 \quad 5.84980-2$ 6.59980-2 8.26560-2 $9.04890-2 \quad 9.54810-2 \quad 1.05360-1$

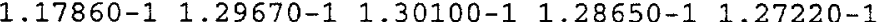
$1.26160-1 \quad 1.25620-1 \quad 1.25270-1 \quad 1.28500-1 \quad 1.36960-1$ $1.45980-1$ 1.54120-1 $1.51160-1$ 1.47220-1 $1.47060-1$ $1.63200-12.00340-1$

fm355 $5.701+10$ $7.86024-6 \quad 1.66401-5 \quad 3.52273-5 \quad 7.45761-5 \quad 1.34159-4$ $2.21191-4 \quad 3.64682-4 \quad 6.01258-4 \quad 9.91309-4 \quad 1.63439-3$ 2.14167-3 2.43063-3 $2.82399-3 \quad 3.37141-3 \quad 4.61914-3$ $7.32483-3$ 1.20766-2 $1.93065-2 \quad 2.41830-2 \quad 2.54229-2$ 2.65293-2 $2.77506-2 \quad 3.14039-2 \quad 4.33909-2 \quad 5.45187-2$ 7.15396-2 1.04645-1 $1.36365-1 \quad 1.66556-1 \quad 2.03432-1$ 2.48472-1 $2.83877-1 \quad 2.95864-1 \quad 2.97851-1 \quad 3.00232-1$ 3.44858-1 $4.42806-1 \quad 5.10634-1 \quad 5.65749-1 \quad 6.75418-1$ 8.24957-1 $1.00760+0 \quad 1.23069+0 \quad 1.50317+0 \quad 1.83598+0$ $\begin{array}{lllll}2.12513+0 & 2.28850+0 & 2.40583+0 & 2.73896+0 & 3.34537+0\end{array}$ $4.08604+0 \quad 4.99070+0 \quad 6.09565+0 \quad 7.44525+0 \quad 9.09365+0$ $1.11070+11.35661+1$

df365 $3.70370-3 \quad 4.37060-3 \quad 4.52500-3 \quad 4.57410-3$ $4.55830-3 \quad 4.48970-3 \quad 4.38170-3 \quad 4.24920-3 \quad 4.12890-3$ $4.03110-3 \quad 3.93550-3 \quad 3.84530-3 \quad 3.76290-3 \quad 3.69080-3$ $3.65280-3 \quad 3.63870-3 \quad 3.62290-3 \quad 3.60620-3 \quad 3.58250-3$ $3.55850-3 \quad 4.08750-3 \quad 5.89750-3 \quad 7.13870-3 \quad 7.42410-3$ $\begin{array}{lllll}7.67700-3 & 7.95180-3 & 8.74210-3 & 1.11490-2 & 1.35030-2\end{array}$ $1.65080-2 \quad 2.25270-2 \quad 2.86610-2 \quad 3.43150-2 \quad 4.10860-2$ $4.92110-2 \quad 5.56070-2 \quad 5.77300-2 \quad 5.80800-2 \quad 5.84980-2$ $6.59980-2 \quad 8.26560-2 \quad 9.04890-2 \quad 9.54810-2 \quad 1.05360-1$

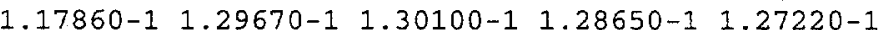
$1.26160-1 \quad 1.25620-1 \quad 1.25270-1 \quad 1.28500-1 \quad 1.36960-1$ $1.45980-1 \quad 1.54120-1 \quad 1.51160-1 \quad 1.47220-1 \quad 1.47060-1$ $1.63200-12.00340-1$

$\operatorname{fm} 365 \quad 5.701+10$

E205:p $\quad 298.958-1524.0 \quad 0.0 \quad 0.0$

de205 $1.50000-2 \quad 3.25000-2 \quad 5.75000-2 \quad 8.50000-2$ $1.25000-1 \quad 2.25000-1 \quad 3.50000-1 \quad 4.55000-1 \quad 5.55000-1$ $\begin{array}{lllll}6.50000-1 & 8.50000-1 & 1.25000+0 & 1.75000+0 & 2.25000+0\end{array}$ $2.75000+0 \quad 3.50000+0 \quad 4.50000+0 \quad 5.50000+0 \quad 6.50000+0$ $7.25000+07.75000+0 \quad 9.00000+0 \quad 1.20000+1$

d£205 $\begin{array}{llll}2.14390-3 & 5.77600-4 & 2.71850-4 & 2.68170-4\end{array}$ $3.27670-4 \quad 5.66760-4 \quad 8.75940-4 \quad 1.08450-3 \quad 1.27970-3$ $\begin{array}{llllll}1.44170-3 & 1.75630-3 & 2.31560-3 & 2.92700-3 & 3.46860-3\end{array}$ $\begin{array}{lllll}3.95960-3 & 4.62210-3 & 5.41370-3 & 6.19090-3 & 6.92650-3\end{array}$ $7.47830-3 \quad 7.84680-3 \quad 8.77160-3 \quad 1.10200-2$ 


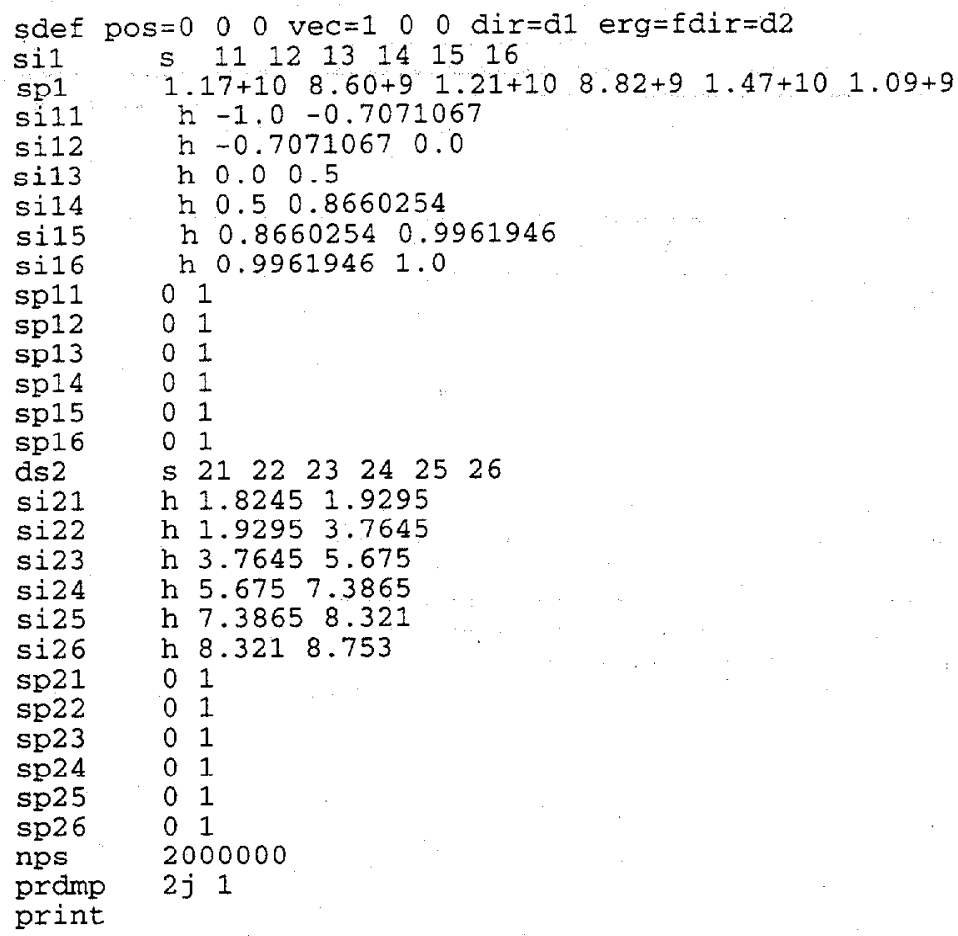


File for MCNP-4B calculation of photon dose rates along the truck lane of the PFNA facility for the source in the horizontal position

message: outp=pfna17.0 mctal $=p$ fna $17 . \mathrm{m}$

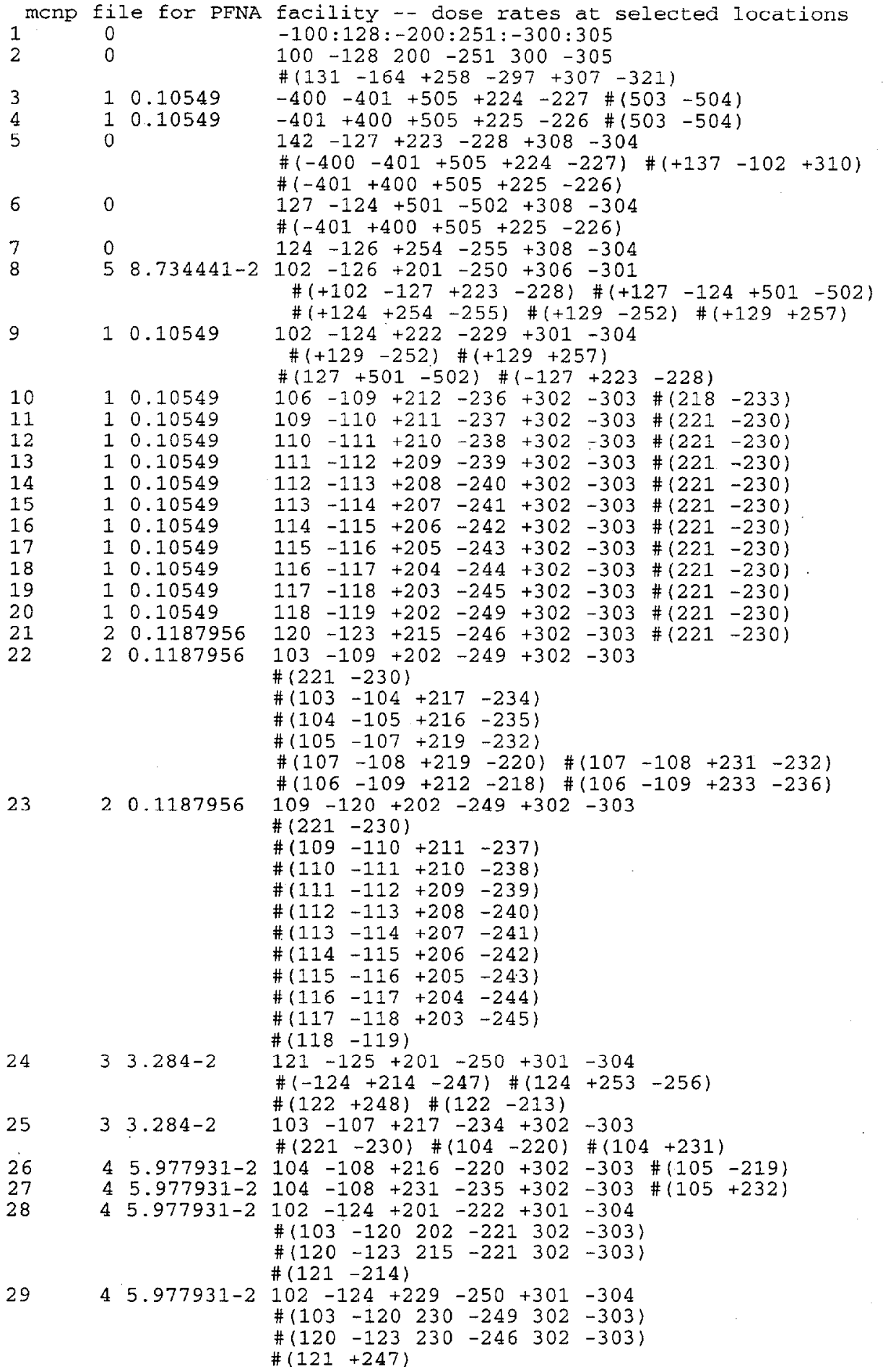


$4 \quad 5.977931-2 \quad 124-126+252-254+301-304$

$\#(-125+213-253)$

$3145.977931-2124-126+255-257+301-304$

\#(-125+256-248)

$32 \quad 0 \quad+167-168+274-281+317-318$

$33 \quad 0+165-166+270-285+308-310$

$34 \quad 0 \quad \begin{array}{ll}\#(+167-168+274-281+317-318) \\ 35 & 400+505+224-227+503-504\end{array}$

$35-0-401+400+505+225-226+503-504$

$3645.977931-2 \quad 122-124+201-250+301-304$

$\#(213-248)$

\# $(129-252)$

$\begin{array}{rrr}37 & 9 & -2.0 \\ 38 & 11 & -1.0 \\ 39 & 12 & -7.86 \\ 40 & 0 & \end{array}$

$\#(129+257)$

$+131-164+258-297+307-308$

$+131-164+258-297+308-320$

$\#(+132-163+259-296)$

$+131-164+258-297+320-321$

$+132-163+259-296+319-320$

$\#(+151-169+271-284+304-315)$

$\#(+151-160+270-271+312-314)$

$\#(+151-160+284-285+312-314)$

$\#(+157-159+274-281+313-315)$

$\#(+158-160+274-281-313)$

$\#(+169-157+274-281+314-316)$

$\begin{array}{lrl}41 & 1 & 0.10549 \\ 42 & 1 & 0.10549 \\ 43 & 1 & 0.10549 \\ 44 & 1 & 0.10549 \\ 45 & 1 & 0.10549 \\ 46 & 1 & 0.10549 \\ 47 & 9 & -2.0 \\ 18 & 10 & -2.0\end{array}$

$+151-169+271-284+304-315$

$+151-160+270-271+312-314$

$+151-160+284-285+312-314$

$+157-159+274-281+313-315$

$+158-160+274-281+308-313$

$+169-157+274-281+314-316$

$+160-162+273-282+308-319$

$+154-161+265-289+308-319$

$\# 47 \#(-160+267-286)$

$+154-160+267-286+308-319 \# 42$ \#43 \#45

$+155-156+259-260+308-319$

$+155-156+295-296+308-319$

$+154-155+259-296+308-319$

$\#(+265-289)$

$+155-163+259-296+308-319$ \#50 \#51

\# $(-161+265-289) \quad \#(+161-162+273-282)$

$+153-154+295-296+308-319$

$+151-126+295-296+308-319$

$+151-126+259-260+308-319$

$+153-154+259-260+308-319$

$+151-152+266-272+308-304$

$+151-152+283-287+308-304$

$+151-154+259-296+308-319$

\#41 \#42 \#43 \#54 \#55 \#56 \#57 \#58 \#59

$\#(+165-166+270-285+308-310)$

$\#(-126+201-250-304)$

\begin{tabular}{|c|c|c|}
\hline $\begin{array}{l}61 \\
62 \\
63\end{array}$ & $\begin{array}{l}1 \\
1 \\
9\end{array}$ & $\begin{array}{l}0.10549 \\
0.10549 \\
-2.0\end{array}$ \\
\hline 64 & 10 & -2.0 \\
\hline $\begin{array}{l}65 \\
66\end{array}$ & $\begin{array}{r}10 \\
0\end{array}$ & -2.0 \\
\hline 67 & 10 & -2.0 \\
\hline $\begin{array}{l}68 \\
69 \\
70\end{array}$ & $\begin{array}{r}10 \\
10 \\
0\end{array}$ & $\begin{array}{l}-2.0 \\
-2.0\end{array}$ \\
\hline
\end{tabular}

$+149-151+272-201+308-304$

$+149-151+250-283+308-304$

$+149-151+264-290+308-319$

$\#(+272-283)$

$+149-151+259-296+308-319$

\# $(+264-290)$

$+146-149+295-296+308-319$

$+132-149+259-296+308-319$

\#65 \# $(+133-294)$

$+133-149+259-294+308-319$

\# $(+142-260-322) \quad \#(+134+260-298)$

$+135-149+261-262+308-319$

$+135-136+262-263+308-319$

$+137-149+268-293+308-311$

$\#(-138-290-310) \quad \#(-102+310)$

$\#(+138+269-290-310) \quad \#(+144+290-292-310)$

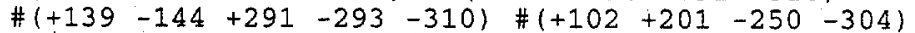

$\begin{array}{lll}71 & 1 & 0.10549 \\ 72 & 1 & 0.10549 \\ 73 & 1 & 0.10549 \\ 74 & 0 & \\ 75 & 1 & 0.10549\end{array}$

$+137-138+268-290+308-310$

$+138-149+269-272+308-310$

$+138-149+288-290+308-310 \#(+140-141)$

$+140-141+288-290+308-310$

$+144-149+290-292+308-310$ 


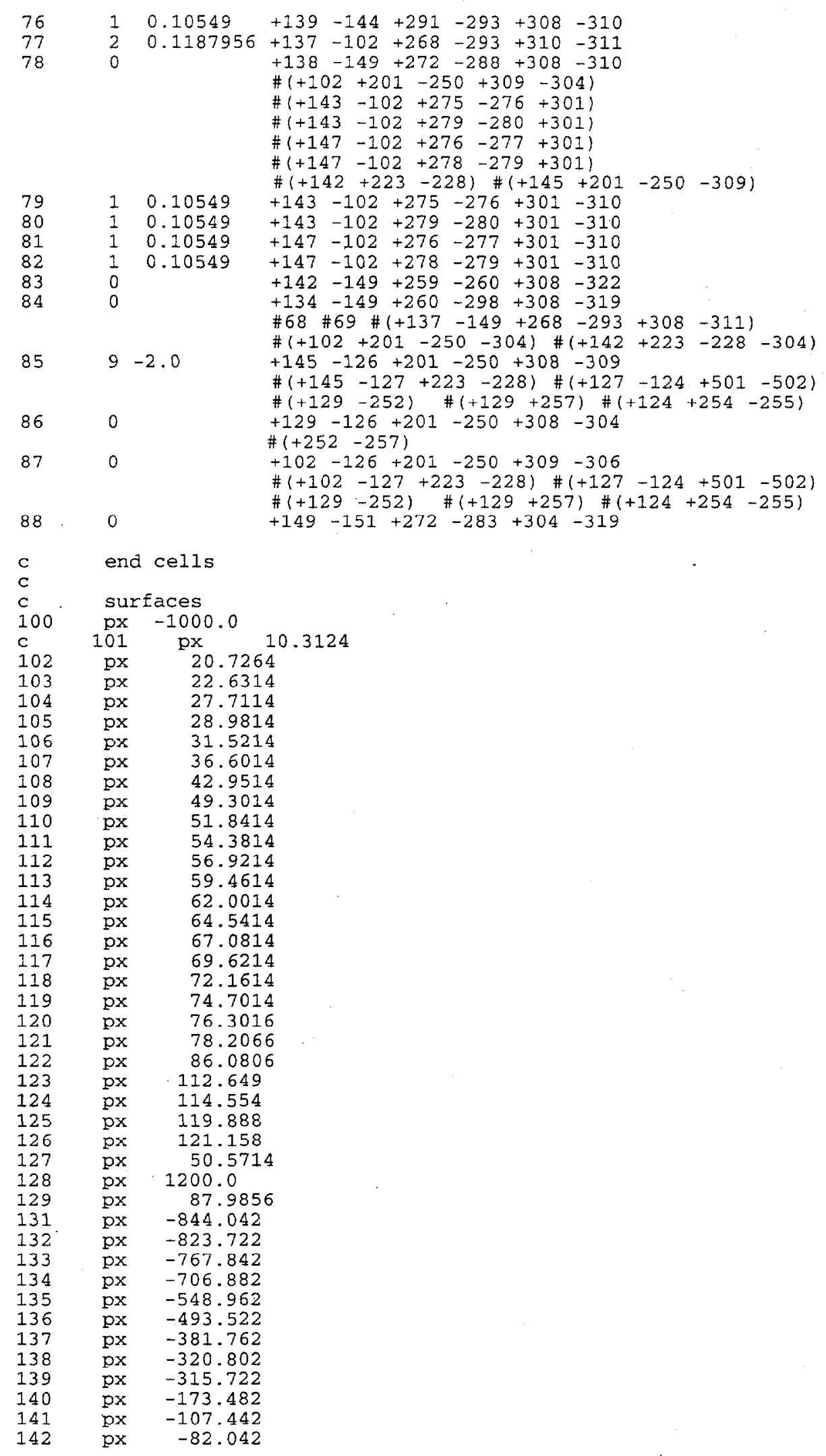




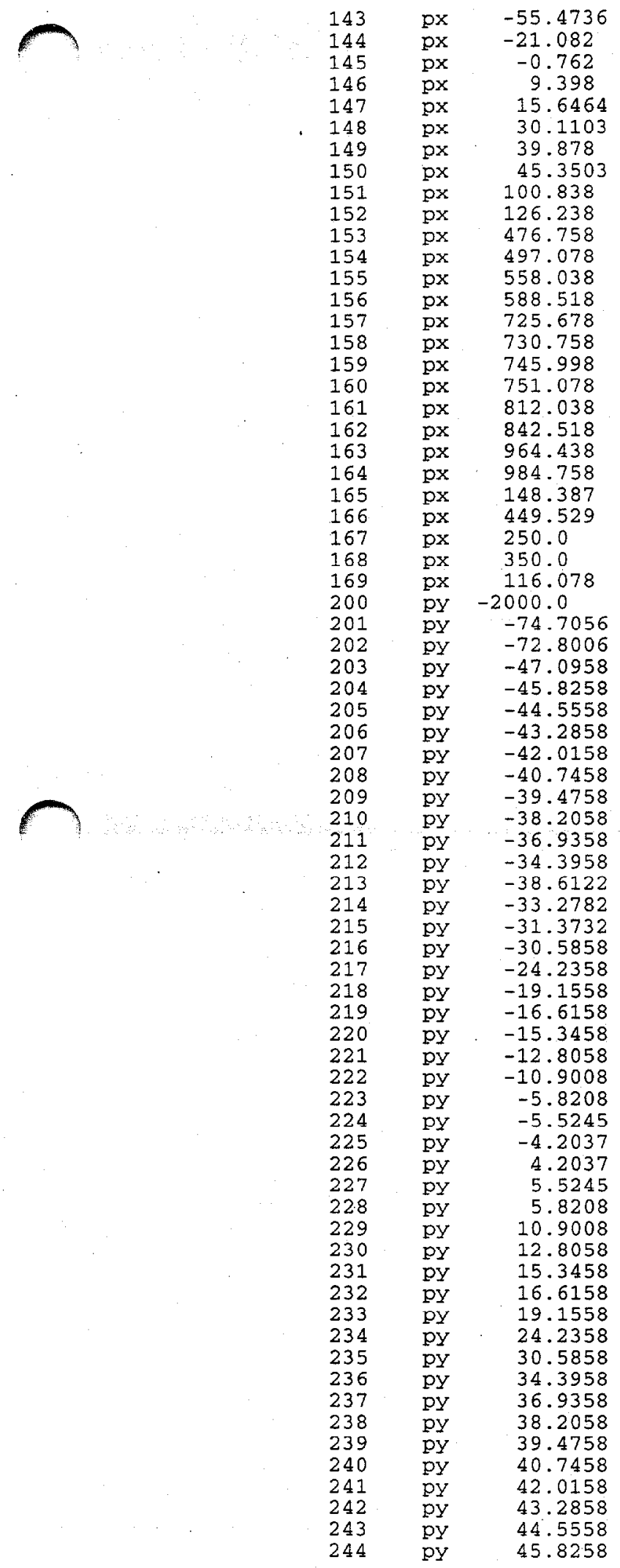




\begin{tabular}{|c|c|c|}
\hline 245 & $\mathrm{PY}$ & 47.0958 \\
\hline 246 & py & 31.3732 \\
\hline 247 & py & 33.2782 \\
\hline 248 & py & 38.6122 \\
\hline 249 & py & 72.8006 \\
\hline 250 & py & 74.7056 \\
\hline 251 & py & 1500.0 \\
\hline 252 & py & -39.8822 \\
\hline 253 & py & -9.8848 \\
\hline 254 & py & -8.6148 \\
\hline 255 & py & 8.6148 \\
\hline 256 & py & 9.8848 \\
\hline 257 & py & 39.8822 \\
\hline 258 & py & -1731.28 \\
\hline 259 & py & -1710.96 \\
\hline 260 & py & -1650.0 \\
\hline 261 & py & -1528.08 \\
\hline 262 & py & -1467.12 \\
\hline 263 & py & -1436.64 \\
\hline 264 & py & -589.28 \\
\hline 265 & py & -241.3 \\
\hline 266 & py & -193.04 \\
\hline 267 & py & -180.34 \\
\hline 268 & py & -167.64 \\
\hline 269 & py & -162.56 \\
\hline 270 & DY & -152.4 \\
\hline 271 & py & -137.16 \\
\hline 272 & py & -101.6 \\
\hline 273 & py & -91.44 \\
\hline 274 & py & -60.96 \\
\hline 275 & py & -45.72 \\
\hline 276 & py & 30.48 \\
\hline 277 & py & -10.16 \\
\hline 278 & py & 10.16 \\
\hline 279 & py & 30.48 \\
\hline 280 & py & 45.72 \\
\hline 281 & py & 60.96 \\
\hline 282 & py & 91.44 \\
\hline 283 & py & 101.6 \\
\hline 284 & py & 137.16 \\
\hline 285 & py & 152.4 \\
\hline 286 & py & 180.34 \\
\hline 287 & py & 193.04 \\
\hline 288 & py & 213.36 \\
\hline 289 & py & 241.3 \\
\hline 290 & py & 274.32 \\
\hline 291 & py & 335.28 \\
\hline 292 & py & 345.44 \\
\hline 293 & $p Y$ & 396.24 \\
\hline 294 & py & 533.4 \\
\hline 295 & py & 1235.44 \\
\hline 296 & pY & 1296.4 \\
\hline 297 & py & 1316.72 \\
\hline 298 & py & 472.44 \\
\hline 300 & $p z$ & -500.0 \\
\hline 301 & $\mathrm{pz}$ & -59.478 \\
\hline 302 & $\mathrm{pz}$ & -57.573 \\
\hline 303 & $\mathrm{pz}$ & 245.957 \\
\hline 304 & $\mathrm{pz}$ & 247.862 \\
\hline 305 & $p z$ & 500.0 \\
\hline 306 & $\mathrm{pz}$ & -60.748 \\
\hline 307 & $\mathrm{pz}$ & -156.0 \\
\hline 308 & $p z$ & -125.095 \\
\hline 309 & $\mathrm{pz}$ & -67.451 \\
\hline 310 & $\mathrm{pz}$ & 235.585 \\
\hline 311 & $\mathrm{pz}$ & 276.225 \\
\hline 312 & $p z$ & 283.845 \\
\hline 313 & $\mathrm{p} z$ & 342.265 \\
\hline 314 & $p z$ & 375.285 \\
\hline 315 & $p z$ & 390.525 \\
\hline 316 & $\mathrm{pz}$ & 395.605 \\
\hline 317 & $p z$ & -50.0 \\
\hline
\end{tabular}




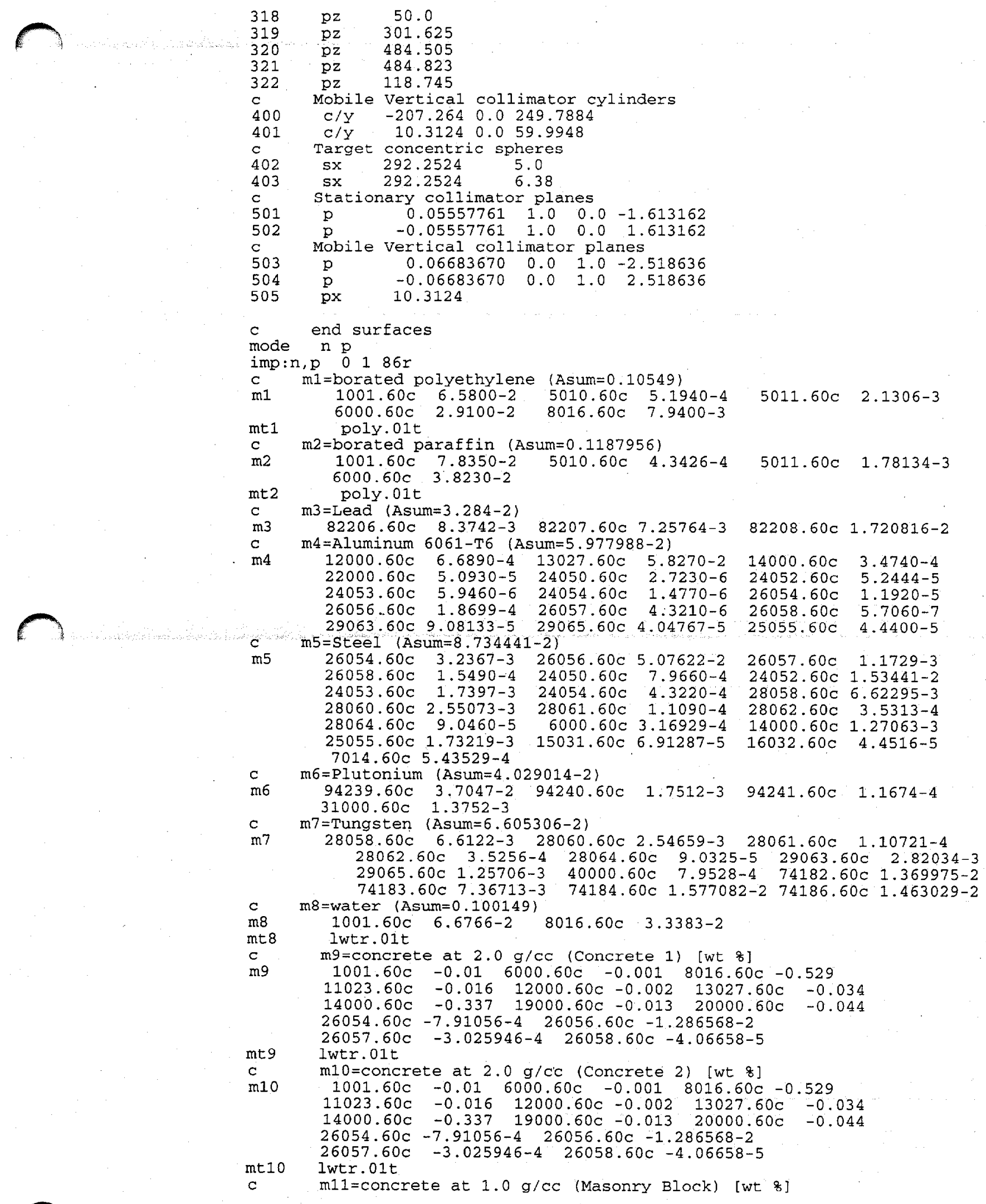




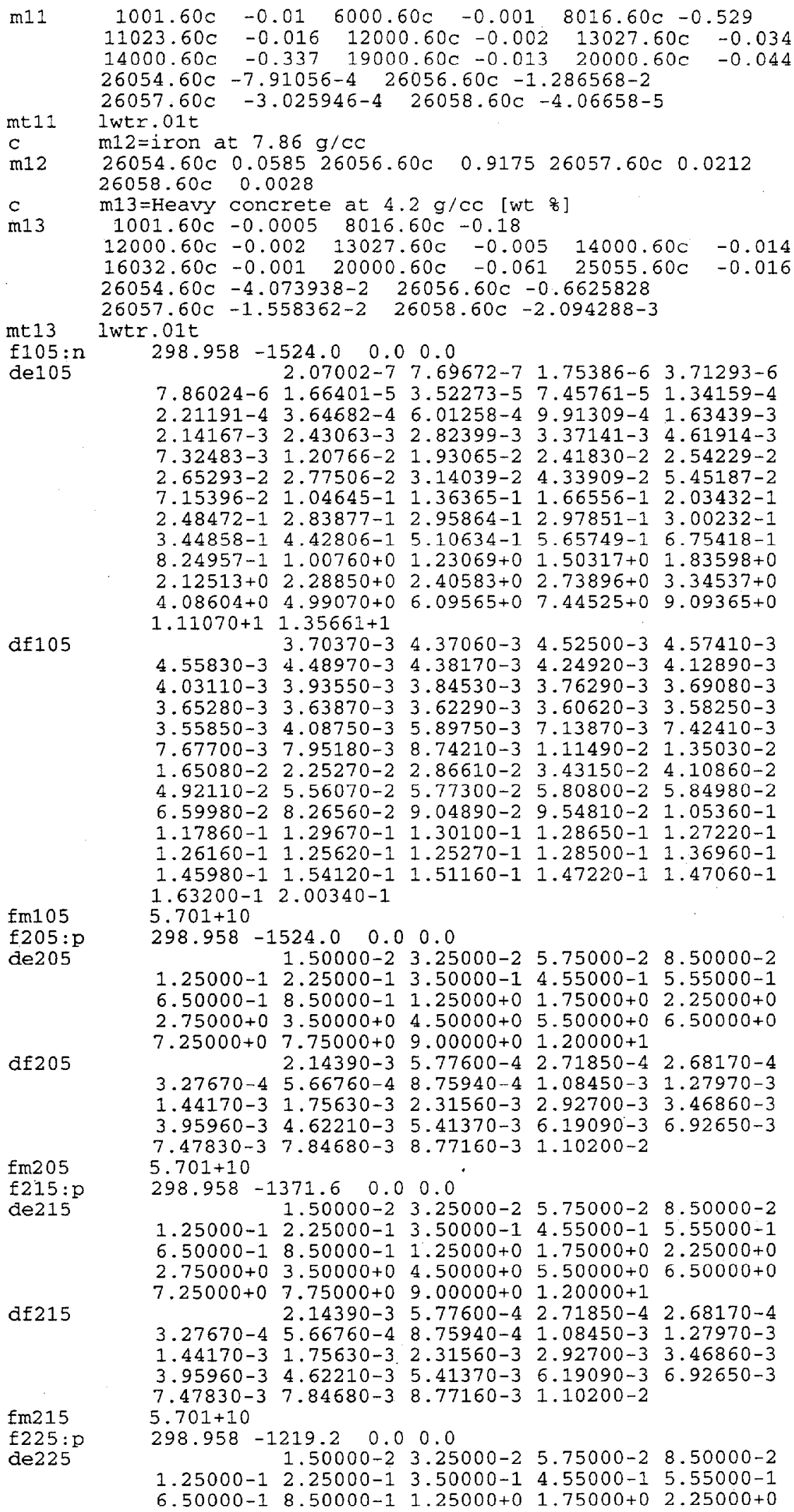


$2.75000+0 \quad 3.50000+0 \quad 4.50000+0 \quad 5.50000+0 \quad 6.50000+0$ $7.25000+0 \quad 7.75000+0 \quad 9.00000+0 \quad 1.20000+1$ $2.14390-3 \quad 5.77600-4 \quad 2.71850-4 \quad 2.68170-4$ $3.27670-4 \quad 5.66760-4 \quad 8.75940-4 \quad 1.08450-3 \quad 1.27970-3$ $1.44170-3 \quad 1.75630-3 \quad 2.31560-3 \quad 2.92700-3 \quad 3.46860-3$ $\begin{array}{llllll}3.95960-3 & 4.62210-3 & 5.41370-3 & 6.19090-3 & 6.92650-3\end{array}$ $\begin{array}{llll}7.47830-3 & 7.84680-3 & 8.77160-3 & 1.10200-2\end{array}$

fm225 $£ 235: p$ de 235

df235

$\mathrm{fm} 235$ de 245

$\operatorname{df} 245$

fm245 f255:p de 255

df 255

$\operatorname{fm} 255$

$\mp 265: \mathrm{p}$

de 265

df 265

fm265

f275:p de 275

$\operatorname{df} 275$

$\mathrm{fm} 275$ f285:p de 285
$5.701+10$

$298.958-1066.8 \quad 0.0 \quad 0.0$

$1.50000-2 \quad 3.25000-2 \quad 5.75000-2 \quad 8.50000-2$ $1.25000-12.25000-13.50000-14.55000-15.55000-1$ $6.50000-1 \quad 8.50000-1 \quad 1.25000+0 \quad 1.75000+0 \quad 2.25000+0$ $2.75000+0 \quad 3.50000+0 \quad 4.50000+0 \quad 5.50000+0 \quad 6.50000+0$ $7.25000+0 \quad 7.75000+0 \quad 9.00000+01.20000+1$ $\begin{array}{lllll}2.14390-3 & 5.77600-4 & 2.71850-4 & 2.68170-4\end{array}$ $3.27670-4 \quad 5.66760-4 \quad 8.75940-4 \quad 1.08450-3 \quad 1.27970-3$ $\begin{array}{llllll}1.44170-3 & 1.75630-3 & 2.31560-3 & 2.92700-3 & 3.46860-3\end{array}$ $3.95960-3 \quad 4.62210-3 \quad 5.41370-3 \quad 6.19090-3 \quad 6.92650-3$ $7.47830-3 \quad 7.84680-3 \quad 8.77160-3 \quad 1.10200-2$ $5.701+10$ $298.958 \quad-914.4 \quad 0.0 \quad 0.0$ $1.50000-2 \quad 3.25000-2 \quad 5.75000-2 \quad 8.50000-2$ $1.25000-1 \quad 2.25000-1 \quad 3.50000-1 \quad 4.55000-1 \quad 5.55000-1$ $6.50000-1 \quad 8.50000-1 \quad 1.25000+0 \quad 1.75000+02.25000+0$ $2.75000+0 \quad 3.50000+0 \quad 4.50000+0 \quad 5.50000+0 \quad 6.50000+0$

$7.25000+0 \quad 7.75000+0 \quad 9.00000+0 \quad 1.20000+1$ $\begin{array}{lllll}2.14390-3 & 5.77600-4 & 2.71850-4 & 2.68170-4\end{array}$ $\begin{array}{lllll}3.27670-4 & 5.66760-4 & 8.75940-4 & 1.08450-3 & 1.27 .970-3\end{array}$ $1.44170-3 \quad 1.75630-3 \quad 2.31560-3 \quad 2.92700-3 \quad 3.46860-3$

$3.95960-3 \quad 4.62210-3 \quad 5.41370-3 \quad 6.19090-3 \quad 6.92650-3$ $7.47830-3 \quad 7.84680-3 \quad 8.77160-3 \quad 1.10200-2$

$5.701+10$

$298.958 \quad-762.0 \quad 0.0 \quad 0.0$

$1.50000-2 \quad 3.25000-2 \quad 5.75000-2 \quad 8.50000-2$

1.25000-1 2.25000-1 3.50000-1 4.55000-1 5.55000-1

$6.50000-18.50000-1 \quad 1.25000+0 \quad 1.75000+0 \quad 2.25000+0$

$2.75000+0 \quad 3.50000+0 \quad 4.50000+0 \quad 5.50000+0 \quad 6.50000+0$

$7.25000+0 \quad 7.75000+0 \quad 9.00000+0 \quad 1.20000+1$ $2.14390-3 \quad 5.77600-4 \quad 2.71850-4 \quad 2.68170-4$

$3.27670-4 \quad 5.66760-4 \quad 8.75940-4 \quad 1.08450-3 \quad 1.27970-3$

$1.44170-3 \quad 1.75630-3 \quad 2.31560-3 \quad 2.92700-3 \quad 3.46860-3$

$\begin{array}{llllll}3.95960-3 & 4.62210-3 & 5.41370-3 & 6.19090-3 & 6.92650-3\end{array}$ $7.47830-3 \quad 7.84680-3 \quad 8.77160-3 \quad 1.10200-2$ $5.701+10$

$298.958 \quad-609.6 \quad 0.0 \quad 0.0$

$1.50000-2 \quad 3.25000-2 \quad 5.75000-2,8.50000-2$

$1.25000-1 \quad 2.25000-1 \quad 3.50000-1 \quad 4.55000-1 \quad 5.55000-1$

$6.50000-1 \quad 8.50000-1 \quad 1.25000+0 \quad 1.75000+0 \quad 2.25000+0$ $2.75000+0 \quad 3.50000+0 \quad 4.50000+0 \quad 5.50000+0 \quad 6.50000+0$ $7.25000+0 \quad 7.75000+0.9 .00000+0 \quad 1.20000+1$

$2.14390-3 \quad 5.77600-4 \quad 2.71850-4 \quad 2.68170-4$

$3.27670-4 \quad 5.66760-4 \quad 8.75940-4.41 .08450-3 \quad 1.27970-3$

$1.44170-3 \quad 1.75630-3 \quad 2.31560-3 \quad 2.92700-3 \quad 3.46860-3$

$3.95960-3 \quad 4.62210-3 \quad 5.41370-3 \quad 6.19090-3 \quad 6.92650-3$ $7.47830-3 \quad 7.84680-3 \quad 8.77160-3 \quad 1.10200-2$

$5.701+10$

$298.958-457.2 \quad 0.0 \quad 0.0$

$1.50000-2 \quad 3.25000-2 \quad 5.75000-2 \quad 8.50000-2$

$1.25000-1 \quad 2.25000-1 \quad 3.50000-1 \quad 4.55000-1 \quad 5.55000-1$

$6.50000-1 \quad 8.50000-1 \quad 1.25000+0 \quad 1.75000+0 \quad 2.25000+0$ $2.75000+0 \quad 3.50000+0 \quad 4.50000+0 \quad 5.50000+0 \quad 6.50000+0$ $7.25000+0 \quad 7.75000+0 \quad 9.00000+0 \quad 1.20000+1$ $\begin{array}{llll}2.14390-3 & 5.77600-4 & 2.71850-4 & 2.68170-4\end{array}$ $3.27670-4 \quad 5.66760-4 \quad 8.75940-4 \quad 1.08450-3 \quad 1.27970-3$ $1.44170-3 \quad 1.75630-3 \quad 2.31560-3 \quad 2.92700-3 \quad 3.46860-3$ $3.95960-3 \quad 4.62210-3 \quad 5.41370-3 \quad 6.19090-3 \quad 6.92650-3$ $7.47830-3 \quad 7.84680-3 \quad 8.77160-3 \quad 1.10200-2$

$5.701+10$

$\begin{array}{lllll}298.958 & -304.8 & 0.0 & 0.0\end{array}$

$1.50000-2 \quad 3.25000-2 \quad 5.75000-2 \quad 8.50000-2$ $1.25000-1 \quad 2.25000-1 \quad 3.50000-1.4 .55000-1 \quad 5.55000-1$ $6.50000-18.50000-1 \quad 1.25000+01.75000+0 \quad 2.25000+0$ 
$2.75000+0 \quad 3.50000+0 \quad 4.50000+0 \quad 5.50000+0 \quad 6.50000+0$ $7.25000+0 \quad 7.75000+0 \quad 9.00000+0 \quad 1.20000+1$

df285

$2.14390-3 \quad 5.77600-4 \quad 2.71850-4 \quad 2.68170-4$

$3.27670-4 \quad 5.66760-4 \quad 8.75940-4 \quad 1.08450-3 \quad 1.27970-3$

$\begin{array}{lllll}1.44170-3 & 1.75630-3 & 2.31560-3 & 2.92700-3 & 3.46860-3\end{array}$

$3.95960-3 \quad 4.62210-3 \quad 5.41370-3 \quad 6.19090-3 \quad 6.92650-3$

$7.47830-3 \quad 7.84680-3 \quad 8.77160-3 \quad 1.10200-2$

fm285

$5.701+10$

f295:p

$298.958-152.4 \quad 0.0 \quad 0.0$

de2 95

$1.25000-1 \quad 2.25000-1 \quad 3.50000-1 \quad 4.55000-1 \quad 5.55000-1$

$6.50000-1 \quad 8.50000-1 \quad 1.25000+0 \quad 1.75000+0 \quad 2.25000+0$

$\begin{array}{lllll}2.75000+0 & 3.50000+0 & 4.50000+0 & 5.50000+0 & 6.50000+0\end{array}$

$7.25000+0 \quad 7.75000+0 \quad 9.00000+0 \quad 1.20000+1$

$\begin{array}{lllll}2.14390-3 & 5.77600-4 & 2.71850-4 & 2.68170-4\end{array}$

$3.27670-4 \quad 5.66760-4 \quad 8.75940-4 \quad 1.08450-3 \quad 1.27970-3$

$\begin{array}{lllll}1.44170-3 & 1.75630-3 & 2.31560-3 & 2.92700-3 & 3.46860-3\end{array}$

$\begin{array}{llllll}3.95960-3 & 4.62210-3 & 5.41370-3 & 6.19090-3 & 6.92650-3\end{array}$

$7.47830-3 \quad 7.84680-3 \quad 8.77160-3 \quad 1.10200-2$

fm295

de 405

$5.701+10$

298.958

$\begin{array}{lll}152.4 & 0.0 & 0.0\end{array}$

$1.50000-2 \quad 3.25000-2 \quad 5.75000-2 \quad 8.50000-2$

$\begin{array}{lllll}1.25000-1 & 2.25000-1 & 3.50000-1 & 4.55000-1 & 5.55000-1\end{array}$

$\begin{array}{lllll}6.50000-1 & 8.50000-1 & 1.25000+0 & 1.75000+0 & 2.25000+0\end{array}$

$2.75000+0 \quad 3.50000+0 \quad 4.50000+0 \quad 5.50000+0 \quad 6.50000+0$

$7.25000+0 \quad 7.75000+0 \quad 9.00000+0 \quad 1.20000+1$

df 405

$\begin{array}{llll}2.14390-3 & 5.77600-4 & 2.71850-4 & 2.68170-4\end{array}$

$3.27670-4 \quad 5.66760-4 \quad 8.75940-4 \quad 1.08450-3 \quad 1.27970-3$

$1.44170-31.75630 .32 .31560-32.92700-3 \quad 3.46860-3$

$3.95960-3 \quad 4.62210-3 \quad 5.41370-3 \quad 6.19090-36.92650-3$

$7.47830-3 \quad 7.84680-3 \quad 8.77160-3 \quad 1.10200-2$

fm405

f $415: p$

$5.701+10$

de 415

298.958

$\begin{array}{lll}304.8 & 0.0 & 0.0\end{array}$

$1.50000-2 \quad 3.25000-2 \quad 5.75000-2 \quad 8.50000-2$

$\begin{array}{llllll}1.25000-1 & 2.25000-1 & 3.50000-1 & 4.55000-1 & 5.55000-1\end{array}$

$\begin{array}{lllll}6.50000-1 & 8.50000-1 & 1.25000+0 & 1.75000+0 & 2.25000+0\end{array}$

$2.75000+0 \quad 3.50000+0 \quad 4.50000+0 \quad 5.50000+0 \quad 6.50000+0$

$7.25000+07.75000+0 \quad 9.00000+01.20000+1$

df 415

$\begin{array}{lllll}2.14390-3 & 5.77600-4 & 2.71850-4 & 2.68170-4\end{array}$

$3.27670-4 \quad 5.66760-4 \quad 8.75940-4 \quad 1.08450-3 \quad 1.27970-3$

$1.44170-3 \quad 1.75630-3 \quad 2.31560-3 \quad 2.92700-3 \quad 3.46860-3$

$\begin{array}{llllll}3.95960-3 & 4.62210-3 & 5.41370-3 & 6.19090-3 & 6.92650-3\end{array}$

$7.47830-3 \quad 7.84680-3 \quad 8.77160-3 \quad 1.10200-2$

fm415 $\quad 5.701+10$

$f 425: \mathrm{p} \quad 298.958 \quad 457.2 \quad 0.0 \quad 0.0$

de425 $1.50000-2 \quad 3.25000-2 \quad 5.75000-2 \quad 8.50000-2$

$1.25000-1 \quad 2.25000-1 \quad 3.50000-1 \quad 4.55000-1 \quad 5.55000-1$

$6.50000-1 \quad 8.50000-1 \quad 1.25000+0 \quad 1.75000+0 \quad 2.25000+0$

$2.75000+0 \quad 3.50000+0 \quad 4.50000+0 \quad 5.50000+0 \quad 6.50000+0$

$7.25000+0 \quad 7.75000+0 \quad 9.00000+0 \quad 1.20000+1$

$\operatorname{df} 425$

$\begin{array}{lllll}2.14390-3 & 5.77600-4 & 2.71850-4 & 2.68170-4\end{array}$

$3.27670-4 \quad 5.66760-4 \quad 8.75940-4 \quad 1.08450-3 \quad 1.27970-3$

$\begin{array}{llllll}1.44170-3 & 1.75630-3 & 2.31560-3 & 2.92700-3 & 3.46860-3\end{array}$

$\begin{array}{lllll}3.95960-3 & 4.62210-3 & 5.41370-3 & 6.19090-3 & 6.92650-3\end{array}$

$7.47830-3 \quad 7.84680-3 \quad 8.77160-3 \quad 1.10200-2$

$\mathrm{fm} 425$

de 435

$01+10$

$298.958 \quad 609.6 \quad 0.0 \quad 0.0$

$1.50000-2 \quad 3.25000-2 \quad 5.75000-2 \quad 8.50000-2$

$\begin{array}{lllll}1.25000-1 & 2.25000-1 & 3.50000-1 & 4.55000-1 & 5.55000-1\end{array}$

$6.50000-18.50000-1 \quad 1.25000+0 \quad 1.75000+0 \quad 2.25000+0$

$2.75000+0 \quad 3.50000+0 \quad 4.50000+0 \quad 5.50000+0 \quad 6.50000+0$

$7.25000+0 \quad 7.75000+0 \quad 9.00000+0 \quad 1.20000+1$

$\begin{array}{llll}2.14390-3 & 5.77600-4 & 2.71850-4 & 2.68170-4\end{array}$

$3.27670-4 \cdot 5.66760-4 \quad 8.75940-4 \quad 1.08450-3 \quad 1.27970-3$

$\begin{array}{lllll}1.44170-3 & 1.75630-3 & 2.31560-3 & 2.92700-3 & 3.46860-3\end{array}$

$\begin{array}{lllll}3.95960-3 & 4.62210-3 & 5.41370-3 & 6.19090-3 & 6.92650-3\end{array}$

$7.47830-3 \quad 7.84580-3 \quad 8.77160-3 \quad 1.10200-2$

fm 435

f $445: p$

$5.701+10$

$298.958 \quad 762.0 \quad 0.0 \quad 0.0$

$1.50000-2 \quad 3.25000-2 \quad 5.75000-2 \quad 8.50000-2$

$1.25000-1 \quad 2.25000-1 \quad 3.50000-1 \quad 4.55000-1 \quad 5.55000-1$

$6.50000-18.50000-1 \quad 1.25000+01.75000+02.25000+0$ 
$2.75000+0 \quad 3.50000+0 \quad 4.50000+0 \quad 5.50000+0 \quad 6.50000+0$ $7.25000+0 \quad 7.75000+0 \quad 9.00000+0 \quad 1.20000+1$

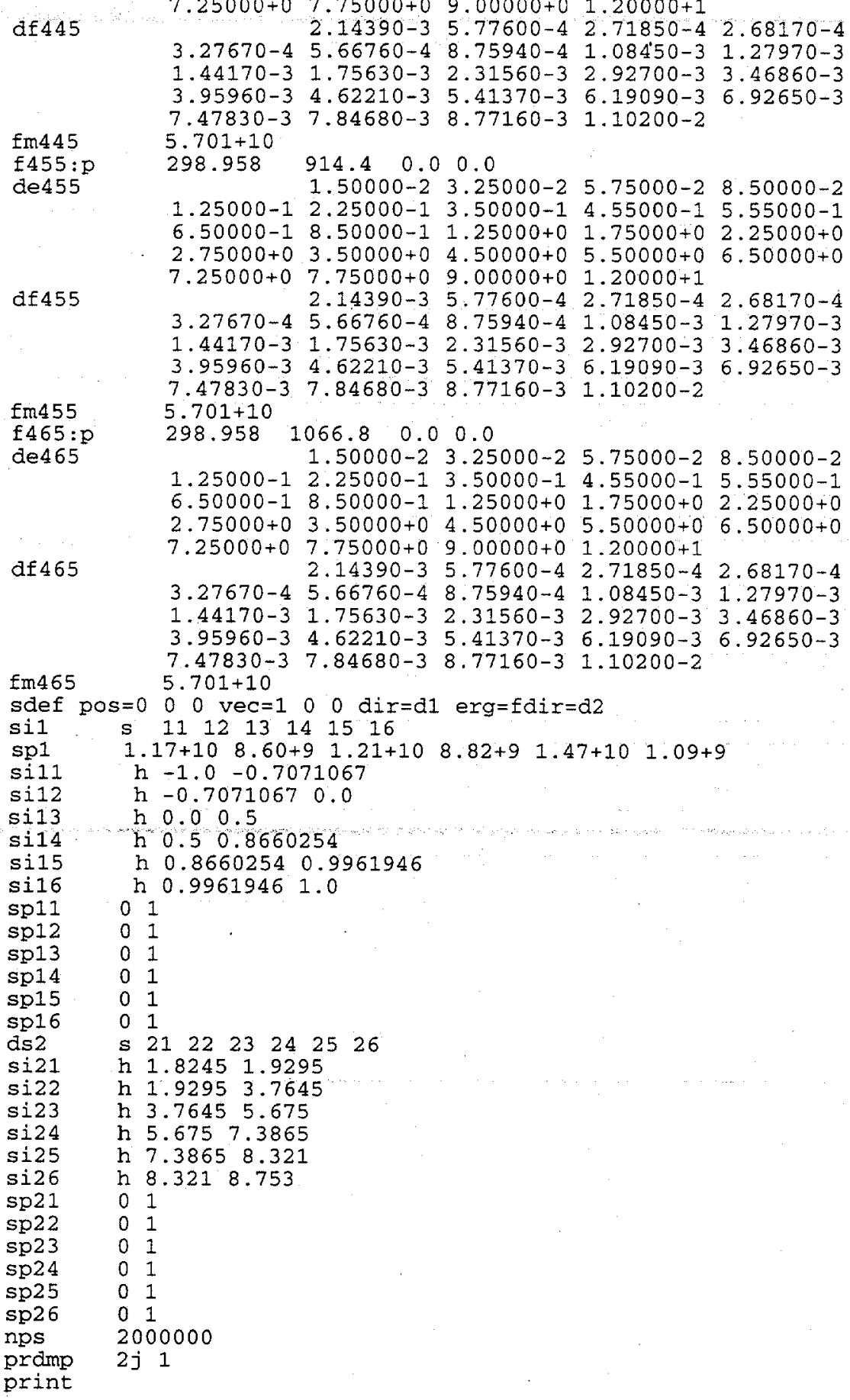


File for MCNP-4B calculation of neutron dose rates along the truck lane of the PFNA facility for the source in the maximum up position

message: outp=pfna18. $\circ$ mctal=pfna18.m

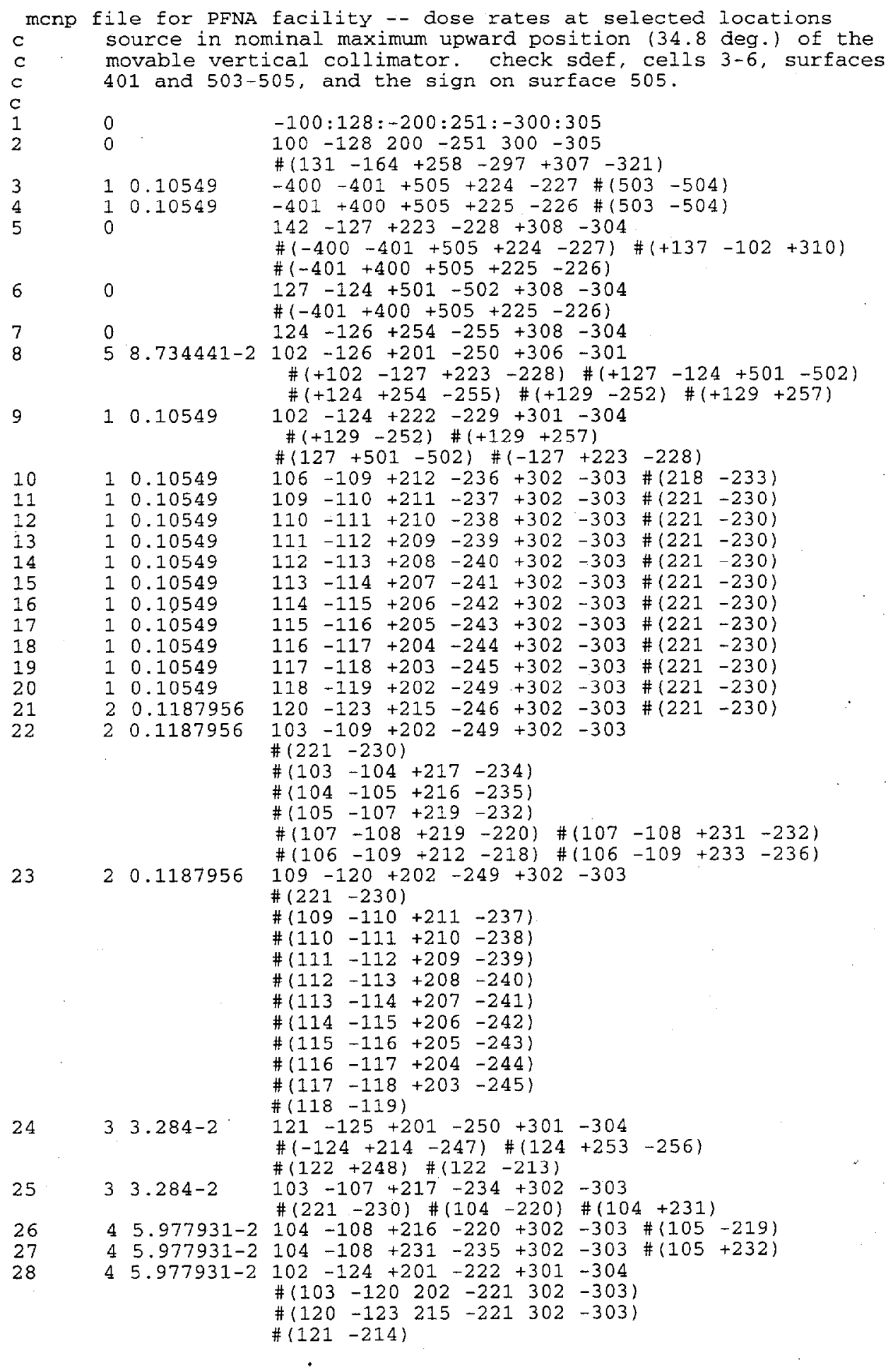




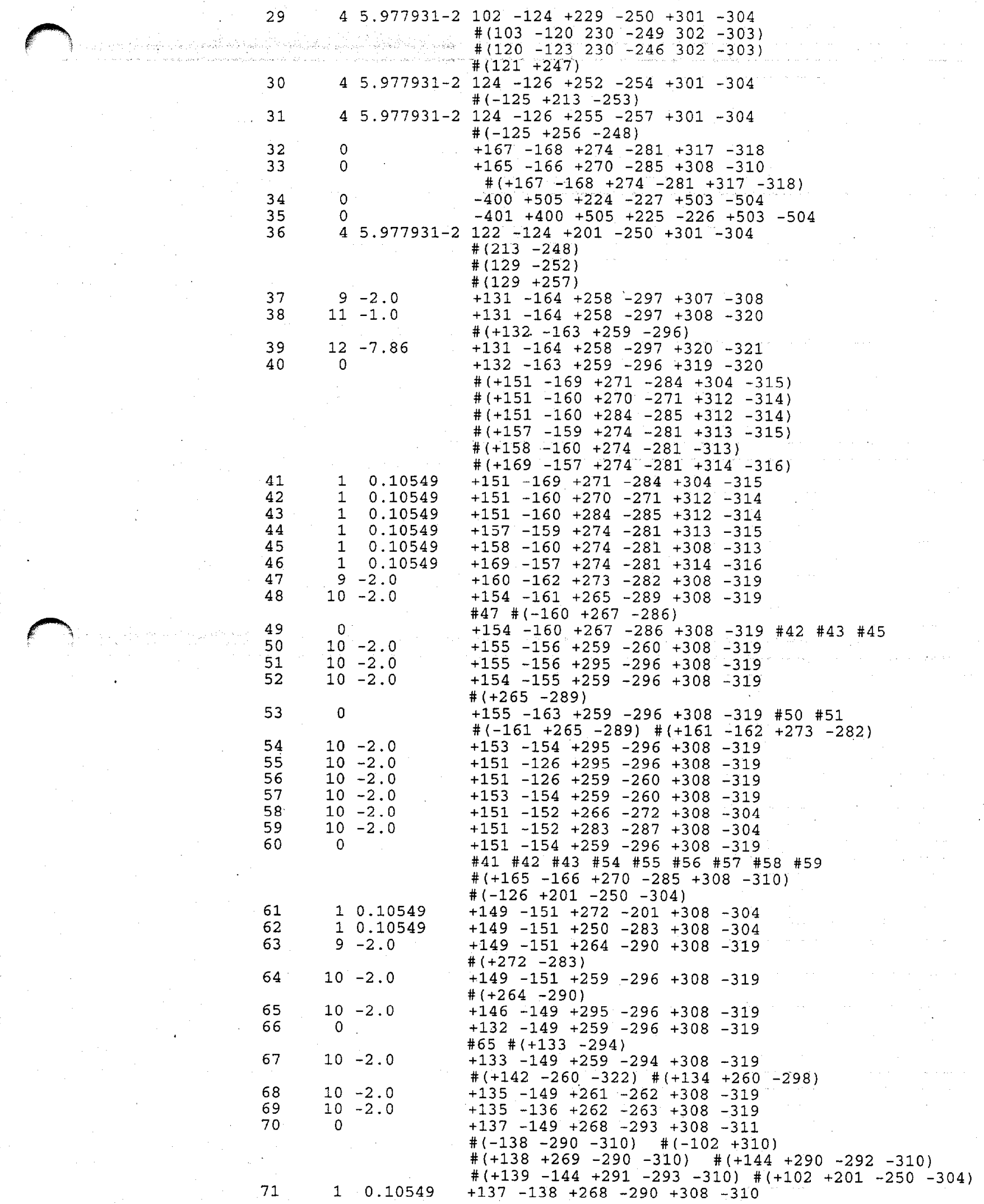




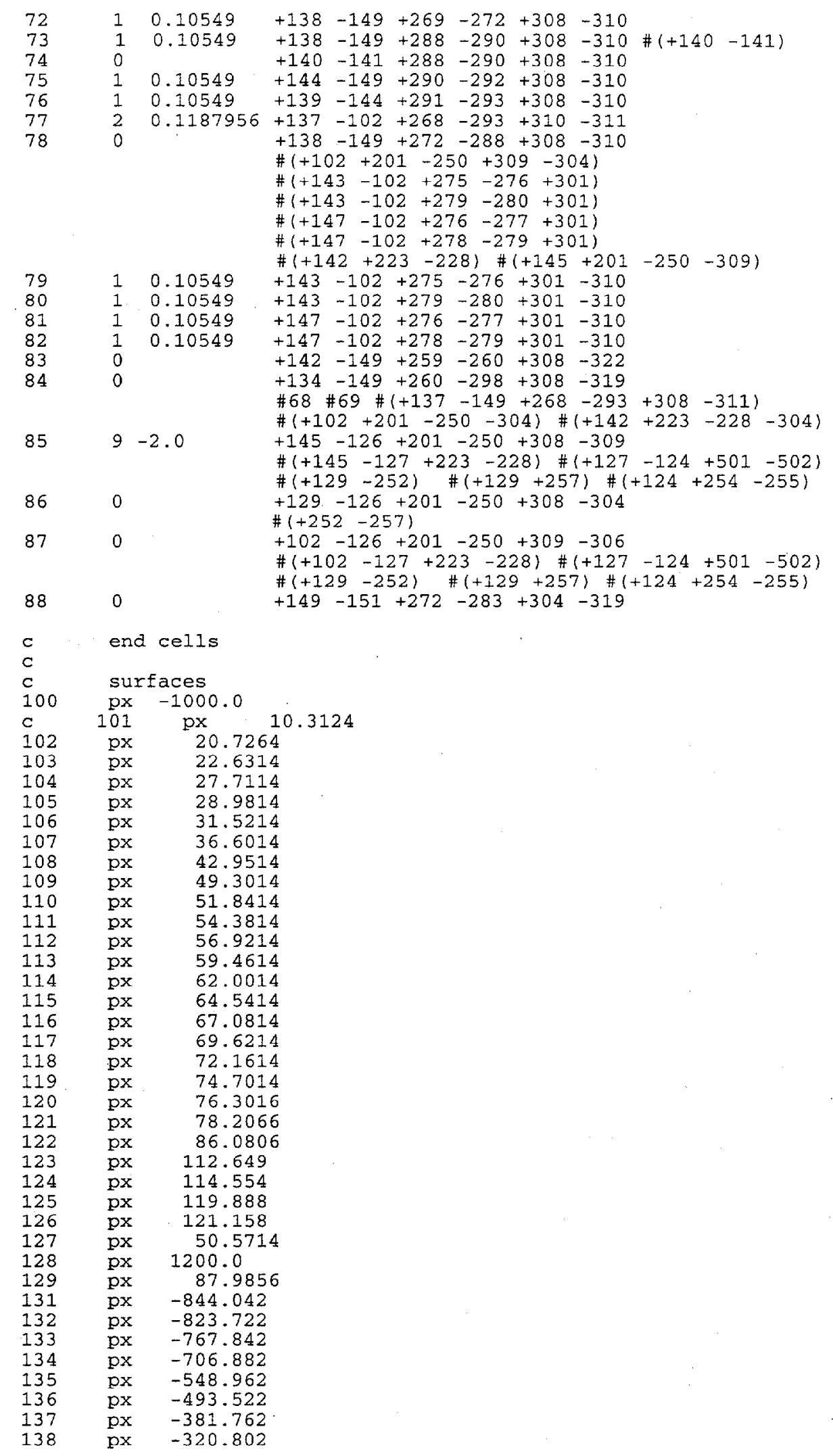




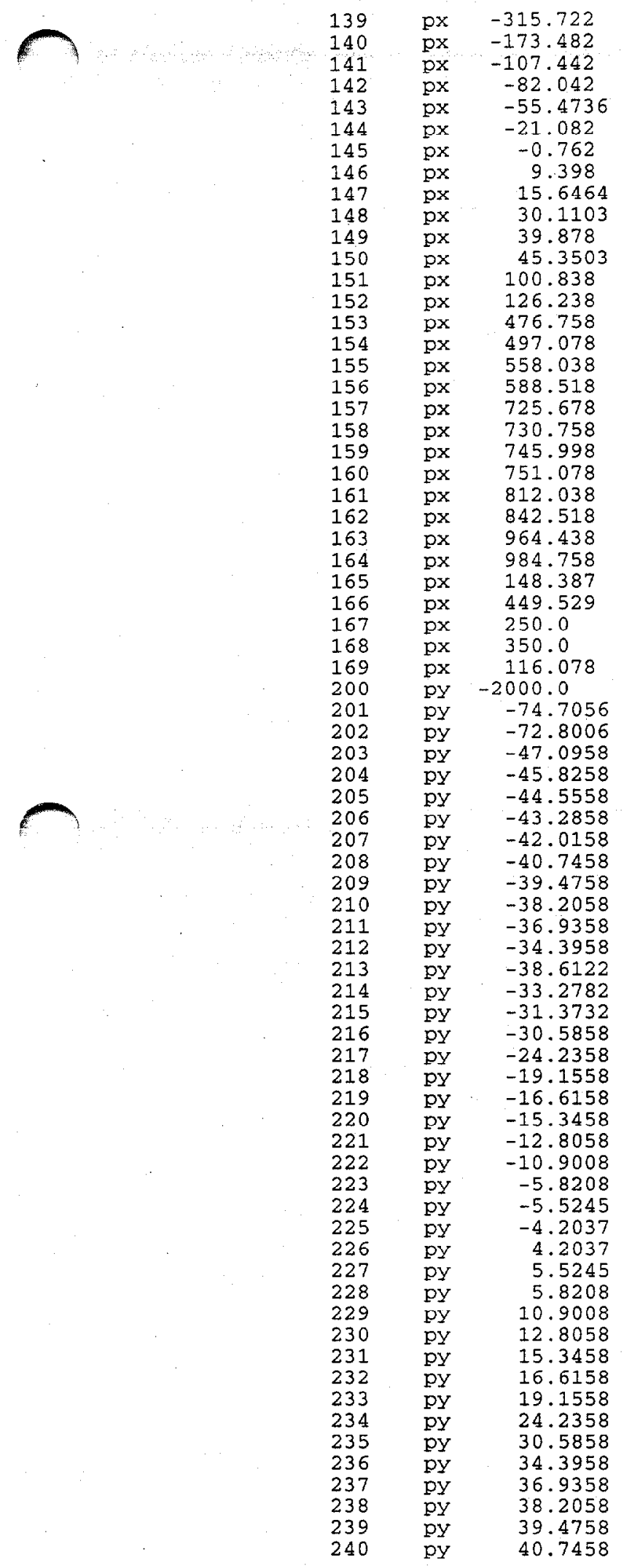




\begin{tabular}{|c|c|c|}
\hline 241 & py & 42.0158 \\
\hline 242 & py & 43.2858 \\
\hline 243 & py & 44.5558 \\
\hline 244 & py & 45.8258 \\
\hline 245 & py & 47.0958 \\
\hline 246 & PY & 31.3732 \\
\hline 247 & py & 33.2782 \\
\hline 248 & py & 38.6122 \\
\hline 249 & py & 72.8006 \\
\hline 250 & py & 74.7056 \\
\hline 251 & py & 1500.0 \\
\hline 252 & py & -39.8822 \\
\hline 253 & py & -9.8848 \\
\hline 254 & py & -8.6148 \\
\hline 255 & py & 8.6148 \\
\hline 256 & py & 9.8848 \\
\hline 257 & py & 39.8822 \\
\hline 258 & py & -1731.28 \\
\hline 259 & py & -1710.96 \\
\hline 260 & PY & -1650.0 \\
\hline 261 & py & -1528.08 \\
\hline 262 & py & -1467.12 \\
\hline 263 & py & -1436.64 \\
\hline 264 & py & -589.28 \\
\hline 265 & py & -241.3 \\
\hline 266 & py & -193.04 \\
\hline 267 & py & -180.34 \\
\hline 268 & py & -167.64 \\
\hline 269 & PY & -162.56 \\
\hline 270 & py & -152.4 \\
\hline 271 & py & -137.16 \\
\hline 272 & py & -101.6 \\
\hline 273 & py & -91.44 \\
\hline 274 & py & -60.96 \\
\hline 275 & py & -45.72 \\
\hline 276 & py & -30.48 \\
\hline 277 & pY & -10.16 \\
\hline 278 & py & 10.16 \\
\hline 279 & py & 30.48 \\
\hline 280 & py & 45.72 \\
\hline 281 & py & 60.96 \\
\hline 282 & py & 91.44 \\
\hline 283 & py & 101.6 \\
\hline 284 & $\mathrm{pY}$ & 137.16 \\
\hline 285 & py & 152.4 \\
\hline 286 & py & 180.34 \\
\hline 287 & py & 193.04 \\
\hline 288 & $\mathrm{pY}$ & 213.36 \\
\hline 289 & py & 241.3 \\
\hline 290 & $\mathrm{pY}$ & 274.32 \\
\hline 291 & py & 335.28 \\
\hline 292 & $\mathrm{pY}$ & 345.44 \\
\hline 293 & py & 396.24 \\
\hline 294 & py & 533.4 \\
\hline 295 & py & 1235.44 \\
\hline 296 & DY & 1296.4 \\
\hline 297 & py & 1316.72 \\
\hline 298 & py & 472.44 \\
\hline 300 & $\mathrm{pz}$ & -500.0 \\
\hline 301 & $\mathrm{pz}$ & -59.478 \\
\hline 302 & $\mathrm{pz}$ & -57.573 \\
\hline 303 & $p z$ & 245.957 \\
\hline 304 & $\mathrm{pz}$ & 247.862 \\
\hline 305 & $\mathrm{pz}$ & 500.0 \\
\hline 306 & $\mathrm{pz}$ & -60.748 \\
\hline 307 & $\mathrm{pz}$ & -156.0 \\
\hline 308 & $\mathrm{pz}$ & -125.095 \\
\hline 309 & $\mathrm{pz}$ & -67.451 \\
\hline 310 & $\mathrm{pz}$ & 235.585 \\
\hline 311 & $p z$ & 276.225 \\
\hline 312 & $p z$ & 283.845 \\
\hline 313 & $\mathrm{pz}$ & 342.265 \\
\hline
\end{tabular}




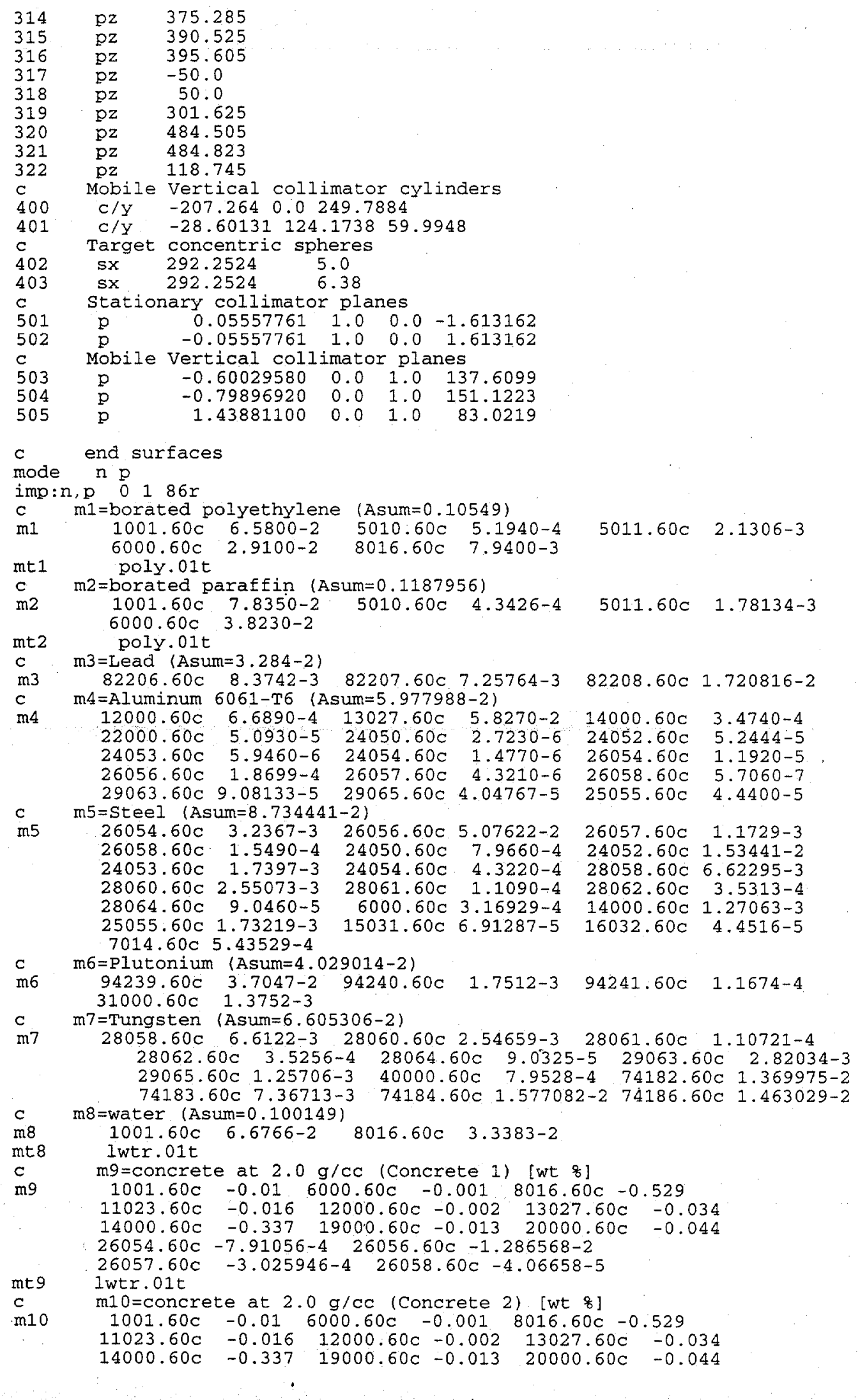


$26054.60 \mathrm{C}-7.91056-4 \quad 26056.60 \mathrm{C}-1.286568-2$

$26057.60 \mathrm{C} \quad-3.025946-4 \quad 26058.60 \mathrm{c}-4.06658-5$

mt 10

1 wtr.01t

c m1l=concrete at $1.0 \mathrm{~g} / \mathrm{cc}$ (Masonry Block) [wt 8]

$\begin{array}{lllllll}\text { m11 } & 1001.60 \mathrm{c} & -0.01 & 6000.60 \mathrm{c} & -0.001 & 8016.60 \mathrm{c} & -0.529\end{array}$

$\begin{array}{lllllll}11023.60 c & -0.016 & 12000.60 c & -0.002 & 13027.60 c & -0.034\end{array}$

$\begin{array}{llllll}14000.60 c & -0.337 & 19000.60 c & -0.013 & 20000.60 c & -0.044\end{array}$

$26054.60 \mathrm{c}-7.91056-4 \quad 26056.60 \mathrm{c}-1.286568-2$

mt11 lwtr.01t

$\begin{array}{llllllll}\mathrm{C} & \mathrm{m} 12=1 \mathrm{ron} & \mathrm{at} & 7.86 & \mathrm{~g} / \mathrm{CC} & & & \\ \mathrm{m} 12 & 26054.60 \mathrm{c} & 0.0585 & 26056.60 \mathrm{C} & 0.9175 & 26057.60 \mathrm{C} & 0.0212\end{array}$

c $\quad 26058.60 \mathrm{c} 0.0028$ m13=Heavy concrete at $4.2 \mathrm{~g} / \mathrm{cc}$ [wt 8 ]

m13 $\quad 1001.60 \mathrm{c}-0.0005 \quad 8016.60 \mathrm{c}-0.18$

$\begin{array}{llllll}12000.60 \mathrm{c} & -0.002 & 13027.60 \mathrm{c} & -0.005 & 14000.60 \mathrm{c} & -0.014\end{array}$

$\begin{array}{llllll}16032.60 c & -0.001 & 20000.60 c & -0.061 & 25055.60 c & -0.016\end{array}$

$26054.60 \mathrm{C}-4.073938-2 \quad 26056.60 \mathrm{C}-0.6625828$

$26057.60 c-1.558362-2 \quad 26058.60 c-2.094288-3$

m.t13

lwtr.01t

f105:n $\quad 298.958-1524.0 \quad 0.00 .0$

de105

df 105

fm105

f $115: n$

de115

$\begin{array}{lllll}7.86024-6 & 2.07002-7 & 7.69672-7 & 1.75386-6 & 3.71293-6\end{array}$ 2.21191-4 $3.64682-4 \quad 6.01258-4 \quad 9.91309-4 \quad 1.63439-3$ 2.14167-3 $2.43063-3 \quad 2.82399-3 \quad 3.37141-3 \quad 4.61914-3$ 7.32483-3 $1.20766-2 \quad 1.93065-2 \quad 2.41830-2 \quad 2.54229-2$ 2.65293-2 2.77506-2 3.14039-2 4.33909-2 5.45187-2 7.15396-2 $1.04645-1 \quad 1.36365-1 \quad 1.66556-1 \quad 2.03432-1$ $\begin{array}{llllll}2.48472-1 & 2.83877-1 & 2.95864-1 & 2.97851-1 & 3.00232-1\end{array}$ 3.44858-1 4.42806-1 $5.10634-1 \quad 5.65749-1 \quad 6.75418-1$ $\begin{array}{lllll}8.24957-1 & 1.00760+0 & 1.23069+0 & 1.50317+0 & 1.83598+0\end{array}$ $\begin{array}{lllll}2.12513+0 & 2.28850+0 & 2.40583+0 & 2.73896+0 & 3.34537+0\end{array}$ $4.08604+0 \quad 4.99070+0 \quad 6.09565+0 \quad 7.44525+0 \quad 9.09365+0$ $1.11070+1 \quad 1.35661+1$ 3.70370-3 $4.37060-3 \quad 4.52500-3 \quad 4.57410-3$ $4.55830-3 \quad 4.48970-3 \quad 4.38170-3 \quad 4.24920-3 \quad 4.12890-3$ $\begin{array}{llllll}4.03110-3 & 3.93550-3 & 3.84530-3 & 3.76290-3 & 3.69080-3\end{array}$ $\begin{array}{llllll}3.65280-3 & 3.63870-3 & 3.62290-3 & 3.60620-3 & 3.58250-3\end{array}$ $\begin{array}{lllllll}3.55850-3 & 4.08750-3 & 5.89750-3 & 7.13870-3 & 7.42410-3\end{array}$ $\begin{array}{lllll}7.67700-3 & 7.95180-3 & 8.74210-3 & 1.11490-2 & 1.35030-2\end{array}$ $1.65080-2 \quad 2.25270-2 \quad 2.86610-2 \quad 3.43150-2 \quad 4.10860-2$ $4.92110-2 \quad 5.56070-2 \quad 5.77300-2 \quad 5.80800-2 \quad 5.84980-2$ $6.59980-2 \quad 8.26560-2 \quad 9.04890-2 \quad 9.54810-2 \quad 1.05360-1$ 1.17860-1 $1.29670-1 \quad 1.30100-1 \quad 1.28650-1 \quad 1.27220-1$ 1.26160-1 $1.25620-1 \quad 1.25270-1 \quad 1.28500-1 \quad 1.36960-1$ $1.45980-1$ 1.54120-1 $1.51160-1$ 1.47220-1 $1.47060-1$ $1.63200-12.00340-1$

$5.701+10$

$298.958-1371.6 \quad 0.0 \quad 0.0$

$2.07002-7 \quad 7.69672-7 \quad 1.75386-6 \quad 3.71293-6$

7.86024-6 $1.66401-5 \quad 3.52273-5 \quad 7.45761-5 \quad 1.34159-4$ $2.21191-4 \quad 3.64682-4 \quad 6.01258-4 \quad 9.91309-4 \quad 1.63439-3$ $2.14167-3 \quad 2.43063-3 \quad 2.82399-3 \quad 3.37141-3 \quad 4.61914-3$ $7.32483-3 \quad 1.20766-2 \quad 1.93065-2 \quad 2.41830-2 \quad 2.54229-2$ 2.65293-2 2.77506-2 $3.14039-2 \quad 4.33909-2 \quad 5.45187-2$ $\begin{array}{llllll}7.15396-2 & 1.04645-1 & 1.363 .65-1 & 1.66556-1 & 2.03432-1\end{array}$ 2.48472-1 2.83877-1 $2.95864-1 \quad 2.97851-1.3 .00232-1$ 3.44858-1 $4.42806-1 \quad 5.10634-1 \quad 5.65749-1 \quad 6.75418-1$

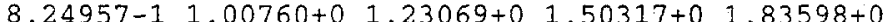
$2.12513+0 \quad 2.28850+0 \quad 2.40583+0 \quad 2.73896+0 \quad 3.34537+0$ $4.08604+0 \quad 4.99070+0 \quad 6.09565+0 \quad 7.44525+0 \quad 9.09365+0$ $1.11070+1 \quad 1.35661+1$

$\operatorname{df} 115$

$3.70370-3 \quad 4.37060-3 \quad 4.52500-3 \quad 4.57410-3$ $4.55830-3 \quad 4.48970-3 \quad 4.38170-3 \quad 4.24920-3 \quad 4.12890-3$ $\begin{array}{llllll}4.03110-3 & 3.93550-3 & 3.84530-3 & 3.76290-3 & 3.69080-3\end{array}$ $3.65280-3 \quad 3.63870-3 \quad 3.62290-3 \quad 3.60620-3 \quad 3.58250-3$ $3.55850-3 \quad 4.08750-3 \quad 5.89750-3 \quad 7.13870-3 \quad 7.42410-3$ $\begin{array}{lllll}7.67700-3 & 7.95180-3 & 8.74210-3 & 1.11490-2 & 1.35030-2\end{array}$ $1.65080-2 \quad 2.25270-2 \quad 2.86610-2 \quad 3.43150-2 \quad 4.10860-2$ $4.92110-2 \quad 5.56070-2 \quad 5.77300-2 \quad 5.80800-2 \quad 5.84980-2$ $6.59980-2.8 .26560-2 \quad 9.04890-2 \quad 9.54810-2.1 .05360-1$ $1.17860-1$ 1.29670-1 $1.30100-1 \quad 1.28650-1 \quad 1.27220-1$ 
$\begin{array}{lllllll}1.26160-1 & 1.25620-1 & 1.25270-1 & 1.28500-1 & 1.36960-1\end{array}$ $1.45980-1$ 1.54120-1 $1.51160-1 \quad 1.47220-1 \quad 1.47060-1$ $1.63200-12.00340-1$

fm115 $5.701+10$

f125:n $\quad 298.958-1219.2 \quad 0.0 \quad 0.0$

$\begin{array}{llllll}\text { de125 } & 2.07002-7 & 7.69672-7 & 1.75386-6 & 3.71293-6\end{array}$ 7.86024-6 $1.66401-5 \quad 3.52273-5 \quad 7.45761-5 \quad 1.34159-4$ 2.21191-4 $3.64682-4 \quad 6.01258-4 \quad 9.91309-4 \quad 1.63439-3$ $2.14167-3 \quad 2.43063-3 \quad 2.82399-3 \quad 3.37141-3 \quad 4.61914-3$ 7.32483-3 $1.20766-2 \quad 1.93065-2 \quad 2.41830-2 \quad 2.54229-2$ 2.65293-2 2.77506-2 $3.14039-2 \quad 4.33909-2 \quad 5.45187-2$ $\begin{array}{lllll}7.15396-2 & 1.04645-1 & 1.36365-1 & 1.66556-1 & 2.03432-1\end{array}$ 2.48472-1 $2.83877-1 \quad 2.95864-1 \quad 2.97851-1 \quad 3.00232-1$ 3.44858-1 4.42806-1 $5.10634-1 \quad 5.65749-1 \quad 6.75418-1$ $8.24957-1 \quad 1.00760+0 \quad 1.23069+0 \quad 1.50317+0 \quad 1.83598+0$ $\begin{array}{llllll}2.12513+0 & 2.28850+0 & 2.40583+0 & 2.73896+0 & 3.34537+0\end{array}$ $4.08604+0 \quad 4.99070+0 \quad 6.09565+0 \quad 7.44525+0 \quad 9.09365+0$ $1.11070+1 \quad 1.35661+1$

df125 $\quad 3.70370-3 \quad 4.37060-3 \quad 4.52500-3 \quad 4.57410-3$ $\begin{array}{lllllll}4.55830-3 & 4.48970-3 & 4.38170-3 & 4.24920-3 & 4.12890-3\end{array}$ $\begin{array}{llllll}4.03110-3 & 3.93550-3 & 3.84530-3 & 3.76290-3 & 3.69080-3\end{array}$ $\begin{array}{llllll}3.65280-3 & 3.63870-3 & 3.62290-3 & 3.60620-3 & 3.58250-3\end{array}$ $3.55850-3 \quad 4.08750-3 \quad 5.89750-3 \quad 7.13870-3 \quad 7.42410-3$ $7.67700-3 \quad 7.95180-3 \quad 8.74210-3 \quad 1.11490-2 \quad 1.35030-2$ $1.65080-2 \quad 2.25270-2 \quad 2.86610-2 \quad 3.43150-2 \quad 4.10860-2$ $4.92110-2 \quad 5.56070-2 \quad 5.77300-2 \quad 5.80800-2 \quad 5.84980-2$ $6.59980-2 \quad 8.26560-2 \quad 9.04890-2 \quad 9.54810-2 \quad 1.05360-1$ $1.17860-1$ 1.29670-1 $1.30100-1 \quad 1.28650-1 \quad 1.27220-1$ $1.26160-1 \quad 1.25620-1 \quad 1.25270-1 \quad 1.28500-1 \quad 1.36960-1$ $1.45980-1 \quad 1.54120-1 \quad 1.51160-1 \quad 1.47220-1 \quad 1.47060-1$ $1.63200-12.00340-1$

fm125 $\quad 5.701+10$

f135:n $\quad 298.958-1066.8 \quad 0.0 \quad 0.0$

de135 $2.07002-7 \quad 7.69672-7 \quad 1.75386-6 \quad 3.71293-6$ $7.86024-6 \quad 1.66401-5 \quad 3.52273-5 \quad 7.45761-5 \quad 1.34159-4$ $2.21191-4 \quad 3.64682-46.01258-4 \quad 9.91309-4 \quad 1.63439-3$ $2.14167-3 \quad 2.43063-3 \quad 2.82399-3 \quad 3.37141-3 \quad 4.61914-3$ $7.32483-3 \quad 1.20766-2 \quad 1.93065-2 \quad 2.41830-2 \quad 2.54229-2$ $2.65293-2$ 2.77505-2 3.14039-2 4.33909-2 5.45187-2 7.15396-2 $1.04645-1 \quad 1.36365-1 \quad 1.66556-1 \quad 2.03432-1$ $2.48472-1 \quad 2.83877-1 \quad 2.95864-1 \quad 2.97851-1 \quad 3.00232-1$ 3.44858-1 $4.42806-1 \quad 5.10634-1 \quad 5.65749-1 \quad 6.75418-1$ $8.24957-1 \quad 1.00760+0 \quad 1.23069+0 \quad 1.50317+0 \quad 1.83598+0$ $\begin{array}{lllll}2.12513+0 & 2.28850+0 & 2.40583+0 & 2.73896+0 & 3.34537+0\end{array}$ $4.08604+0 \quad 4.99070+0 \quad 6.09565+0 \quad 7.44525+0 \quad 9.09365+0$

df135 $\quad \begin{array}{llllll}1.11070+1 & 1.35661+1 & 3.70370-3 & 4.37060-3 & 4.52500-3 & 4.57410-3\end{array}$ $4.55830-3 \quad 4.48970-3 \quad 4.38170-3 \quad 4.24920-3 \quad 4.12890-3$ $4.03110-3 \quad 3.93550-3 \quad 3.84530-3 \quad 3.76290-3 \quad 3.69080-3$ $3.65280-3 \quad 3.63870-3 \quad 3.62290-3 \quad 3.60620-3 \quad 3.58250-3$ $\begin{array}{llllll}3.55850-3 & 4.08750-3 & 5.89750-3 & 7.13870-3 & 7.42410-3\end{array}$ $7.67700-3 \quad 7.95180-3 \quad 8.74210-3 \quad 1.11490-2 \quad 1.35030-2$ $\begin{array}{llllll}1.65080-2 & 2.25270-2 & 2.86610-2 & 3.43150-2 & 4.10860-2\end{array}$ $4.92110-2$ 5.56070-2 5.77300-2 5.80800-2 5.84980-2 $6.59980-2 \quad 8.26560-2 \quad 9.04890-2 \quad 9.54810-2 \quad 1.05360-1$ 1.17860-1 $1.29670-1 \quad 1.30100-1 \quad 1.28650-1 \quad 1.27220-1$ 1.26160-1 1.25620-1 $1.25270-1 \quad 1.28500-1 \quad 1.36960-1$ $1.45980-1$ 1.54120-1 $1.51160-1$ 1.47220-1 $1.47060-1$ $1.63200-12.00340-1$

fm135 $\quad 5.701+10$

$\begin{array}{lllll}\mathrm{E} 145: \mathrm{n} & 298.958 & -914.4 & 0.0 & 0.0\end{array}$

$\begin{array}{lllllll}\operatorname{de} 145 & 2.07002-7 & 7.69672-7 & 1.75386-6 & 3.71293-6\end{array}$ $\begin{array}{lllll}7.86024-6 & 1.66401-5 & 3.52273-5 & 7.45761-5 & 1.34159-4\end{array}$ $2.21191-4 \quad 3.64682-4 \quad 5.01258-4 \quad 9.91309-4 \quad 1.63439-3$ $2.14167-3 \quad 2.43063-3 \quad 2.82399-3 \quad 3.37141-3 \quad 4.61914-3$ $\begin{array}{lllll}7.32483-3 & 1.20766-2 & 1.93065-2 & 2.41830-2 & 2.54229-2\end{array}$ 2.65293-2 2.77506-2 $3.14039-2 \quad 4.33909-2 \quad 5.45187-2$ 7.15396-2 1.04645-1 $1.36365-1 \quad 1.66556-1 \quad 2.03432-1$ 2.48472-1 $2.83877-1 \quad 2.95864-1 \quad 2.97851-1 \quad 3.00232-1$ 3.44858-1 $4.42806-1 \quad 5.10634-1 \quad 5.65749-1 \quad 6.75418-1$ $\begin{array}{llllll}8.24957-1 & 1.00760+0 & 1.23069+0 & 1.50317+0 & 1.83598+0\end{array}$ $\begin{array}{lllll}2.12513+0 & 2.28850+0 & 2.40583+0 & 2.73896+0 & 3.34537+0\end{array}$ 
$4.08604+0 \quad 4.99070+0 \quad 6.09565+0 \quad 7.44525+0 \quad 9.09365+0$ $1.11070+1 \quad 1.35661+1$

df145 $\quad 3.70370-3 \quad 4.37060-3 \quad 4.52500-3 \quad 4.57410-3$ $\begin{array}{llllll}4.55830-3 & 4.48970-3 & 4.38170-3 & 4.24920-3 & 4.12890-3\end{array}$ $4.03110-3.3 .93550-3 \quad 3.84530-3 \quad 3.76290-3 \quad 3.69080-3$ $\begin{array}{llllll}3.65280-3 & 3.63870-3 & 3.62290-3 & 3.60620-3 & 3.58250-3\end{array}$ $\begin{array}{llllll}3.55850-3 & 4.08750-3 & 5.89750-3 & 7.13870-3 & 7.42410-3\end{array}$ $\begin{array}{llllll}7.67700-3 & 7.95180-3 & 8.74210-3 & 1.11490-2 & 1.35030-2\end{array}$ $1.65080-2 \quad 2.25270-2 \quad 2.86610-2 \quad 3.43150-2 \quad 4.10860-2$ $4.92110-2 \quad 5.56070-2 \quad 5.77300-2 \quad 5.80800-2 \quad 5.84980-2$ $6.59980-2 \quad 8.26560-2 \quad 9.04890-2 \quad 9.54810-2 \quad 1.05360-1$ $1.17860-1 \quad 1.29670-1 \quad 1.30100-1 \quad 1.28650-1 \quad 1.27220-1$ $\begin{array}{llllll}1.26160-1 & 1.25620-1 & 1.25270-1 & 1.28500-1 & 1.36960-1\end{array}$ 1.45980-1 1.54120-1 $1.51160-1 \quad 1.47220-1 \quad 1.47060-1$

$\operatorname{fm} 145$ $1.63200-12.00340-1$

f155:n de 155

df 155

$5.701+10$

$298.958-762.0 \quad 0.0 \quad 0.0$

$\begin{array}{lllll}2.07002-7 & 7.69672-7 & 1.75386-6 & 3.71293-6\end{array}$ 7.86024-6 1.66401-5 3.52273-5 7.45761-5 $1.34159-4$ $2.21191-4 \quad 3.64682-4 \quad 6.01258-4 \quad 9.91309-4 \quad 1.63439-3$ 2.14167-3 $2.43063-3 \quad 2.82399-3 \quad 3.37141-3 \quad 4.61914-3$ $7.32483-3$ 1.20766-2 $1.93065-2$ 2.41830-2 2.54229-2 $2.65293-2 \quad 2.77506-2 \quad 3.14039-2 \quad 4.33909-2 \quad 5.45187-2$ 7.15396-2 $1.04645-1 \quad 1.36365-1 \quad 1.66556-1 \quad 2.03432-1$ $\begin{array}{llllll}2.48472-1 & 2.83877-1 & 2.95864-1 & 2.97851-1 & 3.00232-1\end{array}$ 3.44858-1 4.42806-1 $5.10634-1 \quad 5.65749-1 \quad 6.75418-1$ $8.24957-1 \quad 1.00760+0 \quad 1.23069+0 \quad 1.50317+0 \quad 1.83598+0$ $2.12513+0 \quad 2.28850+0 \quad 2.40583+0 \quad 2.73896+0 \quad 3.34537+0$ $4.08604+0 \quad 4.99070+0 \quad 6.09565+0 \quad 7.44525+0 \quad 9.09365+0$ $1.11070+1 \quad 1.35661+1$

$\begin{array}{rllll}3.70370-3 & 4.37060-3 & 4.52500-3 & 4.57410-3\end{array}$ $4.55830-3 \quad 4.48970-3 \quad 4.38170-3 \quad 4.24920-3 \quad 4.12890-3$ $\begin{array}{llllll}4.03110-3 & 3.93550-3 & 3.84530-3 & 3.76290-3 & 3.69080-3\end{array}$ $\begin{array}{llllll}3.65280-3 & 3.63870-3 & 3.62290-3 & 3.60620-3 & 3.58250-3\end{array}$ $\begin{array}{llll}3.55850-3 & 4.08750-3 & 5.89750-3 & 7.13870-3.7 .42410-3\end{array}$ $7.67700-3 \quad 7.95180-3 \quad 8.74210-3 \quad 1.11490-2 \quad 1.35030-2$ $\begin{array}{lllll}1.65080-2 & 2.25270-2 & 2.86610-2 & 3.43150-2 & 4.10860-2\end{array}$ $4.92110-2 \quad 5.56070-2 \quad 5.77300-2 \quad 5.80800-2 \quad 5.84980-2$ $\begin{array}{llllll}6.59980-2 & 8.26560-2 & 9.04890-2 & 9.54810-2 & 1.05360-1\end{array}$ $\begin{array}{lllll}1.17860-1 & 1.29670-1 & 1.30100-1 & 1.28650-1 & 1.27220-1\end{array}$ $1.26160-1 \quad 1.25620-1 \quad 1.25270-1 \quad 1.28500-11.36960-1$ $1.45980-1 \quad 1.54120-1 \quad 1.51160-1 \quad 1.47220-1 \quad 1.47060-1$ $1.63200-12.00340-1$

$\operatorname{fm} 155$

f165:n

de165

$5.701+10$

$298.958 \quad-609.6 \quad 0.0 \quad 0.0$

$2.07002-7 \quad 7.69672-7 \quad 1.75386-6 \quad 3.71293-6$

7.86024-6 $1.66401-5 \quad 3.52273-5 \quad 7.45761-5 \quad 1.34159-4$ 2.21191-4 $3.64682-4 \quad 6.01258-4 \quad 9.91309-4 \quad 1.63439-3$ $2.14167-3 \quad 2.43063-3 \quad 2.82399-3 \quad 3.37141-3 \quad 4.61914-3$ $\begin{array}{llllll}7.32483-3 & 1.20766-2 & 1.93065-2 & 2.41830-2 & 2.54229-2\end{array}$ 2.65293-2 2.77506-2 3.14039-2 $4.33909-2 \quad 5.45187-2$ 7.15396-2 $1.04645-1 \quad 1.36365-1 \quad 1.66556-1 \quad 2.03132-1$ $\begin{array}{lllll}2.48472-1 & 2.83877-1 & 2.95864-1 & 2.97851-1 & 3.00232-1\end{array}$ 3.44858-1 $4.42806-1 \quad 5.10634-1.5 .65749-1 \quad 6.75418-1$ 8.24957-1 $1.00760+0 \quad 1.23069+0 \quad 1.50317+0 \quad 1.83598+0$ $\begin{array}{lllll}2.12513+0 & 2.28850+0 & 2.40583+0 & 2.73896+0 & 3.34537+0\end{array}$ $4.08604+0 \quad 4.99070+0 \quad 6.09565+0 \quad 7.44525+0 \quad 9.09365+0$ $1.11070+1 \quad 1.35661+1$

df 165 $3.70370-3 \quad 4.37060-3 \quad 4.52500-3 \quad 4.57410-3$ $4.55830-3 \quad 4.48970-3 \quad 4.38170-3 \quad 4.24920-3 \quad 4.12890-3$ $\begin{array}{llllll}4.03110-3 & 3.93550-3 & 3.84530-3 & 3.76290-3 & 3.69080-3\end{array}$ $\begin{array}{llllll}3.65280-3 & 3.63870-3 & 3.62290-3 & 3.60620-3 & 3.58250-3\end{array}$ $3.55850-3 \quad 4.08750-3 \quad 5.89750-3 \quad 7.13870-3 \quad 7.42410-3$ $7.67700-3 \quad 7.95180-3 \quad 8.74210-3 \quad 1.11490-2 \quad 1.35030-2$ $1.65080-2 \quad 2.25270-2 \quad 2.86610-2 \quad 3.43150-2 \quad 4.10860-2$ $4.92110-2 \quad 5.56070-2 \quad 5.77300-2 \quad 5.80800-2 \quad 5.84980-2$ 6.59980-2 $8.26560-2 \quad 9.04890-2 \quad 9.54810-2 \quad 1.05360-1$ $1.17860-11.29670-1 \quad 1.30100-1 \quad 1.28650-11.27220-1$ $1.26160-1 \quad 1.25620-1 \quad 1.25270-1 \quad 1.28500-1 \quad 1.36960-1$ 1.45980-1 1.54120-1 1.51160-1 $1.47220-1 \quad 1.47060-1$ $1.63200-12.00340-1$

$\operatorname{fm} 165$

$5.701+10$ 
f175:n de 175

$\mathrm{d} f 175$

$\operatorname{fm} 175$

f185:n de185

df 185

fm185 f195:n de195

af 195
$298.958-457.2 \quad 0.0 \quad 0.0$

$2.07002-7 \quad 7.69672-7.1 .75386-6 \quad 3.71293-6$

$7.86024-6 \quad 1.66401-5 \quad 3.52273-57.45761-51.34159-4$

2.21191-4 3.64682-4 $6.01258-4 \quad 9.913^{\prime} 09-4 \quad 1.63439-3$

$\begin{array}{lllll}2.14167-3 & 2.43063-3 & 2.82399-3 & 3.37141-3 & 4.61914-3\end{array}$

$\begin{array}{lllll}7.32483-3 & 1.20766-2 & 1.93065-2 & 2.41830-2 & 2.54229-2\end{array}$

2.65293-2 2.77506-2 3.14039-2 4.33909-2 5.45187-2

7.15396-2 1.04645-1 $1.36365-1 \quad 1.66556-1 \quad 2.03432-1$

2.48472-1 2.83877-1.2.95864-1 2.97851-1 3.00232-1

3.44858-1 $4.42806-1 \quad 5.10634-1 \quad 5.65749-1 \quad 6.75418-1$

$8.24957-1 \quad 1.00760+0 \quad 1.23069+0 \quad 1.50317+0 \quad 1.83598+0$

$2.12513+0 \quad 2.28850+0 \quad 2.40583+0 \quad 2.73896+0 \quad 3.34537+0$

$4.08604+0 \quad 4.99070+0 \quad 6.09565+0 \quad 7.44525+0 \quad 9.09365+0$

$1.11070+1 \quad 1.35661+1$

$\begin{array}{lllll}3.70370-3 & 4.37060-3 & 4.52500-3 & 4.57410-3\end{array}$

$4.55830-3 \quad 4.48970-3 \quad 4.38170-3 \quad 4.24920-3 \quad 4.12890-3$

$\begin{array}{llllll}4.03110-3 & 3.93550-3 & 3.84530-3 & 3.76290-3 & 3.69080-3\end{array}$

$\begin{array}{llllll}3.65280-3 & 3.63870-3 & 3.62290-3 & 3.60620-3 & 3.58250-3\end{array}$

$3.55850-3 \quad 4.08750-3 \quad 5.89750-3 \quad 7.13870-3 \quad 7.42410-3$

$7.67700-3 \quad 7.95180-3 \quad 8.74210-3 \quad 1.11490-2 \quad 1.35030-2$

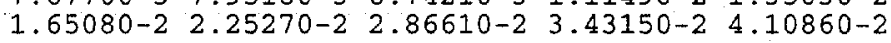

$4.92110-2 \quad 5.56070-2 \quad 5.77300-2 \quad 5.80800-2 \quad 5.84980-2$

6.59980-2 $8.26560-2 \quad 9.04890-2 \quad 9.54810-2 \quad 1.05360-1$

$1.17860-1 \quad 1.29670-1 \quad 1.30100-1$ 1.28650-1 $1.27220-1$

$\begin{array}{lllll}1.26160-1 & 1.25620-1 & 1.25270-1 & 1.28500-1 & 1.36960-1\end{array}$

$1.45980-1$ 1.54120-1 $1.51160-1 \quad 1.47220-1 \quad 1.47060-1$ $1.63200-12.00340-1$

$5.701+10$

$298.958 \quad-304.8 \quad 0.0 \quad 0.0$

$2.07002-7 \quad 7.69672-7 \quad 1.75386-6 \quad 3.71293-6$

7.86024-6 $1.66401-5 \quad 3.52273-5 \quad 7.45761-5 \quad 1.34159-4$ $2.21191-4 \quad 3.64682-4 \quad 6.01258-4 \quad 9.91309-4$ 1.63439-3 2.14167-3 $2.43063-3 \quad 2.82399-3 \quad 3.37141-3 \quad 4.61914-3$

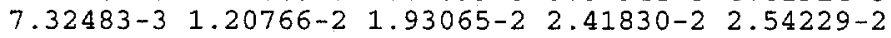
2.65293-2 2.77506-2 3.14039-2 $4.33909-2$ 5.45187-2 7.15396-2 1.04645-1 $1.36365-1 \quad 1.66556-1 \quad 2.03432-1$ $\begin{array}{llllll}2.48472-1 & 2.83877-1 & 2.95864-1 & 2.97851-1 & 3.00232-1\end{array}$ 3.44858-1 $4.42806-1 \quad 5.10634-1 \quad 5.65749-1 \quad 6.75418-1$ 8.24957-1 $1.00760+0 \quad 1.23069+0 \quad 1.50317+0 \quad 1.83598+0$ $\begin{array}{lllll}2.12513+0 & 2.28850+0 & 2.40583+0 & 2.73896+0 & 3.34537+0\end{array}$ $4.08604+0 \quad 4.99070+0 \quad 6.09565+0 \quad 7.44525+0 \quad 9.09365+0$ $1.11070+11.35661+1$

$3.70370-3 \quad 4.37060-3 \quad 4.52500-3 \quad 4.57410-3$ $4.55830-3 \quad 4.48970-3 \quad 4.38170-3 \quad 4.24920-3 \quad 4.12890-3$ $4.03110-3 \quad 3.93550-3 \quad 3.84530-3 \quad 3.76290-3 \quad 3.69080-3$ $\begin{array}{lllll}3.65280-3 & 3.63870-3 & 3.62290-3 & 3.60620-3 & 3.58250-3\end{array}$ $3.55850-3 \quad 4.08750-3 \quad 5.89750-3 \quad 7.13870-3 \quad 7.42410-3$

$\begin{array}{lllll}7.67700-3 & 7.95180-3 & 8.74210-3 & 1.11490-2 & 1.35030-2\end{array}$ $\begin{array}{lllll}1.65080-2 & 2.25270-2 & 2.86610-2 & 3.43150-2 & 4.10860-2\end{array}$ $4.92110-2 \quad 5.56070-2 \quad 5.77300-2 \quad 5.80800-2 \quad 5.84980-2$ $6.59980-2 \quad 8.26560-2 \quad 9.04890-2 \quad 9.54810-2.1 .05360-1$

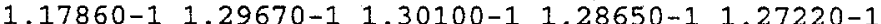
1.26160-1 1.25620-1 1.25270-1 $1.28500-1$ 1.36960-1 1.45980-1 1.54120-1 $1.51160-1$ 1.47220-1 $1.47060-1$ $1.63200-12.00340-1$

$5.701+10$ $298.958 \quad-152.4 \quad 0.0 \quad 0.0$

$2.07002-7 \quad 7.69672-7 \quad 1.75386-6 \quad 3.71293-6$ 7.86024-6 $1.66401-5 \quad 3.52273-5 \quad 7.45761-5 \quad 1.34159-4$ 2.21191-4 $3.64682-4 \quad 6.01258-4 \quad 9.91309-4 \quad 1.63439-3$ $2.14167-3 \quad 2.43063-3 \quad 2.82399-3 \quad 3.37141-3$ 4.61914-3 7.32483-3 1.20766-2 $1.93065-2$ 2.41830-2 $2.54229-2$ 2.65293-2 $2.77506-2 \quad 3.14039-2 \quad 4.33909-2 \quad 5.45187-2$ 7.15396-2 1.04645-1 1.36365-1 $1.66556-1 \quad 2.03432-1$ 2.48472-1 $2.83877-1 \quad 2.95864-1 \quad 2.97851-1 \quad 3.00232-1$ 3.44858-1 $4.42806-1 \quad 5.10634-1 \quad 5.65749-1 \quad 6.75418-1$ $8.24957-1 \quad 1.00760+0 \quad 1.23069+0 \quad 1.50317+0 \quad 1.83598+0$ $2.12513+0 \quad 2.28850+0 \quad 2.40583+0 \quad 2.73896+0 \quad 3.34537+0$ $4.08604+0 \quad 4.99070+0 \quad 6.09565+0 \quad 7.44525+0 \quad 9.09365+0$ $1.11070+11.35661+1$

$3.70370-3 \quad 4.37060-3 \quad 4.52500-3 \quad 4.57410-3$

$4.55830-3 \quad 4.48970-3 \quad 4.38170-3 \quad 4.24920-3 \quad 4.12890-3$ 
fm:195

f305: $\mathrm{n}$

de305

df 305

fm305

E315: $\mathrm{n}$

de 315

$\operatorname{df} 315$

fm315

f $325: n$

de 325
$4.03110-3 \quad 3.93550-3 \quad 3.84530-3 \quad 3.76290-3 \quad 3.69080-3$ $3.65280-3 \quad 3.63870-3 \quad 3.62290-3 \quad 3.60620-3 \quad 3.58250-3$ $3.55850-3 \quad 4.08750-3 \quad 5.89750-3 \quad 7.13870-3 \quad 7.42410-3$ $\begin{array}{llllll}7.67700-3 & 7.95180-3 & 8.74210-3 & 1.11490-2 & 1.35030-2\end{array}$ $1.65080-2 \quad 2.25270-2 \quad 2.86610-2 \quad 3.43150-2 \quad 4.10860-2$ $4.92110-2 \quad 5.56070-2 \quad 5.77300-2 \quad 5.80800 .2 \quad 5.84980-2$ $6.59980-2 \quad 8.26560-2 \quad 9.04890-2 \quad 9.54810-2 \quad 1.05360-1$ $1.17860-1 \quad 1.29670-1 \quad 1.30100-1 \quad 1.28650-1 \quad 1.27220-1$ $1.26160-1 \quad 1.25620-1 \quad 1.25270-1 \quad 1.28500-11.36960-1$ $1.45980-1 \quad 1.54120-1 \quad 1.51160-1 \quad 1.47220-1 \quad 1.47060-1$ $1.63200-12.00340-1$

$5.701+10$

$298.958 \quad 152.4 \quad 0.0 \quad 0.0$

$2.07002-7 \quad 7.69672-7 \quad 1.75386-6 \quad 3.71293-6$

7.86024-6 $1.66401-5 \quad 3.52273-5 \quad 7.45761-5 \quad 1.34159-4$ 2.21191-4 $3.64682-4 \quad 6.01258-4 \quad 9.91309-4 \quad 1.63439-3$ $2.14167-3 \quad 2.43063-3 \quad 2.82399-3 \quad 3.37141-3.4 .61914-3$ $7.32483-3$ 1.20766-2 $1.93065-2 \quad 2.41830-2 \quad 2.54229-2$ 2.65293-2 $2.77506-2 \quad 3.14039-2 \quad 4.33909-2 \quad 5.45187-2$ $\begin{array}{llllll}7.15396-2 & 1.04645-1 & 1.36365-1 & 1.66556-1 & 2.03432-1\end{array}$ $2.48472-1 \quad 2.83877-1 \quad 2.95864-1 \quad 2.97851-1 \quad 3.00232-1$ 3.44858-1 $4.42806-1 \quad 5.10634-1 \quad 5.65749-1 \quad 6.75418-1$ 8.24957-1 $1.00760+0 \quad 1.23069+0 \quad 1.50317+0 \quad 1.83598+0$ $2.12513+0 \quad 2.28850+0 \quad 2.40583+0 \quad 2.73896+0 \quad 3.34537+0$ $4.08604+0 \quad 4.99070+0 \quad 6.09565+0 \quad 7.44525+0 \quad 9.09365+0$ $1.11070+1 \quad 1.35661+1$

$3.70370-3 \quad 4.37060-3 \quad 4.52500-3 \leqslant .57410-3$ $4.55830-3 \quad 4.48970-3 \quad 4.38170-3 \quad 4.24920-3 \quad 4.12890-3$ $\begin{array}{llllll}4.03110-3 & 3.93550-3 & 3.84530-3 & 3.76290-3 & 3.69080-3\end{array}$ $\begin{array}{llllll}3.65280-3 & 3.63870-3 & 3.62290-3 & 3.60520-3 & 3.58250-3\end{array}$ $3.55850-3 \quad 4.08750-3 \quad 5.89750-3 \quad 7.13870-3 \quad 7.42410-3$ $7.67700-3 \quad 7.95180-3 \quad 8.74210-3 \quad 1.11490-2 \quad 1.35030-2$ $\begin{array}{lllll}1.65080-2 & 2.25270-2 & 2.86610-2 & 3.43150-2 & 4.10860-2\end{array}$ $4.92110-2 \quad 5.56070-2 \quad 5.77300-2 \quad 5.80800-2 \quad 5.84980-2$ $6.59980-2 \quad 8.26560-2 \quad 9.04890-2 \quad 9.54810-2 \quad 1.05360-1$ $\begin{array}{lllll}1.17860-1 & 1.29670-1 & 1.30100-1 & 1.28650-1 & 1.27220-1\end{array}$ $1.26160-1 \quad 1.25620-1 \quad 1.25270-1$ 1.28500-1 $1.36960-1$ $1.45980-1$ 1.54120-1 $1.51160-1 \quad 1.47220-1 \quad 1.47060-1$ $1.63200-12.00340-1$

$5.701+10$

$298.958 \quad 304.8 \quad 0.00 .0$

$2.07002-7 \quad 7.69672-7 \quad 1.75386-6 \quad 3.71293-6$

7.86024-6 $1.66401-5 \quad 3.52273-5 \quad 7.45761-5 \quad 1.34159-4$ 2.21191-4 $3.64682-4 \quad 6.01258-4 \quad 9.91309-4 \quad 1.63439-3$ 2.14167-3 $2.43063-3 \quad 2.82399-3 \quad 3.37141-3 \quad 4.61914-3$ $\begin{array}{lllll}7.32483-3 & 1.20766-2 & 1.93065-2 & 2.41830-2 & 2.54229-2\end{array}$ 2.65293-2 $2.77506-2 \quad 3.14039-2 \quad 4.33909-2 \quad 5.45187-2$ 7.15396-2 $1.04645-1 \quad 1.36365-1 \quad 1.66556-1 \quad 2.03432-1$ $\begin{array}{lllll}2.48472-1 & 2.83877-1 & 2.95864-1 & 2.97851-1 & 3.00232-1\end{array}$ 3.44858-1 $4.42806-1 \quad 5.10634-1 \quad 5.65749-1 \quad 6.75418-1$ $8.24957-1 \quad 1.00760+0 \quad 1.23069+0 \quad 1.50317+01.83598+0$ $\begin{array}{llllll}2.12513+0 & 2.28850+0 & 2.40583+0 & 2.73896+0 & 3.34537+0\end{array}$ $4.08604+0 \quad 4.99070+0 \quad 6.09565+0 \quad 7.44525+0 \quad 9.09365+0$ $1.11070+1 \quad 1.35661+1$

$3.70370-34.37060-3 \quad 4.52500-3 \quad 4.57410-3$ $4.55830-3 \quad 4.48970-3 \quad 4.38170-3 \quad 4.24920-3 \quad 4.12890-3$ $4.03110-3 \quad 3.93550-3 \quad 3.84530-3 \quad 3.76290-3 \quad 3.69080-3$ $3.65280-3 \quad 3.63870-3 \quad 3.62290-3 \quad 3.60620-3 \quad 3.58250-3$ $\begin{array}{lllll}3.55850-3 & 4.08750-3 & 5.89750-3 & 7.13870-3 & 7.42410-3\end{array}$ $7.67700-37.95180-3 \quad 8.74210-3 \quad 1.11490-2 \quad 1.35030-2$ $\begin{array}{lllll}1.65080-2 & 2.25270-2 & 2.86610-2 & 3.43150-2 & 4.10860-2\end{array}$ $4.92110-2 \quad 5.56070-2 \quad 5.77300-2 \quad 5.80800-2 \quad 5.84980-2$ 6.59980-2 $8.26560-2 \quad 9.04890-2 \quad 9.54810-2 \quad 1.05360-1$ $1.17860-1 \quad 1.29670-1 \quad 1.30100-1 \quad 1.28650-1 \quad 1.27220-1$ $1.26160-1 \quad 1.25620-1 \quad 1.25270-1 \quad 1.28500-1 \quad 1.36960-1$ 1.45980-1 $1.54120-1$ 1.51160-1 $1.47220-11.47060-1$ $1.63200-12.00340-1$

$5.701+10$

$298.958 \quad 457.2 \quad 0.0 \quad 0.0$

$2.07002-7 \quad 7.69672-7 \quad 1.75386-6 \quad 3.71293-6$

$7.86024-6 \quad 1.66401-5 \quad 3.52273-5 \quad 7.45761-5 \quad 1.34159-4$

$2.21191-4 \quad 3.64682-4 \quad 6.01258-4 \quad 9.91309-4 \quad 1.63439-3$ 
2.14167-3 $2.43063-3 \quad 2.82399-3 \quad 3.37141-3 \quad 4.61914-3$ 7.32483-3 $1.20766-2 \quad 1.93065-2 \quad 2.41830-2 \quad 2.54229-2$ 2.65293-2 2.77506-2 3.14039-2 4.33909-2 5.45187-2 7.15396-2 $1.04645-1 \quad 1.36365-1 \quad 1.66556-1 \quad 2.03432-1$ 2.48472-1 2.83877-1 $2.95864-1 \quad 2.97851-1 \quad 3.00232-1$ 3.44858-1 $4.42806-1 \quad 5.10634-1 \quad 5.65749-1 \quad 6.75418-1$ $8.24957-1 \quad 1.00760+0 \quad 1.23069+0 \quad 1.50317+0 \quad 1.83598+0$ $\begin{array}{lllll}2.12513+0 & 2.28850+0 & 2.40583+0 & 2.73896+0 & 3.34537+0\end{array}$ $4.08604+0 \quad 4.99070+0 \quad 6.09565+0 \quad 7.44525+0 \quad 9.09365+0$ $1.11070+11.35661+1$

df325 $\quad 3.70370-3 \quad 4.37060-3 \quad 4.52500-3 \quad 4.57410-3$ $\begin{array}{llllll}4.55830-3 & 4.48970-3 & 4.38170-3 & 4.24920-3 & 4.12890-3\end{array}$ $\begin{array}{llllll}4.03110-3 & 3.93550-3 & 3.84530-3 & 3.76290-3 & 3.69080-3\end{array}$ $3.65280-3 \quad 3.63870-3 \quad 3.62290-3 \quad 3.60620-3 \quad 3.58250-3$ $3.55850-3 \quad 4.08750-3 \quad 5.89750-3 \quad 7.13870-3 \quad 7.42410-3$ $\begin{array}{lllll}7.67700-3 & 7.95180-3 & 8.74210-3 & 1.11490-2 & 1.35030-2\end{array}$ $1.65080-2 \quad 2.25270-2 \quad 2.86610-2 \quad 3.43150-2 \quad 4.10860-2$ 4.92110-2 5.56070-2 $5.77300-2 \quad 5.80800-2 \quad 5.84980-2$ $6.59980-2 \quad 8.26560-2 \quad 9.04890-2 \quad 9.54810-2 \quad 1.05360-1$ $1.17860-1 \quad 1.29670-1 \quad 1.30100-1$ 1.28650-1 $1.27220-1$ $1.26160-1$ 1.25620-1 $1.25270-1 \quad 1.28500-11.36960-1$ 1.45980-1 1.54120-1 1.51160-1 $1.47220-1$ 1.47060-1 $1.63200-12.00340-1$

$\operatorname{fm} 325$ f335:n $5.701+10$

de 335

df 335

$298.958 \quad 609.6 \quad 0.0 \quad 0.0$

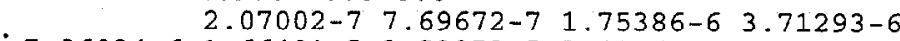

$7.86024-6 \quad 1.66401-5 \quad 3.52273-5 \quad 7.45761-5 \quad 1.34159-4$

2.21191-4 $3.64682-4 \quad 6.01258-4 \quad 9.91309-4 \quad 1.63439-3$

2.14167-3 $2.43063-3 \quad 2.82399-3 \quad 3.37141-3 \quad 4.61914-3$

$\begin{array}{lllll}7.32483-3 & 1.20766-2 & 1.93065-2 & 2.41830-2 & 2.54229-2\end{array}$

2.65293-2 2.77506-2 $3.14039-2 \quad 4.33909-2 \quad 5.45187-2$

7.15396-2 1.04645-1 $1.36365-1 \quad 1.66556-1 \quad 2.03432-1$

$2.48472-1 \quad 2.83877-1 \quad 2.95864-1 \quad 2.97851-1 \quad 3.00232-1$

3.44858-1 4.42806-1 $5.10634-1 \quad 5.65749-1 \quad 6.75418-1$

$8.24957-1 \quad 1.00760+0 \quad 1.23069+0 \quad 1.50317+0 \quad 1.83598+0$

$2.12513+0 \quad 2.28850+0 \quad 2.40583+0 \quad 2.73896+0 \quad 3.34537+0$

$\begin{array}{lllll}4.08604+0 & 4.99070+0 & 6.09565+0 & 7.44525+0 & 9.09365+0\end{array}$

$1.11070+1 \quad 1.35661+1$

$3.70370-3 \quad 4.37060-3 \quad 4.52500-3 \quad 4.57410-3$

$4.55830-3 \quad 4.48970-3 \quad 4.38170-3 \quad 4.24920-3 \quad 4.12890-3$

$4.03110-3 \quad 3.93550-3 \quad 3.84530-3 \quad 3.76290-3 \quad 3.69080-3$

$3.65280-3 \quad 3.63870-3 \quad 3.62290-3 \quad 3.60620-3 \quad 3.58250-3$

$3.55850-3 \quad 4.08750-3 \quad 5.89750-3 \quad 7.13870-3 \quad 7.42410-3$

$7.67700-3 \quad 7.95180-3 \quad 8.74210-3 \quad 1.11490-2 \quad 1.35030-2$

$1.65080-2 \quad 2.25270-2 \quad 2.86610-2 \quad 3.43150-2 \quad 4.10860-2$

4.92110-2 5.56070-2 $5.77300-2 \quad 5.80800-2 \quad 5.84980-2$

6.59980-2 8.26560-2 9.04890-2 9.54810-2 $1.05360-1$

$1.17860-1 \quad 1.29670-1 \quad 1.30100-1 \quad 1.28650-1 \quad 1.27220-1$

$1.26160-1 \quad 1.25620-1 \quad 1.25270-1 \quad 1.28500-11.36960-1$

$1.45980-1$ 1.54120-1 $1.51160-1$ 1.47220-1 $1.47060-1$ $1.63200-12.00340-1$

fm $335 \quad 5.701+10$

$\begin{array}{llllll} & 345: \mathrm{n} & 298.958 & 762.0 & 0.0 & 0.0\end{array}$

de345 $2.07002-7 \quad 7.69672-7 \cdot 1.75386-6 \quad 3.71293-6$ $7.86024-6 \quad 1.66401-5 \quad 3.52273-5 \quad 7.45761-5 \quad 1.34159-4$ $2.21191-4 \quad 3.64682-4 \quad 6.01258-4 \quad 9.91309-4 \quad 1.63439-3$ $2.14167-3 \quad 2.43063-3 \quad 2.82399-3 \quad 3.37141-3 \quad 4.61914-3$ $7.32483-3 \quad 1.20766-2 \quad 1.93065-22.41830-2 \quad 2.54229-2$ 2.65293-2 2.77506-2 $3.14039-2 \quad 4.33909-2 \quad 5.45187-2$ 7.15396-2 1.04645-1 1.36365-1 $1.66556-1 \quad 2.03432-1$ $2.48472-1 \quad 2.83877-12.95864-1 \quad 2.97851-1 \quad 3.00232-1$ 3.44858-1 4.42806-1 5.10634-1 $5.65749-1 \quad 6.75418-1$ $\begin{array}{lllll}8.24957-1 & 1.00760+0 & 1.23069+0 & 1.50317+0 & 1.83598+0\end{array}$ $2.12513+0 \quad 2.28850+0 \quad 2.40583+0 \quad 2.73896+0 \quad 3.34537+0$ $4.08604+0 \quad 4.99070+0 \quad 6.09565+0 \quad 7.44525+0 \quad 9.09365+0$ $1.11070+11.3566 i+1$

df345 $\quad 3.70370-3 \quad 4.37060-3 \quad 4.52500-3 \quad 4.57410-3$ $4.55830-3 \quad 4.48970-3 \quad 4.38170-3 \quad 4.24920-3 \quad 4.12890-3$ $4.03110-3 \quad 3.93550-3 \quad 3.84530-3 \quad 3.76290-3 \quad 3.69080-3$ $3.65280-3 \quad 3.63870-3 \quad 3.62290-3 \quad 3.60620-3 \quad 3.58250-3$ $3.55850-3 \quad 4.08750-3 \quad 5.89750-3 \quad 7.13870-3 \quad 7.42410-3$ $7.67700-3 \quad 7.95180-3 \quad 8.74210-3 \quad 1.11490-2 \quad 1.35030-2$ 


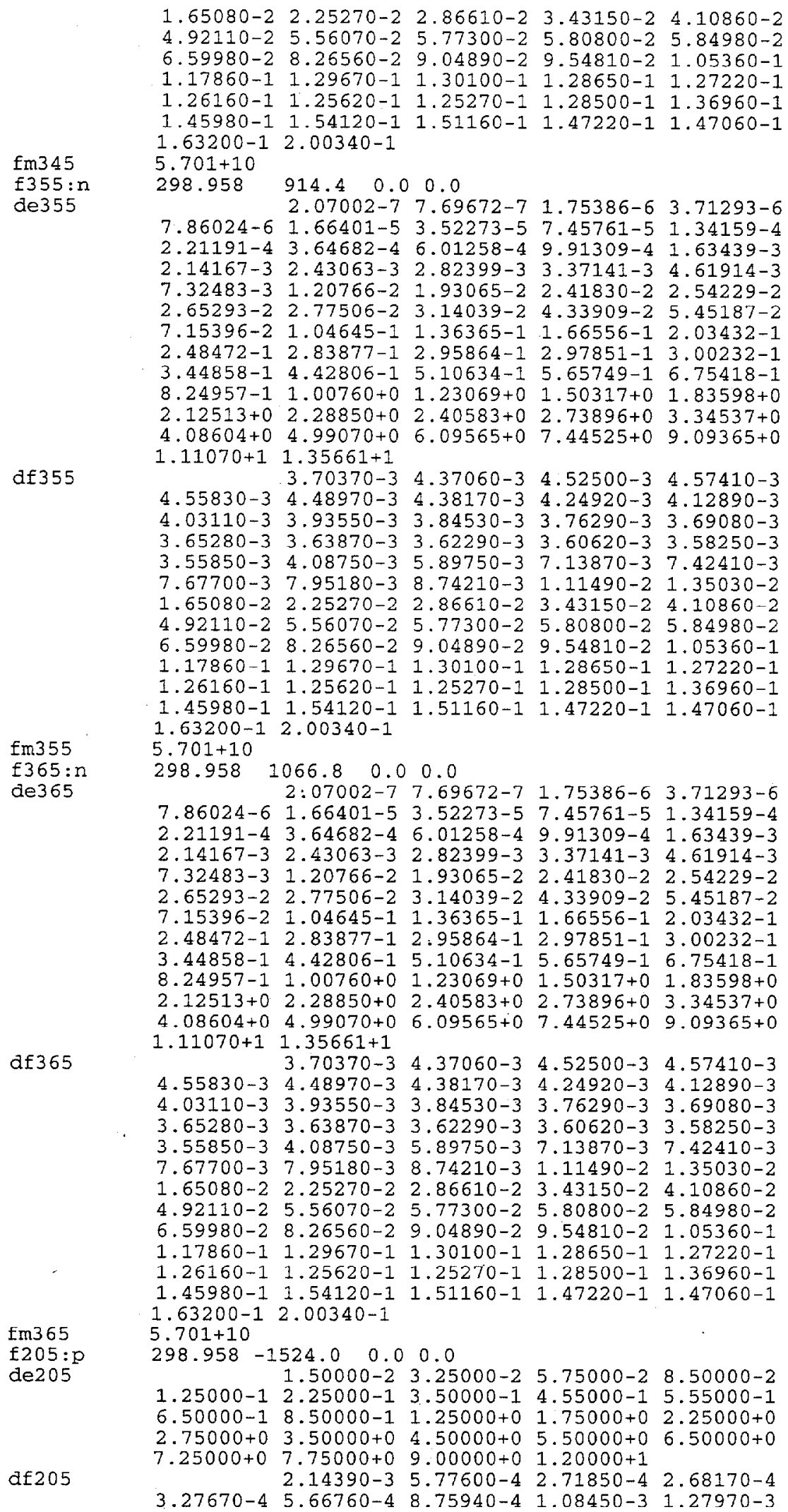




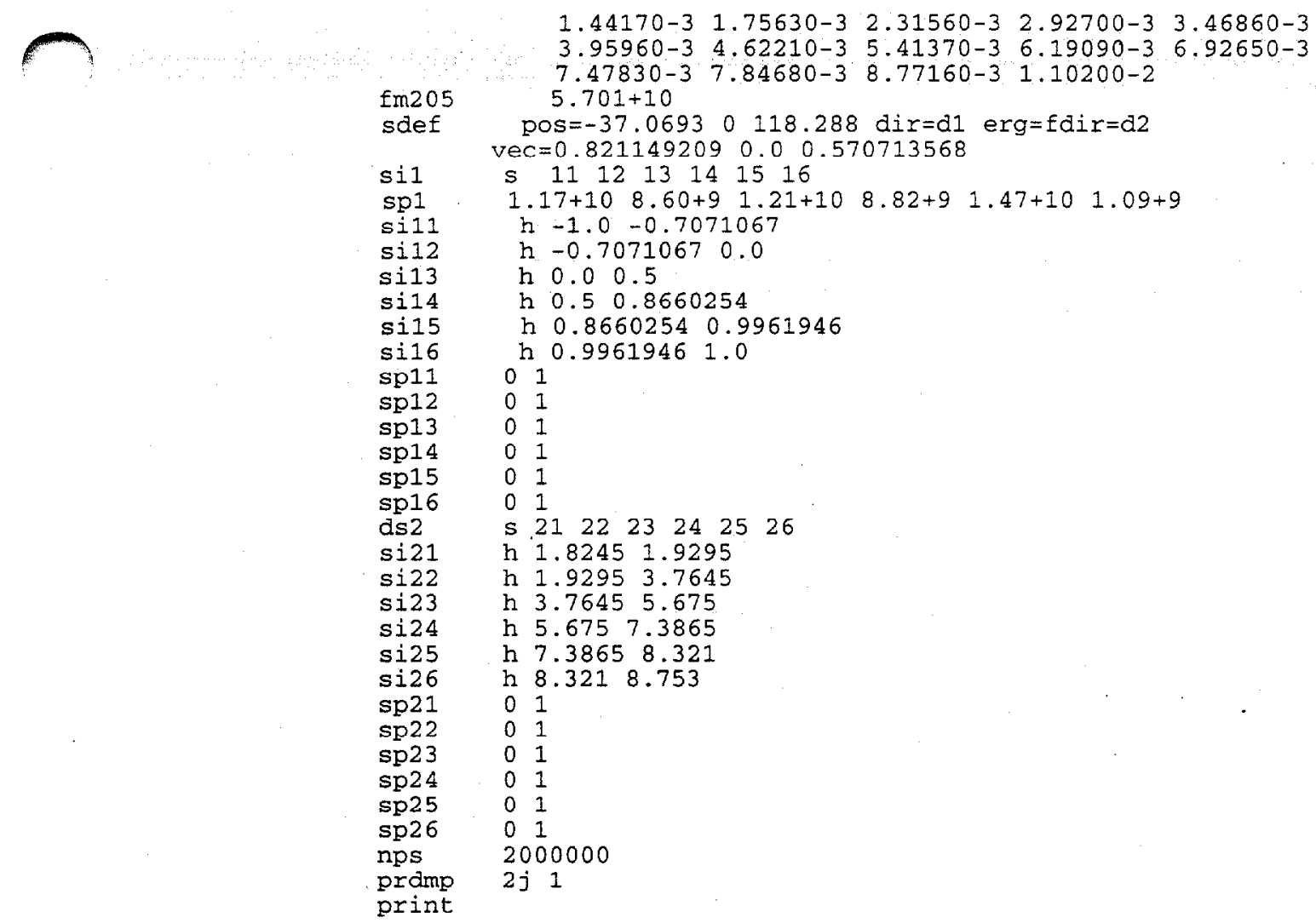


File for MCNP-4B calculation of photon dose rates along the truck lane of the PFNA facility for the source in the maximum up position

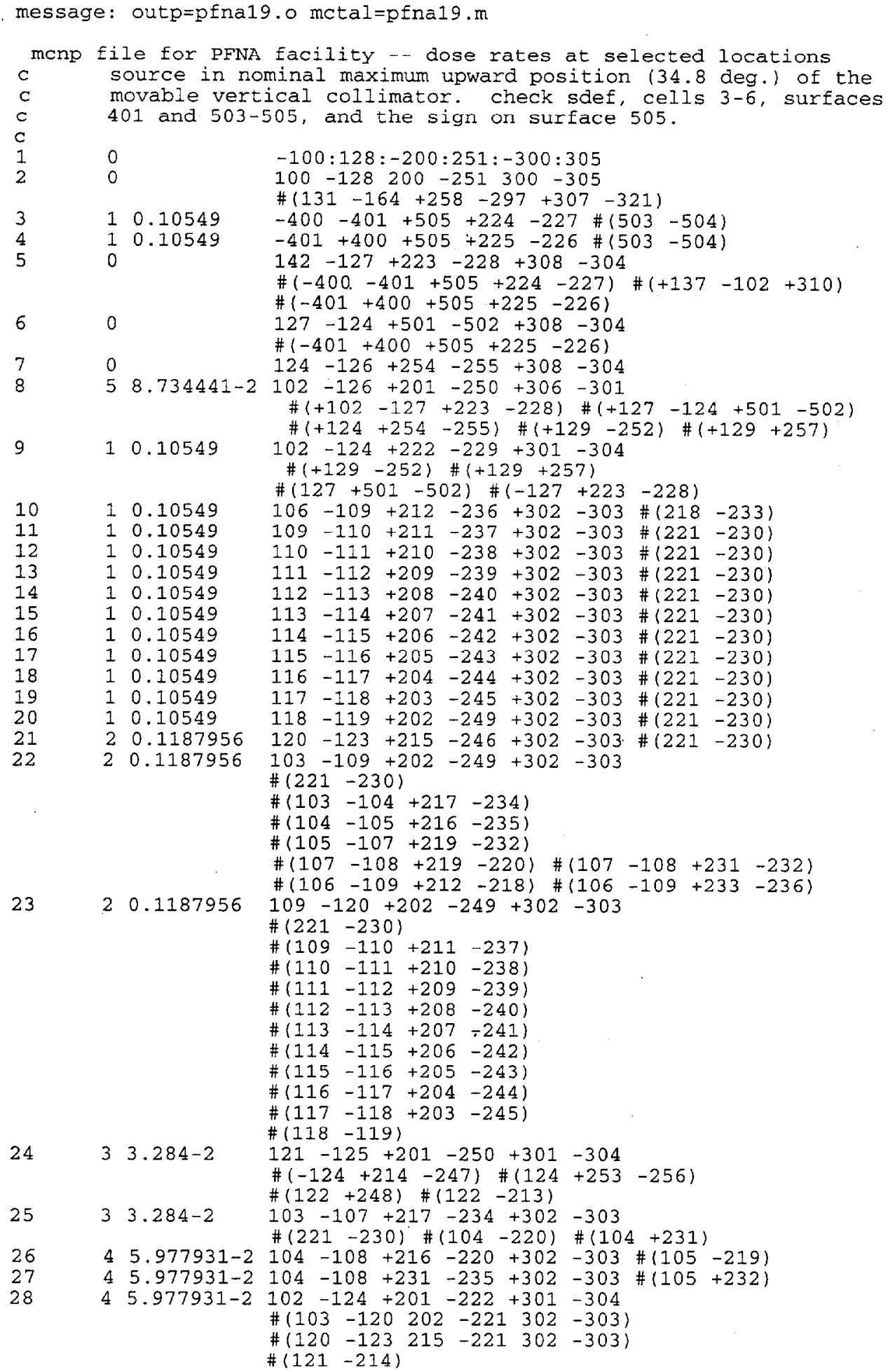




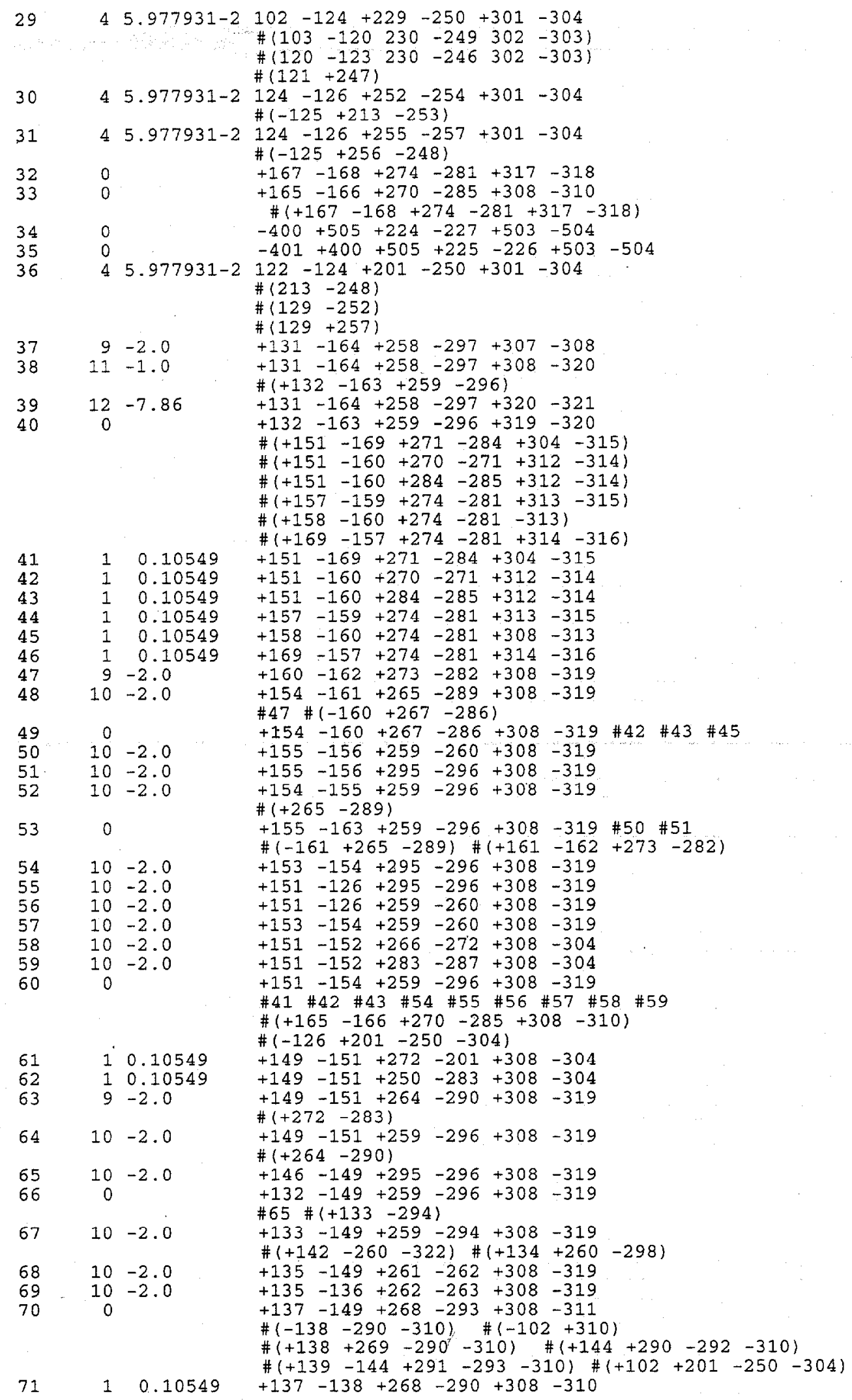




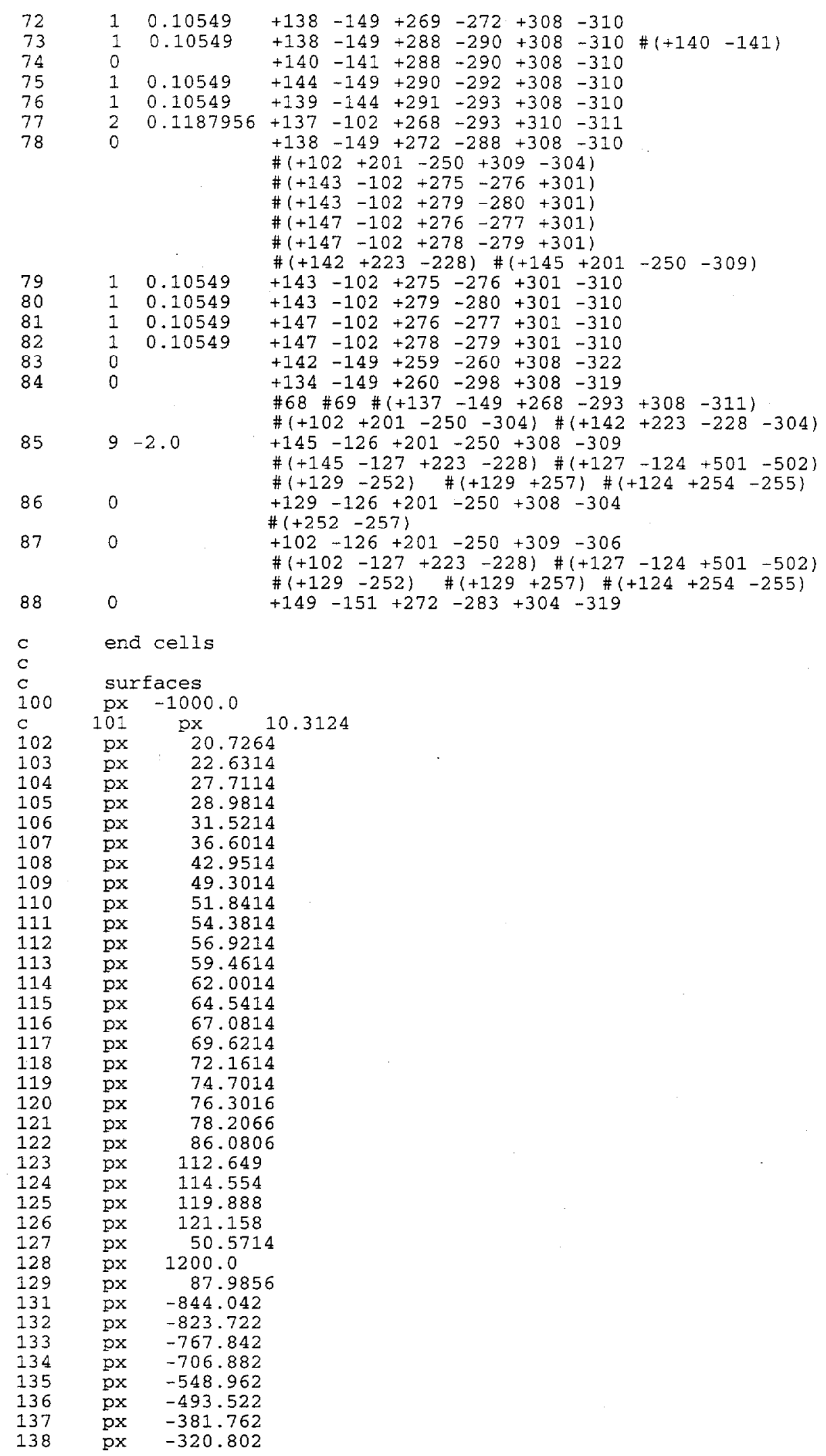




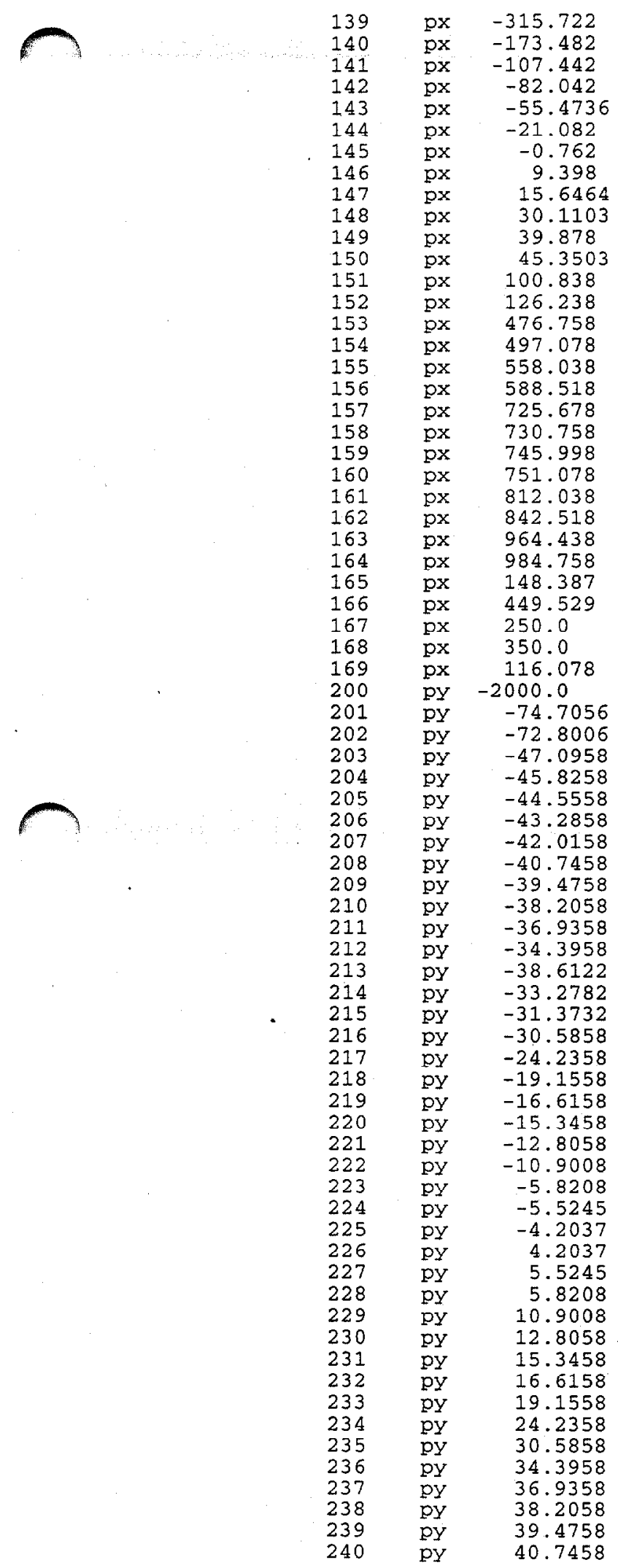




$\begin{array}{llc}241 & \text { py } & 42.0158 \\ 242 & \text { py } & 43.2858 \\ 243 & \text { py } & 44.5558 \\ 244 & \text { py } & 45.8258 \\ 245 & \text { py } & 47.0958 \\ 246 & \text { py } & 31.3732 \\ 247 & \text { py } & 33.2782 \\ 248 & \text { py } & 38.6122 \\ 249 & \text { py } & 72.8006 \\ 250 & \text { py } & 74.7056 \\ 251 & \text { py } & 1500.0 \\ 252 & \text { py } & -39.8822 \\ 253 & \text { py } & -9.8848 \\ 254 & \text { py } & -8.6148 \\ 255 & \text { py } & 8.6148 \\ 256 & \text { py } & 9.8848 \\ 257 & \text { py } & 39.8822 \\ 258 & \text { py } & -1731.28 \\ 259 & \text { py } & -1710.96 \\ 260 & \text { py } & -1650.0 \\ 261 & \text { py } & -1528.08 \\ 262 & \text { py } & -1467.12 \\ 263 & \text { py } & -1436.64 \\ 264 & \text { py } & -589.28 \\ 265 & \text { py } & -241.3 \\ 266 & \text { py } & -193.04 \\ 267 & \text { py } & -180.34 \\ 268 & \text { py } & -167.64 \\ 269 & \text { py } & -162.56 \\ 270 & \text { py } & -152.4 \\ 271 & \text { py } & -137.16 \\ 272 & \text { py } & -101.6 \\ 273 & \text { py } & -91.44 \\ 274 & \text { py } & -60.96 \\ 275 & \text { py } & 45.72 \\ 276 & \text { py } & -30.48 \\ 277 & \text { py } & -10.16 \\ 278 & \text { py } & 10.16 \\ 279 & \text { py } & 30.48 \\ 280 & \text { py } & 45.72 \\ 281 & \text { py } & 60.96 \\ 282 & \text { py } & 91.44 \\ 283 & \text { py } & 101.6 \\ 284 & \text { py } & 137.16 \\ 285 & \text { py } & 152.4 \\ 286 & \text { py } & 180.34 \\ 287 & \text { py } & 193.04 \\ 288 & \text { py } & 213.36 \\ 289 & \text { py } & 241.3 \\ 290 & \text { py } & 274.32 \\ 291 & \text { py } & 335.28 \\ 292 & \text { py } & 345.44 \\ 293 & \text { py } & 396.24 \\ 294 & \text { py } & 533.4 \\ 295 & \text { py } & 1235.44 \\ 296 & \text { py } & 1296.4 \\ 297 & \text { py } & 1316.72 \\ 298 & \text { py } & 472.44 \\ 300 & \text { pz } & -500.0 \\ 301 & \text { pz } & -59.478 \\ 302 & \text { pz } & -57.573 \\ 303 & \text { pz } & 245.957 \\ 304 & \text { pz } & 247.862 \\ 305 & \text { pz } & 500.0 \\ 306 & \text { pz } & -60.748 \\ 307 & \text { pz } & -156.0 \\ 308 & \text { pz } & -125.095 \\ 309 & \text { pz } & -67.451 \\ 310 & \text { pz } & 235.585 \\ 311 & \text { pz } & 276.225 \\ 312 & \text { pz } & 283.845 \\ 313 & \text { pz } & 342.265 \\ & & \end{array}$




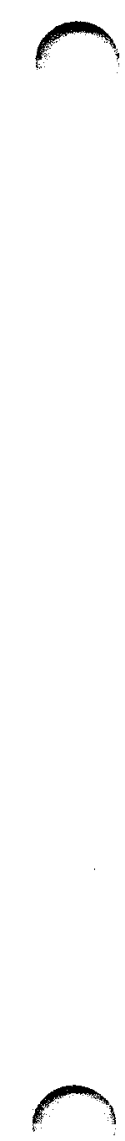

pz $\quad 375.285$

pz $\quad 390.525$

pz $\quad 395.605$

pz $\quad-50.0$

$\mathrm{pz} \quad 50.0$

pz $\quad 301.625$

$\mathrm{pz} \quad 484.505$

$\mathrm{pz} \quad 484.823$

pz 118.745

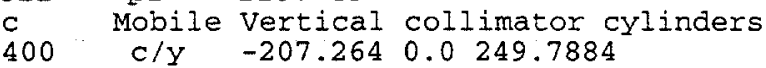

$401 \quad \mathrm{C} / \mathrm{Y} \quad-28.60131 \quad 124.1738 \quad 59.9948$

Target

402 sx 292.2524 spheres

403 sx $292.2524 \quad 6.38$

c Stationary collimator planes

$\begin{array}{llllll}501 & p & 0.05557761 & 1.0 & 0.0 & -1.613162\end{array}$

$\begin{array}{llllll}502 & p & -0.05557761 & 1.0 & 0.0 & 1.613162\end{array}$

c

503

504

505

Mobile Vertical collimator planes

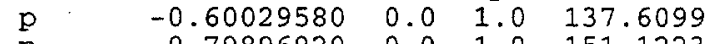

$\begin{array}{lllll}\mathrm{p} & -0.79896920 & 0.0 & 1.0 & 151.1223\end{array}$

$\mathrm{c}$

end surfaces

mode $n \mathrm{p}$

imp:n,p $0186 \mathrm{r}$

c $\mathrm{m} 1=$ borated polyethylene (Asum=0.10549)

mI

$$
\text { m }
$$$$
\text { c }
$$$$
\mathrm{m} 4
$$ 


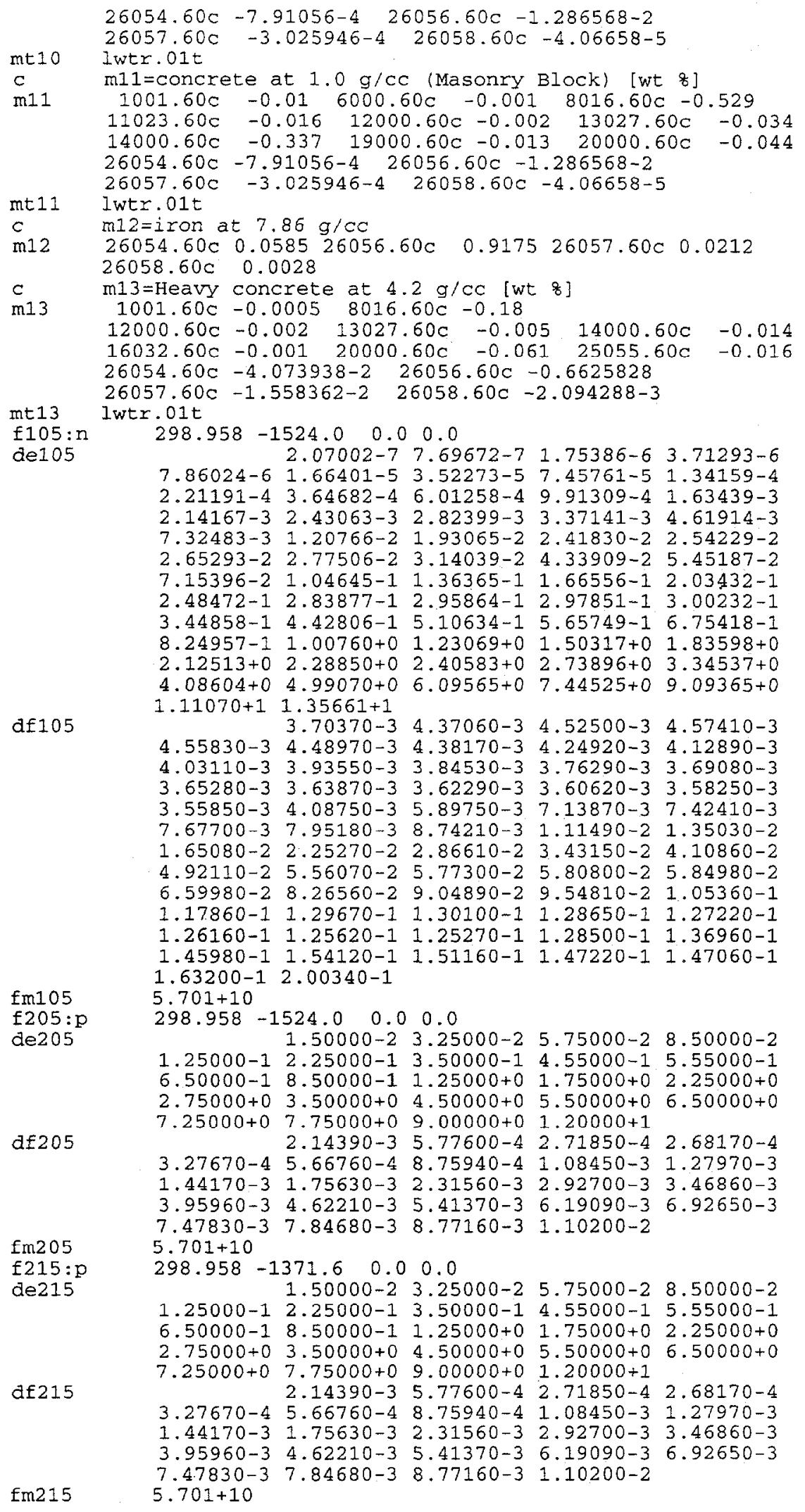




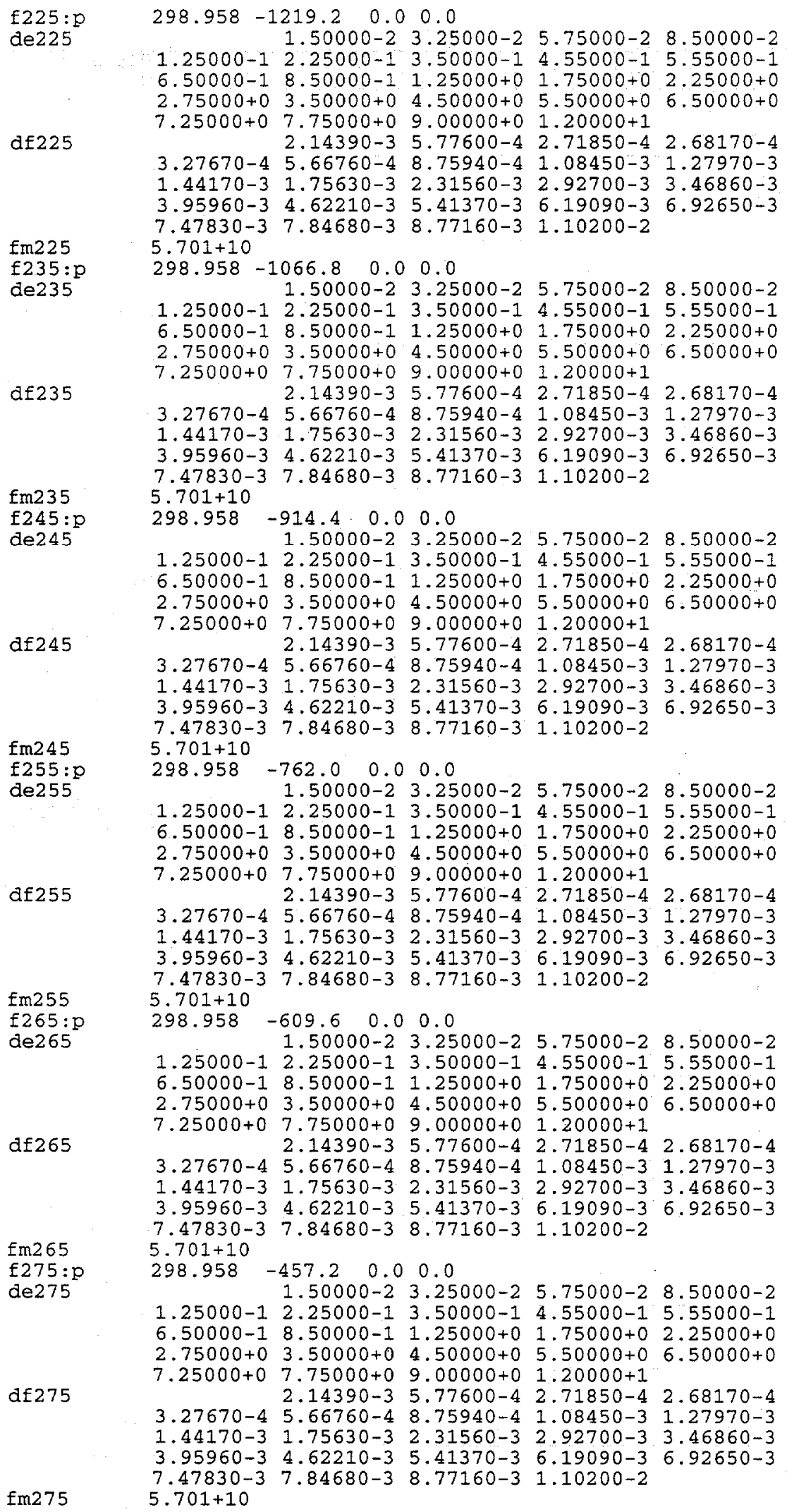




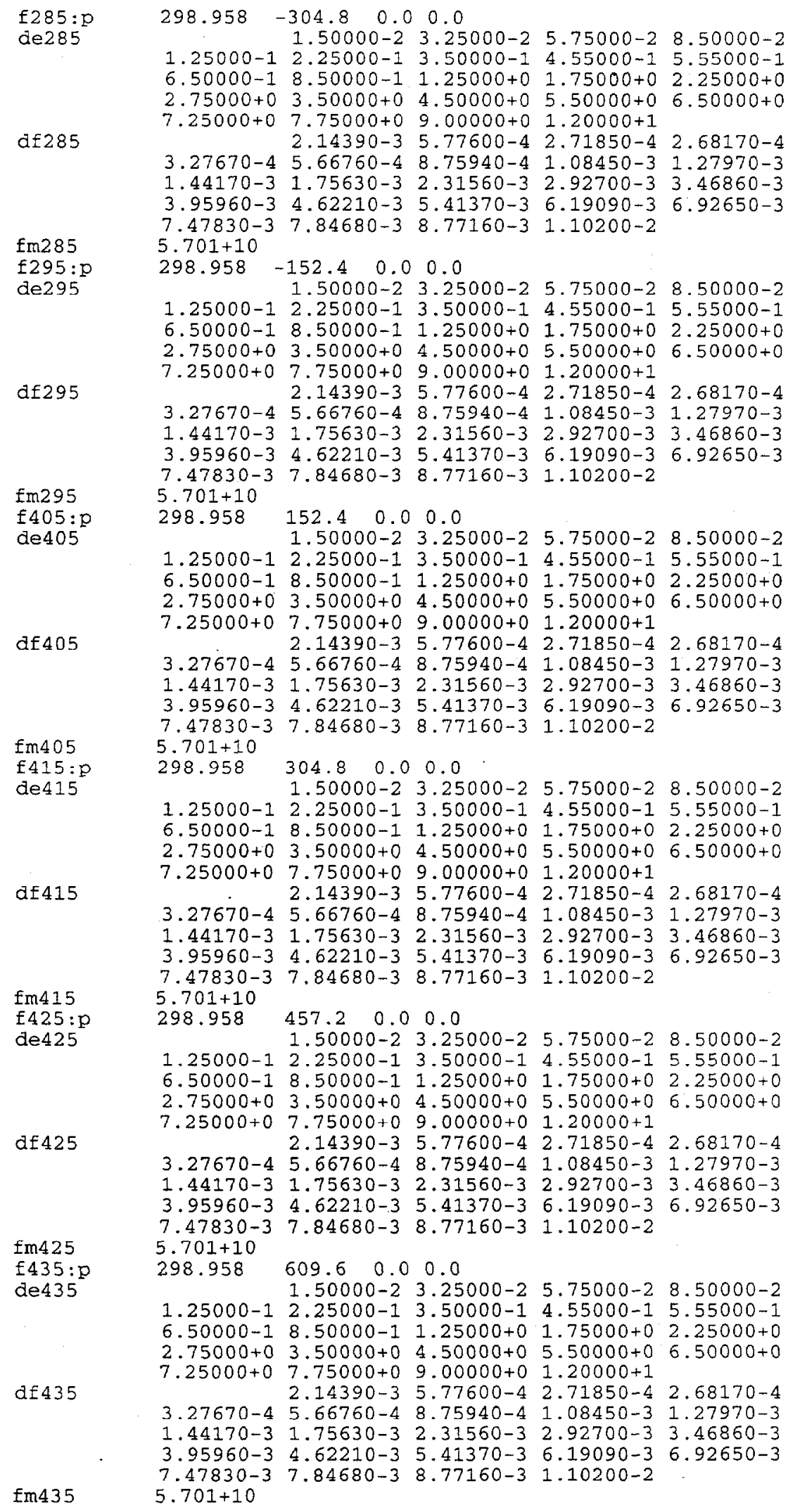




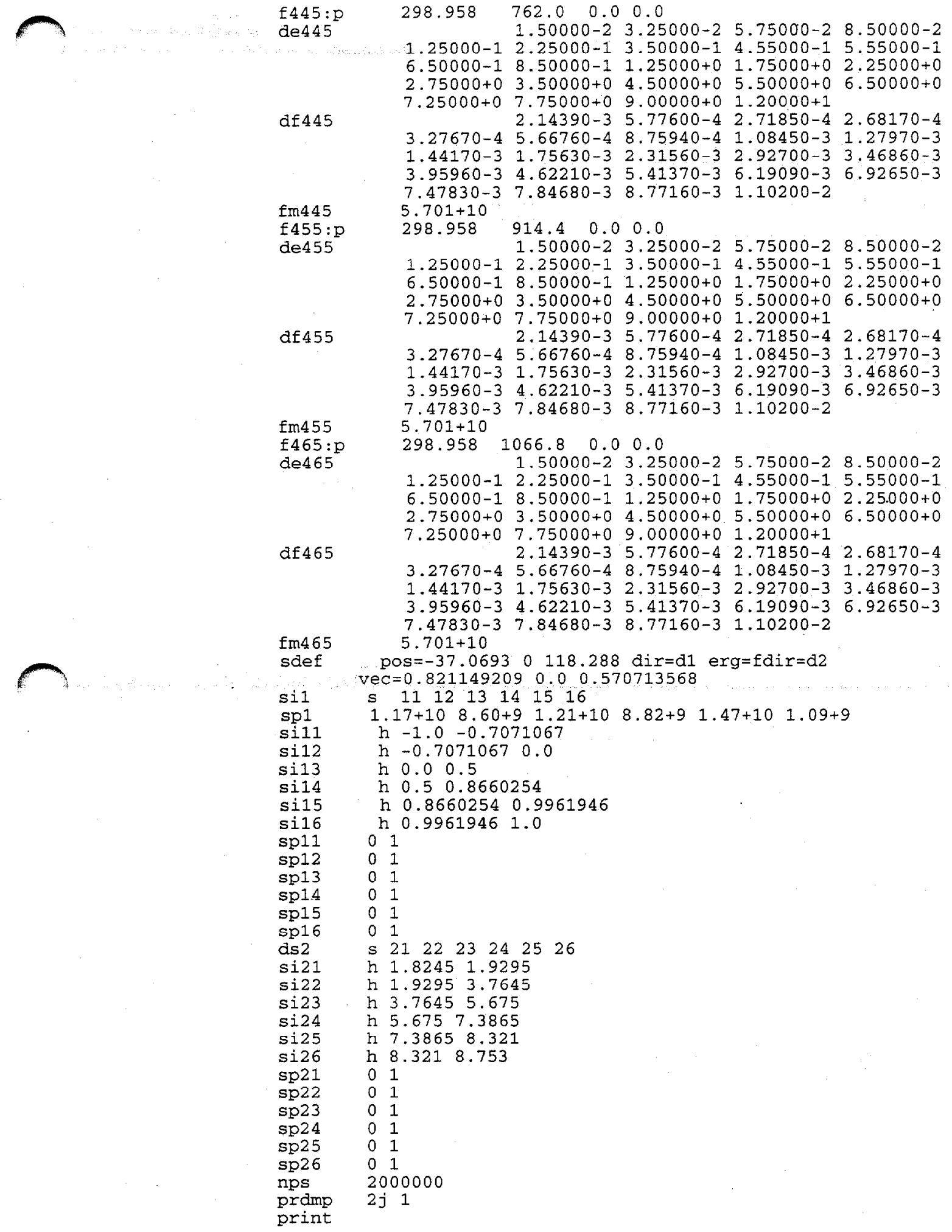


File for MCNP-4B calculation of neutron dose rates along the truck lane of the PFNA facility for the source in the maximum down position

message: outp $=p f n a 20 . \circ$ mctal $=p f n a 20 . m$

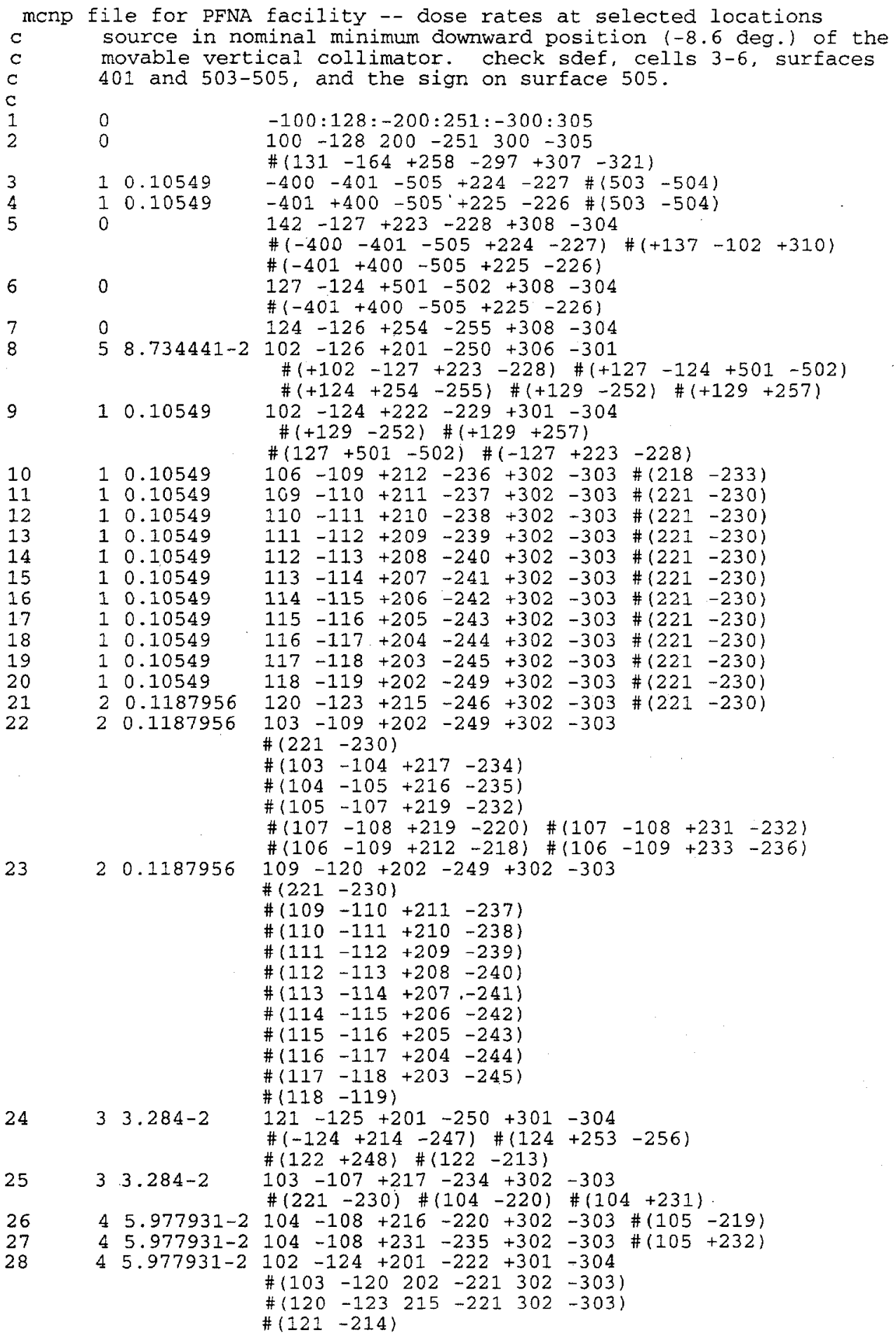


$45.977931-2 \quad 124-126+252-254+301-304$ $\#(-125+213-253)$

$\begin{array}{lll}71 & 1 & 0.10549\end{array}$ 


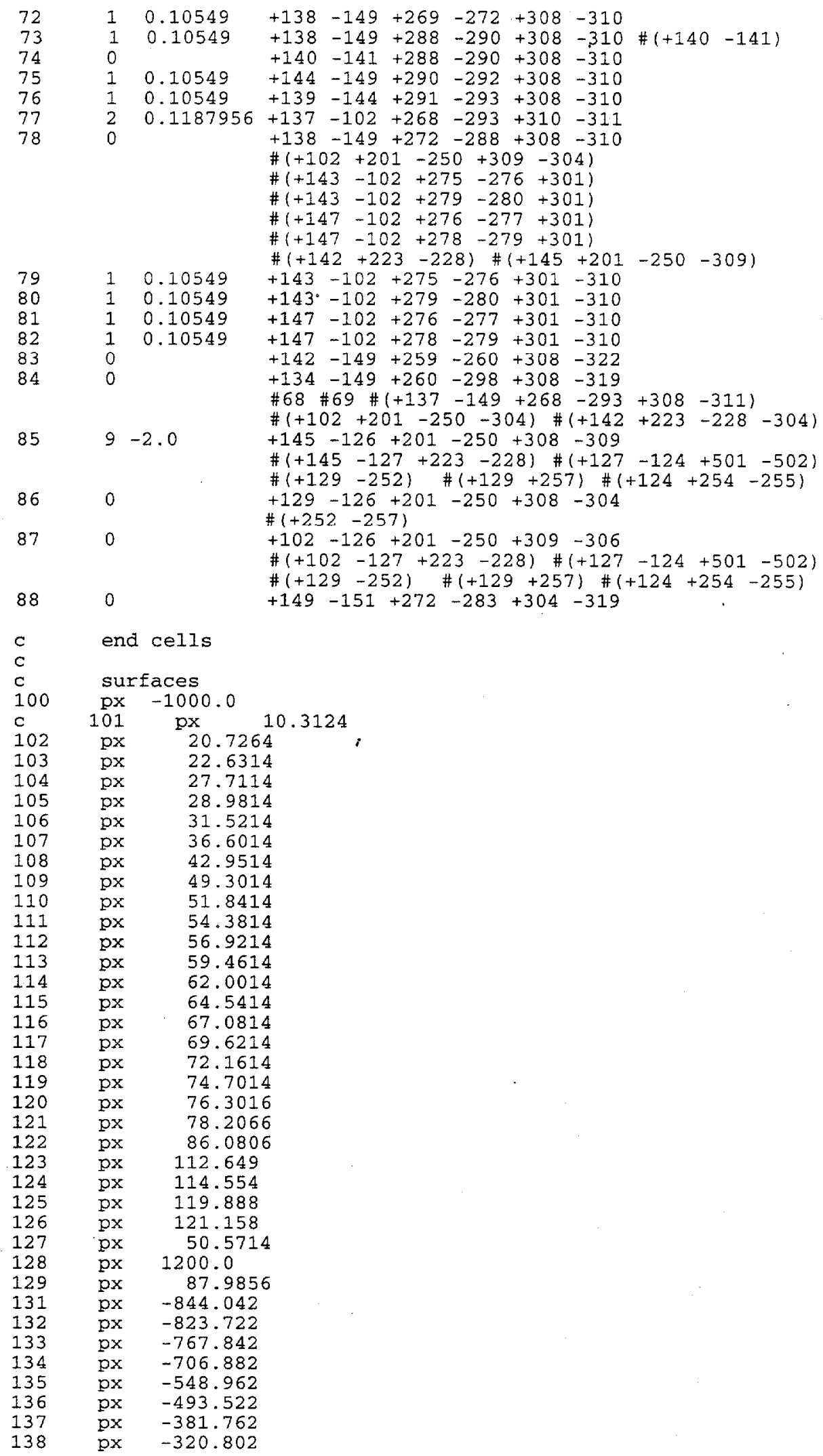




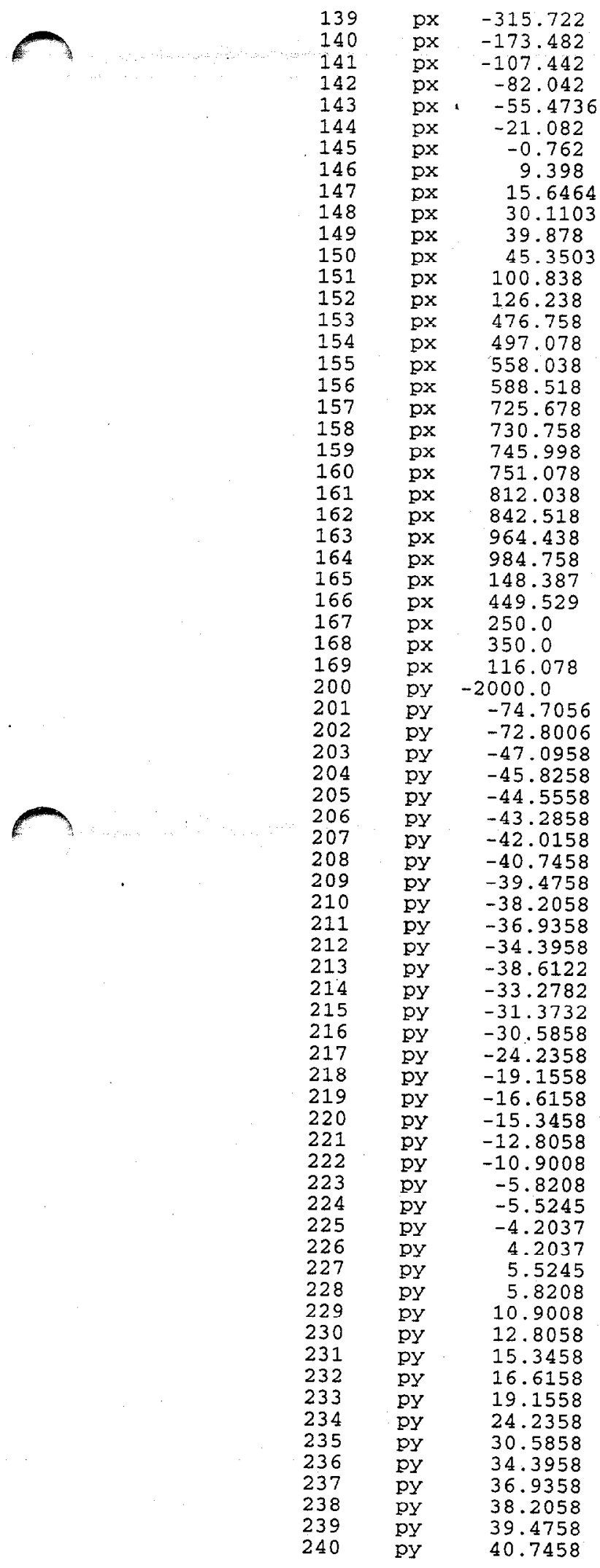




\begin{tabular}{|c|c|c|}
\hline 241 & py & 42.0158 \\
\hline 242 & py & 43.2858 \\
\hline 243 & py & 44.5558 \\
\hline 244 & py & 45.8258 \\
\hline 245 & py & 47.0958 \\
\hline 246 & py & 31.3732 \\
\hline 247 & py & 33.2782 \\
\hline 248 & py & 38.6122 \\
\hline 249 & py & 72.8006 \\
\hline 250 & py & 74.7056 \\
\hline 251 & py & 1500.0 \\
\hline 252 & py & -39.8822 \\
\hline 253 & py & -9.8848 \\
\hline 254 & py & -8.6148 \\
\hline 255 & py & 8.6148 \\
\hline 256 & py & 9.8848 \\
\hline 257 & py & 39.8822 \\
\hline 258 & DY & -1731.28 \\
\hline 259 & py & -1710.96 \\
\hline 260 & py & -1650.0 \\
\hline 261 & PY & -1528.08 \\
\hline 262 & py & -1467.12 \\
\hline 263 & py & -1436.64 \\
\hline 264 & py &.-589.28 \\
\hline 265 & py & -241.3 \\
\hline 266 & py & -193.04 \\
\hline 267 & py & $-180 \cdot 34$ \\
\hline 268 & py & -167.64 \\
\hline 269 & py & -162.56 \\
\hline 270 & py & -152.4 \\
\hline 271 & py & -137.16 \\
\hline 272 & py & -101.6 \\
\hline 273 & py & -91.44 \\
\hline 274 & pY & -60.96 \\
\hline 275 & PY & -45.72 \\
\hline 276 & py & -30.48 \\
\hline 277 & py & -10.16 \\
\hline 278 & py & 10.16 \\
\hline 279 & py & 30.48 \\
\hline 280 & py & 45.72 \\
\hline 281 & py & 60.96 \\
\hline 282 & PY & 91.44 \\
\hline 283 & py & 101.6 \\
\hline 284 & py & 137.16 \\
\hline 285 & py & 152.4 \\
\hline 286 & py & 180.34 \\
\hline 287 & py & 193.04 \\
\hline 288 & py & 213.36 \\
\hline 289 & PY & 241.3 \\
\hline 290 & $\mathrm{pY}$ & 274.32 \\
\hline 291 & py & 335.28 \\
\hline 292 & py & 345.44 \\
\hline 293 & py & 396.24 \\
\hline 294 & py & 533.4 \\
\hline 295 & py & 1235.44 \\
\hline 296 & py & 1296.4 \\
\hline 297 & py & 1316.72 \\
\hline 298 & $\mathrm{pY}$ & 472.44 \\
\hline 300 & $\mathrm{pz}$ & -500.0 \\
\hline 301 & $\mathrm{pz}$ & -59.478 \\
\hline 302 & $\mathrm{pz}$ & -57.573 \\
\hline 303 & $\mathrm{pz}$ & 245.957 \\
\hline 304 & $p z$ & 247.862 \\
\hline 305 & $\mathrm{pz}$ & 500.0 \\
\hline 306 & $p z$ & -60.748 \\
\hline 307 & $\mathrm{pz}$ & -156.0 \\
\hline 308 & $\mathrm{pz}$ & -125.095 \\
\hline 309 & $\mathrm{pz}$ & -67.451 \\
\hline 310 & $\mathrm{pz}$ & 235.585 \\
\hline 311 & $\mathrm{pz}$ & 276.225 \\
\hline 312 & $\mathrm{pz}$ & 283.845 \\
\hline 313 & $\mathrm{pz}$ & 342.265 \\
\hline
\end{tabular}




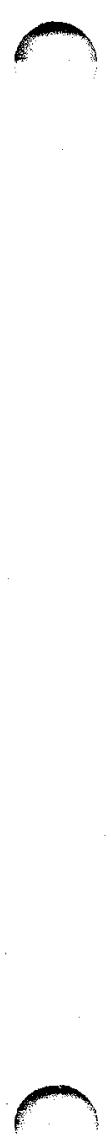

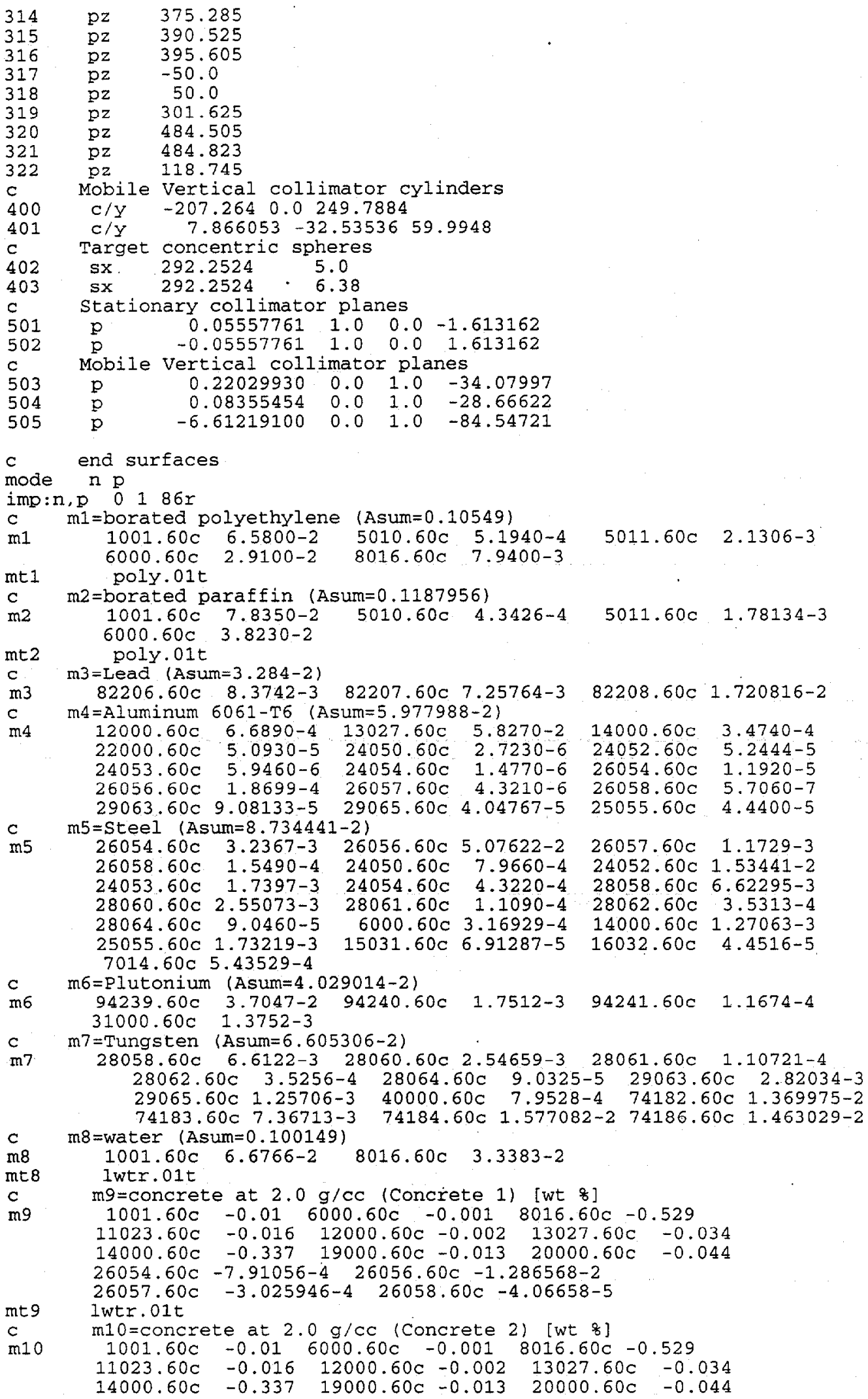




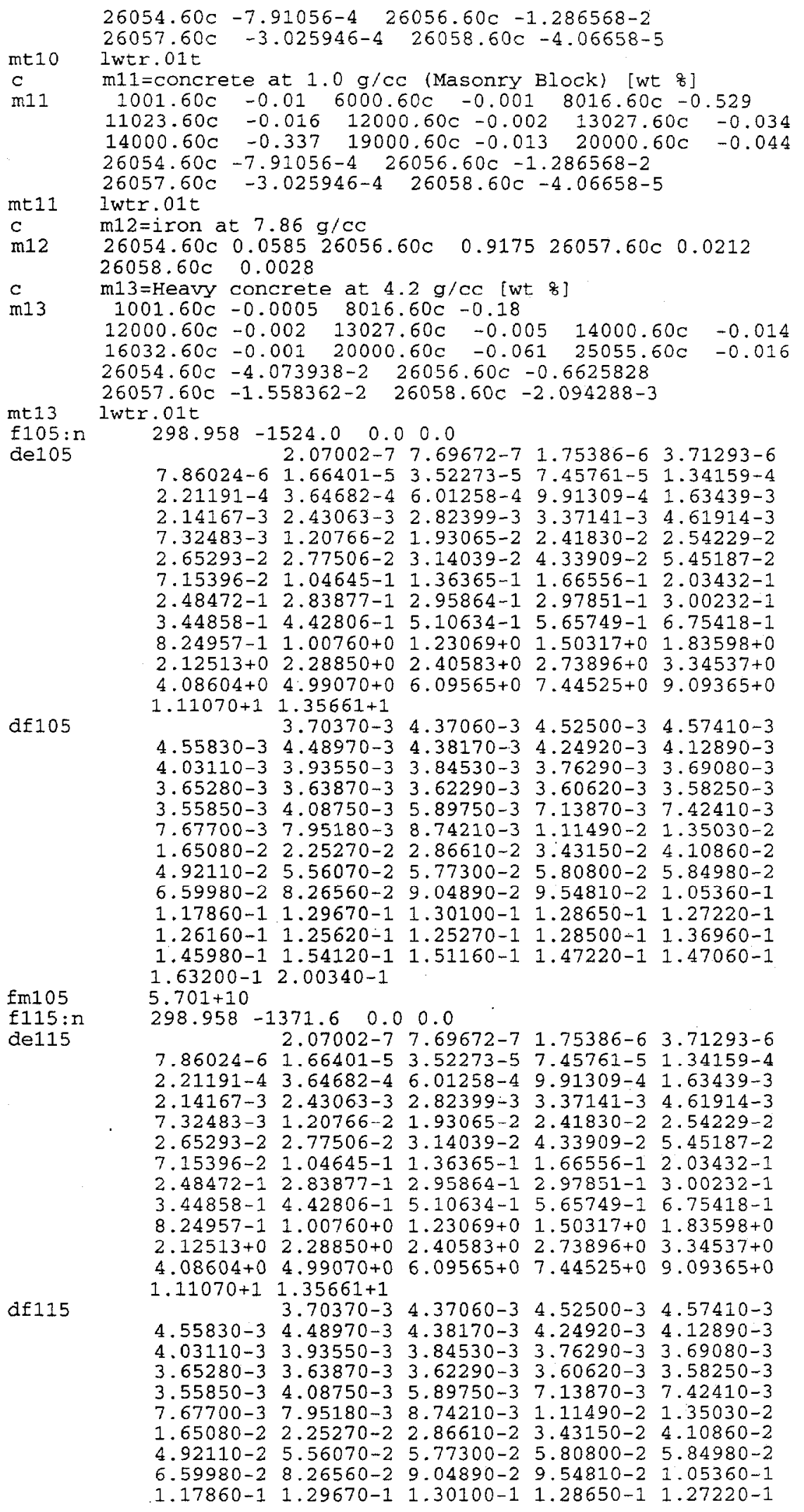


1.26160-1 $1.25620-1 \quad 1.25270-1 \quad 1.28500-1 \quad 1.36960-1$ $1.45980-1 \quad 1.54120-1 \quad 1.51160-1 \quad 1.47220-1 \quad 1.47060-1$ 1. $63200-12.00340-1$

$f \ln 115$ $5.701+10$

f125:n $298.958-1219.2 \quad 0.0 \quad 0.0$

de125

$\begin{array}{llll}07002-7 & 7.69672-7 & 1.75386-6 & 3.71293-6\end{array}$ 1.86024-6 $1.66401-5 \quad 3.52273-5 \quad 7.45761-5 \quad 1.34159-4$ 2.21191-4 $3.64682-4 \quad 6.01258-4 \quad 9.91309-4 \quad 1.63439-3$. $2.14167-3 \quad 2.43063-3 \quad 2.82399-3 \quad 3.37141-3 \quad 4.61914-3$ 7.32483-3 $1.20766-2 \quad 1.93065-2 \quad 2.41830-2 \quad 2.54229-2$ 2.65293-2 2.77506-2 3.14039-2 4.33909-2 5.45187-2 7.15396-2 $1.04645-1 \quad 1.36365-1 \quad 1.66556-1 \quad 2.03432-1$ $2.48472-1 \quad 2.83877-1.2 .95864-1 \quad 2.97851-1 \quad 3.00232-1$ 3.44858-1 $4.42806-1 \quad 5.10634-1 \quad 5.65749-1 \quad 6.75418-1$ $8.24957-1 \quad 1.00760+0 \quad 1.23069+0 \quad 1.50317+0 \quad 1.83598+0$ $2.12513+0 \quad 2.28850+0 \quad 2.40583+0 \quad 2.73896+0 \quad 3.34537+0$ $4.08604+0 \quad 4.99070+0 \quad 6.09565+0 \quad 7.44525+0 \quad 9.09365+0$ $1.11070+11.35661+1$

df125 $\quad 3.70370-3 \quad 4.37060-3 \quad 4.52500-3 \quad 4.57410-3$ $\begin{array}{llllll}4.55830-3 & 4.48970-3 & 4.38170-3 & 4.24920-3 & 4.12890-3\end{array}$ $4.03110-3 \quad 3.93550-3 \quad 3.84530-3 \quad 3.76290-3 \quad 3.69080-3$ $3.65280-3 \quad 3.63870-3 \quad 3.62290-3 \quad 3.60620-3 \quad 3.58250-3$ $\begin{array}{llllll}3.55850-3 & 4.08750-3 & 5.89750-3 & 7.13870-3 & 7.42410-3\end{array}$ $\begin{array}{lllll}7.67700-3 & 7.95180-3 & 8.74210-3 & 1.11490-2 & 1.35030-2\end{array}$ $\begin{array}{lllll}1.65080-2 & 2.25270-2 & 2.86610-2 & 3.43150-2 & 4.10860-2\end{array}$ $4.92110-2 \quad 5.56070-2 \quad 5.77300-2 \quad 5.80800-2 \quad 5.84980-2$ $6.59980-2 \quad 8.26560-2 \quad 9.04890-2 \quad 9.54810-2 \quad 1.05360-1$ $\begin{array}{llllll}1.17860-1 & 1.29670-1 & 1.30100-1 & 1.28650-1 & 1.27220-1\end{array}$ 1.26160-1 $1.25620-1 \quad 1.25270-1 \quad 1.28500-1 \quad 1.36960-1$ $1.45980-1$ 1.54120-1 $1.51160-1$ 1.47220-1 $1.47060-1$ $1.63200-12.00340-1$

$\operatorname{fm} 125 \quad 5.701+10$

f135:n $\quad 298.958-1066.8 \quad 0.0 \quad 0.0$

$\begin{array}{llllll}\text { de135 } & 2.07002-7 & 7.69672-7 & 1.75386-6 & 3.71293-6\end{array}$ $7.86024-6 \quad 1.66401-5 \quad 3.52273-5 \quad 7.45761-5 \quad 1.34159-4$ $2.21191-4 \quad 3.64682-4 \quad 6.01258-4 \quad 9.91309-4 \quad 1.63439-3$ $\begin{array}{llllll}2.14167-3 & 2.43063-3 & 2.82399-3 & 3.37141-3 & 4.61914-3\end{array}$ $7.32483-31.20766-2.1 .93065-2 \quad 2.41830-2 \quad 2.54229-2$ $2.65293-2 \quad 2.77506-2 \quad 3.14039-2 \quad 4.33909-2 \quad 5.45187-2$ 7.15396-2 $1.04645-1 \quad 1.36365-1 \quad 1.66556-1 \quad 2.03432-1$ 2.48472-1 $2.83877-1 \quad 2.95864-1 \quad 2.97851-1 \quad 3.00232-1$ $3.44858-1 \quad 4.42806-1 \quad 5.10634-1 \quad 5.65749-1 \quad 6.75418-1$ 8.24957-1 $1.00760+0 \quad 1.23069+0 \quad 1.50317+0 \quad 1.83598+0$ $2.12513+0 \quad 2.28850+0 \quad 2.40583+0 \quad 2.73896+0 \quad 3.34537+0$ $4.08604+0 \quad 4.99070+0 \quad 6.09565+0 \quad 7.44525+0 \quad 9.09365+0$ $1.11070+11.35661+1$

df135 $3.70370-3 \quad 4.37060-3 \quad 4.52500-3 \quad 4.57410-3$ $4.55830-3 \quad 4.48970-3 \quad 4.38170-3 \quad 4.24920-3 \quad 4.12890-3$ $4.03110-3 \quad 3.93550-3 \quad 3.84530-3 \quad 3.76290-3 \quad 3.69080-3$ $\begin{array}{llllll}3.65280-3 & 3.63870-3 & 3.62290-3 & 3.60620-3 & 3.58250-3\end{array}$ $\begin{array}{llllll}3.55850-3 & 4.08750-3 & 5.89750-3 & 7.13870-3 & 7.42410-3\end{array}$ $\begin{array}{llllll}7.67700-3 & 7.95180-3 & 8.74210-3 & 1.11490-2 & 1.35030-2\end{array}$ $\begin{array}{lllll}1.65080-2 & 2.25270-2 & 2.86610-2 & 3.43150-2 & 4.10860-2\end{array}$ 4.92110-2 5.56070-2 $5.77300-2 \quad 5.80800-2 \quad 5.84980-2$ $\begin{array}{lllll}6.59980-2 & 8.26560-2 & 9.04890-2 & 9.54810-2 & 1.05360-1\end{array}$ 1.17860-1 $1.29670-1 \quad 1.30100-1 \quad 1.28650-1 \quad 1.27220-1$ 1.26160-1 $1.25620-1 \quad 1.25270-1 \quad 1.28500-1 \quad 1.36960-1$ $1.45980-1 \quad 1.54120-1 \quad 1.51160-1 \quad 1.47220-1 \quad 1.47060-1$ $1.63200-12.00340-1$

fm135 $\quad 5.701+10$

$\begin{array}{lllll}\text { f } 145: \mathrm{n} & 298.958 & -914.4 & 0.0 & 0.0\end{array}$

de145 $\quad 2.07002-7 \quad 7.69672-7 \quad 1.75386-6 \quad 3.71293-6$ $7.86024-61.66401-5 \quad 3.52273-5 \quad 7.45761-5 \quad 1.34159-4$ $\begin{array}{llllll}2.21191-4 & 3.64682-4 & 6.01258-4 & 9.91309-4 & 1.63439-3\end{array}$ $2.14167-3 \quad 2.43063-3 \quad 2.82399-3 \quad 3.37141-3 \quad 4.61914-3$ 7.32483-3 $1.20766-2 \quad 1.93065-2 \quad 2.41830-2 \quad 2.54229-2$ 2.65293-2 2.77506-2 $3.14039-2 \quad 4.33909-2 \quad 5.45187-2$ 7.15396-2 $1.04645-1 \quad 1.36365-1 \quad 1.66556-1 \quad 2.03432-1$ $\begin{array}{lllll}2.48472-1 & 2.83877-1 & 2.95864-1 & 2.97851-1 & 3.00232-1\end{array}$ 3.44858-1 $4.42806-1 \quad 5.10634-1 \quad 5.65749-1 \quad 6.75418-1$ $8.24957-1 \quad 1.00760+0 \quad 1.23069+0 \quad 1.50317+0 \quad 1.83598+0$ $\begin{array}{lllll}2.12513+0 & 2.28850+0 & 2.40583+0 & 2.73896+0 & 3.34537+0\end{array}$ 


\begin{tabular}{|c|c|c|c|c|c|}
\hline & $\begin{array}{l}4.08604+0 \\
1.11070+1\end{array}$ & $\begin{array}{l}4.99070+0 \\
1.35661+1\end{array}$ & $6.09565+0$ & $7.44525+0$ & $9.09365+0$ \\
\hline df 145 & $\begin{array}{l}4.55830-3 \\
4.03110-3 \\
3.65280-3 \\
3.55850-3 \\
7.67700-3 \\
1.65080-2 \\
4.92110-2 \\
6.59980-2 \\
1.17860-1 \\
1.26160-1 \\
1.45980-1 \\
1.63200-1\end{array}$ & $\begin{array}{l}3.70370-3 \\
4.48970-3 \\
3.93550-3 \\
3.63870-3 \\
4.08750-3 \\
7.95180-3 \\
2.25270-2 \\
5.56070-2 \\
8.26560-2 \\
1.29670-1 \\
1.25620-1 \\
1.54120-1 \\
2.00340-1\end{array}$ & $\begin{array}{l}4.37060-3 \\
4.38170-3 \\
3.84530-3 \\
3.62290-3 \\
5.89750-3 \\
8.74210-3 \\
2.86610-2 \\
5.77300-2 \\
9.04890-2 \\
1.30100-1 \\
1.25270-1 \\
1.51160-1\end{array}$ & $\begin{array}{l}4.52500-3 \\
4.24920-3 \\
3.76290-3 \\
3.60620-3 \\
7.13870-3 \\
1.11490-2 \\
3.43150-2 \\
5.80800-2 \\
9.54810-2 \\
1.28650-1 \\
1.28500-1 \\
1.47220-1\end{array}$ & $\begin{array}{l}4.57410-3 \\
4.12890-3 \\
3.69080-3 \\
3.58250-3 \\
7.42410-3 \\
1.35030-2 \\
4.10860-2 \\
5.84980-2 \\
1.05360-1 \\
1.27220-1 \\
1.36960-1 \\
1.47060-1\end{array}$ \\
\hline $\begin{array}{l}\mathrm{fm} 145 \\
\mathrm{f} 155: \mathrm{n}\end{array}$ & $\begin{array}{l}5.701+10 \\
298.958\end{array}$ & $-762.0 \quad 0.0$ & 0.0 & & \\
\hline de1 & $\begin{array}{l}7.86024-6 \\
2.21191-4 \\
2.14167-3 \\
7.32483-3 \\
2.65293-2 \\
7.15396-2 \\
2.48472-1 \\
3.44858-1 \\
8.24957-1 \\
2.12513+0 \\
4.08604+0 \\
1.11070+1\end{array}$ & $\begin{array}{l}2.07002-7 \\
1.66401-5 \\
3.64682-4 \\
2.43063-3 \\
1.20766-2 \\
2.77506-2 \\
1.04645-1 \\
2.83877-1 \\
4.42806-1 \\
1.00760+0 \\
2.28850+0 \\
4.9907010 \\
1.35661+1\end{array}$ & $\begin{array}{l}7.69672-7 \\
3.52273-5 \\
6.01258-4 \\
2.82399-3 \\
1.93065-2 \\
3.14039-2 \\
1.36365-1 \\
2.95864-1 \\
5.10634-1 \\
1.23069+0 \\
2.40583+0 \\
6.09565+0\end{array}$ & $\begin{array}{l}1.75386-6 \\
7.45761-5 \\
9.91309-4 \\
3.37141-3 \\
2.41830-2 \\
4.33909-2 \\
1.66556-1 \\
2.97851-1 \\
5.65749-1 \\
1.50317+0 \\
2.73896+0 \\
7.44525+0\end{array}$ & $\begin{array}{l}3.71293-6 \\
1.34159-4 \\
1.63439-3 \\
4.61914-3 \\
2.54229-2 \\
5.45187-2 \\
2.03432-1 \\
3.00232-1 \\
6.75118-1 \\
1.83598+0 \\
3.34537+0 \\
9.09365+0\end{array}$ \\
\hline df155 & $\begin{array}{l}4.55830-3 \\
4.03110-3 \\
3.65280-3 \\
3.55850-3 \\
7.67700-3 \\
1.65080-2 \\
4.92110-2 \\
6.59980-2 \\
1.17860-1 \\
1.26160-1 \\
1.45980-1 \\
1.63200-1\end{array}$ & $\begin{array}{l}3.70370-3 \\
4.48970-3 \\
3.93550-3 \\
3.63870-3 \\
4.08750-3 \\
7.95180-3 \\
2.25270-2 \\
5.56070-2 \\
8.26560-2 \\
1.29670-1 \\
1.25620-1 \\
1.54120-1 \\
2.00340-1\end{array}$ & $\begin{array}{l}4.37060-3 \\
4.38170-3 \\
3.84530-3 \\
3.62290-3 \\
5.89750-3 \\
8.74210-3 \\
2.86610-2 \\
5.77300-2 \\
9.04890-2 \\
1.30100-1 \\
1.25270-1 \\
1.51160-1\end{array}$ & $\begin{array}{l}4.52500-3 \\
4.24920-3 \\
3.76290-3 \\
3.60620-3 \\
7.13870-3 \\
1.11490-2 \\
3.43150-2 \\
5.80800-2 \\
9.54810-2 \\
1.28650-1 \\
1.28500-1 \\
1.47220-1\end{array}$ & $\begin{array}{l}4.57410-3 \\
4.12890-3 \\
3.69080-3 \\
3.58250-3 \\
7.42410-3 \\
1.35030-2 \\
4.10860-2 \\
5.84980-2 \\
1.05360-1 \\
1.27220-1 \\
1.36960-1 \\
1.47060-1\end{array}$ \\
\hline m155 & $\begin{array}{l}5.701+10 \\
298.958\end{array}$ & $-609.6 \quad 0.0$ & & & \\
\hline & $\begin{array}{c}7.86024-6 \\
2.21191-4 \\
2.11167-3 \\
7.32483-3 \\
2.65293-2 \\
7.15396-2 \\
2.48472-1 \\
3.44858-1 \\
8.24957-1 \\
2.12513+0 \\
4.08604+0 \\
1.11070+1\end{array}$ & $\begin{array}{l}2.07002-7 \\
1.66401-5 \\
3.64682-4 \\
2.43063-3 \\
1.20766-2 \\
2.77506-2 \\
1.04645-1 \\
2.83877-1 \\
4.42806-1 \\
1.00760+0 \\
2.28850+0 \\
4.99070+0 \\
1.35661+1\end{array}$ & $\begin{array}{l}7.69672-7 \\
3.52273-5 \\
6.01258-4 \\
2.82399-3 \\
1.93065-2 \\
3.14039-2 \\
1.36365-1 \\
2.95864-1 \\
5.10634-1 \\
1.23069+0 \\
2.40583+0 \\
6.09565+0\end{array}$ & $\begin{array}{l}1.75386-6 \\
7.45761-5 \\
9.91309-4 \\
3.37141-3 \\
2.41830-2 \\
4.33909-2 \\
1.66556-1 \\
2.97851-1 \\
5.65749-1 \\
1.50317+0 \\
2.73896+0 \\
7.44525+0\end{array}$ & $\begin{array}{l}3.71293-6 \\
1.34159-4 \\
1.63439-3 \\
4.61914-3 \\
2.54229-2 \\
5.45187-2 \\
2.03432-1 \\
3.00232-1 \\
6.75418-1 \\
1.83598+0 \\
3.34537+0 \\
9.09365+0\end{array}$ \\
\hline & $\begin{array}{l}4.55830-3 \\
4.03110-3 \\
3.65280-3 \\
3.55850-3 \\
7.67700-3 \\
1.65080-2 \\
4.92110-2 \\
6.59980-2 \\
1.17860-1 \\
1.26160-1 \\
1.45980-1 \\
1.63200-1\end{array}$ & $\begin{array}{l}3.70370-3 \\
4.48970-3 \\
3.93550-3 \\
3.63870-3 \\
4.08750-3 \\
7.95180-3 \\
2.25270-2 \\
5.56070-2 \\
8.26560-2 \\
1.29670-1 \\
1.25620-1 \\
1.54120-1 \\
2.00340-1\end{array}$ & $\begin{array}{l}4.37060-3 \\
4.38170-3 \\
3.84530-3 \\
3.62290-3 \\
5.89750-3 \\
8.74210-3 \\
2.86610-2 \\
5.77300-2 \\
9.04890-2 \\
1.30100-1 \\
1.25270-1 \\
1.51160-1\end{array}$ & $\begin{array}{l}4.52500-3 \\
4.24920-3 \\
3.76290-3 \\
3.60620-3 \\
7.13870-3 \\
1.11490-2 \\
3.43150-2 \\
5.80800-2 \\
9.54810-2 \\
1.28650-1 \\
1.28500-1 \\
1.47220-1\end{array}$ & $\begin{array}{l}4.57410-3 \\
4.12890-3 \\
3.69080-3 \\
3.58250-3 \\
7.42410-3 \\
1.35030-2 \\
4.10860-2 \\
5.84980-2 \\
1.05360-1 \\
1.27220-1 \\
1.36960-1 \\
1.47060-1\end{array}$ \\
\hline & & & & & \\
\hline
\end{tabular}




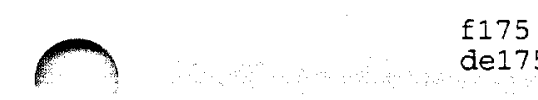

$\begin{array}{llllll}\begin{array}{l}\text { f175:n } \\ \text { de175 }\end{array} & 298.958 & -457.2 & 0.0 & 0.0 \\ & & 2.07002-7 & 7.69672-7 & 1.75386-6 & 3.71293-6 \\ & 7.86024-6 & 1.66401-5 & 3.52273-5 & 7.45761-5 & 1.34159-4 \\ & 2.21191-4 & 3.64682-4 & 6.01258-4 & 9.91309-4 & 1.63439-3 \\ 2.14167-3 & 2.43063-3 & 2.82399-3 & 3.37141-3 & 4.61914-3 \\ & 7.32483-3 & 1.20766-2 & 1.93065-2 & 2.41830-2 & 2.51229-2 \\ & 2.65293-2 & 2.77506-2 & 3.14039-2 & 4.33909-2 & 5.45187-2 \\ & 7.15396-2 & 1.04645-1 & 1.36365-1 & 1.66556-1 & 2.03432-1 \\ & 2.48472-1 & 2.83877-1 & 2.95864-1 & 2.97851-1 & 3.00232-1 \\ & 3.44858-1 & 4.42806-1 & 5.10634-1 & 5.65749-1 & 6.75418-1 \\ & 8.24957-1 & 1.00760+0 & 1.23069+0 & 1.50317+0 & 1.83598+0 \\ & 2.12513+0 & 2.28850+0 & 2.40583+0 & 2.73896+0 & 3.34537+0 \\ & 4.08604+0 & 4.99070+0 & 6.09565+0 & 7.44525+0 & 9.09365+0 \\ 1.11070+1 & 1.35661+1 & & & \end{array}$

$\operatorname{df} 175$

$\begin{array}{llllll}4.55830-3 & 4.48970-3 & 4.38170-3 & 4.24920-3 & 4.12890-3\end{array}$

$4.03110-3 \quad 3.93550-3 \quad 3.84530-3 \quad 3.76290-3 \quad 3.69080-3$

$\begin{array}{lllll}3.65280-3 & 3.63870-3 & 3.62290-3 & 3.60620-3 & 3.58250-3\end{array}$

$\begin{array}{llllll}3.55850-3 & 4.08750-3 & 5.89750-3 & 7.13870-3 & 7.42410-3\end{array}$

$7.67700-3 \quad 7.95180-3 \quad 8.74210-3 \quad 1.11490-2 \quad 1.35030-2$

$1.65080-2 \quad 2.25270-2 \quad 2.86610-2 \quad 3.43150-2 \quad 4.10860-2$

4.92110-2 5.56070-2 5.77300-2 5.80800-2 5.84980-2

$6.59980-2 \quad 8.26560-2 \quad 9.04890-2 \quad 9.54810-2 \quad 1.05360-1$

$1.17860-1 \quad 1.29670-1 \quad 1.30100-1 \quad 1.28650-1 \quad 1.27220-1$

$1.26160-1$ 1.25620-1 $1.25270-1$ 1.28500-1 $1.36960-1$

1.45980-1 1.54120-1 1.51160-1 $1.47220-1 \quad 1.47060-1$

$1.63200-1 \quad 2.00340-1$

f185:n $\quad 298.958 \quad-304.8 \quad 0.0 \quad 0.0$

$\begin{array}{llllll}\operatorname{de} 185 & 2.07002-7 & 7.69672-7 & 1.75386-6 & 3.71293-6\end{array}$

7.86024-6 $1.66401-5 \quad 3.52273-5 \quad 7.45761-5 \quad 1.34159-4$

$2.21191-4 \quad 3.64682-4 \quad 6.01258-4 \quad 9.91309-4 \quad 1.63439-3$

$2.14167-3 \quad 2.43063-3 \quad 2.82399-3 \quad 3.37141-3 \quad 4.61914-3$

7.32483-3 $1.20766-2 \quad 1.93065-2 \quad 2.41830-2 \quad 2.54229-2$

$\begin{array}{lllll}2.65293-2 & 2.77506-2 & 3.14039-2 & 4.33909-2 & 5.45187-2\end{array}$

7.15396-2 $1.04645-1 \quad 1.36365-1 \quad 1.66556-1 \quad 2.03432-1$

2.48472-1 $2.83877-1 \quad 2.95864-1 \quad 2.97851-1.3 .00232-1$

$3.44858-1 \quad 4: 42806-1 \quad 5.10634-1 \quad 5.65749-1 \quad 6.75418-1$

$8.24957-1 \quad 1.00760+0 \quad 1.23069+0 \quad 1.50317+0 \quad 1.83598+0$

$2.12513+0 \quad 2.28850+0 \quad 2.40583+0 \quad 2.73896+0 \quad 3.34537+0$

$4.08604+0 \quad 4.99070+0 \quad 6.09565+0 \quad 7.44525+0 \quad 9.09365+0$

$1.11070+1 \quad 1.35661+1$

df 185

$\begin{array}{llllll}3.70370-3 & 4.37060-3 & 4.52500-3 & 4.57410-3\end{array}$ $4.55830-3 \quad 4.48970-3 \quad 4.38170-3 \quad 4.24920-3 \quad 4.12890-3$ $4.03110-3 \quad 3.93550-3 \quad 3.84530-3 \quad 3.76290-3 \quad 3.69080-3$ $\begin{array}{llllll}3.65280-3 & 3.63870-3 & 3.62290-3 & 3.60620-3 & 3.58250-3\end{array}$ $3.55850-3 \quad 4.08750-3 \quad 5.89750-3 \quad 7.13870-3 \quad 7.42410-3$ $\begin{array}{lllll}7.67700-3 & 7.95180-3 & 8.74210-3 & 1.11490-2 & 1.35030-2\end{array}$ $1.65080-2 \quad 2.25270-2 \quad 2.86610-2$ 3.43150-2 4.10860-2 4.92110-2 5.56070-2 5.77300-2 5.80800-2 5.84980-2 $6.59980-2 \quad 8.26560-2 \quad 9.04890-2 \quad 9.54810-2 \quad 1.05360-1$ $1.17860-1$ 1.29670-1 $1.30100-1 \quad 1.28650-1 \quad 1.27220-1$ 1.26160-1 $1.25620-1 \quad 1.25270-1 \quad 1.28500-1 \quad 1.36960-1$ $1.45980-1$ 1.54120-1 $1.51160-1$ 1.47220-1 $1.47060-1$ $1.63200-12.00340-1$

$\operatorname{fm} 185$ $5.701+10$

f195:n de195 298.958

$\begin{array}{lll}-152.4 & 0.0 & 0.0\end{array}$ $2.07002-7 \quad 7.69672-7 \quad 1.75386-6 \quad 3.71293-6$ 7.86024-6 $1.66401-5 \quad 3.52273-5 \quad 7.45761-5 \quad 1.34159-4$

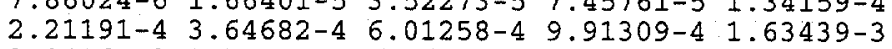
2.14167-3 $2.43063-3 \quad 2.82399-3 \quad 3.37141-3 \quad 4.61914-3$ $7.32483-31.20766-2 \quad 1.93065-2 \quad 2.41830-2 \quad 2.54229-2$ $2.65293-2 \quad 2.77506-2 \quad 3.14039-2 \quad 4.33909-2 \quad 5.45187-2$ 7.15396-2 1.04645-1 $1.36365-1 \quad 1.66556-1 \quad 2.03432-1$ 2.48472-1 2.83877-1 2.95864-1 2.97851-1 3.00232-1 3.44858-1 4.42805-1 5.10634-1 $5.65749-1$. $6.75418-1$ $8.24957-1 \quad 1.00760+0 \quad 1.23069+0 \quad 1.50317+0 \quad 1.83598+0$ $\begin{array}{llllll}2.12513+0 & 2.28850+0 & 2.40583+0 & 2.73896+0 & 3.34537+0\end{array}$ $4.08604+0 \quad 4.99070+0 \quad 6.09565+0 \quad 7.44525+0 \quad 9.09365+0$ $1.11070+1 \quad 1.35661+1$

df195 $\quad \begin{array}{lllll}3.70370-3 & 4.37060-3 & 4.52500-3 & 4.57410-3\end{array}$ 
fm195

f $305: n$

de 305

df 305

fm305

f $315: n$

de 315

$\operatorname{df3} 15$

fm315

f325:n

de 325 $\begin{array}{llllll}4.03110-3 & 3.93550-3 & 3.84530-3 & 3.76290-3 & 3.69080-3\end{array}$ $3.65280-3 \quad 3.63870-3 \quad 3.62290-3 \quad 3.60620-3 \quad 3.58250-3$ $3.55850-3 \quad 4.08750-3 \quad 5.89750-3 \quad 7.13870-3 \quad 7.42410-3$ $7.67700-3 \quad 7.95180-3 \quad 8.74210-3 \quad 1.11490-2 \quad 1.35030-2$ $1.65080-2 \quad 2.25270-2 \quad 2.86610-2 \quad 3.43150-2 \quad 4.10860-2$ $4.92110-2 \quad 5.56070-2 \quad 5.77300-2 \quad 5.80800-2 \quad 5.84980-2$ $6.59980-2 \quad 8.26560-2 \quad 9.04890-2 \quad 9.54810-2 \quad 1.05360-1$ $1.17860-1 \quad 1.29670-1 \quad 1.30100-1 \quad 1.28650-1 \quad 1.27220-1$ $1.26160-1 \quad 1.25620-1 \quad 1.25270-1 \quad 1.28500-1 \quad 1.36960-1$ $1.45980-1$ 1.54120-1 $1.51160-1$ 1.47220-1 $1.47060-1$ 1.63200-1 2.00340-1

$5.701+10$

$298.958 \quad 152.4 \quad 0.0 \quad 0.0$

$2.07002-7 \quad 7.69672-7 \quad 1.75386-6 \quad 3.71293-6$

7.86024-6 $1.66401-5 \quad 3.52273-5 \quad 7.45761-5 \quad 1.34159-4$ $2.21191-4 \quad 3.64682-4 \quad 6.01258-4 \quad 9.91309-4 \quad 1.63439-3$ $2.14167-3 \quad 2.43063-3 \quad 2.82399-3 \quad 3.37141-3 \quad 4.61914-3$ $7.32483-3 \quad 1.20766-2 \quad 1.93065-2 \quad 2.41830-2 \quad 2.54229-2$ $2.65293-2 \quad 2.77506-2 \quad 3.14039-2 \quad 4.33909-2 \quad 5.45187-2$ 7.15396-2 $1.04645-1 \quad 1.36365-1 \quad 1.66556-1 \quad 2.03432-1$ $\begin{array}{llllll}2.48472-1 & 2.83877-1 & 2.95864-1 & 2.97851-1 & 3.00232-1\end{array}$ $3.44858-1 \quad 4.42806-1 \quad 5.10634-1 \quad 5.65749-1 \quad 6.75418-1$ $8.24957-1 \quad 1.00760+0 \quad 1.23069+0 \quad 1.50317+0 \quad 1.83598+0$ $2.12513+0 \quad 2.28850+0 \quad 2.40583+0 \quad 2.73896+0 \quad 3.34537+0$ $4.08604+0 \quad 4.99070+0 \quad 6.09565+0 \quad 7.44525+0 \quad 9.09365+0$ $1.11070+1 \quad 1.35661+1$ $3.70370-3 \quad 4.37060-3 \quad 4.52500-3 \quad 4.57410-3$ $4.55830-3 \quad 4.48970-3 \quad 4.38170-3 \quad 4.24920-3 \quad 4.12890-3$ $\begin{array}{llllll}4.03110-3 & 3.93550-3 & 3.84530-3 & 3.76290-3 & 3.69080-3\end{array}$ $3.65280-3 \quad 3.63870-3 \quad 3.62290-3 \quad 3.60620-3 \quad 3.58250-3$ $\begin{array}{llllll}3.55850-3 & 4.08750-3 & 5.89750-3 & 7.13870-3 & 7.42410-3\end{array}$ $\begin{array}{llllll}7.67700-3 & 7.95180-3 & 8.74210-3 & 1.11490-2 & 1.35030-2\end{array}$ $1.65080-2 \quad 2.25270-2 \quad 2.86610-2 \quad 3.43150-2$ 4.10860-2 $4.92110-2 \quad 5.56070-2 \quad 5.77300-2 \quad 5.80800-2 \quad 5.84980-2$ 6.59980-2 8.26560-2 $9.04890-2 \quad 9.54810-2 \quad 1.05360-1$ $1.17860-1$ 1.29670-1 $1.30100-1 \quad 1.28650-1 \quad 1.27220-1$

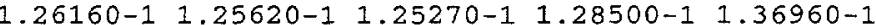
$1.45980-1$ 1.54120-1 $1.51160-1$ 1.47220-1 $1.47060-1$ $1.63200-12.00340-1$

$5.701+10$

$298.958 \quad 304.8 \quad 0.0 \quad 0.0$

$2.07002-7 \quad 7.69672-7 \quad 1.75386-6 \quad 3.71293-6$ 7.86024-6 $1.66401-5 \quad 3.52273-5 \quad 7.45761-5 \quad 1.34159-4$ 2.21191-4 3.64682-4 $6.01258-4 \quad 9.91309-4 \quad 1.63439-3$ $2.14167-3 \quad 2.43063-3 \quad 2.82399-3 \quad 3.37141-3 \quad 4.61914-3$ $\begin{array}{llllll}7.32483-3 & 1.20766-2 & 1.93065-2 & 2.41830-2 & 2.54229-2\end{array}$ 2.65293-2 2.77506-2 3.14039-2 $4.33909-2$ 5.45187-2 $7.15396-2 \quad 1.04645-1 \quad 1.36365-1 \quad 1.66556-1 \quad 2.03432-1$ $\begin{array}{lllll}2.48472-1 & 2.83877-1 & 2.95864-1 & 2.97851-1 & 3.00232-1\end{array}$ 3.44858-1 $4.42806-1 \quad 5.10634-1 \quad 5.65749-1 \quad 6.75418-1$ $8.24957-1 \quad 1.00760+0 \quad 1.23069+0 \quad 1.50317+01.83598+0$ $2.12513+0 \quad 2.28850+0 \quad 2.40583+0 \quad 2.73896+0 \quad 3.34537+0$ $4.08604+0 \quad 4.99070+0 \quad 6.09565+0 \quad 7.44525+0 \quad 9.09365+0$ $1.11070+1 \quad 1.35661+1$

$\begin{array}{lllll}3.70370-3 & 4.37060-3 & 4.52500-3 & 4.57410-3\end{array}$

$4.55830-3 \quad 4.48970-3 \quad 4.38170-3 \quad 4.24920-3 \quad 4.12890-3$ $\begin{array}{lllll}4.03110-3 & 3.93550-3 & 3.84530-3 & 3.76290-3 & 3.69080-3\end{array}$ $3.65280-3 \quad 3.63870-3 \quad 3.62290-3 \quad 3.60620-3 \quad 3.58250-3$ $\begin{array}{llllll}3.55850-3 & 4.08750-3 & 5.89750-3 & 7.13870-3 & 7.42410-3\end{array}$ $7.67700-3 \quad 7.95180-3 \quad 8.74210-3 \quad 1.11490-2 \quad 1.35030-2$ 1.65080-2 2.25270-2 2.86610-2 3.43150-2 $4.10860-2$ $4.92110-2 \quad 5.56070-2 \quad 5.77300-2 \quad 5.80800-2 \quad 5.84980-2$ $6.59980-2 \quad 8.26560-2 \quad 9.04890-2 \quad 9.54810-2 \quad 1.05360-1$ $1.17860-1 \quad 1.29670-1 \quad 1.30100-1 \quad 1.28650-1 \quad 1.27220-1$ $\begin{array}{llllll}1.26160-1 & 1.25620-1 & 1.25270-1 & 1.28500-1 & 1.36960-1\end{array}$ $1.45980-1 \quad 1.54120-1 \quad 1.51160-1 \quad 1.47220-1 \quad 1.47060-1$ $1.63200-12.00340-1$

$5.701+10$

$298.958 \quad 457.2 \quad 0.0 \quad 0.0$

$2.07002-7 \quad 7.69672-7 \quad 1.75386-6 \quad 3.71293-6$

$7.86024-6.1 .66401-5 \quad 3.52273-5 \quad 7.45761 \ldots 5.34159-4$ $2.21191-4 \quad 3.64682-4 \quad 6.01258-4 \quad 9.91309-4 \quad 1.63439-3$ 
2.14167-3 $2.43063-3 \quad 2.82399-3 \quad 3.37141-3 \quad 4.61914-3$ 7.32483-3 1.20766-2 $1.93065-2 \quad 2.41830-2 \quad 2.54229-2$ 2.65293-2 2.77506-2 3.14039-2 4.33909-2 5.45187-2 7.15396-2 $1.04645-1 \quad 1.36365-1 \quad 1.66556-1 \quad 2.03432-1$ $\begin{array}{llllll}2.48472-1 & 2.83877-1 & 2.95864-1 & 2.97851-1 & 3.00232-1\end{array}$ 3.44858-1 $4.42806-1 \quad 5.10634-1 \quad 5.65749-1 \quad 6.75418-1$ $8.24957-1 \quad 1.00760+0 \quad 1.23069+0 \quad 1.50317+0 \quad 1.83598+0$ $2.12513+0 \quad 2.28850+0 \quad 2.40583+0 \quad 2.73896+0 \quad 3.34537+0$ $4.08604+0 \quad 4.99070+0 \quad 6.09565+0 \quad 7.44525+0 \quad 9.09365+0$ $1.11070+1 \quad 1.35661+1$

d£325 $3.70370-3 \quad 4.37060-3 \quad 4.52500-3 \quad 4.57410-3$ $4.55830-3 \quad 4.48970-3 \quad 4.38170-3 \quad 4.24920-3 \quad 4.12890-3$ $4.03110-3 \quad 3.93550-3 \quad 3.84530-3 \quad 3.76290-3 \quad 3.69080-3$ $\begin{array}{lllll}3.65280-3 & 3.63870-3 & 3.62290-3 & 3.60620-3 & 3.58250-3\end{array}$ $\begin{array}{lllll}3.55850-3 & 4.08750-3 & 5.89750-3 & 7.13870-3 & 7.42410-3\end{array}$ $\begin{array}{lllll}7.67700-3 & 7.95180-3 & 8.74210-3 & 1.11490-2 & 1.35030-2\end{array}$ $\begin{array}{lllll}1.65080-2 & 2.25270-2 & 2.86610-2 & 3.43150-2 & 4.10860-2\end{array}$ 4.92110-2 5.56070-2 5.77300-2 5.80800-2 5.84980-2 $6.59980-2 \quad 8.26560-2 \quad 9.04890-2 \quad 9.54810-2 \quad 1.05360-1$

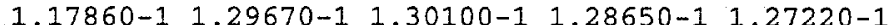
$1.26160-1 \quad 1.25620-1 \quad 1.25270-1 \quad 1.28500-1 \quad 1.36960-1$ 1.45980-1 $1.54120-1$ 1.51160-1 $1.47220-1$ 1.47060-1 $1.63200-1 \quad 2.00340-1$

$\operatorname{fm} 325$ f $335: n$ $5.701+10$

de 335

df 335

fm 335

298.958

$609.6 \quad 0.0 \quad 0.0$

$2.07002-7 \quad 7.69672-7 \quad 1.75386-6 \quad 3.71293-6$

7.86024-6 $1.66401-5 \quad 3.52273-5 \quad 7.45761-5 \quad 1.34159-4$

$\begin{array}{llllll}2.21191-4 & 3.64682-4 & 6.01258-4 & 9.91309-4 & 1.63439-3\end{array}$

2.14167-3 2.43063-3 $2.82399-3 \quad 3.37141-3 \quad 4.61914-3$

$7.32483-31.20766-2 \quad 1.93065-2 \quad 2.41830-2 \quad 2.54229-2$

2.65293-2 2.77506-2 $3.14039-2 \quad 4.33909-2 \quad 5.45187-2$

7.15396-2 $1.04645-1 \quad 1.36365-1 \quad 1.66556-1 \quad 2.03432-1$

2.48472-1 2.83877-1 $2.95864-1 \quad 2.97851-1 \quad 3.00232-1$

3.44858-1 $4.42806-1 \quad 5.10634-1 \quad 5.65749-1 \quad 6.75418-1$ $8.24957-1 \quad 1.00760+0 \quad 1.23069+0 \quad 1.50317+0 \quad 1.83598+0$ $2.12513+0 \quad 2.28850+0 \quad 2.40583+0 \quad 2.73896+0 \quad 3.34537+0$ $4.08604+0 \quad 4.99070+0 \quad 6.09565+0 \quad 7.44525+0 \quad 9.09365+0$ $1.11070+1 \quad 1.35661+1$ $3.70370-3 \quad 4.37060-3 \quad 4.52500-3 \quad 4.57410-3$

4.55830-3 $4.48970-3 \quad 4.38170-3 \quad 4.24920-3 \quad 4.12890-3$ $4.03110-3 \quad 3.93550-3 \quad 3.84530-3 \quad 3.76290-3 \quad 3.69080-3$ $\begin{array}{llllll}3.65280-3 & 3.63870-3 & 3.62290-3 & 3.60620-3 & 3.58250-3\end{array}$ $\begin{array}{llllll}3.55850-3 & 4.08750-3 & 5.89750-3 & 7.13870-3 & 7.42410-3\end{array}$ $7.67700-3 \quad 7.95180-3 \quad 8.74210-3 \quad 1.11490-2 \quad 1.35030-2$ $1.65080-2 \quad 2.25270-2 \quad 2.86610-2 \quad 3.43150-2 \quad 4.10860-2$ $4.92110-2 \quad 5.56070-2 \quad 5.77300-2 \quad 5.80800-2 \quad 5.84980-2$ $6.59980-2 \quad 8.26560-2 \quad 9.04890-2 \quad 9.54810-2 \quad 1.05360-1$ $\begin{array}{llllll}1.17860-1 & 1.29670-1 & 1.30100-1 & 1.28650-1 & 1.27220-1\end{array}$ $1.26160-1 \quad 1.25620-1 \quad 1.25270-1 \quad 1.28500-1 \quad 1.36960-1$ 1.45980-1 $1.54120-1 \quad 1.51160-1 \quad 1.47220-1 \quad 1.47060-1$ $1.63200-12.00340-1$

f $345: \mathrm{n}$ de345

$5.701+10$

$298.958 \quad 762.0 \quad 0.0 \quad 0.0$

$2.07002-7 \quad 7.69672-7 \quad 1.75386-6 \quad 3.71293-6$ $7.86024-6 \quad 1.66401-5 \quad 3.52273-5 \quad 7.45761-5 \quad 1.34159-4$ $\begin{array}{llllll}2.21191-4 & 3.64682-4 & 6.01258-4 & 9.91309-4 & 1.63439-3\end{array}$ 2.14167-3 2.43063-3 $2.82399-3 \quad 3.37141-3 \quad 4.61914-3$ 7.32483-3 $1.20766-2 \quad 1.93065-2 \quad 2.41830-2 \quad 2.54229-2$ 2.65293-2 $2.77506-2 \quad 3.14039-2 \quad 4.33909-2 \quad 5.45187-2$ 7.15396-2 1.04645-1 $1.36365-1 \quad 1.66556-1 \quad 2.03432-1$ $\begin{array}{lllll}2.48472-1 & 2.83877-1 & 2.95864-1 & 2.97851-1 & 3.00232-1\end{array}$ 3.44858-1 4.42806-1 5.10634-1 5.65749-1.6.75418-1 8.24957-1 $1.00760+0 \quad 1.23069+0 \quad 1.50317+0 \quad 1.83598+0$

$2.12513+0 \quad 2.28850+0 \quad 2.40583+0 \quad 2.73896+0 \quad 3.34537+0$ $4.08604+0 \quad 4.99070+0 \quad 6.09565+0 \quad 7.44525+0 \quad 9.09365+0$ $1.11070+1 \quad 1.35661+1$

$3.70370-3 \quad 4.37060-3 \quad 4.52500-3 \quad 4.57410-3$ $4.55830-3 \quad 4.48970-3 \quad 4.38170-3 \quad 4.24920-3 \quad 4.12890-3$ $4.03110-3 \quad 3.93550-3 \quad 3.84530-3 \quad 3.76290-3 \quad 3.69080-3$ $\begin{array}{llllll}3.65280-3 & 3.63870-3 & 3.62290-3 & 3.60620-3 & 3.58250-3\end{array}$ $3.55850-3 \quad 4.08750-3 \quad 5.89750-3 \quad 7.13870-3 \quad 7.42410-3$

$\begin{array}{lllll}7.67700-3 & 7.95180-3 & 8.74210-3 & 1.11490-2 & 1.35030-2\end{array}$ 


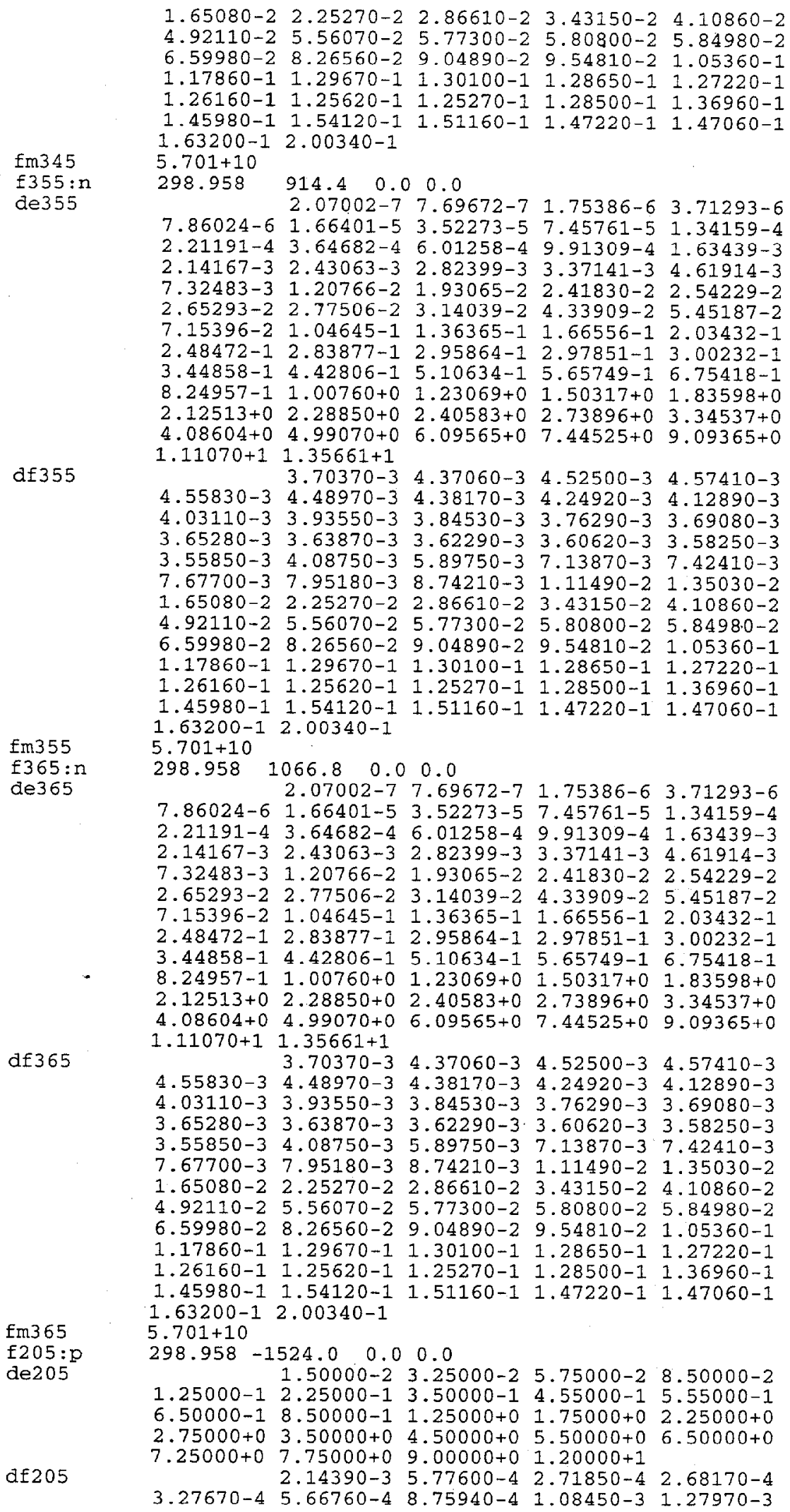




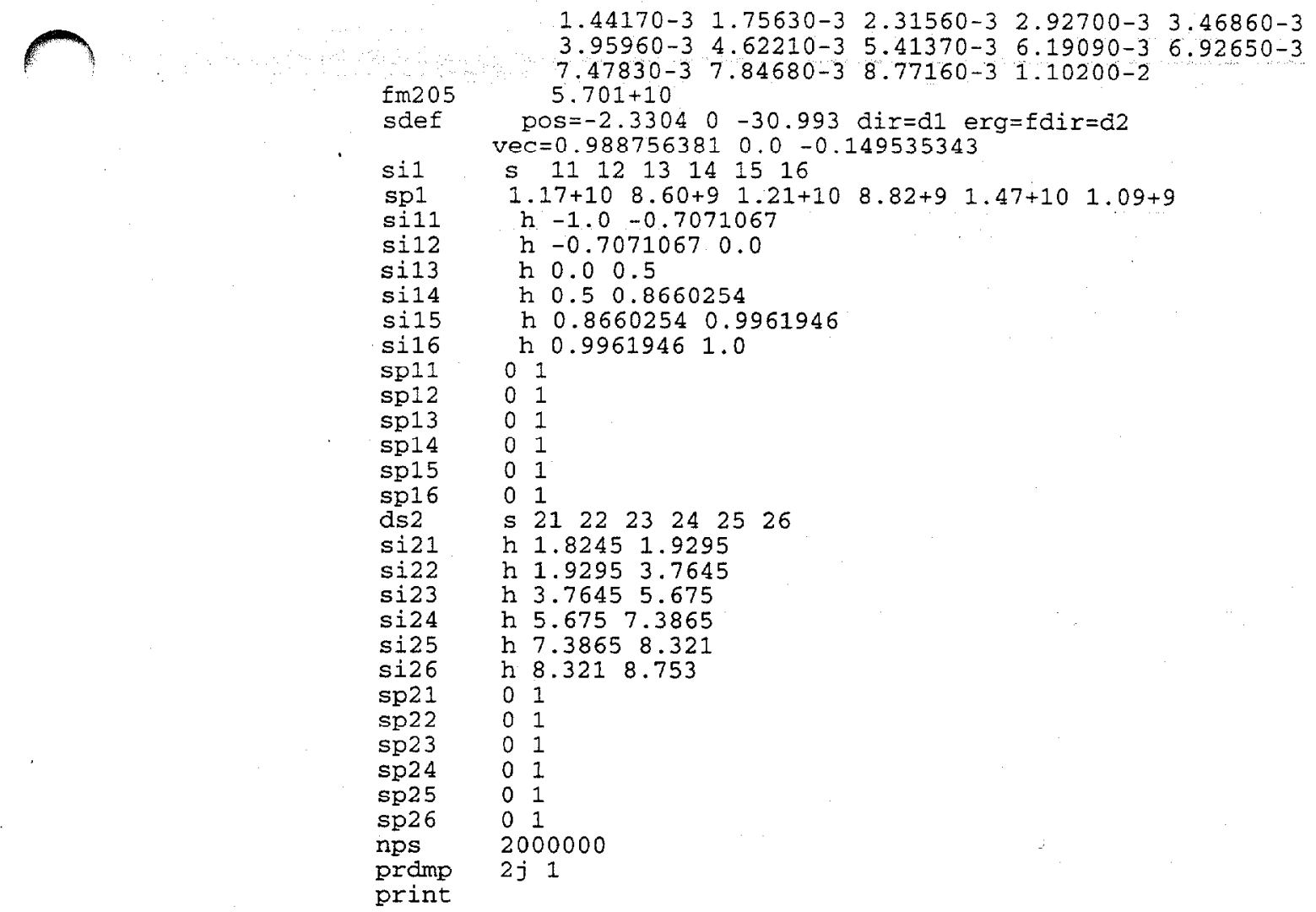


File for MCNP-4B calculation of photon dose rates along the truck lane of the PFNA facility for the source in the maximum down position

message: outp=pfna21.o mctal=pfna21.m

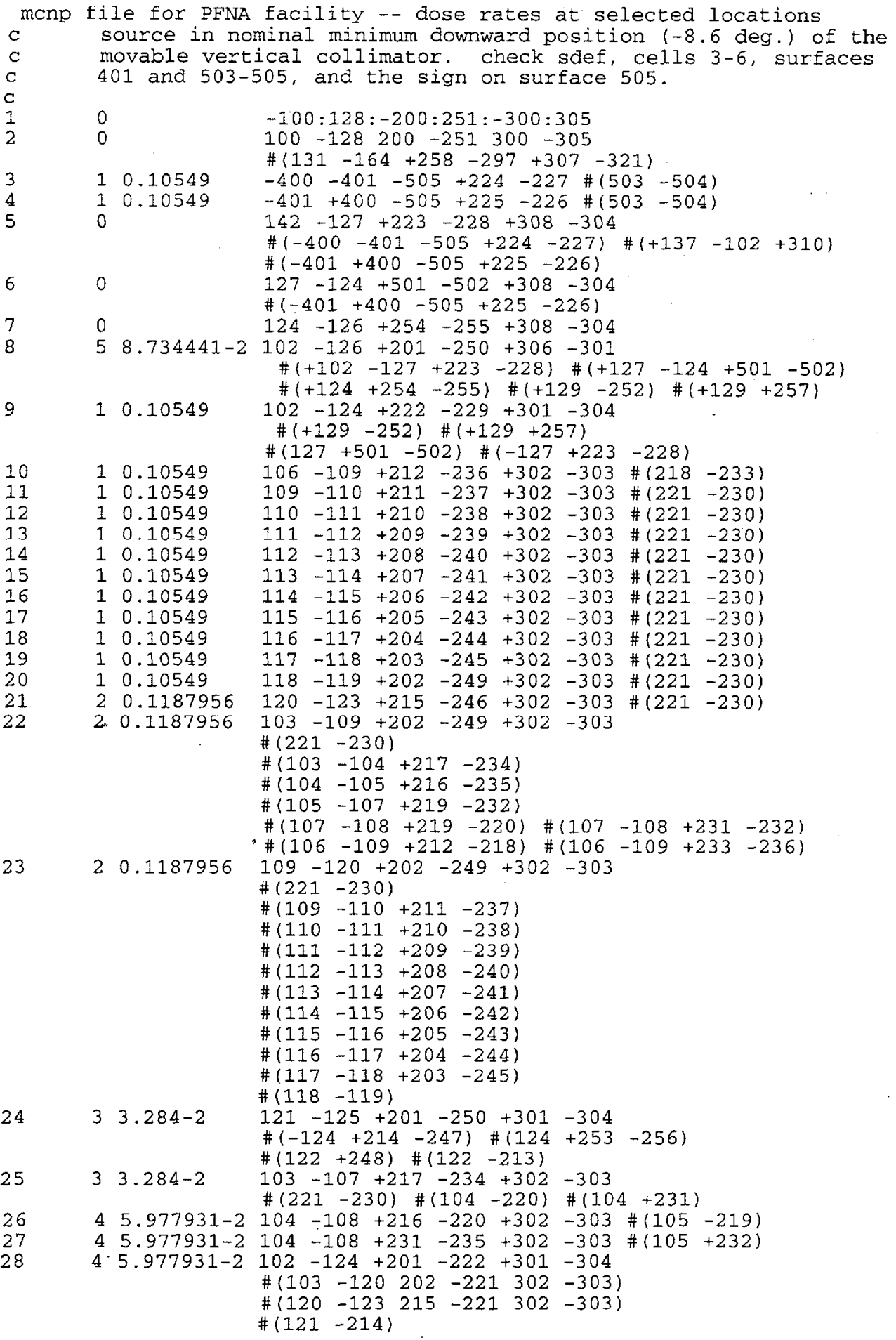



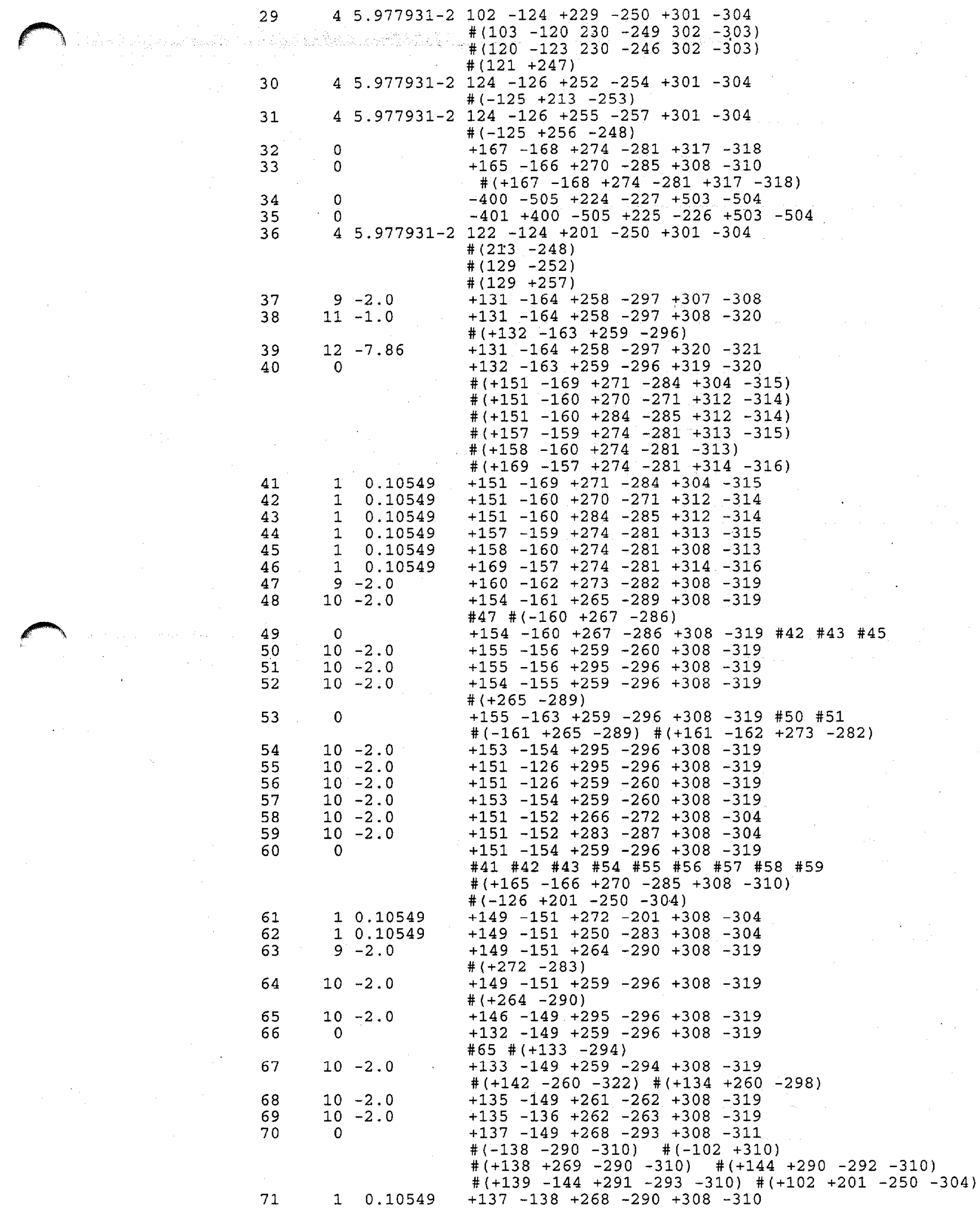


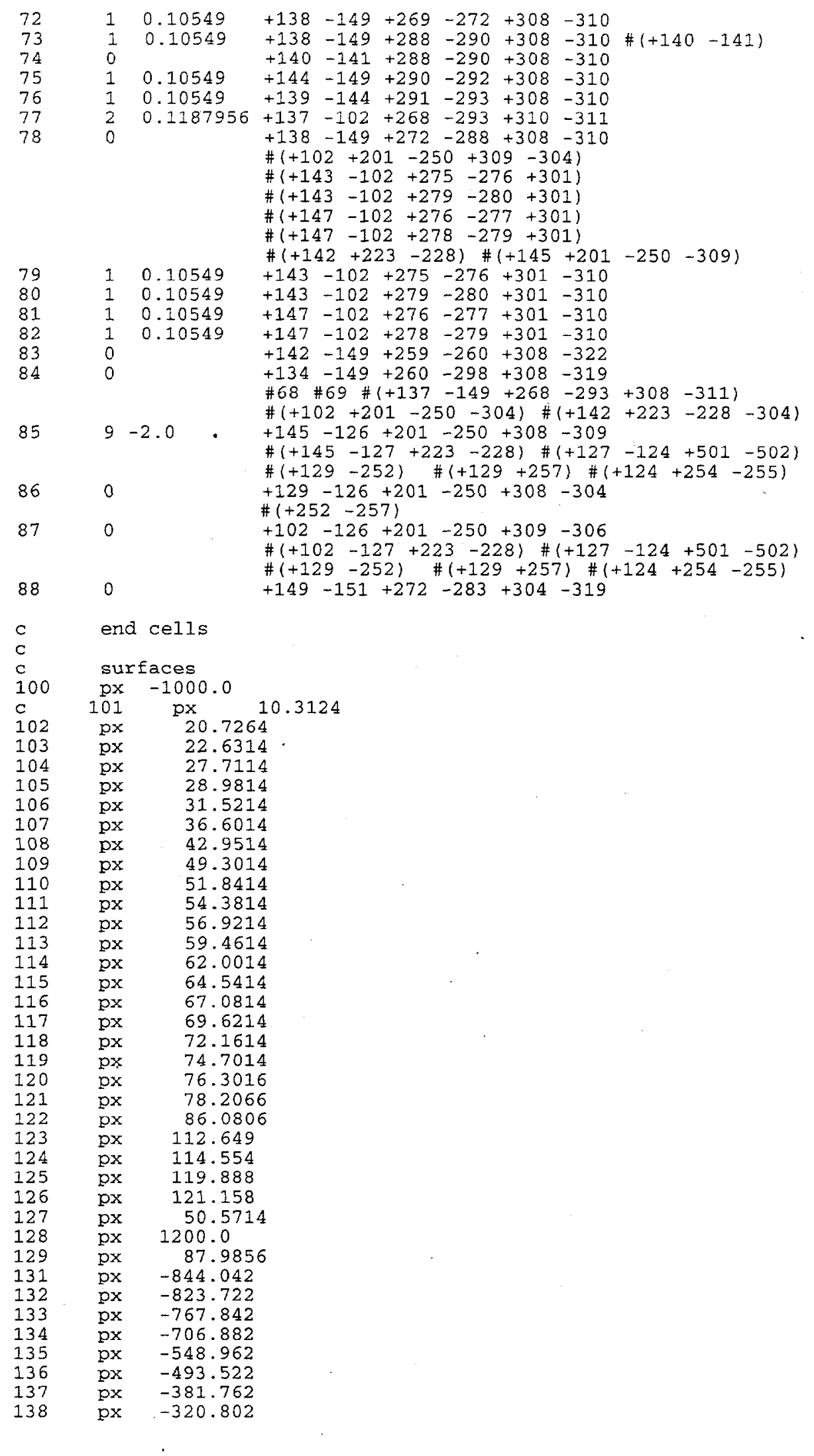




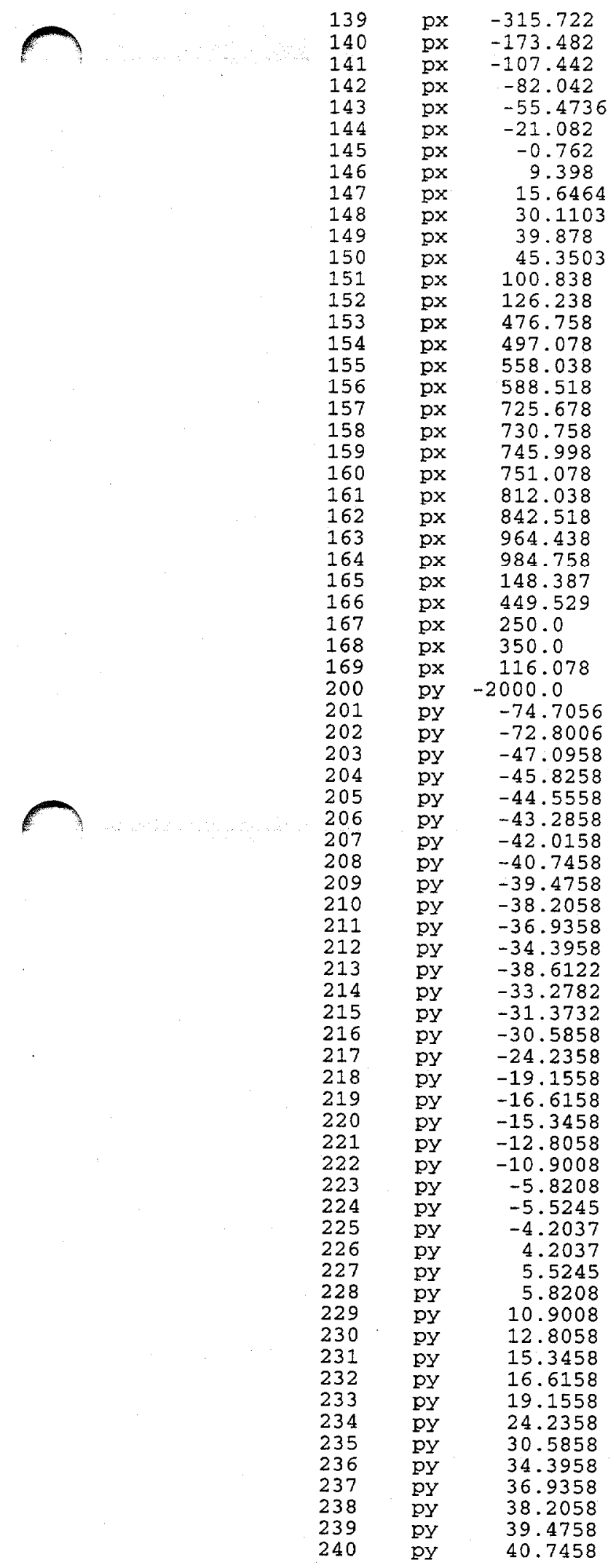




\begin{tabular}{|c|c|c|}
\hline 241 & py & 42.0158 \\
\hline 242 & py & 43.2858 \\
\hline 243 & py & 44.5558 \\
\hline 244 & py & 45.8258 \\
\hline 245 & py & 47.0958 \\
\hline 246 & py & 31.3732 \\
\hline 247 & py & 33.2782 \\
\hline 248 & py & 38.6122 \\
\hline 249 & py & 72.8006 \\
\hline 250 & py & 74.7056 \\
\hline 251 & py & 1500.0 \\
\hline 252 & py & -39.8822 \\
\hline 253 & py & -9.8848 \\
\hline 254 & py & -8.6148 \\
\hline 255 & py & 8.6148 \\
\hline 256 & py & 9.8848 \\
\hline 257 & py & 39.8822 \\
\hline 258 & py & -1731.28 \\
\hline 259 & py & -1710.96 \\
\hline 260 & py & -1650.0 \\
\hline 261 & py & -1528.08 \\
\hline 262 & py & -1467.12 \\
\hline 263 & py & -1436.64 \\
\hline 264 & py & -589.28 \\
\hline 265 & py & -241.3 \\
\hline 266 & py & -193.04 \\
\hline 267 & py & -180.34 \\
\hline 268 & py & -167.64 \\
\hline 269 & py & -162.56 \\
\hline 270 & py & -152.4 \\
\hline 271 & DY & -137.16 \\
\hline 272 & py & -101.6 \\
\hline 273 & py & -91.44 \\
\hline 274 & py & -60.96 \\
\hline 275 & py & -45.72 \\
\hline 276 & py & -30.48 \\
\hline 277 & PY & -10.16 \\
\hline 278 & py & 10.16 \\
\hline 279 & py & 30.48 \\
\hline 280 & py & 45.72 \\
\hline 281 & py & 60.96 \\
\hline 282 & py & 91.44 \\
\hline 283 & py & 101.6 \\
\hline 284 & py & 137.16 \\
\hline 285 & py & 152.4 \\
\hline 286 & py & 180.34 \\
\hline 287 & py & 193.04 \\
\hline 288 & py & 213.36 \\
\hline 289 & py & 241.3 \\
\hline 290 & py & 274.32 \\
\hline 291 & py & 335.28 \\
\hline 292 & py & 345.44 \\
\hline 293 & py & 396.24 \\
\hline 294 & py & 533.4 \\
\hline 295 & py & 1235.44 \\
\hline 296 & py & 1296.4 \\
\hline 297 & py & 1316.72 \\
\hline 298 & py & 472.44 \\
\hline 300 & $\mathrm{pz}$ & -500.0 \\
\hline 301 & $\mathrm{pz}$ & -59.478 \\
\hline 302 & $p z$ & -57.573 \\
\hline 303 & $\mathrm{pz}$ & 245.957 \\
\hline 304 & $\mathrm{pz}$ & 247.862 \\
\hline 305 & $\mathrm{pz}$ & 500.0 \\
\hline 306 & $\mathrm{pz}$ & -60.748 \\
\hline 307 & $\mathrm{pz}$ & -156.0 \\
\hline 308 & $\mathrm{pz}$ & -125.095 \\
\hline 309 & $\mathrm{pz}$ & -67.451 \\
\hline 310 & $p z$ & 235.585 \\
\hline 311 & $\mathrm{pz}$ & 276.225 \\
\hline 312 & $p z$ & 283.845 \\
\hline 313 & $p z$ & 342.265 \\
\hline
\end{tabular}




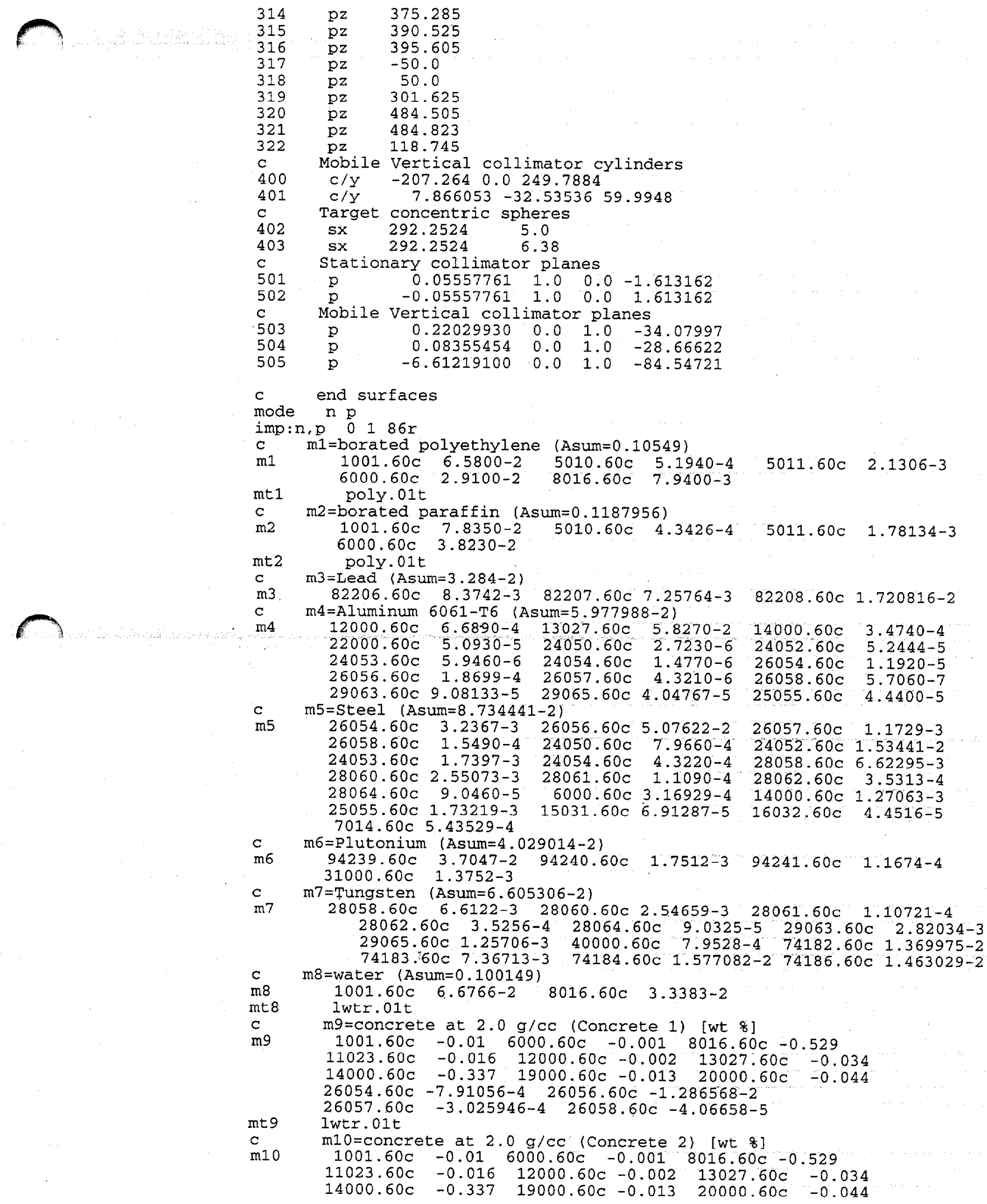




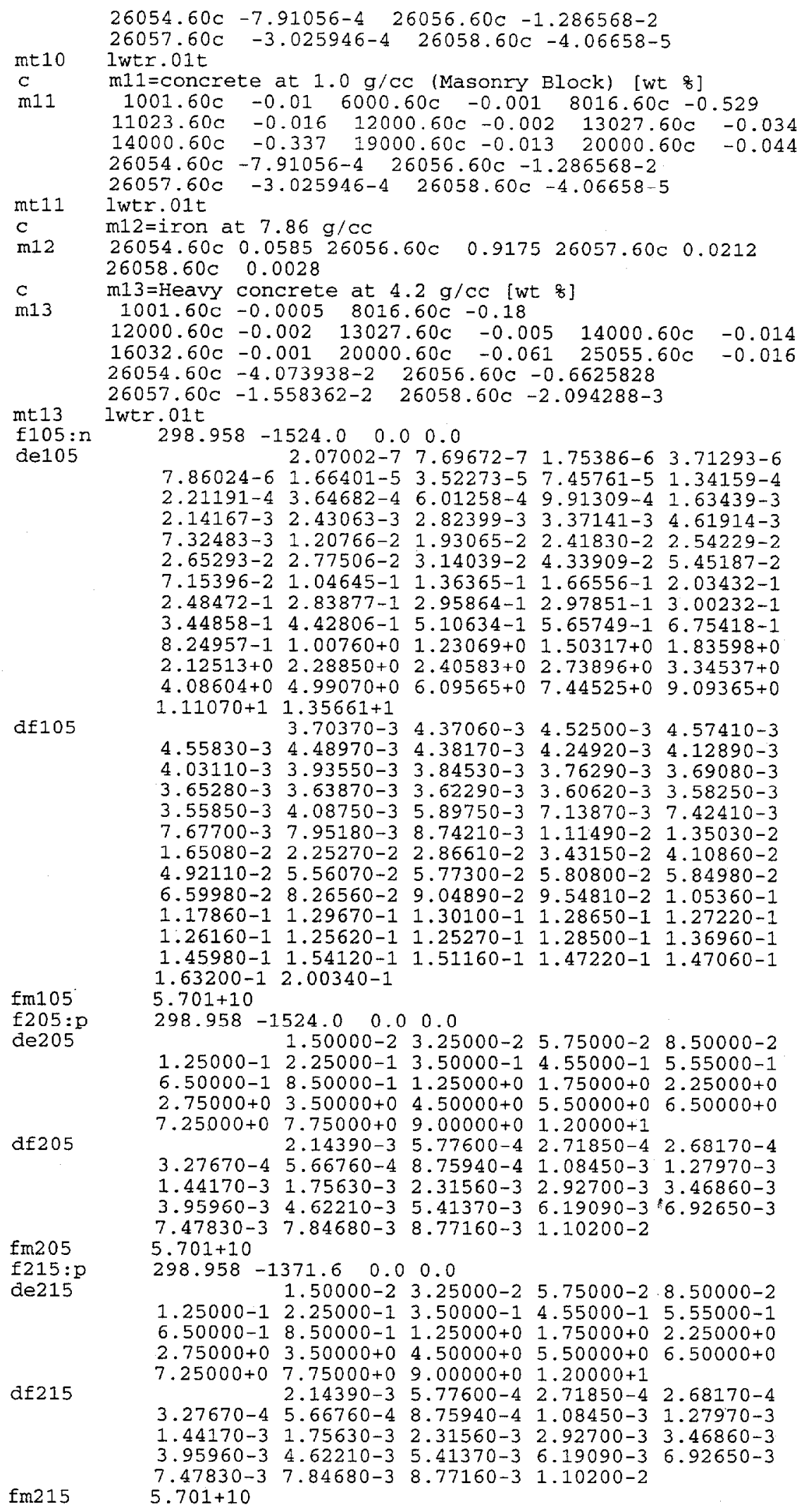


f22 2 :p de 225

df225

fm225

f235:p

de 235

df235

fm235

f245:p

de 245

$\operatorname{df} 245$

fm2 45

f2 55:p

de255

df 255

fm255

f265:p

de 265

df265

$\operatorname{fim} 265$

f275:p

de 275

df 275

fm275
$298.958-1219.2 \quad 0.0 \quad 0.0$

$1.50000-2 \quad 3.25000-2 \quad 5.75000-2 \quad 8.50000-2$ $1.25000-1 \quad 2.25000-1 \quad 3.50000-1 \quad 4.55000-1 \quad 5.55000-1$ $6.50000-18.50000-11.25000+0 \quad 1.75000+02.25000+0$ $2.75000+0 \quad 3.50000+0 \quad 4.50000+0 \quad 5.50000+0 \quad 6.50000+0$ $7.25000+0 \quad 7.75000+0 \quad 9.00000+01.20000+1$

$2.14390-3 \quad 5.77600-4 \quad 2.71850-4 \quad 2.68170-4$ $3.27670-4 \quad 5.66760-4 \quad 8.75940-4 \quad 1.08450-3 \quad 1.27970-3$ $\begin{array}{lllll}1.41170-3 & 1.75630-3 & 2.31560-3 & 2.92700-3 & 3.46860-3\end{array}$ $\begin{array}{llllll}3.95960-3 & 4.62210-3 & 5.41370-3 & 6.19090-3 & 6.92650-3\end{array}$

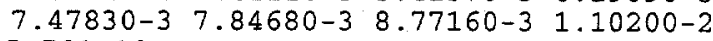
$5.701+10$

$298.958-1066.8 \quad 0.0 \quad 0.0$ $1.50000-2 \quad 3.25000-2 \quad 5.75000-2 \quad 8.50000-2$ $1.25000-1 \quad 2.25000-1 \quad 3.50000-1 \quad 4.55000-1 \quad 5.55000-1$ $6.50000-18.50000-1 \quad 1.25000+01.75000+0.2 .25000+0$ $2.75000+0 \quad 3.50000+0 \quad 4.50000+0 \quad 5.50000+0 \quad 6.50000+0$ $7.25000+0 \quad 7.75000+0 \quad 9.00000+0 \quad 1.20000+1$ $\begin{array}{lllll}2.14390-3 & 5.77600-4 & 2.71850-4 & 2.68170-4\end{array}$ $3.27670-4 \quad 5.667 .60-4 \quad 8.75940-4 \quad 1.08450-3 \quad 1.27970-3$ $1.44170-3 \quad 1.75630-32.31560-32.92700-3 \quad 3.46860-3$ $3.95960-3 \quad 4.62210-3 \quad 5.41370-3 \quad 6.19090-3 \quad 6.92650-3$ $7.47830-3 \quad 7.84680-3 \quad 8.77160-3 \quad 1.10200-2$

$5.701+10$

$298.958 \quad-914.4 \quad 0.0 \quad 0.0$

$1.50000-2 \quad 3.25000-2 \quad 5.75000-2 \quad 8.50000-2$

$1.25000-1 \quad 2.25000-1 \quad 3.50000-1 \quad 4.55000-1 \quad 5.55000-1$ $6.50000-1 \quad 8.50000-1 \quad 1.25000+0 \quad 1.75000+0 \quad 2.25000+0$ $2.75000+0 \quad 3.50000+0 \quad 4.50000+0 \quad 5.50000+0 \quad 6.50000+0$ $7.25000+07.75000+0 \quad 9.00000+01.20000+1$

$2.14390-3 \quad 5.77600-4 \quad 2.71850-4 \quad 2.68170-4$ $3.27670-4 \quad 5.66760-4 \quad 8.75940-4$ 1.08450-3 $1.27970-3$ $1.44170-3.1 .75630-3 \quad 2.31560-3 \quad 2.92700-3 \quad 3.46860-3$ $\begin{array}{llllll}3.95960-3 & 4.62210-3 & 5.41370-3 & 6.19090-3 & 6.92650-3\end{array}$ $\begin{array}{llll}7.47830-3 & 7.84680-3 & 8.77160-3 & 1.10200-2\end{array}$ $5.701+10$

$298.958 \quad-762.0 \quad 0.0 \quad 0.0$

$1.50000-2 \quad 3.25000-2 \quad 5.75000-2 \quad 8.50000-2$ $1.25000-12.25000-1 \quad 3.50000-1 \quad 4.55000-1 \quad 5.55000-1$ $\begin{array}{llllll}6.50000-1 & 8.50000-1 & 1.25000+0 & 1.75000+0 & 2.25000+0\end{array}$ $2.75000+0 \quad 3.50000+0 \quad 4.50000+0 \quad 5.50000+0 \quad 6.50000+0$ $7.25000+0 \quad 7.75000+0 \quad 9.00000+0 \quad 1.20000+1$ $\begin{array}{llll}2.14390-3 & 5.77600-4 & 2.71850-4 & 2.68170-4\end{array}$ $3.27670-4 \quad 5.66760-4 \quad 8.75940-4 \quad 1.08450-3 \quad 1.27970-3$ $1.44170-3 \quad 1.75630-3 \quad 2.31560-3 \quad 2.92700-3 \quad 3.46860-3$ $3.95960-3 \quad 4.62210-3 \quad 5.41370-3 \quad 6.19090-3 \quad 6.92650-3$ $7.47830-3 \quad 7.84680-3 \quad 8.77160-3 \quad 1.10200-2$

$5.701+10$

$298.958-609.6 \quad 0.0 \quad 0.0$

$1.50000-2 \quad 3.25000-2 \quad 5.75000-2 \quad 8.50000-2$

$\begin{array}{llllll}1.25000-1 & 2.25000-1 & 3.50000-1 & 4.55000-1 & 5.55000-1\end{array}$

$6.50000-18.50000-1 \quad 1.25000+0 \quad 1.75000+02.25000+0$

$2.75000+0 \quad 3.50000+0 \quad 4.50000+0 \quad 5.50000+0 \quad 6.50000+0$

$7.25000+07.75000+09.00000+01.20000+1$ $\begin{array}{llll}2.14390-3 & 5.77600-4 & 2.71850-4 & 2.68170-4\end{array}$

$3.27670-4 \quad 5.66760-4 \quad 8.75940-4 \quad 1.08450-3 \quad 1.27970-3$

$\begin{array}{lllll}1.44170-3 & 1.75630-3 & 2.31560-3 & 2.92700-3 & 3.46860-3\end{array}$ $\begin{array}{lllll}3.95960-3 & 4.62210-3 & 5.41370-3 & 6.19090-3 & 6.92650-3\end{array}$ $\begin{array}{lllll}7.47830-3 & 7.84680-3 & 8.77160-3 & 1.10200-2\end{array}$

$5.701+10$ $\begin{array}{lllll}298.958 & -457.2 & 0.0 & 0.0\end{array}$

$1.50000-2 \quad 3.25000-2 \quad 5.75000-2 \quad 8.50000-2$ $1.25000-1 \quad 2.25000-1 \quad 3.50000-1 \quad 4.55000-1 \quad 5.55000-1$ $6.50000-1 \quad 8.50000-1 \quad 1.25000+0 \quad 1.75000+0 \quad 2.25000+0$ $2.75000+0 \quad 3.50000+0 \quad 4.50000+0 \quad 5.50000+0 \quad 6.50000+0$ $7.25000+0 \quad 7.75000+09.00000+01.20000+1$ $\begin{array}{llll}2.14390-3 & 5.77600-4 & 2.71850-4 & 2.68170-4\end{array}$ $3.27670-4 \quad 5.66760-4 \quad 8.75940-4 \quad 1.08450-3 \quad 1.2797 .0-3$ $\begin{array}{lllll}1.44170-3 & 1.75630-3 & 2.31560-3 & 2.92700-3 & 3.46860-3\end{array}$ $3.95960-3 \quad 4.62210-3 \quad 5.41370-3 \quad 6.19090-3 \quad 6.92650-3$ $7.47830-3 \cdot 7.84680-3 \quad 8.77160-3 \quad 1.10200-2$ $5.701+10$ 


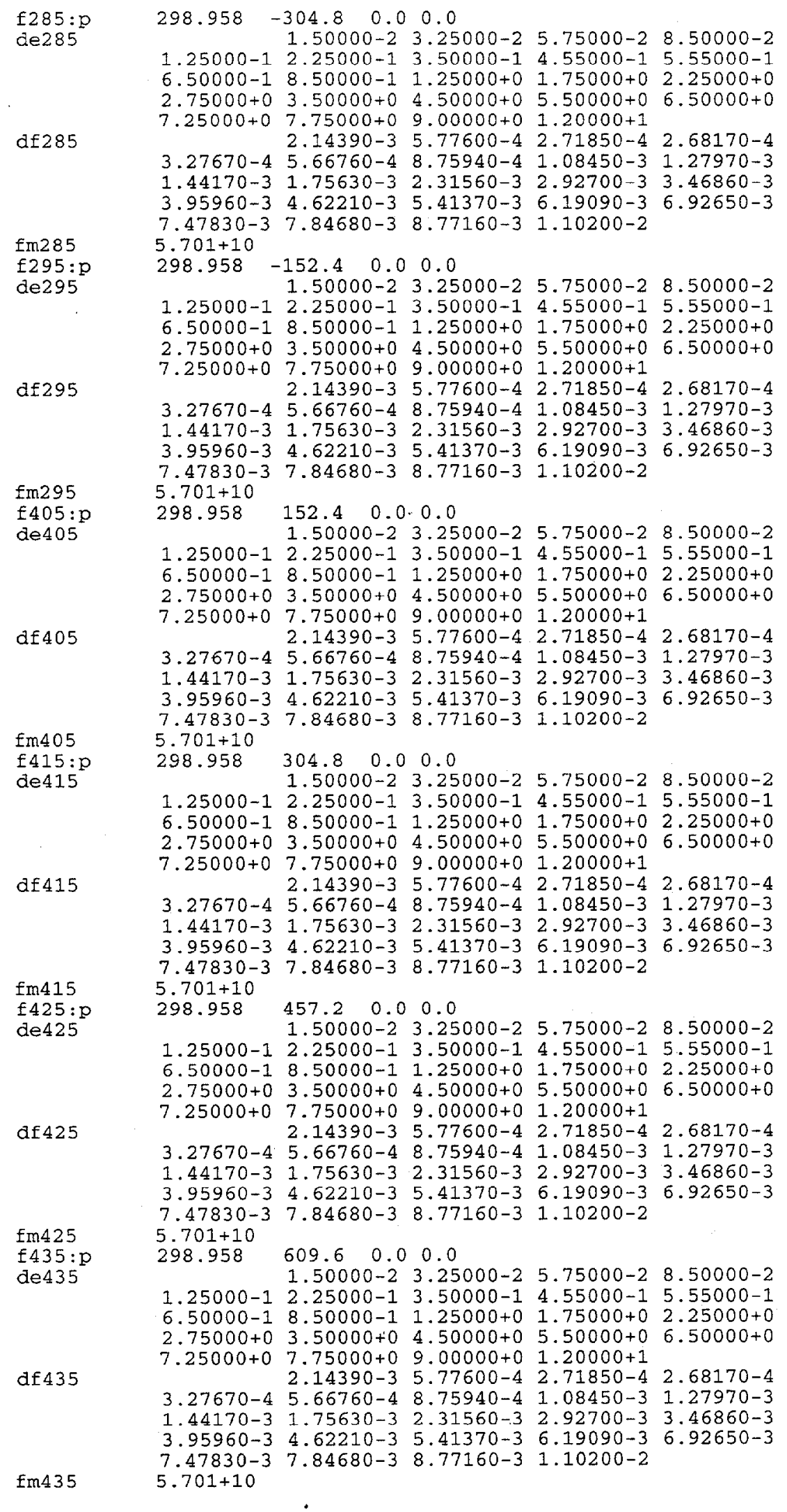




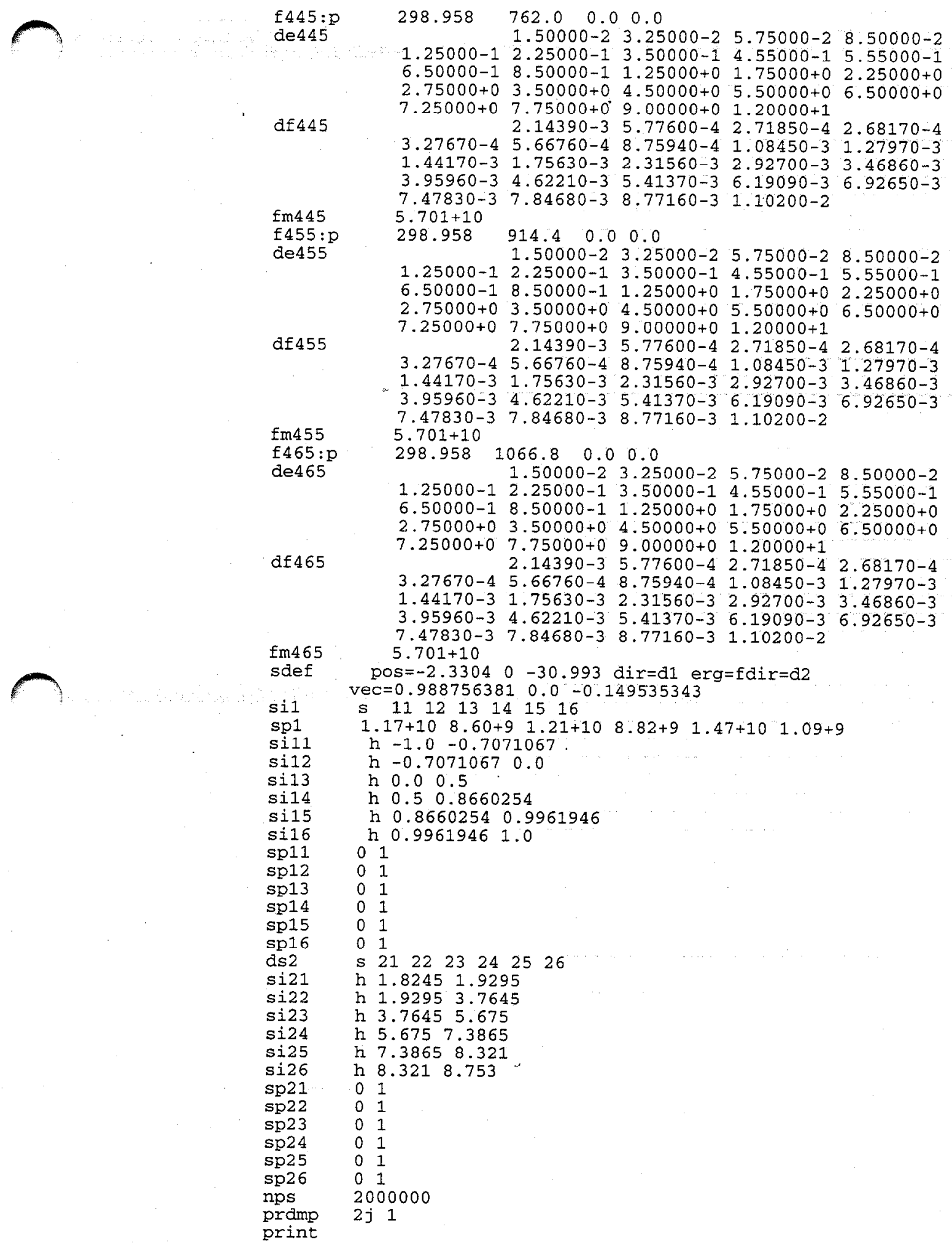


File for MCNP-4B calculation of dose rates at additional points along the truck lane of the PFNA facility for the source in the horizontal. position

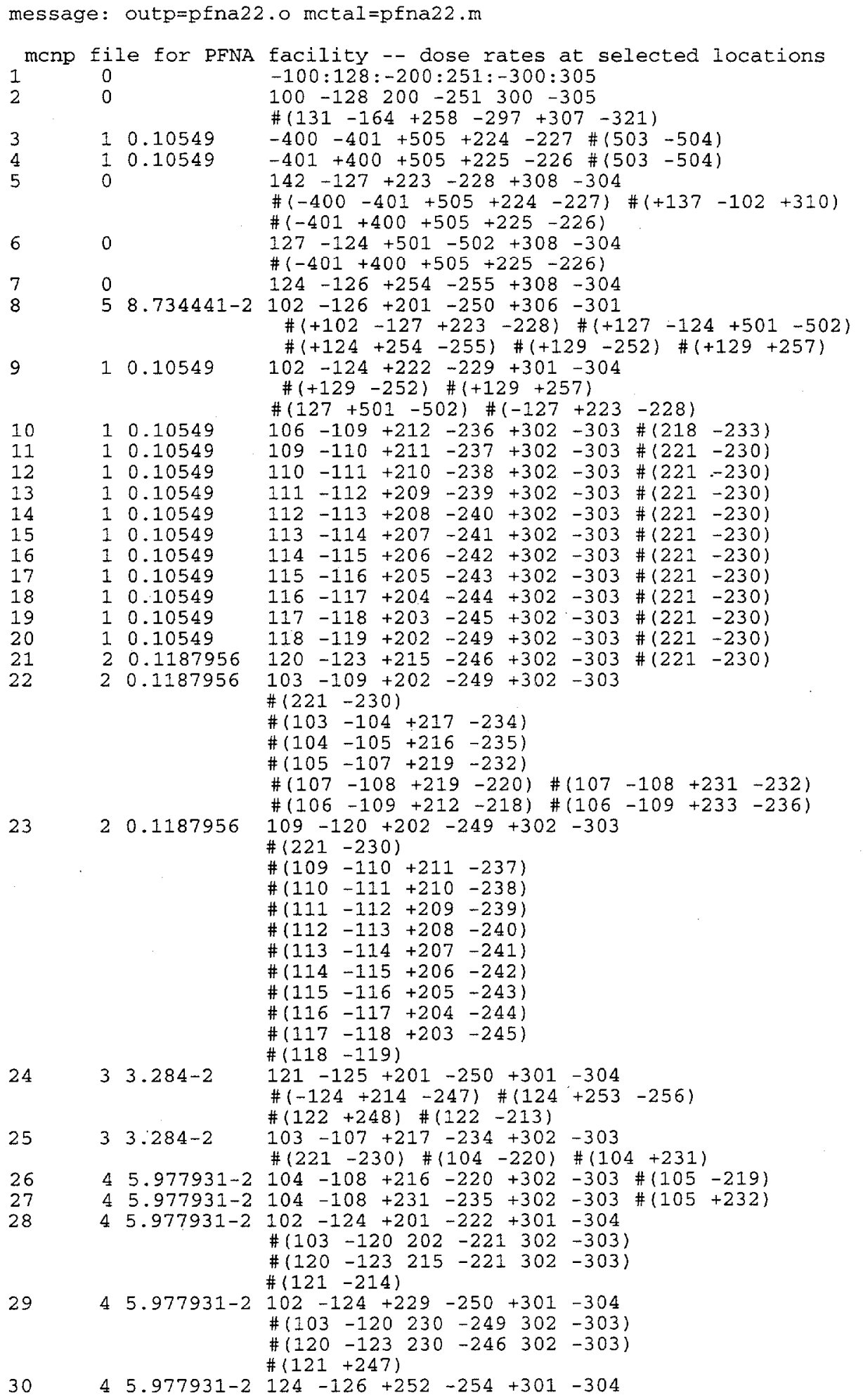




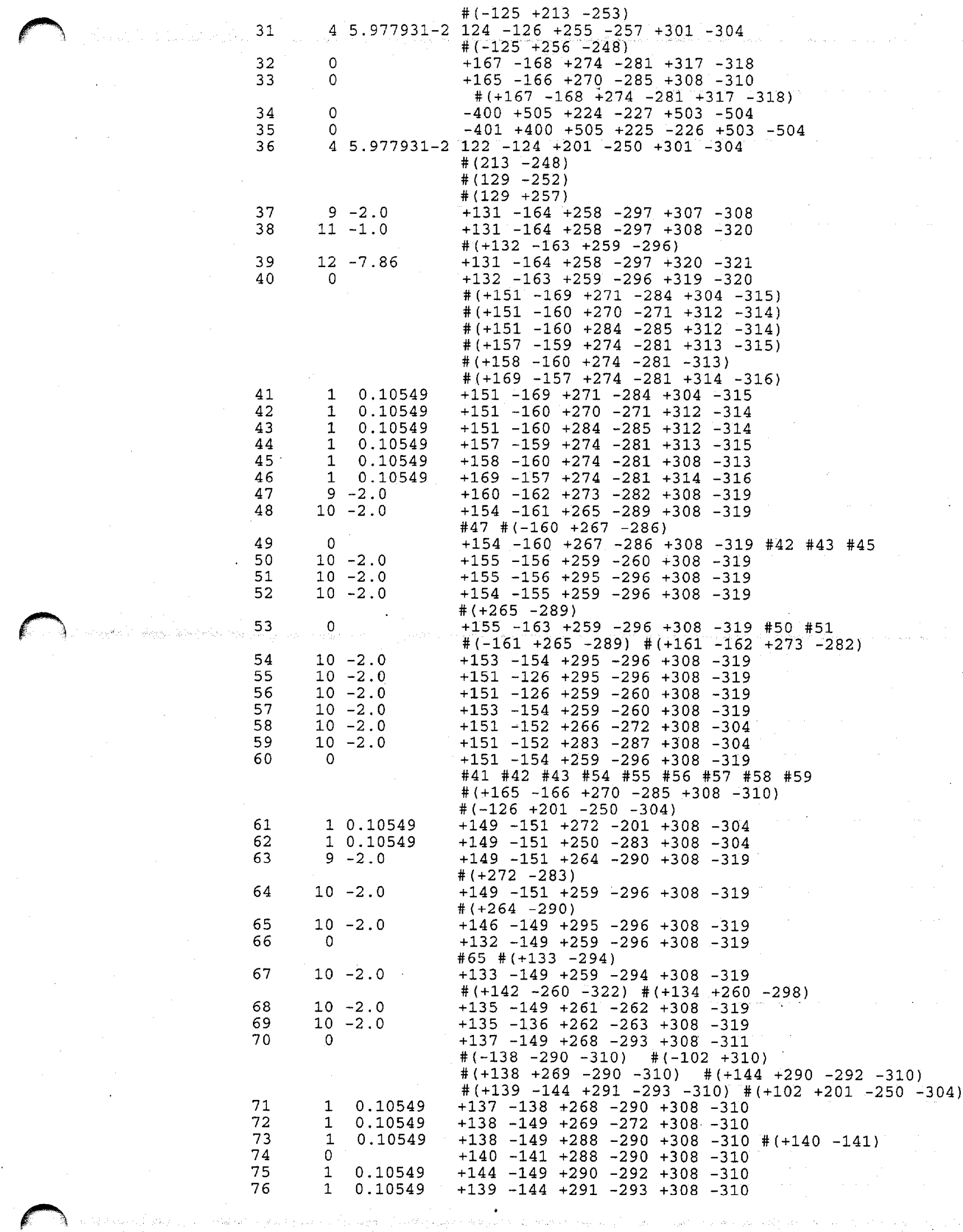




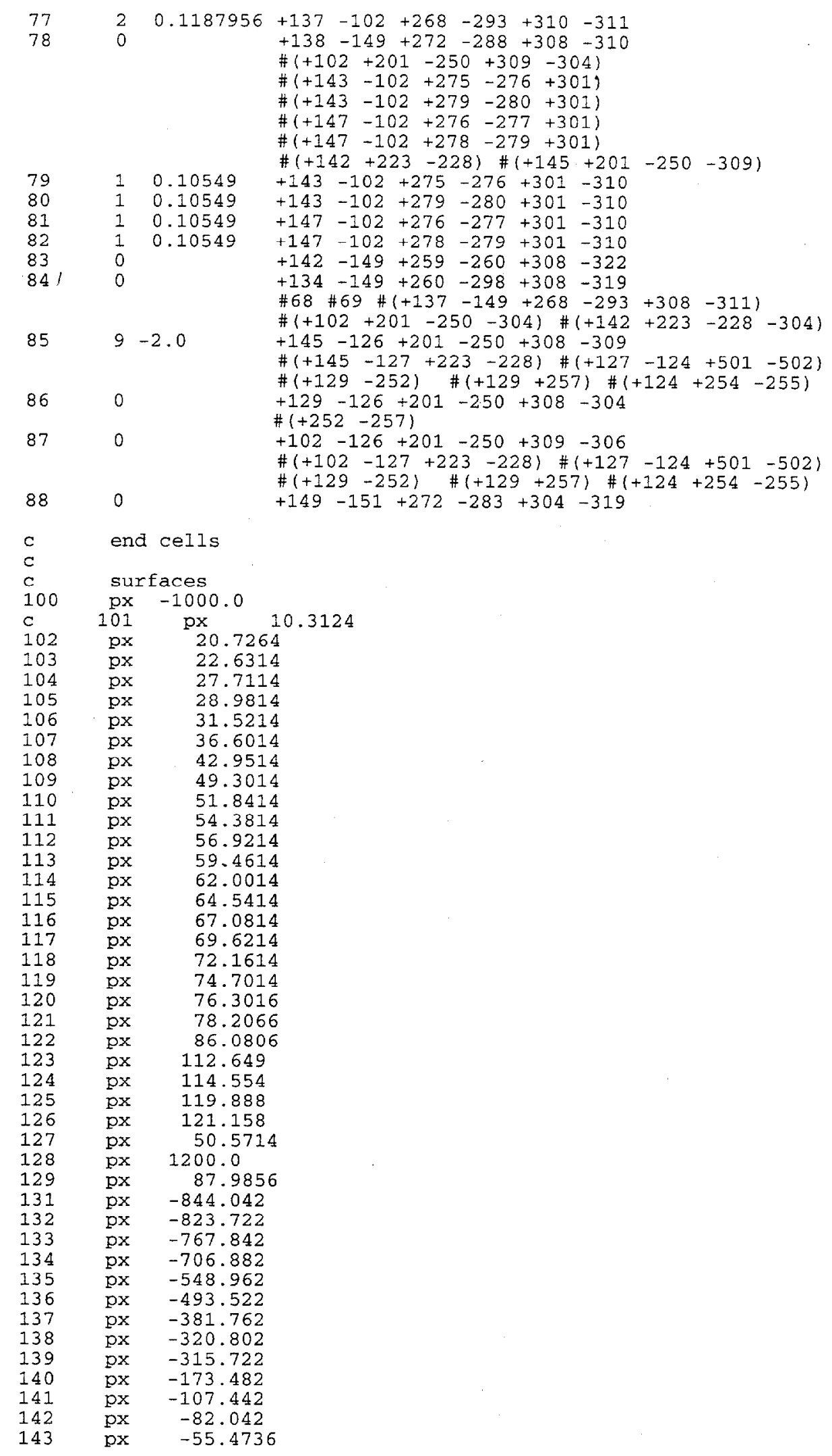




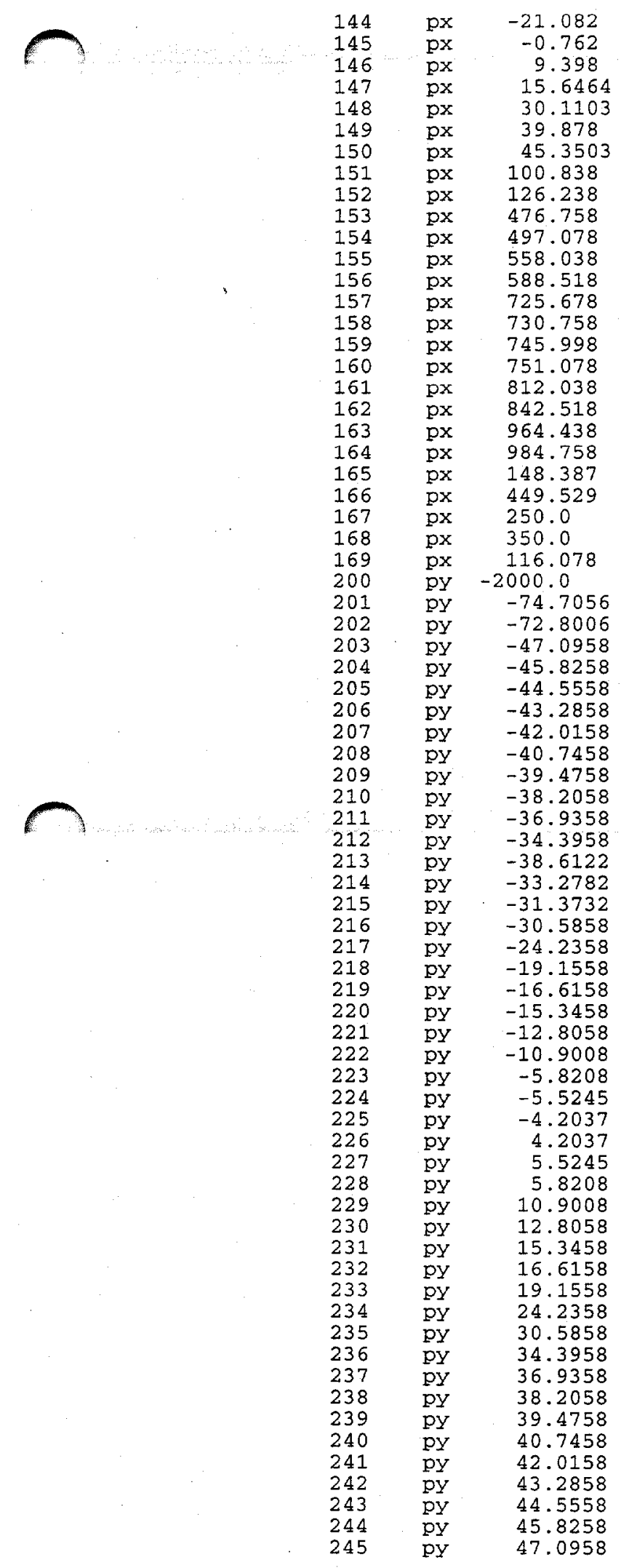




\begin{tabular}{|c|c|c|}
\hline 246 & py & 31.3732 \\
\hline 247 & py & 33.2782 \\
\hline 248 & py & 38.6122 \\
\hline 249 & py & 72.8006 \\
\hline 250 & py & 74.7056 \\
\hline 251 & py & 1500.0 \\
\hline 252 & py & -39.8822 \\
\hline 253 & py & -9.8848 \\
\hline 254 & PY & -8.6148 \\
\hline 255 & py & 8.6148 \\
\hline 256 & py & 9.8848 \\
\hline 257 & py & 39.8822 \\
\hline 258 & py & -1731.28 \\
\hline 259 & py & -1710.96 \\
\hline 260 & py & -1650.0 \\
\hline 261 & py & -1528.08 \\
\hline 262 & py & -1467.12 \\
\hline 263 & py & -1436.64 \\
\hline 264 & py & -589.28 \\
\hline 265 & py & -241.3 \\
\hline 266 & py & -193.04 \\
\hline 267 & py & -180.34 \\
\hline 268 & py & -167.64 \\
\hline 269 & py & -162.56 \\
\hline 270 & py & -152.4 \\
\hline 271 & py & -137.16 \\
\hline 272 & py & -101.6 \\
\hline 273 & py & -91.44 \\
\hline 274 & py & -60.96 \\
\hline 275 & py & -45.72 \\
\hline 276 & py & -30.48 \\
\hline 277 & py & -10.16 \\
\hline 278 & py & 10.16 \\
\hline 279 & py & 30.48 \\
\hline 280 & py & 45.72 \\
\hline 281 & py & 60.96 \\
\hline 282 & py & 91.44 \\
\hline 283 & py & 101.6 \\
\hline 284 & py & 137.16 \\
\hline 285 & py & 152.4 \\
\hline 286 & py & 180.34 \\
\hline 287 & py & 193.04 \\
\hline 288 & py & 213.36 \\
\hline 289 & py & 241.3 \\
\hline 290 & py & 274.32 \\
\hline 291 & py & 335.28 \\
\hline 292 & py & 345.44 \\
\hline 293 & py & 396.24 \\
\hline 294 & py & 533.4 \\
\hline 295 & py & 1235.44 \\
\hline 296 & py & 1296.4 \\
\hline 297 & py & 1316.72 \\
\hline 298 & py & 472.44 \\
\hline 300 & $\mathrm{pz}$ & -500.0 \\
\hline 301 & $\mathrm{pz}$ & -59.478 \\
\hline 302 & $\mathrm{pz}$ & -57.573 \\
\hline 303 & $\mathrm{pz}$ & 245.957 \\
\hline 304 & $\mathrm{pz}$ & 247.862 \\
\hline 305 & $\mathrm{pz}$ & 500.0 \\
\hline 306 & $\mathrm{pz}$ & -60.748 \\
\hline 307 & pz & -156.0 \\
\hline 308 & $\mathrm{pz}$ & -125.095 \\
\hline 309 & $\mathrm{pz}$ & -67.451 \\
\hline 310 & $\mathrm{pz}$ & 235.585 \\
\hline 311 & $\mathrm{pz}$ & 276.225 \\
\hline 312 & $\mathrm{pz}$ & 283.845 \\
\hline 313 & $\mathrm{pz}$ & 342.265 \\
\hline 314 & $\mathrm{pz}$ & 375.285 \\
\hline 315 & $p z$ & 390.525 \\
\hline 316 & $p z$ & 395.605 \\
\hline 317 & $\mathrm{pz}$ & -50.0 \\
\hline 318 & $\mathrm{pz}$ & 50.0 \\
\hline
\end{tabular}




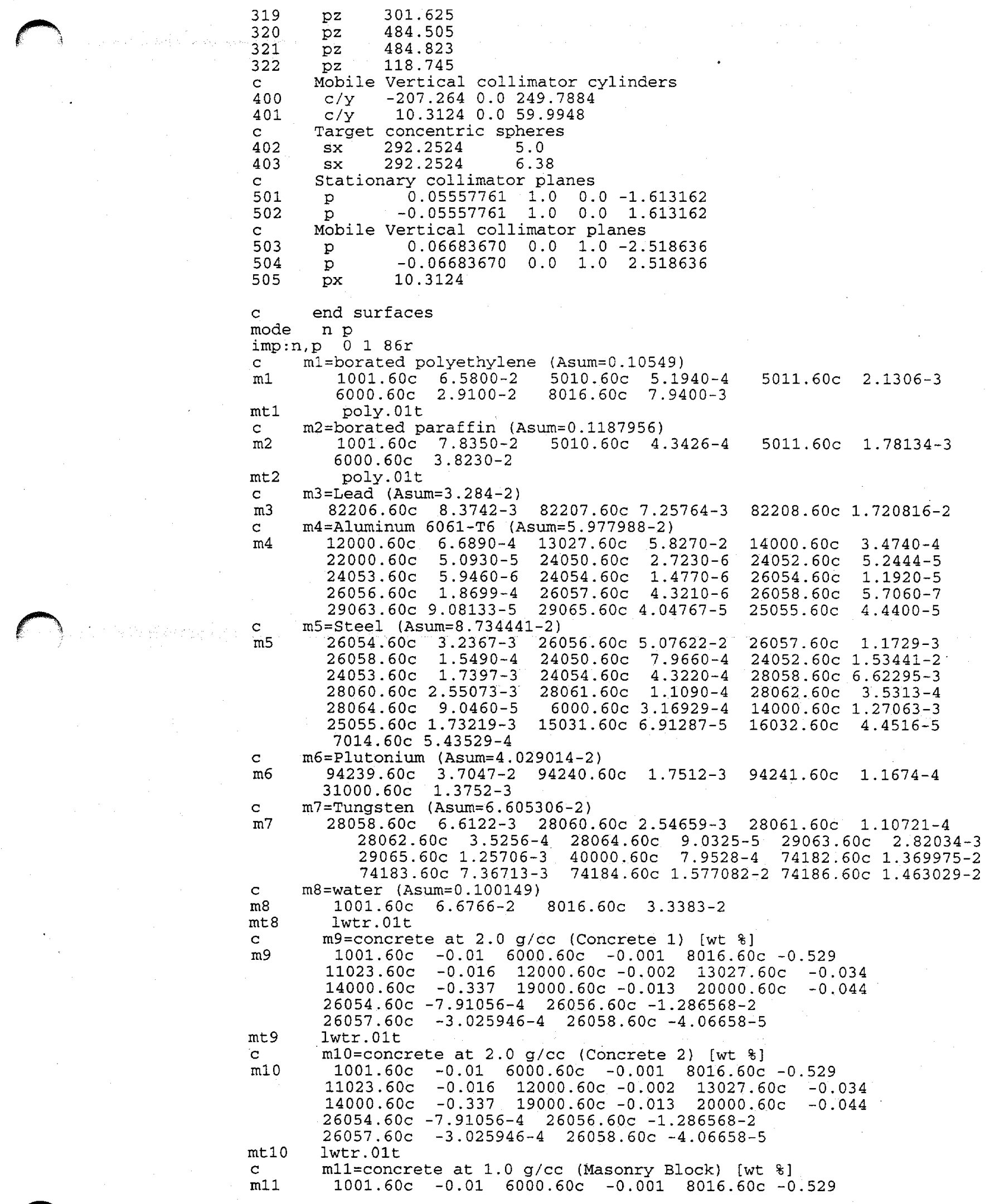




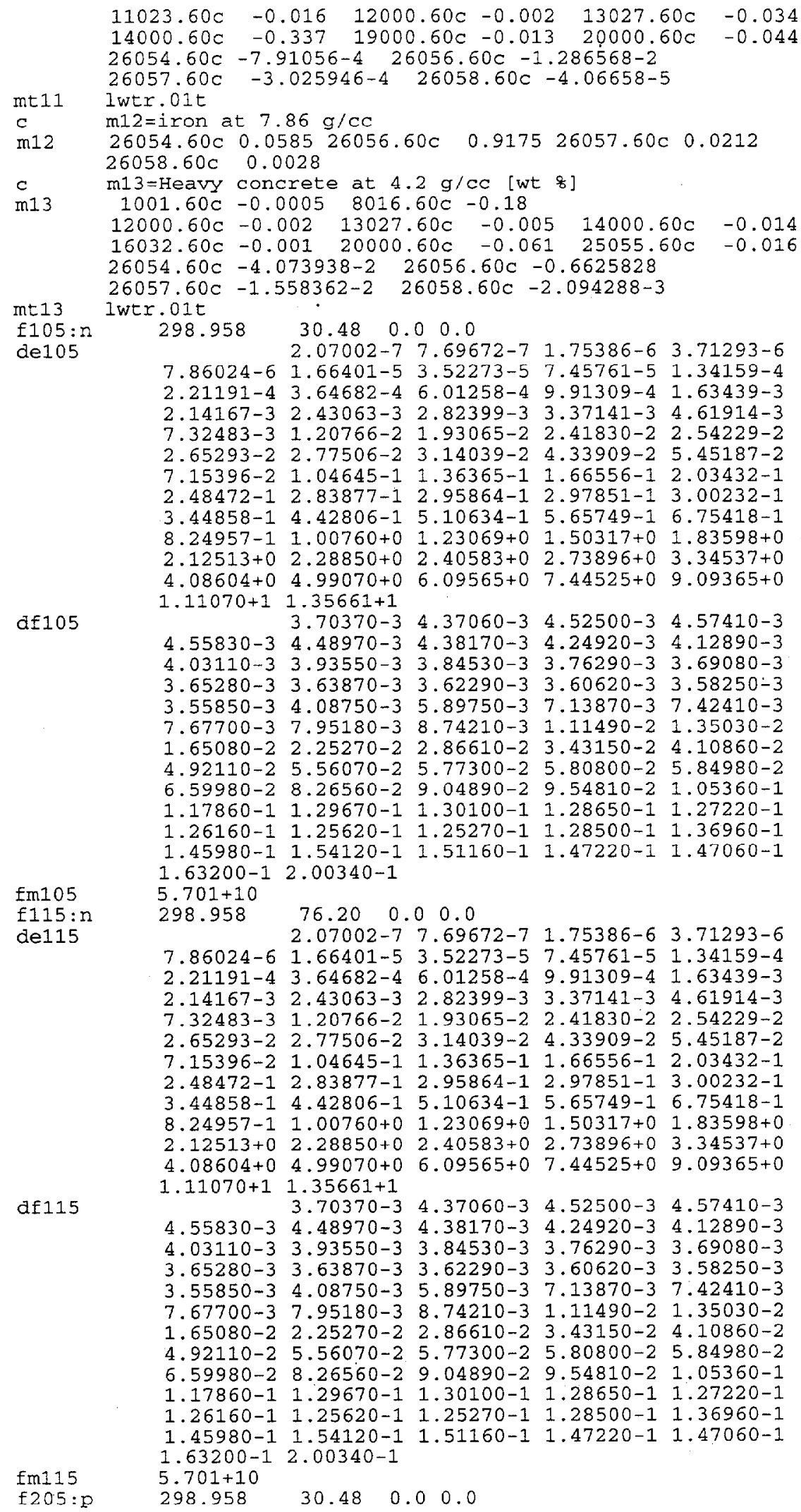




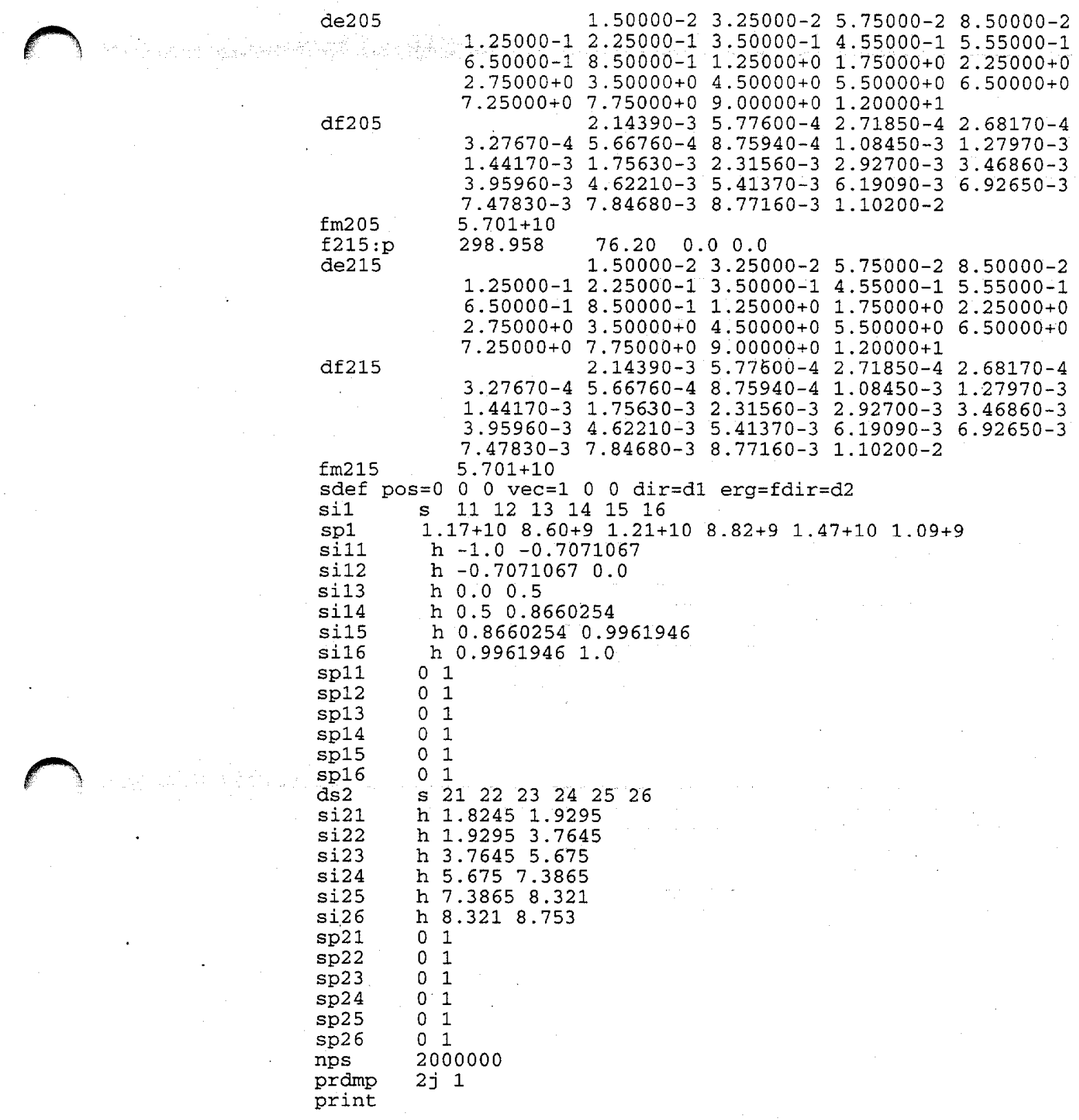


File for MCNP-4B calculation of dose rates at additional points along the truck lane of the PFNA facility for the source in the maximum up position

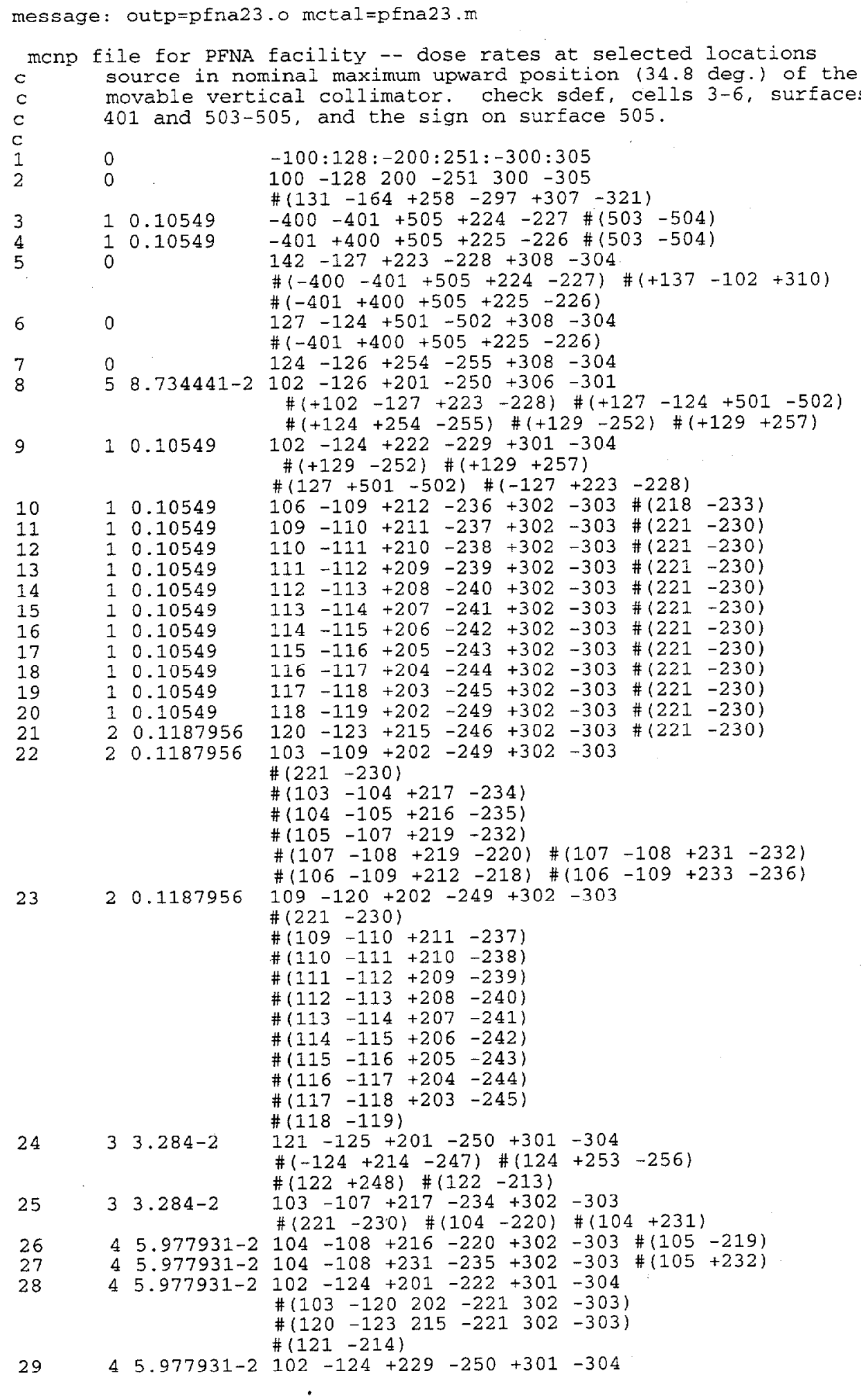




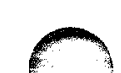

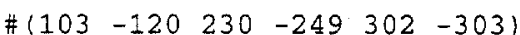

\#( $\left.\begin{array}{llllll}120 & -123 & 230 & -246 & 302 & -3.03\end{array}\right)$

$\#(121+247)$

$45.977931-2124-126+252-254+301-304$

$\#(-125+213-253)$

$3145.977931-2124-126+255-257+301-304$

$\#(-125+256-248)$

$32 \quad 0 \quad+167-168+274-281+317-318$

$330+165-166+270-285+308-310$

$34 \quad \begin{array}{ll}\#(+167-168+274-281+317-318) \\ -400+505+224-227+503-504\end{array}$

$\begin{array}{llll}35 & 0 & -401+400+505+225-226+503-504 \\ 36 & 4 & 5.977931-2 & 122-124+201-250+301-304\end{array}$

$\#(213-248)$

\# $(129-252)$

\begin{tabular}{|c|c|c|}
\hline $\begin{array}{l}37 \\
38\end{array}$ & $\begin{array}{r}9 \\
11\end{array}$ & $\begin{array}{l}-2.0 \\
-1.0\end{array}$ \\
\hline $\begin{array}{l}39 \\
40\end{array}$ & $\begin{array}{r}12 \\
0\end{array}$ & -7.86 \\
\hline
\end{tabular}

$\#(129+257)$

$+131-164+258-297+307-308$

$+131-164+258-297+308-320$

$\#(+132-163+259-296)$

$+131-164+258-297+320-321$

$+132-163+259-296+319-320$

$\#(+151-169+271-284+304-315)$

$\#(+151-160+270-271+312-314)$

$\#(+151-160+284-285+312-314)$

$\#(+157-159+274-281+313-315)$

$\#(+158-160+274-281-313)$

$\#(+169-157+274-281+314-316)$

$\begin{array}{rrr}41 & 1 & 0.10549 \\ 42 & 1 & 0.10549 \\ 43 & 1 & 0.10549 \\ 44 & 1 & 0.10549 \\ 45 & 1 & 0.10549 \\ 46 & 1 & 0.10549 \\ 47 & 9 & -2.0 \\ 48 & 10 & -2.0\end{array}$

$+151-169+271-284+304-315$

$+151-160+270-271+312-314$

$+151-160+284-285+312-314$

$+157-159+274-281+313-315$

$+158-160+274-281+308-313$

$+169-157+274-281+314-316$

$+160-162+273-282+308-319$

$+154-161+265-289+308-319$

$\# 47 \#(-160+267-286)$

$+154-160+267-286+308-319 \# 42 \# 43 \# 45$

$+155-156+259-260+308-319$

$+155-156+295-296+308-319$

$+154-155+259-296+308-319$

\# $(+265-289)$

$+155-163+259-296+308-319 \# 50$ \#51

$\#(-161+265-289) \#(+161-162+273-282)$

$+153-154+295-296+308-319$

$+151-126+295-296+308-319$

$+151-126+259-260+308-319$

$+153-154+259-260+308-319$

$+151-152+266-272+308-304$

$+151-152+283-287+308-304$

$+151-154+259-296+308-319$

\#41 \#42 \#43 \#54 \#55 \#56 \#57 \#58 \#59

$\#(+165-166+270-285+308-310)$

$\#(-126+201-250-304)$

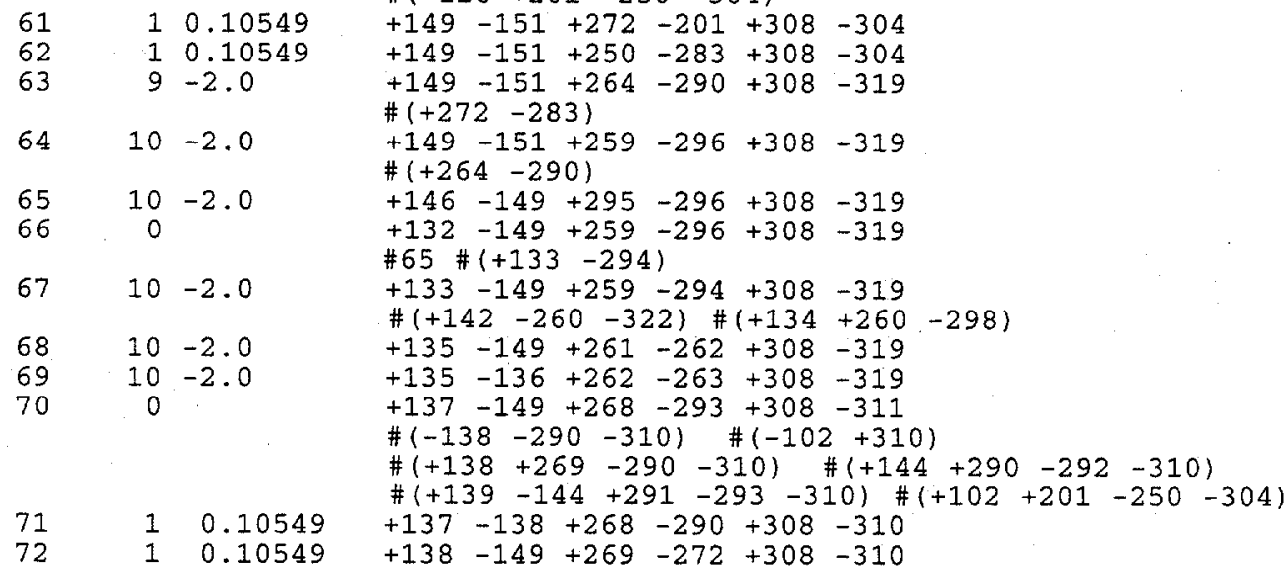




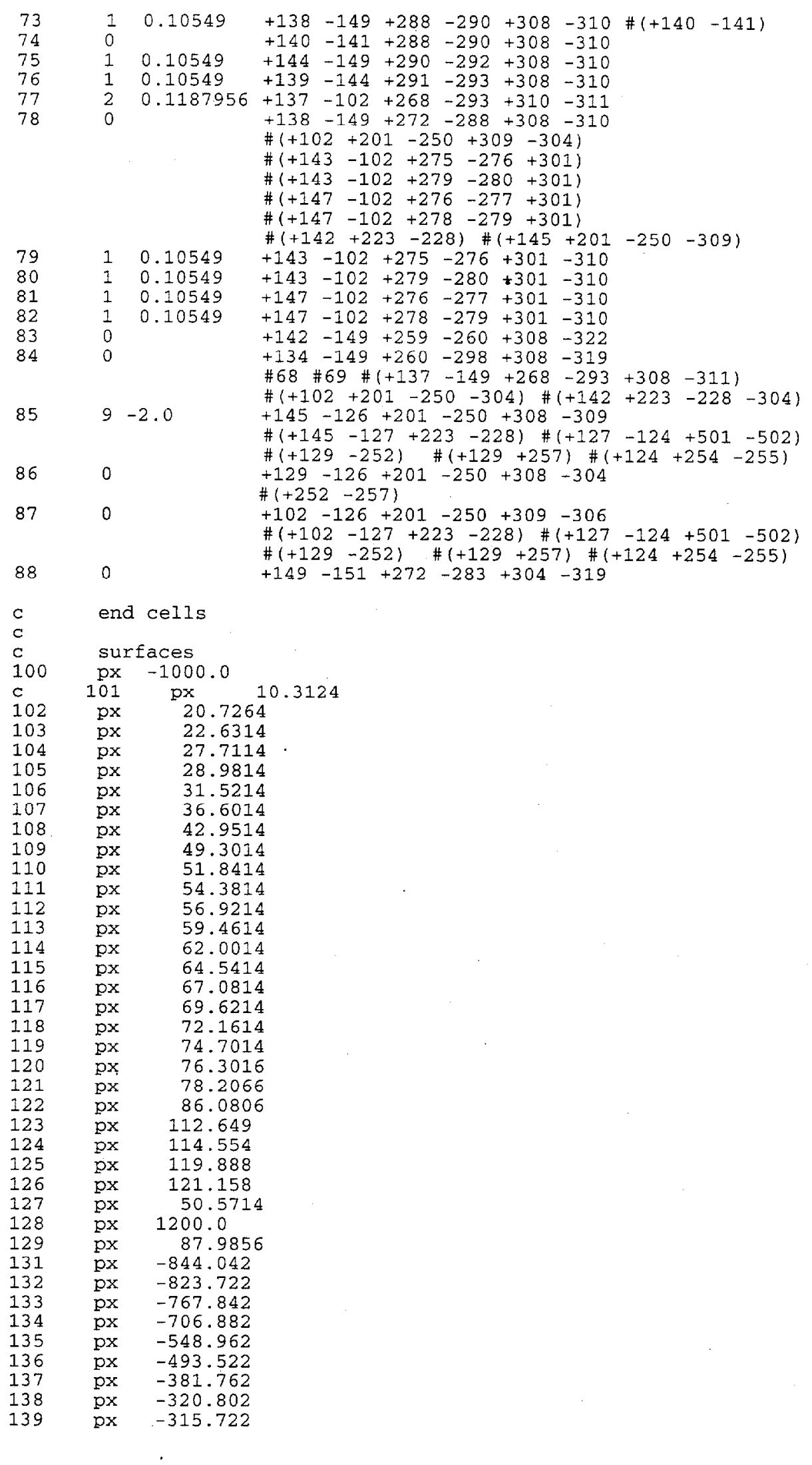




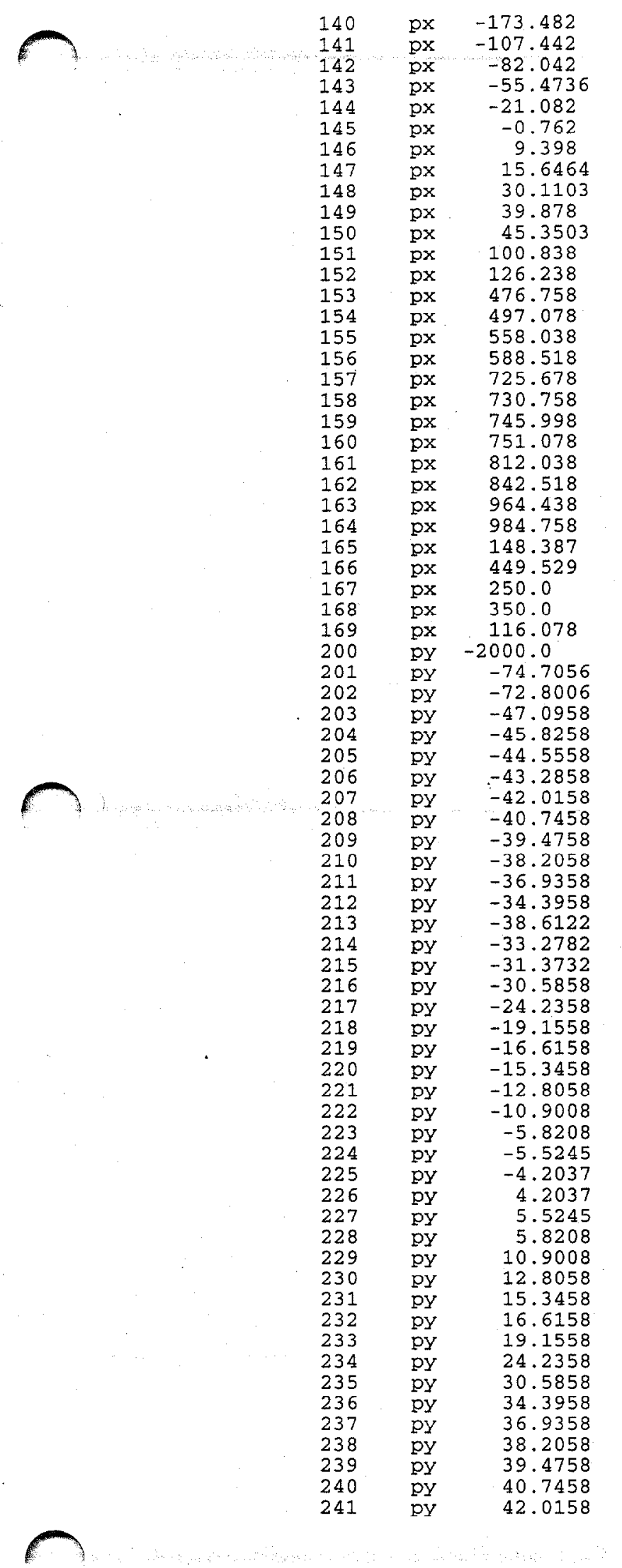




$\begin{array}{llc}242 & \text { py } & 43.2858 \\ 243 & \text { py } & 44.5558 \\ 244 & \text { py } & 45.8258 \\ 245 & \text { py } & 47.0958 \\ 246 & \text { py } & 31.3732 \\ 247 & \text { py } & 33.2782 \\ 248 & \text { py } & 38.6122 \\ 249 & \text { py } & 72.8006 \\ 250 & \text { py } & 74.7056 \\ 251 & \text { py } & 1500.0 \\ 252 & \text { py } & -39.8822 \\ 253 & \text { py } & -9.8848 \\ 254 & \text { py } & -8.6148 \\ 255 & \text { py } & 8.6148 \\ 256 & \text { py } & 9.8848 \\ 257 & \text { py } & 39.8822 \\ 258 & \text { py } & -1731.28 \\ 259 & \text { py } & -1710.96 \\ 260 & \text { py } & -1650.0 \\ 261 & \text { py } & -1528.08 \\ 262 & \text { py } & -1467.12 \\ 263 & \text { py } & -1436.64 \\ 264 & \text { py } & -589.28 \\ 265 & \text { py } & -241.3 \\ 266 & \text { py } & -193.04 \\ 267 & \text { py } & -180.34 \\ 268 & \text { py } & -167.64 \\ 269 & \text { py } & -162.56 \\ 270 & \text { py } & -152.4 \\ 271 & \text { py } & -137.16 \\ 272 & \text { py } & -101.6 \\ 273 & \text { py } & -91.44 \\ 274 & \text { py } & -60.96 \\ 275 & \text { py } & -45.72 \\ 276 & \text { py } & -30.48 \\ 277 & \text { py } & -10.16 \\ 278 & \text { py } & 10.16 \\ 279 & \text { py } & 30.48 \\ 280 & \text { py } & 45.72 \\ 281 & \text { py } & 60.96 \\ 282 & \text { py } & 91.44 \\ 283 & \text { py } & 101.6 \\ 284 & \text { py } & 137.16 \\ 285 & \text { py } & 152.4 \\ 286 & \text { py } & 180.34 \\ 287 & \text { py } & 193.04 \\ 288 & \text { py } & 213.36 \\ 289 & \text { py } & 241.3 \\ 290 & \text { py } & 274.32 \\ 291 & \text { py } & 335.28 \\ 292 & \text { py } & 345.44 \\ 293 & \text { py } & 396.24 \\ 294 & \text { py } & 533.4 \\ 295 & \text { py } & 1235.44 \\ 296 & \text { py } & 1296.4 \\ 297 & \text { py } & 1316.72 \\ 298 & \text { py } & 472.44 \\ 300 & \text { pz } & -500.0 \\ 301 & \text { pz } & -59.478 \\ 302 & \text { pz } & -57.573 \\ 303 & \text { pz } & 245.957 \\ 304 & \text { pz } & 247.862 \\ 305 & \text { pz } & 500.0 \\ 306 & \text { pz } & -60.748 \\ 307 & \text { pz } & -156.0 \\ 309 & \text { pz } & -125.095 \\ 310 & \text { pz } & -67.451 \\ 311 & \text { pz } & 235.585 \\ 312 & \text { pz } & 276.225 \\ 313 & \text { pz } & 342.845 \\ 314 & \text { pz } & 375.285 \\ & & \\ 295 & \end{array}$




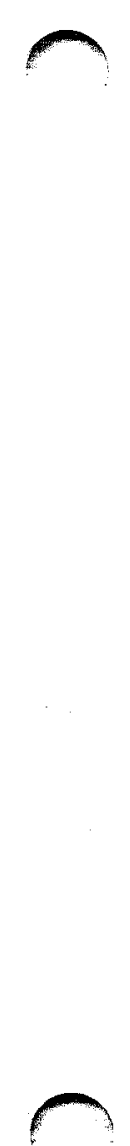

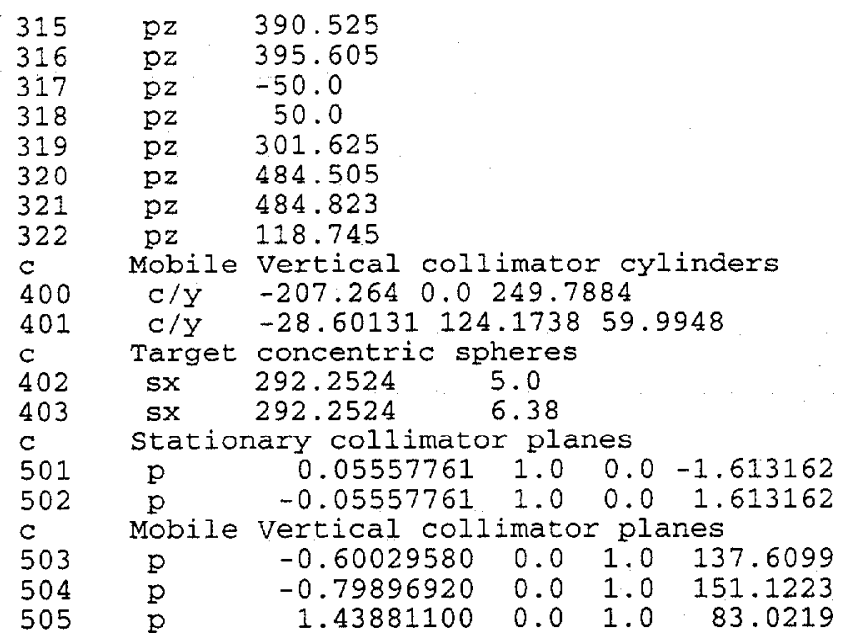

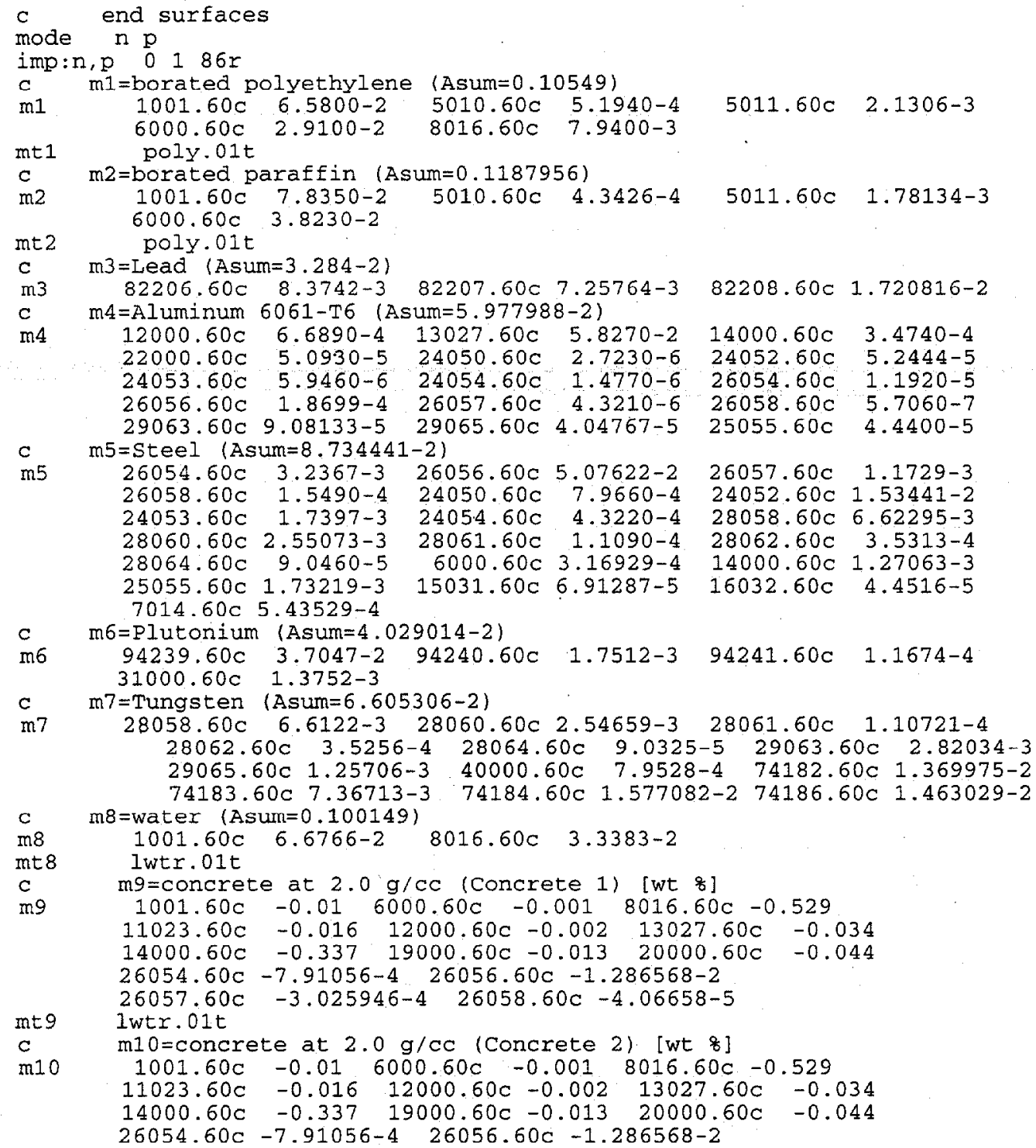




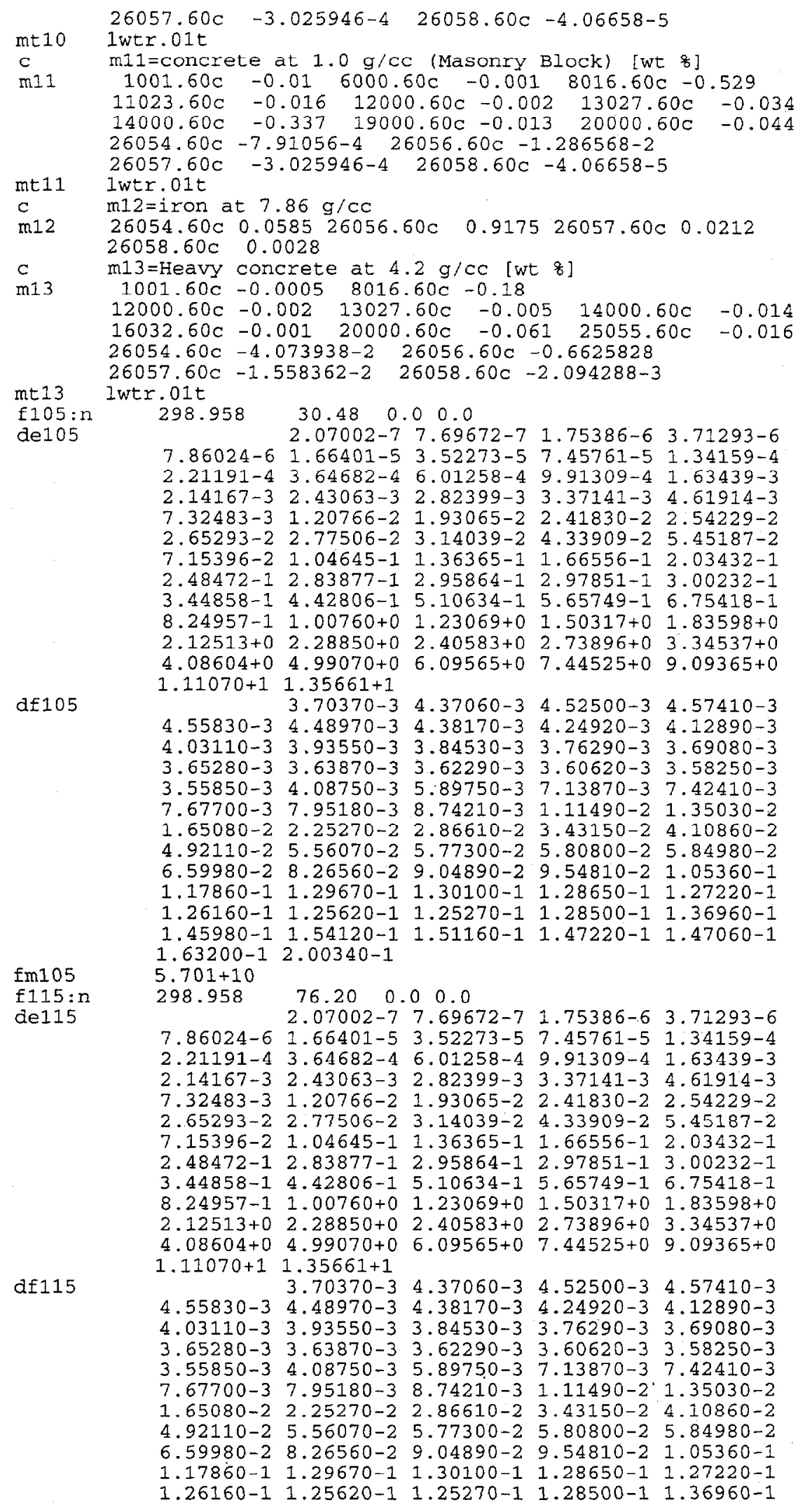


$\begin{array}{llllll}1.45980-1 & 1.54120-1 & 1.51160-1 & 1.47220-1 & 1.47060-1\end{array}$ $1.63200-12.00340-1$

$5.701+10$

fm115

$f 205: p$

298.958

$\begin{array}{lll}30.48 & 0.0 & 0.0\end{array}$

de205

$1.25000-122.25000-1 \quad 3.50000-1 \quad 4.55000-1 \quad 5.55000-1$

$6.50000-18.50000-1 \quad 1.25000+0 \quad 1.75000+0 \quad 2.25000+0$

$2.75000+0 \quad 3.50000+0 \quad 4.50000+0 \quad 5.50000+0 \quad 6.50000+0$

$7.25000+07.75000+0 \quad 9.00000+01.20000+1$

df205

$\begin{array}{llll}2.14390-3 & 5.77600-4 & 2.71850-4 & 2.68170-4\end{array}$

$3.27670-4 \quad 5.66760-4 \quad 8.75940-4 \quad 1.08450-3 \quad 1.27970-3$

$1.44170-3 \quad 1.75630-3 \quad 2.31560-3 \quad 2.92700-3 \quad 3.46860-3$

$\begin{array}{llllll}3.95960-3 & 4.62210-3 & 5.41370-3 & 6.19090-3 & 6.92650-3\end{array}$

$7.47830-3 \quad 7.84680-38.77160-31.10200-2$

$\operatorname{Em} 205 \quad 5.701+10$

E215:p $\quad 298.958 \quad 76.20 \quad 0.0 \quad 0.0$

de215 $1.50000-2 \quad 3.25000-2 \quad 5.75000-2 \quad 8.50000-2$

$\begin{array}{lllll}1.25000-1 & 2.25000-1 & 3.50000-1 & 4.55000-1 & 5.55000-1\end{array}$

$6.50000-1 \quad 8.50000-1 \quad 1.25000+0 \quad 1.75000+0 \quad 2.25000+0$

$2.75000+0 \quad 3.50000+0 \quad 4.50000+0 \quad 5.50000+0 \quad 6.50000+0$

df 215

$7.25000+0 \quad 7.75000+0 \quad 9.00000+0 \quad 1.20000+1$

$\begin{array}{llll}2.14390-3 & 5.77600-4 & 2.71850-4 & 2.68170-4\end{array}$

$3.27670-4 \quad 5.66760-4 \quad 8.75940-4 \quad 1.08450-3 \quad 1.27970-3$

$1.44170-3 \quad 1.75630-3 \quad 2.31560-3 \quad 2.92700-3 \quad 3.46860-3$

$3.95960-3 \quad 4.62210-3 \quad 5.41370-3 \quad 6.19090-3 \quad 6.92650-3$

$\begin{array}{llll}7.47830-3 & 7.84680-3 & 8.77160-3 & 1.10200-2\end{array}$

$\begin{array}{llll}\text { fm215 } & 5.701+10 \\ \text { sdef } & \text { pos }=-37.0693 \quad 0 \quad 118.288 \text { dir=d1 erg=fdir=d2 }\end{array}$

vec $=0.821149209 \quad 0.0 \quad 0.570713568$

si1 S $11121314 \quad 1516$

$\begin{array}{lllllll}\operatorname{sp1} 1 & 1.17+10 & 8.60+9 & 1.21+10 & 8.82+9 & 1.47+10 & 1.09+9\end{array}$

si11 $h-1.0-0.7071067$

$\operatorname{si12} \quad h-0.70710670 .0$

$\operatorname{si13}$ h 0.00 .5

$\begin{array}{llll}\text { sild h } & 0.5 & 0.8660254\end{array}$

si15 h $0.8660254 \quad 0.9961946$

sil6 h 0.99619461 .0

$\operatorname{spl} 11$

$\operatorname{sp} 12$

$\begin{array}{lll}0 & 1 \\ 0 & 1\end{array}$.

$\operatorname{sp} 13$

$\operatorname{sp} 14$

$\operatorname{sp} 15$

sp16

ds2

si 21

si22

$\sin 23$

si 24

si25

si26

$\operatorname{sp} 21$

sp22

$\operatorname{sp} 23$

$\operatorname{sp} 24$

sp25

$\operatorname{sp} 26$

nps

prdmp

$\begin{array}{ll}0 & 1\end{array}$

$\begin{array}{ll}0 & 1\end{array}$

$0 \begin{array}{ll}0 & 1 \\ 1 & 1\end{array}$

$0 \cdot 1$

$\begin{array}{lllllll}\text { s } & 21 & 22 & 23 & 24 & 25 & 26\end{array}$

h $1.8245 \quad 1.9295$

h 1.92953 .7645

h $3.7645 \quad 5.675$

h 5.6757 .3865

h $7.3865 \quad 8.321$

h 8.3218 .753

01

01

$\begin{array}{ll}0 & 1 \\ 0 & 1\end{array}$

$0 \begin{array}{ll}0 & 1 \\ 0 & 1\end{array}$

01

2000000

print

$2 j 1$ 
File for MCNP-4B calculation of dose rates at additional points along the truck lane of the PFNA facility for the source in the maximum down position

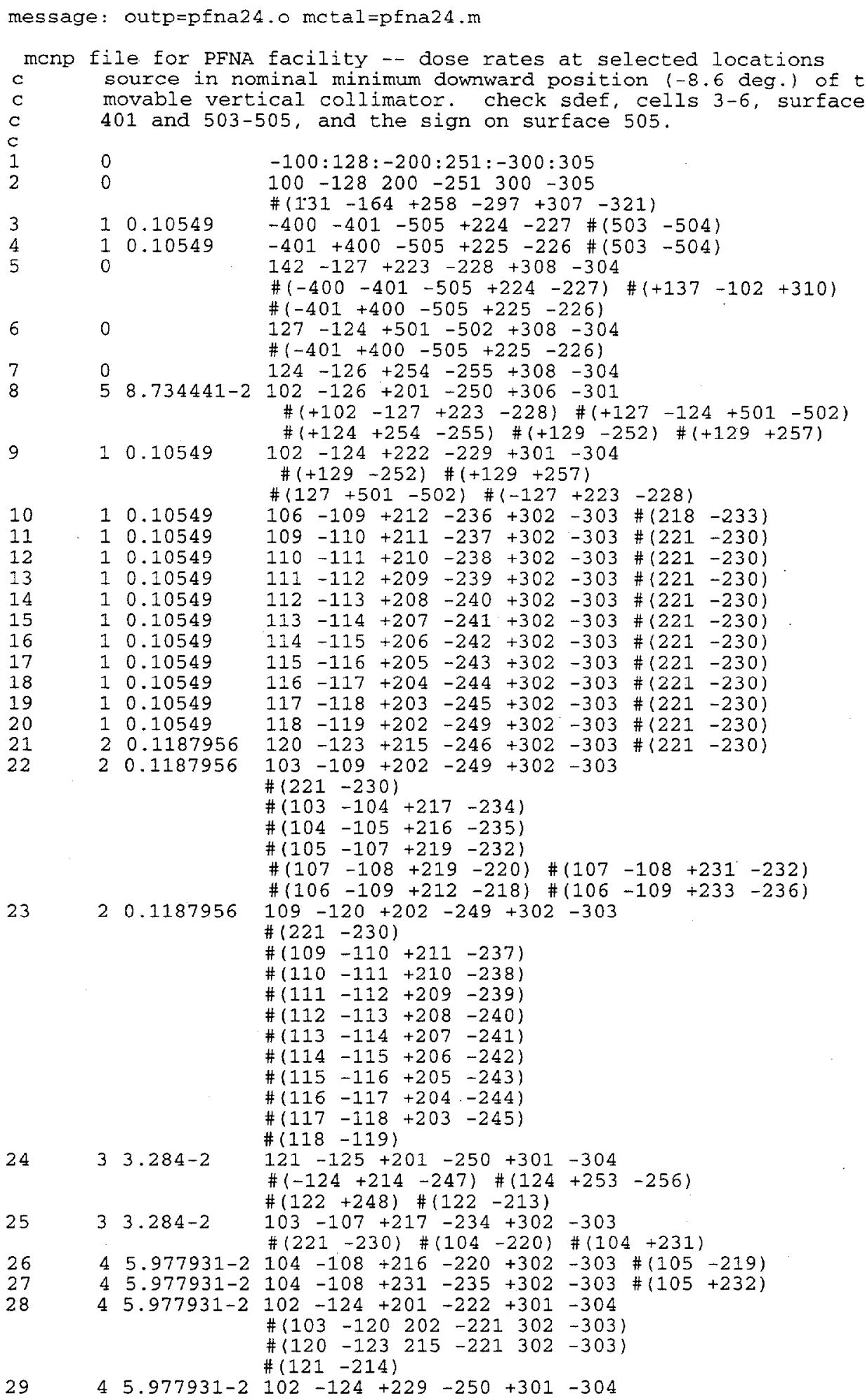




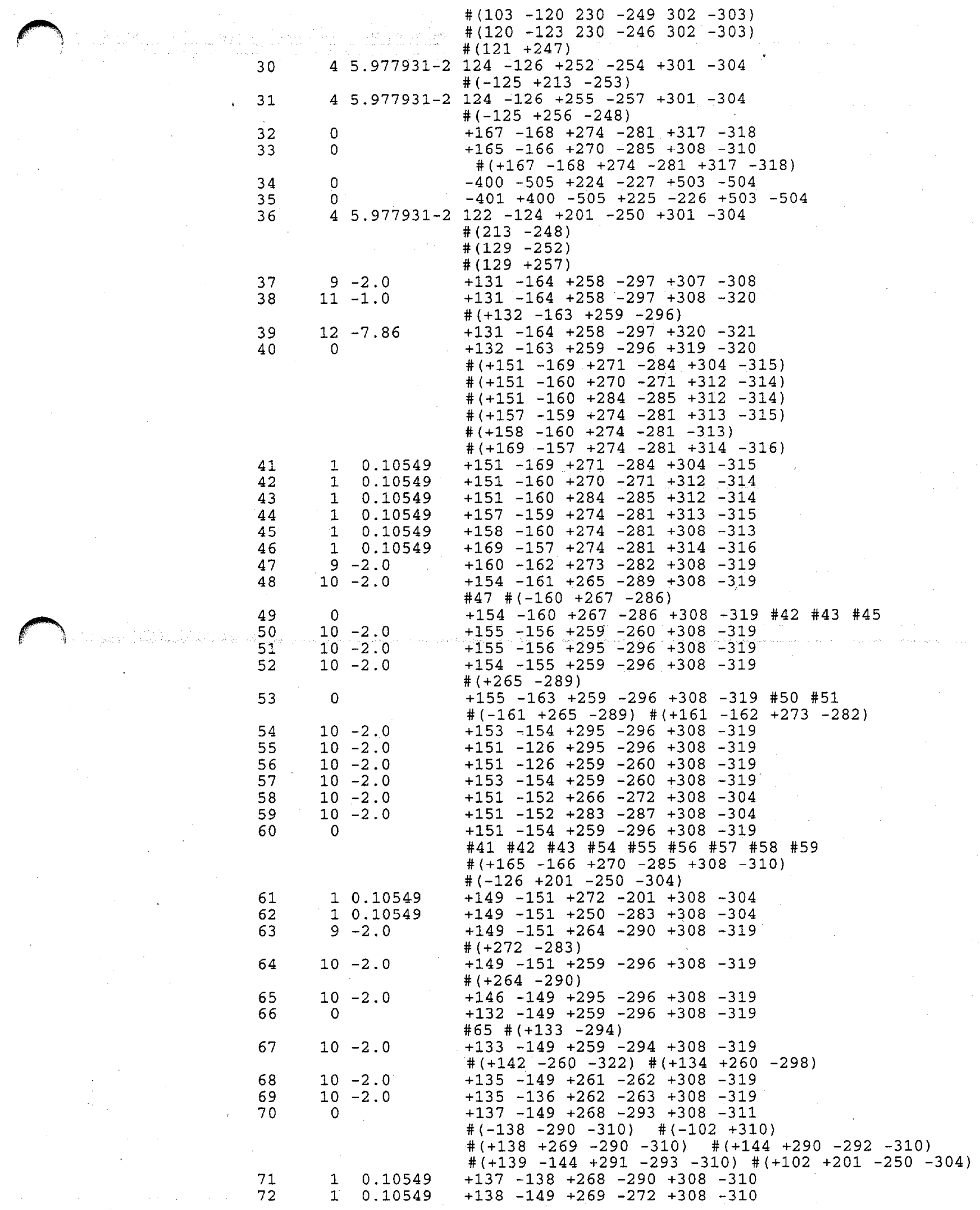




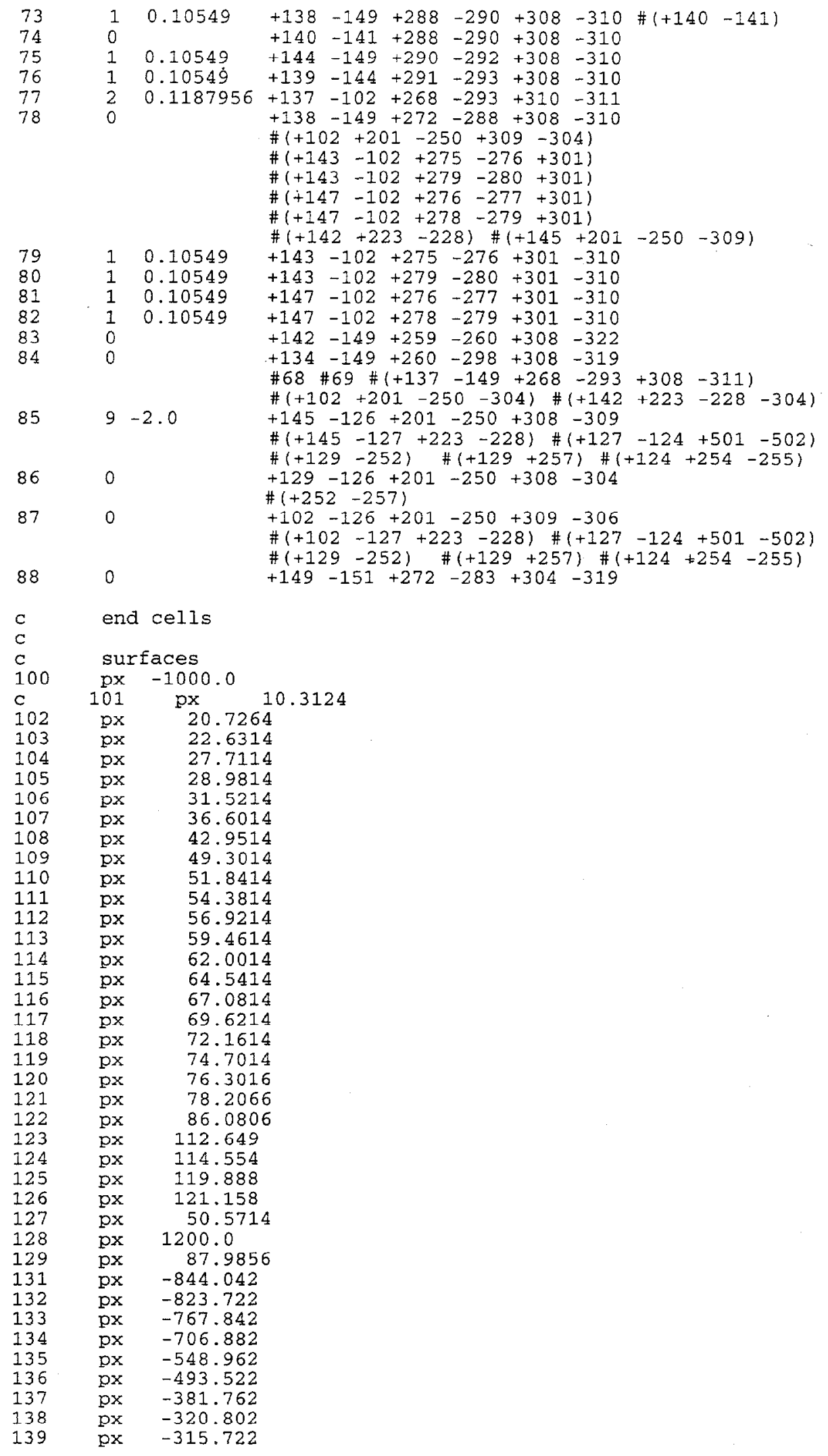




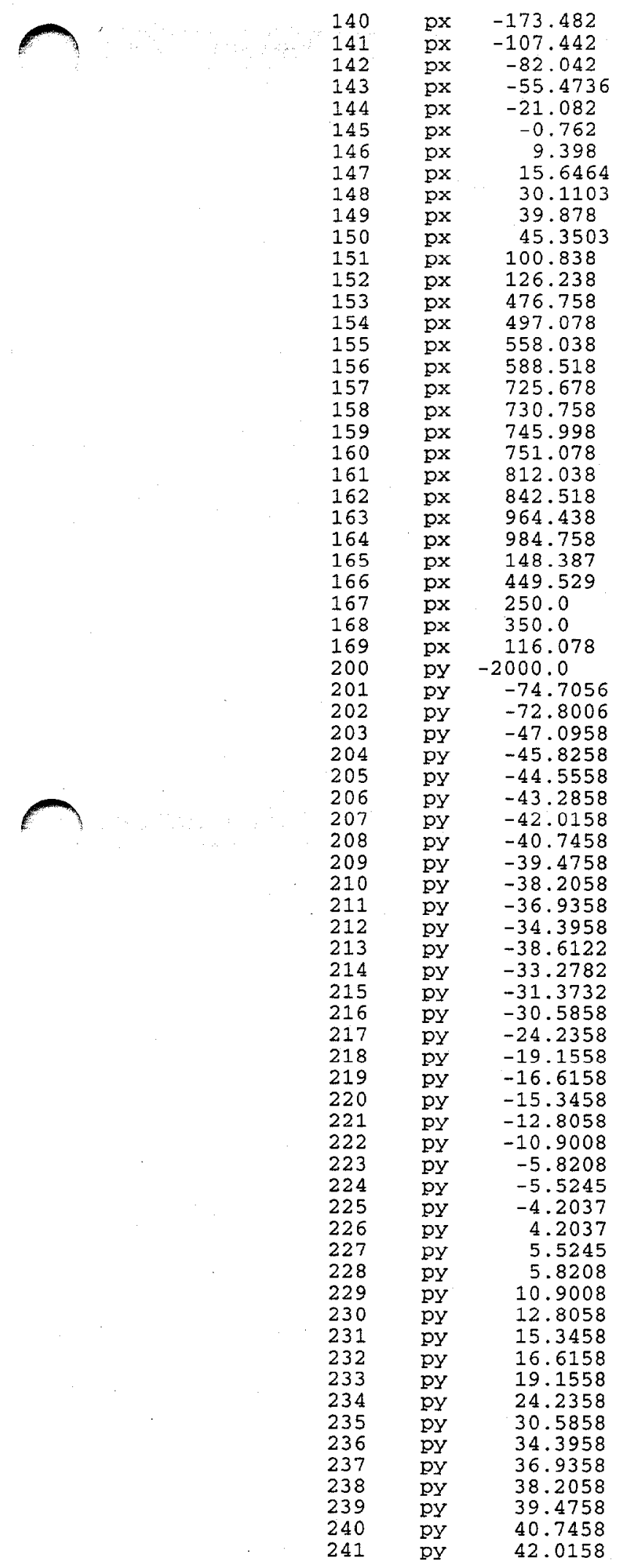




\begin{tabular}{|c|c|c|}
\hline 242 & py & 43.2858 \\
\hline 243 & py & 44.5558 \\
\hline 244 & py & 45.8258 \\
\hline 245 & py & 47.0958 \\
\hline 246 & py & 31.3732 \\
\hline 247 & DY & 33.2782 \\
\hline 248 & py & 38.6122 \\
\hline 249 & py & 72.8006 \\
\hline 250 & py & 74.7056 \\
\hline 251 & py & 1500.0 \\
\hline 252 & py & -39.8822 \\
\hline 253 & py & -9.8848 \\
\hline 254 & py & -8.6148 \\
\hline 255 & pY & 8.6148 \\
\hline 256 & LY & 9.8848 \\
\hline 257 & py & 39.8822 \\
\hline 258 & PY & -1731.28 \\
\hline 259 & py & -1710.96 \\
\hline 260 & py & -1650.0 \\
\hline 261 & py & -1528.08 \\
\hline 262 & py & -1467.12 \\
\hline 263 & py & -1436.64 \\
\hline 264 & py & -589.28 \\
\hline 265 & py & -241.3 \\
\hline 266 & py & -193.04 \\
\hline 267 & py & $-180 \cdot 34$ \\
\hline 2.68 & py & -167.64 \\
\hline 269 & py & -162.56 \\
\hline 270 & py & -152.4 \\
\hline 271 & py & -137.16 \\
\hline 272 & py & -101.6 \\
\hline 273 & PY & $-91 \cdot 44$ \\
\hline 274 & py & -60.96 \\
\hline 275 & py & -45.72 \\
\hline 276 & PY & -30.48 \\
\hline 277 & pY & -10.16 \\
\hline 278 & py & 10.16 \\
\hline 279 & py & 30.48 \\
\hline 280 & py & 45.72 \\
\hline 281 & py & 60.96 \\
\hline 282 & py & 91.44 \\
\hline 283 & py & 101.6 \\
\hline 284 & py & 137.16 \\
\hline 285 & PY & 152.4 \\
\hline 286 & PY & 180.34 \\
\hline 287 & py & 193.04 \\
\hline 288 & py & 213.36 \\
\hline 289 & py & $241 \cdot 3$ \\
\hline 290 & py & 274.32 \\
\hline 291 & py & 335.28 \\
\hline 292 & py & 345.44 \\
\hline 293 & py & 396.24 \\
\hline 294 & py & 533.4 \\
\hline 295 & py & 1235.44 \\
\hline 296 & pY & 1296.4 \\
\hline 297 & PY & 1316.72 \\
\hline 298 & py & 472.44 \\
\hline 300 & $p z$ & -500.0 \\
\hline 301 & $\mathrm{pz}$ & -59.478 \\
\hline 302 & $\mathrm{pz}$ & -57.573 \\
\hline 303 & $p z$ & 245.957 \\
\hline 304 & $\mathrm{pz}$ & 247.862 \\
\hline 305 & $p z$ & 500.0 \\
\hline 306 & $p z$ & -60.748 \\
\hline 307 & $p z$ & -156.0 \\
\hline 308 & $\mathrm{pz}$ & -125.095 \\
\hline 309 & $p z$ & -67.451 \\
\hline 310 & $\mathrm{pz}$ & 235.585 \\
\hline 311 & $p z$ & 276.225 \\
\hline 312 & $p z$ & 283.845 \\
\hline 313 & $\mathrm{pz}$ & 342.265 \\
\hline 314 & $\mathrm{pz}$ & 375.285 \\
\hline
\end{tabular}




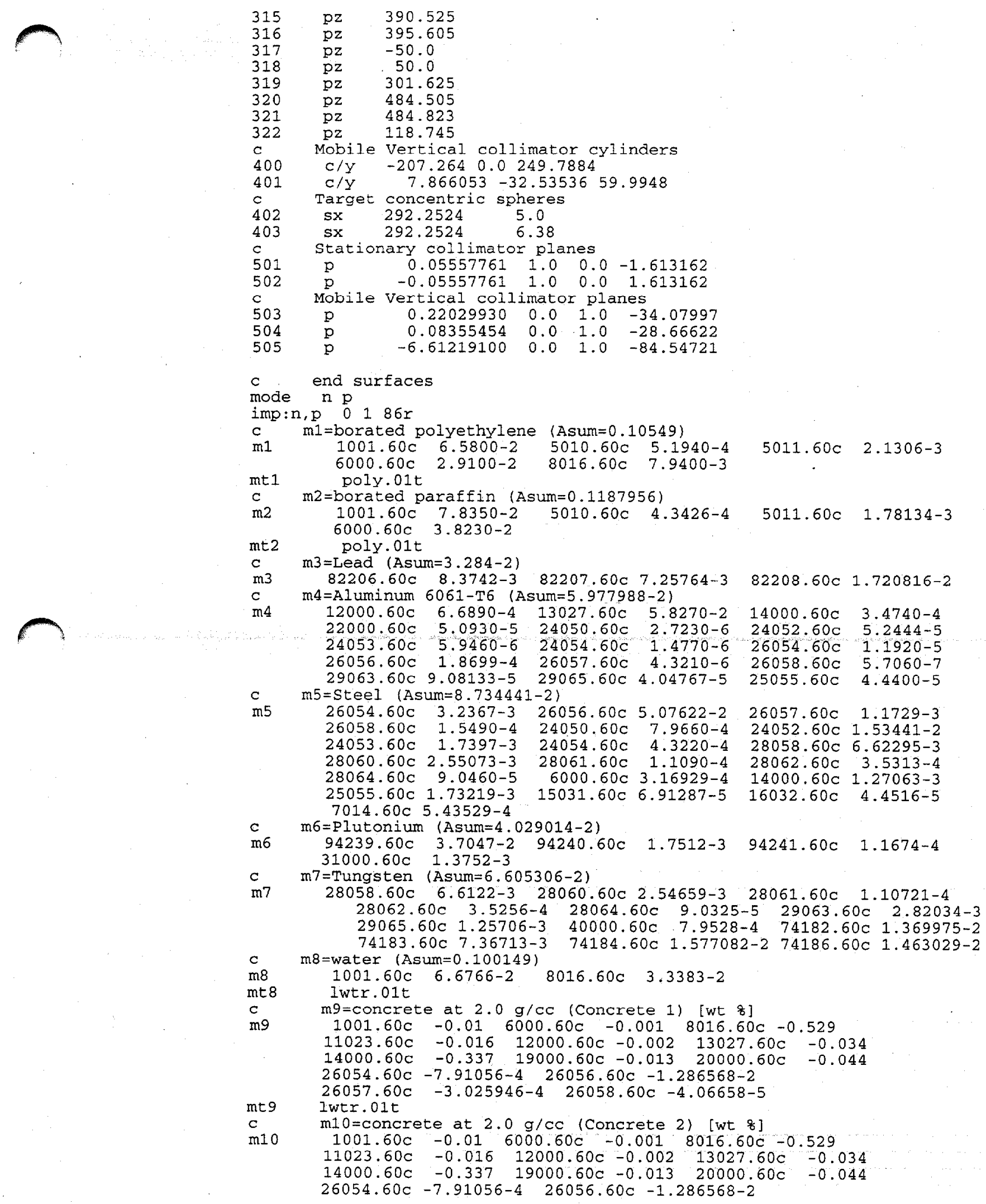




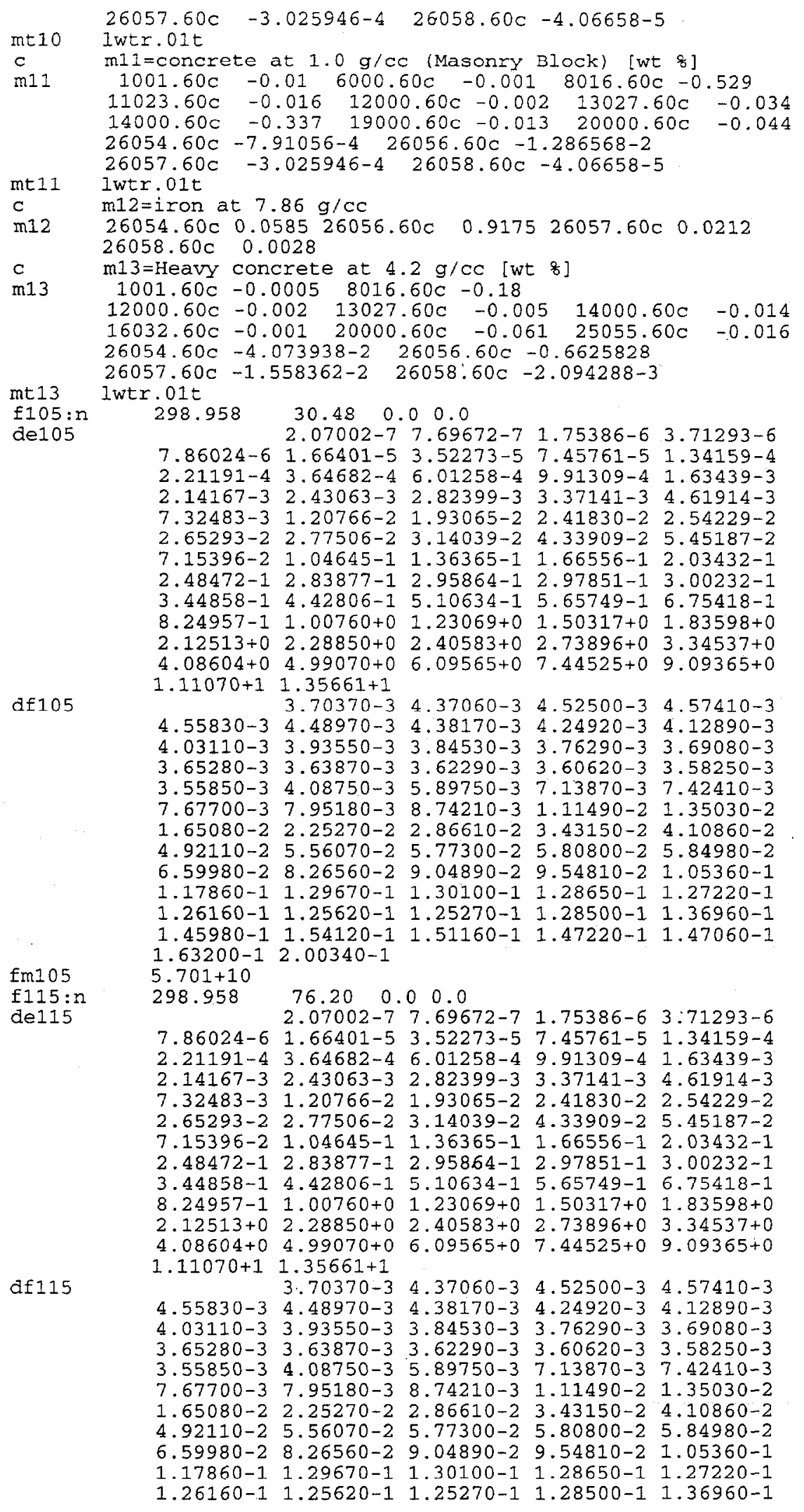


$1.45980-1 \quad 1.54120-1 \quad 1.51160-1 \quad 1.47220-1 \quad 1.47060-1$ $1.63200-12.00340-1$

$\operatorname{fm} 115 \quad 5.701+10$

$f 205: \mathrm{p} \quad 298.958 \quad 30.48 \quad 0.0 \quad 0.0$

de 205

$1.50000-2 \quad 3.25000-2 \quad 5.75000-2 \quad 8.50000-2$

$\begin{array}{llllllll}1.25000-1 & 2.25000-1 & 3.50000-1 & 4.75000-1 & 5.55000-1\end{array}$ $6.50000-1.8 .50000-1 \quad 1.25000+0 \quad 1.75000+0 \quad 2.25000+0$

$2.75000+0 \quad 3.50000+0 \quad 4.50000+0 \quad 5.50000+0 \quad 6.50000+0$

$7.25000+0 \quad 7.75000+0 \quad 9.00000+0 \quad 1.20000+1$

df205

$2.14390-3 \quad 5.77600-4 \quad 2.71850-4 \quad 2.68170-4$

$3.27670-4 \quad 5.66760-4 \quad 8.75940-4 \quad 1.08450-3 \quad 1.27970-3$

$\begin{array}{lllll}1.44170-3 & 1.75630-3 & 2.31560-3 & 2.92700-3 & 3.46860-3\end{array}$

$\begin{array}{lllll}3.95960-3 & 4.62210-3 & 5.41370-3 & 6.19090-3 & 6.92650-3\end{array}$

$\begin{array}{llll}7.47830-3 & 7.84680-3 & 8.77160-3 & 1.10200-2\end{array}$

fm205 $\quad 5.701+10$

f215:p $\quad 298.958 \quad 76.20 \quad 0.0 \quad 0.0$

de 215

$1.25000-1.50000-2 \quad 3.25000-2 \quad 5.75000-2 \quad 8.50000-2$

$6.50000-18.50000-1 \quad 1.25000+0 \quad 1.75000+0 \quad 2.25000+0$

$2.75000+0 \quad 3.50000+0 \quad 4.50000+0 \quad 5.50000+0 \quad 6.50000+0$

df 215

$7.25000+0 \quad 7.75000+0 \quad 9.00000+0 \quad 1.20000+1$

$\begin{array}{lllll}2.14390-3 & 5.77600-4 & 2.71850-4 & 2.68170-4\end{array}$

$3.27670-4 \quad 5.66760-4 \quad 8.75940-4 \quad 1.08450-3 \quad 1.27970-3$

$\begin{array}{lllll}1.44170-3 & 1.75630-3 & 2.31560-3 & 2.92700-3 & 3.46860-3\end{array}$

$\begin{array}{llllll}3.95960-3 & 4.62210-3 & 5.41370-3 & 6.19090-3 & 6.92650-3\end{array}$

fm215 $\quad 5.701+10$

sdef pos $=-2.3304 \quad 0 \quad-30.993$ dir=d1 erg=fdir $=d 2$ vec $=0.988756381 \quad 0.0-0.149535343$

sil $s \quad 11 \quad 12 \quad 13 \quad 14 \quad 15 \quad 16$

$\begin{array}{lllllll}\operatorname{sp} 1 & 1.17+10 & 8.60+9 & 1.21+10 & 8.82+9 & 1.47+10 & 1.09+9\end{array}$

sil1 $h-1.0-0.7071067$

$\operatorname{si12} \quad h-0.70710670 .0$

sil3 h $0.0 \quad 0.5$

si14 h 0.50 .8660254

si15 h $0.8660254 \quad 0.9961946$

si16 h 0.99619461 .0

spl1 01

$\operatorname{sp12} \quad 0 \quad 1$

sp13 01

$\operatorname{sp14} \quad 0 \quad 1$

sp15 01

sp16 01

ds2 s $21 \quad 22 \quad 23 \quad 24 \quad 2526$

si21 h 1.82451 .9295

$\operatorname{si22}$ h $1.9295 \quad 3.7645$

si23 h $3.7645 \quad 5.675$

si24 h 5.6757 .3865

si25 h $7.3865 \quad 8.321$

si26 h 8.3218 .753

$\operatorname{sp} 21 \quad 01$

sp22 01

$\operatorname{sp} 23 \quad 0 \quad 1$

sp24 01

$\operatorname{sp2} 5 \quad 01$

sp26 01

nps 2000000

pramp $2 \mathrm{j} 1$

print 
File for MCNP-4B calculation of dose rates below the overhead source shield and below the roof of the PFNA facility for the source in the maximum up position

message: outp $=$ pfna 28.0 mctal $=p f n a 28 . m$

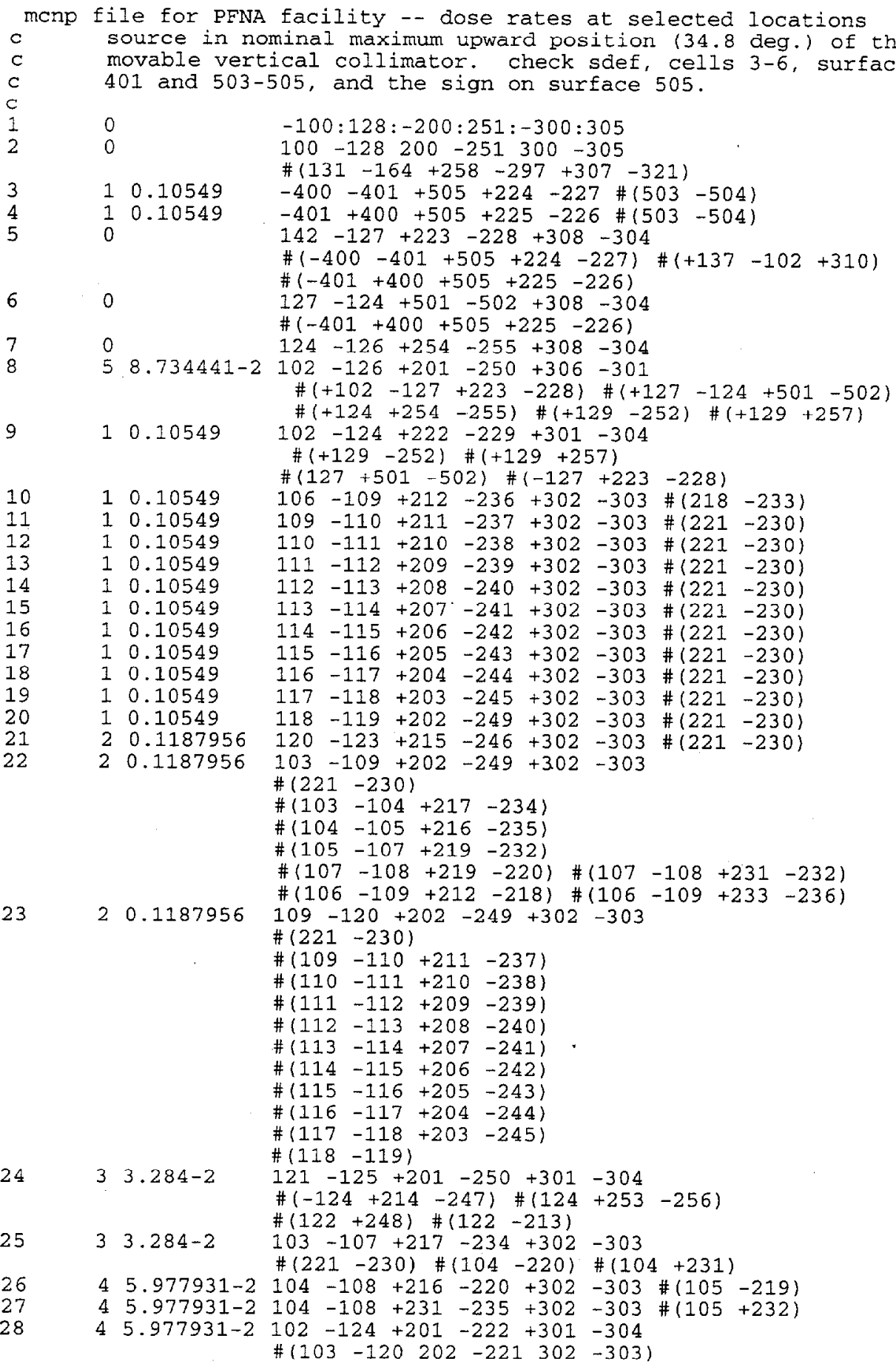




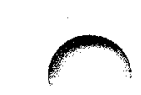

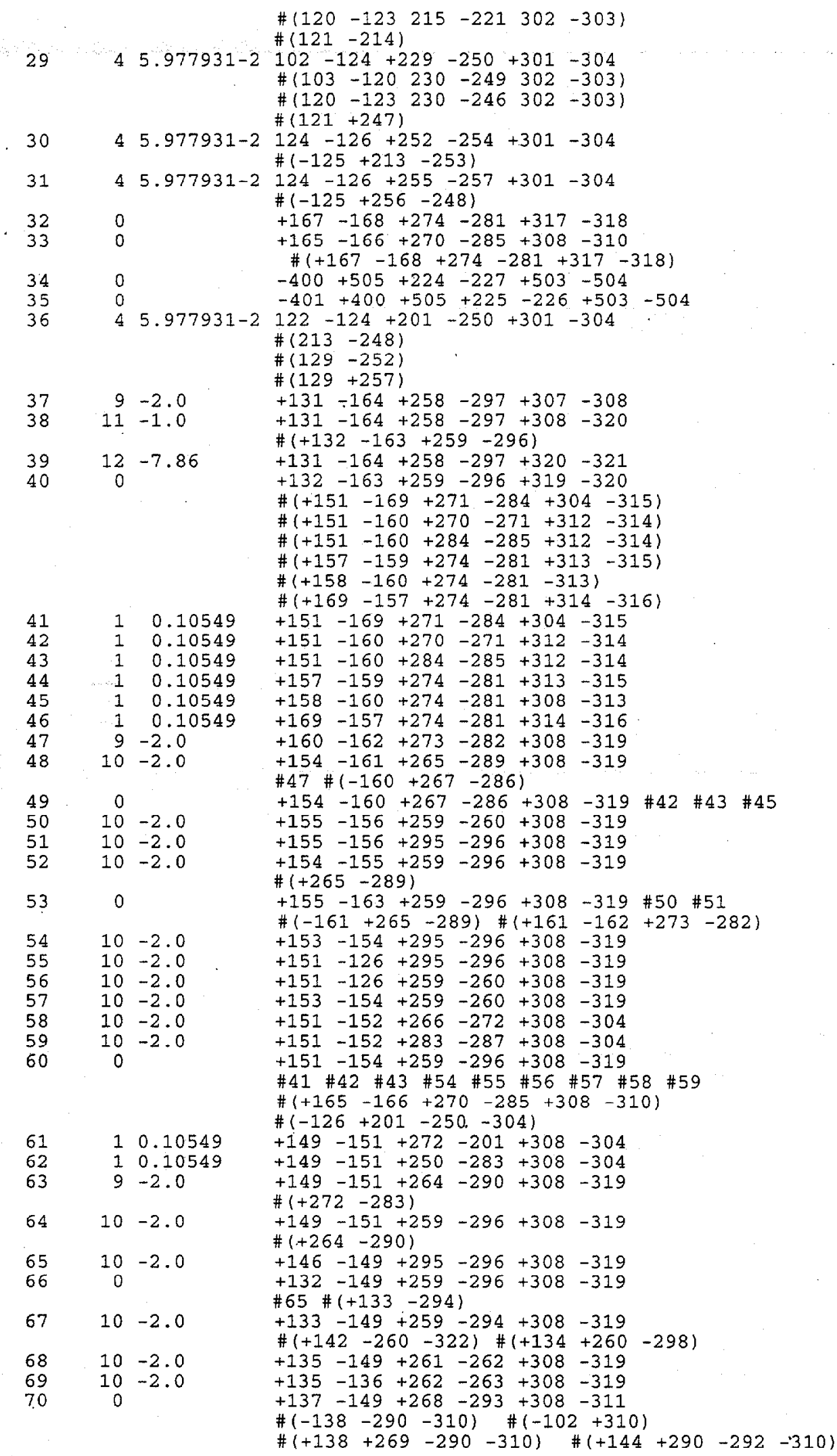




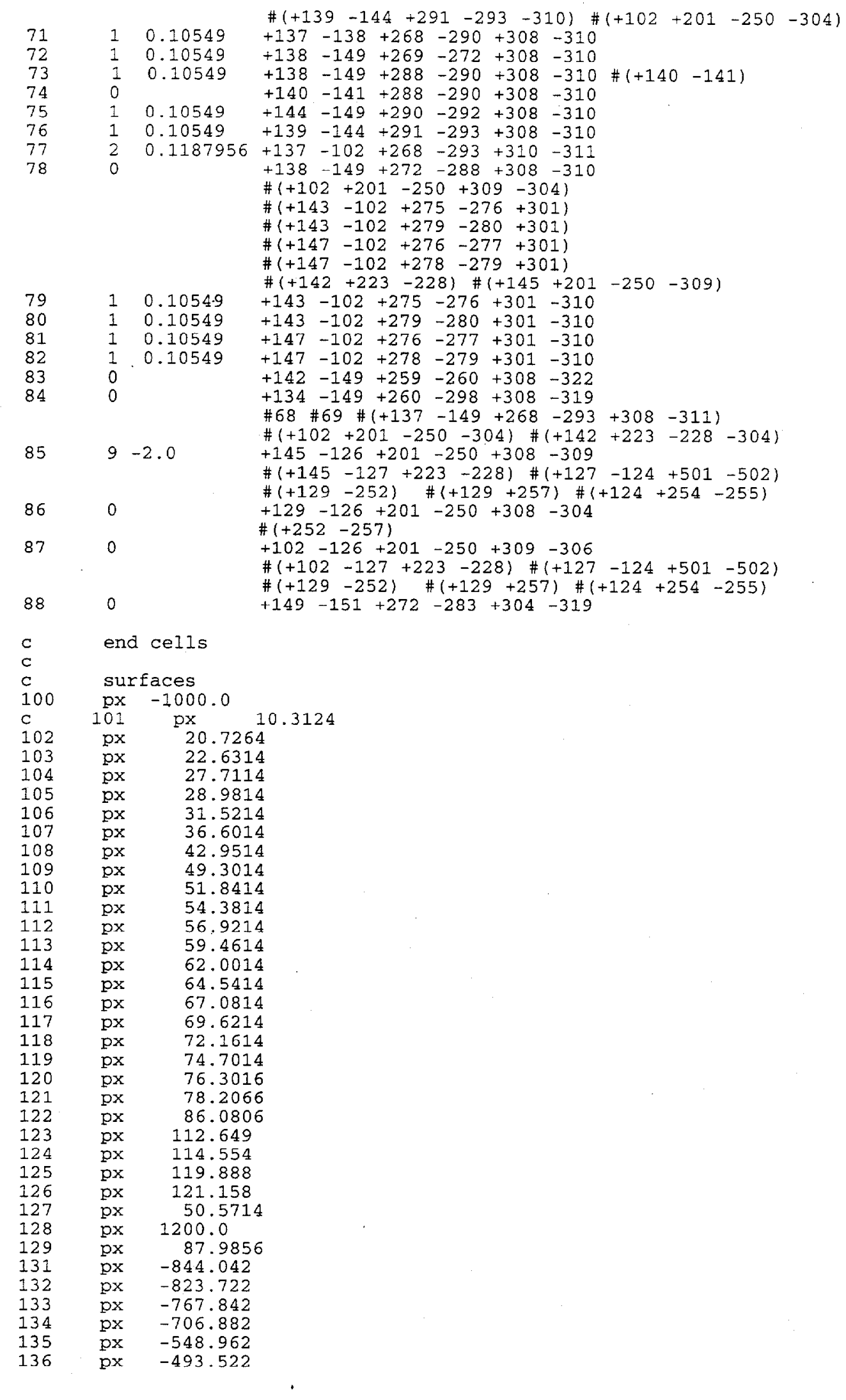




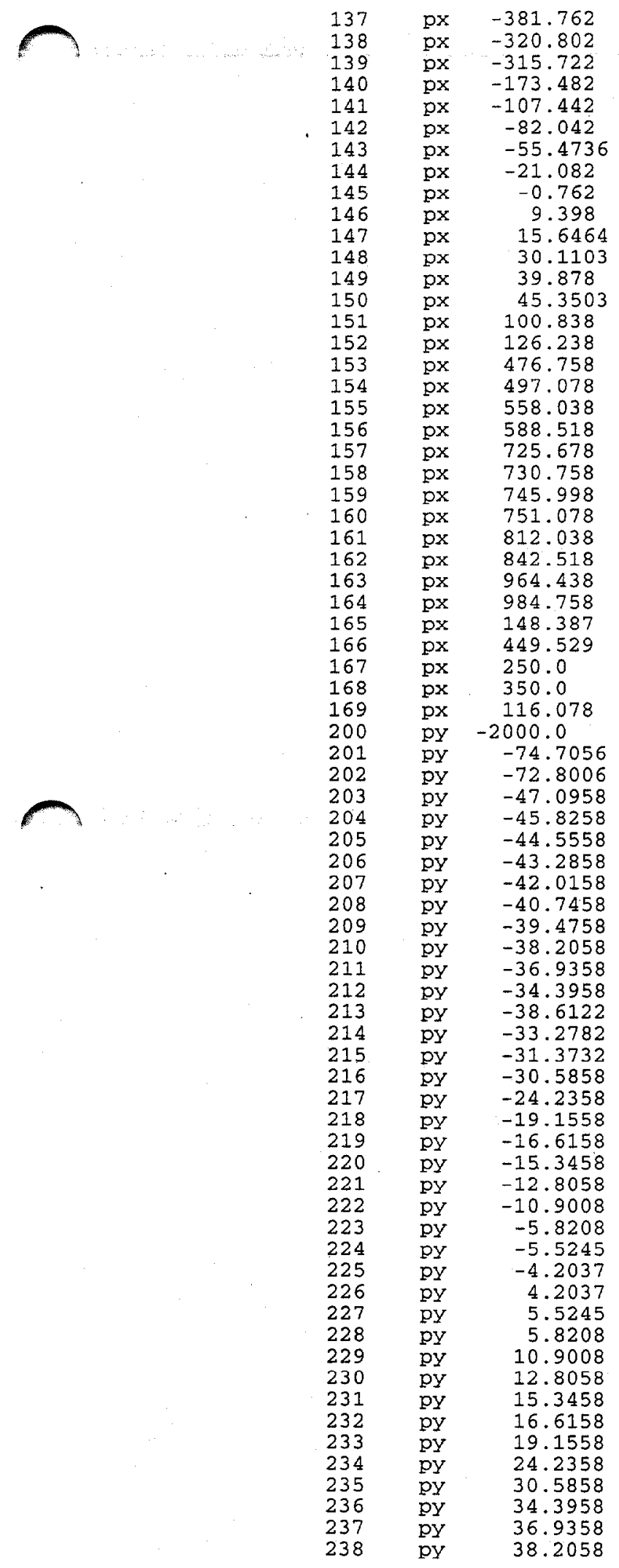




$\begin{array}{llc}239 & \text { py } & 39.4758 \\ 240 & \text { py } & 40.7458 \\ 241 & \text { py } & 42.0158 \\ 242 & \text { py } & 43.2858 \\ 243 & \text { py } & 44.5558 \\ 244 & \text { py } & 45.8258 \\ 245 & \text { py } & 47.0958 \\ 246 & \text { py } & 31.3732 \\ 247 & \text { py } & 33.2782 \\ 248 & \text { py } & 38.6122 \\ 249 & \text { py } & 72.8006 \\ 250 & \text { py } & 74.7056 \\ 251 & \text { py } & 1500.0 \\ 252 & \text { py } & -39.8822 \\ 253 & \text { py } & -9.8848 \\ 254 & \text { py } & -8.6148 \\ 255 & \text { py } & 8.6148 \\ 256 & \text { py } & 9.8848 \\ 257 & \text { py } & 39.8822 \\ 258 & \text { py } & -1731.28 \\ 259 & \text { py } & -1710.96 \\ 260 & \text { py } & -1650.0 \\ 261 & \text { py } & -1528.08 \\ 262 & \text { py } & -1467.12 \\ 263 & \text { py } & -1436.64 \\ 264 & \text { py } & -589.28 \\ 265 & \text { py } & -241.3 \\ 266 & \text { py } & -193.04 \\ 267 & \text { py } & -180.34 \\ 268 & \text { py } & -167.64 \\ 269 & \text { py } & -162.56 \\ 270 & \text { py } & -152.4 \\ 271 & \text { py } & -137.16 \\ 272 & \text { py } & -101.6 \\ 273 & \text { py } & -91.44 \\ 274 & \text { py } & -60.96 \\ 275 & \text { py } & -45.72 \\ 276 & \text { py } & -30.48 \\ 277 & \text { py } & -10.16 \\ 278 & \text { py } & 10.16 \\ 279 & \text { py } & 30.48 \\ 280 & \text { py } & 45.72 \\ 281 & \text { py } & 60.96 \\ 282 & \text { py } & 91.44 \\ 283 & \text { py } & 101.6 \\ 284 & \text { py } & 137.16 \\ 285 & \text { py } & 152.4 \\ 286 & \text { py } & 180.34 \\ 287 & \text { py } & 193.04 \\ 288 & \text { py } & 213.36 \\ 289 & \text { py } & 241.3 \\ 290 & \text { py } & 274.32 \\ 291 & \text { py } & 335.28 \\ 292 & \text { py } & 345.44 \\ 293 & \text { py } & 396.24 \\ 294 & \text { py } & 533.4 \\ 295 & \text { py } & 1235.44 \\ 296 & \text { py } & 1296.4 \\ 297 & \text { py } & 1316.72 \\ 298 & \text { py } & 472.44 \\ 300 & \text { pz } & -500.0 \\ 301 & \text { pz } & -59.478 \\ 303 & \text { pz } & -57.573 \\ 305 & \text { pz } & 245.957 \\ 306 & \text { pz } & 247.862 \\ 307 & \text { pz } & -150.74 .0 \\ 308 & \text { pz } & -125.095 \\ 309 & \text { pz } & -67.451 \\ 310 & \text { pz } & 235.585 \\ 311 & \text { pz } & 276.225 \\ & & \end{array}$




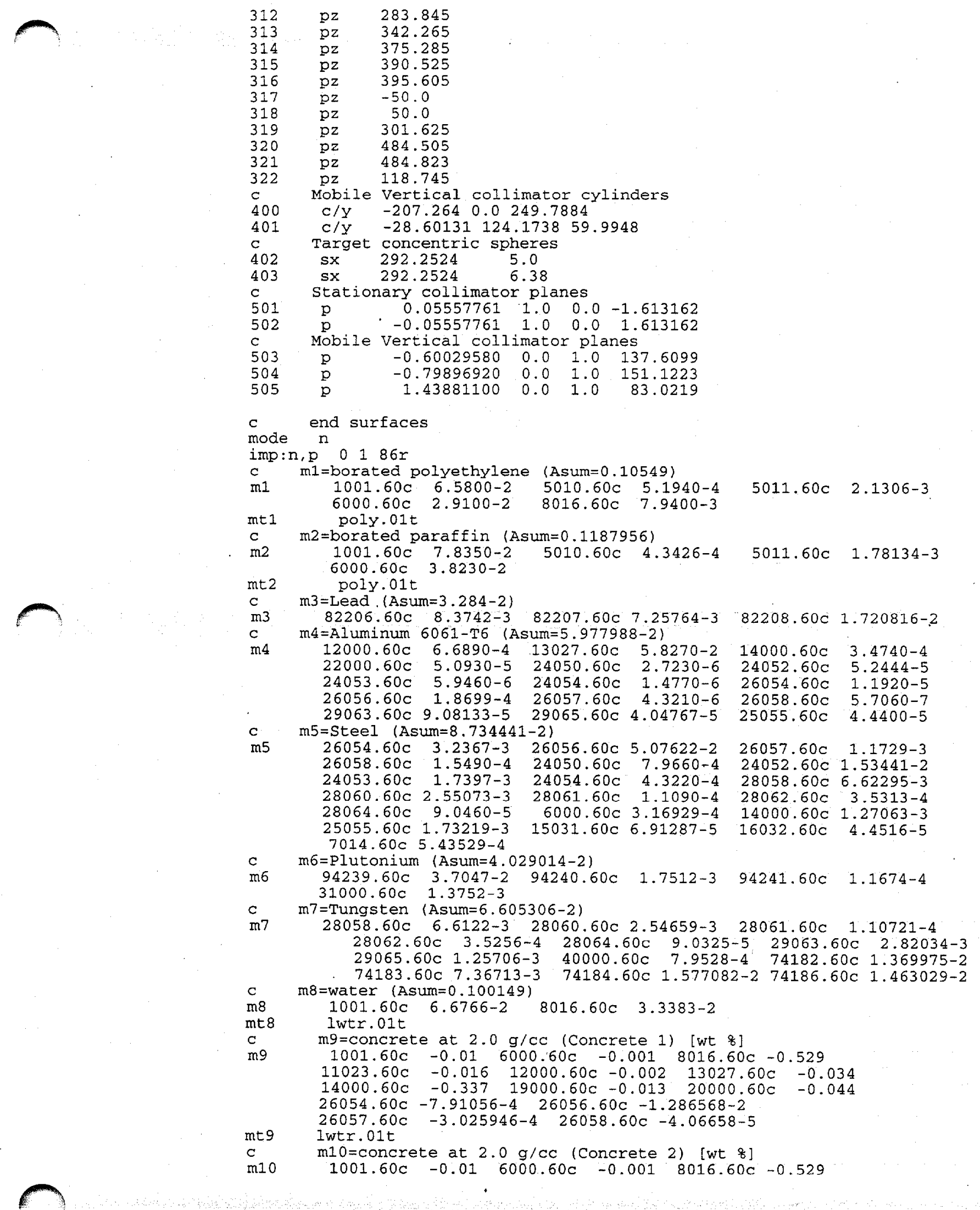




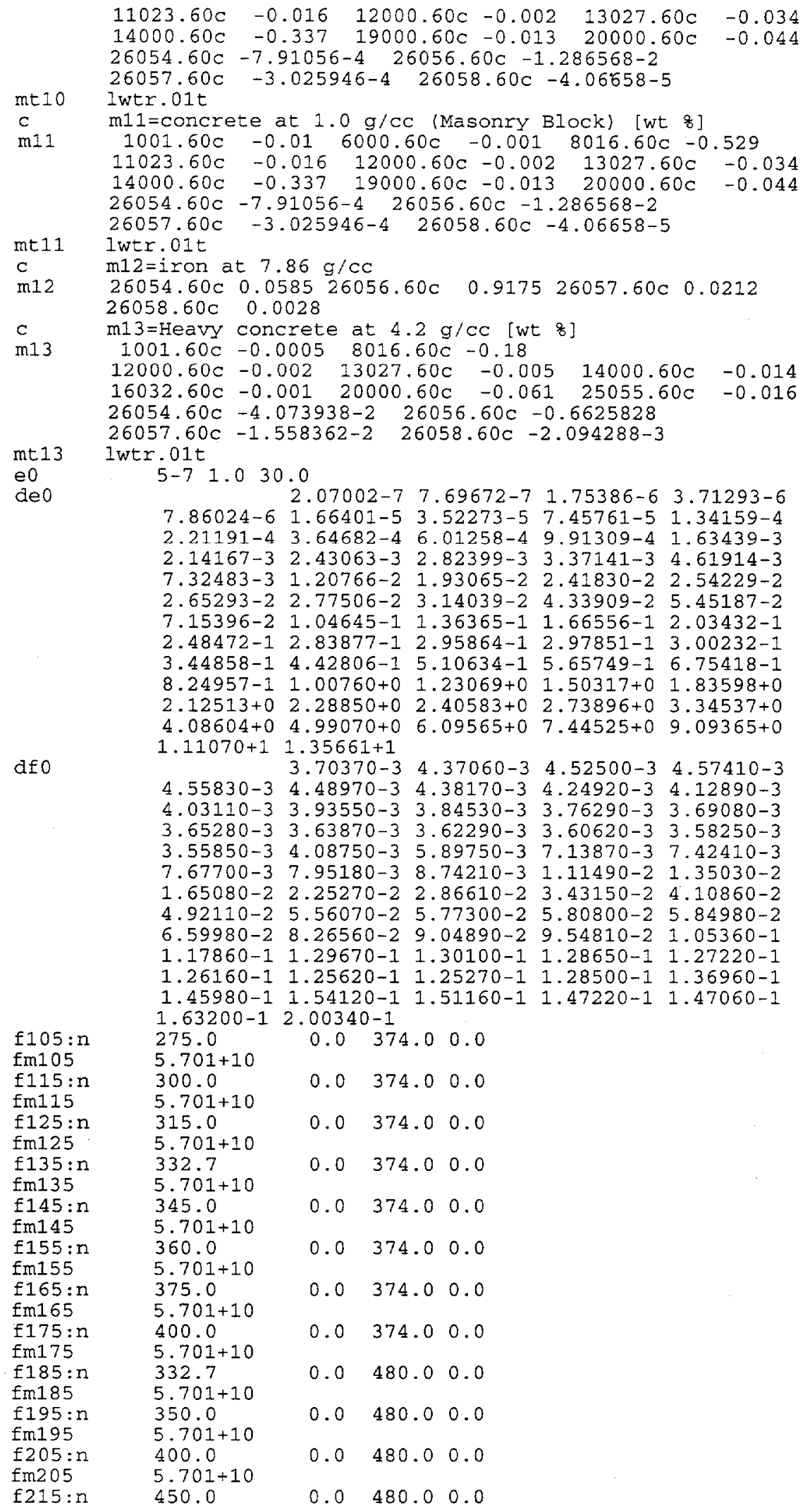




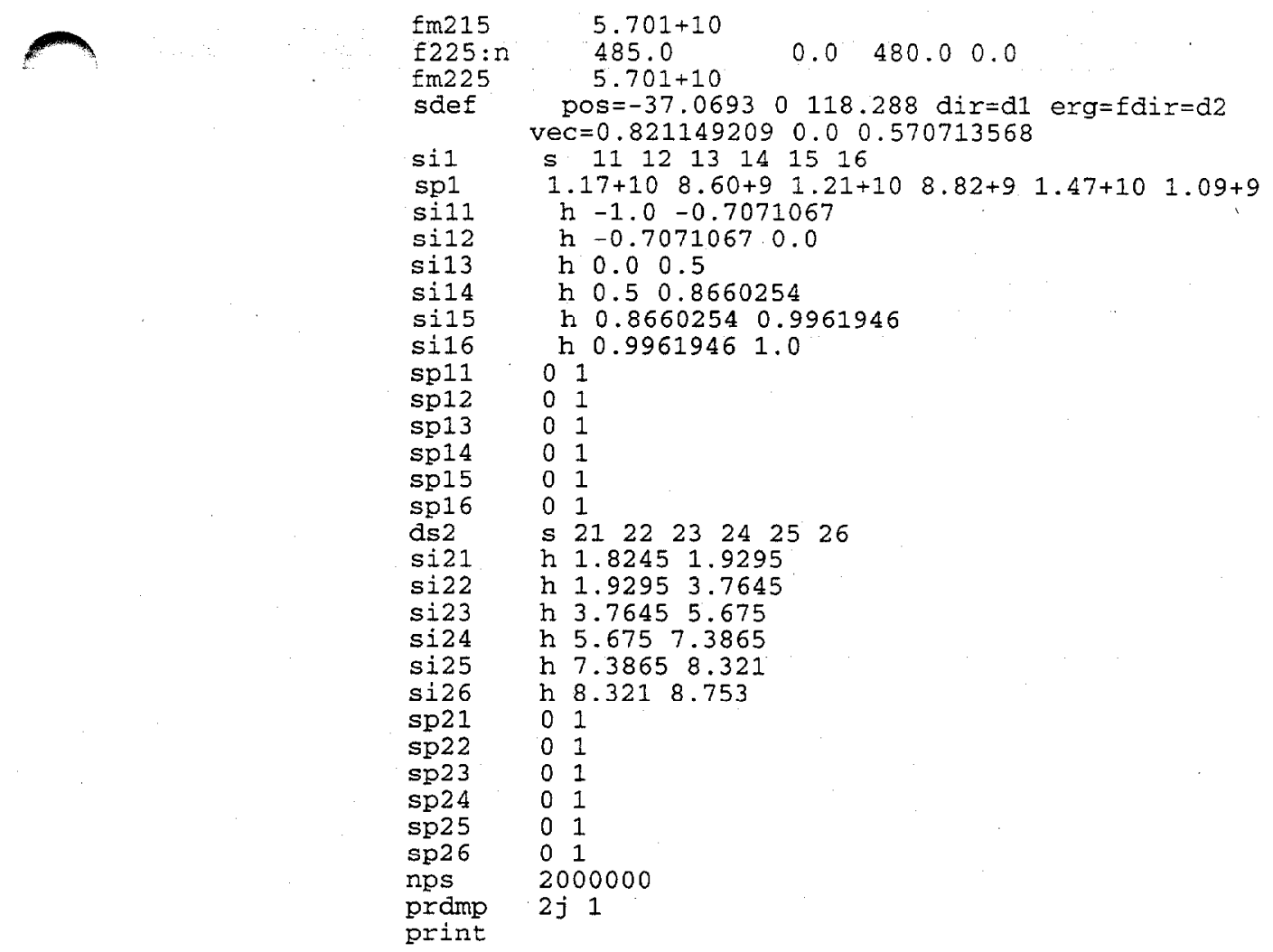


File for MCNP-4B calculation of the dose rate in the PFNA beam and across the truck lane for the source in the horizontal position

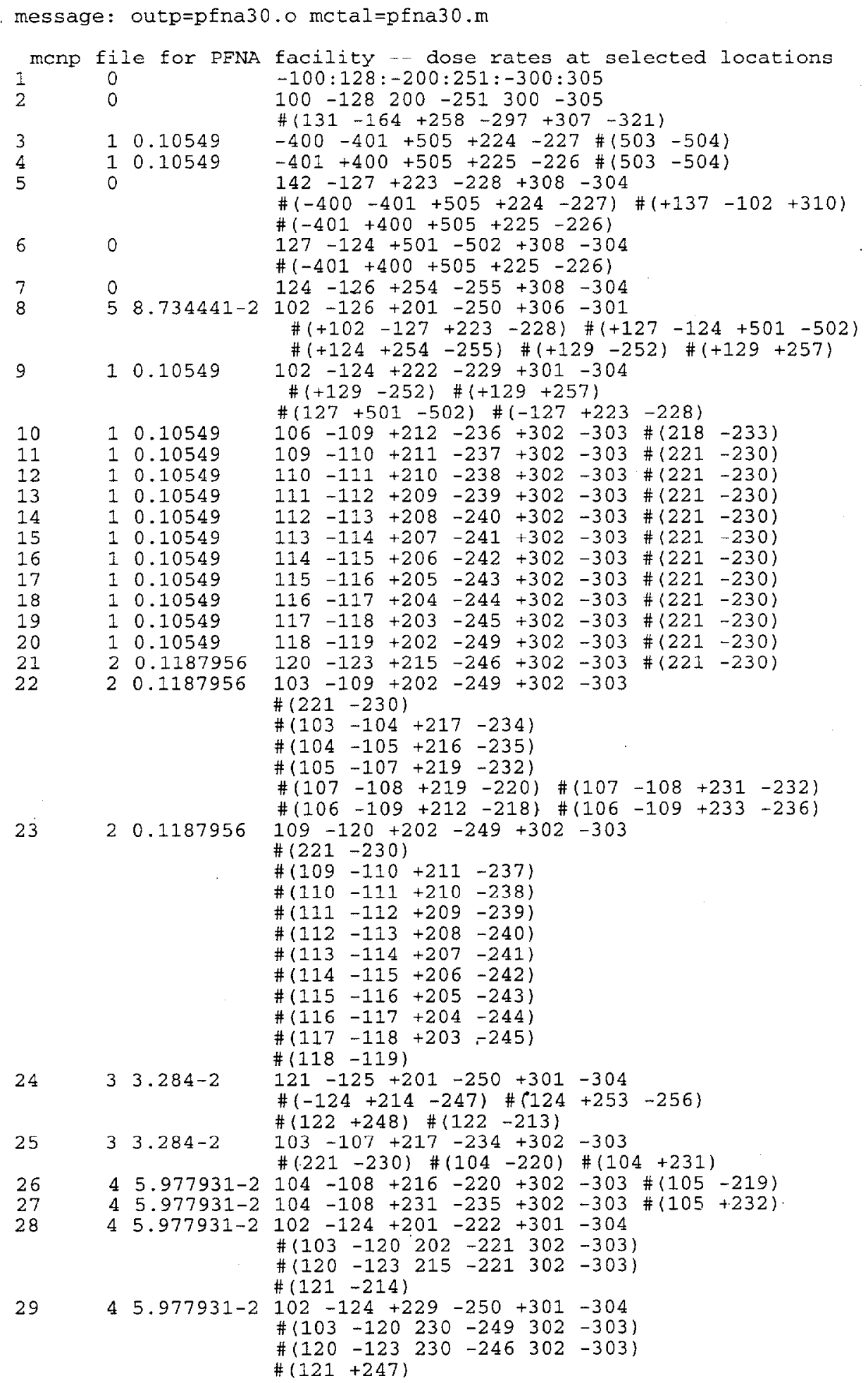




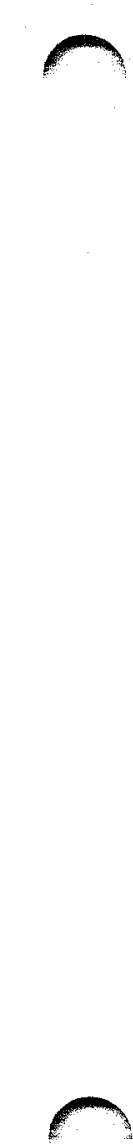

\begin{tabular}{|c|c|c|}
\hline 30 & 4 & $5.977931-2$ \\
\hline 31 & 4 & $5.977931-2$ \\
\hline $\begin{array}{l}32 \\
33\end{array}$ & $\begin{array}{l}0 \\
0\end{array}$ & \\
\hline $\begin{array}{l}34 \\
35 \\
36\end{array}$ & $\begin{array}{l}0 \\
0 \\
4\end{array}$ & $5.977931-2$ \\
\hline $\begin{array}{l}37 \\
38\end{array}$ & $\begin{array}{r}9 \\
11\end{array}$ & $\begin{array}{l}-2.0 \\
-1.0\end{array}$ \\
\hline $\begin{array}{l}39 \\
40\end{array}$ & $\begin{array}{r}12 \\
0\end{array}$ & -7.86 \\
\hline
\end{tabular}

$124-126+252-254+301-304$ $\#(-125+213-253)$

$124-126+255-257+301-304$

$\#(-125+256-248)$

$+167-168+274-281+317-318$

$+165-166+270-285+308-310$

$\#(+167-168+274-281+317-318)$

$-400+505+224-227+503-504$

$-401+400+505+225-226+503-504$

$122-124+201-250+301-304$

\#(213 - 248$)$

\# $(129-252)$

$\#(129+257)$

$+131-164+258-297+307-308$

$+131-164+258-297+308-320$

$\#(+132-163+259-296)$

$+131-164+258-297+320-321$

$+132-163+259-296+319-320$

$\#(+151-169+271-284+304-315)$

$\#(+151-160+270-271+312-314)$

$\#(+151-160+284-285+312-314)$

$\#(+157-159+274-281+313-315)$

$\#(+158-160+274-281-313)$

$\#(+169-157+274-281+314-316)$

$\begin{array}{lrl}41 & 1 & 0.10549 \\ 42 & 1 & 0.10549 \\ 43 & 1 & 0.10549 \\ 44 & 1 & 0.10549 \\ 45 & 1 & 0.10549 \\ 46 & 1 & 0.10549 \\ 47 & 9 & -2.0 \\ 48 & 10 & -2.0\end{array}$

$+151-169+271-284+304-315$

$+151-160+270-271+312-314$

$+151-160+284-285+312-314$

$+157-159+274-281+313-315$

$+158-160+274-281+308-313$

$+169-157+274-281+314-316$

$+160-162+273-282+308-319$

$+154-161+265-289+308-319$

$\# 47 \#(-160+267-286)$

$+154-160+267-286+308-319$ \#42 \#43 \#45

$+155-156+259-260+308-319$

$+155-156+295-296+308-319$

$+154-155+259-296+308-319$

$\#(+265-289)$

$+155-163+259-296+308-319 \# 50$ \#1

$\#(-161+265-289) \cdot \#(+161-162+273-282)$

$+153-154+295-296+308-319$

$+151-126+295-296+308-319$

$+151-126+259-260+308-319$

$+153-154+259-260+308-319$

$+151-152+266-272+308-304$

$+151-152+283-287+308-304$

$+151-154+259-296+308-319$

\#41 \#42 \#43 \#54 \#55 \#56 \#57 \#58 \#59

$\#(+165-166+270-285+308-310)$

$\#\left(\begin{array}{llll}-126+201 & -250-304)\end{array}\right.$

$+149-151+272-201+308-304$

$+149-151+250-283+308-301$

$+149-151+264-290+308-319$

$\#(+272-283)$

$+149-151+259-296+308-319$

$\#(+264-290)$

$+146-149+295-296+308-319$

$+132-149+259-296+308-319$

\#65 \# (+133 -294)

$+133-149+259-294+308-319$

$\#(+142-260-322) \quad \#(+134+260-298)$

$+135-149+261-262+308-319$

$+135-136+262-263+308-319$

$+137-149+268-293+308-311$

$\#(-138-290-310) \quad \#(-102+310)$

$\#(+138+269-290-310) \quad \#(+144+290-292-310)$

$\#(+139-144+291-293-310) \quad \#(+102+201-250-304)$

$+137-138+268-290+308-310$

$+138-149+269-272+308-310$

$+138-149+288-290+308-310 \#(+140-141)$

$+140-141+288-290+308-310$

$+144-149+290-292+308-310$ 


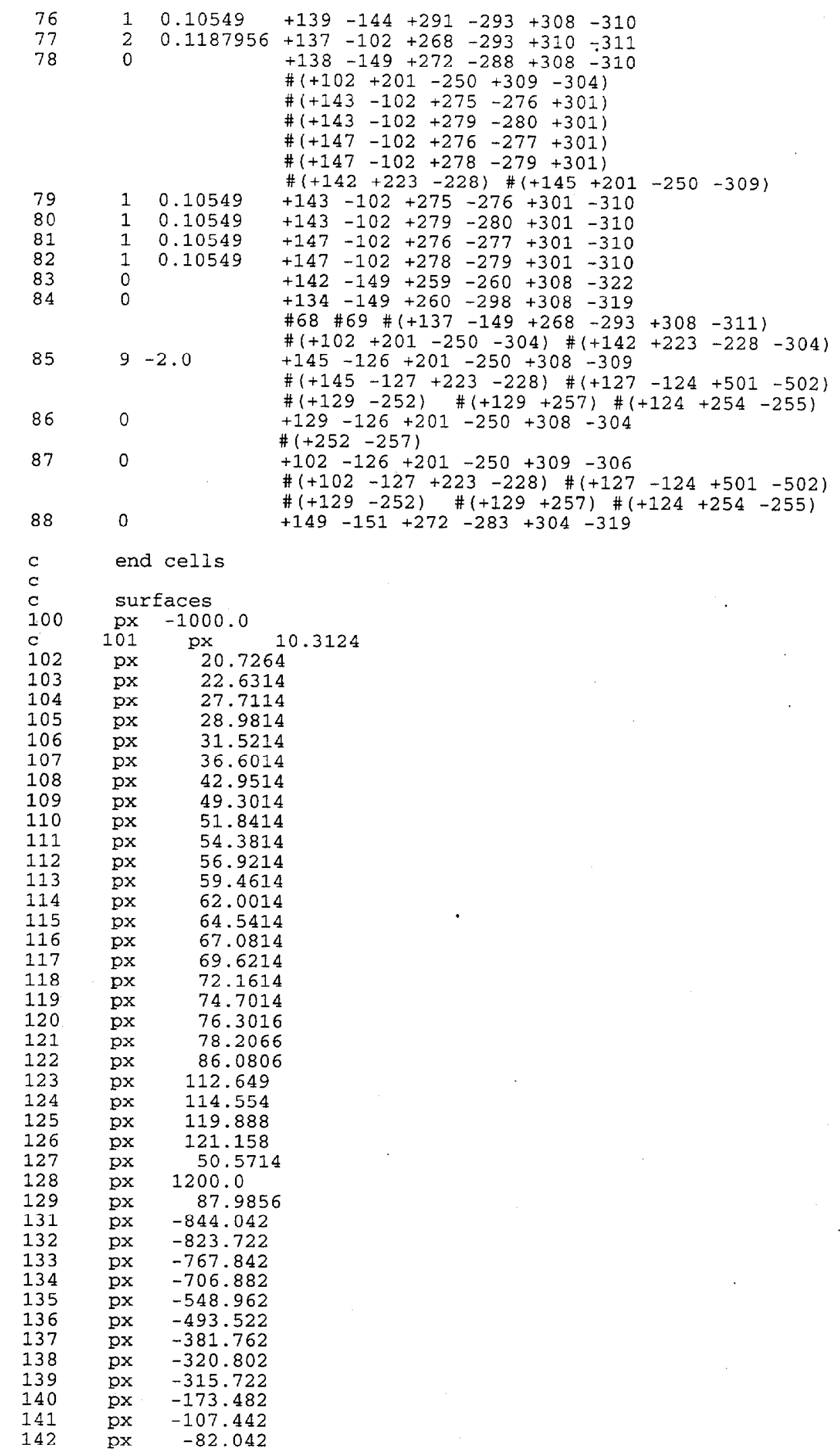




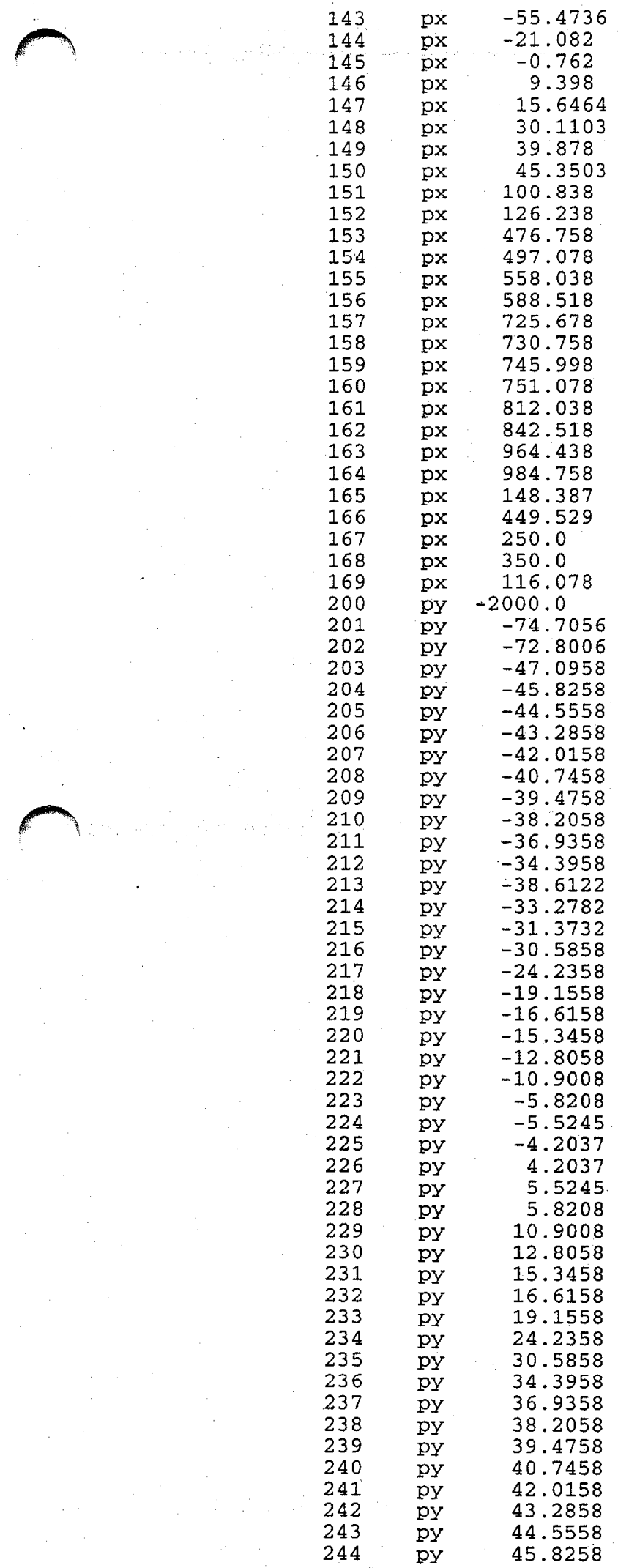




\begin{tabular}{|c|c|c|}
\hline 245 & py & 47.0958 \\
\hline 246 & py & 31.3732 \\
\hline 247 & py & 33.2782 \\
\hline 248 & py & 38.6122 \\
\hline 249 & py & 72.8006 \\
\hline 250 & py & 74.7056 \\
\hline 251 & py & 1500.0 \\
\hline 252 & py & -39.8822 \\
\hline 253 & py & -9.8848 \\
\hline 254 & py & -8.6148 \\
\hline 255 & py & 8.6148 \\
\hline 256 & py & 9.8848 \\
\hline 257 & py & 39.8822 \\
\hline 258 & py & -1731.28 \\
\hline 259 & py & -1710.96 \\
\hline 260 & py & -1650.0 \\
\hline 261 & py & -1528.08 \\
\hline 262 & py & -1467.12 \\
\hline 263 & py & -1436.64 \\
\hline 264 & py & -589.28 \\
\hline 265 & py & -241.3 \\
\hline 266 & py & -193.04 \\
\hline 267 & py & -180.34 \\
\hline 268 & py & -167.64 \\
\hline 269 & py & -162.56 \\
\hline 270 & py & -152.4 \\
\hline 271 & py & -137.16 \\
\hline 272 & py & -101.6 \\
\hline 273 & py & -91.44 \\
\hline 274 & py & -60.96 \\
\hline 275 & py & -45.72 \\
\hline 276 & py & -30.48 \\
\hline 277 & py & -10.16 \\
\hline 278 & py & 10.16 \\
\hline 279 & py & 30.48 \\
\hline 280 & py & 45.72 \\
\hline 281 & py & 60.96 \\
\hline 282 & py & 91.44 \\
\hline 283 & py & 101.6 \\
\hline 284 & py & 137.16 \\
\hline 285 & py & 152.4 \\
\hline 286 & py & 180.34 \\
\hline 287 & py & 193.04 \\
\hline 288 & py & 213.36 \\
\hline 289 & py & 241.3 \\
\hline 290 & py & 274.32 \\
\hline 291 & py & 335.28 \\
\hline 292 & py & 345.44 \\
\hline 293 & py & 396.24 \\
\hline 294 & py & 533.4 \\
\hline 295 & py & 1235.44 \\
\hline 296 & py & 1296.4 \\
\hline 297 & py & 1316.72 \\
\hline 298 & py & 472.44 \\
\hline 300 & $\mathrm{pz}$ & -500.0 \\
\hline 301 & $p z$ & -59.478 \\
\hline 302 & $\mathrm{pz}$ & -57.573 \\
\hline 303 & $\mathrm{pz}$ & 245.957 \\
\hline 304 & $\mathrm{pz}$ & 247.862 \\
\hline 305 & $\mathrm{pz}$ & 500.0 \\
\hline 306 & $\mathrm{pz}$ & -60.748 \\
\hline 307 & $\mathrm{pz}$ & -156.0 \\
\hline 308 & $\mathrm{pz}$ & -125.095 \\
\hline 309 & $\mathrm{pz}$ & -67.451 \\
\hline 310 & $p z$ & 235.585 \\
\hline 311 & $\mathrm{pz}$ & 276.225 \\
\hline 312 & $\mathrm{pz}$ & 283.845 \\
\hline 313 & $\mathrm{pz}$ & 342.265 \\
\hline 314 & $\mathrm{pz}$ & 375.285 \\
\hline 315 & pz & 390.525 \\
\hline 316 & $\mathrm{pz}$ & 395.605 \\
\hline 317 & $\mathrm{pz}$ & -50.0 \\
\hline
\end{tabular}




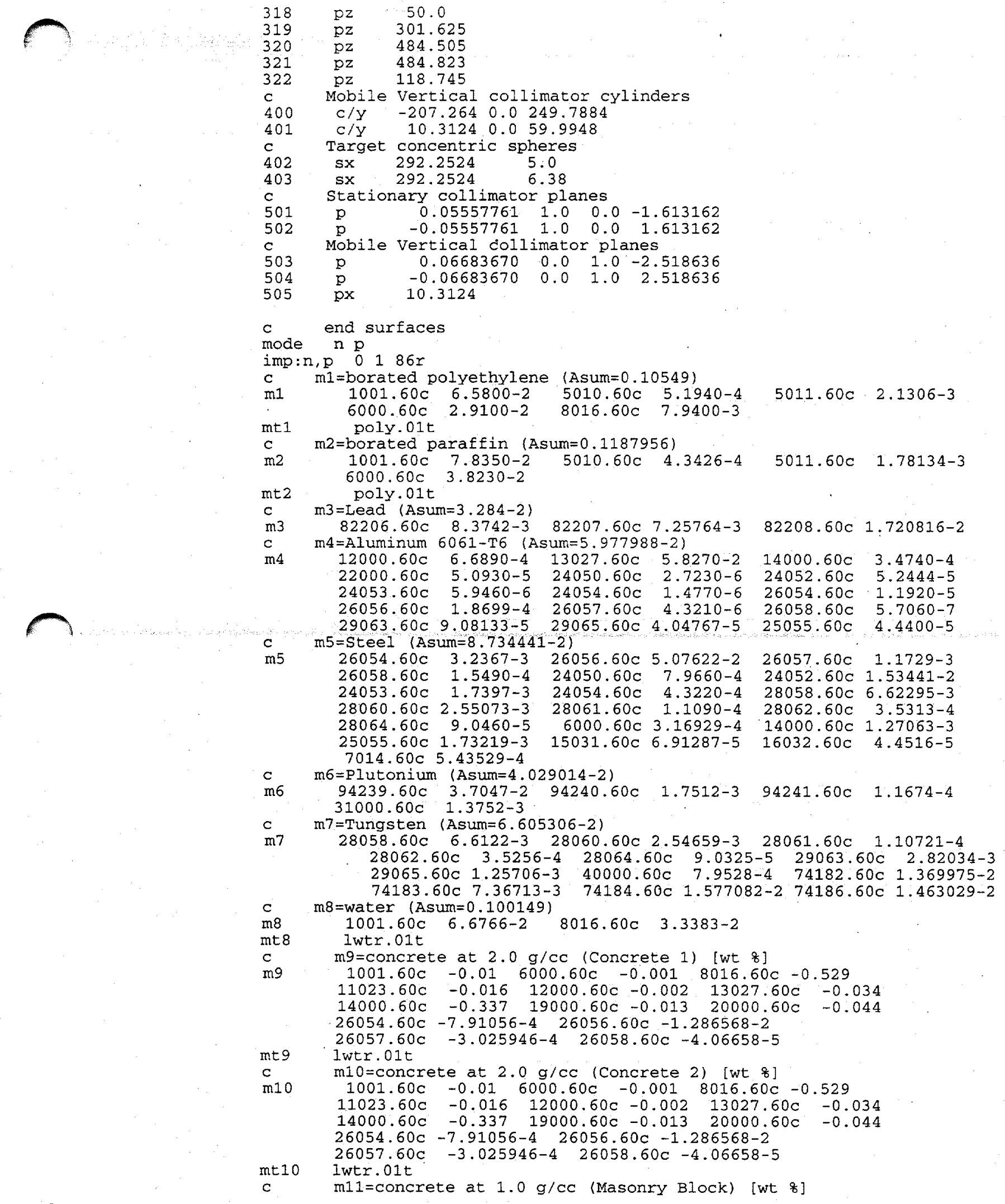




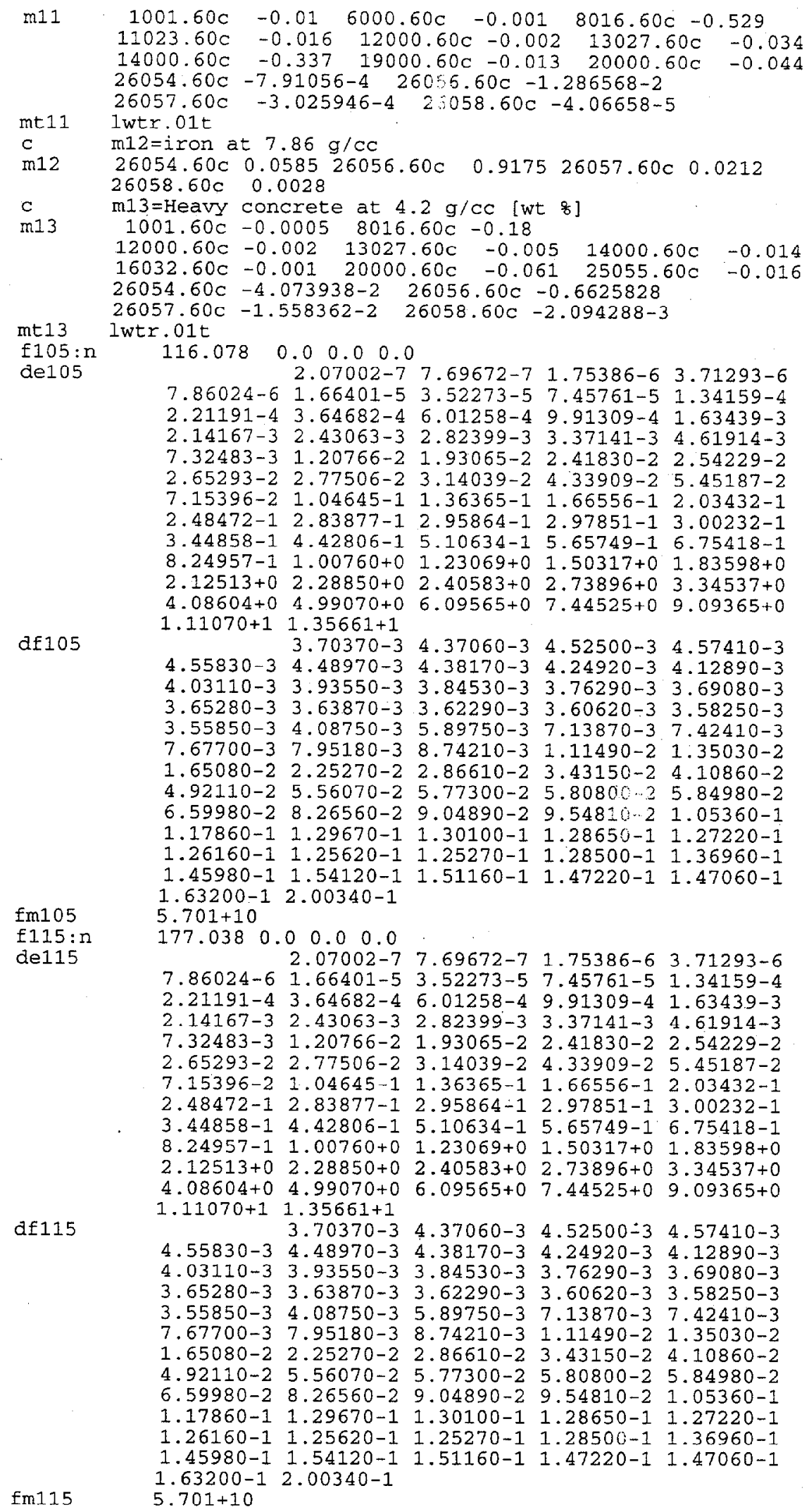


$237.998 \quad 0.0 \quad 0.0 \quad 0.0$

$\begin{array}{cccccc}7.86024-6 & 1.07002-7 & 7.69672-7 & 1.75386-6 & 3.71293-6 \\ & 1.66401-5 & 3.52273-5 & 7.45761-5 & 1 & 34159-4\end{array}$ $2.21191-4 \quad 3.64682-4 \quad 6.01258-4 \quad 9.91309-4 \quad 1.63439-3$ $\begin{array}{llllll}2.14167-3 & 2.43063-3 & 2.82399-3 & 3.37141-3 & 4.61914-3\end{array}$ $7.32483-3 \quad 1.20766-2 \quad 1.93065-2 \quad 2.41830-2 \quad 2.54229-2$ $2.65293-2 \quad 2.77506-2 \quad 3.14039-2 \quad 4.33909-2 \quad 5.45187-2$ $7.15396-2$ 1.04645-1 $1.36365-1.1 .66556-1 \quad 2.03432-1$ $\begin{array}{llllll}2.48472-1 & 2.83877-1 & 2.95864-1 & 2.97851-1 & 3.00232-1\end{array}$ 3.44858-1 4.42806-1 $5.10634-1 \quad 5.65749-1 \quad 6.75418-1$ $8.24957-11.00760+0 \quad 1.23069+0 \quad 1.50317+01.83598+0$ $2.12513+0 \quad 2.28850+0 \quad 2.40583+0 \quad 2.73896+0 \quad 3.34537+0$ $4.08604+0 \quad 4.99070+0 \quad 6.09565+0 \quad 7.44525+0 \quad 9.09365+0$ $1.11070+1 \quad 1.35661+1$

df125 $\quad 3.70370-3 \quad 4.37060-3 \quad 4.52500-3 \quad 4.57410-3$ $\begin{array}{llllll}4.55830-3 & 4.48970-3 & 4.38170-3 & 4.24920-3 & 4.12890-3\end{array}$ $\begin{array}{llllll}4.03110-3 & 3.93550-3 & 3.84530-3 & 3.76290-3 & 3.69080-3\end{array}$ $3.65280-3 \quad 3.63870-3 \quad 3.62290-3 \quad 3.60620-3 \quad 3.58250-3$

$\begin{array}{lllll}3.55850-3 & 4.08750-3 & 5.89750-3 & 7.13870-3 & 7.42410-3\end{array}$

$7.67700-3 \quad 7.95180-3 \quad 8.74210-3 \quad 1.11490-2 \quad 1.35030-2$

$1.65080-2 \quad 2.25270-2 \quad 2.86610-2 \quad 3.43150-2 \quad 4.10860-2$

$4.92110-2 \quad 5.56070-2 \quad 5.77300-2 \quad 5.80800-2 \quad 5.84980-2$. $6.59980-2 \quad 8.26560-2 \quad 9.04890-2 \quad 9.54810-2 \quad 1.05360-1$ $1.17860-1 \quad 1.29670-1 \quad 1.30100-1 \quad 1.28650-1 \quad 1.27220-1$ $1.26160-1 \quad 1.25620-1 \quad 1.25270-1 \quad 1.28500-1$ 1.36960-1 $1.45980-1$ 1.54120-1 $1.51160-1 \quad 1.47220-1 \quad 1.47060-1$ $1.63200-12.00340-1$

$\mathrm{fm} 125 \quad 5.701+10$

f135:n $\quad 359.918 \quad 0.0 \quad 0.0 \quad 0.0$

$\begin{array}{lllllll}\operatorname{de} 135 & 2.07002-7 & 7.69672-7 & 1.75386-6 & 3.71293-6\end{array}$

7.86024-6 $1.66401-5 \quad 3.52273-5 \quad 7.45761-5 \quad 1.34159-4$

$2.21191-4 \quad 3.64682-4 \quad 6.01258-4 \quad 9.91309-4 \quad 1.63439-3$

2.14167-3 2.43063-3 $2.82399-3 \quad 3.37141-3 \quad 4.61914-3$

7.32483-3 $1.20766-2 \quad 1.93065-2 \quad 2.41830-2 \quad 2.54229-2$

2.65293-2 2.77506-2 3.14039-2 4.33909-2 5.45187-2

7.15396-2 $1.04645-1 \quad 1.36365-1 \quad 1.66556-1 \quad 2.03432-1$

$\begin{array}{llllll}2.48472-1 & 2.83877-1 & 2.95864-1 & 2.97851-1 & 3.00232-1\end{array}$

3.44858-1 $4.42806-1 \quad 5.10634-1 \quad 5.65749-1 \quad 6.75418-1$

$8.24957-1 \quad 1.00760+0 \quad 1.23069+0 \quad 1.50317+0 \quad 1.83598+0$

$2.12513+0 \quad 2.28850+0 \quad 2.40583+0 \quad 2.73896+0 \quad 3.34537+0$

$4.08604+0 \quad 4.99070+0 \quad 6.09565+0 \quad 7.44525+0 \quad 9.09365+0$

$1.11070+11.35661+1$

df135 $3.70370-3 \quad 4.37060-3 \quad 4.52500-3 \quad 4.57410-3$

$4.55830-3 \quad 4.48970-3 \quad 4.38170-3 \quad 4.24920-3 \quad 4.12890-3$

$4.03110-3 \quad 3.93550-3 \quad 3.84530-3 \quad 3.76290-3 \quad 3.69080-3$

$\begin{array}{llllll}3.65280-3 & 3.63870-3 & 3.62290-3 & 3.60620-3 & 3.58250-3\end{array}$

$3.55850-3 \quad 4.08750-3 \quad 5.89750-3 \quad 7.13870-3 \quad 7.42410-3$

$7.67700-3 \quad 7.95180-3 \quad 8.74210-3 \quad 1.11490-2 \quad 1.35030-2$

$1.65080-2 \quad 2.25270-2 \quad 2.86610-2 \quad 3.43150-2 \quad 4.10860-2$

$4.92110-2 \quad 5.56070-2 \quad 5.77300-2 \quad 5.80800-2 \quad 5.84980-2$

$6.59980-2 \quad 8.26560-2 \quad 9.04890-2 \quad 9.54810-2 \quad 1.05360-1$

$1.17860-1 \quad 1.29670-1 \quad 1.30100-1 \quad 1.28650-11.27220-1$ $1.26160-1 \quad 1.25620-1 \quad 1.25270-1 \quad 1.28500-1 \quad 1.36960-1$ 1.45980-1 $1.54120-1 \quad 1.51160-1 \quad 1.47220-1 \quad 1.47060-1$ $1.63200-12.00340-1$

$\operatorname{fm} 135: 5.701+10$

f145: $\quad 420.878 \quad 0.0 \quad 0.0 \quad 0.0$

$\begin{array}{llllll}\text { de145 } & 2.07002-7 & 7.69672-7 & 1.75386-6 & 3.71293-6\end{array}$

$7.86024-6 \quad 1.66401-5 \quad 3.52273-5 \quad 7.45761-5 \quad 1.34159-4$

$2.21191-4 \quad 3.64682-4 \quad 6.01258-4 \quad 9.91309-4 \quad 1.63439-3$

2.14167-3 $2.43063-3 \quad 2.82399-3 \quad 3.37141-3 \quad 4.61914-3$

$\begin{array}{llllll}7.32483-3 & 1.20766-2 & 1.93065-2 & 2.41830-2 & 2.54229-2\end{array}$

$2.65293-2 \quad 2.77506-2 \quad 3.14039-2 \quad 4.33909-2 \quad 5.45187-2$

7.15396-2 $1.04645-1 \quad 1.36365-1 \quad 1.66556-1 \quad 2.03432-1$

2.48472-1 $2.83877-1 \quad 2.95864-1 \quad 2.97851-1 \quad 3.00232-1$

3.44858-1 4.42806-1 5.10634-1 $5.65749-1$ 6.75418-1 $8.24957-1 \quad 1.00760+0 \quad 1.23069+0 \quad 1.50317+0 \quad 1.83598+0$ $2.12513+0 \quad 2.28850+0 \quad 2.40583+0 \quad 2.73896+0 \quad 3.34537+0$ $4.08604+0.4 .99070+0 \quad 6.09565+0 \quad 7.44525+0 \quad 9.09365+0$ $1.11070+1 \quad 1.35661+1$

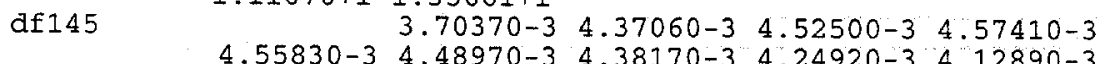


$\begin{array}{llllll}4.03110-3 & 3.93550-3 & 3.84530-3 & 3.76290-3 & 3.69080-3\end{array}$ $3.65280-3 \quad 3.63870-3 \quad 3.62290-3 \quad 3.60620-3 \quad 3.58250-3$ $\begin{array}{llllll}3.55850-3 & 4.08750-3 & 5.89750-3 & 7.13870-3 & 7.42410-3\end{array}$ $\begin{array}{lllll}7.67700-3 & 7.95180-3 & 8.74210-3 & 1.11490-2 & 1.35030-2\end{array}$

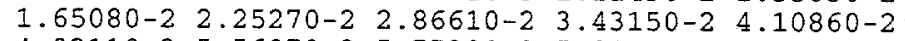
$4.92110-2 \quad 5.56070-2 \quad 5.77300-2 \quad 5.80800-2 \quad 5.84980-2$ $6.59980-2 \quad 8.26560-2 \quad 9.04890-2 \quad 9.54810-2 \quad 1.05360-1$ $1.17860-1 \quad 1.29670-1 \quad 1.30100-1 \quad 1.28650-11.27220-1$ $1.26160-1 \quad 1.25620-1 \quad 1.25270-1 \quad 1.28500-1 \quad 1.36960-1$ $1.45980-1$ 1.54120-1 $1.51160-1$ 1.47220-1 1.47060-1 $1.63200-12.00340-1$

$\operatorname{fm} 145$

$5.701+10$

f155:n 481.838

$\begin{array}{lllll}0.0 & 0.0 & 0.0\end{array}$

de155

df155

$\begin{array}{llll}2.07002-7 & 7.69672-7 & 1.75386-6 & 3.71293-6\end{array}$

$\begin{array}{lllll}1.66401-5 & 3.52273-5 & 7.45761-5 & 1.34159-4\end{array}$

$2.21191-4 \quad 3.64682-4 \quad 6.01258-4 \quad 9.91309-4 \quad 1.63439-3$ 2.14167-3 $2.43063-3 \quad 2.82399-3 \quad 3.37141-3 \quad 4.61914-3$ 7.32483-3 1.20766-2 $1.93065-2 \quad 2.41830-2 \quad 2.54229-2$ $\begin{array}{lllll}2.65293-2 & 2.77506-2 & 3.14039-2 & 4.33909-2 & 5.45187-2\end{array}$ 7.15396-2 $1.04645-1 \quad 1.36365-1 \quad 1.66556-1 \quad 2.03432-1$ $\begin{array}{llllll}2.48472-1 & 2.83877-1 & 2.95864-1 & 2.97851-1 & 3.00232-1\end{array}$ 3.44858-1 $4.42806-1 \quad 5.10634-1 \quad 5.65749-1 \quad 6.75418-1$ $\begin{array}{llllll}8.24957-1 & 1.00760+0 & 1.23069+0 & 1.50317+0 & 1.83598+0\end{array}$ $2.12513+0 \quad 2.28850+0 \quad 2.40583+0 \quad 2.73896+0.3 .34537+0$ $4.08604+0 \quad 4.99070+0 \quad 6.09565+0 \quad 7.44525+0 \quad 9.09365+0$ $1.11070+11.35661+1$ 3.70370-3 $4.37060-3 \quad 4.52500-3 \quad 4.57410-3$ $4.55830-3 \quad 4.48970-3 \quad 4.38170-3.4 .24920-3 \quad 4.12890-3$ $4.03110-3 \quad 3.93550-3 \quad 3.81530-3 \quad 3.76290-3 \quad 3.69080-3$ $3.65280-3 \quad 3.63870-3 \quad 3.62290-3 \quad 3.60620-3 \quad 3.58250-3$ $\begin{array}{llllll}3.55850-3 & 4.08750-3 & 5.89750-3 & 7.13870-3 & 7.42410-3\end{array}$ $\begin{array}{lllll}7.67700-3 & 7.95180-3 & 8.74210-3 & 1.11490-2 & 1.35030-2\end{array}$ $1.65080-2 \quad 2.25270-2 \quad 2.86610-2 \quad 3.43150-2 \quad 4.10860-2$ $4.92110-2 \quad 5.56070-2 \quad 5.77300-2 \quad 5.80800-2 \quad 5.84980-2$ $6.59980-2 \quad 8.26560-2 \quad 9.04890-2 \quad 9.54810-2 \quad 1.05360-1$ 1.17860-1 $1.29670-1 \quad 1.30100-1 \quad 1.28650-1 \quad 1.27220-1$ 1.26160-1 $1.25620-1 \quad 1.25270-1 \quad 1.28500-1 \quad 1.36960-1$ $1.45980-1$ 1.54120-1 $1.51160-1$ 1.47220-1 1.47060-1 $1.63200-12.00340-1$

fm155

$£ 205: p$

$5.701+10$

de205

d. 205

$116.078 \cdot 0.0 \quad 0.0 \quad 0.0$

$1.50000-2 \quad 3.2500$ $8.50000-1 \quad 1.25000+0 \quad 1.75000+0 \quad 2.25000+0$ $\begin{array}{lllll}2.75000+0 & 3.50000+0 & 4.50000+0 & 5.50000+0 & 6.50000+0\end{array}$ $7.25000+0 \quad 7.75000+0 \quad 9.00000+0 \quad 1.20000+1$ $2.14390-3 \quad 5.77600-4 \quad 2.71850-4 \quad 2.68170-4$

$3.27670-4 \quad 5.66760-4 \quad 8.75940-4 \quad 1.08450-3 \quad 1.27970-3$ $\begin{array}{lllll}1.44170-3 & 1.75630-3 & 2.31560-3 & 2.92700-3 & 3.46860-3\end{array}$ $\begin{array}{llllll}3.95960-3 & 4.62210-3 & 5.41370-3 & 6.19090-3 & 6.92650-3\end{array}$ $\begin{array}{llll}7.47830-3 & 7.84680-3 & 8.77160-3 & 1.10200-2\end{array}$

fm205 $\quad 5.701+10$

f215:p $\quad 177.038 \quad 0.0 \quad 0.0 \quad 0.0$

de 215

df 215

$177.038 \quad 0.00 .00 .0$ $\begin{array}{llllll} & & \end{array}$ $6.50000-18.50000-1 \quad 1.25000+0 \quad 1.75000+0 \quad 2.25000+0$ $2.75000+0 \quad 3.50000+0 \quad 4.50000+0.5 .50000+0 \quad 6.50000+0$ $7.25000+0 \quad 7.75000+0 \quad 9.00000+0 \quad 1.20000+1$

$2.14390-3 \quad 5.77600-4 \quad 2.71850-4 \quad 2.68170-4$

$\begin{array}{lllll}3.27670-4 & 5.66760-4 & 8.75940-4 & 1.08450-3 & 1.27970-3\end{array}$ $1.44170-3 \quad 1.75630-3 \quad 2.31560-3 \quad 2.92700-3 \quad 3.46860-3$ $3.95960-3 \quad 4.62210-3 \quad 5.41370-3 \cdot 6.19090-3 \quad 6.92650-3$ $\begin{array}{llll}7.47830-3 & 7.84680-3 & 8.77160-3 & 1.10200-2\end{array}$

$\operatorname{fm} 215 \quad 5.701+10$

f225:p $\quad 237.998 \quad 0.0 \quad 0.0 \quad 0.0$

de225 $1.50000-2 \quad 3.25000-2 \quad 5.75000-2 \quad 8.50000-2$ $1.25000-1 \quad 2.25000-1 \quad 3.50000-1 \quad 4.55000-1 \quad 5.55000-1$ $6.50000-1 \quad 8.50000-1 \quad 1.25000+0 \quad 1.75000+0 \quad 2.25000+0$ $2.75000+0 \quad 3.50000+0 \quad 4.50000+0 \quad 5.50000+0 \quad 6.50000+0$ $7.25000+0 \quad 7.75000+0 \quad 9.00000+0 \quad 1.20000+1$

$\mathrm{d} £ 225$ $\begin{array}{llll}2.14390-3 & 5.77600-4 & 2.71850-4 & 2.68170-4\end{array}$ $3.27670-4 \quad 5.66760-4 \quad 8.75940-4 \quad 08450-3 \quad 1.27970-3$ 
$\begin{array}{llllll}1.44170-3 & 1.75630-3 & 2.31560-3 & 2.92700-3 & 3.46860-3\end{array}$

$\begin{array}{llllll}3.95960-3 & 4.62210-3 & 5.41370-3 & 6.19090-3 & 6.92650-3\end{array}$

$7.47830-3 \quad 7.84680-38.77160-3 \quad 1.10200-2$

fm225 $\quad 5.701+10$

f235:p $\quad 359.918 \quad 0.0 \quad 0.0 \quad 0.0$

de235

$1.50000-2 \quad 3.25000-2 \quad 5.75000-2 \quad 8.50000-2$

$\begin{array}{lllll}1.25000-1 & 2.25000-1 & 3.50000-1 & 4.55000-1 & 5.55000-1\end{array}$

$\begin{array}{llllll}6.50000-1 & 8.50000-1 & 1.25000+0 & 1.75000+0 & 2.25000+0\end{array}$

$2.75000+0 \quad 3.50000+0 \quad 4.50000+0 \quad 5.50000+0 \quad 6.50000+0$

$7.25000+07.75000+0 \quad 9.00000+01.20000+1$

$\begin{array}{llllll}\text { df235 } & & 2.14390-3 & 5.77600-4 & 2.71850-4 & 2.68170-4 \\ & 3.27670-4 & 5.66760-4 & 8.75940-4 & 1.08450-3 & 1.27970-3\end{array}$

$\begin{array}{llllll}1.44170-3 & 1.75630-3 & 2.31560-3 & 2.92700-3 & 3.46860-3\end{array}$

$\begin{array}{lllll}3.95960-3 & 4.62210-3 & 5.41370-3 & 6.19090-3 & 6.92650-3\end{array}$

$\begin{array}{llll}7.47830-3 & 7.84680-3 & 8.77160-3 & 1.10200-2\end{array}$

fm235 $\quad 5.701+10$

f245:p $\quad 420.878 \quad 0.00 .00 .0$

$\operatorname{de} 245 \quad 1.50000-2 \quad 3.25000-2 \quad 5.75000-28.50000-2$

$1.25000-1 \quad 2.25000-1 \quad 3.50000-1 \quad 4.55000-1 \quad 5.55000-1$

$6.50000-1 \quad 8.50000-1 \quad 1.25000+0 \quad 1.75000+0 \quad 2.25000+0$

$2.75000+0 \quad 3.50000+0 \quad 4.50000+0 \quad 5.50000+0 \quad 6.50000+0$

df 245

$2.14390-3 \quad 5.77600-4 \quad 2.71850-4 \quad 2.68170-4$

$\begin{array}{lllll}3.27670-4 & 5.66760-4 & 8.75940-4 & 1.08450-3 & 1.27970-3\end{array}$

$1.44170-3 \quad 1.75630-3 \quad 2.31560-3 \quad 2.92700-3 \quad 3.46860-3$

$\begin{array}{lllll}3.95960-3 & 4.62210-3 & 5.41370-3 & 6.19090-3 & 6.92650-3\end{array}$

$7.47830-3 \quad 7.84680-3 \quad 8.77160-3 \quad 1.10200-2$

fm2 $255 \quad 5.701+10$

f255:D $\quad 481.838 \quad 0.0 \quad 0.0 \quad 0.0$

de255 $1.50000-2 \quad 3.25000-2 \quad 5.75000-2 \quad 8.50000-2$

$\begin{array}{llllll}1.25000-1 & 2.25000-1 & 3.50000-1 & 4.55000-1 & 5.55000-1\end{array}$

$\begin{array}{llllll}6.50000-1 & 8.50000-1 & 1.25000+0 & 1.75000+0 & 2.25000+0\end{array}$

$2.75000+0 \quad 3.50000+0 \quad 4.50000+0 \quad 5.50000+0 \quad 6.50000+0$

$7.25000+0 \quad 7.75000+0 \quad 9.00000+0 \quad 1.20000+1$

$\begin{array}{llllll}\text { df255 } 2.14390-3 & 5.77600-4 & 2.71850-4 & 2.68170-4\end{array}$

$3.27670-4 \quad 5.66760-4 \quad 8.75940-4 \quad 1.08450-3 \quad 1.27970-3$

$\begin{array}{lllll}1.44170-3 & 1.75630-3 & 2.31560-3 & 2.92700-3 & 3.46860-3\end{array}$

$\begin{array}{lllll}3.95960-3 & 4.62210-3 & 5.41370-3 & 6.19090-3 & 6.92650-3\end{array}$

$7.47830-37.84680-38.77160-31.10200-2$

$\operatorname{fm} 255 \quad 5.701+10$

sdef pos $=0$ o 0 vec $=1$ o 0 dir $=d 1$ erg=fdir $=d 2$

$\begin{array}{lllllllll}\text { sil s } & 11 & 12 & 13 & 14 & 15 & 16\end{array}$

$\begin{array}{lllllll}\operatorname{sp} 1 & 1.17+10 & 8.60+9 & 1.21+10 & 8.82+9 & 1.47+10 & 1.09+9\end{array}$

$\operatorname{sill} \quad h-1.0-0.7071067$

si12 h -0.70710670 .0$

$\operatorname{si13} h \quad h .00 .5$

si14. h $0.5 \quad 0.8660254$

si15 h $0.8660254 \quad 0.9961946$

si16 h 0.99619461 .0

sp11. 01

$\operatorname{sp12} \quad 0 \quad 1$

sp13 01

$\operatorname{sp14} \quad 0 \quad 1$

$\operatorname{sp15} \quad 0 \quad 1$

sp16 01

ds2 s $21 \quad 22 \quad 232425 \quad 26$

si21 h 1.82451 .9295

si22 h 1.92953 .7645

si23 h $3.7645 \quad 5.675$

si24 h 5.6757 .3865

si25 h 7.38658 .321

si26 h $8.321 \quad 8.753$

$\operatorname{sp2} 1001$

$\operatorname{sp22} \quad 0 \quad 1$

$\operatorname{sp} 23 \quad 0 \quad 1$

$\operatorname{sp} 24 \quad 0 \quad 1$

$\operatorname{sp25} \quad 0 \quad 1$

sp26 01

nps 2000000

promp $2 j 1$

print 
File for MCNP-4B calculation of the dose rate in the PFNA beam and across

the truck lane for the source in the maximum up position

message: outp=p $\operatorname{ma} 31.0$ metal $=$ pfna31. $\mathrm{m}$

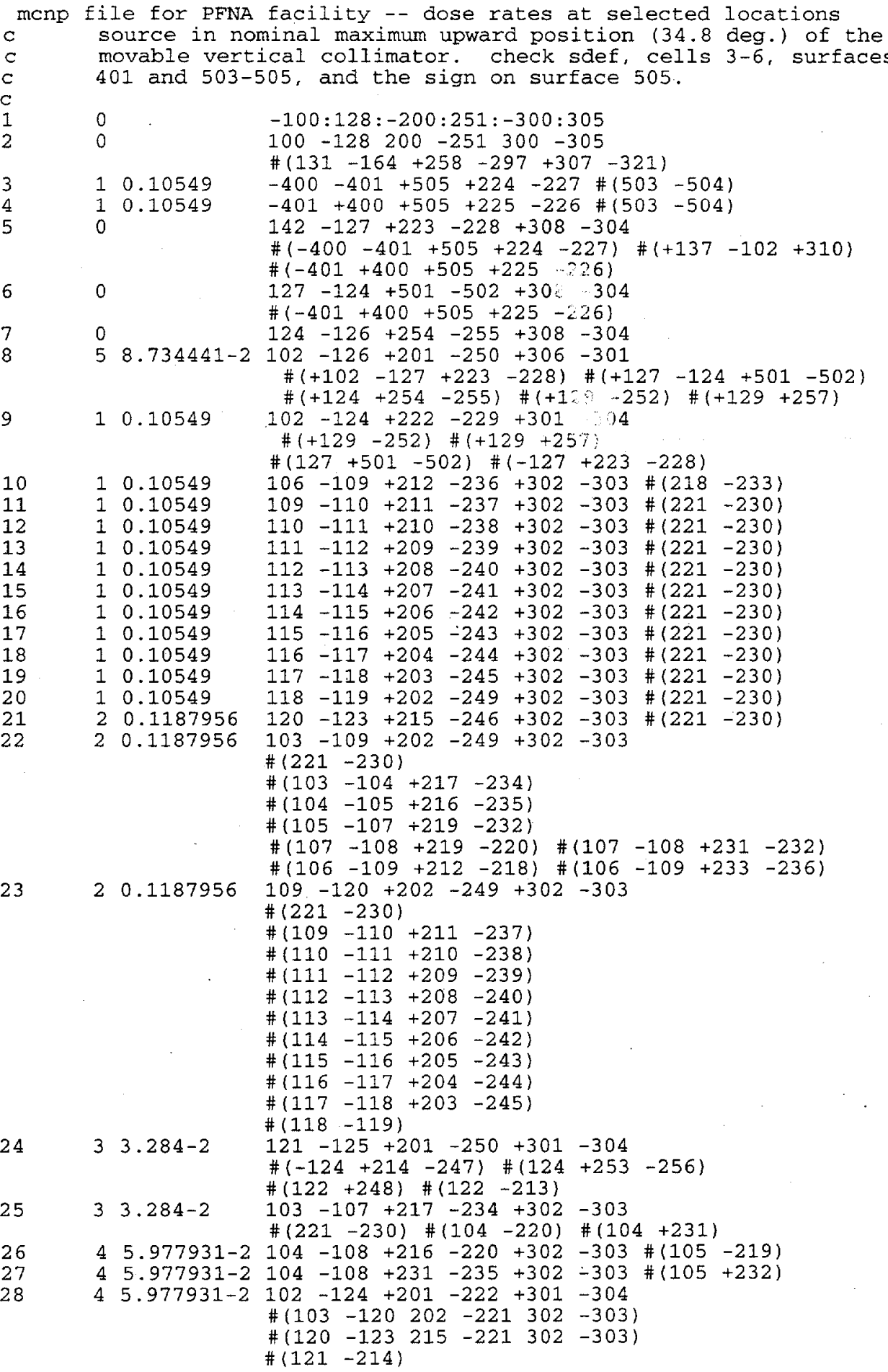




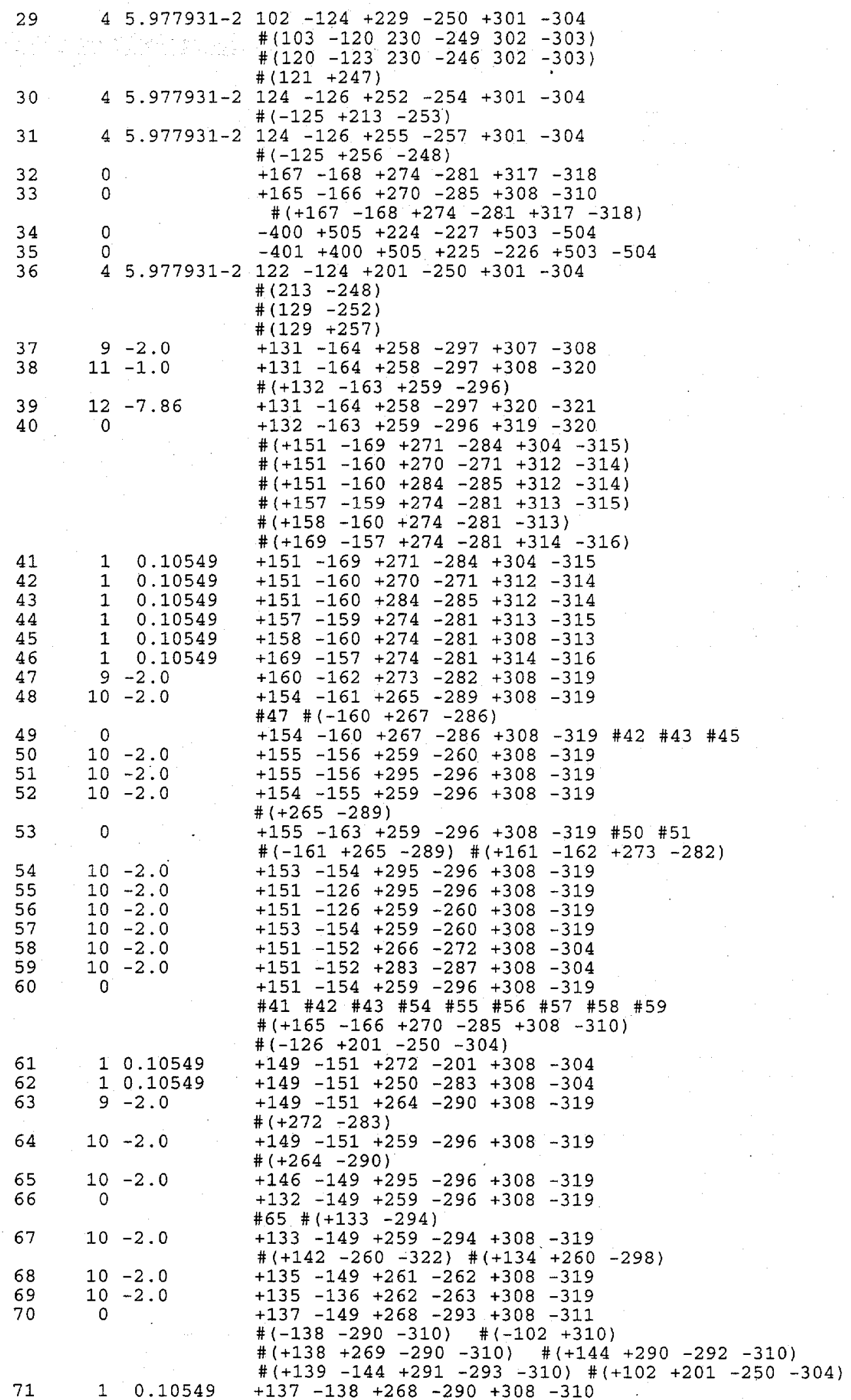




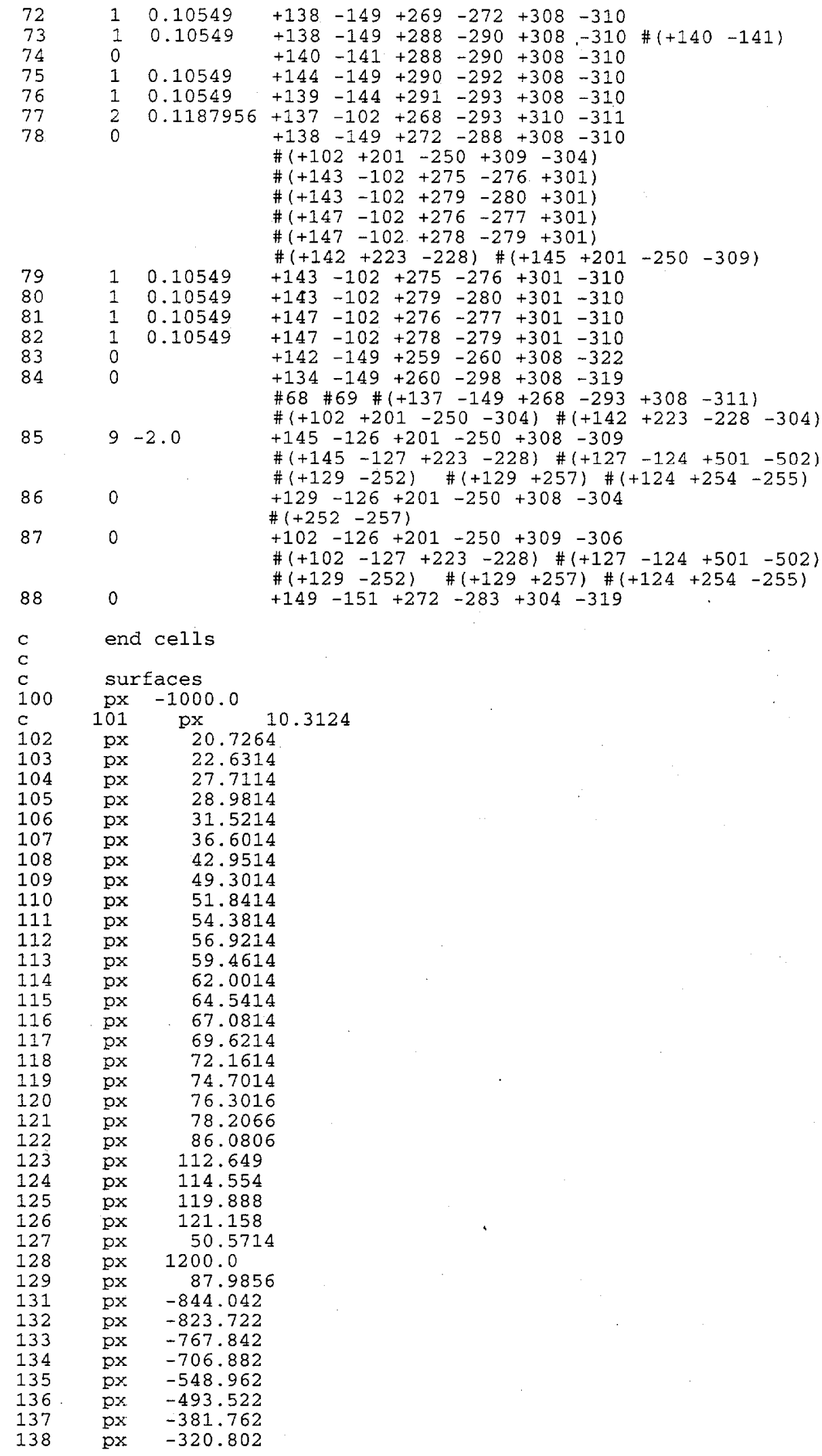




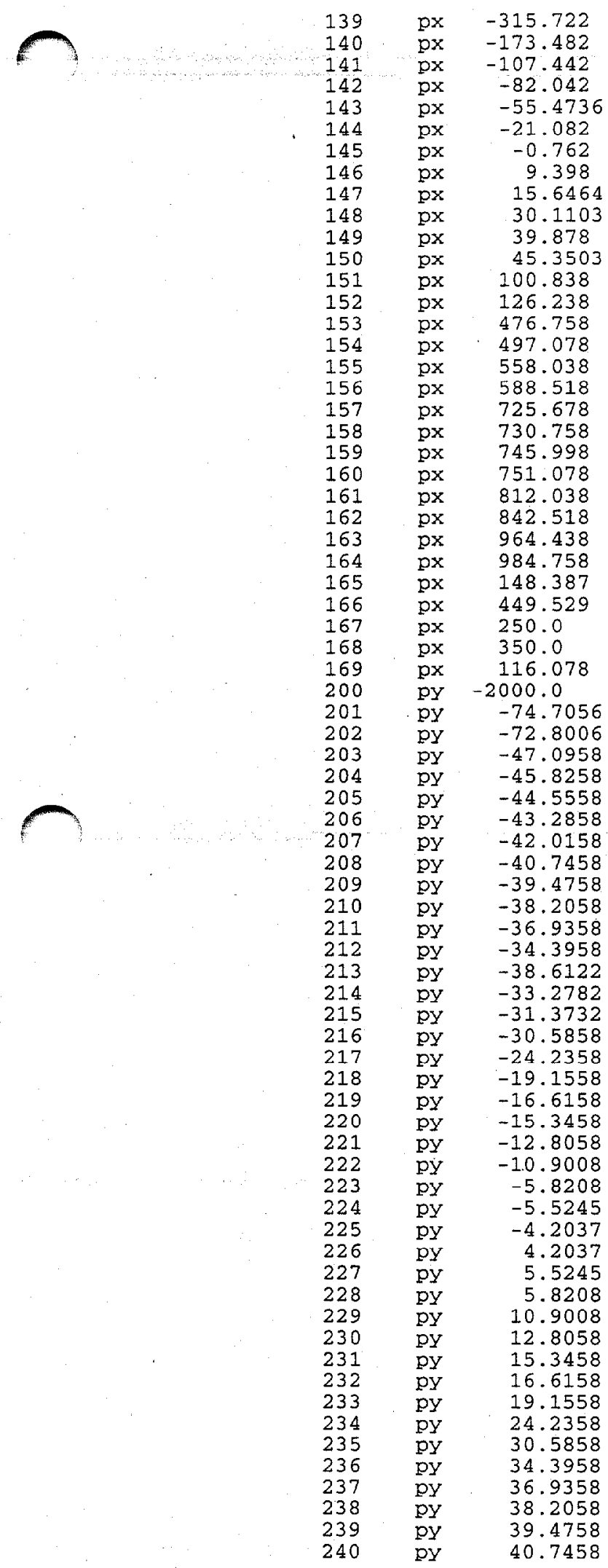




\begin{tabular}{|c|c|c|}
\hline 241 & py & 42.0158 \\
\hline 242 & py & 43.2858 \\
\hline 243 & py & 44.5558 \\
\hline 244 & py & 45.8258 \\
\hline 245 & py & 47.0958 \\
\hline 246 & py & 31.3732 \\
\hline 247 & py & 33.2782 \\
\hline 248 & py & 38.6122 \\
\hline 249 & py & 72.8006 \\
\hline 250 & py & 74.7056 \\
\hline 251 & py & 1500.0 \\
\hline 252 & py & -39.8822 \\
\hline 253 & $\overrightarrow{p y}$ & -9.8848 \\
\hline 254 & py & -8.6148 \\
\hline 255 & py & 8.6148 \\
\hline 256 & PY & 9.8848 \\
\hline 257 & py & 39.8822 \\
\hline 258 & py & -1731.28 \\
\hline 259 & py & -1710.96 \\
\hline 260 & py & -1650.0 \\
\hline 261 & py & -1528.08 \\
\hline 262 & py & -1467.12 \\
\hline 263 & py & -1436.64 \\
\hline 264 & py & -589.28 \\
\hline 265 & py & $-241 \cdot 3$ \\
\hline 266 & PY & -193.04 \\
\hline 267 & py & -180.34 \\
\hline 268 & py. & -167.64 \\
\hline 269 & py & -162.56 \\
\hline 270 & py & -152.4 \\
\hline 271 & py & -137.16 \\
\hline 272 & py & -101.6 \\
\hline 273 & py & -91.44 \\
\hline 274 & py & -60.96 \\
\hline 275 & py & -45.72 \\
\hline 276 & py & -30.48 \\
\hline 277 & py & -10.16 \\
\hline 278 & py & 10.16 \\
\hline 279 & pY & 30.48 \\
\hline 280 & py & 45.72 \\
\hline 281 & pY & 60.96 \\
\hline 282 & py & 91.44 \\
\hline 283 & py & 101.6 \\
\hline 284 & py & 137.16 \\
\hline 285 & py & 152.4 \\
\hline 286 & py & 180.34 \\
\hline 287 & py & 193.04 \\
\hline 288 & py & 213.36 \\
\hline 289 & py & 241.3 \\
\hline 290 & py & 274.32 \\
\hline 291 & py & 335.28 \\
\hline 292 & py & 345.44 \\
\hline 293 & py & 396.24 \\
\hline 294 & py & 533.4 \\
\hline 295 & py & 1235.44 \\
\hline 296 & py & 1296.4 \\
\hline 297 & PY & 1316.72 \\
\hline 298 & py & 472.44 \\
\hline 300 & $\mathrm{pz}$ & -500.0 \\
\hline 301 & $\mathrm{pz}$ & -59.478 \\
\hline 302 & $\mathrm{pz}$ & -57.573 \\
\hline 303 & $\mathrm{pz}$ & 245.957 \\
\hline 304 & $\mathrm{pz}$ & 247.862 \\
\hline 305 & $\mathrm{pz}$ & 500.0 \\
\hline 306 & $\mathrm{pz}$ & -60.748 \\
\hline 307 & $\mathrm{pz}$ & -156.0 \\
\hline 308 & $\mathrm{pz}$ & -125.095 \\
\hline 309 & $p z$ & -67.451 \\
\hline 310 & $\mathrm{pz}$ & 235.585 \\
\hline 311 & $\mathrm{pz}$ & 276.225 \\
\hline 312 & $p z$ & 283.845 \\
\hline 313 & $p z$ & 342.265 \\
\hline
\end{tabular}




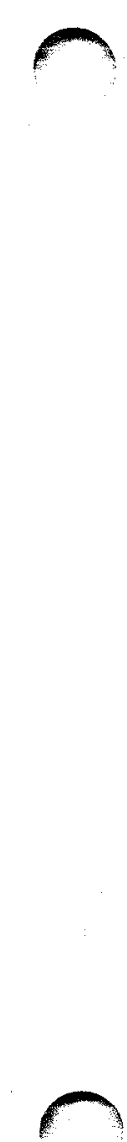

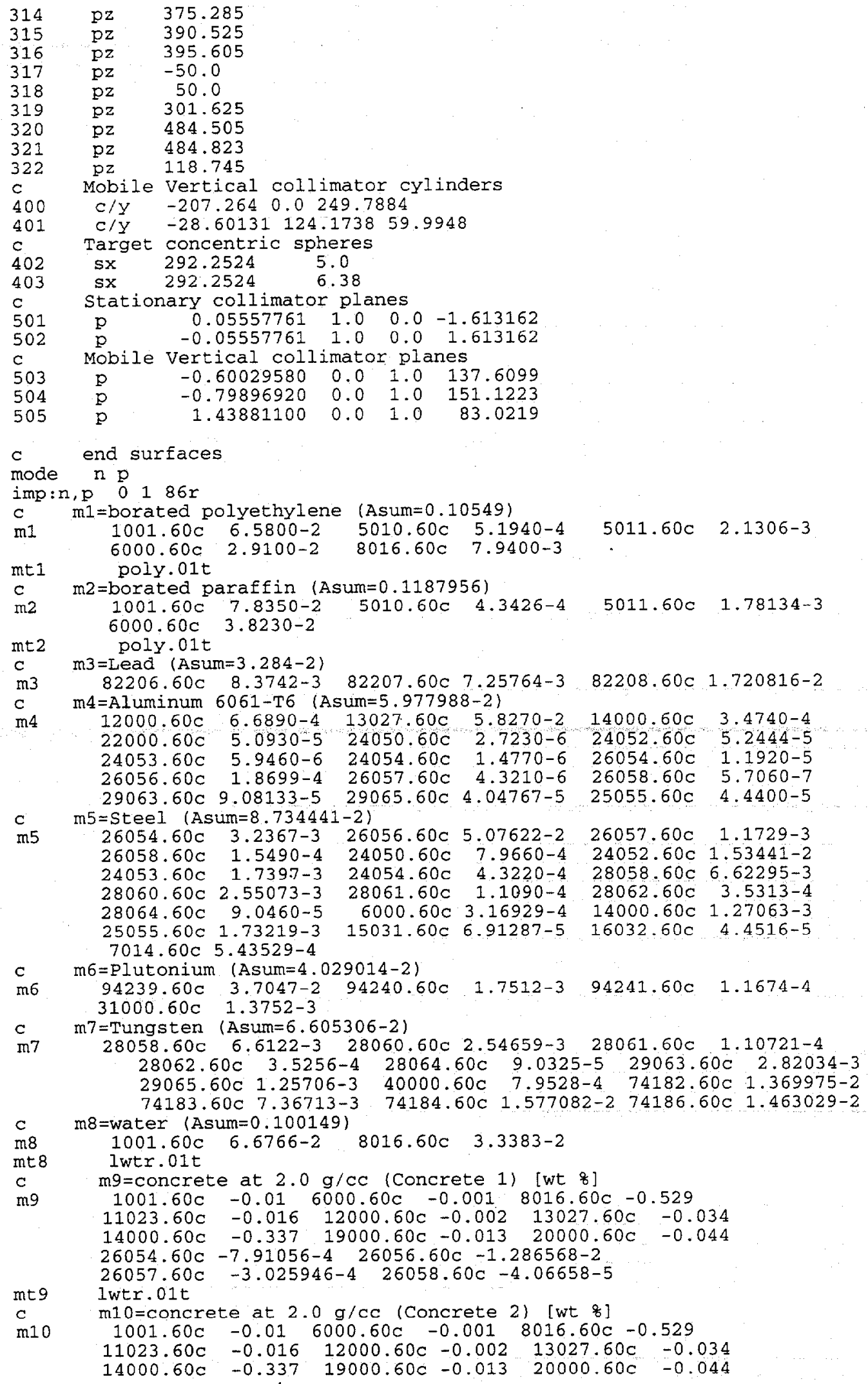




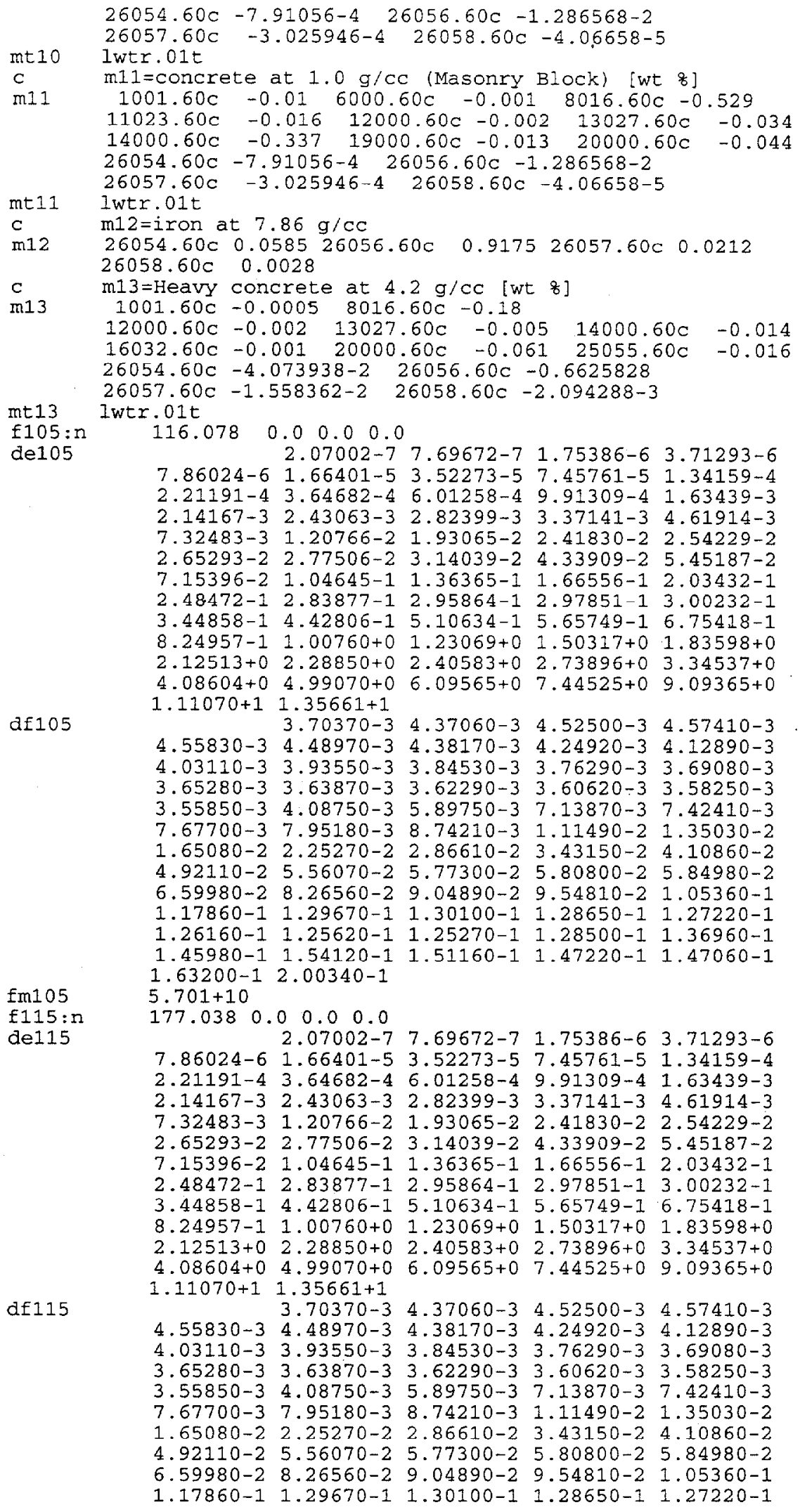


$\begin{array}{lllllll}1.26160-1 & 1.25620-1 & 1.25270-1 & 1.28500-1 & 1.36960-1\end{array}$ $1.45980-1 \quad 1.54120-1 \quad 1.51160-1 \quad 1.47220-1 \quad 1.47060-1$ 1. $63200-12.00340-1$

$\mathrm{fm} 115 \quad 5.701+10$

E125:n $\quad 237.998 \quad 0.0 \quad 0.0 \quad 0.0$

de 125

$2.07002-7 \quad 7.69672-7 \quad 1.75386-6 \quad 3.71293-6$

$7.86024-6 \quad 1.66401-5 \quad 3.52273-5 \quad 7.45761-5 \quad 1.34159-4$

2.21191-4 $3.64682-4 \quad 6.01258-4 \quad 9.91309-4 \quad 1.63439-3$

$2.14167-3 \quad 2.43063-3 \quad 2.82399-3 \quad 3.37141-3$ 4.61914-3

$\begin{array}{llllll}7.32483-3 & 1.20766-2 & 1.93065-2 & 2.41830-2 & 2.54229-2\end{array}$

2.65293-2 2.77506-2 $3.14039-2 \quad 4.33909-2 \quad 5.45187-2$

$\begin{array}{llllll}7.15396-2 & 1.04645-1 & 1.36365-1 & 1.66556-1 & 2.03432-1\end{array}$

2.48472-1 2.83877-1 2.95864-1 2.97851-1 3.00232-1

3.44858-1 4.42806-1 5.10634-1 5.65749-1 6.75418-1

$8.24957-1 \quad 1.0 .0760+0 \quad 1.23069+0 \quad 1.50317+0 \quad 1.83598+0$

$2.12513+0 \quad 2.28850+0 \quad 2.40583+0 \quad 2.73896+0 \quad 3.34537+0$

$4.08604+0 \quad 4.99070+0 \quad 6.09565+0 \quad 7.44525+0 \quad 9.09365+0$

$1.11070+1 \quad 1.35661+1$

df125 $\quad 3.70370-3 \quad 4.37060-3 \quad 4.52500-3 \quad 4.57410-3$

$4.55830-3 \quad 4.48970-3 \quad 4.38170-3 \quad 4.24920-3 \quad 4.12890-3$

$4.03110-3 \cdot 3.93550-3 \quad 3.84530-3 \quad 3.76290-3 \quad 3.69080-3$

$3.65280-3 \quad 3.63870-3 \quad 3.62290-3 \quad 3.60620-3 \quad 3.58250-3$

$3.55850-3 \quad 4.08750-3 \quad 5.89750-3 \quad 7.13870-3 \quad 7.42410-3$

$7.67700-3 \quad 7.95180-3 \quad 8.74210-3 \quad 1.11490-2 \quad 1.35030-2$

$1.65080-22.25270-2.2 .86610-2 \quad 3.43150-2 \quad 4.10860-2$

$4.92110-2 \quad 5.56070-2 \quad 5.77300-2 \quad 5.80800-2 \quad 5.84980-2$

$6.59980-2 \quad 8.26560-29.04890-2 \quad 9.54810-2 \quad 1.05360-1$

$1.17860-1 \quad 1.29670-1 \quad 1.30100-1 \quad 1.28650-1 \quad 1.27220-1$

$\begin{array}{llllll}1.26160-1 & 1.25620-1 & 1.25270-1 & 1.28500-1 & 1.36960-1\end{array}$

$1.45980-1 \quad 1.54120-1 \quad 1.51160-1$ 1.47220-1 $1.47060-1$

$1.63200-12.00340-1$

fm125 $\quad 5.701+10$

f135:n $\quad 359.918 \quad 0.0 \quad 0.0 \quad 0.0$

de135 $\quad 2.07002-7 \quad 7.69672-7 \quad 1.75386-6 \quad 3.71293-6$

$7.86024-6 \quad 1.66401-5 \quad 3.52273-5 \quad 7.45761-5 \quad 1.34159-4$

$2.21191-4 \quad 3.64682-4 \quad 6.01258-4 \quad 9.91309-4 \quad 1.63439-3$

$2.14167-3 \quad 2.43063-3 \quad 2.82399-3 \quad 3.37141-3 \quad 4.61914-3$

$7.32483-3 \quad 1.20766-2 \quad 1.93065-2 \quad 2.41830-2.2 .54229-2$

$2.65293-2 \quad 2.77506-2 \quad 3.14039-2 \quad 4.33909-2 \quad 5.45187-2$

7.15396-2 1.04645-1 $1.36365-1 \quad 1.66556-1 \quad 2.03432-1$ 2.48472-1 $2.83877-1 \quad 2.95864-1 \quad 2.97851-1 \quad 3.00232-1$ 3.44858-1 $4.42806-1 \quad 5.10634-1 \quad 5.65749-1 \quad 6.75418-1$ $8.24957-1 \quad 1.00760+0 \quad 1.23069+0 \quad 1.50317+0 \quad 1.83598+0$ $2.12513+0 \quad 2.28850+0 \quad 2.40583+0 \quad 2.73896+0 \quad 3.34537+0$ $4.08604+0 \quad 4.99070+0 \quad 6.09565+0 \quad 7.44525+0 \quad 9.09365+0$ $1.11070+1 \quad 1.35661+1$

df135 $3.70370-3 \quad 4.37060-3 \quad 4.52500-3 \quad 4.57410-3$ $\begin{array}{lllllll}4.55830-3 & 4.48970-3 & 4.38170-3 & 4.24920-3 & 4.12890-3\end{array}$ $4.03110-3 \quad 3.93550-3 \quad 3.84530-3 \quad 3.76290-3 \quad 3.69080-3$ $\begin{array}{llllll}3.65280-3 & 3.63870-3 & 3.62290-3 & 3.60620-3 & 3.58250-3\end{array}$ $3.55850-3 \quad 4.08750-3 \quad 5.89750-3 \quad 7.13870-3 \quad 7.42410-3$ $7.67700-3 \quad 7.95180-3 \quad 8.74210-31.11490-2 \quad 1.35030-2$ $\begin{array}{llllll}1.65080-2 & 2.25270-2 & 2.86610-2 & 3.43150-2 & 4.10860-2\end{array}$ $4.92110-2 \quad 5.56070-2 \quad 5.77300-2 \cdot 5.80800-2 \quad 5.84980-2$ $6.59980-2 \quad 8.26560-2 \quad 9.04890-2 \quad 9.54810-2 \quad 1.05360-1$ $\begin{array}{lllll}1.17860-1 & 1.29670-1 & 1.30100-1 & 1.28650-1 & 1.27220-1\end{array}$ 1.26160-1 $1.25620-1 \quad 1.25270-1 \quad 1.28500-1 \quad 1.36960-1$ $1.45980-1.1 .54120-1 \quad 1.51160-1 \quad 1.47220-1 \quad 1.47060-1$ $1.63200-12.00340-1$

fm135 $\quad 5.701+10$

f145:n $\quad 420.878 \quad 0.0 \quad 0.0 \quad 0.0$

$\begin{array}{llllll}\operatorname{de} 145 & 2.07002-7 & 7.69672-7 & 1.75386-6 & 3.71293-6\end{array}$ 7.86024-6 $1.66401-5 \quad 3.52273-5 \quad 7.45761-5 \quad 1.34159-4$ 2.21191-4 $3.64682-4 \quad 6.01258-4 \quad 9.91309-4 \quad 1.63439-3$ $2.14167-3 \quad 2.43063-3 \quad 2.82399-3 \quad 3.37141-3 \quad 4.61914-3$ $7.32483-3 \quad 1.20766-2 \quad 1.93065-2 \quad 2.41830-2 \quad 2.54229-2$ $\begin{array}{lllll}2.65293-2 & 2.77506-2 & 3.14039-2 & 4.33909-2 & 5.45187-2\end{array}$ 7.15396-2 $1.04645-1 \quad 1.36365-1 \quad 1.66556-1 \quad 2.03432-1$ 2.48472-1 $2.83877-1 \quad 2.95864-1 \quad 2.97851-1 \quad 3.00232-1$ 3.44858-1 $4.42806-1 \quad 5.10634-1 \quad 5.65749-1 \quad 6.75418-1$ $8.24957-1 \quad 1.00760+0 \quad 1.23069+0 \quad 1.50317+0.1 .83598+0$ $2.12513+0 \quad 2.28850+0 \quad 2.40583+0 \quad 2.73896+0 \quad 3.34537+0$ 
$4.08604+0 \quad 4.99070+0 \quad 6.09565+0 \quad 7.44525+0 \quad 9.09365+0$ $1.11070+1 \quad 1.35661+1$

df145

fm1 145

f155:n

de155

$\operatorname{df155}$

fm155

f205:p

de205

df 205

fin205

$\mp 215: p$

de 215

$4.55830-3 \quad 4.48970$

$4.03110-3 \quad 3.93550-3 \quad 3.84530-3 \quad 3.76290-3 \quad 3.69080-3$

$\begin{array}{llllll}3.65280-3 & 3.63870-3 & 3.62290-3 & 3.60620-3 & 3.58250-3\end{array}$

$3.55850-3 \quad 4.08750-3 \quad 5.89750-3 \quad 7.13870-3 \quad 7.42410-3$

$\begin{array}{llllll}7.67700-3 & 7.95180-3 & 8.74210-3 & 1.11490-2 & 1.35030-2\end{array}$

$1.65080-2 \quad 2.25270-2 \quad 2.86610-2 \quad 3.43150-2 \quad 4.10860-2$

4.92110-2 $5.56070-2 \quad 5.77300-2 \quad 5.80800-2 \quad 5.84980-2$

$6.59980-2 \quad 8.26560-2 \quad 9.04890-2 \quad 9.54810-2 \quad 1.05360-1$

$1.17860-1 \quad 1.29670-1 \quad 1.30100-1 \quad 1.28650-1 \quad 1.27220-1$

$1.26160-1 \quad 1.25620-1 \quad 1.25270-1 \quad 1.28500-1 \quad 1.36960-1$

$1.45980-1$ 1.54120-1 $1.51160-1.1 .47220-1$ 1.47060-1

$1.63200-12.00340-1$

$5.701+10$

$481.838 \quad 0.0 \quad 0.0 \quad 0.0$

$2.07002-7 \quad 7.69672-7 \quad 1.75386-6 \quad 3.71293-6$

7.86024-6 $1.66401-5 \quad 3.52273-5 \quad 7.45761-5 \quad 1.34159-4$

2.21191-4 $3.64682-4 \quad 6.01258-4 \quad 9.91309-4 \quad 1.63439-3$

$\begin{array}{llllll}2.14167-3 & 2.43063-3 & 2.82399-3 & 3.37141-3 & 4.61914-3\end{array}$

$7.32483-3 \quad 1.20766-2 \quad 1.93065-2 \quad 2.41830-2 \quad 2.54229-2$

2.65293-2 $2.77506-2 \quad 3.14039-2 \quad 4.33909-2 \quad 5.45187-2$

7.15396-2 1.04645-1 $1.36365-1 \quad 1.66556-1 \quad 2.03432-1$

$\begin{array}{lllll}2.48472-1 & 2.83877-1 & 2.95864-1 & 2.97851-1 & 3.00232-1\end{array}$

3.44858-1 $4.42806-1 \quad 5.10634-1 \quad 5.65749-1 \quad 6.75418-1$

$8.24957-1 \quad 1.00760+0 \quad 1.23069+0 \quad 1.50317+0 \quad 1.83598+0$

$2.12513+0 \quad 2.28850+0 \quad 2.40583+0 \quad 2.73896+0 \quad 3.34537+0$

$4.08604+0 \quad 4.99070+0 \quad 6.09565+0 \quad 7.44525+0 \quad 9.09365+0$ $1.11070+11.35661+1$

$.70370-3 \quad 4.37060-3 \quad 4.52500-3 \quad 4.57410-3$

$4.55830-3 \quad 4.48970-3 \quad 4.38170-3 \quad 4.24920-3 \quad 4.12890-3$

$4.03110-3 \quad 3.93550-3 \quad 3.84530-3 \quad 3.76290-3 \quad 3.69080-3$

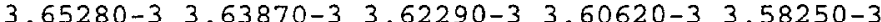

$\begin{array}{llllll}3.55850-3 & 4.08750-3 & 5.89750-3 & 7.13870-3 & 7.42410-3\end{array}$

$7.67700-3 \quad 7.95180-3 \quad 8.74210-3 \quad 1.11490-2 \quad 1.35030-2$

$\begin{array}{lllll}1.65080-2 & 2.25270-2 & 2.86610-2 & 3.43150-2 & 4.10860-2\end{array}$

$4.92110-2 \quad 5.56070-2 \quad 5.77300-2 \quad 5.80800-2 \quad 5.84980-2$

$\begin{array}{llllll}6.59980-2 & 8.26560-2 & 9.04890-2 & 9.54810-2 & 1.05360-1\end{array}$

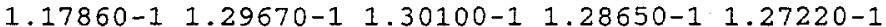

$1.26160-1$ 1.25620-1 $1.25270-1$ 1.28500-1 $1.36960-1$

$1.45980-1 \quad 1.54120-1 \quad 1.51160-1 \quad 1.47220-1 \quad 1.47060-1$ $1.63200-12.00340-1$

$5.701+10$

$116.078 \quad 0.0 \quad 0.0 \quad 0.0$

$1.50000-2 \quad 3.25000-2 \quad 5.75000-2 \quad 8.50000-2$

$1.25000-1 \quad 2.25000-1 \quad 3.50000-1 \quad 4.55000-1 \quad 5.55000-1$ $6.50000-1 \quad 8.50000-1 \quad 1.25000+0 \quad 1.75000+0 \quad 2.25000+0$

$2.75000+0 \quad 3.50000+0 \quad 4.50000+0 \quad 5.50000+0 \quad 6.50000+0$

$7.25000+0 \quad 7.75000+0 \quad 9.00000+0 \quad 1.20000+1$

$\begin{array}{llll}2.14390-3 & 5.77600-4 & 2.71850-4 & 2.68170-4\end{array}$

$3.27670-4 \quad 5.66760-4 \quad 8.75940-4 \quad 1.08450-3 \quad 1.27970-3$

$1.44170-3 \quad 1.75630-3 \quad 2.31560-3 \quad 2.92700-3 \quad 3.46860-3$

$3.95960-3 \quad 4.62210-3 \quad 5.41370-3 \quad 6.19090-3 \quad 6.92650 .3$ $7.47830-3 \quad 7.84680-3 \quad 8.77160-3 \quad 1.10200-2$

$5.701+10$

$177.038 \quad 0.0 \quad 0.0 \quad 0.0$

$1.50000-2 \quad 3.25000-2 \quad 5.75000-2 \quad 8.50000-2$

$1.25000-1 \quad 2.25000-1 \quad 3.50000-1 \quad 4.55000-1 \quad 5.55000-1$

$6.50000-1 \quad 8.50000-1 \quad 1.25000+0 \quad 1.75000+0 \quad 2.25000+0$

$2.75000+0 \quad 3.50000+0 \quad 4.50000+0 \quad 5.50000+0 \quad 6.50000+0$

$7.25000+0 \quad 7.75000+0 \quad 9.00000+0 \quad 1.20000+1$

dE215

$\begin{array}{llll}2.14390-3 & 5.77600-4 & 2.71850-4 & 2.68170-4\end{array}$

$3.27670-4 \quad 5.66760-4 \quad 8.75940-4 \quad 1.08450-3 \quad 1.27970-3$

$1.44170-3 \quad 1.75630-3.2 .31560-3 \quad 2.92700-3 \quad 3.46860-3$

$3.95960-3 \quad 4.62210-3 \quad 5.41370-3 \quad 6.19090-3 \quad 6.92650-3$

$7.47830-3 \quad 7.84680-3 \quad 8.77160-3 \quad 1.10200-2$

fm215 $\quad 5.701+10$

f225:p $\quad 237.998 \quad 0.0 \quad 0.0 \quad 0.0$

de225 $1.50000-2 \quad 3.25000-2 \quad 5.75000-2 \quad 8.50000-2$

$1.25000-1 \quad 2.25000-1 \quad 3.50000-1 \quad 4.55000-1 \quad 5.55000-1$

$6.50000-18.50000-1 \quad 1.25000+01.75000+0 \quad 2.25000+0$ 
$2.75000+0 \quad 3.50000+0 \quad 4.50000+0 \quad 5.50000+0 \quad 6.50000+0$ $7.25000+0.7 .75000+0 \quad 9.00000+0 \quad 1.20000+1$

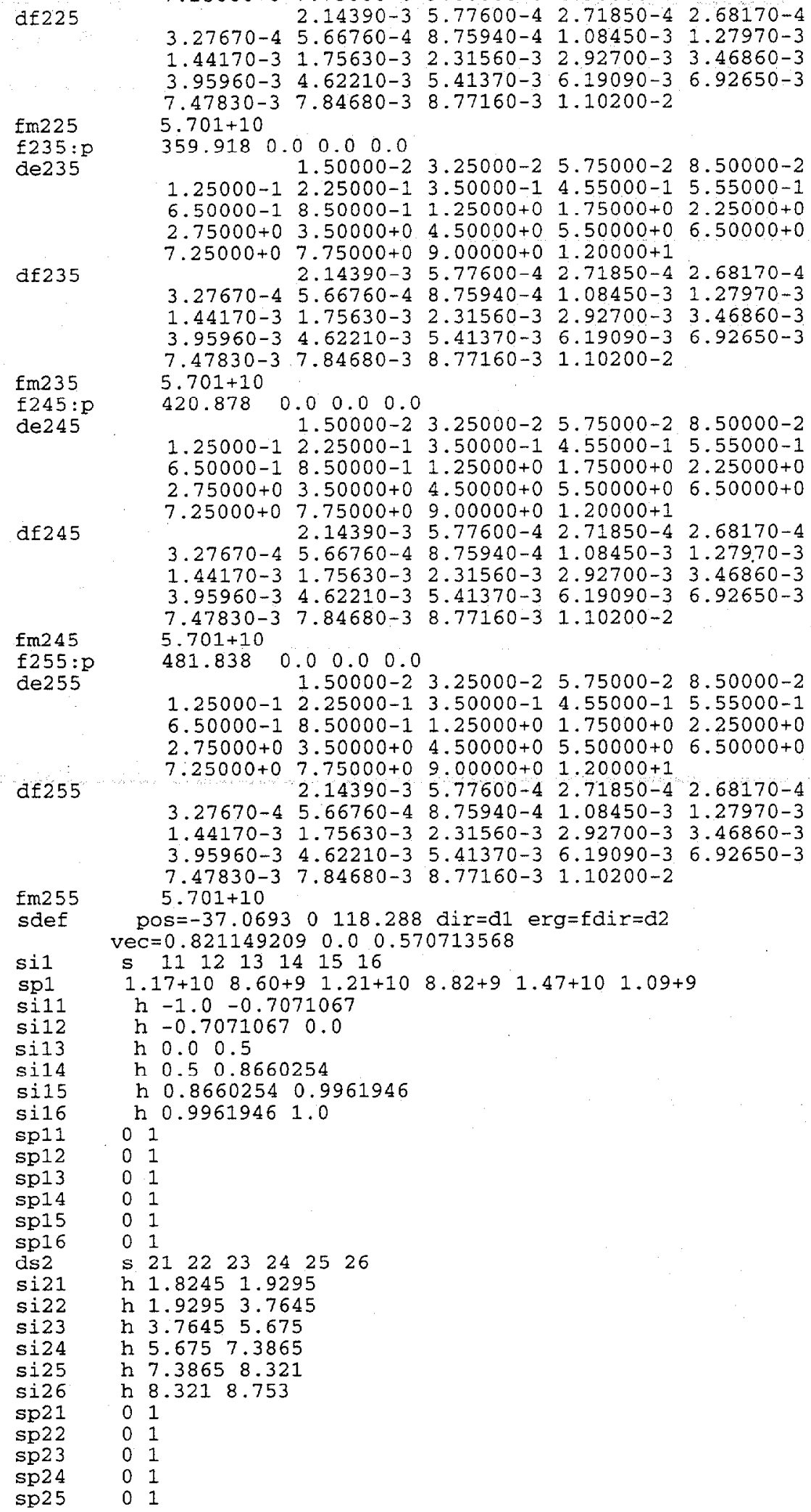




$\begin{array}{ll}\operatorname{sp26} & 01 \\ \text { nps } & 2000000 \\ \text { prdmp } & 2 j 1\end{array}$

print 
File for MCNP-4B calculation of the dose rate in the PFNA beam and across the truck lane and ORIGEN neutron spectra within the stationary collimator for the source in the maximum down position

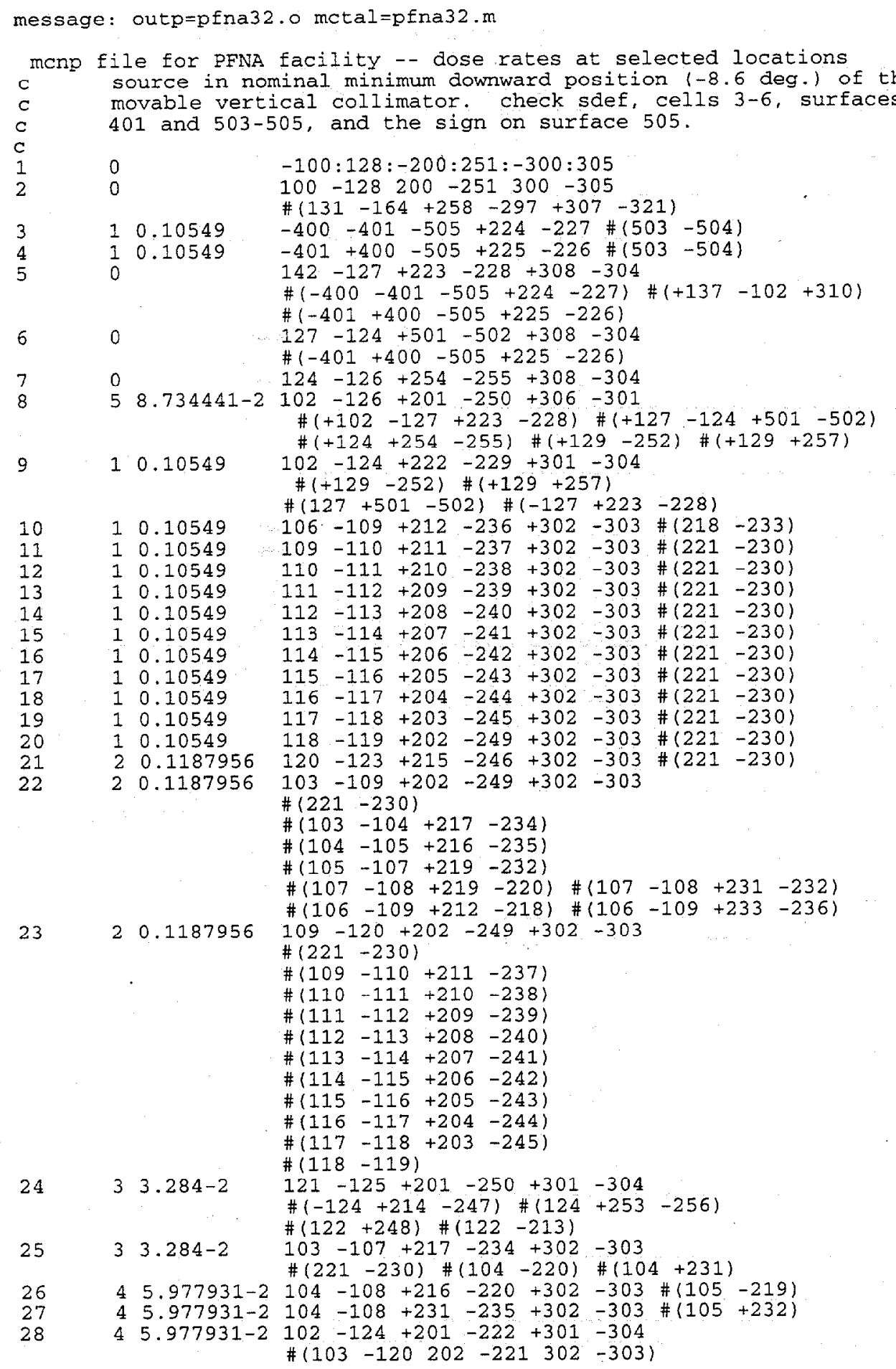




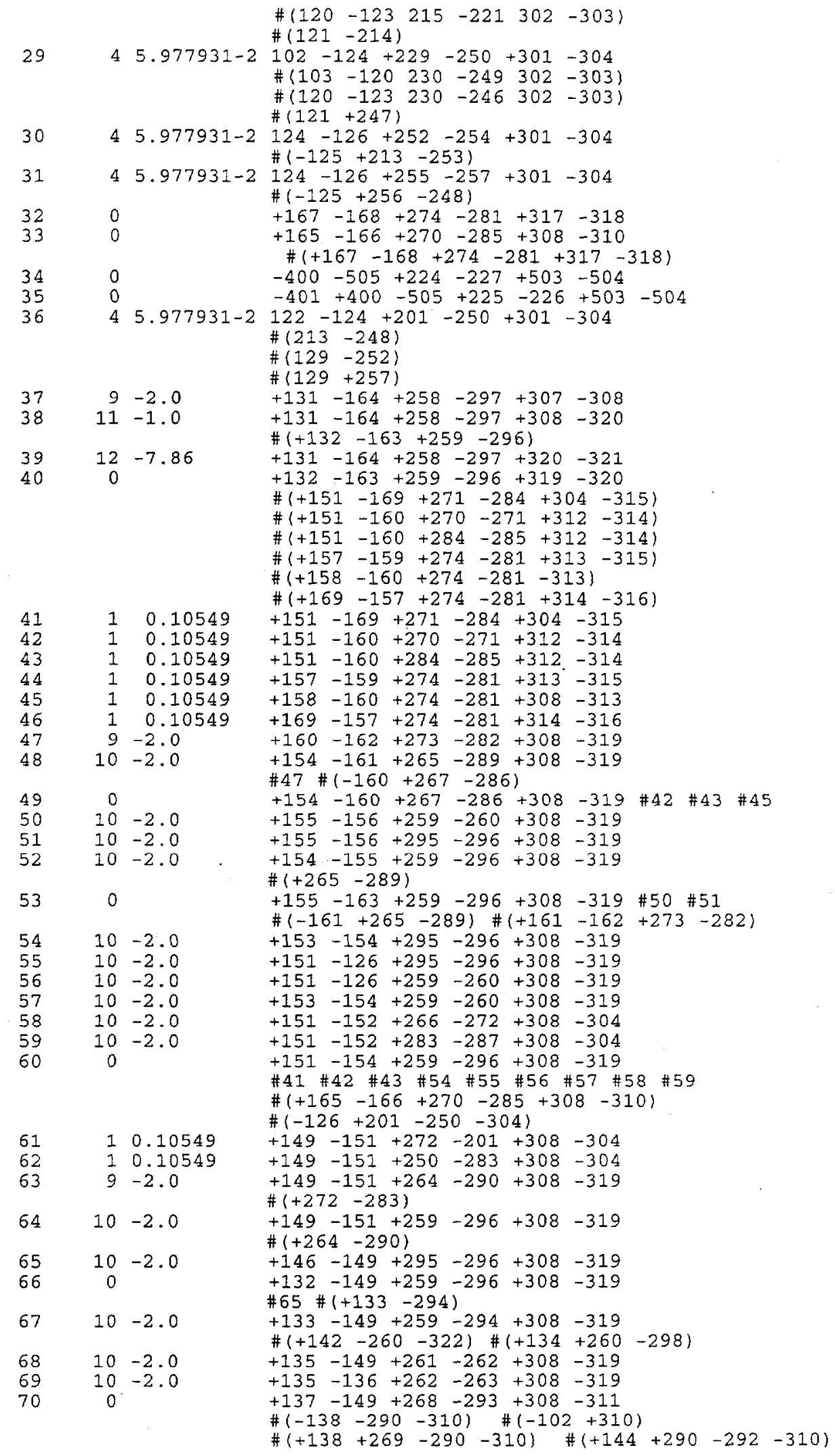




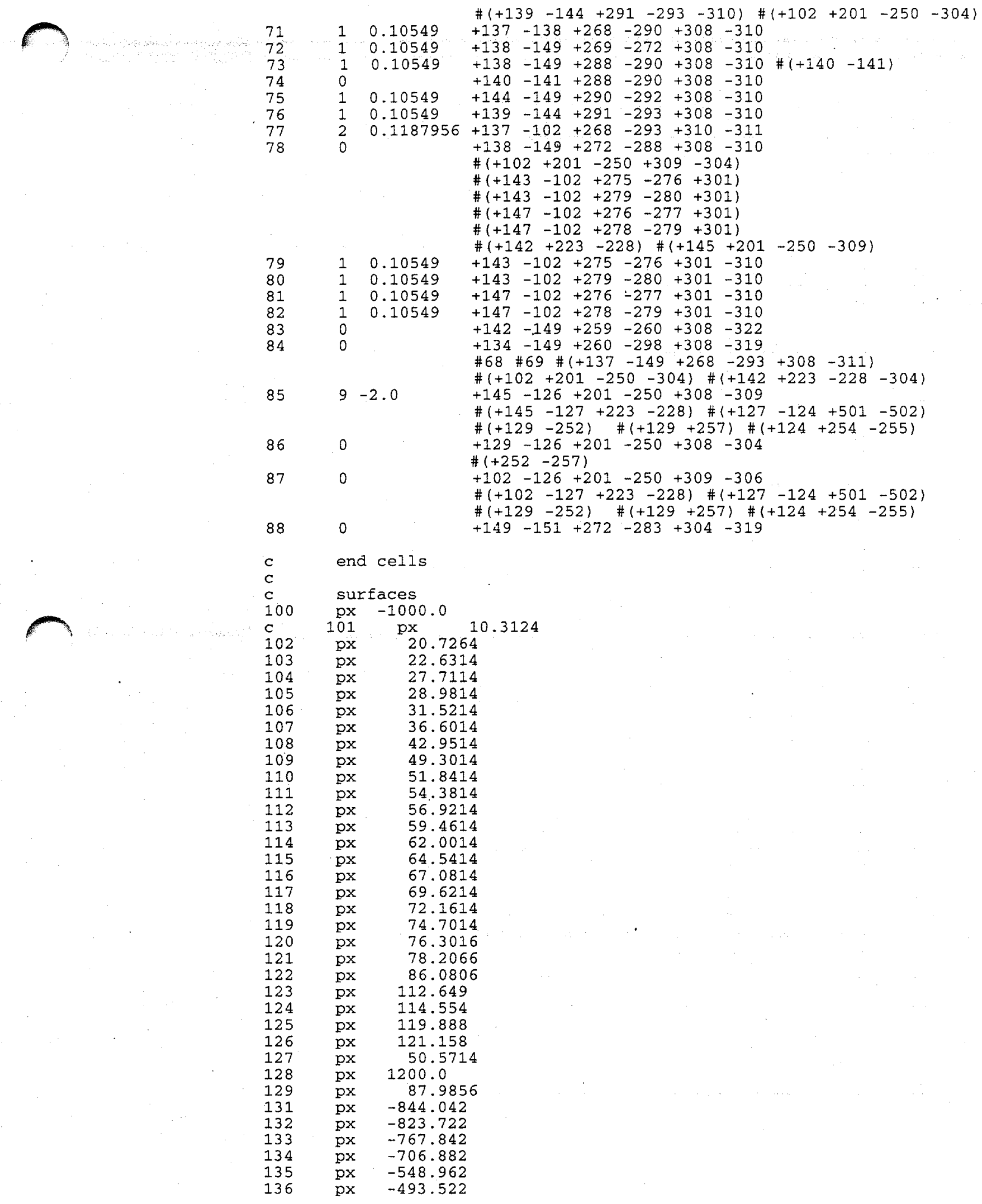




\begin{tabular}{|c|c|c|}
\hline 137 & $\mathrm{px}$ & -381.762 \\
\hline 138 & $p x$ & -320.802 \\
\hline 139 & $\mathrm{px}$ & -315.722 \\
\hline 140 & $\mathrm{px}$ & -173.482 \\
\hline 141 & $p x$ & -107.442 \\
\hline 142 & $\mathrm{px}$ & -82.042 \\
\hline 143 & $p x$ & -55.4736 \\
\hline 144 & px & -21.082 \\
\hline 145 & $p x$ & -0.762 \\
\hline 146 & $p x$ & 9.398 \\
\hline 147 & $\mathrm{px}$ & 15.6464 \\
\hline 148 & $\mathrm{px}$ & 30.1103 \\
\hline 149 & $\mathrm{px}$ & 39.878 \\
\hline 150 & $\mathrm{px}$ & 45.3503 \\
\hline 151 & $\mathrm{px}$ & 100.838 \\
\hline 152 & $\mathrm{px}$ & 126.238 \\
\hline 153 & $\mathrm{px}$ & 476.758 \\
\hline 154 & $p x$ & 497.078 \\
\hline 155 & $p x$ & 558.038 \\
\hline 156 & $p x$ & 588.518 \\
\hline 157 & $\mathrm{px}$ & 725.678 \\
\hline 158 & $p x$ & 730.758 \\
\hline 159 & $\mathrm{px}$ & 745.998 \\
\hline 160 & $p x$ & 751.078 \\
\hline 161 & $\mathrm{px}$ & 812.038 \\
\hline 162 & $p x$ & 842.518 \\
\hline 163 & $\mathrm{px}$ & 964.438 \\
\hline 164 & $\mathrm{px}$ & 984.758 \\
\hline 165 & $\mathrm{px}$ & 148.387 \\
\hline 166 & $\mathrm{px}$ & 449.529 \\
\hline 167 & $\mathrm{px}$ & 250.0 \\
\hline 168 & $p x$ & 350.0 \\
\hline 169 & $p x$ & 116.078 \\
\hline 200 & py & -2000.0 \\
\hline 201 & py & -74.7056 \\
\hline 202 & py & -72.8006 \\
\hline 203 & py & -47.0958 \\
\hline 204 & PY & -45.8258 \\
\hline 205 & py & -44.5558 \\
\hline 206 & $P Y$ & -43.2858 \\
\hline 207 & py & -42.0158 \\
\hline 208 & py & -40.7458 \\
\hline 209 & py & -39.4758 \\
\hline 210 & py & -38.2058 \\
\hline 211 & py & -36.9358 \\
\hline 212 & py & -34.3958 \\
\hline 213 & py & -38.6122 \\
\hline 214 & py & -33.2782 \\
\hline 215 & py & -31.3732 \\
\hline 216 & py & -30.5858 \\
\hline 217 & py & -2.2 .2358 \\
\hline 218 & DY & -19.1558 \\
\hline 219 & py & -16.6158 \\
\hline 220 & py & -1.5 .3458 \\
\hline 221 & py & -12.8058 \\
\hline 222 & py & -10.9008 \\
\hline 223 & py & -5.8208 \\
\hline 224 & py & -5.5245 \\
\hline 225 & py & -4.2037 \\
\hline 226 & py & 4.2037 \\
\hline 227 & PY & 5.5245 \\
\hline 228 & py & 5.8208 \\
\hline 229 & py & 10.9008 \\
\hline 230 & py & 12.8058 \\
\hline 231 & py & 15.3458 \\
\hline 232 & py & 16.6158 \\
\hline 233 & py & 19.1558 \\
\hline 234 & py & 24.2358 \\
\hline 235 & py & 30.5858 \\
\hline 236 & PY & 34.3958 \\
\hline 237 & py & 36.9358 \\
\hline 238 & py & 38.2058 \\
\hline
\end{tabular}




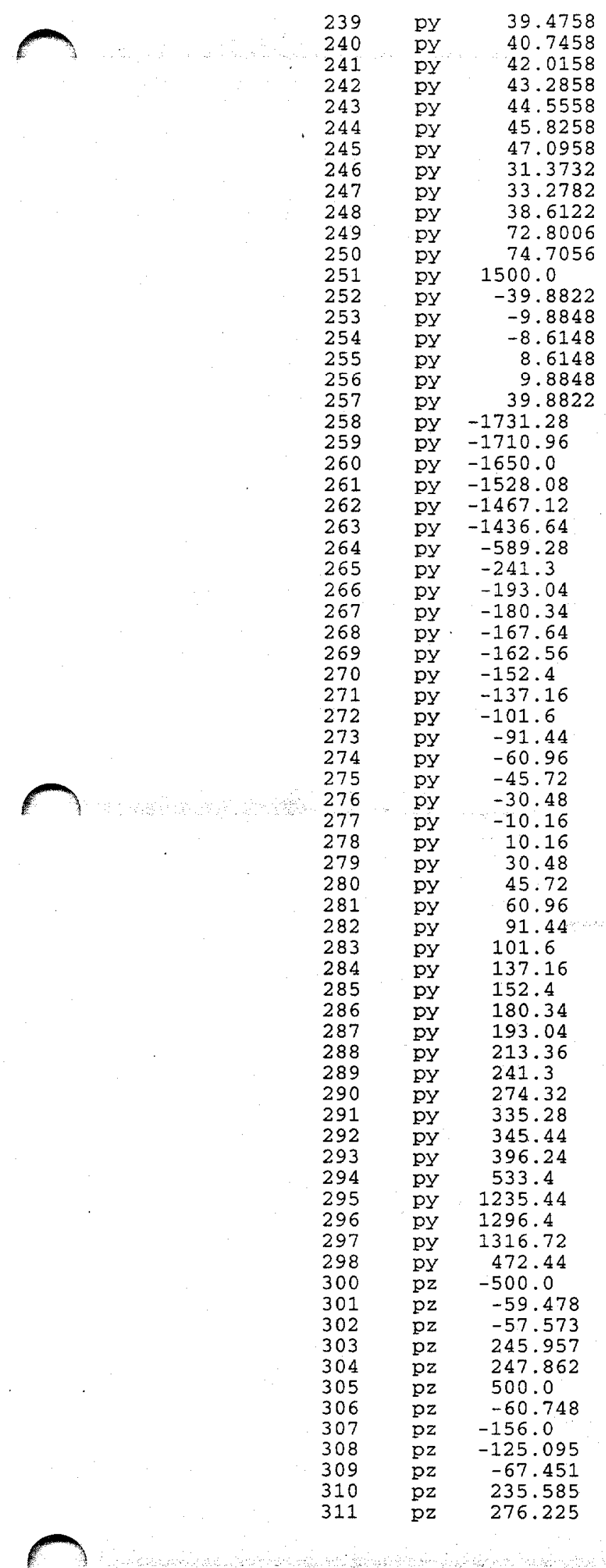




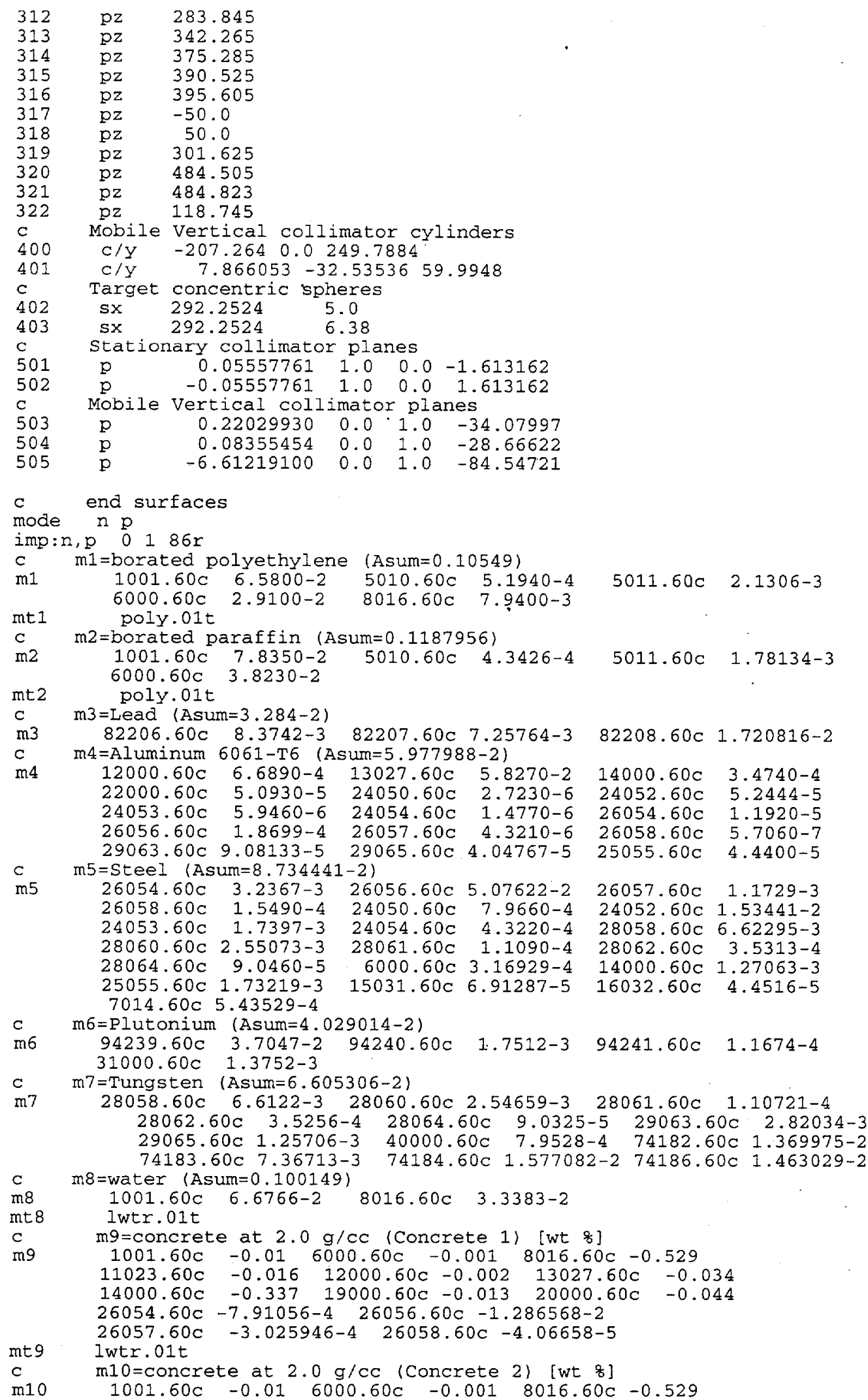


$\begin{array}{llllllll}11023.60 c & -0.016 & 12000.60 c & -0.002 & 13027.60 c & -0.034\end{array}$ $\begin{array}{lllllll}14000.60 \mathrm{c} & -0.337 & 19000.60 \mathrm{c} & -0.013 & 20000.60 \mathrm{c} & -0.044\end{array}$ $26054.60 \mathrm{c}-7.91056-4 \quad 26056.60 \mathrm{c}-1.286568-2$ $26057.60 \mathrm{c} \quad-3.025946-4 \quad 26058.60 \mathrm{c}-4.06658-5$

\section{me10 lwtr.01t}

$\mathrm{c}$ mil=concrete at $1.0 \mathrm{~g} / \mathrm{Cc}$ (Masonry Block) [wt \&]

m11 $1001.60 \mathrm{c}-0.01 \quad 6000.60 \mathrm{c}-0.001 \quad 8016.60 \mathrm{c}-0.529$

$\begin{array}{llllll}11023.60 \mathrm{c} & -0.016 & 12000.60 \mathrm{c} & -0.002 & 13027.60 \mathrm{c} & -0.034\end{array}$ $\begin{array}{lllllll}14000.60 \mathrm{c} & -0.337 & 19000.60 \mathrm{c} & -0.013 & 20000.60 \mathrm{c} & -0.044\end{array}$ $26054.60 \mathrm{c}-7.91056-4 \quad 26056.60 \mathrm{c}-1.286568-2$

$26057.60 \mathrm{C}-3.025946-4 \quad 26058.60 \mathrm{c}-4.06658-5$

mt11 lwtr.01t at $7.86 \mathrm{~g} / \mathrm{CC}$

m12 $26054.60 \mathrm{c} \quad 0.0585 \quad 26056.60 \mathrm{c} \quad 0.9175 \quad 26057.60 \mathrm{c} \quad 0.0212$

$26058.60 \mathrm{C} \quad 0.0028$

c m13=Heavy concrete at $4.2 \mathrm{~g} / \mathrm{cc}$ [wt 8 ]

m13 $1001.60 \mathrm{c}-0.0005 \quad 8016.60 \mathrm{c}-0.18$

$12000.60 \mathrm{c}-0.002 \quad 13027.60 \mathrm{c} \quad-0.005 \quad 14000.60 \mathrm{c} \quad-0.014$

$\begin{array}{llllll}16032.60 \mathrm{c} & -0.001 & 20000.60 \mathrm{c} & -0.061 & 25055.60 \mathrm{c} & -0.016\end{array}$

$26054.60 \mathrm{C}-4.073938-2 \quad 26056.60 \mathrm{c}-0.6625828$

$\begin{array}{lllll}26057.60 \mathrm{c} & -1.558362-2 & 26058.60 \mathrm{c} & -2.094288-3\end{array}$

f105:n $\quad 116.078 \quad 0.0 \quad 0.0 \quad 0.0$

de105

$2.07002-7 \quad 7.69672-7 \quad 1.75386-6 \quad 3.71293-6$

7.86024-6 $1.66401-5 \quad 3.52273-5 \quad 7.45761-5 \quad 1.34159-4$ 2.21191-4 $3.64682-4 \quad 6.01258-4 \quad 9.91309-4 \quad 1.63439-3$ $2.14167-3 \quad 2.43063-3 \quad 2.82399-3 \quad 3.37141-3 \quad 4.61914-3$ $\begin{array}{lllll}7.32483-3 & 1.20766-2 & 1.93065-2 & 2.41830-2 & 2.54229-2\end{array}$ 2.65293-2 2.77506-2 3.14039-2 $4.33909-2 \quad 5.45187-2$ $7.15396-2$ 1.04645-1 $1.36365-1 \quad 1.66556-1 \quad 2.03432-1$ $\begin{array}{llllll}2.48472-1 & 2.83877-1 & 2.95864-1 & 2.97851-1 & 3.00232-1\end{array}$ $\begin{array}{llllll}3.44858-1 & 4.42806-1 & 5.10634-1 & 5.65749-1 & 6.75418-1\end{array}$ $8.24957-1 \quad 1.00760+0 \cdot 1.23069+0 \quad 1.50317+0 \quad 1.83598+0$ $2.12513+0 \quad 2.28850+0 \quad 2.40583+0 \quad 2.73896+0 \quad 3.34537+0$ $\begin{array}{lllll}4.08604+0 & 4.99070+0 & 6.09565+0 & 7.44525+0 & 9.09365+0\end{array}$ $1.11070+1 \quad 1.35661+1$

df105 $\quad 3.70370-3 \quad 4.37060-3 \quad 4.52500-3 \quad 4.57410-3$ $4.55830-3 \quad 4.48970-3 \quad 4.38170-3 \quad 4.24920-3 \quad 4.12890-3$ $4.03110-3 \quad 3.93550-3 \quad 3.84530-3 \quad 3.76290-3 \quad 3.69080-3$ $\begin{array}{llllll}3.65280-3 & 3.63870-3 & 3.62290-3 & 3.60620-3 & 3.58250-3\end{array}$ $3.55850-3 \quad 4.08750-3 \quad 5.89750-3 \quad 7.13870-3 \quad 7.42410-3$ $\begin{array}{llllll}7.67700-3 & 7.95180-3 & 8.74210-3 & 1.11490-2 & 1.35030-2\end{array}$ $1.65080-2 \quad 2.25270-2 \quad 2.86610-2 \quad 3.43150-2 \quad 4.10860-2$ 4.92110-2 $5.56070-2 \quad 5.77300-2 \quad 5.80800-2 \quad 5.84980-2$ $6.59980-2 \quad 8.26560-2 \quad 9.04890-2 \quad 9.54810-2 \quad 1.05360-1$ $1.17860-1$ 1.29670-1 $1.30100-1 \quad 1.28650-1 \quad 1.27220-1$ $\begin{array}{llllll}1.26160-1 & 1.25620-1 & 1.25270-1 & 1.28500-1 & 1.36960-1\end{array}$ 1.45980-1 1.54120-1 $1.51160-1 \quad 1.47220-1 \quad 1.47060-1$ $1.63200-12.00340-1$

$\operatorname{fm} 105 \quad 5.701+10$

f $115: \mathrm{n} \quad 177.038 \quad 0.0 \quad 0.0 \quad 0.0$

$\begin{array}{llllll}\text { de115 } & 2.07002-7 & 7.69672-7 & 1.75386-6 & 3.71293-6\end{array}$

7.86024-6 $1.66401-5 \quad 3.52273-5 \quad 7.45761-5 \quad 1.34159-4$ 2.21191-4 $3.64682-4 \quad 6.01258-4 \quad 9.91309-4 \quad 1.63439-3$ 2.14167-3 2.43063-3 $2.82399-3 \quad 3.37141-3 \quad 4.61914-3$ 7.32483-3 1.20766-2 $1.93065-2 \quad 2.41830-2 \quad 2.54229-2$ 2.65293-2 2.77506-2 3.14039-2 $4.33909-2$ 5.45187-2 7.15396-2 $1.04645-1 \quad 1.36365-1 \quad 1.66556-1.2 .03432-1$ $\begin{array}{lllll}2.48472-1 & 2.83877-1 & 2.95864-1 & 2.97851-1 & 3.00232-1\end{array}$ 3.44858-1 $4.42806-1 \quad 5.10634-1 \quad 5.65749-1 \quad 6.75418-1$ 8.24957-1 $1.00760+0 \quad 1.23069+0 \quad 1.50317+0.1 .83598+0$ $\begin{array}{lllll}2.12513+0 & 2.28850+0 & 2.40583+0 & 2.73896+0 & 3.34537+0\end{array}$ $\begin{array}{lllll}4.08604+0 & 4.99070+0 & 6.09565+0 & 7.44525+0 & 9.09365+0\end{array}$ $1.11070+1 \quad 1.35661+1$ $\begin{array}{llllll}\text { df115 } & & 3.70370-3 & 4.37060-3 & 4.52500-3 & 4.57410-3 \\ & 4.55830-3 & 4.48970-3 & 4.38170-3 & 4.24920-3 & 4.12890-3\end{array}$ $\begin{array}{lllll}4.03110-3 & 3.93550-3 & 3.84530-3 & 3.76290-3 & 3.69080-3\end{array}$ $\begin{array}{lllll}3.65280-3 & 3.63870-3 & 3.62290-3 & 3.60620-3 & 3.58250-3\end{array}$ $\begin{array}{llllll}3.55850-3 & 4.08750-3 & 5.89750-3 & 7.13870-3 & 7.42410-3\end{array}$ $\begin{array}{lllll}7.67700-3 & 7.95180-3 & 8.74210-3 & 1.11490-2 & 1.35030-2\end{array}$ $1.65080-2$ 2.25270-2 2.86610-2 3.43150-2 4.10860-2 $4.92110-2 \quad 5.56070-2 \quad 5.77300-2 \quad 5.80800-2 \quad 5.84980-2$ 
$6.59980-2 \quad 8.26560-2 \quad 9.04890-2 \quad 9.54810-2 \quad 1.05360-1$

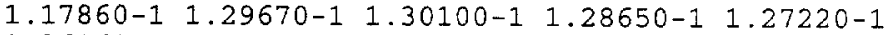
$1.26160-1$ 1.25620-1 $1.25270-1$ 1.28500-1 $1.36960-1$ $1.45980-1 \quad 1.54120-1 \quad 1.51160-1 \quad 1.47220-11.47060-1$ f.m1. $1.5 \quad 5.701+10$

f125:n $\quad 237.998 \quad 0.0 \quad 0.0 \quad 0.0$

de125

df125

$7.86024-61.66401-53.52273-57.75386-6-3.71293-6$ $2.21191-4 \quad 3.64682-4 \quad 6.01258-4 \quad 9.91309-4 \quad 1.63439-3$ $\begin{array}{lllll}2.14107-3 & 2.43063-3 & 2.82399-3 & 3.37141-3 & 4.61914-3\end{array}$ $7.32483-3 \quad 1.20766-2 \quad 1.93065-2 \quad 2.41830-2 \quad 2.54229-2$ 2.65293-2 2.77506-2 $3.14039-2 \quad 4.33909-2 \quad 5.45187-2$ 7.15396-2 1.04645-1 $1.36365-1 \quad 1.66556-1 \quad 2.03432-1$ $\begin{array}{lllll}2.48472-1 & 2.83877-1 & 2.95864-1 & 2.97851-1 & 3.00232-1\end{array}$ 3.44858-1 $4.42806-1 \quad 5.10634-1 \quad 5.65749-1 \quad 6.75418-1$ $8.24957-1 \quad 1.00760+0 \quad 1.23069+0 \quad 1.50317+0 \quad 1.83598+0$ $2.12513+0 \quad 2.28850+0 \quad 2.40583+0 \quad 2.73896+0 \quad 3.34537+0$ $4.08604+0 \quad 4.99070+0 \quad 6.09565+0 \quad 7.44525+0 \quad 9.09365+0$ $1.11070+1 \quad 1.35661+1$

$3.70370-3 \quad 4.37060-3 \quad 4.52500-3 \quad 4.57410-3$ $4.55830-3 \quad 4.48970-3 \quad 4.38170-3 \quad 4.24920-3 \quad 4.12890-3$ $4.03110-3 \quad 3.93550-3 \quad 3.84530-3 \quad 3.76290-3 \quad 3.69080-3$ $3.65280-3 \quad 3.63870-3 \quad 3.62290-3 \quad 3.60620-3 \quad 3.58250-3$ $3.55850-3 \quad 4.08750-3 \quad 5.89750-3 \quad 7.13870-3 \quad 7.42410-3$ $7.67700-3 \quad 7.95180-3 \quad 8.74210-3 \quad 1.11490-2 \quad 1.35030-2$ $1.65080-2 \quad 2.25270-2 \quad 2.86610-2 \quad 3.43150-2 \quad 4.10860-2$ $4.92110-2 \quad 5.56070-2 \quad 5.77300-2 \quad 5.80800-2 \quad 5.84980-2$ $6.59980-2 \quad 8.26560-2 \quad 9.04890-2 \quad 9.54810-2 \quad 1.05360-1$ $1.17860-1 \quad 1.29670-1 \quad 1.30100-1 \quad 1.28650-1 \quad 1.27220-1$

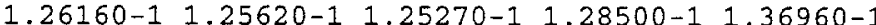
$1.45980-1 \quad 1.54120-1 \quad 1.51160-1 \quad 1.47220-1 \quad 1.47060-1$ $1.63200-12.00340-1$

$\operatorname{fm} 125$ $5.701+10$

f135: $n$ 359.918

de135 $2.07002-7 \quad 7.69672-7 \quad 1.75386-6 \quad 3.71293-6$ 7.86024-6 $1.66401-5 \quad 3.52273-5 \quad 7.45761-5 \quad 1.34159-4$ $2.21191-4 \quad 3.64682-4 \quad 6.01258-4 \quad 9.91309-4$ 1.63439-3 2.14167-3 $2.43063-3 \quad 2.82399-3 \quad 3.37141-3 \quad 4.61914-3$ $\begin{array}{lllll}7.32483-3 & 1.20766-2 & 1.93065-2 & 2.41830-2 & 2.54229-2\end{array}$ 2.65293-2 2.77506-2 3.14039-2 4.33909-2 5.45187-2 7.15396-2 $1.04645-1 \quad 1.36365-1 \quad 1.66556-1 \quad 2.03432-1$ $\begin{array}{llllll}2.48472-1 & 2.83877-1 & 2.95864-1 & 2.97851-1 & 3.00232-1\end{array}$ 3.44858-1 $4.42806-1 \quad 5.10634-1 \quad 5.65749-1 \quad 6.75418-1$ 8.24957-1 $1.00760+0 \quad 1.23069+0 \quad 1.50317+0.1 .83598+0$ $2.12513+0 \quad 2.28850+0 \quad 2.40583+0 \quad 2.73896+0 \quad 3.34537+0$ $4.08604+0 \quad 4.99070+0 \quad 6.09565+0 \quad 7.44525+0 \quad 9.09365+0$ $1.11070+1 \quad 1.35661+1$ $3.70370-3 \quad 4.37060-3 \quad 4.52500-3 \quad 4.57410-3$

df135 $4.55830-3 \quad 4.48970-3 \quad 4.38170-3 \quad 4.24920-3 \quad 4.12890-3$ $\begin{array}{llllll}4.03110-3 & 3.93550-3 & 3.84530-3 & 3.76290-3 & 3.69080-3\end{array}$ $3.65280-3 \quad 3.63870-3 \quad 3.62290-3.3 .60620-3 \quad 3.58250-3$ $\begin{array}{llllll}3.55850-3 & 4.08750-3 & 5.89750-3 & 7.13870-3 & 7.42410-3\end{array}$ $\begin{array}{llllll}7.67700-3 & 7.95180-3 & 8.74210-3 & 1.11490-2 & 1.35030-2\end{array}$

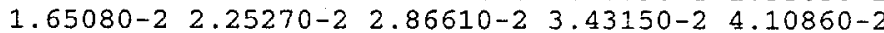
4.92110-2 $5.56070-2 \quad 5.77300-2 \quad 5.80800-2 \quad 5.84980-2$ 6.59980-2 $8.26560-2 \quad 9.04890-2 \quad 9.54810-2 \quad 1.05360-1$

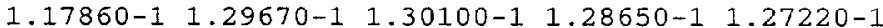
$1.26160-1 \quad 1.25620-1 \quad 1.25270-1 \quad 1.28500-1 \quad 1.36960-1$ $1.45980-1 \quad 1.54120-1$ 1.51160-1 $1.47220-1.1 .47060-1$ $1.63200-12.00340-1$

fm:135 f145:n $5.701+10$

de145

$\begin{array}{llll}420.878 & 0.0 & 0.0 & 0.0\end{array}$ $2.07002-7 \quad 7.69672-7 \quad 1.75386-6 \quad 3.71293-6$

7.86024-6 $1.66401-5 \quad 3.52273-5 \quad 7.45761-5 \quad 1.34159-4$ 2.21191-4 3.64682-4 $6.01258-4 \quad 9.91309-4 \quad 1.63439-3$ 2.14167-3 $2.43063-3 \quad 2.82399-3 \quad 3.37141-3 \quad 4.61914-3$ 7.32483-3 $1.20766-2 \quad 1.93065-2 \quad 2.41830-2 \quad 2.54229-2$ 2.65293-2 2.77506-2 $3.14039-2 \quad 4.33909-2 \quad 5.45187-2$

7.15396-2 $1.04645-1 \quad 1.36365-1 \quad 1.66556-1 \quad 2.03432-1$

$\begin{array}{lllll}2.48472-1 & 2.83877-1 & 2.95864-1 & 2.97851-1 & 3.00232-1\end{array}$

3.44858-1 4.42806-1 5.10634-1 5.65749-1 6.75418-1 
$8.24957-1 \quad 1.00760+0 \quad 1.23069+0 \quad 1.50317+0 \quad 1.83598+0$ $2.12513+0 \quad 2.28850+0 \quad 2.40583+0 \quad 2.73896+0 \quad 3.34537+0$ $4.08604+0 \quad 4.99070+0 \quad 6.09565+0 \quad 7.44525+0 \quad 9.09365+0$ $1.11070+11.35661+1$

def145

$3.70370-3 \quad 4.37060-3 \quad 4.52500-3 \quad 4.57410-3$

$4.55830-3 \quad 4.48970-3 \quad 4.38170-3.4 .24920-3.4 .12890-3$ $4.03110-3 \quad 3.93550-3 \quad 3.84530-3 \quad 3.76290-3 \quad 3.69080-3$

$3.65280-3 \quad 3.63870-3 \quad 3.62290-3 \quad 3.60620-3 \quad 3.58250-3$

$3.55850-3 \quad 4.08750-3 \quad 5.89750-3 \quad 7.13870-3 \quad 7.42410-3$

$7.67700-3 \quad 7.95180-3 \quad 8.74210-3 \quad 1.11490-2 \quad 1.35030-2$

$\begin{array}{lllll}1.65080-2 & 2.25270-2 & 2.86610-2 & 3.43150-2 & 4.10860-2\end{array}$

$4.92110-2 \quad 5.56070-2 \quad 5.77300-2 \quad 5.80800-2 \quad 5.84980-2$

6.59980-2 $8.26560-2 \quad 9.04890-2 \quad 9.54810-2 \quad 1.05360-1$

$1.17860^{-}-1 \quad 1.29670-1 \quad 1.30100-1 \quad 1.28650-1$ 1.27220-1

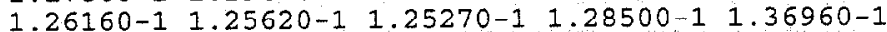

$1.45980-1 \quad 1.54120-1 \quad 1.51160-1 \quad 1.47220-1 \quad 1.47060-1$

$1.63200-12.00340-1$

$\operatorname{fm} 145$

f155:n

de155

$5.701+10$

$481.838: 0.0 \quad 0.0 \quad 0.0$

$2.07002-7 \quad 7.69672-7 \quad 1.75386-6 \quad 3.71293-6$

$\begin{array}{lllll}2.07002-7 & 7.69672-7 & 1.75386-6 & 3.71293-6\end{array}$

$2.21191-4 \quad 3.64682-4 \quad 6.01258-4 \quad 9.91309-4 \quad 1.63439-3$

2.14167-3 2.43063-3 2.82399-3 $3.37141-3$ 4.61914-3

$\begin{array}{lllll}7.32483-3 & 1.20766-2 & 1.93065-2 & 2.41830-2 & 2.54229-2\end{array}$

2.65293-2 $2.77506-2 \quad 3.14039-2 \quad 4.33909-2 \quad 5.45187-2$

7.15396-2 $1.04645-1 \quad 1.36365-1 \quad 1.66556-1 \quad 2.03432-1$

$\begin{array}{lllll}2.48472-1 & 2.83877-1 & 2.95864-1 & 2.97851-1 & 3.00232-1\end{array}$

3.44858-1 $4.42806-1 \quad 5.10634-1,5.65749-1 \quad 6.75418-1$

$8.24957-1 \quad 1.00760+0 \quad 1.23069+0 \quad 1.50317+0 \quad 1.83598+0$

$2.12513+0 \quad 2.28850+0 \quad 2.40583+0 \quad 2.73896+0 \quad 3.34537+0$

$4.08604+0 \quad 4.99070+0 \quad 6.09565+0 \quad 7.44525+0.9 .09365+0$

$1.11070+1 \quad 1.35661+1$

df155 $\quad 3.70370-3 \quad 4.37060-3 \quad 4.52500-3 \quad 4.57410-3$

$4.55830-3 \quad 4.48970-3 \quad 4.38170-3 \quad 4.24920-3 \quad 4.12890-3$

$\begin{array}{llllll}4.03110-3 & 3.93550-3 & 3.84530-3 & 3.76290-3 & 3.69080-3\end{array}$

$3.65280-3 \quad 3.63870-3 \quad 3.62290-3 \quad 3.60620-3 \quad 3.58250-3$

$\begin{array}{llllll}3.55850-3 & 4.08750-3 & 5.89750-3 & 7.13870-3 & 7.42410-3\end{array}$

$7.67700-3 \quad 7.95180-3 \quad 8.74210-3 \quad 1.11490-2 \quad 1.35030-2$

$1.65080-2 \quad 2.25270-2 \quad 2.86610-2 \quad 3.43150-2 \quad 4.10860-2$

$4.92110-2 \quad 5.56070-2 \quad 5.77300-2 \quad 5.80800-2 \quad 5.84980-2$

$6.59980-2 \quad 8.26560-2 \quad 9.04890-2 \quad 9.54810-2 \quad 1.05360-1$

$1.17860-1 \quad 1.29670-1 \quad 1.30100-1 \quad 1.28650-1 \quad 1.27220-1$

$1.26160-1 \quad 1.25620-1 \quad 1.25270-1 \quad 1.28500-1 \quad 1.36960-1$

$1.45980-1$ 1.54120-1 $1.51160-1$ 1.47220-1 1.47060-1

$1.63200-12.00340-1$

$\operatorname{fm} 155$

f165:n

e165

fm165

f175:n

e175

fm175

de175

$5.701+10$

116.078

$5-7 \quad 1.0 \quad 30.0$

$5.701+10$

116.078

$5-7 \quad 1.0 \quad 30.0$

$5.701+10$

$1.0-12 \quad 5 \cdot 0-12 \quad 1 \cdot 0-11 \quad 5 \cdot 0-11 \quad 1 \cdot 0-10 \quad 5 \cdot 0-10$

$\begin{array}{lllllll}1.0-9 & 5.0-9 & 1.0-8 & 2.53-8 & 5.0-8 & 1.0-7 & 5.0-7\end{array}$

$5.00001-720.0$

df175 $\quad 159.0597 \quad 71.1337 \quad 50.2991 \quad 22.4944 \quad 15.906 \quad 7.11337$

$\begin{array}{llllllllll}5.02991 & 2.24944 & 1.5906 & 1.0 & 0.711337 & 0.502991 & 0.224944\end{array}$ $1.0-15 \quad 1.0-15$

$\begin{array}{lllllll}\text { f205:p } & 116.078 & 0.0 & 0.000 .0 & 0 & & \\ \text { de205 } & & 1.50000-2 & 3.25000-2 & 5.75000-2 & 8.50000-2\end{array}$ $1.25000-12.25000-1 \quad 3.50000-1 \quad 4.55000-1 \quad 5.55000-1$ $6.50000-1 \quad 8.50000-1 \quad 1.25000+0 \quad 1.75000+0 \quad 2.25000+0$ $2.75000+0 \quad 3.50000+0 \quad 4.50000+0 \quad 5.50000+0 \quad 6.50000+0$ $7.25000+07.75000+09.00000+01.20000+1$

df205 $2.14390-3 \quad 5.77600-4 \quad 2.71850-4 \quad 2.68170-4$ $3.27670-4 \quad 5.66760-4 \quad 8.75940-4 \quad 1.08450-3 \quad 1.27970-3$ $1.44170-3 \quad 1.75630-3 \quad 2.31560-3 \quad 2.92700-3 \quad 3.46860-3$ $3.95960-3 \quad 4.62210-3 \quad 5.41370-3 \quad 6.19090-3 \quad 6.92650-3$ $7.47830-3 \quad 7.84680-3 \quad 8.77160-3 \quad 1.10200-2$

fm205 $\quad 5.701+10$

f215:p $\quad 177.038 \quad 0.0 \quad 0.0 \quad 0.0$

de 215 $1.50000-2 \quad 3.25000-2 \quad 5.75000-2 \quad 8.50000-2$ 


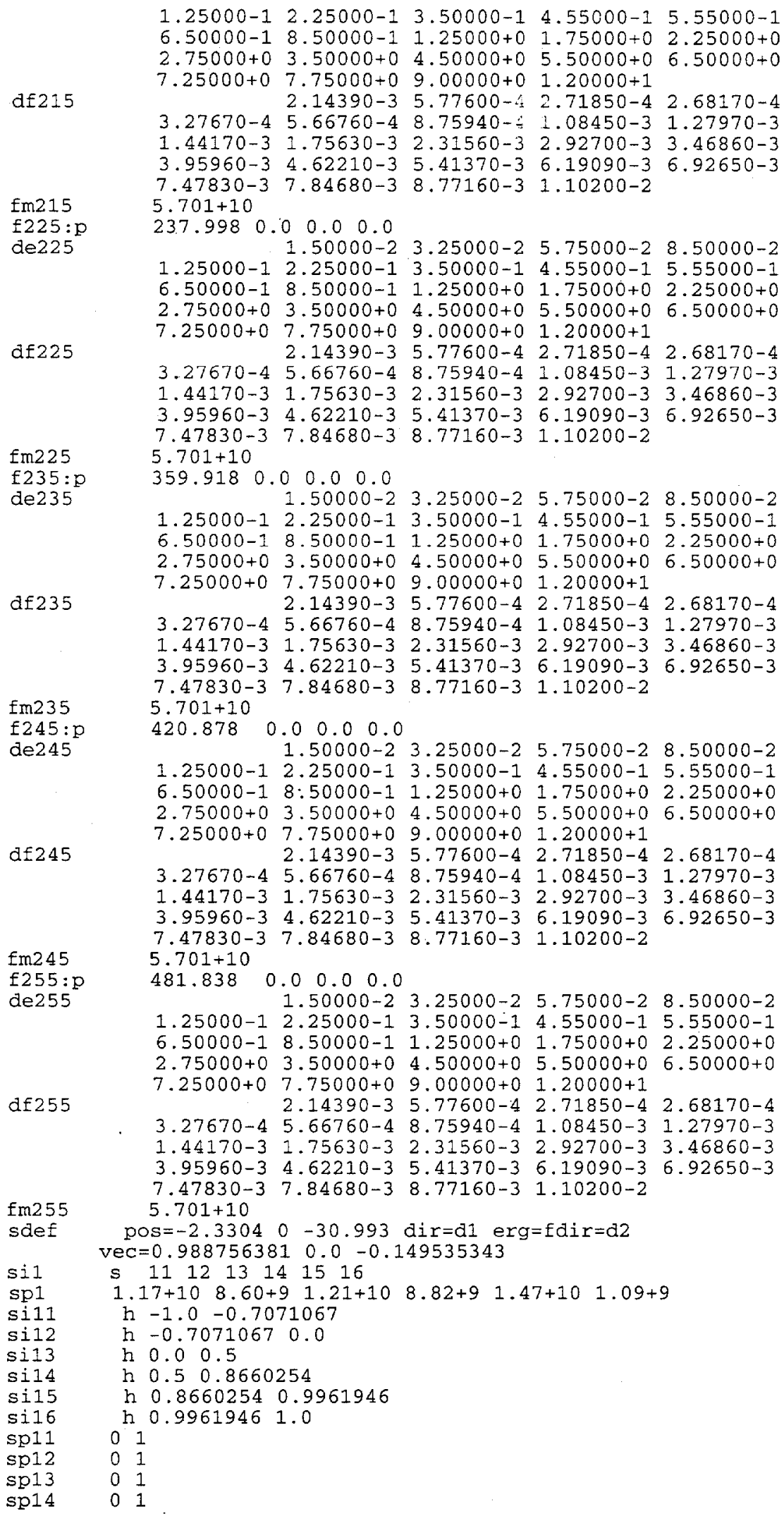




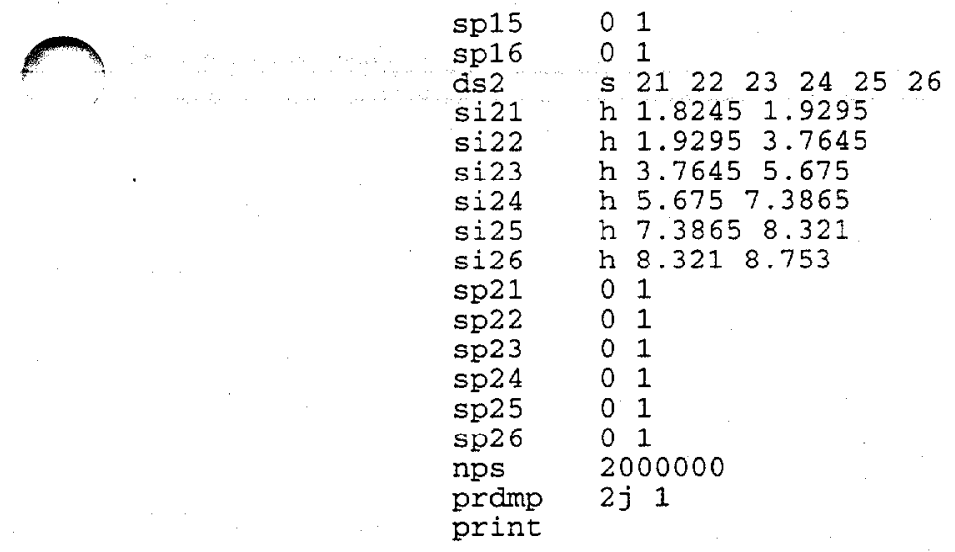


File for MCNP-4B calculation of the dose-rate distribution in the middle of salted beef cargo at the center of the PFNA facility truck lane for the source in the horizontal position

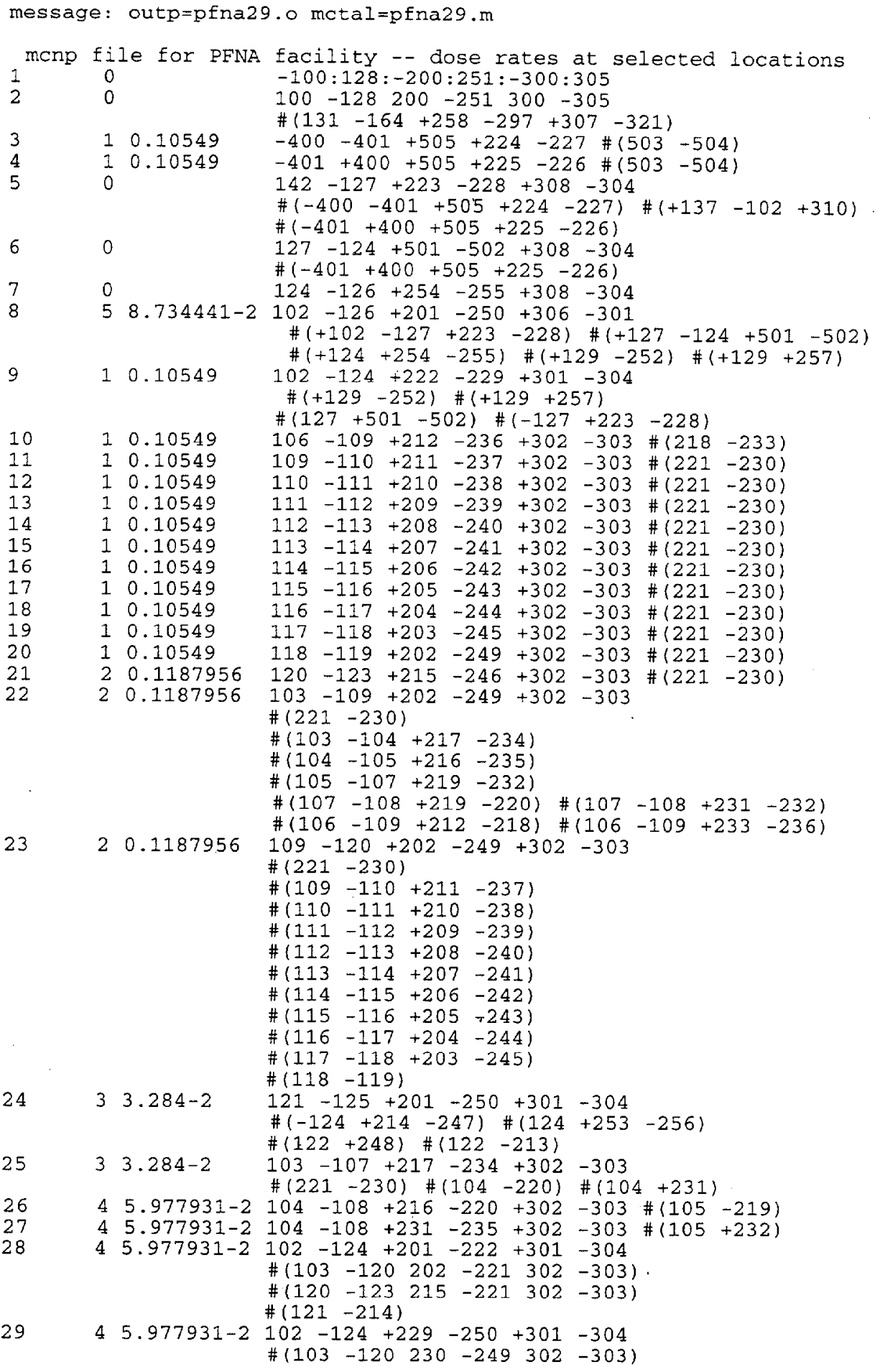




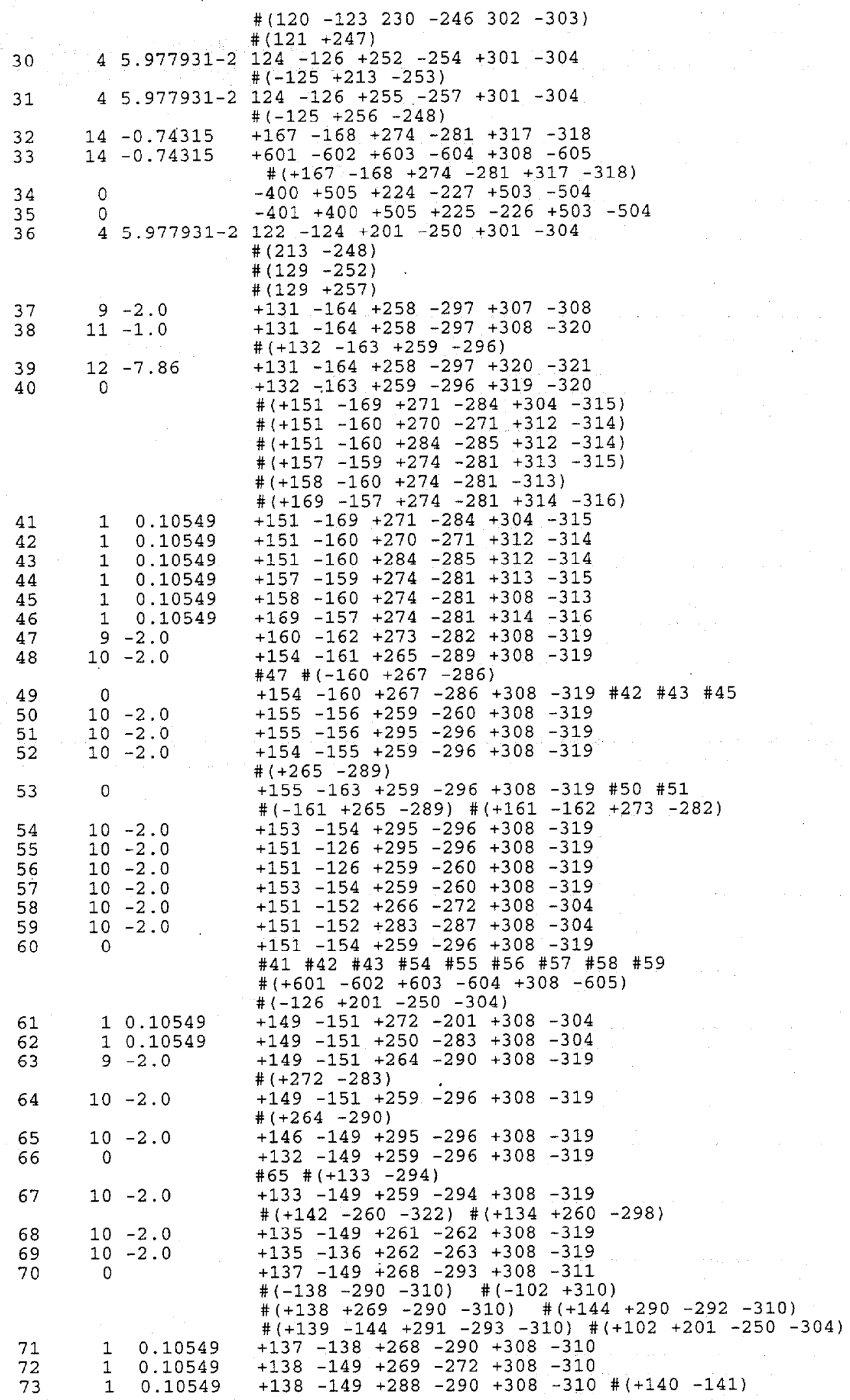




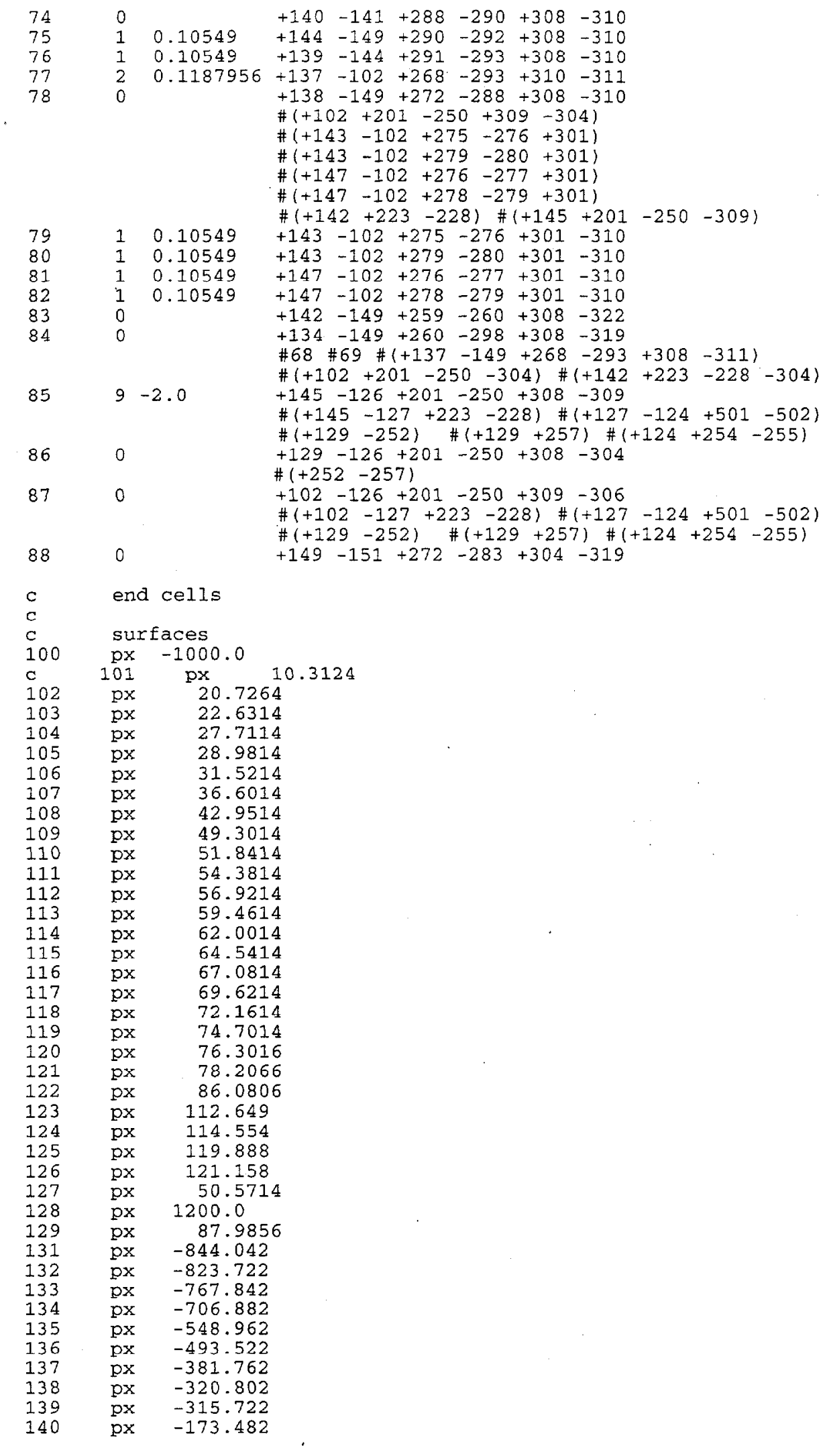




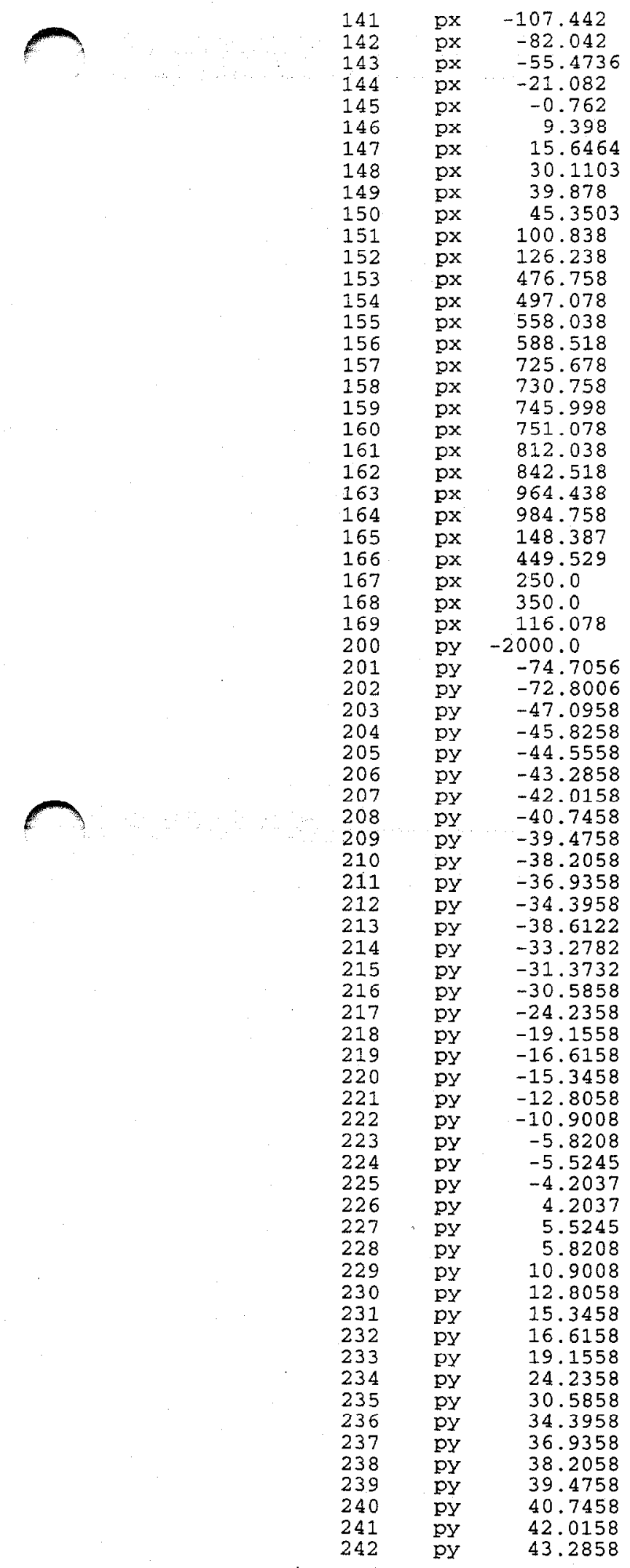




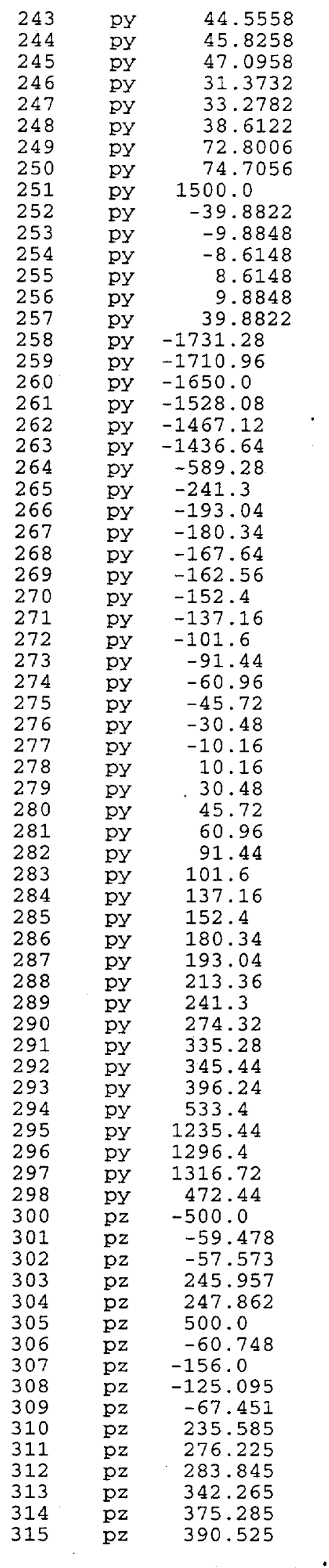




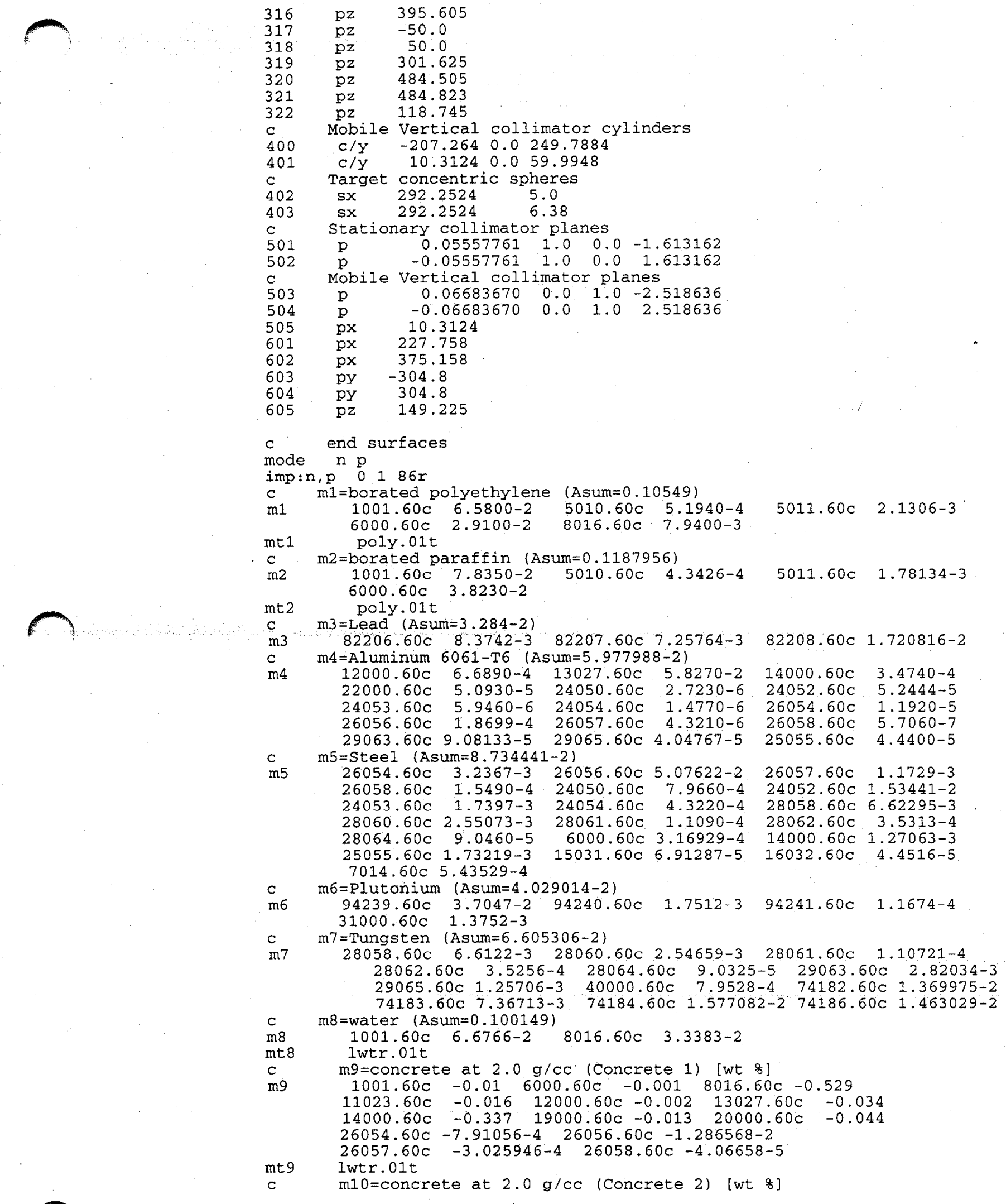




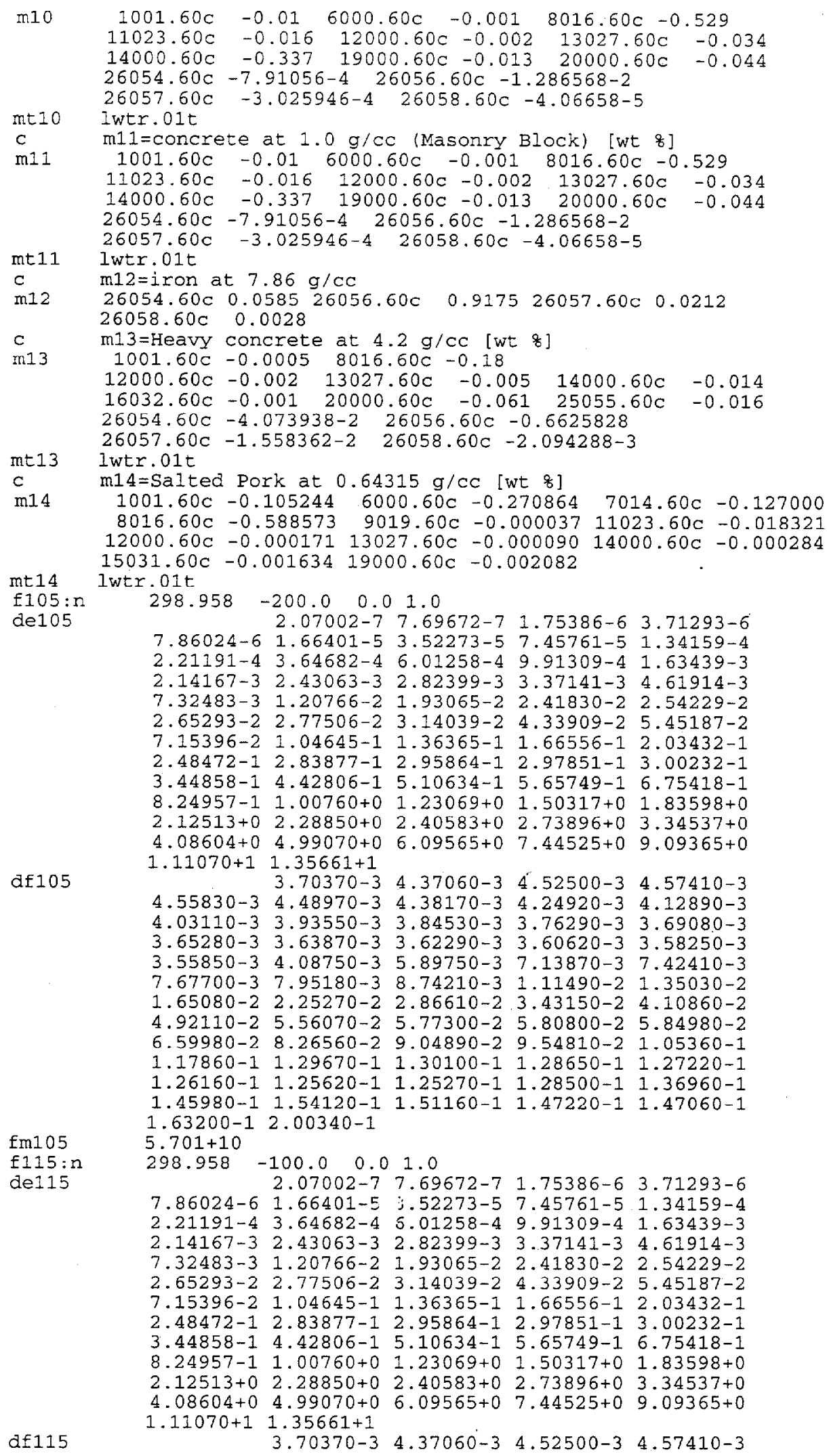


$4.55830-3 \quad 4.48970-3 \quad 4.38170-3 \quad 4.24920-3 \quad 4.12890-3$ $4.03110-3 \quad 3.93550-3 \quad 3.84530-3 \quad 3.76290-3 \quad 3.69080-3$

$3.65280-3 \quad 3.63870-3 \quad 3.62290-3 \quad 3.60620-3 \quad 3.58250-3$

$3.55850-3 \quad 4.08750-3 \quad 5.89750-3 \quad 7.13870-3 \quad 7.42410-3$

$\begin{array}{lllll}7.67700-3 & 7.95180-3 & 8.74210-3 & 1.11490-2 & 1.35030-2\end{array}$

$1.65080-2 \quad 2.25270-2 \quad 2.86610-2 \quad 3.43150-2 \quad 4.10860-2$

$4.92110-2 \quad 5.56070-2 \quad 5.77300-2 \quad 5.80800-2 \quad 5.84980-2$

$6.59980-2 \quad 8.26560-2 \quad 9.04890-2 \quad 9.54810-2 \quad 1.05360-1$

$1.17860-1$ 1.29670-1 $1.30100-1$ 1.28650-1 $1.27220-1$

$1.26160-1 \quad 1.25620-1 \quad 1.25270-1 \quad 1.28500-1 \quad 1.36960-1$

$1.45980-1 \quad 1.54120-1.1 .51160-1 \quad 1.47220-1 \quad 1.47060-1$ $1.63200-12.00340-1$

fm115

f125:n

$5.701+10$

de125

$\operatorname{df} 125$

298.958

$-50.0 \quad 0.0 \quad 1.0$

$2.07002-7 \quad 7.69672-7 \quad 1.75386-6 \quad 3.71293-6$

7.86024-6 $1.66401-5 \quad 3.52273-5 \quad 7.45761-5 \quad 1.34159-4$

$2.21191-4 \quad 3.64682-4 \quad 6.01258-4 \quad 9.91309-4 \quad 1.63439-3$

$2.14167-3 \quad 2.43063-3 \quad 2.82399-3 \quad 3.37141-3 \quad 4.61914-3$

7.32483-3 $1.20766-2 \quad 1.93065-2 \quad 2.41830-2 \quad 2.54229-2$

2.65293-2 2.77506-2 3.14039-2 4.33909-2 5.45187-2

7.15396-2 $1.04645-1 \quad 1.36365-1 \quad 1.66556-1 \quad 2.03432-1$

$\begin{array}{lllll}2.48472-1 & 2.83877-1 & 2.95864-1 & 2.97851-1 & 3.00232-1\end{array}$

$\begin{array}{llllll}3.44858-1 & 4.42806-1 & 5.10634-1 & 5.65749-1 & 6.75418-1\end{array}$

$8.24957-1 \quad 1.00760+0 \quad 1.23069+0 \quad 1.50317+0 \quad 1.83598+0$

$2.12513+0 \quad 2.28850+0 \quad 2.40583+0 \quad 2.73896+0 \quad 3.34537+0$

$4.08604+0 \quad 4.99070+0 \quad 6.09565+0 \quad 7.44525+0 \quad 9.09365+0$

$1.11070+1 \quad 1.35661+1$ $3.70370-3 \quad 4.37060-3 \quad 4.52500-3 \quad 4.57410-3$

$4.55830-3 \quad 4.48970-3 \quad 4.38170-3 \quad 4.24920-3 \quad 4.12890-3$

$4.03110-3 \quad 3.93550-3 \quad 3.84530-3 \quad 3.76290-3 \quad 3.69080-3$

$3.65280-3 \quad 3.63870-3 \quad 3.62290-3 \quad 3.60620-3 \quad 3.58250-3$

$\begin{array}{llllll}3.55850-3 & 4.08750-3 & 5.89750-3 & 7.13870-3 & 7.42410-3\end{array}$

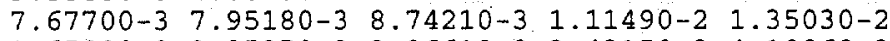

$\begin{array}{lllll}1.65080-2 & 2.25270-2 & 2.86610-2 & 3.43150-2 & 4.10860-2\end{array}$

$4.92110-2 \quad 5.56070-2 \quad 5.77300-2 \quad 5.80800-2 \quad 5.84980-2$

$6.59980-2$ 8.26560-2 $9.04890-2 \quad 9.54810-2.1 .05360-1$

$1.17860-1 \quad 1.29670-1 \quad 1.30100-1 \quad 1.28650-1 \quad 1.27220-1$

$1.26160-1 \quad 1.25620-1 \quad 1.25270-1.1 .28500-1 \quad 1.36960-1$

$1.45980-1$ 1.54120-1 1.51160-1 1.47220-1 1.47060-1

$1.63200-12.00340-1$

$\operatorname{fm} 125 \quad 5.701+10$

f135:n $\quad 298.958 \quad-25.0 \quad 0.01 .0$

de135 $2.07002-7 \quad 7.69672-7 \quad 1.75386-6 \quad 3.71293-6$

$7.86024-61.66401-5 \quad 3.52273-5 \quad 7.45761-51.34159-4$

$2.21191-4 \quad 3.64682-4 \quad 6.01258-4 \quad 9.91309-4 \quad 1.63439-3$

$2.14167-3 \quad 2.43063-3 \quad 2.82399-3 \quad 3.37141-3 \quad 4.61914-3$

$7.32483-3 \quad 1.20766-2 \quad 1.93065-2.2 .41830-2 \quad 2.54229-2$

$2.65293-2 \quad 2.77506-2 \quad 3.14039-2 \quad 4.33909-2 \quad 5.45187-2$

$\begin{array}{llllll}7.15396-2 & 1.04645-1 & 1.36365-1 & 1.66556-1 & 2.03432-1\end{array}$

$\begin{array}{llllll}2.48472-1 & 2.83877-1 & 2.95864-1 & 2.97851-1 & 3.00232-1\end{array}$

3.44858-1 $4.42806-1 \quad 5.10634-1 \quad 5.65749-1 \quad 6.75418-1$

$8.24957-1 \quad 1.00760+01.23069+0.1 .50317+0 \quad 1.83598+0$

$2.12513+0 \quad 2.28850+0 \quad 2.40583+0 \quad 2.73896+0 \quad 3.34537+0$

$4.08604+0 \quad 4.99070+0 \quad 6.09565+0 \quad 7.44525+0 \quad 9.09365+0$ $1.11070+1 \quad 1.35661+1$

$3.70370-3 \quad 4.37060-3 \quad 4.52500-3 \quad 4.57410-3$ 4.55830-3 4.48970-3 $4.38170-3 \quad 4.24920-3 \quad 4.12890-3$ $\begin{array}{llllll}4.03110-3 & 3.93550-3 & 3.84530-3 & 3.76290-3 & 3.69080-3\end{array}$ $\begin{array}{llllll}3.65280-3 & 3.63870-3 & 3.62290-3 & 3.60620-3 & 3.58250-3\end{array}$

$\begin{array}{llllll}3.55850-3 & 4.08750-3 & 5.89750-3 & 7.13870-3 & 7.42410-3\end{array}$

$\begin{array}{lllll}7.67700-3 & 7.95180-3 & 8.74210-3 & 1.11490-2 & 1.35030-2\end{array}$

$\begin{array}{lllll}1.65080-2 & 2.25270-2 & 2.86610-2 & 3.43150-2 & 4.10860-2\end{array}$

4.92110-2 5.56070-2 5.77300-2 5.80800-2 5.84980-2

$6.59980-2 \quad 8.26560-2 \quad 9.04890-2 \quad 9.54810-2 \quad 1.05360-1$

1.17860-1 $1.29670-1 \quad 1.30100-1 \quad 1.28650-1 \quad 1.27220-1$

$1.26160-1$ 1.25620-1 $1.25270-1$ 1.28500-1 $1.36960-1$

$1.45980-1 \quad 1.54120-1 \quad 1.51160-1 \quad 1.47220-1 \quad 1.47060-1$

$1.63200-12.00340-1$

$\operatorname{fm} 135 \quad 5.701+10$

f145:n $\quad 298.958 \quad 0.0 \quad 0.01 .0$

$\begin{array}{lllllll}\text { de145 } & 2.07002-7 & 7.69672-7 & 1.75386-6 & 3.71293-6\end{array}$

$7.86024-6 \quad 1.66401-5 \quad 3.52273-5 \quad 7.45761-5 \quad 1.34159-4$ 
$\begin{array}{llllll}2.21191-4 & 3.64682-4 & 6.01258-4 & 9.91309-4 & 1.63439-3\end{array}$ 2.14167-3 2.43063-3 $2.82399-3 \quad 3.37141-3 \quad 4.61914-3$ 7.32483-3 1.20766-2 $1.93055-2 \quad 2.41830-2 \quad 2.54229-2$ 2.65293-2 2.77506-2 $3.14039-2 \quad 4.33909-2 \quad 5.45187-2$

7.15396-2 $1.04645-1 \quad 1.36365-1 \quad 1.66556-1 \quad 2.03432-1$

2.48472-1 $2.83877-1 \quad 2.95864-1 \quad 2.97851-1 \quad 3.00232-1$

3.44858-1 4.42806-1 $5.10634-1 \quad 5.65749-1 \quad 6.75418-1$

$8.24957-1 \quad 1.00760+0 \quad 1.23069+0 \quad 1.50317+0 \quad 1.83598+0$

$2.12513+0 \quad 2.28850+0 \quad 2.40583+0 \quad 2.73896+0 \quad 3.34537+0$

$4.08604+0 \quad 4.99070+0 \quad 6.09565+0 \quad 7.44525+0 \quad 9.09365+0$

$\operatorname{df145}$

$1.11070+11.35661+1$

$3.70370-3 \quad 4.37060-3 \quad 4.52500-3 \quad 4.57410-3$

$4.55830-3 \quad 4.48970-3 \quad 4.38170-3 \quad 4.24920-3 \quad 4.12890-3$

$4.03110-3 \quad 3.93550-3 \quad 3.84530-3 \quad 3.76290-3 \quad 3.69080-3$

$\begin{array}{llllll}3.65280-3 & 3.63870-3 & 3.62290-3 & 3.60620-3 & 3.58250-3\end{array}$

$\begin{array}{lllll}3.55850-3 & 4.08750-3 & 5.89750-3 & 7.13870-3 & 7.42410-3\end{array}$

$\begin{array}{lllll}7.67700-3 & 7.95180-3 & 8.74210-3 & 1.11490-2 & 1.35030-2\end{array}$

$1.65080-2 \quad 2.25270-2 \quad 2.86610-2 \quad 3.43150-2 \quad 4.10860-2$

$4.92110-2 \quad 5.56070-2 \quad 5.77300-2 \quad 5.80800-2 \quad 5.84980-2$

$6.59980-2 \quad 8.26560-2 \quad 9.04890-2 \quad 9.54810-2 \quad 1.05360-1$

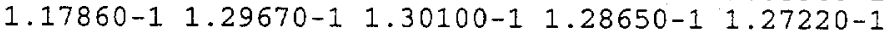

$1.26160-1 \quad 1.25620-1 \quad 1.25270-1 \quad 1.28500-1 \quad 1.36960-1$ $1.45980-1 \quad 1.54120-1 \quad 1.51160-1 \quad 1.47220-1 \quad 1.47060-1$ $1.63200-12.00340-1$

$\operatorname{fm} 145$ $5.701+10$

f155:n 298.958

$25.0 \quad 0.01 .0$

de 155

df155

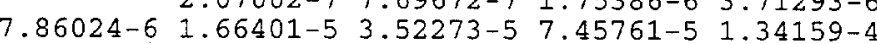
$\begin{array}{llllll}2.21191-4 & 3.64682-4 & 6.01258-4 & 9.91309-4 & 1.63439-3\end{array}$ $\begin{array}{llllll}2.14167-3 & 2.43063-3 & 2.82399-3 & 3.37141-3 & 4.61914-3\end{array}$ $\begin{array}{llllll}7.32483-3 & 1.20766-2 & 1.93065-2 & 2.41830-2 & 2.54229-2\end{array}$ $2.65293-2 \quad 2.77506-2 \quad 3.14039-2 \quad 4.33909-2 \quad 5.45187-2$ 7.15396-2 $1.04645-1 \quad 1.36365-1 \quad 1.66556-1 \quad 2.03432-1$ $2.48472-1 \quad 2.83877-1 \quad 2.95864-1 \quad 2.97851-1 \quad 3.00232-1$ 3.44858-1 $4.42806-1 \quad 5.10634-1 \quad 5.65749-1 \quad 6.75418-1$ $8.24957-11.00760+0 \quad 1.23069+0 \quad 1.50317+0 \quad 1.83598+0$ $\begin{array}{lllll}2.12513+0 & 2.28850+0 & 2.40583+0 & 2.73896+0 & 3.34537+0\end{array}$ $4.08604+0 \quad 4.99070+0 \quad 6.09565+0 \quad 7.44525+0 \quad 9.09365+0$ $1.11070+1 \quad 1.35661+1$

$3.70370-3 \quad 4.37060-3 \quad 4.52500-3 \quad 4.57410-3$ $4.55830-3 \quad 4.48970-3 \quad 4.38170-3 \quad 4.24920-3 \quad 4.12890-3$ $\begin{array}{llllll}4.03110-3 & 3.93550-3 & 3.84530-3 & 3.76290-3 & 3.69080-3\end{array}$ $3.65280-3 \quad 3.63870-3 \quad 3.62290-3 \quad 3.60620-3 \quad 3.58250-3$ $\begin{array}{lllll}3.55850-3 & 4.08750-3 & 5.89750-3 & 7.13870-3 & 7.42410-3\end{array}$ $\begin{array}{llllll}7.67700-3 & 7.95180-3 & 8.74210-3 & 1.11490-2 & 1.35030-2\end{array}$ $1.65080-2 \quad 2.25270-2 \quad 2.86610-2 \quad 3.43150-2 \quad 4.10860-2$ 4.92110-2 5.56070-2 5.77300-2 $5.80800-2 \quad 5.84980-2$ $6.59980-2 \quad 8.26560-2 \quad 9.04890-2 \quad 9.54810-2 \quad 1.05360-1$ $1.17860-1 \quad 1.29670-1 \quad 1.30100-1 \quad 1.28650-1 \quad 1.27220-1$ $1.26160-1 \quad 1.25620-1 \quad 1.25270-1 \quad 1.28500-11.36960-1$ $1.45980-1 \quad 1.54120-1 \quad 1.51160-1 \quad 1.47220-1 \quad 1.47060-1$ $1.63200-12.00340-1$

fm155

f165: $\mathrm{n}$ $5.701+10$

$298.958 \quad 50.0 \quad 0.0 \quad 1.0$

de165

$2.07002-7 \quad 7.69672-7 \quad 1.75386-6 \quad 3.71293-6$ $\begin{array}{lllll} & 07002-7 & 7.5962-7 & 1.75386-6 & 3.71293-6\end{array}$ $2.21191-4 \quad 3.64682-4 \quad 6.01258-4 \quad 9.91309-4 \quad 1.63439-3$ $2.14167-3 \quad 2.43063-3 \quad 2.82399-3 \quad 3.37141-3 \quad 4.61914-3$ $\begin{array}{llllll}7.32483-3 & 1.20766-2 & 1.93065-2 & 2.41830-2 & 2.54229-2\end{array}$ 2.65293-2 2.77506-2 $3.14039-2 \quad 4.33909-2 \quad 5.45187-2$ 7.15396-2 $1.04645-1 \quad 1.36365-1 \quad 1.66556-1 \quad 2.03432-1$ $2.48472-1 \quad 2.83877-1 \quad 2.95864-1 \quad 2.97851-1 \quad 3.00232-1$ 3.44858-1 4.42806-1 $5.10634-1 \quad 5.65749-1 \quad 6.75418-1$ $8.24957-1 \quad 1.00760+0 \quad 1.23069+0 \quad 1.50317+0 \quad 1.83598+0$ $2.12513+0 \quad 2.28850+0 \quad 2.40583+0 \quad 2.73896+0 \quad 3.34537+0$ $4.08604+0 \quad 4.99070+0 \quad 6.09565+0 \quad 7.44525+0 \quad 9.09365+0$ $1.11070+11.35661+1$

df165 $3.70370-3 \quad 4.37060-3 \quad 4.52500-3 \quad 4.57410-3$ $4.55830-3 \quad 4.48970-3 \quad 4.38170-3 \quad 4.24920-3 \quad 4.12890-3$ $\begin{array}{llllll}4.03110-3 & 3.93550-3 & 3.84530-3 & 3.76290-3 & 3.69080-3\end{array}$ $3.65280-3 \quad 3.63870-3 \quad 3.62290-3 \quad 3.60620-3 \quad 3.58250-3$ $3.55850-3 \quad 4.08750-3 \quad 5.89750-3 \quad 7.13870-3 \quad 7.42410-3$ 
$\begin{array}{lllll}7.67700-3 & 7.95180-3 & 8.74210-3 & 1.11490-2 & 1.35030-2\end{array}$ $1.65080-2 \quad 2.25270-2 \quad 2.86610-2 \quad 3.43150-2 \quad 4.10860-2$ $4.92110-2 \quad 5.56070-2 \quad 5.77300-2 \quad 5.80800-2 \quad 5.84980-2$ $6.59980-2 \quad 8.26560-2 \quad 9.04890-2 \quad 9.54810-2 \quad 1.05360-1$ $1.17860-1 \quad 1.29670-1 \quad 1.30100-1 \quad 1.28650-1 \quad 1.27220-1$ $1.26160-1$ 1.25620-1 1.25270-1. $1.28500-1.1 .36960-1$ $1.45980-1 \quad 1.54120-1 \quad 1.51160-1 \quad 1.47220-1 \quad 1.47060-1$ $1.63200-12.00340-1$

$\operatorname{fim} 165$ $5.701+10$

f175: I

$298.958 \quad 100.0 \quad 0.01 .0$ 7.86024-6 $1.66401-5 \quad 3.52273-5 \quad 7.45761-5 \quad 1.34159-4$ 2.21191-4 3.64682-4 $6.01258-4 \quad 9.91309-4 \quad 1.63439-3$ 2.14167-3 $2.43063-3 \quad 2.82399-3 \quad 3.37141-3 \quad 4.61914-3$ 7.32483-3 $1.20765-2 \quad 1.93065-2 \quad 2.41830-2 \quad 2.54229-2$ 2.65293-2 2.77506-2 3.14039-2 4.33909-2 5.45187-2 7.15396-2 $1.04645-1 \quad 1.36365-1 \quad 1.66556-1 \quad 2.03432-1$ $\begin{array}{lllll}2.48472-1 & 2.83877-1 & 2.95864-1 & 2.97851-1 & 3.00232-1\end{array}$ 3.44858-1 4.42806-1 5.10634-1 5.65749-1 6.75418-1 8.24957-1 $1.00760+0 \quad 1.23069+0 \quad 1.50317+0 \quad 1.83598+0$ $2.12513+0 \quad 2.28850+0 \quad 2.40583+0 \quad 2.73896+0 \quad 3.34537+0$ $4.08604+0 \quad 4.99070+0 \quad 6.09565+0 \quad 7.44525+0 \quad 9.09365+0$ $1.11070+11.35661+1$

df175 $\quad 3.70370-3 \quad 4.37060-3 \quad 4.52500-3 \quad 4.57410-3$ $4.55830-3 \quad 4.48970-3 \quad 4.38170-3 \quad 4.24920-3 \quad 4.12890-3$ $4.03110-3 \quad 3.93550-3 \quad 3.84530-3 \quad 3.76290-3 \quad 3.69080-3$ $3.65280-3 \quad 3.63870-3 \quad 3.62290-3 \quad 3.60620-3 \quad 3.58250-3$ $3.55850-3 \quad 4.08750-3 \quad 5.89750-3 \quad 7.13870-3 \quad 7.42410-3$ $\begin{array}{lllll}7.67700-3 & 7.95180-3 & 8.74210-3 & 1.11490-2 & 1.35030-2\end{array}$ $1.65080-22.25270-2 \quad 2.86610-2 \quad 3.43150-2 \quad 4.10860-2$ $4.92110-2 \quad 5.56070-2 \quad 5.77300-2 \quad 5.80800-2 \quad 5.84980-2$ $6.59980-2 \quad 8.26560-2 \quad 9.04890-2 \quad 9.54810-2 \quad 1.05360-1$ $1.17860-1 \quad 1.29670-1 \quad 1.30100-1 \quad 1.28650-1 \quad 1.27220-1$ $1.26160-1 \quad 1.25620-1 \quad 1.25270-1 \quad 1.28500-1 \quad 1.36960-1$ $1.45980-1$ 1.54120-1 $1.51160-1$ 1.47220-1 $1.47060-1$ $1.63200-1 \quad 2.00340-1$

$\operatorname{fm} 175$ $5.701+10$

f185:n $\quad 298.958 \quad 200.0 \quad 0.0 \quad 1.0$

de185 $2.07002-7 \quad 7.69672-7 \quad 1.75386-6 \quad 3.71293-6$ $7.86024-6 \quad 1.66401-5 \quad 3.52273-5 \quad 7.45761-5 \quad 1.34159-4$ $2.21191-4 \quad 3.64682-4 \quad 6.01258-4 \quad 9.91309-4 \quad 1.63439-3$ $\begin{array}{llllll}2.14167-3 & 2.43063-3 & 2.82399-3 & 3.37141-3 & 4.61914-3\end{array}$ $7.32483-3 \quad 1.20766-2 \quad 1.93065-2 \quad 2.41830-2 \quad 2.54229-2$ 2.65293-2 2.77506-2 3.14039-2 $4.33909-2 \quad 5.45187-2$ $7.15396-2 \quad 1.04645-1 \quad 1.36365-1 \quad 1.66556-1 \quad 2.03432-1$ $\begin{array}{llllll}2.48472-1 & 2.83877-1 & 2.95864-1 & 2.97851-1 & 3.00232-1\end{array}$ 3.44858-1 $4.42806-1 \quad 5.10634-1 \quad 5.65749-1 \quad 6.75418-1$ 8.24957-1 $1.00760+0 \quad 1.23069+0 \quad 1.50317+0 \quad 1.83598+0$ $2.12513+0.2 .28850+0 \quad 2.40583+0 \quad 2.73896+0 \quad 3.34537+0$ $4.08604+0 \quad 4.99070+0 \quad 6.09565+0 \quad 7.44525+0.9 .09365+0$ $1.11070+11.35661+1$

df185 $3.70370-3 \quad 4.37060-3 \quad 4.52500-3 \quad 4.57410-3$ $4.55830-3 \quad 4.48970-3 \quad 4.38170-3 \quad 4.24920-3 \quad 4.12890-3$ $4.03110-3 \quad 3.93550-3 \quad 3.84530-3 \quad 3.76290-3.3 .69080-3$ $3.65280-3 \quad 3.63870-3 \quad 3.62290-3 \quad 3.60620-3 \quad 3.58250-3$ $3.55850-3 \quad 4.08750-3 \quad 5.89750-3 \quad 7.13870-3 \quad 7.42410-3$ $7.67700-3 \quad 7.95180-3 \quad 8.74210-3 \quad 1.11490-2 \quad 1.35030-2$

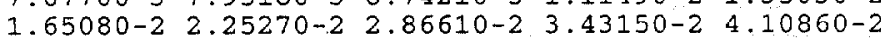
4.92110-2 $5.56070-2 \quad 5.77300-2 \quad 5.80800-2 \quad 5.84980-2$ $6.59980-2 \quad 8.26560-2 \quad 9.04890-2 \quad 9.54810-2 \quad 1.05360-1$ $1.17860-1 \quad 1.29670-1 \quad 1.30100-1.1 .28650-11.27220-1$ $1.26160-1 \quad 1.25620-1 \quad 1.25270-1 \quad 1.28500-1 \quad 1.36960-1$ 1.45980-1 $1.54120-1$ 1.51160-1 $1.47220-1 \quad 1.47060-1$ $1.63200-12.00340-1$

Em185 $5.701+10$

f195:n 298.958

$250.0 \quad 0.01 .0$ $2.07002-7 \quad 7.69672-7 \quad 1.75386-6 \quad 3.71293-6$ $7.86024-6 \quad 1.66401-5 \quad 3.52273-5 \quad 7.45761-5 \quad 1.34159-4$ $2.21191-4 \quad 3.64682-4 \quad 6.01258-4 \quad 9.91309-4 \quad 1.63439-3$ $2.14167-3 \quad 2.43063-3 \quad 2.82399-3 \quad 3.37141-3 \quad 4.61914-3$ $7.32483-3 \quad 1.20766-2 \quad 1.93065-2 \quad 2.41830-2 \quad 2.54229-2$ $2.65293-2$ 2.77506-2 3.14039-2,4.33909-2 5.45187-2 
7.15396-2 $1.04645-1 \quad 1.36365-1 \quad 1.66556-1 \quad 2.03432-1$ $2.48472-1 \quad 2.83877-1 \quad 2.95864-1 \quad 2.97851-1 \quad 3.00232-1$ $3.44858-1 \quad 4.42806-1$ 5. $10634-1 \quad 5.65749-16.75418-1$ $8.24957-1 \quad 1.00760+0 \quad 1.23069+0 \quad 1.50317+0 \quad 1.83598+0$ $2.12513+0 \quad 2.28850+0 \quad 2.40583+0 \quad 2.73896+0 \quad 3.34537+0$ $4.08604+0 \quad 4.99070+0 \quad 6.09565+0 \quad 7.44525+0 \quad 9.09365+0$ $1.11070+11.35661+1$

de195 $3.70370-3 \quad 4.37060-3 \quad 4.52500-3 \quad 4.57410-3$ $4.55830-3 \quad 4.48970-3 \quad 4.38170-3 \quad 4.24920-3 \quad 4.12890-3$ $4.03110-3 \quad 3.93550-3 \quad 3.84530-3 \quad 3.76290-3 \quad 3.69080-3$ $\begin{array}{llllll}3.65280-3 & 3.63870-3 & 3.62290-3 & 3.60620-3 & 3.58250-3\end{array}$ $\begin{array}{llllll}3.55850-3 & 4.08750-3 & 5.89750-3 & 7.13870-3 & 7.42410-3\end{array}$ $\begin{array}{llllll}7.67700-3 & 7.95180-3 & 8.74210-3 & 1.11490-2 & 1.35030-2\end{array}$ $1.65080-2 \quad 2.25270-2 \quad 2.86610-2 \quad 3.43150-2 \quad 4.10860-2$ $4.92110-2 \quad 5.56070-2 \quad 5.77300-2 \quad 5.80800-2 \quad 5.84980-2$ $6.599 .80-2 \quad 8.26560-2 \quad 9.04890-2 \quad 9.54810-2 \quad 1.05360-1$ $1.17860-1 \quad 1.29670-1 \quad 1.30100-1 \quad 1.28650-1 \quad 1.27220-1$ $1.26160-1 \quad 1.25620-1 \quad 1.25270-1 \quad 1.28500-1 \quad 1.36960-1$ 1.45980-1 1.54120-1 1.51160-1 $1.47220-1$ 1.47060-1 $1.63200-12.00340-1$

$\operatorname{fm} 195$ $5.701+10$

f205:p 298.958 $-200.0 \quad 0.0 \quad 1.0$ $1.50000-2 \quad 3.25000-2 \quad 5.75000-2 \quad 8.50000-2$ $\begin{array}{lllll}1.25000-1 & 2.25000-1 & 3.50000-1 & 4.55000-1 & 5.55000-1\end{array}$ $6.50000-1 \quad 8.50000-1 \quad 1.25000+0 \quad 1.75000+0 \quad 2.25000+0$ $2.75000+0 \quad 3.50000+0 \quad 4.50000+0 \quad 5.50000+0 \quad 6.50000+0$ $7.25000+0 \quad 7.75000+0 \quad 9.00000+0 \quad 1.20000+1$

df205 2.14390-3 $5.77600-4 \quad 2.71850-4 \quad 2.68170-4$ $3.27670-4.5 .66760-4 \quad 8.75940-4 \quad 1.08450-3 \quad 1.27970-3$ $\begin{array}{lllll}1.44170-3 & 1.75630-3 & 2.31560-3 & 2.92700-3 & 3.46860-3\end{array}$ $\begin{array}{llllll}3.95960-3 & 4.62210-3 & 5.41370-3 & 6.19090-3 & 6.92650-3\end{array}$ $7.47830-3 \quad 7.84680-3 \quad 8.77160-3 \quad 1.10200-2$

fm205 $5.701+10$

f215:p $298.958-100.0 \quad 0.0 \quad 1.0$

de 215

dE215

fm2 15 f225:p de 225

$\mathrm{d} \mp 225$

$1.50000-2 \quad 3.25000-2 \quad 5.75000-2 \quad 8.50000-2$ $1.25000-12.25000-1 \quad 3.50000-1 \quad 4.55000-1 \quad 5.55000-1$ $6.50000-1 \quad 8.50000-1 \quad 1.25000+0 \quad 1.75000+0 \quad 2.25000+0$ $2.75000+0 \quad 3.50000+0 \quad 4.50000+0 \quad 5.50000+06.50000+0$ $7.25000+0 \quad 7.75000+0 \quad 9.00000+0 \quad 1.20000+1$

$2.14390-3 \quad 5.77600-4 \quad 2.71850-4 \quad 2.68170-4$ $\begin{array}{lllll}3.27670-4 & 5.66760-4 & 8.75940-4 & 1.08450-3 & 1.27970-3\end{array}$ $\begin{array}{lllll}1.44170-3 & 1.75630-3 & 2.31560-3 & 2.92700-3 & 3.46860-3\end{array}$ $\begin{array}{llllll}3.95960-3 & 4.62210-3 & 5.41370-3 & 6.19090-3 & 6.92650-3\end{array}$ $7.47830-3 \quad 7.84680-3 \quad 8.77160-3 \quad 1.10200-2$

$5.701+10$

$298.958 \quad-50.0 \quad 0.01 .0$ $1.50000-2 \quad 3.25000-2 \quad 5.75000-2 \quad 8.50000-2$

$\begin{array}{lllll}1.25000-1 & 2.25000-1 & 3.50000-1 & 4.55000-1 & 5.55000-1\end{array}$ $6.50000-1 \quad 8.50000-1 \quad 1.25000+0 \quad 1.75000+02.25000+0$ $2.75000+0 \quad 3.50000+04.50000+0 \quad 5.50000+0 \quad 6.50000+0$ $7.25000+0 \quad 7.75000+0 \quad 9.00000+0 \quad 1.20000+1$ $\begin{array}{llll}2.14390-3 & 5.77600-4 & 2.71850-4 & 2.68170-4\end{array}$

$3.27670-4 \quad 5.66760-4 \quad 8.75940-4 \quad 1.08450-3 \quad 1.27970-3$

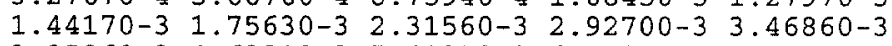
$\begin{array}{llllll}3.95960-3 & 4.62210-3 & 5.41370-3 & 6.19090-3 & 6.92650-3\end{array}$ $7.47830-3 \quad 7.84680-3 \quad 8.77160-3 \quad 1.10200-2$

$\operatorname{fm} 225 \quad 5.701+10$

$f 235: p \quad 298.958 \quad-25.0 \quad 0.01 .0$

de 235

$\operatorname{df} 235$

$\operatorname{fin} 235$

£245:p de 245

$1.50000-23.25000-2 \quad 5.75000-2 \quad 8.50000-2$ $1.25000-1 \quad 2.25000-1 \quad 3.50000-14.55000-15.55000-1$ $6.50000-1 \quad 8.50000-1 \quad 1.25000+0 \quad 1.75000+0 \quad 2.25000+0$ $2.75000+0 \quad 3.50000+0 \quad 4.50000+0 \quad 5.50000+0 \quad 6.50000+0$ $7.25000+0 \quad 7.75000+0 \quad 9.00000+0 \quad 1.20000+1$ $2.14390-3 \quad 5.77600-4 \quad 2.71850-4 \quad 2.68170-4$ $\begin{array}{llllll}3.27670-4 & 5.66760-4 & 8.75940-4 & 1.08450-3 & 1.27970-3\end{array}$ $\begin{array}{lllll}1.44170-3 & 1.75630-3 & 2.31560-3 & 2.92700-3 & 3.46860-3\end{array}$ $3.95960-3 \quad 4.62210-3 \quad 5.41370-3 \quad 6.19090-3 \quad 6.92650-3$ $7.47830-3 \quad 7.84680-3 \quad 8.77160-3 \quad 1.10200-2$ $5.701+10$ $298.958 \quad 0.0 \quad 0.0 \quad 1.0$ $1.50000-23.25000-2 \quad 5.75000-2 \quad 8.50000-2$ $1.25000-12.25000-1 \quad 3.50000-14.55000-15.55000-1$ 
$6.50000-1 \quad 8.50000-1 \quad 1.25000+0 \quad 1.75000+0 \quad 2.25000+0$

$2.75000+0 \quad 3.50000+0 \quad 4.50000+0 \quad 5.50000+0 \quad 6.50000+0$

$7.25000+07.75000+09.00000+01.20000+1$

df245 2.14390-3 $5.77600-4 \quad 2.71850-4 \quad 2.68170-4$

$3.27670-4 \quad 5.66760-4 \quad 8.75940-4 \quad 1.08450-3 \quad 1.27970-3$

$1.44170-3 \quad 1.75630-3 \quad 2.31560-3 \quad 2.92700-3 \quad 3.46860-3$

$3.95960-3 \quad 4.62210-3 \quad 5.41370-3 \quad 6.19090-3 \quad 6.92650-3$

$\begin{array}{llll}7.47830-3 & 7.84680-3 & 8.77160-3 & 1.10200-2\end{array}$

$\operatorname{fm} 245$

f255:p

$5.701+10$

de 255

298.958

$25.0 \quad 0.01 .0$

$1.50000-2 \quad 3.25000-2 \quad 5.75000-2 \quad 8.50000-2$

$1.25000-1 \quad 2.25000-1 \quad 3.50000-1 \quad 4.55000-1 \quad 5.55000-1$

$6.50000-1 \quad 8.50000-1 \quad 1.25000+0 \quad 1.75000+0 \quad 2.25000+0$

$2.75000+0 \quad 3.50000+0 \quad 4.50000+0 \quad 5.50000+0 \quad 6.50000+0$

$7.25000+0 \quad 7.75000+0 \quad 9.00000+0 \quad 1.20000+1$

df255

$2.14390-3 \quad 5.77600-4 \quad 2.71850-4 \quad 2.68170-4$

$3.27670-4 \quad 5.66760-4 \quad 8.75940-4 \quad 1.08450-3 \quad 1.27970-3$

$\begin{array}{llllll}1.44170-3 & 1.75630-3 & 2.31560-3 & 2.92700-3 & 3.46860-3\end{array}$

$3.95960-3 \quad 4.62210-3 \quad 5.41370-3 \quad 6.19090-3 \quad 6.92650-3$

$7.47830-3 \quad 7.84680-3 \quad 8.77160-3 \quad 1.10200-2$

$\operatorname{Im} 255 \quad 5.701+10$

f265:p $\quad 298.958 \quad 50.0 \quad 0.01 .0$

$1.50000-2 \quad 3.25000-2 \quad 5.75000-2 \quad 8.50000-2$

$1.25000-1 \quad 2.25000-1 \quad 3.50000-1 \quad 4.55000-1 \quad 5.55000-1$

$6.50000-18.50000-1 \quad 1.25000+0 \quad 1.75000+0 \quad 2.25000+0$

$2.75000+0 \quad 3.50000+0 \quad 4.50000+0 \quad 5.50000+0 \quad 6.50000+0$

$7.25000+0 \quad 7.75000+0 \quad 9.00000+0 \quad 1.20000+1$

df265 $2.14390-3 \quad 5.77600-4 \quad 2.71850-4 \quad 2.68170-4$

$\begin{array}{llllll}3.27670-4 & 5.66760-4 & 8.75940-4 & 1.08450-3 & 1.27970-3\end{array}$

$\begin{array}{llllll}1.44170-3 & 1.75630-3 & 2.31560-3 & 2.92700-3 & 3.46860-3\end{array}$

$\begin{array}{llllll}3.95960-3 & 4.62210-3 & 5.41370-3 & 6.19090-3 & 6.92650-3\end{array}$

$\begin{array}{llll}7.47830-3 & 7.84680-3 & 8.77160-3 & 1.10200-2\end{array}$

fm265 $\quad 5.701+10$

f275:p $\quad 298.958 \quad 100.0 \quad 0.01 .0$

de275 $1.50000-2 \quad 3.25000-2 \quad 5.75000-2 \quad 8.50000-2$

$1.25000-1 \quad 2.25000-1 \quad 3.50000-1 \quad 4.55000-1 \quad 5.55000-1$

$6.50000-1 \quad 8.50000-1 \quad 1.25000+0 \quad 1.75000+0 \quad 2.25000+0$

$2.75000+0 \quad 3.50000+0 \quad 4.50000+0 \quad 5.50000+0 \quad 6.50000+0$

$7.25000+07.75000+09.00000+01.20000+1$

df275 $2.14390-3 \quad 5.77600-4 \quad 2.71850-4 \quad 2.68170-4$

$3.27670-4 \quad 5.66760-4 \quad 8.75940-4 \quad 1.08450-3 \quad 1.27970-3$

$\begin{array}{llllll}1.44170-3 & 1.75630-3 & 2.31560-3 & 2.92700-3 & 3.46860-3\end{array}$

$\begin{array}{lllll}3.95960-3 & 4.62210-3 & 5.41370-3 & 6.19090-3 & 6.92650-3\end{array}$

$7.47830-3 \quad 7.84680-3 \quad 8.77160-3 \quad 1.10200-2$

fm275 $\quad 5.701+10$

f285:p $\quad 298.958 \quad 200.0 \quad 0.01 .0$

$1.50000-2 \quad 3.25000-2 \quad 5.75000-2 \quad 8.50000-2$

$\begin{array}{llllll}1.25000-1 & 2.25000-1 & 3.50000-1 & 4.55000-1 & 5.55000-1\end{array}$

$6.50000-1 \quad 8.50000-1 \quad 1.25000+0 \quad 1.75000+0 \quad 2.25000+0$

$2.75000+0 \quad 3.50000+0 \quad 4.50000+0 \quad 5.50000+0 \quad 6.50000+0$

$7.25000+0 \quad 7.75000+0 \quad 9.00000+01.20000+1$

$\begin{array}{rllll}\text { df285 } 2.14390-3 & 5.77600-4 & 2.71850-4 & 2.68170-4\end{array}$

$\begin{array}{llllll}3.27670-4 & 5.66760-4 & 8.75940-4 & 1.08450-3 & 1.27970-3\end{array}$

$\begin{array}{lllll}1.44170-3 & 1.75630-3 & 2.31560-3 & 2.92700-3 & 3.46860-3\end{array}$

$3.95960-3 \quad 4.62210-3 \quad 5.41370-3 \quad 6.19090-3 \quad 6.92650-3$

$7.47830-3 \quad 7.84680-3 \quad 8.77160-3 \quad 1.10200-2$

fm285 $\quad 5.701+10$

$\mp 295: \mathrm{p} \quad 298.958 \quad 250.0 \quad 0.01 .0$

de295 $1.50000-2 \quad 3.25000-2 \quad 5.75000-28.50000-2$

$\begin{array}{lllll}1.25000-1 & 2.25000-1 & 3.50000-1 & 4.55000-1 & 5.55000-1\end{array}$

$6.50000-1 \quad 8.50000-1 \quad 1.25000+0 \quad 1.75000+0 \quad 2.25000+0$

$2.75000+0 \quad 3.50000+0 \quad 4.50000+0 \quad 5.50000+0.6 .50000+0$

$7.25000+0 \quad 7.75000+0 \quad 9.00000+0 \quad 1.20000+1$

$\begin{array}{llll}2.14390-3 & 5.77600-4 & 2.71850-4 & 2.68170-4\end{array}$

$3.27670-4 \quad 5.66760-4 \quad 8.75940-4 \quad 1.08450-3 \quad 1.27970-3$

$\begin{array}{llllll}1.44170-3 & 1.75630-3 & 2.31560-3 & 2.92700-3 & 3.46860-3\end{array}$

$3.95960-3 \quad 4.62210-3 \quad 5.41370-3 \quad 6.19090-3 \quad 6.92650-3$

$7,47830-3 \quad 7.84680-3 \quad 8.77160-3 \quad 1.10200-2$

fm295 $\quad 5: 701+10$

sdef pos $=0$ o 0 vec $=1$ o 0 dir=d1 erg=fdir=d2

$\begin{array}{llllllll}\text { sil s } 11 & 12 & 13 & 14 & 15 & 16\end{array}$

$\begin{array}{llllllll}\operatorname{sp} 1 & 1.17+10 & 8.60+9 & 1.21+10 & 8.82+9 & 1.47+10 & 1.09+9\end{array}$ 


\begin{tabular}{|c|c|}
\hline $\operatorname{si11}$ & $h-1.0-0.7071067$ \\
\hline $\operatorname{si1} 12$ & $\mathrm{~h}-0.70710670 .0$ \\
\hline $\sin 3$ & h 0.00 .5 \\
\hline si14 & h 0.50 .8660254 \\
\hline $\operatorname{si15}$ & h 0.86602540 .9961946 \\
\hline $\operatorname{si16}$ & h 0.99619461 .0 \\
\hline $\operatorname{sp11}$ & 01 \\
\hline $\operatorname{sp} 12$ & 01 \\
\hline sp13 & 01 \\
\hline sp14 & 01 \\
\hline $\operatorname{sp15}$ & 01 \\
\hline $\operatorname{spl} 16$ & 01 \\
\hline ds2 & s $\begin{array}{llllll}21 & 22 & 23 & 24 & 25 & 26\end{array}$ \\
\hline $\operatorname{si21}$ & h 1.82451 .9295 \\
\hline $\operatorname{si22}$ & h $1.92953 .7645^{\circ}$ \\
\hline $\operatorname{si23}$ & h $3.7645 \quad 5.675$ \\
\hline $\operatorname{si24}$ & h 5.6757 .3865 \\
\hline si25 & h 7.38658 .321 \\
\hline $\operatorname{si26}$ & h 8.3218 .753 \\
\hline $\operatorname{sp} 21$ & 01 \\
\hline $\operatorname{sp} 22$ & 01 \\
\hline $\operatorname{sp} 23$ & 01 \\
\hline $\operatorname{sp} 24$ & 01 \\
\hline $\operatorname{sp} 25$ & 01 \\
\hline $\operatorname{sp} 26$ & 01 \\
\hline nps & 2000000 \\
\hline $\begin{array}{l}\text { prdmp } \\
\text { print }\end{array}$ & $2 j 1$ \\
\hline
\end{tabular}


File for MCNP-4B calculation of the dose-rate distribution near the source side of salted beef cargo at the center of the PFNA facility truck lane for the source in the horizontal position

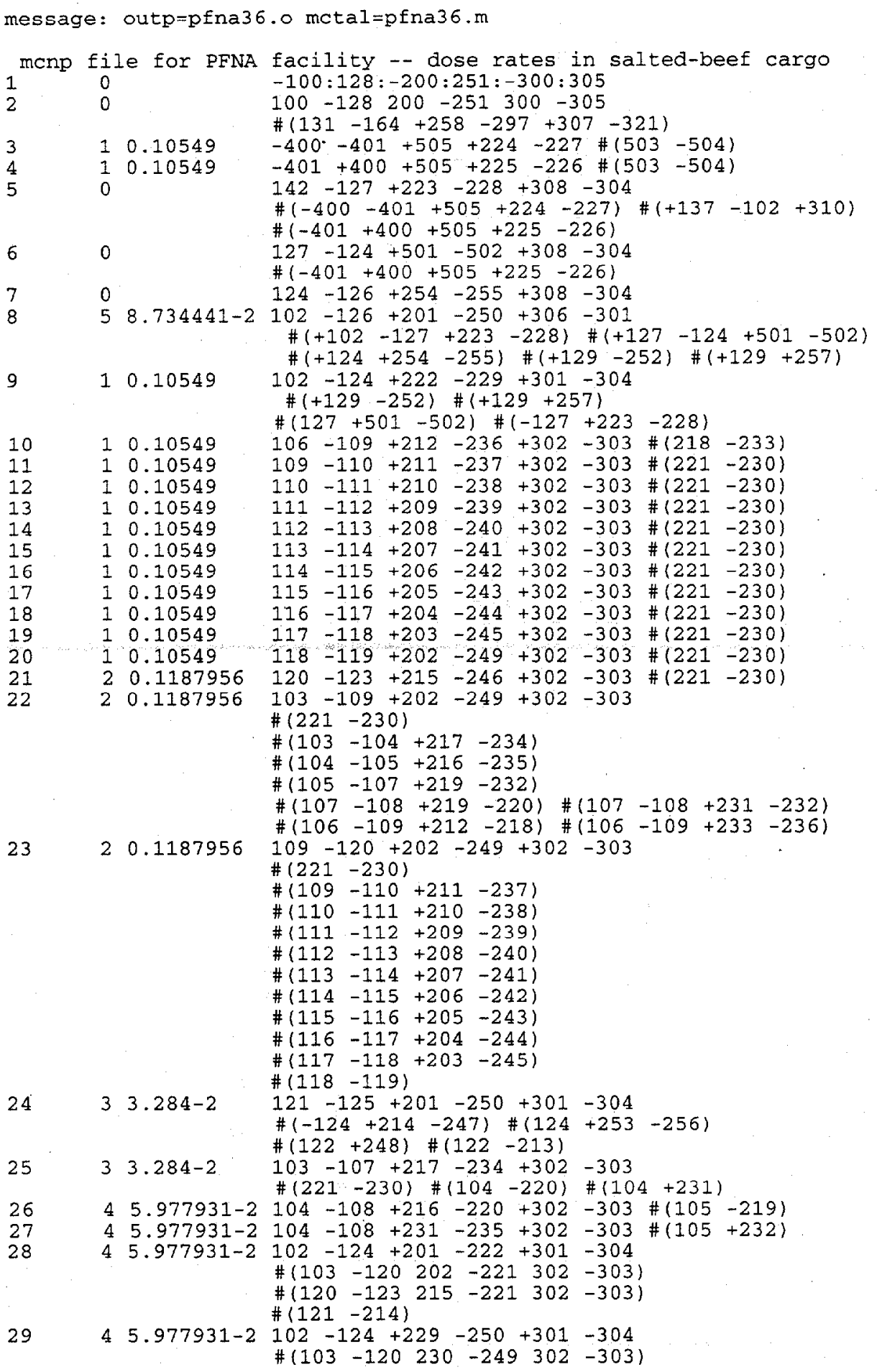




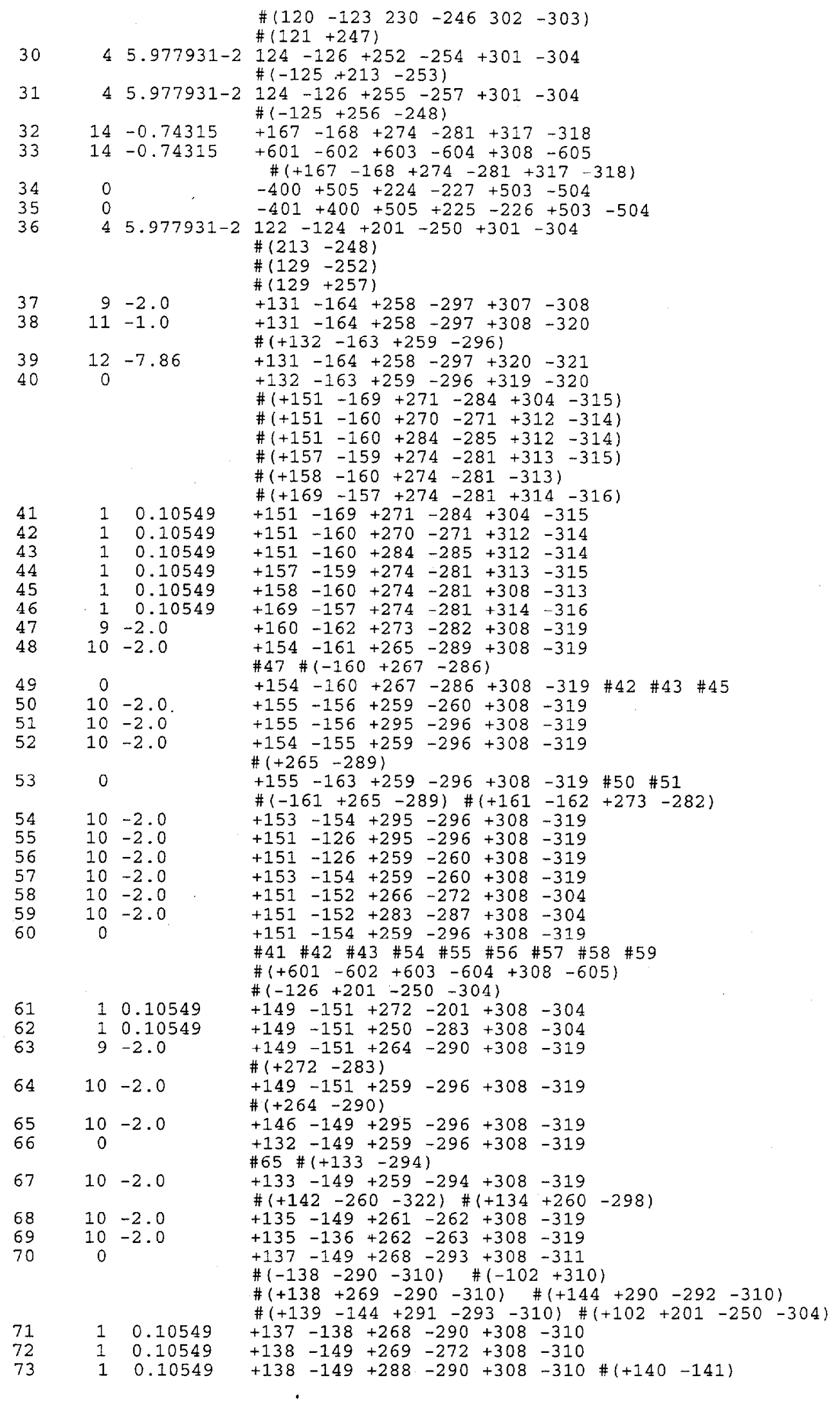




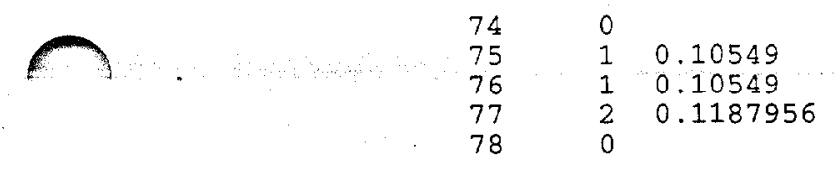

$+140-141+288-290+308-310$

$+144-149+290-292+308-310$

$+139-144+291-293+308-310$

$+137-102+268-293+310-311$

$+138-149+272-288+308-310$

$\#\{+102+201-250+309-304\}$

$\#(+143-102+275-276+301)$

$\#(+143-102+279-280+301)$

$\#(+147-102+276-277+301)$

$\#(+147-102+278-279+301)$

$\#(+142+223-228) \quad \#(+145+201-250-309)$

$\begin{array}{lll}79 & 1 & 0.10549 \\ 80 & 1 & 0.10549 \\ 81 & 1 & 0.10549 \\ 82 & 1 & 0.10549 \\ 83 & 0 & \\ 84 & 0 & \end{array}$

$+143-102+275-276+301-310$

$+143-102+279-280+301-310$

$+147-102+276-277+301-310$

$+147-102+278-279+301-310$

$+142-149+259-260+308-322$

$+134-149+260-298+308-319$

\#68 \#69 \#(+137 $-149+268-293+308-311)$

$859-2.0$

$\#(+102+201-250-304) \quad \#(+142+223-228-304)$

$+145-126+201-250+308-309$

$\#(+145-127+223-228) \#(+127-124+501-502)$

860

$\#(+129-252) \quad \#(+129+257) \quad \#(+124+254-255)$

$+129-126+201-250+308-304$

\# $(+252-257)$

$+102-126+201-250+309-306$

$\#(+102-127+223-228) \#(+127-124+501-502)$

(+129-252) \#(+129+257) \#(+124+254-255)

$88 \quad 0$

$+149-151+272-283+304-319$.

c end cells

c surfaces

100 px -1000.0

100

$\begin{array}{lll}102 & \mathrm{px} & \mathrm{px} \\ 103 & \mathrm{px} & 22.7264\end{array}$

103 px 22.6314

$104 \quad \mathrm{px} \quad 27.7114$

$105 \quad \mathrm{px} \quad 28.9814$

106 px 31.5214

107 px 36.6014

$108 \quad \mathrm{px} \quad 42.9514$

$109 \quad \mathrm{px} \quad 49.3014$

$110 \quad \mathrm{px} \quad 51.8414$

111 px 54.3814

112 px 56.9214

113 px 59.4614

$114 \quad \mathrm{px} \quad 62.0014$

$115 \mathrm{px} \quad 64.5414$

116 px 67.0814

117 px 69.6214

118 px 72.1614

119 px 74.7014

120 px 76.3016

$\begin{array}{lll}121 & \mathrm{px} & 78.2066 \\ 122 & \mathrm{px} & 86.0806\end{array}$

122

123

124

125

126

127

128

129

131

132

133

134

135

136

137

138

139

112.649

114.554

. 119.888

121.158

50.5714

1200.0

$\begin{array}{ll}\mathrm{px} & 87.9856 \\ \mathrm{px} & -844.042\end{array}$

px $\quad-823.722$

px $\quad-767.842$

px $\quad-706.882$

px $\quad-548.962$

px $\quad-493.522$

$\mathrm{px} \quad-381.762$

px $\quad-320.802$

px -315.722

px -173.482 


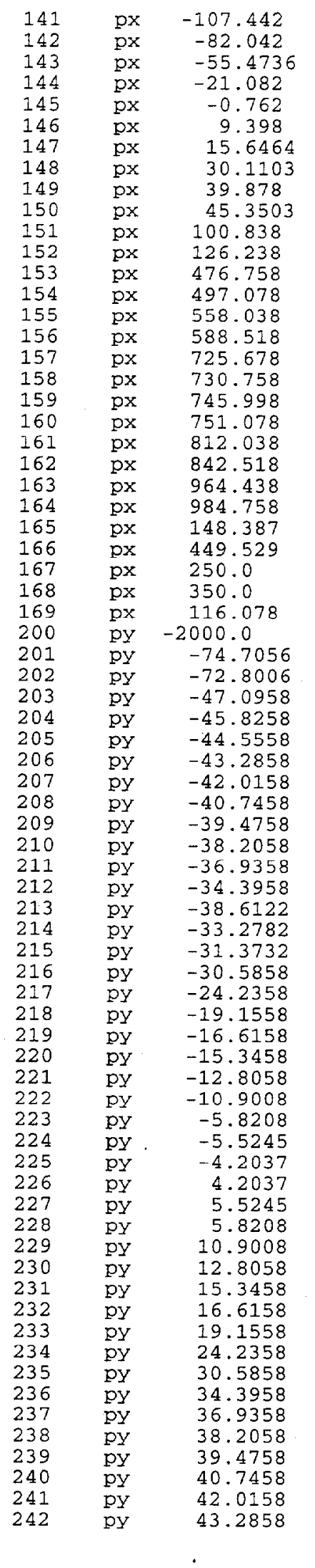




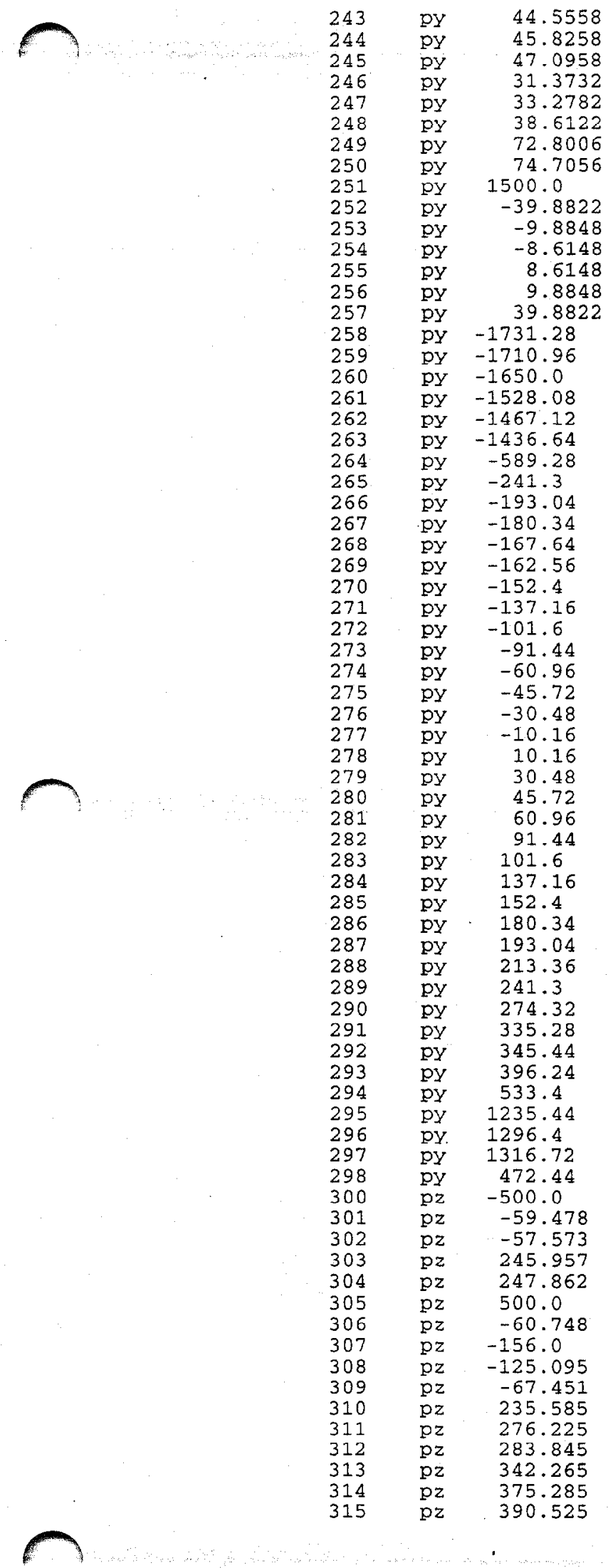




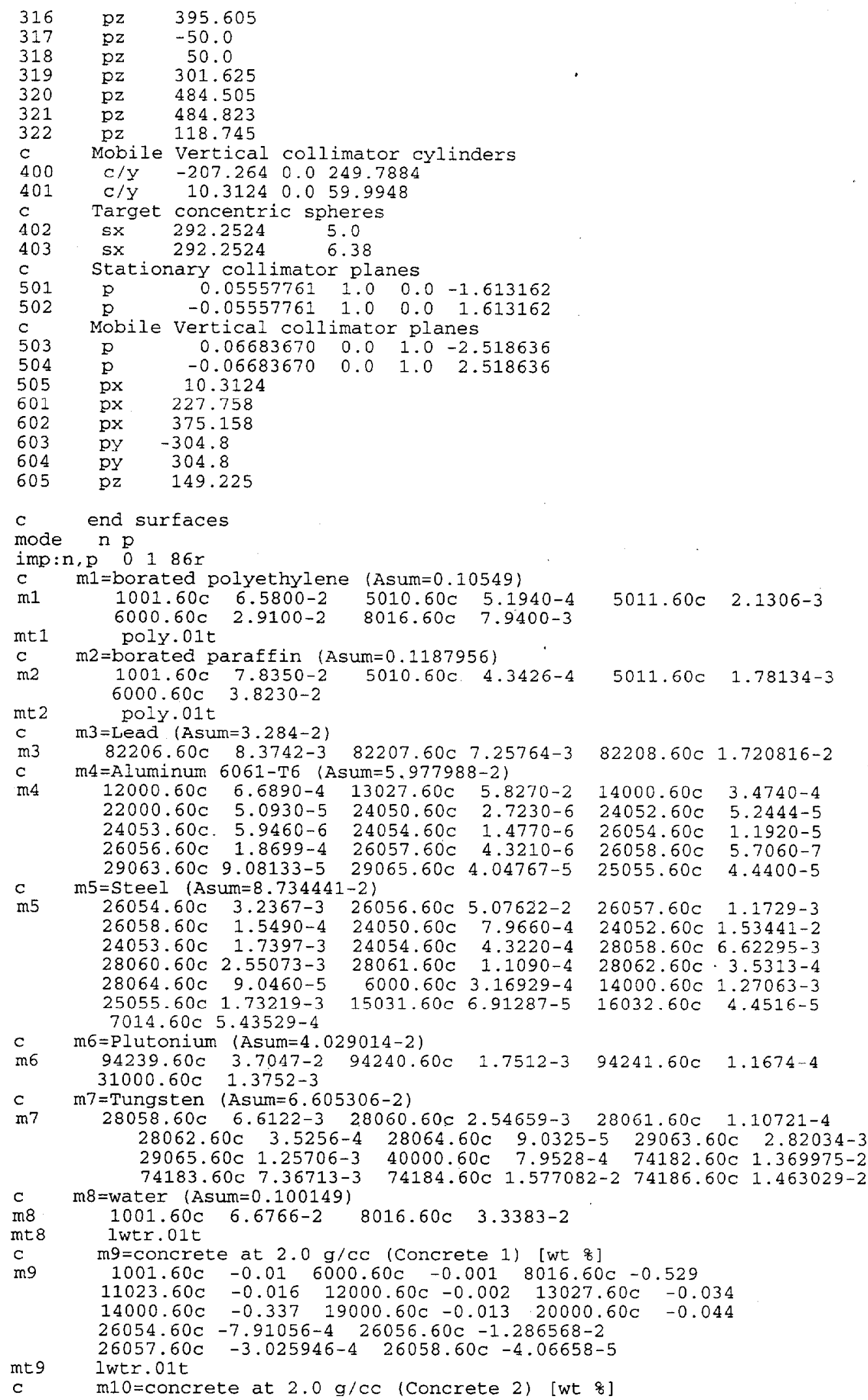



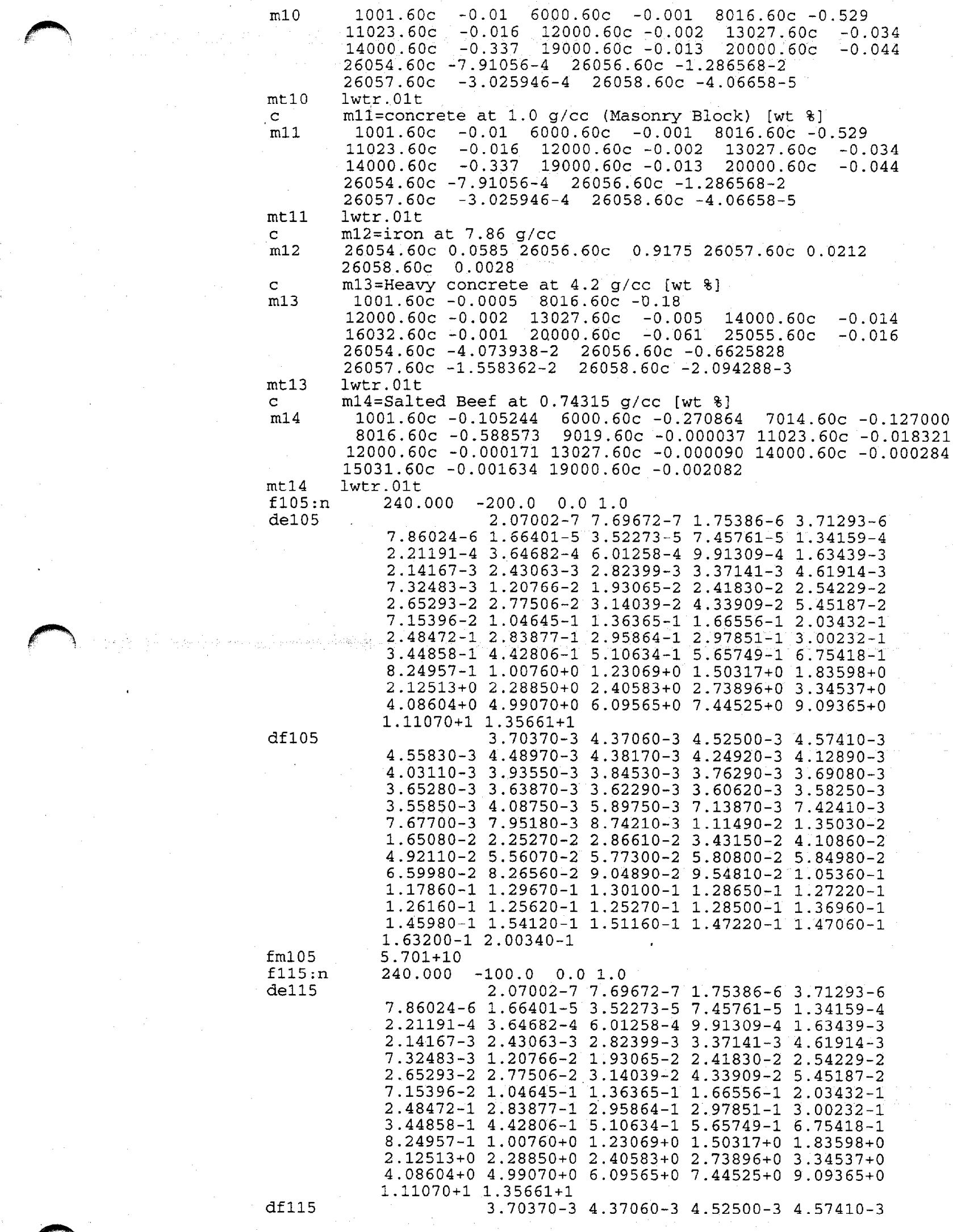
$3.70370-3 \quad 4.37060-3 \quad 4.52500-3 \quad 4.57410-3$

$\operatorname{df} 115$ 
$4.55830-3 \quad 4.48970-3 \quad 4.38170-3 \quad 4.24920-3 \quad 4.12890-3$ $\begin{array}{llllll}4.03110-3 & 3.93550-3 & 3.84530-3 & 3.76290-3 & 3.69080-3\end{array}$ $3.65280-3 \quad 3.63870-3 \quad 3.62290-3 \quad 3.60620-3 \quad 3.58250-3$ $\begin{array}{llllll}3.55850-3 & 4.08750-3 & 5.89750-3 & 7.13870-3 & 7.42410-3\end{array}$

$\begin{array}{lllll}7.67700-3 & 7.95180-3 & 8.74210-3 & 1.11490-2 & 1.35030-2\end{array}$

$\begin{array}{lllll}1.65080-2 & 2.25270-2 & 2.86610-2 & 3.43150-2 & 4.10860-2\end{array}$

$4.92110-2 \quad 5.56070-2 \quad 5.77300-2 \quad 5.80800-2 \quad 5.84980-2$

$\begin{array}{lllll}6.59980-2 & 8.26560-2 & 9.04890-2 & 9.54810-2 & 1.05360-1\end{array}$

$1.17860-1 \quad 1.29670-1 \quad 1.30100-1 \quad 1.28650-1 \quad 1.27220-1$

1.26160-1 $1.25620-1 \quad 1.25270-1 \quad 1.28500-1 \quad 1.36960-1$

$1.45980-11.54120-11.51160-11.47220-1 \quad 1.47060-1$

$1.63200-12.00340-1$

$\operatorname{fm} 115$ $5.701+10$

$\mathrm{f} 125: \mathrm{n}$

240.000

$-50.0 \quad 0.0 \quad 1.0$

$2.07002-7 \quad 7.69672-7 \quad 1.75386-6 \quad 3.71293-6$

7.86024-6 $1.66401-5 \quad 3.52273-5 \quad 7.45761-5 \quad 1.34159-4$

2.21191-4 $3.64682-4 \quad 6.01258-4 \quad 9.91309-4 \quad 1.63439-3$

$2.14167-3 \quad 2.43063-3 \quad 2.82399-3 \quad 3.37141-3 \quad 4.61914-3$

7.32483-3 $1.20766-2 \quad 1.93065-2 \quad 2.41830-2 \quad 2.54229-2$

$2.65293-2 \quad 2.77506-2 \quad 3.14039-2 \quad 4.33909-2 \quad 5.45187-2$

7.15396-2 $1.04645-1 \quad 1.36365-1 \quad 1.66556-1 \quad 2.03432-1$

$2.48472-1 \quad 2.83877-1 \quad 2.95864-1 \quad 2.97851-1 \quad 3.00232-1$

$3.44858-1 \quad 4.42806-1 \quad 5.10634-1 \quad 5.65749-1 \quad 6.75418-1$

$8.24957-1 \quad 1.00760+0 \quad 1.23069+0 \quad 1.50317+0 \quad 1.83598+0$

$2.12513+0 \quad 2.28850+0 \quad 2.40583+0 \quad 2.73896+0 \quad 3.34537+0$

$4.08604+0 \quad 4.99070+0 \quad 6.09565+0 \quad 7.44525+0 \quad 9.09365+0$

df 125

$1.11070+11.35661+1$

$3.70370-3 \quad 4.37060-3 \quad 4.52500-3 \quad 4.57410-3$

1.55830-3 $4.48970-3 \quad 4.38170-3 \quad 4.24920-3 \quad 4.12890-3$

$\begin{array}{llllll}4.03110-3 & 3.93550-3 & 3.84530-3 & 3.76290-3 & 3.69080-3\end{array}$

$\begin{array}{llllll}3.65280-3 & 3.63870-3 & 3.62290-3 & 3.60620-3 & 3.58250-3\end{array}$

$\begin{array}{lllll}3.55850-3 & 4.08750-3 & 5.89750-3 & 7.13870-3 & 7.42410-3\end{array}$

$\begin{array}{lllll}7.67700-3 & 7.95180-3 & 8.74210-3 & 1.11490-2 & 1.35030-2\end{array}$

$1.65080-2 \quad 2.25270-2 \quad 2.86610-2 \quad 3.43150-2 \quad 4.10860-2$

$4.92110-2 \quad 5.56070-2 \quad 5.77300-2 \quad 5.80800-2 \quad 5.84980-2$

$6.59980-2 \quad 8.26560-2 \quad 9.04890-2 \quad 9.54810-2 \quad 1.05360-1$

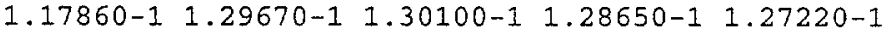

$1.26160-1$ 1.25620-1 $1.25270-1 \quad 1.28500-1 \quad 1.36960-1$

$1.45980-1 \quad 1.54120-1 \quad 1.51160-1 \quad 1.47220-1 \quad 1.47060-1$ $1.63200-12.00340-1$

$\operatorname{Em} 125$ $5.701+10$

f135:n $\quad 240.000 \quad-25.0 \quad 0.01 .0$

de135

df135

$\begin{array}{lllll}2.07002-7 & 7.69672-7 & 1.75386-6 & 3.71293-6\end{array}$

7.86024-6 $1.66401-5 \quad 3.52273-5 \quad 7.45761-5 \quad 1.34159-4$

$2.21191-4 \quad 3.64682-4 \quad 6.01258-4 \quad 9.91309-4 \quad 1.63439-3$

$2.14167-3 \quad 2.43063-3 \quad 2.82399-3 \quad 3.37141-3 \quad 4.61914-3$

$\begin{array}{lllll}7.32483-3 & 1.20766-2 & 1.93065-2 & 2.41830-2 & 2.54229-2\end{array}$

2.65293-2 2.77506-2 3.14039-2 $4.33909-2 \quad 5.45187-2$

$7.15396-2$ 1.04645-1 $1.36365-1 \quad 1.66556-1 \quad 2.03432-1$

$\begin{array}{llllll}2.48472-1 & 2.83877-1 & 2.95864-1 & 2.97851-1 & 3.00232-1\end{array}$

3.44858-1 4.42806-1 5.10634-1 $5.65749-1 \quad 6.75418-1$

$8.24957-1 \quad 1.00760+0 \quad 1.23069+0 \quad 1.50317+0 \quad 1.83598+0$

$\begin{array}{llllll}2.12513+0 & 2.28850+0 & 2.40583+0 & 2.73896+0 & 3.34537+0\end{array}$

$4.08604+0 \quad 4.99070+0 \quad 6.09565+0 \quad 7.44525+0 \quad 9.09365+0$

$1.11070+11.35661+1$

$3.70370-3 \quad 4.37060-3 \quad 4.52500-3 \quad 4.57410-3$

$4.55830-3 \quad 4.48970-3 \quad 4.38170-3 \quad 4.24920-3 \quad 4.12890-3$

$\begin{array}{llllll}4.03110-3 & 3.93550-3 & 3.84530-3 & 3.76290-3 & 3.69080-3\end{array}$

$3.65280-3 \quad 3.63870-3 \quad 3.62290-3 \quad 3.60620-3 \quad 3.58250-3$

$3.55850-3 \quad 4.08750-3 \quad 5.89750-3 \quad 7.13870-3 \quad 7.42410-3$

$7.67700-3 \quad 7.95180-3 \quad 8.74210-3 \quad 1.11490-2 \quad 1.35030-2$

$1.65080-2 \quad 2.25270-2 \quad 2.86610-2 \quad 3.43150-2 \quad 4.10860-2$

$4.92110-2 \quad 5.56070-2 \quad 5.77300-2 \quad 5.80800-2 \quad 5.84980-2$

$6.59980-2 \quad 8.26560-2 \quad 9.04890-2 \quad 9.54810-2 \quad 1.05360-1$

1.17860-1 $1.29670-1 \quad 1.30100-1 \quad 1.28650-1 \quad 1.27220-1$

$1.26160-1 \quad 1.25620-1$ 1.25270-1 $1.28500-11.36960-1$

$1.45980-1 \quad 1.54120-1 \quad 1.51160-1 \quad 1.47220-1 \quad 1.47060-1$

$1.63200-12.00340-1$

fm135 $\quad 5.701+10$

f145:n $\quad 240.000 \quad 0.0 \quad 0.01 .0$

$\begin{array}{llllll}\operatorname{de} 145 & 2.07002-7 & 7.69672-7 & 1.75386-6 & 3.71293-6\end{array}$

$7.86024-6 \quad 1.66401-5 \quad 3.52273-5 \quad 7.45761-5 \quad 1.34159-4$ 
$2.21191-4 \quad 3.64682-4 \quad 6.01258-4 \quad 9.91309-4 \quad 1.63439-3$ $2.14167-3 \quad 2.43063-3 \quad 2.82399-3 \quad 3.37141-3 \quad 4.61914-3$ $7.32483-31.20766-21.93065-2 \quad 2.41830-2 \quad 2.54229-2$ 2.65293-2 2.77506-2 3.14039-2 4.33909-2 5.45187-2 $7.15396-2$ 1.04645-1 $1.36365-1$ 1.66556-1. 2.03432-1 $2.48472-1 \quad 2.83877-1$ 2.95864-1 2.97851-1 3.00232-1 3.44858-1 4.42806-1 5.10634-1 5.65749-1 6.75418-1 $8.24957-1 \quad 1.00760+0 \quad 1.23069+01.50317+0.1 .83598+0$ $2.12513+0 \quad 2.28850+0 \quad 2.40583+0 \quad 2.73896+0 \quad 3.34537+0$ $4.08604+0 \quad 4.99070+0 \quad 6.09565+0 \quad 7.44525+0 \quad 9.09365+0$ $1.11070+1 \quad 1.35661+1$

df145 $3.70370-3 \quad 4.37060-3.4 .52500-34.57410-3$ $\begin{array}{lllllll}4.55830-3 & 4.48970-3 & 4.38170-3 & 4.24920-3 & 4.12890-3\end{array}$ $4.03110-3 \quad 3.93550-3 \quad 3.84530-3 \quad 3.76290-3 \quad 3.69080-3$ $3.65280-3 \quad 3.63870-3 \quad 3.62290-3 \quad 3.60620-3 \quad 3.58250-3$ $3.55850-3 \quad 4.08750-3 \quad 5.89750-3 \quad 7.13870-3 \quad 7.42410-3$ $\begin{array}{llllll}7.67700-3 & 7.95180-3 & 8.74210-3 & 1.11490-2 & 1.35030-2\end{array}$ $1.65080-2 \quad 2.25270-2 \quad 2.86610-2 \quad 3.43150-2 \quad 4.10860-2$ $4.92110-2 \quad 5.56070-2 \quad 5.77300-2 \quad 5.80800-2 \quad 5.84980-2$ $6.59980-2 \quad 8.26560-2 \quad 9.04890-2 \quad 9.54810-2 \quad 1.05360-1$ $1.17860-1 \quad 1.29670-1$ 1.30100-1 $1.28650-1$ 1.27220-1 $1.26160-1 \quad 1.25620-1 \quad 1.25270-1 \quad 1.28500-1 \quad 1.36960-1$ $1.45980-1$ 1.54120-1 $1.51160-1$ 1.47220-1 $1.47060-1$ 1. $63200-12.00340-1$

fm145 $\quad 5.701+10$

$\begin{array}{lllll}\text { E155:n } & 240.000 & 25.0 & 0.0 & 1.0\end{array}$

$\begin{array}{llllll}\text { de155 } & 2.07002-7 & 7.69672-7 & 1.75386-6 & 3.71293-6\end{array}$

$7.86024-6 \quad 1.66401-5.3 .52273-5 \quad 7.45761-5 \quad 1.34159-4$

$2.21191-4 \quad 3.64682-4 \quad 6.01258-4 \quad 9.91309-4 \quad 1.63439-3$

$2.14167-3 \quad 2.43063-3 \quad 2.82399-3 \quad 3.37141-3 \quad 4.61914-3$

$7.32483-3 \quad 1.20766-2 \quad 1.93065-2 \quad 2.41830-2 \quad 2.54229-2$

$2.65293-2 \quad 2.77506-2 \quad 3.14039-2 \quad 4.33909-2 \quad 5.45187-2$

$7.15396-2 \quad 1.04645-1 \quad 1.36365-1$ 1.66556-1 $2.03432-1$

$2.48472-1 \quad 2.83877-1 \quad 2.95864-1 \quad 2.97851-1 \quad 3.00232-1$

$3.44858-1 \quad 4.42806-1 \quad 5.10634-1 \quad 5.65749-1 \quad 6.75418-1$ $8.24957-1 \quad 1.00760+0 \quad 1.23069+0 \quad 1.50317+01.83598+0$ $2.12513+0 \quad 2.28850+0 \quad 2.40583+0 \quad 2.73896+0 \quad 3.34537+0$ $4.08604+0 \quad 4.99070+0 \quad 6.09565+0 \quad 7.44525+0 \quad 9.09365+0$ $1.11070+1 \quad 1 \cdot 35661+1$

df155 $\quad 3.70370-3 \quad 4.37060-3 \quad 4.52500-3 \quad 4.57410-3$ $\begin{array}{llllll}4.55830-3 & 4.48970-3 & 4.38170-3 & 4.24920-3 & 4.12890-3\end{array}$ $4.03110-3 \quad 3.93550-3 \quad 3.84530-3 \quad 3.76290-3 \quad 3.69080-3$ $3.65280-3 \quad 3.63870-3 \quad 3.62290-3 \quad 3.60620-3 \quad 3.58250-3$ $3.55850-3 \quad 4.08750-3 \quad 5.89750-3 \quad 7.13870-3 \quad 7.42410-3$ $7.67700-3 \quad 7.95180-3 \quad 8.74210-3 \quad 1.11490-2 \quad 1.35030-2$ $\begin{array}{lllll}1.65080-2 & 2.25270-2 & 2.86610-2 & 3.43150-2 & 4.10860-2\end{array}$ $4.92110-2 \quad 5.56070-2 \quad 5.77300-2 \quad 5.80800-2 \quad 5.84980-2$ 6.59980-2 8.26560-2 $9.04890-2 \quad 9.54810-2 \quad 1.05360-1$ $1.17860-1.1 .29670-1 \quad 1.30100-1 \quad 1.28650-1 \quad 1.27220-1$ $1.26160-1 \quad 1.25620-1 \quad 1.25270-1 \quad 1.28500-1 \quad 1.36960-1$ $1.45980-1 \quad 1.54120-1 \quad 1.51160-1 \quad 1.47220-1 \quad 1.47060-1$ $1.63200-12.00340-1$

$5.701+10$

fm155

$\mathrm{f} 165: \mathrm{n}$ de 165 240.0 .00

$50.0 \quad 0.01 .0$ $2.07002-7 \quad 7.69672-7 \quad 1.75386-6 \quad 3.71293-6$ 7.86024-6 $1.66401-5 \quad 3.52273-5 \quad 7.45761-5 \quad 1.34159-4$ $2.21191-4 \quad 3.64682-4 \quad 6.01258-4 \quad 9.91309-4 \quad 1.63439-3$ $2.14167-3 \quad 2.43063-3 \quad 2.82399-3 \quad 3.37141-3 \cdot 4.61914-3$ $7.32483-3 \quad 1.20766-2 \quad 1.93065-2 \quad 2.41830-2 \quad 2.54229-2$ 2. $65293-2 \quad 2.77506-2 \quad 3.14039-2 \quad 4.33909-2 \quad 5.45187-2$ 7.15396-2 $1.04645-1 \quad 1.36365-1 \quad 1.66556-1 \quad 2.03432-1$ $2.48472-1 \quad 2.83877-1 \quad 2.95864-1 \quad 2.97851-1 \quad 3.00232-1$ $3.44858-1 \quad 4.42806-1 \quad 5.10634-1 \quad 5.65749-1 \quad 6.75418-1$ $8.24957-1 \quad 1.00760+0 \quad 1.23069+0 \quad 1.50317+0 \quad 1.83598+0$ $2.12513+0 \quad 2.28850+0 \quad 2.40583+0 \quad 2.73896+0.3 .34537+0$ $4.08604+0 \quad 4.99070+0 \quad 6.09565+0 \quad 7.44525+0 \quad 9.09365+0$ $1.11070+11.35661+1$

df165 $\quad 3.70370-3 \quad 4.37060-3 \quad 4.52500-3 \quad 4.57410-3$ $4.55830-3 \quad 4.48970-3 \quad 4.38170-3 \quad 4.24920-3 \quad 4.12890-3$ $4.03110-3 \quad 3.93550-3 \quad 3.84530-3 \quad 3.76290-3 \quad 3.69080-3$ $3.65280-3 \quad 3.63870-3 \quad 3.62290-3 \quad 3.60620-3 \quad 3.58250-3$ $3.55850-3 \quad 4.08750-3 \quad 5.89750-3 \quad 7.13870-37.42410-3$ 
$\begin{array}{llllll}7.67700-3 & 7.95180-3 & 8.74210-3 & 1.11490-2 & 1.35030-2\end{array}$ $1.65080-2 \quad 2.25270-2 \quad 2.86610-2 \quad 3.43150-2 \quad 4.10860-2$ $4.92110-2 \quad 5.56070-2 \quad 5.77300-2 \quad 5.80800-2 \quad 5.84980-2$ $6.59980-2 \quad 8.26560-2 \quad 9.04890-2 \quad 9.54810-2 \quad 1.05360-1$ 1.17860-1 $1.29670-1 \quad 1.30100-1 \quad 1.28650-1 \quad 1.27220-1$ $1.26160-1$ 1.25620-1 $1.25270-1$ 1.28500-1 $1.36960-1$ $1.45980-1 \quad 1.54120-1 \quad 1.51160-1 \quad 1.47220-1 \quad 1.47060-1$ $1.63200-12.00340-1$

f175: n de175

df175

$\operatorname{fm} 175$

f185: de185

df185

$\operatorname{fm} 185$ f195:n de195

$5.701+10$

$240.000 \quad 100.0 \quad 0.01 .0$

$\begin{array}{cccc}2.07002-7 & 7.69672-7 & 1.75386-6 & 3.71293-6\end{array}$ $7.86024-6 \quad 1.66401-5 \quad 3.52273-5 \quad 7.45761-5 \quad 1.34159-4$ 2.21191-4 $3.64682-4 \quad 6.01258-4 \quad 9.91309-4 \quad 1.63439-3$ 2.14167-3 2.43063-3 $2.82399-3 \quad 3.37141-3$ 4.61914-3 $7.32483-3 \quad 1.20766-2 \quad 1.93065-2 \quad 2.41830-2 \quad 2.54229-2$ 2.65293-2 2.77506-2 $3.14039-2 \quad 4.33909-2 \quad 5.45187-2$ 7.15396-2 $1.04645-1 \quad 1.36365-1 \quad 1.66556-1 \quad 2.03432-1$ $\begin{array}{lllll}2.48472-1 & 2.83877-1 & 2.95864-1 & 2.97851-1 & 3.00232-1\end{array}$ 3.44858-1 4.42806-1 $5.10634-1 \quad 5.65749-1 \quad 6.75418-1$ $\begin{array}{lllll}8.24957-1 & 1.00760+0 & 1.23069+0 & 1.50317+0 & 1.83598+0\end{array}$ $2.12513+0 \quad 2.28850+0 \quad 2.40583+0 \quad 2.73896+0 \quad 3.34537+0$ $4.08604+0 \quad 4.99070+0 \quad 6.09565+0 \quad 7.44525+0 \quad 9.09365+0$ $1.11070+11.35661+1$

$3.70370-3 \quad 4.37060-3 \quad 4.52500-3 \quad 4.57410-3$ $4.55830-3 \quad 4.48970-3 \quad 4.38170-3 \quad 4.24920-3 \quad 4.12890-3$ $4.03710-3 \quad 3.93550-3 \quad 3.84530-3 \quad 3.76290-3 \quad 3.69080-3$ $\begin{array}{llllll}3.65280-3 & 3.63870-3 & 3.62290-3 & 3.60620-3 & 3.58250-3\end{array}$ $3.55850-3 \quad 4.08750-3 \quad 5.89750-3 \quad 7.13870-3 \quad 7.42410-3$ $\begin{array}{llllll}7.67700-3 & 7.95180-3 & 8.74210-3 & 1.11490-2 & 1.35030-2\end{array}$ $1.65080-2 \quad 2.25270-2 \quad 2.86610-2 \cdot 3.43150-2 \quad 4.10860-2$ $4.92110-2 \quad 5.56070-2 \quad 5.77300-2 \quad 5.80800-2 \quad 5.84980-2$ $\begin{array}{ccccc}6.59980-2 & 8.26560-2 & 9.04890-2 & 9.54810-2 & 1.05360-1\end{array}$ $1.17860-1 \quad 1.29670-1 \quad 1.30100-1 \quad 1.28650-1 \quad 1.27220-1$

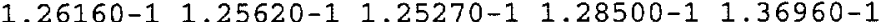
$1.45980-1$ 1.54120-1 $1.51160-1$ 1.47220-1 $1.47060-1$ $1.63200-12.00340-1$

$5.701+10$

$240.000 \quad 200.0 \quad 0.01 .0$

$2.07002-7 \quad 7.69672-7 \quad 1.75386-6 \quad 3.71293-6$ 7.86024-6 $1.66401-5 \quad 3.52273-5 \quad 7.45761-5 \quad 1.34159-4$ 2.21191-4 $3.64682-4 \quad 6.01258-4 \quad 9.91309-4 \quad 1.53439-3$ $2.14167-3 \quad 2.43063-3 \quad 2.82399-3 \quad 3.37141-3 \quad 4.61914-3$ 7.32483-3 $1.20766-2$ 1.93065-2 $2.41830-2 \quad 2.54229-2$ 2.65293-2 2.77506-2 $3.14039-2$ 4.33909-2 $5.45187-2$ $\begin{array}{lllll}7.15396-2 & 1.04645-1 & 1.36365-1 & 1.66556-1 & 2.03432-1\end{array}$ 2.48472-1 2.83877-1 $2.95864-1 \quad 2.97851-1 \quad 3.00232-1$ 3.44858-1 $4.42806-1 \quad 5.10634-1 \quad 5.65749-1 \quad 6.75418-1$

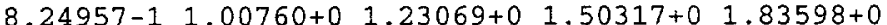
$2.12513+0 \quad 2.28850+0 \quad 2.40583+0 \quad 2.73896+0 \quad 3.34537+0$ $4.08604+0 \quad 4.99070+0 \quad 6.09565+0 \quad 7.44525+0 \quad 9.09365+0$ $1.11070+1 \cdot 1 \cdot 35661+1$ 3.70370-3 $4.37060-3 \quad 4.52500-3 \quad 4.57410-3$ $\begin{array}{lllllll}4.55830-3 & 4.48970-3 & 4.38170-3 & 4.24920-3 & 4.12890-3\end{array}$ $4.03110-3 \quad 3.93550-3 \quad 3.84530-3 \quad 3.76290-3 \quad 3.69080-3$ $3.65280-3 \quad 3.63870-3 \quad 3.62290-3 \quad 3.60620-3 \quad 3.58250-3$ $3.55850-3 \quad 4.08750-3 \quad 5.89750-3 \quad 7.13870-3 \quad 7.42410-3$ $7.67700-3 \quad 7.95180-3 \quad 8.74210-3 \quad 1.11490-2 \quad 1.35030-2$ $1.65080-2$ 2.25270-2 $2.86610-2 \quad 3.43150-2 \quad 4.10860-2$ $4.92110-2 \quad 5.56070-2 \quad 5.77300-2 \quad 5.80800-2 \quad 5.84980-2$ 6.59980-2 8.26560-2 $9.04890-2 \quad 9.54810-2 \quad 1.05360-1$ $\begin{array}{lllll}1.17860-1 & 1.29670-1 & 1.30100-1 & 1.28650-1 & 1.27220-1\end{array}$

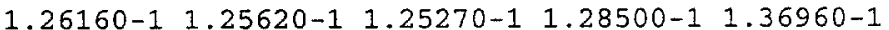
$1.45980-1$ 1.54120-1 $1.51160-1 \quad 1.47220-1 \quad 1.47060-1$ $1.63200-12.00340-1$

$5.701+10$

$240.000 \quad 250.0 \quad 0.01 .0$

$2.07002-7 \quad 7.69672-7 \quad 1.75386-6 \quad 3.71293-6$

7.86024-6 $1.66401-5 \quad 3.52273-5 \quad 7.45761-5 \quad 1.34159-4$ $2.21191-4 \quad 3.64682-4 \quad 6.01258-4 \quad 9.91309-4 \quad 1.63439-3$ $2.14167-3 \quad 2.43063-3 \quad 2.82399-3 \quad 3.37141-3 \quad 4.61914-3$ 7.32483-3 1.20766-2 $1.93065-2 \quad 2.41830-2 \quad 2.54229-2$ $2.65293-2$ 2.77506-2 3.14039-2 4.33909-2 5.45187-2 
7.15396-2 $1.04645-1 \quad 1.36365-1 \quad 1.66556-1 \quad 2.03432-1$ 2.48472-1 $2.83877-1 \quad 2.95864-1 \quad 2.97851-1 \quad 3.00232-1$ 3.44858-1 4.42806-1 5.10634-1 5.65749-1 $6.75418-1$ $8.24957-1 \quad 1.00760+0 \quad 1.23069+0 \quad 1.50317+0 \quad 1.83598+0$ $\begin{array}{llllll}2.12513+0 & 2.28850+0 & 2.40583+0 & 2.73896+0 & 3.34537+0\end{array}$

$4.08604+0 \quad 4.99070+0 \quad 6.09565+0 \quad 7.44525+0 \quad 9.09365+0$ $1.11070+11.35661+1$

$\operatorname{df} 195$ $3.70370-3 \quad 4.37060-3 \quad 4.52500-3 \quad 4.57410-3$ $4.55830-3 \quad 4.48970-3 \quad 4.38170-3 \quad 4.24920-3 \quad 4.12890-3$ $4.03110-3 \quad 3.93550-3 \quad 3.84530-3 \quad 3.76290-3 \quad 3.69080-3$

$3.65280-3 \quad 3.63870-3 \quad 3.62290-3 \quad 3.60620-3 \quad 3.58250-3$ $3.55850-3 \quad 4.08750-3 \quad 5.89750-3 \quad 7.13870-3.7 .42410-3$ $\begin{array}{llllll}7.67700-3 & 7.95180-3 & 8.74210-3 & 1.11490-2 & 1.35030-2\end{array}$

$1.65080-2 \quad 2.25270-2 \quad 2.86610-2 \quad 3.43150-2 \quad 4.10860-2$ 4.92110-2 5.56070-2 $5.77300-2 \quad 5.80800-2 \quad 5.84980-2$ $6.59980-2 \quad 8.26560-2 \quad 9.04890-2 \quad 9.54810-2 \quad 1.05360-1$ 1.17860-1 1.29670-1 $1.30100-1$ 1.28650-1 $1.27220-1$

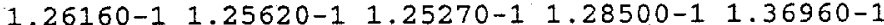
$1.45980-1$ 1.54120-1 $1.51160-1 \quad 1.47220-1 \quad 1.47060-1$ $1.63200-12.00340-1$

fm195. $\quad 5.701+10$

f205:p $\quad 240.000 \quad-200.0 \quad 0.01 .0$

de205

$1.50000-2 \quad 3.25000-2 \quad 5.75000-2 \quad 8.50000-2$ $\begin{array}{llllll}1.25000-1 & 2.25000-1 & 3.50000-1 & 4.55000-1 & 5.55000-1\end{array}$ $6.50000-1 \quad 8.50000-1 \quad 1.25000+0 \quad 1.75000+0 \quad 2.25000+0$ $2.75000+0 \quad 3.50000+0 \quad 4.50000+0 \quad 5.50000+0 \quad 6.50000+0$ $7.25000+0 \quad 7.75000+0 \quad 9.00000+0 \quad 1.20000+1$

df 205 $2.14390-3 \quad 5.77600-4 \quad 2.71850-4 \quad 2.68170-4$ $\begin{array}{llllll}3.27670-4 & 5.66760-4 & 8.75940-4 & 1.08450-3 & 1.27970-3\end{array}$ $\begin{array}{lllll}1.44170-3 & 1.75630-3 & 2.31560-3 & 2.92700-3 & 3.46860-3\end{array}$ $3.95960-3 \quad 4.62210-3 \quad 5.41370-3 \quad 6.19090-3 \quad 6.92650-3$ $7.47830-3 \quad 7.84680-3 \quad 8.77160-3 \quad 1.10200-2$

$\mathrm{fm} 205 \quad 5.701+10$

f215:p $\quad 240.000 \quad-100.0 \quad 0.0 \quad 1.0$

de215 $1.50000-2 \quad 3.25000-2 \quad 5.75000-2 \quad 8.50000-2$

$\begin{array}{lllll}1.25000-1 & 2.25000-1 & 3.50000-1 & 4.55000-1 & 5.55000-1\end{array}$ $6.50000-1.8 .50000-1 \quad 1.25000+0 \quad 1.75000+0.2 .25000+0$

$2.75000+0 \quad 3.50000+0 \quad 4.50000+0 \quad 5.50000+0 \quad 6.50000+0$

$7.25000+0 \quad 7.75000+0 \quad 9.00000+01.20000+1$

$\operatorname{df} 215$

$2.14390-3 \quad 5.77600-4 \quad 2.71850-4 \quad 2.68170-4$ $3.27670-4 \quad 5.66760-4 \quad 8.75940-4 \quad 1.08450-3 \quad 1.27970-3$ $1.44170-3 \quad 1.75630-3 \quad 2.31560-3.2 .92700-3.3 .46860-3$ $3.95960-3 \quad 4.62210-3 \quad 5.41370-3 \quad 6.19090-3 \quad 6.92650-3$ $7.47830-3 \quad 7.84680-38.77160-3.1 .10200-2$

$\operatorname{fm} 215 \quad 5.701+10$

f225:p 240.000

$-50.0 \quad 0.01 .0$

$1.50000-2 \quad 3.25000-2 \quad 5.75000-2 \quad 8.50000-2$ $\begin{array}{llllll}1.25000-1 & 2.25000-1 & 3.50000-1 & 4.55000-1 & 5.55000-1\end{array}$ $6.50000-1 \quad 8.50000-1 \quad 1.25000+0 \quad 1.75000+0 \quad 2.25000+0$ $2.75000+0 \quad 3.50000+0 \quad 4.50000+0 \quad 5.50000+0 \quad 6.50000+0$ $7.25000+0 \quad 7.75000+0 \quad 9.00000+0 \quad 1.20000+1$

df225

$\begin{array}{llll}2.14390-3 & 5.77600-4 & 2.71850-4 & 2.68170-4\end{array}$ $\begin{array}{llllll}3.27670-4 & 5.66760-4 & 8.75940-4 & 1.08450-3 & 1.27970-3\end{array}$ $\begin{array}{llllll}1.44170-3 & 1.75630-3 & 2.31560-3 & 2.92700-3 & 3.46860-3\end{array}$ $3.95960-3 \quad 4.62210-3 \quad 5.41370-3 \quad 6.19090-3 \quad 6.92650-3$ $7.47830-3 \quad 7.84680-3 \quad 8.77160-3 \quad 1.10200-2$

$\mathrm{fm} 225 \quad 5.701+10$

f235:p $\quad 240.000 \quad-25.0 \quad 0.01 .0$

de235 $1.50000-2 \quad 3.25000-2 \quad 5.75000-28.50000-2$

$1.25000-1 \quad 2.25000-1 \quad 3.50000-1 \quad 4.55000-1 \quad 5.55000-1$ $6.50000-18.50000-1 \quad 1.25000+0.1 .75000+0 \quad 2.25000+0$ $2.75000+0 \quad 3.50000+0 \quad 4.50000+0 \quad 5.50000+0 \quad 6.50000+0$ $7.25000+0 \quad 7.75000+0 \quad 9.00000+0.1 .20000+1$

$2.14390-3 \quad 5.77600-4 \quad 2.71850-4 \quad 2.68170-4$

df 235

$3.27670-4 \quad 5.66760-4 \quad 8.75940-4 \quad 1.08450-3 \quad 1.27970-3$ $\begin{array}{lllll}1.44170-3 & 1.75630-3 & 2.31560-3 & 2.92700-3 & 3.46860-3\end{array}$ $3.95960-3 \quad 4.62210-3 \quad 5.41370-3 \quad 6.19090-3 \quad 6.92650-3$ $7.47830-3 \quad 7.84680-3 \quad 8.77 .160-3 \quad 1.10200-2$

fm235 $\quad 5.701+10$

f245:p $\quad 240.000 \quad 0.0 \quad 0.01 .0$

de245 $1.50000-2 \quad 3.25000-2 \quad 5.75000-2 \quad 8.50000-2$ $1.25000-12.25000-1 \quad 3.50000-1.4 .55000-1 \quad 5.55000-1$ 
$6.50000-1 \quad 8.50000-1 \quad 1.25000+0 \quad 1.75000+0 \quad 2.25000+0$ $2.75000+0 \quad 3.50000+0 \quad 4.50000+0 \quad 5.50000+0 \quad 6.50000+0$ $7.25000+0 \quad 7.75000+09.00000+01.20000+1$

df 265 $2.14390-3 \quad 5.77600-42.71850-4 \quad 2.68170-4$ $3.27670-4 \quad 5.66760-4 \quad 8.75940-4 \quad 1.08450-3 \quad 1.27970-3$ $1.44170-3 \quad 1.75630-3 \quad 2.31560-32.92700-3 \quad 3.46860-3$ $3.95960-3 \quad 4.62210-3 \quad 5.41370-3 \quad 6.19090-3 \quad 6.92650-3$ $7.47830-3 \quad 7.84680-38.77160 \cdots 31.10200-2$

$\operatorname{fm} 265$

f275:p $5.701+10$

240.000

$100.0 \quad 0.01 .0$

$1.50000-2 \quad 3.25000-2 \quad 5.75000-2 \quad 8.50000-2$ $1.25000-1 \quad 2.25000-1 \quad 3.50000-1 \quad 4.55000-1 \quad 5.55000-1$ $6.50000-18.50000-1 \quad 1.25000+0 \quad 1.75000+02.25000+0$ $2.75000+0 \quad 3.50000+0 \quad 4.50000+0 \quad 5.50000+0 \quad 6.50000+0$ $7.25000+0 \quad 7.75000+0 \quad 9.00000+01.20000+1$

df 275 $2.14390-3 \quad 5.77600-4 \quad 2.71850-4 \quad 2.68170-4$ $3.27670-4 \quad 5.66760-4 \quad 8.75940-4 \quad 1.08450-3 \quad 1.27970-3$ $1.44170-3 \quad 1.75630-3 \quad 2.31560-3 \quad 2.92700-3 \quad 3.46860-3$ $\begin{array}{lllll}3.95960-3 & 4.62210-3 & 5.41370-3 & 6.19090-3 & 6.92650-3\end{array}$ $\begin{array}{llll}7.47830-3 & 7.84580-3 & 8.77160-3 & 1.10200-2\end{array}$

$\operatorname{fm} 275$ f285:p $5.701+10$

de 285

240.000

$200.0 \quad 0.01 .0$ $1.50000-2 \quad 3.25000-2 \quad 5.75000-2 \quad 8.50000-2$ $\begin{array}{lllll}1.25000-1 & 2.25000-1 & 3.50000-1 & 4.55000-1 & 5.55000-1\end{array}$ $6.50000-1 \quad 8.50000-1 \quad 1.25000+0 \quad 1.75000+0 \quad 2.25000+0$ $2.75000+0 \quad 3.50000+0 \quad 4.50000+0 \quad 5.50000+0 \quad 6.50000+0$ $7.25000+0 \quad 7.75000+0 \quad 9.00000+0 \quad 1.20000+1$

$\operatorname{df} 285$ $2.14390-3 \quad 5.77600-1 \quad 2.71850-4 \quad 2.68170-4$

$3.27670-4 \quad 5.66760-4 \quad 8.75940-4 \quad 1.08450-3 \quad 1.27970-3$ $\begin{array}{lllll}1.44170-3 & 1.75630-3 & 2.31560-3 & 2.92700-3 & 3.46860-3\end{array}$ $3.95960-3 \quad 4.62210-3 \quad 5.41370-3 \quad 6.19090-3 \quad 6.92650-3$ $7.47830-3 \quad 7.84680-3 \quad 8.77160-3 \quad 1.10200-2$

$\mathrm{fm} 285 \quad 5.701+10$

f295:p $240.000 \quad 250.0 \quad 0.01 .0$

de295 $1.50000-2 \quad 3.25000-2 \quad 5.75000-28.50000-2$ $1.25000-1 \quad 2.25000-1 \quad 3.50000-1 \quad 4.55000-1 \quad 5.55000-1$ $6.50000-1 \quad 8.50000-1 \quad 1.25000+0 \quad 1.75000+02.25000+0$ $2.75000+0 \quad 3.50000+0 \quad 4.50000+0 \quad 5.50000+0 \quad 6.50000+0$ $7.25000+0 \quad 7.75000+0 \quad 9.00000+0 \quad 1.20000+1$

df295 $\begin{array}{llll}2.14390-3 & 5.77600-4 & 2.71850-4 & 2.68170-4\end{array}$ $3.27670-4 \quad 5.66760-4 \quad 8.75940-4 \quad 1.08450-3 \quad 1.27970-3$ $\begin{array}{llllll}1.44170-3 & 1.75630-3 & 2.31560-3 & 2.92700-3 & 3.46860-3\end{array}$ $\begin{array}{lllll}3.95960-3 & 4.62210-3 & 5.41370-3 & 6.19090-3 & 6.92650-3\end{array}$ $7.47830-3 \quad 7.84680-3 \quad 8.77160-3 \quad 1.10200-2$ fm295 $5.701+10$ sdef pos $=0$ o 0 vec=1 0 o dir=d1 erg=fdir=d2 si1 s $11 \quad 121314 \quad 15 \quad 16$

$\begin{array}{lllllll}\operatorname{spl} 1 & 1.17+10 & 8.60+9 & 1.21+10 & 8.82+9 & 1.47+10 & 1.09+9\end{array}$ 


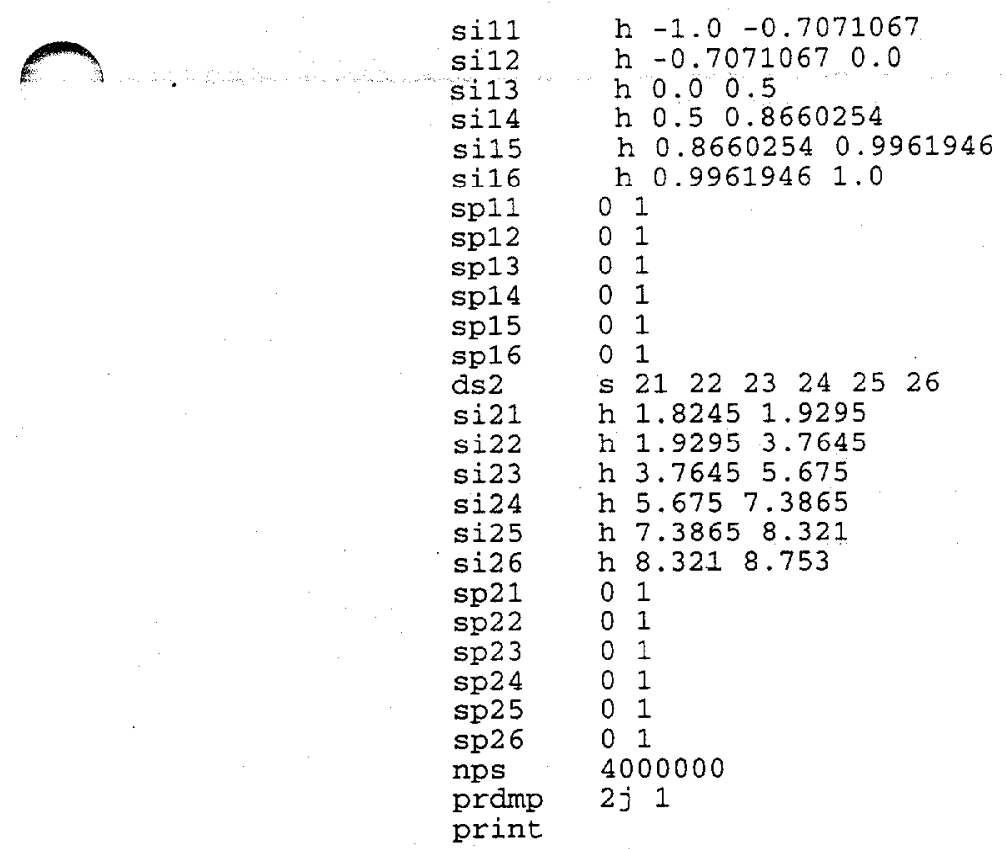




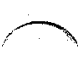




\section{APPENDIX F}

TABLES OF ORIGEN-CALCULATED RADIOACTIVITIES OF SELECTED CARGOES AND STRUCTURES IRRADIATED IN THE PFNA FACILITY 


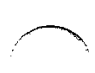

$\longrightarrow$ 


\section{APPENDIX F}

\section{TABLES OF ORIGEN-CALCULATED RADIOACTIVITIES OF SELECTED CARGOES AND STRUCTURES IRRADIATED IN THE PFNA FACILITY}

Table F1. Radioactivity (Ci) After 8-s Irradiation of 1-kg Salted Beef Cargo (Flux Parameter Set 1).

\begin{tabular}{|c|c|c|c|c|c|c|c|c|c|c|c|}
\hline & & & & Time & Aftex $\mathrm{Di}$ & harge & & & & & \\
\hline & & $1 \mathrm{E}-15 \mathrm{sec}$ & & 10.0 sec & $20.0 \mathrm{sec}$ & $30.0 \mathrm{sec}$ & $60.0 \mathrm{sec}$ & $120.0 \mathrm{sec}$ & 300.0 sec & $600.0 \mathrm{sec}$ & $1200.0 \mathrm{sec}$ \\
\hline b 12 & $5.85 \mathrm{E}-13$ & $5.85 \mathrm{E}-13$ & $6.59 \mathrm{E}-14$ & $2.75 \mathrm{E}-14$ & $1.04 \mathrm{E}-14$ & $3.93 E-15$ & $2.13 \mathrm{E}-16$ & $6.24 E-19$ & 1. $57 \mathrm{E}-26$ & $0.00 \mathrm{E}+00$ & $0.00 E+00$ \\
\hline & $8.84 \mathrm{E}-18$ & $8.84 E-18$ & $.84 \mathrm{E}-18$ & $8.84 \mathrm{E}-18$ & $8.84 \mathrm{E}-18$ & $8.84 \mathrm{E}-18$ & $8.84 \mathrm{E}-18$ & $8.84 E-18$ & $8.84 \mathrm{E}-18$ & $8.84 \mathrm{E}-18$ & $8.84 E-18$ \\
\hline C 15 & $1.19 \mathrm{E}-09$ & 1. $19 \mathrm{E}-09$ & $8.99 \mathrm{E}-10$ & $7.04 \mathrm{E}-11$ & $4.15 E-12$ & $2.45 E-13$ & $5.03 \mathrm{E}-17$ & $2.12 E-24$ & $0.00 E+00$ & $0.00 E+00$ & $0.00 \mathrm{E}+00$ \\
\hline n 16 & $6.04 \mathrm{E}-09$ & $6.04 \mathrm{E}-09$ & $5.48 E-09$ & $2.28 \mathrm{E}-09$ & $8.61 E-10$ & $3.27 E-10$ & $77 E-11$ & $5.18 E-14$ & $1.30 \mathrm{E}-21$ & $0.00 \mathrm{E}+00$ & $0.00 \mathrm{E}+00$ \\
\hline 19 & & $4.62 \mathrm{E}-12$ & & & $2.76 \mathrm{E}-12$ & $2.14 \mathrm{E}-12$ & & EE-13 & & $8.98 \mathrm{E}-19$ & $74 E-25$ \\
\hline f 20 & E-09 & $E-09$ & $3 E-09$ & $1.09 \mathrm{E}-09$ & $5.82 \mathrm{E}-10$ & -10 & & $E-12$ & 1. & DE-26 & $0 E+00$ \\
\hline ne 23 & $E-10$ & -10 & 8.5 & 7.6 & 6.3 & 5.2 & & 9. & & $9 E-14$ & $3 E-19$ \\
\hline na 24 & 4.7 & -13 & $E-13$ & -13 & $E-13$ & $E-13$ & $E-13$ & $2 E-13$ & -13 & $0 E-13$ & $6 E-13$ \\
\hline na $24 m$ & 1.8 & & 2. & 0. & $0.00 \mathrm{E}+00$ & 0 . & & & & +00 & $8+00$ \\
\hline na 25 & 1.4 & $E-12$ & -12 & 1.3 & E-12 & -12 & -13 & -13 & & -15 & $E-18$ \\
\hline $\mathrm{mg} 27$ & 1.1 & & -12 & 1.7 & 1.1 & 1. & & & & & -13 \\
\hline a1 28 & 5.0 & -11 & -11 & $1 E-11$ & $4.57 E-11$ & $4 E-11$ & $1 E-11$ & -11 & -11 & $\mathrm{E}-12$ & $4 \mathrm{E}-13$ \\
\hline a) 29 & & & & & & & & & & & \\
\hline al & $1.15 \mathrm{E}-1.4$ & E-14 & $E-15$ & 1. $68 \mathrm{E}-15$ & $2.45 E-16$ & $3.57 E-17$ & $1.11 \mathrm{E}-19$ & $E-24$ & 0.0 & +00 & $E+00$ \\
\hline si 31 & $1.14 \mathrm{E} 11$ & 1.14 & 1.1 & $1.13 \mathrm{E}-11$ & $1.13 \mathrm{E}-11$ & -11 & & & & & $E-11$ \\
\hline p 32 & $2.63 \mathrm{E}-16$ & $2.63 \mathrm{E}-16$ & $2.63 \mathrm{E}-16$ & $2.63 \mathrm{E}-16$ & $2.63 \mathrm{E}-16$ & $2.63 \mathrm{E}-16$ & 2.6 & $2.63 \mathrm{E}-16$ & & & \\
\hline $\operatorname{ar} 39$ & $2.76 \mathrm{E}-17$ & $2.76 E-17$ & $2.76 E-17$ & $2.76 \mathrm{E}-17$ & $2.76 \mathrm{E}-17$ & $2.76 \mathrm{E}-17$ & $2.76 \mathrm{E}-17$ & $2.76 E-17$ & $2.76 \mathrm{E}-17$ & $2.76 \mathrm{E}-17$ & $2.76 \mathrm{E}-17$ \\
\hline k 40 & $1.72 \mathrm{E}-09$ & $1.72 \mathrm{E}-09$ & $1.72 \mathrm{E}-09$ & $1.72 E-0.9$ & $1.72 \mathrm{E}-09$ & $1.72 \mathrm{E}-09$ & $1.72 \mathrm{E}-09$ & & & & $1.72 \mathrm{E}-09$ \\
\hline & $4.87 \mathrm{E}-15$ & $4.87 E-15$ & $4.87 \mathrm{E}-15$ & $4.87 E-15$ & $4.87 \mathrm{E}-15$ & $4.87 E-15$ & $4.87 \mathrm{E}-15$ & & 4.85 & $4.83 \mathrm{E}-15$ & $4.78 \mathrm{E}-15$ \\
\hline & $1.39 \mathrm{E}-08$ & $1.39 E-08$ & $1.10 E-08$ & $5.99 \mathrm{E}-09$ & $3.86 \mathrm{E}-09$ & $2.94 \mathrm{E}-09$ & $2.13 \mathrm{E}-09$ & $1.86 E-09$ & $1.74 \mathrm{E}-09$ & $1.73 \mathrm{E}-09$ & $1.73 E-09$ \\
\hline
\end{tabular}


Table F2. Radioactivity (Ci) After 16-s Irradiation of 1-kg Salted Beef Cargo (Flux Parameter Set 1).

\begin{tabular}{|c|c|c|c|c|c|c|c|c|c|c|c|}
\hline & & & & Time & After $D i$ & Harge & & & & & \\
\hline & initial & $1 \mathrm{E}-15 \mathrm{sec}$ & $1.0 \mathrm{sec}$ & $10.0 \mathrm{sec}$ & $20.0 \mathrm{sec}$ & $30.0 \mathrm{sec}$ & $60.0 \mathrm{sec}$ & $120.0 \mathrm{sec}$ & $300.0 \mathrm{sec}$ & $600.0 \mathrm{sec}$ & $1200.0 \mathrm{sec}$ \\
\hline b 12 & $6.18 \mathrm{E}-13$ & $6.18 \mathrm{E}-13$ & $9.62 \mathrm{E}-14$ & $4.01 \mathrm{E}-14$ & $1.52 \mathrm{E}-14$ & $5.74 E-15$ & $3.11 \mathrm{E}-16$ & $9.10 \mathrm{E}-19$ & $2.29 \mathrm{E}-26$ & $0.00 E+00$ & $0.00 \mathrm{E}+00$ \\
\hline C 14 & $1.77 \mathrm{E}-17$ & $1.77 \mathrm{E}-17$ & $1.77 \mathrm{E}-17$ & $1.77 \mathrm{E}-17$ & $1.77 \mathrm{E}-17$ & $1.77 \mathrm{E}-17$ & $1.77 \mathrm{E}-17$ & $1.77 \mathrm{E}-17$ & $1.77 \mathrm{E}-17$ & $1.77 \mathrm{E}-17$ & $1.77 E=17$ \\
\hline c 15 & $1.32 \mathrm{E}-09$ & $1.32 \mathrm{E}-09$ & $9.92 \mathrm{E}-10$ & $7.77 E-11$ & $4.58 \mathrm{E}-12$ & $2.70 \mathrm{E}-13$ & $5.55 \mathrm{E}-17$ & $2.34 \mathrm{E}-24$ & $0.00 \mathrm{E}+00$ & $0.00 \mathrm{E}+00$ & $0.00 \mathrm{E}+00$ \\
\hline n 16 & $8.81 E-09$ & $8.81 E 09$ & $8.00 \mathrm{E}-09$ & $3.33 \mathrm{E}-09$ & $1.26 \mathrm{E}-09$ & $4.77 E-10$ & & $7.56 \mathrm{E}-14$ & $1.90 \mathrm{E}-21$ & $0.00 \mathrm{E}+00$ & $0.00 E+00$ \\
\hline ० 19 & $8.39 E-12$ & $8.39 E-12$ & $8.17 \mathrm{E}-12$ & $6.48 \mathrm{E}-12$ & $5.01 \mathrm{E}-12$ & $3.87 \mathrm{E}-12$ & $1.79 \mathrm{E}-12$ & $3.81 E-13$ & $3.70 \mathrm{E}-15$ & $1.63 \mathrm{E}-18$ & $3.16 \mathrm{E}-25$ \\
\hline f. 20 & $3.29 \mathrm{E}-09$ & $3.29 \mathrm{E}-09$ & $3.09 E-09$ & $1.75 E-09$ & $9.33 \mathrm{E}-10$ & $4.97 E-10$ & $7.51 \mathrm{E}-11$ & $1.71 E-12$ & $2.03 E-17$ & $1.25 \mathrm{E}-25$ & $0.00 \mathrm{E}+00$ \\
\hline ne 23 & $1.71 E-09$ & LE-09 & $7 E-09$ & $1.42 E-09$ & $1.18 E-09$ & $9.76 \mathrm{E}-10$ & $5.58 \mathrm{E}-10$ & $1.83 E-10$ & $6.41 \mathrm{E}-12$ & $2.41 \mathrm{E}-14$ & 3. $40 \mathrm{E}-19$ \\
\hline na 24 & $9.46 E-13$ & $9.46 \mathrm{E}-13$ & $9.46 \mathrm{E}-13$ & $9.46 \mathrm{E}-13$ & $9.46 \mathrm{E}-13$ & $9.46 F-1.3$ & $9.46 \mathrm{E}-13$ & $9.45 E-13$ & $9.43 E-13$ & $9.39 \mathrm{E}-13$ & $9.32 \mathrm{E}-13$ \\
\hline na $24 \mathrm{~m}$ & $1.88 \mathrm{E}-09$ & $1.88 \mathrm{E}-09$ & $2.27 E-24$ & $0.00 E+00$ & $0.00 E+00$ & $0.00 \mathrm{E}+00$ & $0.00 \mathrm{E}+00$ & $0.00 E+00$ & $0.00 \mathrm{E}+00$ & $0.00 E+00$ & $0.00 \mathrm{E}+00$ \\
\hline na 25 & $2.81 E-12$ & $2.81 \mathrm{E}-12$ & $E-12$ & $E-12$ & $2.23 E-12$ & $E-12$ & & $6.97 \mathrm{E}-13$ & $9 E-14$ & $2.62 \mathrm{E}-15$ & $44 \mathrm{~F}-18$ \\
\hline $\mathrm{mg} 27$ & $2.36 \mathrm{E}-12$ & -12 & -12 & $2.34 \mathrm{E}-12$ & $2.31 \mathrm{E}-12$ & $2.28 \mathrm{E}-12$ & $2.20 \mathrm{E}-12$ & $2.04 \mathrm{E}-12$ & $1.64 \mathrm{E}-12$ & $1.14 \mathrm{E}-12$ & $5.46 \mathrm{E}-13$ \\
\hline al 28 & $9.92 \mathrm{E}-11$ & -11 & $9.87 E-11$ & $9.42 \mathrm{E}-11$ & $8.95 \mathrm{E}-11$ & $E-11$ & 7.2 & $5.34 \mathrm{E}-11$ & $2.11 \mathrm{E}-11$ & $4.49 \mathrm{E}-12$ & $2.04 \mathrm{E}-13$ \\
\hline al 29 & $4.19 E-13$ & -13 & $4.18 \mathrm{E}-13$ & $4.12 \mathrm{E}-13$ & $4.04 \mathrm{E}-13$ & $3.97 E-13$ & $3.77 \mathrm{E}-13$ & $3.39 \mathrm{E}-13$ & $2.47 \mathrm{E}-13$ & $5 \mathrm{E}-13$ & $7 E-14$ \\
\hline al 30 & $1.40 \mathrm{E}-14$ & -14 & $1.15 \mathrm{E}-14$ & $2.04 E-15$ & $2.97 \mathrm{E}-16$ & $4.33 E-17$ & $1.34 \mathrm{E}-19$ & $1.29 E-24$ & $0.00 E+00$ & $0.00 \mathrm{E}+00$ & $0.00 \mathrm{E}+00$ \\
\hline si 31 & $2.27 \mathrm{E}-11$ & $2.27 \mathrm{E}-11$ & $2.27 \mathrm{E}-11$ & $2.27 \mathrm{E}-11$ & $2.27 \mathrm{E}-11$ & $2.27 E-11$ & & $2.25 E-11$ & & $2.17 \mathrm{E}-11$ & $2.08 \mathrm{E}-11$ \\
\hline & $5.27 E-16$ & $5.27 \mathrm{E}-16$ & $5.27 \mathrm{E}-16$ & $5.27 E-16$ & $5.27 \mathrm{E}-16$ & $5.27 E-16$ & $5.27 \mathrm{E}-16$ & $5.27 E-16$ & $5.27 E-16$ & $5.27 \mathrm{E}-16$ & $5.26 E-16$ \\
\hline cl 36 & $1.17 E-20$ & $1.17 \mathrm{E}-20$ & $1.17 \mathrm{E}-20$ & $1.17 \mathrm{E}-20$ & $1.17 \mathrm{E}-20$ & $1.17 \mathrm{E}-20$ & $1.17 \mathrm{E}-20$ & $1.17 \mathrm{E}-20$ & $1.17 \mathrm{E}-20$ & $1.17 E-20$ & $1.17 E-20$ \\
\hline & $5.51 \mathrm{E}-17$ & $5.51 \mathrm{E}-17$ & $5.51 \mathrm{E}-17$ & $5.51 E-17$ & $5.51 \mathrm{E}-17$ & $5.51 E-17$ & $5.51 \mathrm{E}-17$ & $5.51 \mathrm{E}-17$ & $5.51 \mathrm{E}-17$ & $5.51 \mathrm{E}-17$ & $5.51 E-17$ \\
\hline & $1.72 E-09$ & $1.72 \mathrm{E}-09$ & $1.72 \mathrm{E}-09$ & $1.72 \mathrm{E}-09$ & $1.72 \mathrm{E}-09$ & $1.72 \mathrm{E}-09$ & $1.72 \mathrm{E}-09$ & $1.72 \mathrm{E} \quad 09$ & $1.72 \mathrm{E}-09$ & $1.72 \mathrm{E}-09$ & 1. $72 \mathrm{E}-09$ \\
\hline $\mathrm{k}$ & $9.74 \mathrm{E}-15$ & $9.74 \mathrm{E}-15$ & $9.74 \mathrm{E}-15$ & $9.74 E-15$ & $9.74 \mathrm{E}-15$ & $9.74 \mathrm{E}-15$ & $9.73 E-15$ & $9.72 \mathrm{E}-15$ & $9.70 E-15$ & $9.65 \mathrm{E}-15$ & $9.56 \mathrm{E}-15$ \\
\hline & $1.89 \mathrm{E}-08$ & $1.89 \mathrm{E}-08$ & $1.56 \mathrm{E}-08$ & $8.43 E-09$ & $5.22 \mathrm{E}-09$ & $3.78 E-09$ & 2. $48 F ;-09$ & $1.98 E-09$ & $1.77 E-09$ & $1.75 \mathrm{E}-09$ & $1.71 \mathrm{E}-09$ \\
\hline
\end{tabular}


Table F3. Radioactivity (Ci) After 24-s Irradiation of 1-kg Salted Beef Cargo (Flux Parameter Set 1).

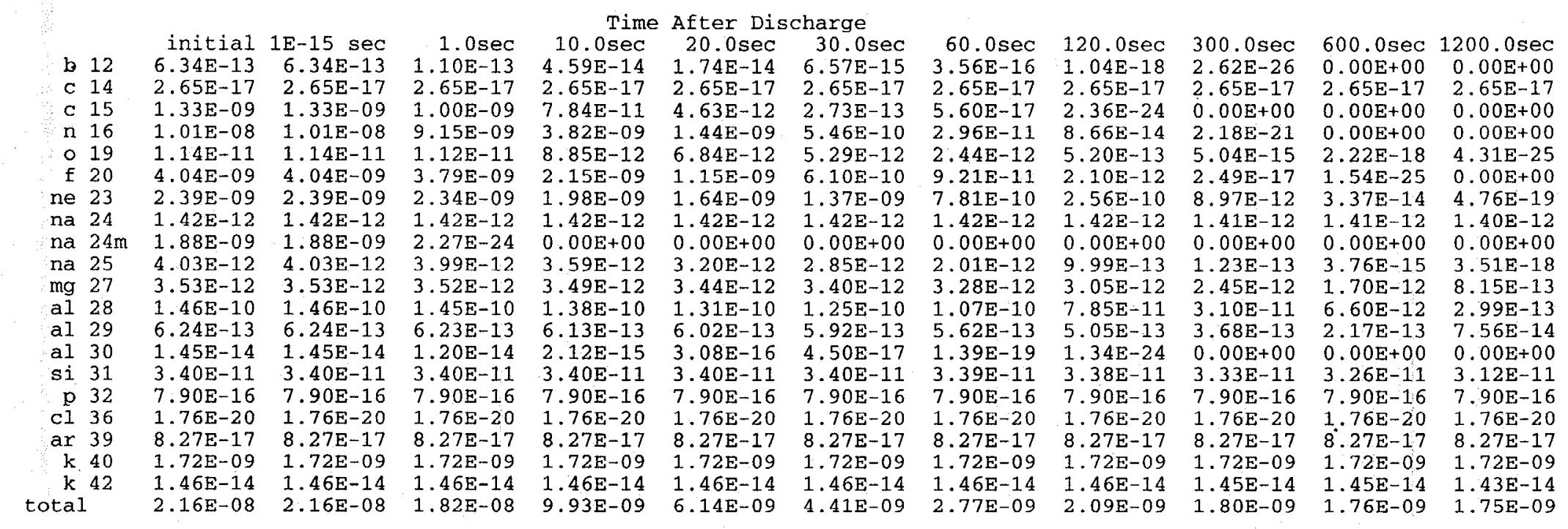


Table F4. Radioactivity (Ci) After 8-s Irradiation of 1-kg Ball Bearings Cargo (Flux Parameter Set 1).

\begin{tabular}{|c|c|c|c|c|c|c|c|c|c|c|c|}
\hline & & & & Time & After & & & & & & \\
\hline & & & & $10.0 \mathrm{sec}$ & $20.0 \mathrm{sec}$ & $30.0 \mathrm{sec}$ & & $120.0 \mathrm{sec}$ & $300.0 \mathrm{sec}$ & $600.0 \mathrm{sec}$ & $1200.0 \mathrm{sec}$ \\
\hline b 12 & $1.87 \mathrm{E}-18$ & $1.87 \mathrm{E}-18$ & $1.70 \mathrm{E}-18$ & $7.07 E-19$ & $2.67 E-19$ & $1.01 \mathrm{E}-19$ & $5.48 \mathrm{E}-21$ & $1.60 \mathrm{E}-23$ & $7.83 E-31$ & $0.00 \mathrm{E}+00$ & $0.00 \mathrm{E}+00$ \\
\hline c 15 & 3. $16 \mathrm{E}-14$ & 3. $16 E-14$ & $2.38 E-14$ & $1.87 \mathrm{E}-15$ & 1. $10 \mathrm{E}-16$ & $6.50 \mathrm{E}-18$ & $1.33 \mathrm{E}-21$ & $5.62 \mathrm{E}-29$ & $0.00 E+00$ & $0.00 \mathrm{E}+00$ & $0.00 E+00$ \\
\hline n 16 & $1.55 \mathrm{E}-13$ & $1.55 \mathrm{E}-13$ & $1.41 \mathrm{E}-13$ & $5.88 \mathrm{E}-14$ & $2.22 \mathrm{E}-14$ & $8.41 \mathrm{E}-15$ & $4.55 \mathrm{E}-16$ & 1. $33 \mathrm{E}-18$ & $3.35 \mathrm{E}-26$ & $0.00 \mathrm{E}+00$ & $0.00 E+00$ \\
\hline o 19 & & & 1. $02 \mathrm{E}-18$ & $9 p-19$ & $6.25 E-19$ & $4.83 \mathrm{E}-19$ & $2.23 \mathrm{E}-19$ & $4.76 \mathrm{E}-20$ & $4.61 F-22$ & $2.03 \mathrm{E}-2.5$ & 3. $94 \mathrm{E}-32$ \\
\hline na 24 & $5 E-14$ & $1.55 \mathrm{E}-14$ & $5 E-14$ & $1.55 \mathrm{E}-14$ & 1. $55 \mathrm{E}-14$ & $1.55 E-14$ & $1.55 \mathrm{E}-14$ & 1. $55 \mathrm{E}-14$ & $1.55 \mathrm{E}-14$ & $1.54 \mathrm{E}-14$ & 1. $53 \mathrm{E}-14$ \\
\hline mg 27 & $6.91 E-12$ & $6.91 E-12$ & $O E-12$ & $6.82 \mathrm{E}-12$ & $6.74 \mathrm{E}-12$ & $6.66 \mathrm{E}-12$ & $6.42 \mathrm{E}-12$ & $5.97 \mathrm{E}-12$ & $4.79 E-12$ & 3. $32 \mathrm{E}-12$ & $1.60 \mathrm{E}-12$ \\
\hline al 28 & $1.70 \mathrm{E}-10$ & $1.70 \mathrm{E}-10$ & $9 \mathrm{E}-10$ & & 1. $53 \mathrm{E}-10$ & $1.45 \mathrm{E}-10$ & $1.25 \mathrm{E}-10$ & $9.14 \mathrm{E}-11$ & $3.61 E-11$ & $7.69 \mathrm{E}-12$ & 3. $48 \mathrm{E}-13$ \\
\hline al 29 & $1.93 E-12$ & $1.93 \mathrm{E}-12$ & $1.93 \mathrm{E}-12$ & $1.90 \mathrm{E}-12$ & $1.86 \mathrm{E}-12$ & $1.83 \mathrm{E}-12$ & 1. $74 \mathrm{E}-12$ & 1. $56 \mathrm{E}-12$ & $1.14 E-12$ & $6.72 \mathrm{E}-13$ & $2.34 \mathrm{E}-13$ \\
\hline al 30 & $1.05 \mathrm{E}-13$ & $1.05 \mathrm{E}-13$ & $8.70 \mathrm{E}-14$ & & $2.24 \mathrm{E}-15$ & $3.27 E-16$ & $1.01 \mathrm{E}-18$ & $9.74 \mathrm{E}-24$ & $0.00 E+00$ & $0.00 E+00$ & $0 E+00$ \\
\hline si 31 & $1.81 \mathrm{E}-12$ & $1.81 \mathrm{E}-12$ & $1.81 \mathrm{E}-12$ & $1.81 \mathrm{E}-12$ & $1.81 \mathrm{E}-12$ & $1.81 \mathrm{E}-12$ & $1.81 \mathrm{E}-12$ & $1.80 \mathrm{E}-12$ & $1.77 \mathrm{E}-12$ & 1. $74 \mathrm{E}-12$ & $6 \mathrm{E}-12$ \\
\hline p 32 & $1.48 \mathrm{E}-14$ & $1.48 \mathrm{E}-14$ & $1.48 \mathrm{E}-14$ & $1.48 \mathrm{E}-14$ & 1. $48 \mathrm{E}-14$ & $1.48 \mathrm{E}-14$ & $1.48 \mathrm{E}-14$ & $8 \mathrm{E}-14$ & & $48 \mathrm{E}-14$ & $1.48 \mathrm{E}-14$ \\
\hline p 33 & $7.10 \mathrm{E}-14$ & $7.10 \mathrm{E}-14$ & $7.10 \mathrm{E}-14$ & & $7.10 \mathrm{E}-14$ & $7.10 \mathrm{E}-14$ & $7.10 \mathrm{E}-14$ & & & & -14 \\
\hline p 34 & $5.49 \mathrm{E}-13$ & 5.4 & -13 & $\mathrm{E}-13$ & $1.80 \mathrm{E}-13$ & $1.03 \mathrm{E}-13$ & 1.93 & & $2.98 \mathrm{E}-20$ & $2 E-27$ & \\
\hline s & 1.83 & & & & 1. $83 \mathrm{E}-19$ & & $1.83 \mathrm{E}$ & & & & \\
\hline s 37 & 1.14 & 1.1 & 1.3 & 1.1 & $1.09 \mathrm{E}-17$ & & $9.93 \mathrm{E}$ & & & & \\
\hline ti 51 & 6.13 & 6.1 & 6.1 & 6.0 & $5.89 \mathrm{E}-14$ & $5.77 E-14$ & 5.44 & -14 & -14 & -14 & -15 \\
\hline y 52 & & 6.04 & & & & & -11 & -11 & -11 & & \\
\hline v 53 & $1.87 E-12$ & 1. $87 \mathrm{E}-12$ & 1. $85 \mathrm{E}-12$ & 1.7 & 1.62 & $z-12$ & 1.2 & 13 & & -14 & $E-16$ \\
\hline v 54 & $3.53 \mathrm{E}-14$ & $3.53 \mathrm{E}-14$ & 3.4 & & 2.67 & -14 & 1.5 & & & & $E-21$ \\
\hline cr 51 & 7.1 & 7.1 & 7.1 & 7.1 & 7.13 & $E-14$ & 7.1 & & & & \\
\hline cr 55 & $8.76 E-12$ & 8.7 & 8.7 & 8.4 & 8.20 & 7.5 & 7.1 & & & & -13 \\
\hline $\mathrm{mn} 54$ & $1.6 \% \mathrm{E}-13$ & 1.6 & & & 1.67 & 1.6 & & & & & \\
\hline $\mathrm{mn}$ & 1.05 & 1.0 & -10 & 1.0 & 1.05 & -10 & 1.0 & & & -10 & -11 \\
\hline min 57 & 1.1 & 1.1 & & & & & 7.0 & & & & -15 \\
\hline $\operatorname{mn} 58$ & 5.0 & 5.0 & $5 E-13$ & 4.5 & $4.04 \mathrm{E}-13$ & $E-13$ & 2.6 & & -14 & -16 & $E-18$ \\
\hline fe & & & & & 9.6 & 9.6 & 9.8 & & -16 & & -16 \\
\hline fe 59 & 6.59 & $6.59 \mathrm{E}-16$ & $E-16$ & 6.5 & 6.59 & 6.5 & 6.5 & & & & $E-16$ \\
\hline $\mathrm{co}$ & $3.62 E-14$ & 3.6 & -14 & 3.8 & 3.62 & 3.6 & 3.6 & -14 & -14 & -14 & $E-14$ \\
\hline $\mathrm{co}$ & 1.88 & 1.8 & 1.88 & & 1.88 & & & & -17 & -17 & $1.88 \mathrm{E}-17$ \\
\hline $\mathrm{CO}$ & 9.40 & 9.4 & $9.4 \mathrm{C}$ & & 9.37 & 9.3 & 9.3 & & -15 & & $7 E-15$ \\
\hline co & 1.72 & 1.7 & & & 1.47 & & & 6.8 & -13 & -14 & $E-16$ \\
\hline ni & 6.68 & 6.6 & 6.6 & 6.6 & 6.60 & 6.6 & 6.6 & & & & \\
\hline ni & & 4.2 & & & 4.19 & & 4.1 & & -14 & -14 & $3.83 \mathrm{E}-14$ \\
\hline $\mathrm{cu}$ & 1.5 & 1.5 & & & 1.59 & 1.5 & 1.5 & & & & $57 \mathrm{E}-13$ \\
\hline $\mathrm{cu}$ & & 4.6 & & & & & 4.0 & & 2 & & \\
\hline $89 \mathrm{~m}$ & 1.79 & 1.7 & 2.2 & & 7.46 & 8.91 & & & & $1.16 \mathrm{E}-17$ & 1. $16 \mathrm{E}-17$ \\
\hline $2 r$ & 1.1 & & & 1.1 & 1.16 & 1.1 & & & & & \\
\hline 95 & $2.05 \mathrm{E}-18$ & 2.05 & 2.05 & & & 2.05 & 2.05 & $2.05 E-18$ & $2.05 \mathrm{E}-18$ & $2.04 \mathrm{E}-18$ & $2.04 \mathrm{E}-18$ \\
\hline $2 x$ & $4.27 E-18$ & 4.27 & 4.27 & $4.26 \mathrm{E}-18$ & $4.26 \mathrm{E}-18$ & $4.26 \mathrm{E}-18$ & $4.26 \mathrm{E}-18$ & $4.26 \mathrm{E}-18$ & & & \\
\hline 95 & $4.87 E-18$ & $4.87 \mathrm{E}-18$ & $4.87 \mathrm{E}-18$ & $4.87 \mathrm{E}-18$ & $4.87 \mathrm{E}-18$ & $4.87 E-18$ & $4.87 E-18$ & $4.87 E-18$ & $E-18$ & -18 & $87 E-18$ \\
\hline$n b$ & $2.69 \mathrm{E}-16$ & $2.69 E-16$ & $2.69 E-16$ & & $2.69 \mathrm{E}-16$ & & & & & & \\
\hline $\mathrm{nb}$ & $2.05 \mathrm{E}-15$ & $2.05 E-15$ & $2.05 E-15$ & $2.05 \mathrm{E}-15$ & $2.05 E-15$ & 2.04 & 2.03 & 2 . & $1.96 \mathrm{E}-15$ & $1.86 \mathrm{E}-15$ & $1.69 \mathrm{E}-15$ \\
\hline $\mathrm{nb} 97 \mathrm{~m}$ & $1.81 \mathrm{E}-19$ & $1.81 \mathrm{E}-19$ & $2.26 \mathrm{E}-19$ & $6.03 \mathrm{E}-19$ & $9.78 \mathrm{E}-19$ & 1. $31 \mathrm{E}-18$ & $2.11 \mathrm{~F}-18$ & $3.08 \mathrm{E}-18$ & $3.91 E-18$ & $4.02 E-18$ & $3.99 \mathrm{E}$ \\
\hline
\end{tabular}




\section{Table F4. (continued)}

\begin{tabular}{|c|c|c|c|c|c|c|c|c|c|c|c|}
\hline & & & & Time & After Di & charge & & & & & \\
\hline & initial & $1 \mathrm{E}-1$ & $1.0 \mathrm{sec}$ & $10.0 \mathrm{sec}$ & $20.0 \mathrm{sec}$ & $30.0 \mathrm{sec}$ & $60.0 \mathrm{sec}$ & $120.0 \mathrm{sec}$ & $300.0 \mathrm{sec}$ & $600.0 \mathrm{sec}$ & $1200.0 \mathrm{sec}$ \\
\hline & $2.11 \mathrm{E}-13$ & $E-13$ & $.66 \mathrm{E}-13$ & $1.87 \mathrm{E}-14$ & $1.66 \mathrm{E}-15$ & $1.47 \mathrm{E}-16$ & & & & & \\
\hline 100 & 1.2 & 1.2 & 7.55 & 1. & 1. & 1. & $1.09 \mathrm{E}-26$ & $0 E+00$ & $0 E+00$ & 0. & 0. \\
\hline & 9.8 & 9.8 & 9.81 & $9.81 \mathrm{E}-16$ & 9.8 & 9. & 9.8 & & & & \\
\hline & & 6.7 & & 6. & 6. & -14 & $E-14$ & & & 4. & $E-14$ \\
\hline & 2.2 & 2.2 & & & & & & & & & \\
\hline & $E-10$ & $4.78 \mathrm{E}-10$ & & $E-1$ & $3 E-10$ & 4. $21 \mathrm{E}-10$ & $4 E-10$ & $3.02 E-10$ & $1.88 \mathrm{E}-10$ & 1. $28 \mathrm{E}-10$ & $03 E-10$ \\
\hline
\end{tabular}


Table F5. Radioactivity (Ci) After 16-s Irradiation of 1-kg Ball Bearings Cargo (Flux Parameter Set 1).

\begin{tabular}{|c|c|c|c|c|c|c|c|c|c|c|c|}
\hline & & & & & After Di & & & & & & \\
\hline & & $1 \mathrm{E}-15 \mathrm{sec}$ & 1.000 & $10.0 \mathrm{sec}$ & $20.0 \mathrm{sec}$ & $30.0 \mathrm{sec}$ & & $120.0 \mathrm{sec}$ & $300.0 \mathrm{sec}$ & $600.0 \mathrm{sec}$ & $1200.0 \mathrm{sec}$ \\
\hline b 12 & $2.73 E-18$ & $2.73 E-18$ & $2.48 \mathrm{E}-18$ & $1.03 \mathrm{E}-18$ & $3.90 \mathrm{E}-19$ & $1.48 E-19$ & $7.99 \mathrm{E}-21$ & $2.34 \mathrm{E}-23$ & $7.83 E-31$ & $0.00 \mathrm{E}+00$ & $0.00 \mathrm{E}+00$ \\
\hline C 15 & $3.49 \mathrm{E}-14$ & 3. $49 \mathrm{E}-14$ & $2.63 \mathrm{E}-14$ & $2.06 \mathrm{E}-15$ & $1.22 \mathrm{E}-16$ & $7.17 \mathrm{E}-18$ & $1.47 \mathrm{E}-21$ & $6.21 \mathrm{E}-29$ & $0.00 \mathrm{E}+00$ & $0.00 \mathrm{E}+00$ & $0.00 \mathrm{E}+00$ \\
\hline I 16 & $2.27 E-13$ & $2.27 E-13$ & $2.06 \mathrm{E}-13$ & $8.57 \mathrm{E}-14$ & $3.24 \mathrm{E}-14$ & $1.23 E-14$ & $6.64 \mathrm{E}-16$ & $1.95 E-18$ & $4.89 \mathrm{E}-26$ & $0.00 \mathrm{E}+00$ & $0.00 \mathrm{E}+00$ \\
\hline ○ 19 & $1.90 \mathrm{E}-18$ & $1.90 \mathrm{E}-18$ & $1.85 \mathrm{E}-18$ & $1.47 \mathrm{E}-18$ & 1. $13 E-18$ & $8.76 \mathrm{E}-19$ & $4.05 E-19$ & $8.63 E-20$ & $8.36 \mathrm{E}-22$ & $3.68 \mathrm{E}-25$ & $7.17 \mathrm{E}-32$ \\
\hline na 24 & $3.11 \mathrm{E}-14$ & $3.11 \mathrm{E}-14$ & $3.11 \mathrm{E}-14$ & $3.11 \mathrm{E}-14$ & $3.11 \mathrm{E}-14$ & $3.11 \mathrm{E}-14$ & $3.11 \mathrm{E}-14$ & $3.10 \mathrm{E}-14$ & $3.10 \mathrm{E}-14$ & $3.08 \mathrm{E}-14$ & $3.06 \mathrm{E}-14$ \\
\hline $\mathrm{mg} 27$ & $1.37 \mathrm{E}-11$ & $1.37 E-11$ & $1.37 \mathrm{E}-11$ & 1. $.36 \mathrm{E}-11$ & $1.34 \mathrm{E}-11$ & $1.33 \mathrm{E}-11$ & $1.28 \mathrm{E}-11$ & 1. $19 \mathrm{E}-11$ & $9.53 \mathrm{E}-12$ & $6.61 \mathrm{E}-12$ & 3. $18 \mathrm{~F}-12$ \\
\hline al 28 & $3.33 \mathrm{E}-10$ & $3.33 E-10$ & $3.31 E-10$ & $3.16 \mathrm{E}-10$ & $3.00 \mathrm{E}-10$ & $2.85 E-10$ & $2.44 \mathrm{E}-10$ & $1.79 \mathrm{E}-10$ & $7.08 \mathrm{E}-11$ & $1.51 \mathrm{E}-11$ & $6.83 \mathrm{E}-13$ \\
\hline al 29 & $3.84 \mathrm{E}-12$ & $3.84 E-12$ & $3.83 \mathrm{E}-12$ & $3.77 \mathrm{E}-12$ & $3.70 \mathrm{E}-12$ & 3. $64 \mathrm{E}-12$ & $3.45 E-12$ & $3.11 \mathrm{E}-12$ & $2.26 \mathrm{E}-12$ & $1.34 \mathrm{E}-12$ & $4.65 \mathrm{E}-13$ \\
\hline al 30 & $1.28 \mathrm{E}-13$ & $1.28 \mathrm{E}-13$ & $1.06 \mathrm{E}-13$ & $1.87 \mathrm{E}-14$ & $2.72 \mathrm{E}-15$ & $3.97 \mathrm{E}-16$ & $1.23 E-18$ & $\cdot 1.18 \mathrm{E}-23$ & $0.00 \mathrm{E}+00$ & $0.00 E+00$ & $0.00 \mathrm{E}+00$ \\
\hline si 31 & $3.63 E-12$ & $3.63 E-12$ & $3.63 \mathrm{E}-12$ & $3.62 \mathrm{E}-12$ & $3.62 \mathrm{E}-12$ & 3. $62 \mathrm{E}-12$ & $3.61 \mathrm{E}-12$ & $3.59 \mathrm{E}-12$ & $3.55 E-12$ & $3.47 E-12$ & $3.32 \mathrm{E}-12$ \\
\hline p 32 & $2.96 \mathrm{E}-14$ & $2.96 E-14$ & $2.96 \mathrm{E}-14$ & $2.96 \mathrm{E}-14$ & $2.96 \mathrm{E}-14$ & $2.96 \mathrm{E}-14$ & $2.96 \mathrm{E}-14$ & $2.96 E-14$ & $2.96 E-14$ & $2.96 E-14$ & $2.96 \mathrm{E}-14$ \\
\hline p 33 & $1.42 \mathrm{E}-13$ & $1.42 \mathrm{E}-13$ & $1.42 \mathrm{E}-13$ & $1.42 \mathrm{E}-13$ & $1.42 \mathrm{E}-13$ & $1.42 \mathrm{E}-13$ & $1.42 \mathrm{E}-13$ & $1.42 \mathrm{E}-13$ & $2 E-13$ & $1.42 \mathrm{E}-13$ & $E-13$ \\
\hline p 34 & $9.00 E-13$ & $9.00 \mathrm{E}-13$ & $8.52 E-13$ & $5.16 \mathrm{E}-13$ & $2.95 \mathrm{E}-13$ & $1.69 \mathrm{t}-13$ & $3.17 \mathrm{E}-14$ & $1.12 \mathrm{E}-15$ & $9 E-20$ & $5 \mathrm{E}-27$ & $00 \mathrm{E}+00$ \\
\hline s 35 & $3.65 E-19$ & & $3.65 E-19$ & $5 E-19$ & & & & & & & \\
\hline 37 & $2.26 \mathrm{E}-17$ & $2.26 \mathrm{E}-17$ & $2.25 \mathrm{E}-17$ & $2.21 \mathrm{E}-17$ & $2.16 \mathrm{E}-17$ & $E-17$ & $E-17$ & $1.72 \mathrm{E}-17$ & E-17 & -18 & $45 \mathrm{E}-18$ \\
\hline ti 51 & 1. $22 \mathrm{E}-13$ & $1.22 \mathrm{E}-13$ & $1.21 \mathrm{E}-13$ & $1.19 \mathrm{E}-13$ & $1.17 \mathrm{E}-13$ & $E-13$ & 1.0 & $9.56 \mathrm{E}-14$ & & & $0 E-14$ \\
\hline v 52 & $1.19 \mathrm{E}-10$ & $1.19 \mathrm{E}-10$ & $1.19 \mathrm{E}-10$ & $1.16 \mathrm{E}-10$ & $2 \mathrm{E}-10$ & $E-10$ & E-11 & $4 \mathrm{E}-11$ & & $8 E-11$ & $6 E-12$ \\
\hline v 53 & $3.63 \mathrm{E}-12$ & $3.63 E-12$ & $3.60 \mathrm{E}-12$ & $3.38 \mathrm{E}-12$ & $4 \mathrm{E}-12$ & $2.92 \mathrm{E}-12$ & 2.3 & $1.53 E-12$ & E-13 & $9 E-14$ & $6.61 \mathrm{E}-16$ \\
\hline v 54 & $6.69 E-14$ & 6.6 & $6.59 \mathrm{~F}-14$ & $5.82 \mathrm{E}-14$ & $5.06 \mathrm{E}-14$ & 4. $40 F-14$ & & $E-14$ & & & -21 \\
\hline cr 51 & $1.43 \mathrm{E}-13$ & 1.43 & $1.43 \mathrm{E}-13$ & $1.43 \mathrm{E}-13$ & $1.43 \mathrm{E}-13$ & $1.43 E-13$ & $1.43 \mathrm{E}-13$ & $1.43 \mathrm{E}-13$ & $E-13$ & $E-13$ & $E-13$ \\
\hline cr 55 & 1.7 & & 1.7 & $1.67 \mathrm{E}-11$ & 1. & -11 & 1. & -11 & 6 . & & -13 \\
\hline $\operatorname{mn} 54$ & 3.35 & 3.3 & 3.3 & $5 E-13$ & -13 & 3. & & -13 & & -13 & $:-13$ \\
\hline $\mathrm{mn} 56$ & 2.10 & 2.1 & 2.1 & $E-10$ & 2.1 & 2 . & & -10 & -10 & -10 & -10 \\
\hline $\mathrm{mn} 57$ & 2.1 & & 2.1 & 2.0 & 1.8 & & & & & -12 & -14 \\
\hline $\mathrm{mn} 58$ & 9.59 & 9.5 & 9.4 & $3 E-13$ & 7.76 & 6.9 & 5. & $E-13$ & -14 & $4 E-15$ & -18 \\
\hline fe 55 & 1.9 & & 1.9 & 1.9 & & & & & & & \\
\hline fe 59 & 1.32 & -15 & 1.3 & 1. $32 \mathrm{E}-15$ & -15 & -15 & 1. & $2 E-1.5$ & & -15 & -15 \\
\hline co 58 & 7.2 & & 7.2 & 7.2 & & & & & & $E-14$ & $E-14$ \\
\hline co 60 & 3.75 & -17 & 3.7 & $3.75 E-17$ & & 3. & & 3. & 3. & $E-17$ & E-17 \\
\hline co 61 & $1.88 \mathrm{E}-11$ & $1.88 \mathrm{E}-14$ & 1.88 & $1.88 E-14$ & $1.8^{\circ}$ & 1. & 1.8 & $\mathrm{~g}-14$ & $E-14$ & $E-11$ & E-14 \\
\hline co 62 & $3.33 \mathrm{E}-12$ & $3.33 \mathrm{E}-12$ & $3.3 c$ & $3.08 \mathrm{E}-12$ & 2.85 & 2.6 & 2.1 & 1. $32 \mathrm{E}-12$ & 3.30 & $E-14$ & $3 E-16$ \\
\hline ni & $\mathrm{E}-17$ & E-17 & 1.32 & $1.32 \mathrm{E}-17$ & 1. $32 \mathrm{E}-17$ & 1. $32 \mathrm{E}-17$ & & 1. $32 \mathrm{E}-17$ & 1. $32 \mathrm{E}-17$ & $2 F-17$ & 1. 32. F-17 \\
\hline ni & $8.40 \mathrm{E}-14$ & 8.40 & 8.4 & 8.3 & & & & & & & \\
\hline $\mathrm{Cu}$ & 3.1 & & 3.1 & -13 & -13 & & 3.1 & $3.18 \mathrm{E}-13$ & & $5-13$ & -13 \\
\hline $\mathrm{cu}$ & $9.12 \mathrm{E}-12$ & & 9.1 & 8.9 & & & & & & & $z-13$ \\
\hline & $6.44 \mathrm{E}-18$ & & & 1.2 & 1.6 & 1.8 & 2.1 & 2.31 & & E-17 & $\Xi-17$ \\
\hline $\mathrm{zr}$ & $2.32 \mathrm{E}-17$ & $2.32 \mathrm{E}-17$ & 2.32 & $2.32 \mathrm{E}-17$ & $2.32 \mathrm{E}-17$ & 2. $32 \mathrm{E}-17$ & $2.32 \mathrm{E}-17$ & $2.32 \mathrm{E}-17$ & & $2.32 \mathrm{E}-17$ & $2.31 \mathrm{E}-17$ \\
\hline $2 r$ & $4.09 \mathrm{E}-18$ & $4.09 \mathrm{E}-18$ & $4.09 \mathrm{E}-18$ & $4.09 \mathrm{E}-18$ & $4.09 \mathrm{E}-18$ & $4.09 \mathrm{E}-18$ & $4.09 \mathrm{E}-18$ & $4.09 \mathrm{E}-18$ & $4.09 E-18$ & $4.09 \mathrm{E}-18$ & $4.09 \mathrm{E}-18$ \\
\hline zr & $8.53 E-18$ & $8.53 E-18$ & $8.53 \mathrm{E}-18$ & $8.53 E-18$ & $8.53 E-18$ & $8.53 \mathrm{E}-18$ & $8.52 \mathrm{E}-18$ & $8.52 E-18$ & $8.50 E-18$ & $8.47 \mathrm{E}-18$ & $8.41 \mathrm{E}-18$ \\
\hline$n b$ & $9.75 \mathrm{E}-18$ & & $9.75 E-18$ & $9.75 \mathrm{E}-18$ & $9.75 \mathrm{E}-18$ & $9.75 E-18$ & $9.75 \mathrm{E}-18$ & $9.75 E-18$ & & $9.75 \mathrm{E}-18$ & $9.75 \mathrm{E}-18$ \\
\hline nb & $5.37 \mathrm{E}-16$ & $5.37 E-16$ & E-16 & $5.37 E-16$ & $5.37 \mathrm{E}-16$ & $5.37 \mathrm{E}-16$ & $5.37 \mathrm{E}-16$ & $5.37 \mathrm{E}-1$ & $5.36 \mathrm{E}-16$ & $5.34 E-16$ & $5.32 \mathrm{E}-16$ \\
\hline 97 & $4.10 \mathrm{E}-15$ & & & $4.09 \mathrm{E}-15$ & & & & & & $3.73 E-15$ & $3.39 \mathrm{E}-15$ \\
\hline 97 & $7.03 \mathrm{E}-19$ & $7.03 E-19$ & $7.88 \mathrm{E}-19$ & $1.51 \mathrm{E}-18$ & $2.23 E-18$ & $2.87 \mathrm{E}-18$ & 1. $39 E-18$ & $6.21 \mathrm{E}-18$ & $7.81 \mathrm{E}-18$ & $8.03 E-18$ & $7.98 E-18$ \\
\hline
\end{tabular}



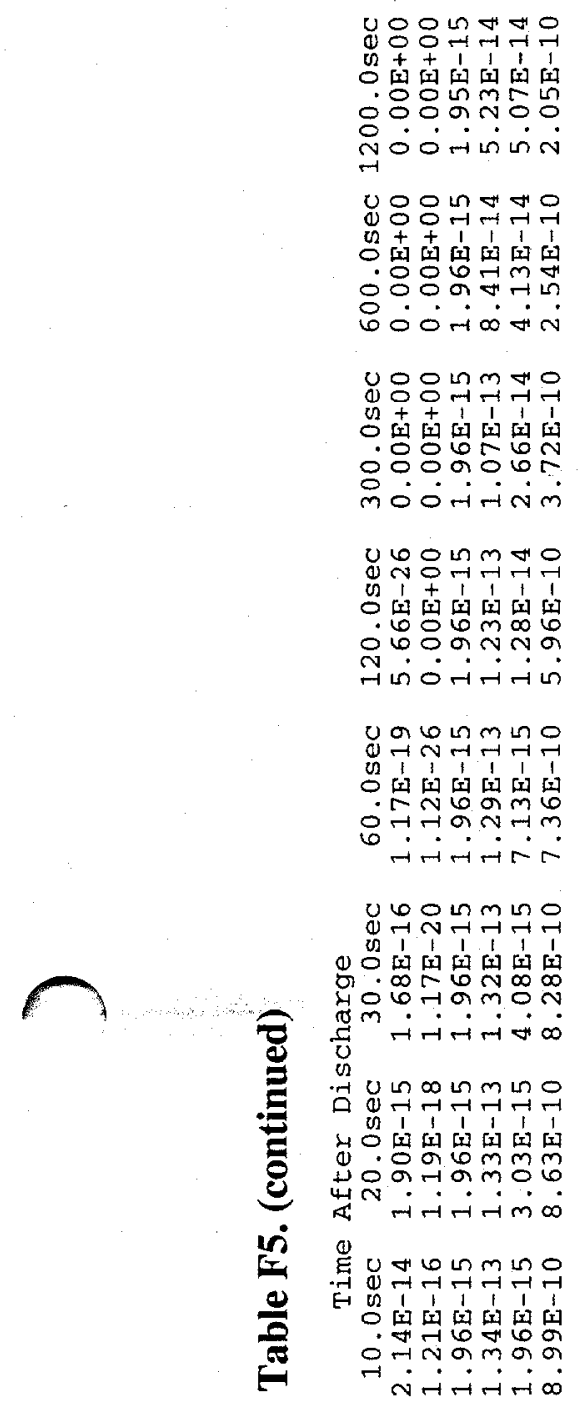

U

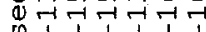

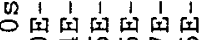

- कर $\mathrm{m} \infty \mathrm{m}$ तirनiकo

um 6 $\begin{array}{lllll}1 & 1 & 1 & 1 & 1 \\ 1 & 1\end{array}$

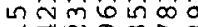
In 페 $n m \infty$ - $\begin{array}{lllll}1 & 1 & 1 & 1 & 1\end{array}$

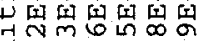
\#ก 0 लनंत्मक

क염ㄷㅁ

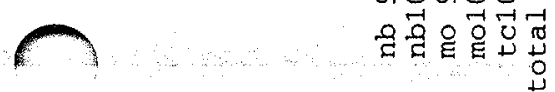


Table F6. Radioactivity (Ci) After 24-s Irradiation of 1-kg Ball Bearings Cargo (Flux Parameter Set 1).

\begin{tabular}{|c|c|c|c|c|c|c|c|c|c|c|c|}
\hline & & & & & $n i$ & harro & & & & & \\
\hline & initial & $1 \mathrm{E}-15 \mathrm{sec}$ & 1.0 sed & $10.0 \mathrm{sec}$ & $20.0 \mathrm{sec}$ & $30.0 \mathrm{sec}$ & $60.0 \mathrm{sec}$ & $120.0 \mathrm{sec}$ & $300.0 \mathrm{sec}$ & $600.0 \mathrm{sec}$ & $1200.0 \mathrm{sec}$ \\
\hline b 12 & $3.12 \mathrm{E}-18$ & $3.12 \mathrm{E}-18$ & $2.83 \mathrm{E}-18$ & 1. $18 \mathrm{E}-18$ & $4.47 \mathrm{E}-19$ & $1.69 \mathrm{E}-19$ & $9.15 \mathrm{E}-21$ & $2.68 E-23$ & $7.83 E-31$ & $0.00 \mathrm{E}+00$ & $0.00 \mathrm{E}+00$ \\
\hline C 15 & $3.53 \mathrm{E}-14$ & $3.53 \mathrm{E}-14$ & 2. $66 \mathrm{E}-14$ & $2.08 \mathrm{E}-15$ & $1.23 \mathrm{E}-16$ & $7.24 \mathrm{E}-18$ & $1.49 \mathrm{E}-21$ & $6.27 E-29$ & $0.00 \mathrm{E}+00$ & $0.00 \mathrm{E}+00$ & $0.00 \mathrm{E}+00$ \\
\hline n 16 & $2.59 \mathrm{E}-13$ & $2.59 \mathrm{E}-13$ & $2.35 \mathrm{E}-13$ & $9.81 \mathrm{E}-14$ & & $1.40 \mathrm{E}-14$ & $7.60 \mathrm{E}-16$ & $2.23 E-18$ & $5.60 \mathrm{E}-26$ & $0.00 \mathrm{E}+00$ & $0.00 E+00$ \\
\hline - 19 & $2.59 \mathrm{E}-18$ & $2.59 \mathrm{E}-18$ & $2.53 E-18$ & $2.00 \mathrm{E}-18$ & $1.55 \mathrm{E}-18$ & 1. $20 \mathrm{E}-18$ & $5.52 \mathrm{E}-19$ & $1.18 \mathrm{E}-19$ & 1. $14 \mathrm{E}-21$ & $5.03 E-25$ & $9.76 \mathrm{E}-32$ \\
\hline na 24 & $4.66 \mathrm{E}-14$ & $4.66 \mathrm{E}-14$ & $4.66 \mathrm{E}-14$ & $4.66 \mathrm{E}-14$ & $4.66 E-14$ & $4.66 \mathrm{E}-14$ & $4.66 \mathrm{E}-14$ & $4.66 E-14$ & $4.64 \mathrm{E}-14$ & $4.63 \mathrm{E}-14$ & $4.59 \mathrm{E}-14$ \\
\hline mg 27 & $2.05 E-11$ & $2.05 E-11$ & $2.05 E-11$ & $2.03 E-11$ & $2.00 \mathrm{E}-11$ & $1.98 \mathrm{E}-11$ & $1.91 \mathrm{E}-11$ & $1.77 \mathrm{E}-11$ & 1. $42 \mathrm{E}-11$ & $9.86 \mathrm{E}-12$ & $4.74 \mathrm{E}-12$ \\
\hline al 28 & $4.89 E-10$ & $4.89 E-10$ & $4.86 \mathrm{E}-10$ & $4.64 E-10$ & $4.41 \mathrm{E}-10$ & $4.19 \mathrm{E}-10$ & $3.59 \mathrm{E}-10$ & $2.63 \mathrm{E}-10$ & $1.04 \mathrm{E}-10$ & $2.21 E-11$ & $1.00 \mathrm{E}-12$ \\
\hline al 29 & $5.71 \mathrm{E}-12$ & $5.71 \mathrm{E}-12$ & $5.70 E-12$ & $5.61 F-12$ & $5.52 \mathrm{E}-12$ & $5.42 \mathrm{E}-12$ & 5. $14 \mathrm{~F}-12$ & $4.63 F-12$ & $3.37 \mathrm{~F}-12$ & $1.99 \mathrm{~F}-12$ & $6.92 \mathrm{E}-13$ \\
\hline a 30 & 1. $33 \mathrm{E}-13$ & $1.33 E-13$ & $1.10 \mathrm{E}-13$ & $1.94 \mathrm{E}-14$ & $2.82 \mathrm{E}-15$ & $4.12 \mathrm{E}-16$ & $1.28 \mathrm{E}-18$ & $1.23 \mathrm{E}-23$ & $0.00 \mathrm{E}+00$ & $0.00 \mathrm{E}+00$ & $00 E+00$ \\
\hline si 31 & $5.44 \mathrm{E}-12$ & & $5.44 \mathrm{E}-12$ & & & $5.42 \mathrm{E}-12$ & $5.41 \mathrm{E}-12$ & $5.39 E-12$ & $5.32 \mathrm{E}-12$ & $5.20 E-12$ & $8 E-12$ \\
\hline p 32 & $4.44 \mathrm{E}-14$ & $4.44 \mathrm{E}-14$ & $4.44 \mathrm{E}-14$ & $4.44 \mathrm{E}-14$ & $4.44 \mathrm{E}-14$ & $4.44 \mathrm{E}-14$ & $4.44 \mathrm{E}-14$ & $4.44 \mathrm{E}-14$ & $4.44 \mathrm{E}-14$ & $4.44 E-14$ & $4.44 \mathrm{E}-14$ \\
\hline p 33 & $2.13 E-13$ & & $2.13 \mathrm{E}-13$ & & & $2.13 \mathrm{E}-13$ & $2.13 \mathrm{E}-13$ & & & & $2.13 E-13$ \\
\hline p 34 & $1.13 E-12$ & 1. $13 \mathrm{E}-12$ & $1.06 \mathrm{E}-12$ & $6.44 E-13$ & $9 E-13$ & 2.11E-13 & $3.96 \mathrm{E}-14$ & $1.40 \mathrm{E}-15$ & $6.11 \mathrm{E}-20$ & $1 E-27$ & $\mathrm{E}+00$ \\
\hline s 35 & $5.48 \mathrm{E}-19$ & $5.48 \mathrm{E}-19$ & $5.48 \mathrm{E}-19$ & $5.48 \mathrm{E}-19$ & $5.48 \mathrm{E}-19$ & $5.48 \mathrm{E}-19$ & $5.48 \mathrm{E}-19$ & $5.48 \mathrm{E}-19$ & $5.48 \mathrm{E}-19$ & & E-19 \\
\hline s 37 & $3.35 E-1 \%$ & & $3.35 \mathrm{E}-17$ & $3.28 \mathrm{E}-17$ & $3.20 \mathrm{E}-17$ & $3.13 E-17$ & $2.92 \mathrm{E}-17$ & $2.55 \mathrm{E}-17$ & $1.69 \mathrm{E}-17$ & $8.50 \mathrm{E}-18$ & $E-18$ \\
\hline ti 51 & $1.81 \mathrm{E}-13$ & $1.81 \mathrm{E}-13$ & $1.81 \mathrm{E}-13$ & $1.77 \mathrm{E}-13$ & $1.74 \mathrm{E}-13$ & $1.70 \mathrm{E}-13$ & $1.60 \mathrm{E}-13$ & $1.42 \mathrm{E}-13$ & $9.92 \mathrm{E}-14$ & $5.43 \mathrm{E}-14$ & $1.63 \mathrm{E}-14$ \\
\hline v 52 & $1.77 \mathrm{E}-10$ & & 1. $76 \mathrm{E}-10$ & $1.71 \mathrm{E}-10$ & & $1.61 \mathrm{E}-10$ & -10 & $1.22 \mathrm{E}-10$ & $7.01 \mathrm{E}-11$ & & -12 \\
\hline v 53 & $5.29 \mathrm{E}-12$ & 5.2 & $5.25 E-12$ & $4.92 \mathrm{E}-12$ & $4.58 \mathrm{E}-12$ & $4.27 \mathrm{E}-12$ & $3.44 \mathrm{E}-12$ & $2.24 E-12$ & $6.15 E-13$ & $7.14 \mathrm{E}-14$ & E-16 \\
\hline V 54 & 9.5 & & & $8 E-14$ & -14 & -14 & -14 & $9 \mathrm{E}-14$ & -15 & -17 & -21 \\
\hline $\operatorname{cr} 51$ & -13 & -13 & -13 & -13 & -13 & -13 & -13 & -13 & -13 & -13 & -13 \\
\hline cr $5 \overline{5}$ & -11 & & 2.5 & 2. $48 F-11$ & 2.40 & $2.32 F-11$ & -11 & -11 & 9. & -12 & -13 \\
\hline $\mathrm{mn} 54$ & 5.0 & & 5.0 & $2 E-13$ & 5.0 & -13 & 5.0 & -13 & & & -13 \\
\hline$m n 56$ & $E-10$ & & & 3.1 & & 3.14 & 3.1 & -10 & & & -10 \\
\hline $\mathrm{mn} 57$ & 3.1 & & & $E-10$ & 2.7 & 2.5 & 1.9 & & & & -14 \\
\hline $\mathrm{mn} 58$ & -12 & & -12 & 1.2 & 1.1 & 1.0 & 7.3 & 3.86 & & & $E-18$ \\
\hline fe 55 & & & & & & & & & & & -15 \\
\hline fe 59 & $\mathrm{E}-15$ & 1.9 & $8 E-15$ & $1.98 \mathrm{E}-15$ & 1.98 & $1.98 \mathrm{E}-15$ & 1.9 & $E-15$ & 1. & -15 & $8 E-15$ \\
\hline co 58 & $1.09 \mathrm{E}-13$ & 1.0 & $1.09 E-13$ & 1.09 & 1.09 & $1.09 \mathrm{E}-13$ & -13 & -13 & 1. & 1. & -13 \\
\hline CO 60 & 5.63 & & 5.6 & $5.63 \mathrm{E}-17$ & $E-17$ & $5.63 \mathrm{E}-17$ & -17 & -17 & & & -17 \\
\hline co 61 & 2.82 & 2.8 & 2.8 & $2.81 \mathrm{E}-14$ & -14 & $2.81 \mathrm{E}-14$ & -14 & -14 & -14 & -14 & -14 \\
\hline Co 62 & $4.85 \mathrm{E}$ & 4.8 & 4.8 & -12 & 4.1 & & $E-12$ & & & & \\
\hline ni 63 & 1.9 & 1.5 & 1.9 & -17 & $E-17$ & $1.98 \mathrm{E}-17$ & $E-17$ & 1.9 & 1. & & -17 \\
\hline ni 65 & 1.26 & 1.2 & & & & & & & & & -13 \\
\hline $\mathrm{cu} \quad 64$ & 4.78 & & 4.7 & $4.78 \mathrm{~F}-13$ & $E-13$ & $4.78 \mathrm{~F}-13$ & 4.7 & -13 & 4.78 & $E-13$ & $O E-13$ \\
\hline cu 66 & $1.36 \mathrm{E}-11$ & 1. $36 \mathrm{E}-11$ & 1.3 & $1.33 E-11$ & $E-11$ & 1. $27 \mathrm{E}-11$ & 1.18 & & & -12 & $5 E-13$ \\
\hline$y 89 \mathrm{~m}$ & $1.31 \mathrm{E}-17$ & $1.31 \mathrm{E}-17$ & $1.40 \mathrm{E}-17$ & $2.07 \mathrm{E}-17$ & $2.56 \mathrm{E}-17$ & $2.88 \mathrm{E}-17$ & 3.31 & 3.4 & 3.4 & -17 & $3.47 \mathrm{E}-17$ \\
\hline $\operatorname{zr} 89$ & $3.48 \mathrm{E}-17$ & $3.48 \mathrm{E}-17$ & $3.48 \mathrm{E}-17$ & $3.48 E-17$ & $3.48 \mathrm{E}-17$ & $3.48 \mathrm{E}-17$ & $3.48 \mathrm{E}-17$ & $3.48 \mathrm{E}-17$ & $3.48 \mathrm{E}-17$ & $3.48 \mathrm{E}-17$ & $3.47 \mathrm{E}-17$ \\
\hline zr 95 & $6.14 \mathrm{E}-18$ & $6.14 \mathrm{E}-18$ & & $6.14 \mathrm{E}-18$ & $6.14 \mathrm{E}-18$ & $6.14 \mathrm{E}-18$ & $6.14 \mathrm{E}-18$ & $6.14 \mathrm{E}-18$ & & & $6.13 \mathrm{E}-18$ \\
\hline $\operatorname{lr} 97$ & 1. $28 \mathrm{E}-17$ & & & $1.28 \mathrm{E}-17$ & 1. $28 \mathrm{E}-17$ & 1. $28 \mathrm{E}-17$ & 1. $28 \mathrm{E}-17$ & 1. $28 \mathrm{E}-17$ & & & E-17 \\
\hline $\mathrm{nb} 95$ & 1. $46 \mathrm{E}-17$ & $1.46 \mathrm{E}-17$ & $1.46 \mathrm{E}-17$ & $1.46 \mathrm{E}-17$ & $1.46 \mathrm{E}-17$ & $1.46 \mathrm{E}-17$ & 1. $46 \mathrm{E}-17$ & $1.46 \mathrm{E}-17$ & 1.4 & 1.46 & 1. $46 \mathrm{E}-17$ \\
\hline $\mathrm{nb}$ & $8.06 \mathrm{E}-16$ & 8.06 & 8.06 & $8.06 E-16$ & $8.06 \mathrm{E}-16$ & $8.05 E-16$ & $8.05 E-16$ & $8.05 t-16$ & 8.0 & 8.02 & $7.98 E-16$ \\
\hline$n b$ & $6.15 E-15$ & $6.15 E-15$ & $6.15 \mathrm{E}-15$ & $6.14 E-15$ & $6.13 E-15$ & $6.12 \mathrm{E}-15$ & $6.09 E-15$ & & & & $5.07 \mathrm{E}-15$ \\
\hline nb & $1.54 \mathrm{E}-18$ & $1.54 \mathrm{E}-18$ & 1. $66 \mathrm{E}-18$ & $2.69 \mathrm{E}-18$ & 3. $72 E-18$ & $4.64 \mathrm{E}-18$ & $6.83 E-18$ & $9.47 \mathrm{E}-18$ & 1. $18 \mathrm{E}-17$ & 1. $20 \mathrm{E}-17$ & $1.20 \mathrm{E}-17$ \\
\hline
\end{tabular}




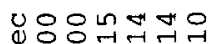

n

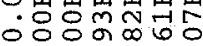
róoritim

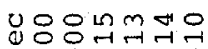

v +1111 요윰ำ ठ००णनंधि

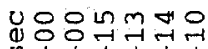

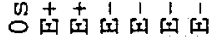

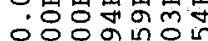
लि0ंसंता

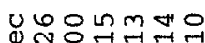

ह1

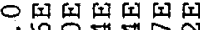
눙요에

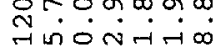

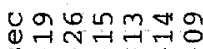

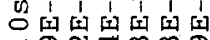

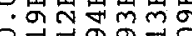

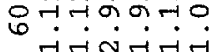

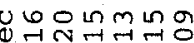
यू को सो सो

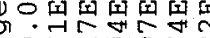

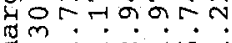

ठु马ु

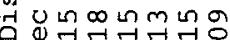
on

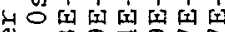

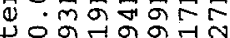

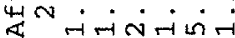

๑ \& y

Q

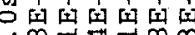

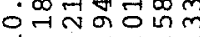
तंजinimi

UMเกนกนก

बुन

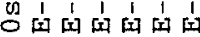

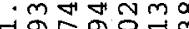
नiिंत्रि

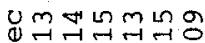
का 1111111

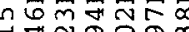
जित्रत्र म

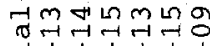

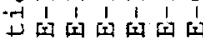

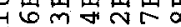
द तूOOO

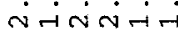

응엉두

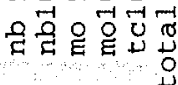


Table F7. Radioactivity (Ci) After 8-s Irradiation of 1-kg Surgical Implant Composition A Cargo (Flux Parameter Set 1).

\begin{tabular}{|c|c|c|c|c|c|c|c|c|c|c|c|c|}
\hline & & & & & & & & & & & & \\
\hline & & initial & $1 \mathrm{E}-15 \mathrm{sec}$ & $1.0 \mathrm{sec}$ & $10.0 \mathrm{sec}$ & $20.0 \mathrm{sec}$ & $30.0 \mathrm{sec}$ & $60.0 \mathrm{sec}$ & $120.0 \mathrm{sec}$ & $300.0 \mathrm{sec}$ & $600.0 \mathrm{sec}$ & 1200 \\
\hline $1 i$ & 8 & $3.80 \mathrm{E}-12$ & $3.80 \mathrm{E}-12$ & 1. $66 \mathrm{E}-12$ & $9.73 E-16$ & $2.49 E-19$ & $6.36 \mathrm{E}-23$ & $0.00 \mathrm{E}+00$ & $0.00 \mathrm{E}+00$ & $0.00 \mathrm{E}+00$ & $0.00 \mathrm{E}+00$ & \\
\hline & 8 & $3.80 \mathrm{E}-12$ & $3.80 \mathrm{E}-12$ & $1.66 \mathrm{E} \quad 12$ & & $2.49 E-19$ & $6.36 \mathrm{E}-23$ & $0.00 \mathrm{E}+00$ & & $.00 \mathrm{E}+00$ & $0.00 \mathrm{E}+00$ & \\
\hline & 11 & $1.08 E-14$ & $1.08 \mathrm{E}-14$ & $1.03 E-14$ & $56 \mathrm{E}-15$ & $3.97 \mathrm{E}-15$ & $2.41 \mathrm{E}-15$ & $.34 E-16$ & $2.63 \mathrm{E}-17$ & $3.13 E-21$ & $9.04 \mathrm{E}-28$ & $00 \mathrm{E}+00$ \\
\hline b & 12 & $3.49 E-13$ & $3.49 E-13$ & & & & $2.91 \mathrm{E}-23$ & & & $.00 \mathrm{E}+00$ & & \\
\hline c & 14 & $1.81 \mathrm{E}-18$ & $1.81 \mathrm{E}-18$ & $1.81 \mathrm{E}-18$ & $1.81 \mathrm{E}-18$ & $1.81 \mathrm{E}-18$ & $1.81 \mathrm{E}-18$ & $1.81 \mathrm{E}-18$ & $1.81 \mathrm{E}-18$ & $1.81 E-18$ & $1.81 \mathrm{E}-18$ & $1.81 \mathrm{E}-18$ \\
\hline $\mathrm{c}$ & 15 & & & & & & & & & & $0.00 \mathrm{E}+00$ & \\
\hline $\mathrm{n}$ & 16 & $4.47 E-17$ & $4.47 \mathrm{E}-17$ & $4.05 E-1.7$ & & $39 E-18$ & $2.42 \mathrm{E}-18$ & $1.31 \mathrm{E}-19$ & & $9.64 \mathrm{E}-30$ & $0.00 \mathrm{E}+00$ & $E+00$ \\
\hline$n$ & 24 & $E-14$ & $E-14$ & 9.7 & $9.33 \mathrm{E}-14$ & -14 & $2 \mathrm{E}-14$ & 9. & & & & \\
\hline & 27 & 4.1 & & & & & & & & & & \\
\hline & 28 & & & 6. & & & & & & & & \\
\hline & 29 & & & & & & & & & & & \\
\hline al & 30 & 4.2 & & & & & & & & & & \\
\hline si & 31 & 1.4 & -12 & -12 & E-12 & & -12 & & & & & \\
\hline $\mathrm{p}$ & 32 & 9.8 & & 9.8 & $E-15$ & & & & & & & \\
\hline $\mathrm{p}$ & 33 & 4.7 & & 4. & & & & & & & & \\
\hline $\mathrm{p}$ & 34 & 3.6 & & & & & & & & & & \\
\hline $\mathrm{s}$ & 35 & 1.2 & & & & & & & & & & \\
\hline $\mathrm{s}$ & 37 & 7.5 & & 7. & & & & & & & & \\
\hline$t i$ & 51 & 1.2 & & 1.2 & & & & & & & & \\
\hline $\mathrm{v}$ & 52 & 1.1 & & & & & & & & & & \\
\hline $\mathrm{v}$ & 53 & & & & & & & & & & & \\
\hline $\mathrm{v}$ & 54 & 7.0 & & 6. & -13 & & & & & & & \\
\hline $\mathrm{cr}$ & 51 & 6.0 & & & & & & & & & & \\
\hline & 55 & 3.1 & & & & & & & & & & \\
\hline $\mathrm{mn}$ & 54 & 1.3 & & & & & & & & & & \\
\hline & 56 & 1.2 & & & & & & & & & & \\
\hline & 57 & 8.7 & & .7 & & & 6. & & & & & \\
\hline & 58 & & & & & & & & & & & \\
\hline $\mathrm{fe}$ & 55 & 2.8 & & .8 & 2. & & & & & & & \\
\hline $\mathrm{fe}$ & 59 & & & & & & & & & & & \\
\hline co & 58 & & & & 1. & & & & & & & \\
\hline co & 60 & & & & & & & & & & & \\
\hline co & $60 \mathrm{~m}$ & & & & & & & & & & & \\
\hline $\mathrm{co}$ & 61 & & & & & & & & & & & \\
\hline $\mathrm{CO}$ & 62 & & & & & & & & & & & \\
\hline ni & 63 & & & & & & & & & & & \\
\hline ni & 65 & $E-14$ & 1.0 & 1.01 & $1.01 \mathrm{E}-14$ & 1.0 & 1.01 & & & & & \\
\hline$y$ & $89 m$ & & & & & & & & & & & \\
\hline $\mathrm{zr}$ & 89 & & & & & & & & & & & -16 \\
\hline $\mathrm{zr}$ & 95 & & & & $1.43 \mathrm{E}-16$ & & & & & & & \\
\hline $2 r$ & 97 & & & & E- 16 & & & & & & & \\
\hline $\mathrm{nb}$ & 95 & $3.41 \mathrm{E}-16$ & & 3.41 & $3.41 \mathrm{E}-16$ & & $3.41 \mathrm{E}-1$ & 3.4 & $3.41 \mathrm{E}-16$ & $E-16$ & & $3.41 \mathrm{E}-16$ \\
\hline $\mathrm{nb}$ & 96 & 1. $88 \mathrm{E}-14$ & & & $1.88 \mathrm{E}-14$ & & $1.88 \mathrm{E}-14$ & $1.88 \mathrm{E}-14$ & & $1.88 \mathrm{E}-11$ & $1.87 \mathrm{E}-14$ & $1.86 \mathrm{E}-14$ \\
\hline
\end{tabular}


Table F7. (continued)

\begin{tabular}{|c|c|c|c|c|c|c|c|c|c|c|c|}
\hline & & & & Time & After D1 & harge & & & & & \\
\hline & initial & $1 \mathrm{E}-15 \mathrm{sec}$ & $1.0 \mathrm{sec}$ & $10.0 \mathrm{sec}$ & $20.0 \mathrm{sec}$ & $30.0 \mathrm{sec}$ & $60.0 \mathrm{sec}$ & $120.0 \mathrm{sec}$ & $300.0 \mathrm{sec}$ & $600.0 \mathrm{sec}$ & $1200.0 \mathrm{sec}$ \\
\hline nb 97 & $1.44 \mathrm{E}-13$ & 1. $44 \mathrm{E}-13$ & $1.44 \mathrm{E}-13$ & $1.43 \mathrm{E}-13$ & 1. $43 \mathrm{E}-13$ & $1.43 \mathrm{E}-13$ & $1.42 \mathrm{E}-13$ & $1.41 \mathrm{E}-13$ & $1.37 \mathrm{E}-13$ & $1.30 E-13$ & $1.19 \mathrm{E}-13$ \\
\hline $\mathrm{nb} 97 \mathrm{~m}$ & $1.27 \mathrm{E}-17$ & $1.27 \mathrm{E}-17$ & $1.58 \mathrm{E}-17$ & $4.22 \mathrm{E}-17$ & $6.85 \mathrm{E}-17$ & -17 & $1.48 \mathrm{E}-16$ & $2.15 \mathrm{E}-16$ & $2.74 \mathrm{E}-16$ & $1 E-16$ & $9 E-16$ \\
\hline & $1.48 \mathrm{E}-11$ & $1.48 \mathrm{E}-11$ & $1.16 \mathrm{E}-11$ & $E-12$ & $6 \mathrm{E}-13$ & $3 \mathrm{E}-14$ & $7.16 \mathrm{E}-18$ & & $0.00 \mathrm{E}+00$ & $0 E+00$ & $0 E+00$ \\
\hline 100 & -13 & -13 & 5.2 & & & 8. & & & & & \\
\hline 99 & 6.8 & -14 & 6.87 & -14 & & 6.8 & & & & & -14 \\
\hline 101 & -12 & 4.75 & 4.74 & -12 & & 4.6 & & & 3. & & 1. \\
\hline tc101 & $1.54 \mathrm{E}-14$ & $1.54 \mathrm{E}-14$ & $1.93 \mathrm{E}-14$ & $E-14$ & 9.1 & $:-13$ & $E-13$ & & $E-13$ & -12 & -12 \\
\hline w181 & $4.01 E-18$ & $4.01 \mathrm{E}-18$ & 4.01 & 4.0 & & & & & 4. & & \\
\hline & $1.30 \mathrm{E}-16$ & $1.30 \mathrm{E}-16$ & $1.30 \mathrm{E}-16$ & $E-16$ & $E-16$ & $0 E-16$ & 1. $30 \mathrm{E}-16$ & DE-16 & $1.30 \mathrm{E}-16$ & $0 \mathrm{E}-16$ & 1. $30 \mathrm{E}-16$ \\
\hline w185m & 9.0 & -15 & -15 & E-15 & & 7. & & & -15 & & $E-18$ \\
\hline w187 & $2.99 \mathrm{E}-13$ & $2.99 \mathrm{E}-13$ & -13 & $E-13$ & $2.99 \mathrm{E}-13$ & $2.99 \mathrm{E}-13$ & -13 & $2.99 \mathrm{E}-13$ & $2.99 \mathrm{E}-13$ & $2-13$ & $2.96 \mathrm{E}-13$ \\
\hline tal & $2.03 E-08$ & $2.03 \mathrm{E}-08$ & $2.02 \mathrm{E}-08$ & $2.00 \mathrm{E}-08$ & $1.97 \mathrm{E}-08$ & $1.94 \mathrm{E}-08$ & $1.87 E-08$ & $1.73 \mathrm{E}-08$ & $1.38 E-08$ & $9.68 \mathrm{E}-09$ & $4.92 \mathrm{E}-09$ \\
\hline
\end{tabular}


Table F8. Radioactivity (Ci) After 16-s Irradiation of 1-kg Surgical Implant Composition A Cargo (Flux Parameter Set 1).

\begin{tabular}{|c|c|c|c|c|c|c|c|c|c|c|c|c|}
\hline & & & & & & & & & & & & \\
\hline & & initial & $1 \mathrm{E}-15 \mathrm{sec}$ & $1.0 \mathrm{sec}$ & $10.0 \mathrm{sec}$ & $20.0 \mathrm{sec}$ & $30.0 \mathrm{sec}$ & $60.0 \mathrm{sec}$ & $120.0 \mathrm{sec}$ & $300.0 \mathrm{sec}$ & $600.0 \mathrm{sec}$ & $1200.0 \mathrm{sec}$ \\
\hline & 8 & & & $1.67 \mathrm{E}-12$ & $9.74 \mathrm{E}-16$ & $2.49 \mathrm{E}-19$ & $6.37 \mathrm{E}-23$ & $0.00 \mathrm{E}+00$ & $0.00 \mathrm{E}+00$ & $0.00 \mathrm{E}+00$ & $0.00 \mathrm{E}+00$ & $00 \mathrm{E}+00$ \\
\hline & 8 & 3. $81 E-12$ & 3. $81 \mathrm{E}-12$ & $1.67 \mathrm{E}-12$ & $9.74 E-16$ & $2.49 \mathrm{E}-19$ & $6.37 \mathrm{E}-23$ & $0.00 \mathrm{E}+00$ & $0.00 \mathrm{E}+00$ & $0.00 \mathrm{E}+00$ & $0.00 \mathrm{E}+00$ & $0.00 \mathrm{E}+00$ \\
\hline & 11 & $1.81 \mathrm{E}-14$ & $1.81 \mathrm{E}-14$ & $1.72 \mathrm{E}-14$ & $1.10 \mathrm{E}-14$ & $6.63 E-15$ & $4.01 \mathrm{E}-15$ & $8.91 E-16$ & $4.38 \mathrm{E}-17$ & $5.23 E-21$ & $1.51 E-27$ & $0.00 \mathrm{E}+00$ \\
\hline & 12 & $3.49 \mathrm{E}-13$ & $3.49 \mathrm{E}-13$ & $7.12 \mathrm{E}-22$ & $2.97 \mathrm{E}-22$ & I. $12 \mathrm{E}-22$ & $4.25 E-23$ & $2.30 E-24$ & $6.73 E-27$ & $0.00 \mathrm{E}+00$ & $0.00 \mathrm{E}+00$ & $0.00 \mathrm{E}+00$ \\
\hline c & 14 & 3. $62 \mathrm{E}-18$ & $3.62 \mathrm{E}-18$ & 3. $62 \mathrm{E}-18$ & $3.62 \mathrm{E}-18$ & 3. $62 \mathrm{E}-18$ & 3. $62 \mathrm{E}-18$ & $3.62 \mathrm{E}-18$ & $3.62 \mathrm{E}-18$ & 3. $62 \mathrm{E}-18$ & 3. $62 \mathrm{E}-18$ & $3.62 \mathrm{E}-18$ \\
\hline & 15 & $1.55 E-14$ & $1.55 \mathrm{E}-14$ & $1.17 \mathrm{E}-14$ & $9.16 E-16$ & $5.40 \mathrm{E}-17$ & $3.19 \mathrm{E}-18$ & $6.54 \mathrm{E}-22$ & $2.76 \mathrm{E}-29$ & $0.00 \mathrm{E}+00$ & $0.00 \mathrm{E}+00$ & $0.00 \mathrm{E}+00$ \\
\hline $\mathrm{n}$ & 16 & $6.52 \mathrm{E}-17$ & $6.52 \mathrm{E}-17$ & $5.91 \mathrm{E}-17$ & $2.47 \mathrm{E}-17$ & $9.33 \mathrm{E}-18$ & $3.53 \mathrm{E}-18$ & $1.91 \mathrm{E}-19$ & $5.59 \mathrm{E}-22$ & 1. $41 \mathrm{E}-29$ & $0.00 \mathrm{E}+00$ & $0.00 \mathrm{E}+00$ \\
\hline & 24 & $1.87 \mathrm{E}-13$ & $1.87 \mathrm{E}-13$ & $1.87 E-13$ & $1.86 \mathrm{E}-13$ & $1.86 \mathrm{E}-13$ & $1.86 \mathrm{E}-13$ & $1.86 \mathrm{E}-13$ & $1.86 \mathrm{E}-13$ & $1.86 \mathrm{E}-13$ & $1.85 \mathrm{E}-13$ & $1.84 \mathrm{E}-13$ \\
\hline & 27 & $8: 23 E-11$ & $8.23 \mathrm{E}-11$ & $8.22 E-11$ & $8.13 \mathrm{E}-11$ & $8.04 \mathrm{E}-11$ & $7.94 \mathrm{E}-11$ & 7. $65 \mathrm{E}-11$ & $7.11 \mathrm{E}-11$ & $5.71 \mathrm{E}-11$ & $3.96 \mathrm{E}-11$ & $1.90 \mathrm{E}-11$ \\
\hline & 28 & $1.30 \mathrm{E}-09$ & $1.30 \mathrm{E}-09$ & $1.30 \mathrm{E}-09$ & $1.24 \mathrm{E}-09$ & $1.18 \mathrm{E}-09$ & $1.12 \mathrm{E}-09$ & $9.56 \mathrm{E}-10$ & $7.02 \mathrm{E}-10$ & $2.77 \mathrm{E}-10$ & $5.90 \mathrm{E}-11$ & $2.67 \mathrm{E}-12$ \\
\hline & 29 & $1.53 E-11$ & $1.53 \mathrm{E}-11$ & $1.53 \mathrm{E}-11$ & $1.51 \mathrm{E}-11$ & $1.48 \mathrm{E}-11$ & $1.46 \mathrm{E}-11$ & 1. $38 \mathrm{E}-11$ & $1.24 \mathrm{E}-11$ & $05 E-12$ & $5.34 \mathrm{E}-12$ & $1.86 \mathrm{E}-12$ \\
\hline & 30 & $5.12 \mathrm{E}-1.3$ & $5.12 \mathrm{E}-13$ & $4.22 \mathrm{E}-13$ & $7.47 \mathrm{E}-14$ & $1.09 \mathrm{E}-14$ & $1.59 \mathrm{E}-15$ & $4.92 E-18$ & $73 E-23$ & $00 E+00$ & $0.00 E+00$ & $00 E+00$ \\
\hline & 31 & $2.91 E-12$ & $2.91 \mathrm{E}-12$ & $2.91 E-12$ & $2.91 \mathrm{E}-12$ & $2.90 \mathrm{E}-12$ & $2.90 E-12$ & $2.90 \mathrm{E}-12$ & $88 E-12$ & $2.84 \mathrm{E}-12$ & -12 & $6 E-12$ \\
\hline & 32 & $1.98 \mathrm{E}-14$ & $1.98 \mathrm{E}-14$ & $1.98 \mathrm{E}-14$ & $1.98 \mathrm{E}-14$ & $1.98 \mathrm{E}-14$ & $1.98 \mathrm{E}-14$ & $1.98 \mathrm{E}-14$ & $1.98 \mathrm{E}-14$ & $1.98 \mathrm{E}-14$ & -14 & $E-14$ \\
\hline & 33 & $9.47 \mathrm{E}-14$ & $9.47 \mathrm{E}$ & $9.47 E-14$ & & & & 9.4. & -14 & -14 & -14 & -14 \\
\hline & 34 & $E-13$ & 6.0 & $5.68 \mathrm{E}-13$ & $3.44 \mathrm{E}-13$ & $E-13$ & $E-13$ & -14 & -16 & -20 & -27 & $E+00$ \\
\hline $\mathrm{s}$ & 35 & 2.43 & 2.4 & $2.43 E-19$ & $2.43 \mathrm{E}-19$ & & & & & & & \\
\hline & 37 & $1.50 \mathrm{E}-17$ & E-17 & $1.50 \mathrm{E}-17$ & $1.47 \mathrm{E}-17$ & 1. $44 \mathrm{E}-17$ & $0 \mathrm{E}-17$ & -17 & -17 & -18 & -18 & -19 \\
\hline$t i$ & 51 & $2.43 \mathrm{E}-12$ & $2.43 E-12$ & $2.43 E-12$ & $2.38 E-12$ & $2.34 \mathrm{E}-12$ & 2.2 & 2. & & & & -13 \\
\hline $\mathrm{v}$ & 52 & E-09 & E-09 & & & & & 1.9 & -09 & -10 & & -11 \\
\hline $\mathrm{v}$ & 53 & $7.25 \mathrm{E}-11$ & $5 E-11$ & $7.20 \mathrm{E}-11$ & $6.75 \mathrm{E}-11$ & 6.2 & 5. & 4.7 & -11 & & & -14 \\
\hline & 54 & 1. $34 \mathrm{E}-12$ & 1. $34 \mathrm{E}-12$ & 1. $32 \mathrm{E}-12$ & $1.16 \mathrm{E}-12$ & 1.0 & & & & & & -20 \\
\hline $\mathrm{cr}$ & 51 & E-13 & E-13 & 1. $21 \mathrm{E}-13$ & $1.21 \mathrm{E}-13$ & 1.2 & 1.2 & -13 & -13 & -13 & -13 & -13 \\
\hline $\mathrm{Cr}$ & 55 & $6.27 \mathrm{E}-11$ & 6.2 & $6.25 \mathrm{E}-11$ & $6.06 \mathrm{E}-11$ & 5.8 & 5.6 & -11 & -11 & -11 & -12 & -12 \\
\hline & 54 & 2.6 & & 2.611 & 2.6 & & & & -15 & & & -15 \\
\hline & 56 & $2.47 \mathrm{E}-11$ & -11 & $2.47 \mathrm{E}-11$ & $2.46 \mathrm{E}-11$ & 2.4 & 2.4 & 2. & -11 & -11 & -11 & $E-11$ \\
\hline & 57 & $1.70 \mathrm{E}$ & 1.7 & $1.69 \mathrm{E}-12$ & 1.5 & & & & & & & \\
\hline $\mathrm{mn}$ & 58 & $7.48 \mathrm{E}$ & & 7.40 & -15 & & 5.4 & -15 & -15 & -16 & & -20 \\
\hline & 55 & $5.63 \mathrm{E}-17$ & & $5.63 E-17$ & 5.6 & & & & & & & \\
\hline $\mathrm{fe}$ & 59 & $2.44 \mathrm{E}-17$ & & $2.44 \mathrm{E}-17$ & E-17 & 2.4 & 2.4 & 2.4 & -17 & -17 & & -17 \\
\hline Co & 58 & $2.89 \mathrm{E}-13$ & 2.8 & 2.8 & $2.89 \mathrm{E}-13$ & 2.8 & & & & & & -13 \\
\hline co & 60 & $1.19 \mathrm{E}-13$ & 1.1 & $9 E-13$ & $1.21 \mathrm{E}-13$ & 1.2 & 1.2 & 1. & -13 & & & $8-13$ \\
\hline $\mathrm{Co}$ & $60 \mathrm{~m}$ & $3.64 \mathrm{E}-08$ & $E-08$ & $3.63 \mathrm{E}-08$ & $3.60 \mathrm{E}-08$ & $6 E-08$ & $3.52 \mathrm{E}-08$ & $E-08$ & -08 & -08 & $E-08$ & $:-09$ \\
\hline $\mathrm{co}$ & 61 & $7.51 \mathrm{E}-14$ & 7.5 & $7.51 \mathrm{E}-14$ & $7.51 \mathrm{E}-14$ & $0 E-14$ & & & & & & \\
\hline $\mathrm{Co}$ & 62 & $8.72 E-13$ & & & $7 E-13$ & 7.4 & & -13 & -13 & -14 & & -17 \\
\hline & 59 & $1.63 \mathrm{E}-20$ & & 1.6 & 1.6 & $1.63 \mathrm{E}-20$ & & & & & & $E-20$ \\
\hline $\mathrm{n}$ & 63 & 1. $98 \mathrm{E}-18$ & & 1.9 & $3 E-18$ & $1.98 \mathrm{E}-1$ & 1.9 & 1.9 & 1.9 & -18 & -18 & $E-18$ \\
\hline & 65 & $2.02 \mathrm{E}-14$ & $2.02 \mathrm{E}-14$ & $2.02 \mathrm{E}-14$ & $2.02 \mathrm{E}-14$ & $2.02 \mathrm{E}-14$ & $2.02 \mathrm{E}$ & & & & & $5 E-14$ \\
\hline$y$ & $89 m$ & & & & $8.62 E-16$ & $1.13 \mathrm{E}-15$ & 1. $30 \mathrm{E}-$ & & $1.62 \mathrm{E}-15$ & E-15 & $E-15$ & \\
\hline $\mathrm{zr}$ & 89 & $1.63 \mathrm{E}-15$ & $1.63 \mathrm{E}-15$ & $1.63 \mathrm{E}-15$ & $1.63 \mathrm{E}-15$ & $1.62 \mathrm{E}-15$ & $1.62 \mathrm{E}-1$ & 1. $62 \mathrm{E}-15$ & 1.6 & & & 1. $62 \mathrm{E}-15$ \\
\hline zr & 95 & $2.86 \mathrm{E}-16$ & $2.86 \mathrm{E}-16$ & $2.86 \mathrm{E}-16$ & $2: 86 \mathrm{E}-16$ & $2.86 \mathrm{E}-1$ & & & & & & \\
\hline $\mathrm{zr}$ & 97 & $5.97 \mathrm{E}-16$ & $5.97 \mathrm{E}-16$ & $5.97 \mathrm{E}-16$ & $5.97 \mathrm{E}-16$ & $5.97 \mathrm{E}-16$ & $5.97 \mathrm{E}-16$ & $5.97 E-16$ & $5.96 \mathrm{E}-16$ & $5.95 E-16$ & $5.93 \mathrm{E}-16$ & $5.89 \mathrm{E}-16$ \\
\hline nb & 94 & $1.20 \mathrm{E}-20$ & $1.20 \mathrm{E}-20$ & $1.20 \mathrm{E}-20$ & $1.20 \mathrm{E}-20$ & $1.20 \mathrm{E}-20$ & $1.20 \mathrm{E}-20$ & $1.20 \mathrm{E}-20$ & $1.20 \mathrm{E}-20$ & $1.20 \mathrm{E}-20$ & $1.20 \mathrm{E}-20$ & $1.20 \mathrm{E}-20$ \\
\hline
\end{tabular}


Table F8. (continued)

\begin{tabular}{|c|c|c|c|c|c|c|c|c|c|c|c|}
\hline & & & & $\operatorname{Tim}$ & After & large & & & & & \\
\hline & initial & $1 \mathrm{E}-15 \mathrm{sec}$ & 1. $0 \mathrm{sec}$ & $10.0 \mathrm{sec}$ & $20.0 \mathrm{sec}$ & $30.0 \mathrm{sec}$ & $60.0 \mathrm{sec}$ & $120.0 \mathrm{sec}$ & $300.0 \mathrm{sec}$ & $600.0 \mathrm{sec}$ & $1200.0 \mathrm{sec}$ \\
\hline nb 95 & $6.82 \mathrm{E}-16$ & $6.82 \mathrm{E}-16$ & $6.82 \mathrm{E}-16$ & $6.82 E-16$ & $6.82 \mathrm{E}-16$ & $6.82 \mathrm{E}-16$ & $6.82 \mathrm{E}-16$ & $6.82 \mathrm{E}-16$ & $6.82 \mathrm{E}-16$ & $6.82 \mathrm{E}-16$ & $6.82 \mathrm{E}-16$ \\
\hline $\mathrm{nb} 96$ & $3.76 \mathrm{E}-14$ & $3.76 \mathrm{E}-14$ & $3.76 \mathrm{E}-14$ & & $3.76 \mathrm{E}-14$ & & $3.76 \mathrm{E}-14$ & $3.76 \mathrm{E}-14$ & $3.75 \mathrm{E}-14$ & $3.74 \mathrm{E}-14$ & $3.72 \mathrm{E}-14$ \\
\hline nb 97 & $2.87 \mathrm{E}-13$ & $2.87 \mathrm{E}-13$ & $2.87 \mathrm{E}-13$ & $2.87 E-13$ & $2.86 \mathrm{E}-13$ & $2.86 \mathrm{E}-13$ & $2.84 \mathrm{E}-13$ & $2.82 E-13$ & $2.74 \mathrm{E}-13$ & 2. $61 \mathrm{E}-13$ & $2.37 \mathrm{E}-13$ \\
\hline $\mathrm{nb} 97 \mathrm{~m}$ & $4.92 \mathrm{E}-17$ & $4.92 \mathrm{E}-17$ & $5.52 \mathrm{E}-17$ & $1.06 E-16$ & $1.56 \mathrm{E}-16$ & $2.01 \mathrm{E}-16$ & $3.08 \mathrm{E}-16$ & $4.36 \mathrm{E}-16$ & $5.48 \mathrm{E}-16$ & $5.62 \mathrm{E}-16$ & $5.59 \mathrm{E}-16$ \\
\hline & $1.69 \mathrm{E}-11$ & $1.69 \mathrm{E}-11$ & $1.33 \mathrm{E}-11$ & 1. $50 \mathrm{E}-12$ & -13 & $1.18 \mathrm{E}-14$ & $9 E-18$ & $6 E-24$ & $00 \mathrm{E}+00$ & $00 \mathrm{E}+00$ & $0.00 \mathrm{E}+00$ \\
\hline nb100 & $E-13$ & E-13 & $5.42 \mathrm{E}-1$ & $8.46 E-15$ & & $2-19$ & $7.82 \mathrm{E}-25$ & $0 \mathrm{E}+00$ & $00 \mathrm{E}+00$ & $0.00 \mathrm{E}+00$ & $0.00 \mathrm{E}+00$ \\
\hline mo 93 & $1.72 E-20$ & $1.72 \mathrm{E}-20$ & $1.72 \mathrm{E}-20$ & $1.72 \mathrm{E}-20$ & $1.72 \mathrm{E}-20$ & $1.72 \mathrm{E}-20$ & 1. $72 \mathrm{E}-20$ & $1.72 \mathrm{E}-20$ & $1.72 \mathrm{E}-20$ & $1.72 \mathrm{E}-20$ & $1.72 \mathrm{E}-20$ \\
\hline mo 99 & $\mathrm{E}-13$ & $7 E-13$ & $7 E-13$ & $7 E-13$ & 1.3 & $7 E-13$ & 1. & $7 E-13$ & $1.37 E-13$ & $1.37 \mathrm{E}-13$ & $1.37 \mathrm{E}-13$ \\
\hline $\operatorname{mol} 101$ & $9.47 \mathrm{E}-12$ & $9.47 \mathrm{E}-12$ & $9.46 \mathrm{E}-12$ & $9 E-12$ & $9.32 \mathrm{E}-12$ & $9.24 E-12$ & $9.03 \mathrm{E}-12$ & $8.61 \mathrm{E}-12$ & $7.47 \mathrm{E}-12$ & $5.89 \mathrm{E}-12$ & $3.66 \mathrm{E}-12$ \\
\hline tc101 & $6.15 \mathrm{E}-14$ & $6.15 \mathrm{E}-14$ & $6.91 \mathrm{E}-14$ & $1.37 \mathrm{E}-13$ & $2.12 \mathrm{E}-13$ & $2.86 \mathrm{E}-13$ & $4.99 \mathrm{E} 13$ & E-13 & $1.86 \mathrm{E}-12$ & $2.89 \mathrm{E}-12$ & $3.55 \mathrm{E}-12$ \\
\hline w181 & 8.0 & 8.0 & 8.0 & $E-18$ & & -18 & $8.03 \mathrm{E}-18$ & $3 E-18$ & $8.03 E-18$ & $8.03 \mathrm{E}-18$ & $8.03 E-18$ \\
\hline w185 & $2.60 \mathrm{E}-16$ & $2.60 \mathrm{E}-16$ & $2.60 \mathrm{E}-16$ & $2.60 \mathrm{E}-16$ & $2.60 \mathrm{E}-16$ & $2.60 \mathrm{E}-16$ & $2.60 \mathrm{E}-16$ & $2.61 \mathrm{E}-16$ & $2.61 \mathrm{~F}-16$ & $2.61 \mathrm{E}-16$ & $2.61 \mathrm{E}-16$ \\
\hline & $1.76 \mathrm{E}-14$ & $1.76 \mathrm{E}-14$ & $1.75 \mathrm{E}-14$ & $1.64 \mathrm{E}-14$ & & $1.43 \mathrm{E}-14$ & $1.16 \mathrm{E}-14$ & $6 E-15$ & $2.21 E-15$ & $2.77 \mathrm{E}-16$ & $4.36 \mathrm{E}-18$ \\
\hline w18 & $5.99 \mathrm{E}-13$ & $5.99 \mathrm{E}-13$ & $5.99 \mathrm{E}-13$ & $5.99 \mathrm{E}-13$ & $5.98 \mathrm{E}-13$ & $5.98 \mathrm{E}-13$ & $5.98 \mathrm{E}-13$ & $5.98 \mathrm{E}-13$ & $5.97 E-13$ & $5.96 \mathrm{E}-13$ & $5.93 \mathrm{E}-13$ \\
\hline & $4.03 E-08$ & $4.03 E-08$ & $4.02 \mathrm{E}-08$ & $3.97 \mathrm{E}-08$ & $3.92 \mathrm{E}-08$ & $3.86 \mathrm{E}-08$ & $3.71 E-08$ & $3.43 \mathrm{E}-08$ & $2,74 \mathrm{E}-08$ & $1.93 \mathrm{E}-08$ & $9.79 \mathrm{E}-09$ \\
\hline
\end{tabular}


Table F9. Radioactivity (Ci) After 24-s Irradiation of 1-kg Surgical Implant Composition A Cargo (Flux Parameter Set 1).

\begin{tabular}{|c|c|c|c|c|c|c|c|c|c|c|c|c|}
\hline & & & & & & & & & & & & \\
\hline & & lnitlal & sec & 1. $0 \mathrm{sec}$ & $.0 \mathrm{sec}$ & $20.0 \mathrm{sec}$ & 30.0 sec & $60.0 \mathrm{sec}$ & 120.0 sec & $300.0 \mathrm{sec}$ & & \\
\hline $1 i$ & 8 & $3.81 \mathrm{E}-12$ & $3.81 \mathrm{E}-12$ & $1.67 \mathrm{E}-12$ & $9.74 E-16$ & $2.49 \mathrm{E}-19$ & $6.37 \mathrm{E}-23$ & $0.00 E+00$ & $0.00 \mathrm{E}+00$ & $0.00 \mathrm{E}+00$ & $0.00 \mathrm{E}+00$ & $0.00 \mathrm{E}+00$ \\
\hline & 8 & $3.81 \mathrm{E}-12$ & $3.81 \mathrm{E}-12$ & $1.67 \mathrm{E} \quad 12$ & $9.74 \mathrm{E}-16$ & $2.49 \mathrm{E}-19$ & $6.37 \mathrm{E}-23$ & $0.00 \mathrm{E}+00$ & $0.00 \mathrm{E}+00$ & $0.00 \mathrm{E}+00$ & $0.00 E+00$ & \\
\hline & 11 & $2.30 \mathrm{E}-14$ & $2.30 \mathrm{E}-14$ & $.18 \mathrm{E}-14$ & $1.39 \mathrm{E}-14$ & $8.41 \mathrm{E}-15$ & $5.09 \mathrm{E}-15$ & $.13 E-15$ & $5.56 \mathrm{E}-17$ & $6.63 \mathrm{E}-21$ & $1.91 E-27$ & $0.00 \mathrm{E}+00$ \\
\hline $\mathrm{b}$ & 12 & $3.49 \mathrm{E}-13$ & & & & & & $2.63 \mathrm{E}-24$ & $7.71 \mathrm{E}-27$ & $0.00 \mathrm{E}+00$ & $0.00 \mathrm{E}+00$ & \\
\hline $\mathrm{c}$ & 14 & $5.44 \mathrm{E}-18$ & & $5.44 \mathrm{E}-18$ & $5.44 \mathrm{E}-18$ & $5.44 \mathrm{E}-18$ & $5.44 \mathrm{E}-18$ & $5.44 \mathrm{E}-18$ & $5.44 E-18$ & $5.44 \mathrm{E}-18$ & $5.44 \mathrm{E}-18$ & $5.44 \mathrm{E}-18$ \\
\hline $\mathrm{C}$ & 15 & & & $1.18 \mathrm{E}-14$ & & & & $6.61 \mathrm{E}-22$ & $2.78 \mathrm{E}-29$ & $0.00 \mathrm{E}+00$ & $0.00 \mathrm{~F}+00$ & $0.00 \mathrm{E}+00$ \\
\hline $\mathrm{n}$ & 16 & 7.4 & & $6.77 \mathrm{E}-1.7$ & $2.82 \mathrm{E}-17$ & $1.07 \mathrm{E}-17$ & $4.04 \mathrm{E}-18$ & $2.19 E-19$ & $6.40 \mathrm{E}-22$ & $1.61 \mathrm{E}-29$ & $0.00 \mathrm{E}+00$ & $00 E+00$ \\
\hline & 24 & E-13 & & 2.8 & $2.80 E-13$ & 2 . & $12=13$ & 12 & & & & \\
\hline & 27 & E-10 & & & $1.21 \mathrm{E}-10$ & $E-10$ & $8 E-10$ & $E-10$ & & & & \\
\hline al & 28 & E-09 & 1.9 & 1.9 & $2 E-09$ & $3 E-09$ & $1.64 \mathrm{E}-09$ & $E-09$ & & & & \\
\hline & 29 & 2.2 & & & & & & & & & & \\
\hline al & 30 & 5.3 & & & $7.75 \mathrm{E}-14$ & & & & & $0.00 \mathrm{E}+00$ & +00 & \\
\hline $\mathrm{si}$ & 31 & & & & & & & -12 & -12 & $\div-12$ & & \\
\hline $\mathrm{p}$ & 32 & 2.9 & & & $2.96 \mathrm{E}-14$ & & & & & & & \\
\hline $\mathrm{p}$ & 33 & 1.4 & 1.4 & 1.4 & $2 E-13$ & & 1. & & & & & \\
\hline $\mathrm{p}$ & 34 & 7.5 & & & & & & & & & & \\
\hline$s$ & 35 & 3.6 & & & & & & & & & & \\
\hline $\mathrm{s}$ & 37 & 2.2 & & 2. & -17 & & & & & & & \\
\hline ti & 51 & 3.6 & & & & & & & & & & \\
\hline $\mathrm{v}$ & 52 & 3.4 & & & & & & & & & & \\
\hline $\mathrm{v}$ & 53 & 1.0 & & & & & & & & & & \\
\hline $\mathrm{v}$ & 54 & 1.9 & 1.5 & 1.8 & $6 E-12$ & & & & & & & \\
\hline cr & 51 & 1.8 & 1.8 & & & & & & & & & \\
\hline & 55 & 9.2 & & 9.2 & 8.5 & & & & & & & \\
\hline & 54 & 3.9 & & 3.9 & 3.9 & & & & & & & \\
\hline & 56 & 3.7 & & & & & & & & & & \\
\hline & 57 & 2.4 & & 2.4 & -12 & & & & & & & \\
\hline & 58 & 1.0 & & & & & & & & & & \\
\hline fe & 55 & 8.4 & & & 8.4 & & & & & & & \\
\hline fe & 59 & & & 3.6 & & & & & & & & -17 \\
\hline co & 58 & 4.3 & & & & & & & & & & \\
\hline co & 60 & & & & & & & & & & & \\
\hline $\mathrm{CO}$ & $60 \mathrm{~m}$ & 5.4 & & 5.4 & & & & & & & & \\
\hline co & 61 & & & & & & & & & & & \\
\hline co & 62 & & & & & & & & & & & -16 \\
\hline$n i$ & 59 & 2.44 & & 2.4 & $2.44 \mathrm{E}-20$ & & & & & & & \\
\hline$n i$ & 63 & & & & $2.97 \mathrm{E}-18$ & & $8-18$ & & & & -18 & $E-18$ \\
\hline $\mathrm{ni}$ & 65 & & & & & & & & & & & \\
\hline$y$ & $89 \mathrm{r}$ & & & & 1.4 & & & & & & & \\
\hline$z r$ & 89 & & & & $2.44 \mathrm{E}-15$ & & & & & & & -15 \\
\hline $\mathrm{zr}$ & 95 & & & & $4.29 \mathrm{E} \quad 16$ & & & & & & & \\
\hline$z r$ & 97 & & & & $8.95 E-16$ & & & & & $8.93 E-16$ & 8.8 & $E-16$ \\
\hline$n b$ & 94 & $1.80 \mathrm{~F}-20$ & & 1. $80 \mathrm{E}-20$ & $1.80 \mathrm{E}-20$ & $1.80 \mathrm{E}-20$ & $1.80 \mathrm{E}-20$ & $1.80 \mathrm{E}-20$ & $1.80 E-20$ & 1. $80 \mathrm{E}-20$ & $1.80 E 20$ & $1.80 \mathrm{E}-20$ \\
\hline
\end{tabular}


Table F9. (continued)

\begin{tabular}{|c|c|c|c|c|c|c|c|c|c|c|c|}
\hline & & & & Time & After Di & harge & & & & & \\
\hline & initial & $1 \mathrm{E}-15 \mathrm{sec}$ & $1.0 \mathrm{sec}$ & $10.0 \mathrm{sec}$ & 20.0 sec & $30.0 \mathrm{sec}$ & $60.0 \mathrm{sec}$ & $120.0 \mathrm{sec}$ & $300.0 \mathrm{sec}$ & $600.0 \mathrm{sec}$ & $1200.0 \mathrm{sec}$ \\
\hline nb 95 & $1.02 \mathrm{E}-15$ & $1.02 \mathrm{E}-15$ & $.02 E-15$ & $1.02 \mathrm{E}-15$ & $1.02 \mathrm{E}-15$ & $1.02 \mathrm{E}-15$ & $1.02 \mathrm{E}-15$ & $1.02 \mathrm{E}-1.5$ & $1.02 \mathrm{E}-1$ & $1.02 \mathrm{E}-15$ & $1.02 \mathrm{E}-15$ \\
\hline $\mathrm{nb} 96$ & $4 E-14$ & $4 \mathrm{E}-14$ & $4 E-1$ & $4 \mathrm{E}-14$ & $4 \mathrm{E}-14$ & $4 \mathrm{~F}-14$ & $4 \mathrm{E}-14$ & $3 E-14$ & $35-1$ & & \\
\hline nb 97 & -13 & $9-13$ & & & & & & & & & \\
\hline $97 \mathrm{~m}$ & 1.0 & & 1.1 & $3 E-16$ & & & & & & & \\
\hline $\mathrm{nb} 98$ & & & & & & & & & & & \\
\hline nb100 & 8.6 & 8.6 & & & & & & +00 & & & \\
\hline mo 93 & 2.5 & & & 2 . & & & & & & & \\
\hline & 2.0 & & & -13 & & & & 13 & & & \\
\hline 101 & & & & & & & & & & & \\
\hline to & 1.3 & & 1. & 2 & & & & & & & \\
\hline & & & & & & & & & & & \\
\hline & $E-16$ & 3.9 & 3.9 & 3.9 & & & & & & & \\
\hline & $2.57 \mathrm{E}-14$ & $2.57 E-14$ & $2.55 E-14$ & $2.39 \mathrm{E}-14$ & & $2.09 \mathrm{E}-14$ & & & & & $6.37 \mathrm{E}-18$ \\
\hline & & & $8.98 \mathrm{E}-13$ & $8.98 E-13$ & & & & & & & \\
\hline & $6.01 \mathrm{E}-08$ & $6.01 \mathrm{E}-08$ & $6.00 \mathrm{E}-08$ & $5.92 E-08$ & $5.84 \mathrm{E}-08$ & $5.77 \mathrm{E}-08$ & $5.54 \mathrm{E}-08$ & $5.13 \mathrm{E}-08$ & 4.10E-08 & $2.88 \mathrm{E}-08$ & 1. $46 \mathrm{E}-08$ \\
\hline
\end{tabular}


Table F10. Radioactivity (Ci) After 8-s Irradiation of 1-kg Surgical Implant Composition B Cargo (Flux Parameter Set 1).

\begin{tabular}{|c|c|c|c|c|c|c|c|c|c|c|c|}
\hline & & & & Time & After Di & arqe & & & & & \\
\hline & & & & $10.0 \mathrm{sec}$ & $20.0 \mathrm{sec}$ & $30.0 \mathrm{sec}$ & & & & & 1200. Usec \\
\hline ti 51 & $1.10 \mathrm{E}-1$ & $E-12$ & $0 E-12$ & $1.08 \mathrm{E}-12$ & $1.06 \mathrm{E}-12$ & $1.04 \mathrm{E}-12$ & $9.78 \mathrm{E}-13$ & $8.67 E-13$ & $6.05 E-13$ & $3.31 \mathrm{E}-13$ & $9.94 \mathrm{E}-14$ \\
\hline & & $E-00$ & $04 \mathrm{~F}-09$ & & $9.79 \mathrm{E}-10$ & & & 7. $20 \mathrm{E}-10$ & $4.13 \mathrm{E}-10$ & $1.64 \mathrm{E}-10$ & $2.58 \mathrm{E}-11$ \\
\hline v 53 & $6 E-11$ & $6 \mathrm{E}-11$ & $33 E-11$ & $3 E-11$ & $2.91 \mathrm{E}-11$ & $1 \mathrm{E}-11$ & $2.18 \mathrm{E}-$ & $1.42 \mathrm{E}-11$ & & & $2 \mathrm{E}-15$ \\
\hline v 54 & & & & & $4.81 E_{-}-13$ & $4.18 \mathrm{E}-13$ & & & & & \\
\hline cr 51 & & & & & & & & & & & \\
\hline cr 55 & & & & & & & & & & & \\
\hline co 60 & & & & & & & & & & & \\
\hline $\mathrm{co} 60 \mathrm{~m}$ & & & & & & & & & & & \\
\hline y $89 \mathrm{~m}$ & & & & & & & & & & & \\
\hline zt 89 & & & & & & & & & & & \\
\hline zI 95 & & & & & & & & & & & \\
\hline zr 97 & & & & & & & & & & & \\
\hline & & & & & & & & & & & \\
\hline nb 96 & .3 & & & & & & & & & & \\
\hline & .0 & & & & & & & & & & \\
\hline & & & & 3.0 & & & & & & & \\
\hline & & -11 & & & & & 5.1 & & & & \\
\hline & & & & & & & & & & & \\
\hline & & & & & & & & & & & \\
\hline & & & & & & & & & & & \\
\hline$t c 10$ & 1.10 & & & $E-14$ & & & 1.6 & & & & \\
\hline & 750 & $2.25 E-08$ & $2.24 \mathrm{E}-08$ & $2.22 \mathrm{E}-08$ & $2.19 \mathrm{E}-08$ & $2.17 \mathrm{E}-08$ & $2.09 \mathrm{E}-08$ & $1.95 \mathrm{E}-08$ & $1.58 \mathrm{E}-08$ & $1.12 \mathrm{E}-08$ & $3.71 \mathrm{E}-0$ \\
\hline
\end{tabular}


Table F11. Radioactivity (Ci) After 16-s Irradiation of 1-kg Surgical Implant Composition B Cargo (Flux Parameter Set 1).

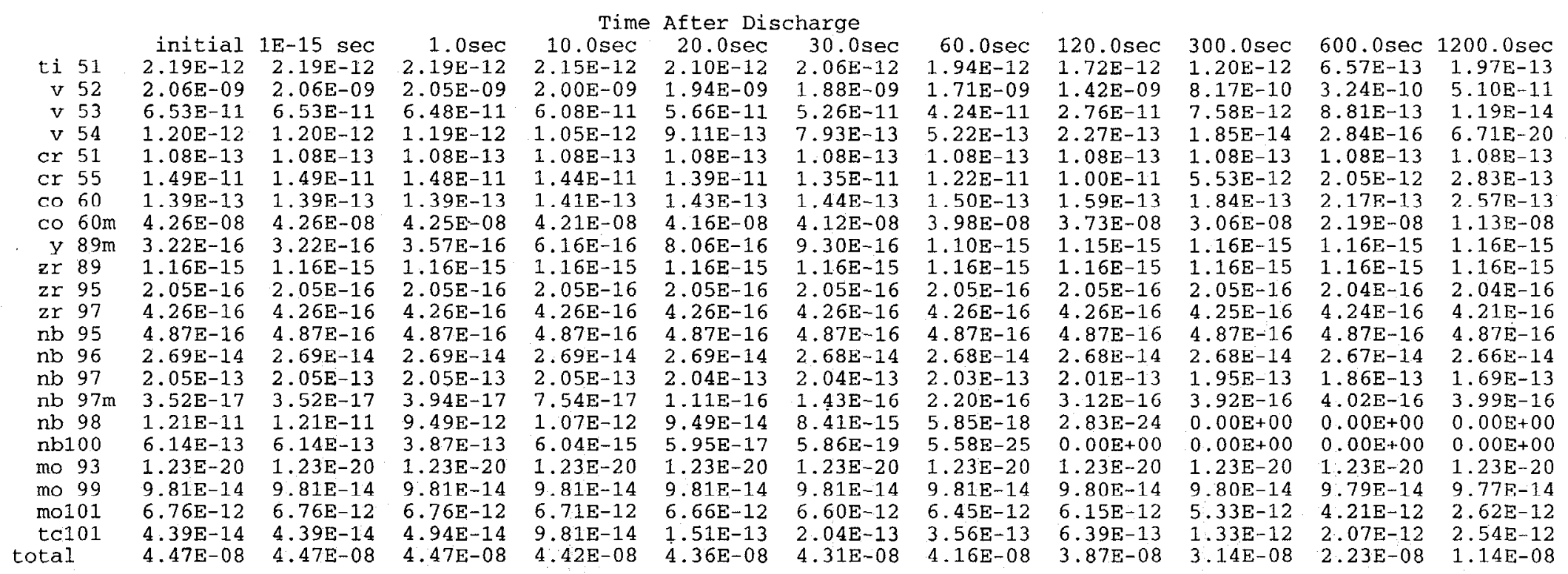


Table F12. Radioactivity (Ci) After 24-s Irradiation of 1-kg Surgical Implant Composition B Cargo (Flux Parameter Set 1).

\begin{tabular}{|c|c|c|c|c|c|c|c|c|c|c|c|}
\hline & & & & & & & & & & & \\
\hline & initial & $1 \mathrm{E}-15 \mathrm{sec}$ & $1.0 \mathrm{sec}$ & $10.0 \mathrm{sec}$ & $20.0 \mathrm{sec}$ & $30.0 \mathrm{sec}$ & $60.0 \mathrm{sec}$ & $120.0 \mathrm{sec}$ & $300.0 \mathrm{sec}$ & $600.0 \mathrm{sec}$ & $1200.0 \mathrm{se}$ \\
\hline ti 51 & & $3.26 \mathrm{E}-12$ & & $3.19 \mathrm{E}-12$ & $3.13 E-12$ & $3.07 \mathrm{E}-12$ & $2.89 \mathrm{E}-12$ & 2. $56 E-12$ & 1. $79 \mathrm{E}-12$ & $9.78 \mathrm{E}-13$ & $2.94 \mathrm{E}-13$ \\
\hline v 52 & $5 E-09$ & $5 E-09$ & $.04 E-09$ & $.96 \mathrm{E}-09$ & $2.87 E-09$ & $2.78 \mathrm{E}-09$ & $.54 \mathrm{E}-09$ & $2.11 \mathrm{E}-09$ & $1.21 \mathrm{E}-09$ & $4.80 E-10$ & $7.56 \mathrm{E}-11$ \\
\hline v 53 & & & & & & $7.68 \mathrm{E}-11$ & & & & & \\
\hline v 54 & $E-12$ & $E-12$ & & $.49 E-12$ & $1.30 \mathrm{E}-12$ & $1.13 \mathrm{E}-12$ & $7.43 \mathrm{E}-13$ & & & & $4 E-20$ \\
\hline cr 51 & $1.62 \mathrm{E}-13$ & & & & & & & & & & \\
\hline & $.20 E-11$ & 2.2 & & $3 E-11$ & & $2.00 \mathrm{E}$ & & & & & \\
\hline co 60 & 13 & & & & & 2 . & & & & & \\
\hline co $60 \mathrm{~m}$ & & & & & & & & & & & \\
\hline & .5 & & & & & & & & & & \\
\hline $\operatorname{zr} 89$ & 1.7 & & & & 1.7 & 1.7 & & & & & \\
\hline zI 95 & & & & & & & & & & & \\
\hline zx 97 & 6.4 & & & 6.4 & 6.4 & 6. & & & & & \\
\hline $\mathrm{nb} 94$ & 1.2 & & & 1.25 & & $1.2+x-5$ & & & & & \\
\hline & 7.3 & & & & & & & & & & \\
\hline & 4.0 & & & 4.0 & & & & & & & \\
\hline & & & & & & & & & & & \\
\hline & 7.6 & & & 1.3 & 1.8 & & & & & & \\
\hline & 1.23 & & & & & & & & & & \\
\hline nb100 & 6.14 & & & & 5.9 & & & & & & \\
\hline & 1.841 & 1.8 & 1.84 & $1.84 \mathrm{E}-20$ & 1.84 & & & & & & \\
\hline & 1.471 & & & & & & & & & & \\
\hline molo & $1.01 \mathrm{E}-11$ & 1.011 & 1.01 & $1.00 \mathrm{E}-$ & $9.95 \mathrm{E}-12$ & $9.87 \mathrm{E}-12$ & $9.64 \mathrm{E}-$ & 9.2 & $7.97 \mathrm{E}-12$ & $\bar{E}-12$ & $3.91 \mathrm{E}-12$ \\
\hline & & 9.8 & 1.0 & $1.79 \mathrm{E}-13$ & 2.59 & & 5.6 & & & & \\
\hline & $6.67 E-08$ & $6.67 E-08$ & $6.67 \mathrm{E}-08$ & $6.59 E-08$ & $6.51 E-08$ & $6.44 \mathrm{E}-08$ & $6.21 E-08$ & $5.78 \mathrm{E}-08$ & $4.69 E-08$ & $3.33 E-08$ & $1.70 \mathrm{E}-08$ \\
\hline
\end{tabular}


Table F13. Radioactivity (Ci) After 8-s Irradiation of 1-kg 16-16-16 Fertilizer Cargo (Flux Parameter Set 1).

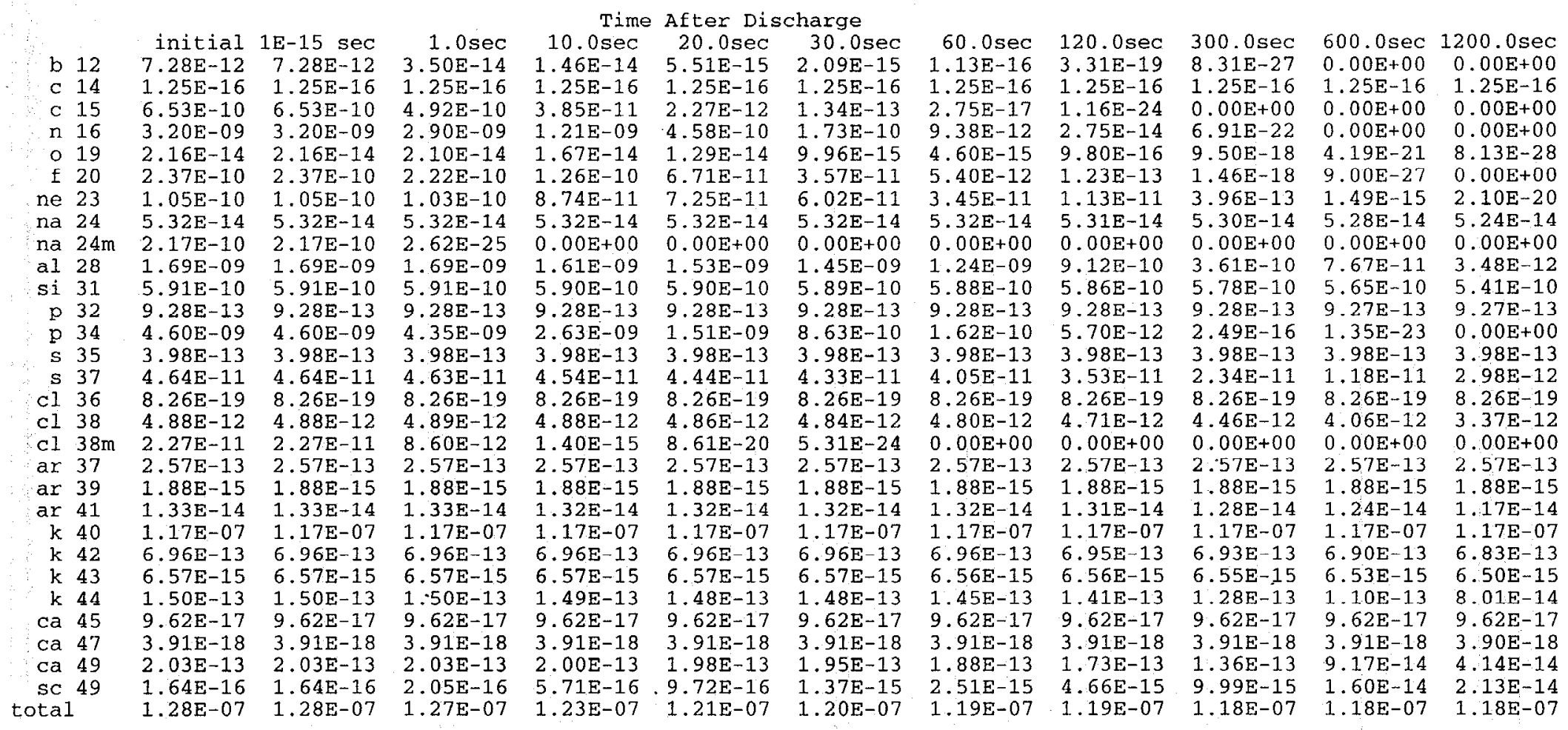


Table F14. Radioactivity (Ci) After 16-s Irradiation of 1-kg 16-16-16 Fertilizer Cargo (Flux Parameter Set 1).

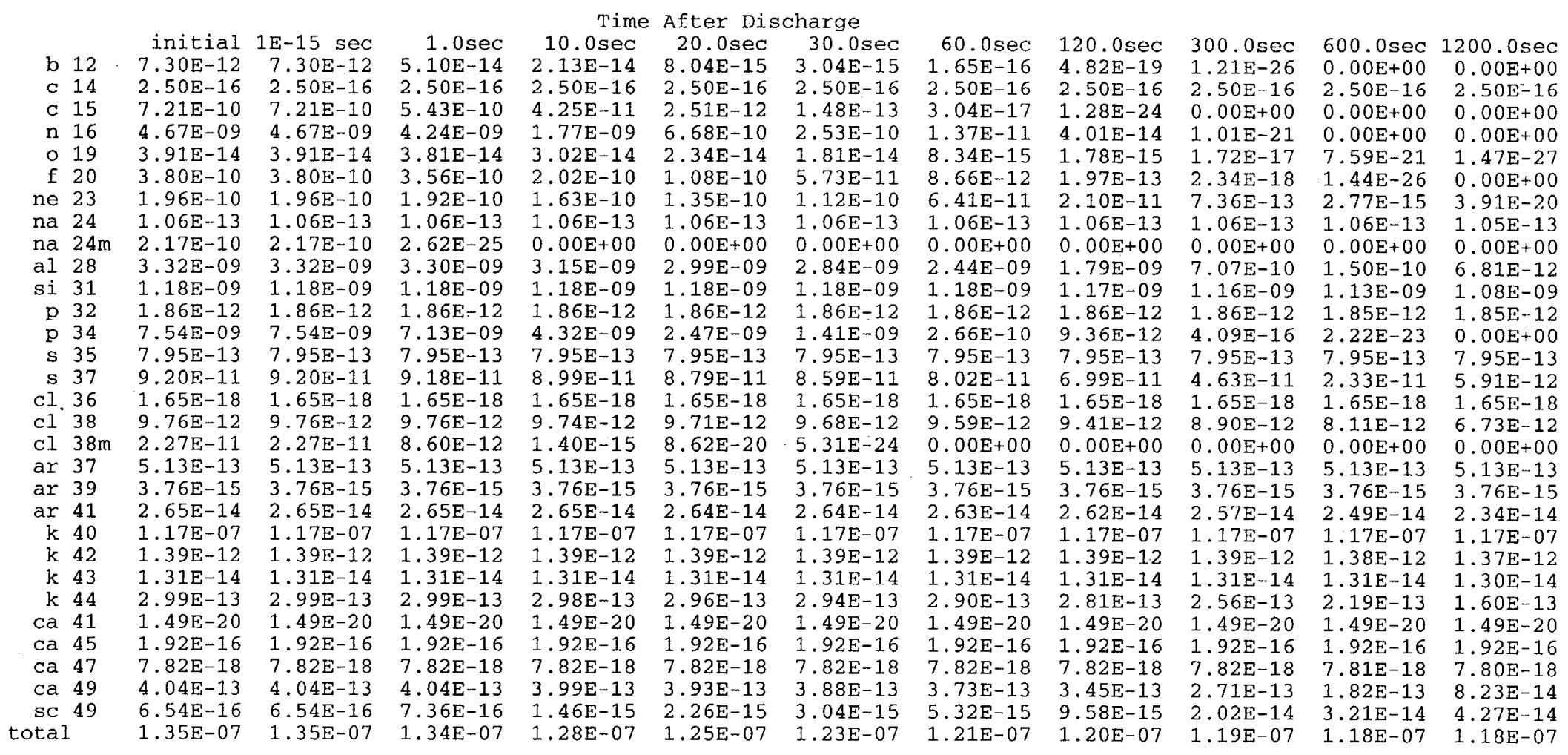


Table F15. Radioactivity (Ci) After 24-s Irradiation of 1-kg 16-16-16 Fertilizer Cargo (Flux Parameter Set 1).

\begin{tabular}{|c|c|c|c|c|c|c|c|c|c|c|c|}
\hline & & & & & & & & & & & \\
\hline & & $\mathrm{E}-15 \mathrm{sec}$ & $1.0 \mathrm{sec}$ & $10.0 \mathrm{sec}$ & 20.0 sec & $30.0 \mathrm{sec}$ & $60.0 \mathrm{sec}$ & $120.0 \mathrm{sec}$ & $300.0 \mathrm{sec}$ & $600.0 \mathrm{sec}$ & $1200.0 \mathrm{sec}$ \\
\hline b 12 & & & & $2.43 E-14$ & $9.21 \mathrm{E}-15$ & $3.48 \mathrm{E}-15$ & & & & $0.00 \mathrm{E}+00$ & $0.00 \mathrm{E}+00$ \\
\hline & $.75 E-16$ & $3.75 E-16$ & $.75 E-16$ & & & & & & & $75 E-16$ & $3.75 \mathrm{E}-16$ \\
\hline & $.28 \mathrm{E}-10$ & $7.28 \mathrm{E}-10$ & $.49 \mathrm{E}-10$ & $F-11$ & $3 E-12$ & $49 \mathrm{E}-13$ & $7=17$ & $29 E-24$ & $.00 \mathrm{E}+00$ & $0.00 \mathrm{E}+00$ & $0.00 \mathrm{E}+00$ \\
\hline n 16 & $5.35 \mathrm{E}-09$ & $5.35 \mathrm{E}-09$ & $.85 \mathrm{E}-09$ & & & & & & & & \\
\hline & & & 71 & E-14 & $3.19 \mathrm{E}-14$ & & 1514 & & & & $2.01 \mathrm{E}-27$ \\
\hline & & & & $2.48 \mathrm{E}-10$ & & & & & & & \\
\hline ne 23 & $2.74 \mathrm{E}-10$ & $2.74 \mathrm{E}-10$ & $2.69 \mathrm{E}-10$ & & & & & & & & \\
\hline & -13 & & & -13 & & & & & & & \\
\hline $24 \mathrm{~m}$ & -10 & $8-10$ & 5 & +00 & & +00 & & & & & \\
\hline al 28 & $.8 \varepsilon$ & & & 4. & & & & & & & \\
\hline si 31 & 09 & & & & & & & & & & \\
\hline p 32 & .7 & & & & & & & & & & \\
\hline & & & & 5.3 & & & & & & & \\
\hline $\mathrm{s}$ & & & & & & & & & & & \\
\hline $\mathrm{s}$ & 10 & & & 1.3 & & & & & & & \\
\hline & & & & & & & & & & & \\
\hline Cl 38 & 11 & & & & & & & & & & \\
\hline & .2 & 2.2 & & -15 & & & & & & & \\
\hline ar 37 & .7 & & & & & & & & & & \\
\hline ar 39 & .6 & & & & 5. & & & & & & \\
\hline ar 41 & & & & & & & & & & & \\
\hline$k$ & .1 & & & 1.1 & 1.1 & & & & & & \\
\hline $\mathrm{k}$ & & & & & & & & & & & \\
\hline $\mathrm{k}$ & $1.9^{\prime \prime}$ & & & & & & & & & & \\
\hline $\mathrm{k}$ & $i-13$ & 4.4 & & & & & & & & & \\
\hline ca 41 & & & & & & & & & & & \\
\hline & & & & & & & & & & & \\
\hline ca 47 & .17 & & & $1.1^{\prime}$ & $1.17 \mathrm{E}-17$ & & & & & & \\
\hline ca 49 & & & 6.0 & 5.9 & $5.87 \mathrm{E}-13$ & & & & & & \\
\hline & $.4 / \mathrm{E}$ & & & 2.67 & & & & & & & $6.41 \mathrm{E}-14$ \\
\hline & טת & $1.40 \mathrm{E}-07$ & $1.39 \mathrm{E}-07$ & $1.31 \mathrm{E}-07$ & $1.27 \mathrm{E}-07$ & $1.25 \mathrm{E}-07$ & $1.23 E-07$ & 1. $22 \mathrm{E}-07$ & $1.20 \mathrm{E}-07$ & $1.19 \mathrm{E}-07$ & $1.19 \mathrm{E}-07$ \\
\hline
\end{tabular}


Table F16. Radioactivity (Ci) After One-Day Irradiation of 1-kg Concrete (Flux Parameter Set 1).

\begin{tabular}{|c|c|c|c|c|c|c|c|c|c|c|c|}
\hline & & & & & & & & & & & \\
\hline & initial & & $1.0 \mathrm{hr}$ & $2.0 \mathrm{hr}$ & $4.0 \mathrm{hr}$ & $6.0 \mathrm{hr}$ & $8.0 \mathrm{hr}$ & $10.0 \mathrm{hr}$ & $12.0 \mathrm{hr}$ & $14.0 \mathrm{hr}$ & $16.0 \mathrm{hr}$ \\
\hline h 3 & $2.64 \mathrm{E}-19$ & $2.64 \mathrm{E}-19$ & $2.64 \mathrm{E}-19$ & $2.64 \mathrm{E}-19$ & $2.64 \mathrm{E}-19$ & $2.64 \mathrm{E}-19$ & $2.64 E-19$ & $2.64 \mathrm{E}-19$ & $2.64 \mathrm{E}-19$ & $2.64 \mathrm{E}-19$ & 2. $64 \mathrm{E}-19$ \\
\hline be 10 & $1.17 \mathrm{E}-20$ & $1.17 E-20$ & $1.17 E-20$ & $1.17 \mathrm{E}-20$ & $1.17 \mathrm{E}-20$ & $1.17 \mathrm{E}-20$ & $1.17 E-20$ & $1.17 \mathrm{E}-20$ & 1. $17 \mathrm{E}-20$ & $1.17 \mathrm{E}-20$ & 1. $17 \mathrm{E}-20$ \\
\hline C 14 & $2.09 E-18$ & $2.09 \mathrm{E}-18$ & $2.09 \mathrm{E}-18$ & $2.09 E-18$ & $09 E-18$ & $2.09 E-18$ & $2.09 E-18$ & $2.09 \mathrm{E}-18$ & $2.09 E-18$ & $2.09 \mathrm{E}-18$ & $2.09 E-18$ \\
\hline na 24 & $4.75 E-09$ & $4.64 E-09$ & $4.53 \mathrm{E}-09$ & $4.32 \mathrm{E}-09$ & $3 \mathrm{E}-09$ & $3.58 \mathrm{E}-09$ & 3. $25 \mathrm{E}-09$ & $2.96 \mathrm{E}-09$ & $2.69 E-09$ & $2.45 \mathrm{E}-09$ & $2.23 \mathrm{E}-09$ \\
\hline na 25 & $2.02 E-10$ & $4 E-19$ & $3 \mathrm{~F}-28$ & $O E+0 O$ & $O E+00$ & $0.00 \mathrm{E}+00$ & $0.00 E+00$ & $.00 \mathrm{E}+00$ & $0.00 \mathrm{E}+00$ & $0.00 E+00$ & $0.00 E+00$ \\
\hline mg 27 & $5 E-08$ & $9 E 09$ & 5. & & & & & E-27 & & $8.36 E-35$ & $0 E+00$ \\
\hline al 28 & E-07 & $3 E-11$ & $E-15$ & $4.08 \mathrm{E}-23$ & $E-28$ & $0 E-29$ & -29 & $E-29$ & $7.87 \mathrm{E}-29$ & $6 E-29$ & $6.89 E-29$ \\
\hline al 29 & & & & & & & & & & & \\
\hline si 31 & 2.7 & E-10 & 2.1 & $1.61 \mathrm{E}-10$ & & 5.6 & & & $E-11$ & $6.76 \mathrm{E}-12$ & $9 \mathrm{E}-12$ \\
\hline cl 36 & 1.3 & 1.3 & & & & & & & & & \\
\hline ar 37 & 5.7 & 5.7 & & & & & & & & & \\
\hline ar 39 & 6.5 & 6.5 & & $E-13$ & & & & & & & -13 \\
\hline ar 41 & & 7. & & -12 & & & & & & & \\
\hline k 40 & 1.1 & 1.1 & & E-08 & & & & & & -08 & -08 \\
\hline k 42 & 7.5 & 7.3 & & -10 & & & & & & 3. & \\
\hline $\mathrm{k} 43$ & 1.3 & 1.2 & & & & & & & & & \\
\hline k 44 & 2.2 & 8.82 & & $z-13$ & & & & & & & \\
\hline ca 41 & 1.6 & 1. & & & & & & & & & \\
\hline $\operatorname{ca} 45$ & 13 & 2 & & -13 & & & & & & & 2 . \\
\hline ca 47 & 8.6 & 8.5 & & & & 8.2 & & & & & \\
\hline ca 49 & & 1.1 & & -16 & & 4.4 & & & & +00 & +00 \\
\hline sc 47 & 2.93 & $3.29 \mathrm{E}-16$ & & 4.3 & & $7.05 \mathrm{E}-16$ & & 9.5 & & $E-15$ & -15 \\
\hline sc 49 & & & & & & & & & & & \\
\hline cr 51 & & & & & & & & & & & -12 \\
\hline cr 55 & & & & & & & & & & & \\
\hline & & & & -12 & -12 & & & & & 8.7 & 8.7 \\
\hline $\mathrm{mn} 56$ & & & & & & & & & & & \\
\hline $\mathrm{mn} 57$ & 2.6 & $1.63 \mathrm{E}-$ & & $i+00$ & $E+00$ & $8+00$ & & & & & +00 \\
\hline fe 55 & & $5.04 \mathrm{E}-14$ & & $5.04 \mathrm{E}-14$ & & & & & & & \\
\hline fe 59 & $3.44 \mathrm{E}-14$ & 3. $44 \mathrm{E}-14$ & & $3.43 E-14$ & 3. $43 \mathrm{E}-14$ & $3.42 \mathrm{E}-14$ & & 3.41 & 3.4 & $E-14$ & 3. $40 \mathrm{E}-14$ \\
\hline , & $6.58 \mathrm{E}-07$ & $2.55 E-08$ & $1.95 \mathrm{E}-08$ & $1.82 \mathrm{E}-08$ & $1.71 \mathrm{E}-08$ & $1.64 \mathrm{E}-08$ & $1.58 \mathrm{E}-08$ & $1.53 E-08$ & $1.49 \mathrm{E}-08$ & $1.46 \mathrm{E}-08$ & $1.43 E-08$ \\
\hline
\end{tabular}


Table F17. Radioactivity (Ci) After Ten-Day Irradiation of 1-kg Concrete (Flux Parameter Set 1).

\begin{tabular}{|c|c|c|c|c|c|c|c|c|c|c|c|}
\hline & & & & & After & harge & & & & & \\
\hline & initial & & $1.0 \mathrm{hr}$ & $2.0 \mathrm{hr}$ & $4.0 \mathrm{~h}$ & $6.0 \mathrm{hr}$ & $8.0 \mathrm{hr}$ & & & & $16.0 \mathrm{hr}$ \\
\hline 3 & & $.64 \mathrm{E}-18$ & & $2.64 E-18$ & & $2.63 \mathrm{E}-18$ & $2.63 E-18$ & $2.63 E-18$ & $2.63 \mathrm{E}-18$ & $2.63 \mathrm{E}-18$ & $2.63 \mathrm{E}-18$ \\
\hline 10 & $.17 E-19$ & $.17 \mathrm{E}-19$ & $.17 \mathrm{E}-19$ & $1.17 \mathrm{E}-19$ & 1. $17 \mathrm{E}-19$ & $1.17 \mathrm{E}-19$ & $1.17 \mathrm{E}-19$ & $1.17 E-19$ & & $1.17 \mathrm{E}-19$ & 1.1 \\
\hline & & & & $09 \mathrm{E}-17$ & & & & $2.09 E-17$ & 2. .09E-17 & 2.09E-17 & $2.09 \mathrm{E}-17$ \\
\hline a 24 & OE-09 & $6.83 E-09$ & & $37 E-09$ & $5.79 \mathrm{E}-09$ & $5.27 \mathrm{E}-09$ & 4.79E-09 & $6 \mathrm{E}-09$ & $3.97 E-09$ & $3.61 E-09$ & 3. $28 E-09$ \\
\hline 25 & $E-10$ & -19 & $E-28$ & $0 E+00$ & $0.00 E+00$ & 0. & $10 E+00$ & & & $E+00$ & $0.00 E+00$ \\
\hline 27 & $z-08$ & & -10 & $8 E-12$ & & & $10 E-23$ & & & & $0.00 E+00$ \\
\hline 28 & & & & $E-23$ & & 1 . & & & & & $6 E-28$ \\
\hline 29 & & & & & & & & & 00 & $E+00$ & $0.00 E+00$ \\
\hline 31 & & & & & & & & & & & \\
\hline 3 & & 1.3 & & & & & & & & & -15 \\
\hline 37 & & & & & & & & & $8-09$ & & -09 \\
\hline 39 & 5 & & & -12 & & & & & & & \\
\hline $\operatorname{ar}$ & & & & & & & & & & & -14 \\
\hline 40 & .1 & & & -08 & & & & & & & \\
\hline$k$ & .0 & & & & & & & & & & \\
\hline $\mathrm{k}$ & & & & & & & & & & & \\
\hline 44 & 2.2 & 8.8 & & $z-13$ & & & & & & & \\
\hline & & & & & & & & & & & \\
\hline 4 & 2.1 & 2.1 & 2.1 & 2. & & & & & & & \\
\hline 4 & & & & & & & & & & & \\
\hline ca 49 & & & & & & & & & & & \\
\hline SC 4 & & & & 2.5 & & & & & & & \\
\hline 49 & & & & & & & & & & & \\
\hline 51 & 3.1 & & & & & $E-11$ & & & & & \\
\hline 55 & 8.9 & & & & & & & & & & \\
\hline & 8.67 & & & & & & & & & & \\
\hline & 2.18 & $1.91 \mathrm{E}-0$ & 1.6 & $E-09$ & & & & & & & \\
\hline & & & & & & & & & & & $0.00 \mathrm{E}+00$ \\
\hline & & & 5.0 & 5.0 & & & & & & & \\
\hline & & & & & & & & & & & $3.17 \mathrm{E}-13$ \\
\hline & 65 & & & -08 & & $2.31 \mathrm{E}-08$ & $2.23 \mathrm{E}-08$ & $2.17 \mathrm{E}-08$ & $2.11 \mathrm{E}-08$ & $2.07 E-08$ & $2.03 \mathrm{E}-08$ \\
\hline
\end{tabular}


Table F18. Radioactivity (Ci) After One-Day Irradiation of 1-kg Concrete (Flux Parameter Set 2).

\begin{tabular}{|c|c|c|c|c|c|c|c|c|c|c|c|}
\hline & & & & & & & & & & & \\
\hline & initial & .5 hr & $1.0 \mathrm{hr}$ & $2.0 \mathrm{hr}$ & $4.0 \mathrm{hr}$ & $6.0 \mathrm{hr}$ & 8.0 hir & $10.0 \mathrm{hr}$ & $12.0 \mathrm{hr}$ & $14.0 \mathrm{hr}$ & $16.0 \mathrm{~F}$ \\
\hline h 3 & & $.65 \mathrm{E}-18$ & & $.65 E-18$ & $2.65 E-18$ & $2.65 E-18$ & $2.65 E-18$ & $2.65 \mathrm{E}-18$ & $2.65 \mathrm{E}-18$ & $2.65 E-18$ & \\
\hline be 10 & $.17 \mathrm{E}-20$ & $.17 \mathrm{E}-20$ & $8.1 \mathrm{E}-20$ & $.17 E-20$ & $8.17 E-20$ & $8.17 E \quad 20$ & $8.17 E-20$ & $8.17 \mathrm{E}-20$ & $8.17 \mathrm{E}-20$ & $8.17 \mathrm{E}-20$ & $8.17 \mathrm{E}-20$ \\
\hline C 14 & & & & $.00 \mathrm{E}-17$ & $2.00 E-17$ & $2.00 E-17$ & $2.00 \mathrm{E}-17$ & $00 \mathrm{E}-17$ & & $2.00 \mathrm{E}-17$ & $0 E-17$ \\
\hline na 24 & $2 E-08$ & $.43 F-08$ & $.35 E-08$ & $.20 E-08$ & $2.91 E-08$ & $2.65 E-08$ & $2.41 \mathrm{E}-08$ & $19 \mathrm{E}-08$ & $.99 E-08$ & $1.81 \mathrm{E}-08$ & \\
\hline na 25 & & $4 \mathrm{E}-18$ & & $0 E+00$ & $0.00 \mathrm{E}+00$ & $E+00$ & $0.00 \mathrm{E}+00$ & $0 \mathrm{E}+00$ & $0 \mathrm{E}+00$ & $0.00 \mathrm{E}+00$ & $0.00 \mathrm{E}+00$ \\
\hline $\mathrm{mg} 27$ & & & & & & & & & & & \\
\hline 28 & & & & $2-22$ & & & & & & & $E-27$ \\
\hline a) 29 & & & & & & & & & & & \\
\hline si 31 & & & & & & & & & & & \\
\hline cl 36 & & & 16 & -16 & & 9.5 & & & & & \\
\hline $\operatorname{ar} 37$ & & & & & & & & & & & \\
\hline ar 39 & & & & ; -12 & & & & & & & \\
\hline ar 41 . & & & & -11 & & & & & & & \\
\hline 40 & & & & -08 & & 1.1 & & & & & \\
\hline 42 & & & & -09 & & 4.1 & & & & & \\
\hline k 43 & & & & & & & & & & & \\
\hline k 44 & & & 11 & -12 & & 2.0 & & & & & \\
\hline $\mathrm{ca} 41$ & & & & & & & & & & & \\
\hline ca 45 & & & & & & & & & & & \\
\hline ca 47 & & & & & & & & & & & \\
\hline ca 49 & & & & & & & & & & & \\
\hline sc 47 & & & & & & & & & & & \\
\hline sc 49 & & & & & & & & & & & \\
\hline cr 51 & & & & & & & & & & & \\
\hline cr 55 & & & & -23 & & 0.0 & & & & & .00 \\
\hline $\mathrm{mn} 54$ & & & & $2-11$ & & & & & & & \\
\hline $\mathrm{mn} 56$ & 1.5 & E-08 & & $9 F-09$ & & & & & & & \\
\hline $\mathrm{mn} 57$ & & & & & & & & & & & \\
\hline $\mathrm{fe}$ & & & & 1 & & 5.15 & & & & & \\
\hline & & & & & & & & & & 4.5 & $4.57 \mathrm{E}-13$ \\
\hline & & .07 & & $.37 \mathrm{E}-08$ & & $4.98 E-0$ & & $4.19 E-08$ & $3.90 \mathrm{E}-08$ & $3.65 \mathrm{E}-08$ & $3.44 E-0$ \\
\hline
\end{tabular}


Table F19. Radioactivity (Ci) After Ten-Day Irradiation of 1-kg Concrete (Flux Parameter Set 2).

\begin{tabular}{|c|c|c|c|c|c|c|c|c|c|c|c|c|}
\hline & & & & & & & & & & & & \\
\hline & & initial & $.5 \mathrm{hr}$ & $1.0 \mathrm{hr}$ & $2.0 \mathrm{hr}$ & & $6.0 \mathrm{hr}$ & $8.0 \mathrm{hr}$ & $10.0 \mathrm{hr}$ & & & \\
\hline & 3 & $2.65 \mathrm{E}-17$ & $2.65 \mathrm{E}-17$ & $2.65 \mathrm{E}-17$ & $2.65 E-17$ & $2.65 \mathrm{E}-17$ & 2. $65 \mathrm{~F}-17$ & $2.65 \mathrm{E}-17$ & $2.65 \mathrm{E}-17$ & $5 E-17$ & $55 E-17$ & $2.65 E-17$ \\
\hline & 10 & $.17 \mathrm{E}-19$ & $8.17 \mathrm{E}-19$ & $8.17 \mathrm{E}-19$ & $8.17 \mathrm{E}-19$ & $8.17 \mathrm{E}-19$ & $8.17 \mathrm{E}-19$ & $17 \mathrm{E}-19$ & & & & \\
\hline$c$ & & & & $2.00 \mathrm{E}-16$ & $2.00 \mathrm{E}-16$ & $2.00 \mathrm{E}-16$ & $2.00 E-16$ & $00 E-16$ & $2.00 \mathrm{E}-16$ & $00 E-16$ & $0 \mathrm{E}-$ & $E-$ \\
\hline na & 24 & 5.1 & & & & $4.29 \mathrm{E}-08$ & & & $3.23 \mathrm{E}-08$ & 2. & & \\
\hline na & 25 & 1.41 & $1.14 \mathrm{E}-18$ & & $0.00 E+00$ & $0.00 \mathrm{E}+00$ & $0.00 \mathrm{E}+00$ & $0.00 E+00$ & $0.00 \mathrm{E}+00$ & $00 \mathrm{E}+00$ & & \\
\hline $\mathrm{mg} 2$ & 27 & & & & . & 7.85 & & & & & & \\
\hline al & 28 & 3.8 & & & $36 E-22$ & & & & -27 & 5. & & \\
\hline al & 29 & 1.3 & 5.4 & 2.3 & $E-13$ & & & & & & & \\
\hline si & 31 & 3.6 & & & & & & & & & & \\
\hline cl & 36 & 9.5 & & & & & & & & & & \\
\hline ar & 37 & & & & -08 & 3.6 & 3. & & 3 . & & & \\
\hline ax & & & & & & & & & & & & \\
\hline ar 4 & 11 & 6.5 & & 4.4 & -11 & 1.4 & -12 & -12 & -12 & & & \\
\hline $\mathrm{k}$ & 40 & & & & & & & & & & & \\
\hline $\mathrm{k}$ & 12 & 7.8 & 7.8 & 7.4 & & & & & & & & \\
\hline $\mathrm{k}$ & 7 & 1.7 & & & & & & & & & & \\
\hline$k$ & 14 & 1.5 & & 2.4 & & & & & & & & \\
\hline $\mathrm{ca}$ & 11 & 1.6 & & 1.6 & & & & & & & & \\
\hline $\mathrm{ca}$ & & & & & & & & & & & & \\
\hline ca 4 & 17 & 4.5 & & 4.5 & -13 & & & & & & & \\
\hline $\mathrm{ca}$ & 19 & & & & & & & & & & & \\
\hline $\mathrm{sc}$ & 47 & 2.83 & & & 2.8 & & & & & & & \\
\hline$s c$ & 49 & & & & & & & & & & & \\
\hline $\mathrm{Cr}$ & 51 & 2.2 & 2.2 & 2.2 & 2.2 & & & & & & & \\
\hline & & & & & & & & & & & & \\
\hline $\mathrm{mn} 5$ & 4 & 6.0 & & 6.06 & & & & & & & & $E-10$ \\
\hline & 6 & & & & & & & & & & & \\
\hline & & & & & & & & & & & & \\
\hline & & & & & & & & & & & & \\
\hline & & & & & & & & & & & & \\
\hline & & $4.60 \mathrm{E}-0.6$ & $1.68 \mathrm{E}-07$ & $1.25 \mathrm{E}-07$ & $1.14 \mathrm{E}-07$ & $1.05 E-07$ & $9.75 \mathrm{E}-08$ & $.1 / 2-$ & & & $7.95 E-08$ & \\
\hline
\end{tabular}


Table F20. Radioactivity (Ci) After One-Day Irradiation of 1-kg Aluminum 6061-T6 (Flux Parameter Set 1).

\begin{tabular}{|c|c|c|c|c|c|c|c|c|c|c|c|}
\hline & & & & & & & & & & & \\
\hline & initial & & & $2.0 \mathrm{hr}$ & & $6.0 \mathrm{hr}$ & $8.0 \mathrm{hr}$ & $10.0 \mathrm{hr}$ & & $14.0 \mathrm{hr}$ & $16.0 \mathrm{hr}$ \\
\hline 24 & $9.17 \mathrm{E}-08$ & $8.96 \mathrm{E}-08$ & $8.75 E-08$ & $8.35 E-08$ & $7.59 \mathrm{E}-08$ & $6.91 E-08$ & $6.28 \mathrm{E}-08$ & $5.72 \mathrm{E}-08$ & $5.20 E-08$ & $4.73 E-08$ & $4.31 \mathrm{E}-08$ \\
\hline 25 & $1.01 \mathrm{E}-09$ & $8.18 \mathrm{E}-19$ & $6.63 E-28$ & $0.00 \mathrm{E}+00$ & $0.00 E+00$ & $0.00 \mathrm{E}+00$ & $0.00 E+00$ & $0.00 \mathrm{E}+00$ & $0.00 \mathrm{E}+00$ & $0.00 \mathrm{E}+00$ & $0.00 \mathrm{E}+00$ \\
\hline $\mathrm{mg} 27$ & $1.37 \mathrm{E}-06$ & $1.52 \mathrm{E}-07$ & $1.69 \mathrm{E}-08$ & $2.08 \mathrm{E}-10$ & $3.16 \mathrm{E}-14$ & $4.81 \mathrm{E}-18$ & 7. $32 \mathrm{E}-22$ & $1.11 \mathrm{E}-25$ & $1.69 \mathrm{E}-29$ & $2.56 \mathrm{E}-33$ & $0.00 \mathrm{E}+00$ \\
\hline al 28 & $6.88 \mathrm{E}-08$ & $6.40 E-12$ & $5.95 \mathrm{E}-16$ & $5.15 \mathrm{E}-24$ & $2.89 E-27$ & $2.70 E-27$ & $2.53 E-27$ & $2.37 E-27$ & $2.22 \mathrm{E}-27$ & $2.07 E-27$ & $1.94 \mathrm{E}-27$ \\
\hline al 29 & 3. $32 \mathrm{E}-10$ & $1.40 \mathrm{E}-11$ & $5.89 \mathrm{E}-13$ & $1.05 \mathrm{E}-15$ & $3.30 \mathrm{E}-21$ & $1.04 E-26$ & $3.28 \mathrm{E}-32$ & $0.00 \mathrm{E}+00$ & $0.00 \mathrm{E}+00$ & $0.00 \mathrm{E}+00$ & $0.00 \mathrm{E}+00$ \\
\hline si 31 & 4. $88 \mathrm{E}-12$ & 4. $27 \mathrm{E}-12$ & $3.74 E-12$ & $2.87 \mathrm{~F}-12$ & 1. $69 \mathrm{~F}-12$ & & $5.88 \mathrm{E}-13$ & $3.47 \mathrm{~F}-13$ & & 1. 20F-13 & $7.10 \mathrm{~F}-14$ \\
\hline ca 45 & $4.43 \mathrm{E}-15$ & $4.42 \mathrm{E}-15$ & $4.42 E-15$ & $4.42 \mathrm{E}-15$ & $4.42 \mathrm{E}-15$ & $4.42 \mathrm{E}-15$ & $4.42 \mathrm{E}-15$ & $4.42 \mathrm{E}-15$ & $4.42 \mathrm{E}-15$ & $4.41 \mathrm{E}-15$ & $4.41 E-15$ \\
\hline ca 47 & $4.01 \mathrm{E}-16$ & $4.00 \mathrm{E}-16$ & $3.99 \mathrm{E}-16$ & $3.96 \mathrm{E}-16$ & $3.91 \mathrm{E}-16$ & $3.86 \mathrm{E}-16$ & $3.81 \mathrm{E}-16$ & $3.77 \mathrm{E}-16$ & $3.72 \mathrm{E}-16$ & $3.67 \mathrm{E}-16$ & $3.62 \mathrm{E}-16$ \\
\hline sc 46 & $1.12 \mathrm{E}-12$ & 1. $12 \mathrm{E}-12$ & 1. $12 \mathrm{E}-12$ & 1. $12 \mathrm{E}-12$ & 1. $12 \mathrm{E}-12$ & 1. $12 \mathrm{E}-12$ & 1. $12 \mathrm{E}-12$ & $1.12 \mathrm{E}-12$ & 1.121 & 1.12 & $2 E-12$ \\
\hline sc 47 & $4.22 \mathrm{E}-11$ & $4.20 \mathrm{E}-11$ & $4.18 \mathrm{E}-11$ & $4.14 \mathrm{E}-11$ & $4.07 E-11$ & $4.00 \mathrm{E}-11$ & $3.93 E-1 I$ & $7 E-11$ & 3. & $E-11$ & $E-11$ \\
\hline sc 48 & $7.11 \mathrm{E}-12$ & $7.05 \mathrm{E}-12$ & $7.00 \mathrm{E}-12$ & $6.89 \mathrm{E}-12$ & & & & & & & -12 \\
\hline sc 49 & $4.78 \mathrm{E}-11$ & 3. $32 \mathrm{E}-11$ & $2.31 \mathrm{E}-11$ & $1.12 \mathrm{E}-11$ & $2.61 E-12$ & $6.09 \mathrm{E}-13$ & $1.42 \mathrm{E}-13$ & $E-14$ & E-15 & $1 E-15$ & $3 E-16$ \\
\hline sc 50 & $1.00 \mathrm{E}-12$ & $5.18 \mathrm{E}-18$ & & $1.72 \mathrm{E}-34$ & $0.00 \mathrm{E}+00$ & $0.00 \mathrm{E}+00$ & & & & $E+00$ & $0 E+00$ \\
\hline ti 51 & $2.55 \mathrm{E}-12$ & $6.89 E-14$ & & $1.36 \mathrm{E}-18$ & $9 E-25$ & 31 & $O E+00$ & $E+00$ & 0 . & +00 & $E+00$ \\
\hline V 52 & $3.61 \mathrm{E}-10$ & 1. $41 \mathrm{E}-12$ & $5.50 \mathrm{E}-15$ & $8.40 \mathrm{E}-20$ & 1. $95 \mathrm{E}-29$ & +00 & & $E+00$ & & $8+00$ & $E+00$ \\
\hline v 53 & $4.46 \mathrm{E}-12$ & 1. $10 \mathrm{E}-17$ & 2.7 & $1.64 \mathrm{E}-34$ & $E+00$ & $2+00$ & +00 & $0 \mathrm{E}+00$ & 0.0 & +00 & +00 \\
\hline cr 51 & $3.22 \mathrm{E} 12$ & $3.22 \mathrm{E}-12$ & 3.2 & $3.21 \mathrm{E}-12$ & $g-12$ & $e-12$ & $E-12$ & -12 & 12 & -12 & -12 \\
\hline cr 55 & $1.37 E-10$ & $3.57 \mathrm{E}-13$ & $3 E-16$ & $6.37 \mathrm{E}-21$ & $3 E-31$ & $0.00 \mathrm{E}+00$ & +00 & $8+00$ & 0. & +00 & +00 \\
\hline $\mathrm{mn} 54$ & $4.38 E-12$ & $4.38 \mathrm{E}-12$ & -12 & 4. $38 \mathrm{E}-12$ & $E-12$ & -12 & -12 & & & -12 & -12 \\
\hline mn 56 & 3.65 & $3.19 \mathrm{E}-09$ & $2.79 \mathrm{E}-09$ & $2.13 \mathrm{E}-09$ & $1.25 \mathrm{E}-09$ & -10 & $4.25 \mathrm{E}$ & -10 & 1.4 & -11 & $4.95 \mathrm{E}-11$ \\
\hline $\mathrm{mn} 57$ & 1.3 & $8.14 \mathrm{~F}$ & & $0.00 \mathrm{E}+00$ & 0.0 & & & & & & +00 \\
\hline $\mathrm{fe}$ & 2.52 & .52 & 2.5 & $E-14$ & $e-14$ & 2.5 & -14 & -14 & 2. & -14 & $2 \mathrm{E}-14$ \\
\hline fe 59 & & $1.72 \mathrm{~F}$ & & 1.7 & & & & & & & \\
\hline Co 60 & 3.12 & 3. $12 \mathrm{E}-14$ & 3.12E-14 & $3.12 \mathrm{E}-14$ & $2 \mathrm{E}-14$ & & $E-14$ & 3. & 3. & -14 & $E-14$ \\
\hline co 62 & $2.42 \mathrm{E}-11$ & $2.30 \mathrm{E}-17$ & -23 & $0.00 \mathrm{E}+00$ & $0.00 \mathrm{E}+00$ & $0.00 \mathrm{E}+00$ & +00 & & & +00 & $E+00$ \\
\hline ni 63 & $2.06 \mathrm{~b}-14$ & $2.06 \mathrm{E}-14$ & $2.06 \mathrm{E}-14$ & $2.06 \mathrm{E}-14$ & $2.06 \mathrm{E}-14$ & $2.06 \mathrm{E}-14$ & & 2. & & -14 & ti-14 \\
\hline ni 65 & $5.17 \mathrm{E}-11$ & $4.50 E-11$ & $3.93 E-11$ & $2.98 \mathrm{E}-11$ & $1.72 \mathrm{E}-11$ & $9.92 \mathrm{E}-12$ & 5.72 & -12 & 1.91 & 1.1 & $6.34 \mathrm{E}-13$ \\
\hline cu 64 & $4.18 \mathrm{E}-10$ & $4.07 \mathrm{E}-10$ & $3.96 \mathrm{E}-10$ & $3.75 E-10$ & 3. $36 \mathrm{E}-10$ & & & & & & \\
\hline $\mathrm{Cu}$ & 2.31 & 3. $91 \mathrm{E}-12$ & 6.6 & $1.90 \mathrm{E}-17$ & $1.57 \mathrm{E}-$ & $E-28$ & $3.85 \mathrm{E}-28$ & 3.7 & 3. & 3.5 & -28 \\
\hline zn 65 & 1. $36 \mathrm{E}-13$ & $1.36 \mathrm{E}-13$ & 1.36 & $1.36 \mathrm{E}-13$ & $1.36 \mathrm{E}-13$ & & & & & & -13 \\
\hline $\operatorname{zn} 69$ & $9.66 \mathrm{E}-11$ & $6.75 \mathrm{E}-11$ & 4.731 & $2.37 E-11$ & $6.90 \mathrm{E}-12$ & $2.92 \mathrm{E}-12$ & 1.88 & 1.53 & 1.34 & 1.20 & $E-12$ \\
\hline zn $69 \mathrm{~m}$ & 2. $2.7 \mathrm{~F}-12$ & 2. $21 \mathrm{E}-12$ & 2. $16 \mathrm{~F}-12$ & $2.05 \mathrm{E}-12$ & 1. $85 \mathrm{E}-12$ & 1. $68 \mathrm{E}-12$ & 1. $52 \mathrm{~F}-12$. & $1.37 \mathrm{~F}-12$ & & & $1.01 \mathrm{E}-12$ \\
\hline 71 & $1.04 \mathrm{E}-13$ & $2.13 \mathrm{E}-17$ & $4.39 \mathrm{E}-21$ & $1.87 E-28$ & $0.00 \mathrm{E}+00$ & $0.00 \mathrm{E}+00$ & $0.00 \mathrm{E}+00$ & $0.00 \mathrm{E}+00$ & $0.00 E+00$ & $0.00 \mathrm{E}+00$ & $0.00 \mathrm{E}+00$ \\
\hline & & $7.49 \mathrm{E}-15$ & & $5.76 E-15$ & & & & 1. $42 \mathrm{E}-15$ & 1.00E-15 & $7.06 \mathrm{E}-16$ & $4.97 \mathrm{E}-16$ \\
\hline & $1.54 \mathrm{E}-06$ & $2.45 \mathrm{E}-07$ & $1.08 \mathrm{E}-07$ & $8.63 E-08$ & $7.76 \mathrm{E}-08$ & $7.02 \mathrm{E}-08$ & $6.36 \mathrm{E}-08$ & $5.77 \mathrm{E}-08$ & $5.24 \mathrm{E}-08$ & $4.77 \mathrm{E}-08$ & $4.33 \mathrm{E}-08$ \\
\hline
\end{tabular}


Table F21. Radioactivity (Ci) After Ten-Day Irradiation of 1-kg Aluminum 6061-T6 (Flux Parameter Set 1).

\begin{tabular}{|c|c|c|c|c|c|c|c|c|c|c|c|}
\hline & & & & Time & After Dis & harge & & & & & \\
\hline & initial & & & $2.0 \mathrm{hr}$ & $4.0 \mathrm{hr}$ & $6.0 \mathrm{hr}$ & $8.0 \mathrm{hr}$ & $10.0 \mathrm{hr}$ & $12.0 \mathrm{hr}$ & $14.0 \mathrm{hr}$ & $16.0 \mathrm{hr}$ \\
\hline na 24 & $1.35 \mathrm{E}-07$ & 1. $32 \mathrm{E}-07$ & 1. $29 \mathrm{E}-07$ & $1.23 \mathrm{E}-07$ & 1. $12 \mathrm{E}-07$ & $1.02 \mathrm{E}-07$ & $9.26 \mathrm{E}-08$ & $8.43 E-08$ & $7.67 \mathrm{E}-08$ & $6.97 \mathrm{E}-08$ & $6.34 \mathrm{E}-08$ \\
\hline na 25 & $1.01 \mathrm{E}-09$ & $8.18 E-19$ & $6.63 E-28$ & $0.00 \mathrm{E}+00$ & $0.00 \mathrm{E}+00$ & $0.00 \mathrm{E}+00$ & $0.00 \mathrm{E}+00$ & $0.00 \mathrm{E}+00$ & $0.00 \mathrm{E}+00$ & $0.00 E+00$ & $0.00 E+00$ \\
\hline $\mathrm{mg} 27$ & $1.37 \mathrm{E}-06$ & $1.52 \mathrm{E}-07$ & $1.69 \mathrm{E}-08$ & $2.08 \mathrm{E}-10$ & $3.16 \mathrm{E}-14$ & $4.81 \mathrm{E}-18$ & $7.32 \mathrm{E}-22$ & $1.11 \mathrm{E}-25$ & $1.69 \mathrm{E}-29$ & $2.56 \mathrm{E}-33$ & $0.00 \mathrm{E}+00$ \\
\hline al 28 & $6.88 E-08$ & $6.40 \mathrm{E}-12$ & $5.95 \mathrm{E}-16$ & $5.15 E-24$ & $5.26 \mathrm{E}-27$ & $4.92 \mathrm{E}-27$ & $4.61 \mathrm{E}-27$ & $4.31 \mathrm{E}-27$ & $4.04 E-27$ & $3.78 E-27$ & $3.54 \mathrm{E}-27$ \\
\hline al 29 & $3.32 \mathrm{E}-10$ & $1.40 \mathrm{E}-11$ & $5.89 \mathrm{E}-13$ & $1.05 E-15$ & $3.30 \mathrm{E}-21$ & $1.04 \mathrm{E}-26$ & $3.28 E-32$ & $0.00 E+00$ & $0.00 \mathrm{E}+00$ & $0.00 \mathrm{E}+00$ & $0.00 \mathrm{E}+00$ \\
\hline si 31 & $4.89 E-12$ & $4.28 \mathrm{E}-12$ & 3. $75 E-12$ & $2.88 \mathrm{E}-12$ & $1.70 \mathrm{E}-12$ & $1.00 \mathrm{E}-12$ & $5.89 E-13$ & $3.47 \mathrm{E}-13$ & $2.05 E-13$ & 1. $21 \mathrm{E}-13$ & $7.11 \mathrm{E}-14$ \\
\hline ca 45 & 4. $34 \mathrm{E}-14$ & $4.34 \mathrm{E}-14$ & $4.34 E-14$ & $4.34 \mathrm{E}-14$ & $4.34 \mathrm{E}-14$ & $4.34 \mathrm{E}-14$ & $4.34 E-14$ & $4.33 E-14$ & $4.33 E-14$ & $4.33 \mathrm{E}-14$ & $4.33 \mathrm{E}-14$ \\
\hline ca 47 & $2.22 \mathrm{E}-15$ & $2.21 \mathrm{E}-15$ & $2.20 \mathrm{E}-15$ & $2.19 \mathrm{E}-15$ & $2.16 \mathrm{E}-15$ & $2.13 E-15$ & $2.11 E-15$ & $2.08 \mathrm{E}-15$ & $2.05 \mathrm{E}-15$ & $2.03 \mathrm{E}-15$ & $2.00 \mathrm{E}-15$ \\
\hline sc 46 & $1.08 \mathrm{E}-11$ & 1. $08 \mathrm{E}-11$ & $1.08 \mathrm{E}-11$ & $1.08 \mathrm{E}-11$ & $1.08 \mathrm{E}-11$ & 1. $.08 \mathrm{E}-11$ & $1.08 \mathrm{E}-11$ & $1.08 \mathrm{E}-11$ & $1.08 \mathrm{E}-11$ & $1.08 \mathrm{E}-11$ & $1.08 \mathrm{E}-11$ \\
\hline sc 47 & $1.97 E-10$ & $1.96 \mathrm{E}-10$ & $1.95 E-10$ & $1.91 \mathrm{E}-10$ & $1.90 \mathrm{E}-10$ & $1.87 E-10$ & $1.84 \mathrm{E}-10$ & $1.81 \mathrm{E} \cdot 10$ & & $1.74 \mathrm{E}-10$ & $1.72 \mathrm{E}-10$ \\
\hline Sc 48 & $2.20 \mathrm{E}-11$ & $2.18 \mathrm{E}-11$ & $2.16 \mathrm{E}-11$ & $2.13 \mathrm{E}-11$ & $2.06 \mathrm{E}-11$ & $2.00 E-11$ & $1.93 \mathrm{E}-11$ & $1.87 \mathrm{E}-11$ & $1.81 \mathrm{E}-11$ & $1.76 \mathrm{E}-11$ & $1.70 \mathrm{E}-11$ \\
\hline sc 49 & $4.78 \mathrm{E}-11$ & 3. $32 \mathrm{E}-11$ & 2. $31 \mathrm{E}-11$ & $1.12 \mathrm{~F}-11$ & 2. $61 \mathrm{E}-12$ & $6.09 E-13$ & 1. $42 \mathrm{E}-13$ & $3.32 \mathrm{E}-14$ & $7.76 \mathrm{E}-15$ & $1.81 E-15$ & $4.23 \mathrm{E}-16$ \\
\hline sc 50 & $1.00 \mathrm{E}-12$ & $5.18 \mathrm{E}-18$ & $2.68 \mathrm{E}-23$ & $7.72 \mathrm{E}-34$ & $0.00 \mathrm{E}+00$ & $0.00 E+00$ & $0.00 E+00$ & $0.00 E+00$ & $0.00 \mathrm{E}+00$ & $0.00 \mathrm{E}+00$ & $E+00$ \\
\hline ti 51 & $2.55 \mathrm{E}-12$ & $6.89 \mathrm{E}-14$ & $1.86 E-15$ & 1. $36 \mathrm{E}-18$ & $7.29 \mathrm{E}-25$ & $3.90 E-31$ & $0.00 \mathrm{E}+00$ & $0.00 E+00$ & $0.00 \mathrm{E}+00$ & $0.00 \mathrm{E}+00$ & $0.00 \mathrm{E}+00$ \\
\hline v 52 & $3.61 E-10$ & $1.41 \mathrm{E}-12$ & $5.50 \mathrm{E}-15$ & $8.40 \mathrm{E}-20$ & $1.95 \mathrm{E}-29$ & $0.00 E+00$ & $0.00 \mathrm{E}+00$ & $0.00 \mathrm{E}+00$ & $0.00 \mathrm{E}+00$ & $0.00 \mathrm{E}+00$ & +00 \\
\hline v 53 & $4.46 \mathrm{E}-12$ & $1.10 \mathrm{E}-17$ & $2.70 \mathrm{E}-23$ & $1.64 \mathrm{E}-34$ & $0.00 \mathrm{E}+00$ & $0.00 E+00$ & $0.00 \mathrm{E}+00$ & $0.00 E+00$ & $0.00 \mathrm{E}+00$ & $0.00 \mathrm{E}+00$ & $+\infty 0$ \\
\hline 51 & $2.88 \mathrm{E}-11$ & $2.88 \mathrm{E}-11$ & $2.88 E-11$ & $2.88 \mathrm{E}-11$ & $2.87 \mathrm{E}-11$ & $2.87 \mathrm{E}-11$ & $2.86 E-11$ & $2.85 E-11$ & $2.85 \mathrm{E}-11$ & $2.84 \mathrm{E}-11$ & $E-11$ \\
\hline 55 & $1.37 E-10$ & $3.57 \mathrm{E}-13$ & $9.33 \mathrm{E}-16$ & $6.37 \mathrm{E}-21$ & $2.98 \mathrm{E}-31$ & $0.00 \mathrm{E}+00$ & $0.00 \mathrm{E}+00$ & $0.00 \mathrm{E}+00$ & $0.00 \mathrm{E}+00$ & $0.00 E+00$ & $0.00 E+00$ \\
\hline mai 5 & $4.34 E-11$ & $4.34 \mathrm{E}-11$ & $4.34 \mathrm{E}-11$ & $4.34 \mathrm{E}-11$ & $4.34 \mathrm{E}-11$ & $4.33 \mathrm{E}-11$ & $4.33 \mathrm{E}-11$ & $4.33 E-11$ & $4.33 \mathrm{E}-11$ & $8-11$ & $E-11$ \\
\hline $\mathrm{mn} 56$ & $3.66 E-09$ & $3.20 \mathrm{E}-09$ & $2.79 E-09$ & $2.14 \mathrm{E}-09$ & $1.25 \mathrm{E}-$ & $7.29 \mathrm{E}-10$ & $4.26 \mathrm{E}-10$ & $2.49 E-10$ & $1.45 \mathrm{E}-10$ & $8.48 \mathrm{E}-11$ & $4.96 \mathrm{E}-11$ \\
\hline $\mathrm{mn} 57$ & $1.33 E-11$ & $8.14 \mathrm{E}-18$ & $4.97 E-24$ & $0.00 \mathrm{E}+00$ & $0.00 \mathrm{E}+00$ & $0.00 E+00$ & $0.00 \mathrm{E}+00$ & $0.00 \mathrm{E}+00$ & 0.00 & +00 & $E+b 0$ \\
\hline fe 55 & $2.51 \mathrm{E}-13$ & $2.51 \mathrm{E}-13$ & $2.51 \mathrm{E}-13$ & $2.51 E-13$ & $2.51 \mathrm{E}-13$ & $2.51 E-13$ & $2.51 \mathrm{E}-13$ & $2.51 E-13$ & $2.51 \mathrm{E}-13$ & $2.51 \mathrm{E}-13$ & $2.51 E-13$ \\
\hline fe 59 & $1.60 E-13$ & $1.60 \mathrm{E}-13$ & $1.60 \mathrm{E}-13$ & $1.60 \mathrm{E}-13$ & $1.60 \mathrm{E}-13$ & $1.60 E-13$ & $1.60 \mathrm{E}-13$ & $1.59 \mathrm{E}-13$ & $1.59 \mathrm{E}-13$ & 1.59 & -13 \\
\hline co 60 & 3. $12 \mathrm{E}-13$ & $3.12 \mathrm{E}-13$ & $3.12 \mathrm{E}-13$ & 3. $12 \mathrm{E}-13$ & $3.12 \mathrm{E}-13$ & $3.12 \mathrm{E}-13$ & $3.12 \mathrm{E}-13$ & $3.12 \mathrm{E}-13$ & $3.12 \mathrm{E}-13$ & $3.12 \mathrm{E}-13$ & $3.12 \mathrm{E}-13$ \\
\hline co 62 & $2.42 \mathrm{E}-11$ & $2.30 \mathrm{E}-17$ & $2.20 \mathrm{E}-23$ & $0.00 E+00$ & $0.00 \mathrm{E}+00$ & $0.00 \mathrm{~F}+00$ & $0.00 \mathrm{E}+00$ & +00 & $0.00 \mathrm{E}+00$ & $0.00 E+00$ & $0.00 E+00$ \\
\hline 63 & $2.06 E-13$ & $2.06 \mathrm{E}-13$ & $2.06 \mathrm{E}-13$ & $2.06 \mathrm{E}-13$ & $2.06 \mathrm{E}-13$ & $2.06 \mathrm{E}-13$ & $2.06 \mathrm{E}-13$ & $2.06 \mathrm{E}-13$ & $2.06 \mathrm{E}-13$ & $2.06 \mathrm{E}-13$ & $6 E-13$ \\
\hline ni 65 & $5.18 E-11$ & $4.51 \mathrm{E}-11$ & $3.93 E-11$ & $2.99 E-11$ & $1.72 \mathrm{E}-11$ & $9.94 \mathrm{E}-12$ & $5.73 \mathrm{E}-12$ & $3.31 E-12$ & $1.91 \mathrm{E}-12$ & 1. $10 \mathrm{E}-12$ & $6.35 E-13$ \\
\hline cu 64 & $5.73 E-10$ & $5.58 \mathrm{E}-10$ & $5.43 \mathrm{E}-10$ & $5.14 \mathrm{E}-10$ & $4.61 \mathrm{E}-10$ & $4.13 E-10$ & $3.70 \mathrm{E}-10$ & $3.32 \mathrm{E}-10$ & $2.98 \mathrm{E}-10$ & $2.67 \mathrm{E}-10$ & $2.39 \mathrm{E}-10$ \\
\hline cu 66 & $2.31 \mathrm{E}-10$ & $3.91 \mathrm{E}-12$ & $6.63 E-14$ & $1.90 \mathrm{E}-17$ & $1.57 \mathrm{E}-24$ & 1. $44 \mathrm{E}-27$ & $1.41 \mathrm{E}-27$ & $1.37 \mathrm{E}-27$ & $1.34 \mathrm{E}-27$ & $1.30 \mathrm{E}-27$ & $1.27 \mathrm{E}-27$ \\
\hline zn 65 & $1.34 \mathrm{E}-12$ & $1.34 \mathrm{E}-12$ & $1.34 \mathrm{E}-12$ & $1.34 \mathrm{E}-12$ & $1.34 \mathrm{E}-12$ & $1.34 \mathrm{E}-12$ & $1.34 \mathrm{E}-12$ & $1.34 E-12$ & 1. $34 \mathrm{E}-12$ & $1.34 \mathrm{E}-12$ & $1.34 \mathrm{E}-12$ \\
\hline $\operatorname{zn} 69$ & $9.76 \mathrm{E}-11$ & $6.85 E-11$ & $4.83 E-11$ & $2.46 \mathrm{E}-11$ & $7.75 E-12$ & $3.69 E-12$ & $2.57 \mathrm{E}-12$ & $2.15 E-12$ & $1.91 \mathrm{E}-12$ & $1.72 \mathrm{E}-12$ & $1.55 \mathrm{E}-12$ \\
\hline$z n 69 m$ & $3.23 \mathrm{E}-12$ & $3.15 \mathrm{E}-12$ & $3.07 \mathrm{E}-12$ & $2.92 \mathrm{E}-12$ & $2.64 \mathrm{E}-12$ & $2.39 \mathrm{E}-12$ & $2.16 \mathrm{E}-12$ & $1.95 \mathrm{E}-12$ & $1.77 \mathrm{E}-12$ & $1.60 \mathrm{E}-12$ & $1.44 \mathrm{E}-12$ \\
\hline zn 71 & 1.04E-13 & $2.13 \mathrm{E}-17$ & $4.39 \mathrm{E}-21$ & 1. $87 \mathrm{E}-28$ & $0.00 \mathrm{E}+00$ & $0.00 \mathrm{E}+00$ & $0.00 \mathrm{E}+00$ & $0.00 \mathrm{E}+00$ & $0.00 E+00$ & $0.00 E+00$ & $0.00 E+00$ \\
\hline & $8.30 E \cdot 15$ & $7.61 E-15$ & $6.97 \mathrm{E}-15$ & $5.85 E-15$ & $4.12 \mathrm{E}-15$ & $2.91 E-15$ & $2.05 \mathrm{E}-15$ & 1. $44 \mathrm{E}-15$ & $1.02 E-15$ & $7.17 E-16$ & $5.05 \mathrm{E}-16$ \\
\hline & $1.58 E-06$ & $2.88 \mathrm{E}-07$ & $1.50 \mathrm{E}-07$ & $1.26 \mathrm{E}-07$ & $1.14 \mathrm{E}-07$ & $1.03 \mathrm{E}-07$ & $9.37 \mathrm{E}-08$ & $8.51 E-08$ & $7.74 \mathrm{E}-08$ & $7.04 \mathrm{E}-08$ & $6.40 \mathrm{E}-08$ \\
\hline
\end{tabular}


Table F22. Radioactivity (Ci) After One-Day Irradiation of 1-kg Aluminum 6061-T6 (Flux Parameter Set 2).

\begin{tabular}{|c|c|c|c|c|c|c|c|c|c|c|c|c|}
\hline & & & & & & & & & & & & \\
\hline & & initial & $.5 \mathrm{hr}$ & $1.0 \mathrm{hr}$ & $2.0 \mathrm{hr}$ & $4.0 \mathrm{hr}$ & $6.0 \mathrm{hr}$ & $8.0 \mathrm{hr}$ & $10.0 \mathrm{hr}$ & $12.0 \mathrm{hr}$ & $14.0 \mathrm{hr}$ & $16.0 \mathrm{hr}$ \\
\hline na & 24 & $6.41 \mathrm{E}-07$ & $6.26 E-07$ & $6.12 \mathrm{E}-07$ & $5.83 E-07$ & $5.31 E-07$ & $4.83 E-07$ & $4.39 \mathrm{E}-07$ & $4.00 \mathrm{E}-07$ & $3.64 \mathrm{E}-07$ & $3.31 \mathrm{E}-07$ & $3.01 \mathrm{E}-07$ \\
\hline na & 25 & $7.06 \mathrm{E}-09$ & $5.72 \mathrm{E}-18$ & $4.63 E-27$ & $0.00 \mathrm{E}+00$ & $0.00 \mathrm{E}+00$ & $0.00 E+00$ & $0.00 \mathrm{E}+00$ & $0.00 \mathrm{E}+00$ & $0.00 \mathrm{E}+00$ & $0.00 E+00$ & $0.00 \mathrm{E}+00$ \\
\hline $\mathrm{mg}$ & 27 & $9.56 E-06$ & $1.06 \mathrm{E}-06$ & $1.18 \mathrm{E}-07$ & $1.45 \mathrm{E}-09$ & $2.21 \mathrm{E}-13$ & $3.36 \mathrm{E}-17$ & $5.11 \mathrm{E}-21$ & $7.78 \mathrm{E}-25$ & $1.18 \mathrm{E}-28$ & $1.80 E-32$ & $0.00 \mathrm{E}+00$ \\
\hline a1. & 2.8 & $7.59 \mathrm{E}-07$ & $7.05 \mathrm{E}-11$ & $6.56 \mathrm{E}-15$ & $5.68 \mathrm{E}-2.3$ & $1.02 \mathrm{E}-2.5$ & $9.59 F-26$ & $8.97 F-26$ & $8.40 E-26$ & $7.86 \mathrm{E}-26$ & $7.35 E-26$ & $6.88 \mathrm{E}-26$ \\
\hline al & 29 & $2.32 \mathrm{E}-09$ & $9.77 \mathrm{E}-11$ & $4.12 \mathrm{E}-12$ & $7.31 \mathrm{E}-15$ & $2.31 \mathrm{E}-20$ & $7.28 \mathrm{E}-26$ & $2.30 \mathrm{E}-31$ & $0.00 E+00$ & $0.00 \mathrm{E}+00$ & $0.00 E+00$ & $0.00 \mathrm{E}+00$ \\
\hline si & 31 & $6.43 \mathrm{E}-11$ & $5.64 \mathrm{E}-11$ & $4.94 \mathrm{E}-11$ & $3.79 \mathrm{E}-11$ & $2.23 \mathrm{E}-11$ & 1. $32 \mathrm{E}-11$ & $7.76 \mathrm{E}-12$ & $4.57 E-12$ & $2.70 \mathrm{E}-12$ & & $9.36 \mathrm{E}-13$ \\
\hline $\mathrm{ca}$ & 45 & $3.09 \mathrm{E}-14$ & $3.09 \mathrm{E}-14$ & $3.09 \mathrm{E}-14$ & $3.09 E-14$ & $3.09 E-14$ & $3.09 \mathrm{E}-14$ & $3.09 \mathrm{E}-14$ & $3.09 \mathrm{E}-14$ & $3.09 \mathrm{E}-14$ & $3.09 E-14$ & $3.08 \mathrm{E}-14$ \\
\hline $\mathrm{ca}$ & 47 & $2.80 E-15$ & $2.80 \mathrm{E}-15$ & $2.79 E-15$ & $2.77 \mathrm{E}-15$ & $2.73 \mathrm{E}-15$ & $2.70 \mathrm{E}-15$ & $2.67 \mathrm{E}-15$ & $2.63 E-15$ & $2.60 \mathrm{E}-15$ & $2.57 \mathrm{E}-15$ & $2.53 \mathrm{E}-15$ \\
\hline sc & 46 & $7.85 \mathrm{E}-12$ & $7.85 E-12$ & $7.85 \mathrm{E}-12$ & $7.85 \mathrm{E}-12$ & $7.84 \mathrm{E}-12$ & $7.84 \mathrm{E}-12$ & $7.83 \mathrm{E}-12$ & $7.83 \mathrm{E}-12$ & $7.82 \mathrm{E}-12$ & $7.82 \mathrm{E}-12$ & $7.81 \mathrm{E}-12$ \\
\hline $\mathrm{sc}$ & 47 & $2.95 \mathrm{E}-10$ & $2.93 E-10$ & $2.92 \mathrm{E}-10$ & $2.90 \mathrm{E}-10$ & $2.85 \mathrm{E}-10$ & $2.80 E-10$ & $2.75 \mathrm{E}-10$ & $2: 70 \mathrm{E}-10$ & $2.66 \mathrm{E}-10$ & $2.61 \mathrm{E}-10$ & $2.57 \mathrm{E}-10$ \\
\hline $\mathrm{sc}$ & 48 & $4.97 \mathrm{E}-11$ & $4.93 \mathrm{E}-11$ & $4.89 \mathrm{E}-11$ & $4.81 \mathrm{E}-11$ & $4.66 \mathrm{E}-11$ & $4.52 \mathrm{E}-11$ & $4.38 \mathrm{E}-11$ & $4.24 \mathrm{E}-11$ & $4.11 \mathrm{E}-11$ & $3.98 \mathrm{E}-11$ & $b E-11$ \\
\hline sc & 49 & $3.34 \mathrm{E}-10$ & $2.32 \mathrm{E}-10$ & $1.61 \mathrm{E}-10$ & $7.80 E-11$ & $1.82 \mathrm{E}-11$ & $4.25 E-12$ & $9.94 \mathrm{E}-13$ & $2.32 \mathrm{E}-13$ & $5.42 \mathrm{E}-14$ & $1.27 \mathrm{E}-14$ & $2.96 \mathrm{E}-15$ \\
\hline $\mathrm{sc}$ & 50 & $7.00 \mathrm{E}-12$ & $3.62 \mathrm{E}-17$ & $1.87 \mathrm{E}-22$ & $4.94 E-33$ & $0.00 \mathrm{E}+00$ & $0.00 \mathrm{E}+00$ & $0.00 \mathrm{E}+00$ & $0.00 E+00$ & $0.00 \mathrm{E}+00$ & $0.00 \mathrm{E}+00$ & $0.00 \mathrm{E}+00$ \\
\hline ti & 51 & $2.62 \mathrm{E}-11$ & $7.09 E-13$ & $1.92 \mathrm{E}-14$ & $1.40 \mathrm{E}-17$ & $7.51 E-24$ & $4.02 \mathrm{E}-30$ & $0.00 \mathrm{E}+00$ & $0.00 E+00$ & $0.00 E+00$ & $0.00 \mathrm{E}+00$ & $0.00 \mathrm{E}+00$ \\
\hline $\mathrm{v}$ & 52 & $2.52 \mathrm{E}-09$ & $9.84 \mathrm{E}-12$ & $3.85 \mathrm{E} 14$ & $5.87 \mathrm{E}-19$ & $1.37 \mathrm{E}-28$ & $0.00 \mathrm{E}+00$ & $0.00 \mathrm{E}, 00$ & $0.00 E+00$ & $0.00 \mathrm{E}+00$ & $0.00 \mathrm{E}+00$ & $0.00 \mathrm{E}+00$ \\
\hline $\mathrm{v}$ & 53 & $3.12 \mathrm{E}-11$ & $7.66 \mathrm{E}-17$ & $1.88 \mathrm{E}-22$ & 1. $15 \mathrm{E}-33$ & $0.00 \mathrm{E}+00$ & $0.00 E+00$ & $0.00 \mathrm{E}+00$ & $0.00 E+00$ & $0 \mathrm{E}+00$ & $0.00 \mathrm{E}+00$ & $0.00 \mathrm{E}+00$ \\
\hline cr & 51 & $2.65 E-11$ & $2.65 E-11$ & $2.65 E-11$ & $2.65 \mathrm{E}-11$ & $2.64 \mathrm{E}-11$ & $2.64 \mathrm{E}-11$ & $2.63 E-11$ & $2.62 \mathrm{E}-11$ & $2 \mathrm{E}-11$ & $E-11$ & $2.61 \mathrm{E}-11$ \\
\hline $\mathrm{cr}$ & 55 & $9.61 \mathrm{E}-10$ & $2.51 \mathrm{E}-12$ & $6.56 \mathrm{E}-15$ & $4.48 \mathrm{E}-20$ & $2.09 E-30$ & $0.00 E+00$ & $0.00 \mathrm{E}+00$ & $0.00 \mathrm{E}+00$ & $0.00 \mathrm{E}+00$ & $0.00 \mathrm{E}+00$ & \pm+00 \\
\hline $\mathrm{mn}$ & 54 & $3.06 \mathrm{E}-11$ & $3.06 \mathrm{E}-11$ & $3.06 \mathrm{E}-11$ & $3.06 \mathrm{E}-11$ & $3.06 \mathrm{E}-11$ & $3.06 \mathrm{E}-11$ & $3.06 E-11$ & $6 E-11$ & & -11 & -11 \\
\hline $\mathrm{mn}$ & 56 & $4.23 \mathrm{E}-08$ & $3.69 \mathrm{E}-08$ & $3.23 E-08$ & $2.47 \mathrm{E}-08$ & $1.44 \mathrm{E}-08$ & $8.42 \mathrm{E}-09$ & $4.92 \mathrm{E}-09$ & $2.87 \mathrm{E}-09$ & $1.68 \mathrm{E}-09$ & $9.81 \mathrm{E}-10$ & $5.73 \mathrm{E}-10$ \\
\hline $\mathrm{mn}$ & 57 & $9.30 \mathrm{E}-11$ & $5.69 \mathrm{E}-17$ & $3.47 E-23$ & $0.00 E+00$ & $0.00 \mathrm{E}+00$ & $0.00 E+00$ & & $E+00$ & +00 & +00 & $E+00$ \\
\hline $\mathrm{Ee}$ & 55 & $2.58 \mathrm{E}-13$ & $2.58 \mathrm{E}-13$ & $2.58 \mathrm{E}-13$ & $2.58 E-13$ & $2.58 \mathrm{E}-13$ & $2.58 \mathrm{E}-13$ & $2.58 \mathrm{E}-13$ & $2.58 \mathrm{E}-13$ & $2.58 E-13$ & -13 & $2.58 \mathrm{E}-13$ \\
\hline $\mathrm{fe}$ & 59 & $2.31 E-13$ & $2.31 \mathrm{E}-13$ & $2.31 E-13$ & $2.30 \mathrm{E}-13$ & $2.30 \mathrm{E}-13$ & $2.30 E-13$ & 2.3 & 2.2 & & 2. & -13 \\
\hline co & 60 & $2.18 \mathrm{E}-13$ & $2.18 E-13$ & $2.18 \mathrm{E}-13$ & $2.18 \mathrm{E}-13$ & $2.18 \mathrm{E}-13$ & $2.18 t-13$ & $2.18 \mathrm{E}-13$ & $2.18 \mathrm{E}-13$ & $2.18 \mathrm{E}-13$ & $2.18 \mathrm{E}-13$ & $2.18 \mathrm{E}-13$ \\
\hline CO & 62 & $1.69 \mathrm{E}-10$ & $1.61 \mathrm{E}-16$ & 1.541 & $1.76 \mathrm{E}-34$ & $0.00 \mathrm{E}+00$ & $0.00 \mathrm{E}+00$ & & 0.0 & & 0. & \\
\hline ni & 63 & $1.44 \mathrm{E}-13$ & $1.44 \mathrm{E}-13$ & $1.44 \mathrm{E}-13$ & $1.44 \mathrm{E}-13$ & $1.44 \mathrm{E}-13$ & $1.44 \mathrm{E}-13$ & $1.44 \mathrm{E}-13$ & $1.44 \mathrm{E}-13$ & 1.4 & -13 & -13 \\
\hline $\mathrm{ni}$ & 65 & $3.61 \mathrm{E}-10$ & $3.15 \mathrm{E}-10$ & $2.74 \mathrm{E}-10$ & $2.08 \mathrm{E}-10$ & $1.20 \mathrm{E}-10$ & $6.93 \mathrm{E}-11$ & $4.00 \mathrm{E}-11$ & $2.31 \mathrm{E}-11$ & 1. $33 E-11$ & $7.68 \mathrm{E}-12$ & $4.43 \mathrm{E}-12$ \\
\hline $\mathrm{cu}$ & 64 & $5.47 \Xi-09$ & $5.32 \mathrm{E}-09$ & $5.18 \mathrm{E}-09$ & $4.90 \mathrm{E}-09$ & $4.40 \mathrm{E}-09$ & $3.94 \mathrm{E}-09$ & $3.53 E-09$ & $3.17 \mathrm{E}-09$ & & & $2.28 \mathrm{E}-09$ \\
\hline $\mathrm{Cu}$ & 66 & $3.18 \mathrm{E}-09$ & $5.40 \mathrm{E}-11$ & $9.15 \mathrm{E}-13$ & $2.63 E-16$ & $2.17 E-23$ & $2.65 \mathrm{E}-26$ & $2.58 \mathrm{E}-26$ & $2.52 E-26$ & $2.45 E-26$ & $2.39 \mathrm{E}-26$ & $2.33 E-26$ \\
\hline $7 n$ & 65 & 2. $2.7 F,-12$ & $2.27 \mathrm{~F}-12$ & 2. $27 \mathrm{E}-12$ & 2. . .7E-12 & $2.27 F-12$ & 2. $2.7 F-12$ & 2. $27 \mathrm{E}-12$ & 2. $27 E-12$ & & 2.26 & \\
\hline $\mathrm{zn}$ & 69 & $1.93 \mathrm{E}-09$ & $1.35 \mathrm{E}-09$ & $9.46 E-10$ & $4.74 \mathrm{E}-10$ & $1.38 \mathrm{E}-10$ & $5.84 \mathrm{E}-11$ & $3.77 \mathrm{E}-11$ & $3.06 \mathrm{E}-11$ & $2.69 E-11$ & $2.41 \mathrm{E}-11$ & $2.18 \mathrm{E}-11$ \\
\hline $\mathrm{zn}$ & $69 \mathrm{~m}$ & $4.54 \mathrm{E}-11$ & $4.42 \mathrm{E}-11$ & $4.31 \mathrm{E}-11$ & $4.10 \mathrm{E}-11$ & $3.71 \mathrm{E}-11$ & $3.35 E-11$ & $3.03 E-11$ & $2.74 \mathrm{E}-11$ & $2.48 E-11$ & $2.24 \mathrm{E}-11$ & $2.03 \mathrm{E}-11$ \\
\hline $\mathrm{zn}$ & 71 & $5.25 \mathrm{E}-13$ & $1.08 \mathrm{E}-16$ & $2.23 E-20$ & $9.47 \mathrm{E}-28$ & $0.00 \mathrm{E}+00$ & $0.00 E+00$ & $0.00 \mathrm{E}+00$ & $0.00 \mathrm{E}+00$ & $0.00 \mathrm{E}+00$ & $0.00 \mathrm{E}+00$ & $0.00 \mathrm{E}+00$ \\
\hline & & $4.15 \mathrm{E}-14$ & $3.80 E-14$ & $3.48 E-14$ & $2.93 \mathrm{E}-14$ & $2.06 \mathrm{E}-14$ & $1.45 \mathrm{E}-14$ & $1.02 \mathrm{E}-14$ & $7.21 \mathrm{E}-15$ & $5.08 E-15$ & $3.58 \mathrm{E}-15$ & $2.52 \mathrm{E}-15$ \\
\hline & & $1.10 \mathrm{E}-05$ & $1.73 \mathrm{E}-06$ & $7.69 E-07$ & $6.16 \mathrm{E}-07$ & $5.50 \mathrm{E}-07$ & $4.96 \mathrm{E}-07$ & $4.48 \mathrm{E}-07$ & $4.06 \mathrm{E}-07$ & $3.68 \mathrm{E}-07$ & $3.35 \mathrm{E}-07$ & $3.04 \mathrm{E}-07$ \\
\hline
\end{tabular}


Table F23. Radioactivity (Ci) After Ten-Day Irradiation of 1-kg Aluminum 6061-T6 (Flux Parameter Set 2).

\begin{tabular}{|c|c|c|c|c|c|c|c|c|c|c|c|}
\hline & & & & & & & & & & & \\
\hline & initial & . & $1.0 \mathrm{hr}$ & $2.0 \mathrm{hr}$ & $4.0 \mathrm{hr}$ & $6.0 \mathrm{hr}$ & $8.0 \mathrm{hr}$ & $10.0 \mathrm{hr}$ & $12.0 \mathrm{hr}$ & $14.0 \mathrm{hr}$ & $16.0 \mathrm{hr}$ \\
\hline na 24 & $9.45 \mathrm{E}-07$ & $9.23 \mathrm{E}-07$ & $9.01 \mathrm{E}-07$ & $8.60 E-07$ & $7.82 \mathrm{E}-07$ & $7.11 \mathrm{E}-07$ & $6.47 \mathrm{E}-07$ & $5.89 \mathrm{E}-07$ & $5.36 E-07$ & $4.87 E-07$ & $4.43 E-07$ \\
\hline na 25 & $7.06 \mathrm{E}-09$ & $5.72 \mathrm{E}-18$ & $4.63 \mathrm{E}-27$ & $0.00 \mathrm{E}+00$ & $0.00 \mathrm{E}+00$ & $0.00 \mathrm{E}+00$ & $0.00 \mathrm{E}+00$ & $0.00 \mathrm{E}+00$ & $0.00 \mathrm{E}+00$ & $0.00 \mathrm{E}+00$ & $0.00 \mathrm{E}+00$ \\
\hline mg 27 & $9.56 \mathrm{E}-06$ & $1.06 \mathrm{E}-06$ & $1.18 \mathrm{E}-07$ & & & $3.36 \mathrm{E}-17$ & $.11 \mathrm{E}-21$ & $7.78 E-25$ & $1.18 E-28$ & & $0.00 \mathrm{E}+00$ \\
\hline a1 28 & $7.59 \mathrm{E}-07$ & $7.05 E-11$ & $6.56 \mathrm{E}-15$ & $5.69 \mathrm{E}-23$ & $1.87 \mathrm{E}-25$ & $1.75 \mathrm{E}-25$ & $.63 E-25$ & $1.53 E-25$ & 1. $43 \mathrm{E}-25$ & $1.34 \mathrm{E}-25$ & $1.25 \mathrm{E}-25$ \\
\hline al & & & $4.12 \mathrm{E}-12$ & $7.31 \mathrm{E}-15$ & $2.31 \mathrm{E}-20$ & $7.28 \mathrm{E}-26$ & $2.30 \mathrm{E}-31$ & $0.00 \mathrm{E}+00$ & $0.00 \mathrm{E}+00$ & $0.00 \mathrm{E}+00$ & $0.00 \mathrm{E}+00$ \\
\hline si & $6.44 \mathrm{E}-11$ & $5.65 \mathrm{E}-11$ & $4.95 E-11$ & $3.80 E-11$ & $2.24 \mathrm{E}-11$ & $32 E-11$ & $7.78 \mathrm{E}-12$ & $58 \mathrm{E}-12$ & $2.70 \mathrm{E}^{3}-12$ & $1.59 \mathrm{E}-12$ & $9.38 \mathrm{E}-13$ \\
\hline $\mathrm{ca}$ & $3.03 \mathrm{E}-13$ & $3.03 \mathrm{E}-13$ & $3.03 \mathrm{E}-13$ & $3 E-13$ & $3.03 E-13$ & $3 E-13$ & $3 E-13$ & $3 E-13$ & $3.03 E-13$ & $3.03 E-13$ & $3.03 E-13$ \\
\hline $\mathrm{ca}$ & $1.55 \mathrm{E}-14$ & $1.54 \mathrm{E}-14$ & $1.54 \mathrm{E}-14$ & $1.53 \mathrm{E}-14$ & $1.51 \mathrm{E}-14$ & $1.49 E-14$ & & & & & \\
\hline sc 46 & $7.57 \mathrm{E}-11$ & $7.57 \mathrm{E}-11$ & $7.57 \mathrm{E}-11$ & $7.56 \mathrm{E}-11$ & $7.56 \mathrm{E}-11$ & $7.55 E-11$ & $7.55 \mathrm{E}-11$ & $7.54 \mathrm{E}-11$ & $7.54 \mathrm{E}-11$ & 7. $53 \mathrm{E}-11$ & $7.53 \mathrm{E}-11$ \\
\hline sc 47 & & $1.37 \mathrm{E}-09$ & $1.36 E-09$ & $1.35 E-09$ & $1.33 \mathrm{E}-09$ & $1.31 \mathrm{E}-09$ & & & & & \\
\hline sc 48 & $1.53 \mathrm{E}-10$ & 1. $52 \mathrm{E}-10$ & $1.51 \mathrm{E}-10$ & $1.49 \mathrm{E}-10$ & $1.44 \mathrm{E}-10$ & $1.39 \mathrm{E}-10$ & $35 E-10$ & -10 & 1.2 & -10 & \\
\hline sc & 3.34 & 2. $32 \mathrm{E}-10$ & $1.61 \mathrm{E}$ & $E-11$ & -11 & & & -13 & -14 & -14 & -15 \\
\hline $\mathrm{sc}$ & 7.00 & $3.62 \mathrm{E}-17$ & $1.87 \mathrm{E}$ & & $8+00$ & & & +00 & +00 & 0.8 & \\
\hline ti & $2.62 \mathrm{E}-11$ & $7.09 \mathrm{E}-13$ & $1.92 \mathrm{E}-14$ & $1.40 \mathrm{E}-17$ & -24 & -30 & -00 & 00 & 0.0 & 0.0 & \\
\hline $\mathrm{v}$ & & $9.84 E-12$ & 3.851 & $8-19$ & -28 & $O E+00$ & 00 & .00 & 0.0 & & \\
\hline 53 & 3.1 & 7.6 & 1.8 & -33 & +00 & $E+00$ & & & & & \\
\hline $\mathrm{Cr}$ & 2.3 & 2.3 & 2.37 & -10 & -10 & -10 & & & 2.3 & 2.3 & -10 \\
\hline cr 55 & -10 & 2.5 & 6.5 & & & +00 & & & +00 & +00 & \\
\hline mn 54 & 3.0 & 3.0 & 3.0 & -10 & 3. & & & & 3. & 3.0 & \\
\hline ח & & & & & & & & & 1.6 & 9.8 & -10 \\
\hline $\mathrm{mn}$ & 9.38 & 5.691 & 3.4 & $8+00$ & 0.0 & .00 & & & & & \\
\hline $\mathrm{fe}$ & & $2.57 \mathrm{E}-12$ & 2.5 & -12 & & & & & 2.5 & & \\
\hline fe 59 & -12 & $2.15 E-12$ & 2.1 & -12 & & & & & & & \\
\hline co 60 & $.18 \mathrm{E}-12$ & $.18 \mathrm{E}-12$ & 2.1 & -12 & 2.1 & -12 & & & 2.1 & 2.1 & \\
\hline 62 & 1.691 & & 1.54 & & & & & & & & \\
\hline 63 & 1.44 & $1.44 \mathrm{E}-12$ & 1.44 & $E-12$ & 1.4 & 1. & & 1.44 & 1.4 & 1.6 & \\
\hline 65 & 3.6 & & & & & & & & -11 & $5-12$ & \\
\hline & 7.4 & $7.29 \mathrm{E}$ & 7.0 & $2 E-09$ & 6.0 & & & & & & \\
\hline 66 & 3.18 & $5.40 \mathrm{E}$ & 9.15 & $2.63 E-16$ & 2.1 & $7 E-26$ & & & & & \\
\hline zn 65 & & & $2.24 \mathrm{E}-11$ & $2.24 \mathrm{E}-11$ & & & & & & & -11 \\
\hline $\mathrm{zn} 69$ & $1.95 \mathrm{E}-09$ & $1.37 \mathrm{E}-09$ & $9.66 \mathrm{E}-10$ & $4.93 E-10$ & $1.55 \mathrm{E}-10$ & $7.37 \mathrm{E}-11$ & -11 & 4.31 & 3.8 & $3.43 \mathrm{E}-11$ & $0 \mathrm{E}-11$ \\
\hline $\operatorname{zn} 69 \mathrm{~m}$ & $6.47 \mathrm{E}-11$ & $6.31 E-11$ & $6.15 \mathrm{E}-11$ & $5.85 E-11$ & $5.29 \mathrm{E}-11$ & & & & & & \\
\hline 71 & $5.25 \mathrm{E}-13$ & $1.08 \mathrm{E}-16$ & $2.23 \mathrm{E}-20$ & $9.47 E-28$ & 0.0 & $D E+00$ & & & 0.001 & & \\
\hline & & 386511 & 3.54 & $2.97 E \cdot 14$ & $2.09 \mathrm{E}-14$ & $1.47 \mathrm{E}-14$ & & & $5.16 \mathrm{E}-15$ & 3. $64 \mathrm{E}-15$ & $2.56 \mathrm{E}-15$ \\
\hline & $1.13 E-05$ & $2.03 E-06$ & $1.06 \mathrm{E}-06$ & $8.96 E-07$ & $8.05 E-07$ & $7.28 \mathrm{E}-07$ & $6.59 \mathrm{E}-07$ & $5.98 \mathrm{E}-07$ & $5.43 E-07$ & $4.94 E-07$ & $4.49 \mathrm{E}-07$ \\
\hline
\end{tabular}


Table F24. Radioactivity (Ci) After One-Day Irradiation of 1-kg Steel Plate (Flux Parameter Set 1).

\begin{tabular}{|c|c|c|c|c|c|c|c|c|c|c|c|}
\hline & & & & Tim & After $\mathrm{Di}$ & harge & & & & & \\
\hline & initial & $.5 \mathrm{hr}$ & $1.0 \mathrm{hr}$ & 2.0 hr & 4.0 hr & $\begin{array}{r}6.0 \mathrm{hr} \\
4\end{array}$ & $\begin{array}{l}8.0 \mathrm{hr} \\
4.17 \mathrm{E}-15\end{array}$ & $\begin{array}{r}10.0 \mathrm{hr} \\
417 \mathrm{~F}-15\end{array}$ & $\begin{array}{r}12.0 \mathrm{hr} \\
4.17 \mathrm{E}-15\end{array}$ & $\begin{array}{r}14.0 \mathrm{hr} \\
4.17 \mathrm{E}-15\end{array}$ & $\begin{array}{r}16.0 \mathrm{hr} \\
4.17 \mathrm{E}-15\end{array}$ \\
\hline & -15 & $17 \mathrm{E}-15$ & $.17 \mathrm{E}-15$ & & $\begin{array}{r}17 E-15 \\
36 E-19\end{array}$ & $4.17 \mathrm{E}-15$ & $4.17 \mathrm{E}-15$ & $4.17 E-15$ & $4.17 \mathrm{E}-15$ & $4.17 E-15$ & $17 E-15$ \\
\hline $\begin{array}{l}\text { Iny } \\
\text { aI }\end{array}$ & -11 & $3 E-12$ & $.26 \mathrm{E}-13$ & $.55 E-15$ & & $58 \mathrm{E}-23$ & $45 E-27$ & $.29 E-31$ & 1. $39 \mathrm{E}-34$ & $0.00 \mathrm{E}+00$ & $00 E+00$ \\
\hline aI & & & & $.23 \mathrm{E}-25$ & & $2.28 \mathrm{E}-3$ & $28 E-32$ & $28 \mathrm{E}-32$ & $.28 E-32$ & $2.28 E-32$ & \\
\hline al 29 & E- 10 & $E \cdot 11$ & $36 E-13$ & $.31 E-15$ & $3 E-21$ & 1. $30 \mathrm{E}-26$ & $11 E-32$ & $00 \mathrm{E}+00$ & $.00 E+00$ & $0.00 \mathrm{E}+00$ & $0.00 E+00$ \\
\hline si 31 & E-09 & $3 E-09$ & -09 & $.88 \mathrm{E}-09$ & $1.70 \mathrm{E}-09$ & $1.00 \mathrm{E}-09$ & & $3 E-10$ & $E-10$ & & \\
\hline p 32 & & & & $5 E-10$ & I. $05 \mathrm{E}-10$ & & & & & & \\
\hline p 33 & & & & $E-10$ & 5.0 & & & & & & \\
\hline s 35 & & & & & & & & & & & \\
\hline s 37 & & & & & & & & & & & \\
\hline ti 51 & 5. & & & -17 & 1.47 & 7.8 & & & & & \\
\hline & & & & & & & & & & & \\
\hline v 53 & & & & $z-32$ & & & & & & & \\
\hline cr 51 & & & & -10 & & & & & & & \\
\hline cr 55 & & & & & & & & & & & \\
\hline $\operatorname{mn} 54$ & & & & $8-10$ & 4. & & & & & & \\
\hline & & & & & & & & & & & \\
\hline $\mathrm{mn} 57$ & & & & & & & & & & & \\
\hline $\operatorname{mn} 58$ & & & & & & & & & & & \\
\hline fe 55 & & & & & & & & & & & \\
\hline fe 59 & & & & $E-12$ & & & & & & & \\
\hline & & & & & & & & & & & \\
\hline $\mathrm{co} 6 \mathrm{C}$ & & & & 1.5 & & & & & & & \\
\hline & & & & & & & & & & & \\
\hline & & & & & & & & & & & \\
\hline & -16 & 3.5 & & 3.5 & $3.52 \mathrm{E}-1$ & $3.52 \mathrm{E}-16$ & & & & & $3.52 \mathrm{E}-16$ \\
\hline & & & & & & & & & & & \\
\hline & & & & $1.02 \mathrm{E}-10$ & $5.88 \mathrm{E}-$ & $3.39 \mathrm{E}-1$ & & & 6.52 & & $2.17 \mathrm{E}-12$ \\
\hline & $1.97 \mathrm{E}-07$ & 31 & $.15 E-07$ & $8.97 \mathrm{E}-08$ & & & $2.39 \mathrm{E}-08$ & $1.71 \mathrm{E}-08$ & $1.31 \mathrm{E}-08$ & $1.08 \mathrm{E}-08$ & $9.43 E-0$ \\
\hline
\end{tabular}


Table F25. Radioactivity (Ci) After Ten-Day Irradiation of 1-kg Steel Plate (Flux Parameter Set 1).

\begin{tabular}{|c|c|c|c|c|c|c|c|c|c|c|c|}
\hline & & & & & & & & & & & \\
\hline & initial & $.5 \mathrm{hr}$ & $1.0 \mathrm{hr}$ & $2.0 \mathrm{hr}$ & $4.0 \mathrm{hr}$ & $6.0 \mathrm{hr}$ & $8.0 \mathrm{hr}$ & $10.0 \mathrm{hr}$ & $12.0 \mathrm{hr}$ & $14.0 \mathrm{hr}$ & $16.0 \mathrm{hr}$ \\
\hline $\begin{aligned} \text { be } 10 \\
\text { c } 14\end{aligned}$ & $\begin{array}{l}9.36 \mathrm{E}-20 \\
4.17 \mathrm{E}-14\end{array}$ & $\begin{array}{r}9.36 \mathrm{E}-20 \\
4.17 \mathrm{E}-14\end{array}$ & $\begin{array}{l}9 \cdot 36 \mathrm{E}-20 \\
4\end{array}$ & $9 \cdot 36 \mathrm{E}-20$ & $\begin{array}{l}9.36 \mathrm{E}-20 \\
4 \\
17 \mathrm{E}-14\end{array}$ & $9.36 \mathrm{E}-20$ & $\begin{array}{l}9.36 \mathrm{E}-20 \\
17 \mathrm{E}-14\end{array}$ & $9.36 \mathrm{E}-20$ & $9.36 \mathrm{E}-20$ & $\begin{array}{l}9.36 \mathrm{E}-20 \\
4 \\
17 \mathrm{E}-14\end{array}$ & $\begin{array}{l}9.36 \mathrm{E}-20 \\
4.17 \mathrm{E}-14\end{array}$ \\
\hline mg 27 & $1.02 \mathrm{E}-11$ & & $1.26 \mathrm{E}-13$ & $1.55 \mathrm{E}-15$ & & & 4 & $.29 \mathrm{E}-31$ & $\begin{array}{l}4.1 \\
1.3\end{array}$ & $\begin{array}{l}4.17 \mathrm{E}-14 \\
0.00 \mathrm{E}+00\end{array}$ & $\begin{array}{l}4.17 \mathrm{E}-14 \\
0.00 \mathrm{E}+00\end{array}$ \\
\hline 28 & $.24 \mathrm{E}-08$ & $1.15 \mathrm{E}-12$ & $1.07 \mathrm{E}-16$ & $9.23 E-25$ & & $4.57 \mathrm{E}-32$ & $.57 E-32$ & $.28 \mathrm{E}-32$ & $2.28 E-32$ & $2.28 \mathrm{E}-32$ & $.28 E-32$ \\
\hline 29 & $.15 E-10$ & $5 E-11$ & $7.36 \mathrm{E}-13$ & $1.31 \mathrm{E}-15$ & $4.13 E-21$ & $1.30 \mathrm{E}-26$ & $.11 \mathrm{E}-32$ & $.00 E+00$ & $0.00 \mathrm{E}+00$ & $0.00 \mathrm{E}+00$ & \\
\hline 31 & $.90 \mathrm{E}-09$ & 4.2 & & $2.89 E-09$ & & & & & & & \\
\hline 32 & -10 & $8.57 E-10$ & $8.56 E-10$ & $5 E-10$ & $1 \mathrm{E}-10$ & $8 E-10$ & & $E-10$ & & $E-10$ & $E-10$ \\
\hline $\mathrm{p}$ & & & & & & & & & & & \\
\hline 35 & $1.27 E-14$ & $7 E-14$ & & $7 E-14$ & & & & & & & \\
\hline 37 & 1.2 & E-17 & 3.3 & $3 E-23$ & 6. & +00 & +00 & +00 & +00 & $8+00$ & \\
\hline 51 & 5.1 & 1.3 & & -17 & & & & & & & \\
\hline v 52 & 3.23 & 1.2 & 4.5 & E-18 & & & & & & & \\
\hline v 53 & 4.46 & 1.1 & 2.7 & -32 & & & & & & & \\
\hline$=51$ & 2.7 & & & & & & & & & & \\
\hline 55 & 2.0 & 5.2 & & -20 & & & & & & & \\
\hline & & & & & & & & & & & \\
\hline $\mathrm{mn} 56$ & 1.3 & 1.1 & 1.0 & E-08 & & & & & & & \\
\hline $\mathrm{mn} 57$ & 1.2 & 7.5 & 4.6 & $1 E-34$ & & & & & & & \\
\hline & 4.15 & & & 0.0 & & & & & & & \\
\hline & 3.22 & 3.2 & 3.2 & -11 & & & & & & & \\
\hline & & & & & & & & & & & \\
\hline & 5.9 & & 5.9 & $2-08$ & 5.9 & & & & & & \\
\hline 060 & 1.57 & & & $1.57 \mathrm{E} \cdot 11$ & 1.5 & & & & & & \\
\hline & 4.67 & 3.78 & 3.07 & $2.01 \mathrm{E}-10$ & 8.6 & & & & & & \\
\hline & $9.03 \mathrm{E}-11$ & $8.61 \mathrm{E}-17$ & $8.21 E-23$ & $0.00 \mathrm{E}+00$ & $0.00 \mathrm{E}+00$ & & & & $8+00$ & 0.0 & $E+00$ \\
\hline & 3.5 & & & $E-15$ & & & & & & & \\
\hline & & & 4.2 & -13 & 4.2 & & & & & & \\
\hline & 1.77 & & $1.34 \mathrm{E}-1$ & $1.02 \mathrm{E}-10$ & & & & & & & \\
\hline & $2.62 \mathrm{E}-07$ & E-07 & $1.80 \mathrm{E}-07$ & $1.54 \mathrm{E}-07$ & $1.20 \mathrm{E}-07$ & $9.98 \mathrm{E}-08$ & $8.81 E-08$ & $E-08$ & $7.72 \mathrm{E}-08$ & $7.48 \mathrm{E}-08$ & $7.34 \mathrm{E}-0$ \\
\hline
\end{tabular}


Table F26. Radioactivity (Ci) After One-Day Irradiation of 1-kg Steel Plate (Flux Parameter Set 2).

\begin{tabular}{|c|c|c|c|c|c|c|c|c|c|c|c|}
\hline & & & & Time & After Di & charge & & & & & \\
\hline & initial & & & $2.0 \mathrm{hr}$ & & $6.0 \mathrm{hr}$ & $8.0 \mathrm{hr}$ & $10.0 \mathrm{hr}$ & $12.0 \mathrm{hr}$ & $14.0 \mathrm{hr}$ & $16.0 \mathrm{hr}$ \\
\hline & & $6.54 \mathrm{E}-20$ & $.54 \mathrm{E}-20$ & $6.54 \mathrm{E}-20$ & $6.54 \mathrm{E}-20$ & $6.54 \mathrm{E}-20$ & $6.54 \mathrm{E}-20$ & $6.54 \mathrm{E}-20$ & $6.54 \mathrm{E}-20$ & $6.54 \mathrm{E}-20$ & $6.54 \mathrm{E}-20$ \\
\hline & $.94 E \quad 14$ & $94 F-14$ & $2.94 \mathrm{E}-14$ & $.94 E-14$ & $2.94 \mathrm{E}-14$ & $2.94 \mathrm{E}-14$ & $.94 \mathrm{E}-14$ & $2.94 \mathrm{E}-14$ & $2.94 \mathrm{E}-14$ & $2.94 E-14$ & $2.94 \mathrm{E}-14$ \\
\hline mg 27 & $.12 E-11$ & $7.91 \mathrm{E}-12$ & $8.78 \mathrm{E}-13$ & $.08 \mathrm{E}-14$ & 1. $65 \mathrm{E}-18$ & $2.51 E-22$ & $.81 \mathrm{E}-26$ & $5.79 \mathrm{E}-30$ & $8.92 \mathrm{E}-34$ & $0.00 \mathrm{E}+00$ & $00 \mathrm{E}+00$ \\
\hline al & $63 E-08$ & $8.03 \mathrm{E}-12$ & $7.46 E-16$ & $.45 E-24$ & $7.53 \mathrm{E}-31$ & $7.07 \mathrm{E}-31$ & $6.62 \mathrm{E}-31$ & $6.16 \mathrm{E}-31$ & $5.93 \mathrm{E} 31$ & $5.48 \mathrm{E}-31$ & $2 E-31$ \\
\hline al 29 & $.90 \mathrm{E}-09$ & $1.22 \mathrm{E}-10$ & $5.15 \mathrm{E}-12$ & $.14 E-15$ & & & $2.87 \mathrm{E}-31$ & $0.00 \mathrm{E}+00$ & $0.00 \mathrm{E}+00$ & & $0 E+00$ \\
\hline si 31 & $3.42 \mathrm{E}-08$ & $3.00 \mathrm{E}-08$ & $2.63 \mathrm{E}-08$ & $2.02 \mathrm{E}-08$ & $1.19 \mathrm{E}-08$ & $7.00 \mathrm{E}-09$ & 4. $13 \mathrm{~F}-09$ & $2.43 \mathrm{E}-09$ & $1.43 \mathrm{E}-09$ & $8.45 E-10$ & \\
\hline $\mathrm{p}$ & 7. $40 \mathrm{E}-10$ & $7.39 \mathrm{E}-10$ & $8 E-10$ & $7.37 \mathrm{E}-10$ & -10 & $1 E-10$ & & -10 & E-10 & & $6 E_{-1}$ \\
\hline$p$ & $3.56 E-09$ & & $5 E-09$ & $3.55 \mathrm{E}-09$ & & & & & & & \\
\hline 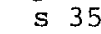 & 6.6 & 6. & 6.6 & $E-15$ & 6.6 & 6. & & & & & \\
\hline s 37 & 6.3 & & & -22 & 3. & 0. & & & +00 & & \\
\hline ti 51 & 3.55 & & & & 1.03 & $0 E-29$ & & & & & \\
\hline V 52 & 2.26 & 8.8 & & -17 & $1.22 \mathrm{E}-26$ & $0.00 \mathrm{E}+00$ & $8+00$ & +00 & +00 & & \\
\hline $\mathrm{v}:$ & 3.1 & & 1.8 & & & & & & & & \\
\hline cr 51 & $2.56 \mathrm{~F}$ & 2 . & 2 . & -09 & 2 . & & & & -09 & & \\
\hline cr 55 & 1.4 & & & -19 & & 0 . & & & & 0. & \\
\hline $\mathrm{mn} 5$ & 2.8 & & & & & & & & & & \\
\hline $\mathrm{mn} 5$ & 1.17 & 1.0 & 8. & $E-07$ & 3.9 & $5-07$ & & & .08 & & 1. \\
\hline $\mathrm{mn} 57$ & 8.6 & & & & & $E+00$ & & & +00 & 0. & 0 . \\
\hline $\mathrm{mn} 5$ & 2.9 & 1.4 & & $+\infty 0$ & 0. & 0. & & & & & \\
\hline fe & & & & & 3.0 & $E-11$ & & & & & \\
\hline fe 5 & 2.3 & & & & & & & & & & \\
\hline co & & & & $E-08$ & & & & & & & \\
\hline co 6 & 1.1 & & & & & & & & & 1.1 & 1.10 \\
\hline co 6 & & & & -09 & 6.0 & 2.621 & & & & & \\
\hline co 62 & 6.3 & & & 5.27 & $0.00 \mathrm{~F}$ & $0.00 \mathrm{E}+00$ & & $0.00 \mathrm{E}+00$ & $0.00 \mathrm{E}+00$ & 0.0 & 0 . \\
\hline ni 5 & 3.4 & & & $E-15$ & $3.45 \mathrm{E}-15$ & $3.45 \mathrm{E}-15$ & & & & & \\
\hline & $4.20 \mathrm{E}-13$ & & & $E-13$ & $4.20 \mathrm{E}-13$ & $4.20 E-13$ & & $4: 2$ & -13 & & $4.20 \mathrm{E}-$ \\
\hline & & & & $E-09$ & & $3.96 \mathrm{E}-1$ & & & & $4.39 \mathrm{E}-11$ & \\
\hline & & & $9.16 \mathrm{E}-$ & $E-07$ & $5-07$ & $2.94 \mathrm{E}-07$ & $1.94 \mathrm{E}-07$ & $1.35 \mathrm{E}-07$ & $1.01 \mathrm{E}-07$ & $8.10 \mathrm{E}-08$ & $6.93 \mathrm{E}-\mathrm{C}$ \\
\hline
\end{tabular}


Table F27. Radioactivity (Ci) After Ten-Day Irradiation of 1-kg Steel Plate (Flux Parameter Set 2).

\begin{tabular}{|c|c|c|c|c|c|c|c|c|c|c|c|}
\hline & & & & & after & narge & & & & & \\
\hline be 10 & $\begin{array}{r}\text { initial } \\
6.54 \mathrm{E}-19\end{array}$ & $\begin{array}{r}.5 \mathrm{hr} \\
.54 \mathrm{E}-19\end{array}$ & $\begin{array}{r}1.0 \mathrm{hr} \\
.54 \mathrm{E}-19\end{array}$ & $\begin{array}{r}2.0 \mathrm{hr} \\
6.54 \mathrm{E}-19\end{array}$ & & $6.0 \mathrm{hr}$ & $8.0 \mathrm{hr}$ & $\begin{array}{r}10.0 \mathrm{hr} \\
6.54 \mathrm{E}-19\end{array}$ & $\begin{array}{r}12.0 \mathrm{hr} \\
6.54 \mathrm{~F}-19\end{array}$ & $14.0 \mathrm{hr}$ & $\begin{array}{r}16.0 \mathrm{hr} \\
654 \mathrm{E}-19\end{array}$ \\
\hline C 14 & $2.94 \mathrm{E}-13$ & $2.94 \mathrm{E}-13$ & $2.94 \mathrm{E}-13$ & $2.94 \mathrm{E}-13$ & $2.94 \mathrm{E}-13$ & $2.94 \mathrm{E}-13$ & $2.94 \mathrm{E}-13$ & $2.94 \mathrm{E}-13$ & $2.94 \mathrm{E}-13$ & $2.94 \mathrm{E}-13$ & $2.94 \mathrm{E}-13$ \\
\hline $\mathrm{mg} 27$ & $7.12 \mathrm{E}-11$ & $7.91 \mathrm{E}-12$ & $8.78 E-13$ & $1.08 \mathrm{E}-14$ & $1.65 \mathrm{E}-18$ & $2.51 \mathrm{E}-22$ & $3.81 \mathrm{E}-26$ & $5.79 E-30$ & $8.92 \mathrm{E}-34$ & $0.00 \mathrm{E}+00$ & $0.00 \mathrm{E}+00$ \\
\hline 28 & $8.63 E-08$ & $8.03 \mathrm{E}-12$ & $7.46 \mathrm{E}-16$ & $6.45 \mathrm{E}-24$ & & & $21 E-30$ & $14 \mathrm{E}-30$ & & $1.00 \mathrm{E}-30$ & $9.36 E-31$ \\
\hline al 29 & $2.90 \mathrm{E}-09$ & $1.22 \mathrm{E}-10$ & $5.15 \mathrm{E}-12$ & $9.14 \mathrm{E}-15$ & $2.88 E-20$ & $9.10 \mathrm{E}-26$ & $2.87 E-31$ & $0.00 \mathrm{E}+00$ & $0.00 \mathrm{E}+00$ & $0.00 \mathrm{E}+00$ & $0.00 \mathrm{E}+00$ \\
\hline si 31 & $3 E-08$ & $3.00 \mathrm{E}-08$ & $2.63 E-08$ & $2.02 \mathrm{E}-08$ & $1.19 \mathrm{E}-08$ & $7.02 \mathrm{E}-09$ & $4.14 \mathrm{E}-09$ & $2.44 \mathrm{E}-09$ & $1.44 \mathrm{E}-09$ & $8.46 \mathrm{E}-10$ & $4.99 \mathrm{E}-10$ \\
\hline 32 & $0 \mathrm{E}-09$ & $6.00 \mathrm{E}-09$ & $5.99 E-09$ & $5.98 \mathrm{E}-09$ & $5.95 E-09$ & $5.93 \mathrm{E}-09$ & $5.91 \mathrm{E}-09$ & $5.88 \mathrm{E}-09$ & $5.86 \mathrm{E}-09$ & $5.84 \mathrm{E}-09$ & $5.81 E-09$ \\
\hline 33 & $3.16 \mathrm{E}-08$ & $3.15 \mathrm{E}-08$ & $3.15 E-08$ & $3.15 \mathrm{E}-08$ & $3.14 \mathrm{E}-08$ & $3.13 \mathrm{E}-08$ & $3 E-08$ & E-08 & $1 \mathrm{E}-08$ & $1 E-08$ & $3.10 \mathrm{E}-08$ \\
\hline 35 & $6.43 \mathrm{E}-14$ & $6.43 \mathrm{E}-14$ & $6.43 E-14$ & & & & & -14 & $E-14$ & & \\
\hline s 37 & -15 & 1.0 & 1. $69 \mathrm{E}-18$ & $4.48 \mathrm{E}-22$ & $3.15 \mathrm{E}-29$ & $0.00 \mathrm{E}+00$ & $0 \mathrm{E}+00$ & $E+00$ & $0.00 \mathrm{E}+00$ & $0 \mathrm{E}+00$ & $0 E+00$ \\
\hline 51 & & & & $1.92 \mathrm{E}-16$ & $1.03 E-22$ & & & & & & \\
\hline 52 & E-07 & $8.81 \mathrm{E}-10$ & $3.44 \mathrm{E}-12$ & $5.25 \mathrm{E}-17$ & 1. $22 \mathrm{E}-26$ & $E+00$ & 00 & $\mathrm{~F}+00$ & $E+00$ & & $0 E+00$ \\
\hline 53 & $2 E-09$ & $6 E-15$ & 1. $88 E-20$ & $1.14 \mathrm{E}-31$ & $0.00 \mathrm{E}+00$ & $0.00 \mathrm{E}+00$ & 0.0 & & & & +00 \\
\hline 51 & E-08 & 2.2 & 2.2 & $2.29 \mathrm{E}-$ & E-08 & 2.2 & & & & & \\
\hline 55 & E-08 & 3.8 & 1.0 & E-19 & 3.2 & 0.0 & & -00 & & & 0. \\
\hline 54 & 2.8 & 2.8 & 2.8 & $E-08$ & 2.8 & 2.8 & & & & & \\
\hline 56 & 1.17 & 1.02 & 8.9 & $6.83 E-07$ & & & & & & & \\
\hline 57 & $8.63 \mathrm{~F}$ & 5.27 & 3.2 & $1.27 \mathrm{E}-33$ & $E+00$ & $E+00$ & & & & 0.0 & +00 \\
\hline 58 & 2.9 & 1.46 & & $0.00 \mathrm{E}+00$ & +00 & 0. & & -00 & & & +00 \\
\hline & $:-10$ & $3.00 \mathrm{E}-10$ & 3.0 & $3.00 \mathrm{E}-10$ & -10 & & & & & & \\
\hline & & 2.2 & 2.19 & $2.19 \mathrm{E}-10$ & $E-10$ & 2.19 & & -10 & -10 & 2 . & -10 \\
\hline 58 & 4.1 & $4.18 \mathrm{E}-07$ & 4.17 & $4.17 \mathrm{E}-07$ & 4.1 & & & & & & \\
\hline 60 & -10 & $1.10 \mathrm{E}-10$ & 1.10 & $1.10 \mathrm{E}-10$ & -10 & 1.10 & & 10 & 1.1 & 1.1 & 1.1 \\
\hline 61 & 3.2 & 2.6 & & $1.41 \mathrm{E}-09$ & -10 & 2.62 & & & & & $\mathrm{E}-12$ \\
\hline 62 & 6.3 & 6.0 & 5.74 & $5.27 \mathrm{E}-34$ & & & & 00 & & 0.0 & $\mathrm{~F}+00$ \\
\hline & $3.45 \mathrm{E}-14$ & $3.45 \mathrm{E}-14$ & $3.45 E-14$ & $3.45 E-14$ & $3.45 \mathrm{E}-14$ & $3.45 \mathrm{E}-14$ & $3.45 \mathrm{E}-14$ & $3.45 \mathrm{E}-14$ & $3.45 \mathrm{E}-14$ & $3.45 \mathrm{E}-1$ & $3.45 E-14$ \\
\hline & & & & $4.19 \mathrm{E}-12$ & $4.19 \mathrm{E}-12$ & $4.19 E-12$ & & & & & \\
\hline & $2.07 \mathrm{E}-09$ & $1.80 \mathrm{E}-09$ & $1.57 \mathrm{E}-09$ & $1.19 \mathrm{E}-09$ & $6.88 \mathrm{E}-10$ & $3.97 E-10$ & $2.29 \mathrm{E}-10$ & 1. $32 \mathrm{E}-10$ & $7.62 \mathrm{E}-11$ & $4.39 \mathrm{E}-11$ & $2.54 \mathrm{E}-11$ \\
\hline & $2.06 \mathrm{E}-06$ & $1.56 \mathrm{E}-06$ & $1.43 E-06$ & 1. $21 \mathrm{E}-06$ & $9.18 \mathrm{E}-07$ & $7.46 E-07$ & $6.46 \mathrm{E}-07$ & $5.87 E-07$ & $5.52 \mathrm{E}-07$ & $5.32 \mathrm{E}-07$ & $5.19 \mathrm{E}-07$ \\
\hline
\end{tabular}


Table F28. Radioactivity (Ci) After One-Day Irradiation of 1-kg Havar Foil (Flux Parameter Set 1).

\begin{tabular}{|c|c|c|c|c|c|c|c|c|c|c|c|}
\hline & & & & & & & & & & & \\
\hline & initial & $5 \mathrm{hr}$ & $1.0 \mathrm{hr}$ & $2.0 \mathrm{hr}$ & & $6.0 \mathrm{hr}$ & $8.0 \mathrm{hr}$ & $10.0 \mathrm{hr}$ & $12.0 \mathrm{hr}$ & $14.0 \mathrm{hr}$ & $16.0 \mathrm{hr}$ \\
\hline be 10 & $2.34 \mathrm{E}-20$ & $.34 \mathrm{E}-20$ & $2.34 \mathrm{E}-20$ & $2.34 \mathrm{E}-20$ & $2.34 E-20$ & $2.34 E-20$ & 2. $34 \mathrm{E}-20$ & $2.34 \mathrm{E}-20$ & $2.34 \mathrm{E}-20$ & $2.34 \mathrm{E}-20$ & $2.34 \mathrm{E}-20$ \\
\hline ti 51 & $5.01 \mathrm{E}-11$ & $1.35 \mathrm{E}-12$ & $3.66 \mathrm{E}-14$ & $2.68 \mathrm{E}-17$ & $1.43 \mathrm{E}-23$ & $7.68 \mathrm{E}-30$ & $0.00 \mathrm{E}+00$ & $0.00 \mathrm{E}+00$ & $0.00 \mathrm{E}+00$ & $0.00 \mathrm{E}+00$ & $0.00 \mathrm{E}+00$ \\
\hline v 52 & $4 \mathrm{E}-08$ & $3 \mathrm{E}-10$ & $9 \mathrm{E}-13$ & $7.30 \mathrm{E}-18$ & $1.70 E-27$ & $0.00 \mathrm{E}+00$ & $.00 \mathrm{E}+00$ & $0.00 \mathrm{E}+00$ & $0.00 \mathrm{E}+00$ & $0.00 \mathrm{E}+00$ & $.00 E+00$ \\
\hline v 53 & $5 E-10$ & $7 E-15$ & $3 E-21$ & 1. $59 \mathrm{E}-32$ & $0.00 \mathrm{E}+00$ & $0.00 \mathrm{E}+00$ & $.00 \mathrm{E}+00$ & $0.00 \mathrm{E}+00$ & $0.00 \mathrm{E}+00$ & $0.00 \mathrm{E}+00$ & \\
\hline cr 51 & $9 \mathrm{E}-10$ & $1.89 \mathrm{E}-10$ & $1.88 \mathrm{E}-10$ & $1.88 E-10$ & $1.88 \mathrm{E}-10$ & $1.87 \mathrm{E}-10$ & $1.87 \mathrm{E}-10$ & $1.87 \mathrm{E}-10$ & & & $E-10$ \\
\hline cr 55 & $4 \mathrm{~F}-09$ & $0 \mathrm{~F}-12$ & $2 E-14$ & & & $0.00 \mathrm{E}+00$ & & $0.00 E+00$ & & & $E+00$ \\
\hline $\mathrm{mn} 54$ & $E-10$ & 1. $20 \mathrm{E}-10$ & $E-10$ & 1. $20 \mathrm{E}-10$ & $9 E-10$ & $1.19 \mathrm{E}-10$ & $1.19 \mathrm{E}-10$ & $1.19 \mathrm{E}-10$ & $1.19 \mathrm{E}-10$ & $1.19 \mathrm{E}-$ & $1.19 \mathrm{E}-10$ \\
\hline $\mathrm{mn} 56$ & 5.7 & $9 E-08$ & & & & & & & & & \\
\hline $\mathrm{mn} 57$ & $E-10$ & $2.22 \mathrm{E}-16$ & & $0 E+00$ & & & & & & & \\
\hline fe 55 & & & & $3 E-12$ & & & & & & & \\
\hline & & $E-13$ & & $9 \mathrm{E}-13$ & & & & & & & \\
\hline co 58 & & ח & & & & & & & & & \\
\hline $\mathrm{CO} \cdot 60$ & & & & & & & & & & & \\
\hline co $60 \mathrm{~m}$ & .9 & & & & & & & & & & \\
\hline co 61 & & & & & & & & & & & \\
\hline co 62 & & & & & & & & & & & \\
\hline ni 59 & 3 & & & & & & & & & & \\
\hline ni 63 & & & & & & & & & & & \\
\hline ni. 65 & & & & -10 & & & & & & & \\
\hline y $89 \mathrm{~m}$ & 13 & & & -13 & & & & & & & \\
\hline $\operatorname{zr} 89$ & & & & & & & & & & & \\
\hline zr 93 & & & & & & & & & & & \\
\hline zr 95 & & & & & & & & & & & \\
\hline zr 97 & & & & & & & & & & & \\
\hline nb 92 & & & & & & & & & & & \\
\hline $\mathrm{nb} 9$ & & & & & & & & & & & \\
\hline nb 95 & & & & & & & & & & & \\
\hline & & & & & & & & & & & \\
\hline & & $1.87 \mathrm{E}$ & & & & & & & & & \\
\hline nb 97 & -11 & & & -11 & & & & & & & \\
\hline & & & & & & & & & & & \\
\hline mo 93 & & & & & & & & & & & \\
\hline & & & & & & & & & & & \\
\hline & & & & & & & & & & & \\
\hline & & & & & & & & 4.5 & & & 6.2 \\
\hline & & 1. $36 \mathrm{E}-10$ & & & & .30 & & & & & \\
\hline & & & & & & 1.95 & & & & & \\
\hline & $6.32 \mathrm{E}-12$ & $6.32 \mathrm{E}-12$ & 6.325 & $6.32 \mathrm{E}-12$ & $6.32 \mathrm{E}-12$ & $6.31 \mathrm{E}-12$ & 6.31 & 6.3 & 6.30 & 6.2 & $6.29 \mathrm{E}-12$ \\
\hline & & & & & & & & & & & \\
\hline & & $1.28 \mathrm{E}-08$ & $1.26 \mathrm{E}-08$ & $1.23 \mathrm{E}-08$ & $1.16 E-08$ & $1.09 \mathrm{E}-08$ & & $9.71 \mathrm{E}-09$ & $9.17 \mathrm{E}-09$ & & \\
\hline & $1.61 \mathrm{E}-06$ & $2.77 \mathrm{E}-07$ & $9.24 \mathrm{E}-08$ & $5.38 \mathrm{E}-08$ & $3.85 \mathrm{E}-08$ & $2.97 \mathrm{E}-08$ & $2.43 E-08$ & $2.09 \mathrm{E}-08$ & $1.87 \mathrm{E}-08$ & $1.73 \mathrm{E}-08$ & 1. $62 \mathrm{E}-08$ \\
\hline
\end{tabular}


Table F29. Radioactivity (Ci) After Ten-Day Irradiation of 1-kg Havar Foil (Flux Parameter Set 1).

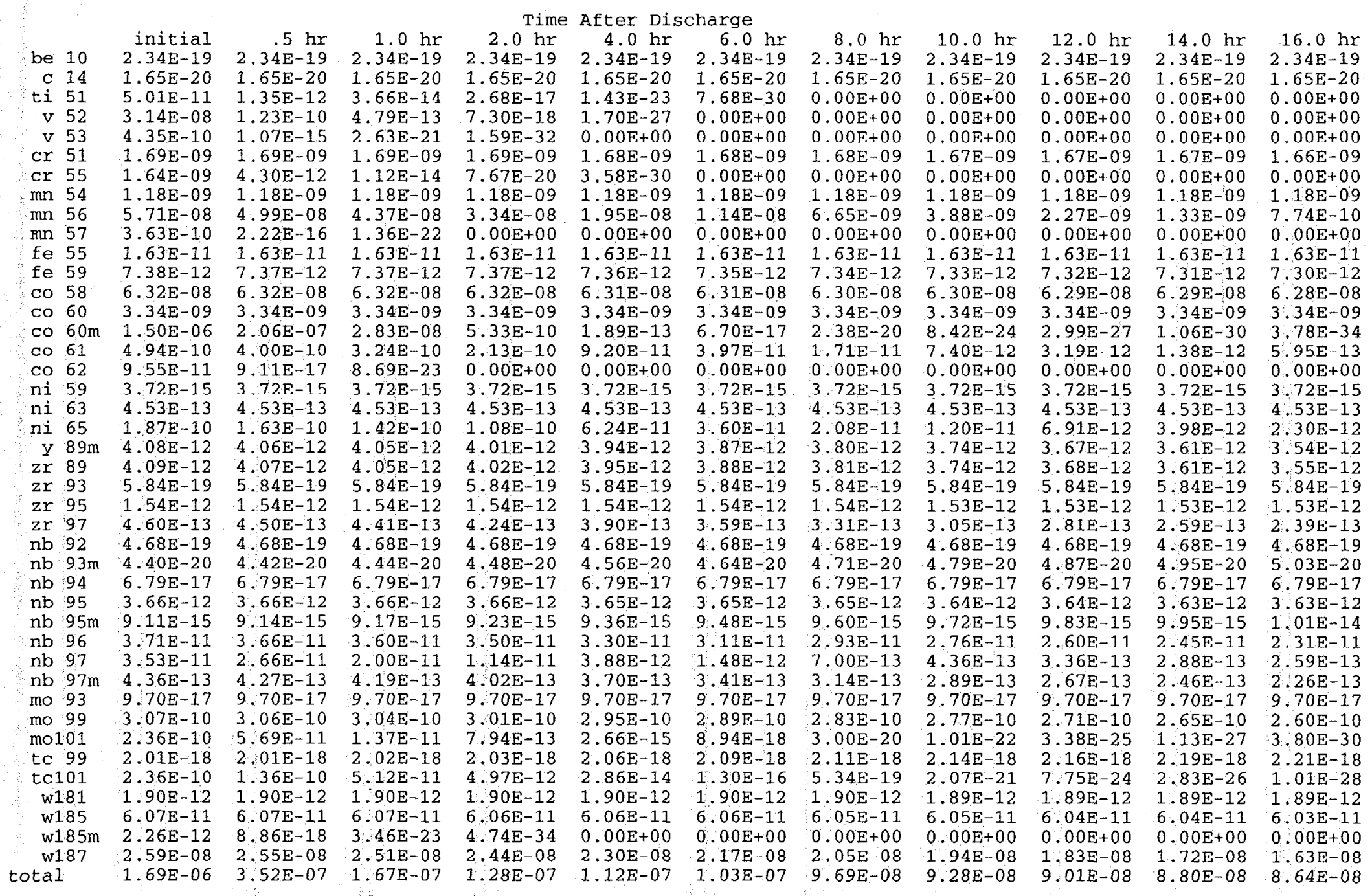


Table F30. Radioactivity (Ci) After One-Day Irradiation of 1-kg Havar Foil (Flux Parameter Set 2).

\begin{tabular}{|c|c|c|c|c|c|c|c|c|c|c|c|}
\hline & & & & Time & After & $\arg$ & & & & & \\
\hline & initial & & & 2.0111 & & 0.011 & $8.0 \mathrm{hr}$ & $10.0 \mathrm{hr}$ & & $14.0 \mathrm{hr}$ & $16.0 \mathrm{hr}$ \\
\hline be 10 & $1.63 \mathrm{E}-19$ & 1. $63 \mathrm{E}-19$ & 1. $63 \mathrm{E}-19$ & $1.63 \mathrm{E}-19$ & $1.63 E-19$ & $1.63 \mathrm{E}-19$ & $1.63 \mathrm{E}-19$ & $1.63 \mathrm{E}-19$ & $1.63 E-19$ & $1.63 E-19$ & $1.63 E-19$ \\
\hline C 14 & $2.54 \mathrm{E}-20$ & $2.54 E-20$ & $2.54 E-20$ & $2.54 E-20$ & $2.54 \mathrm{E}-20$ & $2.54 \mathrm{E}-20$ & $2.54 \mathrm{E}-20$ & $2.54 \mathrm{E}-20$ & $2.54 \mathrm{E}-20$ & $2.54 \mathrm{E}-20$ & $2.54 E-20$ \\
\hline ti 51 & $3.50 \mathrm{E}-10$ & $9.47 \mathrm{E}-12$ & $2.56 \mathrm{E}-13$ & $1.87 \mathrm{E}-16$ & $1.00 \mathrm{E}-22$ & $5.36 \mathrm{E}-29$ & $4.58 E-35$ & $0.00 \mathrm{E}+00$ & $0.00 E+00$ & $0.00 \mathrm{E}+00$ & $0.00 E+00$ \\
\hline v 52 & $9 E-07$ & $8.57 \mathrm{E}-10$ & $3.35 E-12$ & $5.10 \mathrm{E}-17$ & $1.19 E-26$ & $0.00 \mathrm{E}+00$ & $0.00 \mathrm{E}+00$ & $0.00 \mathrm{E}+00$ & $0.00 \mathrm{E}+00$ & $0.00 \mathrm{E}+00$ & $0.00 E+00$ \\
\hline v 53 & $3.04 \mathrm{E}-09$ & $7.47 \mathrm{E}-15$ & $1.84 E-20$ & $1.11 \mathrm{E}-31$ & $0.00 \mathrm{E}+00$ & $0.00 \mathrm{E}+00$ & $0.00 \mathrm{E}+00$ & $0.00 \mathrm{E}+00$ & $0.00 \mathrm{E}+00$ & $0.00 \mathrm{E}+00$ & $0.00 \mathrm{E}+00$ \\
\hline cr 51 & $1.71 \mathrm{E}-09$ & $1.71 \mathrm{E}-09$ & $1.71 E-09$ & $1.71 \mathrm{E}-09$ & $1.70 \mathrm{E}-09$ & $1.70 \mathrm{E}-09$ & $1.70 \mathrm{E}-09$ & $1.69 \mathrm{E}-09$ & $1.69 \mathrm{E}-09$ & $1.69 \mathrm{E}-09$ & $1.68 \mathrm{E}-09$ \\
\hline cr 55 & $1.21 \mathrm{E}-08$ & 3. $16 \mathrm{E}-11$ & $8.27 E-14$ & $5.65 \mathrm{E}-19$ & 2. $64 \mathrm{E}-29$ & $0.00 \mathrm{E}+00$ & $0.00 \mathrm{E}+00$ & $0.00 \mathrm{E}+00$ & $0.00 \mathrm{E}+00$ & $0.00 \mathrm{E}+00$ & $0.00 \mathrm{E}+00$ \\
\hline $\mathrm{mn} 54$ & $8.35 E-10$ & $8.35 E-10$ & $8.35 E-10$ & $8.35 E-10$ & $8.35 E-10$ & $8.35 \mathrm{E}-10$ & $8.35 \mathrm{E}-10$ & $8.35 E-10$ & $8.34 \mathrm{E}-10$ & $8.31 \mathrm{E}-10$ & $8.34 \mathrm{E}-10$ \\
\hline $\mathrm{mn} 56$ & $5.77 \mathrm{E}-07$ & $5.05 \mathrm{E}-07$ & $4.41 \mathrm{E}-07$ & $3.37 \mathrm{E}-07$ & & $1.15 \mathrm{E}-07$ & $6.72 \mathrm{E}-08$ & $3.93 \mathrm{E}-08$ & $2.29 \mathrm{E}-08$ & & $7.82 \mathrm{E}-09$ \\
\hline $\operatorname{mn} 57$ & $2.54 \mathrm{E}-09$ & 1. $55 \mathrm{E}-15$ & $9.48 \mathrm{E}-22$ & $3.63 \mathrm{E}-34$ & $0.00 \mathrm{E}+00$ & $0.00 \mathrm{E}+00$ & $0.00 \mathrm{E}+00$ & $0.00 \mathrm{E}+00$ & $0.00 E+00$ & $0.00 \mathrm{E}+00$ & $0.00 E+00$ \\
\hline $\operatorname{mn} 58$ & & & & $0.00 \mathrm{E}+00$ & & & $0.00 \mathrm{E}+00$ & $0.00 \mathrm{E}+00$ & & & \\
\hline fe 55 & $1.36 E-11$ & $1.36 \mathrm{E}-11$ & $6 \mathrm{E}-11$ & $1.36 \mathrm{E}-11$ & 1. $36 \mathrm{E}-11$ & $1.36 \mathrm{E}-11$ & 1. $36 \mathrm{E}-11$ & $1.36 \mathrm{E}-11$ & & & 1. $36 \mathrm{E}-11$ \\
\hline fe 59 & $8.55 E-12$ & $8.54 \mathrm{E}-12$ & & & & & $8.50 E-12$ & & & & $8.46 \mathrm{E}-12$ \\
\hline co 58 & $4.62 \mathrm{E}-08$ & $4.62 \mathrm{E}-08$ & $2 E-08$ & $61 E-08$ & & $4.61 \mathrm{E}-08$ & $4.60 \mathrm{E}-08$ & $4.60 E-08$ & $8-08$ & -08 & $E-08$ \\
\hline co 60 & 5.7 & $5.82 E-09$ & $5.83 \mathrm{E}-09$ & $34 \mathrm{E}-09$ & $5.84 E-09$ & $5.84 \mathrm{E}-09$ & 5.84 & -09 & & & $8-09$ \\
\hline $\mathrm{co} 60 \mathrm{~m}$ & & $3.60 \mathrm{E}-06$ & $4.95 \mathrm{E}-07$ & $31 E-09$ & 3. $30 \mathrm{E}-12$ & $1.17 \mathrm{E}-15$ & -19 & -22 & & & -33 \\
\hline co 61 & 3.4 & $30 E-09$ & -09 & $9 E-09$ & -10 & 2.78 & $:-10$ & 5. & 2. & & -12 \\
\hline 62 & 6.6 & 6.3 & 6. & $7 E-34$ & +00 & 0.0 & 0. & & & & \\
\hline 59 & 3.8 & $3.65 \mathrm{E}-1.5$ & 3.6 & $3.65 E-15$ & -15 & -15 & 3.6 & & & & \\
\hline 63 & & $4.44 \mathrm{E}-13$ & 4.44 & $4.44 \mathrm{E}-1.3$ & 4.4 & $4.44 \mathrm{E}-13$ & 4.4 & 4.4 & 4.4 & & 4. \\
\hline ni 65 & 2.1 & $1.90 \mathrm{E}-09$ & 1.6 & $1.26 \mathrm{E}-09$ & $E-10$ & $4.19 \mathrm{E}-10$ & 2.4 & 1.4 & & & -11 \\
\hline y $89 \mathrm{~m}$ & 6.2 & $6.17 E-12$ & & $6.09 \mathrm{E}-12$ & & 5.8 & 5.7 & & & & \\
\hline $\operatorname{zr} 89$ & 6.2 ( & & & & & & & & & & \\
\hline zr 93 & 4.0 & $4.08 \mathrm{E}$ & 4.0 & $E-19$ & & 4.0 & 4.0 & 4.0 & 4. & 4. & $E-19$ \\
\hline zr 95 & $1.13 E-12$ & $1.13 E-$ & & $3 E-12$ & & 1.1 & 1.1 & & $1 .$. & & -12 \\
\hline zr 97 & 2.0 & $7 E-12$ & 1.9 & $5 E-12$ & $E-12$ & 1.5 & 1.4 & & & & -12 \\
\hline nb 92 & 3.2 & $27 E-19$ & 3.2 & $7 E-19$ & -19 & $3.27 \mathrm{E}-19$ & 3. & 3.2 & & & \\
\hline nb 94 & 4.7 & 4.74 & & $4.74 \mathrm{E}-17$ & & & & & & & \\
\hline nb 95 & 2.69 & $2.69 \mathrm{E}-12$ & 2.69 & 2. $69 \mathrm{E}-12$ & -12 & $2.68 \mathrm{E}-12$ & 2.68 & 2.6 & 2.6 & 2. & -12 \\
\hline $\mathrm{nb} 95 \mathrm{~m}$ & 3.9 & 4.4 & & $6 E-16$ & & & & & & & \\
\hline nb 96 & 1.3 & 1.3 & 1.28 & $5 E-10$ & -10 & 1.1 & 1. & 9.8 & 9.2 & 8.7 & $8.23 E-11$ \\
\hline $\mathrm{nb} 97$ & & 1.8 & 1.39 & & & & & & & & \\
\hline$n b 97 m$ & -12 & 1.8 & 1.83 & $5 E-12$ & -12 & 1.49 & 1.3 & 1.2 & 1.1 & 1. & -13 \\
\hline mo 93 & $2.54 \mathrm{E}-16$ & $2.54 \mathrm{E}-16$ & 2.54 & $2.54 E-16$ & & & & 2. & 2.5 & & $2.54 \mathrm{E}-16$ \\
\hline mo 99 & $2.15 E-09$ & $2.14 \mathrm{E}-09$ & 2.13 & 2.11E-09 & $6 E-09$ & $2.02 \mathrm{E}-09$ & & & & & \\
\hline & & $1.56 \mathrm{E}-09$ & & $2.18 E-11$ & & $2.45 \mathrm{E}-16$ & & & & & 1.0 \\
\hline & 3.92 & $4.40 \mathrm{E}-19$ & 4.88 & $5.83 E-19$ & & $9.54 \mathrm{E}-19$ & & & & & \\
\hline tc10 & 6.49 & 3.741 & 1.41 & 1. $36 \mathrm{E}-10$ & $7.86 \mathrm{E}-13$ & $3.57 \mathrm{E}-15$ & 1.47 & 5. & & 7.7 & $E-27$ \\
\hline & & $5.65 E-12$ & 5.65 & & & $5.65 E-12$ & $5.64 \mathrm{E}-12$ & & & & $5.63 E-12$ \\
\hline & 1. $56 \mathrm{E}-10$ & 1. $56 \mathrm{E}-10$ & 1.56 & 1. $56 \mathrm{E}-10$ & $1.56 \mathrm{E}-10$ & 1. $56 \mathrm{E}-10$ & 1. $56 \mathrm{E}-10$ & $1.56 \mathrm{E}-10$ & $1.56 \mathrm{E}-10$ & & $1.55 \mathrm{E}-10$ \\
\hline & $5.60 \mathrm{E}-11$ & $2.19 E-16$ & $8.56 \mathrm{E}-22$ & 1. $31 \mathrm{E}-32$ & $0.00 E+00$ & $0.00 \mathrm{E}+00$ & $0.00 \mathrm{E}+00$ & $0.00 \mathrm{E}+00$ & $0.00 \mathrm{E}+00$ & $0.00 E+00$ & $0.00 E+00$ \\
\hline & $3.45 \mathrm{E}-07$ & $3.40 \mathrm{E}-07$ & $3.35 \mathrm{E}-07$ & $3.25 E-07$ & $3.07 \mathrm{E}-07$ & $2.90 \mathrm{E}-07$ & $2.73 E-07$ & $2.58 E-07$ & $2.44 \mathrm{E}-07$ & $2.30 \mathrm{E}-07$ & $2.17 \mathrm{E}-07$ \\
\hline & $2.75 \mathrm{E}-05$ & $4.52 \mathrm{E}-06$ & $1.33 \mathrm{E}-06$ & $7.32 \mathrm{E}-07$ & $5.62 \mathrm{E}-07$ & $4.62 \mathrm{E}-07$ & $3.98 \mathrm{E}-07$ & $3.54 \mathrm{E}-07$ & $3.23 \mathrm{E}-07$ & $3.00 E-07$ & $2.81 E-07$ \\
\hline
\end{tabular}


Table F31. Radioactivity (Ci) After Ten-Day Irradiation of 1-kg Havar Foil (Flux Parameter Set 2).

\begin{tabular}{|c|c|c|c|c|c|c|c|c|c|c|c|}
\hline & & & & & & & & & & & \\
\hline & initial & & & & & & & & & & 16 \\
\hline be 10 & $1.63 \mathrm{E}-18$ & $.63 \mathrm{E}-18$ & $.63 \mathrm{E}-18$ & 1. $63 \mathrm{E}-18$ & 1. $63 \mathrm{E}-18$ & 1. $63 \mathrm{E}-18$ & 1. $63 \mathrm{E}-18$ & 1. $63 \mathrm{E}-18$ & $1.63 \mathrm{E}-18$ & $1.63 \mathrm{E}-18$ & $63 \mathrm{E}-18$ \\
\hline & & & $.54 \mathrm{E}-19$ & $.54 \mathrm{E}-19$ & $2.54 \mathrm{E}-19$ & $2.54 \mathrm{E}-19$ & $2.54 \mathrm{E}-19$ & $2.54 \mathrm{E}-19$ & $2.54 \mathrm{E}-19$ & $.54 \mathrm{E}-19$ & $2.54 \mathrm{E}-19$ \\
\hline & $3.50 \mathrm{E}-10$ & $9.47 \mathrm{E}-12$ & $.56 \mathrm{E}-13$ & $.87 E-16$ & $1.00 \mathrm{E}-22$ & $5.36 \mathrm{E}-29$ & $4.58 \mathrm{E}-35$ & $0.00 \mathrm{E}+00$ & $0.00 \mathrm{E}+00$ & $0.00 \mathrm{E}+00$ & $0.00 \mathrm{E}+00$ \\
\hline 52 & & & & & $1.19 \mathrm{E}-26$ & $0.00 \mathrm{E}+00$ & & $0.00 \mathrm{E}+00$ & $0.00 \mathrm{E}+00$ & $.00 \mathrm{E}+00$ & $00 E+00$ \\
\hline 53 & $3.04 \mathrm{E}-09$ & $7.47 \mathrm{E}-15$ & $1.84 \mathrm{E}-20$ & $1.11 \mathrm{E}-31$ & $0.00 \mathrm{E}+00$ & $0.00 \mathrm{E}+00$ & $0.00 \mathrm{E}+00$ & $0.00 \mathrm{E}+00$ & $0.00 \mathrm{E}+00$ & $0.00 \mathrm{E}+00$ & $0.00 \mathrm{E}+00$ \\
\hline 51 & & $1.53 E-08$ & & & $1.53 \mathrm{E}-08$ & $1.52 \mathrm{E}-08$ & & $1.52 \mathrm{E}-08$ & & & \\
\hline 55 & $1.21 \mathrm{E}-08$ & $6 E-11$ & $8.27 \mathrm{E}-14$ & $.65 E-19$ & $2.64 \mathrm{E}-29$ & $0.00 \mathrm{E}+00$ & $0.00 \mathrm{E}+00$ & $0.00 \mathrm{E}+00$ & $0 E+00$ & & $0 \mathrm{E}+00$ \\
\hline 54 & $8.27 E-09$ & $7 E-09$ & -09 & $.27 E-09$ & $8.27 E-09$ & $8.27 \mathrm{E}-09$ & & $2-09$ & E-09 & & 2-09 \\
\hline 56 & $5.78 \mathrm{E}-07$ & $5 E-07$ & & $3 E-07$ & $1.97 E-07$ & & & & & & -09 \\
\hline 57 & 2.5 & 1. & & $63 E-34$ & $0.00 E+00$ & $0.00 \mathrm{E}+00$ & & & +00 & & $E+00$ \\
\hline 58 & & & & & & & & & & & \\
\hline$r$ & & & & & & & & & & & \\
\hline 59 & 7.9 & & & -11 & $E-11$ & 7.5 & & & & & \\
\hline 58 & 4.4 & 4.4 & & & -07 & & & & & & \\
\hline 60 & 5.8 & 5. & & & 5.8 & & & & & & \\
\hline $60 \mathrm{~m}$ & & & & & & & & & & & \\
\hline 61 & & & & & & & & & & & \\
\hline 62 & 6. & & & & & & & & & & \\
\hline 59 & & & & & & & & & & & \\
\hline 63 & 4.4 & 4.4 & $\therefore$ & & 4.4 & 4. & & & & & \\
\hline 65 & & & & & & & & & & & \\
\hline $89 \mathrm{~m}$ & 2.8 & & & & -11 & & & & & & -11 \\
\hline 89 & 2.8 & & & & $:-11$ & & & & & & \\
\hline 93 & 4.0 & 4. & & & 4.0 & & & & & & \\
\hline 95 & & & & & & & & & & & \\
\hline 97 & & & & & & & & & & & \\
\hline 92 & 3.2 & & & & & & & & & & \\
\hline & .1 & . & & & & & & & & & \\
\hline 94 & 4 & & & -16 & 4.7 & 4. & & & & & \\
\hline & & & & & & & & & & & \\
\hline $95 \mathrm{~m}$ & & & & & & & & & & & \\
\hline 96 & & & & -10 & & & & & & & \\
\hline 97 & & & & & & & & & & & \\
\hline $97 \mathrm{~m}$ & & & & & & & & & & & \\
\hline 93 & 2.5 & 2.5 & & -15 & & & & & & & \\
\hline & & & & & & & & & & & \\
\hline & & & & -11 & 7.3 & 2.4 & & & & & \\
\hline & & & & & & & & & & & \\
\hline & & 3.7 & & & & & & & & & \\
\hline & 5.5 & & & & $5.51 \mathrm{E}-11$ & & & & & & $E-11$ \\
\hline & & & & & & & & & & & \\
\hline & 5.6 & $2.19 \mathrm{E}-16$ & 8.56 & $1.31 E-32$ & $0.00 \mathrm{E}+00$ & $0.00 \mathrm{E}+00$ & 0.0 & & & & $E+00$ \\
\hline & $6.87 \mathrm{E}-07$ & $6.77 \mathrm{E}-07$ & $.67 \mathrm{E}-07$ & $6.48 \mathrm{E}-07$ & $6.12 \mathrm{E}-07$ & $5.77 \mathrm{E}-07$ & $5.45 \mathrm{E}-07$ & $5.14 \mathrm{E}-07$ & $4.85 \mathrm{E}-07$ & $4.58 \mathrm{E}-07$ & $4.32 E-07$ \\
\hline
\end{tabular}




\section{Table F31. (continued)}

\begin{tabular}{|c|c|c|c|c|c|c|c|c|c|c|c|}
\hline & & & & Time & After Di & charge & & & & & \\
\hline & initial & $.5 \mathrm{hr}$ & $1.0 \mathrm{hr}$ & $2.0 \mathrm{hr}$ & $4.0 \mathrm{hr}$ & $6.0 \mathrm{hr}$ & $8.0 \mathrm{hr}$ & $10.0 \mathrm{hr}$ & $12.0 \mathrm{hr}$ & $14.0 \mathrm{hr}$ & $16.0 \mathrm{hr}$ \\
\hline w188 & $2.56 \mathrm{E}-20$ & $2.56 \mathrm{E}-20$ & $2.56 \mathrm{E}-20$ & $2.56 \mathrm{E}-20$ & $2.55 \mathrm{E}-20$ & $2.55 \mathrm{E}-20$ & $2.55 \mathrm{E}-20$ & $2.55 \mathrm{E}-20$ & $2.54 \mathrm{E}-20$ & $2.54 \mathrm{E}-20$ & $2.54 \mathrm{E}-20$ \\
\hline re187 & $1.73 \mathrm{E}-19$ & $1.74 \mathrm{E}-19$ & $1.74 E-19$ & $1.75 E-19$ & $1.77 \mathrm{E}-19$ & $1.79 \mathrm{E}-19$ & $1.81 \mathrm{E}-19$ & $1.83 \mathrm{E}-19$ & $1.84 \mathrm{E}-19$ & $1.86 \mathrm{E}-19$ & $1.87 \mathrm{E}-19$ \\
\hline re188 & $2.61 \mathrm{E}-19$ & $2.64 \mathrm{E}-19$ & $2.61 \mathrm{E}-19$ & $2.53 E-19$ & $2.35 E-19$ & $2.19 \mathrm{E}-19$ & $2.04 E-19$ & $1.90 \mathrm{E}-19$ & $1.77 \mathrm{E}-19$ & $1.65 \mathrm{E}-19$ & 1. $54 \mathrm{E}-19$ \\
\hline re188m & $5.95 E-19$ & $1.95 E-19$ & $6.36 \mathrm{E}-20$ & $6.80 E-21$ & $7.77 \mathrm{E}-23$ & $8.87 E-25$ & $1.01 \mathrm{E}-26$ & $1.16 \mathrm{E}-28$ & $1.32 \mathrm{E}-30$ & $1.51 \mathrm{E}-32$ & $1.70 \mathrm{E}-34$ \\
\hline otal & $2.83 E-05$ & $5.33 \mathrm{E}-06$ & $2.14 \mathrm{E}-06$ & $1.53 \mathrm{E}-06$ & $1.34 \mathrm{E}-06$ & 1. $23 \mathrm{E}-06$ & $1.15 \mathrm{E}-06$ & $1.09 \mathrm{E}-06$ & $1.04 \mathrm{E}-06$ & $1.00 \mathrm{E}-06$ & $9.70 \mathrm{E}-07$ \\
\hline
\end{tabular}


Table F32. Radioactivity (Ci) After One-Day Irradiation of 1-kg Concrete (Flux Parameter Set 3).

\begin{tabular}{|c|c|c|c|c|c|c|c|c|c|c|c|c|}
\hline \multicolumn{13}{|c|}{ Time After I } \\
\hline & & initial & & $1.0 \mathrm{hr}$ & $2.0 \mathrm{hr}$ & $4.0 \mathrm{hr}$ & $6.0 \mathrm{hr}$ & & $10.0 \mathrm{hr}$ & $12.0 \mathrm{hr}$ & $14.0 \mathrm{hr}$ & $16.0 \mathrm{~h}$ \\
\hline & 3 & $1.66 \mathrm{E}-18$ & $1.66 \mathrm{E}-18$ & $1.66 \mathrm{E}-18$ & $1.66 \mathrm{E}-18$ & $1.66 \mathrm{E}-18$ & $1.66 \mathrm{E}-18$ & $1.66 \mathrm{E}-18$ & $.66 E-18$ & $1.66 \mathrm{E}-18$ & $1.66 \mathrm{E}-18$ & 1. $66 \mathrm{E}-18$ \\
\hline C & 14 & 1. $30 \mathrm{E}-17$ & $1.30 \mathrm{E}-17$ & $1.30 \mathrm{E}-17$ & $1.30 \mathrm{E}-17$ & $1.30 \mathrm{E}-17$ & $1.30 \mathrm{E}-17$ & $1.30 \mathrm{E}-17$ & $.30 \mathrm{E}-17$ & $1.30 \mathrm{E}-17$ & 1. $30 \mathrm{E}-17$ & \\
\hline na & 24 & $7.82 \mathrm{E}-09$ & $7.63 E-09$ & $7.45 E-09$ & & $6.47 E-09$ & & & $7 E-09$ & $4.43 E-09$ & $03 E-09$ & $7 E-09$ \\
\hline na & 25 & $1.76 \mathrm{E}-11$ & $1.42 \mathrm{E}-20$ & $1.15 \mathrm{E}-29$ & $0.00 \mathrm{E}+00$ & $0.00 \mathrm{E}+00$ & $0.00 \mathrm{E}+00$ & $.00 \mathrm{E}+00$ & $.00 \mathrm{E}+00$ & $0.00 \mathrm{E}+00$ & $0.00 \mathrm{E}+00$ & $0.00 \mathrm{E}+00$ \\
\hline $\mathrm{mg}$ & 27 & $E-09$ & $4.71 \mathrm{E}-10$ & $5.23 \mathrm{E}-11$ & $5 E-13$ & & 1. & $27 E-24$ & & & & \\
\hline al & 28 & -08 & $5.68 E-12$ & $3.28 \mathrm{E}-16$ & $7 E-24$ & $E-29$ & & & & & & $3 E-29$ \\
\hline al & 29 & 1.6 & g-11 & 2.88 & $E-15$ & $E-20$ & & & 0 & & & \\
\hline si. & 31 & & & & & & & & & & & \\
\hline cl & 7 & & & 1.2 & & & & & & & & \\
\hline ar & 37 & 5.0 & & & & & & & & & & \\
\hline ar & 39 & & & & & & & & & & & \\
\hline ar & 41 & 8 & & 5.6 & -13 & & & & & & & \\
\hline$k$ & 40 & & & & & & & & & & & \\
\hline$k$ & 42 & 6.8 & & 6.8 & 6 . & & & & & & & \\
\hline$k$ & 43 & 1.1 & & & & & & & & & & \\
\hline $\mathrm{k}$ & & & & & & & & & & & & \\
\hline ca & 41 & 1.0 & & 1.0 & 1.0 & & & & & & & \\
\hline ca & 45 & & & & & & & & & & & \\
\hline $\mathrm{ca}$ & 47 & 5.3 & 5.3 & & & & & & & & & \\
\hline $\mathrm{ca}$ & 49 & & 5.9 & & & & & & & & & \\
\hline sc & 47 & & & 2.2 & & & & & & & & \\
\hline $\mathrm{sc}$ & 49 & & & & & & & & & -14 & & \\
\hline cr & 51 & & & & & & & & & & & \\
\hline cr & & & & & & & & & & & & \\
\hline $\mathrm{mn}$ & 54 & 7.6 & 7.6 & & & & & & & & & \\
\hline $\mathrm{mn}$ & & & 1.6 & 1.4 & 1.1 & & & & & 7. & & 2.5 \\
\hline & & & 1. $42 \mathrm{E}-18$ & & 0.0 & 0.0 & & & & & & \\
\hline & & & & & & & & & & & & \\
\hline & & $2.37 \mathrm{E}-13$ & $2.37 \mathrm{E}-13$ & & $2.37 E-13$ & $2.37 \mathrm{E}-1$ & & $2.36 \mathrm{E}-$ & $2.36 \mathrm{E}-1$ & & & \\
\hline & & $1.01 \mathrm{E}-07$ & $2.19 \mathrm{E}-08$ & $2.10 \mathrm{E}-08$ & $2.02 \mathrm{E}-08$ & & & $1.73 E-08$ & $1.66 \mathrm{E}-08$ & $1.61 \mathrm{E}-08$ & 1. $56 \mathrm{E}-08$ & $1.52 \mathrm{E}-\mathrm{C}$ \\
\hline
\end{tabular}


Table F33. Radioactivity (Ci) After Ten-Day Irradiation of 1-kg Concrete (Flux Parameter Set 3).

\begin{tabular}{|c|c|c|c|c|c|c|c|c|c|c|c|}
\hline & & & & & & & & & & & \\
\hline & InI & .0111 & 1.0111 & $2.0 \mathrm{~h}$ & & & $8.0 \mathrm{hr}$ & $10.0 \mathrm{hr}$ & $12.0 \mathrm{hr}$ & $14.0 \mathrm{hr}$ & $16.0 \mathrm{~h}$ \\
\hline h 3 & & 1. $66 \mathrm{E}-17$ & $.66 \mathrm{E}-17$ & $.66 \mathrm{E}-17$ & $1.66 \mathrm{E}-17$ & 1. $66 \mathrm{E}-17$ & 1. $66 \mathrm{E}-17$ & $1.66 \mathrm{E}-17$ & $1.66 \mathrm{E}-17$ & $1.66 \mathrm{E}-17$ & $1.66 \mathrm{E}-17$ \\
\hline be 10 & $1.02 \mathrm{E}-20$ & $1.02 \mathrm{E}-20$ & $.02 \mathrm{E}-20$ & $1.02 \mathrm{E}-20$ & $1.02 \mathrm{E}-20$ & $1.02 \mathrm{E}-20$ & $1.02 \mathrm{E}-20$ & $1.02 \mathrm{E}-20$ & $1.02 \mathrm{E}-20$ & $1.02 E-20$ & $1.02 \mathrm{E}-20$ \\
\hline & & & & & & & & & & & \\
\hline na 24 & $5 E-08$ & $2 E-08$ & $0 \mathrm{E}-08$ & $5 E-08$ & $3 E-09$ & $8.67 \mathrm{E}-09$ & $7.89 \mathrm{E}-09$ & $7.18 \mathrm{E}-09$ & & $5.94 \mathrm{E}-09$ & $5.40 \mathrm{E}-09$ \\
\hline na 25 & E-11 & $E-20$ & 20 & $0 E+00$ & $E+00$ & $0.00 E+00$ & $0.00 \mathrm{E}+00$ & $0.00 E+00$ & $0.00 \mathrm{E}+00$ & $0.00 \mathrm{E}+00$ & $0.00 \mathrm{E}+00$ \\
\hline $\mathrm{mg} 27$ & $E-09$ & -10 & -11 & $5 E-13$ & $E-17$ & $1.19 \mathrm{E}-20$ & $7 E-21$ & -28 & & $O E+00$ & $E+00$ \\
\hline al 28 & -08 & 5.6 & $9-16$ & $7 E-24$ & & $E-29$ & & & & & -29 \\
\hline 29 & & & & & & & & & & & \\
\hline si 31 & & & & LE-09 & & $6 \mathrm{E}-10$ & & & & & $E-11$ \\
\hline Cl 36 & & & & & & & & & & & \\
\hline ar & -10 & & & & & & & & & & \\
\hline ar 39 & & & & $E-13$ & & & & & & & \\
\hline ar 41 & & & & & & & & & & & \\
\hline k 40 & .1 & & & -08 & & & & & & & \\
\hline k 42 & 10 & & & & & 6.6 & & & & & \\
\hline k 43 & & & & & & & & & & & \\
\hline k 44 & 12 & 13 & & & & 2.4 & & & & & \\
\hline ca 41 &. & & & & & & & & & & \\
\hline ca 45 & 1 & & & -11 & & 1.2 & & & & & \\
\hline ca 47 & -13 & & & & & & & & & & \\
\hline ca 49 & & & & -15 & & 2.3 & & & & & \\
\hline sc 47 & 13 & & & & & $1.8 \varepsilon$ & & & & & \\
\hline sc 49 & & & & & & & & & & & \\
\hline cr 51 & 2.7 & & & & & & & & & & \\
\hline cr 55 & & & & & & & & & & & \\
\hline $\mathrm{mn} 54$ & & & & & & 7.55 & & & & & -12 \\
\hline $\operatorname{mn} 56$ & 1.90 & $1.66 \mathrm{E}-10$ & & & & $3.79 \mathrm{E}-11$ & & & & & \\
\hline mn 57 & -12 & & & & & $0.00 \mathrm{E}+00$ & & & & & \\
\hline & 3.17 & $3.17 \mathrm{E}-12$ & & & & 3.17 & & & & & $\mathrm{E}-12$ \\
\hline & 2.22 & $2.21 \mathrm{E}-12$ & & & & 2. $21 \mathrm{E}-12$ & & & & & \\
\hline & $1.06 \mathrm{E}-07$ & $2.62 E-08$ & $2.52 \mathrm{E}-08$ & $2.42 \mathrm{E}-08$ & $2.27 \mathrm{E}-08$ & $2.14 \mathrm{E}-08$ & $2.04 \mathrm{E}-08$ & $1.95 E-08$ & $1.87 E-08$ & $1.81 \mathrm{E}-08$ & $1.75 \mathrm{E}-0$ \\
\hline
\end{tabular}




\section{APPENDIX G}

\section{Tables of ORIGEN-Calculated Decay Photon Spectra for Selected Cargoes}

and Structures Irradiated in the PFNA Facility 
ح

$\curvearrowright$

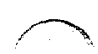




\section{APPENDIX G}

Tables of ORIGEN-Calculated Decay Photon Spectra for Selected Cargoes and Structures Irradiated in the PFNA Facility

Table G1. Decay Photon Spectrum (/kg.s) After 8-s Irradiation of 1-kg Salted Beef Cargo (Flux Parameter Set 1).

\begin{tabular}{|c|c|c|c|c|c|c|c|c|c|c|c|}
\hline emean & & & & 2 after & charge & & & & & & \\
\hline$(\mathrm{mev})$ & ll11 121 & - & $1.0 \mathrm{sec}$ & $10.0 \mathrm{sec}$ & 20.0 sec & $30.0 \mathrm{sec}$ & 60.0 sec & $120.0 \mathrm{sec}$ & $300.0 \mathrm{sec}$ & $600.0 \mathrm{sec}$ & $1200.0 \mathrm{~s}$ \\
\hline $3.00 \mathrm{E}-01$ & & $E+01$ & $.00 E+01$ & $2.48 \mathrm{E}+01$ & $1.15 \mathrm{E}+01$ & & & & & & \\
\hline & +01 & +01 & +01 & & & $E+$ & 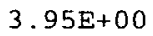 & & & & $7 E-01$ \\
\hline & & & & & & & & & & & \\
\hline & & & & & & & & & & & \\
\hline & & & & & & & & & & & \\
\hline & & & & & & & & & & & \\
\hline & & & & & & & & & & & \\
\hline & & & & & & & & & & & \\
\hline 3.7 & 1.1 & & & & & & & & & & \\
\hline & & & & & & & & & & & -07 \\
\hline & & & & & & & & & & & $D E+00$ \\
\hline & & & & & & & & & & & $0.00 \mathrm{E}+00$ \\
\hline & $1.71 \mathrm{E}+02$ & & & $7.05 E+01$ & $3.85 E+01$ & $2.57 E+01$ & & $1.032+01$ & $7.96 \mathrm{E}+00$ & $7.49 \mathrm{E}+00$ & $7.37 E+00$ \\
\hline & 1. $38 \mathrm{E}+02$ & $1.38 \mathrm{E}+02$ & ר & $5.96 E+01$ & $3.46 E+01$ & $2.47 E+01$ & 1. $68 \mathrm{E}+01$ & $1.34 E+01$ & 1. $12 E+01$ & $1.06 \mathrm{E}+01$ & $1.04 E+01$ \\
\hline
\end{tabular}


Table G2. Decay Photon Spectrum (/kg.s) After 16-s Irradiation of 1-kg Salted Beef Cargo (Flux Parameter Set 1).

\begin{tabular}{|c|c|c|c|c|c|c|c|c|c|c|c|}
\hline emean & & & & after & charge & & & & & & \\
\hline (mev) & initial & $1 \mathrm{E}-15 \mathrm{sec}$ & $1.0 \mathrm{sec}$ & $10.0 \mathrm{sec}$ & $20.0 \mathrm{sec}$ & 30.0 sec & $60.0 \mathrm{sec}$ & $120.0 \mathrm{sec}$ & $300.0 \mathrm{sec}$ & $600.0 \mathrm{sec}$ & $1200.0 \mathrm{sec}$ \\
\hline $3.00 \mathrm{E}-01$ & $9.62 \mathrm{E}+01$ & $9.62 \mathrm{E}+01$ & $8.62 \mathrm{E}+01$ & $3.73 \mathrm{E}+01$ & $1.79 E+01$ & $1.02 E+01$ & $4.16 E+00$ & $1.93 E+00$ & $9.10 \mathrm{E}-01$ & $8.29 E-01$ & $8.15 \mathrm{E}-01$ \\
\hline $6.50 E-01$ & $2 \mathrm{E}+01$ & $72 E+01$ & $.92 \mathrm{E}+01$ & $3.94 \mathrm{E}+01$ & $2.25 E+01$ & $1.50 E+01$ & $7.14 E+00$ & $.52 \mathrm{E}+00$ & $76 \mathrm{E}-01$ & $2.57 \mathrm{E}-01$ & $2.29 \mathrm{E}-01$ \\
\hline $1.12 \mathrm{E}+00$ & $2.23 \mathrm{E}+01$ & $2.23 E+01$ & 1. $99 \mathrm{E}+01$ & $8.20 \mathrm{E}+00$ & $3.64 E+00$ & $88 E+00$ & $97 E-01$ & $11 E-01$ & $6 E-02$ & $16 E-02$ & \\
\hline $1.58 E+00$ & $E+01$ & $E+01$ & $6 \mathrm{E}+01$ & $1.46 \mathrm{E}+01$ & $1.22 \mathrm{E}+01$ & $1.10 \mathrm{E}+01$ & $O E+00$ & $\mathrm{OE}+0 \mathrm{O}$ & $2 \mathrm{E}+00$ & $52 \mathrm{E}+00$ & $6.34 \mathrm{E}+00$ \\
\hline $2.00 E+00$ & $5.25 \mathrm{E}+00$ & $\mathrm{E}+00$ & $E+00$ & $1.89 \mathrm{E}+00$ & $7.98 \mathrm{E}-01$ & $1 E-01$ & $4 E-02$ & $4 \mathrm{E}-02$ & & & $6 E-05$ \\
\hline $2.40 E+00$ & $3.50 \mathrm{E}+00$ & $E+00$ & $E+00$ & $1.22 \mathrm{E}+00$ & $4.88 \mathrm{E}-01$ & $5 E-01$ & $9 \mathrm{E}-02$ & 1. $11 \mathrm{E}-02$ & $7 \mathrm{E} 04$ & $9-04$ & \\
\hline $2.80 \mathrm{E}+00$ & $4.67 \mathrm{E}+00$ & $7 E+00$ & $E+00$ & $1.72 \mathrm{E}+00$ & $9 \mathrm{E}-01$ & $1 E-01$ & $E-02$ & $8 E-02$ & $E-02$ & $E-02$ & 3 . \\
\hline 3. $25 E+00$ & $\mathrm{E}+00$ & +00 & $E+00$ & $4.74 \mathrm{E}-01$ & $E-01$ & -02 & $2-03$ & $1.11 \mathrm{E}-03$ & $5 E-05$ & $1 E-07$ & \\
\hline 00 & 1.54 & 1.5 & +00 & 5.22 & & & & & & & $E-05$ \\
\hline $4.25 E+00$ & 5.471 & 5.4 & -01 & 1.86 & 6.9 & 2.6 & 1.4 & $2.26 \mathrm{E}-05$ & $9.29 E-07$ & $E-07$ & $\mathrm{~F}-07$ \\
\hline & $4.71 \mathrm{E}-01$ & & & $1.61 \mathrm{E}-01$ & & & $1.22 \mathrm{E}-03$ & $7 E-06$ & $8.98 \mathrm{E}-14$ & $0.00 E+00$ & $0.00 \mathrm{E}+00$ \\
\hline $5.50 E+00$ & $7.95 \mathrm{E}-01$ & $7.95 \mathrm{E}-01$ & & $2.73 \mathrm{E}-01$ & $1.02 \mathrm{E}-01$ & $3.84 \mathrm{E}-02$ & $2.08 \mathrm{E}-03$ & $6.08 \mathrm{E}-06$ & $1.53 \mathrm{E}-13$ & $0.00 \mathrm{E}+00$ & $0.00 \mathrm{E}+00$ \\
\hline total & $2.46 \mathrm{E}+02$ & $2.46 \mathrm{E}+02$ & $2.22 \mathrm{E}+02$ & $1.06 \mathrm{E}+02$ & $5.88 \mathrm{E}+01$ & $3.92 \mathrm{E}+01$ & $2.19 E+01$ & $1.34 \mathrm{E}+01$ & $8.59 \mathrm{E}+00$ & $7.67 \mathrm{E}+00$ & $7.43 \mathrm{E}+00$ \\
\hline $\mathrm{mev} / \mathrm{sec}$ & $1.96 \mathrm{E}+02$ & $1.96 \mathrm{E}+02$ & $1.78 \mathrm{E}+02$ & $8.71 \mathrm{E}+01$ & $5.04 \mathrm{E}+01$ & $3.54 \mathrm{E}+01$ & $2.25 \mathrm{E}+01$ & $1.64 \mathrm{E}+01$ & $1.20 \mathrm{E}+01$ & $1.08 E+01$ & $1.05 E+01$ \\
\hline
\end{tabular}

Table G3. Decay Photon Spectrum (/kg.s) After 24-s Irradiation of 1-kg Salted Beef Cargo (Flux Parameter Set 1).

\begin{tabular}{|c|c|c|c|c|c|c|c|c|c|c|c|}
\hline emean & & & & after & charge & & & & & & \\
\hline (mev) & & & $1.0 \mathrm{sec}$ & & $20.0 \mathrm{sec}$ & $30.0 \mathrm{sec}$ & $60.0 \mathrm{sec}$ & $120.0 \mathrm{sec}$ & $300.0 \mathrm{sec}$ & $600.0 \mathrm{sec}$ & $1200.0 \mathrm{se}$ \\
\hline $3.00 \mathrm{E}-01$ & 1. $11 \mathrm{E}+02$ & $E+02$ & $.97 E+01$ & $4.44 \mathrm{E}+01$ & $2.20 \mathrm{E}+01$ & $29 \mathrm{E}+\mathrm{C}$ & $5.46 \mathrm{E}+\mathrm{C}$ & $2.39 \mathrm{E}+00$ & $9.61 \mathrm{E}-01$ & $44 \mathrm{E}-01$ & $24 \mathrm{E}-$ \\
\hline & $4 \mathrm{E}+02$ & $\mathrm{E}+02$ & $48 E+01$ & & & & & & & & \\
\hline 00 & $E+01$ & & +01 & 9.7 & & & & $E-01$ & & & \\
\hline $8+00$ & $E+01$ & $E+01$ & +01 & & & & & & & & \\
\hline & & & & 2. & & & & & & & \\
\hline 00 & & & & & & & & & & & \\
\hline $2 . \varepsilon$ & 5. & 5. & 4. & 1.9 & & & & & & & \\
\hline & & & & & & & & & & & \\
\hline & 1.7 & 1. & & 5. & & & & & & & \\
\hline & 6.1 & & & & & & & & & & \\
\hline & & & & & & & & & & & \\
\hline & & 8. & E-01 & 3.1 & & & & & & & \\
\hline & $2.86 \mathrm{E}+02$ & $2.86 \mathrm{E}+02$ & $2.60 E+02$ & $1.28 \mathrm{E}+0$ & & $4.99 \mathrm{E}+01$ & & & & & \\
\hline & $2.28 \mathrm{E}+02$ & $2.28 E+02$ & $2.07 \mathrm{E}+02$ & $1.05 \mathrm{E}+02$ & $6.18 \mathrm{E}+01$ & $4.39 \mathrm{E}+01$ & $2.77 \mathrm{E}+01$ & 1. $90 \mathrm{E}+01$ & 1. $29 \mathrm{E}+01$ & $1.11 \mathrm{E}+01$ & $1.06 \mathrm{E}+01$ \\
\hline
\end{tabular}


Table G4. Decay Photon Spectrum (/kg.s) After 8-s Irradiation of 1-kg Ball Bearings Cargo (Flux Parameter Set 1).

\begin{tabular}{|c|c|c|c|c|c|c|c|c|c|c|c|}
\hline emean & & & & me after & scharge & & & & & & \\
\hline (mev) & initial & $1 \mathrm{E}-15 \mathrm{sec}$ & $1.0 \mathrm{sec}$ & $10.0 \mathrm{sec}$ & $20.0 \mathrm{sec}$ & $30.0 \mathrm{sec}$ & $60.0 \mathrm{sec}$ & $120.0 \mathrm{sec}$ & $300.0 \mathrm{sec}$ & $600.0 \mathrm{sec}$ & $1200.0 \mathrm{sec}$ \\
\hline $3.00 \mathrm{E}-01$ & $1.20 \mathrm{E}+00$ & $1.20 \mathrm{E}+00$ & $1.19 \mathrm{E}+00$ & $1.14 \mathrm{E}+00$ & $1.08 \mathrm{E}+00$ & $1.03 \mathrm{E}+00$ & $8.98 \mathrm{E}-01$ & $6.93 \mathrm{E}-01$ & $3.78 \mathrm{E}-01$ & $2.22 \mathrm{E}-01$ & $1.66 \mathrm{E}-01$ \\
\hline $6.50 \mathrm{E}-01$ & $6.09 E+00$ & $6.09 E+00$ & $6.09 E+00$ & $6.04 E+00$ & $5.99 E+00$ & $5.94 E+00$ & $5.82 \mathrm{E}+00$ & $5.62 E+00$ & $5.29 E+00$ & $5.03 E+00$ & $4.73 E+00$ \\
\hline $1.12 \mathrm{E}+00$ & $4.36 \mathrm{E}-01$ & $4.36 \mathrm{E}-01$ & 1.34E-01 & $4.14 \mathrm{E}-01$ & $3.96 \mathrm{E}-01$ & $3.79 \mathrm{E}-01$ & $3.34 \mathrm{E}-01$ & $2.66 \mathrm{E}-01$ & $57 \mathrm{E}-01$ & $8.99 E-02$ & $53 E-02$ \\
\hline $1.58 \mathrm{E}+00$ & $9.79 \mathrm{E}+00$ & $9.79 \mathrm{E}+00$ & $9.74 \mathrm{E}+00$ & $9.37 E+00$ & $8.96 E+00$ & $8.58 \mathrm{E}+00$ & $54 E+00$ & $5.85 \mathrm{E}+00$ & $2.92 \mathrm{E}+00$ & $1.23 \mathrm{E}+00$ & $25 E-01$ \\
\hline $2.00 \mathrm{E}+00$ & $1.08 \mathrm{~F}+00$ & 1. $.08 \mathrm{E}+00$ & $1.08 \mathrm{E}+00$ & $1.07 \mathrm{~F}+00$ & $1.07 \mathrm{E}+00$ & $7 E+00$ & $7 \mathrm{~F}+00$ & 1. $06 \mathrm{~F}+00$ & 1. $.05 \mathrm{E}+\mathrm{C}$ & $1.02 F+00$ & $77 \mathrm{E}-0]$ \\
\hline $2.40 \mathrm{E}+00$ & $5.81 \mathrm{E}-02$ & $5.81 \mathrm{E}-02$ & $5.76 \mathrm{E}-02$ & $5.50 \mathrm{E}-02$ & $5.39 \mathrm{E}-02$ & $5.31 E-02$ & $5.10 \mathrm{E}-02$ & $4.80 E-02$ & $34 \mathrm{E}-02$ & $06 \mathrm{E}-02$ & $9 E-02$ \\
\hline $2.80 E+00$ & $3.84 \mathrm{E}-02$ & $3.84 \mathrm{E}-02$ & $3.84 E-02$ & $3.82 \mathrm{E}-02$ & $0 E-02$ & $3.80 \mathrm{E}-02$ & & & & $E-02$ & $4 \mathrm{E}-02$ \\
\hline $3.25 E+00$ & $8.39 \mathrm{E}-03$ & $8.39 \mathrm{E}-03$ & $8.26 \mathrm{E}-03$ & $7.72 \mathrm{E}-03$ & $7.56 \mathrm{E}-03$ & $7.48 \mathrm{E}-03$ & $2 E-03$ & $7.07 \mathrm{E}-03$ & $6.72 \mathrm{E}-03$ & $6.49 \mathrm{E}-03$ & $20 E-03$ \\
\hline $3.75 E+00$ & E- 04 & $7.16 \mathrm{E}-04$ & $6.09 \mathrm{E}-04$ & $1.89 \mathrm{E}-04$ & $1.02 \mathrm{E}-04$ & $8.17 \mathrm{E}-05$ & & & & E-07 & \\
\hline $5 E+00$ & $1.93 \mathrm{E}-04$ & $1.93 \mathrm{E}-04$ & $1.90 \mathrm{E}-04$ & $1.72 \mathrm{E}-04$ & $1.57 \mathrm{E}-04$ & $1.45 \mathrm{E}-04$ & $1.15 \mathrm{E}-04$ & $7.23 \mathrm{E}-05$ & $1.81 \mathrm{E}-05$ & $1.80 \mathrm{E}-06$ & $2.24 \mathrm{E}-08$ \\
\hline $5 E+00$ & $9.75 \mathrm{E}$ & $9.75 E-05$ & -05 & 2. & 7.8 & $E-06$ & & & & & \\
\hline $5.50 \mathrm{E}+00$ & $1.47 \mathrm{E}-05$ & $1.47 \mathrm{E}-05$ & $1.30 \mathrm{E}-05$ & $4.86 \mathrm{E}-06$ & $1.80 \mathrm{E}-06$ & $6.78 \mathrm{E}-07$ & $6 \mathrm{E}-08$ & $7.59 E-10$ & $1.63 \mathrm{E}-10$ & $1.62 \mathrm{E}-11$ & $1.59 \mathrm{E}^{2}-1$ \\
\hline total & $1.87 \mathrm{E}+01$ & $1.87 E+01$ & $1.86 \mathrm{E}+01$ & $1.81 \mathrm{E}+01$ & $1.76 \mathrm{E}+01$ & $1.71 \mathrm{E}+01$ & $1.58 \mathrm{E}+01$ & 1. $36 \mathrm{E}+01$ & $9.88 \mathrm{E}+00$ & $7.68 \mathrm{E}+00$ & $6.62 \mathrm{E}+00$ \\
\hline $\mathrm{mev} / \mathrm{sec}$ & $2.27 \mathrm{E}+01$ & $2.27 \mathrm{E}+01$ & $2.26 \mathrm{E}+01$ & $2.19 \mathrm{E}+01$ & $2.12 E+01$ & $2.05 \mathrm{E}+01$ & $1.87 E+01$ & $1.57 E+01$ & $1.07 \mathrm{E}+01$ & $7.64 E+00$ & $6.32 \mathrm{E}+0$ \\
\hline
\end{tabular}

Table G5. Decay Photon Spectrum (/kg.s) After 16-s Irradiation of 1-kg Ball Bearings Cargo (Flux Parameter Set 1).

\begin{tabular}{|c|c|c|c|c|c|c|c|c|c|c|c|}
\hline emean & & & & & & & & & & & \\
\hline (mev & initial & $1 \mathrm{E}-15 \mathrm{sec}$ & $1.0 \mathrm{sec}$ & $10.0 \mathrm{sec}$ & $20.0 \mathrm{sec}$ & $30.0 \mathrm{sec}$ & $60.0 \mathrm{sec}$ & $120.0 \mathrm{sec}$ & $300.0 \mathrm{sec}$ & $600.0 \mathrm{sec}$ & $1200.0 \mathrm{sec}$ \\
\hline$E-01$ & & $2.35 E+00$ & $.33 E+00$ & $3 E+00$ & $2.13 E+00$ & & & & & & $3.32 E-01$ \\
\hline$E-01$ & $1 E+01$ & $1 E+01$ & $1 E+01$ & & & $8 E+01$ & & & & $01 \mathrm{E}+01$ & $9.46 \mathrm{E}+00$ \\
\hline+00 & & & & & & & & & & & $9.03 F-02$ \\
\hline & 1.9 & & & & & & & & & & +00 \\
\hline & & & & & & & & & & & \\
\hline & & & & & & & & & & & \\
\hline & & & & & & & & & & & \\
\hline & & & & & & & & & & & \\
\hline & & & & & & & & & & & \\
\hline & 3.6 & & & & & & & & & & \\
\hline & & & & & & & & & & & \\
\hline & & & & & & & & & & & \\
\hline total & $3.69 \mathrm{E}+01$ & $3.69 E+01$ & $3.68 \mathrm{E}+01$ & $3.58 \mathrm{E}+01$ & $3.48 \mathrm{E}+01$ & $3.38 \mathrm{E}+01$ & $3.12 \mathrm{E}+01$ & $2.69 \mathrm{E}+01$ & $1.97 \mathrm{E}+01$ & $1.53 \mathrm{E}+01$ & $1.32 \mathrm{E}+01$ \\
\hline mev/sec & $4.47 \mathrm{E}+01$ & $4.47 E+01$ & $4.45 E+01$ & $4.32 E+01$ & $4.18 E+01$ & $4.05 E+01$ & $3.69 \mathrm{E}+01$ & $3.12 \mathrm{E}+01$ & $.12 \mathrm{E}+01$ & 1. $52 \mathrm{E}+01$ & $.26 \mathrm{E}+0$ \\
\hline
\end{tabular}


Table G6. Decay Photon Spectrum (/kg.s) After 24-s Irradiation of 1-kg Ball Bearings Cargo (Flux Parameter Set 1).

\begin{tabular}{|c|c|c|c|c|c|c|c|c|c|c|c|}
\hline emean & & & & aIce & scharge & & & & & & \\
\hline (mev) & initial & $1 \mathrm{E}-15 \mathrm{sec}$ & $1.0 \mathrm{sec}$ & $10.0 \mathrm{sec}$ & $20.0 \mathrm{sec}$ & $30.0 \mathrm{sec}$ & $60.0 \mathrm{sec}$ & $120.0 \mathrm{sec}$ & $300.0 \mathrm{sec}$ & $600.0 \mathrm{sec}$ & $1200.0 \mathrm{sec}$ \\
\hline $3.00 \mathrm{E}-01$ & $3.45 \mathrm{E}+00$ & $3.45 E+00$ & $3.43 E+00$ & $3.28 \mathrm{E}+00$ & $3.13 E+00$ & $2.98 E+00$ & $2.60 \mathrm{E}+00$ & $2.01 \mathrm{E}+00$ & $11 \mathrm{E}+00$ & $.61 \mathrm{E}-01$ & $4.98 \mathrm{E}-01$ \\
\hline $6.50 \mathrm{E}-01$ & $1.81 \mathrm{E}+01$ & $1.81 \mathrm{E}+01$ & $1.81 \mathrm{E}+01$ & $1.80 E+01$ & $1.79 \mathrm{E}+01$ & $1.77 \mathrm{E}+01$ & $1.74 \mathrm{E}+01$ & $1.68 \mathrm{E}+01$ & $58 E+01$ & 1. $51 \mathrm{E}+01$ & $42 E+01$ \\
\hline $1.12 \mathrm{E}+00$ & 1. $26 \mathrm{E}+00$ & $1.26 \mathrm{E}+00$ & $1.25 E+00$ & $1.20 \mathrm{E}+00$ & $1: 15 E+00$ & $1.10 \mathrm{E}+00$ & $9.70 \mathrm{E}-01$ & $7.75 \mathrm{E}-01$ & $4.62 \mathrm{E}-01$ & $2.67 \mathrm{E}-01$ & $1.35 \mathrm{E}-01$ \\
\hline 1. $58 \mathrm{E}+00$ & $2.84 \mathrm{E}+01$ & $2.84 \mathrm{E}+01$ & $2.82 E+01$ & $2.71 \mathrm{E}+01$ & $2.60 \mathrm{E}+01$ & $2.49 E+01$ & $2.19 \mathrm{E}+01$ & $1.70 E+01$ & $8.52 E+00$ & 3. $62 \mathrm{E}+00$ & $1.87 \mathrm{E}+00$ \\
\hline $2.00 \mathrm{E}+00$ & $3.23 E+00$ & $3.23 \mathrm{E}+00$ & $3.23 \mathrm{E}+00$ & $3.22 \mathrm{E}+00$ & $3.22 \mathrm{E}+00$ & $3.22 \mathrm{E}+00$ & $3.21 \mathrm{E}+00$ & $3.19 \mathrm{E}+00$ & $3.14 \mathrm{E}+00$ & $3.07 E+00$ & $2.93 E+00$ \\
\hline $2.40 \mathrm{E}+00$ & $1.68 \mathrm{E}-01$ & $1.68 \mathrm{E}-01$ & $1.67 \mathrm{E}-01$ & $1.62 \mathrm{E}-01$ & $1.60 \mathrm{E}-01$ & $1.57 \mathrm{E}-01$ & $1.52 \mathrm{E}-01$ & 1. $43 \mathrm{E}-01$ & $.30 \mathrm{E}-01$ & $22 \mathrm{E}-01$ & $1.14 \mathrm{E} \quad 01$ \\
\hline $2.80 E+00$ & $1.15 \mathrm{E}-01$ & $1.15 \mathrm{E}-01$ & $1.15 \mathrm{E}-01$ & $1.14 \mathrm{E}-01$ & $1.14 \mathrm{E}-01$ & $1.14 \mathrm{E}-01$ & $1.13 \mathrm{E}-01$ & $1.12 \mathrm{E}-01$ & $1.10 \mathrm{E}-01$ & $1.08 \mathrm{E}-01$ & $1.03 \mathrm{E}-0.1$ \\
\hline $3.25 E+00$ & $2.38 E-02$ & $2.38 \mathrm{E}-02$ & $2.36 E-02$ & $2.28 \mathrm{E}-02$ & $2.25 \mathrm{E}-02$ & $2.23 \mathrm{E}-02$ & $2.18 \mathrm{E}-02$ & $2.11 \mathrm{E}-02$ & $2.01 E-02$ & $1.95 E-02$ & $1.86 \mathrm{E}-02$ \\
\hline $3.75 E+00$ & $1.07 E-03$ & $1.07 \mathrm{E}-03$ & $9.33 \mathrm{E}-04$ & $3.86 \mathrm{E}-04$ & $2.62 \mathrm{E}-04$ & $2.24 \mathrm{E}-04$ & $1.67 \mathrm{E}-04$ & $9.77 \mathrm{E}-05$ & $2.09 E-05$ & $2.70 \mathrm{E}-06$ & $1.10 \mathrm{E}-06$ \\
\hline $4.25 \mathrm{E}+00$ & $5.31 \mathrm{E}-04$ & $5.31 \mathrm{E}-04$ & $5.25 \mathrm{E}-04$ & $4.82 E-04$ & $4.43 \mathrm{E}-04$ & 4.09 & $3.24 \mathrm{E}-04$ & & & & $6.40 \mathrm{E}-08$ \\
\hline $4.75 \mathrm{E}+00$ & $1.35 \mathrm{E}-04$ & $1.35 \mathrm{E}-04$ & $1.15 E-04$ & $3.54 \mathrm{E}-05$ & $1.79 \mathrm{E}-05$ & $1.35 \mathrm{E}-05$ & $9.14 \mathrm{E}-06$ & $4.81 \mathrm{E}-06$ & $7.12 \mathrm{E}-07$ & $2.95 \mathrm{E}-08$ & $5.10 \mathrm{E}-11$ \\
\hline $5.50 \mathrm{E}+00$ & $2.33 E-05$ & $2.33 \mathrm{E}-05$ & $2.08 \mathrm{E}-05$ & $8.05 \mathrm{E}-06$ & $3.00 \mathrm{E}-06$ & $1.13 \mathrm{E}-06$ & $6.40 \mathrm{E}-08$ & $2.02 \mathrm{E}-09$ & $4.61 \mathrm{E}-10$ & $4.57 E-11$ & $4.50 \mathrm{E}-13$ \\
\hline total & $5.47 E+01$ & $5,47 \mathrm{E}+01$ & $5.46 \mathrm{E}+01$ & $5.31 E+01$ & $5.16 \mathrm{E}+01$ & $5.02 \mathrm{E}+01$ & $4.63 E+01$ & $4.01 \mathrm{E}+01$ & $2.93 \mathrm{E}+01$ & $2.29 \mathrm{E}+\mathrm{C}$ & \\
\hline $\mathrm{mev} / \mathrm{sec}$ & $6.62 \mathrm{E}+01$ & $6.62 \mathrm{E}+01$ & $6.59 E+01$ & $6.40 \mathrm{E}+01$ & $6.20 \mathrm{E}+01$ & $6.00 \mathrm{E}+01$ & $5.47 \mathrm{E}+01$ & $4.63 E+01$ & $3.15 \mathrm{E}+01$ & $2.28 \mathrm{E}+01$ & $1.89 \mathrm{E}+0$ \\
\hline
\end{tabular}

\section{Table G7. Decay Photon Spectrum (/kg.s) After 8-s Irradiation of 1-kg Surgical Implant Composition A Cargo} (Flux Parameter Set 1).

\begin{tabular}{|c|c|c|c|c|c|c|c|c|c|c|c|}
\hline emean & & & & atter & scharge & & & & & & \\
\hline (mev) & initial & $1 \mathrm{E}-15 \mathrm{sec}$ & $1.0 \mathrm{sec}$ & $10.0 \mathrm{sec}$ & $20.0 \mathrm{sec}$ & 30.0 sec & $60.0 \mathrm{sec}$ & $120.0 \mathrm{sec}$ & $300.0 \mathrm{sec}$ & $600.0 \mathrm{sec}$ & 1200.0 see \\
\hline $3.00 \mathrm{E}-01$ & $4.87 \mathrm{E}+00$ & $4.87 \mathrm{E}+00$ & $4.79 E+00$ & $4.53 E+00$ & $4.35 E+00$ & $4.19 E+00$ & $3.73 E+00$ & $2.98 \mathrm{E}+00$ & $1.57 E+00$ & $6.16 \mathrm{E}-01$ & 1. $70 \mathrm{E}-01$ \\
\hline $6.50 \mathrm{E}-01$ & $4.91 \mathrm{E}+00$ & $4.91 \mathrm{E}+00$ & $4.83 E+00$ & $4.61 E+00$ & $4.49 \mathrm{E}+00$ & $4.38 \mathrm{E}+00$ & $4.08 E+00$ & $3.56 \mathrm{E}+00$ & $52 E+00$ & 1. $66 \mathrm{E}+00$ & $00 E+00$ \\
\hline $1.12 \mathrm{E}+00$ & $4.85 \mathrm{E}+00$ & $4.85 \mathrm{E}+00$ & $4.82 \mathrm{E}+00$ & $4.65 \mathrm{E}+00$ & $4.50 \mathrm{E}+00$ & $4.37 E+00$ & $4.01 E+00$ & $3.43 E+00$ & $2.35 E+00$ & $1.49 \mathrm{E}+00$ & $7.09 \mathrm{E}-01$ \\
\hline $1.58 \mathrm{E}+00$ & $6.72 \mathrm{E}+01$ & $6.72 \mathrm{E}+01$ & $6.70 E+01$ & $6.46 \mathrm{E}+01$ & $6.21 \mathrm{E}+01$ & $5.98 \mathrm{E}+01$ & $5.32 \mathrm{E}+01$ & $4.22 \mathrm{E}+01$ & $2.16 \mathrm{E}+01$ & $7.54 \mathrm{E}+00$ & $1.11 \mathrm{E}+00$ \\
\hline $2.00 \mathrm{E}+00$ & $1.83 \mathrm{E}-01$ & $1.83 \mathrm{E}-01$ & $1.78 \mathrm{E}-01$ & $1.71 \mathrm{E}-01$ & $1.70 \mathrm{E}-01$ & $1.69 \mathrm{E}-01$ & $56 E-01$ & $1.62 \mathrm{E}-01$ & $1.51 \mathrm{E}-01$ & 1. $39 \mathrm{E}-01$ & $.25 E-01$ \\
\hline $2.40 E+00$ & $4.30 \mathrm{E}-02$ & $4.30 \mathrm{E}-02$ & $3.72 \mathrm{E}-02$ & $2.62 \mathrm{E}-02$ & $2.44 \mathrm{E}-02$ & $2.37 \mathrm{E}-02$ & 2. $22 \mathrm{E}-02$ & $1.99 \mathrm{E}-02$ & $50 \mathrm{E}-02$ & $1.04 \mathrm{E}-02$ & $6.44 E-03$ \\
\hline $2.80 \mathrm{E}+00$ & $1.33 \mathrm{E}-02$ & $1.33 E-02$ & $1.05 \mathrm{E}-02$ & $7.87 \mathrm{E}-03$ & $7.77 \mathrm{E}-03$ & $7.75 \mathrm{E}-03$ & $7.73 \mathrm{E}-03$ & $7.70 \mathrm{E}-03$ & $7.63 \mathrm{E}-03$ & $7.52 \mathrm{E}-03$ & $7.31 \mathrm{E}-03$ \\
\hline $3.25 \mathrm{E}+00$ & $7.14 \mathrm{E}-03$ & $7.14 \mathrm{E}-03$ & $0 E-03$ & $1.42 \mathrm{E}-03$ & $1.05 \mathrm{E}-03$ & $9.86 \mathrm{E}-04$ & 9.3 & $g-04$ & $E-04$ & $7.62 \mathrm{E}-04$ & $7.27 \mathrm{E}-04$ \\
\hline $3.75 \mathrm{E}+00$ & $6.38 \mathrm{E}-03$ & $6.38 E-03$ & $3.72 E-03$ & $3.60 E-04$ & $6.34 \mathrm{E}-05$ & $1.98 \mathrm{E}-05$ & $1.04 E-05$ & $7.34 \mathrm{E}-06$ & $3.47 \mathrm{E}-06$ & $2.31 \mathrm{E}-06$ & $2.17 E-06$ \\
\hline $4.25 E+00$ & $1.53 \mathrm{E}-03$ & $1.53 \mathrm{E}-03$ & $6.98 \mathrm{E}-04$ & $3-05$ & $4.10 \mathrm{E}-05$ & $3.79 E-05$ & $3.01 E-05$ & 1.9 & E-06 & $E-07$ & $31 E-08$ \\
\hline $4.75 \mathrm{E}+00$ & $1.75 \mathrm{E}-03$ & $1.75 \mathrm{E}-03$ & $8.91 E-04$ & $4.86 E-05$ & $7.07 E-06$ & 1. $06 \mathrm{E}-06$ & $2.92 \mathrm{E}-08$ & $1.38 \mathrm{E}-08$ & $2.06 \mathrm{E}-09$ & $8.81 \mathrm{E} \cdot 11$ & $1.91 \mathrm{E}-13$ \\
\hline $5.50 \mathrm{E}+00$ & $2.65 \mathrm{E}-03$ & $2.65 E-03$ & $1.16 \mathrm{E}-03$ & $8.04 \mathrm{E}-07$ & $1.45 \mathrm{E}-08$ & $2.21 \mathrm{E}-09$ & $2.86 \mathrm{E}-10$ & $1.71 \mathrm{E}-10$ & $4.27 E-11$ & $4.24 \mathrm{E}-12$ & $4.17 \mathrm{E}-14$ \\
\hline total & $8.21 \mathrm{E}+01$ & $8.21 \mathrm{E}+01$ & $3.16 \mathrm{E}+01$ & $7.86 \mathrm{E}+01$ & $7.57 \mathrm{E}+01$ & $7.29 \mathrm{E}+01$ & $6.52 \mathrm{E}+01$ & $5.24 \mathrm{E}+01$ & $2.82 \mathrm{E}+01$ & $1.15 E+01$ & $3.13 \mathrm{E}+00$ \\
\hline $\mathrm{mev} / \mathrm{se}$ & $1.17 \mathrm{E}+02$ & $1.17 E+02$ & $1.16 \mathrm{E}+02$ & $1.12 \mathrm{E}+02$ & $1.08 E+02$ & $1.04 \mathrm{E}+02$ & $9.25 E+01$ & $7.40 \mathrm{E}+01$ & $3.92 E+01$ & 1. $51 \mathrm{E}+01$ & $3.54 \mathrm{E}+\mathrm{C}$ \\
\hline
\end{tabular}


Table G8. Decay Photon Spectrum (/kg.s) After 16-s Irradiation of 1-kg Surgical Implant Composition A Cargo (Flux Parameter Set 1).

\begin{tabular}{|c|c|c|c|c|c|c|c|c|c|c|c|}
\hline emean & & & & e after & scharge & & & & & & \\
\hline $\begin{array}{l}\text { (mev) } \\
3.00 \mathrm{E}-01\end{array}$ & $\begin{array}{r}\text { initial } \\
94 \mathrm{E}+00\end{array}$ & $\begin{array}{r}1 E-15 \mathrm{sec} \\
9.44 \mathrm{E}+00\end{array}$ & $\begin{array}{r}1.0 \mathrm{sec} \\
9.34 \mathrm{E}+00\end{array}$ & $\begin{array}{r}10.0 \mathrm{sec} \\
8.92 \mathrm{E}+00\end{array}$ & $\begin{array}{r}20.0 \mathrm{sec} \\
8.57 \mathrm{E}+00\end{array}$ & $\begin{array}{r}30.0 \mathrm{sec} \\
8.25 \mathrm{E}+00\end{array}$ & $\begin{array}{r}60.0 \mathrm{sec} \\
7.35 \mathrm{E}+00\end{array}$ & $120.0 \mathrm{sec}$ & $300.0 \mathrm{sec}$ & 600.0 sec & $\begin{array}{r}1200.0 \mathrm{sec} \\
3.39 \mathrm{E}-01\end{array}$ \\
\hline $6.50 \mathrm{E}-01$ & $9.55 \mathrm{E}+00$ & $9.55 \mathrm{E}+00$ & $9.46 E+00$ & $9.13 \mathrm{E}+00$ & $8.89 \mathrm{E}+00$ & $8.68 \mathrm{E}+00$ & $8.08 E+00$ & $7.07 \mathrm{E}+00$ & $5.01 E+00$ & $\begin{array}{l}\text { 1. } 2 \angle E+00 \\
\text { 3. } 31 E+00\end{array}$ & $2.00 \mathrm{E}+00$ \\
\hline 1. $12 \mathrm{E}+00$ & $9.53 \mathrm{E}+00$ & $9.53 \mathrm{E}+00$ & $9.48 \mathrm{E}+00$ & $9.18 \mathrm{E}+00$ & $8.90 \mathrm{E}+00$ & $8.64 \mathrm{E}+00$ & $7.93 \mathrm{E}+00$ & $79 \mathrm{E}+00$ & $.67 \mathrm{E}+00$ & $.96 \mathrm{E}+00$ & $.41 \mathrm{E}+00$ \\
\hline $1.58 \mathrm{E}+00$ & $1: 32 \mathrm{E}+02$ & $1.32 E+02$ & 1. $32 E+02$ & $1.27 \mathrm{E}+02$ & 1. $22 E+02$ & 1. $18 \mathrm{E}+02$ & 1. $0.5 E+02$ & $32 \mathrm{E}+01$ & $4.26 \mathrm{E}+0.1$ & 1. $49 \mathrm{E}+01$ & $2.20 E+00$ \\
\hline $.00 \mathrm{E}+00$ & & $3.55 \mathrm{E}-01$ & $9 E-01$ & $3.41 \mathrm{E}-01$ & $3.39 E-01$ & 3.3 & $3.32 \mathrm{E}-01$ & $23 E-0$ & & & $2.50 \mathrm{E}-01$ \\
\hline $2.40 \mathrm{E}+00$ & $1 E-02$ & $1 E-02$ & $8 E-02$ & $8 \mathrm{E}-02$ & $2 E-02$ & $4.70 \mathrm{E}-02$ & $41 \mathrm{E}-02$ & $4 E-02$ & $98 E-02$ & $2.08 \mathrm{E}-02$ & $1.28 E-02$ \\
\hline $2.80 \mathrm{E}+00$ & $2 E-02$ & $2 E-02$ & $1 E-02$ & $6 \mathrm{E}-02$ & $5 E-02$ & $1.55 \mathrm{E}-02$ & $5 \mathrm{E}-02$ & $\mathrm{E}-02$ & $3 E-02$ & ;0E-02 & $1.46 \mathrm{E}-02$ \\
\hline$E+00$ & $6 \mathrm{E}-03$ & $6 E-03$ & $E-03$ & $E-03$ & $2.04 \mathrm{E}-03$ & $E-03$ & $1.87 \mathrm{E}-03$ & $5 E-03$ & $E-03$ & $2 E-03$ & $1.45 \mathrm{E}-03$ \\
\hline $\mathrm{E}+00$ & $1 \mathrm{E}-03$ & -03 & -03 & $z-04$ & -05 & & & & & & $4.34 \mathrm{E}-06$ \\
\hline $4.25 \mathrm{E}+00$ & $1.58 \mathrm{E}-03$ & $3 E-03$ & $5 E-04$ & $8.72 E-05$ & $7.95 E-05$ & $7.35 E-05$ & $5.84 \mathrm{E}-05$ & $8 E-05$ & $4 E-06$ & $9.68 \mathrm{E}-07$ & $6.59 E-08$ \\
\hline & 1 & 1.8 & & & & & & & & & $3.67 \mathrm{E}-13$ \\
\hline $5.50 \mathrm{E}+00$ & -1 & 26 & -03 & 8. & $6 \mathrm{E}-08$ & 2.96 & 5.47 & $2 E-10$ & 8.2 & 8. & $8.09 \mathrm{E}-14$ \\
\hline total & $1.61 \mathrm{E}+02$ & $1.61 \mathrm{E}+02$ & $1.61 \mathrm{E}+02$ & $1.55 \mathrm{E}+02$ & $1.49 \mathrm{E}+0.2$ & $1.44 \mathrm{E}+02$ & $1.29 E+02$ & $1.03 E+02$ & $5.57 \mathrm{E}+01$ & $2.27 \mathrm{E}+01$ & $6.23 \mathrm{E}+00$ \\
\hline mev/sec & $2.29 E+02$ & $2.29 E+02$ & $2.28 \mathrm{E}+02$ & $2.20 E+02$ & $2.12 \mathrm{E}+02$ & $2.04 E+02$ & $1.82 E+02$ & $1.46 \mathrm{E}+02$ & $7.73 \mathrm{E}+01$ & $2.99 \mathrm{E}+01$ & $7.03 \mathrm{E}+00$ \\
\hline
\end{tabular}

\section{Table G9. Decay Photon Spectrum (/kg.s) After 24-s Irradiation of 1-kg Surgical Implant Composition A Cargo} (Flux Parameter Set 1).

\begin{tabular}{|c|c|c|c|c|c|c|c|c|c|c|c|}
\hline emean & & & & after & scharge & & & & & & \\
\hline (mev) & initial & $1 \mathrm{E}-15 \mathrm{sec}$ & $1.0 \mathrm{sec}$ & $10.0 \mathrm{sec}$ & $20.0 \mathrm{sec}$ & $30.0 \mathrm{sec}$ & $60.0 \mathrm{sec}$ & $120.0 \mathrm{sec}$ & $300.0 \mathrm{sec}$ & $600.0 \mathrm{sec}$ & $1200.0 \mathrm{sec}$ \\
\hline $3.00 \mathrm{E}-01$ & $1.39 \mathrm{E}+01$ & $1.39 \mathrm{E}+01$ & $1.37 E+01$ & 1. $32 \mathrm{E}+01$ & 1. $27 \mathrm{E}+01$ & 1. $22 \mathrm{E}+01$ & 1. $09 \mathrm{E}+01$ & $8.68 E+00$ & $4.59 \mathrm{E}+00$ & $1.81 \mathrm{E}+00$ & $5.05 \mathrm{E}-01$ \\
\hline $6.50 \mathrm{E}-01$ & $1.41 \mathrm{E}+01$ & $1.41 E+01$ & $1.40 \mathrm{E}+01$ & $1.35 \mathrm{E}+01$ & $1.32 E+01$ & $1.29 E+01$ & $1.20 \mathrm{E}+01$ & $1.05 \mathrm{E}+01$ & $7.47 E+00$ & $4.94 \mathrm{E}+00$ & $2.99 \mathrm{E}+00$ \\
\hline $1.12 \mathrm{E}+00$ & $1.41 \mathrm{E}+01$ & $1.41 E+01$ & $1.40 \mathrm{E}+01$ & $1.36 \mathrm{E}+01$ & $1.32 \mathrm{E}+01$ & 1. $28 \mathrm{E}+01$ & $1.18 \mathrm{E}+01$ & $01 E+01$ & $96 \mathrm{E}+00$ & $11 E+00$ & $11 \mathrm{E}+00$ \\
\hline $\mathrm{E}+00$ & $1.96 \mathrm{E}+02$ & $1.96 E+02$ & $1.95 \mathrm{E}+02$ & $1.88 E+02$ & $1.81 E+02$ & $1.74 \mathrm{E}+02$ & $1.55 \mathrm{E}+02$ & $3 E+02$ & $0 \mathrm{E}+01$ & $0 E+01$ & $3.26 \mathrm{E}+00$ \\
\hline$E+00$ & $5.25 \mathrm{E}-01$ & $5.25 \mathrm{E}-01$ & $5.19 \mathrm{E}-01$ & $5.11 \mathrm{E}-01$ & & $5.05 \mathrm{E}-01$ & $4.97 \mathrm{E}-0$ & & & & $3.75 \mathrm{E}-01$ \\
\hline $2.40 \mathrm{E}+00$ & $9 \mathrm{E}-02$ & $9.49 E-02$ & $\mathrm{E}-02$ & $7.48 \mathrm{E}-02$ & $7.16 E-02$ & $6.98 \mathrm{E}-02$ & $7 \mathrm{E}-02$ & -02 & -02 & 1E-02 & $1.92 \mathrm{E}-02$ \\
\hline$E+00$ & $E-02$ & $0 E-02$ & E-02 & -02 & -02 & -02 & 2 . & -02 & -02 & -02 & $9 E-02$ \\
\hline$E+00$ & $E-03$ & $9 E-03$ & $7.41 \mathrm{E}-03$ & $E-03$ & E-03 & $2.91 \mathrm{E}-03$ & 2.7 & -03 & -03 & -03 & $18 E-03$ \\
\hline$E+00$ & 7.0 & $7.03 E-03$ & $4.26 \mathrm{E}-03$ & & & & & & & & $2 E-06$ \\
\hline$E+00$ & $E-03$ & $1.62 \mathrm{E}-03$ & $7.87 \mathrm{E}-04$ & $1.26 \mathrm{E}-04$ & $1.16 \mathrm{E}-04$ & $1.07 \mathrm{E}-04$ & 8.4 & & -05 & $1.41 \mathrm{E}-06$ & $9.84 \mathrm{E}-08$ \\
\hline & 1.8 & $1.83 E-03$ & $9.63 \mathrm{E}-04$ & $6.13 \mathrm{E}-0$ & & & 7.5 & & & & $30 E-13$ \\
\hline $5.50 \mathrm{E}+00$ & $2.66 \mathrm{E}-03$ & $2.66 \mathrm{E}-03$ & $1.16 \mathrm{E}-03$ & $8.31 E-07$ & $1.85 \mathrm{E}-08$ & $3.38 \mathrm{E}-09$ & $7.90 \mathrm{E}-10$ & $4.83 \mathrm{E}-10$ & 1. $21 \mathrm{E}-10$ & $1.20 \mathrm{E}-11$ & $1.18 \mathrm{E}-13$ \\
\hline total & $2.38 \mathrm{E}+02$ & $2.38 E+02$ & $2.37 \mathrm{E}+02$ & $2.29 \mathrm{E}+02$ & $2.20 \mathrm{E}+02$ & $2.12 \mathrm{E}+02$ & $1.90 \mathrm{E}+02$ & $1.53 E+02$ & $8.25 E+01$ & $3.36 \mathrm{E}+01$ & $9.28 \mathrm{E}+00$ \\
\hline mev/sec & $3.39 \mathrm{E}+02$ & $3.39 E+02$ & $3.37 E+02$ & $3.25 E+02$ & $3.13 E+02$ & $3.02 \mathrm{E}+02$ & $2.69 \mathrm{E}+02$ & $2.16 \mathrm{E}+02$ & $1.14 \mathrm{E}+02$ & $4.44 \mathrm{E}+01$ & $1.05 \mathrm{E}+01$ \\
\hline
\end{tabular}


Table G10. Decay Photon Spectrum (/kg.s) After 8-s Irradiation of 1-kg Surgical Implant Composition B Cargo (Flux Parameter Set 1).

\begin{tabular}{|c|c|c|c|c|c|c|c|c|c|c|c|}
\hline emean & & & & after & charge & & & & & & \\
\hline (mev) & initial & 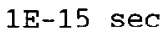 & 1.0 sec & $10.0 \mathrm{sec}$ & 20.0 sec & 30.0 sec & 60.0 sec & 120.0 sec & $300.0 \mathrm{sec}$ & $600.0 \mathrm{sec}$ & $1200.0 \mathrm{sec}$ \\
\hline $.00 \mathrm{E}-01$ & $2.49 \mathrm{E}+00$ & $2.49 \mathrm{E}+00$ & $2.47 \mathrm{E}+00$ & $2.36 \mathrm{E}+00$ & $2.29 E+00$ & $2.22 \mathrm{E}+00$ & $2.02 E+00$ & $1.68 \mathrm{E}+00$ & $7 E-01$ & $4.18 \mathrm{E}-01$ & $1.08 \mathrm{E}-01$ \\
\hline-01 & $.51 \mathrm{E}+00$ & $1 E+00$ & $9 E+00$ & 1. $40 \mathrm{E}+00$ & $1.35 \mathrm{E}+00$ & $L E+00$ & & $1 E+00$ & & $88 \mathrm{E}-01$ & $8.79 \mathrm{E}-02$ \\
\hline & & & & & & & & & & & \\
\hline & & & & & & & & & & & \\
\hline & -02 & & & & & & & & & & .03 \\
\hline & -03 & & & & & & & & & & 05 \\
\hline & & & & & & & & & & & \\
\hline & & 1. & & & & & & & & & \\
\hline & & & & & & & & & & & \\
\hline & 4.6 & & & 4. & & & & & & & -29 \\
\hline & & & & & & & & & & & \\
\hline & & 2. & 1.6 & 1.9 & & & & & & & $E-31$ \\
\hline total & $4.33 E+01$ & $4.33 E+01$ & $4.31 \mathrm{E}+01$ & $4.18 \mathrm{E}+01$ & $4.05 E+01$ & $3.93 \mathrm{E}+01$ & $3.59 E+01$ & $2.99 \mathrm{E}+01$ & $1.76 \mathrm{E}+01$ & $7.61 E+00$ & $1.75 \mathrm{E}+00$ \\
\hline mev/sec & $6.18 E+01$ & $6.18 \mathrm{E}+01$ & $6.16 E+01$ & $5.98 \mathrm{E}+01$ & $5.80 E+01$ & $5.62 \mathrm{E}+01$ & $5.13 E+01$ & $4.28 \mathrm{E}+01$ & $2.50 \mathrm{E}+01$ & $1.06 \mathrm{E}+01$ & $2.24 \mathrm{E}+00$ \\
\hline
\end{tabular}

Table G11. Decay Photon Spectrum (/kg.s) After 16-s Irradiation of 1-kg Surgical Implant Composition B Cargo (Flux Parameter Set 1).

\begin{tabular}{|c|c|c|c|c|c|c|c|c|c|c|c|}
\hline emean & & & & after & charge & & & & & & \\
\hline (mev) & initial & $1 \mathrm{E}-15 \mathrm{sec}$ & $1.0 \mathrm{sec}$ & $10.0 \mathrm{sec}$ & $20.0 \mathrm{sec}$ & $30.0 \mathrm{sec}$ & $60.0 \mathrm{sec}$ & $120.0 \mathrm{sec}$ & $300.0 \mathrm{sec}$ & $600.0 \mathrm{sec}$ & 1200.0 \\
\hline $3.00 E-01$ & $4.88 E+00$ & $8 \mathrm{E}+00$ & $4.85 \mathrm{E}+00$ & $4.67 \mathrm{E}+00$ & $4.52 \mathrm{E}+00$ & $4.38 \mathrm{E}+\mathrm{C}$ & 00 & & & & \\
\hline & & $2 \mathrm{E}+00$ & $.89 E+00$ & $2.75 E+00$ & $2.67 \mathrm{E}+00$ & $.59 E+00$ & & 00 & 10 & & 01 \\
\hline $\mathrm{E}+00$ & 100 & $E+00$ & $E+00$ & $7.75 \mathrm{E}+\mathrm{C}$ & & & & & & & $2 \mathrm{E}+00$ \\
\hline+00 & & 6. & & & & & & & & & \\
\hline+00 & & & & & & & & & & & \\
\hline 2. & & & & & & & & & & & \\
\hline 2.8 & & & & 3. & & & & & & & \\
\hline & & & & & & & & & & & \\
\hline 3.75 & 3. & 3.9 & 3. & & & & & & & & \\
\hline & & & 4.1 & & & & & & & & \\
\hline & & & & & & & & & & & \\
\hline & & & & & & & & & & & \\
\hline & $8.54 \mathrm{E}+01$ & $8.54 \mathrm{E}+01$ & $8.51 \mathrm{E}+01$ & $8.26 \mathrm{E}+01$ & $8.01 \mathrm{E}+01$ & $7.77 \mathrm{E}+01$ & $7.09 E+01$ & $3.92 \mathrm{E}+0 \perp$ & $3.49 \mathrm{E}+01$ & L.SAT & \\
\hline & $1.22 \mathrm{E}+02$ & $1.22 \mathrm{E}+02$ & $1.22 \mathrm{E}+02$ & $1.18 E+02$ & $1.15 \mathrm{E}+02$ & $1.11 \mathrm{E}+02$ & $1.01 \mathrm{E}+02$ & $8.46 E+01$ & $4.95 E+01$ & $2.09 E+01$ & $4.43 E+00$ \\
\hline
\end{tabular}


Table G12. Decay Photon Spectrum (/kg.s) After 24-s Irradiation of 1-kg Surgical Implant Composition B Cargo (Flux Parameter Set 1).

\begin{tabular}{|c|c|c|c|c|c|c|c|c|c|c|c|}
\hline emean & & & & atter & & & & & & & \\
\hline (mev) & initial & $1 \mathrm{E}-15 \mathrm{sec}$ & $1.0 \mathrm{sec}$ & $10.0 \mathrm{sec}$ & 20.0 sec & $30.0 \mathrm{sec}$ & $60.0 \mathrm{sec}$ & $120.0 \mathrm{sec}$ & $300.0 \mathrm{sec}$ & $600.0 \mathrm{sec}$ & $0.0 \mathrm{se}$ \\
\hline & & $.19 \mathrm{E}+00$ & $.16 \mathrm{E}+00$ & & & & & & & & \\
\hline$E-01$ & $E+00$ & $E+00$ & $\mathrm{E}+00$ & $3 E+00$ & & & & & & & \\
\hline$E+00$ & +01 & & $\mathrm{E}+01$ & $E+01$ & & & & & & & \\
\hline & & & & & & & & & & & \\
\hline & & & & & & & & & & & \\
\hline & & & & & & & & & & & \\
\hline+00 & & -04 & -04 & 3.1 & & & & & & & \\
\hline & & & & & & & & & & & \\
\hline & 4.0 & & 3.1 & 3.5 & & & & & & & \\
\hline & & & & & & & & & & & \\
\hline & & & & & & & & & & & \\
\hline & $2.51 \mathrm{E}-10$ & 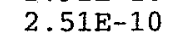 & $1.97 \mathrm{E}-10$ & $2.23 \mathrm{E}-11$ & $1.97 \mathrm{E}-12$ & $1.75 E-13$ & 1. $22 \mathrm{E}-16$ & & & $2.34 \mathrm{E}-30$ & \\
\hline total & $1.26 \mathrm{E}+02$ & $1.26 \mathrm{E}+02$ & $1.26 E+02$ & $1.22 E+02$ & $1.19 \mathrm{E}+02$ & $1.15 \mathrm{E}+02$ & $1.05 \mathrm{E}+02$ & $8.77 \mathrm{E}+01$ & $5.17 \mathrm{E}+01$ & $2.23 \mathrm{E}+01$ & $3.130+0$ \\
\hline mev & $1.81 E+02$ & $1.81 E+02$ & $1.80 \mathrm{E}+02$ & $1.75 E+02$ & $1.70 E+02$ & $1.65 E+02$ & $1.50 \mathrm{E}+02$ & $1.25 \mathrm{E}+02$ & $7.34 \mathrm{E}+01$ & $3.11 \mathrm{E}+01$ & $6.59 \mathrm{E}+0 \mathrm{t}$ \\
\hline
\end{tabular}

Table G13. Decay Photon Spectrum (/kg.s) After 8-s Irradiation of 1-kg 16-16-16 Fertilizer Cargo (Flux Parameter Set 1).

\begin{tabular}{|c|c|c|c|c|c|c|c|c|c|c|c|}
\hline emean & & & & le after & scharge & & & & & & \\
\hline (mev) & initial & $1 \mathrm{E}-15 \mathrm{sec}$ & $1.0 \mathrm{sec}$ & $10.0 \mathrm{sec}$ & 20.0 sec & 30.0 sec & $60.0 \mathrm{sec}$ & $120.0 \mathrm{sec}$ & 300.0 sec & $600.0 \mathrm{sec}$ & $1200.0 \mathrm{sec}$ \\
\hline $3.00 \mathrm{E}-01$ & $9.29 \mathrm{E}+01$ & $9.29 \mathrm{E}+01$ & $8.89 E+01$ & $7.02 \mathrm{E}+01$ & $6.32 \mathrm{E}+01$ & $6.05 \mathrm{E}+01$ & $5.85 \mathrm{E}+01$ & $5.73 \mathrm{E}+01$ & $5.57 \mathrm{E}+01$ & $5.49 \mathrm{E}+01$ & $5.47 \mathrm{E}+01$ \\
\hline $6.50 \mathrm{E}-01$ & $4.43 E+01$ & $4.43 E+01$ & $4.06 \mathrm{E}+01$ & $2.57 \mathrm{E}+01$ & $2.03 \mathrm{E}+01$ & $1.82 \mathrm{E}+01$ & $1.65 \mathrm{E}+01$ & $1.56 \mathrm{E}+01$ & $1.46 \mathrm{E}+01$ & $1.12 \mathrm{E}+01$ & $1.41 \mathrm{E}+01$ \\
\hline $1.12 \mathrm{E}+00$ & $8.70 \mathrm{E}+00$ & $8.70 \mathrm{E}+00$ & $7.74 \mathrm{E}+00$ & $3.29 \mathrm{E}+00$ & $1.65 \mathrm{E}+00$ & $1.04 \mathrm{E}+00$ & $6.30 \mathrm{E}-01$ & $5.04 \mathrm{E}-01$ & $3.47 \mathrm{E}-01$ & $2.70 \mathrm{E}-01$ & $2.49 \mathrm{E}-01$ \\
\hline 1. $58 \mathrm{E}+00$ & $5.04 \mathrm{E}+02$ & $5.04 E+02$ & $5.03 E+02$ & $4.98 E+02$ & $4.94 \mathrm{E}+02$ & $4.90 \mathrm{E}+02$ & $4.81 E+02$ & $4.68 E+02$ & $4.44 \mathrm{E}+02$ & $4.33 E+02$ & $30 \mathrm{E}+02$ \\
\hline $2,00 \mathrm{E}+00$ & $2.03 E+00$ & $2.03 E+00$ & $1.80 \mathrm{E}+00$ & $7.30 \mathrm{E}-01$ & $3.39 \mathrm{E}-01$ & $1.95 \mathrm{E}-01$ & $1.08 \mathrm{E}-01$ & & & $7.29 \mathrm{E}-02$ & $99 E-02$ \\
\hline $2.40 E+00$ & 1. $31 E+00$ & 1. $31 E+00$ & $1.15 \mathrm{E}+00$ & $4.25 \mathrm{E}-01$ & $E-01$ & $6.45 \mathrm{E}-02$ & $8.03 E-03$ & E-03 & -03 & $64 \mathrm{E}-04$ & E-04 \\
\hline $2.80 \mathrm{E}+00$ & $1.70 E+00$ & $1.70 E+00$ & $1.52 \mathrm{E}+00$ & $6.02 \mathrm{E}-01$ & $2.28 \mathrm{E}-01$ & $8.85 \mathrm{E}-02$ & $7.90 \mathrm{E}-03$ & $2.53 \mathrm{E}-03$ & $2.10 E-03$ & $2.06 \mathrm{E}-03$ & $2.02 \mathrm{E}-03$ \\
\hline $3.25 E+00$ & $5.28 \mathrm{E}-01$ & $5.28 E-01$ & $4.66 \mathrm{E}-01$ & $1.71 \mathrm{E}-01$ & $6.34 \mathrm{E}-02$ & $2.41 E-02$ & $1.55 E-03$ & $1.36 \mathrm{E}-04$ & $6.34 E-05$ & $5.56 \mathrm{E}-05$ & $4.62 \mathrm{E}-0.5$ \\
\hline $\mathrm{E}+00$ & $5.85 \mathrm{E}-01$ & $5.85 \mathrm{~F}$ & -01 & $1.91 \mathrm{E}-01$ & -02 & $2.66 \mathrm{E}-02$ & & & & & $E-05$ \\
\hline $4.25 E+00$ & $2.08 E-01$ & $2.08 \mathrm{E}-01$ & $1.83 \mathrm{E}-01$ & $6.79 E-02$ & $2.51 E-02$ & $9.45 E-03$ & $5.20 \mathrm{E}-04$ & $8.40 \mathrm{E}-06$ & $5.51 E-06$ & $4.99 E-06$ & $4.14 E-06$ \\
\hline $4.75 E+00$ & $1.78 \mathrm{E}-01$ & $1.78 \mathrm{E}-01$ & $1.58 \mathrm{E}-01$ & $5.88 \mathrm{E}-02$ & $2.17 \mathrm{E}-02$ & $8.19 E-03$ & $4.43 \mathrm{~b}-04$ & $1.38 \mathrm{E}-06$ & $\mathrm{E}-08$ & $7.32 \mathrm{E}-08$ & $8 \mathrm{E}-08$ \\
\hline $5.50 \mathrm{E}+00$ & $3.00 \mathrm{E}-01$ & $3.00 \mathrm{E}-01$ & $2.66 \mathrm{E}-01$ & $9.98 \mathrm{E}-02$ & $3.70 \mathrm{E}-02$ & $1.39 \mathrm{E}-02$ & $7.54 \mathrm{E}-04$ & $2.28 \mathrm{E}-06$ & $6.94 \mathrm{E}-08$ & $6.32 \mathrm{E}-08$ & $5.25 \mathrm{E}-08$ \\
\hline total & $6.57 \Xi+02$ & $6.57 \mathrm{E}+02$ & $6.47 E+02$ & $6.00 \mathrm{E}+02$ & $5.80 E+02$ & $5.70 E+02$ & $5.57 E+02$ & $5.41 \mathrm{E}+02$ & $5.15 \mathrm{E}+02$ & $5.02 \mathrm{E}+02$ & $4.99 \mathrm{E}+02$ \\
\hline $\mathrm{mev} / \mathrm{sec}$ & $8.80 E+02$ & $8.80 E+02$ & $8.72 \mathrm{E}+02$ & $8.32 E+02$ & $8.14 E+02$ & $8.05 E+02$ & $7.87 E+02$ & $7.65 \mathrm{E}+02$ & $7.27 E+02$ & $7.08 E+02$ & $7.02 \mathrm{E}+02$ \\
\hline
\end{tabular}


Table G14. Decay Photon Spectrum (/kg.s) After 16-s Irradiation of 1-kg 16-16-16 Fertilizer Cargo (Flux Parameter Set 1).

\begin{tabular}{|c|c|c|c|c|c|c|c|c|c|c|c|}
\hline emean & initial & $1 \mathrm{~F}-15 \mathrm{sec}$ & $0<e^{t}$ & e after & scharge & 30000 & $600 \mathrm{cos}$ & 12000 & & & \\
\hline $.00 E-01$ & & 1 & $106 \mathrm{~F}+0 ?$ & 10.0sec & 6 USt & $30.0 \mathrm{sec}$ & bu.use & {$[20.0$ se } & & & \\
\hline & & & & & & BE+ & E- & $3 E+01$ & $2 \mathrm{E}+$ & $6 E+01$ & $2 E+01$ \\
\hline & & $5.80 \mathrm{E}+01$ & +01 & & & & & $1.72 \mathrm{E}+01$ & $.53 E+01$ & $45 \mathrm{E}+01$ & $42 \mathrm{E}+01$ \\
\hline 00 & +01 & $1.24 E+01$ & $1.11 \mathrm{E}+01$ & $4.92 \mathrm{E}+00$ & $2.55 E+00$ & $1.64 \mathrm{E}+00$ & $1.01 \mathrm{E}+00$ & $7.71 \mathrm{E}-01$ & $65 \mathrm{E}-01$ & $15 \mathrm{E}-01$ & $4 E-01$ \\
\hline $1.58 \mathrm{E}+00$ & $5.74 \mathrm{E}+02$ & $5.74 \mathrm{E}+02$ & $5.73 \mathrm{E}+02$ & $5.63 \mathrm{E}+02$ & $5.55 \mathrm{E}+02$ & $5.49 \mathrm{E} \cdot 02$ & $5.31 \mathrm{E}+02$ & $5.04 \mathrm{E}+02$ & $59 E+02$ & $6 \mathrm{E}+02$ & $30 E+02$ \\
\hline $2.00 E+00$ & $2.90 \mathrm{E}+00$ & $2.90 \mathrm{E}+00$ & $2.59 \mathrm{E}+00$ & $1.12 \mathrm{E}+00$ & $5.53 \mathrm{E}-01$ & $3.43 \mathrm{E}-01$ & $2.12 \mathrm{E}-01$ & $1.89 \mathrm{E}-01$ & 01 & $6 \mathrm{E}-01$ & $0 E-01$ \\
\hline $2.40 \mathrm{E}+00$ & 1. $83 E+00$ & $1.83 \mathrm{E}+00$ & $1.62 \mathrm{E}+00$ & $6.20 \mathrm{E}-01$ & $E-01$ & $2 E-02$ & $1.40 \mathrm{E}-02$ & $5.66 \mathrm{E}-03$ & 2 & $2 E-04$ & $1 E-04$ \\
\hline $2.80 E+00$ & $2.44 \mathrm{E}+00$ & $2.44 \mathrm{E}+00$ & $2.19 \mathrm{E}+00$ & $8.78 \mathrm{E}-01$ & $3.34 \mathrm{E}-01$ & $1.31 \mathrm{E}-01$ & $1.32 \mathrm{E}-02$ & $4.99 \mathrm{E}-03$ & $20 E-03$ & $1 \mathrm{E}-03$ & $4.03 E-03$ \\
\hline $3.25 \mathrm{E}+00$ & $7.39 \mathrm{E}-01$ & $7.39 \mathrm{E}-01$ & $6.55 E-01$ & -01 & -02 & & 2.3 & $:-04$ & 1. & $1 E-04$ & $3 E-05$ \\
\hline $3.75 \mathrm{E}+00$ & $8.19 \mathrm{E}-01$ & $8.19 \mathrm{E}-01$ & $7.27 \mathrm{E}-01$ & $2.76 \mathrm{E}-01$ & $1.03 \mathrm{E}-01$ & $3.89 \mathrm{E}-02$ & $2.33 \mathrm{E}-03$ & $1.85 \mathrm{E}-04$ & $1.43 \mathrm{E}-04$ & -04 & -04 \\
\hline$E+00$ & -01 & -01 & $2.59 \mathrm{E}-01$ & $9.84 \mathrm{E}-02$ & $3.65 \mathrm{E}-02$ & $1.38 \mathrm{E}-02$ & $3 E-04$ & $1.58 \mathrm{E}-05$ & 1.1 & -06 & E-06 \\
\hline $4.75 \mathrm{E}+00$ & $2.51 \mathrm{E}-01$ & $2.51 \mathrm{E}-01$ & $2.23 \mathrm{E}-01$ & $8.53 E-02$ & $3.17 \mathrm{E}-02$ & & 6.47 & & & $6 \mathrm{E}-07$ & $1.21 E-07$ \\
\hline $5.50 \mathrm{E}+00$ & $4.23 \mathrm{E}-01$ & $4.23 \mathrm{E}-01$ & $3.76 \mathrm{E}-01$ & $1.45 \mathrm{E}-01$ & $5.39 \mathrm{E}-02$ & $2.03 \mathrm{E}-02$ & $1.10 \mathrm{E}-03$ & $3.37 E-06$ & $1.39 \mathrm{E}-07$ & $1.26 \mathrm{E}-07$ & $1.05 \mathrm{E}-07$ \\
\hline total. & $7.65 E+02$ & $7.65 \mathrm{E}+02$ & $7.52 \mathrm{E}+02$ & $6.85 \mathrm{E}+02$ & $6.54 \mathrm{E}+02$ & $6.39 \mathrm{E}+02$ & $6.14 \mathrm{E}+02$ & $5.83 E+02$ & $5.32 E+02$ & $5.06 E+02$ & $5.00 E+02$ \\
\hline $\mathrm{mev} / \mathrm{sec}$ & $1.02 \mathrm{E}+03$ & $1.02 \mathrm{E}+03$ & $1.00 \mathrm{E}+03$ & $9.48 E+02$ & $9.19 \mathrm{E}+02$ & $9.02 E+02$ & $8.70 \mathrm{E}+02$ & $8.25 E+02$ & $7.51 E+02$ & $7.13 E+02$ & $7.03 E+02$ \\
\hline
\end{tabular}

\section{Table G15. Decay Photon Spectrum (/kg.s) After 24-s Irradiation of 1-kg 16-16-16 Fertilizer Cargo (Flux Parameter Set 1).}

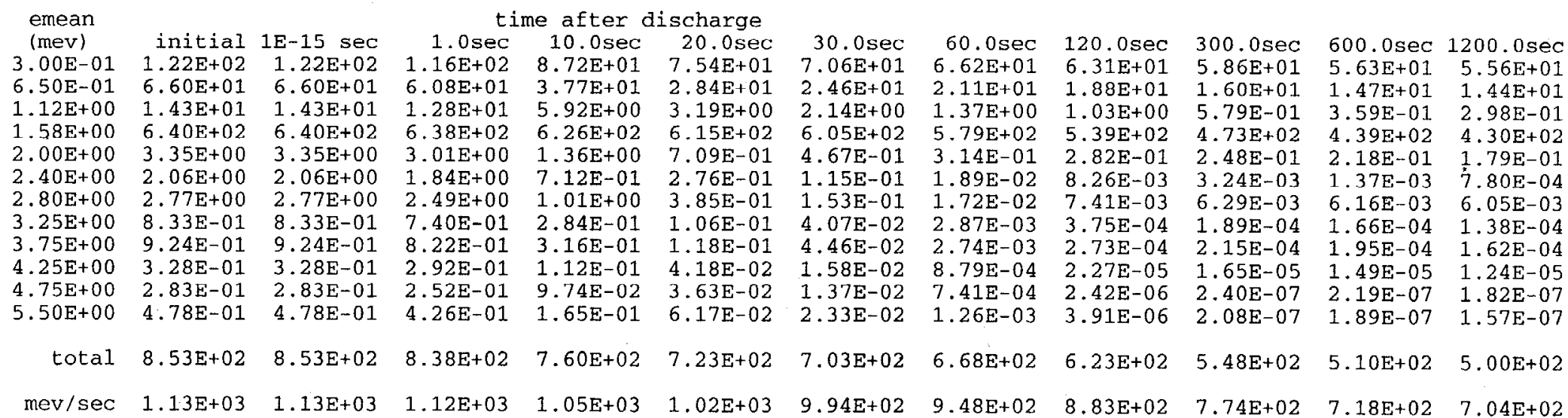


Table G16. Decay Photon Spectrum (/kg.s) After One-Day Irradiation of 1-kg Concrete (Flux Parameter Set 1).

$\mathrm{hr}$

\begin{tabular}{|c|c|c|c|c|c|c|c|c|c|c|c|}
\hline $\begin{array}{l}\text { emean } \\
\text { (mev) }\end{array}$ & initial & $.5 \mathrm{hr}$ & $1.0 \mathrm{tim}$ & $\begin{array}{l}\text { after di } \\
2.0 \mathrm{hr}\end{array}$ & $\begin{array}{l}\text { harge } \\
4.0 \mathrm{hr}\end{array}$ & $6.0 \mathrm{hr}$ & 8.0 & 10.0 & 12.0 & 14.0 & 16.0 \\
\hline $\begin{array}{l}.00 \mathrm{E}-01 \\
.50 \mathrm{E}-01 \\
.12 \mathrm{E}+00 \\
58 \mathrm{E}+00 \\
.00 \mathrm{E}+00 \\
40 \mathrm{E}+00 \\
80 \mathrm{E}+00 \\
25 \mathrm{E}+00 \\
75 \mathrm{E}+00 \\
.25 \mathrm{E}+00 \\
.75 \mathrm{E}+00 \\
.50 \mathrm{E}+00\end{array}$ & $\begin{array}{l}1.75 \mathrm{E}+03 \\
2.81 \mathrm{E}+03 \\
1.35 \mathrm{E}+03 \\
2.31 \mathrm{E}+04 \\
5.83 \mathrm{E}+01 \\
4.49 \mathrm{E}+01 \\
1.79 \mathrm{E}+02 \\
1.85 \mathrm{E}+00 \\
1.95 \mathrm{~F}+00 \\
6.18 \mathrm{E}-01 \\
5.44 \mathrm{E}-01 \\
8.96 \mathrm{E}-01\end{array}$ & $\begin{array}{l}2.22 \mathrm{E}+01 \\
2.86 \mathrm{E}+02 \\
8.19 \mathrm{E}+01 \\
2.08 \mathrm{E}+02 \\
2.04 \mathrm{E}+01 \\
2.37 \mathrm{E}+00 \\
1.69 \mathrm{E}+02 \\
1.23 \mathrm{E}-01 \\
1.10 \mathrm{E}-01 \\
1.44 \mathrm{E}-03 \\
5.60 \mathrm{E}-15 \\
8.33 \mathrm{E}-24\end{array}$ & $\begin{array}{l}1.50 \mathrm{E}+01 \\
1.06 \mathrm{E}+02 \\
7.88 \mathrm{E}+00 \\
2.01 \mathrm{E}+02 \\
1.70 \mathrm{E}+01 \\
7.34 \mathrm{E}-01 \\
1.65 \mathrm{E}+02 \\
1.08 \mathrm{E}-01 \\
1.07 \mathrm{E}-01 \\
1.40 \mathrm{E}-03 \\
3.37 \mathrm{E}-23 \\
4.77 \mathrm{E}-24\end{array}$ & $\begin{array}{l}1.33 \mathrm{E}+01 \\
6.64 \mathrm{E}+01 \\
8.86 \mathrm{E}-01 \\
1.92 \mathrm{E}+02 \\
1.30 \mathrm{E}+01 \\
5.12 \mathrm{E}-01 \\
1.57 \mathrm{E}+02 \\
8.23 \mathrm{E}-02 \\
1.02 \mathrm{E}-01 \\
1.34 \mathrm{E}-03 \\
1.81 \mathrm{E}-24 \\
1.56 \mathrm{E}-24\end{array}$ & $\begin{array}{l}1.19 \mathrm{E}+01 \\
4.02 \mathrm{E}+01 \\
5.73 \mathrm{E}-01 \\
1.76 \mathrm{E}+02 \\
7.58 \mathrm{E}+00 \\
3.04 \mathrm{E}-01 \\
1.43 \mathrm{E}+02 \\
4.81 \mathrm{E}-02 \\
9.29 \mathrm{E}-02 \\
1.22 \mathrm{E}-03 \\
1.93 \mathrm{E}-25 \\
1.67 \mathrm{E}-25\end{array}$ & $\begin{array}{l}1.08 \mathrm{E}+01 \\
2.50 \mathrm{E}+01 \\
4.32 \mathrm{E}-01 \\
1.62 \mathrm{E}+02 \\
4.44 \mathrm{E}+00 \\
1.82 \mathrm{E}-01 \\
1.30 \mathrm{E}+02 \\
2.82 \mathrm{E}-02 \\
8.46 \mathrm{E}-02 \\
1.11 \mathrm{E}-03 \\
2.07 \mathrm{E}-26 \\
1.79 \mathrm{E}-26\end{array}$ & $\begin{array}{l}1.00 \mathrm{E}+01 \\
1.61 \mathrm{E}+01 \\
3.44 \mathrm{E}-01 \\
1.50 \mathrm{E}+02 \\
2.60 \mathrm{E}+00 \\
1.11 \mathrm{E}-01 \\
1.18 \mathrm{E}+02 \\
1.65 \mathrm{E}-02 \\
7.69 \mathrm{E}-02 \\
1.01 \mathrm{E}-03 \\
2.22 \mathrm{E}-27 \\
1.92 \mathrm{E}-27\end{array}$ & $\begin{array}{l}9.45 \mathrm{E}+00 \\
1.08 \mathrm{E}+01 \\
2.87 \mathrm{E}-01 \\
1.40 \mathrm{E}+02 \\
1.53 \mathrm{E}+00^{\circ} \\
6.85 \mathrm{E}-02 \\
1.08 \mathrm{E}+02 \\
9.72 \mathrm{E}-03 \\
7.00 \mathrm{E}-02 \\
9.17 \mathrm{E}-04 \\
2.38 \mathrm{E}-28 \\
2.05 \mathrm{E}-28\end{array}$ & $\begin{array}{l}8.97 \mathrm{E}+00 \\
7.60 \mathrm{E}+00 \\
2.46 \mathrm{E}-01 \\
1.31 \mathrm{E}+02 \\
9.01 \mathrm{E}-01 \\
4.33 \mathrm{E}-02 \\
9.78 \mathrm{E}+01 \\
5.73 \mathrm{E}-03 \\
6.37 \mathrm{E}-02 \\
8.34 \mathrm{E}-04 \\
2.54 \mathrm{E}-29 \\
2.19 \mathrm{E}-29\end{array}$ & $\begin{array}{l}8.57 \mathrm{E}+00 \\
5.70 \mathrm{E}+00 \\
2.16 \mathrm{E}-01 \\
1.22 \mathrm{E}+02 \\
5.33 \mathrm{E}-01 \\
2.83 \mathrm{E}-02 \\
8.90 \mathrm{E}+01 \\
3.40 \mathrm{E}-03 \\
5.79 \mathrm{E}-02 \\
7.59 \mathrm{E}-04 \\
2.68 \mathrm{E}-30 \\
2.31 \mathrm{E}-30\end{array}$ & $\begin{array}{l}8.23 \mathrm{E}+00 \\
4.54 \mathrm{E}+00 \\
1.92 \mathrm{E}-01 \\
1.15 \mathrm{E}+02 \\
3.17 \mathrm{E}-01 \\
1.92 \mathrm{E}-02 \\
8.10 \mathrm{E}+01 \\
2.03 \mathrm{E}-03 \\
5.27 \mathrm{E}-02 \\
6.90 \mathrm{E}-04 \\
2.55 \mathrm{E}-31 \\
2.20 \mathrm{E}-31\end{array}$ \\
\hline total & $2.93 E+04$ & $7.90 \mathrm{E}+02$ & $5.13 E+02$ & $4.43 E+02$ & $3.79 \mathrm{E}+02$ & $3.33 \mathrm{E}+02$ & $2.98 \mathrm{E}+02$ & $2.70 \mathrm{E}+02$ & $2.46 \mathrm{E}+02$ & $2.26 \mathrm{E}+02$ & 2.09E+02 \\
\hline ev/sed & $4.10 E+04$ & $1.13 E+03$ & $8.98 E+02$ & $8.18 \mathrm{E}+02$ & $7.24 \mathrm{E}+02$ & $6.49 \mathrm{E}+02$ & $5.87 \mathrm{E}+02$ & $5.35 E+02$ & $4.90 \mathrm{E}+02$ & $4.50 \mathrm{E}+02$ & $4.14 \mathrm{E}+02$ \\
\hline
\end{tabular}

\section{Table G17. Decay Photon Spectrum (/kg.s) After Ten-Day Irradiation of 1-kg Concrete (Flux Parameter Set 1).}

\begin{tabular}{|c|c|c|c|c|c|c|c|c|c|c|c|}
\hline $\begin{array}{l}\text { emean } \\
\text { (mev) }\end{array}$ & initial & $.5 \mathrm{hr}$ & $1.0 \mathrm{hr}$ time & $\begin{array}{l}\text { after di } \\
2.0 \mathrm{hr}\end{array}$ & harge & & & & & & \\
\hline $3.00 \mathrm{E}-01$ & $1.75 E+03$ & $2.53 \mathrm{E}+01$ & $1.80 \mathrm{Er}+01$ & $\begin{array}{c}2.0 \mathrm{nr} \\
1.62 \mathrm{E}+01\end{array}$ & $\begin{array}{c}4.0 \mathrm{hr} \\
1.45 \mathrm{E}+01\end{array}$ & $\begin{array}{c}6.0 \mathrm{hr} \\
1.32 \mathrm{E}+01\end{array}$ & $\begin{array}{c}8.0 \mathrm{hr} \\
1.22 \mathrm{E}+01\end{array}$ & $10.0 \mathrm{hr}$ & $\begin{array}{l}12.0 \mathrm{hr} \\
1.08 \mathrm{t}+01\end{array}$ & $\begin{array}{l}14.0 \mathrm{hr} \\
1.02 \mathrm{E}+01\end{array}$ & $\begin{array}{r}16.0 \mathrm{hr} \\
9.77 \mathrm{E}+00\end{array}$ \\
\hline $6.50 \mathrm{E}-01$ & $2.82 \mathrm{E}+03$ & $2.91 \mathrm{E}+02$ & $1.11 \mathrm{E}+02$ & 7. $15 \mathrm{E}+01$ & $4.52 \mathrm{E}+01$ & $2.98 \mathrm{E}+01$ & $2.08 \mathrm{E}+01$ & $1.54 \mathrm{E}+01$ & $1.21 E+01$ & $1.01 E+01$ & $8.92 E+00$ \\
\hline $1.12 \mathrm{E}+00$ & $1.35 E+03$ & $8.20 E+01$ & $8.02 \mathrm{E}+00$ & $1.02 \mathrm{E}+00$ & $6.92 \mathrm{E}-01$ & $5.39 \mathrm{E}-01$ & $4.41 \mathrm{E}-01$ & $3.74 \mathrm{E}-01$ & $3.24 \mathrm{E}-01$ & $2.86 \mathrm{E}-01$ & $2.55 \mathrm{E}-01$ \\
\hline $1.58 \mathrm{E}+00$ & $2.32 \mathrm{E}+04$ & $2.80 E+02$ & $2.71 \mathrm{E}+02$ & $2.59 \mathrm{E}+02$ & $2.37 \mathrm{E}+02$ & $2.18 \mathrm{E}+02$ & $2.01 \mathrm{E}+02$ & $1.86 \mathrm{E}+02$ & $1.72 \mathrm{E}+02$ & $1.60 \mathrm{E}+02$ & $1.49 E+02$ \\
\hline $2.00 E+00$ & $5.84 \mathrm{E}+01$ & $2.04 E+01$ & $1.70 \mathrm{E}+01$ & 1. $30 \mathrm{E}+01$ & $7.61 \mathrm{E}+00$ & $4.46 \mathrm{E}+00$ & $2.62 \mathrm{E}+00$ & $1.54 \mathrm{E}+00$ & $9.10 \mathrm{E}-01$ & $5.41 \mathrm{E}-01$ & $3.24 \mathrm{E}-01$ \\
\hline $2.40 E+00$ & $4.50 E+01$ & $2.38 \mathrm{E}+00$ & $7.41 \mathrm{E}-01$ & $5.19 \mathrm{E}-01$ & $3.10 \mathrm{E}-01$ & $1.87 \mathrm{E}-01$ & $1.15 \mathrm{E}-01$ & $7.24 \mathrm{E}-02$ & $4.68 \mathrm{E}-02$ & $3.14 \mathrm{E}-02$ & $2.20 \mathrm{E}-02$ \\
\hline $2.80 E+00$ & $2.60 \mathrm{E}+02$ & $2.49 E+02$ & $2.43 E+02$ & $2.32 \mathrm{E}+02$ & 2. $11 \mathrm{E}+02$ & $1.92 \mathrm{E}+02$ & $1.74 \mathrm{E}+02$ & 1. $58 \mathrm{E}+02$ & 1. $44 \mathrm{E}+02$ & 1. $31 \mathrm{E}+02$ & 1. $19 \mathrm{E}+02$ \\
\hline $3.25 E+00$ & $1.85 \mathrm{E}+00$ & $1.23 \mathrm{E}-01$ & $1.08 \mathrm{E}-01$ & $8.25 \mathrm{E}-02$ & $4.83 \mathrm{E}-02$ & $2.83 \mathrm{E}-02$ & $1.66 \mathrm{E}-02$ & $9.80 \mathrm{E}-03$ & $5.80 \mathrm{E}-03$ & $3.45 \mathrm{E}-03$ & $2.08 E-03$ \\
\hline $3.75 \mathrm{E}+00$ & $2.00 \mathrm{E}+00$ & $1.62 E-01$ & $1.58 \mathrm{E}-01$ & $1.51 \mathrm{E}-01$ & $1.37 \mathrm{E}-01$ & $1.25 \mathrm{E}-01$ & $1.13 \mathrm{E}-01$ & & $8 \mathrm{E}-02$ & $4 \mathrm{E}-02$ & $7.77 \mathrm{E}-02$ \\
\hline $4.25 E+00$ & $6.18 \mathrm{E}-01$ & 2. $12 \mathrm{E}-03$ & $2.07 \mathrm{E}-03$ & $1.97 \mathrm{E}-03$ & $1.79 \mathrm{E}-03$ & $1.63 \mathrm{E}-03$ & $1.49 \mathrm{E}-03$ & $1.35 \mathrm{E}-03$ & $1.23 \mathrm{E}-03$ & $1.12 \mathrm{E}-03$ & $1.02 \mathrm{E}-03$ \\
\hline $4.75 E+00$ & $5.44 \mathrm{E}-01$ & $5.60 E-15$ & $1.83 E-21$ & $5.89 \mathrm{E}-22$ & & $6.76 \mathrm{E}-24$ & $7.24 \mathrm{E}-25$ & $7.75 E-26$ & 8.3 & 8.8 & $9.52 \mathrm{E}-29$ \\
\hline $5.50 \mathrm{E}+00$ & $8.96 \mathrm{E}-01$ & $2.72 \mathrm{E}-21$ & $1.55 \mathrm{E}-21$ & $5.09 \mathrm{E}-22$ & & $5.84 \mathrm{E}-24$ & $6.25 \mathrm{E}-25$ & $6.69 \mathrm{E}-26$ & $7.17 \mathrm{E}-27$ & $7.68 \mathrm{E}-28$ & $8.22 \mathrm{E}-29$ \\
\hline total & $2.95 \mathrm{E}+04$ & $9.51 \mathrm{E}+02$ & $6.70 \mathrm{E}+02$ & $5.93 \mathrm{E}+02$ & $5.16 \mathrm{E}+02$ & $4.58 E+02$ & $4.11 E+02$ & $3.73 E+02$ & $3.41 E+02$ & $3.13 E+02$ & $2.88 \mathrm{E}+02$ \\
\hline mev/sec & $4.14 \mathrm{E}+04$ & $1.48 \mathrm{E}+03$ & $1.23 E+03$ & $1.14 \mathrm{E}+03$ & $1.01 \mathrm{E}+03$ & $9.13 E+02$ & $8.28 \mathrm{E}+02$ & $7.54 E+02$ & $6.89 \mathrm{E}+02$ & $6.31 E+02$ & $5.79 E+02$ \\
\hline
\end{tabular}


Table G18. Decay Photon Spectrum (/kg.s) After One-Day Irradiation of 1-kg Concrete (Flux Parameter Set 2).

\begin{tabular}{|c|c|c|c|c|c|c|c|c|c|c|c|}
\hline $\begin{array}{l}\text { emean } \\
\text { (mev) }\end{array}$ & initial & & $1.0 \mathrm{hr}$ & $\begin{array}{l}\text { after di } \\
2.0 \mathrm{hr}\end{array}$ & $\begin{array}{l}\text { tharge } \\
4.0 \mathrm{hr}\end{array}$ & $6.0 \mathrm{hr}$ & & & & & \\
\hline $3,00 E-01$ & $1.22 \mathrm{E}+04$ & 1.29E+02 & $7.80 \mathrm{E}+01$ & $6.60 \mathrm{E}+01$ & $5.50 \mathrm{E}+01$ & $4.73 E+01$ & $4.16 \mathrm{E}+01$ & $3.71 \mathrm{E}+01$ & $3.36 \mathrm{E}+01$ & $\begin{array}{l}14.0 \mathrm{hr} \\
3.06 \mathrm{E}+01\end{array}$ & $\begin{array}{r}16.0 \mathrm{hr} \\
2.80 \mathrm{E}+01\end{array}$ \\
\hline $6.50 \mathrm{E}-01$ & $1.97 E+04$ & $1.99 \mathrm{E}+03$ & $7.36 \mathrm{E}+02$ & $4.58 \mathrm{E}+02$ & $2.75 E+02$ & $1.68 E+02$ & $1.05 E+02$ & $6.82 \mathrm{E}+01$ & $4.60 \mathrm{E}+01$ & 3. $26 E+01$ & $2.44 E+01$ \\
\hline $1.12 \mathrm{E}+00$ & $9.44 \mathrm{E}+03$ & $5.72 \mathrm{E}+02$ & $5.53 \mathrm{E}+01$ & $6.35 E+00$ & $4.12 E+00$ & $3.10 \mathrm{E}+00$ & $2.46 \mathrm{E}+00$ & $2.04 \mathrm{E}+00$ & 1. $74 \mathrm{E}+00$ & $1.51 \mathrm{E}+00$ & $1.33 \mathrm{E}+00$ \\
\hline $1.58 \mathrm{E}+00$ & $1.62 E+05$ & $1.27 \mathrm{E}+03$ & $1.22 E+03$ & $1.15 \mathrm{E}+03$ & $1.04 \mathrm{E}+03$ & $9.37 \mathrm{E}+02$ & $8.50 E+02$ & $7.73 \mathrm{E}+02$ & $7.05 E+02$ & $6.43 E+02$ & $5.88 E+02$ \\
\hline $2.00 \mathrm{E}+00$ & $4.08 \mathrm{E}+02$ & $1.43 \mathrm{E}+02$ & $1.19 E+02$ & $9.06 \mathrm{E}+01$ & $5.30 \mathrm{E}+01$ & $3.11 \mathrm{E}+01$ & $1.82 \mathrm{E}+01$ & $1.07 \mathrm{E}+01$ & $6.31 \mathrm{E}+00$ & $4 \mathrm{E}+00$ & $2.23 \mathrm{E}+00$ \\
\hline $2.40 \mathrm{E}+00$ & $3.14 \mathrm{E}+02$ & $1.66 \mathrm{~F}+01$ & $5.14 E+00$ & $3.59 \mathrm{E}+00$ & $2.14 \mathrm{E}+00$ & 1.29F+00 & $7.83 \mathrm{~F}-01$ & $6 \mathrm{E}-01$ & $3.10 \mathrm{E}-01$ & $4 E-01$ & $0 E-01$ \\
\hline $2.80 E+00$ & $1.32 \mathrm{E}+03$ & $1.25 E+03$ & $1.22 \mathrm{E}+03$ & $1.17 E+03$ & $1.06 \mathrm{E}+03$ & $9.63 E+02$ & $8.76 \mathrm{E}+02$ & $7.97 E+02$ & $7.25 \mathrm{E}+02$ & $9 E+02$ & $6.00 \mathrm{E}+02$ \\
\hline $3.25 \mathrm{E}+00$ & $1.29 \mathrm{E}+01$ & 8.60 & $7.52 \mathrm{E}-01$ & 5.7 & & & 1.1 & & & & \\
\hline $75 E+00$ & $1.37 \mathrm{E}+01$ & $8.12 \mathrm{E}-01$ & $7.93 \mathrm{E}-01$ & $7.57 \mathrm{E}-01$ & $6.88 \mathrm{E}-01$ & $6.26 \mathrm{E}-01$ & $5.70 \mathrm{E}-01$ & $5.18 \mathrm{E}-01$ & $4.72 \mathrm{E}-01$ & $4.29 \mathrm{E}-01$ & $3.90 \mathrm{E}-01$ \\
\hline $4.25 \mathrm{E}+00$ & $4.32 E+00$ & 1.06 & $1.04 \mathrm{E}-02$ & & & 8.2 & 7.4 & & & & \\
\hline $\mathrm{E}+00$ & $3.80 \mathrm{E}+00$ & $3.92 \mathrm{E}-14$ & $3.93 E-22$ & $6.40 \mathrm{E}-23$ & $6.86 \mathrm{E}-24$ & $7.35 E-25$ & $7.87 \mathrm{E}-26$ & $8.43 E-27$ & $9.02 E-28$ & 9.66 & -29 \\
\hline & $6.26 \mathrm{E}+00$ & $2.95 \mathrm{E}-22$ & $1.69 \mathrm{E}-22$ & $5.53 \mathrm{E}-23$ & $5.92 \mathrm{E}-24$ & $6.34 \mathrm{E}-25$ & $6.80 \mathrm{E}-26$ & $7.28 \mathrm{E}-27$ & $7.79 \mathrm{E}-28$ & $8.34 \mathrm{E}-29$ & $8.93 E-30$ \\
\hline & $2.05 E+05$ & $5.38 \mathrm{E}+03$ & $3.44 E+03$ & $2.95 \mathrm{E}+03$ & $2.49 E+03$ & $2.15 \mathrm{E}+03$ & $1.89 \mathrm{E}+03$ & $1.69 \mathrm{E}+03$ & $1.52 \mathrm{E}+03$ & $1.37 E+03$ & $1.24 E+C$ \\
\hline $\mathrm{mev} / \mathrm{sec}$ & $2.87 E+05$ & $7.82 \mathrm{E}+03$. & $6.17 E+03$ & $5.60 \mathrm{E}+03$ & $4.91 E+03$ & $4.37 E+03$ & $3.92 E+03$ & $3.53 \mathrm{E}+03$ & $3.20 \mathrm{E}+03$ & $2.90 \mathrm{E}+03$ & $2.64 \mathrm{E}+0$ \\
\hline
\end{tabular}

Table G19. Decay Photon Spectrum (/kg.s) After Ten-Day Irradiation of 1-kg Concrete (Flux Parameter Set 2).

\begin{tabular}{|c|c|c|c|c|c|c|c|c|c|c|c|}
\hline emean & & & time & after di & harge & & & & & & \\
\hline (mev) & hilial & $.5 \mathrm{hr}$ & $1.0 \mathrm{hr}$ & $2.0 \mathrm{hr}$ & 4.0 hr & $6.0 \mathrm{hr}$ & & & & & 16.0 \\
\hline $3.00 \mathrm{E}-01$ & $1.22 \mathrm{E}+04$ & $1.52 \mathrm{E}+02$ & $1.00 \mathrm{E}+02$ & $8.72 E+01$ & $7.44 \mathrm{E}+01$ & $6.50 \mathrm{E}+01$ & $5.77 \mathrm{E}+01$ & $19 \mathrm{E}+01$ & $71 E+01$ & $29 \mathrm{E}+01$ & $93 E+01$ \\
\hline $6.50 \mathrm{E}-01$ & $1.97 \mathrm{E}+04$ & $2.03 E+03$ & $7.73 E+02$ & $4.94 E+02$ & $3.10 \mathrm{E}+02$ & $2.02 \mathrm{E}+02$ & $1.39 \mathrm{E}+02$ & $1.01 \mathrm{E}+02$ & $7.79 E+01$ & $6.40 \mathrm{E}+01$ & $53 \mathrm{E}+01$ \\
\hline $1.12 \mathrm{E}+00$ & $9.44 \mathrm{E}+03$ & $5.74 E+02$ & $5.63 \mathrm{E}+01$ & $7.37 \mathrm{E}+00$ & $5.03 E+00$ & $3.92 E+00$ & $3.20 E+00$ & $2.70 \mathrm{E}+00$ & $2.34 \mathrm{E}+00$ & $2.05 E+00$ & $1.81 E+00$ \\
\hline $1.58 \mathrm{E}+00$ & $1.62 \mathrm{E}+05$ & $1.81 E+03$ & $1.75 \mathrm{E}+03$ & $1.65 E+03$ & $1.49 E+03$ & 1. $35 E+03$ & 1. $23 \mathrm{E}+03$ & $11 E+03$ & $1.02 E+03$ & $9.26 \mathrm{E}+02$ & $5 E+02$ \\
\hline $2.00 E+00$ & $4.08 \mathrm{E}+02$ & $1.43 \mathrm{E}+02$ & $1.19 \mathrm{E}+02$ & $9.09 \mathrm{E}+01$ & $5.32 \mathrm{E}+01$ & $3.12 \mathrm{E}+01$ & $1.83 \mathrm{E}+01$ & $1.08 \mathrm{E}+01$ & $6.38 \mathrm{E}+00$ & $3.80 \mathrm{E}+00$ & $8 \mathrm{E}+00$ \\
\hline $2.40 E+00$ & $4 E+02$ & $1.67 \mathrm{E}+01$ & $5.20 \mathrm{E}+00$ & $E+00$ & 2.1 & $1.32 \mathrm{E}$ & 8. & & & 2. & $51 E-01$ \\
\hline $2.80 \mathrm{E}+00$ & $3 E+03$ & $1.84 E+03$ & $1.80 \mathrm{E}+03$ & $E+03$ & $1.56 \mathrm{E}+03$ & $1.42 \mathrm{E}+03$ & $1.29 \mathrm{E}+03$ & $1.17 \mathrm{E}+03$ & $1.07 E+03$ & $9.71 \mathrm{E}+02$ & $8.84 E+02$ \\
\hline $25 E+00$ & $9 E+01$ & -01 & $7.54 \mathrm{E}-01$ & 5.7 & 3.3 & 1.9 & 1.1 & & & $E-02$ & $E-02$ \\
\hline $3.75 E+00$ & $1.41 E+01$ & $1.20 \mathrm{E}+00$ & $1.17 \mathrm{E}+00$ & $1.12 \mathrm{E}+00$ & $1.01 \mathrm{E}+00$ & $9.23 \mathrm{E}-01$ & $8.40 \mathrm{E}-01$ & $7.64 \mathrm{E}-01$ & $6.95 \mathrm{E}-01$ & $6.32 \mathrm{E}-01$ & $5.75 \mathrm{E}-01$ \\
\hline $25 E+00$ & $2 \mathrm{E}+00$ & $1.57 \mathrm{E}-02$ & $1.53 \mathrm{E}-02$ & $1.46 \mathrm{E}-02$ & $1.33 \mathrm{E}-02$ & $1.21 \mathrm{E}-02$ & $1.10 \mathrm{E}-02$ & 1. & & $8 \mathrm{E}-03$ & $E-03$ \\
\hline $4.75 E+00$ & $0 E+00$ & $3.92 \mathrm{E}-14$ & $6.40 \mathrm{E}-20$ & & $2.24 \mathrm{E}-21$ & $2.40 \mathrm{E}-22$ & & & & & \\
\hline $5.50 \mathrm{E}+00$ & $6 \mathrm{E}+00$ & $9.64 \mathrm{E}-20$ & $5.51 \mathrm{E}-20$ & $1.80 \mathrm{E}-20$ & $1.93 \mathrm{E}-21$ & $2.07 \mathrm{E}-22$ & $2.22 \mathrm{E}-23$ & $2.37 \mathrm{E}-24$ & $2.54 \mathrm{E}-$ & $2.72 \mathrm{E}-26$ & $2.92 \mathrm{E}-27$ \\
\hline total & $2.06 \mathrm{E}+05$ & $6.57 \mathrm{E}+03$ & $4.60 \mathrm{E}+03$ & $4.05 E+03$ & $3.50 \mathrm{E}+03$ & $3.07 \mathrm{E}+03$ & $2.74 \mathrm{E}+03$ & $2.46 \mathrm{E}+03$ & $2.22 \mathrm{E}+03$ & $2.01 E+03$ & $1.83 E+03$ \\
\hline mev/sec & $2.90 \mathrm{E}+05$ & $1.04 \mathrm{E}+04$ & $8.64 E+03$ & $7.96 \mathrm{E}+03$ & $7.06 \mathrm{E}+03$ & $6.32 \mathrm{E}+03$ & $5.70 \mathrm{E}+03$ & $5.15 \mathrm{E}+03$ & $4.67 E+03$ & $4.25 E+03$ & $3.86 \mathrm{E}+0$ \\
\hline
\end{tabular}


Table G20. Decay Photon Spectrum (/kg.s) After One-Day Irradiation of 1-kg Aluminum 6061-T6 (Flux Parameter Set 1).

\begin{tabular}{|c|c|c|c|c|c|c|c|c|c|c|c|}
\hline emean & & & time & after dis & charge & & & & & & \\
\hline $\begin{array}{l}(\mathrm{mev}) \\
3.00 \mathrm{E}-01\end{array}$ & $1.74 \mathrm{E}+03$ & $2.38 \mathrm{E}+02$ & $\begin{array}{l}1.0 \mathrm{hr} \\
8.20 \mathrm{E}+01\end{array}$ & $2.0 \mathrm{hr}$ & $\begin{array}{c}4.0 \mathrm{hr} \\
5.36 \mathrm{E}+01\end{array}$ & $\begin{array}{c}6.0: n r \\
4.81 E+01\end{array}$ & $\begin{array}{c}8.0 \mathrm{hr} \\
4.34 \mathrm{E}+01\end{array}$ & $10.0 \mathrm{hr}$ & $12.0 \mathrm{hr}$ & $14.0 \mathrm{hr}$ & $16.0 \mathrm{hr}$ \\
\hline $6.50 \mathrm{E}-01$ & $4.80 E+04$ & $5.47 E+03$ & $7.44 E+02$ & $1.31 E+02$ & $7.87 \mathrm{E}+01$ & $5.19 \mathrm{E}+01$ & $3.57 \mathrm{E}+01$ & $2.58 \mathrm{E}+01$ & $1.95 \mathrm{E}+01$ & $1.54 \mathrm{E}+01$ & $\begin{array}{l}1.27 \mathrm{E}+01 \\
1\end{array}$ \\
\hline $1.12 \mathrm{E}+00$ & $1.28 E+04$ & $1.42 E+03$ & $1.60 E+02$ & $3.79 E+00$ & $1.54 \mathrm{E}+00$ & 1. $33 E+00$ & $1.20 \mathrm{E}+00$ & $1.10 \mathrm{E}+00$ & 1. $.02 \mathrm{E}+00$ & $9.58 \mathrm{E}-01$ & $9.07 \mathrm{E}-01$ \\
\hline $1.58 \mathrm{E}+00$ & $5.87 \mathrm{E}+03$ & $2.90 E+03$ & $2.83 E+03$ & $2.70 \mathrm{E}+03$ & $2.45 E+03$ & $2.22 \mathrm{E}+03$ & $2.02 \mathrm{E}+03$ & $1.84 \mathrm{E}+03$ & $1.67 \mathrm{E}+03$ & $1.52 \mathrm{E}+03$ & $1.38 E+03$ \\
\hline $2.00 E+00$ & $3.86 \mathrm{E}+01$ & $3.24 E+01$ & $2.83 E+01$ & $2.17 \mathrm{E}+01$ & 1. $27 \mathrm{E}+01$ & $7.39 E+00$ & $4.32 \mathrm{E}+00$ & $2.52 \mathrm{E}+00$ & $1.47 \mathrm{E}+00$ & $8.61 \mathrm{E}-01$ & $5.03 \mathrm{E}-01$ \\
\hline $2.40 \mathrm{E}+00$ & $4 \mathrm{E}+00$ & $1.27 E+00$ & $1.09 \mathrm{E}+00$ & $8.30 E-01$ & $4.85 \mathrm{E}-01$ & $2.83 E-01$ & $1.66 \mathrm{E}-01$ & $9.67 E-02$ & $5.65 \mathrm{E}-02$ & $3.30 \mathrm{E}-02$ & $1.93 \mathrm{E}-02$ \\
\hline $2.80 \mathrm{E}+00$ & $3.33 E+03$ & $3.26 E+03$ & $3.18 E+03$ & $3.03 E+03$ & $2.76 \mathrm{E}+03$ & $2.51 E+03$ & $2.28 \mathrm{E}+03$ & $2.08 E+03$ & $1.89 E+03$ & $1.72 \mathrm{E}+03$ & 1. $56 \mathrm{E}+03$ \\
\hline $3.25 \mathrm{E}+00$ & $2.61 \mathrm{E}-01$ & $2.06 \mathrm{E}-01$ & $1.80 \mathrm{E}-01$ & $1.37 \mathrm{E}-01$ & $8.03 E-02$ & $1.69 \mathrm{E}-02$ & $2.74 \mathrm{E}-02$ & $1.60 \mathrm{E}-02$ & $9.36 \mathrm{E}-03$ & $5.47 \mathrm{E}-03$ & $3.20 \mathrm{E}-03$ \\
\hline $3.75 E+00$ & $2.17 \mathrm{E}+00$ & $2.12 E+00$ & $2.07 E+00$ & $1.97 E+00$ & $1.80 E+00$ & $1.63 E+00$ & $1.49 E+00$ & $1.35 E+00$ & $1.23 E+00$ & $2 \mathrm{E}+00$ & $1.02 \mathrm{E}+00$ \\
\hline $25 \mathrm{E}+00$ & $3.12 \mathrm{E}-02$ & $2.78 \mathrm{E}-02$ & $2.71 \mathrm{E}-02$ & $2.59 \mathrm{E}-02$ & $2.35 \mathrm{E}-02$ & $2.14 \mathrm{E}-02$ & $1.95 \mathrm{~F}-02$ & $1.77 \mathrm{~F}-02$ & $1.61 \mathrm{E}-02$ & $1.47 \mathrm{~F}-02$. & 1. $3.3 \mathrm{E}-02$ \\
\hline $4.75 \mathrm{E}+00$ & $2.53 E-04$ & $2.84 E-14$ & $2.44 E-20$ & $0.00 \mathrm{E}+00$ & $0.00 \mathrm{E}+00$ & $0.00 E+00$ & $E+00$ & $E+00$ & $E+00$ & $0.00 \mathrm{E}+00$ & $0.00 E+00$ \\
\hline $5.50 \mathrm{E}+00$ & $3.89 \mathrm{E}-07$ & $2.21 \mathrm{E}-14$ & $2.10 \mathrm{E}-20$ & $0.00 \mathrm{E}+00$ & $0.00 E+00$ & $0.00 \mathrm{E}+00$ & $0.00 \mathrm{E}+00$ & $0.00 \mathrm{E}+00$ & $0.00 \mathrm{E}+00$ & $0.00 \mathrm{E}+00$ & $0.00 \mathrm{E}+00$ \\
\hline total. & $7.18 \mathrm{E}+04$ & $1.33 E+04$ & $7.03 E+03$ & $5.95 E+03$ & $5.36 E+03$ & $4.85 E+03$ & $4.39 E+03$ & $3.99 \mathrm{E}+03$ & $3.62 E+03$ & $3.29 \mathrm{E}+03$ & $2.99 E+03$ \\
\hline $\mathrm{mev} / \mathrm{sec}$ & $6.48 \mathrm{E}+04$ & $1.90 E+04$ & $1.41 E+04$ & 1. $29 E+04$ & $1.17 E+04$ & 1. $06 E+04$ & $9.63 E+03$ & $8.75 E+03$ & $7.96 E+03$ & $7.24 E+03$ & $6.58 \mathrm{E}+03$ \\
\hline
\end{tabular}

Table G21. Decay Photon Spectrum (/kg.s) After Ten-Day Irradiation of 1-kg Aluminum 6061-T6 (Flux Parameter Set 1).

\begin{tabular}{|c|c|c|c|c|c|c|c|c|c|c|c|}
\hline emean & & & time & after dis & charge & & & & & & \\
\hline (mev) & initial & $.5 \mathrm{hr}$ & $1.0 \mathrm{hr}$ & $2.0 \mathrm{hr}$ & $4.0 \mathrm{hr}$ & $6.0 \mathrm{hr}$ & $8.0 \mathrm{hr}$ & $10.0 \mathrm{hr}$ & $12.0 \mathrm{hr}$ & $14.0 \mathrm{hr}$ & $16.0 \mathrm{hr}$ \\
\hline $3.00 \mathrm{E}-01$ & $1.77 \mathrm{E}+03$ & $2.57 \mathrm{E}+02$ & $1.10 \mathrm{E}+02$ & $8.74 \mathrm{E}+01$ & $7.81 E+01$ & $7.04 E+01$ & $6.37 E+01$ & $5.77 E+01$ & $5.24 E+01$ & $4.76 E+01$ & $4.32 E+01$ \\
\hline $6.50 \mathrm{E}-01$ & $4.80 \mathrm{E}+04$ & $5.48 \mathrm{E}+03$ & $7.56 \mathrm{E}+02$ & $1.42 \mathrm{E}+02$ & $8.91 \mathrm{E}+01$ & $6.15 E+01$ & $4.46 \mathrm{E}+01$ & $3.40 \mathrm{E}+01$ & $2.72 \mathrm{E}+01$ & $2.26 E+01$ & $1.94 \mathrm{E}+01$ \\
\hline $1.12 \mathrm{E}+00$ & $1.28 \mathrm{E}+04$ & $1.43 \mathrm{E}+03$ & $1.62 \mathrm{E}+02$ & $6.12 \mathrm{E}+00$ & $3.80 E+00$ & $3.53 E+00$ & $3.33 E+00$ & $3.17 E+00$ & $3.04 \mathrm{E}+00$ & $2.92 E+00$ & $2.82 \mathrm{E}+00$ \\
\hline $1.58 \mathrm{E}+00$ & $7.27 \mathrm{E} .03$ & $4.26 \mathrm{E}+03$ & $4.16 \mathrm{E}+03$ & $3.97 \mathrm{E}+03$ & $3.60 \mathrm{E}+03$ & $3.28 \mathrm{E}+03$ & $2.98 E+03$ & $2.71 E+03$ & $2.46 \mathrm{E}+03$ & $2.24 E+03$ & $2.04 \mathrm{E}+03$ \\
\hline $2.00 \mathrm{E}+00$ & $3.87 \mathrm{E}+01$ & $3.25 \mathrm{E}+01$ & $2.84 E+01$ & $2.17 E+01$ & $1.27 E+01$ & $7.40 E+00$ & $4.33 E+00$ & $2.53 E+00$ & $1.48 E+00$ & $8.62 E-01$ & $5.04 \mathrm{E}-01$ \\
\hline $2.40 E+00$ & $2.55 E+00$ & $1.27 \mathrm{E}+00$ & $1.09 \mathrm{E}+00$ & $8.32 \mathrm{E}-01$ & $4.86 \mathrm{E}-01$ & $2.84 \mathrm{E}-01$ & $1.66 \mathrm{E}-01$ & $9.69 \mathrm{E}-02$ & $5.67 \mathrm{E}-02$ & $3.31 \mathrm{E}-02$ & $1.94 \mathrm{E}-02$ \\
\hline $2.80 E+00$ & $4.91 \mathrm{E}+03$ & $4.80 \mathrm{E}+03$ & $4.69 E+03$ & $4.47 E+03$ & $4.07 E+03$ & $3.70 E+03$ & $3.37 E+03$ & $3.06 E+03$ & $2.79 E+03$ & $2.53 E+03$ & $2.31 E+03$ \\
\hline $3.25 E+00$ & $2.61 \mathrm{E}-01$ & $2.06 \mathrm{E}-01$ & $1.80 \mathrm{E}-01$ & $1.38 \mathrm{E}-01$ & $8.04 \mathrm{E}-02$ & $4.70 \mathrm{E}-02$ & $2.75 \mathrm{E}-02$ & 1. $60 \mathrm{E}-02$ & $9.37 \mathrm{E}-03$ & $5.48 \mathrm{E}-03$ & $3.20 E-03$ \\
\hline $3.75 E+00$ & $3.20 \mathrm{E}+00$ & $3.12 \mathrm{E}+00$ & $3.05 E+00$ & $2.91 \mathrm{E}+00$ & $2.65 E+00$ & $2.41 E+00$ & $2.19 E+00$ & $1.99 \bar{E}+00$ & $1.81 E+00$ & $1.65 \mathrm{E}+00$ & $1.50 \mathrm{E}+00$ \\
\hline $4.25 E+00$ & $4.46 \mathrm{E}-02$ & $4.09 \mathrm{E}-02$ & $4.00 \mathrm{E}-02$ & $3.81 E-02$ & $3.47 \mathrm{E}-02$ & $3.15 \mathrm{E}-02$ & $2.87 E-02$ & $2.61 \mathrm{E}-02$ & $2.38 \mathrm{E}-02$ & $2.16 \mathrm{E}-02$ & $1.97 \mathrm{E}-02$ \\
\hline $4.75 E+00$ & $2.53 \mathrm{E}-04$ & $4 \mathrm{E}-14$ & $2.44 \mathrm{E}-20$ & $0.00 \mathrm{E}+00$ & $0.00 E+00$ & $0.00 E+00$ & $0.00 E+00$ & $0.00 \mathrm{E}+00$ & $0.00 E+00$ & $0.00 \mathrm{E}+00$ & $0.00 \mathrm{E}+00$ \\
\hline $5.50 E+00$ & $3.89 \mathrm{E}-07$ & $1 E-14$ & $2.10 \mathrm{E}-20$ & $0.00 \mathrm{E}+00$ & $0.00 \mathrm{E}+00$ & $0.00 \mathrm{E}+00$ & $0.00 E+00$ & $0.00 \mathrm{E}+.00$ & $0.00 \mathrm{E}+00$ & $0.00 \mathrm{E}+00$ & $0.00 \mathrm{E}+00$ \\
\hline total & $7.49 \mathrm{E}+04$ & $1.63 \mathrm{E}+04$ & $9.91 \mathrm{E}+03$ & $8.70 E+03$ & $7.86 E+03$ & $7.12 E+03$ & $6.46 \mathrm{E}+03$ & $5.87 E+03$ & $5.34 E+03$ & $4.85 E+03$ & $4.41 \mathrm{E}+03$ \\
\hline $\mathrm{mev} / \mathrm{sec}$ & $7.15 E+04$ & $2.55 E+04$ & $2.05 E+04$ & $1.89 \mathrm{E}+04$ & $1.72 \mathrm{E}+04$ & $1.56 \mathrm{E}+04$ & 1. $42 \mathrm{E}+04$ & $1.29 \mathrm{E}+04$ & $1.17 E+04$ & $1.07 E+04$ & $9.70 \mathrm{E}+03$ \\
\hline
\end{tabular}


Table G22. Decay Photon Spectrum (/kg.s) After One-Day Irradiation of 1-kg Aluminum 6061-T6 (Flux Parameter Set 2).

\begin{tabular}{|c|c|c|c|c|c|c|c|c|c|c|c|}
\hline emean & & & & & & & & & & & \\
\hline (mev) & initial & $.5 \mathrm{hr}$ & $1.0 \mathrm{hr}$ & $2.0 \mathrm{hr}$ & $4.0 \mathrm{hr}$ & $6.0 \mathrm{hr}$ & $8.0 \mathrm{hr}$ & $10.0 \mathrm{hr}$ & $12.0 \mathrm{hr}$ & $14.0 \mathrm{hr}$ & $16.0 \mathrm{hr}$ \\
\hline $3.00 \mathrm{E}-01$ & 1. $29 \mathrm{E}+04$ & $1.62 \mathrm{E}+03$ & $5.94 E+02$ & $4.39 \mathrm{E}+02$ & $3.84 \mathrm{E}+02$ & $3.42 \mathrm{E}+02$ & $3.06 \mathrm{E}+02$ & $2.76 \mathrm{E}+02$ & $2.50 \mathrm{E}+02$ & $2.26 \mathrm{E}+02$ & $2.05 \mathrm{E}+02$ \\
\hline $6.50 \Sigma-01$ & $3.37 \mathrm{E}+05$ & $3.90 \mathrm{E}+04$ & $5.85 E+03$ & $1.41 \mathrm{E}+03$ & $8.49 \mathrm{E}+02$ & $5.44 \mathrm{E}+02$ & $3.62 \mathrm{E}+02$ & $2.51 \mathrm{E}+02$ & $1.83 \mathrm{E}+02$ & 1. $39 \mathrm{E}+02$ & $1.11 \mathrm{E}+02$ \\
\hline $1.12 \mathrm{E}+00$ & $8.98 E+04$ & $9.96 \mathrm{E}+03$ & $1.12 \mathrm{E}+03$ & $2.87 \mathrm{E}+01$ & $1.21 \mathrm{E}+01$ & $1.02 \mathrm{E}+01$ & $8.95 E+00$ & $8.07 \mathrm{E}+00$ & $7.43 E+00$ & $6.93 \mathrm{E}+00$ & $6.52 \mathrm{E}+00$ \\
\hline $1.58 E+00$ & $5.27 E+04$ & $2.03 E+04$ & $1.98 E+04$ & $1.89 \mathrm{E}+04$ & $1.71 \mathrm{E}+04$ & $1.56 \mathrm{E}+04$ & $1.41 \mathrm{E}+04$ & $1.29 \mathrm{E}+04$ & $1.17 E+04$ & $1.06 \mathrm{E}+04$ & $67 E+03$ \\
\hline $2.00 \mathrm{E}+00$ & $4.43 \mathrm{E}+02$ & $3.76 \mathrm{E}+02$ & 3. $28 \mathrm{E}+02$ & $2.51 \mathrm{E}+02$ & $1.47 \mathrm{E}+02$ & $8.56 E+01$ & $5.00 \mathrm{E}+01$ & $2.92 \mathrm{E}+01$ & $1.71 \mathrm{E}+01$ & $9.97 \mathrm{E}+00$ & $82 \mathrm{E}+00$ \\
\hline $2.40 \mathrm{E}+00$ & $2.49 \mathrm{E}+01$ & $1.46 \mathrm{E}+01$ & $1.26 \mathrm{E}+01$ & $9.61 \mathrm{E}+00$ & $5.61 \mathrm{~F}+00$ & $3.28 \mathrm{E}+00$ & 1. $92 \mathrm{E}+00$ & $1.12 \mathrm{E}+00$ & $6.54 \mathrm{E}-01$ & $3.82 \mathrm{E}-01$ & $2.23 E-01$ \\
\hline $2.80 \mathrm{E}+00$ & $2.33 \mathrm{E}+04$ & $2.28 \mathrm{E}+04$ & $2.22 \mathrm{E}+04$ & $2.12 \mathrm{E}+04$ & $1.93 \mathrm{E}+04$ & $1.75 E+04$ & $1.60 E+04$ & $1.45 \mathrm{E}+04$ & 1. $32 \mathrm{E}+04$ & $1.20 E+04$ & $1.09 \mathrm{E}+04$ \\
\hline $3.25 E+00$ & $2.90 \mathrm{E}+00$ & $2.38 E+00$ & $2.08 E+00$ & $1.59 \mathrm{E}+00$ & $9.30 \mathrm{E}-01$ & $5.43 \mathrm{E}-01$ & $3.17 \mathrm{E}-01$ & $1.85 \mathrm{E}-01$ & $1.08 \mathrm{E}-01$ & $6.33 \mathrm{E}-02$ & $3.70 \mathrm{~F}-02$ \\
\hline $3.75 \mathrm{E}+00$ & $1.52 \mathrm{E}+01$ & $1.48 \mathrm{E}+01$ & $1.45 \mathrm{E}+01$ & $1.38 \mathrm{E}+01$ & $1.26 \mathrm{E}+01$ & $1.14 \mathrm{E}+01$ & 1. $04 \mathrm{E}+01$ & $9.45 \mathrm{E}+00$ & $8.60 \mathrm{E}+00$ & $7.82 \mathrm{E}+00$ & $7.12 \mathrm{E}+00$ \\
\hline $4.25 \mathrm{E}+00$ & $2.18 \mathrm{E}-01$ & $1.94 \mathrm{E}-01$ & $1.90 E-01$ & $1.81 \mathrm{E}-01$ & $1.64 \mathrm{E}-01$ & $1.50 \mathrm{E}-01$ & $1.36 \mathrm{E}-01$ & $1.24 \mathrm{E}-01$ & $1.13 \mathrm{E}-01$ & 1. $02 \mathrm{E}-01$ & $9.32 \mathrm{E}-02$ \\
\hline $4.75 \mathrm{E}+00$ & $1.77 \mathrm{E}-03$ & $1.98 \mathrm{E}-13$ & $1.70 \mathrm{E}-19$ & 1. $95 \mathrm{E}-31$ & $0.00 \mathrm{E}+00$ & $0.00 E+00$ & $0.00 \mathrm{E}+00$ & $0.00 \mathrm{E}+00$ & $0.00 \mathrm{E}+00$ & $0.00 \mathrm{E}+00$ & $0.00 \mathrm{E}+00$ \\
\hline $5.50 \mathrm{E}+00$ & $2.72 \mathrm{E}-06$ & $1.54 \mathrm{E}-13$ & $1.47 E-19$ & $1.68 E-31$ & $0.00 \mathrm{E}+00$ & $0.00 \mathrm{E}+00$ & $0.00 \mathrm{E}+00$ & $0.00 \mathrm{E}+00$ & $0.00 \mathrm{E}+00$ & $0.00 \mathrm{E}+00$ & $0.00 \mathrm{E}+00$ \\
\hline total & $5.16 \mathrm{E}+05$ & $9.41 \mathrm{E}+04$ & $5.00 \mathrm{E}+04$ & $4.23 E+04$ & $3.78 E+04$ & $3.41 E+04$ & $3.08 \mathrm{E}+04$ & $2.80 \mathrm{E}+04$ & $2.54 \mathrm{E}+04$ & $2.30 \mathrm{E}+04$ & $2.09 \mathrm{E}+04$ \\
\hline $\mathrm{mev} / \mathrm{sec}$ & $4.73 E+05$ & $1.34 E+05$ & $9.95 \mathrm{E}+04$ & $9.08 \mathrm{E}+04$ & $8.20 E+04$ & $7.43 \mathrm{E}+04$ & $6.74 \mathrm{E}+04$ & $6.13 \mathrm{E}+04$ & $5.57 \mathrm{E}+04$ & $5.06 E+04$ & $4.60 \mathrm{E}+04$ \\
\hline
\end{tabular}

\section{Table G23. Decay Photon Spectrum (/kg.s) After Ten-Day Irradiation of 1-kg Aluminum 6061-T6 (Flux Parameter Set 2).}

\begin{tabular}{|c|c|c|c|c|c|c|c|c|c|c|c|}
\hline mean & & & time & after di & harge & & & & & & \\
\hline & initial & & $1.0 \mathrm{hr}$ & & $4.0 \mathrm{hr}$ & $6.0 \mathrm{hr}$ & $8.0 \mathrm{hr}$ & $10.0 \mathrm{hIF}$ & $12.0 \mathrm{hr}$ & $14.0 \mathrm{hr}$ & $16.0 \mathrm{hr}$ \\
\hline $3.00 \mathrm{E}-01$ & & $1.82 \mathrm{E}+03$ & $7.92 \mathrm{E}+02$ & $6.27 \mathrm{E}+02$ & $5.56 \mathrm{E}+02$ & $4.98 \mathrm{E}+02$ & $4.49 E+02$ & $4.06 \mathrm{E}+02$ & $3.67 \mathrm{E}+02$ & $3.33 \mathrm{E}+02$ & $3.03 \mathrm{E}+02$ \\
\hline $6.50 E-01$ & $3.37 \mathrm{E}+05$ & $3.91 E+04$ & $5.94 \mathrm{E}+03$ & 1. $50 \mathrm{E}+03$ & $9.30 \mathrm{E}+02$ & $6.19 \mathrm{E}+02$ & 4. $30 \mathrm{E}+02$ & $3.15 \mathrm{E}+02$ & $2.42 \mathrm{E}+02$ & $1.94 \mathrm{E}+02$ & 1. $62 \mathrm{E}+02$ \\
\hline$E+00$ & $8.99 \mathrm{E}+04$ & $9.98 \mathrm{E}+03$ & $1.14 \mathrm{E}+03$ & $4.53 \mathrm{E}+01$ & $2.82 \mathrm{E}+01$ & $2.58 \mathrm{E}+01$ & $2.41 \mathrm{E}+01$ & $2.28 \mathrm{E}+01$ & $2.18 \mathrm{E}+01$ & $09 \mathrm{E}+01$ & $2.01 \mathrm{E}+01$ \\
\hline$E+00$ & $6.25 E+04$ & $2.99 E+04$ & $2.92 \mathrm{E}+04$ & $2.78 \mathrm{E}+04$ & $2.52 \mathrm{~F}+04$ & 2. $29 \mathrm{E}+04$ & $2.08 \mathrm{E}+04$ & $1.89 \mathrm{E}+04$ & $1.72 E+04$ & $1.57 \mathrm{E}+04$ & $13 E+04$ \\
\hline$E+00$ & $4.44 \mathrm{E}+02$ & $3.76 \mathrm{E}+02$ & $3.29 \mathrm{E}+02$ & $2.51 \mathrm{E}+02$ & $1.47 \mathrm{E}+02$ & $8.57 \mathrm{E}+01$ & $5.01 \mathrm{E}+01$ & $2.93 \mathrm{E}+01$ & $1 \mathrm{E}+01$ & 9 & $3 \mathrm{E}+00$ \\
\hline $8+00$ & & $1.46 \mathrm{E}+01$ & $1.26 \mathrm{E}+01$ & $9.63 \mathrm{E}+00$ & $5.62 \mathrm{E}+00$ & $3.29 \mathrm{E}+00$ & $1.92 \mathrm{E}+00$ & $2 \mathrm{E}+00$ & $5 E-01$ & E-01 & $E-01$ \\
\hline $2.80 E+00$ & $3 E+04$ & $3.35 \mathrm{E}+04$ & $3.28 \mathrm{E}+04$ & $3.12 \mathrm{E}+04$ & $4 E+04$ & $E+04$ & $5 E+04$ & $4 \mathrm{E}+04$ & & $7 \mathrm{E}+04$ & $1 \mathrm{E}+04$ \\
\hline$E+00$ & $1 \mathrm{E}+00$ & $9 \mathrm{E}+00$ & $2.09 \mathrm{E}+00$ & $9 \mathrm{E}+00$ & $1 \mathrm{E}-01$ & $4 E-01$ & $3 E-01$ & $6 \mathrm{E}-01$ & 1. & $4 \mathrm{E}-02$ & $0 E-02$ \\
\hline $3.75 E+00$ & $2.24 \mathrm{E}+01$ & $2.18 \mathrm{E}+01$ & $2.13 E+01$ & $2.03 \mathrm{E}+01$ & $E+01$ & & & & & & $1.05 \mathrm{E}+01$ \\
\hline$E+00$ & $3.12 \mathrm{E}-01$ & $2.86 \mathrm{E}-01$ & $2.79 \mathrm{E}-01$ & $2.66 \mathrm{E}-01$ & $2.42 \mathrm{E}-01$ & $2.20 \mathrm{E}-01$ & $2.01 \mathrm{E}-01$ & $1.82 \mathrm{E}-01$ & $1.66 \mathrm{E}-01$ & $1 E-01$ & $7 E-01$ \\
\hline $4.75 \mathrm{E}+00$ & & 1.98 & $1.70 \mathrm{E}-19$ & $1.95 \mathrm{E}-$ & & & $\mathrm{E}+00$ & $0 E+00$ & $0.00 \mathrm{E}+00$ & $0 \mathrm{E}+00$ & $0.00 \mathrm{E}+00$ \\
\hline $5.50 \mathrm{E}+00$ & $2.72 \mathrm{E}-06$ & $\mathrm{E}-13$ & $1.47 \mathrm{E}-19$ & $1.68 \mathrm{E}-31$ & $E+00$ & $0 \mathrm{E}+00$ & $0.00 \mathrm{E}+00$ & $0.00 E+00$ & $0.00 \mathrm{E}+00$ & $0.00 \mathrm{E}+00$ & $0.00 \mathrm{E}+00$ \\
\hline total & $5.37 E+05$ & $1.15 \mathrm{E}+05$ & $7.01 \mathrm{E}+04$ & $6.15 E+04$ & $5.53 E+04$ & $5.00 \mathrm{E}+04$ & $4.53 \mathrm{E}+04$ & $4.11 \mathrm{E}+04$ & $3.74 E+04$ & $3.39 \mathrm{E}+04$ & $3.09 E+04$ \\
\hline $\mathrm{mev} / \mathrm{sec}$ & $5.20 \mathrm{E}+05$ & $1.79 E+05$ & $1.44 \mathrm{E}+05$ & $1.33 E+05$ & $1.20 \mathrm{E}+05$ & $1.09 \mathrm{E}+05$ & $9.93 E+04$ & $9.02 \mathrm{E}+04$ & $8.20 \mathrm{E}+04$ & $7.46 \mathrm{E}+04$ & $6.78 E+04$ \\
\hline
\end{tabular}


Table G24. Decay Photon Spectrum (/kg.s) After One-Day Irradiation of 1-kg Steel Plate (Flux Parameter Set 1).

\begin{tabular}{|c|c|c|c|c|c|c|c|c|c|c|c|}
\hline $\begin{array}{l}\text { emean } \\
\text { (mev) }\end{array}$ & & & $\begin{array}{l}\text { time } \\
1.0 \mathrm{hr}\end{array}$ & $\begin{array}{c}\text { after dis } \\
2.0 \mathrm{hr}\end{array}$ & $\begin{array}{l}\text { harge } \\
4.0 \mathrm{hr}\end{array}$ & & & & & & \\
\hline $3.00 \mathrm{E}-01$ & $3.44 \mathrm{E}+02$ & $1.998 \mathrm{Er}+02$ & $1.73 E+02$ & $1.33 \mathrm{E}+02$ & $7.85 E+01$ & $4.67 \mathrm{E}+01$ & $2.82 \mathrm{E}+01$ & $1.73 \mathrm{E}+01$ & $1.10 \mathrm{E}+01$ & $7.30 \mathrm{E}+00$ & $5.14 \mathrm{E}+00$ \\
\hline $6.50 \mathrm{E}-01$ & $6.98 \mathrm{E}+03$ & $6.09 \mathrm{E}+03$ & $5.37 E+03$ & $4.19 \mathrm{E}+03$ & $2.60 \mathrm{E}+03$ & $1.67 \mathrm{E}+03$ & $1.12 \mathrm{E}+03$ & $8.07 E+02$ & $6.21 \mathrm{E}+02$ & $5.13 \mathrm{E}+02$ & $4.49 E+02$ \\
\hline 1. $12 \mathrm{E}+00$ & $8.27 \mathrm{E}+01$ & $2.54 \mathrm{E}+01$ & $2.15 E+01$ & $1.65 \mathrm{E}+01$ & $9.66 \mathrm{E}+00$ & $5.69 \mathrm{E}+00$ & $3.38 \mathrm{E}+00$ & $2.03 E+00$ & $1.24 \mathrm{E}+00$ & $7.85 \mathrm{E}-01$ & $5.17 \mathrm{E}-01$ \\
\hline $1.58 \mathrm{E}+00$ & $2.39 \mathrm{E}+03$ & $6.93 \mathrm{E}+02$ & $6.03 \mathrm{E}+02$ & $4.61 \mathrm{E}+02$ & $2.70 \mathrm{E}+02$ & $1.58 \mathrm{E}+02$ & $9.29 \mathrm{E}+01$ & $5.48 \mathrm{E}+01$ & $3.25 E+01$ & $1.95 \mathrm{E}+01$ & $.19 \mathrm{E}+01$ \\
\hline $2.00 \mathrm{E}+00$ & $1.37 E+03$ & $1.20 \mathrm{E}+03$ & $1.05 E+03$ & $8.02 \mathrm{E}+02$ & $4.69 E+02$ & $2.74 E+02$ & $1.60 \mathrm{E}+02$ & $9.34 \mathrm{E}+01$ & $5.46 \mathrm{E}+01$ & $3.19 \mathrm{E}+01$ & $1.86 \mathrm{E}+01$ \\
\hline $2.40 \mathrm{E}+00$ & $5.40 \mathrm{E}+01$ & $4.60 \mathrm{E}+01$ & $4.02 E+01$ & $3.07 \mathrm{E}+01$ & $1.80 \mathrm{E}+01$ & $1.05 \mathrm{E}+01$ & $6.13 \mathrm{E}+00$ & $3.58 \mathrm{E}+00$ & $2.09 E+00$ & $1.22 \mathrm{E}+00$ & $7.13 E-01$ \\
\hline $2.80 \mathrm{E}+00$ & $4.76 \mathrm{E}+01$ & $4.16 \mathrm{E}+01$ & $3.64 E+01$ & $2.78 E+01$ & $1.62 \mathrm{E}+01$ & $9.49 E+00$ & $5.54 E+00$ & $3.24 E+00$ & $1.89 E+00$ & $1.10 \mathrm{E}+00$ & $6.45 \mathrm{E}-01$ \\
\hline $3.25 E+00$ & $8.76 \mathrm{E}+00$ & $7.62 \mathrm{E}+00$ & $6.66 \mathrm{E}+00$ & $5.09 \mathrm{E}+00$ & $2.97 \mathrm{E}+00$ & $1.74 \mathrm{E}+00$ & $1 E+00$ & $5.92 \mathrm{E} 01$ & $3.46 \mathrm{E}-01$ & $2.02 \mathrm{E}-01$ & $1.18 \mathrm{E}-01$ \\
\hline $3.75 \mathrm{E}+00$ & $5.22 \mathrm{E}-03$ & $2.42 \mathrm{E}-09$ & $2.31 E-15$ & $4.91 \mathrm{E}-22$ & $4.47 \mathrm{E}-22$ & $4.07 \mathrm{E}-22$ & $0 E-22$ & $3.37 \mathrm{E}-22$ & $3.06 \mathrm{E}-22$ & $2.79 \mathrm{E}-22$ & $2.53 \mathrm{E}-22$ \\
\hline $25 E+00$ & $9.58 \mathrm{E}-03$ & $9.13 \mathrm{E}-09$ & $8.71 E-15$ & $6.44 \mathrm{E}-24$ & $5.86 \mathrm{E}-24$ & $5.33 \mathrm{E}-24$ & $4.85 E-24$ & $4.41 \mathrm{E}-24$ & $4.01 \mathrm{E}-24$ & $3.65 \mathrm{E}-24$ & $3.32 \mathrm{E}-24$ \\
\hline $4.75 E+00$ & $3.68 \mathrm{E}-04$ & $3.55 \mathrm{E}-13$ & $9.24 \mathrm{E}-20$ & $8.55 E-30$ & $8.93 \mathrm{E}-31$ & $3 E-31$ & $E+00$ & $0 E+00$ & $0 \mathrm{E}+00$ & $0.00 \mathrm{E}+00$ & $0.00 \mathrm{E}+00$ \\
\hline $5.50 \mathrm{E}+00$ & $1.21 \mathrm{E}-06$ & $8.25 \mathrm{E}-14$ & $7.86 \mathrm{E}-20$ & $7.39 \mathrm{E}-30$ & $7.72 \mathrm{E}-31$ & $1.10 \mathrm{E}-31$ & $0.00 \mathrm{E}+00$ & $0.00 \mathrm{E}+00$ & $0.00 \mathrm{E}+00$ & $0.00 \mathrm{E}+00$ & $6.60 \mathrm{E}+00$ \\
\hline total & $1.13 E+04$ & $8.31 \mathrm{E}+03$ & $7.30 \mathrm{E}+03$ & $5.67 \mathrm{E}+03$ & $3.46 \mathrm{E}+03$ & $2.17 E+03$ & $1.42 \mathrm{E}+03$ & $9.82 \mathrm{E}+02$ & $7.25 \mathrm{E}+02$ & $5.75 E+02$ & $4.87 E+02$ \\
\hline $\mathrm{mev} / \mathrm{sec}$ & $1.15 \mathrm{E}+04$ & $7.79 \mathrm{E}+03$ & $6.84 \mathrm{E}+03$ & $5.28 \mathrm{E}+03$ & $3.18 \mathrm{E}+03$ & $1.96 \mathrm{E}+03$ & 1. $24 \mathrm{E}+03$ & $8.25 \mathrm{E}+02$ & $5.80 E+02$ & $4.37 \mathrm{E}+02$ & $3.54 \mathrm{E}+02$ \\
\hline
\end{tabular}

Table G25. Decay Photon Spectrum (/kg.s) After Ten-Day Irradiation of 1-kg Steel Plate (Flux Parameter Set 1).

\begin{tabular}{|c|c|c|c|c|c|c|c|c|c|c|c|}
\hline emean & & & time & after dis & harge & & & & & & \\
\hline $\begin{array}{l}\text { (mev) } \\
3.00 \mathrm{E}-01\end{array}$ & initial & $2.5 \mathrm{hr}$ & $1.0 \mathrm{hr}$ & $2.0 \mathrm{hr}$ & $4.0 \mathrm{hr}$ & $6.0 \mathrm{hr}$ & $\begin{array}{c}8.0 \mathrm{hr} \\
455 \mathrm{E}+01\end{array}$ & $10.0 \mathrm{hr}$ & 12.0 hr & $14.0 \mathrm{hr}$ & $16.0 \mathrm{hr}$ \\
\hline $6.50 \mathrm{E}-01$ & $1.01 \mathrm{E}+04$ & $9.22 \mathrm{E}+03$ & $8.49 \mathrm{E}+03$ & $7.31 \mathrm{E}+03$ & $5.71 \mathrm{E}+03$ & $4.78 \mathrm{E}+03$ & $4.23 E+03$ & $3.91 \mathrm{E}+03$ & $3.72 \mathrm{E}+03$ & $31 \mathrm{E}+03$ & 3. $54 \mathrm{E}+03$ \\
\hline 1. $12 \mathrm{E}+00$ & $8.40 \mathrm{E}+01$ & $2.67 \mathrm{E}+01$ & $2.28 \mathrm{E}+01$ & $1.77 \mathrm{E}+01$ & $1.09 \mathrm{E}+01$ & $6.96 \mathrm{E}+00$ & $4.65 \mathrm{E}+00$ & $3.29 \mathrm{E}+00$ & $2.51 \mathrm{E}+00$ & $2.05 \mathrm{E}+00$ & $1.78 \mathrm{E}+00$ \\
\hline 1. $58 \mathrm{E}+00$ & $2.41 \mathrm{E}+03$ & $7.05 \mathrm{E}+02$ & $6.14 \mathrm{E}+02$ & $4.72 \mathrm{E}+02$ & $2.81 \mathrm{E}+02$ & $1.69 \mathrm{E}+02$ & $1.04 \mathrm{E}+02$ & $6.57 \mathrm{E}+01$ & 4. $34 \mathrm{E}+01$ & $3.04 \mathrm{E}+01$ & $2.28 \mathrm{E}+01$ \\
\hline $2.00 \mathrm{E}+00$ & $1.38 \mathrm{E}+03$ & $1.20 \mathrm{E}+03$ & $1.05 E+03$ & $8.03 E+02$ & $4.69 E+02$ & $2.74 E+02$ & $1.60 \mathrm{E}+02$ & $9.35 E+01$ & $5.46 \mathrm{E}+01$ & $3.19 \mathrm{E}+01$ & $1.86 \mathrm{E}+01$ \\
\hline $2.40 \mathrm{E}+00$ & $5.41 \mathrm{E}+01$ & $4.61 \mathrm{E}+01$ & $4.03 \mathrm{E}+01$ & $3.08 \mathrm{E}+01$ & $1.80 \mathrm{E}+01$ & $1.05 \mathrm{E}+01$ & $6.13 \mathrm{E}+00$ & $3.58 \mathrm{E}+00$ & $2.09 \mathrm{E}+00$ & $1.22 \mathrm{E}+00$ & $7.14 \mathrm{E}-01$ \\
\hline $2.80 \mathrm{E}+00$ & $4.77 \mathrm{E}+01$ & $4.17 \mathrm{E}+01$ & $3.64 E+01$ & $2.78 \mathrm{E}+01$ & $1.63 \mathrm{E}+01$ & $9.50 \mathrm{E}+00$ & $5.55 E+00$ & $3.24 \mathrm{E}+00$ & $1.89 \mathrm{E}+00$ & $1.11 \mathrm{E}+00$ & $6.46 \mathrm{E}-01$ \\
\hline $3.25 \mathrm{E}+00$ & $8.77 \mathrm{E}+00$ & $7.63 \mathrm{E}+00$ & $6.67 \mathrm{E}+00$ & $5.10 \mathrm{E}+00$ & $2.98 \mathrm{E}+00$ & $1.74 \mathrm{E}+00$ & $1.02 \mathrm{E}+00$ & $3 E-01$ & $3.47 \mathrm{E}-01$ & $2.02 \mathrm{E}-01$ & 1. $18 \mathrm{E}-01$ \\
\hline $3.75 E+00$ & $5.22 \mathrm{E}-03$ & $2.42 \mathrm{E}-09$ & $2.31 \mathrm{E}-15$ & 1. $30 \mathrm{E}-20$ & $1.18 \mathrm{E}-20$ & $1.07 \mathrm{E}-20$ & $9.78 \mathrm{E}-21$ & $8.89 \mathrm{E}-21$ & $8.09 \mathrm{E}-21$ & $7.36 \mathrm{E}-21$ & $6.70 \mathrm{E}-21$ \\
\hline $4.25 E+00$ & $9.58 \mathrm{E}-03$ & $9.13 \mathrm{E}-09$ & $8.71 \mathrm{E}-15$ & $1.70 \mathrm{E}-22$ & $1.55 \mathrm{E}-22$ & $1.41 \mathrm{E}-22$ & 1.28 & $1.17 \mathrm{E}-22$ & $1.06 \mathrm{E}-22$ & -23 & $8.77 \mathrm{E}-23$ \\
\hline $4.75 E+00$ & $3.68 E-04$ & $3.55 \mathrm{E}-13$ & $9.24 \mathrm{E}-20$ & $9.66 \mathrm{E}-29$ & $1.03 \mathrm{E}-29$ & $1.15 \mathrm{E}-30$ & $1.28 \mathrm{E}-31$ & $0.00 \mathrm{E}+00$ & $0.00 \mathrm{E}+00$ & $0.00 \mathrm{E}+00$ & $0.00 \mathrm{E}+00$ \\
\hline $5.50 \mathrm{E}+00$ & $1.21 \mathrm{E}-06$ & $8.25 \mathrm{E}-14$ & $7.86 \mathrm{E}-20$ & $8.34 \mathrm{E}-29$ & $8.93 \mathrm{E}-30$ & $9.92 \mathrm{E}-31$ & $1.10 \mathrm{E}-31$ & $0.00 \mathrm{E}+00$ & $0.00 \mathrm{E}+00$ & $0.00 \mathrm{E}+00$ & $0.00 \mathrm{E}+00$ \\
\hline total & $1.44 \mathrm{E}+04$ & $1.15 \mathrm{E}+04$ & $1.05 \mathrm{E}+04$ & $8.82 \mathrm{E}+03$ & $6.61 \mathrm{E}+03$ & $5.31 \mathrm{E}+03$ & $4.56 \mathrm{E}+03$ & $4.11 \mathrm{E}+03$ & $3.85 E+03$ & $3.70 \mathrm{E}+03$ & $3.61 \mathrm{E}+0$ \\
\hline $\mathrm{mev} / \mathrm{sec}$ & $1.36 \mathrm{E}+04$ & $9.85 \mathrm{E}+03$ & $8.89 \mathrm{E}+03$ & $7.34 \mathrm{E}+03$ & $5.23 \mathrm{E}+03$ & $4.00 \mathrm{E}+03$ & $3.29 \mathrm{E}+03$ & $2.87 E+03$ & $2.62 \mathrm{E}+03$ & $2.47 E+03$ & $2.39 E+0$ \\
\hline
\end{tabular}


Table G26. Decay Photon Spectrum (/kg.s) After One-Day Irradiation of 1-kg Steel Plate (Flux Parameter Set 2).

\begin{tabular}{|c|c|c|c|c|c|c|c|c|c|c|c|}
\hline $\begin{array}{l}\text { emean } \\
\text { (mev) }\end{array}$ & & & time & after dis & harge & & & & & & \\
\hline $3.00 \mathrm{E}-01$ & $2.77 \mathrm{E}+03$ & $1.71 \mathrm{E}+03$ & $1.49 \mathrm{E}+03$ & $1.14 \mathrm{E}+03$ & $\begin{array}{l}4.0 \mathrm{hr} \\
6.75 \mathrm{E}+02\end{array}$ & $4.01 \mathrm{E}+02$ & $\begin{array}{c}8.0 h x \\
2.41 E+02\end{array}$ & $\begin{array}{c}10.0 \mathrm{hrr} \\
1.48 \mathrm{E}+02\end{array}$ & $\begin{array}{c}12.0 \mathrm{hr} \\
9.29 \mathrm{E}+01\end{array}$ & $14.0 \mathrm{hr}$ & $\begin{array}{r}16.0 \mathrm{hr} \\
4.24 \mathrm{E}+01\end{array}$ \\
\hline $6.50 E-01$ & $5.96 E+04$ & $5.21 E+04$ & $4.58 E+04$ & $3.56 \mathrm{E}+04$ & $2.19 E+04$ & $1.38 E+04$ & $9.12 \mathrm{E}+03$ & $6.38 \mathrm{E}+03$ & $4.77 \mathrm{E}+03$ & $3.83 \mathrm{E}+03$ & $\begin{array}{l}4.24 \mathrm{E}+01 \\
3.29 \mathrm{E}+03\end{array}$ \\
\hline $1.12 E+00$ & $7 E+02$ & $2.20 \mathrm{E}+02$ & $1.88 \mathrm{E}+02$ & $1.43 E+02$ & $8.40 \mathrm{E}+01$ & $4.94 \mathrm{E}+01$ & $2.92 \mathrm{E}+01$ & $1.75 \mathrm{E}+01$ & $1.06 \mathrm{E}+01$ & $6.60 \mathrm{E}+00$ & $4.27 E+00$ \\
\hline $1.58 E+00$ & $1.80 E+04$ & $5.99 \mathrm{E}+03$ & $5.21 \mathrm{E}+03$ & $3.98 \mathrm{~F}+03$ & $2.33 E+03$ & $1.36 \mathrm{E}+03$ & $8.01 E+02$ & $4.71 E+02$ & $2.79 E+02$ & $1.67 \mathrm{E}+02$ & $1.01 \mathrm{E}+02$ \\
\hline$E+00$ & $9 E+04$ & $1.04 \mathrm{E}+04$ & $9.07 \mathrm{E}+03$ & $6.93 E+03$ & $4.05 E+03$ & $2.36 \mathrm{E}+03$ & $1.38 \mathrm{E}+03$ & $8.07 \mathrm{E}+02$ & $E+02$ & $5 E+02$ & $1 E+02$ \\
\hline$E+00$ & $4.64 \mathrm{E}+02$ & $3.98 \mathrm{E}+02$ & $3.47 E+02$ & $2.66 \mathrm{~F}$ & $5 E+02$ & & & $9 \mathrm{E}+01$ & $1.81 \mathrm{E}+01$ & 1. $0.5 \mathrm{E}+01$ & $6 E+00$ \\
\hline$E+00$ & $4.11 \mathrm{E}+02$ & $3.60 \mathrm{E}+02$ & $3.14 \mathrm{E}+02$ & $2.40 \mathrm{E}+02$ & $1.40 \mathrm{E}+02$ & $\mathrm{E}+01$ & $8+01$ & 2. & $1.63 \mathrm{E}+01$ & $9.54 \mathrm{E}+00$ & $5.57 \mathrm{E}+00$ \\
\hline 3.2 & 7.5 & 6.58 & $5.75 E+01$ & $4.40 \mathrm{E}+01$ & $2.57 \mathrm{E}+01$ & $E+01$ & 8. & 5.12 & & +00 & $2 \mathrm{E}+00$ \\
\hline $3.75 \mathrm{E}+00$ & $3.65 \mathrm{E}-02$ & $1.69 \mathrm{E}-08$ & $1.61 \mathrm{E}-14$ & $2.40 \mathrm{E}-20$ & $2.18 \mathrm{E}-20$ & $1.99 \mathrm{E}-20$ & $1.81 \mathrm{E}-20$ & $1.64 \mathrm{E}-20$ & 1.5 & & $1 \mathrm{E}-20$ \\
\hline$E+00$ & $6.70 E-02$ & 6.38 & $6.09 \mathrm{E}-14$ & $3.15 \mathrm{E}-22$ & $2.86 \mathrm{E}-22$ & $2.60 \mathrm{E}-22$ & $2.37 \mathrm{E}-22$ & $E-22$ & 1.9 & $E-22$ & $1.62 \mathrm{E}-22$ \\
\hline $4.75 E+00$ & $2.57 \mathrm{E}-03$ & $2.48 \mathrm{E}-12$ & $6.46 \mathrm{E}-19$ & $2.21 \mathrm{E}-28$ & $2.36 E-29$ & & & & & $0.00 \mathrm{E}+00$ & $0.00 \mathrm{E}+00$ \\
\hline & $8.43 E-06$ & $5.76 \mathrm{E}-13$ & $5.50 E-19$ & $1.91 \mathrm{E}-28$ & $2.04 \mathrm{E}-29$ & $2.20 E-30$ & 2.20 & $0.00 \mathrm{E}+00$ & 0.0 & $0.00 \mathrm{E}+00$ & $0.00 \mathrm{E}+00$ \\
\hline total & $9.39 \mathrm{E}+04$ & $7.12 \mathrm{E}+04$ & $6.25 \mathrm{E}+04$ & $4.84 \mathrm{E}+04$ & $2.93 \mathrm{E}+04$ & $1.82 \mathrm{E}+04$ & $1.17 \mathrm{E}+04$ & $7.88 E+03$ & $5.66 \mathrm{E}+03$ & $4.37 \mathrm{E}+03$ & $3.61 \mathrm{E}+03$ \\
\hline $\mathrm{mev} / \mathrm{sec}$ & $9.50 \mathrm{E}+04$ & $6.69 \mathrm{E}+04$ & $5.87 E+04$ & $4.52 \mathrm{E}+04$ & $2.71 \mathrm{E}+04$ & $1.65 \mathrm{E}+04$ & $1.03 \mathrm{E}+04$ & $6.73 \mathrm{E}+03$ & $4.62 \mathrm{E}+03$ & $3.39 \mathrm{E}+03$ & $2.67 \mathrm{E}+03$ \\
\hline
\end{tabular}

Table G27. Decay Photon Spectrum (/kg.s) After Ten-Day Irradiation of 1-kg Steel Plate (Flux Parameter Set 2).

\begin{tabular}{|c|c|c|c|c|c|c|c|c|c|c|c|}
\hline $\begin{array}{c}\text { emean } \\
\text { (mev) }\end{array}$ & & & time & after di & harge & & & & & & \\
\hline $\begin{array}{l}(\mathrm{mev}) \\
3.00 \mathrm{E}-01\end{array}$ & $\begin{array}{l}\text { initial } \\
2.91 E+03\end{array}$ & $\begin{array}{c}.5 \mathrm{hr} \\
.84 \mathrm{E}+03\end{array}$ & $\begin{array}{c}1.0 \mathrm{hr} \\
1.63 \mathrm{E}+03\end{array}$ & $\begin{array}{c}2.0 \mathrm{hr} \\
1.28 \mathrm{E}+03\end{array}$ & $\begin{array}{c}4.0 \mathrm{hr} \\
8.09 \mathrm{E}+02\end{array}$ & $6.0 \mathrm{hr}$ & $8.0 \mathrm{hr}$ & 10.0 & $12.0 \mathrm{hr}$ & $14.0 \mathrm{hr}$ & $16.0 \mathrm{ln}$ \\
\hline$O E-01$ & $8.15 E+04$ & $739 E+04$ & $77 E+04$ & $\begin{array}{l}1.28 \mathrm{E}+03 \\
5.74 \mathrm{~F}+04\end{array}$ & $\begin{array}{l}8.09 \mathrm{E}+02 \\
4.36 \mathrm{~F}+04\end{array}$ & $5.35 \mathrm{E}+02$ & $3.74 \mathrm{E}+02$ & $2.81 \mathrm{E}+02$ & $2.26 \mathrm{E}+02$ & 1. $94 \mathrm{E}+02$ & $1.75 \mathrm{E}+02$ \\
\hline $\mathrm{E}+00$ & $6.36 \mathrm{E}+02$ & $2.29 \mathrm{E}+02$ & $197 \mathrm{E}+02$ & $1.52 \mathrm{~F}+02$ & $6 E+04$ & $3.55 \mathrm{E}+04$ & $3.08 \mathrm{E}+04$ & $2.81 E+04$ & $4 \mathrm{E}+04$ & $5 \mathrm{E}+04$ & $2.49 \mathrm{E}+04$ \\
\hline & & & & $\begin{array}{l}1.3 \\
4.0\end{array}$ & $9.30 \mathrm{E}+01$ & $5.83 \mathrm{E}+01$ & $3.81 \mathrm{E}+01$ & $2.63 \mathrm{E}+01$ & $1.94 \mathrm{E}+01$ & $1.54 \mathrm{E}+01$ & $1.31 \mathrm{E}+01$ \\
\hline & & & & & 2.4 & & & 2 & $E+02$ & $2.43 \mathrm{E}+02$ & $1.77 \Xi+02$ \\
\hline & & & & & $4.06 \mathrm{E}+03$ & $2.37 \mathrm{E}+03$ & $1.38 \mathrm{E}+03$ & $8.08 \mathrm{E}+02$ & $4.72 \mathrm{E}+02$ & $2.76 \mathrm{E}+02$ & $1.61 \mathrm{E}$ \\
\hline ton & $4.03 E+0 L$ & 3.5 & 3.48 & $0 \mathrm{E}+\mathrm{U} 2$ & 1.5 & 9. & $E+01$ & $3.10 \mathrm{E}+01$ & $1 E+01$ & $1.06 \mathrm{E}+01$ & $6.17 \mathrm{E}+00$ \\
\hline+00 & 4.12E+02 & $3.60 E+02$ & $3.15 \mathrm{E}+02$ & $2.41 \mathrm{E}+02$ & $1.41 \mathrm{E}+02$ & $8.21 \mathrm{E}+01$ & $4.80 \mathrm{E}+01$ & $2.80 \mathrm{E}+01$ & $1.64 \mathrm{E}+01$ & 9.5 & \\
\hline & & & & & $E+01$ & $E+01$ & $8.78 \mathrm{E}+00$ & $5.13 E+00$ & $3.00 \mathrm{E}+00$ & 1.7 & $1.02 \mathrm{E}+00$ \\
\hline $3.75 \mathrm{E}+00$ & $3.65 E-02$ & $E-08$ & $1.61 \mathrm{E}-14$ & $6.34 \mathrm{E}-19$ & $5.77 E-19$ & $5.25 \mathrm{E}-19$ & $4.77 \mathrm{E}-19$ & $4.34 E-19$ & -19 & & $7 E-19$ \\
\hline $4.25 \mathrm{E}+00$ & $6.70 \mathrm{E}-02$ & ;-08 & $E-14$ & $E-21$ & $6 \mathrm{E}-21$ & $6.88 \mathrm{E}-21$ & $6.26 \mathrm{E}-21$ & $9 E-21$ & $E-21$ & -21 & $4.29 E-21$ \\
\hline $4.75 \mathrm{E}+00$ & $2.57 \mathrm{E}-03$ & $8 E-12$ & $6.46 \mathrm{E}-19$ & $2.49 \mathrm{E}-27$ & $2.66 \mathrm{E}-28$ & $2.86 E-29$ & 3.061 & 3.8 & $0.00 \mathrm{E}+00$ & $0.00 \mathrm{E}+00$ & $0.00 \mathrm{E}+00$ \\
\hline & $8.43 E-06$ & $6 E-13$ & $5.50 \mathrm{E}-19$ & $2.15 \mathrm{E}-27$ & $2.30 \mathrm{E}-28$ & $2.47 E-29$ & $2.65 E-30$ & $3.31 \mathrm{E}-31$ & $0.00 \mathrm{E}+00$ & $0.00 E+00$ & $0.00 \mathrm{E}+00$ \\
\hline total & $1.16 \mathrm{E}+05$ & $.33 E+04$ & $8.46 \mathrm{E}+04$ & $7.04 \mathrm{E}+04$ & $5.13 \mathrm{E}+04$ & $4.01 \mathrm{E}+04$ & $3.36 \mathrm{E}+04$ & $2.98 E+04$ & $2.75 \mathrm{E}+04$ & $2.62 \mathrm{E}+04$ & $2.55 \mathrm{E}+04$ \\
\hline $\mathrm{mev} / \mathrm{sec}$ & $1.09 \mathrm{E}+05$ & $8.14 \mathrm{E}+04$ & $7.31 \mathrm{E}+04$ & $5.96 \mathrm{E}+04$ & $4.15 \mathrm{E}+04$ & $3.08 \mathrm{E}+04$ & $2.46 \mathrm{E}+04$ & $2.10 E+04$ & $1.89 \mathrm{E}+04$ & $1.76 E+04$ & $1.69 E+04$ \\
\hline
\end{tabular}


Table G28. Decay Photon Spectrum (/kg.s) After One-Day Irradiation of 1-kg Havar Foil (Flux Parameter Set 1).

\begin{tabular}{|c|c|c|c|c|c|c|c|c|c|c|c|}
\hline emean & & & time & after di & charge & & & & & & \\
\hline (mev) & initial & & $1.0 \mathrm{hr}$ & $2.0 \mathrm{hr}$ & $4.0 \mathrm{hr}$ & $6.0 \mathrm{hr}$ & $8.0 \mathrm{hr}$ & $10.0 \mathrm{hr}$ & $12.0 \mathrm{hr}$ & $14.0 \mathrm{hr}$ & $16.0 \mathrm{hr}$ \\
\hline $3.00 \mathrm{E}-01$ & $1.85 \mathrm{E}+02$ & $9.21 \mathrm{E}+01$ & $7.84 \mathrm{E}+01$ & $5.98 \mathrm{E}+01$ & 3. $68 \mathrm{E}+01$ & $2.34 \mathrm{E}+01$ & $1.55 \mathrm{E}+01$ & $1.08 E+01$ & $7.99 \mathrm{E}+00$ & $6.30 \mathrm{E}+00$ & $5.25 E+00$ \\
\hline $6.50 \mathrm{E}-01$ & $52 \mathrm{E}+03$ & $3.11 \mathrm{E}+03$ & $2.80 \mathrm{E}+03$ & $2.29 \mathrm{E}+03$ & $1.60 \mathrm{E}+03$ & $1.19 \mathrm{E}+03$ & $9.49 \mathrm{E}+02$ & $8.00 E+02$ & $7.08 \mathrm{E}+02$ & $6.49 \mathrm{E}+02$ & $6.09 E+02$ \\
\hline $1.12 \mathrm{E}+00$ & $42 \mathrm{E}+02$ & $6.23 E+01$ & $4.09 \mathrm{E}+01$ & $54 \mathrm{E}+01$ & $3.23 E+01$ & $3.05 E+01$ & $2.94 \mathrm{E}+01$ & $2.88 \mathrm{E}+01$ & $2.85 \mathrm{E}+01$ & $2.82 \mathrm{E}+01$ & $81 E+01$ \\
\hline 1. $58 \mathrm{E}+00$ & $1.39 \mathrm{E}+03$ & $2.97 \mathrm{E}+02$ & $2.56 \mathrm{E}+02$ & $1.96 \mathrm{E}+02$ & $1.15 \mathrm{E}+02$ & $6.77 \mathrm{E}+01$ & $4.01 \mathrm{E}+01$ & $2.40 E+01$ & $1.46 \mathrm{E}+01$ & $9.08 \mathrm{E}+00$ & $5.86 \mathrm{E}+00$ \\
\hline $2.00 \mathrm{E}+00$ & $5.81 \mathrm{E}+02$ & $5.07 E+02$ & $4.43 E+02$ & $3.38 E+02$ & $1.98 \mathrm{E}+02$ & $1.15 E+02$ & $.75 E+01$ & $3.94 \mathrm{E}+01$ & $2.30 \mathrm{E}+01$ & $1.34 \mathrm{E}+01$ & $7.86 \mathrm{E}+00$ \\
\hline $2.40 \mathrm{E}+00$ & $2.28 \mathrm{E}+01$ & $1.94 \mathrm{E}+01$ & $1.70 \mathrm{E}+01$ & $1.30 \mathrm{E}+01$ & $7.57 \mathrm{E}+00$ & $4.42 \mathrm{E}+00$ & $2.58 \mathrm{E}+00$ & $.51 \mathrm{E}+00$ & $8.82 \mathrm{E}-01$ & $5.15 \mathrm{E}-01$ & $3.01 \mathrm{E}-01$ \\
\hline $2.80 \mathrm{E}+00$ & $2.01 \mathrm{E}+01$ & $1.76 \mathrm{E}+01$ & $1.53 \mathrm{E}+01$ & $1.17 \mathrm{E}+01$ & $6.85 \mathrm{E}+00$ & $4.00 \mathrm{E}+00$ & $2.34 \mathrm{E}+00$ & $1.37 \mathrm{E}+00$ & $7.98 \mathrm{E}-01$ & $4.66 \mathrm{E}-01$ & $2.72 \mathrm{E}-01$ \\
\hline $3.25 \mathrm{E}+00$ & $3.73 E+00$ & $3.21 \mathrm{E}+00$ & $2.81 E+00$ & $2.15 E+00$ & $1.25 \mathrm{E}+00$ & 7. $33 \mathrm{E}-01$ & $4.28 \mathrm{E}-01$ & $2.50 E-01$ & $1.46 \mathrm{E}-01$ & $8.53 \mathrm{E}-02$ & $4.98 \mathrm{E}-02$ \\
\hline $3.75 E+00$ & $2.84 E-03$ & $2.56 \mathrm{E}-09$ & $2.44 \mathrm{E}-15$ & $2.03 \mathrm{E}-23$ & $1.25 \mathrm{E}-23$ & $E-24$ & $E-24$ & $4 E-24$ & & -24 & $18 E-24$ \\
\hline $4.25 E+00$ & $1.01 E-02$ & $9.67 \mathrm{E}-09$ & $9.22 \mathrm{E} \quad 15$ & $3.33 E-25$ & $3.89 E-27$ & $4.43 \mathrm{E}-29$ & $0.00 E+00$ & $0.00 \mathrm{E}+00$ & $0.00 \mathrm{E}+00$ & $0.00 \mathrm{E}+00$ & $0.00 \mathrm{E}+00$ \\
\hline $4.75 E+00$ & $1.53 E-05$ & $1.78 \mathrm{E}-13$ & $9.68 \mathrm{E}-20$ & $2.97 \mathrm{E}-27$ & $3.47 \mathrm{E}-29$ & $3.95 \mathrm{E}-31$ & $0.00 \mathrm{E}+00$ & $0.00 \mathrm{E}+00$ & $0.00 \mathrm{E}+00$ & $0.00 \mathrm{E}+00$ & $0.00 \mathrm{E}+00$ \\
\hline $5.50 \mathrm{E}+00$ & $9.16 \mathrm{E}-08$ & $8.73 \mathrm{E}-14$ & $8.32 E-20$ & $2.56 \mathrm{E}-27$ & $3.00 \mathrm{E}-29$ & $3.41 \mathrm{E}-31$ & $0.00 \mathrm{E}+00$ & $0.00 \mathrm{E}+00$ & $0.00 \mathrm{E}+00$ & $0.00 E+00$ & $0.00 \mathrm{~F}+00$ \\
\hline total & $5.96 \mathrm{E}+03$ & $4.11 E+03$ & $3.65 \mathrm{E}+03$ & $2.95 \mathrm{E}+03$ & $2.00 \mathrm{E}+03$ & $1.44 \mathrm{E}+03$ & $1.11 E+03$ & $9.06 \mathrm{E}+02$ & $7.84 \mathrm{E}+02$ & $7.07 \mathrm{E}+02$ & $6.57 E+02$ \\
\hline $\mathrm{mev} / \mathrm{sec}$ & $6.09 \mathrm{E}+03$ & $3.71 \mathrm{E}+03$ & $3.27 \mathrm{E}+03$ & $2.60 \mathrm{E}+03$ & $1.71 \mathrm{E}+03$ & $1.18 \mathrm{E}+03$ & $8.67 \mathrm{E}+02$ & $6.80 E+02$ & $5.68 \mathrm{E}+02$ & $4.99 \mathrm{E}+02$ & $4.56 \mathrm{E}+02$ \\
\hline
\end{tabular}

Table G29. Decay Photon Spectrum (/kg.s) After Ten-Day Irradiation of 1-kg Havar Foil (Flux Parameter Set 1).

\begin{tabular}{|c|c|c|c|c|c|c|c|c|c|c|c|}
\hline $\begin{array}{l}\text { emean } \\
\text { (mev) }\end{array}$ & initial & $.5 \mathrm{hr}$ & $1.0 \mathrm{hr}$ & $\begin{array}{l}\text { after dis } \\
2.0 \mathrm{hr}\end{array}$ & $\begin{array}{l}=4 a r g e \\
4.0 \mathrm{hr}\end{array}$ & $6.0 \mathrm{hr}$ & $8.0 \mathrm{hr}$ & $10.0 \mathrm{hr}$ & $12.0 \mathrm{hr}$ & $14.0 \mathrm{hr}$ & $16.0 \mathrm{hr}$ \\
\hline $3.00 \mathrm{E}-01$ & & $1.09 \mathrm{E}+02$ & $9.56 \mathrm{~F}+01$ & $7.70 \mathrm{E}+01$ & $5.36 \mathrm{E}+01$ & $.00 \mathrm{E}+01$ & $.19 E+01$ & $2.70 \mathrm{E}+01$ & $2.40 \mathrm{E}+01$ & $2.22 \mathrm{E}+01$ & $2.10 \mathrm{E}+01$ \\
\hline $6.50 \mathrm{E}-01$ & $7.01 \mathrm{E}+03$ & $6.60 \mathrm{E}+03$ & $6.28 \mathrm{E}+03$ & $5.76 \mathrm{E}+03$ & $5.05 E+03$ & $4.63 \mathrm{E}+03$ & $4.36 E+03$ & $4.20 \mathrm{E}+03$ & $4.09 \mathrm{E}+03$ & $4.01 \mathrm{E}+03$ & $3.96 \mathrm{E}+03$ \\
\hline $1.12 E+00$ & $4.90 E+02$ & $3.11 \mathrm{E}+02$ & $2.89 \mathrm{E}+02$ & $2.84 \mathrm{E}+02$ & $2.81 E+02$ & $2.79 E+02$ & $2.78 \mathrm{E}+02$ & $2.77 \mathrm{E}+02$ & $2.77 E+02$ & $2.76 \mathrm{E}+02$ & $2.76 \mathrm{E}+02$ \\
\hline $1.58 \mathrm{E}+00$ & $1.41 E+03$ & $3.09 \mathrm{E}+02$ & $2.68 \mathrm{E}+02$ & $2.08 E+02$ & $1.27 \mathrm{E}+02$ & $7.93 E+01$ & $5.17 \mathrm{E}+01$ & $3.55 \mathrm{E}+01$ & $2.61 E+01$ & $2.06 \mathrm{E}+01$ & $1.73 E+01$ \\
\hline $2.00 \mathrm{E}+00$ & $5.82 \mathrm{E}+02$ & $5.08 \mathrm{E}+02$ & $4.44 E+02$ & $3.39 E+02$ & $1.98 \mathrm{E}+02$ & $1.16 E+02$ & $6.76 \mathrm{E}+01$ & $3.95 E+01$ & $2.31 E+01$ & $1.35 \mathrm{E}+01$ & $7.87 \mathrm{E}+00$ \\
\hline $2.40 \mathrm{E}+00$ & $2.28 \mathrm{E}+01$ & $1.94 \mathrm{E}+01$ & $1.70 \mathrm{E}+01$ & 1. $30 \mathrm{E}+01$ & $7.59 E+00$ & $4.43 \mathrm{E}+00$ & $2.59 \mathrm{E}+00$ & $1.51 \mathrm{E}+00$ & $8.83 \mathrm{E}-01$ & $5.16 E-01$ & $3.01 \mathrm{E}-01$ \\
\hline $2.80 \mathrm{E}+00$ & $2.01 \mathrm{E}+01$ & $1.76 \mathrm{E}+01$ & $1.54 \mathrm{E}+01$ & $1.18 \mathrm{E}+01$ & $6.86 \mathrm{E}+00$ & $4.01 \mathrm{E}+00$ & $2.34 E+00$ & $1.37 \mathrm{E}+00$ & $7.99 \mathrm{E}-01$ & $4.67 E-01$ & $2.73 \mathrm{E}-01$ \\
\hline $3.25 E+00$ & $3.73 E+00$ & $3.22 \mathrm{E}+00$ & $2.81 E+00$ & $2.15 E+00$ & $1.26 E+00$ & $4 \mathrm{E}-01$ & 4. $29 \mathrm{E}-01$ & $2.50 \mathrm{E}-01$ & $E-01$ & $8.54 E-02$ & $4.99 \mathrm{E}-02$ \\
\hline $3.75 \mathrm{E}+00$ & $2.84 \mathrm{E}-03$ & $2.56 \mathrm{E}-09$ & $2.44 \mathrm{E}-15$ & $3.05 E-22$ & $1.93 E-22$ & $1.30 \mathrm{E}-22$ & $8.81 E-23$ & $5.95 \mathrm{E}-23$ & $4.02 \mathrm{E}-23$ & $2.72 \mathrm{E}-23$ & $1.84 \mathrm{E}-23$ \\
\hline $4.25 E+00$ & 1. $01 \mathrm{E}-02$ & $9.67 \mathrm{E}-09$ & $9.22 \mathrm{E}-15$ & 3. $50 \mathrm{E}-24$ & $4.10 E-26$ & $4.81 E-28$ & $5.53 E-30$ & $0.00 \mathrm{E}+00$ & $0.00 \mathrm{E}+00$ & $0.00 \mathrm{E}+00$ & $0.00 \mathrm{E}+00$ \\
\hline $4.75 E+00$ & $1.53 \mathrm{E}-05$ & $1.78 \mathrm{E}-13$ & $9.68 \mathrm{E}-20$ & $3.13 \mathrm{E}-26$ & $3.66 E-28$ & $4.29 \mathrm{E}-30$ & $4.93 E-32$ & $0.00 \mathrm{E}+00$ & $0.00 \mathrm{E}+00$ & $0.00 E+00$ & $0.00 \mathrm{E}+00$ \\
\hline $5.50 \mathrm{E}+00$ & $9.16 \mathrm{E}-08$ & $8.73 \mathrm{E}-14$ & $8.32 \mathrm{E}-20$ & $2.70 \mathrm{E}-26$ & $3.16 \mathrm{E}-28$ & $3.71 E-30$ & $4.26 \mathrm{E}-32$ & $0.00 \mathrm{E}+00$ & $0.00 \mathrm{E}+00$ & $0.00 \mathrm{E}+00$ & $0.00 \mathrm{E}+00$ \\
\hline total & $9.74 E+03$ & $7.88 \mathrm{E}+03$ & $7.42 \mathrm{E}+03$ & $6.70 E+03$ & $5.73 \mathrm{E}+03$ & $5.15 \mathrm{E}+03$ & $4.80 E+03$ & $4.58 \mathrm{E}+03$ & $4.44 \mathrm{E}+03$ & $4.35 E+03$ & $4.28 \mathrm{E}+03$ \\
\hline $\mathrm{mev} / \mathrm{sec}$ & $8.67 E+03$ & $6.28 \mathrm{E}+03$ & $5.84 E+03$ & $5.17 E+03$ & $4.25 E+03$ & $3.71 E+03$ & $3.39 \mathrm{E}+03$ & $3.19 E+03$ & $3.07 E+03$ & $2.99 E+03$ & $2.93 \mathrm{E}+03$ \\
\hline
\end{tabular}


Table G30. Decay Photon Spectrum (/kg.s) After One-Day Irradiation of 1-kg Havar Foil (Flux Parameter Set 2).

\begin{tabular}{|c|c|c|c|c|c|c|c|c|c|c|c|}
\hline emean & & & time & after di & harge & & & & & & \\
\hline & initial & & $1.0 \mathrm{hr}$ & $2.0 \mathrm{hr}$ & $4.0 \mathrm{hr}$ & $6.0 \mathrm{hr}$ & & $10.0 \mathrm{hr}$ & $12.0 \mathrm{hr}$ & $14.0 \mathrm{hr}$ & $16.0 \mathrm{hr}$ \\
\hline $3.00 \mathrm{E}-01$ & $1.84 E+03$ & $1.07 \mathrm{E}+03$ & $8.80 E+02$ & $6.63 E+02$ & $4.23 E+02$ & $2.84 \mathrm{E}+02$ & $2.01 E+02$ & $1.51 \mathrm{E}+02$ & $1.21 \mathrm{E}+02$ & $1.01 \mathrm{E}+02$ & $8.82 \mathrm{E}+01$ \\
\hline $6.50 \mathrm{E}-01$ & $3.98 E+04$ & $3.56 \mathrm{E}+04$ & $3.24 \mathrm{E}+04$ & $2.71 \mathrm{E}+04$ & $1.98 \mathrm{E}+04$ & $1.54 \mathrm{E}+04$ & $1.27 \mathrm{E}+04$ & $1.09 \mathrm{E}+04$ & $9.78 E+03$ & $8.97 E+03$ & $8.38 \mathrm{E}+03$ \\
\hline $1.12 \mathrm{E}+00$ & $3.80 \mathrm{E}+03$ & $1.01 E+03$ & $6.41 \mathrm{E}+02$ & $5.60 \mathrm{E}+02$ & $5.28 \mathrm{E}+02$ & $5.09 \mathrm{E}+02$ & $4.99 \mathrm{E}+02$ & $4.92 \mathrm{E}+02$ & $4.89 E+02$ & $86 \mathrm{E}+02$ & $35 E+02$ \\
\hline $1.58 \mathrm{E}+00$ & $1.08 \mathrm{E}+04$ & $3.00 \mathrm{E}+03$ & $2.59 E+03$ & $1.98 \mathrm{E}+03$ & $1.16 \mathrm{E}+03$ & $6.82 \mathrm{E}+02$ & $4.02 \mathrm{E}+02$ & $2.39 \mathrm{E}+02$ & $1.43 E+02$ & $7 E+01$ & $51 E+01$ \\
\hline $2.00 \mathrm{E}+00$ & $5.90 \mathrm{E}+03$ & $5.13 \mathrm{E}+03$ & $4.48 E+03$ & $3.43 E+03$ & $2.00 E+03$ & $.7 E+03$ & $6.83 E+02$ & $3.99 E+02$ & 2. $33 E+02$ & $6 \mathrm{E}+$ & \\
\hline $2.10 \mathrm{E}+00$ & $2.29 \mathrm{E}+02$ & $1.97 \mathrm{E}+02$ & $1.72 E+02$ & $1.31 E+02$ & $7.67 E+01$ & $4.48 \mathrm{E}+01$ & $2.62 \mathrm{E}+01$ & $1.53 E+01$ & $8.93 E+00$ & $5.21 \mathrm{E}+00$ & $3.05 E+00$ \\
\hline $2.80 \mathrm{E}+00$ & $2.03 E+02$ & $1.78 \mathrm{E}+02$ & $1.55 E+02$ & 1. $19 E+02$ & $6.94 \mathrm{E}+01$ & $4.05 E+01$ & $2.37 \mathrm{E}+01$ & 1. $38 E+01$ & $8 E+00$ & $4.72 E+00$ & $6 \mathrm{E}+0$ \\
\hline $3.25 \mathrm{~F}+00$ & $3.76 \mathrm{E}+01$ & 3. $25 \mathrm{E}+01$ & $2.84 \mathrm{E}+01$ & $2.17 \mathrm{E}+01$ & 1. $27 \mathrm{E}+01$ & $7.42 \mathrm{E}+00$ & $4.33 \mathrm{E}+00$ & $2.53 E+00$ & $1.48 E+00$ & $8.63 \mathrm{~F}-01$ & $4 E-01$ \\
\hline $3.75 \mathrm{E}+00$ & $1.98 \mathrm{E}-02$ & $1.79 E-08$ & $1.71 E-14$ & $9.91 E-22$ & $8 E-22$ & -22 & $2.77 \mathrm{E}-22$ & $7 E-22$ & $7 E-22$ & & 5 \\
\hline $25 \mathrm{E}+00$ & $7.09 \mathrm{E}-02$ & $6.76 \mathrm{E}-08$ & $6.44 \mathrm{E}-14$ & $1.63 \mathrm{E}-23$ & $0 E-25$ & $2.22 \mathrm{E}-27$ & $2.77 \mathrm{E}-29$ & $0.00 \mathrm{E}+00$ & $0.00 \mathrm{E}+00$ & $0.00 \mathrm{E}+00$ & $0.00 \mathrm{E}+0$ \\
\hline $4.75 \mathrm{E}+00$ & $1.07 \mathrm{E}-04$ & $1.24 E-12$ & $6.76 \mathrm{E}$ & 1.4 & & 1.98 & & & & & \\
\hline $5.50 \mathrm{E}+00$ & $6.40 \mathrm{E}-07$ & $6.10 \mathrm{E}-13$ & $5.82 \mathrm{E}-19$ & $1.25 \mathrm{E}-25$ & $6 \mathrm{E}-27$ & $1.71 \mathrm{E}-29$ & $2.13 E-31$ & $0.00 \mathrm{E}+00$ & $0.00 \mathrm{E}+00$ & $0.00 \mathrm{E}+00$ & $0.00 \mathrm{E}$ \\
\hline total & $6.26 \mathrm{E}+04$ & $4.63 E+04$ & $4.13 E+04$ & $3.40 \mathrm{E}+04$ & $2.41 E+04$ & $1.82 \mathrm{E}+04$ & $1.45 \mathrm{E}+04$ & 1. $23 \mathrm{E}+04$ & 1. $.08 \mathrm{E}+04$ & $9.79 \mathrm{E}+03$ & $9.09 \mathrm{E}+0$ \\
\hline $\mathrm{mev} / \mathrm{sec}$ & $6.08 \mathrm{E}+04$ & $4.07 \mathrm{E}+04$ & $3.60 \mathrm{E}+04$ & $2.91 E+04$ & $1.99 \mathrm{E}+04$ & $1.43 E+04$ & $1.10 \mathrm{E}+04$ & $8.97 E+03$ & $7.68 \mathrm{E}+03$ & $6.85 \mathrm{E}+03$ & $6.28 \mathrm{E}+\mathrm{C}$ \\
\hline
\end{tabular}

Table G31. Decay Photon Spectrum (/kg.s) After Ten-Day Irradiation of 1-kg Havar Foil (Flux Parameter Set 2).

\begin{tabular}{|c|c|c|c|c|c|c|c|c|c|c|c|}
\hline nean & & & $10 \mathrm{~h}$ & after di & rae & & & & & & \\
\hline$(\mathrm{mev})$ & initial & $.5 \mathrm{hr}$ & $\begin{array}{l}1.0 \mathrm{hr} \\
1.09 \mathrm{E}+03\end{array}$ & $\begin{array}{c}2.0 \mathrm{hr} \\
8.66 \mathrm{E}+02\end{array}$ & $4.0 \mathrm{hr}$ & $6.0 \mathrm{hr}$ & & $10.0 \mathrm{hr}$ & $12.0 \mathrm{hr}$ & $\mathrm{hr}$ & 16 \\
\hline $\begin{array}{l}E-01 \\
E-01\end{array}$ & & $\begin{array}{l}.28 \mathrm{E}+ \\
.63 \mathrm{E}+\end{array}$ & & $\begin{array}{l}8.66 \mathrm{E}+02 \\
5.73 \mathrm{E}+04\end{array}$ & $\begin{array}{l}E+02 \\
E+04\end{array}$ & $\mathrm{E}+\mathrm{C}_{-1}$ & $\begin{array}{l}.89 \mathrm{E}+02 \\
.16 \mathrm{E}+04\end{array}$ & $\begin{array}{l}4 \mathrm{E}+ \\
4 \mathrm{E}+\end{array}$ & $\mathrm{E}+$ & $\begin{array}{c}8+02 \\
+04\end{array}$ & \\
\hline & & $.33 \mathrm{E}+$ & $6 \mathrm{E}+03$ & $4.88 \mathrm{E}+03$ & 4. & & $2 \mathrm{E}+03$ & & & & \\
\hline+00 & +04 & 3 & & +03 & 1. & & 2 & & & & \\
\hline & & & & & & & & & & & \\
\hline & & & & & & & & & & & \\
\hline & & 1. & & & & & & & & & \\
\hline & & & & & & & & & & & \\
\hline 3. & & 1.7 & & 1.2 ( & 9. & & & & & & \\
\hline & & & & & & & & & & & \\
\hline & & & & & & & & & & & \\
\hline & & $6.10 \mathrm{E}$ & & 1. $32 \mathrm{E}-24$ & 1.5 & & & & & & \\
\hline & $9.80 \mathrm{E}+04$ & & & & $5.85 \mathrm{E}+04$ & $5.21 E+04$ & $4.80 E+04$ & $4.53 E+04$ & & & \\
\hline $\mathrm{mev} / \mathrm{sec}$ & $8.59 E+04$ & $6.57 \mathrm{E}+04$ & $6.09 \mathrm{E}+04$ & $5.39 \mathrm{E}+04$ & $4.43 E+04$ & $3.84 \mathrm{E}+04$ & $3.48 E+04$ & $3.25 E+04$ & $3.10 E+04$ & $2.99 E+04$ & $2.91 E+0$ \\
\hline
\end{tabular}


Table G32. Decay Photon Spectrum (/kg.s) After One-Day Irradiation of 1-kg Concrete (Flux Parameter Set 3).

\begin{tabular}{|c|c|c|c|c|c|c|c|c|c|c|c|}
\hline $\begin{array}{l}\text { emean } \\
\text { (mev) }\end{array}$ & initial & $.5 \mathrm{hr}$ & $\begin{array}{l}\text { time } \\
1.0 \mathrm{hr}\end{array}$ & $\begin{array}{c}\text { after di } \\
2.0 \mathrm{hr}\end{array}$ & $\begin{array}{l}\text { harge } \\
4.0 \mathrm{hr}\end{array}$ & $6.0 \mathrm{hr}$ & & $10.0 \mathrm{hr}$ & $12.0 \mathrm{hr}$ & $14.0 \mathrm{hr}$ & $16.0 \mathrm{hr}$ \\
\hline $3.00 \mathrm{E}-01$ & $2.03 E+02$ & $1.51 \mathrm{E}+01$ & $1.41 \mathrm{E}+01$ & $1.33 E+01$ & $1.22 \mathrm{E}+01$ & $1.14 \mathrm{E}+01$ & $1.07 \mathrm{E}+01$ & $1.01 \mathrm{E}+01$ & $9.57 \mathrm{E}+00$ & $9.13 E+00$ & $8.75 E+00$ \\
\hline $6.50 \mathrm{E}-01$ & $2.71 \mathrm{E}+02$ & $2.93 E+01$ & 1. $34 \mathrm{E}+01$ & $9.76 \mathrm{E}+00$ & $7.12 \mathrm{E}+00$ & $5.51 \mathrm{E}+00$ & $4.50 \mathrm{E}+00$ & $3.83 \mathrm{E}+00$ & $3.38 \mathrm{E}+00$ & $3.07 \mathrm{E}+00$ & $2.83 \mathrm{E}+00$ \\
\hline $1.12 \mathrm{E}+00$ & $1.22 \mathrm{E}+02$ & $7.55 \mathrm{E}+00$ & $1.08 E+00$ & $4.35 E-01$ & $3.60 \mathrm{E}-01$ & $3.10 E-01$ & $2.72 \mathrm{E}-01$ & $2.41 \mathrm{E}-01$ & $2.16 \mathrm{E}-01$ & $1.94 \mathrm{E}-01$ & $1.76 E-01$ \\
\hline 1. $58 \mathrm{E}+00$ & $2.86 \mathrm{E}+03$ & $2.92 \mathrm{E}+02$ & $2.86 \mathrm{E}+02$ & $2.74 \mathrm{E}+02$ & $2.53 \mathrm{E}+02$ & 2. $34 \mathrm{E}+02$ & $2.16 \mathrm{E}+02$ & $2.00 \mathrm{E}+02$ & $1.86 \mathrm{E}+02$ & $1.73 \mathrm{E}+02$ & $1.61 \mathrm{E}+02$ \\
\hline $2.00 \mathrm{E}+00$ & $5.26 \mathrm{E}+00$ & $1.81 \mathrm{E}+00$ & $1.51 \mathrm{E}+00$ & $1.16 \mathrm{E}+00$ & $6.88 \mathrm{E}-01$ & $4.12 \mathrm{E}-01$ & $2.49 \mathrm{E}-01$ & 1. $53 \mathrm{E}-01$ & $9.64 \mathrm{E}-02$ & $6.24 \mathrm{E}-02$ & $4.20 \mathrm{E}-02$ \\
\hline $2.40 \mathrm{E}+00$ & $3.96 \mathrm{E}+00$ & $2.22 \mathrm{E}-01$ & $7.86 \mathrm{E}-02$ & $5.85 \mathrm{E}-02$ & $3.90 \mathrm{E}-02$ & $2.70 \mathrm{E}-02$ & $1.96 \mathrm{E}-02$ & $1.49 \mathrm{E}-02$ & $1.17 \mathrm{E}-02$ & $9.57 \mathrm{E}-03$ & $8.03 \mathrm{E}-03$ \\
\hline $2.80 \mathrm{E}+00$ & $2.84 \mathrm{E}+02$ & $2.77 \mathrm{E}+02$ & $2.71 \mathrm{E}+02$ & $2.58 \mathrm{E}+02$ & $5 E+02$ & $2.14 \mathrm{E}+02$ & $5 E+02$ & $1.77 \mathrm{E}+02$ & $1.61 \mathrm{E}+02$ & $1.46 \mathrm{E}+02$ & ]. $33 E+02$ \\
\hline $3.25 \mathrm{E}+00$ & $1.61 \mathrm{E}-01$ & $1.10 \mathrm{E}-02$ & $9.61 \mathrm{E}-03$ & $7.39 \mathrm{E}-03$ & $4.40 \mathrm{E}-03$ & $2.64 \mathrm{E}-03$ & $1.60 \mathrm{E}-03$ & $9.92 \mathrm{E}-04$ & $6.29 \mathrm{E}-04$ & $4.12 \mathrm{E}-04$ & $2.81 \mathrm{E}-04$ \\
\hline $3.75 \mathrm{E}+00$ & $3.45 \mathrm{E}-01$ & $1.81 \mathrm{E}-01$ & $1.76 \mathrm{E}-01$ & $1.68 \mathrm{E}-01$ & $1.53 \mathrm{E}-01$ & I. $39 \mathrm{E}-01$ & $1.27 \mathrm{E}-01$ & $1.15 \mathrm{E}-01$ & $1.05 E-01$ & $9.53 \mathrm{E}-02$ & $8.67 \mathrm{E}-02$ \\
\hline $4.25 E+00$ & $5.61 \mathrm{E}-02$ & $2.37 \mathrm{E}-03$ & $2.31 \mathrm{E}-03$ & $2.20 \mathrm{E}-03$ & $2.00 \mathrm{E}-03$ & $1.82 \mathrm{E}-03$ & $1.66 \mathrm{E}-03$ & 1.51 & 1.3 & & $4 \mathrm{E}-03$ \\
\hline $4.75 \mathrm{E}+00$ & $4.74 \mathrm{E}-02$ & $4.88 \mathrm{E}-16$ & $5.11 \mathrm{E}-24$ & $8.67 \mathrm{E}-25$ & $9.29 \mathrm{E}-26$ & $9.95 \mathrm{E}-27$ & $1.07 E-27$ & $1.14 \mathrm{E}-28$ & $1.23 E-29$ & $1.28 \mathrm{E}-30$ & $1.28 \mathrm{E}-31$ \\
\hline $5.50 \mathrm{E}+00$ & $7.80 \mathrm{E}-02$ & $4.00 E-24$ & $2.29 \mathrm{E}-24$ & $7.49 \mathrm{E}-25$ & $8.02 \mathrm{E}-26$ & $8.59 \mathrm{E}-27$ & $9.20 \mathrm{~F}-28$ & $9.85 \mathrm{E}-29$ & ]. $06 \mathrm{~F}-29$ & 1. $10 \mathrm{E}-30$ & 1. 1.0F,-31 \\
\hline total & $3.75 E+03$ & $6.23 E+02$ & $5.87 E+02$ & $5.57 \mathrm{E}+02$ & $5.08 \mathrm{E}+02$ & $4.65 \mathrm{E}+02$ & $4.26 \mathrm{E}+02$ & $3.92 \mathrm{E}+02$ & $3.60 \mathrm{E}+02$ & $3.32 E+02$ & $3.06 \mathrm{E}+02$ \\
\hline $\mathrm{mev} / \mathrm{sec}$ & $5.69 \mathrm{E}+03$ & $1.27 \mathrm{E}+03$ & $1.23 E+03$ & $1.17 \mathrm{E}+03$ & $1.07 \mathrm{E}+03$ & $9.75 E+02$ & $8.92 \mathrm{E}+02$ & $8.17 \mathrm{E}+02$ & $7.49 \mathrm{E}+02$ & $6.87 E+02$ & $6.31 \mathrm{E}+\mathrm{C}$ \\
\hline
\end{tabular}

Table G33. Decay Photon Spectrum (/kg.s) After Ten-Day Irradiation of 1-kg Concrete (Flux Parameter Set 3).

\begin{tabular}{|c|c|c|c|c|c|c|c|c|c|c|c|}
\hline $\begin{array}{l}\text { emean } \\
\text { (mev) }\end{array}$ & initial & $.5 \mathrm{hr}$ & $\begin{array}{l}\text { time } \\
1.0 \mathrm{hr}\end{array}$ & $\begin{array}{c}\text { after di } \\
2.0 \mathrm{hr}\end{array}$ & $\begin{array}{l}\text { harge } \\
4.0 \mathrm{hr}\end{array}$ & $6.0 \mathrm{hr}$ & & $10.0 \mathrm{hr}$ & $12.0 \mathrm{hr}$ & $14.0 \mathrm{hr}$ & \\
\hline $3.00 \mathrm{E}-01$ & $2.07 E+02$ & $1.84 \mathrm{E}+01$ & $1.74 \mathrm{E}+01$ & $1.65 \mathrm{E}+01$ & $1.51 \mathrm{E}+01$ & 1.39E+01 & $1.30 \mathrm{E}+01$ & $1.22 \mathrm{E}+01$ & $15 \mathrm{E}+01$ & $1.09 \mathrm{E}+01$ & $1.03 \mathrm{E}+01$ \\
\hline $6.50 \mathrm{E}-01$ & $2.72 \mathrm{E}+02$ & $3.08 \mathrm{E}+01$ & $1.50 \mathrm{E}+01$ & $1.12 \mathrm{E}+01$ & $8.49 \mathrm{E}+00$ & $6.78 \mathrm{E}+00$ & $5.67 \mathrm{E}+00$ & $4.92 \mathrm{E}+00$ & $4.40 \mathrm{E}+00$ & $4.02 \mathrm{E}+00$ & $3.72 \mathrm{E}+00$ \\
\hline $1.12 \mathrm{E}+00$ & $1.22 E+02$ & $7.69 \mathrm{E}+00$ & $1.21 \mathrm{E}+00$ & $5.62 \mathrm{E}-01$ & $4.75 \mathrm{E}-01$ & $4.14 \mathrm{E}-01$ & $3.65 \mathrm{E}-01$ & $3.26 \mathrm{E}-01$ & $2.92 \mathrm{E}-01$ & $2.63 \mathrm{E}-01$ & $2.38 \mathrm{E}-01$ \\
\hline $1.58 \mathrm{E}+00$ & $2.98 \mathrm{E}+03$ & $4.10 \mathrm{E}+02$ & $4.01 E+02$ & $3.84 \mathrm{E}+02$ & $3.53 \mathrm{E}+02$ & $3.24 \mathrm{E}+02$ & $2.99 \mathrm{E}+02$ & $2.75 \mathrm{E}+02$ & $2.54 \mathrm{E}+02$ & $2.35 \mathrm{E}+02$ & $2.17 \mathrm{E}+02$ \\
\hline $2.00 \mathrm{E}+00$ & $5.27 \mathrm{E}+00$ & $1.83 \mathrm{E}+00$ & $1.53 \mathrm{E}+00$ & 1. $17 \mathrm{E}+00$ & $7.00 \mathrm{E}-01$ & $4.22 \mathrm{E}-01$ & $2.58 \mathrm{E}-01$ & $1.61 \mathrm{E}-01$ & $1.03 \mathrm{E}-01$ & $6.87 \mathrm{E}-02$ & $4.76 \mathrm{E}-02$ \\
\hline $2.40 \mathrm{E}+00$ & $3.96 \mathrm{E}+00$ & $2.28 \mathrm{E}-01$ & $8.45 \mathrm{E}-02$ & $6.40 \mathrm{E}-02$ & $4.38 \mathrm{E}-02$ & $3.14 \mathrm{E}-02$ & $2.35 \mathrm{E}-02$ & $1.83 \mathrm{E}-02$ & $1.48 \mathrm{E}-02$ & 1. $23 \mathrm{E}-02$ & $1.05 \mathrm{E}-02$ \\
\hline $2.80 \mathrm{E}+00$ & $4.19 \mathrm{E}+02$ & $4.09 \mathrm{E}+02$ & $3.99 \mathrm{E}+02$ & $3.81 \mathrm{E}+02$ & $3.46 \mathrm{E}+02$ & $3.15 \mathrm{E}+02$ & $2.87 \mathrm{E}+02$ & $2.61 \mathrm{E}+02$ & $2.37 \mathrm{E}+02$ & $2.16 \mathrm{E}+02$ & $1.96 \mathrm{E}+02$ \\
\hline $3.25 \mathrm{E}+00$ & $1.62 \mathrm{E}-01$ & $1.11 \mathrm{E}-02$ & $9.72 \mathrm{E}-03$ & $7.49 \mathrm{E}-03$ & $4.48 \mathrm{E}-03$ & $2.71 \mathrm{E}-03$ & $1.67 \mathrm{E}-03$ & $1.05 \mathrm{E}-03$ & $6.81 \mathrm{E}-04$ & $4.58 \mathrm{E}-04$ & $3.21 \mathrm{E}-04$ \\
\hline $3.75 \mathrm{E}+00$ & $4.32 \mathrm{E}-01$ & $2.66 \mathrm{E}-01$ & $2.60 \mathrm{E}-01$ & $2.48 \mathrm{E}-01$ & $2.25 \mathrm{E}-01$ & $2.05 \mathrm{E}-01$ & $1.87 \mathrm{E}-01$ & $1.70 \mathrm{E}-01$ & $1.54 \mathrm{E}-01$ & $1.41 \mathrm{E}-01$ & $1.28 \mathrm{E}-01$ \\
\hline $4.25 E+00$ & $5.72 \mathrm{E}-02$ & $3.49 \mathrm{E}-03$ & $3.40 \mathrm{E}-0.3$ & $3.25 \mathrm{E}-03$ & $2.95 \mathrm{~F}-03$ & 2.6 & 2. & 2.2 & 2 . & & -03 \\
\hline $4.75 \mathrm{E}+00$ & $4.74 \mathrm{E}-02$ & $4.88 \mathrm{E}-16$ & $8.52 \mathrm{E}-22$ & $2.78 \mathrm{E}-22$ & $2.98 \mathrm{E}-23$ & $3.19 \mathrm{E}-24$ & $3.41 \mathrm{E}-25$ & $3.66 \mathrm{E}-26$ & $3.92 \mathrm{E}-27$ & $4.19 \mathrm{E}-28$ & $4.49 \mathrm{E}-29$ \\
\hline $5.50 \mathrm{E}+00$ & $7.80 \mathrm{E}-02$ & 1. $28 \mathrm{E}-21$ & 7. $33 E-22$ & $2.40 \mathrm{E}-22$ & $2.57 \mathrm{E}-23$ & $2.75 E-24$ & $2.95 \mathrm{E}-25$ & $3.16 \mathrm{E}-26$ & $3.38 \mathrm{E}-27$ & $3.62 \mathrm{E}-28$ & $3.88 \mathrm{E}-29$ \\
\hline total & $4.01 E+03$ & $8.78 \mathrm{E}+02$ & $8.35 E+02$ & $7.94 \mathrm{E}+02$ & $7.24 \mathrm{E}+02$ & $6.61 E+02$ & $6.05 E+02$ & $5.54 \mathrm{E}+02$ & $5.08 \mathrm{E}+02$ & $4.66 \mathrm{E}+02$ & 4.28 \\
\hline $\mathrm{mev} / \mathrm{sec}$ & $6.26 \mathrm{E}+03$ & $1.83 \mathrm{E}+03$ & $1.77 E+03$ & $1.69 \mathrm{E}+03$ & $1.54 \mathrm{E}+03$ & $1.40 \mathrm{E}+03$ & $1.28 \mathrm{E}+03$ & $1.17 \mathrm{E}+03$ & $1.07 \mathrm{E}+03$ & $9.81 \mathrm{E}+02$ & $8.98 E+0$ \\
\hline
\end{tabular}




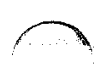

$\curvearrowright$ 


\section{APPENDIX H}

LISTINGS OF THE MCNP-4B INPUT FILES USED TO CALCULATE NEUTRON SPECTRA FOR THE ORIGEN

ACTIVATION CALCULATIONS 


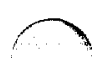

$\curvearrowleft$

$\infty$ 


\title{
APPENDIX H
}

\section{LISTINGS OF THE MCNP-4B INPUT FILES USED TO CALCULATE NEUTRON SPECTRA FOR THE ORIGEN ACTIVATION CALCULATIONS}

\author{
File for MCNP-4B calculation of ORIGEN neutron spectra at selected points \\ and dose rates near the roof of the PFNA facility for the source in the \\ horizontal position
}

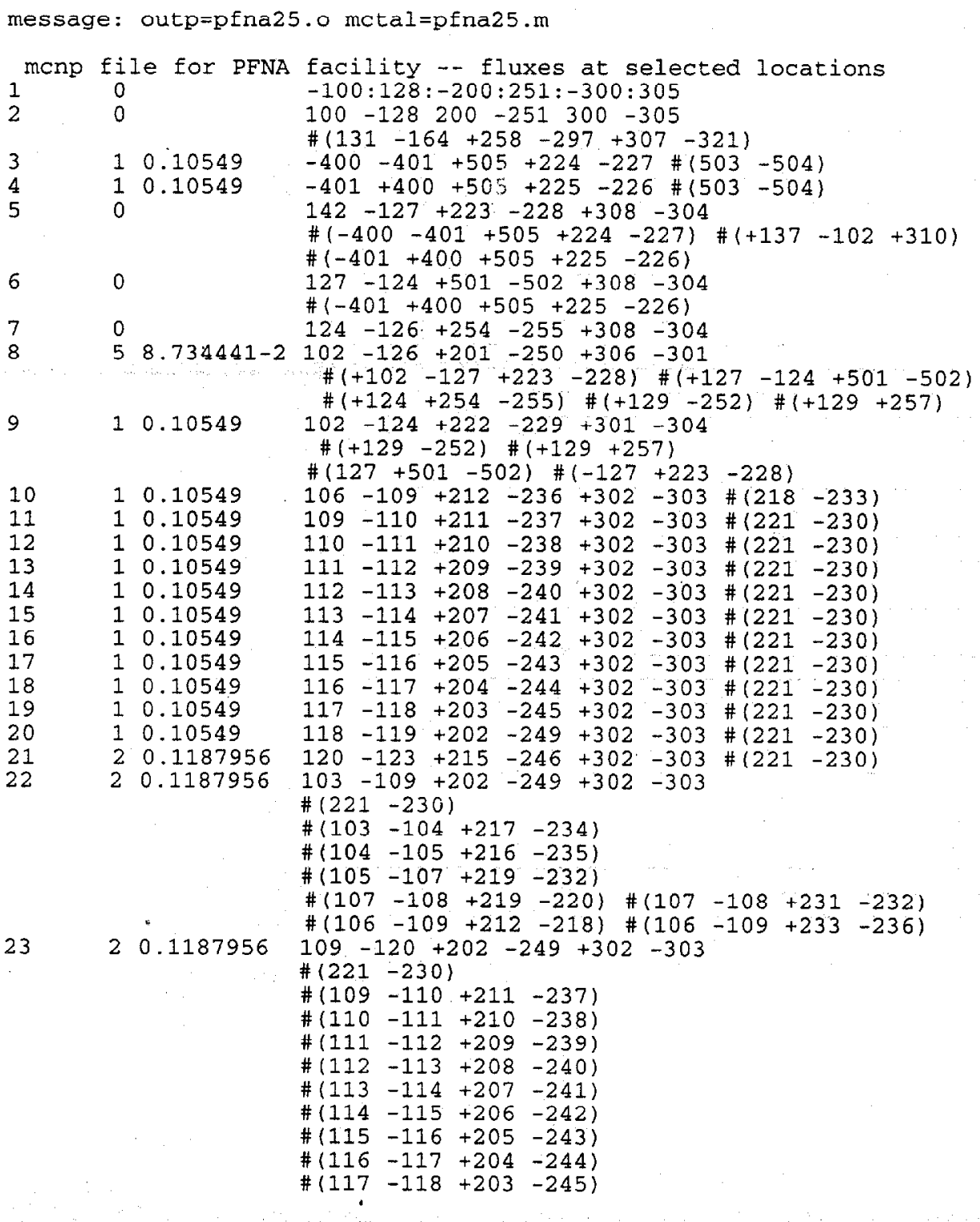




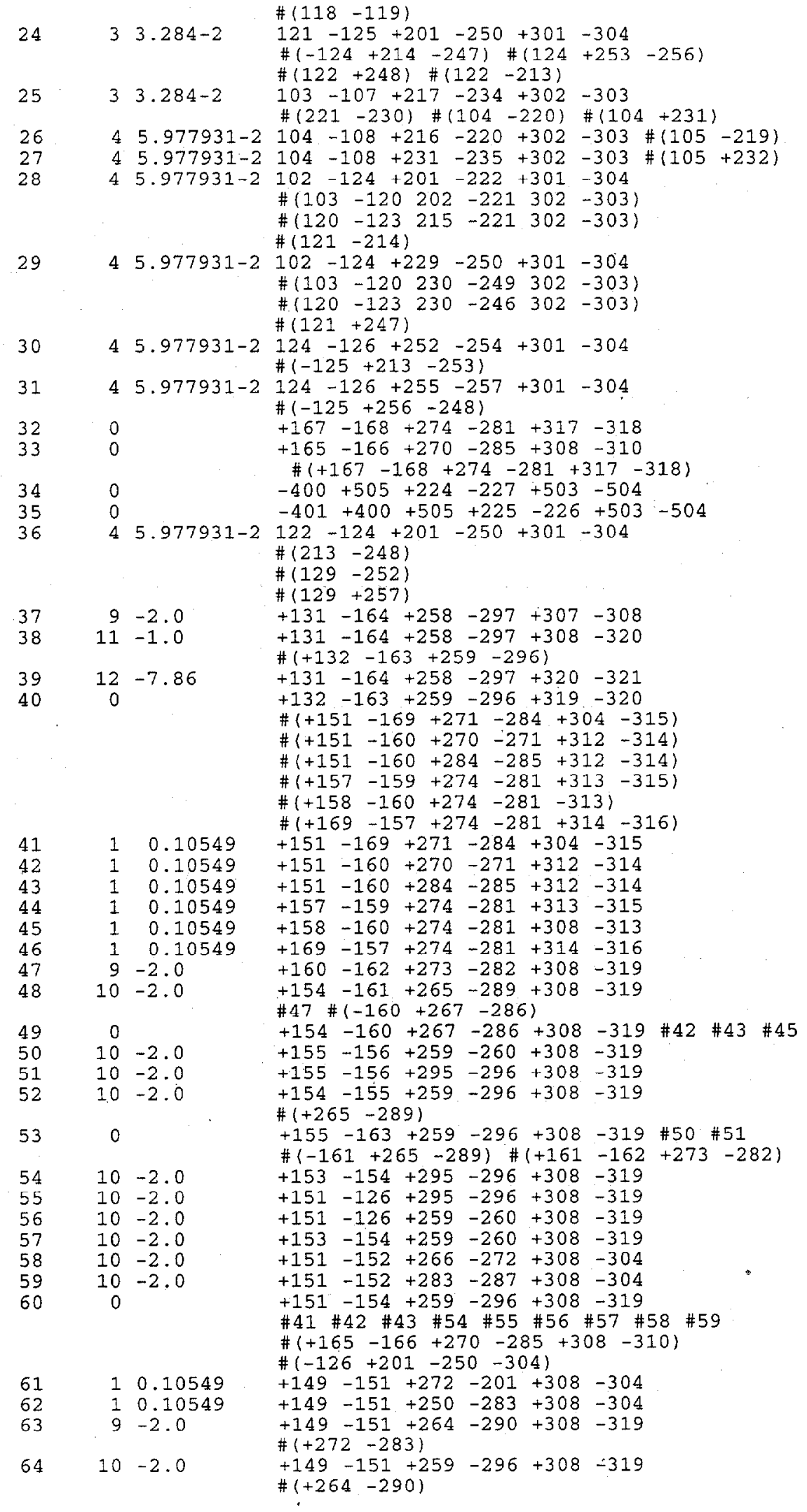




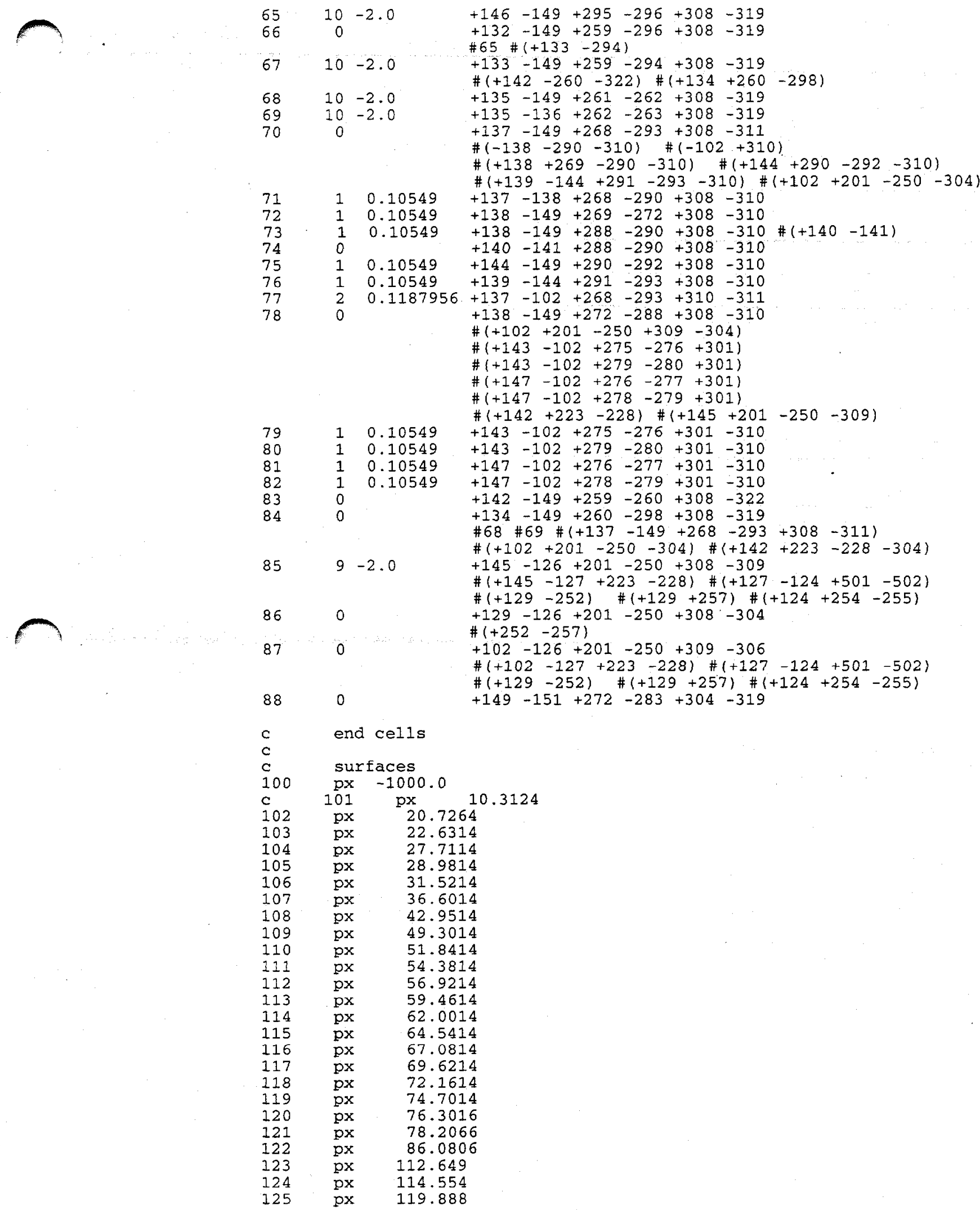




$\begin{array}{llc}126 & \mathrm{px} & 121.158 \\ 127 & \mathrm{px} & 50.5714 \\ 128 & \mathrm{px} & 1200.0 \\ 129 & \mathrm{px} & 87.9856 \\ 131 & \mathrm{px} & -844.042 \\ 132 & \mathrm{px} & -823.722 \\ 133 & \mathrm{px} & -767.842 \\ 134 & \mathrm{px} & -706.882 \\ 135 & \mathrm{px} & -548.962 \\ 136 & \mathrm{px} & -493.522 \\ 137 & \mathrm{px} & -381.762 \\ 138 & \mathrm{px} & -320.802 \\ 139 & \mathrm{px} & -315.722 \\ 140 & \mathrm{px} & -173.482 \\ 141 & \mathrm{px} & -107.442 \\ 142 & \mathrm{px} & -82.042 \\ 143 & \mathrm{px} & -55.4736 \\ 144 & \mathrm{px} & -21.082 \\ 145 & \mathrm{px} & -0.762 \\ 146 & \mathrm{px} & 9.398 \\ 147 & \mathrm{px} & 15.6464 \\ 148 & \mathrm{px} & 30.1103 \\ 149 & \mathrm{px} & 39.878 \\ 150 & \mathrm{px} & 45.3503 \\ 151 & \mathrm{px} & 100.838 \\ 152 & \mathrm{px} & 126.238 \\ 153 & \mathrm{px} & 476.758 \\ 154 & \mathrm{px} & 497.078 \\ 155 & \mathrm{px} & 558.038 \\ 156 & \mathrm{px} & 588.518 \\ 157 & \mathrm{px} & 725.678 \\ 158 & \mathrm{px} & 730.758 \\ 159 & \mathrm{px} & 745.998 \\ 160 & \mathrm{px} & 751.078 \\ 161 & \mathrm{px} & 812.038 \\ 162 & \mathrm{px} & 842.518 \\ 163 & \mathrm{px} & 964.438 \\ 164 & \mathrm{px} & 984.758 \\ 165 & \mathrm{px} & 148.387 \\ 166 & \mathrm{px} & 449.529 \\ 167 & \mathrm{px} & 250.0 \\ 168 & \mathrm{px} & 350.0 \\ 169 & \mathrm{px} & 116.078 \\ 200 & \mathrm{py} & -2000.0 \\ 201 & \mathrm{py} & -74.7056 \\ 202 & \mathrm{py} & -72.8006 \\ 203 & \mathrm{py} & -47.0958 \\ 204 & \mathrm{py} & -45.8258 \\ 205 & \mathrm{py} & -44.5558 \\ 206 & \mathrm{py} & -43.2858 \\ 207 & \mathrm{py} & -42.0158 \\ 208 & \mathrm{py} & -40.7458 \\ 209 & \mathrm{py} & -39.4758 \\ 210 & \mathrm{py} & -38.2058 \\ 211 & \mathrm{py} & -36.9358 \\ 212 & \mathrm{py} & -34.3958 \\ 213 & \mathrm{py} & -38.6122 \\ 214 & \mathrm{py} & -33.2782 \\ 215 & \mathrm{py} & -31.3732 \\ 216 & \mathrm{py} & -30.5858 \\ 217 & \mathrm{py} & -24.2358 \\ 218 & \mathrm{py} & -19.1558 \\ 219 & \mathrm{py} & -16.6158 \\ 225 & \mathrm{py} & -15.3458 \\ 226 & \mathrm{py} & -12.8058 \\ 227 & \mathrm{py} & -10.9008 \\ 228 & \mathrm{py} & -5.8208 \\ & \mathrm{py} & -4.2035 \\ & \mathrm{py} & 5.2037 \\ 228 & 5.8208 \\ 218 & \end{array}$




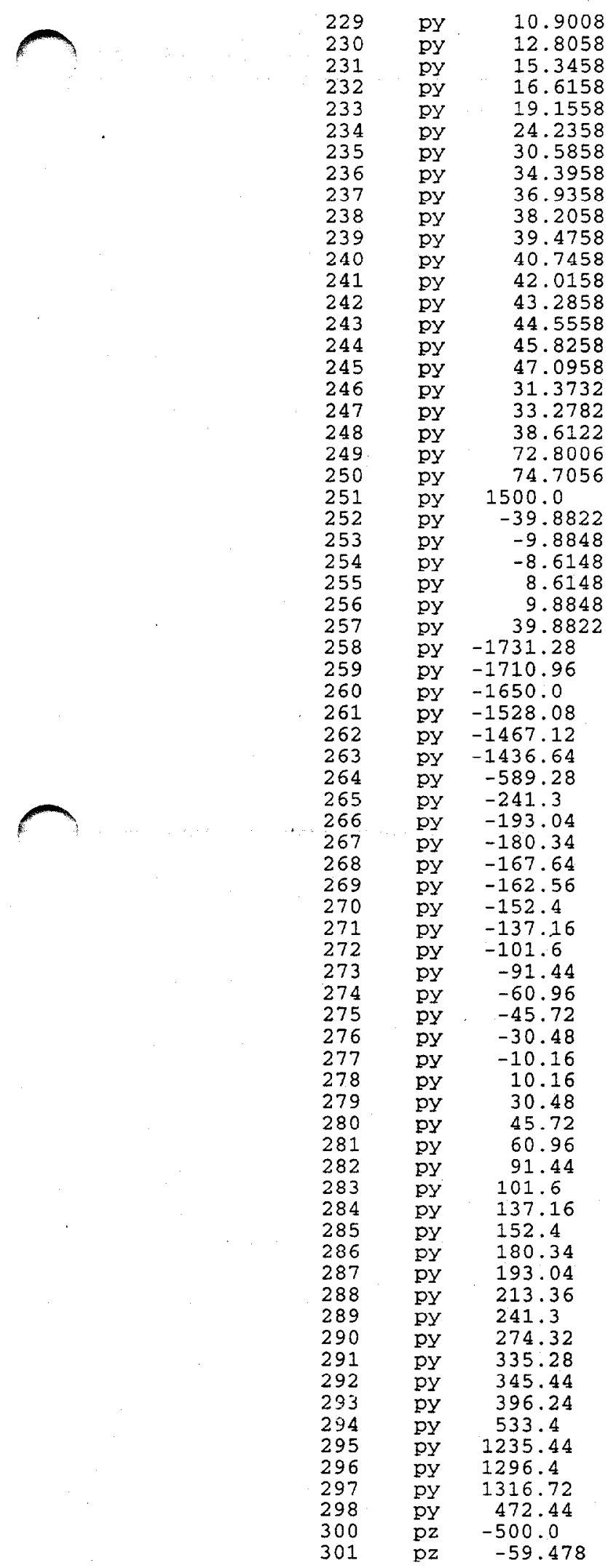




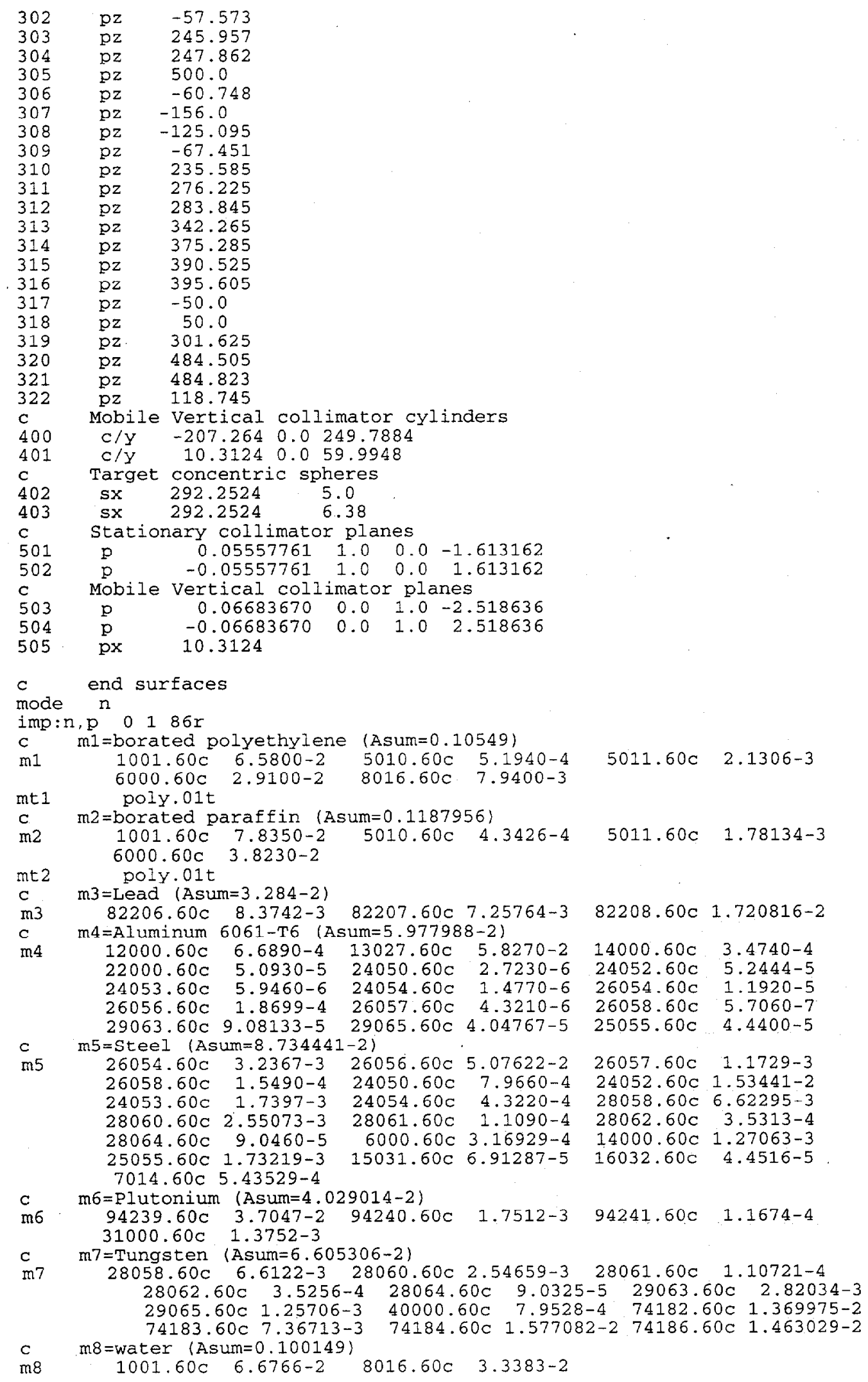




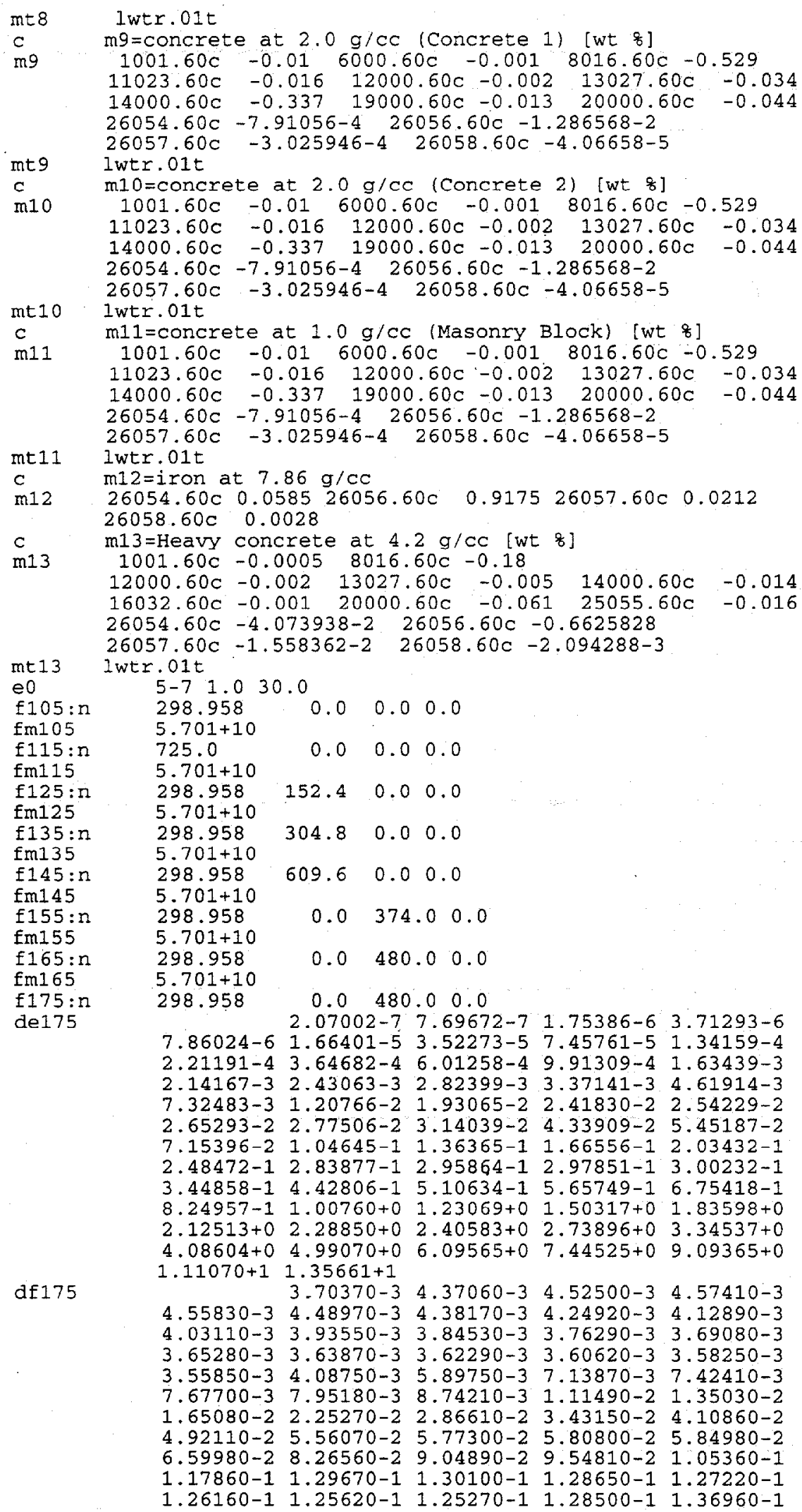

df 175

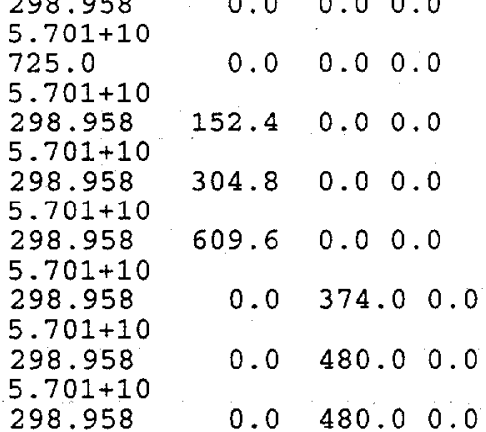




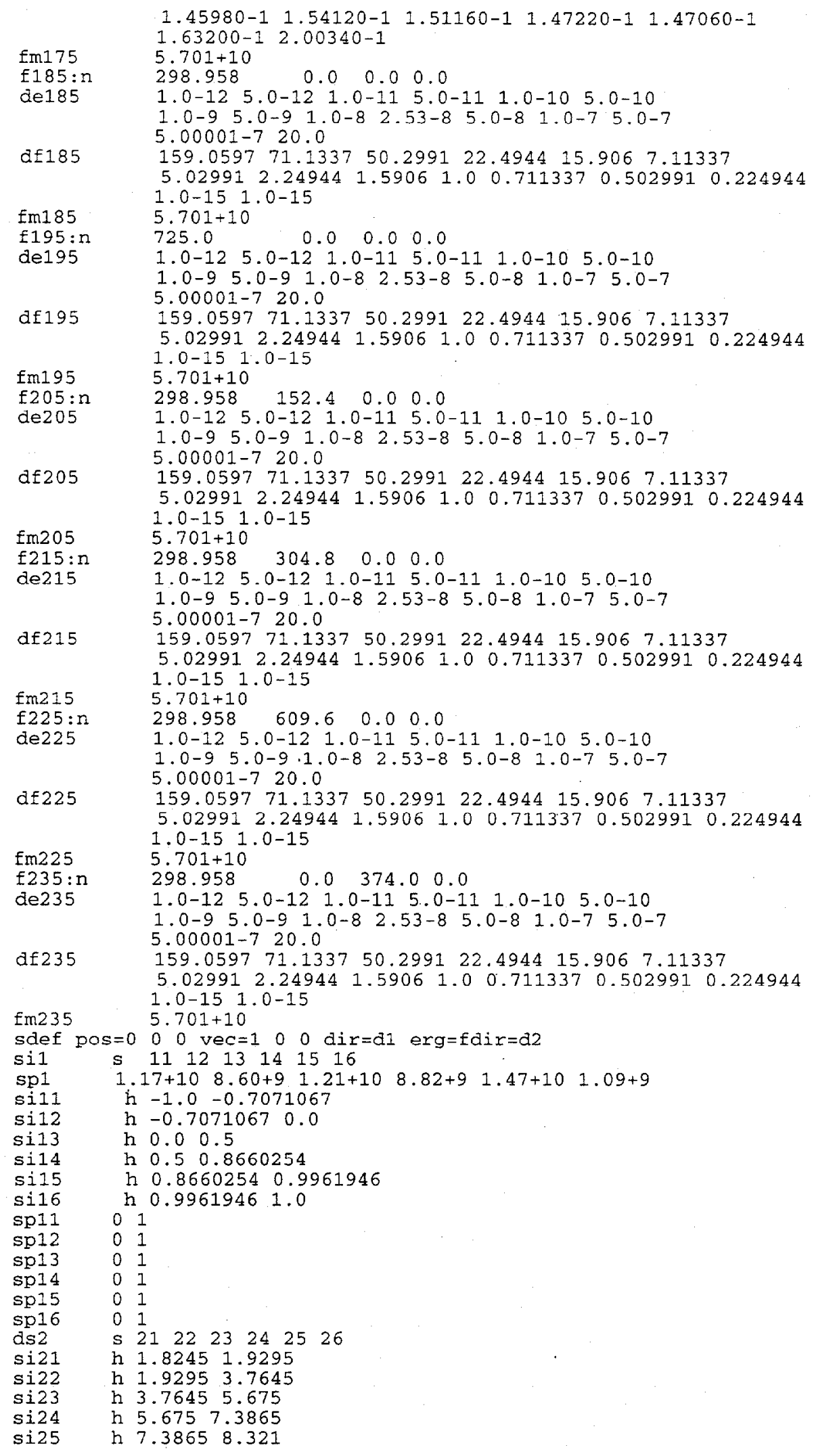




$\begin{array}{lll}\operatorname{si26} & \text { h } 8.3218 .753 \\ \operatorname{sp} 21 & 0 & 1 \\ \operatorname{sp} 22 & 0 & 1 \\ \operatorname{sp} 23 & 0 & 1 \\ \operatorname{sp} 24 & 0 & 1 \\ \operatorname{sp} 25 & 0 & 1 \\ \text { sp26 } & 0 & 1 \\ \text { nps } & 2000000 \\ \text { prdmp } & 2 j 1 \\ \text { print } & \end{array}$


File for MCNP-4B calculation of ORIGEN neutron spectra at selected points and dose rates near the roof of the PFNA facility for the source in the maximum up position

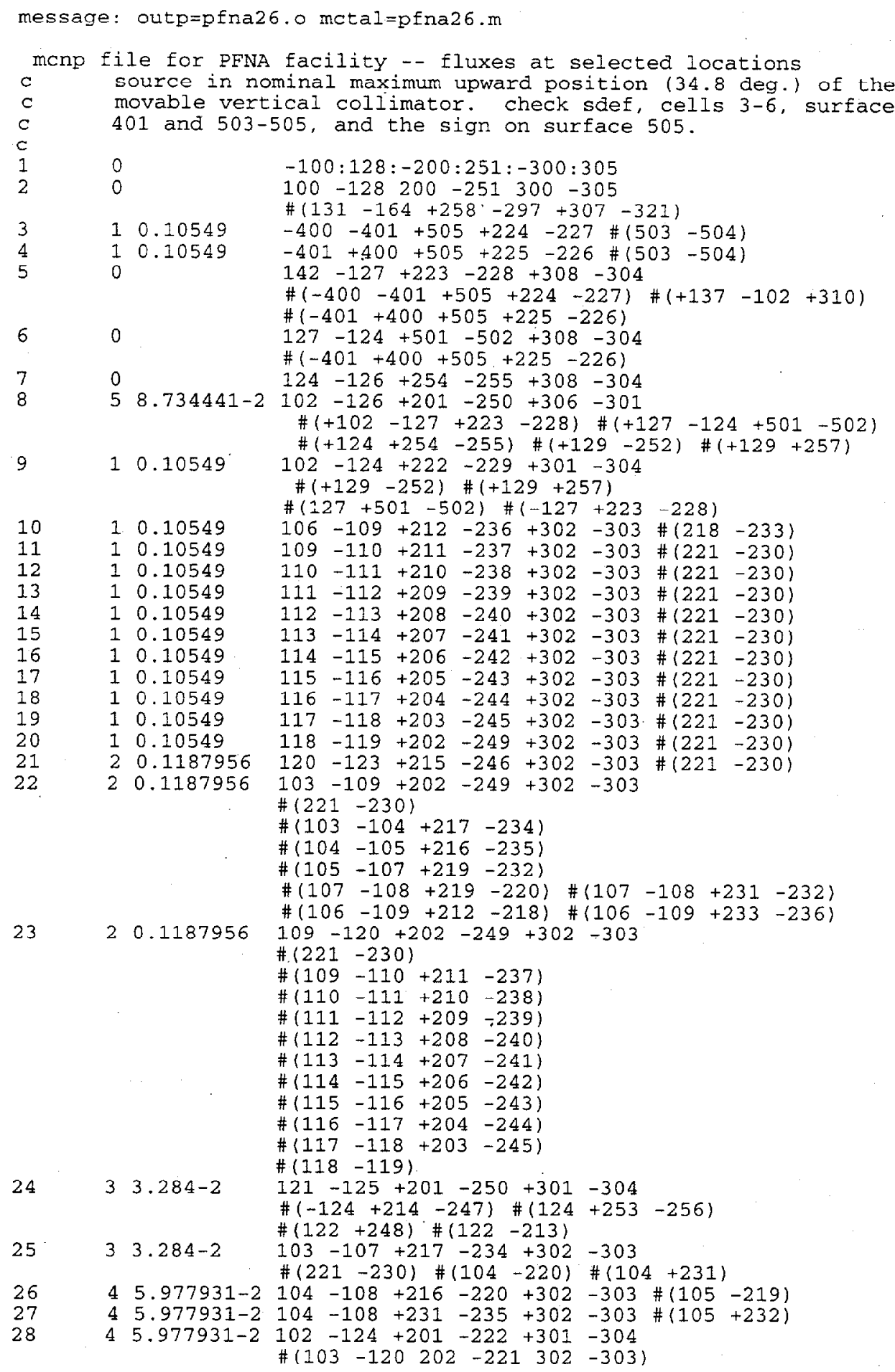


\#(120 $\left.\begin{array}{llllll}123 & 215 & -221 & 302 & -303\end{array}\right)$ \#(121-214)

$45.977931-2102-124+229-250+301-304$

\#( $\left(\begin{array}{llllll}103 & -120 & 230 & -249 & 302 & -303\end{array}\right)$

\#( $\left(\begin{array}{llllll}120 & -123 & 230 & -246 & 302 & -303\end{array}\right)$ $\#(121+247)$

$45.977931-2 \quad 124-126+252-254+301-304$ $\#(-125+213-253)$ 


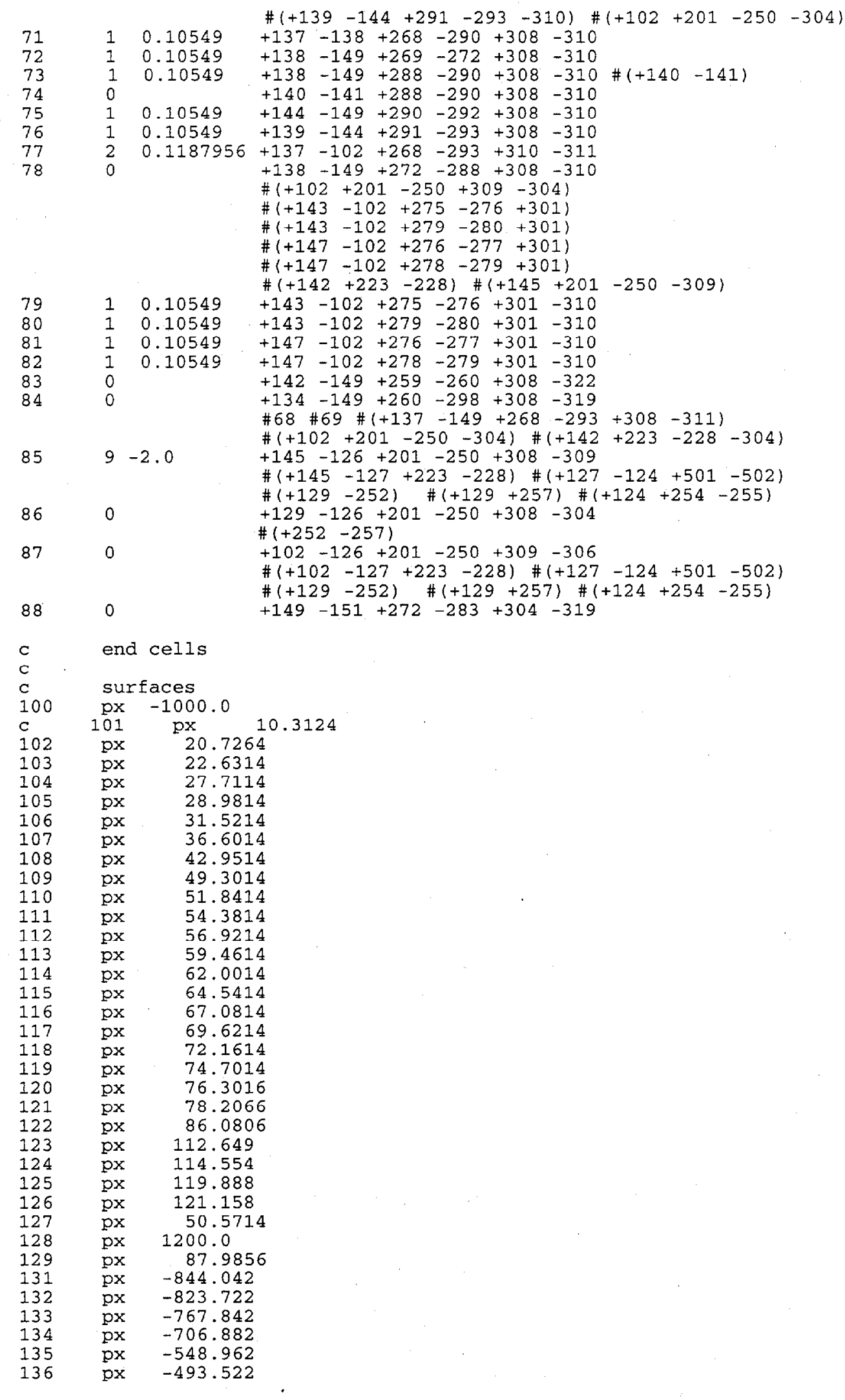




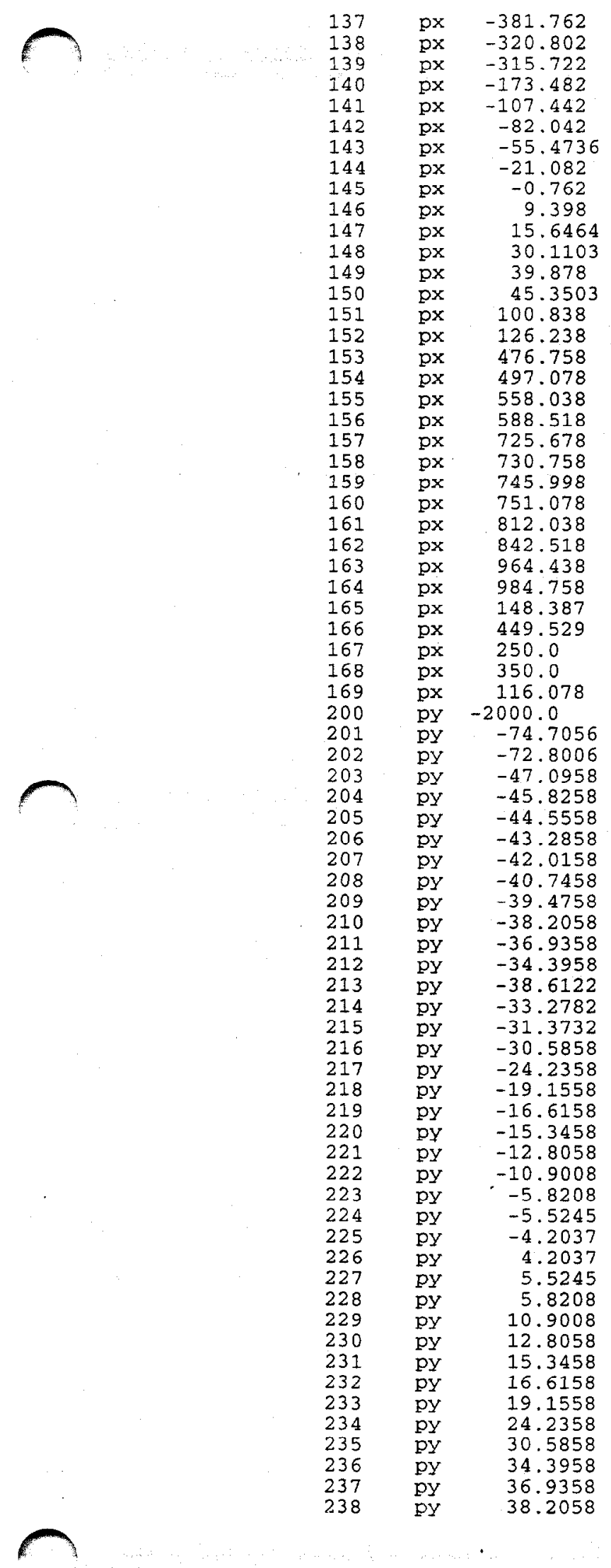




$\begin{array}{llc}239 & \text { py } & 39.4758 \\ 240 & \text { py } & 40.7458 \\ 241 & \text { py } & 42.0158 \\ 242 & \text { py } & 43.2858 \\ 243 & \text { py } & 44.5558 \\ 244 & \text { py } & 45.8258 \\ 245 & \text { py } & 47.0958 \\ 246 & \text { py } & 31.3732 \\ 247 & \text { py } & 33.2782 \\ 248 & \text { py } & 38.6122 \\ 249 & \text { py } & 72.8006 \\ 250 & \text { py } & 74.7056 \\ 251 & \text { py } & 1500.0 \\ 252 & \text { py } & -39.8822 \\ 253 & \text { py } & -9.8848 \\ 254 & \text { py } & -8.6148 \\ 255 & \text { py } & 8.6148 \\ 256 & \text { py } & 9.8848 \\ 257 & \text { py } & 39.8822 \\ 258 & \text { py } & -1731.28 \\ 259 & \text { py } & -1710.96 \\ 260 & \text { py } & -1650.0 \\ 261 & \text { py } & -1528.08 \\ 262 & \text { py } & -1467.12 \\ 263 & \text { py } & -1436.64 \\ 264 & \text { py } & -589.28 \\ 265 & \text { py } & -241.3 \\ 266 & \text { py } & -193.04 \\ 267 & \text { py } & -180.34 \\ 268 & \text { py } & -167.64 \\ 269 & \text { py } & -162.56 \\ 270 & \text { py } & -152.4 \\ 271 & \text { py } & -137.16 \\ 272 & \text { py } & -101.6 \\ 273 & \text { py } & -91.44 \\ 274 & \text { py } & -60.96 \\ 275 & \text { py } & -45.72 \\ 276 & \text { py } & -30.48 \\ 277 & \text { py } & -10.16 \\ 278 & \text { py } & 10.16 \\ 279 & \text { py } & 30.48 \\ 280 & \text { py } & 45.72 \\ 281 & \text { py } & 60.96 \\ 282 & \text { py } & 91.44 \\ 283 & \text { py } & 101.6 \\ 284 & \text { py } & 137.16 \\ 285 & \text { py } & 152.4 \\ 286 & \text { py } & 180.34 \\ 287 & \text { py } & 193.04 \\ 288 & \text { py } & 213.36 \\ 289 & \text { py } & 241.3 \\ 290 & \text { py } & 274.32 \\ 291 & \text { py } & 335.28 \\ 292 & \text { py } & 345.44 \\ 293 & \text { py } & 396.24 \\ 294 & \text { py } & 533.4 \\ 295 & \text { py } & 1235.44 \\ 296 & \text { py } & 1296.4 \\ 297 & \text { py } & 1316.72 \\ 298 & \text { py } & 472.44 \\ 300 & \text { pz } & -500.0 \\ 301 & \text { pz } & -59.478 \\ 302 & \text { pz } & -57.573 \\ 305 & \text { pz } & 245.957 \\ 306 & \text { pz } & 247.862 \\ 307 & \text { pz } & -60.74 \\ 308 & \text { pz } & -156.0 \\ 309 & \text { pz } & -125.095 \\ 310 & \text { pz } & 235.558 \\ 311 & \text { pz } & 276.225 \\ & & \\ 230 & \end{array}$



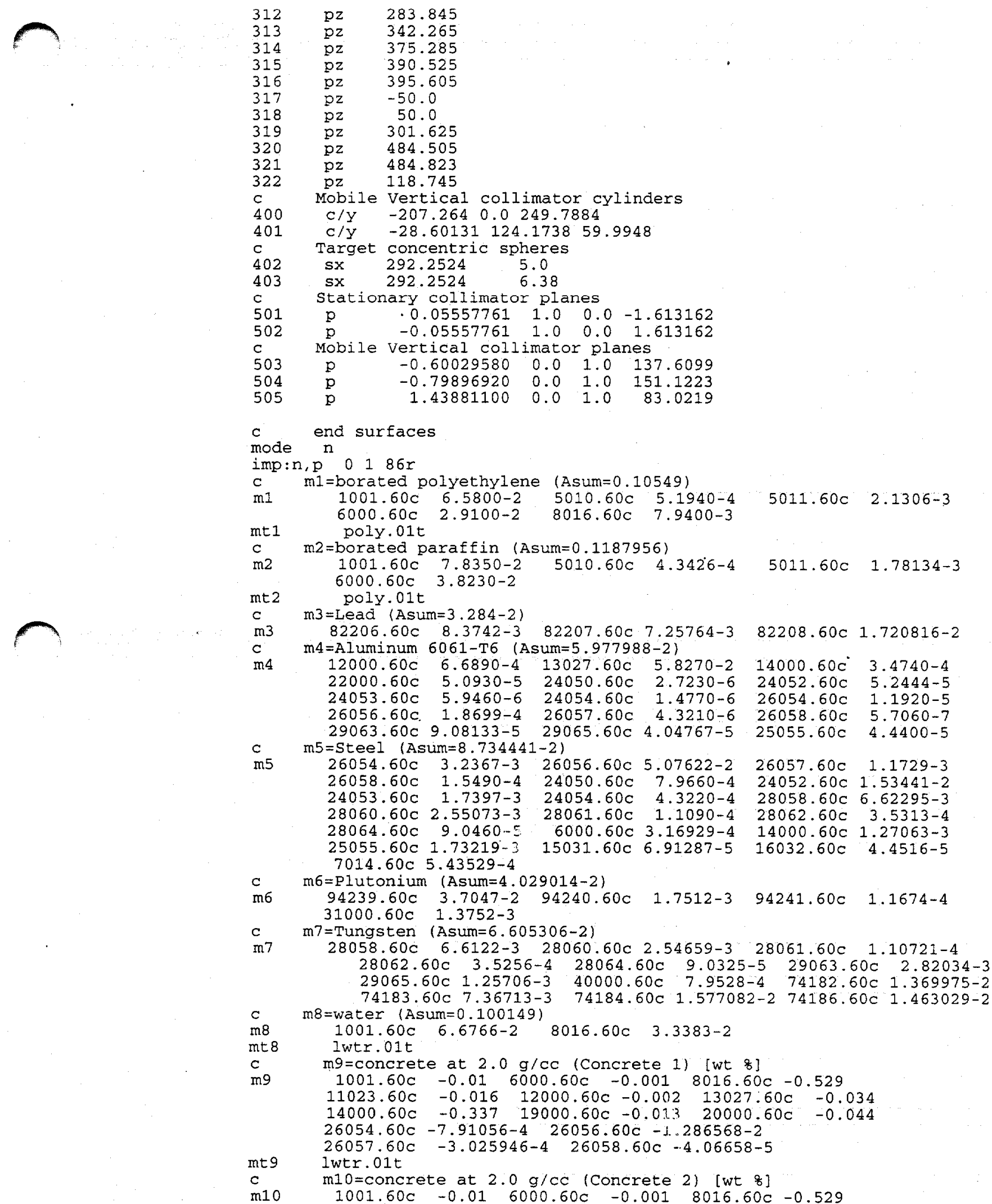


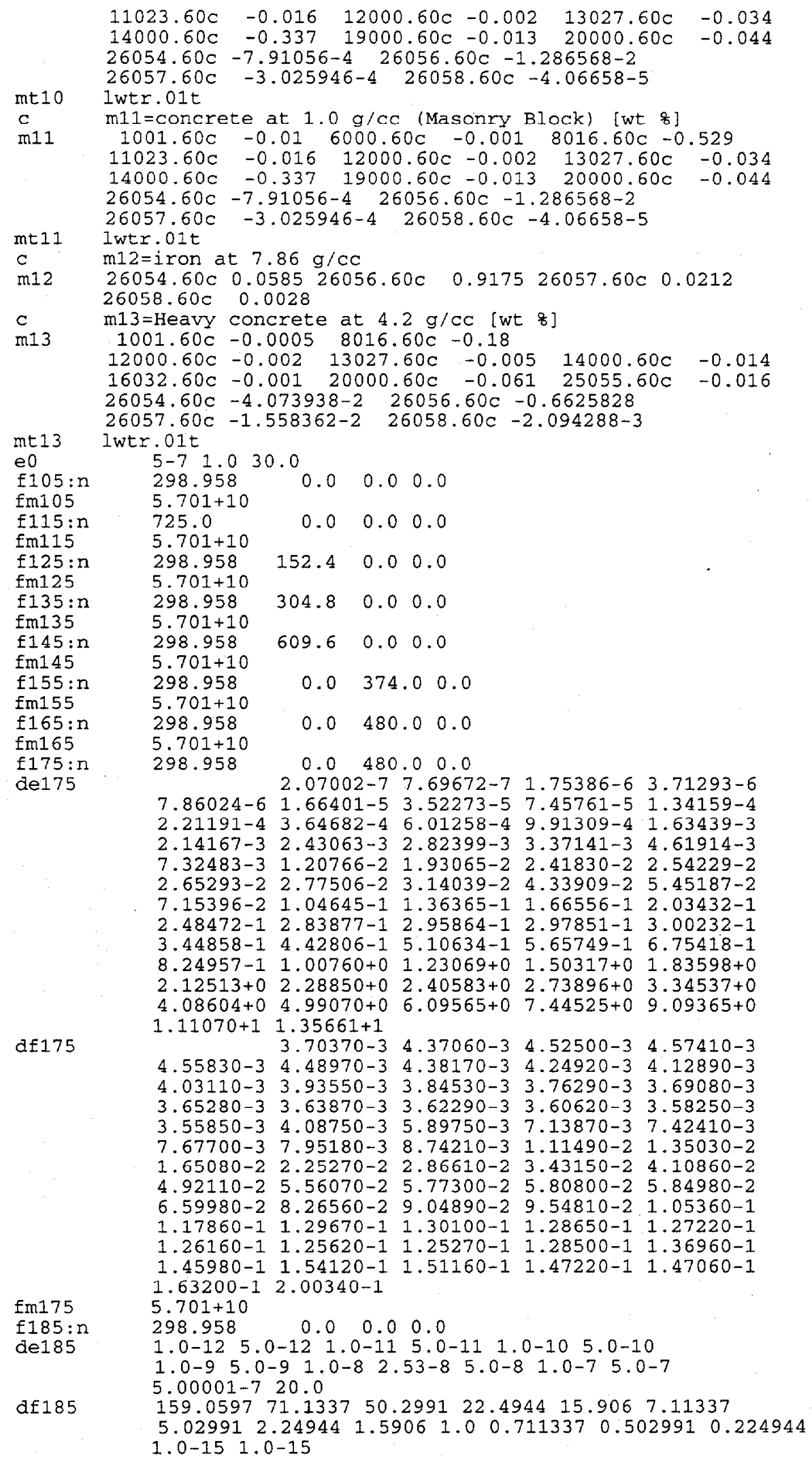




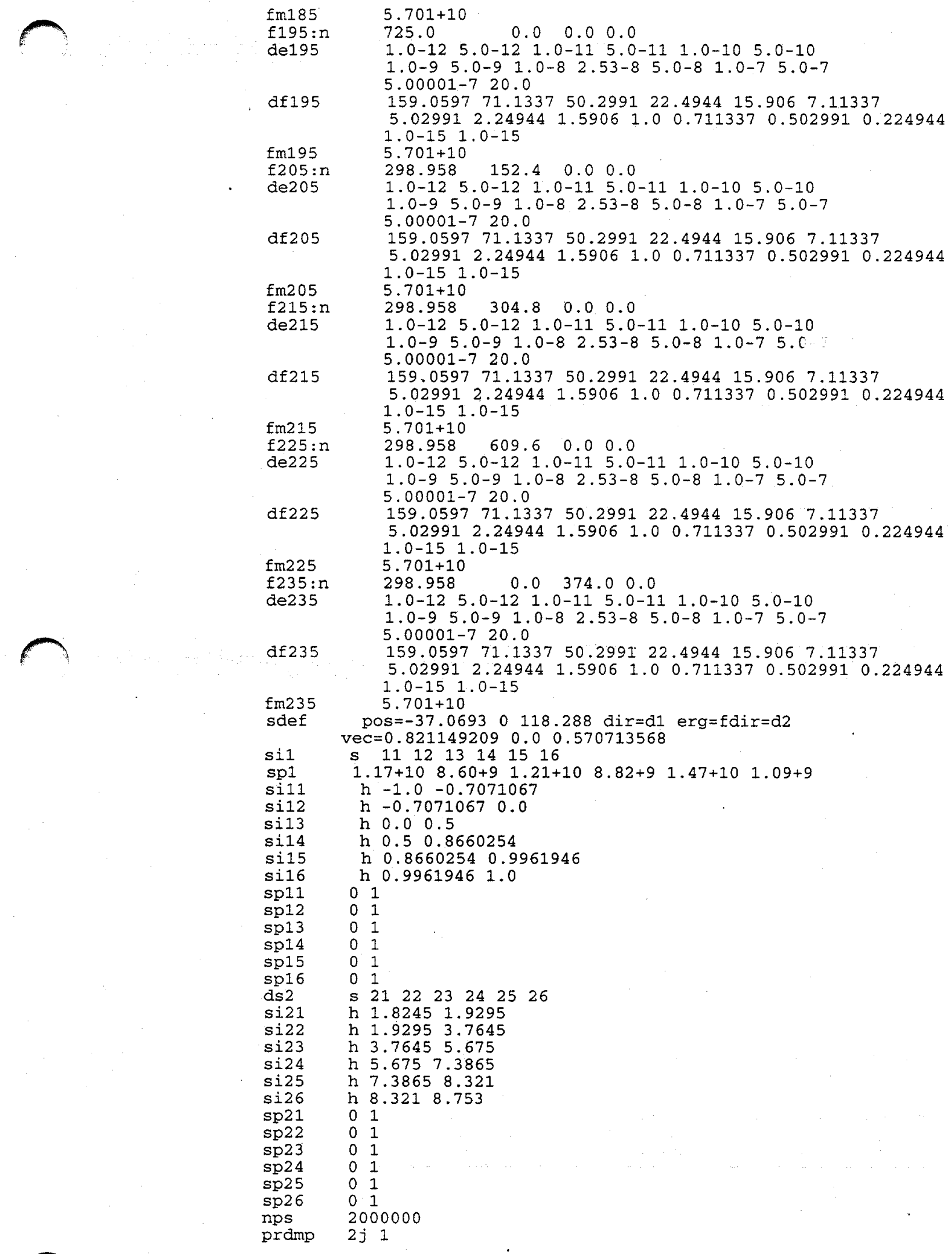


print 
File for MCNP-4B Calculation of ORIGEN neutron spectra at selected points and dose rates near the roof of the PFNA facility for the source in the maximum down position

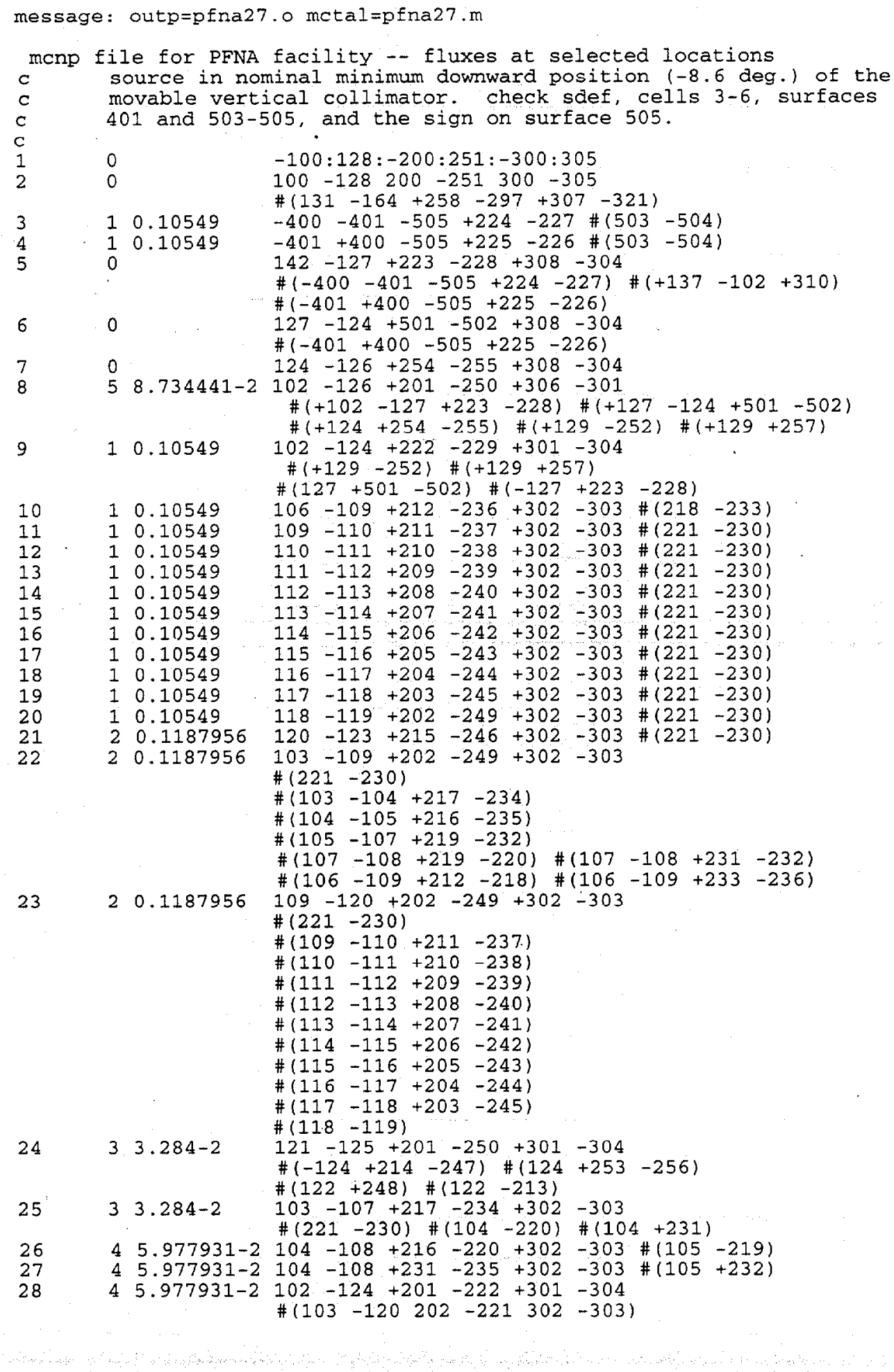




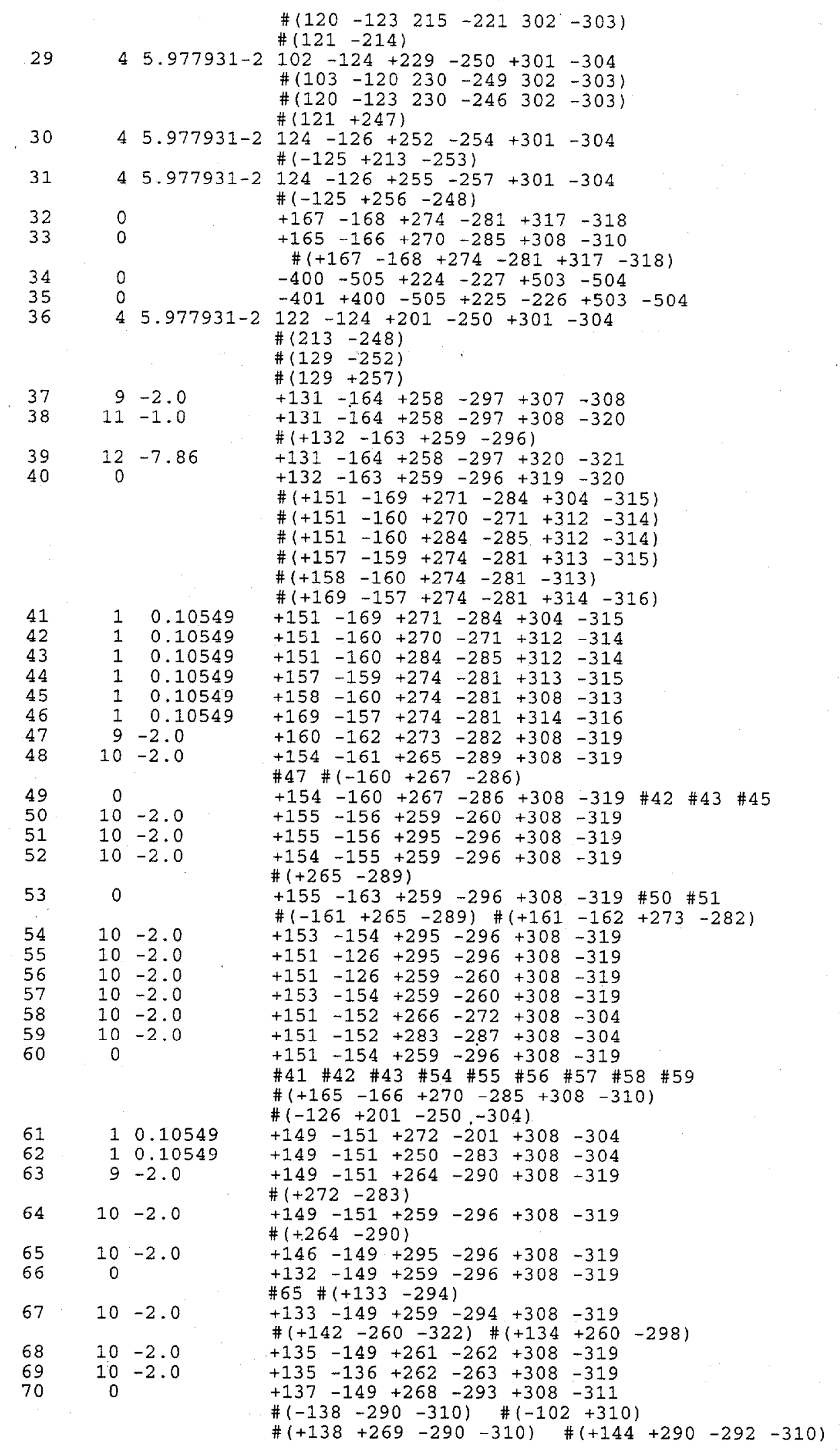




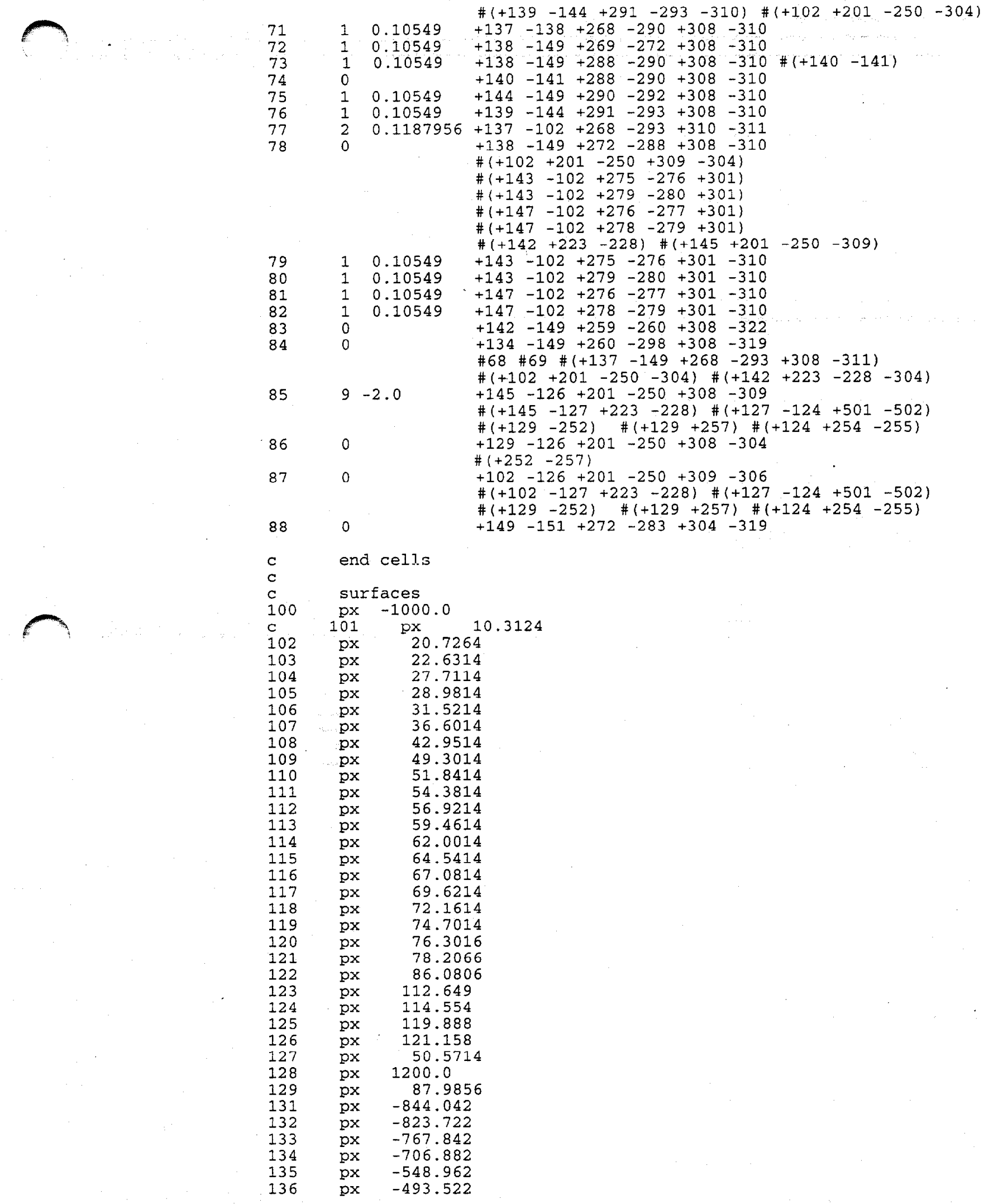




\begin{tabular}{|c|c|c|}
\hline 137 & $\mathrm{px}$ & -381.762 \\
\hline 138 & $\mathrm{px}$ & -320.802 \\
\hline 139 & $\mathrm{px}$ & -315.722 \\
\hline 140 & $p x$ & -173.482 \\
\hline 141 & $\mathrm{px}$ & -107.442 \\
\hline 142 & $p x$ & -82.042 \\
\hline 143 & $\mathrm{px}$ & -55.4736 \\
\hline 144 & $\mathrm{px}$ & -21.082 \\
\hline 145 & $\mathrm{px}$ & -0.762 \\
\hline 146 & $\mathrm{px}$ & 9.398 \\
\hline 147 & $\mathrm{px}$ & 15.6464 \\
\hline 148 & $\mathrm{px}$ & 30.1103 \\
\hline 149 & $\mathrm{px}$ & 39.878 \\
\hline 150 & $\mathrm{px}$ & 45.3503 \\
\hline 151 & $\mathrm{px}$ & 100.838 \\
\hline 152 & $p x$ & 126.238 \\
\hline 153 & $\mathrm{px}$ & 476.758 \\
\hline 154 & $\mathrm{px}$ & 497.078 \\
\hline 155 & $\mathrm{px}$ & 558.038 \\
\hline 156 & $\mathrm{px}$ & 588.518 \\
\hline 157 & $\mathrm{px}$ & 725.678 \\
\hline 158 & $\mathrm{px}$ & 730.758 \\
\hline 159 & $\mathrm{px}$ & 745.998 \\
\hline 160 & $\mathrm{px}$ & 751.078 \\
\hline 161 & $\mathrm{px}$ & 812.038 \\
\hline 162 & $p x$ & 842.518 \\
\hline 163 & $\mathrm{px}$ & 964.438 \\
\hline 164 & $\mathrm{px}$ & 984.758 \\
\hline 165 & $\mathrm{px}$ & 148.387 \\
\hline 166 & $p x$ & 449.529 \\
\hline 167 & $\mathrm{px}$ & 250.0 \\
\hline 168 & $p x$ & 350.0 \\
\hline 169 & $\mathrm{px}$ & 116.078 \\
\hline 200 & py & -2000.0 \\
\hline 201 & py & -74.7056 \\
\hline 202 & py & -72.8006 \\
\hline 203 & py & -47.0958 \\
\hline 204 & py & -45.8258 \\
\hline 205 & py & -44.5558 \\
\hline 206 & py & -43.2858 \\
\hline 207 & py & -42.0158 \\
\hline 208 & py & -40.7458 \\
\hline 209 & DY & -39.4758 \\
\hline 210 & py & -38.2058 \\
\hline 211 & py & -36.9358 \\
\hline 212 & py & -34.3958 \\
\hline 213 & py. & -38.6122 \\
\hline 214 & py & -33.2782 \\
\hline 215 & py & -31.3732 \\
\hline 216 & py & -30.5858 \\
\hline 217 & py & -24.2358 \\
\hline 218 & py & -19.1558 \\
\hline 219 & PY & -16.6158 \\
\hline 220 & py & -15.3458 \\
\hline 221 & PY & -12.8058 \\
\hline 222 & py & -10.9008 \\
\hline 223 & py & -5.8208 \\
\hline 224 & py & -5.5245 \\
\hline 225 & py & -4.2037 \\
\hline 226 & py & 4.2037 \\
\hline 227 & py & 5.5245 \\
\hline 228 & py & 5.8208 \\
\hline 229 & py & 10.9008 \\
\hline 230 & py & 12.8058 \\
\hline 231 & py & 15.3458 \\
\hline 232 & py & 16.6158 \\
\hline 233 & py & 19.1558 \\
\hline & py & 24.2358 \\
\hline 235 & py & 30.5858 \\
\hline 236 & PY & 34.3958 \\
\hline & py & 36.9358 \\
\hline & py & 38.2058 \\
\hline
\end{tabular}




$\begin{array}{rllr}239 & \text { py } & 39.4758 \\ 240 & \text { py } & 40.7458 \\ 241 & \text { py } & 42.0158 \\ 242 & \text { py } & 43.2858 \\ 243 & \text { py } & 44.5558 \\ 244 & \text { py } & 45.8258 \\ 245 & \text { py } & 47.0958 \\ 246 & \text { py } & 31.3732 \\ 247 & \text { py } & 33.2782 \\ 248 & \text { py } & 38.6122 \\ 249 & \text { py } & 72.8006 \\ 250 & \text { py } & 74.7056 \\ 251 & \text { py } & 1500.0 \\ 252 & \text { py } & -39.8822 \\ 253 & \text { py } & -9.8848\end{array}$




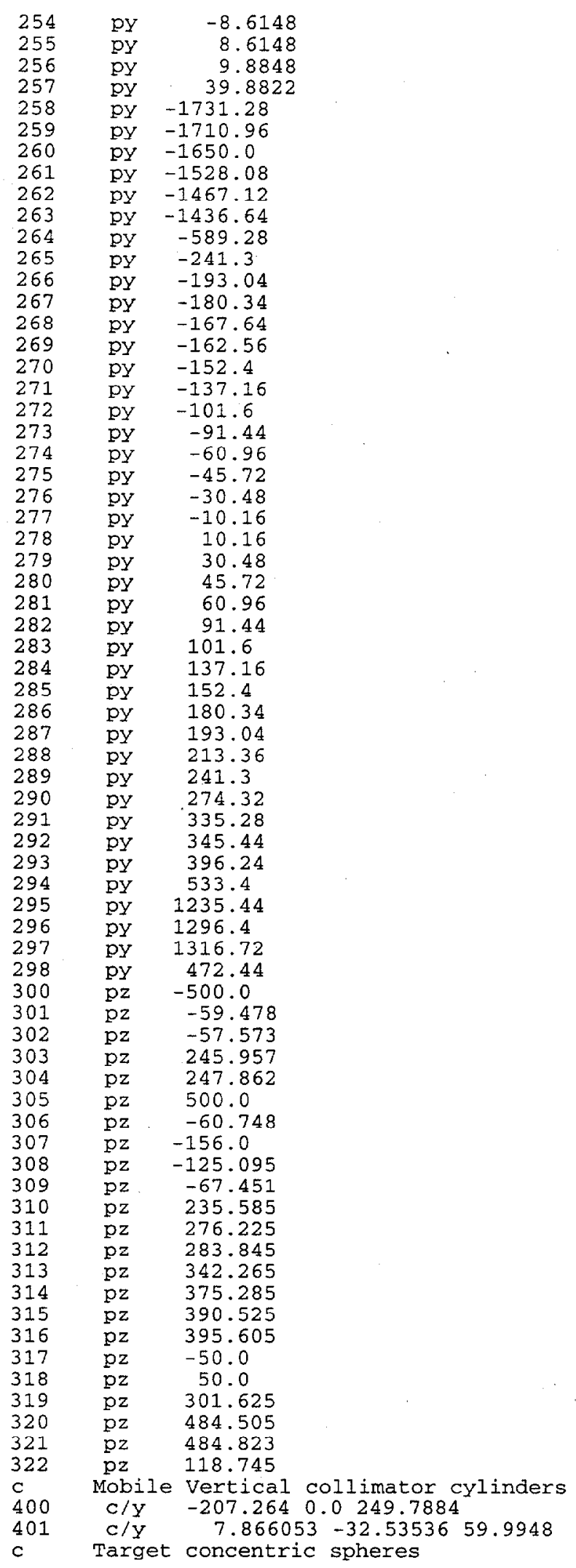




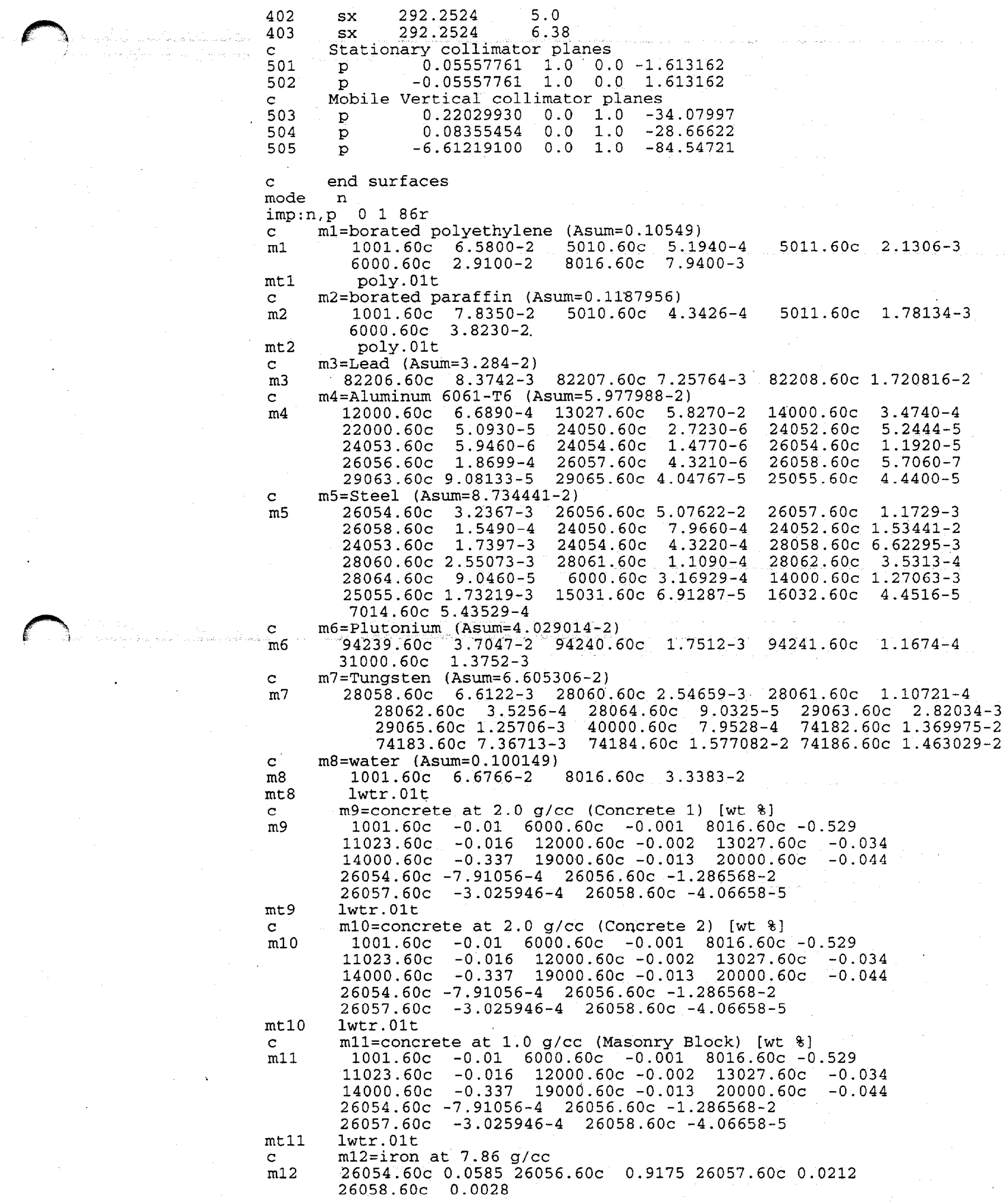




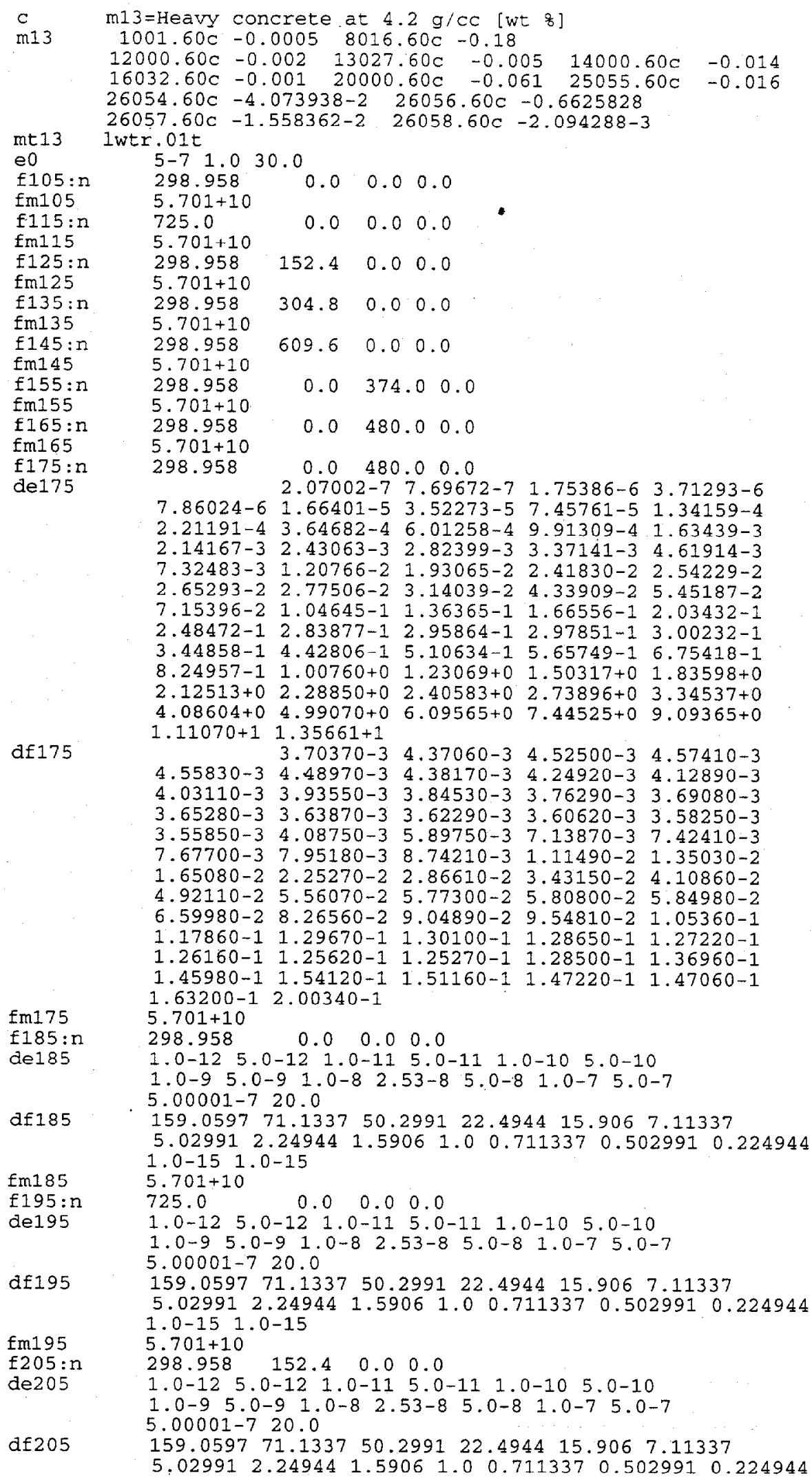

df205$$
\begin{array}{llll}
725.0 & 0.0 & 0.0 & 0.0
\end{array}
$$

1.0-12 5.0-12 1.0-11 5.0-11 $1.0-10 \quad 5.0-10$ $\begin{array}{lllllll}1.0-9 & 5.0-9 & 1.0-8 & 2.53-8 & 5.0-8 & 1.0-7 & 5.0-7\end{array}$ $5.00001-720.0$

$\begin{array}{lllllll}159.0597 & 71.1337 & 50.2991 & 22.4944 & 15.906 & 7.11337\end{array}$ $\begin{array}{llllllll}5.02991 & 2.24944 & 1.5906 & 1.0 & 0.711337 & 0.502991 & 0.224944\end{array}$ $1.0-15 \quad 1.0-15$ $5.701+10$ $\begin{array}{lllll}298.958 & 152.4 & 0.0 & 0.0\end{array}$

$1.0-125 \cdot 0-12 \quad 1.0-115.0-11 \quad 1.0-10 \quad 5 \cdot 0-10$

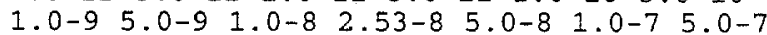
$5.00001-7 \quad 20.0$ $\begin{array}{llllll}159.0597 & 71.1337 & 50.2991 & 22.4944 & 15.906 & 7.11337\end{array}$ $\begin{array}{llllllll}5.02991 & 2.24944 & 1.5906 & 1.0 & 0.711337 & 0.502991 & 0.224944\end{array}$ 


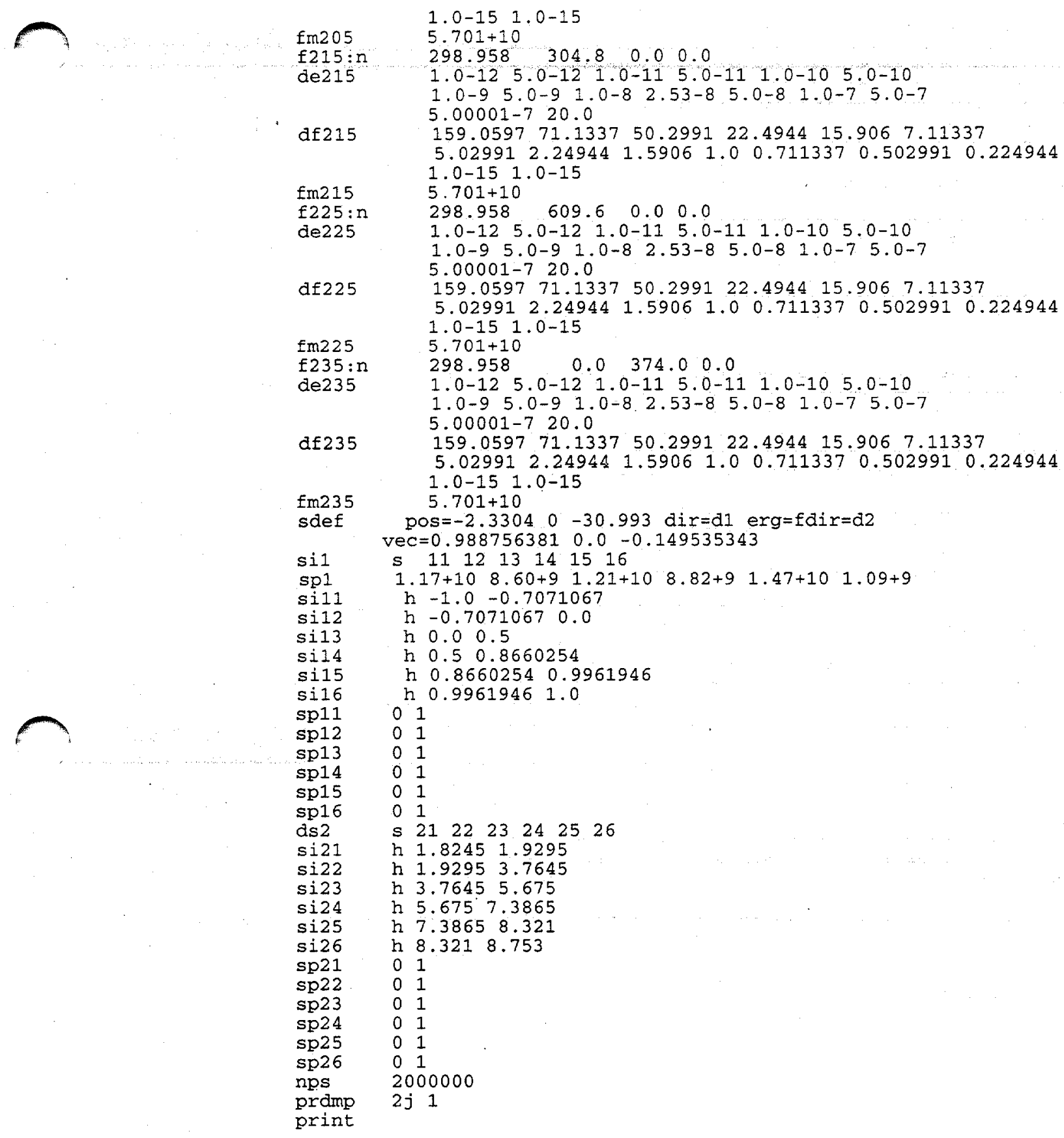


File for MCNP-4B calculation of the dose rate in the PFNA beam and across the truck lane and ORIGEN neutron spectra within the stationary collimator for the source in the maximum down position

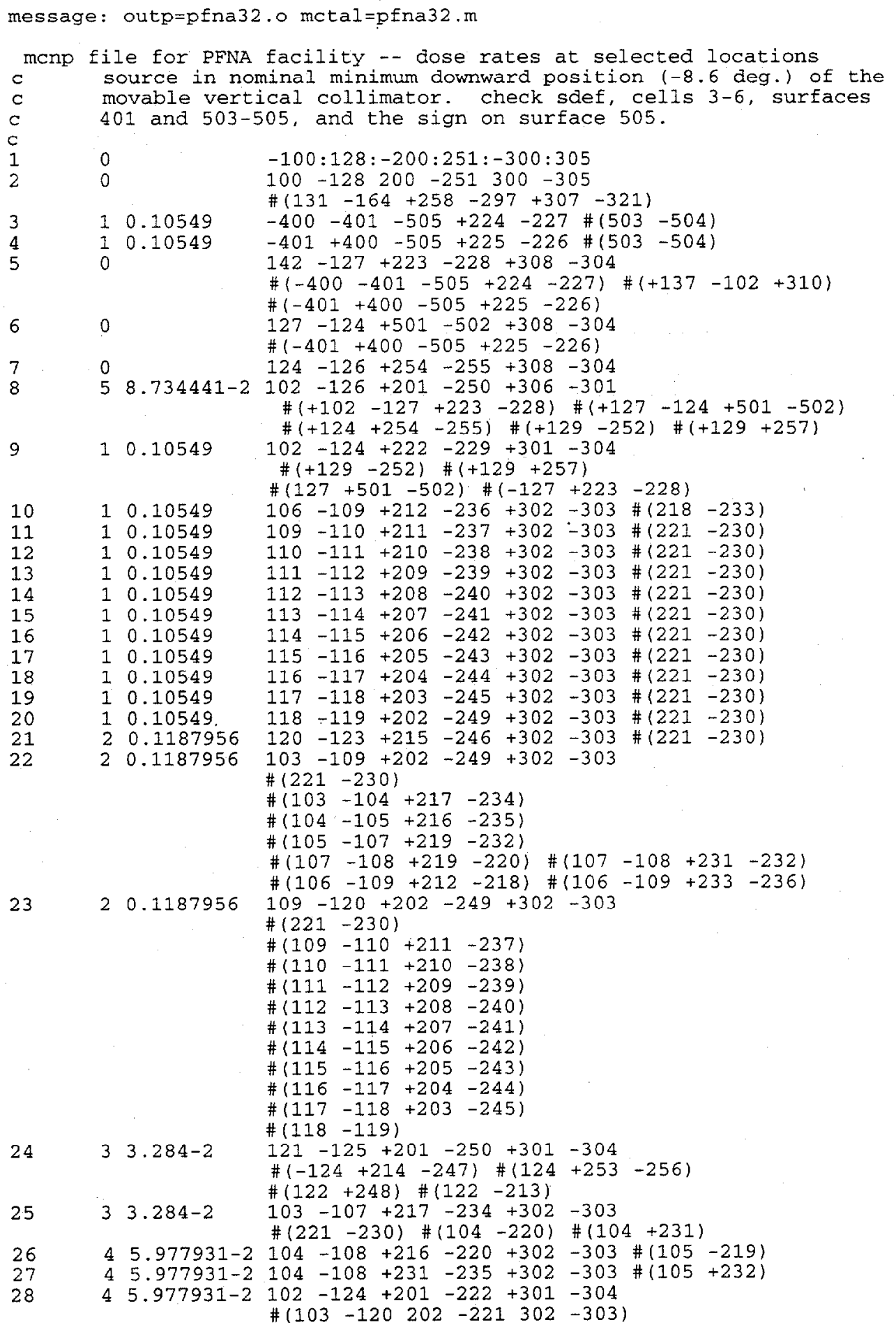


\#( $\left.\begin{array}{llllll}120 & -123 & 215 & -221 & 302 & -303\end{array}\right)$

\# $(121-214)$

$2945.977931-2 \quad 102-124+229-250+301-304$

$\#(103-120 \quad 230-243 \quad 302-303)$

\#( $\left.\begin{array}{llllll}120 & -123 & 230 & -245 & 302 & -303\end{array}\right)$

$\#(121+247)$

$3045.977931-2 \quad 124-126+252-254+301-304$

$31445.977931-2 \quad 124-126+255-257+301-304$

$32 \quad 0 \quad+167-168+274-281+317-318$

$33 \quad 0 \quad+165-166+270-285+308-310$

\# $(+167-168+274-281+317-318)$

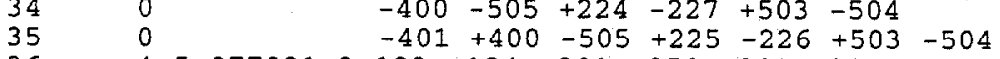

$3645.977931-2122-124+201-250+301-304$

$\#(213-248)$

$\#(129-252)$

$37 \quad 9-2.0 \quad+131-164+258-297+307-308$

$38 \quad 11-1.0 \quad+131-164+258-297+308-320$

$\begin{array}{lllll}39 & 12-7.86 & \#(+132-1.63+259-296) \\ & +131-164+258-297+320 & -321\end{array}$

$40+0 \quad+132-163+259-296+319-320$

$\#(+151-169+271-284+304-315)$

$\#(+151-160+270-271+312-314)$

$\#(+151-160+284-285+312-314)$

$\#(+157-159+274-281+313-315)$

$\#(+158-160+274-281-313)$

$41 \quad 1 \quad 0.10549 \quad \begin{array}{lll} & & \\ 4 & 151-169-157+274-281+314-316)\end{array}$

42. $10.10549+151-160+70-271+312-314$

$43 \quad 1 \quad 0.10549+151-160+284-285+312-314$

$44 \quad 1 \quad 0.10549+157-159+274-281+313-315$

$45 \quad 1 \quad 0.10549+158-160+274-281+308-313$

$46 \quad 1 \quad 0.10549+169-157+274-281+314-316$

$47 \quad 9-2.0+160-162+273-282+308-319$

$48 \quad 10-2.0^{\circ}+154-161+265-289+308-319$

$\begin{array}{lll}49 & 0 & \\ 50 & 10 & -2.0 \\ 51 & 10 & -2.0\end{array}$

$51 \quad 10-2.0$

530

$\# 47 \#(-160+267-286)$

$+154-160+267-286+308-319 \# 42 \# 43 \# 45$

$+155-156+259-260+308-319$

$+155-156+295-296+308-319$

$+154-155+259-296+308-319$

$\#(+265-289)$

$+155-163+259-296+308-319 \# 50$ \#51

$\#\left(\begin{array}{llllll}-161+265 & -289) & \#(+161-162+273-282\end{array}\right)$

$+153-154+295-296+308-319$

$+151-126+295-296+308-319$

$+151-126+259-260+308-319$

$+153-154+259-260+308-319$

$+151-152+266-272+308-304$

$+151-152+283-287+308-304$

$+151-154+259-296+308-319$

\#41 \#42 \#43 \#54 \#55 \#56 \#57 \#58 \#59

$\#(+165-166+270-285+308-310)$

$\#\left(\begin{array}{llll}-126+201 & -250 & -304\end{array}\right)$

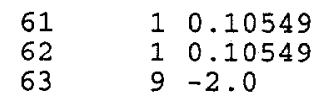

$+149-151+272-201+308-304$

$+149-151+250-283+308-304$

$+149-151+264-290+308-319$

$\#(+272-283)$

$64 \quad 10-2.0$

$+149-151+259-296+308-319$

\# $(+264-290)$

$\begin{array}{rrr}65 & 10 & -2.0 \\ 65 & 0 & \end{array}$

$+146-149+295-296+308-319$

$+132-149+259-296+308-319$

\#65 \# $(+133 \cdot-294)$

$67 \quad 10-2.0$

$\begin{array}{lll}68 & 10 & -2.0\end{array}$

$\begin{array}{rrr}69 & 10 & -2.0 \\ 70 & 0 & \end{array}$

$+133-149+259-294+308-319$

\# $\left(\begin{array}{lll}+142 & -260 & -322)\end{array} \#(+134+260-298)\right.$

$+135-149+261-262+308-319$

$+135-136+262-263+308-319$

$+137-149+268-293+308-311$

$\#(-138-290-310)$ \# $(-102+310)$

$\#(+138+269-290-310) \quad \#(+144+290-292-31)$

. 


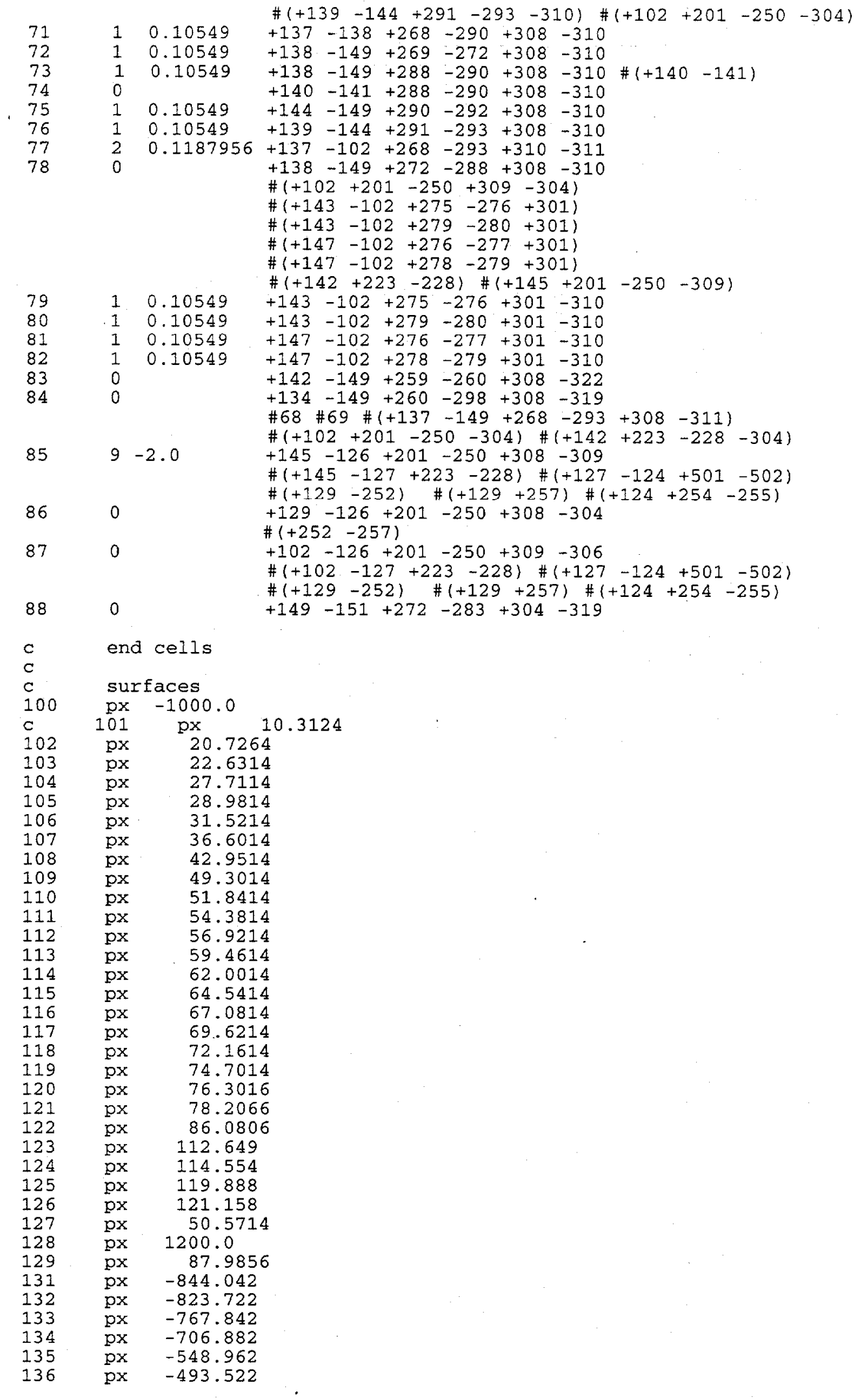




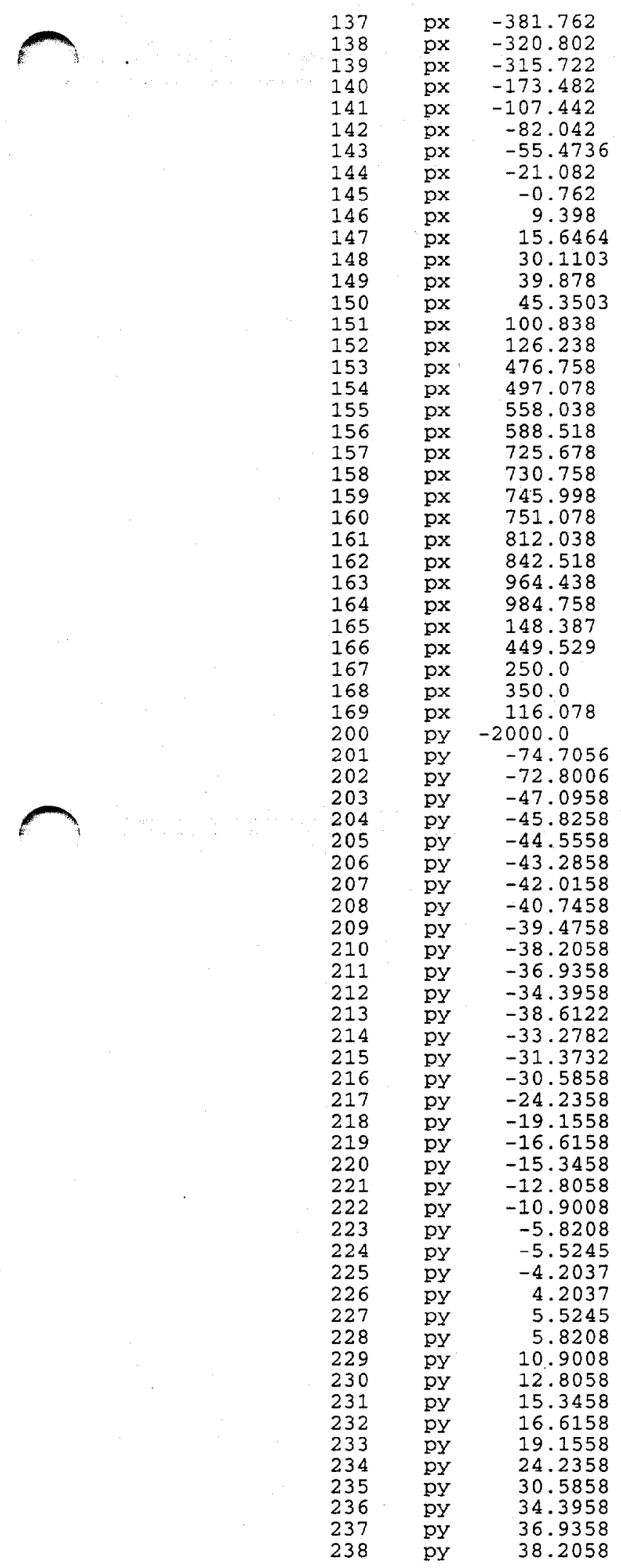




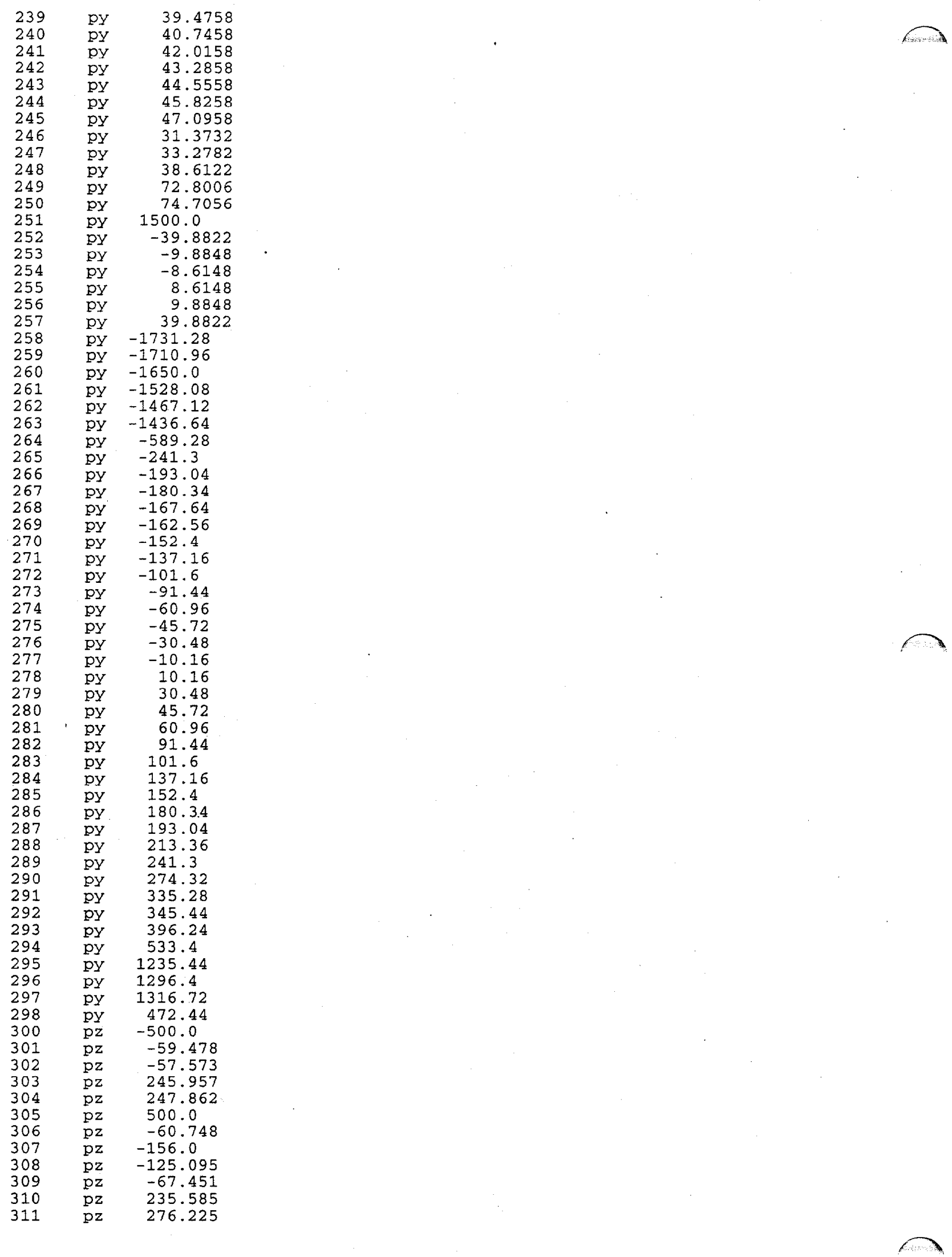




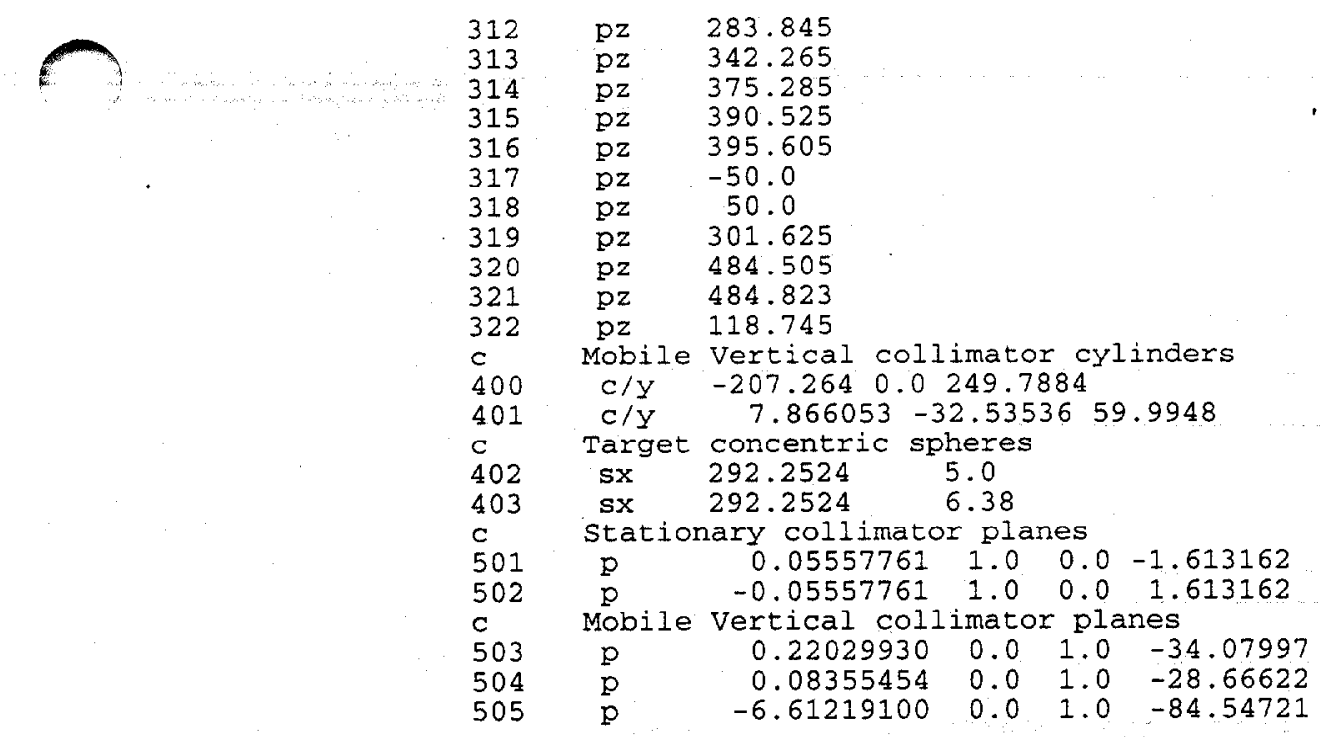

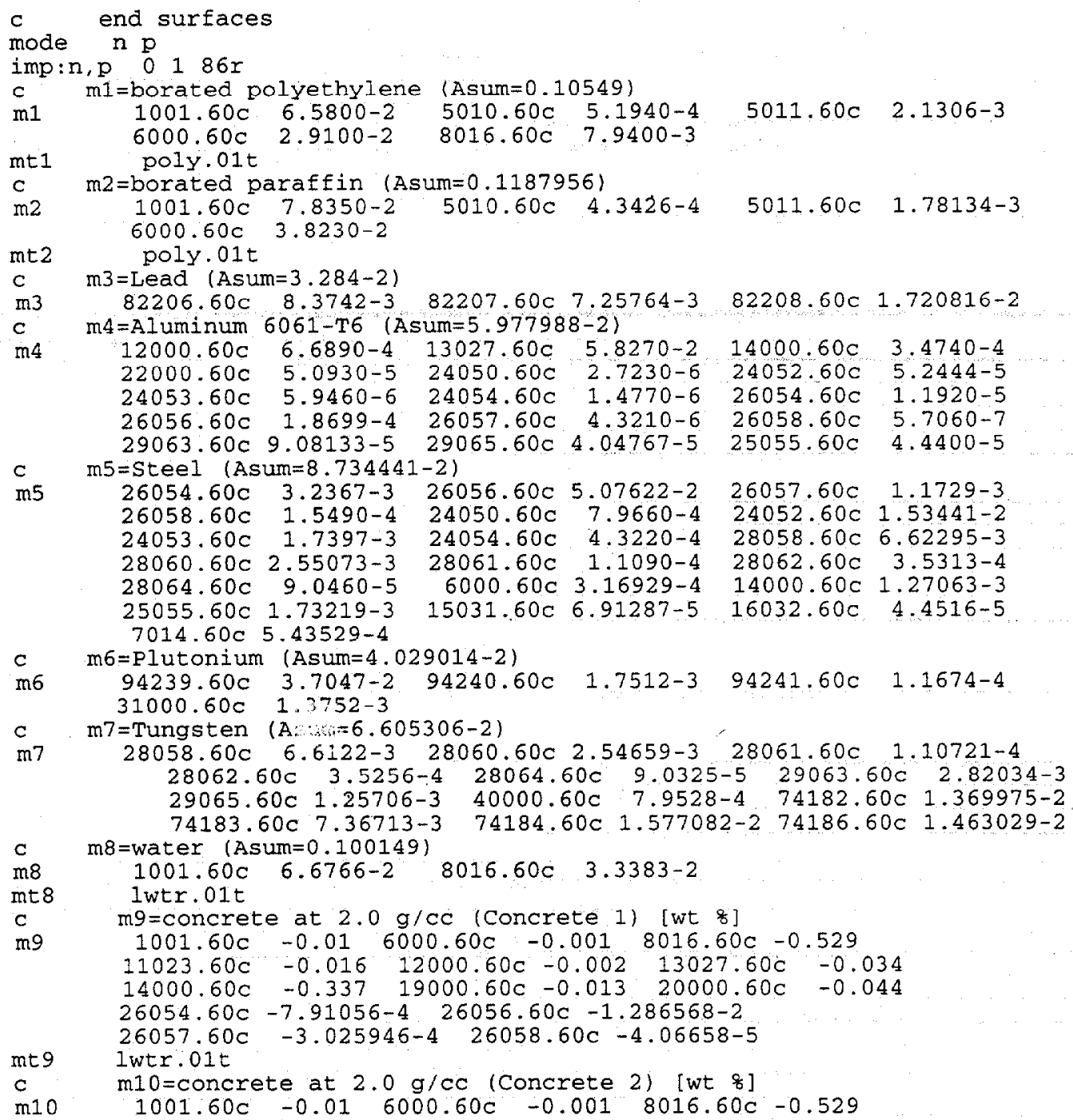




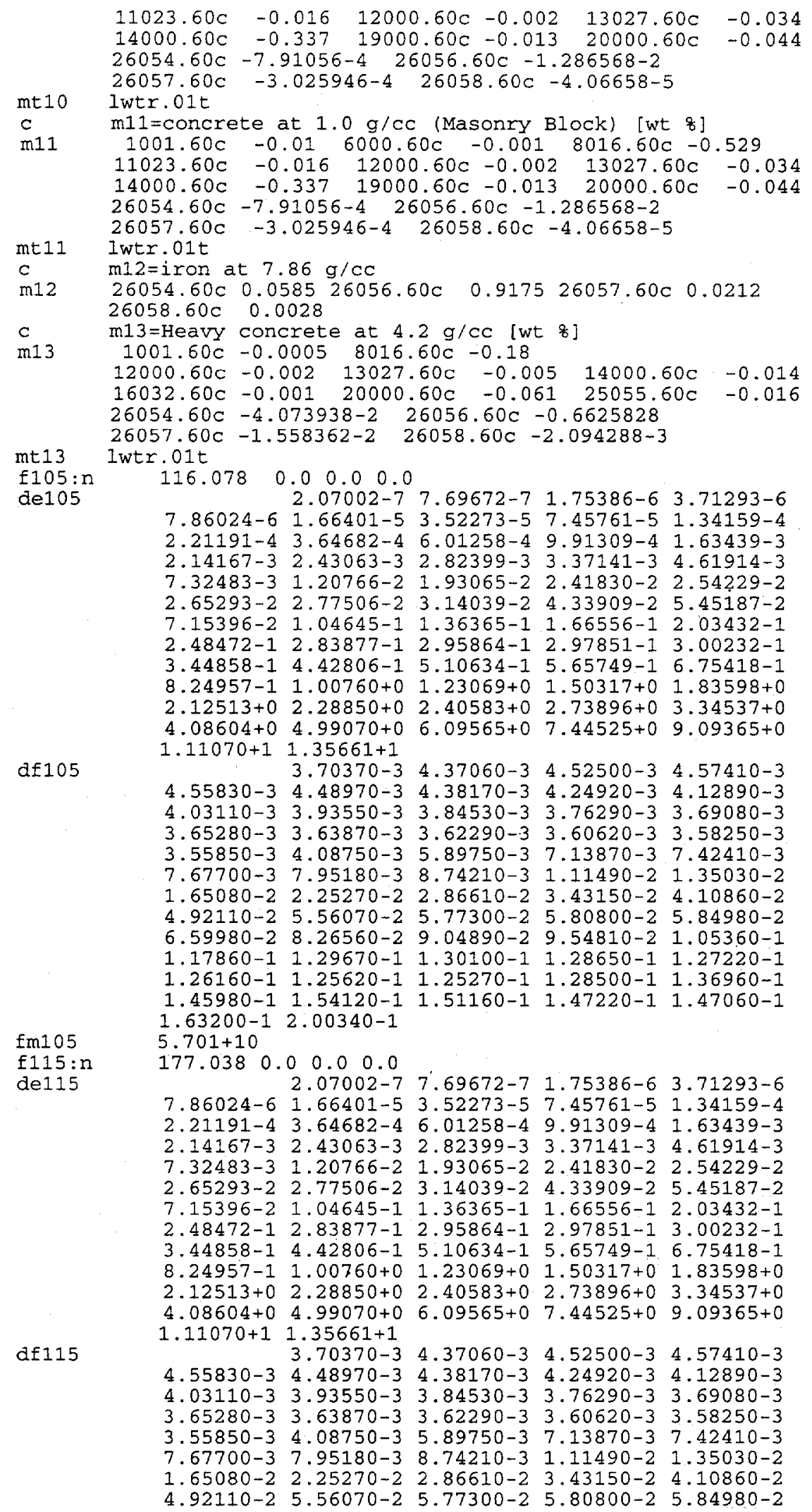


6.59980-2 $8.26560-2 \quad 9.04890-2 \quad 9.54810-2 \quad 1.05360-1$ $1.17860-11.29670-11.30100-1 \quad 1.28650-1.1 .27220-1$ $\begin{array}{lllllll}1.26160-1 & 1.25620-1 & 1.25270-1 & 1.28500-1 & 1.36960-1\end{array}$ $1.45980-1$ 1.54120-1 1.51160-1 $1.47220-1$ 1.47060-1

fm115 $125: n$ de125

df 125

$\operatorname{df} 135$

$\operatorname{fm} 135$ f145:n de145

\section{$1.63200-12.00340-1$}

$5.701+10$

$237.998 \quad 0.0 \quad 0.0 \quad 0.0$

$2.07002-7 \quad 7.69672-7 \quad 1.75386-6 \quad 3.71293-6$

$7.86024-6 \quad 1.66401-5 \quad 3.52273-5 \quad 7.45761-5 \quad 1.34159-4$

2.21191-4 $3.64682-4 \quad 6.01258-4 \quad 9.91309-4 \quad 1.63439-3$

$\begin{array}{llllll}2.14167-3 & 2.43063-3 & 2.82399-3 & 3.37141-3 & 4.61914-3\end{array}$

$7.32483-3 \quad 1.20766-2 \quad 1.93065-2 \quad 2.41830-2 \quad 2.54229-2$

2.65293-2 2.77506-2 $3.14039-2 \quad 4.33909-2 \quad 5.45187-2$

$7.15396-2$ 1.04645-1 1.36365-1 1.66556-1 2.03432-1

$2.48472-1 \quad 2.83877-1 \quad 2.95064-1 \quad 2.97051-1 \quad 3.00232-1$

3.44858-1 4.42806-1 5.10634-1 5.65749-1 6.75418-1

$8.24957-1 \quad 1.00760+0 \quad 1.23069+0 \quad 1.50317+0.1 .83598+0$

$2.12513+0 \quad 2.28850+0 \quad 2.40583+0 \quad 2.73896+0 \quad 3.34537+0$

$4.08604+0 \quad 4.99070+0 \quad 6.09565+0 \quad 7.44525+0 \quad 9.09365+0$ $1.11070+11.356 .51+1$

3. $70370-3 \quad 4.37060-3 \quad 4.52500-3 \quad 4.57410-3$

$\begin{array}{llllll}4.55830-3 & 4.48970-3 & 4.38170-3 & 4.24920-3 & 4.12890-3\end{array}$

$\begin{array}{llllll}4.03110-3 & 3.93550-3 & 3.84530-3 & 3.76290-3 & 3.69080-3\end{array}$

$\begin{array}{llllll}3.65280-3 & 3.63870-3 & 3.62290-3 & 3.60620-3 & 3.58250-3\end{array}$

$3.55850-3 \quad 4.08750-3 \quad 5.89750-3 \quad 7.13870-3 \quad 7.42410-3$

$7.67700-3 \quad 7.95180-3 \quad 8.74210-3 \quad 1.11490-2 \quad 1.35030-2$

$1.65080-2 \quad 2.25270-2 \quad 2.86610-2 \quad 3.43150-2 \quad 4.10860-2$

4.92110-2 $5.56070-2 \quad 5.77300-2 \quad 5.80800-2 \quad 5.84980-2$

6.59980-2 $8.26560-2 \quad 9.04890-2 \quad 9.54810-2 \quad 1.05360-1$

$1.17860-1 \quad 1.29670-1 \quad 1.30100-1 \quad 1.28650-1 \quad 1.27220-1$

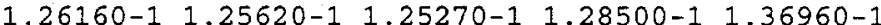

$1.45980-1$ 1.54120-1 $1.51160-1$ 1.47220-1 1.47060-1 $1.63200-12.00340-1$

$\operatorname{fm} 125 \quad 5.701+10$

E135:n $\quad 359.918 \quad 0.0 \quad 0.0 \quad 0.0$

$\begin{array}{lllll}2.07002-7 & 7.69672-7 & 1.75386-6 & 3.71293-6\end{array}$

$\begin{array}{llllll}7.86024-6 & 1.66401-5 & 3.52273-5 & 7.45761-5 & 1.34159-4\end{array}$

2.21191-4 3.64682-4 $6.01258-4 \quad 9.91309-4 \quad 1.63439-3$

$2.14167-3 \quad 2.43063-3 \quad 2.82399-3 \quad 3.37141-3 \quad 4.61914-3$

7.32483-3 $1.20766-2 \quad 1.93065-2 \quad 2.41830-2 \quad 2.54229-2$

2.65293-2 2.77506-2 $3.14039-2 \quad 4.33909-2 \quad 5.45187-2$

7.15396-2 $1.04645-1 \quad 1.36355-1 \quad 1.66556-1 \quad 2.03432-1$

$\begin{array}{lllll}2.48472-1 & 2.83877-1 & 2.95864-1 & 2.97851-1 & 3.00232-1\end{array}$

3.44858-1 4.42806-1 5.10634-1 $5.65749-1 \quad 6.75418-1$

$8.24957-1 \quad 1.00760+0 \quad 1.23069+0 \quad 1.50317+0 \quad 1.83598+0$

$\begin{array}{lllll}2.12513+0 & 2.28850+0 & 2.40583+0 & 2.73896+0 & 3.34537+0\end{array}$

$4.08604+0 \quad 4.99070+0 \quad 6.09565+0 \quad 7.44525+0 \quad 9.09365+0$

$1.11070+11.35661+1$

$3.70370-3 \quad 4.37060-3 \quad 4.52500-3 \quad 4.57410-3$

$4.55830-3 \quad 4.48970-3 \quad 4.38170-3 \quad 4.24920-3 \quad 4.12890-3$

$4.03110-3 \quad 3.93550-3 \quad 3.84530-3 \quad 3.76290-3 \quad 3.69080-3$

$3.65280-3 \quad 3.63870-3 \quad 3.62290-3 \quad 3.60620-3 \quad 3.58250-3$

$\begin{array}{llllll}3.55850-3 & 4.08750-3 & 5.89750-3 & 7.13870-3 & 7.42410-3\end{array}$

$\begin{array}{lllll}7.67700-3 & 7.95180-3 & 8.74210-3 & 1.11490-2 & 1.35030-2\end{array}$

$1.65080-2 \quad 2.25270-2 \quad 2.86610-2 \quad 3.43150-2 \quad 1.10860-2$

4.92110-2 $5.56070-2 \quad 5.77300-2 \quad 5.80800-2 \quad 5.84980-2$

$6.59980-2 \quad 8.26560-2 \quad 9.04890-2 \quad 9.54810-2 \quad 1.05360-1$

$1.17860-1$ 1.29670-1 $1.30100-1 \quad 1.28650-1 \quad 1.27220-1$

$1.26160-1 \quad 1.25620-1 \quad 1.25270-1 \quad 1.28500-1 \quad 1.36960-1$

$1.45980-1$ 1.54120-1 $1.51160-1 \quad 1.47220-1 \quad 1.47060-1$ $1.63200-1 \quad 2.00340-1$

$5.701+10$

$420.878 \quad 0.0 \quad 0.0 \quad 0.0$

$2.07002-7 \quad 7.69672-7 \quad 1.75386-6 \quad 3.71293-6$

$7.86024-6$ 1.66401-5 $3.52273-5 \quad 7.45761-51.34159-4$

$2.21191-4 \quad 3.64682-4 \cdot 6.01258-4 \quad 9.91309-4 \quad 1.63439-3$

$2.14167-3 \quad 2.43063-3 \quad 2.82399-3 \quad 3.37141-3 \quad 4.61914-3$

$7.32483-3$ 1.20766-2 $1.93065-2 \quad 2.41830-2 \quad 2.54229-2$

2.65293-2 $2.77506-2 \quad 3.14039-2 \quad 4.33909-2 \quad 5.45187-2$

$7.15396-2$ 1.04645-1 $1.36365-1 \quad 1.66556-1 \quad 2.03432-1$

2.48472-1 $2.83877-1 \quad 2.95864-1 \quad 2.97851-1 \quad 3.00232-1$

3.44858-1 4.42806-1 5.10634-1 5.65749-1 6.75418-1 
8.24957-1 $1.00760+0 \quad 1.23069+0 \quad 1.50317+0 \quad 1.83598+0$ $2.12513+0 \quad 2.28850+0 \quad 2.40583+0 \quad 2.73896+0 \quad 3.34537+0$ $4.08604+0 \quad 4.99070+0 \quad 6.09565+0 \quad 7.44525+0 \quad 9.09365+0$ $1.11070+11.35661+1$

df145 $3.70370-3 \quad 4.37060-3 \quad 4.52500-3 \quad 4.57410-3$ $4.55830-3 \quad 4.48970-3 \quad 4.38170-3 \quad 4.24920-3 \quad 4.12890-3$ $4.03110-3 \quad 3.93550-3 \quad 3.84530-3 \quad 3.76290-3 \quad 3.69080-3$ $3.65280-3 \quad 3.63870-3 \quad 3.62290-3 \quad 3.60620-3 \quad 3.58250-3$ $\begin{array}{llllll}3.55850-3 & 4.08750-3 & 5.89750-3 & 7.13870-3 & 7.42410-3\end{array}$ $\begin{array}{lllll}7.67700-3 & 7.95180-3 & 8.74210-3 & 1.11490-2 & 1.35030-2\end{array}$ $1.65080-2 \quad 2.25270-2 \quad 2.86610-2 \quad 3.43150-2 \quad 4.10860-2$ $4.92110-2 \quad 5.56070-2 \quad 5.77300-2 \quad 5.80800-2 \quad 5.84980-2$ $6.59980-2 \quad 8.26560-2 \quad 9.04890-2 \quad 9.54810-2 \quad 1.05360-1$ $1.17860-1 \quad 1.29670-1 \quad 1.30100-1 \quad 1.28650-11.27220-1$

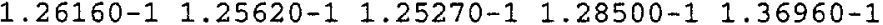
$1.45980-1$ 1.54120-1 $1.51160-1 \quad 1.47220-11.47060-1$ 1.63200-1 2.00340-1

$\operatorname{fm} 145$ $5.701+10$

E155:n de155

$\mathrm{d} \mp 155$

$\operatorname{fm} 155$

f165:n

e165

fm165

f175:n

e175

$\operatorname{fm} 175$

de175

$\operatorname{df} 175$ 481.838

$0.0 \quad 0.0 \quad 0.0$

$2.07002-7 \quad 7.69672-7 \quad 1.75386-6 \quad 3.71293-6$ $7.86024-6 \quad 1.66401-5 \quad 3.52273-5 \quad 7.45761-5 \quad 1.34159-4$ 2.21191-4 $3.64682-4 \quad 6.01258-4 \quad 9.91309-4$ 1.63439-3 $\begin{array}{llllll}2.14167-3 & 2.43063-3 & 2.82399-3 & 3.37141-3 & 4.61914-3\end{array}$ 7.32483-3 $1.20765-2 \quad 1.93065-2 \quad 2.41830-2 \quad 2.54229-2$ 2.65293-2 2.77506-2 $3.14039-2 \quad 4.33909-2 \quad 5.45187-2$ 7.15396-2 $1.04645-1 \quad 1.36365-1 \quad 1.65556-1 \quad 2.03432-1$ 2.48472-1 2.83877-1 $2.95864-1 \quad 2.97851-1 \quad 3.00232-1$ $\begin{array}{llllll}3.44858-1 & 4.42806-1 & 5.10634-1 & 5.65749-1 & 6.75418-1\end{array}$ $8.24957-1 \quad 1.00760+0 \quad 1.23069+0 \quad 1.50317+0 \quad 1.83598+0$ $2.12513+0 \quad 2.28850+0 \quad 2.40583+0 \quad 2.73896+0 \quad 3.34537+0$ $4.08604+0 \quad 4.99070+0 \quad 6.09565+0 \quad 7.44525+0 \quad 9.09365+0$ $1.11070+1 \quad 1.35661+1$

$3.70370-3 \quad 4.37060-3 \quad 4.52500-3 \quad 4.57410-3$ $\begin{array}{llllll}4.55830-3 & 4.48970-3 & 4.38170-3 & 4.24920-3 & 4.12890-3\end{array}$ $4.03110-3 \quad 3.93550-3 \quad 3.84530-3 \quad 3.76290-3 \quad 3.69080-3$ $3.65280-3 \quad 3.63870-3 \quad 3.62290-3 \quad 3.60620-3 \quad 3.58250-3$ $3.55850-3 \quad 4.08750-3 \quad 5.89750-3 \quad 7.13870-3 \quad 7.42410-3$ $7.67700-3 \quad 7: 95180-3 \quad 8.74210-3 \quad 1.11490-2 \quad 1.35030-2$ $1.65080-2 \quad 2.25270-2 \quad 2.86610-2 \quad 3.43150-2 \quad 4.10860-2$ 4.92110-2 $5.56070-2 \quad 5.77300-2 \quad 5.80800-2 \quad 5.84980-2$ $6.59980-2$ 8.26560-2 $9.04890-2$ 9.54810-2 $1.05360-1$ $\begin{array}{lllll}1.17860-1 & 1.29670-1 & 1.30100-1 & 1.28650-1 & 1.27220-1\end{array}$ $1.26160-1 \quad 1.25620-1 \quad 1.25270-1 \quad 1.28500-1 \quad 1.36960-1$ $1.45980-11.54120-1 \quad 1.51160-1 \quad 1.47220-1 \quad 1.47060-1$ 1.63200-1 2.00340-1

$5.701+10$

$116.078 \quad 0.0 \quad 0.0 \quad 0.0$

$5-7 \quad 1.0 \quad 30.0$

$5.701+10$

$\begin{array}{lllll}116.078 & 0.0 & 0.0 & 0.0\end{array}$

$5-7 \quad 1.0 \quad 30.0$

$5.701+10$

1. $0-12 \quad 5 \cdot 0-12$ 1. $0-11 \quad 5 \cdot 0-11$ 1. $0-10,5 \cdot 0-10$

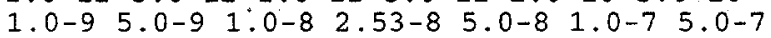

$5.00001-720.0$

$\begin{array}{llllll}159.0597 & 71.1337 & 50.2991 & 22.4944 & 15.906 & 7.11337\end{array}$

$\begin{array}{llllllll}5.02991 & 2.24944 & 1.5906 & 1.0 & 0.711337 & 0.502991 & 0.224944\end{array}$ $1.0-15 \quad 1.0-15$

f205:p

$\begin{array}{llll}116.078 & 0.0 & 0.0 & 0.0\end{array}$

de205

df205

$1.50000-2 \quad 3.25000-2 \quad 5.75000-2 \quad 8.50000-2$ $\begin{array}{llllll}1.25000-1 & 2.25000-1 & 3.50000-1 & 4.55000-1 & 5.55000-1\end{array}$ $6.50000-18.50000-1 \quad 1.25000+0 \quad 1.75000+0 \quad 2.25000+0$ $2.75000+0 \quad 3.50000+0 \quad 4.50000+0 \quad 5.50000+0 \quad 6.50000+0$ $7.25000+0 \quad 7.75000+0 \quad 9.00000+0 \quad 1.20000+1$ $\begin{array}{llll}2.14390-3 & 5.77600-4 & 2.71850-4 & 2.68170-4\end{array}$ $3.27670-4 \quad 5.66760-4 \quad 8.75940-4 \quad 1.08450-3 \quad 1.27970-3$ $\begin{array}{llllll}1.44170-3 & 1.75630-3 & 2.31560-3 & 2.92700-3 & 3.46860-3\end{array}$ $\begin{array}{lllll}3.95960-3 & 4.62210-3 & 5.41370-3 & 6.19090-3 & 6.92650-3\end{array}$ $7.47830-3 \quad 7.84680-3 \quad 8.77160-3 \quad 1.10200-2$

fm205 $\quad 5.701+10$

f215:p $\quad 177.038 \quad 0.0 \quad 0.0 \quad 0.0$

de $215 \quad 1.50000-2 \quad 3.25000-2 \quad 5.75000-2 \quad 8.50000-2$ 
$1.25000-1 \quad 2.25000-1 \quad 3.50000-1 \quad 4.55000-1 \quad 5.55000-1$ $6.50000-1 \quad 8.50000-1 \quad 1.25000+0 \quad 1.75000+0 \quad 2.25000+0$ $2.75000+0 \quad 3.50000+0 \quad 4.50000+0 \quad 5.50000+0 \quad 6.50000+0$ $7.25000+07.75000+09.00000+01.20000+1$

dE215 $2.14390-3 \quad 5.77600-42.71850-4 \quad 2.68170-4$ $3.27670-4 \quad 5.66760-4 \quad 8.75940-4 \quad 1.08450-3 \quad 1.27970-3$ $1.44170-3 \quad 1.75630-3 \quad 2.31560-3 \quad 2.92700-3 \quad 3.46860-3$ $\begin{array}{llllll}3.95960-3 & 4.62210-3 & 5.41370-3 & 6.19090-3 & 6.92650-3\end{array}$ $7.47830-3 \quad 7.84680-3 \quad 8.77160-3 \quad 1.10200-2$

Em215 $5.701+10$

f225:p

de225

$237.998 \quad 0.0 \quad 0.0 \quad 0.0$

$1.50000-2 \quad 3.25000-25.75000-2.8 .50000-2$

$\begin{array}{llllll}1.25000-1 & 2.25000-1 & 3.50000-1 & 4.55000-1 & 5.55000-1\end{array}$

$6.50000-1 \quad 8.50000-1 \quad 1.25000+0 \quad 1.75000+0 \quad 2.25000+0$ $2.75000+0 \quad 3.50000+0 \quad 4.50000+0 \quad 5.50000+0 \quad 6.50000+0$ $7.25000+0 \quad 7.75000+0 \quad 9.00000+01.20000+1$

df225 $2.14390-3 \quad 5.77600-42.71850-42.68170-4$ $3.27670-4 \quad 5.66760-4 \quad 8.75940-4 \quad 1.08450-3 \quad 1.27970-3$ $\begin{array}{lllll}1.44170-3 & 1.75630-3 & 2.31560-3 & 2.92700-3 & 3.46860-3\end{array}$ $\begin{array}{llllll}3.95960-3 & 4.62210-3 & 5.41370-3 & 6.19090-3 & 6.92650-3\end{array}$ $7.47830-3 \quad 7.84680-3 \quad 8.77160-3 \quad 1.10200-2$

$\operatorname{fm} 225 \quad 5.701+10$

f235:p $\quad 359.918 \quad 0.0 \quad 0.0 \quad 0.0$

de 235

$1.50000-2 \quad 3.25000-2 \quad 5.75000-2 \quad 8.50000-2$

$1.25000-1 \quad 2.25000-1 \quad 3.50000-1 \quad 4.55000-1 \quad 5.55000-1$ $6.50000-18.50000-1 \quad 1.25000+0.1 .75000+0 \quad 2.25000+0$ $2.75000+0 \quad 3.50000+0 \quad 4.50000+0 \quad 5.50000+0 \quad 6.50000+0$ $7.25000+07.75000+09.00000+01.20000+1$

$d £ 235$ $2.14390-3 \quad 5.77600-4 \quad 2.71850-4 \quad 2.68170-4$ $3.27670-4 \quad 5.66760-4 \quad 8.75940-4 \quad 1.08450-3 \quad 1.2797 .0-3$ $1.44170-3$
3 $\begin{array}{llllll}3.95960-3 & 4.62210-3 & 5.41370-3 & 6.19090-3 & 6.92650-3\end{array}$ $7.47830-3 \quad 7.84680-3 \quad 8.77160-3 \quad 1.10200-2$

fm 235 $5.701+10$

f245:p

de 245

$\operatorname{df} 245$

$\begin{array}{llll}120.878 & 0.0 & 0.0 & 0.0\end{array}$

$1.50000-2 \quad 3.25000-2 \quad 5.75000-2 \quad 8.50000-2$ $\begin{array}{lllll}1.25000-1 & 2.25000-1 & 3.50000-1 & 4.55000-1 & 5.55000-1\end{array}$ $6.50000-1 \quad 8.50000-1 \quad 1.25000+0 \quad 1.75000+0 \quad 2.25000+0$ $2.75000+0 \quad 3.50000+0 \quad 4.50000+0.5 .50000+0 \quad 6.50000+0$ $7.25000+0 \quad 7.75000+0 \quad 9.00000+0 \quad 1.20000+1$

$2.14390-3 \quad 5.77600-4 \quad 2.71850-4 \quad 2.68170-4$ $3.27670-4 \quad 5.66760-4 \quad 8.75940-4 \quad 1.08450-3 \quad 1.27970-3$ $\begin{array}{lllll}1.44170-3 & 1.75630-3 & 2.31560-3 & 2.92700-3 & 3.46860-3\end{array}$ $3.95960-3 \quad 4.62210-3 \quad 5.41370-3 \quad 6.19090-3 \quad 6.92650-3$ $\begin{array}{llll}7.47830-3 & 7.84680-3 & 8.77160-3 & 1.10200-2\end{array}$

fm245 $\quad 5.701+10$

f255:p $\quad 481.838 \quad 0.0 \quad 0.0 \quad 0.0$

de255 $1.50000-2 \quad 3.25000-2 \quad 5.75000-28.50000-2$

$1.25000-1 \quad 2.25000-1 \quad 3.50000-1 \quad 4.55000-1 \quad 5.55000-1$ $6.50000-18.50000-1 \quad 1.25000+01.75000+0 \quad 2.25000+0$ $2.75000+0 \quad 3.50000+0 \quad 4.50000+0 \quad 5.50000+0 \quad 6.50000+0$ $7.25000+07.75000+0 \quad 9.00000+0 \quad 1.20000+1$ $2.14390-3 \quad 5.77600-4 \quad 2.71850-4 \quad 2.68170-4$

df 255 $3.27670-4 \quad 5.66760-4 \quad 8.75940-4 \quad 1.08450-3 \quad 1.27970-3$ $1.44170-3 \quad 1.75630-3 \quad 2.31560-3 \quad 2.92700-3 \quad 3.46860-3$ $3.95960-3 \quad 4.62210-3 \quad 5.41370-3 \quad 6.19090-3 \quad 6.92650-3$ $7.47830-3 \quad 7.84680-3 \quad 8.77160-3 \quad 1.10200-2$

fm255 $5.701+10$

sdef pos $=-2.3304 \quad 0 \quad-30.993$ dir=d1 erg=fdir=d2

sil vec $=0.988756381 \quad 0.0 \quad-0$

$\begin{array}{llllllll}\mathrm{sp1} & 1.17+10 & 8.60+9 & 1.21+10 & 8.82+9 & 1.47+10 & 1.09+9\end{array}$

si11 $h-1.0-0.7071067$

si12 h $-0.7071067 \quad 0.0$

si13 h 0.00 .5

$\begin{array}{llll}\text { si14 } & \text { h } & 0.5 & 0.8660254\end{array}$

sil5 h $0.8660254 \quad 0.9961946$

sil6 h 0.99619461 .0

spII

$\operatorname{sp} 12$

sp13

01

01

$0 \begin{array}{ll}0 & 1 \\ 0 & 1\end{array}$

01 


\begin{tabular}{|c|c|}
\hline $\operatorname{sp15}$ & 01 \\
\hline $\operatorname{sp16}$ & 01 \\
\hline ds2 & $\begin{array}{lllllll}\mathbf{S} & 21 & 22 & 23 & 24 & 25 & 26\end{array}$ \\
\hline $\operatorname{si21}$ & h $1.8245 \quad 1.9295$ \\
\hline $\operatorname{si22}$ & h $1.9295 \quad 3.7645$ \\
\hline $\operatorname{si23}$ & h $3.7645 \quad 5.675$ \\
\hline $\operatorname{si24}$ & h $5.675 \quad 7.3865$ \\
\hline $\operatorname{si25}$ & h $7.3865 \quad 8.321$ \\
\hline $\operatorname{si26}$ & h 8.3218 .753 \\
\hline $\operatorname{sp21}$ & 01 \\
\hline $\operatorname{sp} 22$ & 01 \\
\hline $\operatorname{sp23}$ & 01 \\
\hline $\operatorname{sp24}$ & 01 \\
\hline $\operatorname{sp25}$ & 01 \\
\hline $\operatorname{sp26}$ & 01 \\
\hline nps & 2000000 \\
\hline ramp & $2 j 1$ \\
\hline
\end{tabular}


File for MCNP-4B calculation of ORIGEN neutron spectra within the stationary collimator for the source in the horizontal position

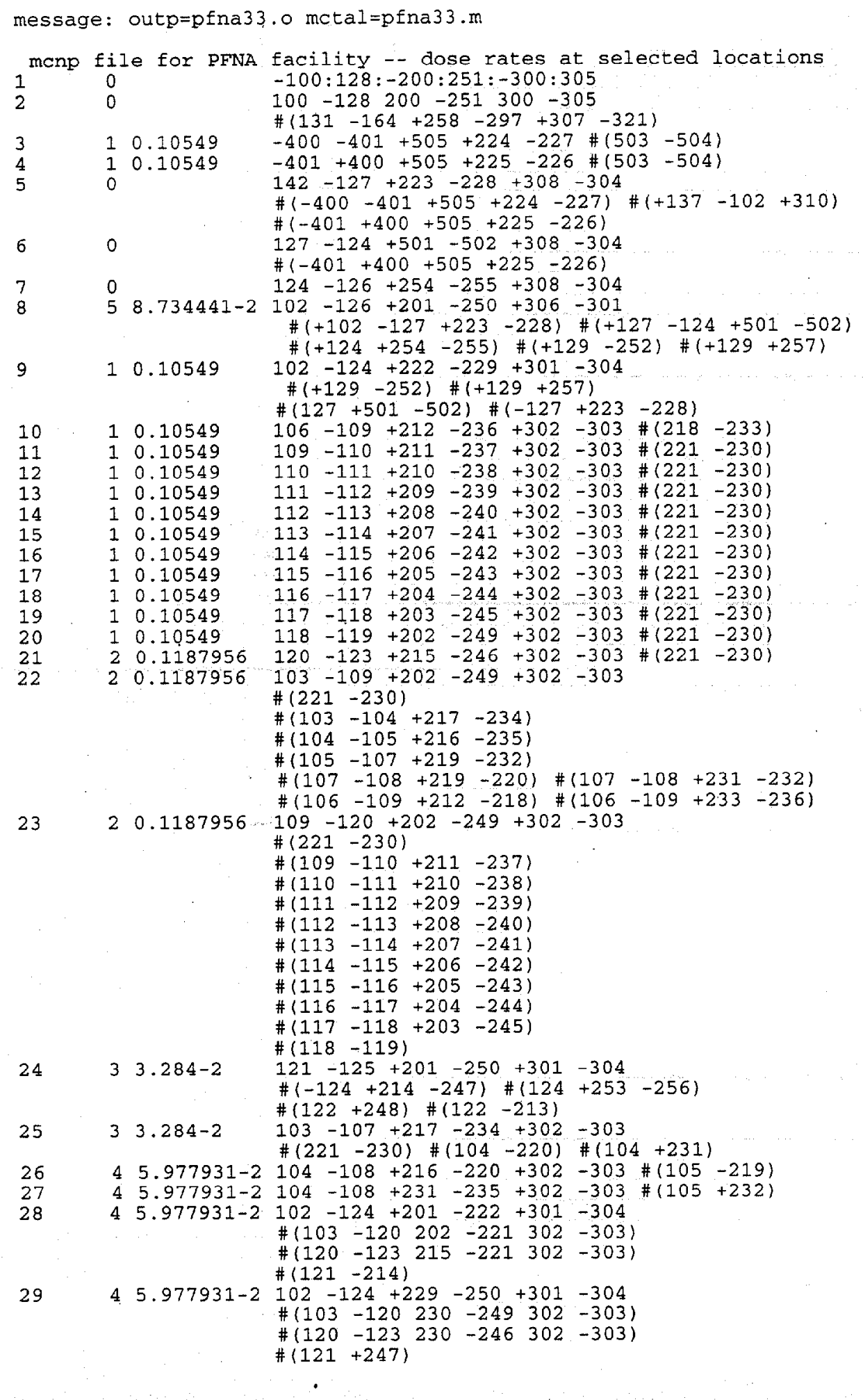




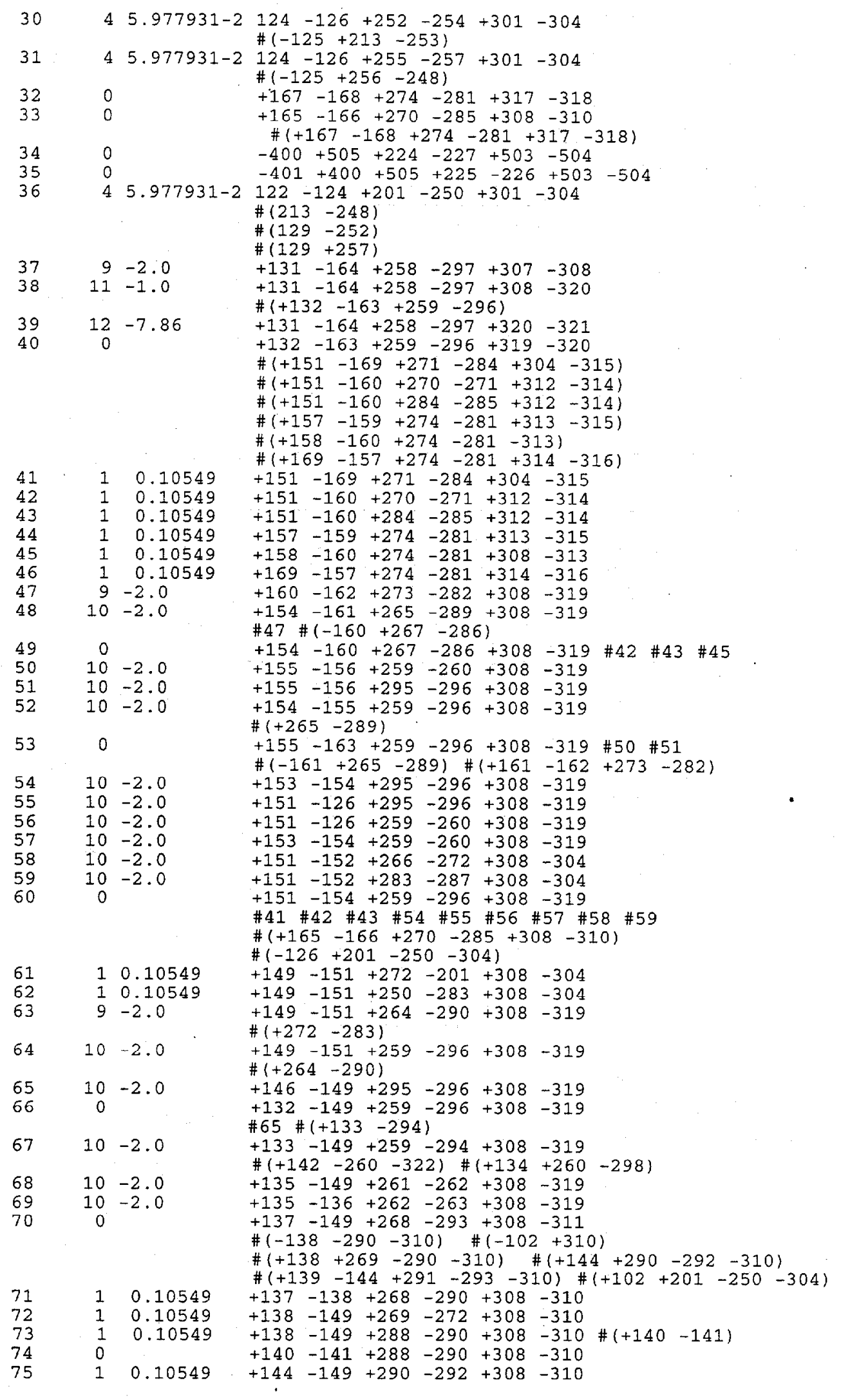




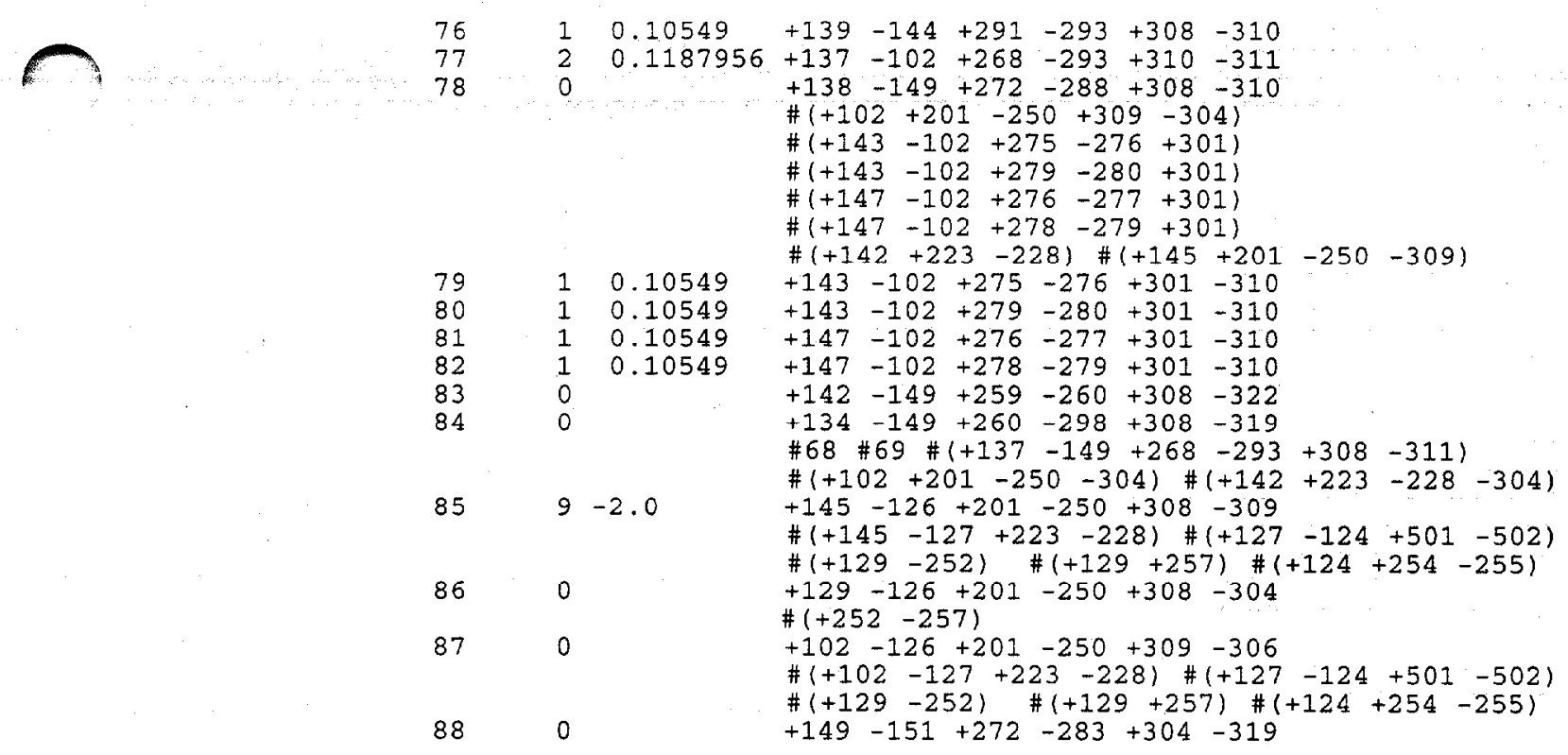

c end cells

c surfaces

100 px -1000.0

$\begin{array}{lll}101 & 10.3124\end{array}$

102 px 20.7264

103 px 22.6314

104 px 27.7114

105 px 28.9814

106 px 31.5214

107 px 36.6014 .

px 42.9514

109 px 49.3014

$110 \quad \mathrm{px} \quad 51.8414$

$111 \mathrm{px} \quad 54.3814$

112 px 56.9214

113 px 59.4614

114 px 62.0014

115 px 64.5414

116 px $\quad 67.0814$

$117 \quad \mathrm{px} \quad 69.6214$

118 px 72.1614

119 px 74.7014

$120 \quad \mathrm{px} \quad 76.3016$

121 px 78.2066

$122 \quad \mathrm{px} \quad 86.0806$

123 px 112.649

124 px 114.554

$125 \quad \mathrm{px} \quad 119.888$

126 px 121.158

127 px $\quad 50.5714$

128 px 1200.0

129 px 87.9856

$131 \quad \mathrm{px} \quad-844.042$

$132 \quad \mathrm{px} \quad-823.722$

$133 \quad \mathrm{px} \quad-767.842$

$134 \quad \mathrm{px} \quad-706.882$

$135 \quad \mathrm{px} \quad-548.962$

136 px -493.522

137 ox -381.762

$138 \quad \mathrm{px} \quad-320.802$

139 px -315.722

$140 \quad \mathrm{px} \quad-173.482$

141 px -107.442

142 px -82.042 


\begin{tabular}{|c|c|c|}
\hline 143 & $\mathrm{px}$ & -55.4736 \\
\hline 144 & $\mathrm{px}$ & -21.082 \\
\hline 145 & $p x$ & -0.762 \\
\hline 146 & $\mathrm{px}$ & 9.398 \\
\hline 147 & $\mathrm{px}$ & 15.6464 \\
\hline 148 & $p x$ & 30.1103 \\
\hline 149 & $\mathrm{px}$ & 39.878 \\
\hline 150 & $p x$ & 45.3503 \\
\hline 151 & $p x$ & 100.838 \\
\hline 152 & $p x$ & 126.238 \\
\hline 153 & px & 476.758 \\
\hline 154 & px & 497.078 \\
\hline 155 & $p x$ & 558.038 \\
\hline 156 & $\mathrm{px}$ & 588.518 \\
\hline 157 & $p x$ & 725.678 \\
\hline 158 & $\mathrm{px}$ & 730.758 \\
\hline 159 & $\mathrm{px}$ & 745.998 \\
\hline 160 & $\mathrm{px}$ & 751.078 \\
\hline 161 & $\mathrm{px}$ & 812.038 \\
\hline 162 & $\mathrm{px}$ & 842.518 \\
\hline 163 & $\mathrm{px}$ & 964.438 \\
\hline 164 & $p x$ & 984.758 \\
\hline 165 & px & 148.387 \\
\hline 166 & $\mathrm{px}$ & 449.529 \\
\hline 167 & $\mathrm{px}$ & 250.0 \\
\hline 168 & $\mathrm{px}$ & 350.0 \\
\hline 169 & $\mathrm{px}$ & 116.078 \\
\hline 200 & py & -2000.0 \\
\hline 201 & py & -74.7056 \\
\hline 202 & py & -72.8006 \\
\hline 203 & py & -47.0958 \\
\hline 204 & py & -45.8258 \\
\hline 205 & py & -44.5558 \\
\hline 206 & py & -43.2858 \\
\hline 207 & py & -42.0158 \\
\hline 208 & py & -40.7458 \\
\hline 209 & py & -39.4758 \\
\hline 210 & $\mathrm{py}$ & -38.2058 \\
\hline 211 & py & -36.9358 \\
\hline 212 & py & -34.3958 \\
\hline 213 & py & -38.6122 \\
\hline 214 & py & -33.2782 \\
\hline 215 & py & -31.3732 \\
\hline 216 & py & -30.5858 \\
\hline 217 & py & -24.2358 \\
\hline 218 & py & -19.1558 \\
\hline 219 & py & -16.6158 \\
\hline 220 & py & -15.3458 \\
\hline 221 & py & -12.8058 \\
\hline 222 & py & -10.9008 \\
\hline 223 & py & -5.8208 \\
\hline 224 & py & -5.5245 \\
\hline 225 & py & -4.2037 \\
\hline 226 & py & 4.2037 \\
\hline 227 & py & 5.5245 \\
\hline 228 & py & 5.8208 \\
\hline 229 & py & 10.9008 \\
\hline 230 & py & 12.8058 \\
\hline 231 & py & 15.3458 \\
\hline 232 & py & 16.6158 \\
\hline 233 & py & 19.1558 \\
\hline 234 & py & 24.2358 \\
\hline 235 & py & 30.5858 \\
\hline 236 & py & 34.3958 \\
\hline 237 & py & 36.9358 \\
\hline 238 & py & 38.2058 \\
\hline 239 & py & 39.4758 \\
\hline 240 & py & 40.7458 \\
\hline 241 & py & 42.0158 \\
\hline 242 & py & 43.2858 \\
\hline 243 & py & 44.5558 \\
\hline 244 & $\mathrm{py}$ & 45.8258 \\
\hline
\end{tabular}




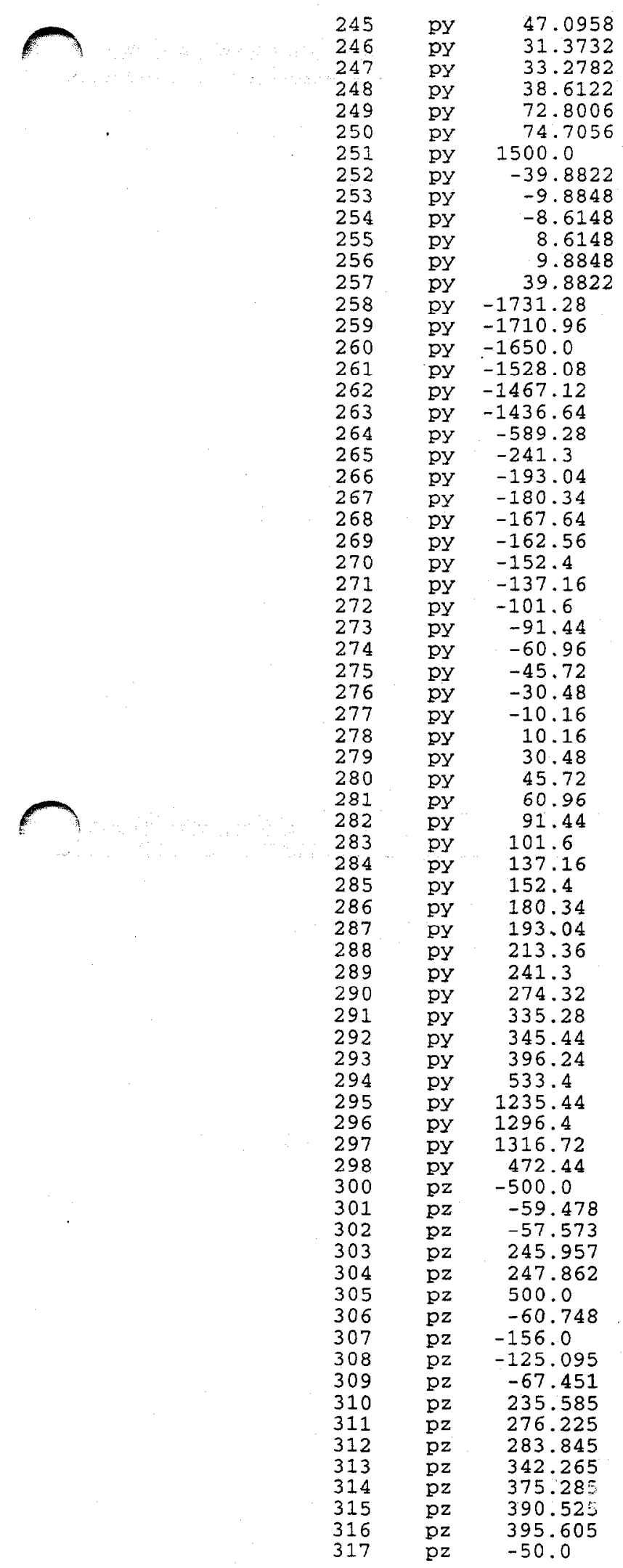




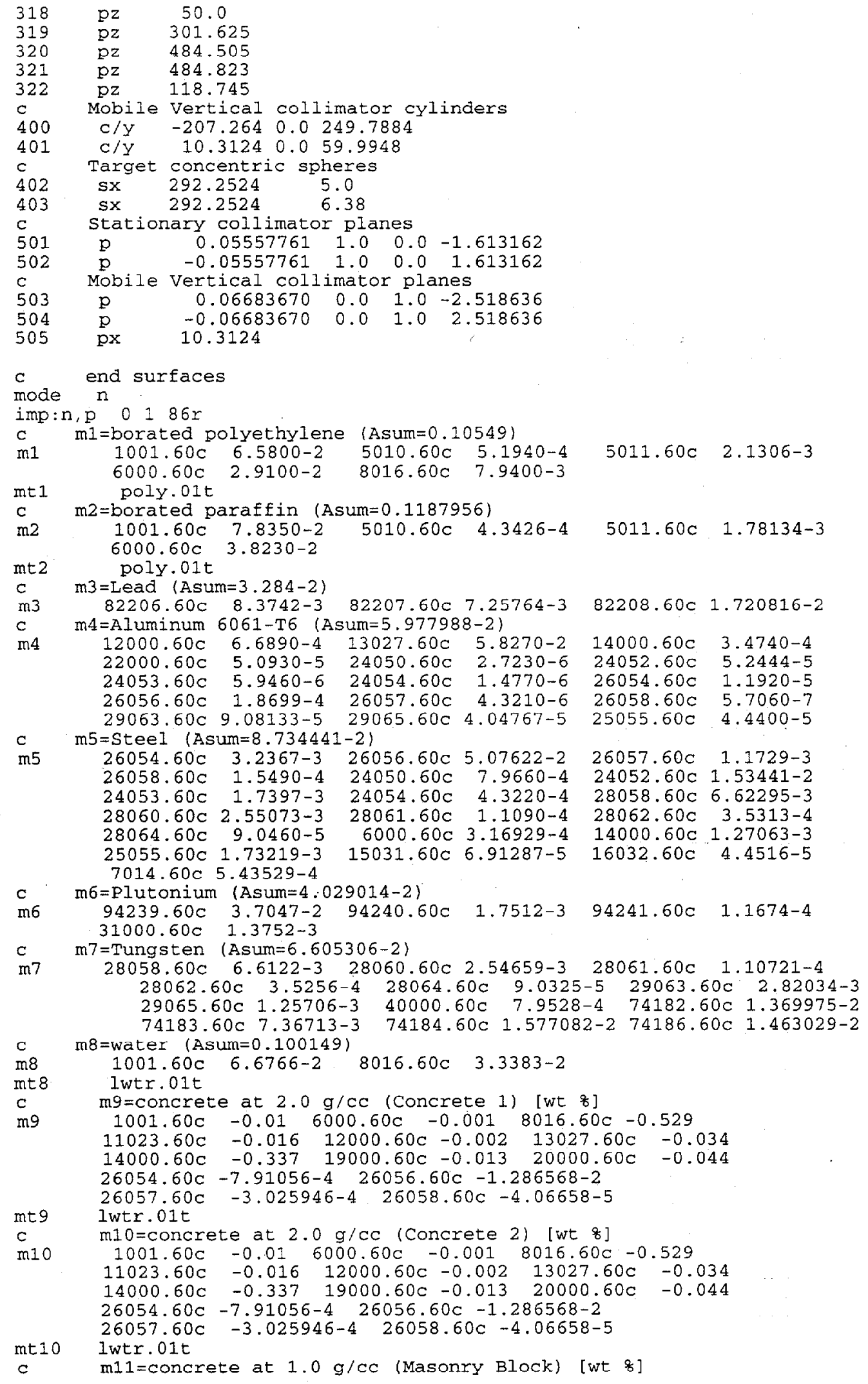




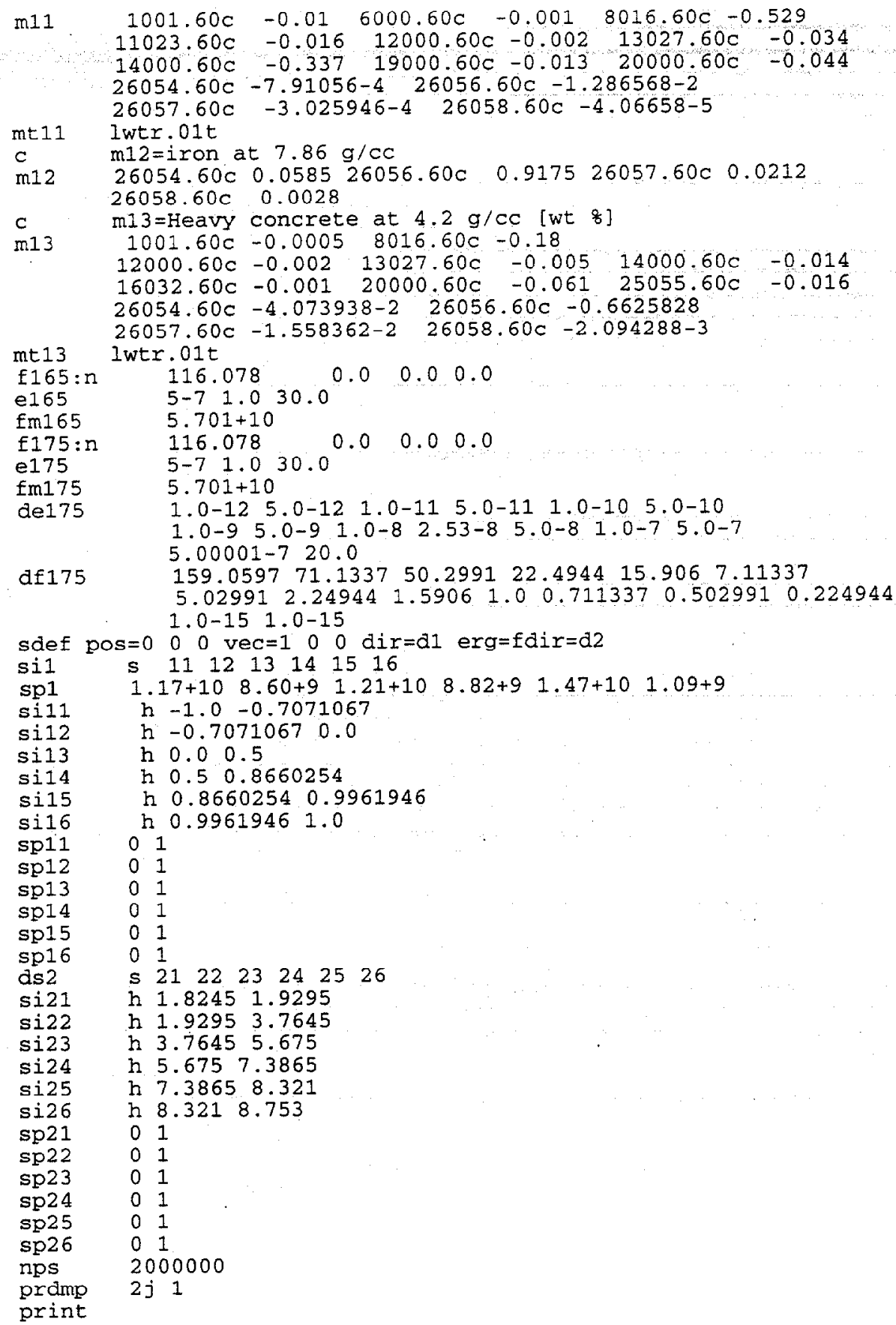


File for MCNP-4B calculation of ORIGEN neutron spectra within the stationary collimator for the source in the maximum up position

message: outp=pfna34.o mctal=pfna $34 . \mathrm{m}$

menp file for PFNA facility -- dose rates at sclected locations
$c$ source in nominal maximum upward position ( 34.8 deg.) of the
$c$ movable vertical collimator. check sdef, cells $3-6$, surfaces

c 401 and 503-505, and the sign on surface 505 .

$10-100: 128:-200: 251:-300: 305$

$2 \quad 0 \quad 100-128 \quad 200-251300-305$

\#(131-164+258-297+307-321)

$3 \quad 10.10549 \quad-400-401+505+224-227 \#(503-504)$

$\begin{array}{llll}1 & 0.10549 & -401+400+505+225-226 \#(503-504)\end{array}$

$0 \quad 142-127+223-228+308-304$

$\#(-400-401+505+224-227)$

$\#(-401+400+505+225-226)$

$6 \quad 0 \quad 127-124+501-502+308-304$

$\#(-401+400+505+225-226)$

$7 \quad 0 \quad 124-126+254-255+308-304$

$85 \quad 58.734441-2102-126+201-250+306-301$

$\#(+102-127+223-228) \quad \#(+127-124+501-502)$

$9 \quad \begin{array}{lllllll} & 1 & 0.10549 & \#(+124+254 & -255) & \#(+129-252) & \#(+129+257)\end{array}$

$\#(+129-252) \quad \#(+129+257)$

$\#(127+501-502) \#(-127+223-228)$

$1 \cdot 0.10549$

10.10549

10.10549

10.10549

10.10549

10.10549

10.10549

10.10549

10.10549

10.10549

10.10549

20.1187956

20.1187956

$106-109+212-236+302-303 \#(218-233)$

$109-110+211-237+302-303 \#(221-230)$

$110-111+210-238+302-303 \#(221-230)$

$111-112+209-239+302-303 \#(221-230)$

$112-113+208-240+302-303 \#(221-230)$

$113-114+207-241+302-303 \#(221-230)$

$114-115+206-242+302-303 \#(221-230)$

$115-116+205-243+302-303 \#(221-230)$

$116-117+204-244+302-303 \#(221-230)$

$117-118+203-245+302-303 \#(221-230)$

$118-119+202-249+302-303 \#(221-230)$

$120-123+215-246+302-303 \#(221-230)$

$103-109+202-249+302-303$

$\#(221-230)$

$\#(103-104+217-234)$

$\#(104-105+216-235)$

$\#(105-107+219-232)$

$\#(107-108+219-220) \quad \#(107-108+231-232)$

$\#(106-109+212-218) \#(106-109+233-236)$

$109-120+202-249+302-303$

$\#(221-230)$

$\#(109-110+211-237)$

$\#(110-111+210-238)$

$\#(111-112+209-239)$

$\#(112-113+208-240)$

$\#(113-114+207-241)$

$\#(114-115+206-242)$

$\#(115-116+205-243)$

\# $(116-117+204-244)$

$\#(117-118+203-245)$

\#(118-119)

$2433.284-2 \quad 121-125+201-250+301-304$

$\#(-124+214-247) \#(124+253-256)$

$\#(122+248) \#(122-213)$

25

$33.284-2$

$103-107+217-234+302-303$

$\#(221-230) \#(104-220) \#(104+231)$

$45.977931-2104-108+216-220+302-303 \#(105-219)$

$45.977931-2 \cdot 104-108+231-235+302-303 \#(105+232)$

$45.977931-2 \quad 102-124+201-222+301-304$

$\#\left(\begin{array}{llllll}103 & -120 & 202 & -221 & 302 & -303\end{array}\right)$

\#( $\left.\begin{array}{llllll}120 & -123 & 215 & -221 & 302 & -303\end{array}\right)$

\# $(121-214)$ 
$45.977931-2124-126+252-254+301-304$ $\#(-125+213-253)$ 


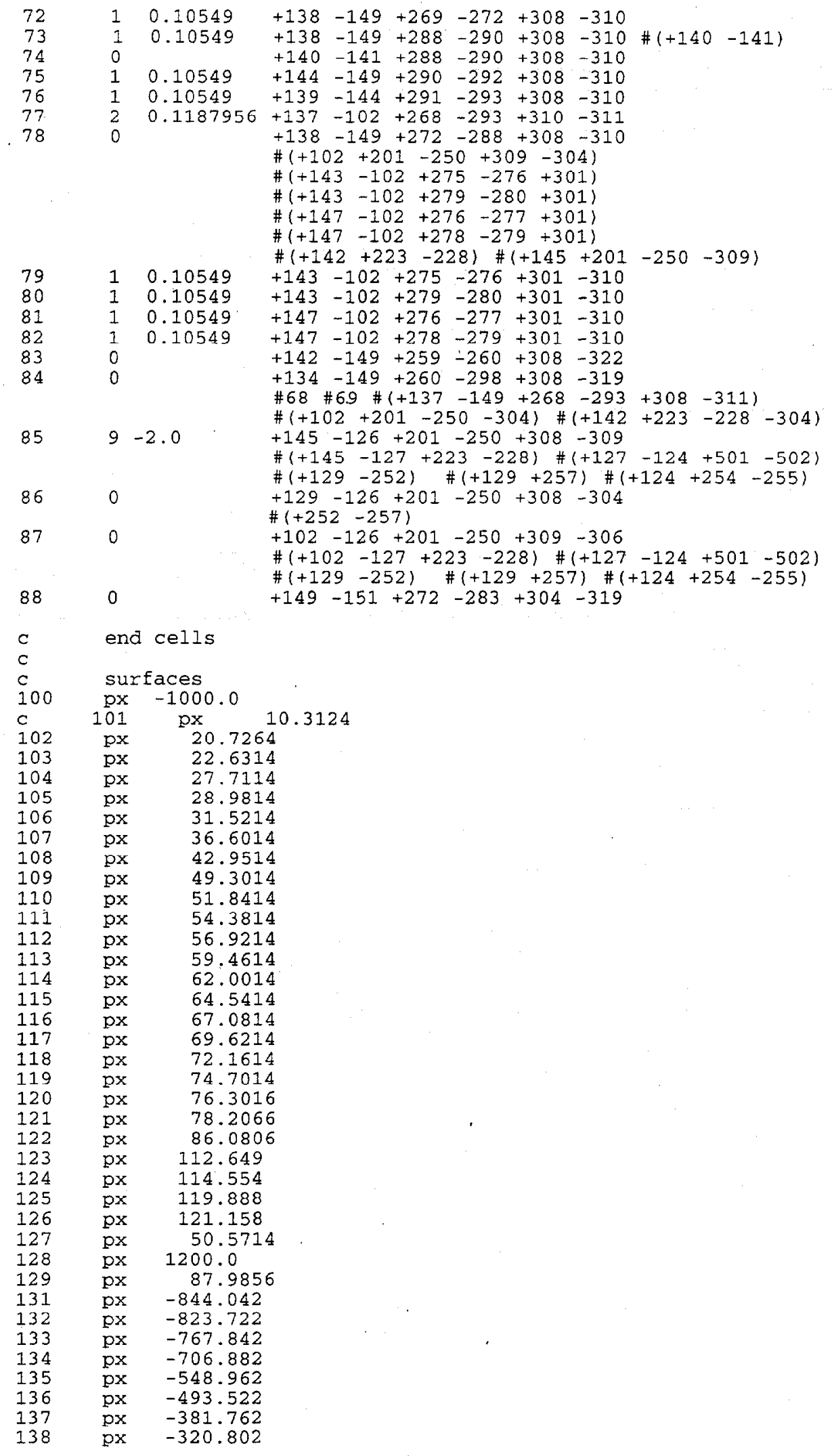




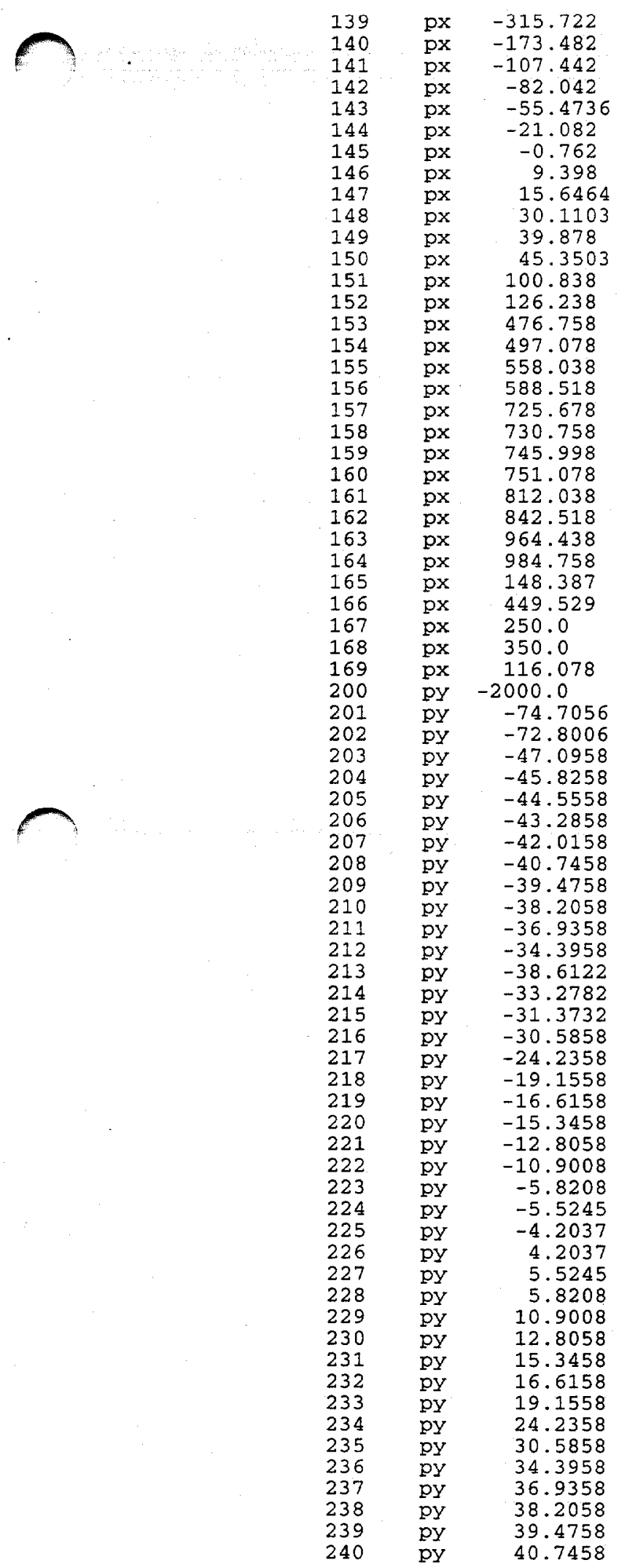




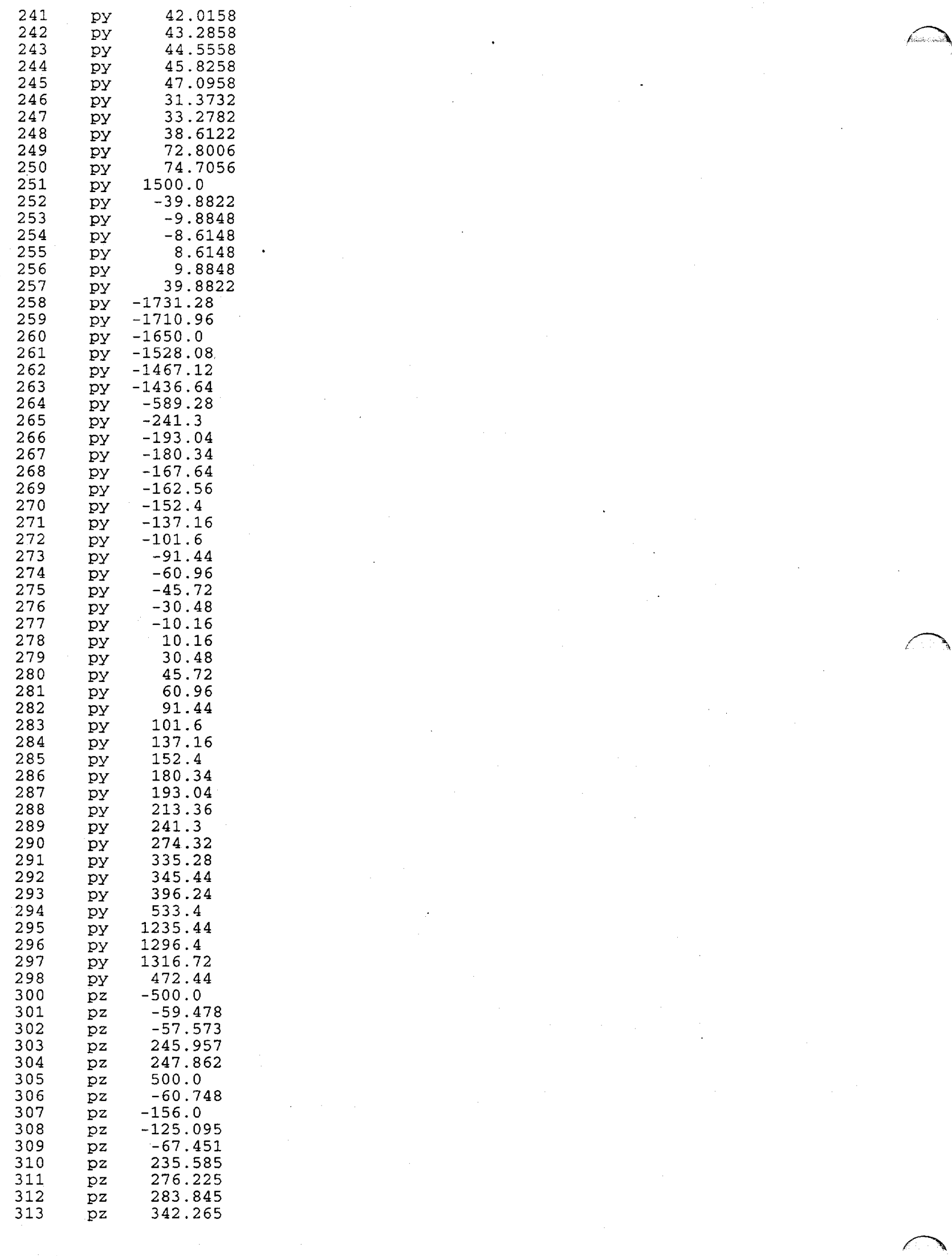




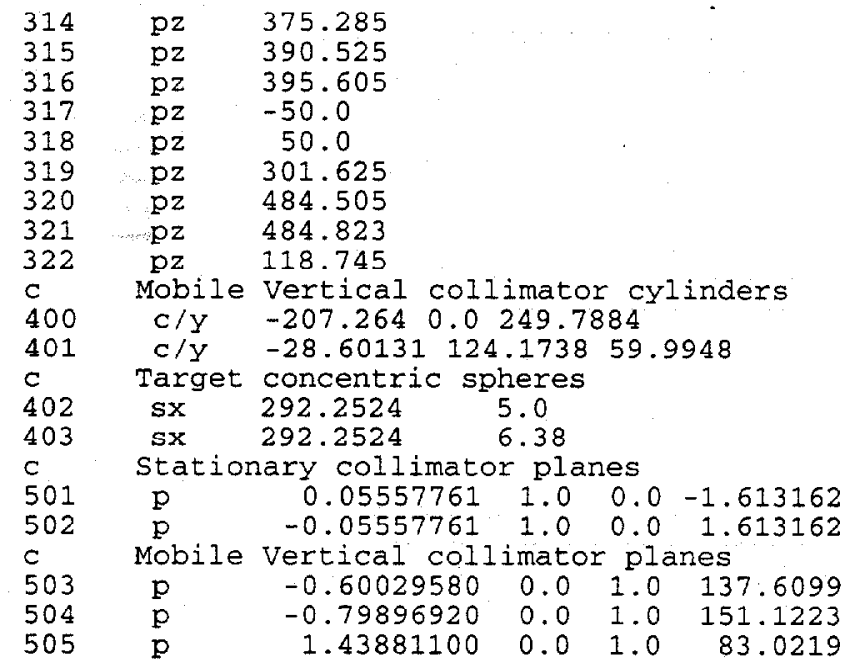

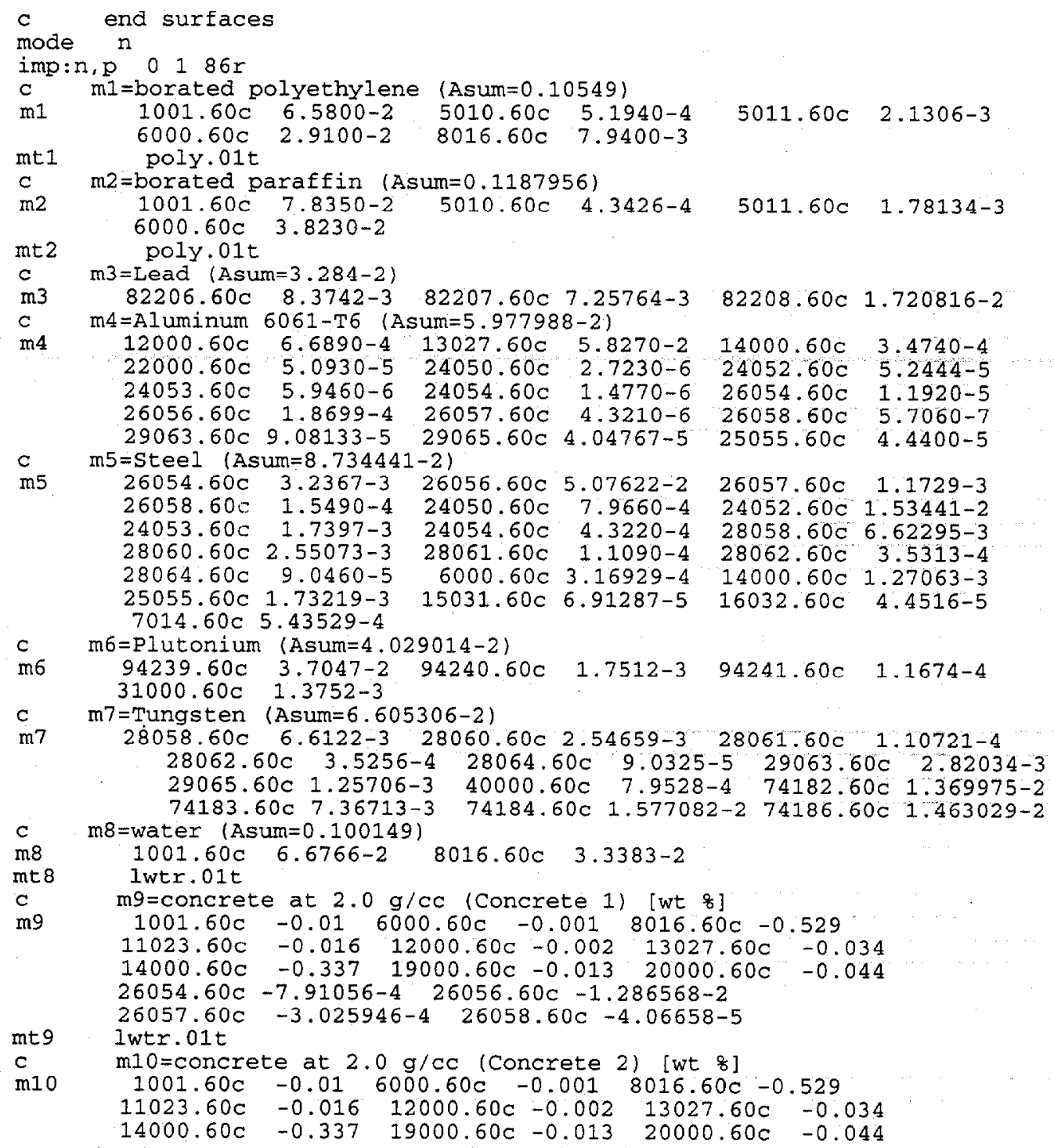




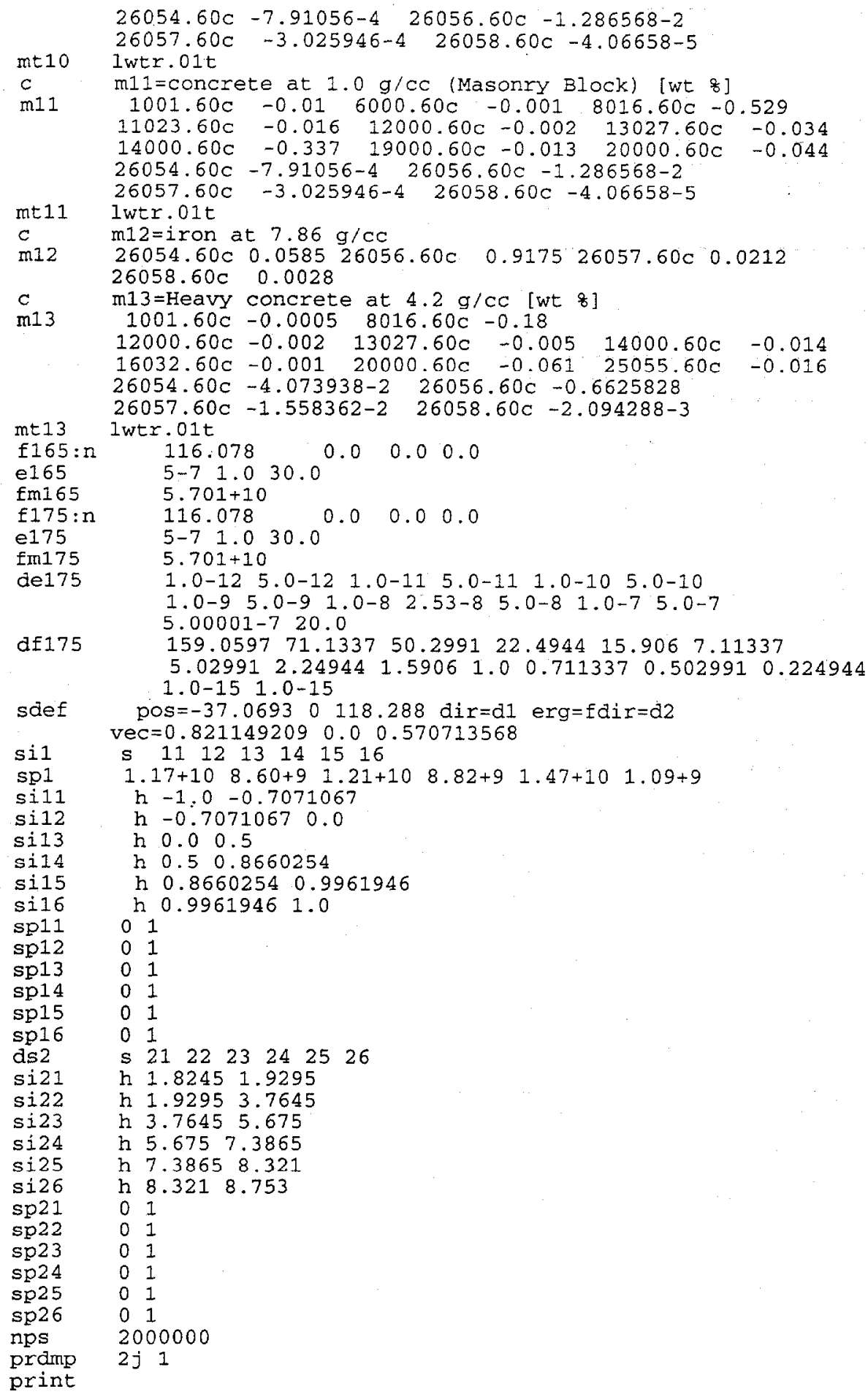


File for MCNP-4B calculation of ORIGEN neutron spectra within the concrete beam stop for the source in the horizontal position

message: outp=pfna35.o mctal=pfna35.m

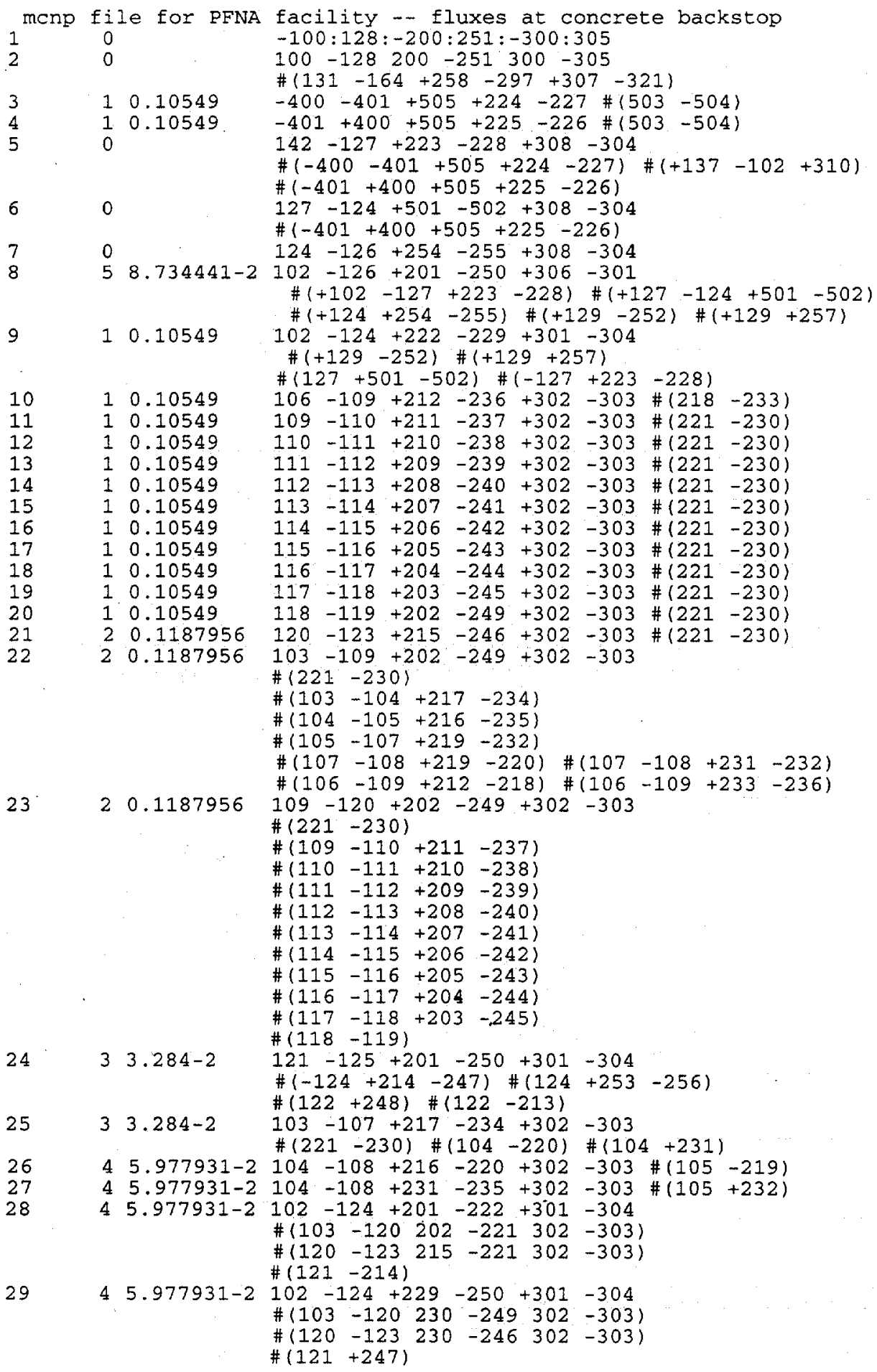




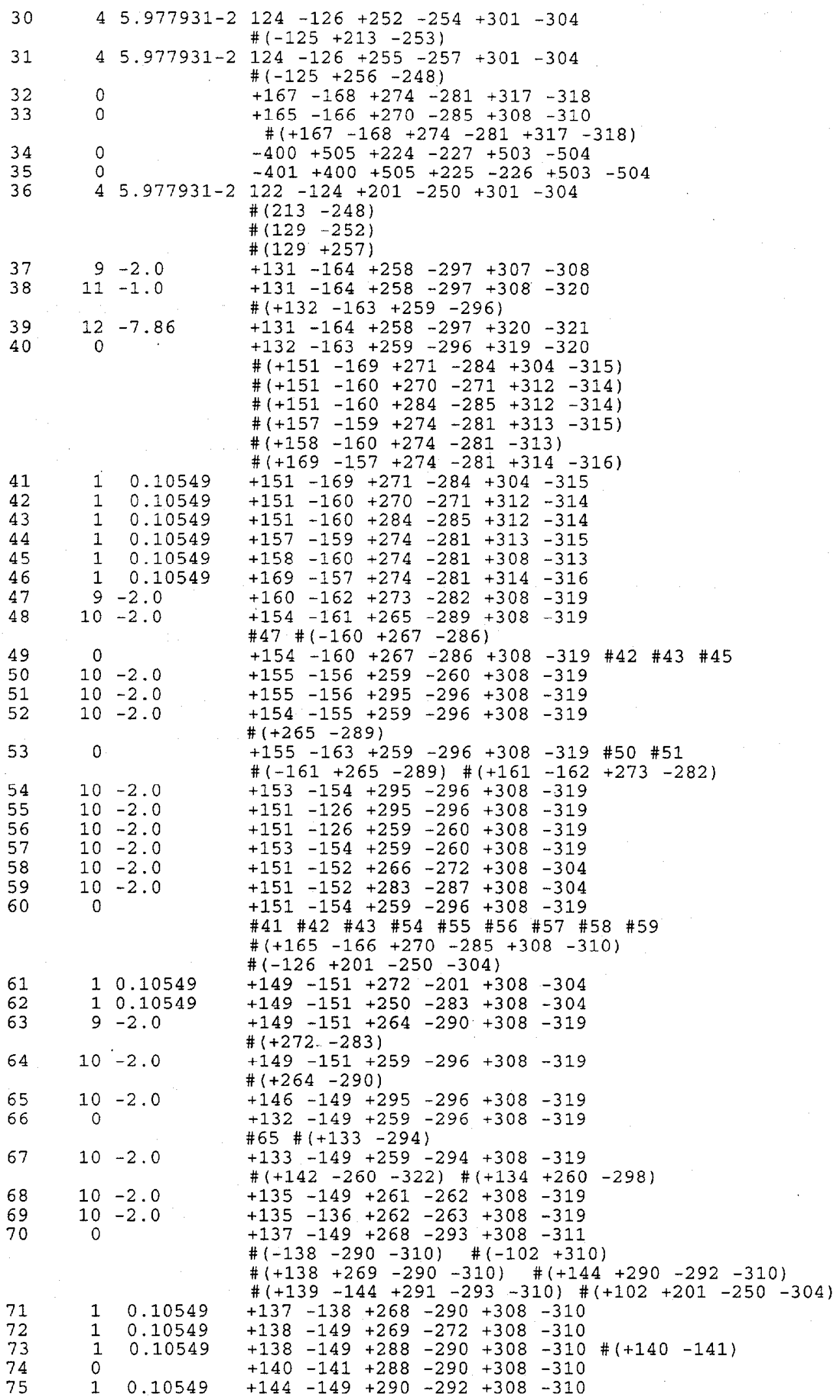




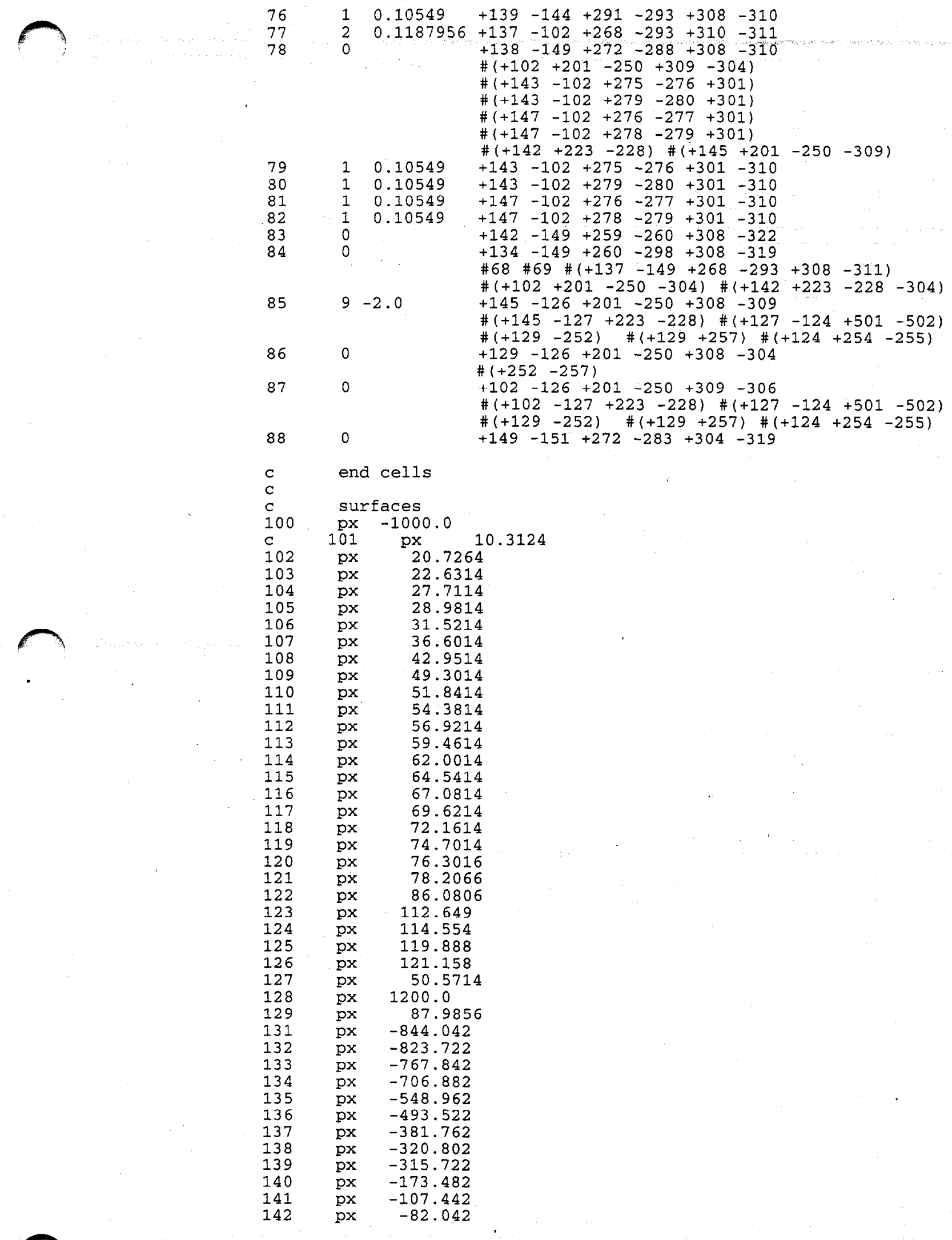




$\begin{array}{llc} & & \\ 143 & \text { px } & -55.4736 \\ 144 & \text { px } & -21.082 \\ 145 & \text { px } & -0.762 \\ 146 & \text { px } & 9.398 \\ 147 & \text { px } & 15.6464 \\ 148 & \text { px } & 30.1103 \\ 149 & \text { px } & 39.878 \\ 150 & \text { px } & 45.3503 \\ 151 & \text { px } & 100.838 \\ 152 & \text { px } & 126.238 \\ 153 & \text { px } & 476.758 \\ 154 & \text { px } & 497.078 \\ 155 & \text { px } & 558.038 \\ 156 & \text { px } & 588.518 \\ 157 & \text { px } & 725.678 \\ 158 & \text { px } & 730.758 \\ 159 & \text { px } & 745.998 \\ 160 & \text { px } & 751.078 \\ 161 & \text { px } & 812.038 \\ 162 & \text { px } & 842.518 \\ 163 & \text { px } & 964.438 \\ 164 & \text { px } & 984.758 \\ 165 & \text { px } & 148.387 \\ 166 & \text { px } & 449.529 \\ 167 & \text { px } & 250.0 \\ 168 & \text { px } & 350.0 \\ 169 & \text { px } & 116.078 \\ 200 & \text { py } & -2000.0 \\ 201 & \text { py } & -74.7056 \\ 202 & \text { py } & -72.8006 \\ 203 & \text { py } & -47.0958 \\ 204 & \text { py } & -45.8258 \\ 205 & \text { py } & -44.5558 \\ 206 & \text { py } & -43.2858 \\ 207 & \text { py } & -42.0158 \\ 208 & \text { py } & -40.7458 \\ 209 & \text { py } & -39.4758 \\ 210 & \text { py } & -38.2058 \\ 211 & \text { py } & -36.9358 \\ 212 & \text { py } & -34.3958 \\ 213 & \text { py } & -38.6122 \\ 214 & \text { py } & -33.2782 \\ 215 & \text { py } & -31.3732 \\ 216 & \text { py } & -30.5858 \\ 217 & \text { py } & -24.2358 \\ 218 & \text { py } & -19.1558 \\ 219 & \text { py } & -16.6158 \\ 220 & \text { py } & -15.3458 \\ 221 & \text { py } & -12.8058 \\ 222 & \text { py } & -10.9008 \\ 223 & \text { py } & -5.8208 \\ 224 & \text { py } & -5.5245 \\ 225 & \text { py } & -4.2037 \\ 226 & \text { py } & 4.2037 \\ 227 & \text { py } & 5.5245 \\ 228 & \text { py } & 5.8208 \\ 229 & \text { py } & 10.9008 \\ 230 & \text { py } & 12.8058 \\ 231 & \text { py } & 15.3458 \\ 232 & \text { py } & 16.6158 \\ 233 & \text { py } & 19.1558 \\ 234 & \text { py } & 24.2358 \\ 235 & \text { py } & 30.5858 \\ 236 & \text { py } & 34.3958 \\ 237 & \text { py } & 36.9358 \\ 238 & \text { py } & 38.2058 \\ 239 & \text { py } & 39.4758 \\ 240 & \text { py } & 40.7458 \\ 241 & \text { py } & 42.0158 \\ 242 & \text { py } & 43.2858 \\ 243 & \text { py } & 44.5558 \\ 244 & \text { py } & 45.8258 \\ & & \end{array}$




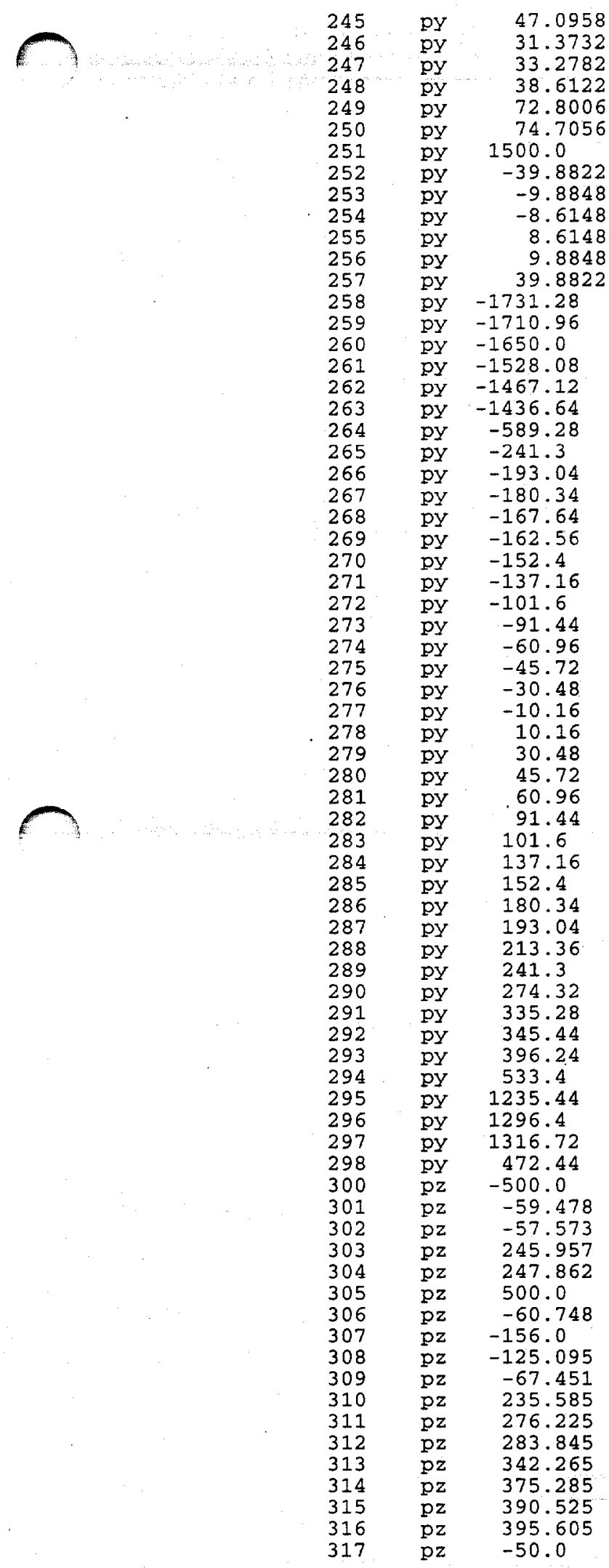




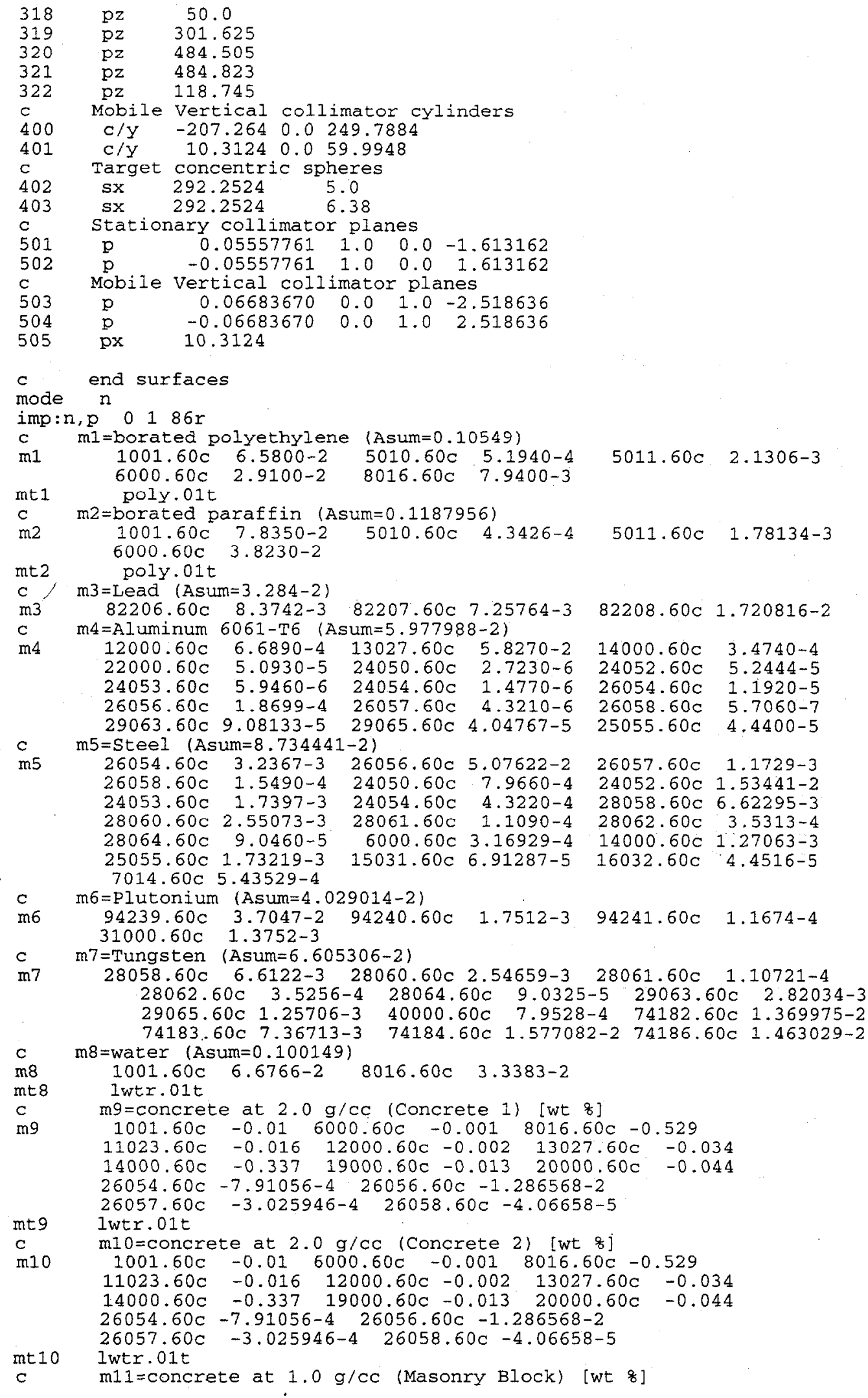




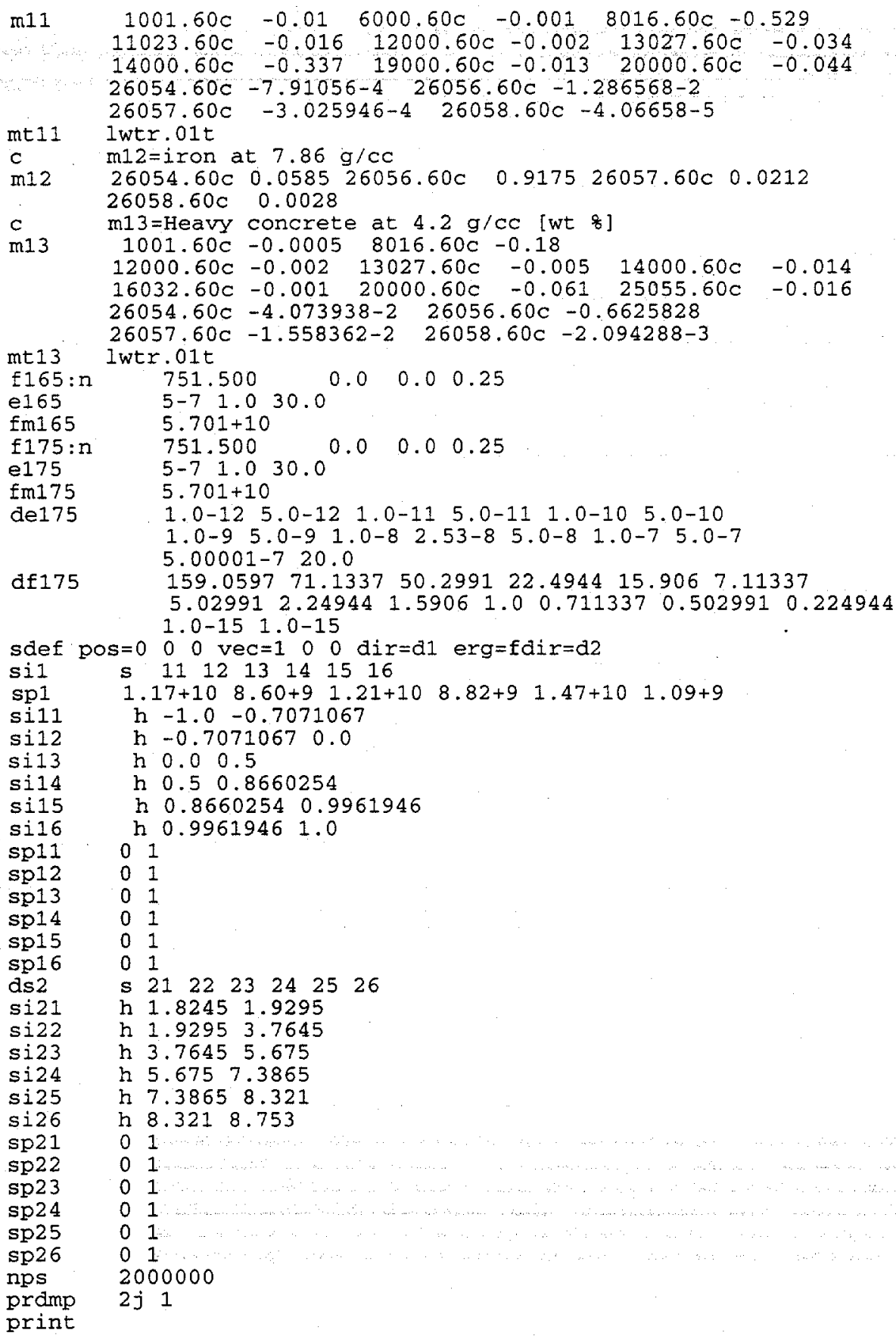




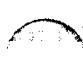

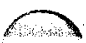

$\gg$ 


\section{APPENDIX I .}

\section{LISTINGS OF THE ORIGEN INPUT FILES FOR CALCULATING NEUTRON ACTIVATION OF SELECTED MATERIALS}




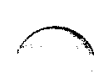

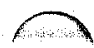

9 


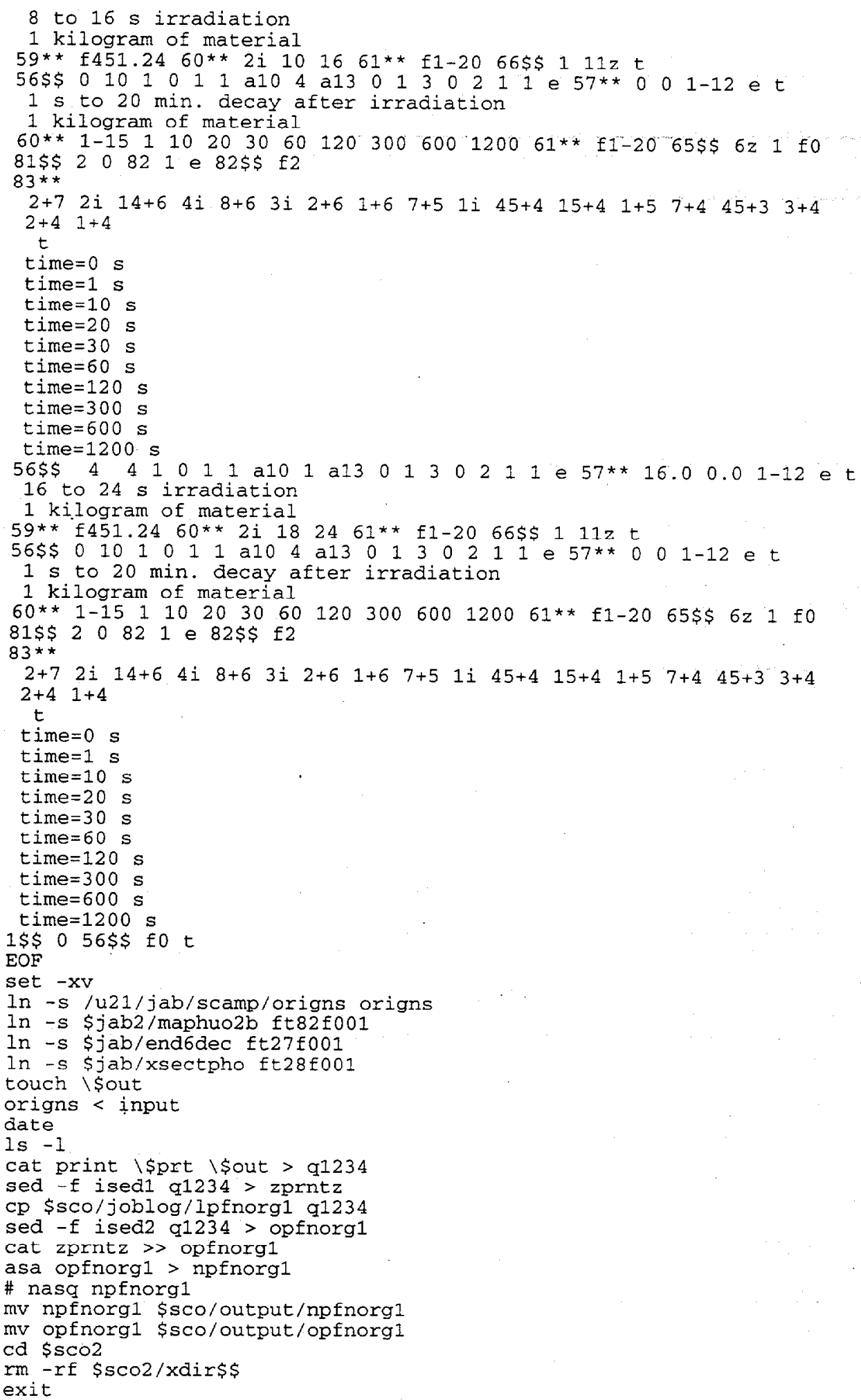


File for ORIGEN calculation of the activation of ball bearings using neutron spectrum 1

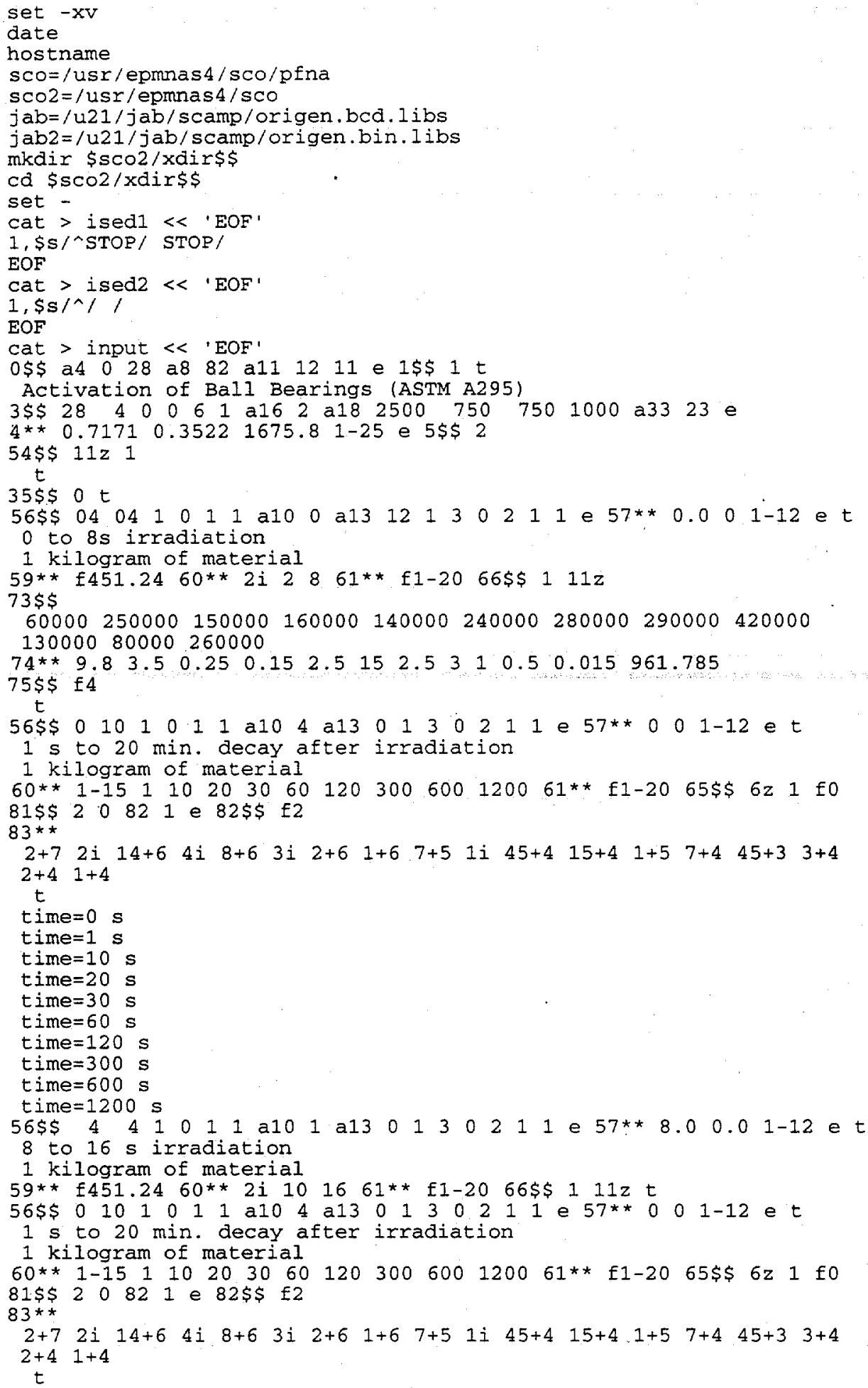




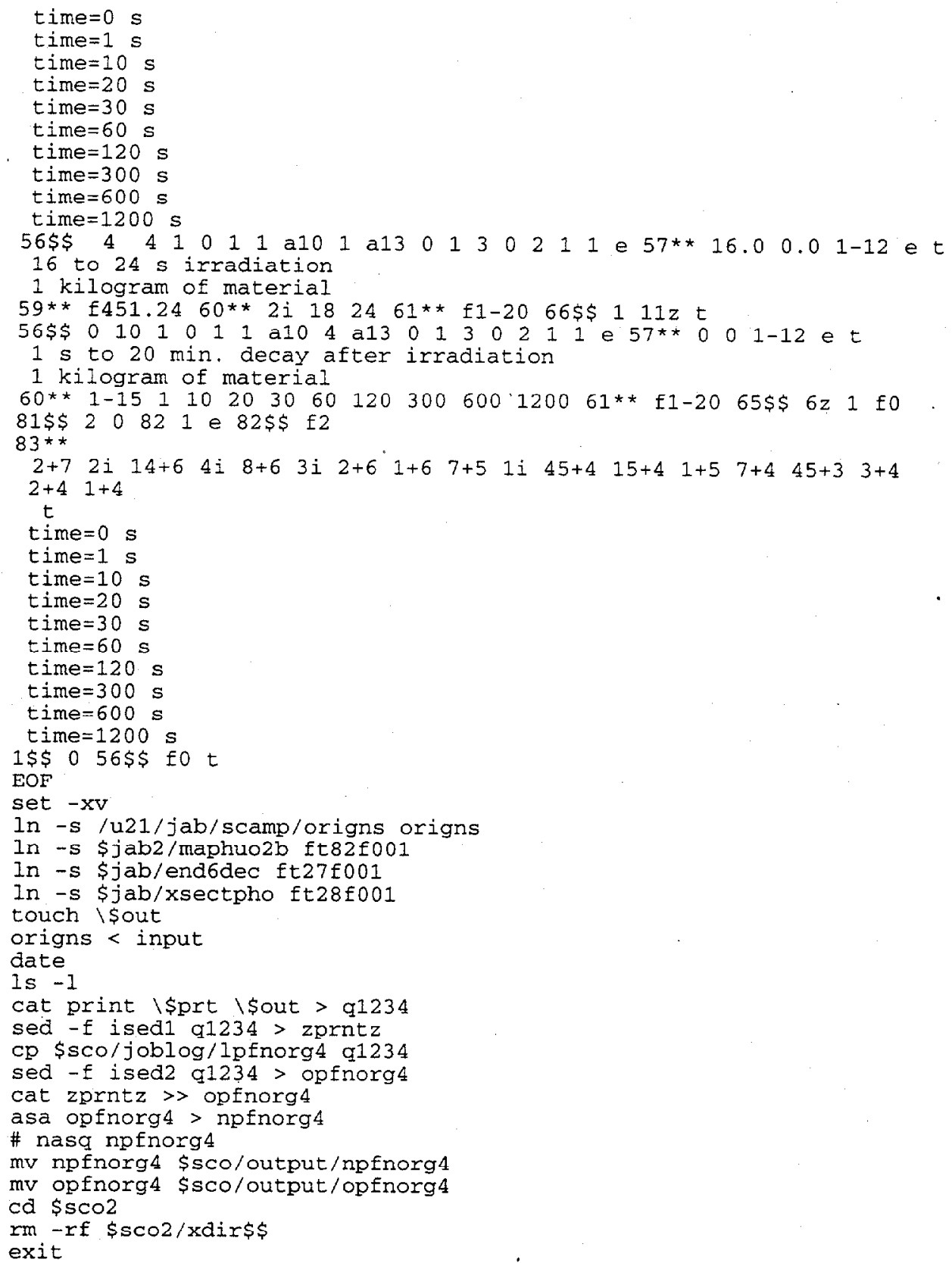


File for ORIGEN calculation of the activation of surgical implant with composition $A$ using neutron spectrum 1

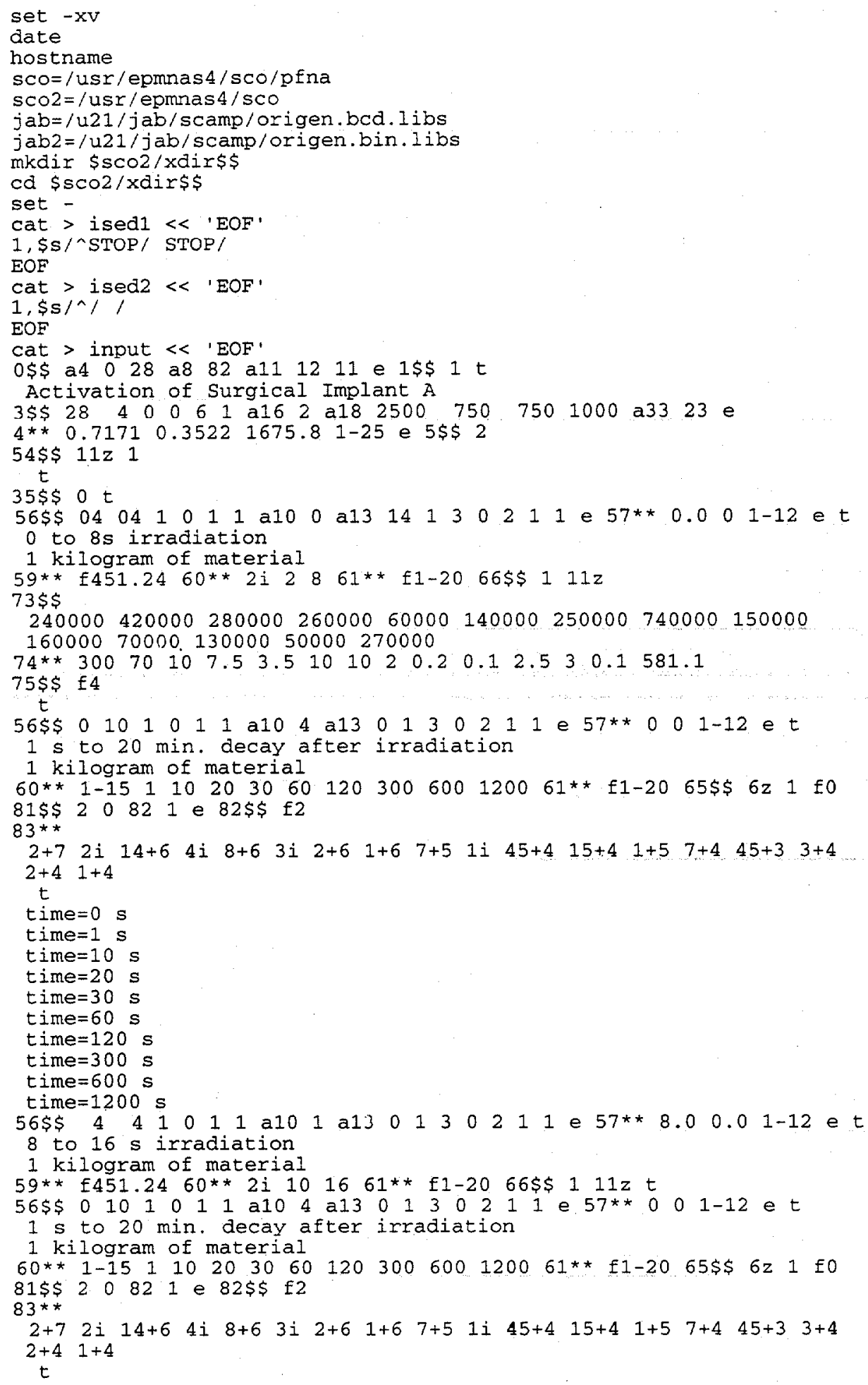




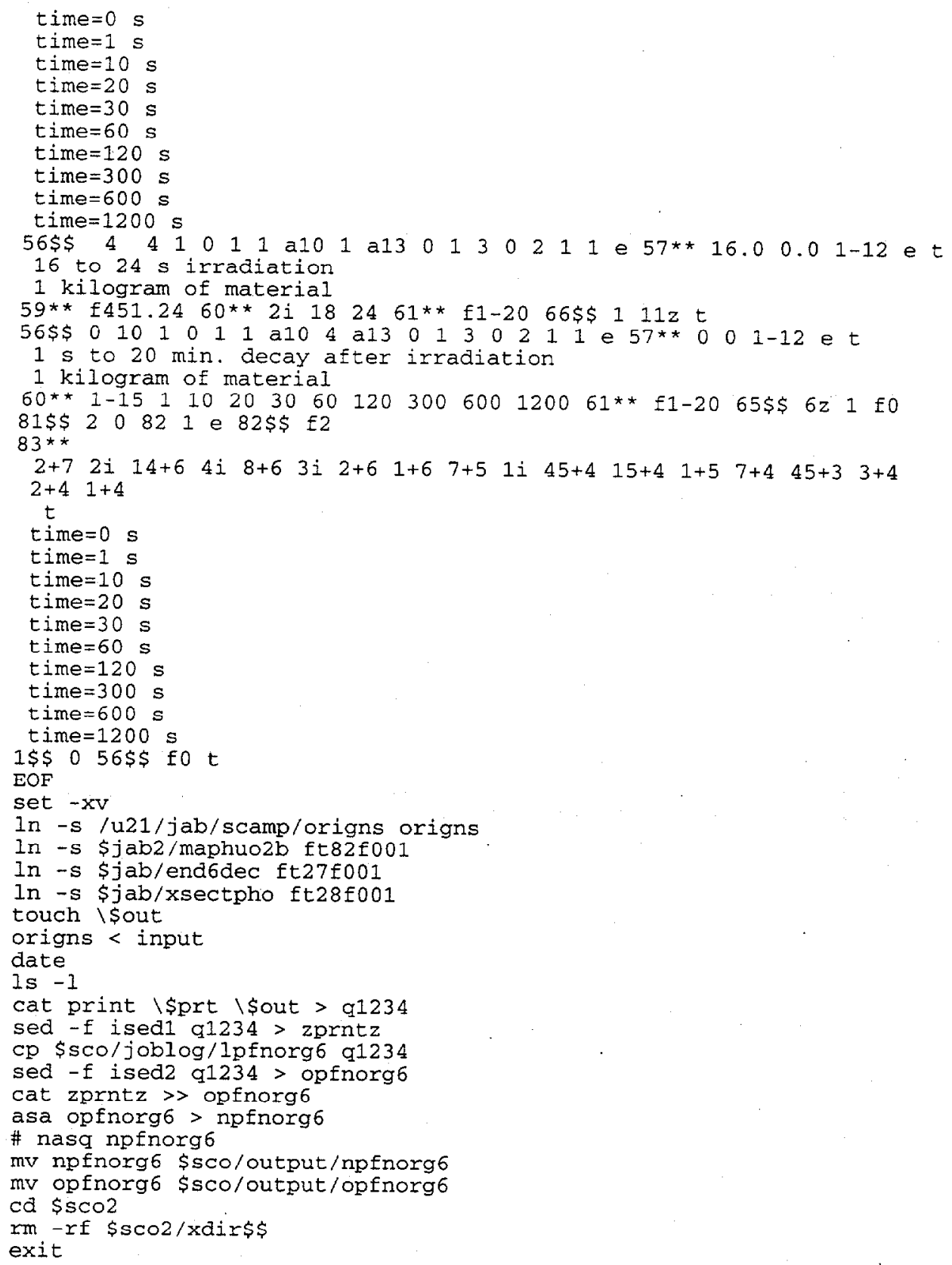


File for ORIGEN calculation of the activation of surgical implant with composition $B$ using neutron spectrum 1

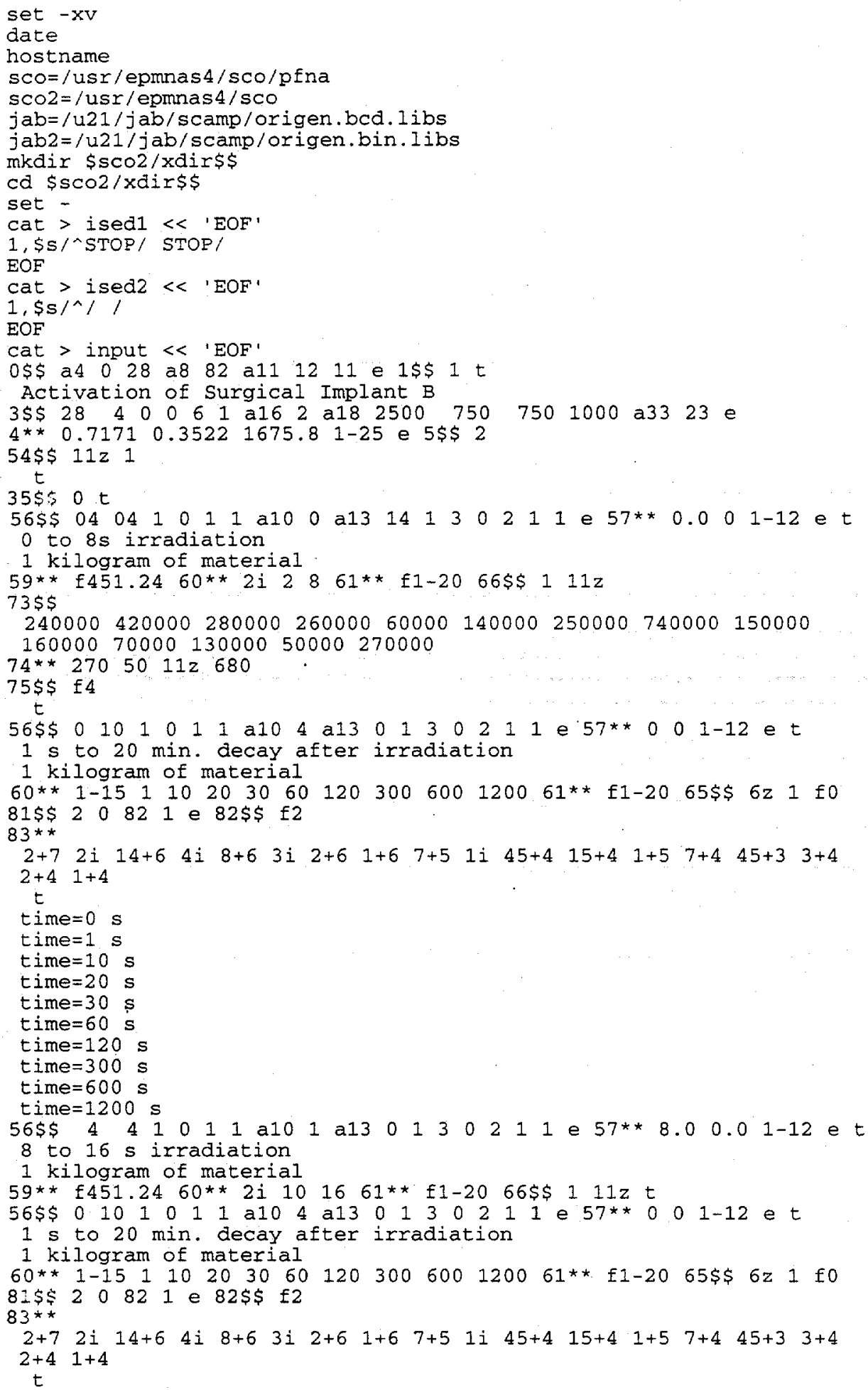




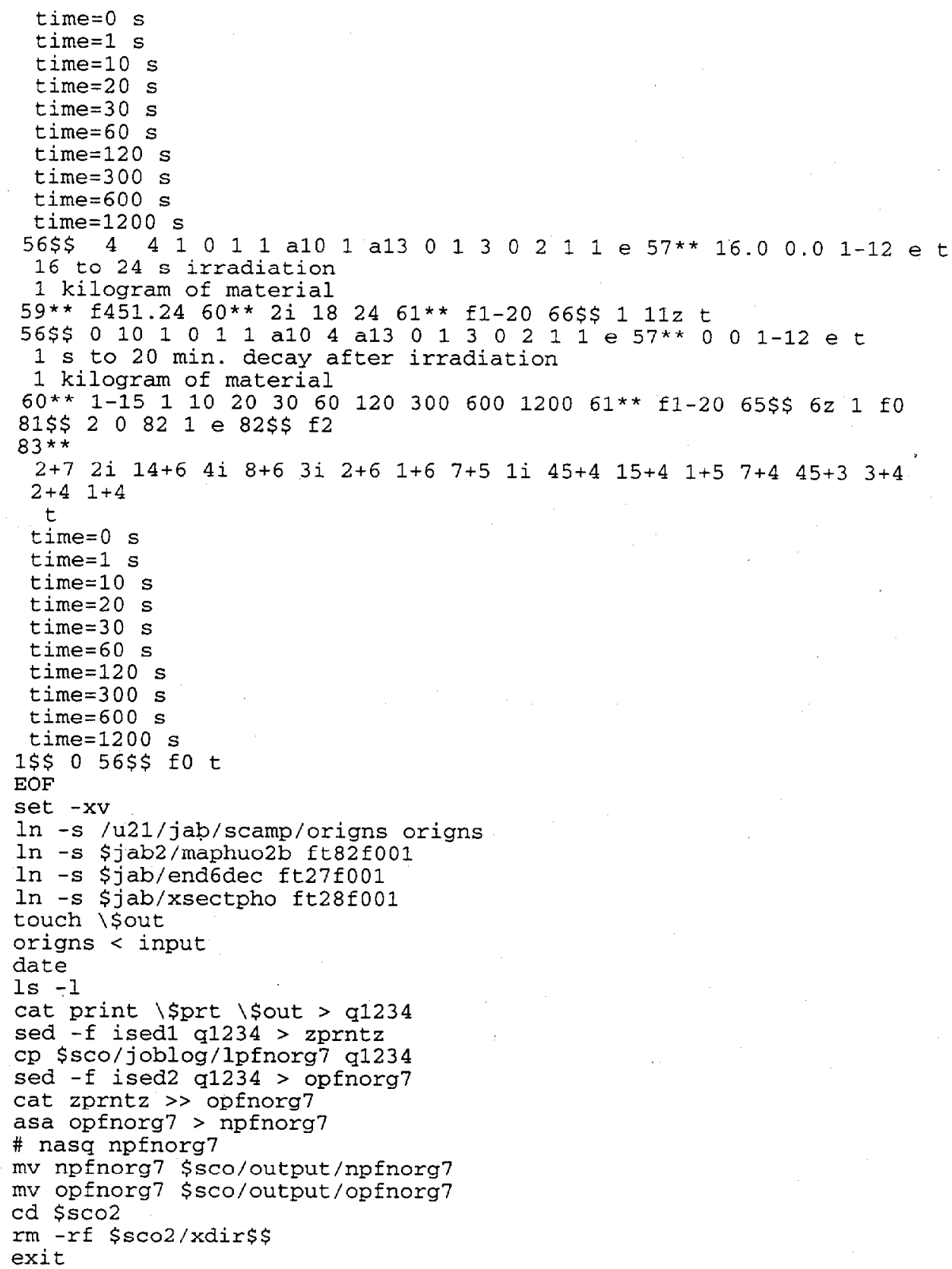


File for ORIGEN calculation of the activation of 16-16-16 fertilizer cargo using neutron spectrum 1

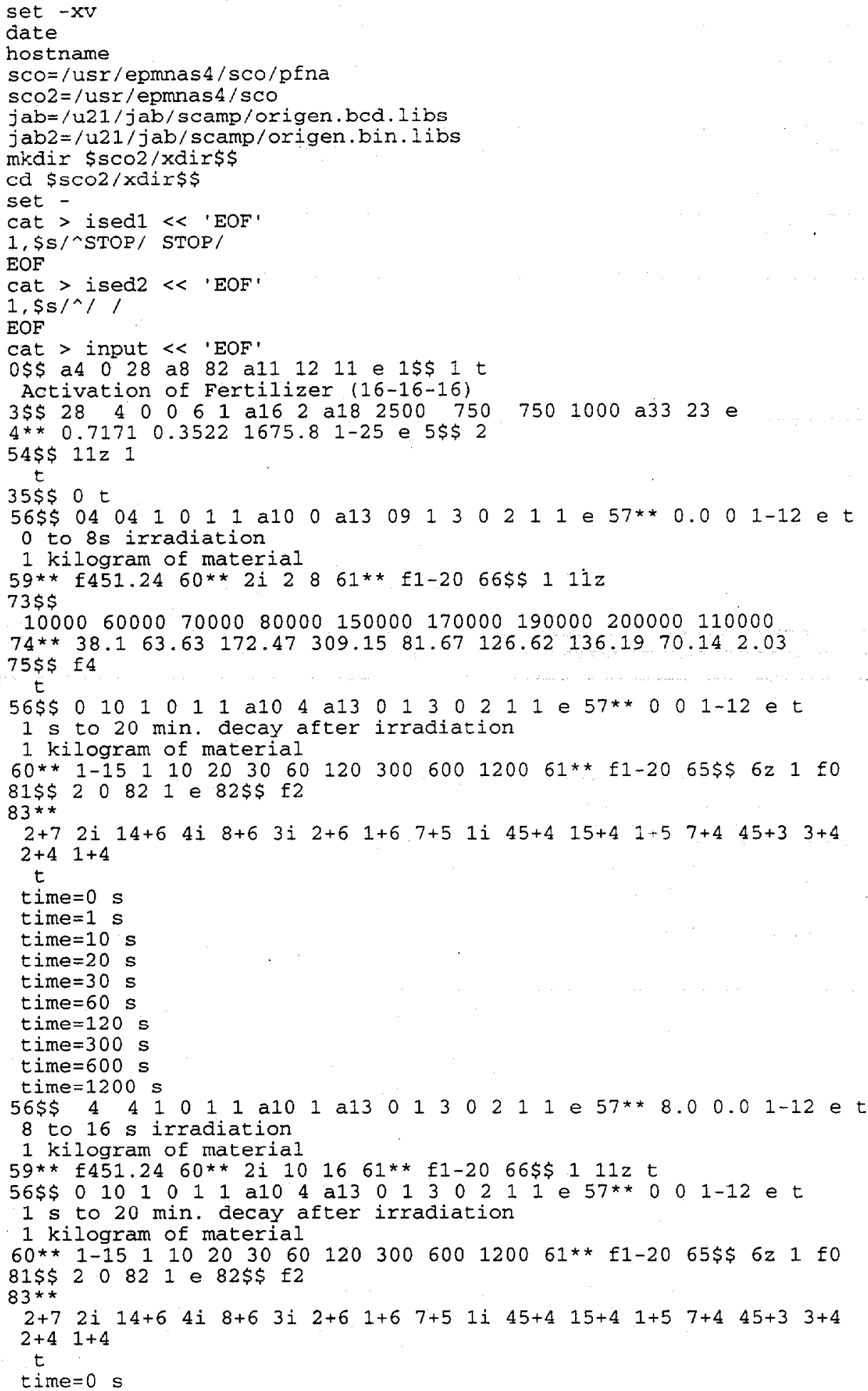




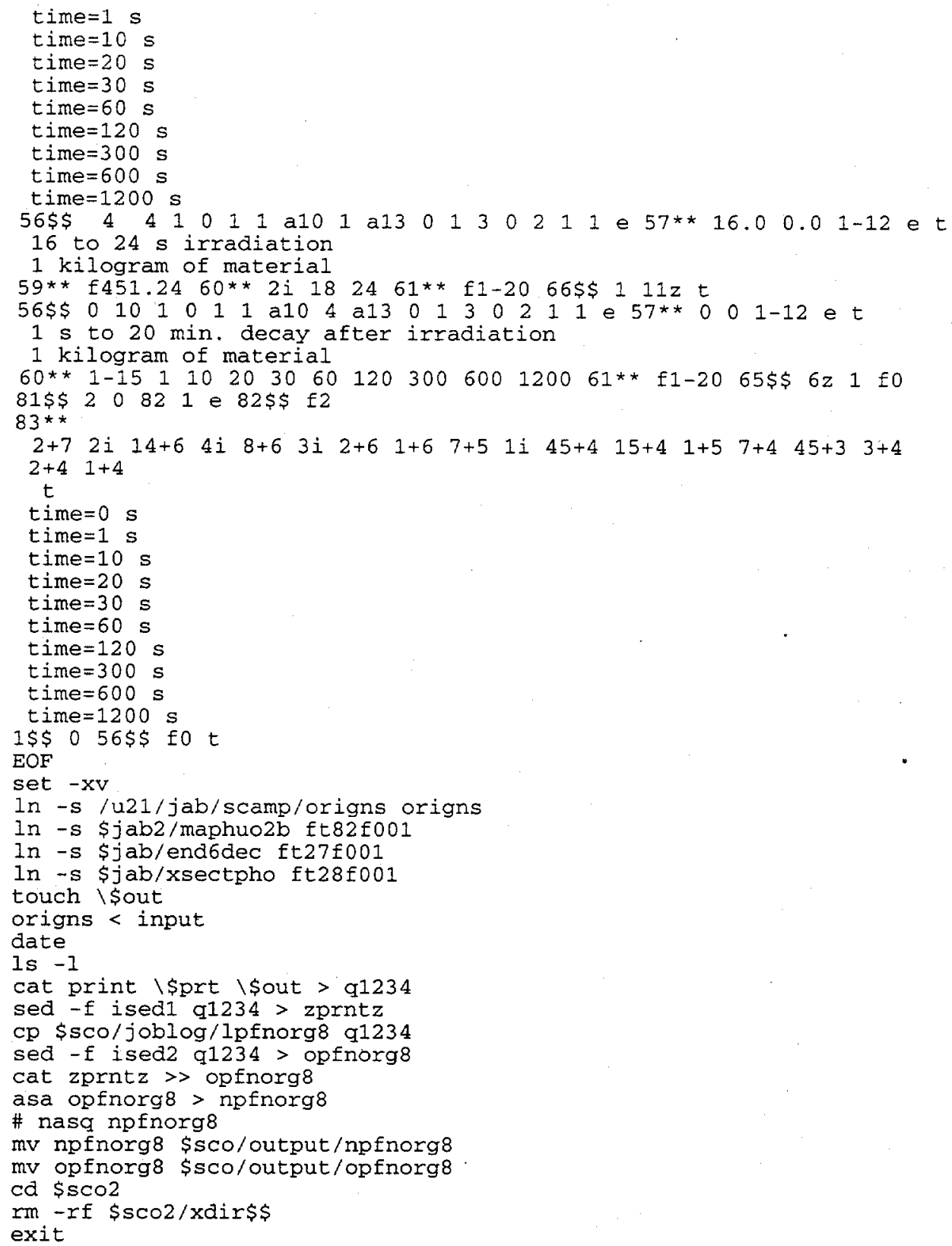


File for ORIGEN calculation of the activation of the concrete structure using neutron spectrum 1

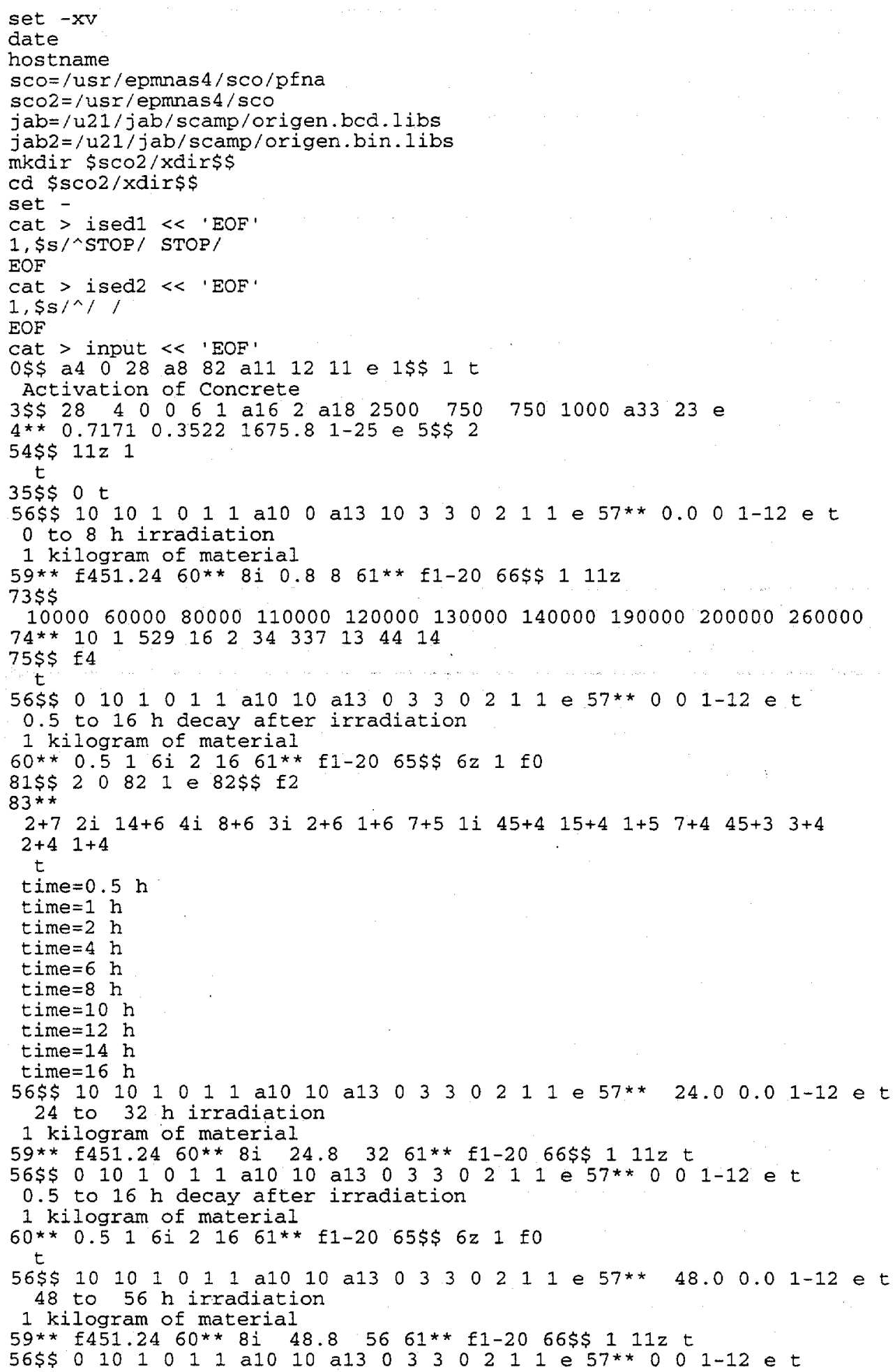


0.5 to $16 \mathrm{~h}$ decay after irradiation

1 kilogram of material

$60 * * 0.516 i 216 \quad 61 * *$ f1-20 $65 \$ \$ 6 z \quad 1$ fo

$t$

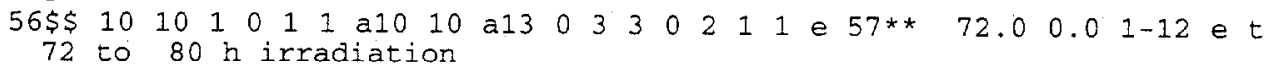

1 kilogram of material

$59 * *$ f $451.2460 * * 8 i \quad 72.8 \quad 8061 * *$ f1-20 66\$\$ $111 z t$

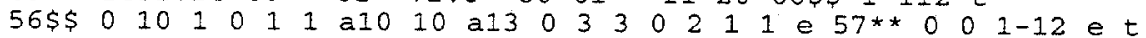

0.5 to $16 \mathrm{~h}$ decay after irradiation

1 kilogram of material

$60 * * 0.516 i 21661 * \star$ f1-20 65\$\$ $6 z 1$ fo

t

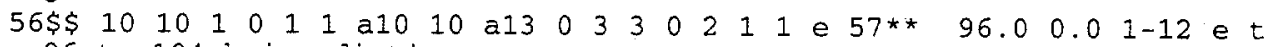
96 to $104 \mathrm{~h}$ irradiation

1 kilogram of material

$59 * \star$ f451.24 60** 8i $96.810461 * *$ f1-20 66\$\$ $111 \mathrm{z} t$

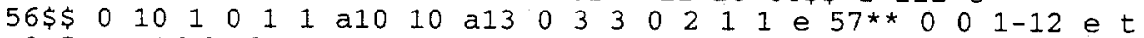

0.5 to $16 \mathrm{~h}$ decay after irradiation

1 kilogram of material

$60 * * 0.516 i 216 \quad 61 * *$ f1-20 65\$\$ $6 z 1$ fo

$t$

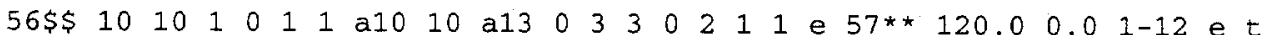

120 to $128 \mathrm{~h}$ irradiation

1 kilogram of material

$59 *$ f $451.2460 *$ * 8 i $120.812861 *$ f $1-20$ 66\$\$ $111 z t$

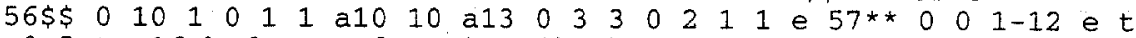

0.5 to $16 \mathrm{~h}$ decay after irradiation

1 kilogram of material

$60 * * 0.516 i 216 \quad 61 * *$ f $1-20 \quad 65 \$ \$ 6 z \quad 1$ fo

$t$

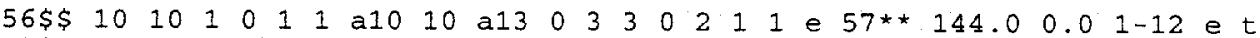

144 to 152 h irradiation

1 kilogram of material

$59 * *$ f451.24 60** 8i $144.815261 * *$ f1-20 66\$\$ I $11 \mathrm{z} t$

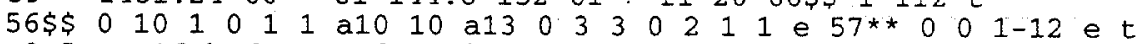

0.5 to $16 \mathrm{~h}$ decay after irradiation

1 kilogram of material

$60 * * 0.516 i 216 \quad 61 * \star \quad f 1-20 \quad 65 \$ \$ 6 z \quad 1$ fo

$t$

$56 \$ \$ 101010$ I I a 1010 a13 03330211 e $57 * * 168.00 .01-12$ e $t$

168 to $176 \mathrm{~h}$ irradiation

1 kilogram of material

$59 * \star$ f451.24 60** 8i $158.8 \quad 176 \quad 61 * *$ f1-20 $66 \$ \$ 111 z t$

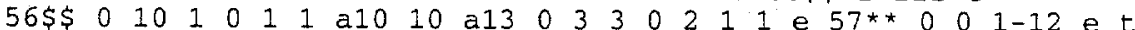

0.5 to $16 \mathrm{~h}$ decay after irradiation

1 kilogram of material

$60 * * 0.516 \mathrm{i} 21661 * *$ f1-20 $65 \$ \$ 6 z \quad 1$ fo

$t$

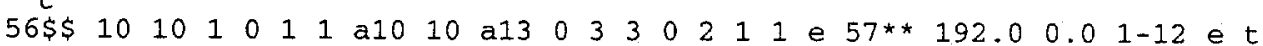

192 to $200 \mathrm{~h}$ irradiation

1 kilogram of material

$59 *$ f $451.2460 * * 8$ i $192.820061 *$ f $1-20$ 66\$\$ $111 z t$

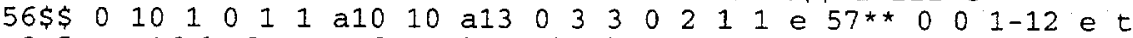

0.5 to $16 \mathrm{~h}$ decay after irradiation

1 kilogram of material

$60 * \star 0.516 i 216 \quad 61 * \star \quad f 1-20 \quad 65 \$ \$ 6 z \quad 1$ fo

$t$

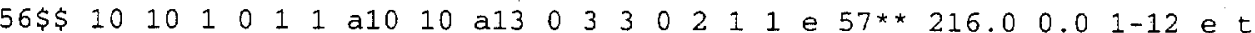

216 to 224 h irradiation

1 kilogram of material

59 * f $451.24 \quad 60 * *$ 8i $216.8 \quad 224$ 61** f1-20 66\$\$ $111 z t$

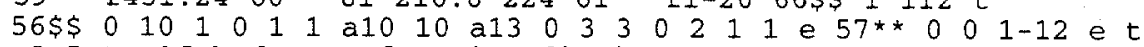

0.5 to $16 \mathrm{~h}$ decay after irradiation

1 kilogram of material

$60 * * 0.516 i 21661^{* *} £ 1-2065 \$ \$ 6 z \quad 1$ f0

$81 \$ \$ 2 \quad 0 \quad 821$ e $82 \$ \$$ f2

$83 * *$

$\begin{array}{llllllllllllllll}2+7 & 2 i & 14+6 & 4 i & 8+6 & 3 i & 2+6 & 1+6 & 7+5 & 1 i & 45+4 & 15+4 & 1+5 & 7+4 & 45+3 & 3+4\end{array}$

$2+41+4$

$t$

time $=0.5 \mathrm{~h}$ 


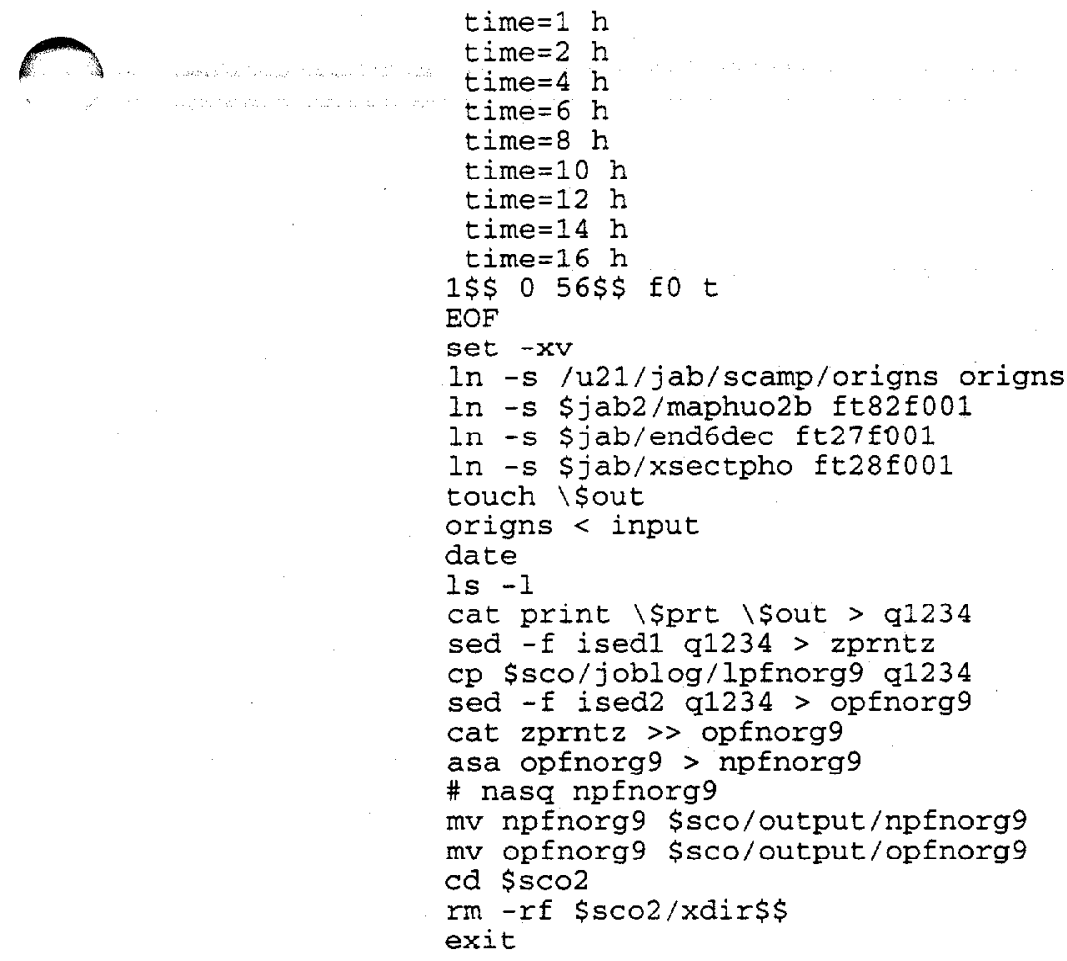


File for ORIGEN calculation of the activation of the aluminum (6061-T6) structure using neutron spectrum 1

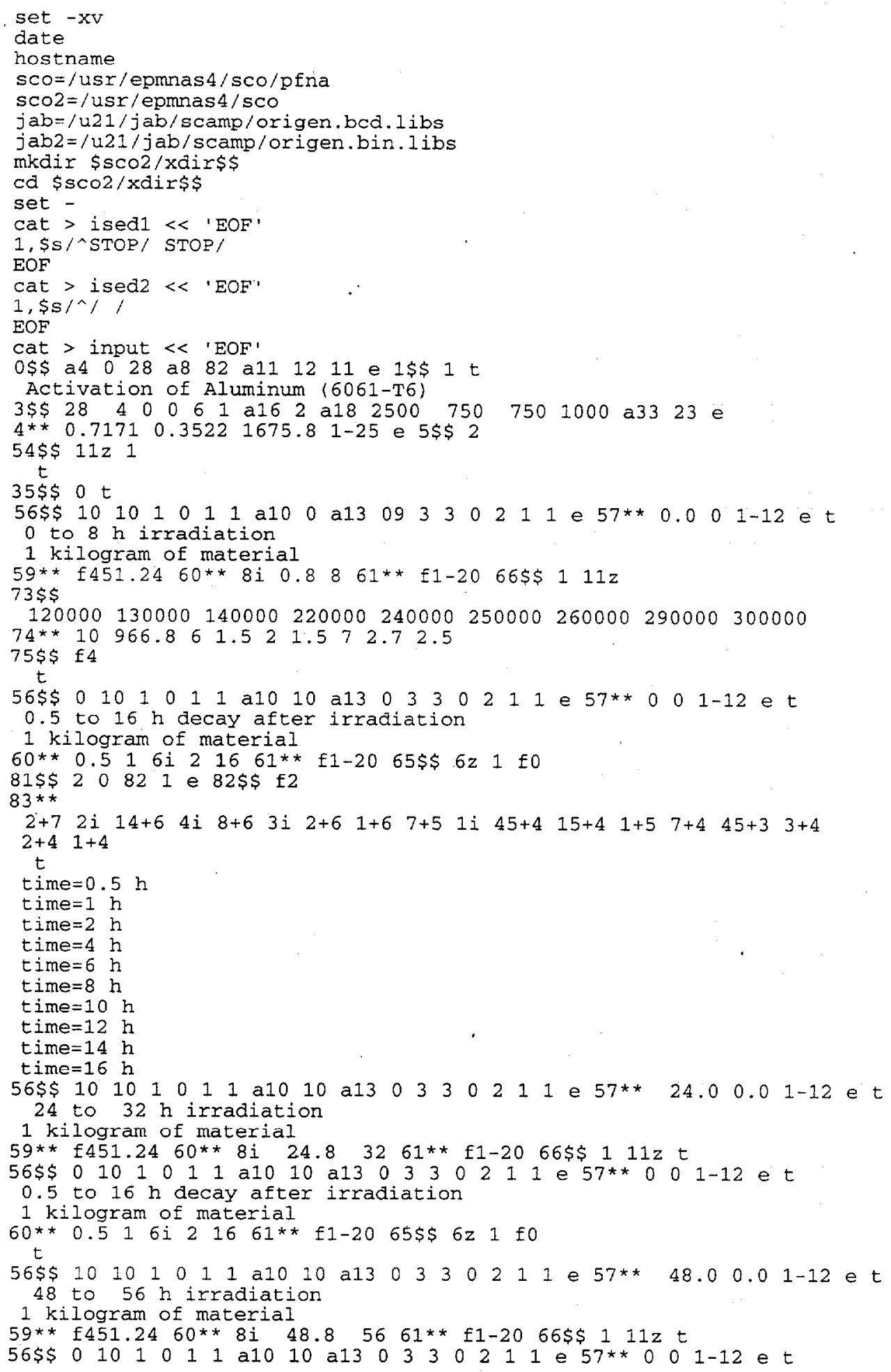


0.5 to $16 \mathrm{~h}$ decay after irradiation

1 kilogram of material

$60 * * 0.516 i 216 \quad 61 * *$ f $1-20 \quad 65 \$ \$ \quad 6 z \quad 1$ fo

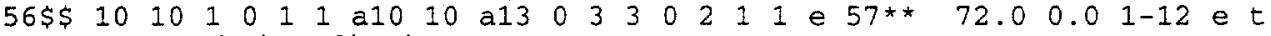

72 to $80 \mathrm{~h}$ irradiation

1 kilogram of material

$59 *$ f451.24 $60 * * 8 i \quad 72.8 \quad 8061 * \star$ f1-20 $66 \$ \$ 111 z t$

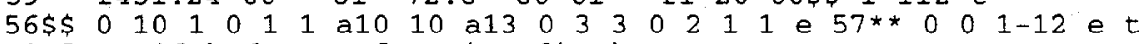

0.5 to $16 \mathrm{~h}$ decay after irradiation

1 kilogram of material

$60 * * 0.516 i 21661 * *$ f $1-20 \quad 65 \$ \$ 6 z \quad 1$ f

$t$

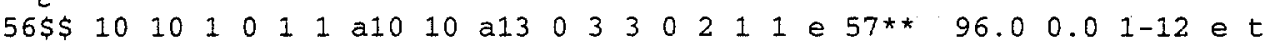

96 to $104 \mathrm{~h}$ irradiation

1 kilogram of material

$59 * *$ f $451.24 \quad 60 * * 8 i \quad 96.8 \quad 10461 * \star \quad 11-20 \quad 66 \$ \$ 111 z t$

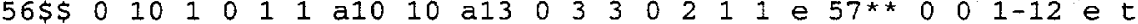

0.5 to $16 \mathrm{~h}$ decay after irradiation

1 kilogram of material

$60 * * 0.516 i \quad 2 \quad 16 \quad 61 * * \pm 1-20 \quad 65 \$ \$ 6 z \quad 1$ fo

$t$

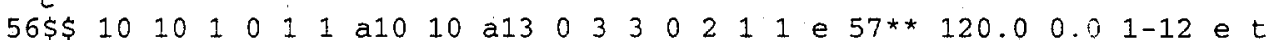
120 to $128 \mathrm{~h}$ irradiation

1 kilogram of material

$59 *$ f $451.24 \quad 60 * * 8 i \quad 120.8 \quad 128 \quad 61 * *$ f1-20 $66 \$ \$ 111 \mathrm{z}$

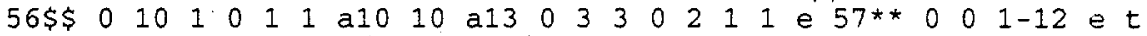

0.5 to 16 h decay after irradiation

1 kilogram of material

$60 * * 0.516 i 216$ 61** $21-20 \quad 65 \$ \$ 6 z \quad 1$ f0

$t$

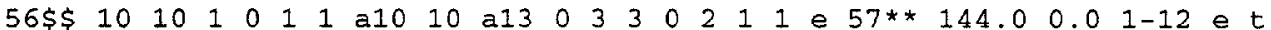

144 to 152 h irradiation

1 kilogram of material

$59 *$ f $451.2460 *$ * 8 i $144.8 \quad 152 \quad 61 * *$ f1-20 $66 \$ \$ 111 z t$

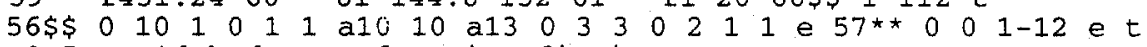

0.5 to $16 \mathrm{~h}$ decay after irradiation

1 kilogram of material

$60 * * 0.516 i 21661 * *$ f1-20 65\$ $6 z 1$ fO

$t$

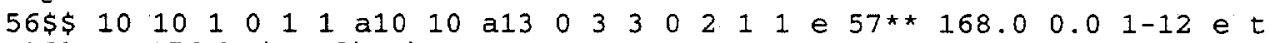

168 to 176 h irradiation

1 kilogram of material

$59 * \star$ E451.24 60** 8i $168.8 \quad 176 \quad 61 * *$ f1-20 66\$\$ $1112 \mathrm{t}$

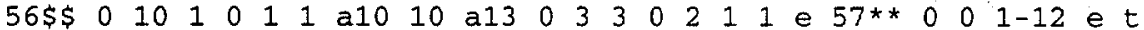

0.5 to $16 \mathrm{~h}$ decay after irradiation

1 kilogram of material

$60 * \star 0.516 i 216 \quad 61 * \star \quad f 1-20 \quad 65 \$ \$ 6 z \quad 1$ fo

$t$

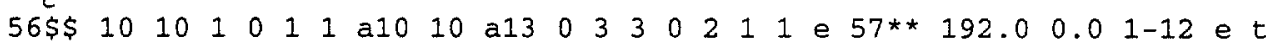

192 to $200 \mathrm{~h}$ irradiation

1 kilogram of material

$59 * *$ E451.24 60** 8i $192.8 \quad 20061 * \star E 1-20 \quad 66 \$ \$ 111 z$

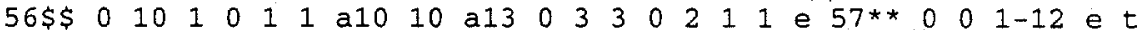

0.5 to $16 \mathrm{~h}$ decay after irradiation

1 kilogram of material

$60 * \star 0.516 i 216 \quad 61 * \star \quad f 1-20 \quad 65 \$ \$ 6 z \quad 1$ fo

$t$

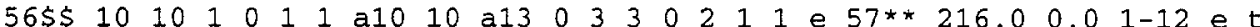

216 to $224 \mathrm{~h}$ irradiation

1 kilogram of material

$59 * \star$ f451.24 60** 8i 216.8 224 61** f1-20 66\$\$ $111 \mathrm{z} t$

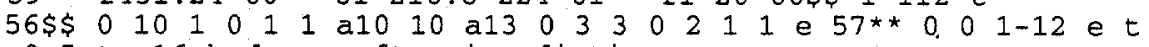

0.5 to $16 \mathrm{~h}$ decay after irradiation

1 kilogram of material

$60 * * 0.516 i 21661 * *$ f1-20 65\$\$ $6 \mathrm{z} 1$ fo

$81 \$ \$ 20821$ e $82 \$ \$$ f2

$83 * *$

$\begin{array}{llllllllllllllll}2+7 & 2 i & 14+6 & 4 i & 8+6 & 3 i & 2+6 & 1+6 & 7+5 & 1 i & 45+4 & 15+4 & 1+5 & 7+4 & 45+3 & 3+4\end{array}$

$2+4 \quad 1+4$

$t$

time $=0.5 \mathrm{~h}$ 


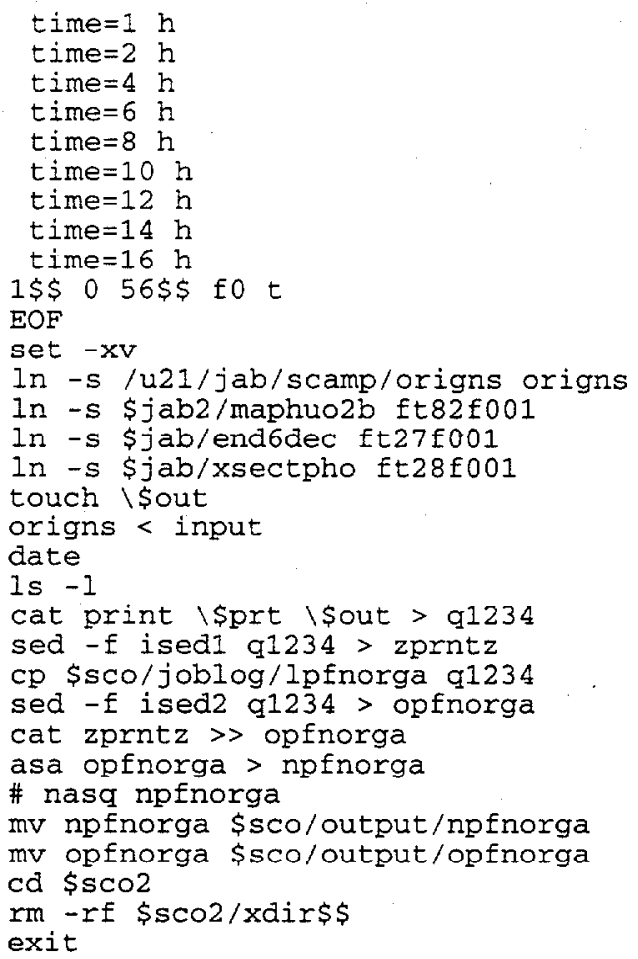


File for ORIGEN calculation of the activation of the steel plate using neutron spectrum 1

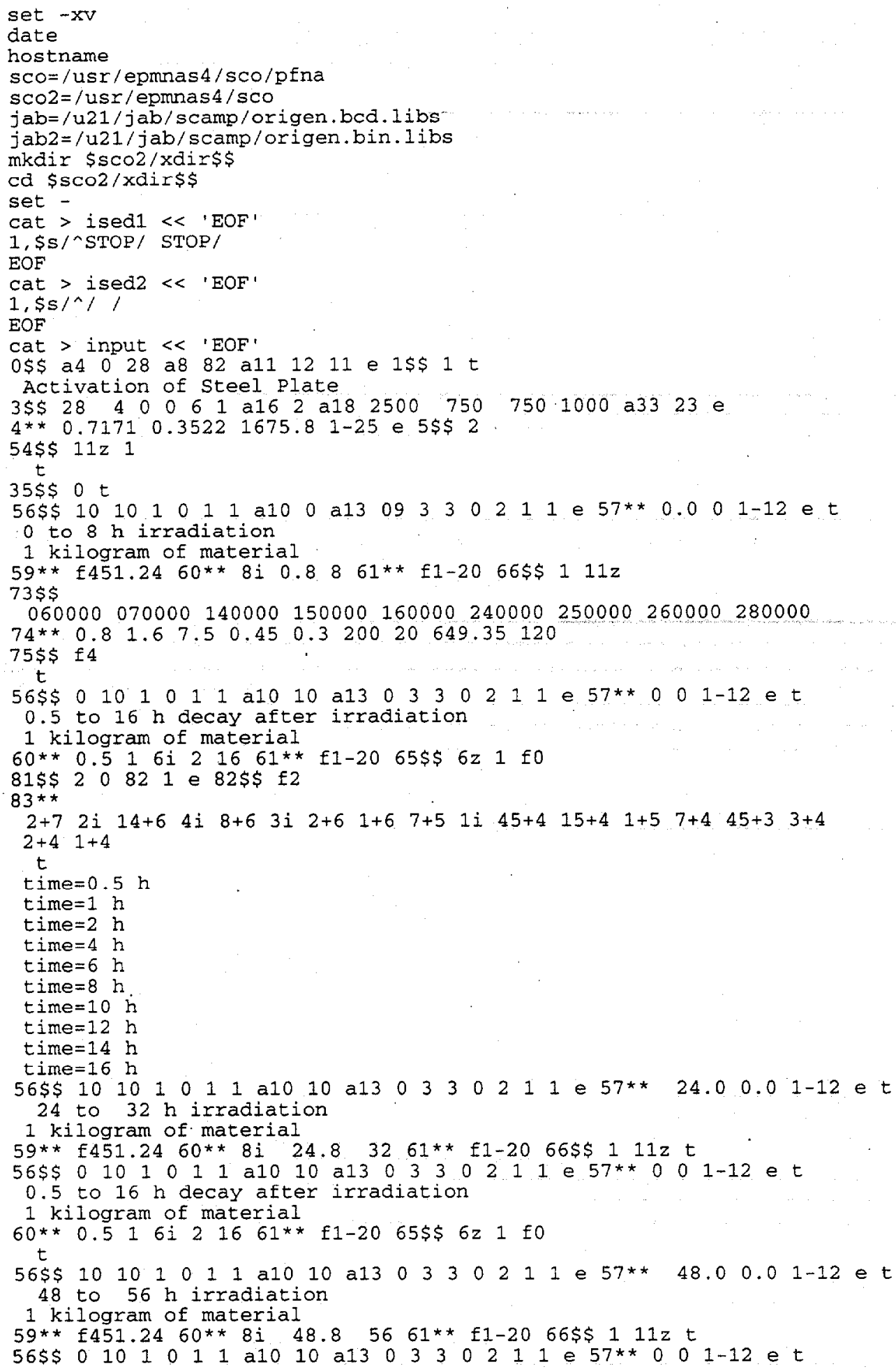




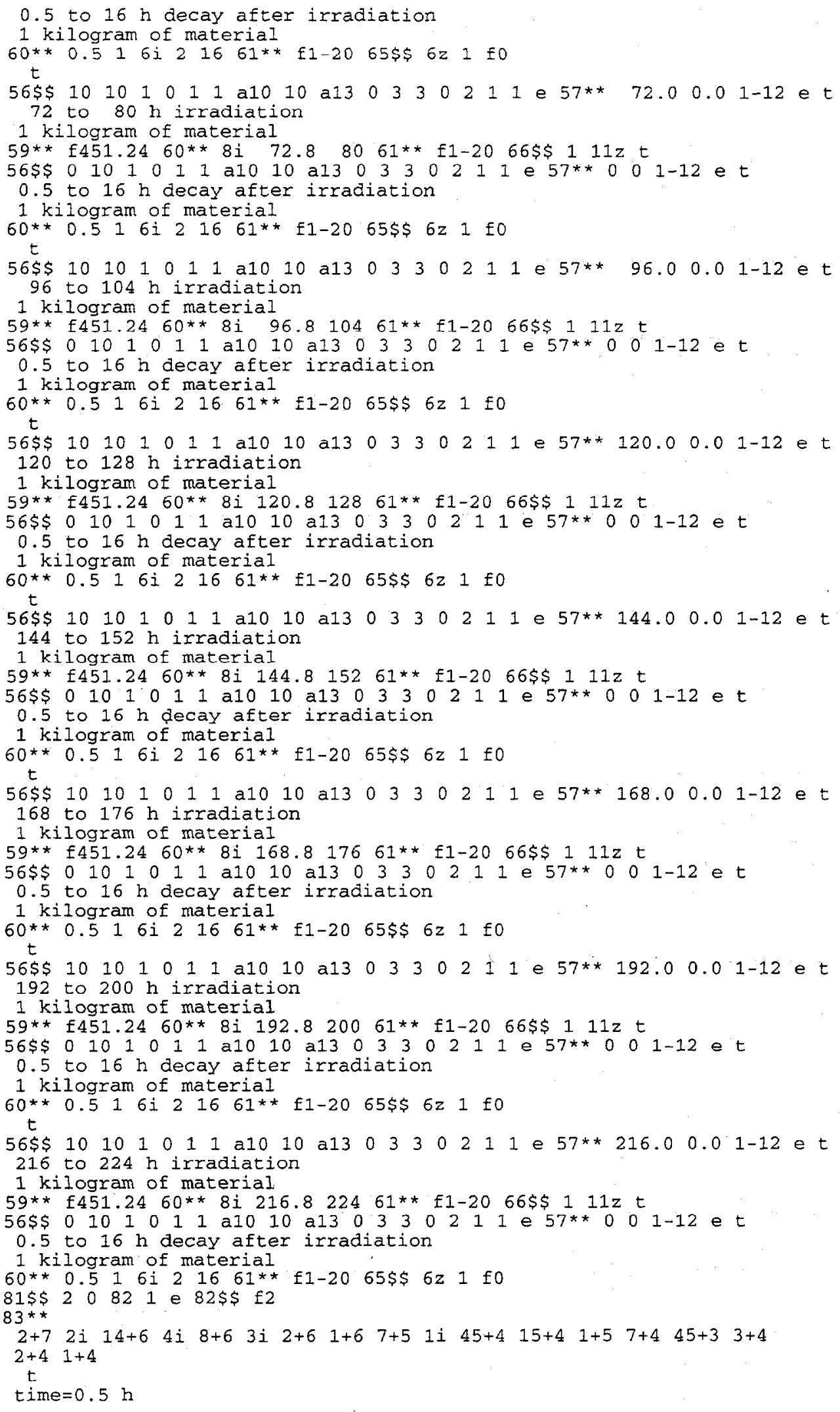




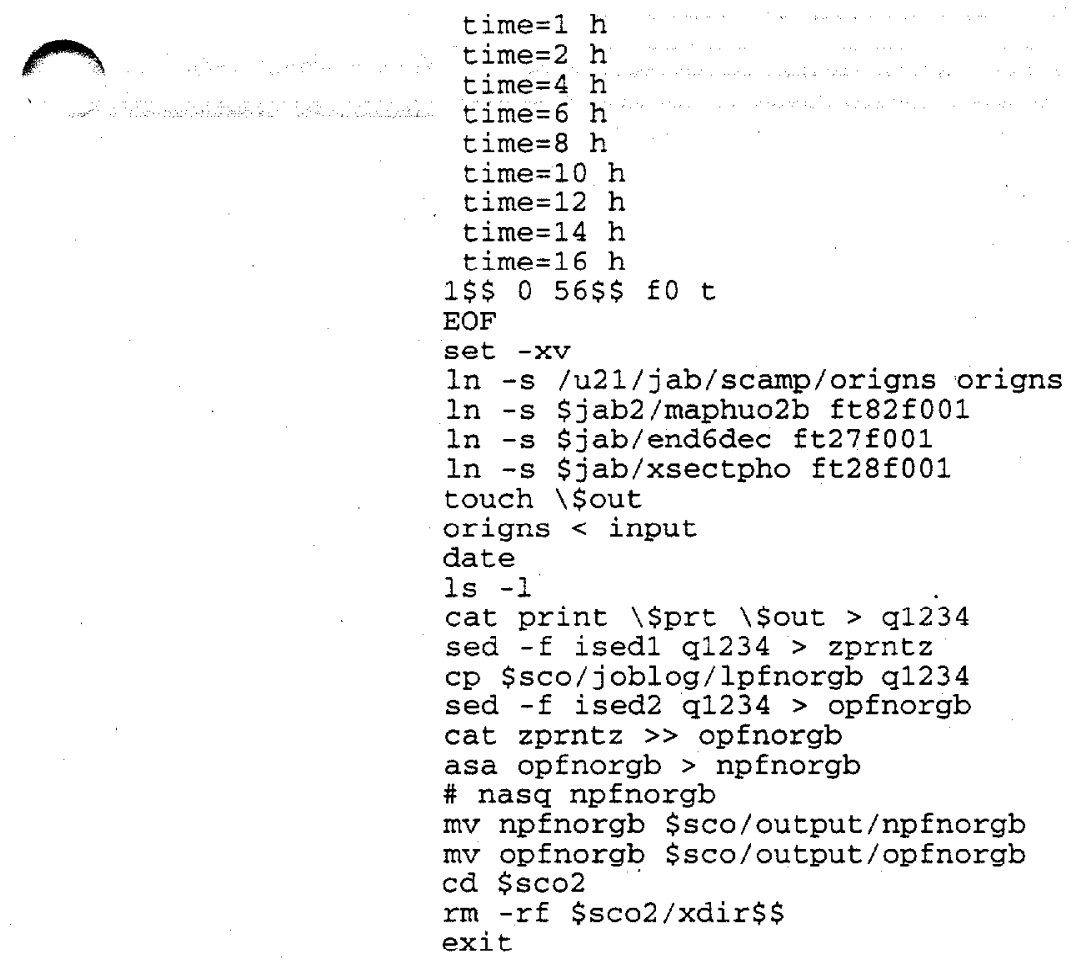


File Eor ORIGEN calculation of the activation of Havar foil using neutron spectrum 1

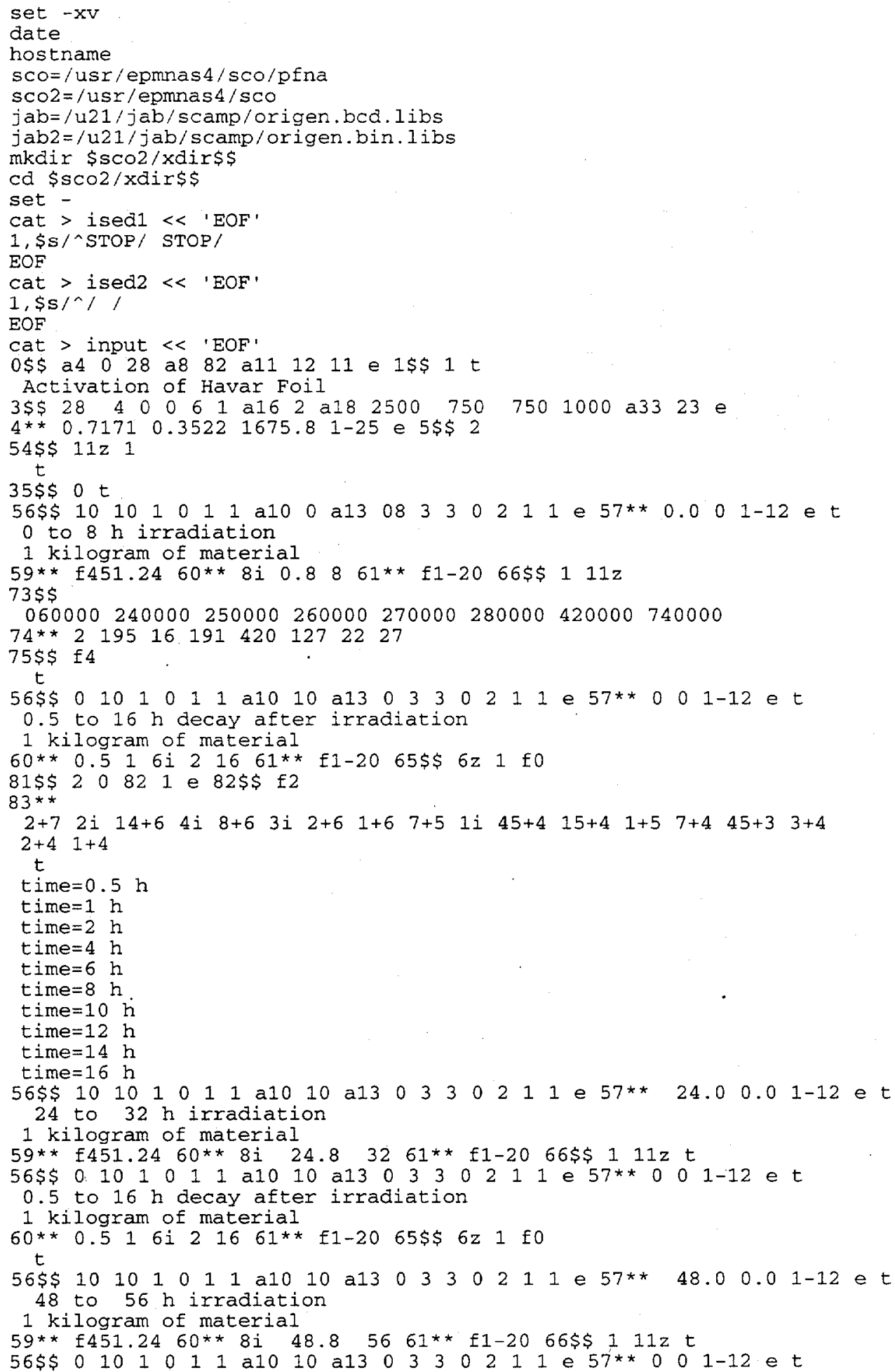


0.5 to 16 h decay after irradiation

1 kilogram of material

$60 * \star 0.51$ 6i $2 \quad 16 \quad 61 * *$ f $1-20 \quad 65 \$ \$ 6 z 1$ f0

$t$

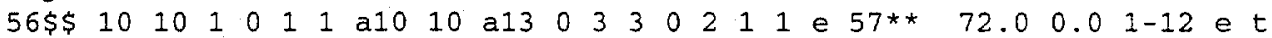

72 to $80 \mathrm{~h}$ irradiation

1 kilogram of material

$59 * \star$ f $451.2460 * \star$ 8i $72.8 \quad 80 \quad 61 * *$ f1-20 $66 \$ \$ 111 z t$

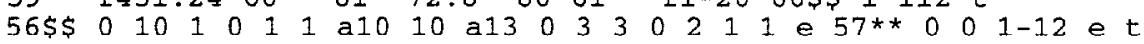

0.5 to 16 h decay after irradiation

1 kilogram of material

$60 * \star 0.516 i 216 \quad 61 * \star \pm 1-20 \quad 65 \$ \$ 6 z \quad 1 . \pm 0$

$t$

$56 \$ \$ 101010011$ a 1010 a13 033300211 e $57^{* *} 96.00 .01-12$ e $t$

96 to $104 \mathrm{~h}$ irradiation

1 kilogram of material

$59 * \star$ f $451.2460 * \star 8 i \quad 96.810461 * \star$ f $1-2066 \$ \$ 111 z t$

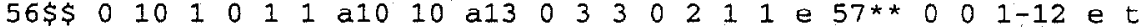

0.5 to $16 \mathrm{~h}$ decay after irradiation

1 kilogram of material

$60 * * 0.516 i 216 \quad 61 * * \pm 1-2065 \$ \$ 6 z \quad 1$ fo

$t$

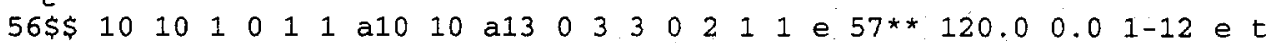
120 to $128 \mathrm{~h}$ irradiation

1 kilogram of material

$59 * \star$ f $451.2460 * * 8 i \quad 120.812861 * *$ f $1-20$ 66\$\$ $111 \mathrm{zt}$

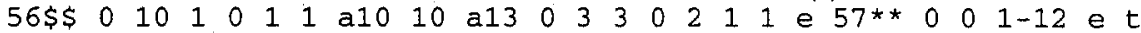

0.5 to 16 h decay after irradiation

1 kilogram of material

$60 * * 0.516 i 216$ 61** f1-20 65\$\$ $6 \mathrm{z} 1 \mathrm{fo}$ $t$

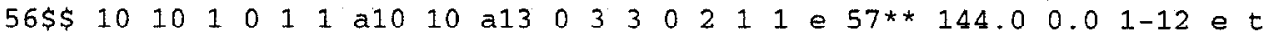

144 to $152 \mathrm{~h}$ irradiation

1 kilogram of material

59 ** f451.24 60** 8i $144.8 \quad 152 \quad 61 * *$ f1-20 $66 \$ \$ 111 \mathrm{z} t$

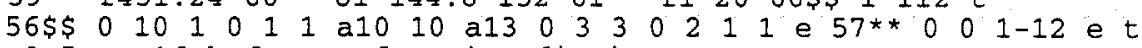

0.5 to $16 \mathrm{~h}$ decay after irradiation

1 kilogram of material

$60 * * 0.516 i 21661 * *$ f1-20 65\$\$ $6 z 1$ fo

$t$

$56 \$ \$ 10101011$ a10 10 a13 03330211 e $57 * * 168.00 .01-12$ e t

168 to $176 \mathrm{~h}$ irradiation

1 kilogram of material

$59 * *$ f $451.2460 * * 81 \quad 168.8176 \quad 61 *$ fl-20 $66 \$ \$ 111 z t$

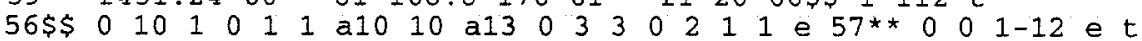

0.5 to $16 \mathrm{~h}$ decay after irradiation

1 kilogram of material

$60 * * 0.516 i 21661 * *$ fl-20 $65 \$ \$ 6 z 1$ fo

$t$

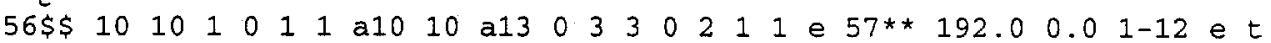
192 to $200 \mathrm{~h}$ irradiation

1 kilogram of material

$59 * \star f 451.2460 * * 8 i \quad 192.8 \quad 20061 * *$ f1-20 66\$\$ $111 \mathrm{z} t$

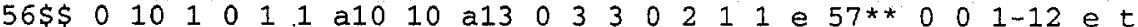

0.5 to $16 \mathrm{~h}$ decay after irradiation

1 kilogram of material

$60 * \star 0.516 i 216 \quad 61 * * 11-20 \quad 65 \$ \$ 6 z \quad 1$ fo

$t$

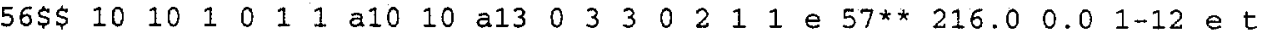
216 to $224 \mathrm{~h}$ irradiation

1 kilogram of material

$59 *$ f $451.24 \quad 60 * *$ 8i $216.8 \quad 22461 * *$ f1-20 $66 \$ \$ 111 z t$

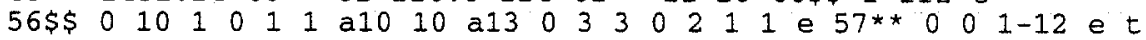

0.5 to $16 \mathrm{~h}$ decay after irradiation

1 kilogram of material

$60 * * 0.5 \quad 1 \quad 6 i 216 \quad 61 * * \quad f 1-20 \quad 65 \$ \$ 6 z \quad 1$ fo

$81 \$ \$ 20821$ e $82 \$ \$$ f2

$83 * \star$

$2+7 \quad 2 i \quad 14+6 \quad 4 i \quad 8+6 \quad 3 i \quad 2+6 \quad 1+6 \quad 7+5 \quad 1 i \quad 45+4 \quad 15+4 \quad 1+5 \quad 7+4 \quad 45+3 \quad 3+4$

$2+4 \quad 1+4$

$t$

time $=0.5 \mathrm{~h}$ 


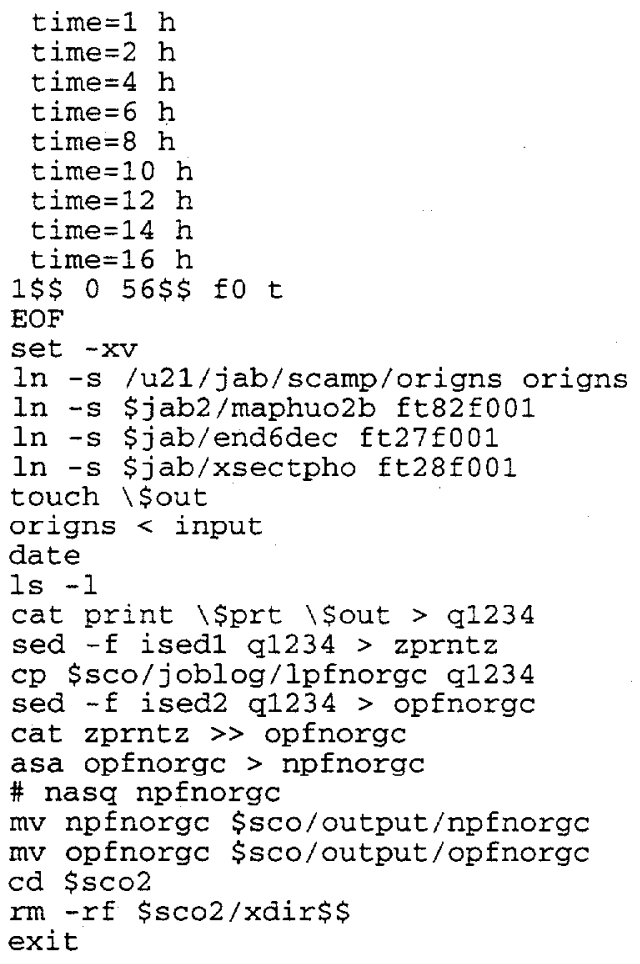


File for ORIGEN calculation of the activation of the aluminum (6061-T6) structure using neutron spectrum 2

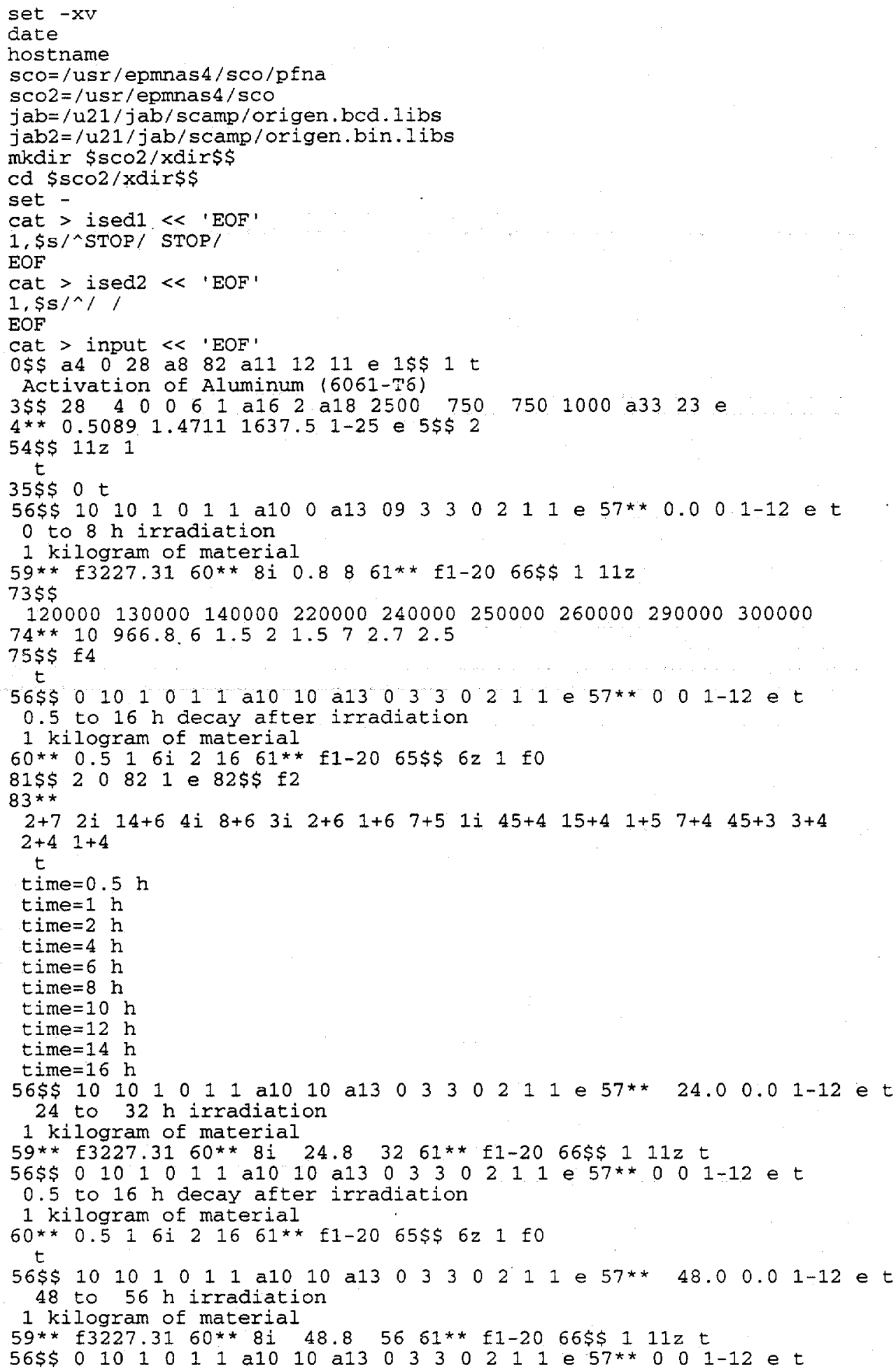




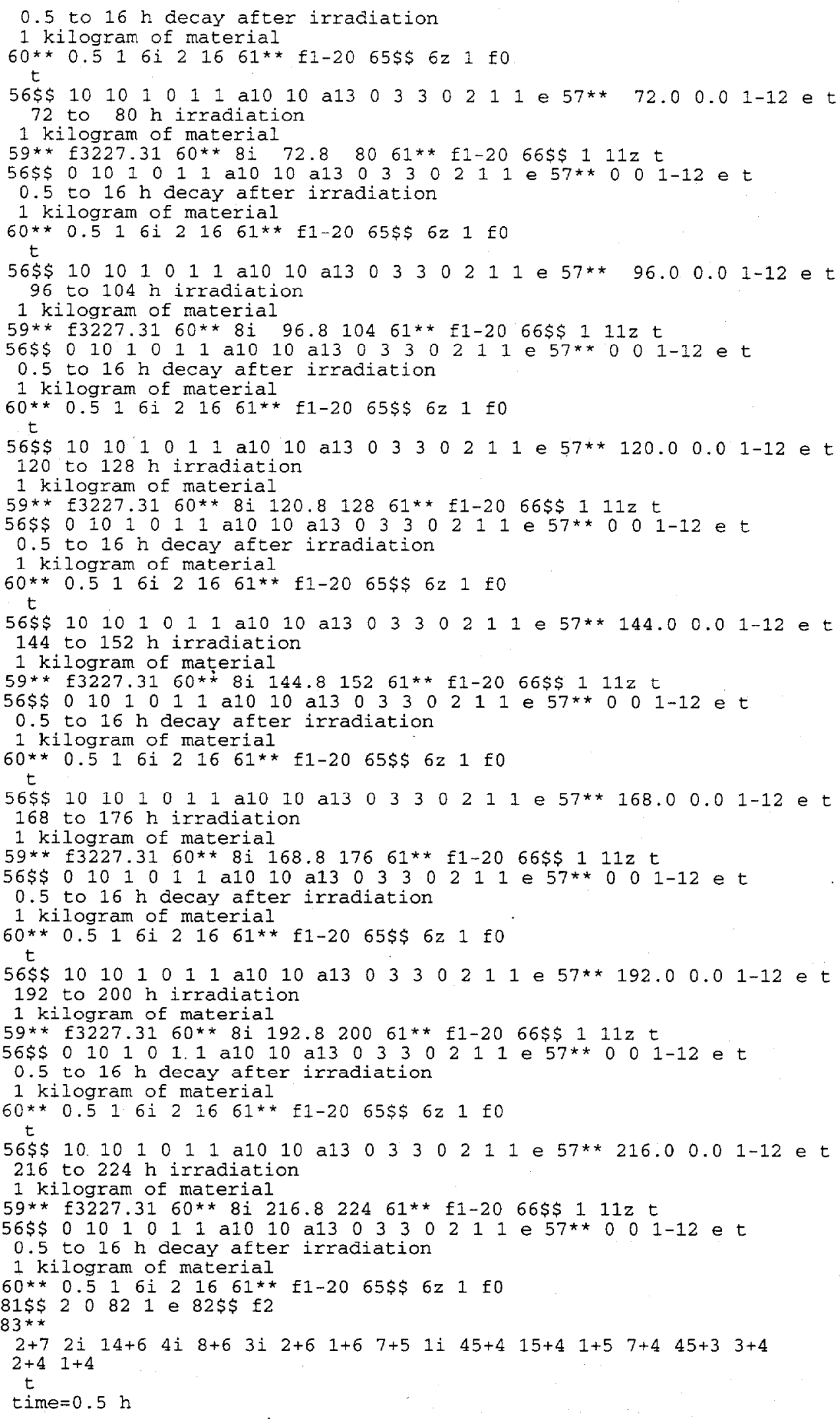




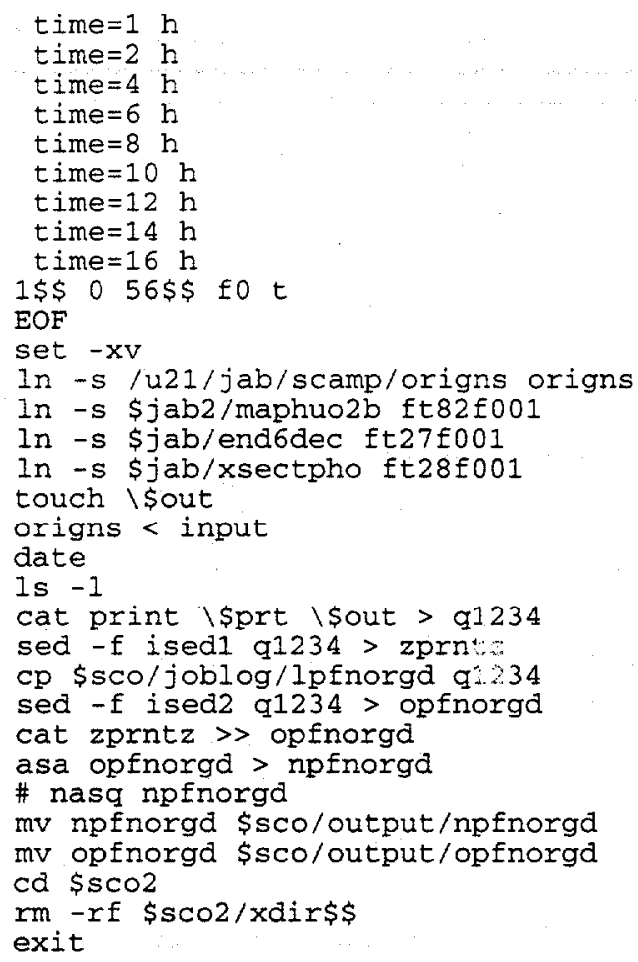


File for ORIGEN calculation of the activation of the steel plate using neutron spectrum 2

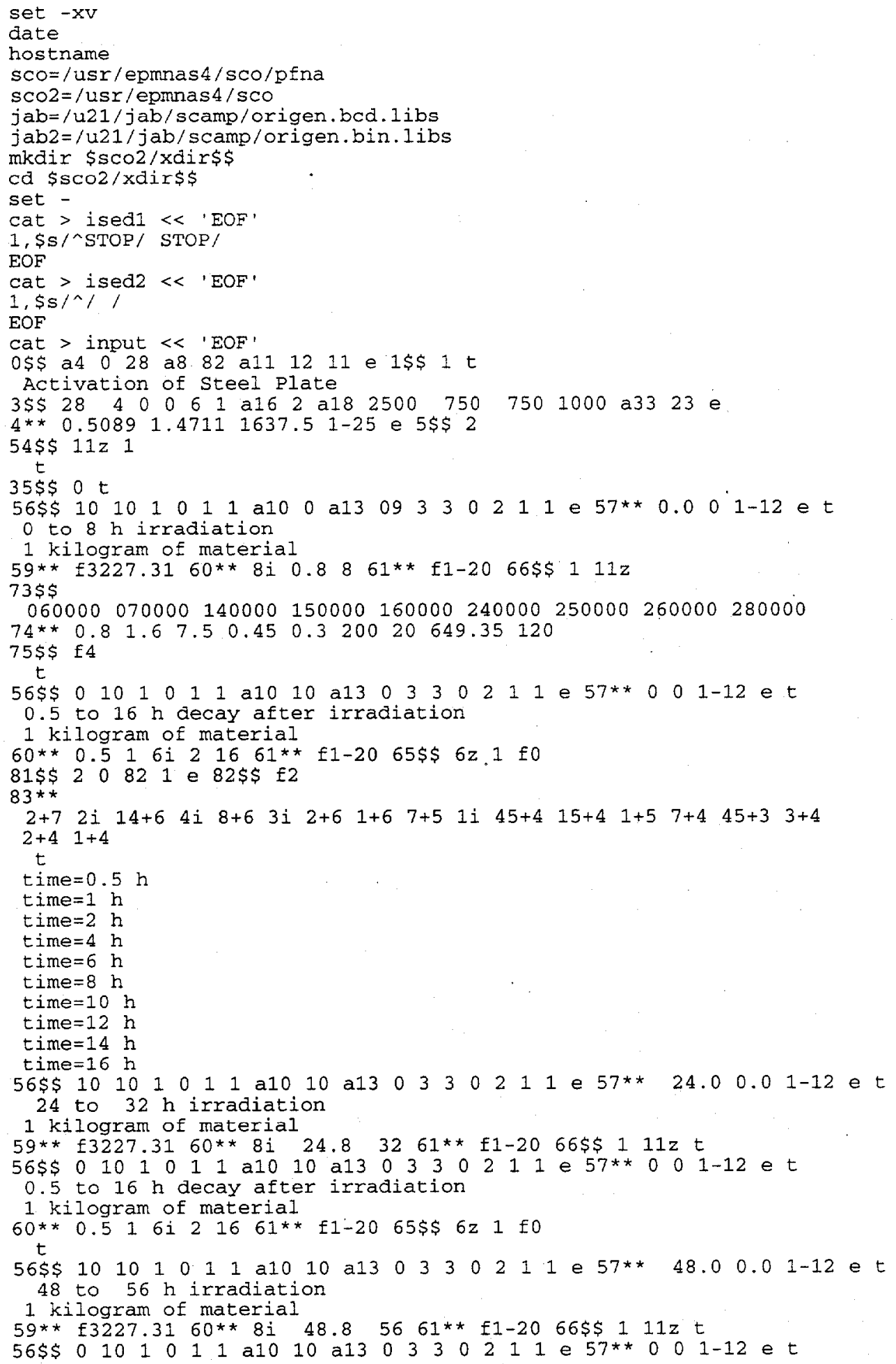


0.5 to 16 h decay after irradiation

1 kilogram of material

$60 * \star 0.516 i 216 \quad 61 * *$ f $1-20$ 65\$\$ 6 z 1 f0

$t$

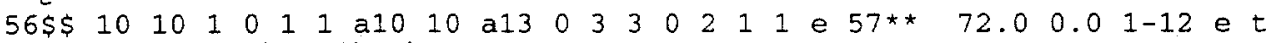

72 to $80 \mathrm{~h}$ irradiation

1 kilogram of material

$59 * * f 3227.3160 * * 8 i \quad 72.8 \quad 80 \quad 61 * *$ f1-20 $66 \$ \$ 111 z t$

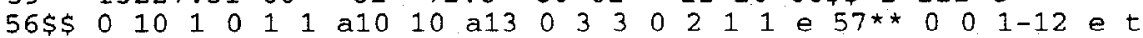

0.5 to 16 h decay after irradiation.

1 kilogram of material

$60 * * 0.516 i 216 \quad 61 * * f 1-20 \quad 65 \$ \$ 6 z \quad 1$ f0

$t$

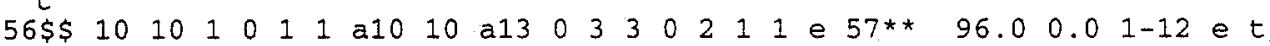
96 to $104 \mathrm{~h}$ irradiation

1 kilogram of material

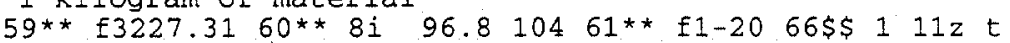

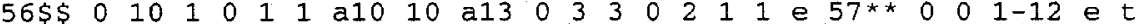

0.5 to 16 h decay after irradiation

1 kilogram of material

$60 * * 0.516 i 216$ 6I** fl-20 65\$\$ $6 z$ I fO

$t$

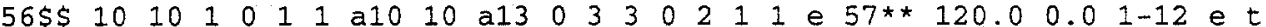

120 to $128 \mathrm{~h}$ irradiation

1 kilogram of material

$59 *$ * $3227.3160 *$ * $8 i \quad 120.8128$ 61** f1-20 66\$\$ $111 z t$

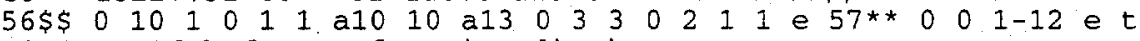

0.5 to $16 \mathrm{~h}$ decay after irradiation

1 kilogram of material

$60 * * 0.516 i 216 \quad 61 * * f 1-20 \quad 65 \$ \$ 6 z 1$ fo

$t$

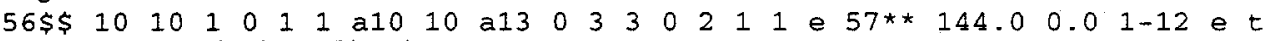

144 to $152 \mathrm{~h}$ irradiation

1 kilogram of material

$59 *$ f3227.31 60** 8i $144.8 \quad 152 \quad 61 * *$ f1-20 $66 \$ \$ 111 \mathrm{z}$

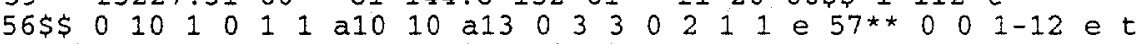

0.5 to 16 h decay after irradiation

1 kilogram of material

$60 * \star 0.516 i 21661 * \star \pm 1-2065 \$ \$ 6 z \quad 1 f 0$

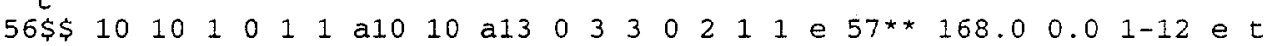
168 to $176 \mathrm{~h}$ irradiation

1 kilogram of material

$59 * *$ f $3227.3160 * \star$ i $168.8176 \quad 61 *$ f $1-20$ 66\$\$ $111 \mathrm{z} t$

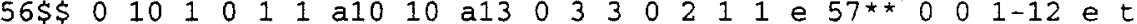

0.5 to $16 \mathrm{~h}$ decay after irradiation

1 kilogram of material

$60 * * 0.5 \quad 1 \quad 6 i \quad 2 \quad 16 \quad 61 * * \quad f 1-20 \quad 65 \$ \$ 6 z \quad 1$ f0

$t$

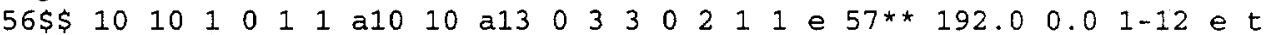

192 to $200 \mathrm{~h}$ irradiation

1 kilogram of material

$59 * *$ f3227.31 60** 8 i $192.8 \quad 200$ 61** f1-20 66\$\$ $111 z$

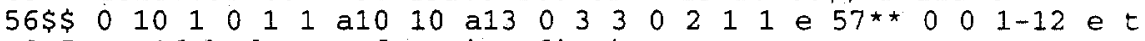

0.5 to 16 h decay after irradiation

1 kilogram of material

$60 * \star 0.516 i 2$ 2 16 61** f1-20 65\$\$ $6 z 1$ fo

$t$

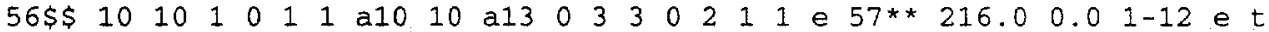

216 to 224 h irradiation

1 kilogram of material

$59 *$ f $3227.3160 * * 8 i 216.8224 \quad 61 * *$ f1-20 $66 \$ \$ 111 z t$

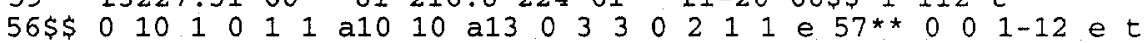

0.5 to $16 \mathrm{~h}$ decay after irradiation

1 kilogram of material

$60 * * 0.516 i 21661 * *$ f $1-2065 \$ \$ 6 z 1$ fo

$81 \$ \$ 20821$ e $82 \$ \$ f 2$

$83 *$ *

$\begin{array}{llllllllllllllll}2+7 & 2 i & 14+6 & 4 i & 8+6 & 3 i & 2+6 & 1+6 & 7+5 & 1 i & 45+4 & 15+4 & 1+5 & 7+4 & 45+3 & 3+4\end{array}$

$2+4 \quad 1+4$

$t$

time $=0.5 \mathrm{~h}$ 


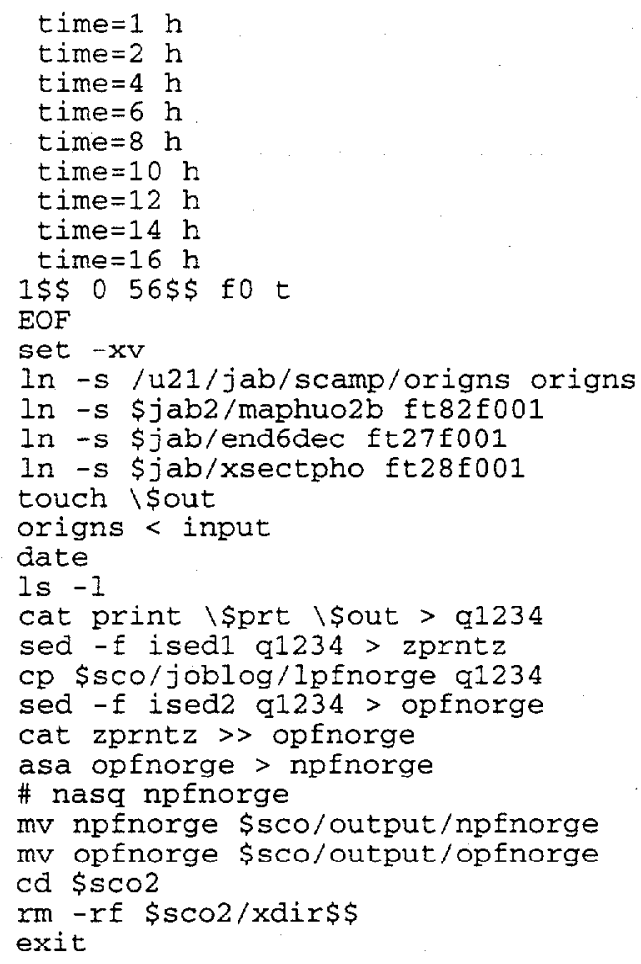


File for ORIGEN calculation of the activation of Havar foil using neutron spectrum 2

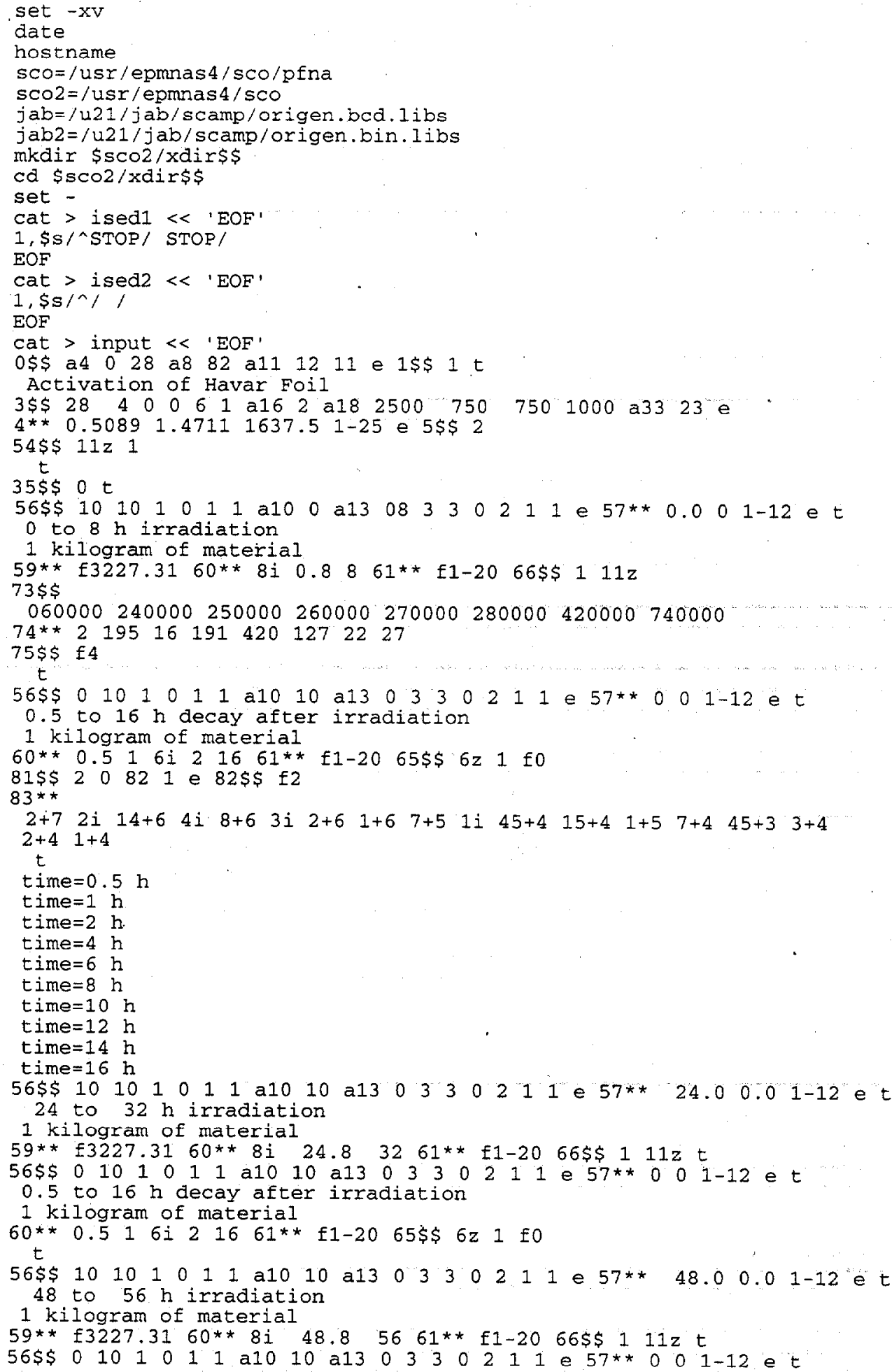


0.5 to 16 h decay after irradiation

1 kilogram of material

$60 * * 0.516 i 21661 * \star \mathrm{fl}-20$ 65\$ $6 \mathrm{z} 1 \mathrm{fo}$

$t$

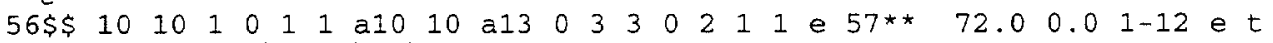
72 to $80 \mathrm{~h}$ irradiation

$1 \mathrm{kilogram}$ of material

$59 * *$ f $3227.3160 * * 8 i \quad 72.8 \quad 80$ 61** f1-20 66\$\$ $111 z t$

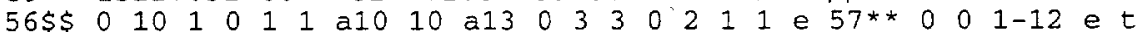

0.5 to 16 h decay after irradiation

1 kilogram of material

$60 * * 0.516 i 21661 * \star$ f $1-20$ 65\$\$ $6 \mathrm{z} 1 \mathrm{fo}$

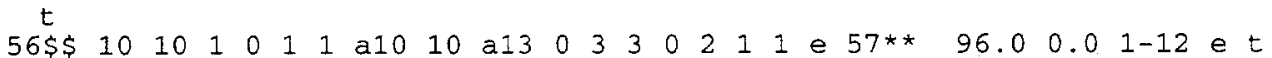
96 Lo 104 li irradiation

1 kilogram of material

$59 * *$ E3227.31 60** $8 i$ 96.8 104 61** f1-20 66\$\$ $111 z t$

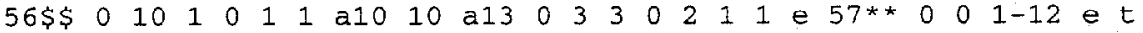

0.5 to $16 \mathrm{~h}$ decay after irradiation

1 kilogram of material

$60 * \star 0.516 i 21661 * \star \pm 1-2065 \$ \$ 6 z \quad 1 \quad 0$

$t$

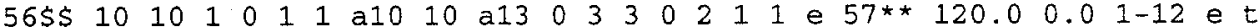
120 to $128 \mathrm{~h}$ irradiation

1 kilogram of material

$59 * *=3227.3160 * *$ 8i 120.8128 61** f1-20 66\$\$ $111 z \mathrm{t}$

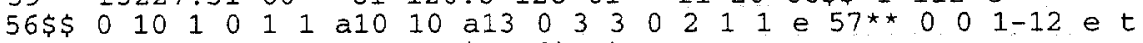

0.5 to 16 h decay after irradiation

1 kilogram of material

$60 * * 0.516 i 21661 *$ $61-2065 \$ \$ 6 z \quad 1$ fo

$t$

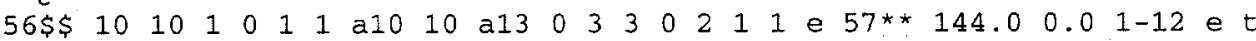

144 to $152 \mathrm{~h}$ irradiation

1 kilogram of material

$59 * * \pm 3227.3160^{* *} 8 i \quad 144.8152$ 61** f1-20 66\$\$ $111 \mathrm{z}$

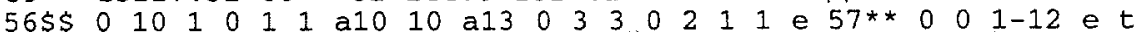

0.5 to 16 h decay after irradiation

1 kilogram of material

$60 * \star 0.516 i 21661 * * f 1-2065 \$ \$ 6 z 1 \mathrm{fO}$

$t$

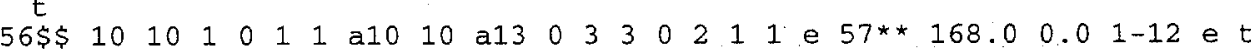
168 to $176 \mathrm{~h}$ irradiation

1 kilogram of material

$59 * *$ E3227.31 60** 8i 168.8176 61** f1-20 66\$\$ $111 z t$

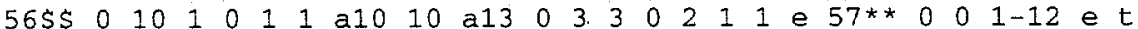

0.5 to $16 \mathrm{~h}$ decay after irradiation

1 kilogram of material

$60 * * 0.516 i 21661 * *$ f1-20 $65 \$ \$ 6 z \quad$ fo

$t$

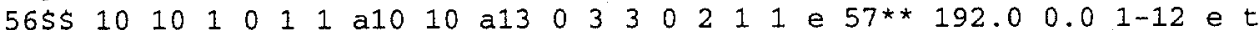
192 to $200 \mathrm{~h}$ irradiation

1 kilogram of material

$59 *$ f $3227.3160 * * 8 i$ 192.8 200 61** ff $1-2066 \$ \$ 111 z t$

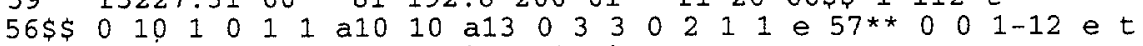

0.5 to $16 \mathrm{~h}$ decay after irradiation

$1 \mathrm{kilogram}$ of material

$60 * * 0.516 i 21661 * *$ f1-20 65\$\$ $6 z 1$ fo

$t$

$56 \$ \$ 10101011$ a10 10 a13 033302111 e $57 * * 216.00 .01-12$ e

216 to $224 \mathrm{~h}$ irradiation

1 kilogram of material

$59 * \star f 3227.3160 * *$ 8i $216.8 \quad 22461 * *$ f1-20 $66 \$ \$ 111 z t$

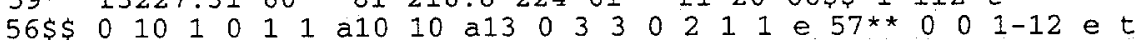

0.5 to $16 \mathrm{~h}$ decay after irradiation

1 kilogram of material

$60 * * 0.516 i 21661 * *$ f1-20 65\$\$ $6 z 1 \mathrm{zO}$

$81 \$ \$ 20821$ e $82 \$ \$ \mathrm{f} 2$

$83 * *$

$\begin{array}{llllllllllllllll}2+7 & 2 i & 14+6 & 4 i & 8+6 & 3 i & 2+6 & 1+6 & 7+5 & 1 i & 45+4 & 15+4 & 1+5 & 7+4 & 45+3 & 3+4\end{array}$

$2+4 \quad 1+4$

$\mathrm{t}$

time $=0.5 \mathrm{~h}$ 


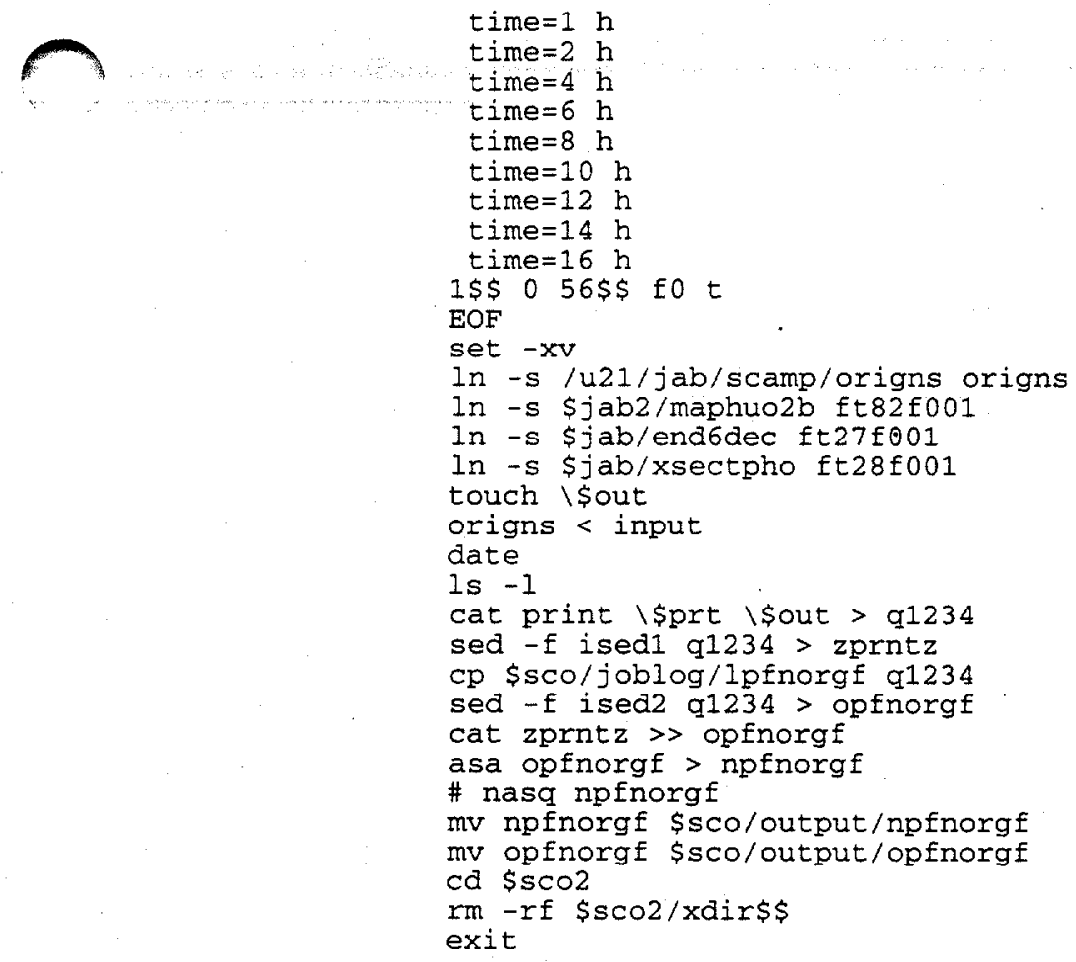


File for ORIGEN calculation of the activation of the concrete structure using neutron spectrum 2

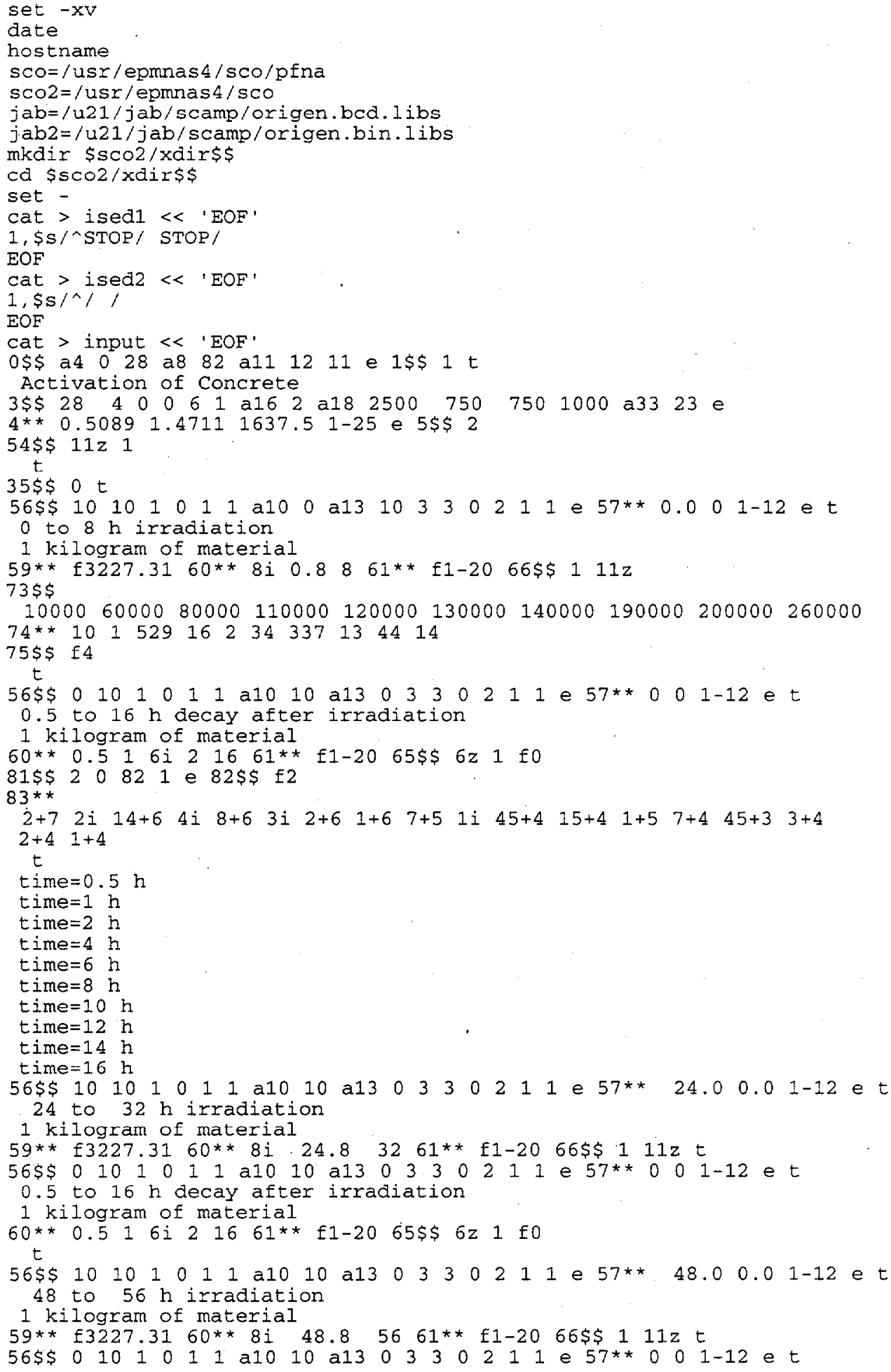




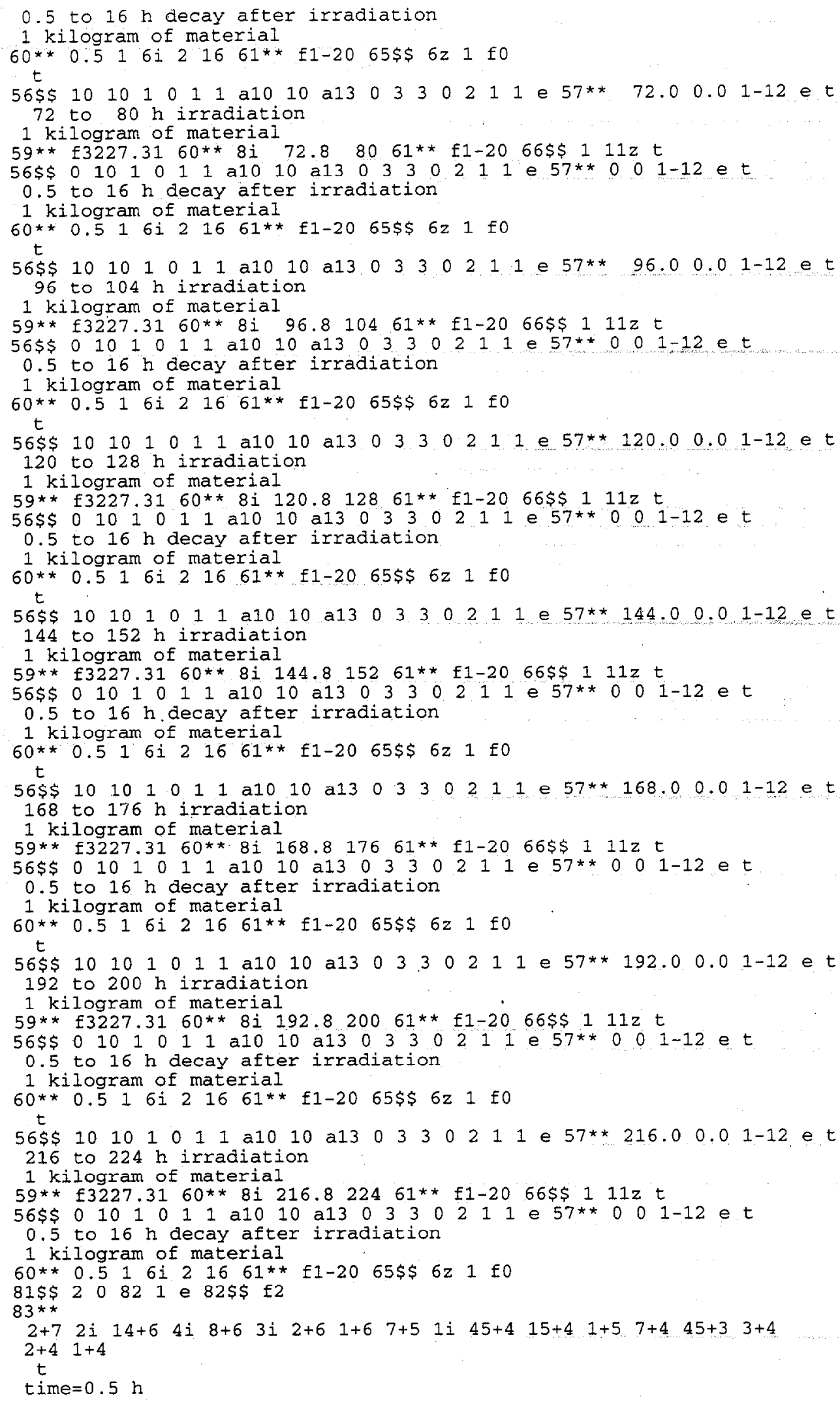




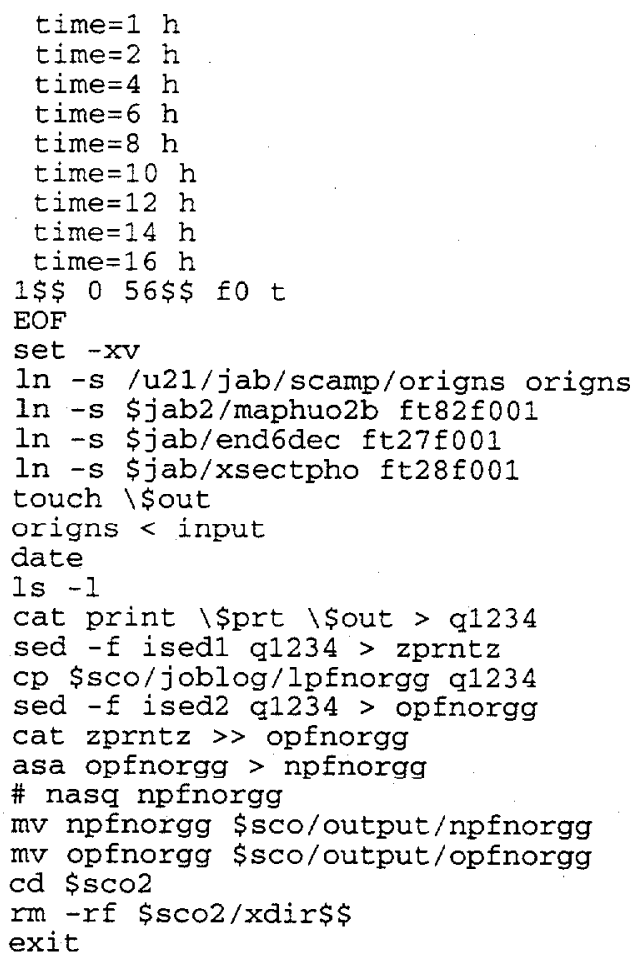


File for ORIGEN calcuiation of the activation of the concrete structure using neutron spectrum 3

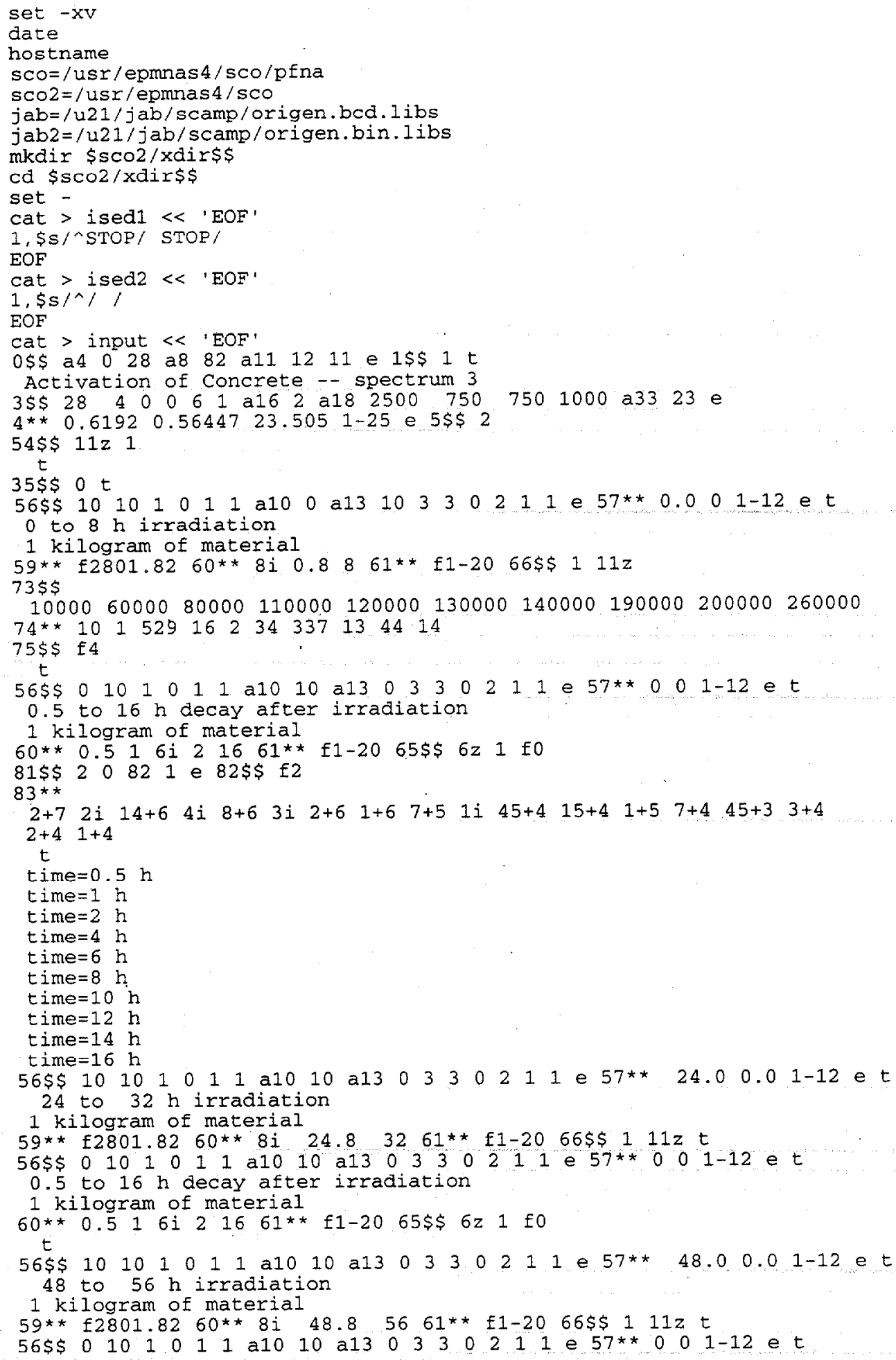




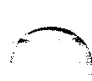


0.5 to $16 \mathrm{~h}$ decay after irradiation

1 kilogram of material

$60 * * 0.516 i 216 \quad 61 * *$ f $1-20 \quad 65 \$ \$ 6 z \quad 1 \quad f 0$

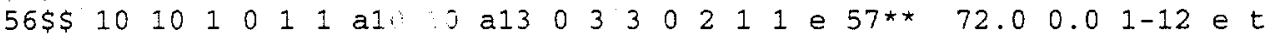
72 to $80 \mathrm{~h}$ irradiat: $\mathrm{s}$

1 kilogram of material

$59 * \star f 2801.8260^{* \star} 8 i \quad 72.8 \quad 8061 * \star$ f1-20 $66 \$ \$ 111 z t$

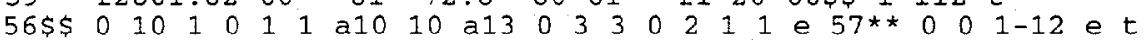

0.5 to $16 \mathrm{~h}$ decay after irradiation

1 kilogram of material

$60 * * 0.516 i 21661 * \star$ fl-20 $65 \$ \$ 6 z \quad 1$ fo

$t$

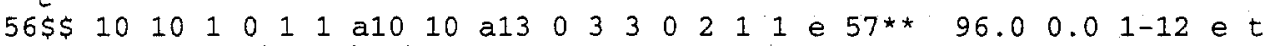
96 to $104 \mathrm{~h}$ irradiation

1 kilogram of material

$59 * \star$ f2801.82 $60 * \star 8 i \quad 96.8 \quad 104 \quad 61 * *$ f1-20 $66 \$ \$ 111 z t$

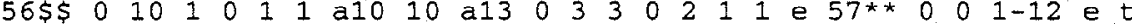

0.5 to $16 \mathrm{~h}$ decay after irradiation

1 kilogram of material

$60 * * 0.516 i 216 \quad 61 * *$ f1-20 65\$\$ $6 z 1$ f0

$t$

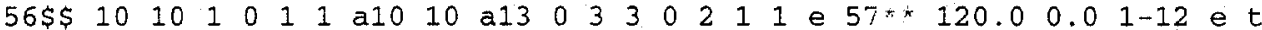

120 to $128 \mathrm{~h}$ irradiation

1 kilogram of material

$59 * *$ f2801.82 $60 * *$ 8i 120.8128 61** f1-20 66\$\$ $111 z t$

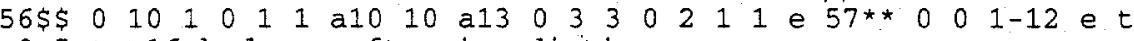

0.5 to $16 \mathrm{~h}$ decay after irradiation

1 kilogram of material

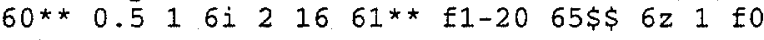

$t$

$56 \$ \$ 10101011$ a10 10 a13 03330211 e $57 * * 144.00 .01-12$ et

144 to $152 \mathrm{~h}$ irradiation

1 kilogram of material

59 ** $2801.8260 * *$ 8i 144.8 152 61 ** f1-20 $66 \$ \$ 111 z t$

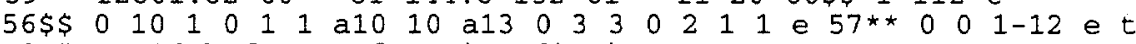

0.5 to 16 h decay after irradiation

1 kilogram of material

$60 * * 0.516 i 21661 * *$ I-20 $65 \$ \$ 6 z \quad 1$ fo

$\mathrm{t}$

$56 \$ \$ 10101001121010$ a13 03330211 e $57 * * 168.00 .01-12$ e t 168 to $176 \mathrm{~h}$ irradiation

1 kilogram of material

$59^{* *} \mathrm{f} 2801.8260^{* *} 8 \mathrm{i} 168.8176 \quad 61 * * \mathrm{f} 1-2066 \$ \$ 111 \mathrm{t}$

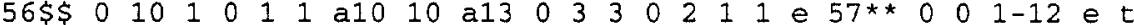

0.5 to 16 h decay after irradiation

1 kilogram of material

$60 * * 0.516 i 21661 * *$ f1-20 $65 \$ \$ 6 z \quad 1$ fo

$\mathrm{t}$

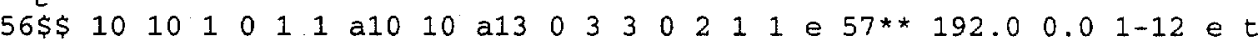
192 to 200 h irradiation

1 kilogram of material

$59 *$ f $2801.8260 * \star 8 i \quad 192.820061 * *$ f1-20 $66 \$ \$ 111 z$

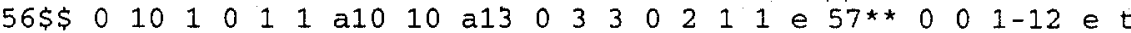

0.5 to $16 \mathrm{~h}$ decay after irradiation

1 kilogram of material

$60 * * 0.516 i 216 \quad 61 * *$ fl-20 $65 \$ \$ 6 z 1$ fo

$t$

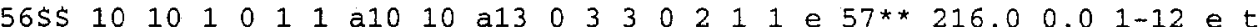

216 to $224 \mathrm{~h}$ irradiation

$1 \mathrm{kilogram}$ of material

$59 *$ f2801.82 60** 8i 216.8 224 61** f1-20 66\$\$ $111 \mathrm{zt}$

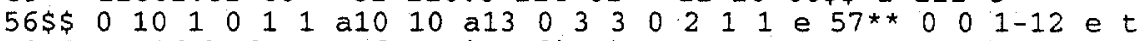

0.5 to $16 \mathrm{~h}$ decay after irradiation

1 kilogram of material

$60^{* *} 0.516 i 21661^{* *}$ f1-20 65\$\$ $6 \mathrm{z} 1 \mathrm{fO}$

$81 \$ \$ 20821$ e $82 \$ \$$ f2

83 **

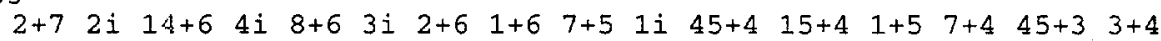

$2+4 \quad 1+4$

$t$

time $=0.5 \mathrm{~h}$ 


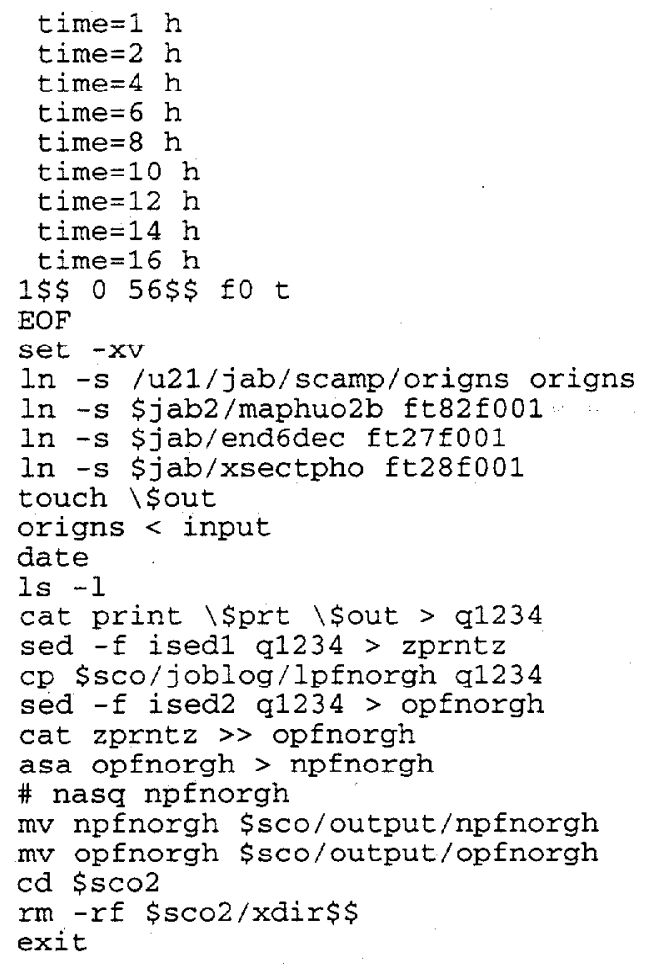




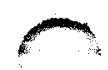<smiles>C1CCCCC1</smiles>

$+$

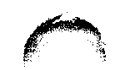


$m$

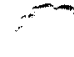

